WSRC-TR-99-00013

Part I

\title{
H-Area Hazardous Waste Management Facility Correction Action Report, Third and Fourth Quarter 1998, Volumes I and II
}

by

J. Chase

RECORDS ADMINISTRATION

Westinghouse Savannah River Company

$\mathrm{R} 0124633$

Savannah River Site

Aiken, South Carolina 29808

This paper was prepared in connection with work done under the above contract number with the U.S. Department of Energy. By acceptance of this paper, the publisher and/or recipient acknowledges the U. S. Government's right to retain a nonexclusive, royalty-free license in and to any copyright covering this paper, along with the right to reproduce and to authorize others to reproduce all or part of the copyrighted paper. 


\title{
H-AREA HAZARDOUS WASTE MANAGEMENT FACILITY CORRECTIVE ACTION REPORT (U)
}

THIRD AND FOURTH QUARTER 1998

\author{
Volume I
}

Publication Date: March 1999

Authorized Derivative Classifier and Reviewing Official:

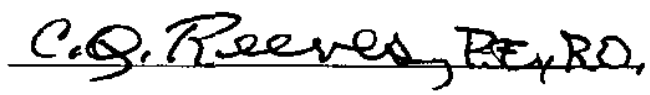

UNCLASSIFIED

Does Not Contain Unclassified Controlled Nuclear Information

Westinghouse Savannah River Company

Savannah River Site

Aiken, SC 29808 


\title{
H-AREA HAZARDOUS WASTE MANAGEMENT FACILITY CORRECTIVE ACTION REPORT (U)
}

\section{THIRD AND FOURTH QUARTER 1998}

\author{
Volume I
}

Publication Date: March 1999

Authorized Derivative Classifier and Reviewing Official:

UNCLASSIFIED

Does Not Contain Unclassified

Controlled Nuclear Information

Westinghouse Savannah River Company

Savannah River Site

Aiken, SC 29808 


\section{DISCLAIMER}

This report was prepared as an account of work sponsored by an agency of the United States Government. Neither the United States Government nor any agency thereof, nor any of their employees, makes any warranty, express or implied, or assumes any legal liability or responsibility for the accuracy, completeness, or usefulness of any information, apparatus, product, or process disclosed, or represents that its use would not infringe privately owned rights. Reference herein to any specific commercial product, process, or service by trade name, trademark, manufacturer, or otherwise does not necessarily constitute or imply its endorsement, recommendation, or favoring by the United States Government or any agency thereof. The views and opinions of authors expressed herein do not necessarily state or reflect those of the United States Government or any agency thereof.

This report has been reproduced directly from the best available copy.

Available to DOE and DOE contractors from the Office of Scientific and Technical Information, P.O. Box 62, Oak Ridge, TN 37831; prices available from (615) 576-8401.

Available to the public from the National Technical Information Service, U.S. Department of Commerce; 5285 Port Royal Road, Springfield, VA 22161. 


\section{Contents}

Volume I

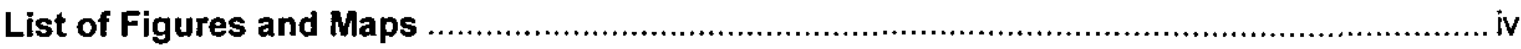

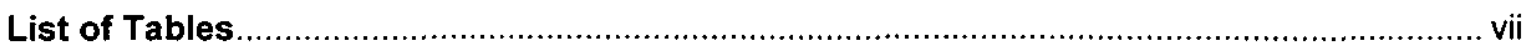

Executive Summary

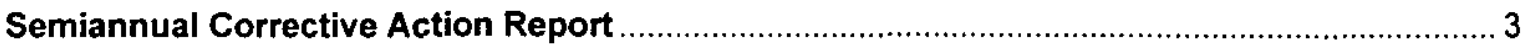

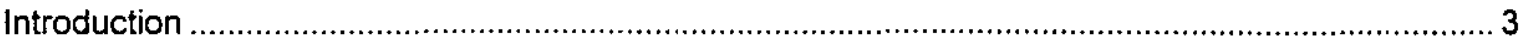

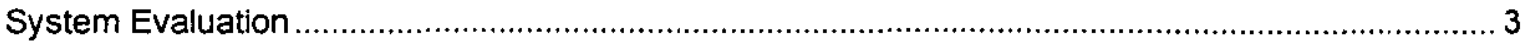

Water Quality and Elevation Data Tables (IIID.H.11.c.i) ................................................................ 3

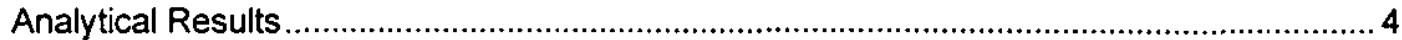

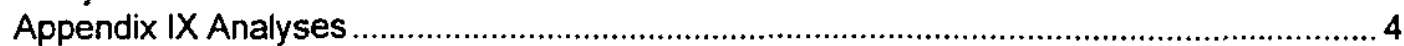

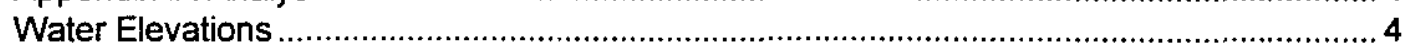

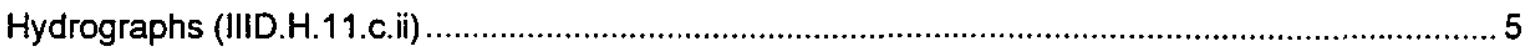

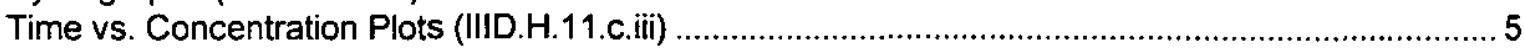

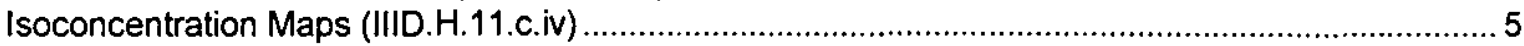

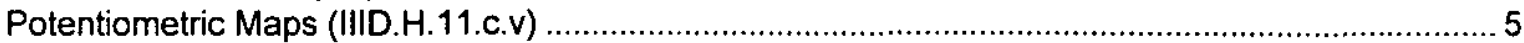

Potentiometric Cross Sections and Isoconcentration Cross-Sections (IIID.H.11.c.vi) .........................5

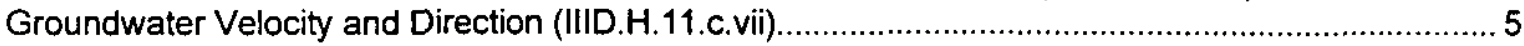

Extent and Severity of Groundwater Contamination (IIID.H.11.C.viii).............................................6

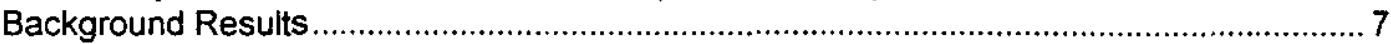

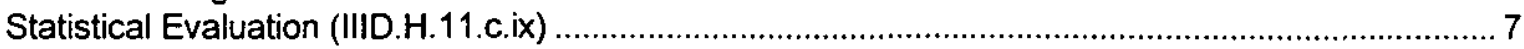

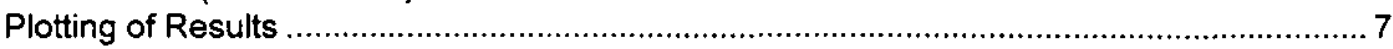

Evaluation of Water Quality and Water Elevation Data (IIID.H.11.c.x) ........................................... 8

Volume and Rates of Groundwater Pumped (IIID.H.11.c.xi) ......................................................... 8

Volume and Rates of Treated Groundwater Injected (IIID.H.11.C.xii) ........................................... 8

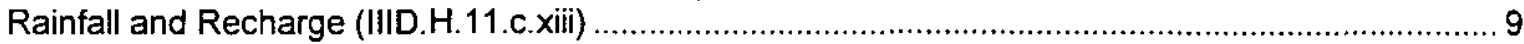

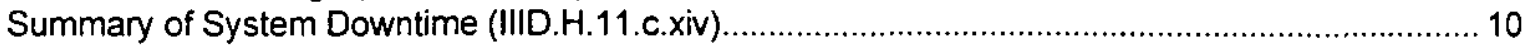

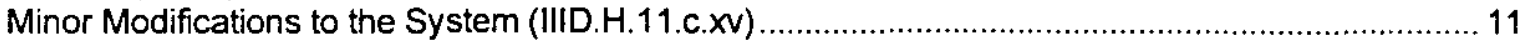

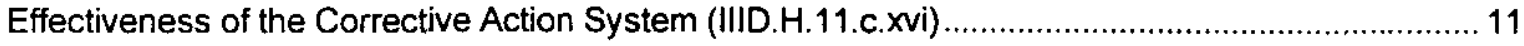

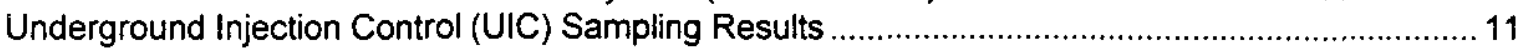

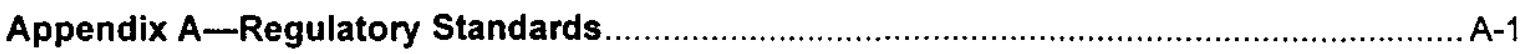

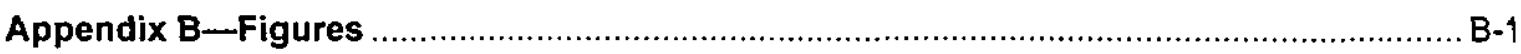

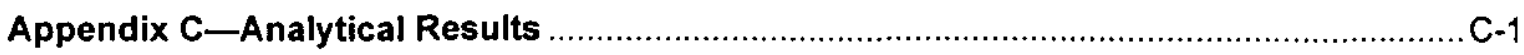

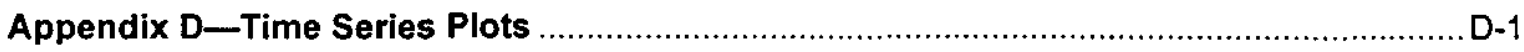

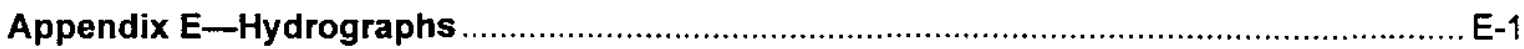

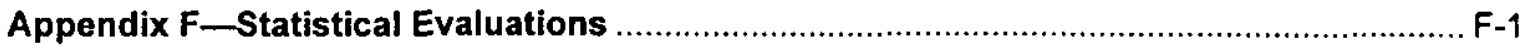

Appendix G-Operational Activities and Downtime ........................................................

Volume II

Appendix H-General Separations Area Maps for the F-and H-Area Hazardous Waste Management Facilities Semiannual Corrective Action Reports (U) 


\section{List of Figures and Maps}

Volume I-In-text Figure

Page

1. Rainfall Data for H Area During Third and Fourth Quarter 1998.

\section{Appendix B-Figures}

1. Location of the H-Area HWMF at the Savannah River Site.

2. Location of the Groundwater Monitoring Wells at the H-Area HWMF...

3. Location of Aquifer Zone ${\| B_{2}}_{2}$ (Water Table) Groundwater Monitoring Wells at the H-Area HWMF

4. Location of Aquifer Zone IIB, (Barnwell/McBean) Groundwater Monitoring Wells at the H-Area HWMF

1. Location of Aquifer Unit IIA (Congaree) Groundwater Monitoring Wells at the H-Area HWMF.

1. Hydrogeologic Cross-Section Showing Potentiometric Surfaces at the H-Area HWMF, Fourth Quarter 1998.

2. Hydrogeologic Cross-Section Showing Mercury Concentrations at the H-Area HWMF, Fourth Quarter 1998.

3. Hydrogeologic Cross-Section Showing Nitrate Concentrations at the H-Area HWMF, Fourth Quarter 1998

4. Hydrogeologic Cross-Section Showing Tritium Activities at the H-Area HWMF, Fourth Quarter 1998

5. Hydrogeologic Cross-Section Showing lodine-129 Activities at the H-Area HWMF, Third Quarter 1998.

6. Hydrogeologic Cross-Section Showing Strontium-90 Activities at the H-Area HWMF, Third Quarter 1998

7. Hydrogeologic Cross-Section Showing Gross Alpha Activities at the H-Area HWMF, Fourth Quarter 1998

8. Hydrogeologic Cross-Section Showing Nonvolatile Beta Activities at the H-Area HWMF, Fourth Quarter 1998.

\section{Volume II}

Appendix H-Maps

1. Cadmium Concentrations in Aquifer Zone $\| \mathrm{B}_{2}$ (Water Table) at the General Separations Area, Fourth Quarter 1998.

2. Cadmium Concentrations in Aquifer Zone $\| B_{1}$ (Barnwell/McBean) at the General Separations Area, Fourth Quarter 1998

3. Cadmium Concentrations in Aquifer Unit IIA (Congaree) at the General Separations Area, Fourth Quarter 1998.

4. Gross Alpha Activities in Aquifer Zone $\| B_{2}$ (Water Table) at the General Separations Area, Fourth Quarter 1998. 
5. Gross Alpha Activities in Aquifer Zone $\| B_{1}$ (Barnwell/McBean) at the General Separations Area, Fourth Quarter 1998.

6. Gross Alpha Activities in Aquifer Unit IIA (Congaree) at the General Separations Area, Fourth Quarter 1998

7. Iodine-129 Activities in Aquifer Zone $\| \mathrm{B}_{2}$ (Water Table) at the General Separations Area, Third Quarter 1998

8. lodine-129 Activities in Aquifer Zone IIB $_{1}$ (Barnwell/McBean) at the General Separations Area, Third Quarter 1998

9. lodine-129 Activities in Aquifer Unit IIA (Congaree) at the General Separations Area, Third Quarter 1998

10. Lead Concentrations in Aquifer Zone $\| I B_{2}$ (Water Table) at the General Separations Area, Fourth Quarter 1998

11. Lead Concentrations in Aquifer Zone $\| B_{1}$ (Barnwell/McBean) at the General Separations Area, Fourth Quarter 1998.

12. Lead Concentrations in Aquifer Unit IIA (Congaree) at the General Separations Area, Fourth Quarter 1998

13. Mercury Concentrations in Aquifer Zone $\mathrm{II}_{2}$ (Water Table) at the General Separations Area, Fourth Quarter 1998

14. Mercury Concentrations in Aquifer Zone $\|_{1} B_{1}$ (Barnwell/McBean) at the General Separations Area, Fourth Quarter 1998

15. Mercury Concentrations in Aquifer Unit IIA (Congaree) at the General Separations Area, Fourth Quarter 1998

16. Nitrate Concentrations in Aquifer Zone $\| B_{2}$ (Water Table) at the General Separations Area, Fourth Quarter 1998

17. Nitrate Concentrations in Aquifer Zone liB, (Barnwell/McBean) at the General Separations Area, Fourth Quarter 1998

18. Nitrate Concentrations in Aquifer Unit IIA (Congaree) at the General Separations Area, Fourth Quarter 1998.

19. Nonvolatile Beta Activities in Aquifer Zone $\| B_{2}$ (Water Table) at the General Separations Area, Fourth Quarter 1998

20. Nonvolatile Beta Activities in Aquifer Zone IIB 1 (Barnwell/McBean) at the General Separations Area, Fourth Quarter 1998

21. Nonvolatile Beta Activities in Aquifer Unit IIA (Congaree) at the General Separations Area, Fourth Quarter 1998

22. Strontium-90 Activities in Aquifer Zone $\|_{2}$ (Water Table) at the General Separations Area, Third Quarter 1998

23. Strontium-90 Activities in Aquifer Zone IIB ${ }_{1}$ (Barnwell/McBean) at the General Separations Area, Third Quarter 1998

24. Strontium-90 Activities in Aquifer Unit IIA (Congaree) at the General Separations Area, Third Quarter 1998

25. Technetium-99 Activities in Aquifer Zone $\mathrm{IIB}_{2}$ (Water Table) at the General Separations Area, Third Quarter 1998 
26. Technetium-99 Activities in Aquifer Zone IIB, (Barnwell/McBean) at the General Separations Area, Third Quarter 1998

27. Technetium-99 Activities in Aquifer Unit IIA (Congaree) at the General Separations Area, Third Quarter 1998

28. Tritium Activities in Aquifer Zone $\mathrm{IIB}_{2}$ (Water Table) at the General Separations Area, Fourth Quarter 1998.

29. Tritium Activities in Aquifer Zone IIB, (Barnwell/McBean) at the General Separations Area, Fourth Quarter 1998.

30. Tritium Activities in Aquifer Unit IIA (Congaree) at the General Separations Area, Fourth Quarter 1998

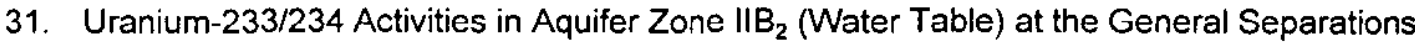
Area, Third Quarter 1998

32. Uranium-233/234 Activities in Aquifer Zone $\|_{1} B_{1}$ (Barnwell/McBean) at the General Separations Area, Third Quarter 1998

33. Uranium-233/234 Activities in Aquifer Unit IIA (Congaree) at the General Separations Area, Third Quarter 1998

34. Uranium-238 Activities in Aquifer Zone $\| B_{2}$ (Water Table) at the General Separations Area, Third Quarter 1998

35. Uranium-238 Activities in Aquifer Zone $\| B_{1}$ (Barnwell/McBean) at the General Separations Area, Third Quarter 1998

36. Uranium-238 Activities in Aquifer Unit IlA (Congaree) at the General Separations Area, Third Quarter 1998

37. Potentiometric Surface Map of Aquifer Zone $\| 1_{2}$ (Water Table) at the General Separations Area, Third Quarter 1998

38. Potentiometric Surface Map of Aquifer Zone $\|_{1} B_{2}$ (Water Table) at the General Separations Area, Fourth Quarter 1998

39. Potentiometric Surface Map of Aquifer Zone IIB, (Barnwell/McBean) at the General Separations Area, Third Quarter 1998

40. Potentiometric Surface Map of Aquifer Zone IIB (Barnwell/McBean) at the General Separations Area, Fourth Quarter 1998

41. Potentiometric Surface Map of Aquifer Unit IIA (Congaree) at the General Separations Area, Third Quarter 1998

42. Potentiometric Surface Map of Aquifer Unit IIA (Congaree) at the General Separations Area, Fourth Quarter 1998 


\section{List of Tables}

In-text

Page

1. Horizontal Groundwater Flow Rates (ft/year) in the Hydrostratigraphic Units beneath the H-Area HWMF .6

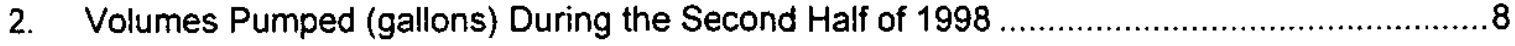

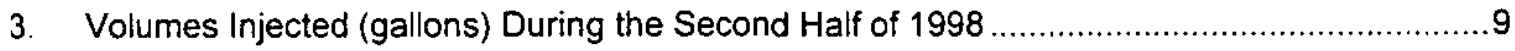
Appendix A

Page

A-1. Groundwater Protection Standard.

A-3

A-2. UIC Limits A-5

Appendix C Page

C-1. Groundwater Monitoring Results for Individual Wells C-6

C-2. UIC Results C-283 
WSRC-TR-99-00013

Unclassified

THIS PAGE LEFT BLANK INTENTIONALLY 


\section{Executive Summary}

The groundwater in the uppermost aquifer beneath the H-Area Hazardous Waste Management Facility (HWMF), also known as the H-Area Seepage Basins, at the Savannah River Site (SRS) is monitored periodically for various hazardous and radioactive constituents as required by Module III, Section D, of the 1995 Resource Conservation and Recovery Act (RCRA) Renewal Permit (South Carolina Hazardous and Mixed Waste Permit SC1-890-008-989), effective October 5, 1995 (hereafter called the RCRA permit). Currently, the H-Area HWMF monitoring network consists of 130 wells of the HSB series and 8 wells of the HSL series screened in the three hydrostratigraphic units that make up the uppermost aquifer beneath the H-Area HWMF (Figures $2-5$, Appendix B). This report presents the results of the required groundwater monitoring program as identified in Section IIID.H.11.C of the RCRA permit and Section C of the Underground Injection Control (UIC) permit application (hereafter referred to as the UIC application).

As shown in Table C-1 (Appendix C), the following constituents or summations exceeded the Groundwater Protection Standards (GWPS) in wells at the H-Area HWMF during the second half of 1998: beta dose, cobalt, lead, mercury, nitrate-nitrite (as N), tin, total radium, tritium, vanadium, bis(2-ethylhexyl) phthalate, gross alpha, nonvolatile beta, and the sums of both alpha- and betaemitting radionuclides.

In addition, the following radionuclides individually exceeded the standard for either sum of alpha emitters or sum of beta emitters: carbon-14, iodine-129, radium-226, strontium-90, technetium-99, and uranium-233/234. Radium-226 alone exceeded the standard for total radium.

Tritium was the primary constituent detected above the GWPS; it was elevated in 91 (65\%) of the wells. Mercury was the only metal that exceeded the GWPS in more than 10 wells. Bis(2ethylhexyl) phthalate in three wells was the only organic constituent that definitively exceeded the GWPS during the second half of 1998.

Isoconcentration maps included in Volume II of this report indicate both the concentration or activity and the extent of the contamination during the second half of 1998 in each of the three hydrostratigraphic units.

Tritium, the radionuclide most frequently above the GWPS at the H-Area HWMF, had a maximum activity of $8,500 \mathrm{pCi} / \mathrm{mL}$ in Aquifer Zone $11 \mathrm{~B}_{1}$ (Barnwell/McBean) well $\mathrm{HSB} 137 \mathrm{C}$ during fourth quarter 1998. Maximum activities for three key radionuclides occurred in Aquifer Zone $\mathrm{IIB}_{2}$ (Water Table) wells: nonvolatile beta, 2,700 pCi/L in well HSB115D during fourth quarter, and iodine-129, $110 \mathrm{pCi} / \mathrm{L}$ in well HSB114D and strontium-90, $930 \mathrm{pCi} / \mathrm{L}$ in well HSB115D, both during third quarter. The maximum activity for gross alpha, $58 \mathrm{pCi} / \mathrm{L}$ in HSB102D, occurred in the fourth quarter.

Nitrate-nitrite (as $N$ ) had a maximum concentration of $44,000 \mu \mathrm{g} / \mathrm{L}$ in Aquifer Zone $\mathrm{IlB}_{1}$ (Barnwell/McBean) well HSB137C during fourth quarter 1998. Mercury had a maximum concentration of $19 \mu \mathrm{g} / \mathrm{L}$ in Aquifer Zone IIB $_{2}$ (Water Table) well HSB106D during third quarter.

Water-level maps indicate that the groundwater velocities and directions at the H-Area HWMF have remained relatively constant since the basins ceased operation in 1988. Groundwater flow in Aquifer Zone $\mathrm{IIB}_{2}$ (Water Table) for third and fourth quarter 1998 was south or southwest and in Aquifer Zone $\| B_{1}$ (Barnwell/McBean) south to southeast toward Fourmile Branch. Flow in Aquifer Unit IIA (Congaree) was northwest toward Upper Three Runs Creek.

The estimated maximum groundwater velocities during the second half of 1998 ranged from approximately 770 to $880 \mathrm{ft} /$ year during third quarter and from 690 to $840 \mathrm{ft} /$ year during fourth quarter in Aquifer Zone $\|_{1} B_{2}$ (Water Table). In Aquifer Zone IIB 1 (Barnwell/McBean), velocity estimates ranged from 230 to $280 \mathrm{ft} /$ year during third quarter and from 210 to $230 \mathrm{ft} /$ year during fourth quarter. The estimated velocity in Aquifer Unit llA (Congaree) was $320 \mathrm{ft} / y e a r$ for third quarter and $310 \mathrm{ft} / \mathrm{year}$ for fourth quarter 1998. 
The H-Area Groundwater Remediation Wastewater Treatment Units (WTU) facility operating permit was received on July 7,1997 , and shakedown operations began on July 8,1997 . The WTUs operated in a shakedown mode through fourth quarter 1998. 
WSRC-TR-99-00013

Unclassified

\section{Semiannual Corrective Action Report}

\section{Introduction}

SRS monitors groundwater quality at the $\mathrm{H}$-Area Hazardous Waste Management Facility (HWMF) and provides results of this monitoring to the South Carolina Department of Health and Environmental Control (SCDHEC) semiannually as required by the Resource Conservation and Recovery Act (RCRA) permit. SRS also performs monthly sampling of the WTU effluent in accordance with Section C of the Underground Injection Control (UIC) application.

The H-Area HWMF is described in the introduction to Module III, Section D, of the RCRA permit. The H-Area HWMF well network monitors three distinct hydrostratigraphic units in the uppermost aquifer beneath the facility. The hydrostratigraphy at the H-Area HWMF is described in RCRA permit Section IIID.H.2, and the groundwater monitoring system is described in IIID.H.4 and Appendix IIID-B. A detailed description of the uppermost aquifer is included in the RCRA Part B post-closure care RCRA permit renewal application for the H-Area HWMF submitted to SCDHEC in October 1993.

Sampling and analysis are conducted as required by Section IIID.H.6 for the constituents and at the intervals specified in Appendix IIID-D of the RCRA permit. Groundwater quality is compared to the Groundwater Protection Standards (GWPS) list described in Appendix IIID-A of the RCRA permit.

\section{System Evaluation}

The H-Area Wastewater Treatment Unit (WTU) continued in a shakedown mode during the third and fourth quarter 1998. Consistent flow rates were limited due to ongoing system testing. Fullscale operation was not initiated due to various problems with the WTU. SRS has initiated a significant effort to identify and correct the problems that have been encountered during shakedown operations.

A statistical evaluation of water quality and water level data was performed and is included in this report (Section IIID.H.11.c.ix). Shewhart-CUSUM charts (Appendix F) are provided in accordance with the RCRA permit application. Due to limited operations during 1998, no significant effects due to remediation system operation were identified. However, several trends in water quality and water levels were identified as discussed in Section IIID.H.11.c.ix.

\section{Water Quality and Elevation Data Tables (IIID.H.11.c.i)}

Water-quality and water-elevation data for the second half of 1998 for all H-Area HWMF wells are presented in Table C-1 (Appendix C). Table C-1 identifies field parameters and modifiers that define laboratory accuracy and precision. Definitions of the abbreviations and the modifiers, as well as descriptions of holding times, data rounding, and data qualification practices are also provided in Appendix C. Constituents that exceeded either the GWPS or United States Environmental Protection Agency (US EPA)-approved holding times are denoted by symbols in columns headed ST and $\mathrm{H}$, respectively.

SRS implemented a data filtering process during third and fourth quarter 1998 to better evaluate the data collected. This process qualifies certain data (" $L$ ", "R", " $U$ ", and "J") for the purposes of regulatory decision making. The data tables in Appendix $C$ include both a column for all data collected and a column for filtered data for comparison purposes. Permission to implement the data filtering process was requested from SCDHEC (Cook to Taylor; November 25, 1997) and granted (Taylor to Cook; April 21, 1998). 
Variable-speed pumps have been installed in wells with a history of elevated analytical results for metals. Samples from wells with variable-speed pumps can be collected at a slower rate to minimize turbidity, which has been associated with elevated metals levels. The chart below identifies those wells that currently have variable-speed pumps as well as those with single-speed pumps.

\begin{tabular}{|c|l|}
\hline Variable-Speed Pumps & Single-Speed Pumps \\
\hline HSB 67, 68,69,84A, 84D, 86C, 86D, & All remaining HSB wells have single-speed \\
101D, 102D, 103D, 104D, 105D, 106D, & pumps \\
107D, 108D, 110D, 111D, 111E, 112D, & \\
$112 E, 113 D, 114 C, 114 D, 116 C, 116 D$, & \\
$117 C, 125 D$, and 136D & \\
HSL 1D, 2D, 3D, 4D,5D,6D,7D, and 8D & \\
\hline
\end{tabular}

\section{Analytical Results}

All data received from the laboratories are validated and verified in accordance with Savannah River Site (SRS) and US EPA guidelines. Due to analytical limitations, curium-245/246 activities are reported as an upper limit of curium-246 activities, and uranium-233/234 activities are reported as an upper limit of uranium-234.

Data received from the laboratories for the following radionuclide analyses were rejected during third quarter due to matrix interference: thorium-228 (in wells HSB102D, HSB112E, HSB113C, and HSB115D) and iodine-129 (in well HSB116D).

Well HSB141C was abandoned on November 2, 1998. Well HSB141CR had previously replaced HSB141C for monitoring purposes.

Well HSB 65A had maintenance problems for the first two attempts at sampling, on July 1 and July 28 . Maintenance was performed, and the well was successfully sampled on August 27. 1998, with no loss of permit-required data.

Well HSB119 was purged through the sample port to lower turbidity for fourth quarter 1998 sampling.

\section{Appendix IX Analyses}

During third quarter 1998, Appendix IX analyses were performed as required by the RCRA renewal permit. Sulfide in well HSB107D was the only constituent not already on the RCRA permit Groundwater Protection Standards list that was reported above its detection limit in $\mathrm{H}$-Area wells. Confirmation sampling was performed during January 1999, and laboratory results are pending.

\section{Water Elevations}

Synchronous water elevations were measured during third and fourth quarter 1998 in compliance with Section IIID.H.7 of the RCRA permit. Potentiometric maps are provided in Volume II. A significant water level increase observed in most wells in the spring of 1998 is likely due to increased precipitation and associated recharge during this time period. Compared to the uppermost aquifer zone, the lower aquifer zones generally have smaller, yet more gradual increases, as would be expected from recharge effects. 


\section{Hydrographs (IIID.H.11.c.ii)}

Hydrographs showing the water elevations for the H-Area HWMF are provided in Appendix E. Hydrograph data include synchronous water level measurements and water level measurements taken during sampling. A significant water level increase in most wells in the spring of 1998 is likely due to increased precipitation and associated recharge during this time period. See also section IIID.H.11.c.xiii. Compared to the uppermost aquifer zone, the lower aquifer zones generally have smaller yet more gradual increases, as would be expected from recharge effects.

\section{Time vs. Concentration Plots (IIID.H.11.c.iii)}

Appendix D contains time series plots of mercury, nitrate/nitrite, gross alpha, iodine-129, nonvolatile beta, strontium-90, and tritium data for selected wells. The wells were selected to delineate the extent of the constituent distribution, to monitor the migration of the constituents, and to follow trends of constituents present at high levels. Constituents reported as below detection were not plotted.

\section{Isoconcentration Maps (IIID.H.11.c.iv)}

Isoconcentration maps for iodine-129 and strontium-90 in the three hydrostratigraphic units during third quarter 1998 and for mercury, nitrate, gross alpha, nonvolatile beta, and tritium during fourth quarter 1998 are presented in Volume II.

\section{Potentiometric Maps (IIID.H.11.c.v)}

Piezometric and potentiometric surface maps for the monitored water-bearing units during third and fourth quarter 1998 are located in Volume ll. The maps illustrate groundwater flow patterns beneath the H-Area HWMF for the third and fourth quarter 1998.

\section{Potentiometric Cross Sections and Isoconcentration Cross-Sections (IIID.H.11.c.vi)}

Potentiometric and isoconcentration cross-sections, required by RCRA permit Section IIID.H.11.c.vi, are located in Appendix B.

\section{Groundwater Velocity and Direction (IIID.H.11.c.vii)}

Horizontal velocity calculations are used to estimate the transport rate of constituents originating from the H-Area HWMF. Velocities in Aquifer Zone $\|_{1} B_{2}$ (Water Table) and Aquifer Zone IIB, (Barnwell/McBean) are calculated along two paths (designated flow paths $A$ and $B$ ) to characterize the approximate maximum and minimum groundwater velocities within these units in areas associated with the basins. The velocity is calculated along a single flow path for Aquifer Unit IIA (Congaree).

Velocities are estimated using the following equation:

$$
\text { Flow }(\mathrm{ft} / \text { day })=\frac{\text { Hydraulic Conductivity }(\mathrm{ft} / \text { day) }}{\text { Porosity (unitless) }} \times \frac{d h(\mathrm{ft})}{d l(\mathrm{ft})}
$$

The value $d h$ is the difference in head, and $d l$ is the length of the flow path. Flow path length is calculated to the nearest $100 \mathrm{ft}$ for path B of Aquifer Zone IIB 1 (Barnwell/ McBean) and to the nearest $50 \mathrm{ft}$ for the remaining flow paths. Maximum velocity per day is calculated and rounded to two significant digits, then multiplied by 365 and rounded to two significant digits to obtain the velocity per year. Velocity estimates vary depending upon the vertical gradient between wells, the size of the area under consideration, the number of data points, and the length and location of the flow path. Because these calculations are based on inferred or estimated parameters, velocity estimates are accurate to an order of magnitude only. 
Maximum horizontal velocity estimates for the hydrostratigraphic units during third and fourth quarter 1998 are provided in Table 1.

Table 1. Horizontal Groundwater Velocities (ft/year) in the H-Area HWMF

\begin{tabular}{|llll|}
\hline & $\begin{array}{l}\text { Aquifer Zone IIB } \\
\text { Water Table) }\end{array}$ & $\begin{array}{l}\text { Aquifer Zone IIB } 1 \\
\text { (Barnwell/McBean) }\end{array}$ & $\begin{array}{l}\text { Aquifer Unit IIA } \\
\text { (Congaree) }\end{array}$ \\
\hline $\mathrm{K}_{\mathrm{h}}$ (ft/day) & $20-40$ & $1.5-10$ & 65 \\
Effective porosity & $20 \%$ & $20 \%$ & $20 \%$ \\
$d h / d l$ & Varies & varies & Varies \\
Velocity (ft/yr) (3Q98) & $770-880$ & & 320 \\
Velocity (f/yr) (4Q98) & $690-840$ & $230-280$ & 310 \\
\hline
\end{tabular}

The hydraulic conductivity $\left(K_{n}\right)$ values represent regional average hydraulic conductivities and may vary locally.

During both third and fourth quarter 1998, flow in Aquifer Zones $\| B_{2}$ (Water Table) and $\| B_{1}$ (Barnwell/McBean) was south along both flow paths $A$ and $B$ toward Fourmile Branch. Flow in Aquifer Unit IIA (Congaree) was west or northwest toward Upper Three Runs Creek during both quarters.

\section{Extent and Severity of Groundwater Contamination (IIID.H.11.c.viii)}

Constituents that exceeded the GWPS (Table A-1, Appendix A) during third and fourth quarter 1998 in the H-Area HWMF wells are denoted in Table C-1 (Appendix C).

As shown in Table $\mathrm{C}-1$, the following constituents or summations exceeded the GWPS in wells at the $\mathrm{H}$-Area HWMF: beta dose, cobalt, lead, mercury, nitrate-nitrite (as $\mathrm{N}$ ), tin, vanadium, bis (2ethylhexyl) phthalate, gross alpha, nonvolatile beta, total radium, tritium, beta dose, and the sums of both alpha- and beta-emitting radionuclides.

In addition, carbon-14, iodine-129, radium-226, strontium-90, technetium-99, and uranium-233/234 individually exceeded the standard for either total alpha emitters or total beta emitters ( $15 \mathrm{pCi} / \mathrm{L}$ and $50 \mathrm{pCi} / \mathrm{L}$, respectively). Radium-226 alone also exceeded the standard for total radium.

The extent and severity of groundwater contamination is delineated by the contours above the GWPS on the isoconcentration maps (Volume If). Tritium is the most widespread contaminant and exceeds the GWPS in all three aquifers (Maps 28-30 in Appendix $\mathrm{H}$ ). Elevated activities north of the basins are probably due to tritium sources in the burial grounds. The highest activities are downgradient of the basins in the Water Table and the Barnwell/McBean aquifer zones. Tritium is also present in the Water Table aquifer zone along the H-Area Inactive Process Sewer Line to the basins. Tritium is elevated in some Congaree wells near the western end of the basins.

Nitrate-nitrite (as N) (Maps 16-18) is present in all the aquifer zones under and south of the basins. Gross alpha (Maps 4-6), uranium-234 (Maps 31-33), uranium-238 (Maps 34-36), and iodine-129 (Maps 7-9) are present in a few wells, mostly near the western end of the basins. Nonvolatile beta and strontium-90 (Maps 19-24) are present in the Water Table aquifer zone below and south of the basins. Nonvolatile beta is also present to a lesser extent in the Barnwell/McBean aquifer zone. Technetium-99 (Maps 25-27) is present in the Water Table and Barnwell/McBean aquifer zones below and south of the western end of the basins. Mercury (Maps 13-15) is present in the Water Table and Barnwell/McBean aquifer zones below and south of the eastern end of the basins. 


\section{Background Results}

Wells HSB 66, 83A, and 858 are the background wells for the H-Area HWMF. They are screened in the Water Table, Middle Congaree, and lower Barnwell/McBean aquifer zones, respectively. No constituents were found to exceed their GWPS in any of the three background wells during third and fourth quarter 1998.

Tritium has historically been above its GWPS of $20 \mathrm{pCi} / \mathrm{mL}$ in well HSB 66 during both third and fourth quarter of every year since 1993 and during second quarter 1995; however, tritium levels fell to 14 in third quarter and 12 in fourth quarter 1998.

\section{Statistical Evaluation (IIID.H.11.c.ix)}

The H-Area RCRA Permit requires an annual statistical evaluation of water quality and water level data to assess significant changes or impacts associated with operation of the H-Area WTU. Constituents listed in Appendix IIID-A of the RCRA permit must be evaluated in all Point of Compliance ( $P O C)$ wells and in a representative number of plume assessment wells.

Control charts have historically been used in industry and laboratories to monitor processes to determine whether the monitoring data shows a constituent either "in control" or "out of control." For groundwater monitoring, control charts can be used to monitor water quality data and to fiag anomalous results. The statistical procedure employed by SRS to assess the effectiveness of corrective action is the Shewhart-CUSUM control chart, detailed in Appendix $F$. The chart will be used to detect both increasing and decreasing trends and to identify either sudden incursions (a contaminant slug) or steady drift (a change in plume concentration or size). Water level data will be evaluated similarly.

The selected wells for this statistical analysis include all POC wells (as listed in Table IIID-B of the RCRA permit) and a representative number of plume wells, as follows:

$\begin{array}{lll}\text { HSB125D } & \text { HSB129D } & \text { HSB137D } \\ \text { HSB126C } & \text { HSB135C } & \text { HSB139C } \\ \text { HSB126D } & \text { HSB135D } & \text { HSB139D } \\ \text { HSB127C } & \text { HSB136C } & \text { HSB145C } \\ \text { HSB127D } & \text { HSB136D } & \text { HSB145D } \\ \text { HSB129C } & \text { HSB137C } & \end{array}$

For this evaluation, background samples consisted of all samples between the first quarter of 1991 and the last quarter of 1995. The end of 1995 was chosen as a background cutoff date to provide adequate background sample numbers and to provide control plot points for at least one year prior to startup of remediation operations.

\section{Plotting of Results}

Shewhart-CUSUM Control Plots are given in Appendix F for those constituent/well pairings that made it through the data qualification and treatment process described above. To maintain clarity and uniformity in all the control plots, the $y$-axis ranges have been set to between -6 and +6 standardized units.

Due to the fimited operation of the WTU and the minimal available trend data since the background time period, the analysis of the control chart statistics cannot be extensive but some general comments can be made concerning the indications of gradual increases/decreases or sudden breakthroughs (or sudden drops). 
In general, the charts do not show any effect from the shakedown operations. There are a number of wells that show statistically significant decreases of tritium, nonvolatile beta, and nitrate-nitrite as $\mathrm{N}$, but at the same time there are also a number of wells that show statistically significant increases of these same constituents. There is no evident spatial correlation to these increases or decreases.

\section{Evaluation of Water Quality and Water Elevation Data (IIID.H.11.C.x)}

Due to the limited remediation operations, the zone of capture and drawdown has not yet been determined. No significant trends or changes have been attributed to the operation of the WTU.

\section{Volume and Rates of Groundwater Pumped (IIID.H.11.c.xi)}

Table 2 summarizes the pump rates, monthly volumes, and total volumes pumped from each extraction well during third and fourth quarter 1998. The pump rates represent the average pump rate for each well. The individual well volumes (and consequently, the average flow rates) are estimated due to instrument errors. The well totals are based on recorded well performance (PLC) and extraction totals. The total volumes extracted and injected are based on the totalizers on the extraction and injection tanks. Data are collected daily from the totalizers.

Table 2. Volume Pumped (gallons) During the Second Half of 1998

\begin{tabular}{|c|c|c|c|c|c|c|c|c|}
\hline Well & $\begin{array}{l}\begin{array}{l}\text { Rate } \\
\text { (gpm) }\end{array} \\
\end{array}$ & $\begin{array}{l}\text { July } \\
1998\end{array}$ & $\begin{array}{c}\text { August } \\
1998\end{array}$ & $\begin{array}{l}\text { Sept. } \\
1998 \\
\end{array}$ & $\begin{array}{c}\text { October } \\
1998 \\
\end{array}$ & $\begin{array}{l}\text { Nov. } \\
1998\end{array}$ & $\begin{array}{l}\text { Dec. } \\
1998\end{array}$ & $\begin{array}{c}\text { 6-Month } \\
\text { Total }\end{array}$ \\
\hline HEX 1 & 4.7 & 34,090 & 146,890 & 90,210 & 186,270 & 6,293 & 314,525 & 778,278 \\
\hline HEX 2 & - & & . & - & - & - & - & \\
\hline HEX 3 & 11.9 & 320,385 & 343,186 & 411,831 & 441,679 & 25,137 & 314,650 & $1,856,868$ \\
\hline HEX 4 & 6.6 & 272,505 & 229,438 & 387,501 & 299,772 & - & 105,710 & $1,294,926$ \\
\hline HEX 9 & 5.4 & 185,937 & 101,002 & 262,787 & 119,209 & 5,870 & 172,155 & 846,960 \\
\hline HEX 12 & 5.2 & 169,632 & 132,176 & 192,188 & 110,551 & 12,483 & 141,014 & 758.044 \\
\hline HEX 16 & 9.3 & 367,992 & 264,353 & 399,268 & 497,793 & 6,994 & 183,395 & $1,719,795$ \\
\hline HEX 17 & 13.1 & 490,838 & 332,462 & 576,563 & 527,470 & 7,011 & 497,139 & $2,431,483$ \\
\hline HEX 18 & 7.8 & 143,093 & 147,140 & & & 16,758 & 309,843 & 616,834 \\
\hline HEX 19 & 5.7 & 156,772 & 109,731 & 172,577 & 114,969 & 9,747 & 38,499 & 602,295 \\
\hline Total & & $2,141,244$ & $1,806,378$ & $2.492,925$ & $2,297,713$ & 90,293 & $2,076,930$ & $10,905,483$ \\
\hline
\end{tabular}

\section{Volume and Rates of Treated Groundwater Injected (IIID.H.11.c.xii)}

Table 3 summarizes the pump rates, monthly volumes, and total volumes pumped to each injection well during third and fourth quarter 1998. The pump rates are the average pump rate for each well. The individual well volumes (and consequently, the average flow rates) are estimated due to instrument errors. The well totals are based on recorded well performance (PLC) and injection totals. The total volumes extracted and injected are based on the totalizers on the extraction and injection tanks. Data are collected daily from the totalizers. The difference in total volume extracted (Table 2) and total volume injected (Table 3 ) is due to the time delay between collecting extraction and injection readings as well as water retention in the WTU. 
Table 3. Volume Injected (gallons) During the Second Half of 1998

\begin{tabular}{|lcccccccc|}
\hline Well & $\begin{array}{c}\text { Rate } \\
\text { (gpm) }\end{array}$ & $\begin{array}{c}\text { July } \\
1998\end{array}$ & $\begin{array}{c}\text { August } \\
\mathbf{1 9 9 8}\end{array}$ & $\begin{array}{c}\text { Sept. } \\
\mathbf{1 9 9 8}\end{array}$ & $\begin{array}{c}\text { October } \\
\text { 1998 }\end{array}$ & $\begin{array}{c}\text { Nov. } \\
1998\end{array}$ & $\begin{array}{c}\text { Dec. } \\
1998\end{array}$ & $\begin{array}{c}\text { 6-Month } \\
\text { Total }\end{array}$ \\
\hline HIN 1 & 5.4 & 157,867 & 127,937 & 235,332 & 320,089 & 5,608 & 145,691 & 992,524 \\
HIN 2 & 6.6 & 105,062 & 179,561 & 341,231 & 389,619 & 9,834 & 159,609 & $1,184,916$ \\
HIN 3 & 5.4 & 190,152 & 105,990 & 235,332 & 287,444 & 8,430 & 123,750 & 951,098 \\
HIN 4 & 11.1 & 481,809 & 177,387 & 439,286 & 255,223 & 32,319 & 181,550 & $1,567,574$ \\
HIN 5 & 7.9 & 388,512 & 179,560 & 352,998 & 288,702 & 7,079 & 194,840 & $1,411,691$ \\
HIN 6 & - & - & - & - & - & - & - & - \\
HIN 7 & - & - & - & - & - & - & - & - \\
HIN 8 & 12.0 & 477,537 & 269,341 & 630,421 & 570,650 & 7,028 & 304,548 & $2,259,525$ \\
HIN 9 & 2.7 & 93,006 & 142,651 & 125,510 & 64,865 & 2,804 & 34,354 & 463,190 \\
HIN 10 & 3.7 & 145,555 & 69,330 & 113,734 & 129,731 & 9,832 & 76,607 & 544,789 \\
HIN 11 & 0.4 & - & - & - & - & - & 66,075 & 66,075 \\
HIN 12 & 1.1 & - & & - & - & - & 174,028 & 174,028 \\
HIN 13 & 0.5 & - & - & - & - & - & 82,751 & 82,751 \\
HIN 14 & 1.1 & - & - & - & - & - & 200,155 & 200,155 \\
HIN 15 & 0.9 & - & - & - & - & - & 170,141 & 170,141 \\
HIN 16 & 0.3 & - & - & - & - & - & 50,779 & 50,779 \\
HIN 17 & 4.0 & - & 508,755 & - & - & - & 116,352 & 625,107 \\
Total & & $2,039,500$ & $1,760,512$ & $2,473,844$ & $2,306,323$ & 82,934 & $2,081,230$ & $10,744,343$ \\
\hline
\end{tabular}

\section{Rainfall and Recharge (IIID.H.11.c.xiii)}

Figure 1 illustrates the rainfall data for $\mathrm{H}$ Area during third and fourth quarter 1998 . Recharge to groundwater is estimated to be $30 \%$ of rainfall in the General Separations Area. 


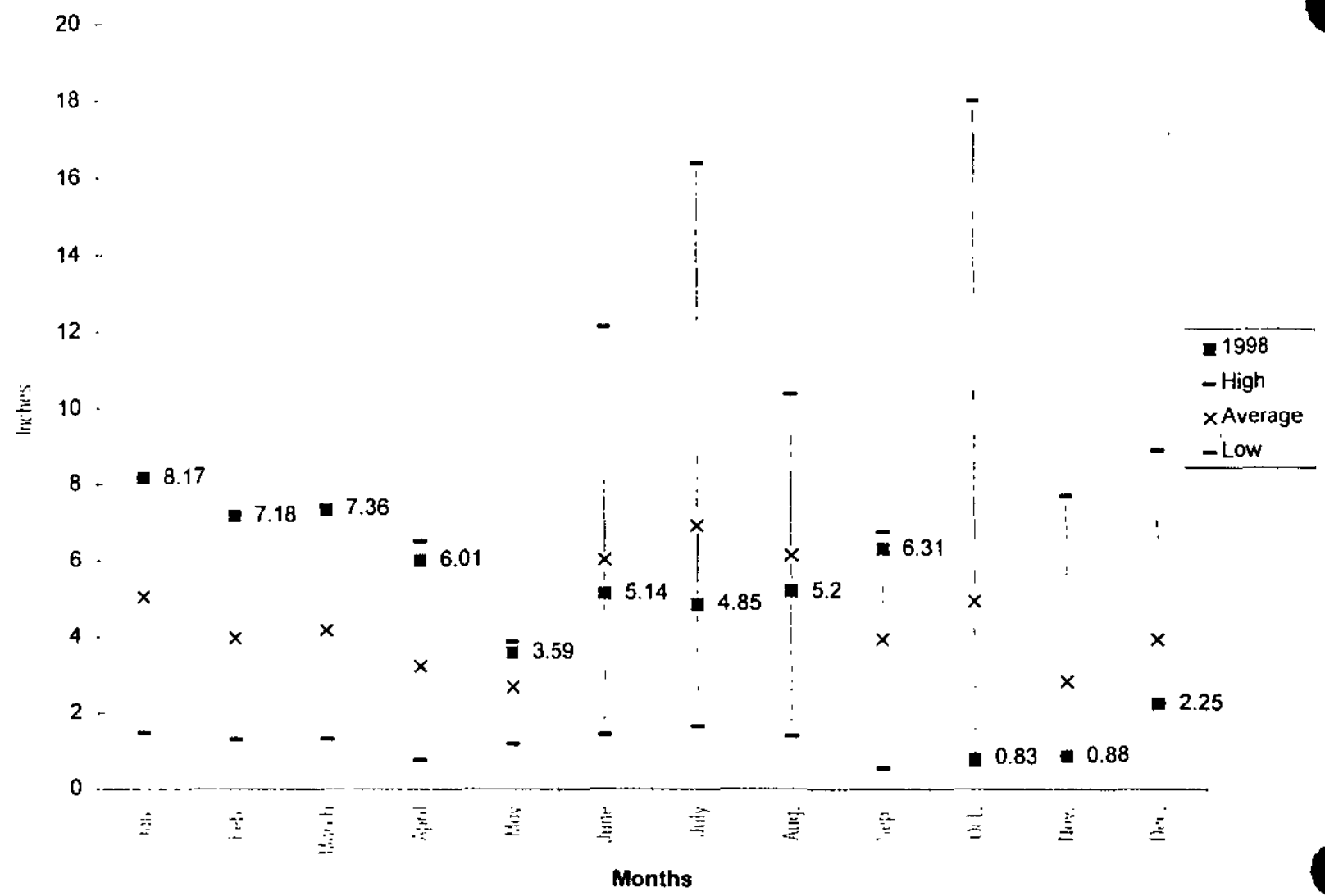

Figure 1. Rainfall Data for H Area During Third And Fourth Quarter 1998

\section{Summary of System Downtime (IIID.H.11.c.xiv)}

Significant downtime for the facility was incurred because of lightning strikes in the well fields or in the vicinity of the control room programmable logic controller (PLC). The electrical surges through the system resulted in damage to well instrumentation, PLC input/output modules, and miscellaneous controls. Initial modifications consisted of the addition of in-line fuses to help protect electrical components. In addition, SRS is currently designing improved lightning protection system. The additional fuses have been effective in reducing much of the electrical damage of lightning strikes. The design is complete and scheduled for installation during second quarter 1999.

Vibration problems that have been identified during this time period will be resolved in the near future. The first area of concern is the vibration induced into the system from the positive displacement, piston-type, reverse osmosis (RO) pumps. To date, three cracks have developed in the "B" RO Pump's stainless steel inlet or outlet piping that may have been aggravated by pump vibration. A vibration analysis is being conducted and will be followed by recommendations for design modifications, as applicable. Completion of the modifications is estimated for third quarter 1999. The second area of concern is the vibration caused by the air-driven diaphragm pumps used for sludge transfer from the sludge collection tanks (flocculation tank, clarifier, or filtrate return tank) to the filter press. This vibration has contributed to problems with the system surge protectors. SRS is currently designing modifications to the sludge transfer system to reduce vibration and improve reliability. Completion of this modification is for third quarter 1999. 
The $\mathrm{H}$-Area facility was limited to one-RO operation due to problems associated with the clarification and sludge removal systems. Most operations and maintenance downtime problems were due to one of the following problems:

- Clogging, pump, or flow problems with the polymer feed system

- Clogging, pump, or flow problems with the ferric chloride feed system

- Clogging, pump, or flow problems with the caustic $(\mathrm{NaOH})$ feed system

- Excessive carryover of the flocculant and precipitants through the clarifier due to incomplete chemical reaction and/or settling

- $\mathrm{pH}$ probe coating or calibration problems

- Removal of process solids caking on top of the medium in the roughing filters

- Fouling or platting of the flow control valve in the chemical feed line due to iron settling out of solution during low-flow or no-flow operating conditions.

SRS assumed operation of the WTU from the subcontractor on January 1, 1999. Upon transition to SRS operation, several modifications to improve process efficiency and reliability are anticipated.

Appendix $G$ summarizes the operating activities and downtime for third and fourth quarter 1998.

\section{Minor Modifications to the System (IIID.H.11.c.xv)}

Subsequent to receiving the wastewater permit to operate the modified treatment system on April 30,1998 , it was determined that numerous process changes would be required to correct the problems associated with the effective operation of the treatment system. These approved temporary process changes included: installation of additional roughing filters, new PolyBlend addition system, and numerous piping reconfigurations. A permit application modification for the $\mathrm{H}$-Area WTU, which incorporated these and other temporary changes, was submitted to SCDHEC on September 30, 1998. A permit to construct was issued on November 10, 1998.

Implementation of the permanent changes will commence upon receipt of the permanent roughing filters.

Permanent access platforms for the ion exchangers were fabricated and installed. In addition, a permanent platform and stairway for the flocculation/sludge tank was fabricated and installed. Eight new injection wells (HIN-11 through HIN-16. HIW-1ID, and HIW-2IC) were added to the HArea injection system and became operational in December 1998.

\section{Effectiveness of the Corrective Action System (IIID.H.11.c.xvi)}

The H-Area WTU operated in a shakedown mode during the third and fourth quarter 1998 but has not commenced full-scale operation. Approximately 10.7 million gallons of groundwater were treated and injected during this period. A statistical analysis was completed on the POC wells and a representative number of plume assessment wells, and this information will serve as a baseline for future reports. Due to the limited operations thus far, no direct impact to groundwater from operation of the WTU has been established. The statistical analysis did identify groundwater trends for a few constituents, and this information is discussed in Section IIIC.H.11.b.ix.

\section{Underground Injection Control (UIC) Sampling Results}

SRS collected samples to determine compliance with UIC standards as specified in the operating permit issued on April 17, 1997. The results of these samples are presented in Appendix C.

The H-Area WTU was sampled on July 30 , August 20 , September 22, October 13 , November 23 , and December 9,1998, for the suite of contaminants specified in the current UIC permit. Results 
were reported to SCDHEC both by electronic submittal and by letter. Whenever an exceedance was confirmed, SRS immediately notified SCDHEC. In each case, SCDHEC granted approval to restart/continue operating the unit based on the proposed corrective actions.

Starting with the October 1998 samples, a change was implemented in the reporting format for UIC sampling results. SRS replaced the AN95 reporting format with the AN98, the most significant change being the implementation of a sample-specific quantitation limit (SSEQL) for radionuclide results. Radionuclides were previously reported using the method detection limit as the quantitation limit. Reported detection limits that appear higher for October, November, and December may be attributable to this change.

The UIC permit divides the compliance suite of constituents into four sections: Section I, Inorganics; Section II, Organics; Section III, Radionuclides; and Section IV, Radionuclides. Section IV includes speciated radionuclide results, and Section III reports results for gross alpha, gross (nonvolatile) beta, and total radium.

No Section I or Section II constituents exceeded their respective regulatory standards during the third and fourth quarters of 1998 . Only barium, mercury, cobalt, nickel, and copper regularly exceeded their respective quantitation limits during the third and fourth quarters of 1998. Mercury results were between $0.5 \mathrm{ppb}$ and $1 \mathrm{ppb}$ during the third and fourth quarters.

Gross alpha did not exceed its limit of $15 \mathrm{pCi} /$; however, the October result showed an elevated quantitation limit of $29.18 \mathrm{pCi} / \mathrm{L}$. The October raw result was only $6.03 \mathrm{pCi} / \mathrm{L}$, and it is highly unlikely that gross alpha was over $15 \mathrm{pCi} / \mathrm{L}$ in that month. Gross (nonvolatile) beta and total radium were below their respective compliance limits of $50 \mathrm{pCi} / L$ and $5 \mathrm{pCi} / \mathrm{L}$ for all six months. Gross beta reported a slightly elevated quantitation limit in October of $52.8 \mathrm{pCi} / \mathrm{L}$; however, the raw result was $15.9 \mathrm{pCi} / \mathrm{L}$, and it is highly unlikely that the sample was higher than $50 \mathrm{pCi} / \mathrm{L}$.

The alpha-emitting radionuclides americium-241, curium-242, curium-243/244, curium-246. plutonium-238, plutonium-239/240, thorium-228, thorium-230, thorium-232, and uranium-235 were all below their respective detection limits during the third and fourth quarters of 1998 . Radium-226 exceeded its detection limit during August but was below the compliance limit of 5 $\mathrm{pCi} / \mathrm{L}$ for total radium in both of those months. Uranium-233/234 and uranium-238 exceeded their respective detection limits in July, but both remained below their respective detection limits for the other five months. The July results for both constituents were well below the sum of the alpha compliance limit of $15 \mathrm{pCi} / \mathrm{L}$. Consequently, the sum of the alpha contributors standard was not exceeded in any month and was above a detection limit only in July and August. Overall, the sum of the alpha contributors remained relatively steady at acceptable levels during all six months of the third and fourth quarters of 1998.

Results for the beta-emitting radionuclides cesium-137, cobalt-60, nickel-63, radium-228, and technetium- 99 were all below their respective detection limits during each of the six months of the third and fourth quarters of 1998. Carbon-14 exceeded its detection limit in July. September, October, and December, but no result exceeded the $50 \mathrm{pCi} / \mathrm{L}$ compliance limit for the sum of the beta contributors. lodine-129 was detected in each month except November but remained well below the $50 \mathrm{pCi} / \mathrm{L}$ compliance limit for the sum of the beta contributors. Strontium-90 exceeded its detection limit in July, August, September, and October. During July and October, it exceeded its compliance limit of $8 \mathrm{pCi} / \mathrm{L}$ but remained below that limit in the other months. Due to the August result of $5.79 \mathrm{pCi} / \mathrm{L}$, there was no confirmed exceedance of strontium-90 during the third and fourth quarters of 1998. Modifications performed on the H-Area WTU appear to have resulted in lower strontium-90 results during the fourth quarter of 1998. The sum of the beta contributors exceeded its compliance limit in October, but there was no confirmed exceedance; all results ranged between $11.04 \mathrm{pCi} / \mathrm{L}$ and $61.49 \mathrm{pCi} / \mathrm{L}$. The most common contributors to that sum were iodine-129 and strontium-90. Modifications to the unit designed to control strontium-90 appear to have been successful relative to the limited operations through the end of 1998. 


\section{Appendix A}

\section{Regulatory Standards}


WSRC-TR-99-00013 Unclassified

THIS PAGE LEFT BLANK INTENTIONALLY. 


\section{Table A-1. Groundwater Protection Standard}

Established for the H-Area Hazardous Waste Management Facility by the 1995 RCRA Renewal Permit (SCDHEC, 1995).

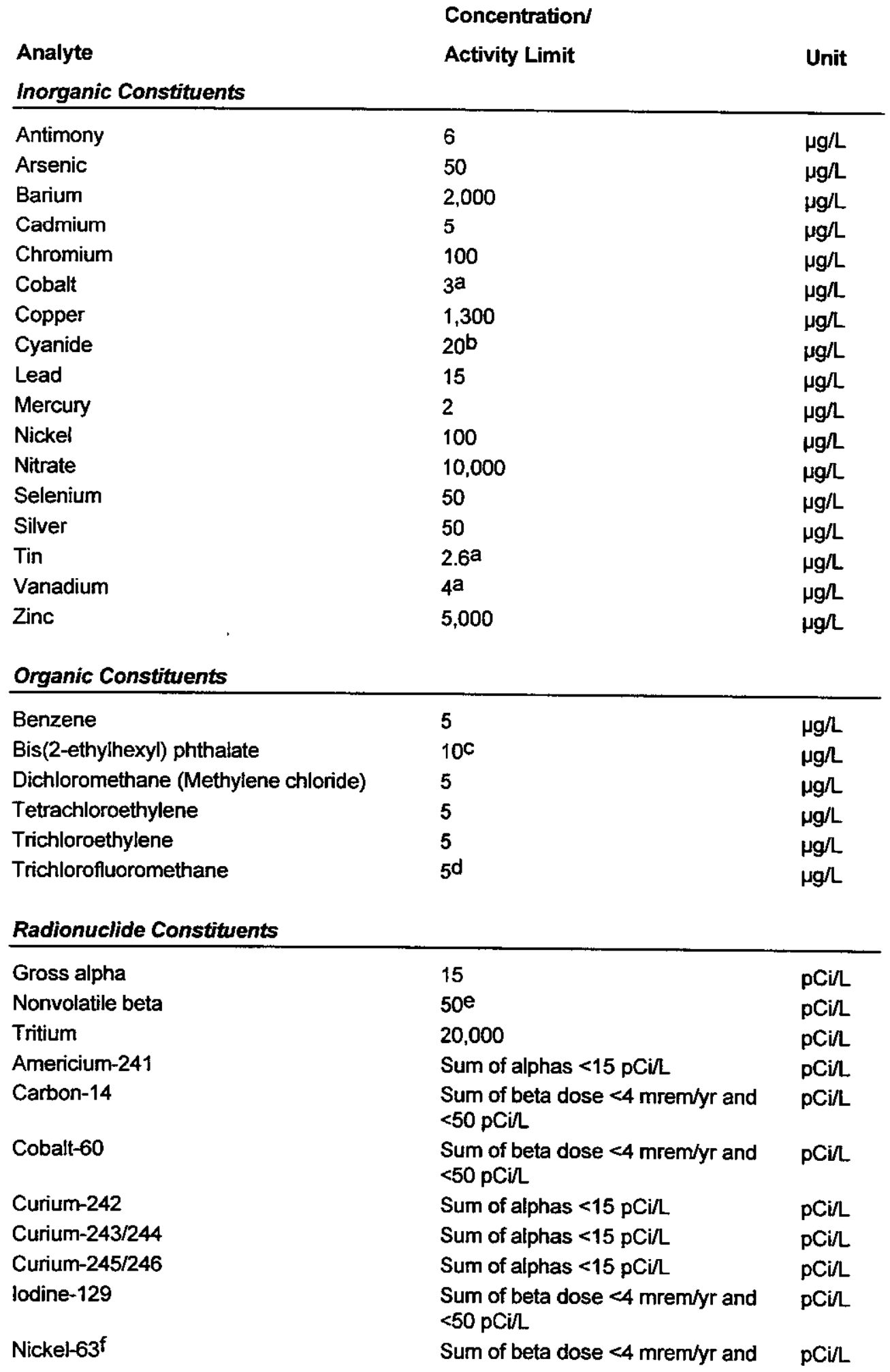




\begin{tabular}{|c|c|c|}
\hline \multirow{2}{*}{ Analyte } & \multicolumn{2}{|l|}{ Concentration/ } \\
\hline & Activity Limit & Unit \\
\hline & $<50 \mathrm{pCi} / \mathrm{L}$ & \\
\hline Plutonium-238 & Sum of alphas $<15 \mathrm{pCi} / \mathrm{L}$ & $\mathrm{pCi} / \mathrm{L}$ \\
\hline Plutonium-239/240 & Sum of alphas $<15 \mathrm{pCi} / \mathrm{L}$ & $\mathrm{pCi} / \mathrm{L}$ \\
\hline Radium-226 & Total radium $<5 \mathrm{pCi} / \mathrm{L}$ & $\mathrm{pCi} / \mathrm{L}$ \\
\hline Radium-228 & $\begin{array}{l}\text { Sum of beta dose }<4 \text { mrem } / y r \text { and } \\
<50 \mathrm{pCi} / \text { and total radium }<5 \\
\mathrm{pCin}\end{array}$ & $\mathrm{pCi} / \mathrm{L}$ \\
\hline Strontium-90 & $\begin{array}{l}\text { Sum of beta dose }<4 \mathrm{mrem} / \mathrm{yr} \text { and } \\
<50 \mathrm{pCi} / \mathrm{L} \text { and strontium }-90 \\
<8 \mathrm{pCi} / \mathrm{L}\end{array}$ & $\mathrm{pCi} / \mathrm{L}$ \\
\hline Technetium-99 & $\begin{array}{l}\text { Sum of beta dose }<4 \mathrm{mrem} / \mathrm{yr} \text { and } \\
<50 \mathrm{pCi} / \mathrm{L}\end{array}$ & $\mathrm{pCin}$ \\
\hline Thorium-228 & Sum of alphas $<15 \mathrm{pCi} / \mathrm{L}$ & $\mathrm{pCi} / \mathrm{L}$ \\
\hline Thorium-230 & Sum of alphas $<15 \mathrm{pCi} / \mathrm{L}$ & $\mathrm{pCi} / \mathrm{L}$ \\
\hline Thorium-232 & Sum of alphas $<15 \mathrm{pCi} / \mathrm{L}$ & $\mathrm{pCi} / \mathrm{L}$ \\
\hline Total alpha-emitting radiumg & 5 & $\mathrm{pCi} / \mathrm{L}$ \\
\hline Total radium (radium-226 and -228) & 5 & $\mathrm{pCin}$ \\
\hline Uranium-233/234 & Sum of alphas $<15 \mathrm{pCi} / \mathrm{L}$ & $\mathrm{pCi} / \mathrm{L}$ \\
\hline Uranium-234 & Sum of alphas $<15 \mathrm{pCi} / \mathrm{L}$ & $\mathrm{pCi} / \mathrm{L}$ \\
\hline Uranium-235 & Sum of alphas $<15 \mathrm{pCi} / \mathrm{L}$ & $\mathrm{pCi} / \mathrm{L}$ \\
\hline Uranium-238 & Sum of alphas $<15 \mathrm{pCi} / \mathrm{L}$ & $\mathrm{pCi} / \mathrm{L}$ \\
\hline
\end{tabular}

a Concentrations are observed background levels.

b Concentration is the practical quantitation limit (PQL) for EPA Method 335.2 (used by WA) and 335.3 (used by GE).

c Concentration is the PQL for EPA Method 8270 as published in 40CFR Part 264, Appendix IX.

d Concentration is the PQL for EPA Method 8240 as published in 40CFR Part 264, Appendix IX.

e This is the screening level above which providers of public drinking water should perform analyses for specific manmade radionuclides. The standard for the total dose equivalent from all such radionuclides is $4 \mathrm{mrem} / \mathrm{yr}$.

f The activity of nickel-63 is calculated based on the activity of cobalt-60 until development of a reliable analytical method as required by permit condition IIIC.H.6.f.

g Results reported by this analysis, which does not distinguish among radium-223, radium-224, and radium-226, are assumed to be primarily radium-226 and are compared to the GWPs for total radium. 
Table A-2. Regulatory Limits for UIC Permitted Constituents

Constituent

Section I, Inorganics

Arsenic

Barium

Cadmium

Chromium

Lead

Mercury

Selenium

Silver

Section II, Organics

\begin{tabular}{|c|c|c|}
\hline Antimony & 6 & $\mu g /$ \\
\hline Cobalt & 140 & $\mu g / L$ \\
\hline Copper & 1,300 & $\mu g /$ \\
\hline Cyanide & 200,000 & $\mu g / L$ \\
\hline Benzene & 5 & $\mu g / L$ \\
\hline Bis(2-ethylhexyl) phthalate & 140 & $\mu g /$ \\
\hline $\begin{array}{l}\text { Methylene chloride } \\
\text { (Dichloromethane) }\end{array}$ & 5 & $\mu g / L$ \\
\hline Nickel & 100 & $\mu g / L$ \\
\hline Tetrachloroethylene & 5 & $\mu g / L$ \\
\hline Tin & 50 & $\mu g / L$ \\
\hline Trichloroethylene & 5 & $\mu g / L$ \\
\hline Trichlorofiuoromethane & 100 & $\mu g / L$ \\
\hline Vanadium & 49 & $\mu g \Omega$ \\
\hline Zinc & 5,000 & $\mu g / L$ \\
\hline \multicolumn{3}{|l|}{ Section III, Radionuclides } \\
\hline Gross Alpha & 15 & $\mathrm{pCi} /$ \\
\hline Gross Beta & 50 & $\mathrm{pCi} / \Omega$ \\
\hline Total Radium $(226+228)$ & 5 & $\mathrm{pCi} / \mathrm{L}$ \\
\hline \multicolumn{3}{|l|}{ Section N, Radionuclides } \\
\hline Americium-241 & SOA & $\mathrm{pCi} / \mathrm{L}$ \\
\hline Cesium-137 & $\mathrm{SOB}$ & $\mathrm{pCi} /$ \\
\hline Curium-242 & SOA & $\mathrm{pCi} / L$ \\
\hline Curium-243/244 & SOA & $\mathrm{pCi} / 2$ \\
\hline Curium-246 & SOA & $\mathrm{pCi} / \mathrm{L}$ \\
\hline
\end{tabular}

Reg. Limit Unit

$50 \mu g / L$

$2,000 \mu g / L$

$5 \mu g /$.

$100 \mu \mathrm{g} / \mathrm{L}$

$50 \mu g / L$

$2 \mu g / L$

$50 \mu g / L$

$50 \mu g / L$

$\mu g / L$

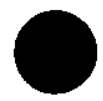




\begin{tabular}{|c|c|c|}
\hline Constituent & Reg. Limit & Unit \\
\hline Carbon-14 & SOB & $\mathrm{pCi} / \mathrm{L}$ \\
\hline Cobalt-60 & SOB & $\mathrm{pCi} / \mathrm{L}$ \\
\hline lodine-129 & SOB & $\mathrm{pCi} / \mathrm{L}$ \\
\hline Plutonium-238 & SOA & $\mathrm{pCi} / 2$ \\
\hline Plutonium-239/240 & SOA & $\mathrm{pCi} / \Omega$ \\
\hline Nickel-63 & SOB & $\mathrm{pCi} / \mathrm{L}$ \\
\hline Radium-226 & SOR & $\mathrm{pCi} / \mathrm{L}$ \\
\hline Radium-228 & SOR & $\mathrm{pCi} / \mathrm{L}$ \\
\hline Strontium-90 & SOB & $\mathrm{pCi} / \mathrm{L}$ \\
\hline Technetium-99 & SOB & $\mathrm{pCi} / \mathrm{L}$ \\
\hline Thorium-228 & SOA & $\mathrm{pCi} / \Omega$ \\
\hline Thorium-230 & SOA & $\mathrm{pCi} / \mathrm{L}$ \\
\hline Thorium-232 & SOA & $\mathrm{pCi} / \mathrm{L}$ \\
\hline Uranium-233/234 & SOA & $\mathrm{pCi} / \mathrm{L}$ \\
\hline Uranium-235 & SOA & $\mathrm{pCi} / \mathrm{L}$ \\
\hline Uranium-238 & SOA & $\mathrm{pCi} / \mathrm{L}$ \\
\hline \multicolumn{3}{|l|}{ Sum of Alphas } \\
\hline Sum of Betas & & \\
\hline
\end{tabular}




\section{Appendix B}

Figures 
THIS PAGE LEFT BLANK INTENTIONALLY. 


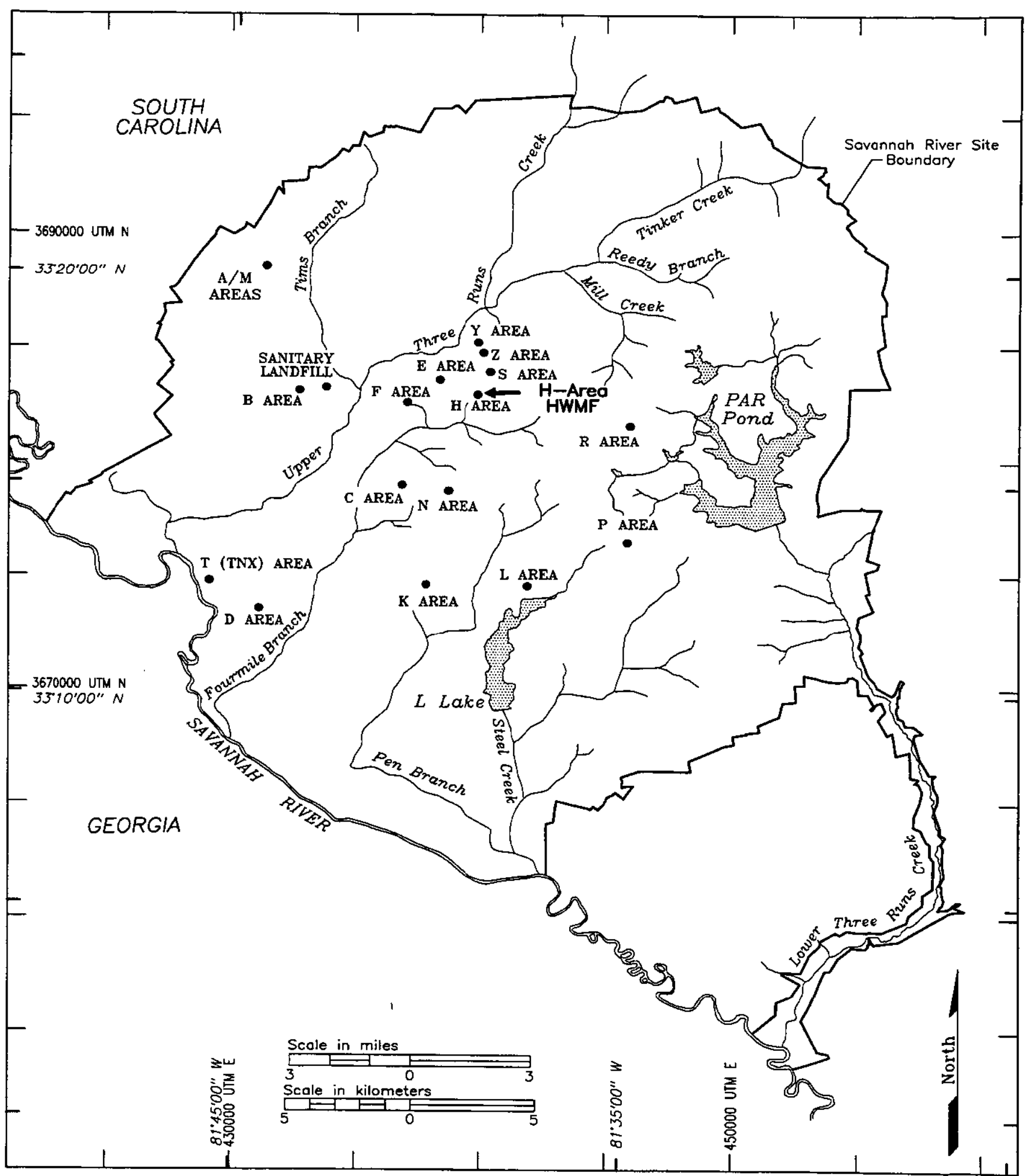

Figure 1. Location of the H-Area HWMF at the Savannah River Site 


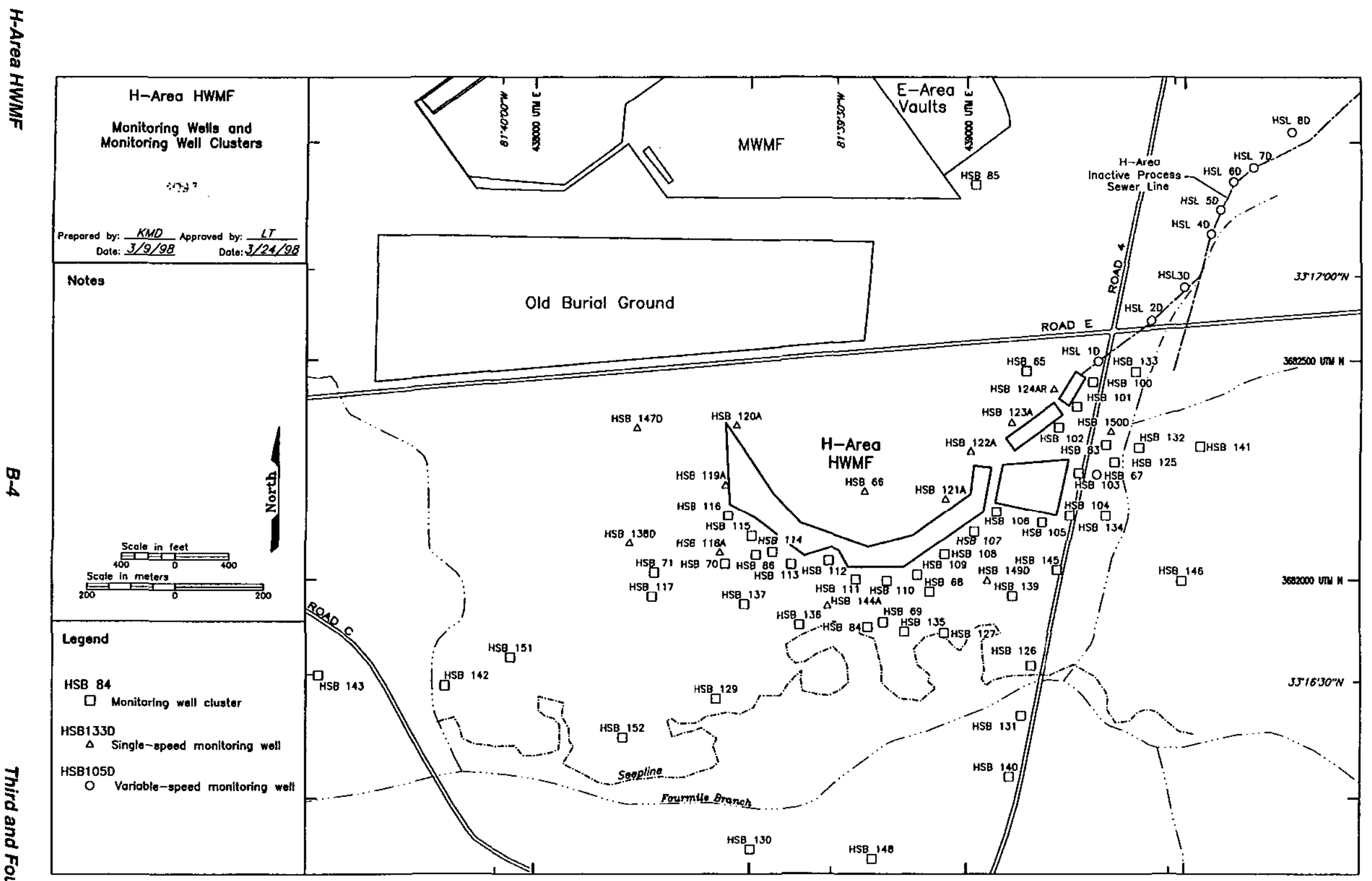

Figure 2. Location of the Groundwater Monitoring Wells at the H-Area HWMF 


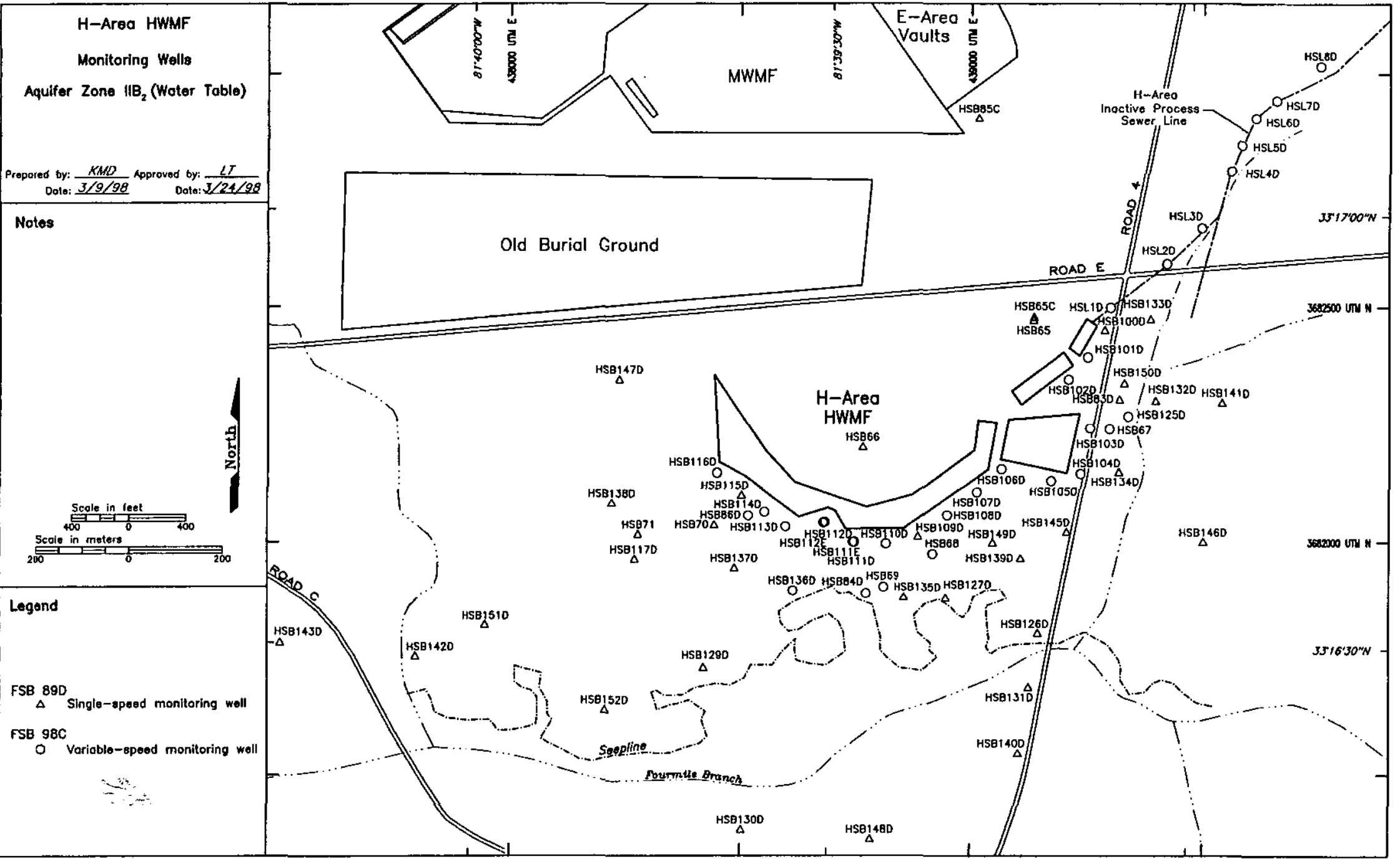

Figure 3. Location of Aquifer Zone IIB2 (Water Table) Groundwater Monitoring Wells at the H-Area HWMF 


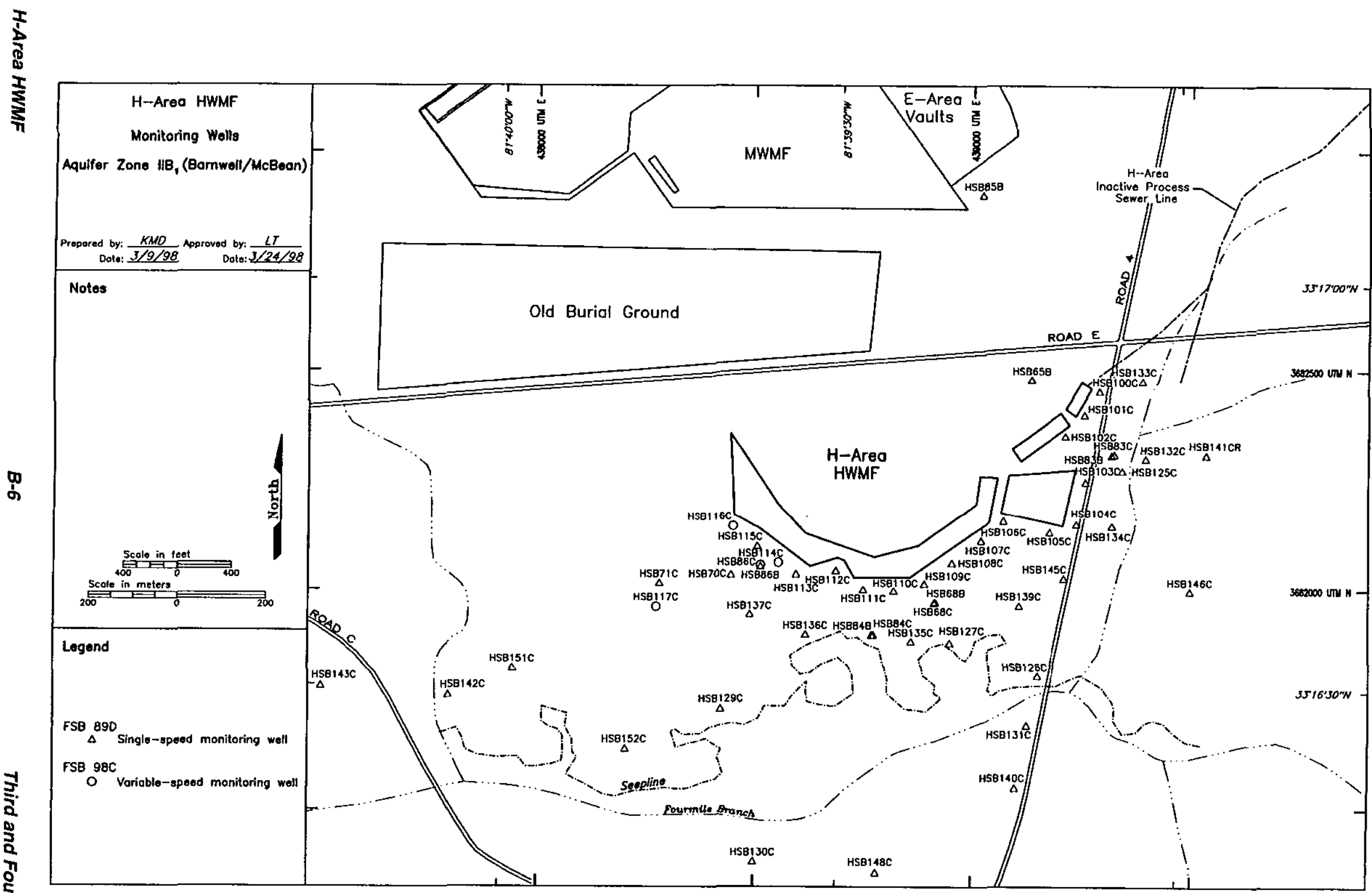

Figure 4. Location of Aquifer Zone IIB1 (Barnwell/McBean) Groundwater Monitoring Wells at the H-Area HWMF 


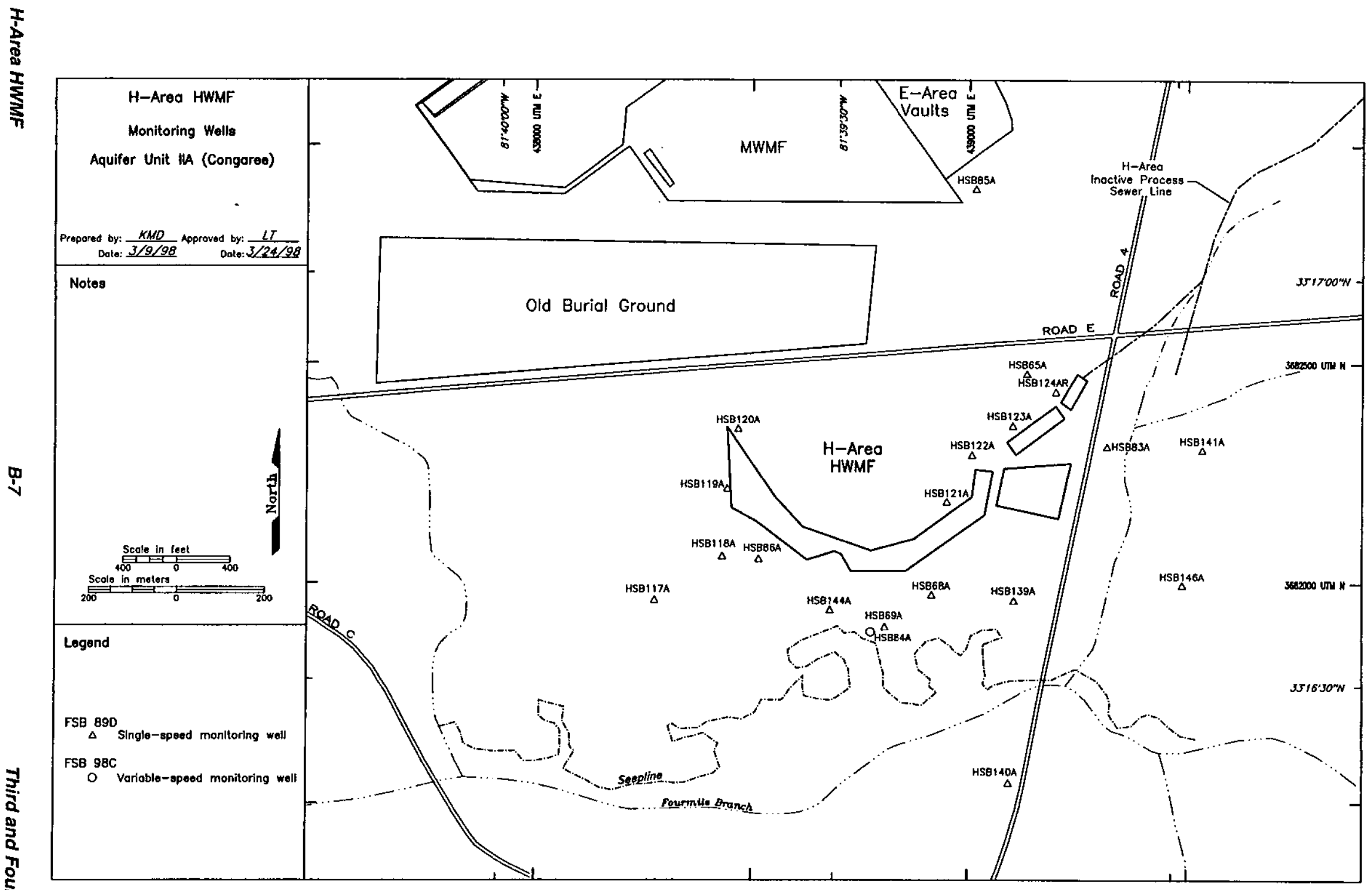

Figure 5. Location of Aquifer Unit IIA (Congaree) Groundwater Monitoring Welis at the H-Area HWMF 
WSRC-TR-99-00013

Unclassified

THIS PAGE LEFT BLANK INTENTIONALLY. 
Figure 6. Hydrogeologic Cross-Section Showing Potentiometric Surfaces at the H-Area HWmF, Fourth Quarter 1998

Figure 7. Hydrogeologic Cross-Section Showing Mercury Concentrations at the H-Area HWmF, Fourth Quarter 1898 


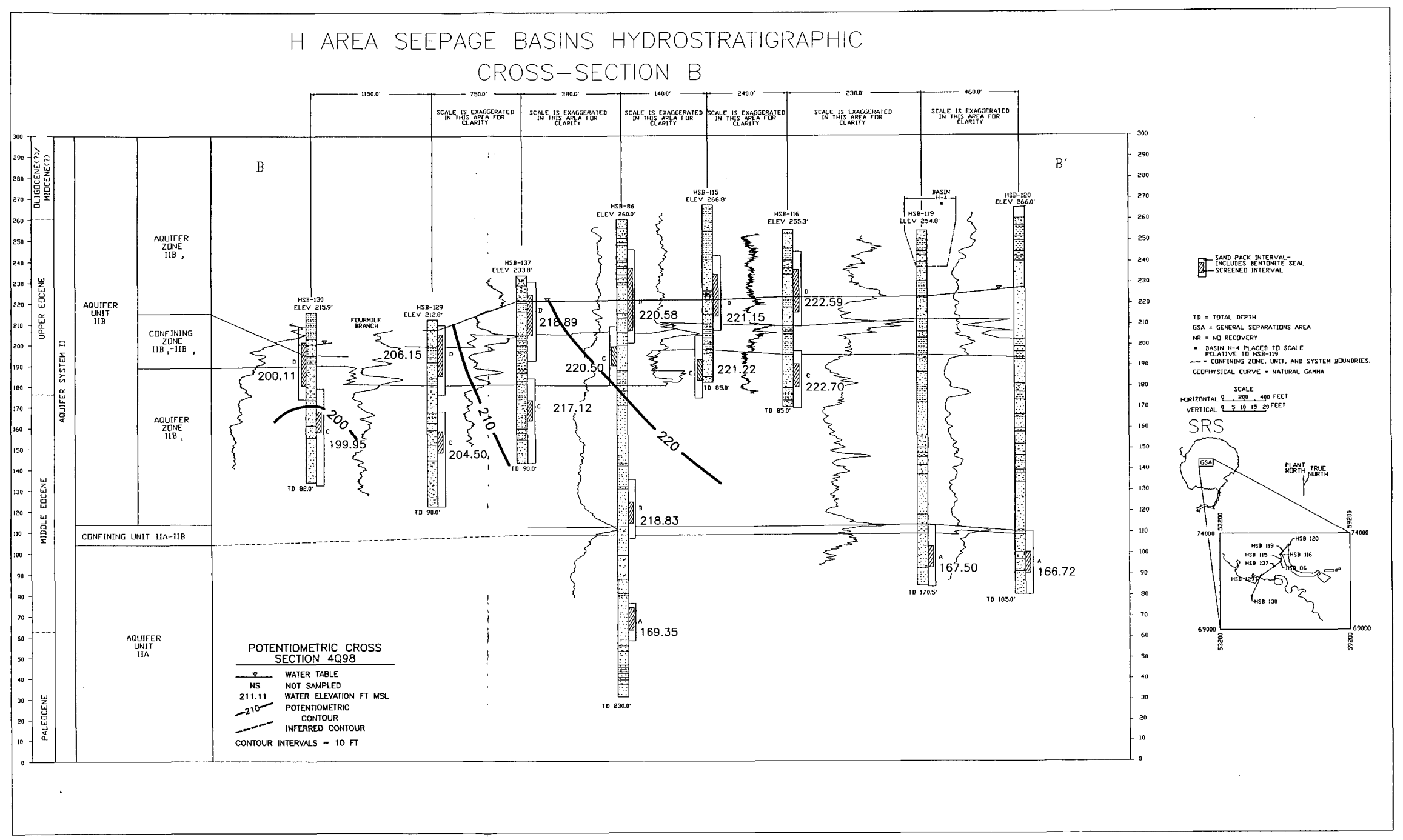




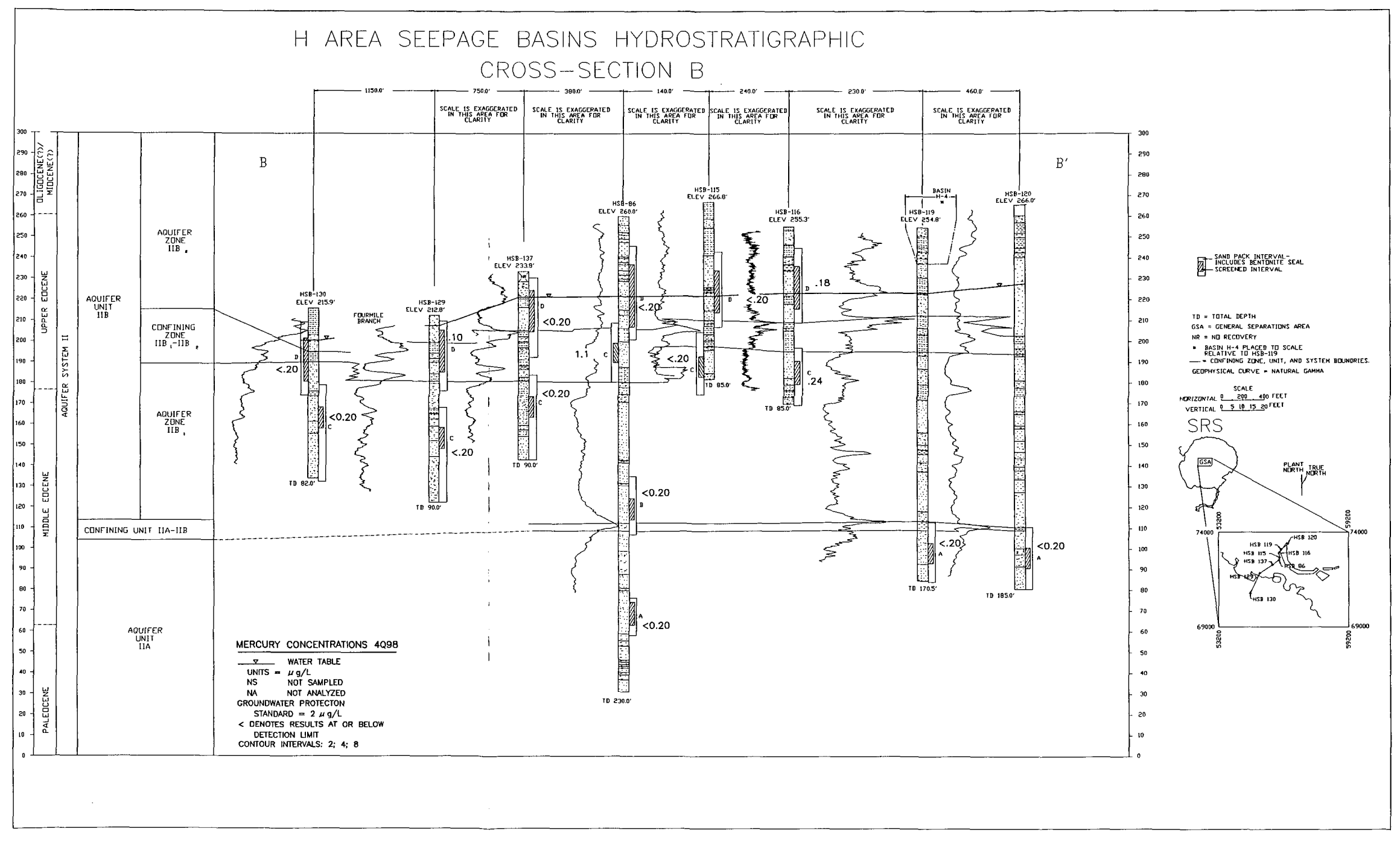


Figure 8. Hydrogeologic Cross-Section Showing Nitrate Concentrations at the H-Area HWMF, Fourth Quarter 1998

Figure 9. Hydrogeologic Cross-Section Showing Tritium Activities at the H-Area HWMF, Fourth Quarter 1998 


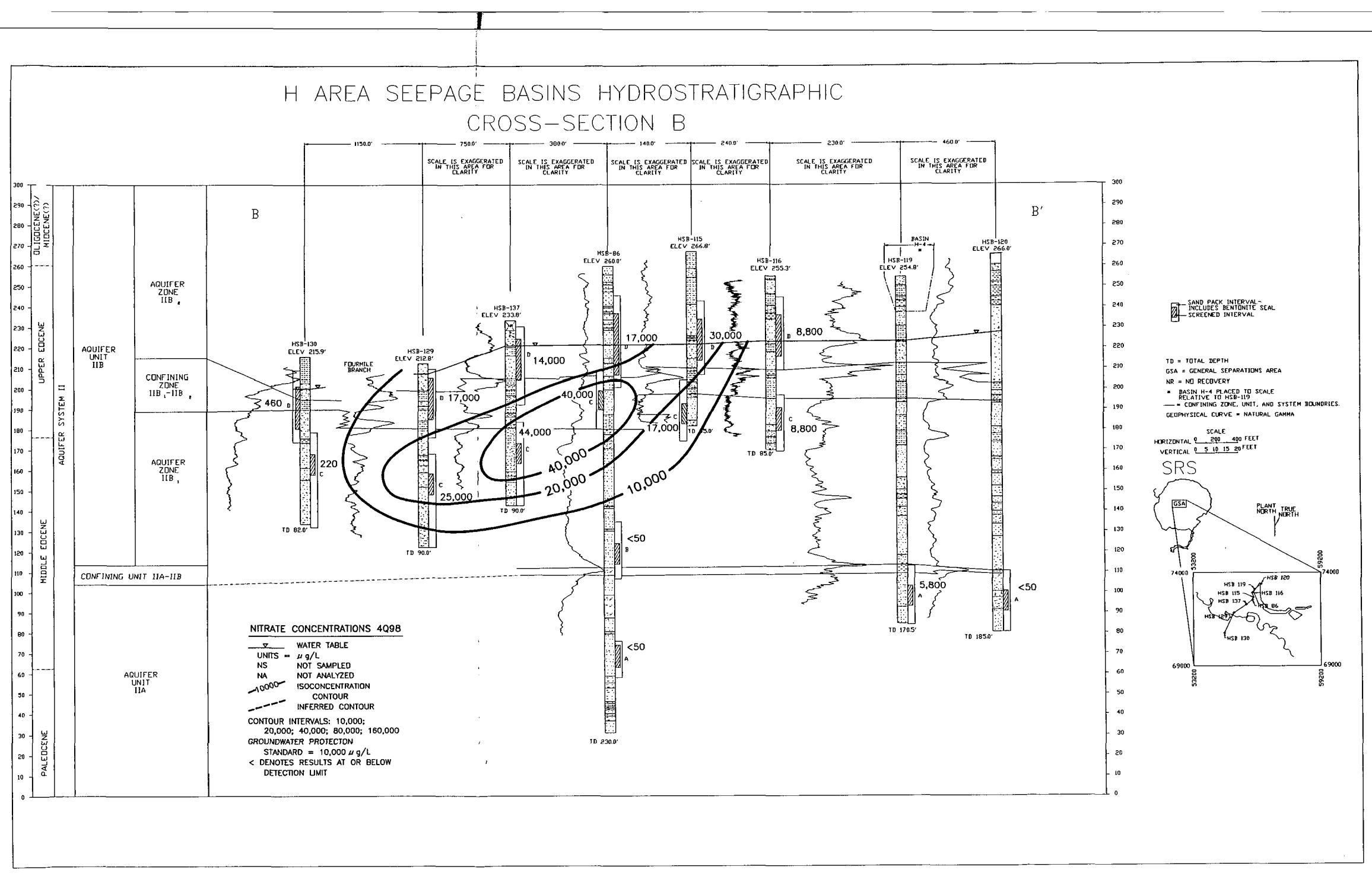




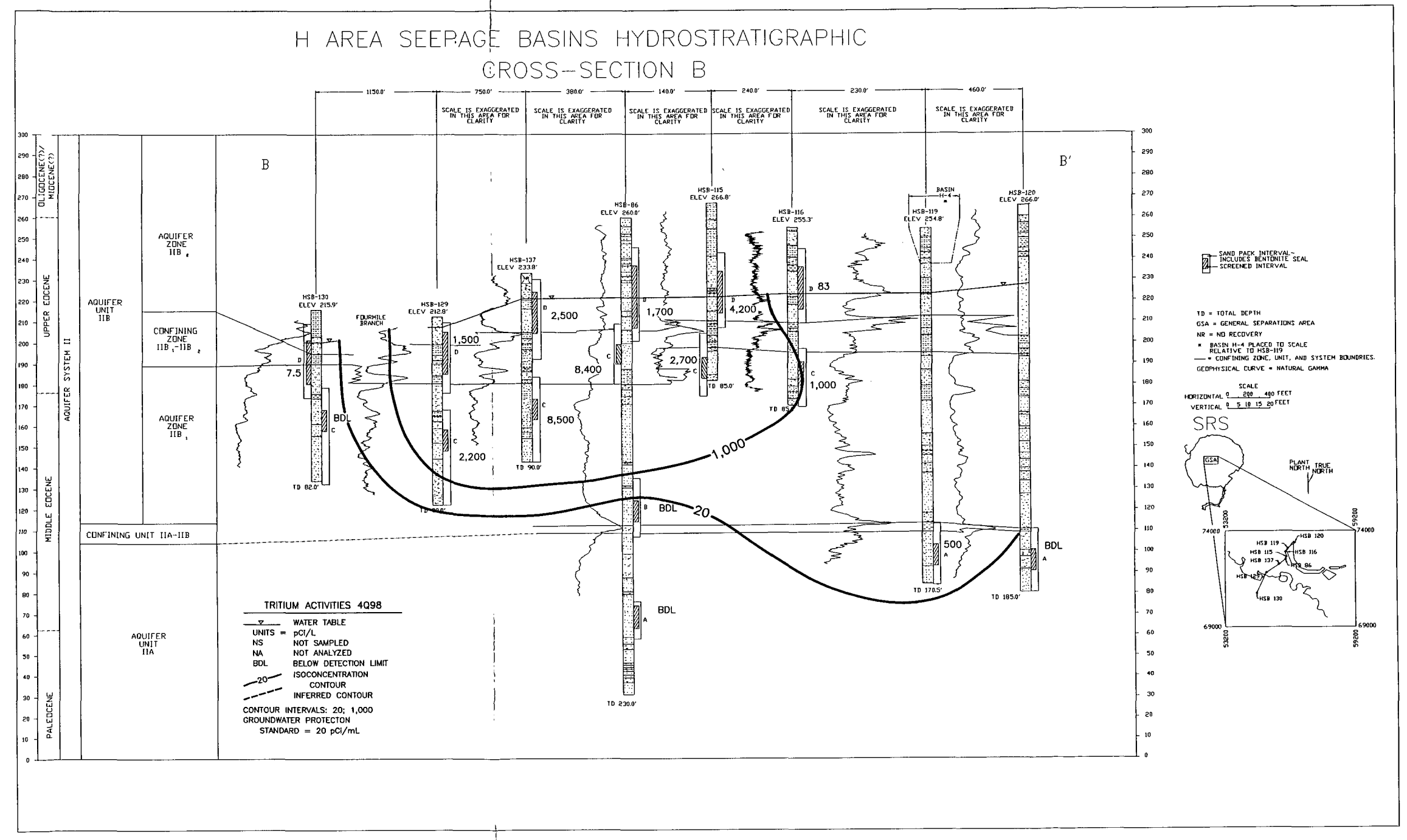


Figure 10. Hydrogeologic Croas-Section Showing lodine-129 Activities at the H-Area HWMF, Third Ouartor 1 ses

Figure 11. Hydrogeologic Cross-section showing Stromium- 80 Activities at the H-Area HWMF, Third Quarter 1998 


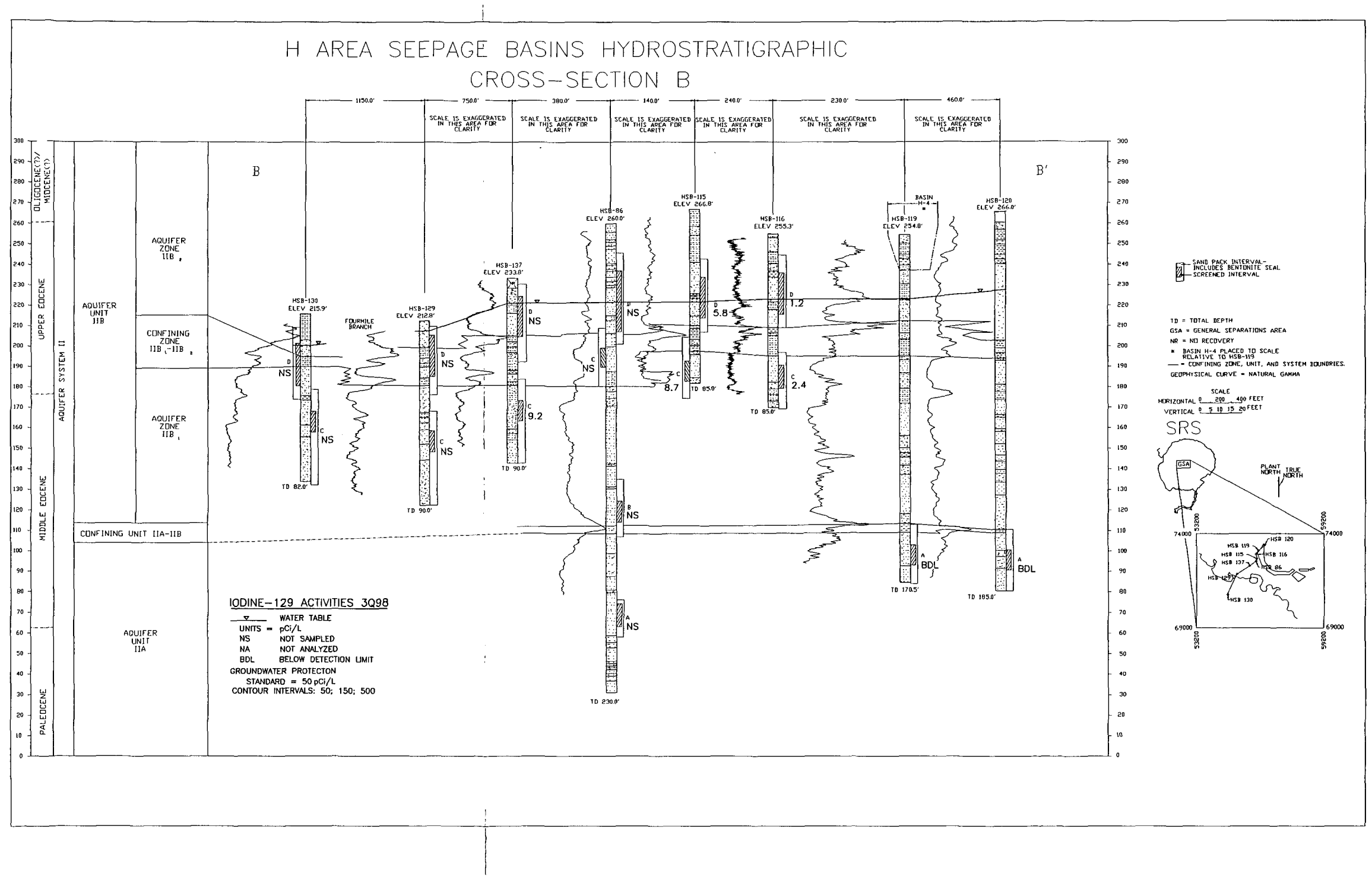




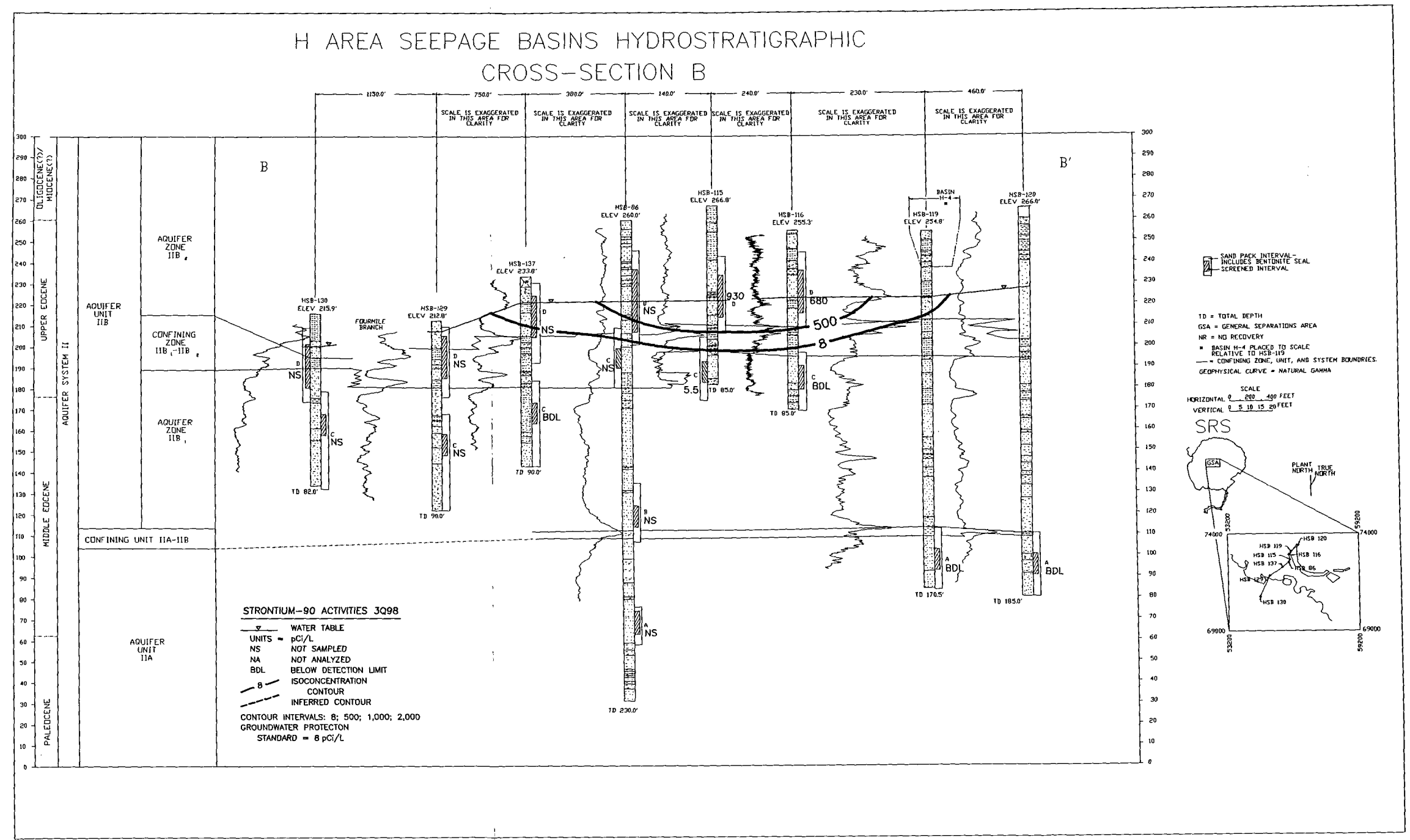


Figure 12. Hydrogeologic Croes-Section Showing Gross Alpha Activities at the H-Area HWMF, Fourth Quarter ises

Figure 13. Hydrogeofogic Crose-Section Showing Nonvolatlle Beta Activities at the H-Area HWMF, Fourth Quarter 1998 


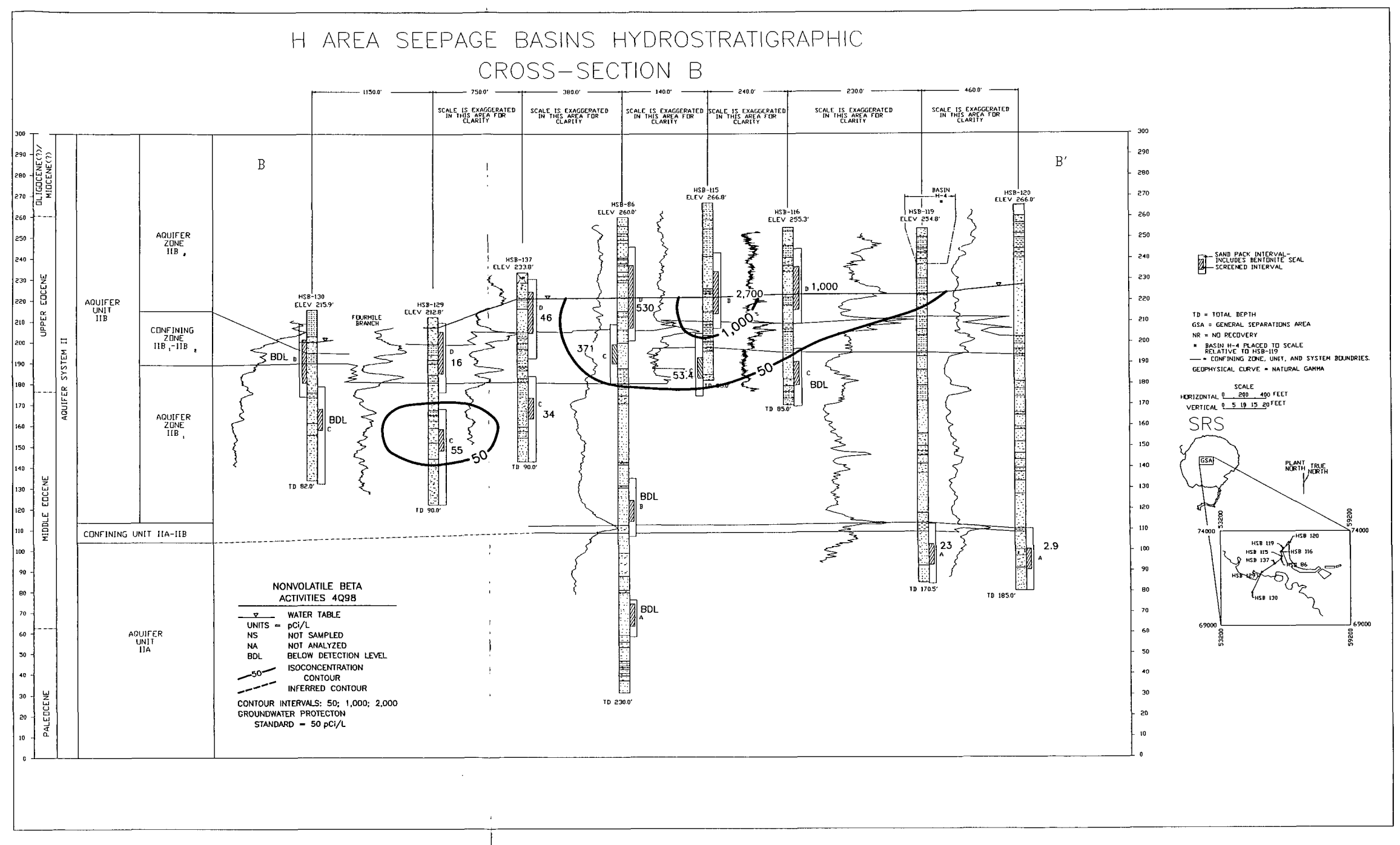




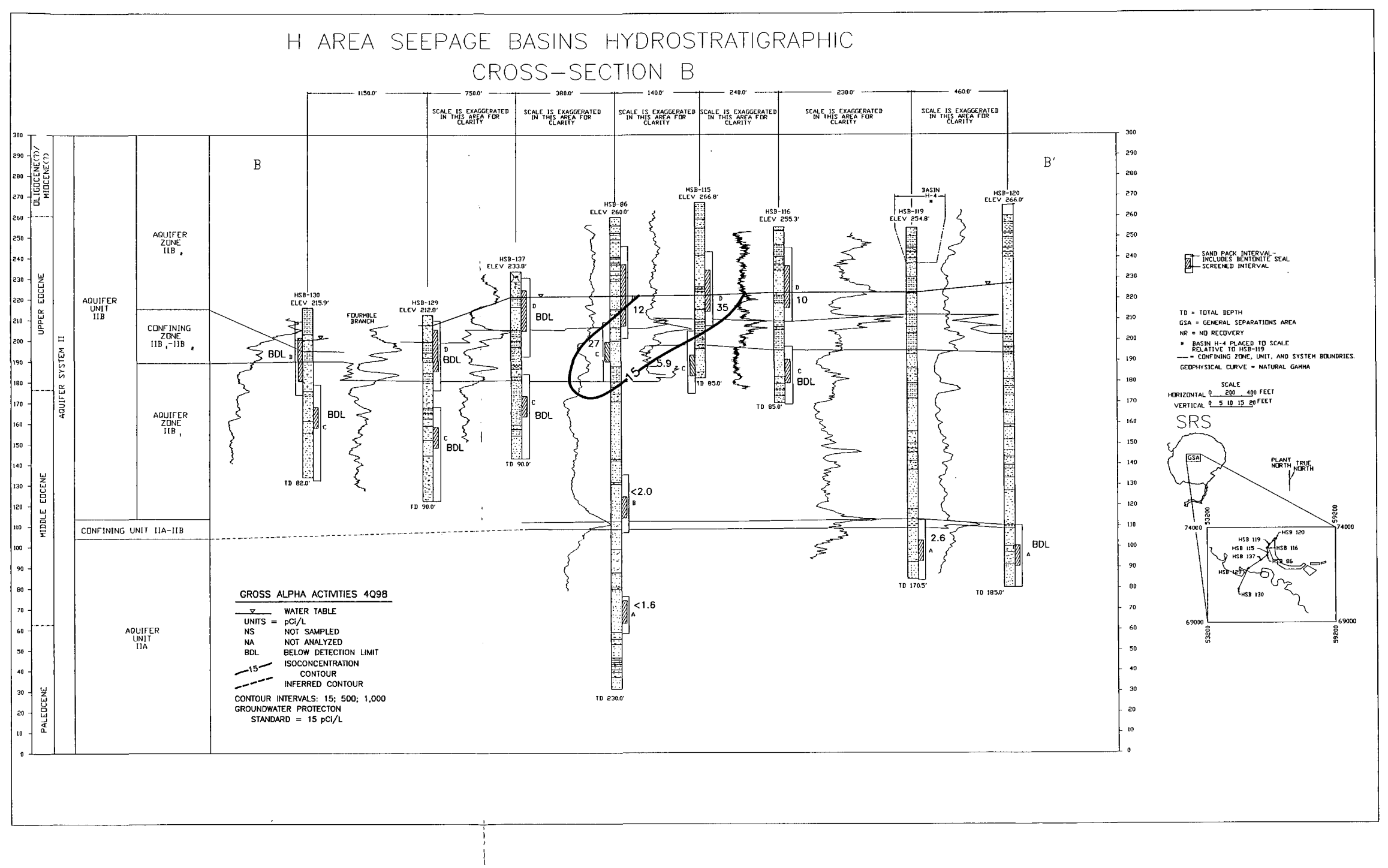


WSRC-TR-99-00013

Unclassified

\section{Appendix C}

Analytical Results 
WSRC-TR-99-00013

Unclassified

THIS PAGE LEFT BLANK INTENTIONALLY. 


\section{Key to Reading the Tables}

The following abbreviations may appear in the data tables:

\section{Constituents}

Sp. conductance

Specific conductance

\section{Laboratories}

$\mathrm{EM}$

GE and GP

TM

WA

Environmental Protection Department/Environmental Monitoring Section (EPD/EMS) Laboratory

General Engineering Laboratories, Inc.

Thermo NUtech

Recra LabNet Philadelphia (was Roy F. Weston, Inc., until June 1997)

\section{Sampling Codes}

$\begin{array}{ll}\text { A } & \text { Pump was surging excessively; aerated } \\ \text { B } & \text { Blank sample was collected } \\ \text { C } & \text { Well was pumping continuously } \\ \text { D } & \text { Well was dry } \\ \text { I } & \text { Well went dry during sampling; insufficient water to collect all samples } \\ \text { L } & \text { Well went dry before sampling began; only depth to water can be } \\ \text { N } & \text { determined } \\ \text { P } & \text { Well was not stabilized before sampling began } \\ \text { S } & \text { Inaccessibility or mechanical failure prevented sample collection and field } \\ \text { T } & \text { analysis of the water } \\ \text { W } & \text { No water in standpipe; for water-level events only } \\ \text { X } & \text { Samples were collected, but some samples were not sent to the } \\ \text { laboratory due to high turbidity }\end{array}$




\section{Data Table Column Headings}

$\begin{array}{ll}\text { DF } & \text { dilution factor } \\ \text { H } & \text { exceeded the holding time } \\ \text { Mod } & \text { modifier } \\ \text { ST } & \text { exceeded the GWPS }\end{array}$

Other

CS

E

PVC

carbon steel

TOC

exponential notation (e.g., 1.1E-09 $=1.1 \times 10^{-9}=0.0000000011$ )

polyvinyl chloride

top of casing

\section{Data Qualification}

The contract laboratories submit sample- or batch-specific quality assurance/quality control information either at the same time as analytical results or in a quarterly summary. Properly defined and used data modifiers (also referred to as qualifiers) can be a key component in assessing data usability. These modifiers appear in the data tables under the column Mod. Data for third quarter 1998 were reported by the laboratories in data format AN95, while data for fourth quarter 1998 were reported in AN98. One major difference between the two formats is the modifiers used. In the data tables, three possible fields for modifiers for each quarter are separated by slashes. For AN95 data, result qualifiers (RQ) may appear before the first slash, analysis qualifiers $(A Q)$ between the two slashes, and bias modifiers $(B)$ after the second. For AN98 data, functional guidance qualifiers (FG) may appear before the first slash, STORET codes (SC) between the two slashes, and EMS codes (EC) after the second slash. For further information on modifiers and their definitions, contact EMS.
AN95 Modifiers
Data are not qualified. Numbers should be interpreted exactly as reported.
$J$
Value is estimated because quantitation in the sample or in associated quality control samples did not meet specifications. (RQ)
L
Value is off-scale high. The actual value is not known but is known to be greater than the value shown. (RQ)
$\mathrm{R}$
Result was rejected because performance requirements in the sample analysis or associated quality control analyses were not met. (RQ)
$U \quad$ Material was analyzed for but not detected. Analytical result reported is less than the sample quantitation limit. (RQ)
E
The detected result is between the sample-specific EQL and the method detection limit. (AQ)
1
The value in the result field is the instrument reading, not the sample quantitation limit. Always used with the result qualifier $U$. (AQ)
0
Surrogate or tracer spike recovery is out of specification. (AQ)
$\checkmark$
Analyte was detected in an associated method blank. (AQ)
Y
Result was obtained from an unpreserved or improperly preserved sample. Data may not be accurate. (AQ)
4 Matrix interference. Value cannot be determined. Used with $R Q R$. (AQ) 


\section{AN98 Modifiers}

(Blank)

J

R

U

C

I

K

L

Q

$\checkmark$

$Y$

A

C

D

H

I

K

L

O

$\mathrm{P}$

$S$

W

$x$

4

5

6

8

9

Data are not qualified. Numbers should be interpreted exactly as reported.

The analyte was positively identified; the associated numerical value is an estimated concentration of the analyte in the sample. (FG)

The sample results are rejected due to serious deficiencies in the ability to analyze the sample and meet quality control criteria. The presence or absence of the analyte cannot be verified. (FG)

Material analyzed for, but not detected. The analyte concentration is <ssEQL. (SC)

The result is calculated. (SC)

The result is less than the SsEQL, but equal to or greater than the MDL. (SC)

The actual concentration is known to be less than the reported result. (SC)

The actual concentration is known to be greater than the reported result. (SC)

The sample was held beyond the normal holding time prior to analysis. (SC)

The analyte was detected in both the method blank and the sample. (SC)

The result is from an unpreserved or incorrectly preserved sample; the data may not be accurate. (SC)

Compound identification criteria were not met. (EC)

LCS or BS criteria were not met. (EC)

ICP serial dilution criteria were not met. (EC)

Internal standard criteria were not met when the IS was used for quantitation.

(EC)

Matrix spike recovery was not within the control limits. (EC)

A tentatively identified compound is a suspected aldol-condensation product.

(EC)

Initial or continuing calibration criteria were not met. (EC)

Surrogate or tracer spike recovery is out of specification. (EC)

Graphite furnace atomic absorption QC

a. Duplicate injection criteria were not met.

b. Post-digestion spike recovery was not within control limits and the sample absorbance is $>50 \%$ of the post-digestion spike absorbance. (EC)

The sample was analyzed by the method of standard additions. (EC)

Graphite furnace atomic absorption QC: the post-digestion spike recovery is not . within control limits and the sample absorbance is $<50 \%$ of the post-digestion spike absorbance. (EC)

The laboratory duplicate RPD or MS/MSD RPD was not within control limits. (EC)

Matrix interference is present. (EC)

Matrix spike concentration was $<0.25 \times$ the sample concentration, and the percent recovery cannot be determined. (EC)

The analyte was detected in both the sample and associated field blank. (EC)

The analyte was detected in both the sample and associated trip blank. (EC)

The field duplicate RPD was not within control limits. (EC) 


\section{Results below Detection}

For radiological analyses, the analytical result field contains the result recorded on the analytical instrument and reported by the laboratory, even if it is negative. For nonradiological analyses, if the analyte is not detected, the sample-specific estimated quantitation limit (EQL) is entered into the result field and is reported with a less than $[<]$ sign. The EQL is defined as the lowest concentration that can be achieved reliably within specified limits of precision and accuracy during routine laboratory operating conditions. The sample-specific EQL is modified for sample concentration or dilution or unusual aliquot size that affects analytical sensitivity.

\section{Data Filtering}

Data in the column headed Filt. have been filtered to clarify their usability. In this column, Rej. is used to indicate rejected data.

For nondetects, this column contains the less-than symbol and the sample-specific method detection limit or sample-specific minimum detectable activity. For chemical analyses, this is the same numeric value as appears in the unfiltered result column with a few exceptions, generally data that were qualified as nondetects during validation because of contamination in an associated blank.

For data qualified with the result qualifier (third quarter 1998) or field guidance code (fourth quarter 1998) $\mathrm{J}$, indicating an estimated quantity, the Filt. column contains the letters $N D D$, meaning that these data are considered not "decision" data.

\section{Holding Times}

Standard analytical methods include a limit, called holding time, on the maximum elapsed time between sample collection and extraction or analysis by the laboratory. In the data tables, a large bullet $(\cdot)$ in the $H$ (holding time) column indicates that holding time was exceeded. Analyses performed beyond holding times may not yield valid results.

The laboratory procedure used for the determination of specific conductance allows one day to elapse between sampling and analysis. Thus, laboratory specific conductance measurements may exceed the holding time criterion.

\section{Data Rounding}

A constituent result in the analytical results tables that is not marked in the $S T$ column but appears to equal an Appendix $A$ standard is below the standard in the database. Values stored in the database contain more significant digits than the reported results. Apparent discrepancies in the tables are due to the rounding of reported results.

\section{Sampling Dates}

Samples for field data are collected once each quarter, but samples for analytical data may be collected more than once each quarter. Because the results tables present the highest analytical results for each quarter, the date of collection for reported analytical results may not coincide with the date of collection for field data. 


\section{Table C-1. Groundwater Monitoring Results for Individual Wells}

\section{WELL HSB 65}

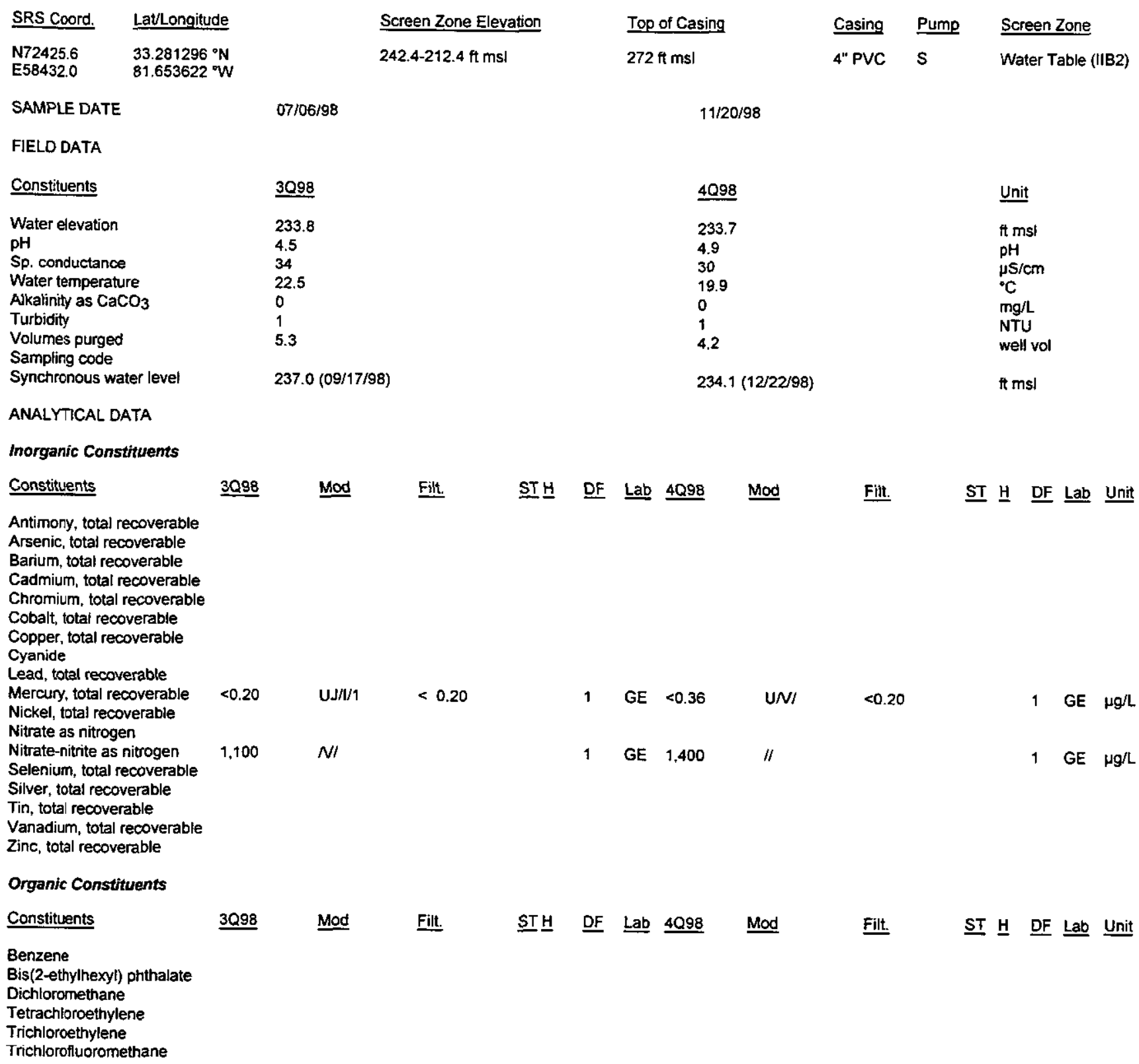

Notes:

$-=$ exceeded holding time

- = exceeded groundwater protection or monitoring constituent standard (See Appendix A.) 
WELL HSB 65 (cont.)

Radioactive Constituents

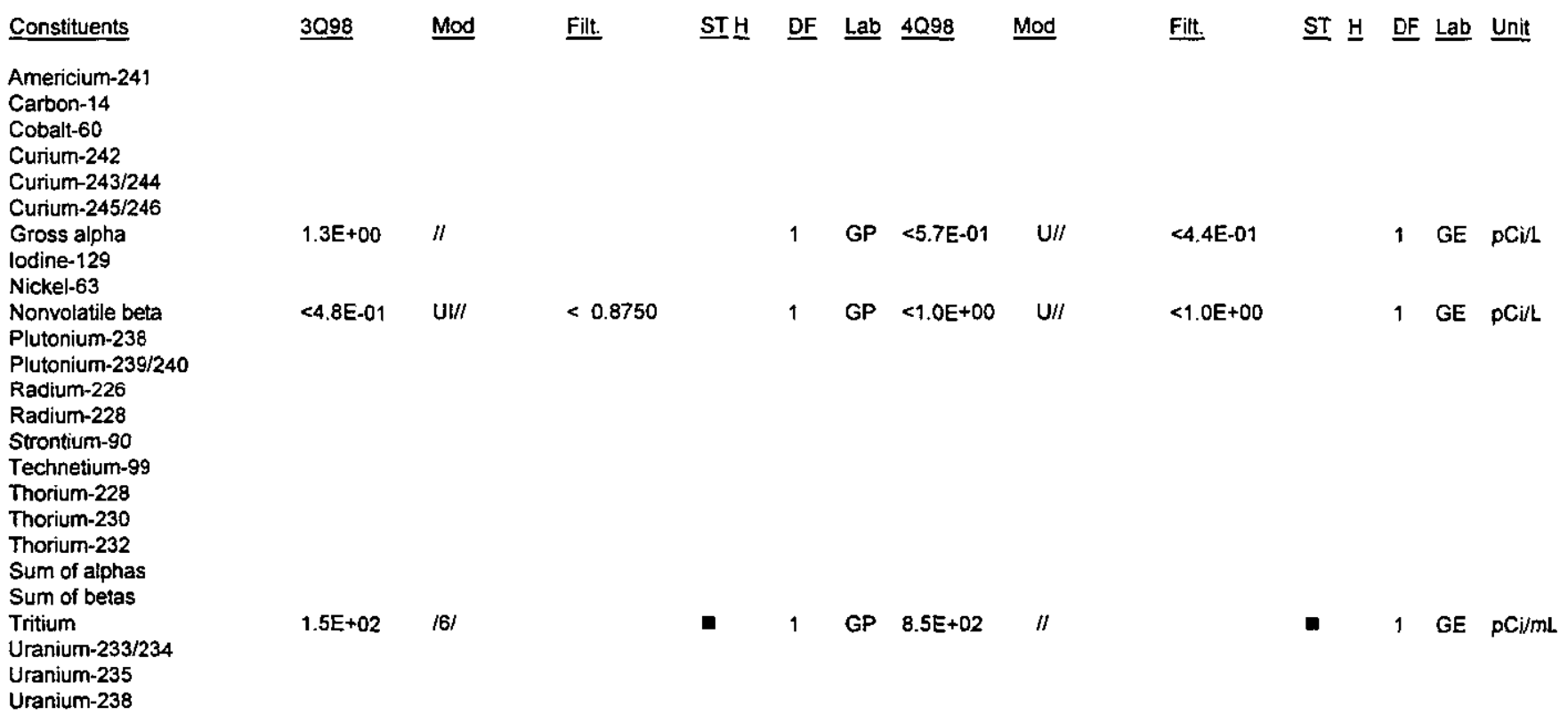

Notes:

- exceeded holding time

- exceeded groundwater protection or monitoring constituent standard (See Appendix A.) 


\section{WELL HSB 65A}

\begin{tabular}{|c|c|c|c|}
\hline SRS Coord. & \multicolumn{2}{|l|}{ LatLongitude } & Screen Zone Elevation \\
\hline $\begin{array}{l}N 72436.2 \\
E 58436.0\end{array}$ & \multicolumn{2}{|l|}{$\begin{array}{l}33.281326^{\circ} \mathrm{N} \\
81.653633^{\circ} \mathrm{W}\end{array}$} & $73.2-62.5 \mathrm{ft} \mathrm{msl}$ \\
\hline \multicolumn{2}{|c|}{ SAMPLE DATE } & $08 / 27 / 98$ & \\
\hline \multicolumn{4}{|c|}{ FIELD DATA } \\
\hline \multicolumn{2}{|l|}{ Constituents } & $\underline{3098}$ & \\
\hline \multicolumn{2}{|c|}{ Water elevation } & 169.4 & \\
\hline \multirow{2}{*}{\multicolumn{2}{|c|}{$\begin{array}{l}\mathrm{pH} \\
\text { Sp. conductance }\end{array}$}} & 7.2 & \\
\hline & & 200 & \\
\hline \multicolumn{2}{|c|}{ Water temperature } & 20.2 & \\
\hline \multirow{2}{*}{\multicolumn{2}{|c|}{ Aikalinity as $\mathrm{CaCO}_{3}$}} & 81 & \\
\hline & & & \\
\hline \multicolumn{2}{|c|}{ Volumes purged } & 2.2 & \\
\hline \multicolumn{2}{|c|}{ Synchronous water level } & $172.1<09$ & \\
\hline
\end{tabular}

$\begin{array}{llll}\text { Top of Casing } & \text { Casing } & \text { Pump } & \text { Screen Zone } \\ 273.6 \mathrm{ft} \mathrm{mst} & 4 \text { " PVC } & \text { S } & \text { L. Congaree (IIA) }\end{array}$

$10 / 05 / 98$

ANALYTICAL DATA

Inorganic Constituents

Constituents

Antimony, total recoverable

Arsenic, total recoverable

Barium, total recoverable

Cadmium, total recoverable

Chromium, total recoverable

Cobalt, total recoverable

Copper, total recoverabie

Cyanide

Lead, total recoverable

Mercury total recoverable

Nickel, total recoverable

Nitrate as nitrogen

Nitrate-nitrite as nitrogen

Selenium, total recoverable

Silver, total recoverable

Tin, total recoverable

Vanadium, total recoverable

Zinc, total recoverable

Organic Constituents

Constituents

Benzene

Bis(2-ethylhexyl) phthalate

Dichloromethane

Tetrachloroethylene

Trichloroethylene

Trichlorofluoromethane

$<50$
Mod

Filt.

ST보

DF 늠

4098

4098

172.0

7.6

160

20.2

90

2.2

$171.9(12 / 22 / 98)$

J/E

NDD

1 GE $<0.20$

$\mathrm{U} / 1$

1 GE $<30$

UN

$\mathrm{U} / \textrm{ } \quad<50$

UN

$1 \mathrm{GE} \mu g / \mathrm{L}$

1 GE $\mu g / L$

Mod $\quad$ Filt.

$\underline{\text { STH}}$

DF Lab $\underline{4 Q 98}$

Mod

Filt.

$\underline{\text { ST }} \underline{H}$ DF Lab Unit

Notes:

- exceeded holding time

- exceeded groundwater protection or monitoring constituent standard (See Appendix A.) 
WELL HSB 65A (cont.)

Radioactive Constituents

Constituents

Americium-241

Carbon-14

Cobalt-60

Curium-242

Curium-243/244

Curium-245/246

Gross alpha

lodine-129

Nickel-63

Nonvolatile beta

Plutonium-238

Plutonium-239/240

Radium-226

Radium-228

Strontium-90

Technetium-99

Thorium-228

Thorium-230

Thorium-232

Sum of alphas

Sum of betas

Tritium

Uranium-233/234

Uranium-235

Uranium-238
3098

Mod

Filt.

STH DF Lab 4Q98 Mod

$1.1 E+00 \quad / /$

$1.6 E+00 \quad / 1$

$1 \quad$ GP $<1.2 \mathrm{E}+00$

U/I

$1 \quad G P<1.3 E+00$

$\mathrm{U} / 1$

$<1.1 E+\infty 0$

$1 \mathrm{GE} \mathrm{pC} / / \mathrm{L}$

1 GE PCin

$<1.9 E-01$

UIII

$<5.8 \mathrm{E}-01$

1 GP $<-2.4 \mathrm{E}-02 \quad \mathrm{U} / /$

$<5.3 E-01$

$1 \mathrm{GE} \quad \mathrm{pCi} / \mathrm{mL}$

Notes:

- = exceeded holding time

- exceeded groundwater protection or monitoring constituent standard (See Appendix A.) 
WELL HSB 65B

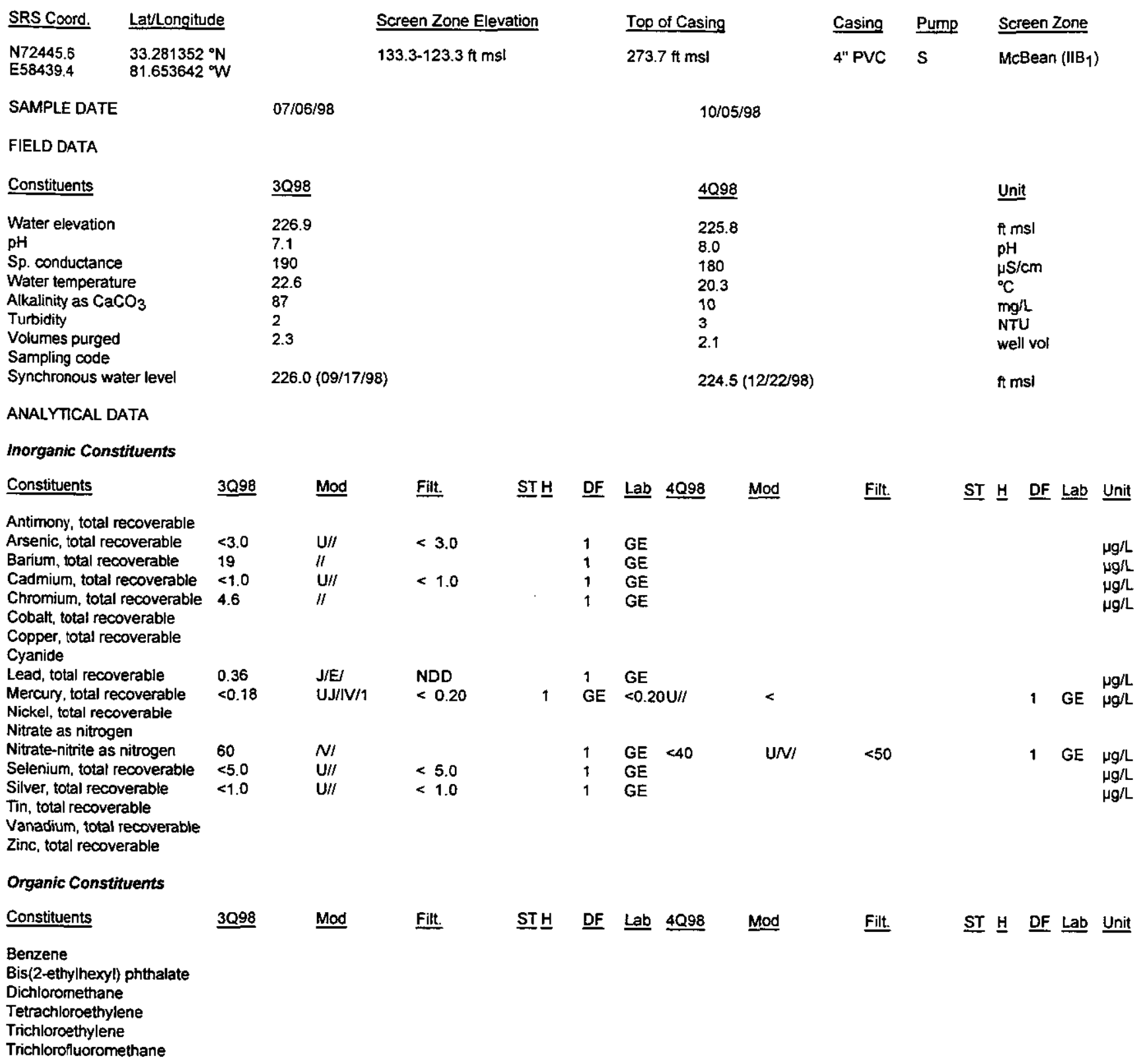

Notes:

$\bullet=$ exceeded holding time

- exceeded groundwater protection or monitoring constituent standard (See Appendix A.) 
WELL HSB 65B (cont.)

Radioactive Constituents

Constituents

Americitsm-241

Carbon-14

Cobalt-60

Curium-242

Curium-243/244

Curium-245/246

Gross alpha

lodine-129

Nickel-63

Nonvolatile beta

Plutonium-238

Plutonium-239/240

Radium-226

Radium-228

Strontium-90

Technetium-99

Thorium-228

Thorium-230

Thorium-232

Sum of alphas

Sum of betas

Tritium

Uranium-233/234

Uranium-235

Uranium-238
3098

Mod

Filt.

$\underline{\text { ST보 }}$

DF Lab 4 QQ98

Mod

Filt.

ST 브 DF Lab Unit

$<4.0 \mathrm{E}-02 \quad \mathrm{UI} / / \quad<0.93$

1 TM $<2.3 \mathrm{E}-01$

$\mathrm{U} / /$

$<5.5 E-01$

$1 \quad$ GP $<2.6 E-01$

$\mathrm{U} / /$

$<1.1 E+00$

1 GE $\mathrm{PCI} / \mathrm{L}$

$<1.3 E-01 \quad$ UII

$<1.4$

$1.8 \mathrm{E}+00$

$1 \mathrm{TM}<-2.8 \mathrm{E}-01$

U/I

$<5.2 \mathrm{E}-01$

$1 \mathrm{GE} \mathrm{pCi} / \mathrm{mL}$

Notes:

- = exceeded holding time

- = exceeded groundwater protection or monitoring constituent standard (See Appendix A.) 


\section{WELL HSB 65C}

$\begin{array}{lll}\text { SRS Coord. } & & \text { Lat/Longitude } \\ \text { N72439.6 } & & 33.281351{ }^{\circ} \mathrm{N} \\ \text { E58447.1 } & & 81.653610^{\circ} \mathrm{W}\end{array}$

SAMPLE DATE

FIELD DATA

\section{Constituents}

Water elevation

pH

Sp. conductance

Water temperature

Alkalinity as $\mathrm{CaCO}_{3}$

Turbidity

Volumes purged

Sampling code

Synchronous water level

\section{ANALYTICAL DATA}

Inorganic Constituents

Constituents

Antimony, total recoverable

Arsenic, total recoverable

Barium, total recoverable

Cadmium, total recoverable

Chromium, total recoverable

Cobalt, total recoverable

Copper, total recoverable

Cyanioe

Lead, total recoverable

Zinc, total recoverable

\section{Screen Zone Elevation}

218.6-207.8 ft ms

Top of Casing

$273.6 \mathrm{ft} \mathrm{ms}$

Casing

Pump

4" PVC S

Screen Zone

Water Table (IIB2)
$07 / 01 / 98$

$10 / 05 / 98$

4Q98

237.2

4.9

21.4

2.3

$234.7(12 / 22 / 98)$

$237.6(09 / 17 / 98)$
Mercury, total recoverable

Nickel, total recoverable

Nitrate as nitrogen

Nitrate-nitrite as nitrogen

Selenium, total recoverable

Silver, total recoverable

Tin, total recoverable

Vanadium, total recoverable

Organic Constituents

Constituents

$<0.20$

1 GE $<0.20$

$\mathrm{U} / /$

Benzene

Bis(2-ethylhexyl) phthalate

Dichloromethane

Tetrachloroethylene

Trichloroethylene

Trichlorofluoromethane

5,100
- 5 GE $\mu g / l$

it ms

$\mathrm{pH}$

uS/cm

${ }^{\circ} \mathrm{C}$

mgl

well vol

ft msl 
WELL HSB 65C (cont.)

Radioactive Constituents

Constituents

Americium-241

Carbon-14

Cobalt-60

Curium-242

Curium-243/244

Curium-245/246

Gross alpha

lodine-129

Nickel-63

Nonvolatile beta

Plutonium-238

Plutonium-239/240

Radium-226

Radium-228

Strontium-90

Technetium-99

Thorium-228

Thorium-230

Thorium-232

Sum of alphas

Sum of betas

Tritium

Uranium-233/234

Uranium-235

Uranium-238
$1.5 \mathrm{E}+00 \quad / /$

$3.0 E+00 \quad / /$

$3.5 \mathrm{E}+02$. $/ /$
ST브 DF Lab $4098 \quad$ Mod

1 GP $4.2 \mathrm{E}+00 \quad / /$

$1 \quad \mathrm{GP} \quad 3.1 \mathrm{E}+00 \quad / /$

a $\quad 1$ GP $2.0 E+03 \quad / /$
Filt.

$\underline{\text { ST }} \underline{H}$ DF Lab Unit

1 GE pCi/L

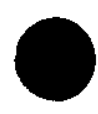

Notes:

- = exceeded holding time

- exceeded groundwater protection or monitoring constituent standard (See Appendix A.) 
WSRC-TR-99-00013

Unclassified

WELL HSB 66

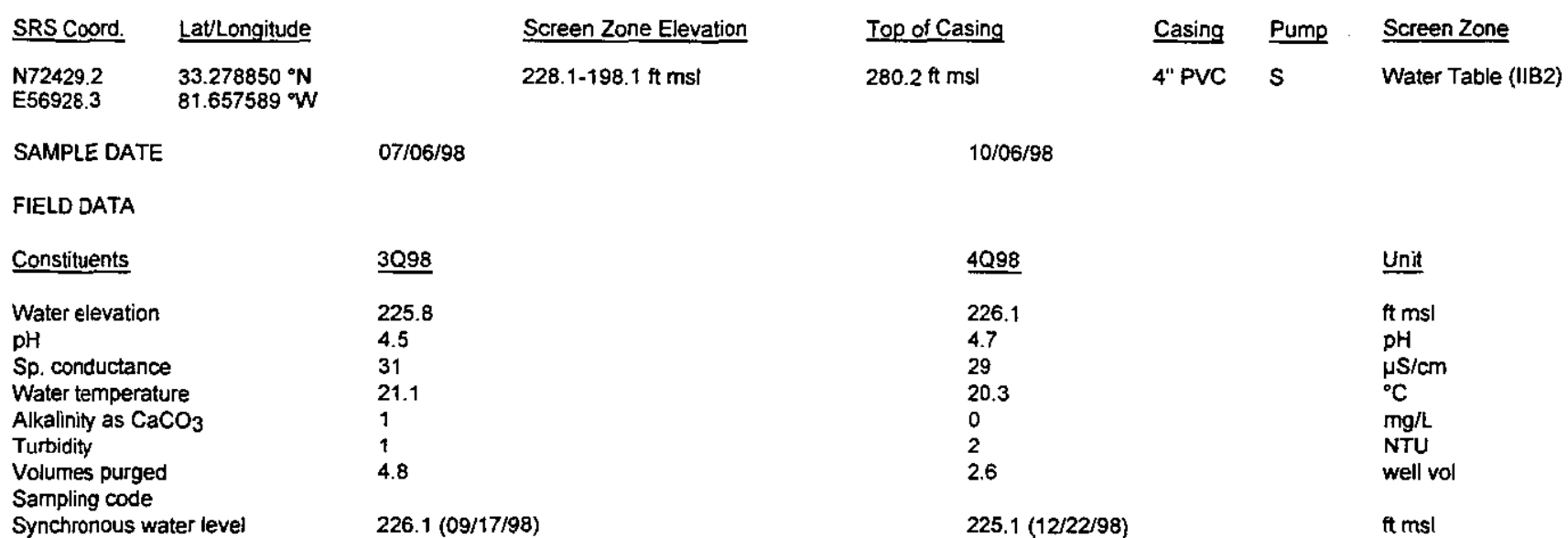

ANALYTICAL DATA

Inorganic Constituents

Constituents

Mod $\quad$ F

Filt. ST브 DF Lab 4098 Mod

Antimony, total recoverable Arsenic total recoverable Barium, total recoverable

Cadmium, total recoverable

Chromium, total recoverable

Cobalt, total recoverable

Copper, total recoverable

Cyanide

Lead, total recoverable

Mercury, total recoverable

Nickel, rotal recoverable

Nitrate as nitrogen

Nitrate-nitrite as nitrogen

Selenium, total recoverable

Silver, total recoverable

Tin, total recoverable

Vanadium, total recoverable

Zinc, total recoverable

Organic Constituents

Constituents

Filt.

SI브

DF

4Q98 Mod

Filt.

$\underline{\text { ST }} \underline{\mathrm{H}}$ DF Lab Unit

Benzene

Bis(2-thylhexyl) phthalate

Dichloromethane

Tetrachbroethylene

Trichloroethylene

Trichlorofluoromethane

\section{Notes:}

- = exceeded holding time

= exceeded groundwater protection or monitoring constituent standard (See Appendix A.) 
WELL HSB 66 (cont.)

Radioactive Constituents

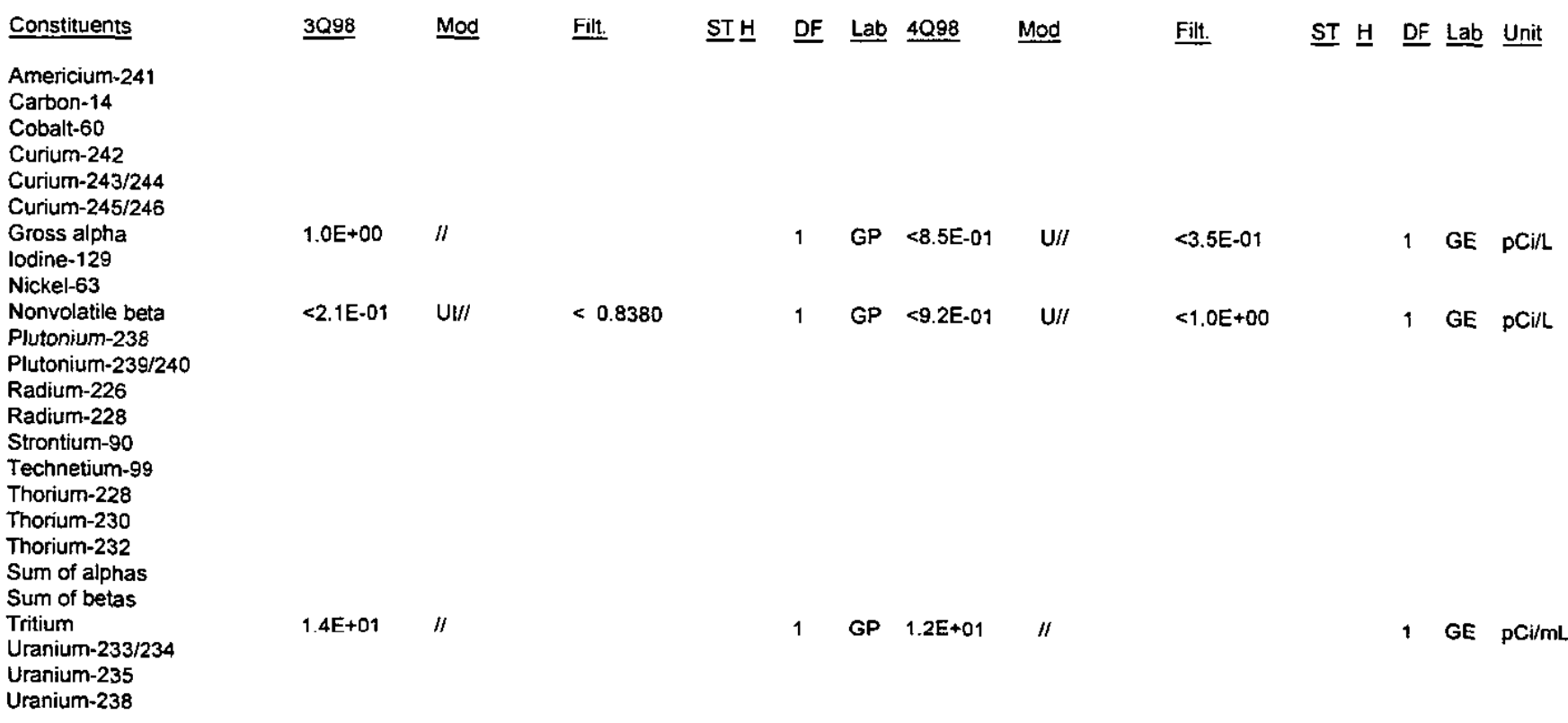

\section{Notes:}

$\bullet$ = exceeded nolding time

- = exceeded groundwater protection or monitoring constituent standard (See Appendix A.) 


\section{WELL HSB 67}

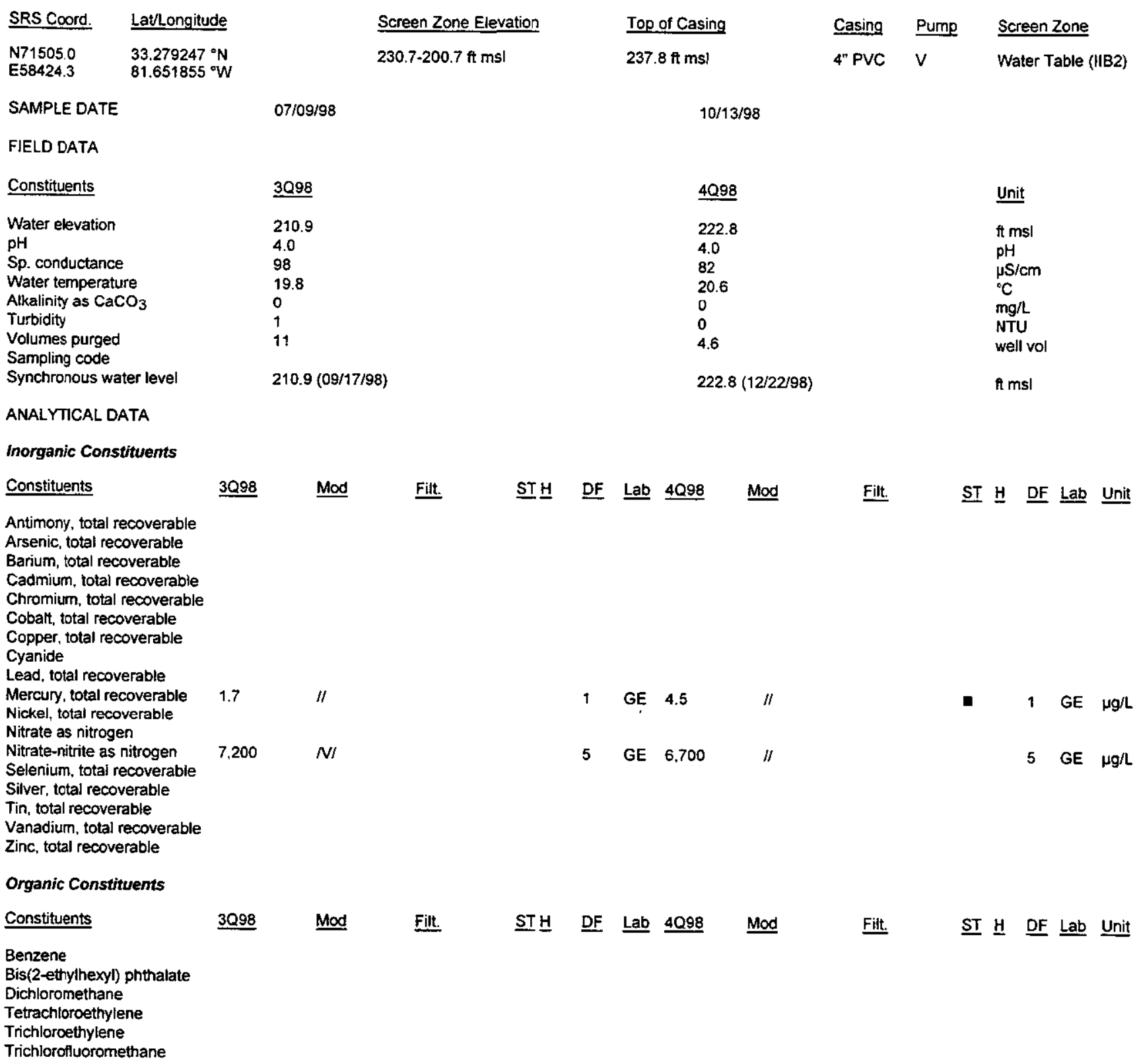

Notes:

- = exceeded holding time

- = exceeded groundwater protection or monitoring constituent standard (See Appendix A.) 
WELL HSB 67 (cont.)

Radioactive Constituents

Constituents

Americium-241

Carbon-14

Cobalt-60

Curium-242

Curium-243/244

Curium-245/246

Gross alpha

lodine-129

Nickel-63

Nonvolatile beta

Plutonium-238

Plutonium-239/240

Radium-226

Radium-228

Strontium-90

Technetium-99

Thorium-228

Thorium-230

Thorium-232

Sum of alphas

Sum of betas

Tritium

Uranium-233/234

Uranium-235

Uranium-238
3Q98

Mod

Filt.

ST브 DF Lab 4 QQ98

Mod

Filt.

ST H DF Lab Unit

$2.8 \mathrm{E}+01$

$6.9 \mathrm{E}+02 \quad /$

$\begin{array}{llll}\text { - } & 1 & \mathrm{GP} & 1.4 \mathrm{E}+01\end{array}$

1 GE PCi/L

- $\quad 1$ GP $5.8 E+02$

-

1 GE $\mathrm{pCi} / \mathrm{L}$

$2.4 \mathrm{E}+02 \quad / /$

-

1 GP $5.1 E+02 \quad / /$

- 1 GE pCi/ml

Notes:

$\because=$ exceeded holding time

- = exceeded groundwater protection or monitoring constituent standard (See Appendix A.) 


\section{WELL HSB 68}

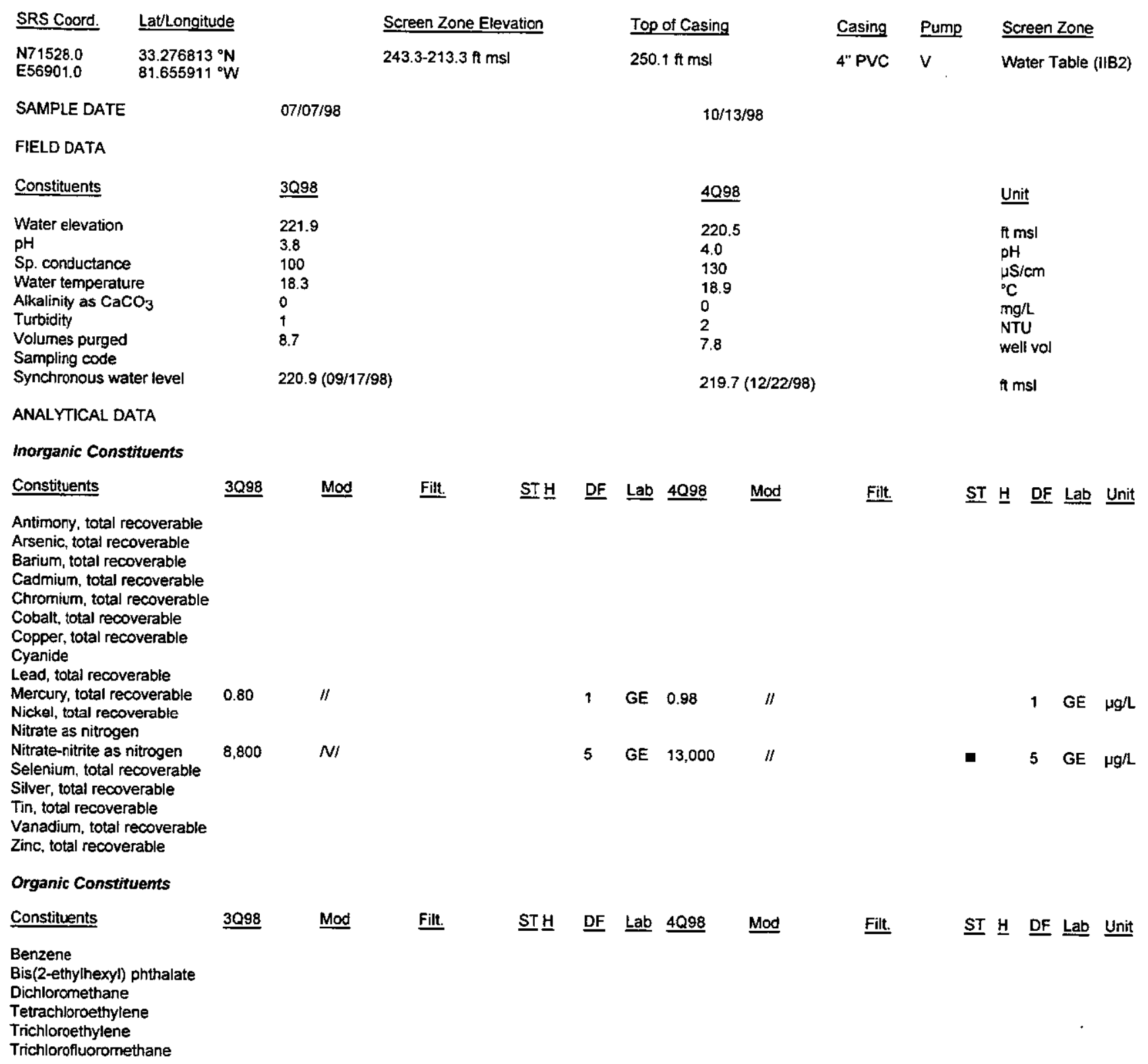

Notes:

- = exceeded holding time

- exceeded groundwater protection or monitoring constituent standard (See Appendix A.) 
WELL HSB 68 (cont.)

Radioactive Constituents

Constituents

Americium-241

Carbon-14

Cobalt-60

Curium-242

Curium-243/244

Curium-245/246

Gross alpha

lodine-129

Nickel-63

Nonvolatile beta

Plutonium-238

Plutonium-239/240

Radium-226

Radium-228

Strontium-90

Technetium-99

Thorium-228

Thorium-230

Thorium-232

Sum of alphas

Sum of betas

Tritium

Uranium-233/234

Uranium-235

Uranium-238
3098

Mod

$1.8 \mathrm{E}+03 \quad / /$

$1.6 \mathrm{E}+02 \quad /$

GP $2.9 \mathrm{E}+01 \quad / /$

-

1

GP $\quad 2.4 \mathrm{E}+03$

- 1 GP $3.4 \mathrm{E}+02$ /I
Filt.

$\underline{\text { ST }} \underline{H} \quad \underline{\mathrm{DF}} \underline{\text { Lab Unit }}$

- 1 GE $\mathrm{pCi} / \mathrm{L}$

- $1 \mathrm{GE} \mathrm{pCi} / \mathrm{l}$

$1 \mathrm{GE} \quad \mathrm{pCi} / \mathrm{mL}$

Notes:

- = exceeded holding time

= exceeded groundwater protection or monitoring constituent standard (See Appendix A.) 
WSRC-TR-99-00013

Unclassified

WELL HSB 68A

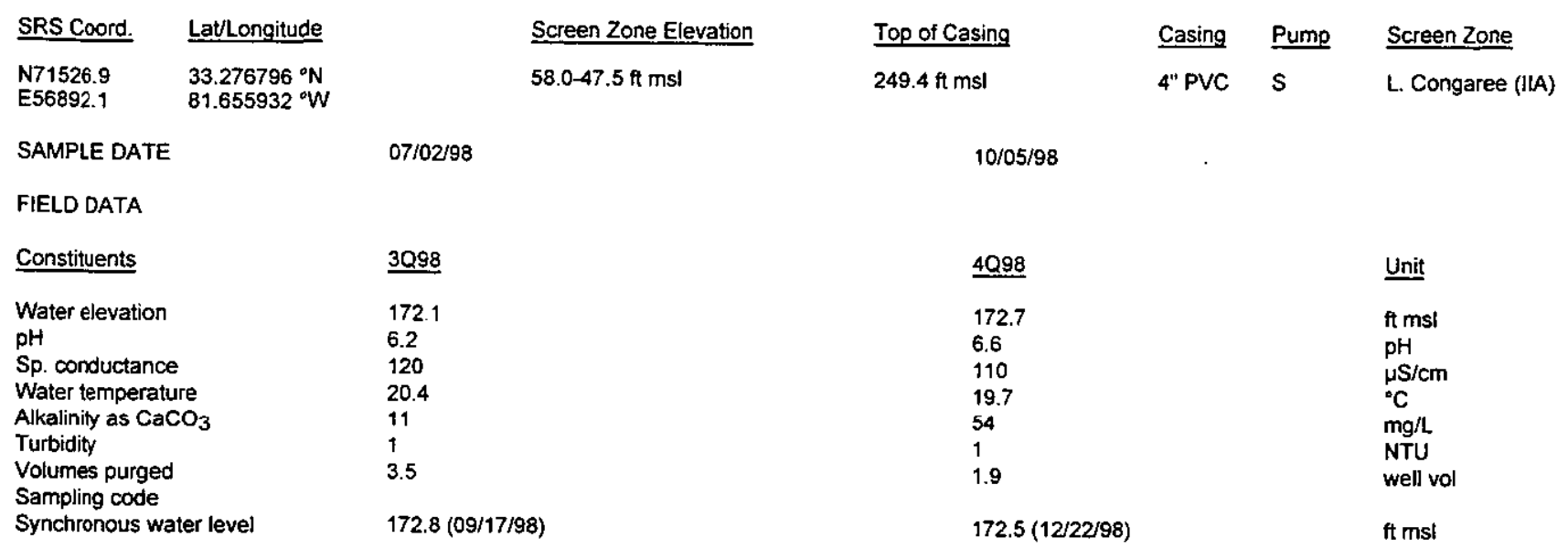

\section{ANALYTICAL DATA}

Inorganic Constituents

Constituents

Antimony, total recoverable

Arsenic total recoverable

Barium, total recoverable

Cadmium, total recoverable

Chromium, total recoverable

Cobalt, total recoverable

Copper, total recoverable

Cyanide

Lead, total recoverable

Mercury, total recoverable

Nickel, lotal recoverable

Nitrate as nitrogen

Nitrate-nitrite as nitrogen

Selenium, total recoverable

Silver, total recoverable

Tin, total recoverable

Vanadium, total recoverable

Zinc, total recoverable

Organic Constituents

Constituents

$\underline{3 Q 98}$

$<20$
$\mathrm{UJ} / / / 1$

$<0.20$

GE

$<0.20$

$\mathrm{U} / I$

UNI $<50$

1

$\mathrm{GE}<30$

ST보

Lab 4Q98

Mod

Filt.

$\underline{\text { ST }} \underline{H} \quad \underline{\text { DF Lab Unit }}$

Benzene

Bis(2-ethylhexyl) phthalate

Dichloromethane

Tetrachloroethylene

Trichloroethylene

Trichlorofluoromethane

Notes:

- = exceeded holding time

- = exceeded groundwater protection or monitoring constituent standard (See Appendix A.) 


\section{WELL HSB 68A (cont.)}

Radioactive Constituents

\section{Constituents}

Americium-241

Carbon-14

Cobalt- 60

Curium-242

Curium-243/244

Curium-245/246

Gross alpha

lodine-129

Nickel-63

Nonvolatile beta

Plutonium-238

Plutonium-239/240

Radium-226

Radium-228

Strontium-90

Technetium-99

Thorium-228

Thorium-230

Thorium-232

Sum of alphas

Sum of betas

Tritium

Uranium-233/234

Uranium-235

Uranium-238
Mod

Filt.

$\underline{\text { ST }} \underline{\mathrm{H}}$

DF LLab $\underline{4098}$

Mod

Filt.

ST $\underline{H}$ DF Lab Unit

$\begin{array}{lll}<2.8 \mathrm{E}-01 \quad \mathrm{U} I / & <0.5770 \quad 1\end{array}$

GP $<4.7 \mathrm{E}-01 \quad \mathrm{U} / 1$

1

GP

$<2.5 E+00$

$\mathrm{U} / I$

$<1.3 E+00$

$1 \mathrm{GE} \mathrm{pCi} / \mathrm{L}$

$2.6 \mathrm{E}+00 \quad / 1$

$<3.2 \mathrm{E}-01$

UIII

$<5.8 \mathrm{E}-01$

1

GP $<6.4 \mathrm{E}-01$

U/I

$<5.3 \mathrm{E}-01$

1 GE $\mathrm{pCi} / \mathrm{mL}$

Notes: 


\section{WELL HSB 68B}

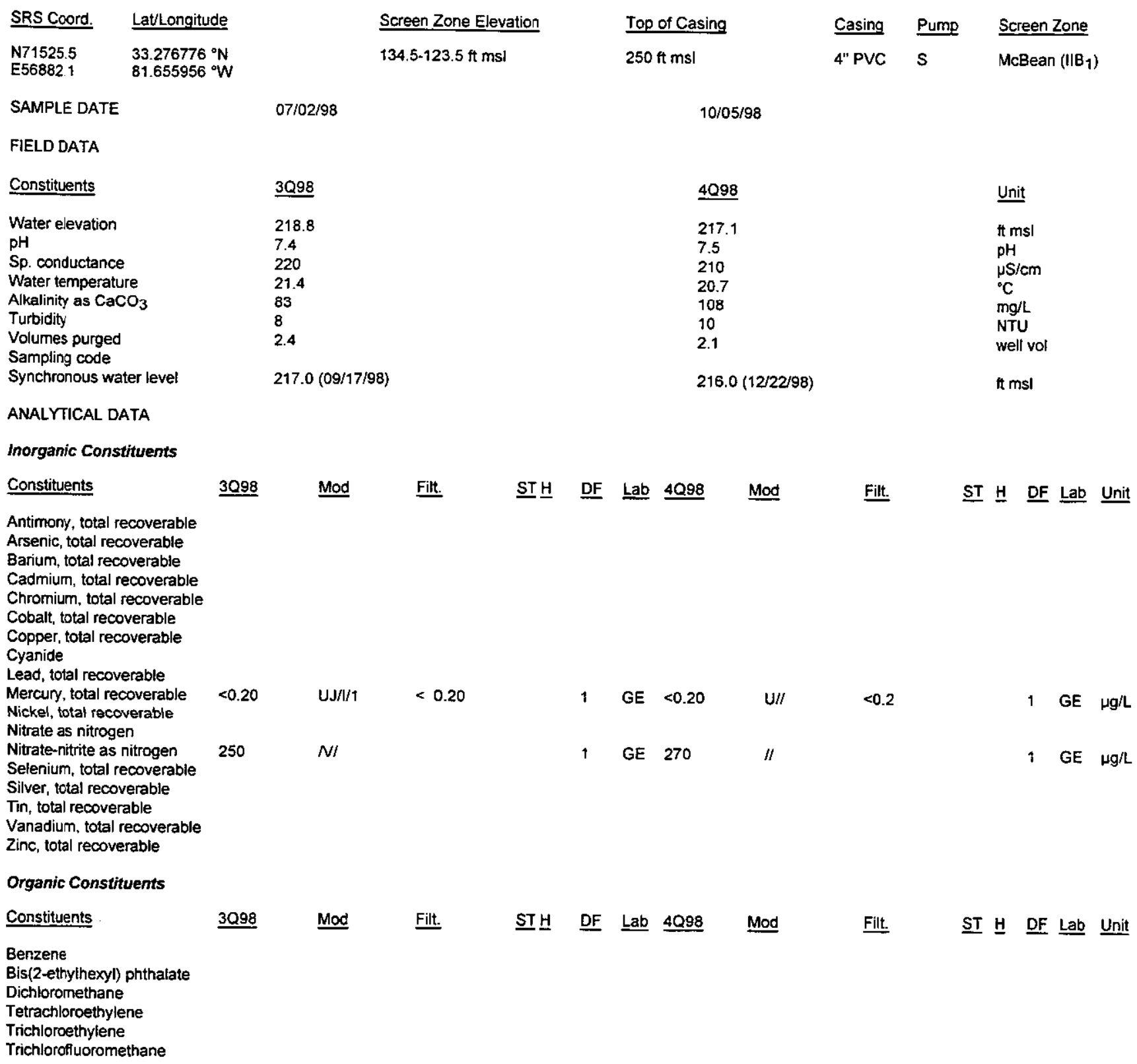

Notes:

- = exceeded holding time

- = exceeded groundwater protection or monitoring constituent standard (See Appendix A.) 


\section{WELL HSB 68B (cont.)}

Radioactive Constituents

Constituents

Americium-241

Carbon-14

Cobalt-60

Curium-242

Curium-243/244

Curium-245/246

Gross alpha

lodine-129

Nickel-63

Nonvolatile beta

Plutonium-238

Plutonium-239/240

Radium-226

Radium-228

Strontium-90

Technetium-99

Thorium-228

Thorium-230

Thorium-232

Sum of alphas

Sum of betas

Tritium

Uranium-233/234

Uranium-235

Uranium-238 $\underline{3 Q 98}$

Mod

Filt.

$\underline{\text { ST }} \underline{H}$

DF Lab 4098

Mod

Filt.

ST $\underline{\text { H }}$ DF Lab Unit

8.4E-01

$2.7 \mathrm{E}+00$

1

$G P<8.3 E-01$

$\mathrm{U} / \mathrm{I}$

1 GP $<7.8 E-01$

U/I

$<1.4 E+00$

$1 \quad$ GP $\quad 1.5 E+01$

/I
1 GE PCi/L

$1 \mathrm{GE} \mathrm{pCi} / \mathrm{L}$
$1.4 \mathrm{E}+01 \quad \|$

1 GE $\mathrm{pCi} / \mathrm{mL}$

Notes: 


\section{WELL HSB 68C}

\begin{tabular}{|c|c|}
\hline SRS Coord. & Lat/Longitude \\
\hline $\begin{array}{l}\text { N71524.1 } \\
\text { E56872.7 }\end{array}$ & $\begin{array}{l}33.276758 \\
81.655978\end{array}$ \\
\hline
\end{tabular}

SAMPLE DATE

FIELD DATA

Constituents

Water elevation

$\mathrm{pH}$

Sp. conductance

Water temperature

Alkalinity as $\mathrm{CaCO}_{3}$

Turbidity

Volumes purged

Sampling code

Synchronous water level

ANALYTICAL DATA

Inorganic Constituents

Constituents

Antimony, total recoverable

Arsenic, total recoverable

Barium, total recoverable

Cadimium, total recoverable

Chromium, total recoverable

Cobalt, total recoverable

Copper, total recoverable

Cyanide

Lead, total recoverable

Mercury, total recoverable

Nickel, total recoverable

Nitrate as nitrogen

Nitrate-nitrite as nitrogen

Selenium, total recoverable

Silver, total recoverable

Tin, total recoverable

Vanadium, total recoverable

Zinc, total recoverable

Organic Constituents

Constituents
$07 / 13 / 98$

Screen Zone Elevation

$179.5-168.4 \mathrm{ft}$ mst

218.4

4.9

130

20.5

25

2

0.030

$\mathrm{XN}$

$217.9(09 / 17 / 98)$
Top of Casing

$250.1 \mathrm{ft} \mathrm{msl}$

$10 / 05 / 98$
Casing Pump Screen Zone

4" PVC S Barnwell (IIB 1$)$

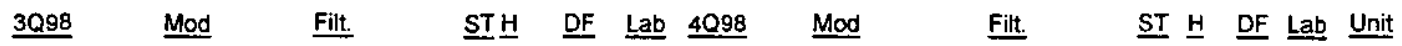

0.42

1 GE $0.40 \quad n$

1 GE $\mu g / L$

$12,000 \quad N$

- 5 GE 12,000 //

- 5 GE $\mu g / L$

\section{Benzene}

Bis(2-ethylhexyl) phthalate

Dichloromethane

Tetrachloroethylene

Trichloroethylene

Trichlorofluoromethane 
WELL HSB $68 \mathrm{C}$ (cont.)

Radioactive Constituents

\begin{tabular}{|c|c|c|c|c|c|c|c|c|c|c|c|c|c|}
\hline Constituents & 3098 & Mad & Filt. & $\underline{\text { STH}}$ & $\underline{\mathrm{OF}}$ & $\underline{L a b}$ & 4Q98 & Mod & Filt. & $\underline{\text { ST }} \underline{H}$ & $\underline{D F}$ & $\underline{\text { Lab }}$ & Unit \\
\hline $\begin{array}{l}\text { Americium-241 } \\
\text { Carbon-14 } \\
\text { Cobalt-60 } \\
\text { Curium-242 } \\
\text { Curium-243/244 } \\
\text { Curium-245/246 }\end{array}$ & & & & & & & & & & & & & \\
\hline $\begin{array}{l}\text { Gross alpha } \\
\text { Jodine-129 } \\
\text { Nickel-63 }\end{array}$ & $<7.7 \mathrm{E}-01$ & $\mathrm{UI} / /$ & $<1.0500$ & & 1 & GP & $<1.4 \mathrm{E}+\infty 0$ & $\mathrm{U} / 1$ & $<6.0 \mathrm{E}-01$ & & 1 & $\mathrm{GE}$ & $\mathrm{pCi} / \mathrm{L}$ \\
\hline $\begin{array}{l}\text { Nonvolatile beta } \\
\text { Plutonium-238 } \\
\text { Plutonium-239/240 } \\
\text { Radium-226 } \\
\text { Radium-228 } \\
\text { Strontium-90 } \\
\text { Technetium-99 } \\
\text { Thorium-228 } \\
\text { Thorium-230 } \\
\text { Thorium-232 } \\
\text { Sum of alphas } \\
\text { Sum of betas } \\
\text { Tritium }\end{array}$ & $7.4 \mathrm{E}+00$ & $n$ & & - & 1 & GP & $6.7 \mathrm{E}+00$ & 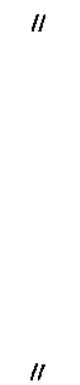 & & n & 1 & GE & $\mathrm{pCi} / \mathrm{L}$ \\
\hline
\end{tabular}

Uranium-238

Notes:

- exceeded holding time

- = exceeded groundwater protection or monitoring constituent standard (See Appendix A.) 
WELL HSB 69

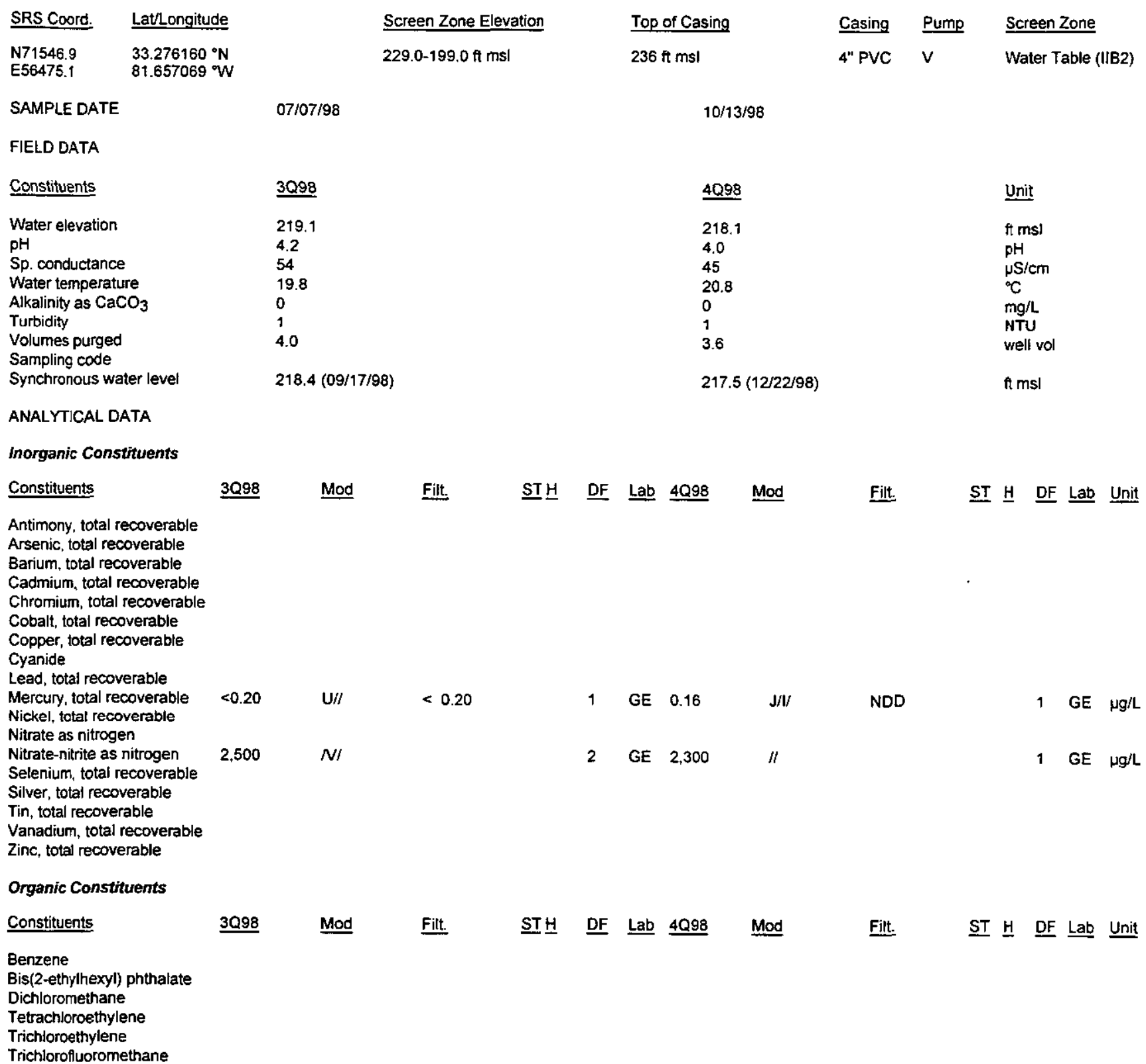

\section{Notes:}

- = exceeded holding time

- = exceeded groundwater protection or monitoring constituent standard (See Appendix A.) 
WELL HSB 69 (cont.)

Radioactive Constituents

Constituents

Americium-241

Carbon-14

Cobalt-60

Curium-242

Curium-243/244

Curium-245/246

Gross alpha

lodine-129

Nickel-63

Nonvolatile beta

Plutonium-238

Plutonium-239/240

Radium-226

Radium-228

Strontium-90

Technetium-99

Thorium-228

Thorium-230

Thorium-232

Sum of alphas

Sum of betas

Tritium

Uranium-233/234

Uranium-235

Uranium-238
3098

$9.9 E+00$

$5.3 E+02$

Mod

Filt.

ST브 DF Lab 4 QQ98

Mod

$1 \quad G P<2.6 E+00 \quad U \prime \prime$

$<1.1 \mathrm{E}+00$

1 GP $5.4 \mathrm{E}+02$ //

2.5E+01 II

-

1 GP 2.6E+01 $/ /$
Filt.

ST $\underline{H}$ DF Lab Unit

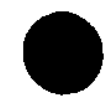

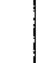

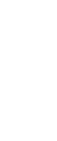


WELL HSB 69A

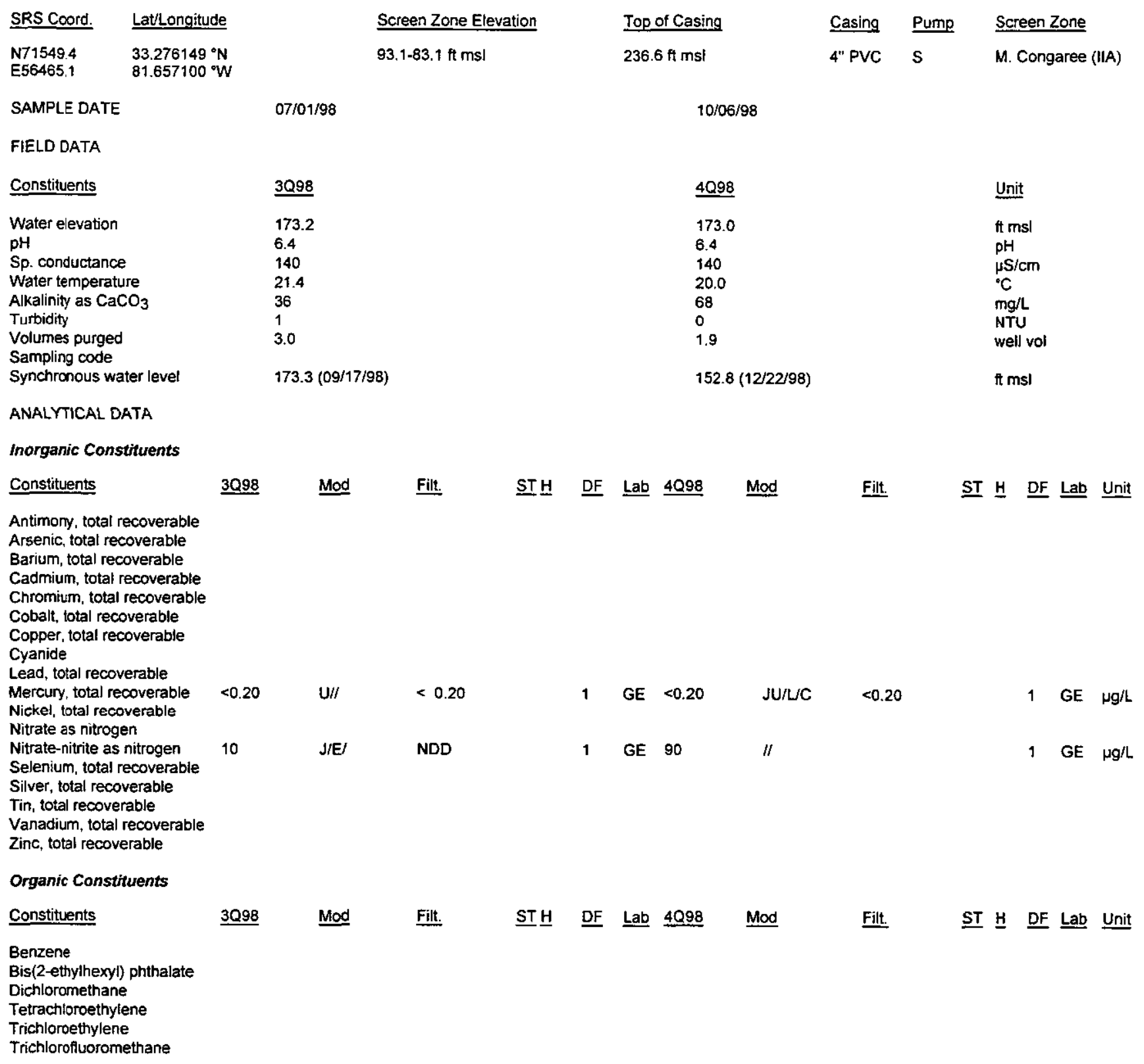

Notes:

$\bullet=$ exceeded holding time

- exceeded groundwater protection or monitoring constituent standard (See Appendix A.) 
WELL HSB 69A (cont.)

Radioactive Constituents

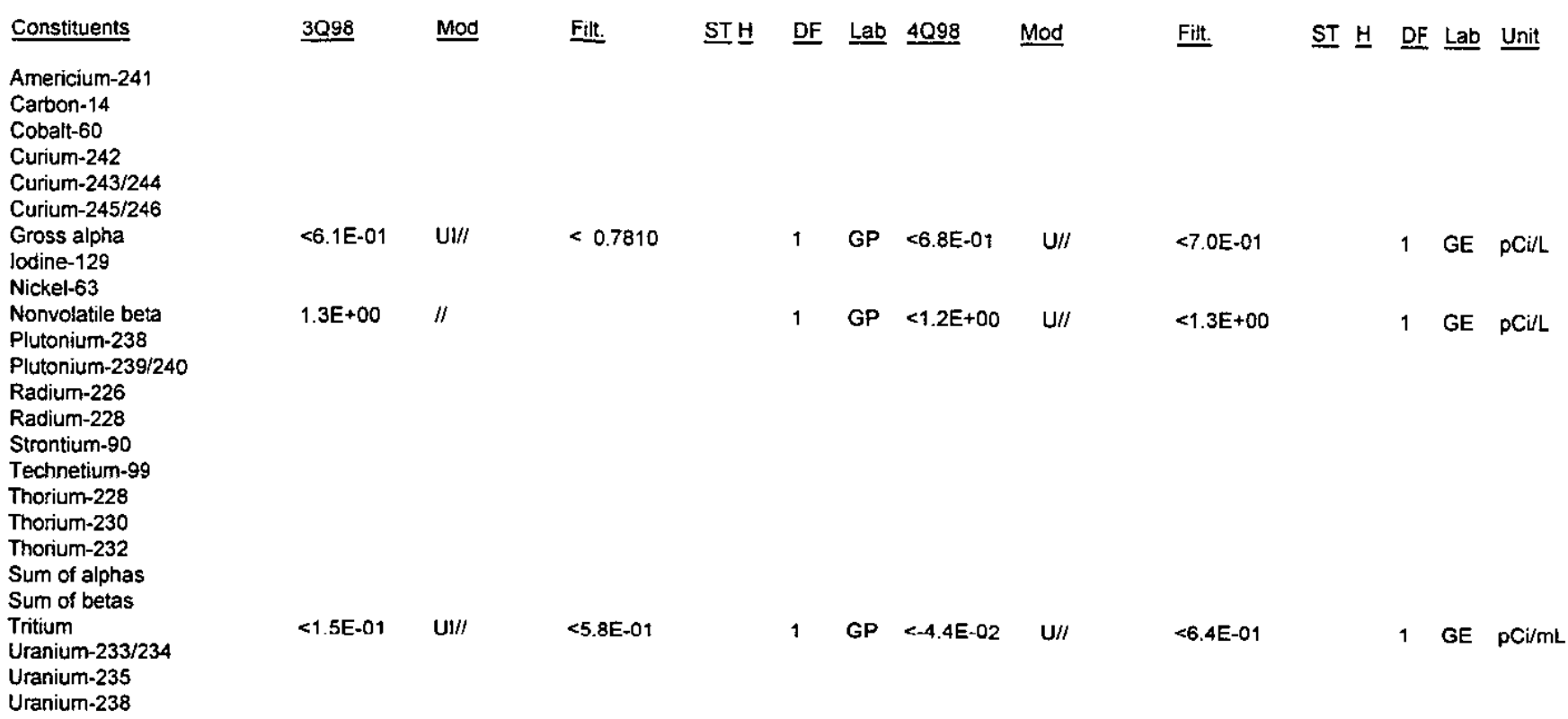


WELL HSB 70

\begin{tabular}{|c|c|}
\hline SRS Coord. & Latlongitude \\
\hline $\begin{array}{l}N 72606.9 \\
\text { E55758.9 }\end{array}$ & $81.661013^{\circ}$ \\
\hline
\end{tabular}

Screen Zone Elevation

235.7-205.7 ft msl

07/06/98

$\underline{3098}$

Constituents

Water elevation

$\mathrm{pH}$

Sp. conductance

Water temperature

Alkalinity as $\mathrm{CaCO}_{3}$

Turbidity

Volumes purged

Sampling code

Synchronous water level

ANALYTICAL DATA

Inorganic Constituents

Constituents

Antimony, total recoverable

Arsenic, total recoverable

Barium, total recoverable

Cadmium, total recoverable

Chromium, total recoverable

Cobalt, total recoverable

Copper, total recoverable

Cyanide

Lead, total recoverable

Mercury, total recoverable

Nickel, total recoverable

Nitrate as nitrogen

Nitrate-nitrite as nitrogen

Selenium, total recoverable

Silver, total recoverable

Tin, total recoverable

Vanadium, total recoverable

Zinc, total recoverable

Organic Constituents

Constituents

Benzene

Bis(2-ethylhexyl) phthalate

Dichloromethane

Tetrachloroethylen

Trichloroethylene

Trichlorofluoromethane

$<0.20$

UJ/I/1

$<0.20$

NI

Mod $\quad$ Filt.
Mod

Casing Pump

4" PVC S

$10 / 05 / 98$

4Q98

222.4

5.0

58

19.0

0

2.9

$221.3(12 / 22 / 98)$

Filt.

$\underline{\text { ST }} \underline{H} \quad \underline{\text { DF Lab Unit }}$

1 GE $<0.20$

$\mathrm{U} / 1$

GE 220

4

Mod

ST브 DF ㄴab 4098

$$
\begin{aligned}
& \text { Unit } \\
& \mathrm{ft} \mathrm{ms} \\
& \mathrm{pH} \\
& \mu \mathrm{S} / \mathrm{cm} \\
& { }^{\circ} \mathrm{C} \\
& \mathrm{mg} / \mathrm{L} \\
& \mathrm{NTU} \\
& \text { well vol } \\
& \text { ft ms! }
\end{aligned}
$$


WSRC-TR-99-00013

Unclassified

WELL HSB 70 (cont.)

Radioactive Constituents

\section{Constituents}

Americium-241

Carbon-14

Cobalt-60

Curium-242

Curium-243/244

Curium-245/246

Gross alpha

lodine-129

Nickel-63

Nonvolatile beta

Plutonium-238

Plutonium-239/240

Radium-226

Radium-228

Strontium-90

Technetium-99

Thorium-228

Thorium-230

Thorium-232

Sum of alphas

Sum of betas

Tritium

Uranium-233/234

Uranium-235

Uranium-238
3098

Mod

Filt.

ST보

DF Lab $4 Q 98$

Mod

Filt.

$\underline{\text { ST }} \underline{H} \quad \underline{\text { DF Lab }} \underline{\text { Unit }}$
1 GP $1.8 E+00$

1 GP $8.5 E+00$

1 GP $2.7 E+01 / /$
$1 \mathrm{GE} \mathrm{pCi} / \mathrm{h}$

$1 \mathrm{GE} \quad \mathrm{pCi} / \mathrm{L}$

2.7E+01
1 GE $\mathrm{pCi} / \mathrm{mL}$

- exceeded groundwater protection or monitoring constituent standard (See Appendix A.) 


\section{WELL HSB 70C}

$\begin{array}{ll}\text { SRS Coord. } & \text { Lat/Longitude } \\ \text { N72597.3 } & 33.277311^{\circ} \mathrm{N} \\ \text { E55757.1 } & 81.660999^{\circ} \mathrm{W}\end{array}$

SAMPLE DATE

$07 / 28 / 98$

Screen Zone Elevation

$174.9-164.9 \mathrm{ft} \mathrm{ms}$
Top of Casing

$243.1 \mathrm{ft} \mathrm{msl}$

$\begin{array}{ll}\text { Casing Pump } & \text { P" PVC }\end{array}$

Screen Zone

Barnwell (IIB 1$)$
FIELD DATA

Constituents

Water elevation

$\mathrm{pH}$

Sp. conductance

Water temperature

Alkalinity as $\mathrm{CaCO}_{3}$

Turbidity

Volumes purged

Sampling code

Synchronous water level
3098

222.8

10.2

480

19.8

56

2.9

0.63

$\mathrm{XN}$

222.02(09/17/98)
$11 / 05 / 98$

$4 Q 98$
221.1
7.5
410
15.7
29
1
0.027
$X N$
$220.3(12 / 22 / 98)$

Unit

ft msl

pH

uS/cm

${ }^{\circ} \mathrm{C}$

mg/L

NTU

well vol

ft msl

ANALYTICAL DATA

Inorganic Constituents

\begin{tabular}{|c|c|c|c|c|c|c|c|c|c|c|c|c|c|}
\hline Constituents & $3 \mathrm{Q99}$ & Mod & Filt. & ST브 & $\underline{\mathrm{DF}}$ & $\underline{\text { Lab }}$ & $\underline{4 Q 98}$ & Mod & Filt. & $\underline{\mathrm{ST}} \underline{\mathrm{H}}$ & DF & $\underline{L a b}$ & Unit \\
\hline Antimony, total recoverable & $<0.20$ & $\mathrm{U} / I$ & $<0.20$ & & 1 & GE & & & & & & & $\mu g / L$ \\
\hline Arsenic, total recoverable & $<3.0$ & $\mathrm{U} / /$ & $<3.0$ & & 1 & GE & & & & & & & $\mu g / L$ \\
\hline Barium, total recoverable & 110 & $\|$ & & & 1 & GE & & & & & & & $\mu g / L$ \\
\hline Cadmium, total recoverable & 0.54 & $\mathrm{~J} / \mathrm{E} /$ & NDD & & 1 & $\mathrm{GE}$ & & & & & & & $\mu g / L$ \\
\hline Chromium, total recoverable & 0.80 & $\mathrm{~J} / \mathrm{E} /$ & NDD & & 1 & GE & & & & & & & $\mu g / L$ \\
\hline Cobalt, total recoverable & 0.74 & $/ /$ & & & 1 & GE & & & & & & & $\mu g / L$ \\
\hline Copper, total recoverable & 0.55 & $\|$ & & & 1 & $\overline{G E}$ & & & & & & & $\mu \mathrm{g} / \mathrm{L}$ \\
\hline Cyanide & $<10$ & $\mathrm{U} / /$ & $<10$ & & 1 & GE & & & & & & & $\mu g / L$ \\
\hline Lead, total recoverable & 0.91 & J/EV/ & NDD & & 1 & GE & & & & & & & $\mu g / L$ \\
\hline Mercury, total recoverable & 0.070 & $\mathrm{~S} / \mathrm{E} /$ & NDD & & 1 & GE & $<0.20$ & $\mathrm{U} / /$ & $<0.2$ & & 1 & GE & $\mu g / L$ \\
\hline $\begin{array}{l}\text { Nickel, total recoverable } \\
\text { Nitrate as nitrogen }\end{array}$ & 4.9 & $\|$ & & & 1 & GE & & & & & , & UL & $\mu g / L$ \\
\hline Nitrate-nitrite as nitrogen & 39,000 & $N \prime$ & & $\mathbf{u}$ & 25 & GE & 38,000 & $/ /$ & & $\mathbf{E}$ & 25 & GE & $\mu g /$ \\
\hline Selenium, total recoverable & $<5.0$ & $\mathrm{U} / /$ & $<5.0$ & & 1 & GE & & & & & & & $\mu g / \mathrm{L}$ \\
\hline Silver, total recoverable & $<1.0$ & $\mathrm{U} / /$ & $<1.0$ & & 1 & $\overline{G E}$ & & & & & & & $\mu g / L$ \\
\hline Tin, total recoverable & $<2.0$ & $\mathrm{U} / /$ & $<2.0$ & & 1 & $\overline{G E}$ & & & & & & & $\mu g / L$ \\
\hline Vanadium, total recoverable & $<2.0$ & $\mathrm{U} / /$ & $<2.0$ & & 1 & GE & & & & & & & $\mu g / L$ \\
\hline Zinc, total recoverable & 18 & $/ 1$ & & & 1 & GE & & & & & & & $\mu g / L$ \\
\hline \multicolumn{14}{|l|}{ Organic Constituents } \\
\hline Constituents & 3098 & Mod & Filt. & $\underline{\text { ST }} \underline{H}$ & $\underline{\mathrm{DF}}$ & $\underline{\text { Lab }}$ & 4Q98 & Mod & Filt. & $\underline{\text { ST }} \underline{H}$ & $\underline{\text { DF }}$ & $\underline{\text { Lab }}$ & Unit \\
\hline Benzene & $<1.0$ & $\mathrm{UJ} / \mathrm{O} / 1$ & $<1.0$ & 1 & & GE & & & & & & & $\mu g / L$ \\
\hline Bis(2-ethylhexyl) phthalate & $<10$ & $\mathrm{U} / 1$ & $<10$ & & 1 & GE & & & & & & & $\mu g / L$ \\
\hline Dichloromethane & 5.8 & $\mathrm{~J} / \mathrm{O} / 1$ & NDD & & 1 & GE & & & & & & & $\mu g / L$ \\
\hline Tetrachloroethylene & $<1.0$ & $\mathrm{UJ} / \mathrm{O} / 1$ & $<1.0$ & 1 & & GE & & & & & & & $\mu g / \mathrm{L}$ \\
\hline Trichloroethylene & $<1.0$ & UJ/O/1 & $<1.0$ & 1 & & GE & & & & & & & $\mu g / L$ \\
\hline Trichlorofluoromethane & $<1.0$ & $\mathrm{UJ} / \mathrm{O} / 1$ & $<1.0$ & 1 & & GE & & & & & & & $\mu g / \mathrm{L}$ \\
\hline
\end{tabular}

Notes:

- = exceeded holding time

- =xceeded groundwater protection or monitoring constituent standard (See Appendix A.) 
WELL HSB 70C (cont.)

Radioactive Constituents

\begin{tabular}{|c|c|c|c|c|c|c|c|c|c|c|c|c|c|}
\hline Constituents & 3098 & Mod & Filt. & ST브 & $\underline{D F}$ & Lab & 4Q98 & Mod & Filt. & $\underline{\text { ST }} \underline{H}$ & $\underline{D F}$ & Lيb & Unit \\
\hline Americium-241 & $<3.7 E-02$ & $\mathrm{UI} / \prime$ & $<0.1670$ & & 1 & GP & & & & & & & $\mathrm{pCi} / \mathrm{L}$ \\
\hline Beta dose & 2.75 & & & - & & & & & & & & & $\mathrm{pCinh}$ \\
\hline Carbon-14 & $3.0 E+02$ & /l & & m & 1 & GP & & & & & & & $\mathrm{pCi} / \mathrm{L}$ \\
\hline Cobalt- 60 & $<2.6 \mathrm{E}-01$ & $\mathrm{UI} / /$ & $<3.5100$ & & 1 & GP & & & & & & & $\mathrm{pCi} / \mathrm{L}$ \\
\hline Curium-242 & $<3.5 \mathrm{E}-02$ & $\mathrm{UI} / /$ & $<0.2550$ & & 1 & GP & & & & & & & $\mathrm{pCi} / \mathrm{L}$ \\
\hline Curium-243/244 & $<8.0 \mathrm{E}-02$ & $\mathrm{UI} / I$ & $<0.1980$ & & 1 & GP & & & & & & & $\mathrm{pCi} / \mathrm{L}$ \\
\hline Curium-245/246 & $<0.0 E+00$ & $\mathrm{U}] \mathrm{l} / \mathrm{I}$ & $<0.0951$ & & 1 & GP & & & & & & & $\mathrm{DCi} / \mathrm{L}$ \\
\hline Gross alpha & $4.9 E+00$ & $/ /$ & & & 1 & GP & $<3.9 E+00$ & $\mathrm{U} / /$ & $<2.6 E+\infty 0$ & & 1 & GE & $\mathrm{pCi} / \mathrm{L}$ \\
\hline lodine-129 & $2.4 E+00$ & $/ /$ & & & 1 & GP & & & & & & & $\mathrm{pCi} / \mathrm{L}$ \\
\hline Nickel-63 & $<2.6 \mathrm{E}-01$ & UI & $<3.5100$ & & & & & & & & & & $\mathrm{pCi} / \mathrm{L}$ \\
\hline Nonvolatile beta & $1.1 E+02$ & "I & & - & 1 & $G P$ & $1.8 \mathrm{E}+01$ & 11 & & & 1 & GE & $\mathrm{pCi} / \mathrm{L}$ \\
\hline Plutonium-238 & $<-7.2 \mathrm{E}-03$ & $\mathrm{UII}$ & $<0.2910$ & & 1 & GP & & & & & & & $\mathrm{pCi} / \mathrm{L}$ \\
\hline Plutonium-239/240 & $<-8.6 \mathrm{E}-03$ & $\mathrm{U} W /$ & $<0.1900$ & & $i$ & GP & & & & & & & $\mathrm{pCil}$ \\
\hline Radium-226 & $4.2 E+00$ & $\|$ & & & 1 & GP & & & & & & & $\mathrm{pCi} / \mathrm{L}$ \\
\hline Radium-228 & $<-2.2 E-01$ & UIII & $<1.2100$ & & 1 & GP & & & & & & & $\mathrm{pCi} / \mathrm{L}$ \\
\hline Strontium-90 & $<4.2 \mathrm{E}-01$ & UIJ/L & & & 1 & GP & & & & & & & $\mathrm{pCi} / \mathrm{L}$ \\
\hline Technetium-99 & $1.8 E+02$ & "l & & $\square$ & 1 & GP & & & & & & & $\mathrm{pCi} / \mathrm{L}$ \\
\hline Thorium-228 & $<0.0 E+00$ & $\mathrm{U} \mid \mathrm{J} / \mathrm{Cl}$ & & & 1 & GP & & & & & & & $\mathrm{pCi} / \mathrm{L}$ \\
\hline Thorium-230 & 7.9E-01 & $\mathrm{J} / \mathrm{C} /$ & NDD & & 1 & GP & & & & & & & pCill \\
\hline Thorium-232 & $<0.0 E+00$ & $\mathrm{U} / \mathrm{J} / \mathrm{Cl}$ & & & 1 & GP & & & & & & & PCin \\
\hline Sum of alphas & 7.9E-01 & & & & & & & & & & & & $\mathrm{pCi} / \mathrm{L}$ \\
\hline $\begin{array}{l}\text { Sum of betas } \\
\text { Total radium }\end{array}$ & $\begin{array}{l}4.8 E+02 \\
4.2 E+00\end{array}$ & & & - & & & & & & & & & $\mathrm{PCi} / \mathrm{L}$ \\
\hline Tritium & $4.5 \mathrm{E}+03$ & $/ 1$ & & घ & 1 & GP & $4.7 \mathrm{E}+03$ & /I & & घ & 1 & GE & $\mathrm{pCi} / \mathrm{mL}$ \\
\hline Uranitum-233/234 & $<1.1 \mathrm{E}-01$ & $\mathrm{U} 1 / /$ & $<0.1800$ & & 1 & GP & & & & & & & $\mathrm{pCi} / \mathrm{L}$ \\
\hline Uranium-235 & $<2.0 \mathrm{E}-02$ & $\mathrm{U} 1 / / /$ & $<0.1370$ & & 1 & GP & & & & & & & pCill \\
\hline Uranium-238 & $<7.2 \mathrm{E}-03$ & $\mathrm{U} 1 / /$ & $<0.1800$ & & 1 & GP & & & & & & & $\mathrm{pCi} \mathrm{L}$ \\
\hline
\end{tabular}

Notes:

- = exceeded holding time

- exceeded groundwater protection or monitoring constituent standard (See Appendix A.) 
WELL HSB 71

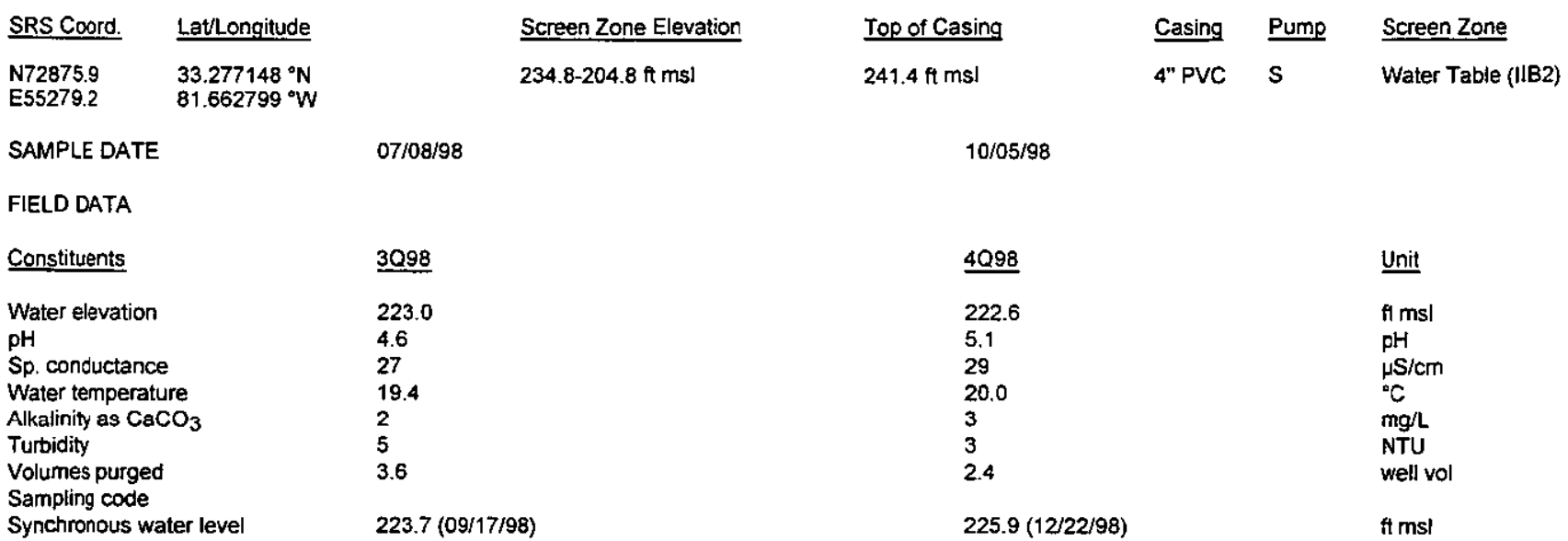

ANALYMCAL DATA

Inorganic Constituents

\section{Constituents}

Antimony, total recoverable

Arsenic, total recoverable

Barium, lotal recoverable

Cadmium, total recoverable

Chromium, total recoverable

Cobalt, total recoverable

Copper, iotal recoverable

Cyanide

Lead, total recoverable

Mercury, total recoverable

Nickel, total recoverable

Nitrate as nitrogen

Nitrate-nitrite as nitrogen

Selenium, total recoverable

Silver, total recoverable

Tin, total recoverable

Vanadium, total recoverable

Zinc, total recoverable

Organic Constituents

Constituents

190
Mod

Filt.

ST보

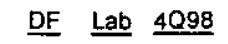

Mod

U/I

$<0.20$

$\uparrow$

GE $<0.20$

U/I

NI

Benzene

Bis(2-ethylhexyl) phthalate

Dichloromethane

Tetrachloroethylene

Trichloroethylene

Trichlorofluoromethane

Notes:

- exceeded hoiding time

- exceeded groundwater protection or monitoring constituent standard (See Appendix A.) 
WELL HSB 71 (cont.)

Radioactive Constituents

\begin{tabular}{|c|c|c|c|c|c|c|c|c|c|c|c|c|c|}
\hline Constituents & 3098 & Mod & Filt. & $\underline{\text { ST}} \underline{H}$ & $\underline{D F}$ & $\underline{L a b}$ & $\underline{4 Q 98}$ & Mod & Filt. & $\underline{\text { ST }} \underline{H}$ & $\underline{\mathrm{DF}}$ & $\underline{\text { Lab }}$ & Unit \\
\hline $\begin{array}{l}\text { Americium-241 } \\
\text { Carbon-14 } \\
\text { Cobalt-60 } \\
\text { Curium-242 } \\
\text { Curium-243/244 } \\
\text { Curium-245/246 }\end{array}$ & & & & & & & & & & & & & \\
\hline $\begin{array}{l}\text { Gross aipha } \\
\text { lodine-129 } \\
\text { Nickel-63 }\end{array}$ & $<5.0 \mathrm{E}-01$ & $\mathrm{Ut} / /$ & $<0.525$ & & 1 & GP & $<-6.3 E-02$ & $\mathrm{U} / /$ & $<5.8 E-01$ & & 1 & GE & $\mathrm{pCi} / \mathrm{L}$ \\
\hline $\begin{array}{l}\text { Nonvolatile beta } \\
\text { Plutonium-238 } \\
\text { Plutonium-239/240 } \\
\text { Radium-226 } \\
\text { Radium-228 } \\
\text { Strontium-90 } \\
\text { Technetium-99 } \\
\text { Thorium-228 } \\
\text { Thorium-230 } \\
\text { Thorium-232 } \\
\text { Sum of alphas } \\
\text { Sum of betas }\end{array}$ & $1.4 E+00$ & $\|$ & & & 1 & GP & $<-5.3 E-01$ & $\mathrm{U} / 1$ & $<5.8 \mathrm{E}-01$ & & 1 & GE & $\mathrm{pCi} / \mathrm{L}$ \\
\hline $\begin{array}{l}\text { Tritium } \\
\text { Uranium-233/234 } \\
\text { Uranium-235 } \\
\text { Uranium-238 }\end{array}$ & $2.4 \mathrm{E}+01$ & 11 & & = & 1 & GP & $3.7 E+01$ & $/ 1$ & & - & 1 & $\mathrm{GE}$ & $\mathrm{pCi} / \mathrm{mL}$ \\
\hline
\end{tabular}

Notes:

- exceeded holding time

- exceeded groundwater protection or monitoring constituent standard (See Appendix A.) 
WELL HSB 71C

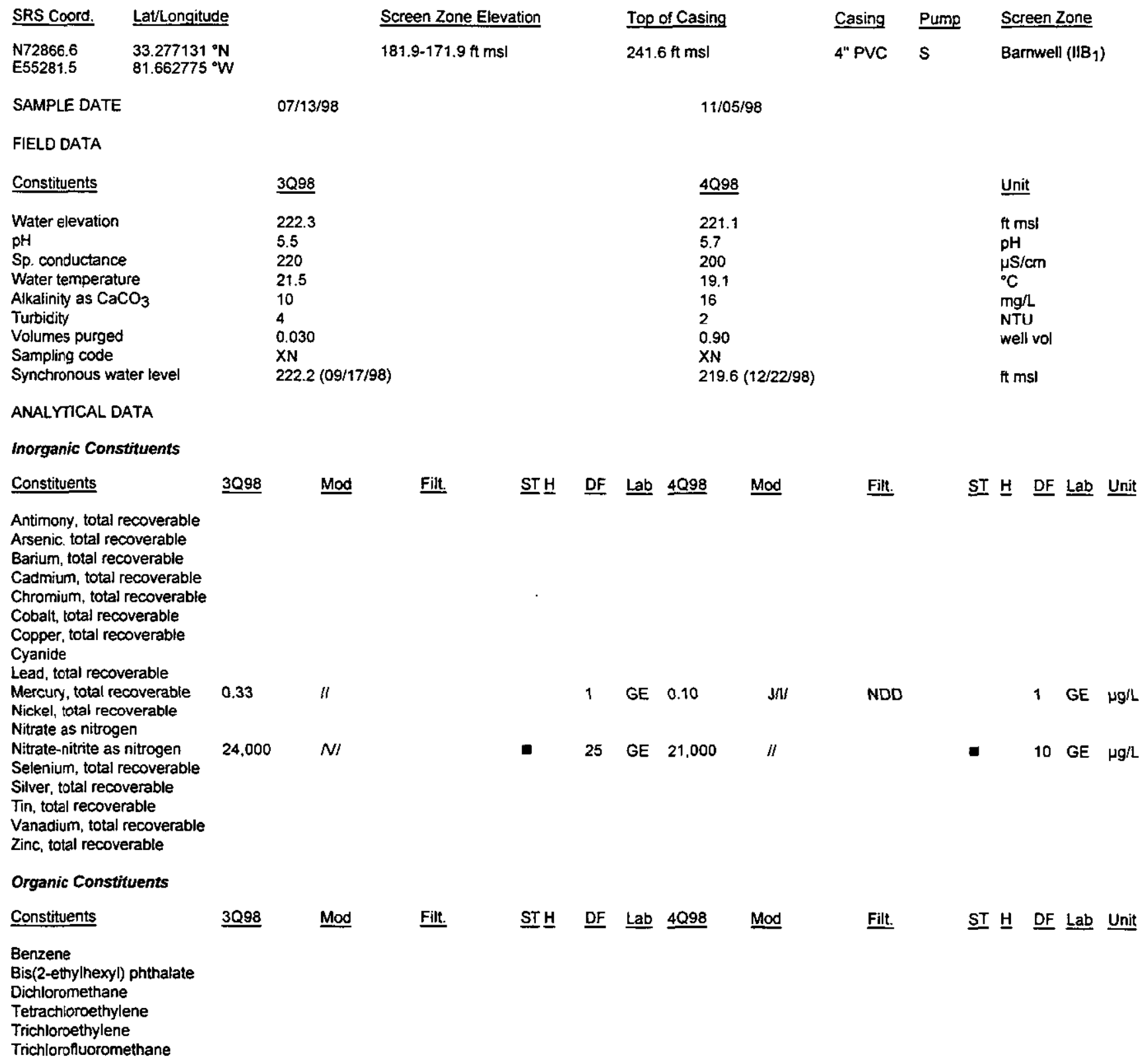

Notes:

$\bullet=$ exceeded holding time

= exceeded groundwater protection or monitoring constituent standard (See Appendix A.) 
WELL HSB 71C (cont.)

Radioactive Constituents

\begin{tabular}{|c|c|c|c|c|c|c|c|c|c|c|c|c|c|}
\hline Constituents & 3098 & Mod & Fijt. & $\underline{\text { ST보 }}$ & DF & $\underline{\text { Lab }}$ & $4 Q 98$ & Mod & Filt. & $\underline{\text { ST }} \underline{H}$ & $\underline{D F}$ & Lab & Unit \\
\hline $\begin{array}{l}\text { Americium-241 } \\
\text { Carbon-14 } \\
\text { Cobalt-60 } \\
\text { Curium-242 } \\
\text { Curium-243/244 } \\
\text { Curium-245/246 }\end{array}$ & & & & & & & & & & & & & \\
\hline $\begin{array}{l}\text { Gross alpha } \\
\text { lodine-129 } \\
\text { Nickel-63 }\end{array}$ & $4.8 \mathrm{E}+00$ & $\|$ & & & 1 & GP & $<4.2 E+00$ & $\mathrm{U} / /$ & $<2.2 \mathrm{E}+00$ & & 1 & GE & $\mathrm{pCi} / \mathrm{L}$ \\
\hline $\begin{array}{l}\text { Nonvolatile beta } \\
\text { Plutonium-238 } \\
\text { Plutonium-239/240 } \\
\text { Radium-226 } \\
\text { Radium-228 } \\
\text { Strontium-90 } \\
\text { Technetium-99 } \\
\text { Thorium-228 } \\
\text { Thorium-230 } \\
\text { Thorium-232 } \\
\text { Sum of alphas } \\
\text { Sum of betas }\end{array}$ & $2.3 E+01$ & $\|$ & & & 1 & GP & $2.7 E+01$ & $1 /$ & & & 1 & GE & $\mathrm{pCi} / \mathrm{L}$ \\
\hline $\begin{array}{l}\text { Tritium } \\
\text { Uranium-233/234 } \\
\text { Uranium-235 } \\
\text { Uranium-238 }\end{array}$ & $2.5 E+03$ & "l & & - & 1 & $\mathrm{GP}$ & $2.4 E+03$ & $\|$ & & - & 1 & GE & $\mathrm{pCi} / \mathrm{mL}$ \\
\hline
\end{tabular}

Notes:

- exceeded holding time

- = exceeded groundwater protection or monitoring constituent standard (See Appendix A.) 
WELL HSB 83A

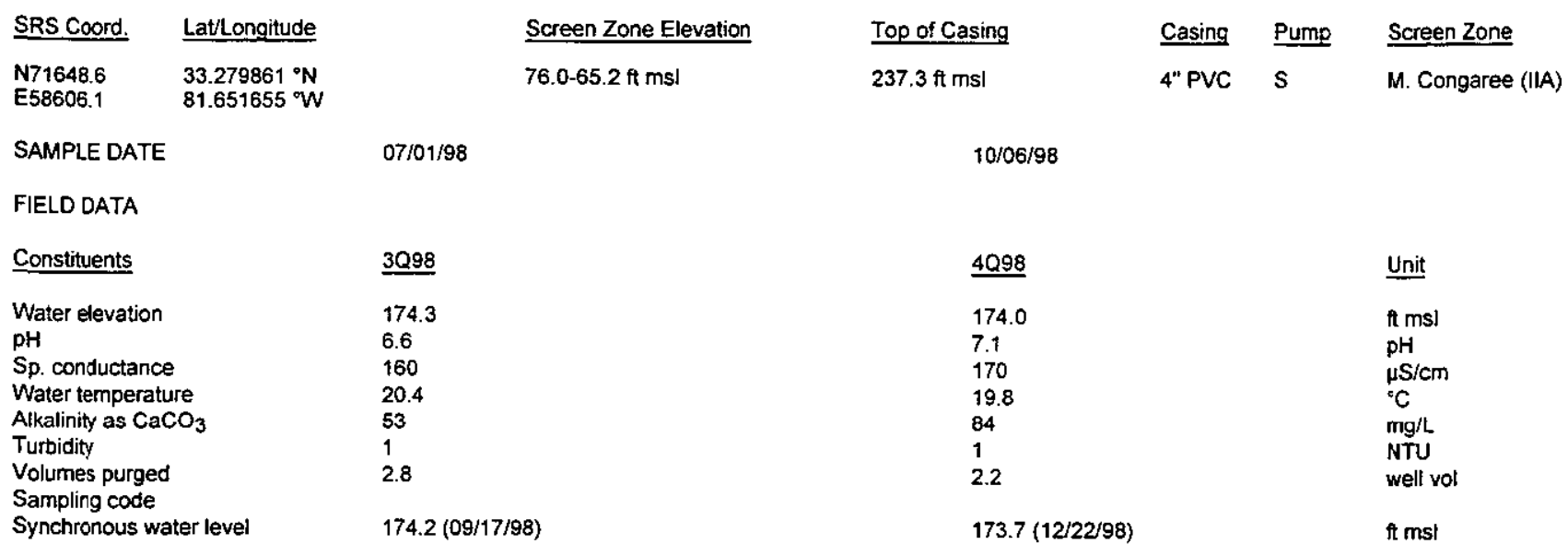

ANALYTICAL DATA

Inorganic Constituents

Constituents

Mod

Filt.

DF Lab $\underline{4 Q 98}$

Mod

Filt.

ST $\underline{H}$ DF Lab Unit

Antimony, total recoverable

Arsenic, total recoverable

Barium, total recoverable

Cadmium, total recoverable

Chromium, total recoverable

Cobalt, total recoverable

Copper, total recoverable

Cyanide

Lead, total recoverable

Mercury, total recoverable

Nickel, total recoverable

Nitrate as nitrogen

Nitrate-nitrite as nitrogen

Selenium, total recoverable

U/I

$<0.20$

1 GE $<0.20$

JU/L/C

$<2.0$

1 GE $\mu g / L$

Silver, total recoverable

20

$J / E$

NDD

1 GE $<20$

UNI

$<50$

$\uparrow$ GE $\mu g / L$

Tin, total recoverable

Vanadium, total recoverable

Zinc, total recoverable

Organic Constituents

Constituents

Filt.

ST브

DF Lab 4098

Mod

Filt.

ST $\underline{H}$ DF Lab Unit

Benzene

Bis(2-ethylhexyl) phthalate

Dichloromethane

Tetrachloroethylene

Trichloroethylene

Trichlorofluoromethane

Notes:

- exceeded holding time

= exceeded groundwater protection or monitoring constituent standard (See Appendix A.) 
WELL HSB 83A (cont.)

Radioactive Constituents

Constituents

Americium-241

Carbon-14

Cobalt-60

Curium-242

Curium-243/244

Curium-245/246

Gross alpha

lodine-129

Nickel-63

Nonvolatile beta

Plutonium-238

Plutonium-239/240

Radium-226

Radium-228

Strontium-90

Technetium-99

Thorium-228

Thorium-230

Thorium-232

Sum of alphas

Sum of betas

Tritium

Uranium-233/234

Uranium-235

Uranium-238 $\underline{3098}$

Mod

Filt.

ST브 DF Lab $\underline{4 Q 98}$ Mod

Filt.

ST 브 DF Lab Unit

$1.0 E+00$

$1.5 \mathrm{E}+00$

$1 \quad G P \quad<4,8 E-01$

U/l

$<8.0 E-01$

U/I

$<1.2 \mathrm{E}+00$

1 GE $\mathrm{pCi} / \mathrm{L}$

1 GE pCi/L

$<2.4 \mathrm{E}-02 \quad \mathrm{U} \mathrm{I} /$

$<5.8 E-01$

$1 \quad$ GP $\quad<-1.4 E-01$

$\mathrm{U} / \prime$

$<6.5 \mathrm{E}-01$

$1 \mathrm{GE} \mathrm{pCi} / \mathrm{mL}$

Notes:

- = exceeded holding time

- = exceeded groundwater protection or monitoring constituent standard (See Appendix A.) 
WELL HSB 83B

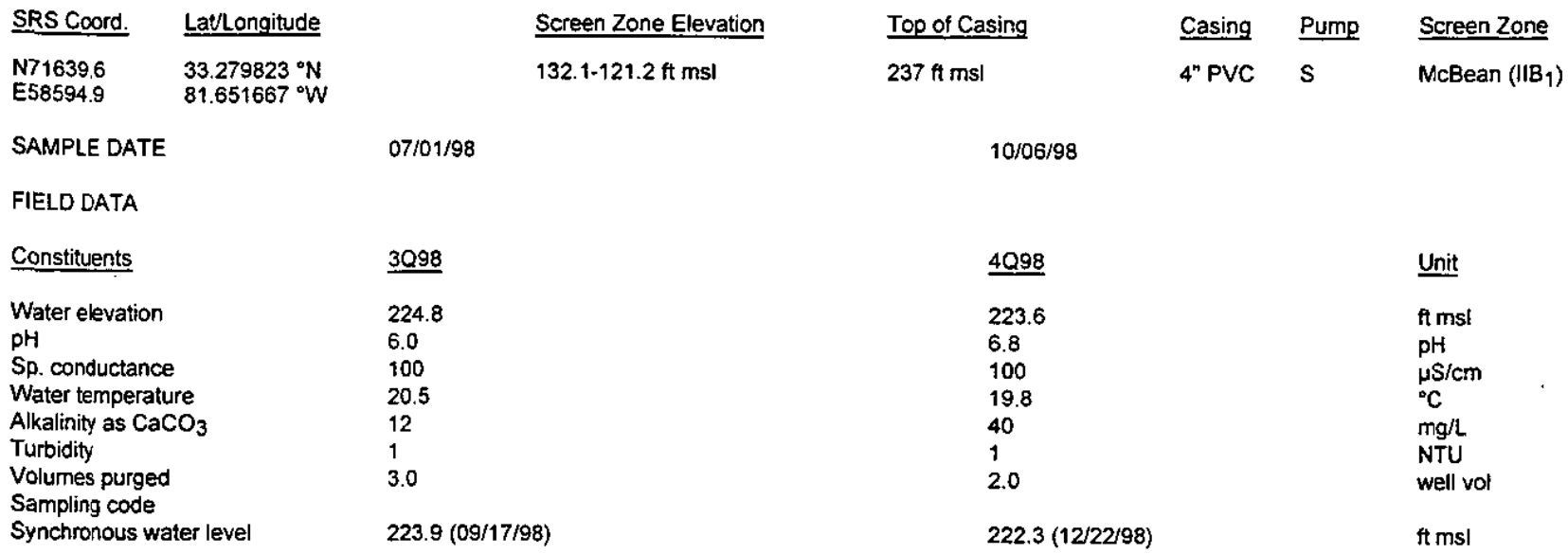

ANALYTICAL DATA

Inorganic Constituents

Constituents

Antimony, total recoverable

Arsenic, total recoverable

Barium, total recoverable

Cadmium, total recoverable

Chromium, total recoverable

Cobalt, total recoverable

Copper, total recoverable

Cyanide

Lead, total recoverable

Mod

Filt.

ST $\underline{\text { H DF Lab Unit }}$

Mercury, total recoverable

Nickel, total recoverable

Nitrate as nitrogen

Nitrate-nitrite as nitrogen

Selenium, total recoverable

Silver, total recoverable

Tin, total recoverable

Vanadium, total recoverable

Zinc, total recoverable

Organic Constituents

Canstituents

NDD

1

GE

$<0.20$

$\mathrm{JU} / \mathrm{L} / \mathrm{C}$

1 GE $<40$

UN/

$<50$

Bis(2-ethylhexyl) phthalate

Dichloromethane

Tetrachtoroethylene

Trichloroethylene

Trichlorofluoromethane

Notes:

- exceeded holding time

= exceeded groundwater protection or monitoring constituent standard (See Appendix A.) 


\section{WELL HSB 83B (cont.)}

Radioactive Constituents

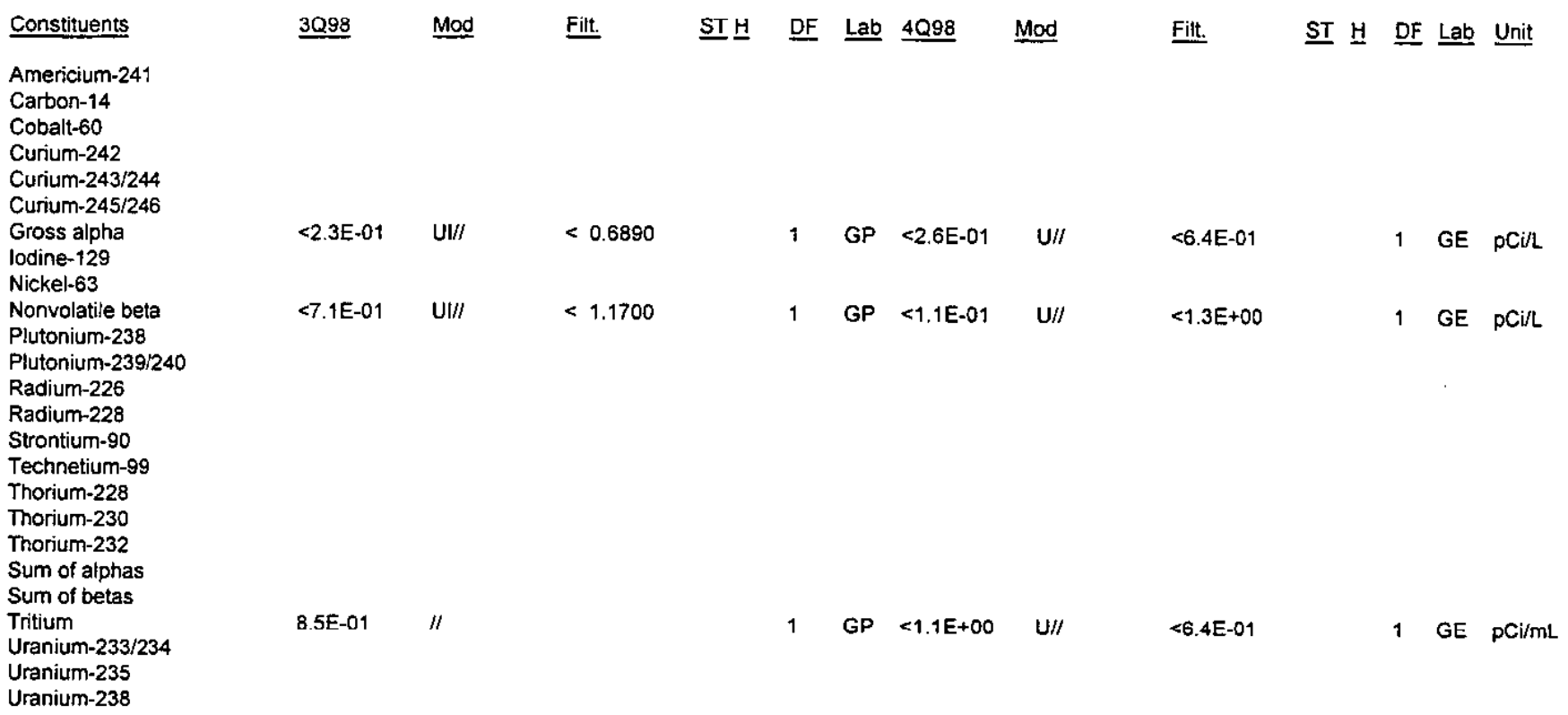

Notes:

- = exceeded holding time

- exceeded groundwater protection or monitoring constituent standard (See Appendix A.) 
WELL HSB 83C

$\begin{array}{lll}\text { SRS Coord. } & & \text { Latlongitude } \\ \text { N71636.9 } & & 33.279849^{\circ} \mathrm{N} \\ \text { E58614.8 } & & 81.651609^{\circ}\end{array}$

SAMPLE DATE

FIELD DATA

Constituents

Water elevation

$\mathrm{pH}$

Sp. conductance

Water temperature

Alkalinity as $\mathrm{CaCO}_{3}$

Turbidity

Volumes purged

Sampling code

Synchronous water level

ANALYTICAL DATA

Inorganic Constituents

\section{Constituents}

Antimony, total recoverable

Arsenic, total recoverable

Barium, total recoverable

Cadmium, total recoverable

Chromium, total recoverable

Cobalt, total recoverable

Copper, total recoverable

Cyanide

Lead, total recoverable

Mercury, total recoverable

Nickel, total recoverable

Nitrate as nitrogen

Nitrate-nitrite as nitrogen

Selenium, total recoverable

Silver, total recoverable

Tin, total recoverable

Vanadium, total recoverable

Zinc, total recoverable

Organic Constituents

Constituents

Benzene

Bis(2-ethylhexyl) phthalate

Dichloromethane

Tetrachloroethylene

Trichloroethylene

Trichlorofluoromethane

DF Lab $\underline{4 Q 98}$
Screen Zone Elevation

$171.2-160.2 \mathrm{ft} \mathrm{ms}$

07/01/98
3098

227.0

4.8

22

21.2

2

3.7

$225.9(09 / 17 / 98)$
Top of Casing

$237.1 \mathrm{ft} \mathrm{msl}$

$10 / 06 / 98$

Mod

Filt.

ST브

$\underline{\text { Mod }}$

1 GE $<0.20$

JU/LC

$<0.20$

1 GE 60

/l

J/E $\quad$ NDD

50

4Q98

225.6

5.1
23

20.3

2

2.2

$224.2(12 / 22 / 98)$

Casing

Pump

4" PVC S

Screen Zone

Barnwell (IIB1)

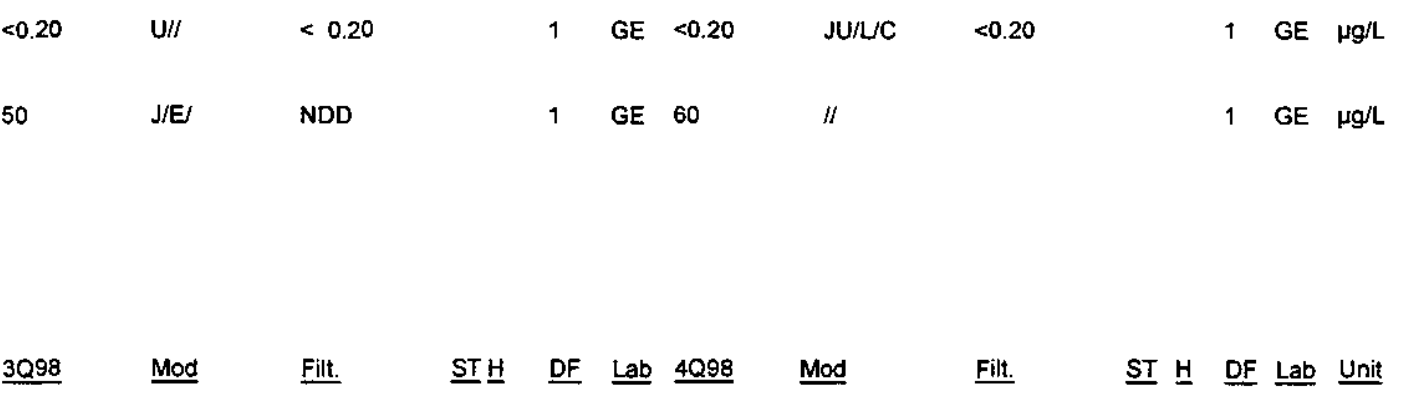

Notes:

- exceeded holding time

- exceeded groundwater protection or monitoring constituent standard (See Appendix A.) 
WELL HSB $83 \mathrm{C}$ (cont.)

Radioactive Constituents

Constituents

Americium-241

Carbon-14

Cobalt-60

Curium-242

Curium-243/244

Curium-245/246

Gross alpha

lodine-129

Nickel-63

Nonvolatile beta

Plutonium-238

Plutonium-239/240

Radium-226

Radium-228

Strontium-90

Technetium-99

Thorium-228

Thorium-230

Thorium-232

Sum of alphas

Sum of betas

Tritium

Uranium-233/234

Uranium-235

Uranium-238
3098

Mod

Filt.

ST브 DF Lab 4 Q998

Mod

1 GP $<4.0 E-01$

U/I

$1 \quad \mathrm{GP}<4.8 \mathrm{E}-01 \quad \mathrm{U} / /$

$<1.0700$

$<8.6 \mathrm{E}-01$

$\mathrm{U} \mathrm{J} /$

1.0700

$<1.3 E+00$

$1 \mathrm{GE} \quad \mathrm{pCi} / \mathrm{L}$

1 GE $\mathrm{pCi} / \mathrm{L}$

$<2.5 E-01 \quad$ Ui $/ 1 \quad<5.9 \mathrm{E}-01$

1 GP $\quad<7.0 E-02$

$\mathrm{u} / /$

$<6.4 \mathrm{E}-01$

$1 \mathrm{GE} \quad \mathrm{pCi} / \mathrm{mL}$

Notes:

- =xceeded holding time

- = exceeded groundwater protection or monitoring constituent standard (See Appendix A.) 
WELL HSB 83D

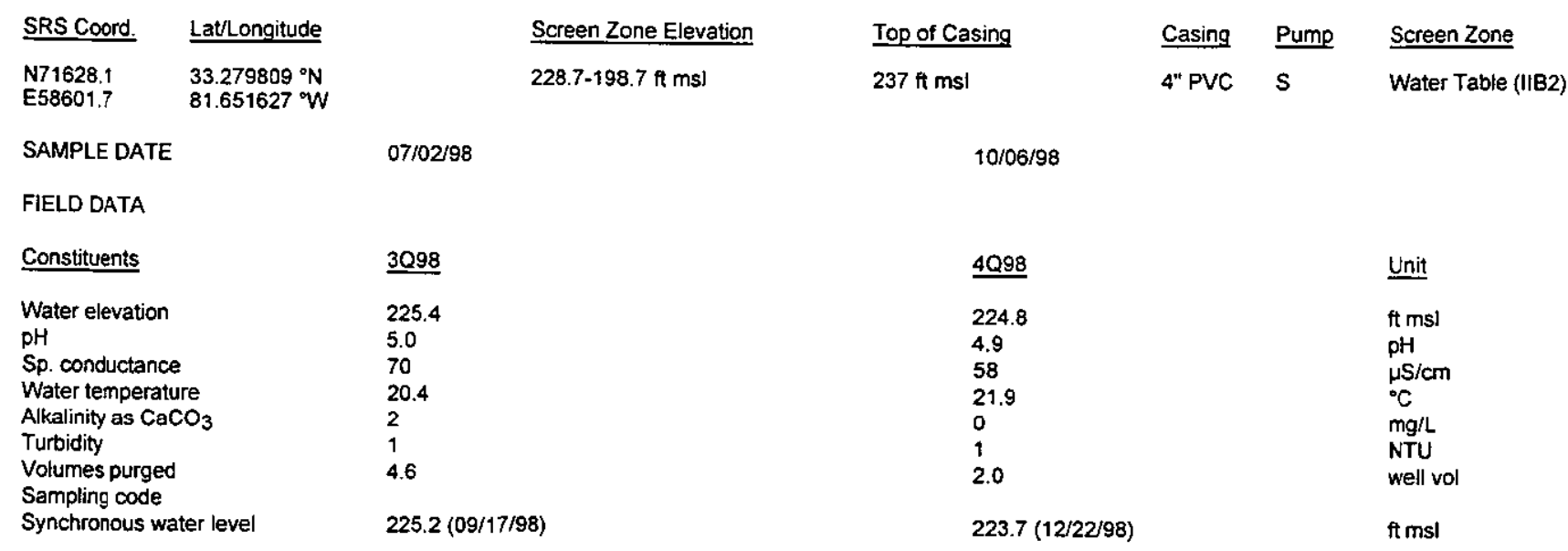

\section{ANALYTICAL DATA}

Inorganic Constituents

\section{Constituents}

Antimony, total recoverable

Arsenic, total recoverable

Barium, total recoverable

Cadmium, total recoverable

Chromium, total recoverable

Cobalt, total recoverable

Copper, total recoverable

Cyanide

Lead, total recoverable

Mercury, total recoverable

Nickel, total recoverable

Nitrate as nitrogen

Nitrate-nitrite as nitrogen

Selenium, total recoverable

Silver, total recoverable

Tin, total recoverable

Vanadium, total recoverable

Zinc, total recoverable

Organic Constituents

Constituents

$\underline{3098}$

Benzene

Bis(2-ethyinexyl) phthalate

Dichloromethane

Tetrachloroethylene

Trichloroethylene

Trichlorolluoromethane

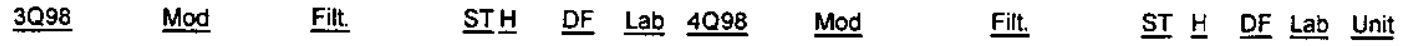

\begin{tabular}{|c|c|c|c|c|c|c|c|c|}
\hline$<0.45$ & $\mathrm{UJ} / \mathrm{V} / 1$ & $<0.20$ & 1 & GE & $0.93 \mathrm{~J} / \mathrm{L} / \mathrm{C}$ & NDD & 1 & GE \\
\hline 3,500 & $N I$ & & & 2 & GE $\quad 4,200$ & "l & 3 & GE \\
\hline
\end{tabular}

Mod

Filt.

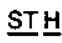

$\underline{\text { DF }} \underline{\text { Lab }} \underline{4 Q 98}$

Mod

Filt.

$\underline{\text { ST }} \underline{H} \underline{\text { DF Lab }} \underline{\text { Unit }}$

Notes:

- exceeded holding time

= exceeded groundwater protection or monitoring constituent standard (See Appendix A.) 


\section{WELL HSB 83D (cont.)}

\section{Radioactive Constituents}

Constituents

Americium-241

Carbon-14

Cobalt-60

Curium-242

Curium-243/244

Curium-245/246

Gross alpha

lodine-129

Nickel-63

Nonvolatile beta

Plutonium-238

Plutonium-239/240

Radium-226

Radium-228

Strontium-90

Technetium-99

Thorium-228

Thorium-230

Thorium-232

Sum of alphas

Sum of betas

Tritium

Uranium-233/234

Uranium-235

Uranium-238
3098

$1.7 \mathrm{E}+00$

4.3E+01 /

3.7E+02
$1 \quad G P<9.4 E-01 \quad U / /$

1 GP $\quad 2.2 \mathrm{E}+01 \quad / /$

GP $1.9 \mathrm{E}+02 \quad / 1$
Filt.

ST 브 DF Lab Unit
$1 \mathrm{GE} \mathrm{PCi/L}$

1 GE $\mathrm{pCi} / \mathrm{L}$

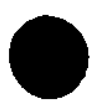

ST브 DF Lab $4098 \quad$ Mod

\author{
Lab Unit
}$$
\mathrm{PCi} / \mathrm{L}
$$

$1.7 \mathrm{E}+02 \quad$




\section{WELL HSB 84A}

\begin{tabular}{|c|c|}
\hline SRS Coord. & Lat/Longitude \\
\hline $\begin{array}{l}\text { N71586.2 } \\
\text { E56359.1 }\end{array}$ & $81.657450^{\circ}$ \\
\hline
\end{tabular}

Screen Zone Elevation

$75.9-64.7 \mathrm{ft} \mathrm{ms}$

07/01/98

$\underline{3098}$

Constituents

Water elevation

pH

Sp. conductance

Water temperature

Alkalinity as $\mathrm{CaCO}_{3}$

Turbidity

Volumes purged

Sampling code

Synchronous water level

ANALYTICAL DATA

Inorganic Constituents

Constituents

Antimony, total recoverable

Arsenic, total recoverable

Barium, total recoverable

Cadmium, total recoverable

Chromium, total recoverable

Cobalt, total recoverable

Copper, total recoverable

Cyanide

Lead, total recoverable

Mercury, total recoverable

Nickel, total recoverable

Nitrate as nitrogen

Nitrate-nitrite as nitrogen

Selenium, total recoverable

$\mathrm{U} / 1$

Silver, total recoverable

Tin, total recoverable

Vanadium, total recoverable

Zinc, total recoverable

Organic Constituents

Constituents

Benzene

Bis(2-ethylhexyl) phthalate

Dichloromethane

Tetrachloroethylene

Trichloroethylene

Trichlorofluoromethane
173.0

5.9

28

$172.9(09 / 17 / 98)$

\section{Top of Casing}

$228.7 \mathrm{ft} \mathrm{ms}$

$10 / 27 / 98$

$\underline{4098}$

172.6

6.5

100

19.8

38

2.4

$172.2(12 / 22 / 98)$

Casing Pump

4"PVC V

Screen Zone

L. Congaree (IIA)
$\mathrm{J} / \mathrm{E}$

NDD

$1 \quad$ GE $\quad 0.062$

J/II

GE 100

/I

Mod $\quad$ Filt.

\section{ST브}


WELL HSB 84A (cont.)

Radioactive Constituents

Constituents

Americium-241

Carbon-14

Cobalt-60

Curium-242

Curium-243/244

Curium-245/246

Gross alpha

lodine-129

Nickel-63

Nonvolatile beta

Plutonium-238

Plutonium-239/240

Radium-226

Radium-228

Strontium-90

Technetium-99

Thorium-228

Thorium-230

Thorium-232

Sum of alphas

Sum of betas

Tritium

Uranium-233/234

Uranium-235

Uranium-238
309

8.6E-01

/I

$1.7 E+01 / /$

1

GP $\quad 2.2 \mathrm{E}+\infty \quad \mathrm{J} / /$

1

GP $\quad 2.6 \mathrm{E}+01 \quad /$

4.5E+00 $/ 1$

1 GP $4.0 \mathrm{OE}+\infty \quad / /$
NDD

$1 \mathrm{TM} \mathrm{pCi/}$

$1 \mathrm{TM} \mathrm{PCi/l}$

Filt. $\quad$ ST $\underline{H}$ DF Lab $\underline{\text { Unit }}$

$\mathrm{TM} \mathrm{pCi} / \mathrm{mL}$

Notes:

- exceeded holding time

E = exceeded groundwater protection or monitoring constituent standard (See Appendix A.) 
WELL HSB 84B

$\begin{array}{ll}\text { SRS Coord. } & \text { Lat/longitude } \\ \text { N71603.3 } & 33.276084^{\circ} \mathrm{N} \\ \text { E56352.4 } & 81.657501^{\circ} \mathrm{W}\end{array}$

SAMPLE DATE

FIELD DATA

Constituents

Water elevation

$\mathrm{pH}$

$\mathrm{Sp}$. conductance

Water temperature

Alkatinity as $\mathrm{CaCO}_{3}$

Turbidity

Volumes purged

Sampling code

Synchronous water level

ANALYTICAL DATA

Inorganic Constituents

Constituents

$\underline{3098}$

211.1

7.0

170

21.6

51

1.2

$210.7(09 / 17 / 98)$
Screen Zone Elevation

$132.9-121.8 \mathrm{ft} \mathrm{ms|}$

Top of Casing

$228.9 \mathrm{ft} \mathrm{msl}$

$10 / 05 / 98$

4Q98

210.7

8.5

150

20.1

73

0.034

$\mathrm{XN}$

209.7 (12/22/98)
Casing Pump

4" PVC S
Screen Zone

McBean ( $\left.\mathrm{IBB}_{1}\right)$

Antimony, total recoverable

Arsenic, total recoverable

Barium, total recoverable

Cadmium, total recoverable

Chromium, total recoverable

Cobalt, total recoverable

Copper, total recoverable

Cyanide

Lead, total recoverable

Mercury, total recoverable

Nickel, total recoverab!e

$<0.20$

UII

$<0.20$

1

GE $<0.20$

JU/LC

1 GE 1,200

/I

Filt.

ST $\underline{H}$ DF Lab Unit

Nitrate-nitrite as nitrogen

Selenium, total recoverable

600

/l

Silver, total recoverable

Tin, total recoverable

Vanadium, total recoverable

Zinc, total recoverable

Organic Constituents

Constituents

Mod

Filt.

STH

DF $\underline{\text { Lab }}$

$4 Q 98$

Mod

Filt.

1 GE $\mu g / L$

1 GE $\mu g / L$

Bis(2ethylhexyl) phthalate

Dichloromethane

Tetrachloroethylene

Trichloroethylene

Trichlorofluoromethane

Notes:

- = exceeded holding time

- exceeded groundwater protection or monitoring constituent standard (See Appendix A.) 
WELL HSB 84B (cont.)

Radioactive Constituents

Constituents

Americium-241

Carbon-14

Cobalt-60

Curium-242

Curium-243/244

Curium-245/246

Gross alpha

lodine-129

Nickel-63

Nonvolatile beta

Plutonium-238

Plutonium-239/240

Radium-226

Radium-228

Strontium-90

Technetium-99

Thorium-228

Thorium-230

Thorium-232

Sum of alphas

Sum of betas

Tritium

Uranium-233/234

Uranium-235

Uranium-238
3Q98

$<3.3 E-01$

$\mathrm{U} \mathrm{I} /$

$<0.7290$

$3.8 E+00$

/l

2.1E+01 /

Mod

Filt.

ST브 DF Lab 4 Q98

Mod

1

GP $<6.6 \mathrm{E}-01 \quad \mathrm{U} / /$

$1 \quad G P \quad 5.2 E+00$

/
1 GE pCi/l

1 GE $\mathrm{PCi} / \mathrm{L}$

$\underline{\text { ST }} \underline{H}$ DF Lab Unit

Filt.

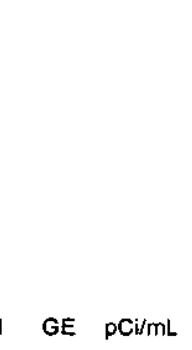

Notes:

- = exceeded holding time

- = exceeded groundwater protection or monitoring constituent standard (See Appendix A.) 
WELL HSB 84C

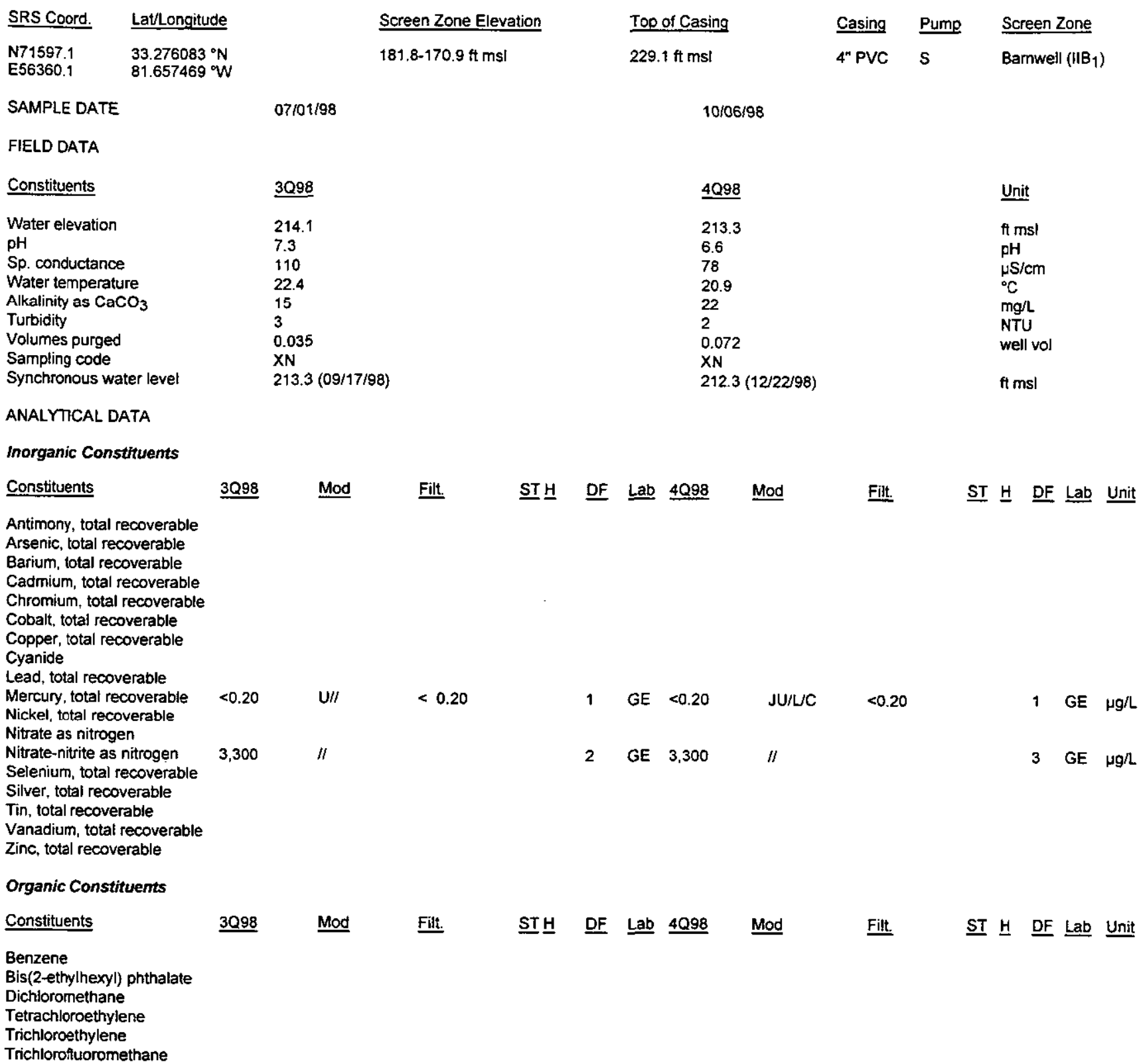




\section{WELL HSB $84 C$ (cont.)}

\section{Radioactive Constituents}

Constituents

3098

Filt.

ST브 DF Lab 4098 Mod

Filt.

ST $\underline{H}$ DF Lab Unit

Americium-241

Carbon-14

Cobait-60

Curium-242

Curium-243/244

Curium-245/246

Gross alpha

lodine-129

Nickel-63

Nonvolatile beta

Plutonium-238

Plutonium-239/240

Radium-226

Radium-228

Strontium-90

Technetium-99

Thorium-228

Thorium-230

Thorium-232

Sum of alphas

Sum of betas

Tritium

Uranium-233/234

$<5.1 \mathrm{E}-01 \quad \mathrm{UI} / l \quad<0.6280$

$9.0 E+00 \quad / 1$

Uranium-235

Uranium-238

Notes:

- exceeded holding time

- exceeded groundwater protection or monitoring constituent standard (See Appendix A.) 
WELL HSB 84D

\begin{tabular}{|c|c|c|c|}
\hline SRS Coord. & Lat/Longitude & & Screen Zone Elevation \\
\hline $\begin{array}{l}\text { N71583.9 } \\
\text { E56349.9 }\end{array}$ & $\begin{array}{l}33.276037^{\circ} \mathrm{N} \\
81.657470^{\circ} \mathrm{W}\end{array}$ & & $219.5-199.5 \mathrm{ft} \mathrm{msl}$ \\
\hline \multicolumn{2}{|c|}{ SAMPLE DATE } & \multicolumn{2}{|l|}{$07 / 08 / 98$} \\
\hline \multicolumn{4}{|c|}{ FIELD DATA } \\
\hline \multicolumn{2}{|l|}{ Constituents } & \multicolumn{2}{|l|}{$\underline{3098}$} \\
\hline \multicolumn{2}{|c|}{ Water elevation } & \multicolumn{2}{|l|}{218.4} \\
\hline \multirow{2}{*}{\multicolumn{2}{|c|}{$\mathrm{pH}$}} & \multicolumn{2}{|l|}{4.2} \\
\hline \multirow{2}{*}{\multicolumn{2}{|c|}{$\begin{array}{l}\text { Sp. conductance } \\
\text { Water temperatire }\end{array}$}} & \multicolumn{2}{|l|}{50} \\
\hline & & \multicolumn{2}{|l|}{19.4} \\
\hline \multicolumn{2}{|c|}{ Alkalinity as $\mathrm{CaCO}_{3}$} & \\
\hline \multicolumn{2}{|c|}{ Turbidity } & \multicolumn{2}{|l|}{1} \\
\hline \multicolumn{2}{|c|}{$\begin{array}{l}\text { Volumes purged } \\
\text { Sampling code }\end{array}$} & \multicolumn{2}{|l|}{4.8} \\
\hline \multicolumn{2}{|c|}{$\begin{array}{l}\text { Sampling coce } \\
\text { Synchronous water level }\end{array}$} & \multicolumn{2}{|c|}{$217.9(09 / 17 / 98)$} \\
\hline
\end{tabular}

$\begin{array}{llll}\text { Top of Casing } & \text { Casing } & \text { Pump } & \text { Screen Zone } \\ 228.8 \mathrm{ft} \mathrm{msl} & \text { 4" PVC } & \text { V } & \text { Water Table (IIB2) }\end{array}$

$10 / 13 / 98$

$\begin{array}{ll}4098 & \text { Unit } \\ 217.6 & \mathrm{ft} \mathrm{msl} \\ 4.1 & \mathrm{pH} \\ 45 & \mu \mathrm{S} / \mathrm{cm} \\ 19.8 & { }^{\circ} \mathrm{C} \\ 0 & \mathrm{mg} / \mathrm{L} \\ 0 & \mathrm{NTU} \\ 3.4 & \text { well vol } \\ 217.2(12 / 22 / 98) & \mathrm{ft} \mathrm{msl}\end{array}$

ANALYTICAL DATA

Inorganic Constituents

\begin{tabular}{|c|c|c|c|c|c|c|c|c|c|c|c|c|c|}
\hline Constituents & $\underline{3098}$ & Mod & Filt. & $\underline{\text { ST }} \underline{H}$ & $\underline{\text { DF }}$ & $\underline{\text { Lab }}$ & 4098 & $\underline{\text { Mod }}$ & Filt. & $\underline{\text { ST }} \underline{H}$ & $\underline{\mathrm{DF}}$ & $\underline{\text { Lab }}$ & $\underline{\text { Unit }}$ \\
\hline Antimony, total recoverable & $<0.20$ & $\mathrm{U} / /$ & $<0.20$ & & 1 & GE & & & & & & & $\mu g / \mathrm{L}$ \\
\hline Arsenic, total recoverable & $<3.0$ & $\mathrm{U} / /$ & $<3.0$ & & 1 & GE & & & & & & & yg/L \\
\hline Barium, total recoverable & 7.3 & $\|$ & & & 1 & GE & & & & & & & $\mu \mathrm{g} / \mathrm{L}$ \\
\hline Cadmium, total recoverable & $<1.0$ & $\mathrm{U} / /$ & $<1.0$ & & 1 & $\mathrm{GE}$ & & & & & & & $\mathrm{Hg} / \mathrm{L}$ \\
\hline Chromium, total recoverable & 0.65 & $J / E J$ & NDD & & 1 & GE & & & & & & & $\mu g / \mathrm{t}$ \\
\hline Cobalt, total recoverable & 1.0 & "I & & & 3 & GE & & & & & & & $\mu g / \mathrm{h}$ \\
\hline Copper, total recoverable & $<3.9$ & UNI & $<0.20$ & & $i$ & GE & & & & & & & $\mu g / L$ \\
\hline Cyanide & $<10$ & $\mathrm{U} / \mathrm{I}$ & $<10$ & & 1 & $\mathrm{GE}$ & & & & & & & Ho/L \\
\hline Lead, total recoverable & $<0.092$ & $\mathrm{U} / N /$ & $<2.0$ & & 1 & GE & & & & & & & $\mu \mathrm{g} / \mathrm{L}$ \\
\hline Mercury, total recoverable & $<0.20$ & $\mathrm{U} / \mathrm{f}$ & $<0.20$ & & 1 & $\mathrm{GE}$ & 0.13 & $\mathrm{~J} / \mathrm{l} /$ & NDD & & 1 & GE & $\mu g / L$ \\
\hline $\begin{array}{l}\text { Nickel, total recoverable } \\
\text { Nitrate as nitrogen }\end{array}$ & $<3.4$ & UNI & $<0.20$ & & 1 & GE & & . & (100 & & , & 는 & $\mu g / 2$ \\
\hline Nitrate-nitrite as nitrogen & 2,800 & NI & & & 1 & GE & 2,000 & $" 1$ & & & 1 & GE & $\mu g / L$ \\
\hline Selenium, total recoverable & $<5.0$ & $\mathrm{U} / /$ & $<5.0$ & & 1 & $\overline{G E}$ & & & & & & & $\mu g / \mathrm{L}$ \\
\hline Silver, total recoverable & $<1.0$ & $\mathrm{U} / \mathrm{I}$ & $<1.0$ & & 1 & $\mathrm{GE}$ & & & & & & & $\mu g / \mathrm{h}$ \\
\hline Tin, total recoverable & $<0.13$ & UNI & $<2.0$ & & 1 & GE & & & & & & & $\mu \mathrm{g} / \mathrm{L}$ \\
\hline Vanadium, total recoverable & $<2.0$ & $\mathrm{U} / /$ & $<2.0$ & & 1 & $\mathrm{GE}$ & & & & & & & $\mu g / \mathrm{L}$ \\
\hline Zinc, total recoverable & $<10$ & $\mathrm{UNI}$ & $<5.0$ & & 1 & $\mathrm{GE}$ & & & & & & & $\mu g / L$ \\
\hline \multicolumn{14}{|l|}{ Organic Constituents } \\
\hline Constituents & $\underline{3098}$ & Mod & Filt. & $\underline{\text { ST }} \underline{H}$ & $\underline{\text { DF }}$ & $\underline{\text { Lab }}$ & $\underline{4 Q 98}$ & Mod & Filt. & $\underline{\text { ST }} \underline{H}$ & $\underline{\mathrm{DF}}$ & Lab & Unit \\
\hline Benzene & $<1.0$ & UJ/O/1 & $<1.0$ & & 1 & $\mathrm{GE}$ & & & & & & & $\mu \mathrm{g} / \mathrm{h}$ \\
\hline Bis(2-ethylhexyl) phthalate & $<9.9$ & $\mathrm{U} / /$ & $<9.9$ & & 1 & GE & & & & & & & $\mu \mathrm{g} / \mathrm{h}$ \\
\hline Dichloromethane & $<2.4$ & UJ/OV/t & $<1.0$ & & 1 & GE & & & & & & & $\mu g / L$ \\
\hline Tetrachloroethylene & 0.76 & $\mathrm{~J} / \mathrm{EO} / 1$ & NDD & & 1 & GE & & & & & & & $\mu \mathrm{g} / \mathrm{L}$ \\
\hline Trichloroethylene & $<1.0$ & UJ $/ O / 1$ & $<1.0$ & & 1 & $\mathrm{GE}$ & & & & & & & $\mu g / L$ \\
\hline Trichlorofluoromethane & $<1.0$ & UJ/O/1 & $<1.0$ & & 1 & $\mathrm{GE}$ & & & & & & & $\mu g / L$ \\
\hline
\end{tabular}

Notes:

- = exceeded holding time

- exceeded groundwater protection or monitoring constituent standard (See Appendix A.) 
WELL HSB 84D (cont.)

Radioactive Constituents

\begin{tabular}{|c|c|c|c|c|c|c|c|c|c|c|c|c|c|}
\hline Constituents & $\underline{3 Q 98}$ & Mod & Filt. & $\underline{\text { ST }} \underline{\underline{H}}$ & $\underline{\mathrm{DF}}$ & $\underline{\text { Lab }}$ & 4Q98 & Mod & Filt. & $\underline{\text { ST }} \underline{H}$ & DF & $\underline{L a b}$ & Unit \\
\hline Americium-241 & $<5.8 \mathrm{E}-02$ & UIII & $<0.1180$ & & 1 & GP & & & & & & & $\mathrm{pCi} / \mathrm{L}$ \\
\hline Beta dose & 16.46 & & & - & & & & & & & & & $\mathrm{pCi} / \mathrm{L}$ \\
\hline Carbon-14 & $<-4.7 E-01$ & $\mathrm{UH} / /$ & $<8.9500$ & & 1 & GP & & & & & & & $\mathrm{pCi} / \mathrm{L}$ \\
\hline Cobalt- 60 & $6.9 E+00$ & $/ 1$ & & & 1 & GP & & & & & & & $\mathrm{PCi} / \mathrm{L}$ \\
\hline Curium-242 & $<3.0 \mathrm{E}-02$ & $\mathrm{UI} / /$ & $<0.1240$ & & 1 & GP & & & & & & & pCi/L \\
\hline Curium-243/244 & $<-9.1 E-03$ & $\mathrm{UI} / /$ & $<0.1180$ & & 1 & GP & & & & & & & $\mathrm{pCi} / \mathrm{L}$ \\
\hline Curium-245/246 & $<0.0 E+00$ & $\mathrm{U}] / /$ & $<0.0570$ & & 1 & GP & & & & & & & $\mathrm{pCi} / \mathrm{L}$ \\
\hline Gross alpha & $5.4 E+00$ & 11 & & & 1 & GP & $<2.4 \mathrm{E}+00$ & $\mathrm{U} / /$ & $<7,4 \mathrm{E}-01$ & & 1 & GE & $\mathrm{pCi} / \mathrm{L}$ \\
\hline lodine-129 & $<8.3 E-01$ & $\mathrm{UI} / /$ & $<1.3100$ & & 1 & GP & & & & & & & $\mathrm{pCi} / \mathrm{L}$ \\
\hline Nickel-63 & $6.9 E+00$ & $\|$ & & & & & & & & & & & $\mathrm{pCi} / \mathrm{L}$ \\
\hline Nonvolatile beta & $3.0 \mathrm{E}+02$ & $\|$ & & $\mathbf{\square}$ & 1 & GP & $2.8 E+02$ & $/ /$ & & $\mathbf{\square}$ & 1 & GE & $\mathrm{pCi} / \mathrm{L}$ \\
\hline Plutonium-238 & $<-9.5 E-02$ & $\mathrm{UI} / /$ & $<0.1910$ & & 1 & GP & & & & & & & $\mathrm{pCi} / \mathrm{L}$ \\
\hline Plutonium-239/240 & $<-9.2 \mathrm{E}-03$ & $\mathrm{UH} /$ & $<0.0989$ & & 1 & GP & & & & & & & $\mathrm{pCi} / \mathrm{L}$ \\
\hline Radium-226 & $1.6 E+00$ & $/ /$ & & & 1 & GP & & & & & & & $\mathrm{pCi} / \mathrm{L}$ \\
\hline Radium-228 & $4.0 E+00$ & $\mathrm{~J} / \mathrm{H} / 2$ & NDD & & 1 & GP & & & & & & & $\mathrm{pCi} / \mathrm{L}$ \\
\hline Strontium-90 & $1.3 \mathrm{E}+02$ & $/ 1$ & & $\mathbf{\square}$ & 1 & GP & & & & & & & $\mathrm{pCi} / \mathrm{L}$ \\
\hline Technetium-99 & $<1.4 E+01$ & $\mathrm{UI} / 1$ & $<20.4000$ & & 1 & GP & & & & & & & $\mathrm{pCi} / \mathrm{L}$ \\
\hline Thorium-228 & $<2.8 \mathrm{E}-01$ & $\mathrm{UI} / 1$ & $<0.9530$ & & 1 & GP & & & & & & & $\mathrm{pCi} / \mathrm{L}$ \\
\hline Thorium-230 & $<7.2 \mathrm{E}-02$ & $\mathrm{UI} / /$ & $<0.5010$ & & 1 & GP & & & & & & & $\mathrm{pCi} / \mathrm{L}$ \\
\hline Thorium-232 & $<0.0 \mathrm{E}+00$ & U1/I & $<0.2850$ & & 1 & GP & & & & & & & pCill \\
\hline Sum of alphas & 1.1E+00 & & & & & & & & & & & & $\mathrm{pCi} / \mathrm{L}$ \\
\hline Sum of betas & $1.5 E+02$ & & & 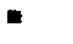 & & & & & & & & & $\mathrm{PCi} / \mathrm{L}$ \\
\hline Total radium & $5.6 E+00$ & & & & & & & & & & & & $\mathrm{pCi} / \mathrm{L}$ \\
\hline Tritium & $1.2 E+02$ & $/ 1$ & & $\boldsymbol{E}$ & 1 & GP & $9.0 \mathrm{E}+01$ & $/ 1$ & & $\mathbf{D}$ & 1 & GE & $\mathrm{pCi} / \mathrm{mL}$ \\
\hline Uranium-233/234 & $6.6 \mathrm{E}-01$ & $N I$ & & & 1 & GP & & & & & & & $\mathrm{pCi} / \mathrm{L}$ \\
\hline Uranium-235 & $<1.1 E-01$ & $\mathrm{UI} / /$ & $<0.1180$ & & 1 & GP & & & & & & & $\mathrm{pCi} / \mathrm{L}$ \\
\hline Uranium-238 & 4.1E-01 & /I & & & 1 & GP & & & & & & & pCill. \\
\hline
\end{tabular}

Notes:

$\bullet=$ exceeded holding time

- = exceeded groundwater protection or monitoring constituent standard (See Appendix A.) 


\section{WELLL HSB 85A}

\begin{tabular}{|c|c|c|c|}
\hline SRS Coord. & Lat/Longitude & & Screen Zone Elevation \\
\hline $\begin{array}{l}N 73791.9 \\
\text { E58943.4 }\end{array}$ & $\begin{array}{l}33.285152^{\circ} \mathrm{N} \\
81.654930^{\circ} \mathrm{W}\end{array}$ & & $71.1-61.1 \mathrm{ft} \mathrm{msl}$ \\
\hline \multicolumn{2}{|c|}{ SAMPLE DATE } & $07 / 08 / 98$ & \\
\hline \multicolumn{4}{|c|}{ FIELD DATA } \\
\hline \multicolumn{2}{|l|}{ Constituents } & $\underline{3098}$ & \\
\hline \multicolumn{2}{|c|}{ Water elevation } & 169.5 & \\
\hline & 7.1 & \\
\hline \multicolumn{2}{|c|}{ Sp. conductance } & 160 & \\
\hline \multicolumn{2}{|c|}{ Water temperature } & 20.9 & \\
\hline \multicolumn{2}{|c|}{ Alkalinity as $\mathrm{CaCO}_{3}$} & 64 & \\
\hline \multicolumn{2}{|c|}{ Turbidity } & 0 & \\
\hline \multicolumn{2}{|c|}{ Volumes purged } & 2.9 & \\
\hline \multicolumn{2}{|c|}{ Synchronous water level } & 169.5109 & \\
\hline
\end{tabular}

$\begin{array}{llll}\text { Top of Casing } & \text { Casing } & \text { Pump } & \text { Screen Zone } \\ 294.4 \mathrm{f} \mathrm{msl} & \text { 4" PVC } & \text { S } & \text { U. Congaree (IIA) }\end{array}$

$11 / 12 / 98$

ANALYTICAL DATA

Inorganic Constituents

\begin{tabular}{|c|c|c|c|c|c|c|c|c|c|c|c|c|c|}
\hline Constituents & $\underline{3098}$ & Mod & Filt. & $\underline{\text { ST }} \underline{H}$ & $\underline{\mathrm{DF}}$ & Lab & $\underline{4 Q 98}$ & Mod & Fill. & ST $\underline{H}$ & $\underline{D F}$ & $\underline{\text { Lab }}$ & $\underline{\text { Unit }}$ \\
\hline Antimony, total recoverable & $<1.0$ & $\mathrm{U} / /$ & $<1.0$ & & 5 & GE & $<27$ & $\mathrm{U} / 1$ & $<27$ & & 1 & WA & $\mu g / L$ \\
\hline Arsenic, total recoverable & & & & & & & $<40$ & $\mathrm{U} / /$ & $<40$ & & 1 & WA & $\mu g / L$ \\
\hline Barium, total recoverable & & & & & & & 29 & "I & & & 1 & WA & $\mu g / L$ \\
\hline Cadmium, total recoverable & $<1.0$ & $\mathrm{U} / /$ & $<1.0$ & & 1 & GE & $<1.0$ & $\mathrm{U} / /$ & $<1.0$ & & 1 & GE & $\mu g / L$ \\
\hline Chromium, total recoverable & & & & & & & $<7.0$ & $\mathrm{U} / /$ & $<7.0$ & & 1 & WA & $\mu g / L$ \\
\hline Cobalt, total recoverable & 0.64 & $\mathrm{~J} / \mathrm{E} /$ & NDD & & 5 & GE & & & & & & & $\mu g / L$ \\
\hline $\begin{array}{l}\text { Copper, total recoverable } \\
\text { Cyanide }\end{array}$ & 5.8 & NI & & & 5 & GE & 3.3 & $\mathrm{~J} / / /$ & NDD & & 1 & WA & $\mu g / L$ \\
\hline Lead, total recoverable & 4.0 & $\mathrm{~J} / \mathrm{EV} /$ & NDD & & 5 & GE & 0.81 & $\mathrm{~J} / \mathrm{I} /$ & NDD & & 1 & GE & $\mu g / L$ \\
\hline Mercury, total recoverable & $<0.20$ & $\mathrm{U} / /$ & $<0.20$ & & 1 & GE & 1.2 & $/ /$ & & & 1 & $\mathrm{GE}$ & $\mu \mathrm{g} / \mathrm{L}$ \\
\hline $\begin{array}{l}\text { Nickel, total recoverable } \\
\text { Nitrate as nitrogen }\end{array}$ & $<2.1$ & UNI & $<1.0$ & & 5 & GE & $<26$ & $\mathrm{U} / \prime$ & $<26$ & & 1 & WA & $\mu g / L$ \\
\hline Nitrate-nitrite as nitrogen & 22 & "I & & & 1 & WA & $<20$ & UNI & $<50$ & & 1 & GE & $\mu g / L$ \\
\hline Selenium, total recoverable & & & & & & & $<66$ & $\mathrm{U} / 1$ & $<66$ & & 1 & WA & a \\
\hline Silver, total recoverable & & & & & & & $<5.0$ & $\mathrm{U} / /$ & $<5.0$ & & 1 & WA & $\mu g / L$ \\
\hline Tin, total recoverable & 7.7 & $\mathrm{~J} / \mathrm{EV} /$ & NDD & & 5 & GE & $<70$ & $\mathrm{U} / /$ & $<70$ & & 1 & WA & Ig \\
\hline Vanadjum, total recoverable & 13 & $N /$ & & - & 5 & $\mathrm{GE}$ & & & & & & & L \\
\hline Zinc, total recoverable & 12 & J/EV/ & NDD & & 5 & GE & $<53$ & $\mathrm{u} / /$ & $<53$ & & & WA & \\
\hline
\end{tabular}

Organic Constituents

$\underline{3098} \quad \underline{M o d} \quad$ Filt.

ST브

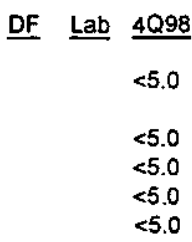

Mod

$\mathrm{U} / I$

Bis(2-ethylhexyl) phthalate

Dichloromethane

Tetrachloroethylene

Trichloroethylene

Trichlorofluoromethane
$<5.0$

$<5.0$

$<5.0$
$\mathrm{U} / 1$
$\mathrm{U} / 1$
$\mathrm{u} / 1$
$\mathrm{U} / /$

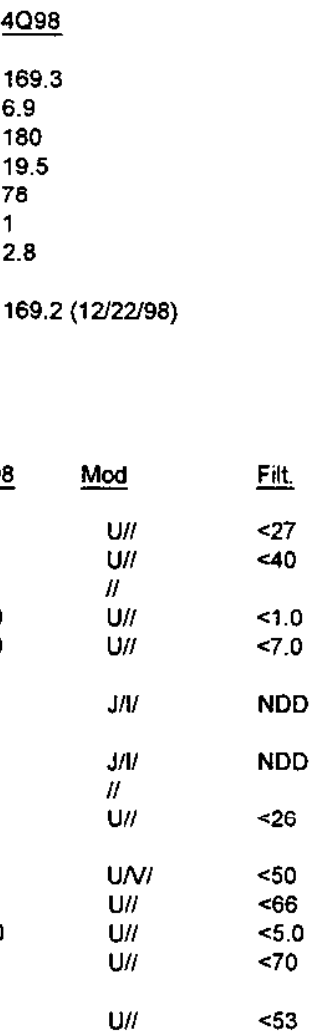

$\underline{\text { Unit }}$

ft ms

$\mathrm{pH}$

$\mu \mathrm{S} / \mathrm{cm}$

${ }^{\circ} \mathrm{C}$

MT/L

well vol

ft msl
$<5.0$

$<5.0$

$<5.0$

$<5.0$

$<5.0$
ST 브 DF Lab Unit

1 WA $\mu g / L$

1 WA $\mu g / L$

1 WA $\mu g / L$

1 WA $\mu g / L$

1 WA $\mu g / L$

Notes:

- = exceeded holding time

- exceeded groundwater protection or monitoring constituent standard (See Appendix A.) 
WELL HSB 85A (cont.)

Radioactive Constituents

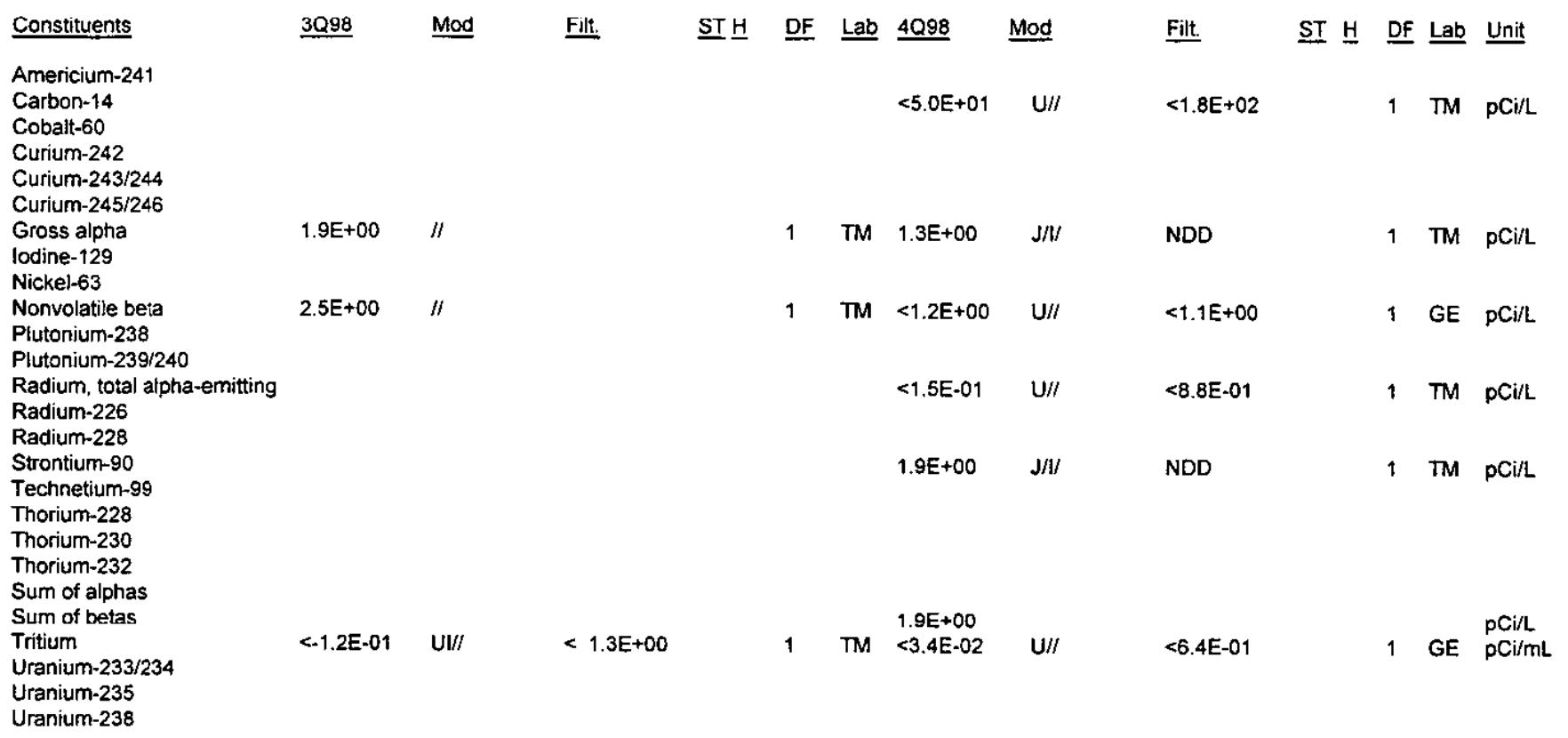

\section{Notes:}

- = exceeded holding time

- exceeded groundwater protection or monitoring constituent standard (See Appendix A.) 
WELL HSB 85B

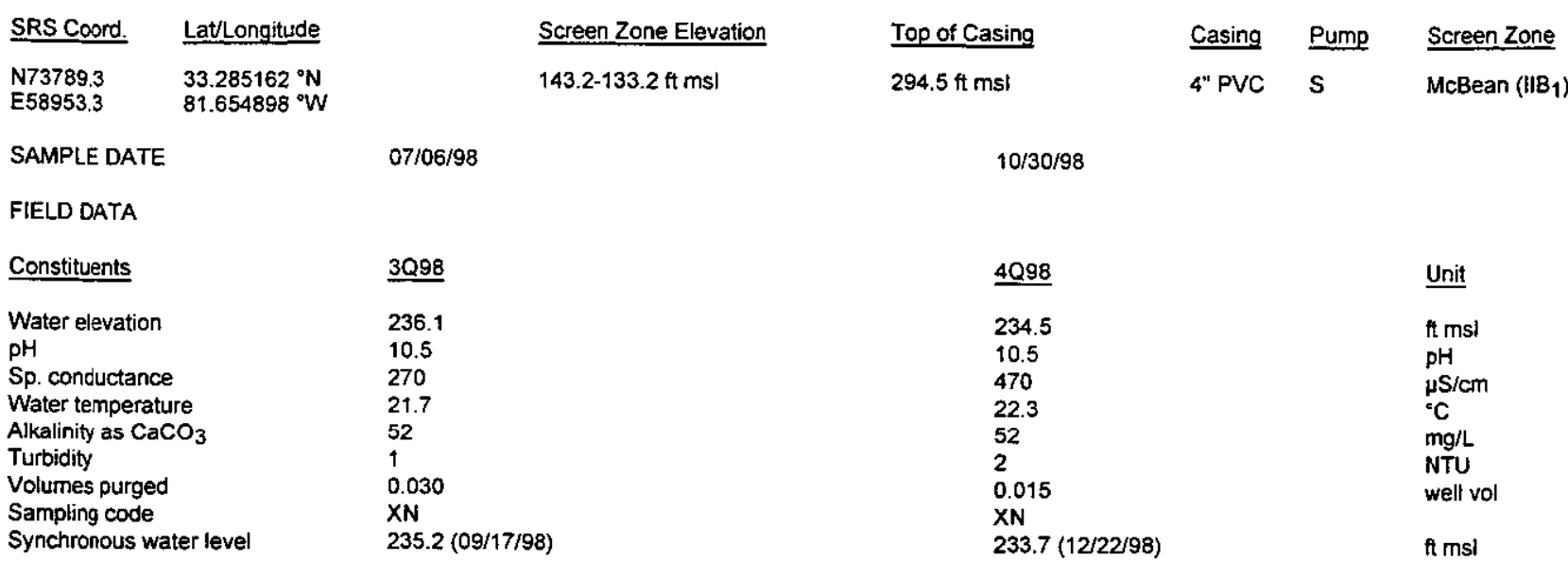

ANALYTICAL DATA

Inorganic Constituents

Constituents

Antimony, total recoverable Arsenic, total recoverable Barium, total recoverable Cadmium, total recoverable Chromitim, total recoverable

Cobalt, total recoverable Copper, total recoverable

Cyanide

Lead, total recoverable Mercury, total recoverable

Nickel, total recoverable

Nitrate as nitrogen

Nitrate-nitrite as nitrogen

Selenium, total recoverable

Silver, total recoverable

Tin, total recoverable

Vanadium, total recoverable

Zinc, total recoverable

\begin{tabular}{|c|c|c|c|c|c|c|c|c|c|c|c|c|}
\hline 3098 & Mod & Filt. & ST$\underline{H}$ & DF & $\underline{\text { Lab }}$ & 4Q98 & Mod & Filt. & $\underline{\text { ST }} \underline{H}$ & DF & $\underline{\text { Lab }}$ & Unit \\
\hline & & & & & & $<27$ & $\mathrm{U} / /$ & $<27$ & & 1 & WA & $\mu g / L$ \\
\hline & & & & & & $<40$ & $\mathrm{U} / /$ & $<40$ & & 1 & WA & $\mu g / L$ \\
\hline & & & & & & 26 & /I & & & 1 & WA & $\mu g / L$ \\
\hline & & & & & & $<4.7$ & $\mathrm{U} / /$ & $<4.7$ & & 1 & WA & $\mu g / L$ \\
\hline & & & & & & 3.4 & $\mathrm{~J} / \mathrm{I}$ & NDD & & 1 & WA & $\mu g / L$ \\
\hline & & & & & & $<15$ & $\mathrm{U} / /$ & $<15$ & & 1 & WA & $\mu g / L$ \\
\hline & & & & & & $<47$ & $\mathrm{U} / / \mathrm{I}$ & $<47$ & & 1 & WA & $\mu g / L$ \\
\hline$<0.20$ & $\mathrm{UJ} / / / 1$ & $<0.20$ & & 1 & GE & $<0.20$ & $\mathrm{U} / /$ & $<0.2$ & & 1 & GE & $\mu g / L$ \\
\hline & & & & & & $<26$ & $\mathrm{U} / \prime$ & $<26$ & & 1 & WA & $\mu g / L$ \\
\hline 700 & $N I$ & & & 1 & $\mathrm{GE}$ & 1,100 & /1 & & & 1 & GE & $\mu g / L$ \\
\hline & & & & & & $<66$ & $\mathrm{U} / /$ & $<66$ & & 1 & WA & $\mu g / L$ \\
\hline & & & & & & $<5.0$ & $\mathbf{U} / I$ & $<5.0$ & & 1 & WA & $\mu g / L$ \\
\hline & & & & & & $<70$ & $\mathrm{U} / \prime$ & $<70$ & & 1 & WA & $\mu g / L$ \\
\hline & & & & & & $<53$ & $\mathbf{U} / /$ & $<53$ & & 1 & WA & $\mu g / L$ \\
\hline 3098 & Mod & Filt. & ST브 & $\underline{D F}$ & $\underline{\text { Lab }}$ & 4098 & Mod & Filt. & $\underline{\text { ST }} \underline{H}$ & $\underline{D F}$ & Lab & Unit \\
\hline & & & & & & $<5.0$ & $\mathrm{U} / /$ & $<5.0$ & & 1 & WA & $\mu g / L$ \\
\hline & & & & & & $<2.8$ & $\mathrm{U} / \mathrm{N} /$ & $<5.0$ & & 1 & WA & $\mu g / L$ \\
\hline & & & & & & $<5.0$ & $\mathrm{U} / /$ & $<5.0$ & & 1 & WA & $\mu g / L$ \\
\hline & & & & & & $<5.0$ & $U / I$ & $<5.0$ & & 1 & WA & $\mu g / L$ \\
\hline & & & & & & $<5.0$ & $\mathrm{U} / /$ & $<5.0$ & & 1 & WA & $\mu g / L$ \\
\hline
\end{tabular}

Notes:

- = exceeded holding time

- = exceeded groundwater protection or monitoring constituent standard (See Appendix A.) 
WELL HSB 85B (cont.)

Radioactive Constituents

Constituents

Americium-241

Carbon-14

Cobalt-60

Curiurn-242

Curium-243/244

Curium-245/246

Gross alpha

lodine-129

Nickel-63

Nonvolatile beta

Plutonium-238

Plutonium-239/240

Radium, total alpha-emitting

Radium-226

Radium-228

Strontium-90

Technetium-99

Thorium-228

Thorium-230

Thorium-232

Sum of alphas

Sum of betas

Tritium

Uranium-233/234

Uranium-235

Uranium-238

\begin{abstract}
Mod
Filt.

ST브 DF L Lab 4098 Mod

Filt.

ST $\underline{H}$ DF Lab Unit
\end{abstract}

$$
<8.7 \mathrm{E}+01 \quad \mathrm{U} / \prime
$$

$<1.7 E+02$

$1 \mathrm{TM} \mathrm{pCil}$

$\begin{array}{lll}<-1.1 \mathrm{E}-02 & \text { UI// } & <0.8580 \\ <7.4 \mathrm{E}-01 \quad \text { UII/ } & <1.1900\end{array}$

1

GP $<2.0 E-01 \quad U / /$

$<1.2 \mathrm{E}+00$

$1 \mathrm{TM} \quad \mathrm{pCi} / \mathrm{L}$

$<7.4 E-01 \quad$ UIII

1

GP $\quad 3.2 \mathrm{E}+00$

$1 \mathrm{GE} \quad \mathrm{pCi} / \mathrm{L}$

$<-1.9 \mathrm{E}-01 \quad \mathrm{U} / /$

$<1.5 E+00$

$1 \mathrm{TM} \quad \mathrm{PC} / \mathrm{L}$

$<2.4 \mathrm{E}-01 \quad \mathrm{U} / \mathrm{I}$

$<1.5 E+00$

$1 \mathrm{TM} \mathrm{PCi} / \mathrm{L}$

1 GP $9.3 \mathrm{E}+00 \quad / 1$

$1 \mathrm{TM} \mathrm{pCi} / \mathrm{mL}$ 
WELL HSB 85C

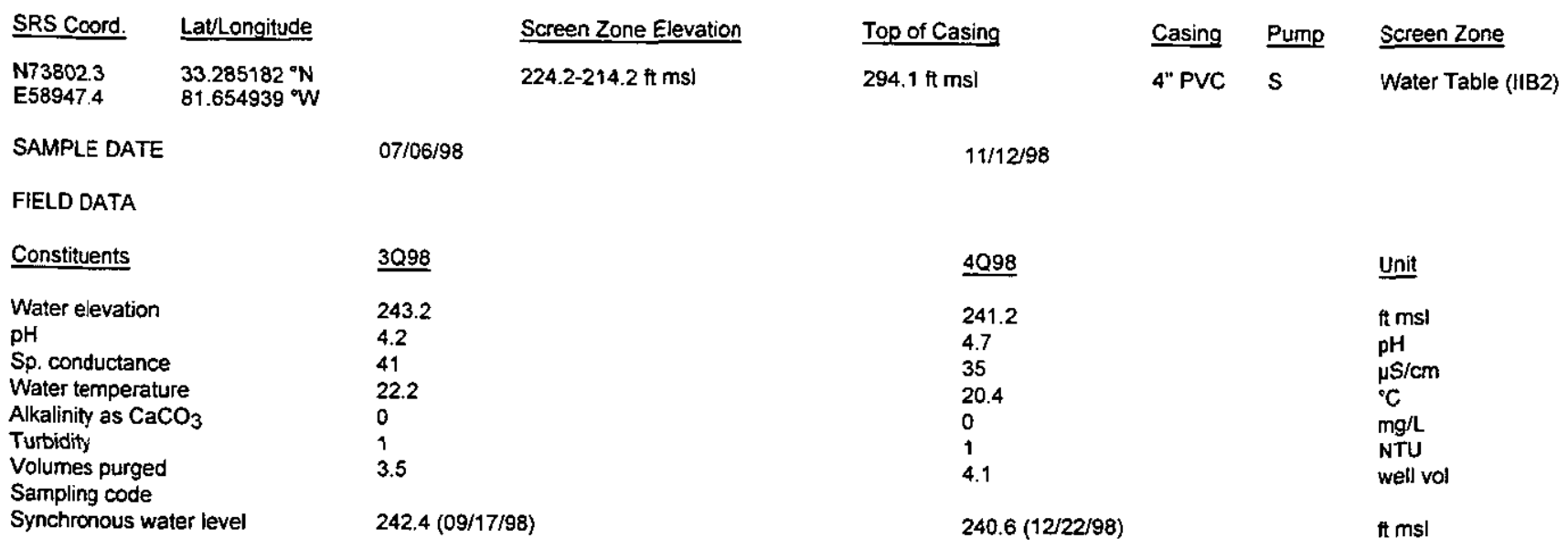

ANALYTICAL DATA

inorganic Constituents

Constituents

\begin{tabular}{|c|c|c|c|c|c|c|c|c|c|c|c|c|}
\hline 3098 & Mod & Filt. & ST브 & $\underline{\mathrm{DF}}$ & $\underline{\text { Lab }}$ & 4Q98 & Mod & Filt. & $\underline{\text { ST }} \underline{H}$ & $\underline{\text { DF }}$ & $\underline{\text { Lab }}$ & $\underline{\text { Unit }}$ \\
\hline & & & & & & $<27$ & $\mathrm{U} / /$ & $<27$ & & 1 & WA & $\mu g / L$ \\
\hline & & & & & & $<40$ & $\mathrm{U} / /$ & $<40$ & & 1 & WA & $\mu g / L$ \\
\hline & & & & & & 12 & "I & & & 1 & WA & $\mu g / L$ \\
\hline & & & & & & 0.56 & $\sqrt{ } / / \mathrm{I}$ & NOD & & 1 & WA & $\mu g / L$ \\
\hline & & & & & & $<7.0$ & $U / I$ & $<7.0$ & & 1 & WA & $\mu g / L$ \\
\hline & & & & & & 80 & /I & & & 1 & WA & $\mu g / L$ \\
\hline & & & & & & $<47$ & $\mathrm{U} / /$ & $<47$ & & 1 & WA & $\mu g / \mathrm{L}$ \\
\hline$<0.20$ & $\mathrm{UJ} / / / 1$ & $<0.20$ & & 1 & $\mathrm{GE}$ & 0.31 & II & & & 1 & GE & $\mu g / L$ \\
\hline & & & & & & $<26$ & $\mathbf{U} / /$ & $<26$ & & 1 & WA & $\mu \mathrm{g} / \mathrm{L}$ \\
\hline 2,600 & $N I$ & & & 1 & GE & 2,600 & /I & & & 1 & $\mathrm{GE}$ & $\mu g / L$ \\
\hline & & & & & & $<66$ & $\mathrm{U} / /$ & $<66$ & & 1 & WA & $\mu g / L$ \\
\hline & & & & & & $<5.0$ & $\mathrm{U} / /$ & $<5.0$ & & 1 & WA & $\mu g / L$ \\
\hline & & & & & & $<70$ & $\mathbf{U} / /$ & $<70$ & & 1 & WA & \\
\hline & & & & & & $<53$ & $\mathrm{U} / I$ & $<53$ & & 1 & WA & $\mu \mathrm{g} / \mathrm{L}$ \\
\hline
\end{tabular}

Organic Constituents

Constituents

Filt.

STI브

DF Lab 4 QQ98

Mod

Filt.

Bis (2-ethylhexyl) phthalate

Dichloromethane

Tetrachloroethylene

Trichloroethylene

Trichlorofluoromethane

$<5.0$

$<5.0$

$<5.0$

$<5.0$

$<5.0$
ST H DF Lab Unit

1 WA $\mu g / 2$

WA $\mu g / L$ WA $\mu g / L$ WA $\mu g / L$

1 WA $\mu g / L$

Notes:

- = exceeded holding time

- exceeded groundwater protection or monitoring constituent standard (See Appendix A.) 
WELL HSB 85C (cont.)

Radioactive Constituents

\begin{tabular}{|c|c|c|c|c|c|c|c|c|c|c|c|c|c|}
\hline Constituents & $3 Q 98$ & Mod & Filt. & ST브 & $\underline{D F}$ & $\underline{\underline{L a b}}$ & 4098 & Mod & Fist. & $\underline{\text { ST }} \underline{H}$ & DF & Lab & Unit \\
\hline Americium-241 & & & & & & & & & & & & & \\
\hline $\begin{array}{l}\text { Carbon-14 } \\
\text { Cobalt-60 } \\
\text { Curium-242 } \\
\text { Curium-243/244 } \\
\text { Curium-245/246 }\end{array}$ & & & & & & & $<.7 .2 E+00$ & $\mathrm{U} / /$ & $<1.8 E+02$ & & 1 & $\mathrm{TM}$ & $\mathrm{pCi} / \mathrm{L}$ \\
\hline $\begin{array}{l}\text { Gross alpha } \\
\text { lodine-129 }\end{array}$ & $3.4 \mathrm{E}+00$ & $\|$ & & & 1 & GP & $3.5 \mathrm{E}+00$ & $1 /$ & & & 1 & $T M$ & $\mathrm{pCi} / \mathrm{L}$ \\
\hline Nickel-63 & & & & , & & & & & & & & & \\
\hline $\begin{array}{l}\text { Nonvolatile beta } \\
\text { Plutonium-238 } \\
\text { Plutonium-239/240 }\end{array}$ & $2.0 \mathrm{E}+00$ & $/ /$ & & & 1 & GP & $3.4 E+00$ & $\mathrm{~J} / \mathrm{I} /$ & NDD & & 1 & $\mathrm{TM}$ & $\mathrm{pCi} / \mathrm{L}$ \\
\hline $\begin{array}{l}\text { Radium, total alpha-emitting } \\
\text { Radium-226 } \\
\text { Radium-228 }\end{array}$ & & & & & & & $2.5 E+00$ & $/ 1$ & & & 1 & $T M$ & $\mathrm{pCi} / \mathrm{L}$ \\
\hline $\begin{array}{l}\text { Strontium-90 } \\
\text { Technetium-99 }\end{array}$ & & & & & & & $<-7.5 E-01$ & $\mathrm{U} / /$ & $<1.5 \mathrm{E}+00$ & & 1 & $T M$ & $p C i / L$ \\
\hline Thorium-228 & & & & & & & & & & & & & \\
\hline $\begin{array}{l}\text { Thorium- } 232 \\
\text { Sum of alphas } \\
\text { Sum of betas }\end{array}$ & & & & & & & & & & & & & \\
\hline $\begin{array}{l}\text { Tritium } \\
\text { Uranium-233/234 } \\
\text { Uranium-235 } \\
\text { Uranium-238 }\end{array}$ & $1.0 E+01$ & // & & & 1 & GP & $1.3 E+01$ & $\|$ & & & 1 & $\mathrm{TM}$ & $\mathrm{pCi} / \mathrm{mL}$ \\
\hline
\end{tabular}

Notes:

- exceeded holding time

- exceeded groundwater protection or monitoring constituent standard (See Appendix A.) 
WELL HSB 86A

\begin{tabular}{|c|c|}
\hline SRS Coord. & Lat/Longitude \\
\hline $\begin{array}{l}\text { N72520.2 } \\
\text { E55985.9 }\end{array}$ & 81.660247 on \\
\hline
\end{tabular}

Screen Zone Elevation

73.9-63.1 $\mathrm{ft} \mathrm{ms|}$

07/01/98

SAMPLE DATE

FIELD DATA

Constituents

Water elevation

$\mathrm{pH}$

Sp. conductance

Water temperature

Alkalinity as $\mathrm{CaCO}_{3}$

Turbidity

Volumes purged

Sampling code

Synchronous water level

3Q98

169.7

8.0

120

20.8

48

1

2.2

$169.6(09 / 17 / 98)$

ANALYTICAL DATA

Inorganic Constituents

Constituents

Antimony, total recoverable

Arsenic, total recoverable

Barium, total recoverable

Cadmium, total recoverable

Chromium, total recoverable

Cobalt, total recoverable

Copper, total recoverable

Cyanide

Lead, total recoverable

Mercury, total recoverable

Nickel, total recoverable

Nitrate as nitrogen

Nitrate-nitrite as nitrogen

Selenium, total recoverable

Silver, total recoverable

Tin, total recoverable

Vanadium, total recoverable

Zinc, total recoverable

Organic Constituents

Constituents

Mod

Benzene

Bis(2-ethylhexyl) phthalate

Dichloromethane

Tetrachloroethylene

Trichloroethylene

Trichlorofluoromethane

$10 / 06 / 98$

Unit

169.4

6.5

110

20.6

51

21

$169.4(12 / 21 / 98)$

$\mathrm{ft} \mathrm{msl}$

$\mathrm{pH}$

$\mu \mathrm{S} / \mathrm{cm}$

${ }^{\circ} \mathrm{C}$

$\mathrm{mg} / \mathrm{h}$

NTU

well vol

$\mathrm{ft} \mathrm{ms}$

\begin{tabular}{|c|c|c|c|c|c|c|c|c|c|c|c|c|}
\hline$<0.20$ & $\mathrm{U} / I$ & $<0.20$ & & 1 & GE & $<0.20$ & JU/UC & & & 1 & GE & $\mu g / L$ \\
\hline 10 & $\mathrm{~J} / \mathrm{E} /$ & NDD & & 1 & GE & $<10$ & UNI & $<50$ & & 1 & GE & $\mu g / L$ \\
\hline 3098 & Mod & Filt. & $\underline{S T} \underline{H}$ & DF & Lab & 4098 & Mod & Filt. & $\underline{\text { ST }} \underline{H}$ & $\underline{\mathrm{DF}}$ & Lab & Unit \\
\hline
\end{tabular}

\section{Notes:}

- = exceeded holding time

- exceeded groundwater protection or monitoring constituent standard (See Appendix A.) 
WELL HSB 86A (cont.)

Radioactive Constituents

Constituents

Americium-241

Carbon-14

Cobalt-60

Curium-242

Curium-243/244

Curium-245/246

Gross alpha

lodine-129

Nickel-63

Nonvolatile beta

Plutonium-238

Plutonium-239/240

Radium-226

Racium-228

Strontium-90

Technetium-99

Thorium-228

Thorium-230

Thorium-232

Sum of alphas

Sum of betas

Tritium

Uranium-233/234

Uranium-235

Uranium-238
$1.2 \mathrm{E}+00 \quad \mathrm{~J} / \mathrm{C} / \quad \mathrm{NDD}$

$1.9 E+00 \quad / /$

7.9E-01 $/ 1$

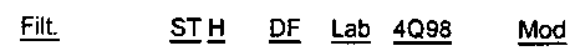

$1 \quad \mathrm{GP} \quad<5.7 \mathrm{E}-01$

$\mathrm{U} / 1$

1 GP $<1.5 E+00$

$\mathrm{U} / /$

$<1.2 \mathrm{E}+\infty 0$

1 GE $\mathrm{pCi} / \mathrm{L}$

1 GE PCill

1 GP $\quad<7.9 E-01$

U/I

$<6.5 \mathrm{E}-01$

$1 \mathrm{GE} \mathrm{pCi} / \mathrm{mL}$ 
WELL HSB 86B

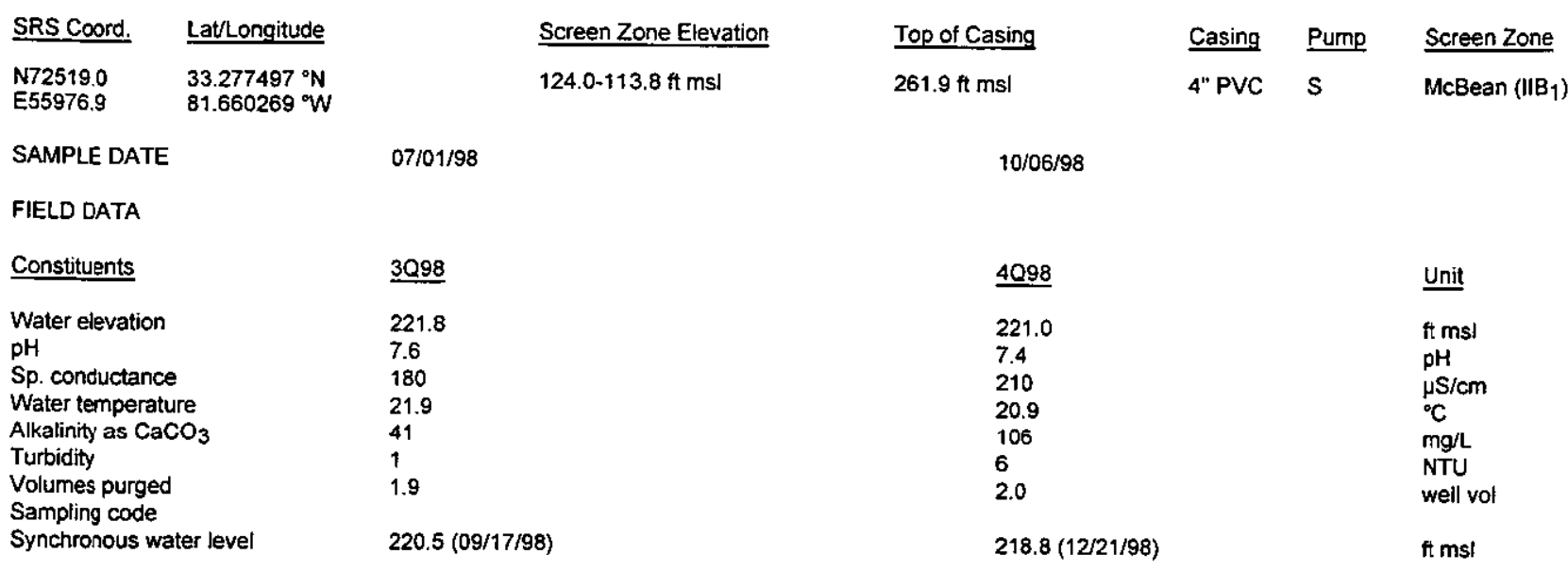

ANALYTICAL DATA

Inorganic Constituents

Constituents

Antimony, total recoverable Arsenic, total recoverable Barium, total recoverable Cadmium, total recoverable Chromium, total recoverable Cobalt, total recoverable Copper, total recoverable Cyanide

Lead, total recoverable Mercury, total recoverable Nickel, total recoverable

Nitrate as nitrogen

Nitrate-nitrite as nitrogen Selenium, total recoverable

Silver, total recoverable

Tin, total recoverable

Vanadium, total recoverable

Zinc, total recoverable

Organic Constituents

Constituents

$J / E J$

NDD

1

GE $<0.20$

$\mathrm{JU} / \mathrm{L} / \mathrm{C}$

40

\section{Benzene}

Bis(2-ethylhexyl) phthalate

Dichloromethane

Tetrachloroethylene

Trichloroethylene

Trichlorofluoromethane

\section{Notes:}

- = exceeded holding time

= exceeded groundwater protection or monitoring constituent standard (See Appendix A.) 
WELL HSB $86 B$ (cont.)

Radioactive Constituents

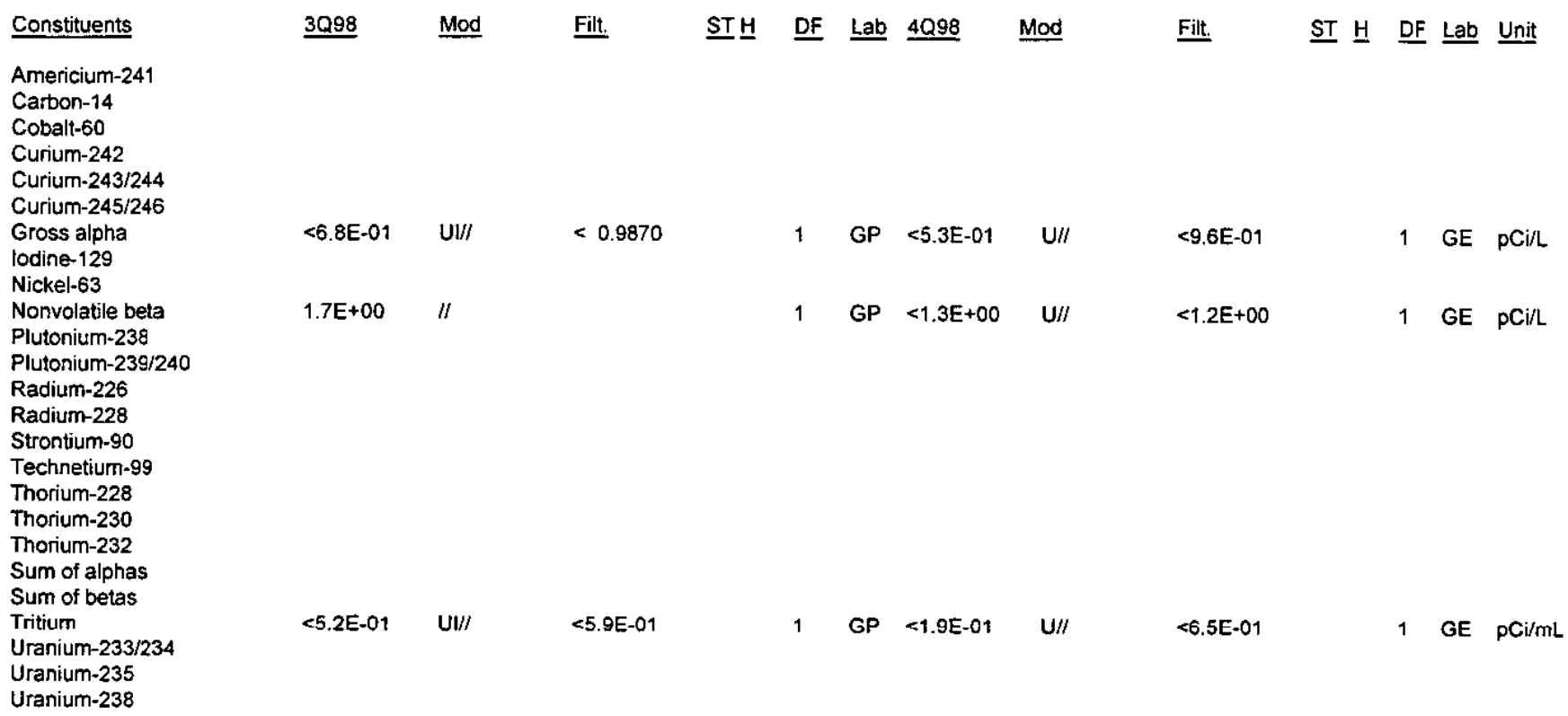

Notes:

- exceeded holding time

- = exceeded groundwater protection or monitoring constituent standard (See Appendix A.) 
WELL HSB 86C

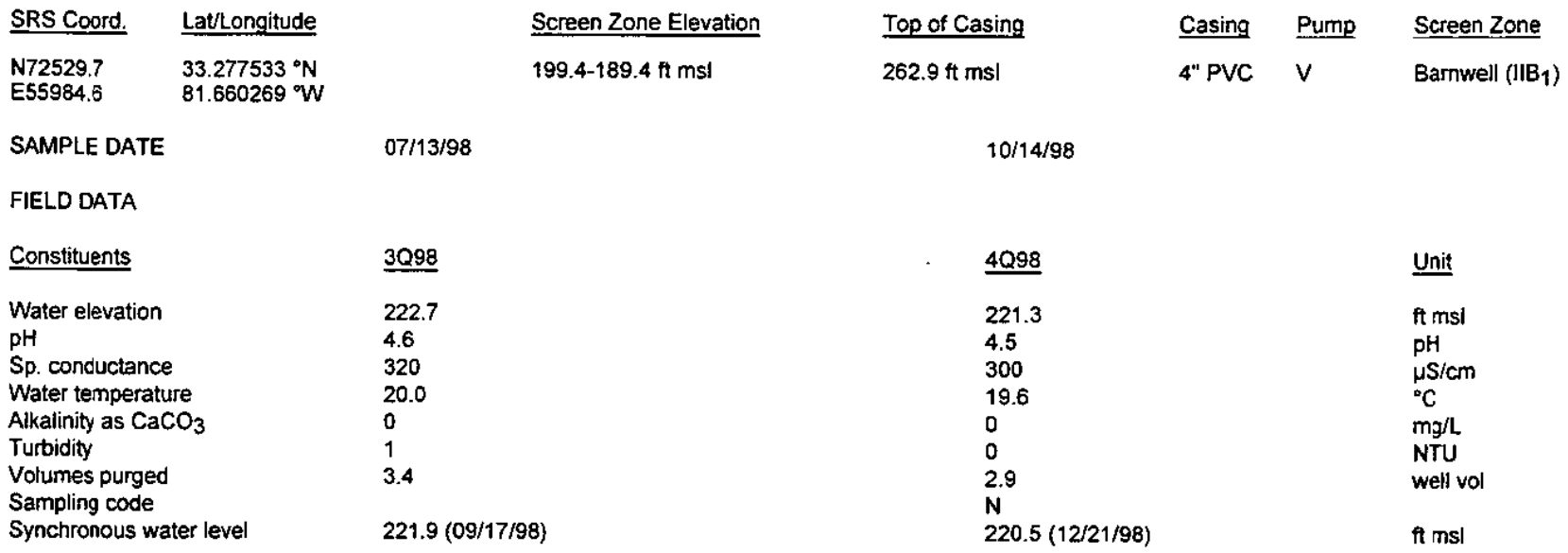

ANALYTICAL DATA

Inorganic Constituents

Constituents

Antimony, total recoverable

Arsenic, total recoverable

Barium, total recoverable

Cadmium, total recoverable

Chromium, total recoverable

Cobalt, total recoverable

Copper, total recoverable

Cyanide

Lead, total recoverable

Mercury, total recoverable

Nickel, total recoverable

Nitrate as nitrogen

Nitrate-nitrite as nitrogen

Selenium, total recoverable

Silver, total recoverable

Tin, total recoverable

Vanadium, total recoverable

Zinc, total recoverable

Organic Constituents

Constituents

$\underline{3098}$

Benzene

Bis(2-ethylhexyl) phthalate

Dichloromethane

Tetrachloroethylene

Trichloroethylene

Trichlorofluoromethane

39,000

Filt.

II

NI

1

GE $\quad 1.1$

II

-

25

GE $\quad 40,000$

ll

Mod Filt.

ST브

DF Lab $4 Q 98$

Mod

Filt.

$\underline{\text { ST }} \underline{\mathrm{H}}$ DF Lab Unit

Notes:

- = exceeded holding time

= exceeded groundwater protection or monitoring constituent standard (See Appendix A.) 
WELL HSB $86 \mathrm{C}$ (cont.)

Radioactive Constituents

\begin{tabular}{|c|c|c|c|c|c|c|c|c|c|c|c|c|c|}
\hline Constituents & 3098 & Mod & Filt. & ST브 & $\underline{\mathrm{DF}}$ & Lab & $\underline{4 Q 98}$ & Mod & Filt. & $\underline{\text { ST }} \underline{H}$ & $\underline{D F}$ & $\underline{\text { Lab }}$ & Unit \\
\hline $\begin{array}{l}\text { Americium-241 } \\
\text { Carbon-14 } \\
\text { Cobalt-60 } \\
\text { Curium-242 } \\
\text { Curium-243/244 } \\
\text { Curium-245/246 }\end{array}$ & & & & & & & & & & & & & \\
\hline $\begin{array}{l}\text { Gross alpha } \\
\text { lodine-129 } \\
\text { Nickel-63 }\end{array}$ & $2.7 E+01$ & $/ /$ & & - & 1 & GP & $2.7 E+01$ & $\|$ & & - & 1 & GE & $\mathrm{pCi} / \mathrm{L}$ \\
\hline $\begin{array}{l}\text { Nonvolatile beta } \\
\text { Plutonium-238 } \\
\text { Plutonium-239/240 } \\
\text { Radium-226 } \\
\text { Radium-228 } \\
\text { Strontium-90 } \\
\text { Technetium-99 } \\
\text { Thorium-228 } \\
\text { Thorium-230 } \\
\text { Thorium-232 } \\
\text { Sum of alphas } \\
\text { Sum of betas }\end{array}$ & $4.7 \mathrm{E}+02$ & $\|$ & & a & 1 & GP & $3.7 \mathrm{E}+02$ & 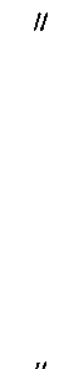 & & 口 & 1 & $G E$ & $\mathrm{pCi} i \mathrm{~L}$ \\
\hline $\begin{array}{l}\text { Tritium } \\
\text { Uranium-233/234 } \\
\text { Uranium-235 } \\
\text { Uranium-238 }\end{array}$ & $8.4 E+03$ & 11 & & ॥ & 1 & GP & $8.4 E+03$ & $/ 1$ & & - & 1 & $\mathrm{GE}$ & $\mathrm{pCi} / \mathrm{mL}$ \\
\hline
\end{tabular}

Notes:

- exceeded holding time

- = exceeded groundwater protection or monitoring constituent standard (See Appendix A.) 
WELL HSB 86D

$\begin{array}{ll}\text { SRS Coord. } & \text { Latllongitude } \\ \text { N72522.1 } & 33.277536^{\circ} \mathrm{N} \\ \text { E55996.5 } & 81.660223^{\circ} \mathrm{W}\end{array}$

SAMPLE DATE

FIELD DATA

Constituents

Water elevation

$\mathrm{pH}$

$\mathrm{Sp}$. conductance

Water temperature

Alkalinity as $\mathrm{CaCO}_{3}$

Turbidity

Volumes purged

Sampling code

Synchronous water level

ANALYTICAL DATA

Inorganic Constituents

Constituents

Antimony, total recoverable Arsenic, total recoverable

Barium, total recoverable

Cadmium, total recoverable

Chromium, total recoverable

Cobalt, total recoverable

Copper, total recoverable

Cyanide

Lead, total recoverable

Mercury, total recoverable

Nickel, total recoverable

Nitrate as nitrogen

Nitrate-nitrite as nitrogen

Selenium, total recoverable

Silver, total recoverable

Tin, total recoverable

Vanadium, total recoverable

Zinc, total recoverable

Organic Constituents

Constituents

Benzene

Bis(2-ethylhexyl) phthalate

Dichloromethane

Tetrachloroethylene

Trichloroethylene

Trichlorofluoromethane
Screen Zone Elevation

$236.6-206.6 \mathrm{ft} \mathrm{ms}$

07/13/98

$\underline{3098}$

4.2

100

19.5

2

$221.9(09 / 17 / 98)$
Top of Casing

$263 \mathrm{ft} \mathrm{msl}$

$10 / 14 / 98$
Casing Pump

4" PVC V
Screen Zone

Water Table (IBB2)
4Q98

221.2

4.4

140

20.6

0

1

3.1

$\mathrm{N}$
$220.6(12 / 21 / 98)$
Unit

$\mathrm{ft}$ msl

$\mathrm{pH}$

$\mu \mathrm{S} / \mathrm{cm}$

$\mathrm{mgh}$

NTU

well vol

ft msl

$3098 \quad$ Mod $\quad$ Filt. $\quad \underline{\text { STH}}$ DF Lab $\underline{4 Q 98} \quad \underline{M o d} \quad \underline{\text { Filt. }} \quad \underline{\text { ST }} \underline{\mathrm{H}} \underline{\mathrm{DF}} \underline{\underline{L}} \underline{\underline{\text { Unit }}}$

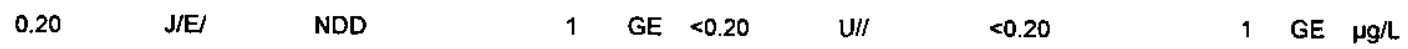

$\begin{array}{lllll}12,000 \quad N / & 5 & \text { GE } & 17,000\end{array}$

- $\quad 10 \mathrm{GE} \mu g / \mathrm{L}$

Mod Filt. $\underline{\text { STH }} \underline{\text { DF Lab }} \underline{4 Q 98}$ Mod $\quad \underline{\text { Filt. }} \underline{\text { ST }} \underline{\text { DF }} \underline{\underline{\text { Lab }}} \underline{\text { Unit }}$

Notes:

- = exceeded holding time

- = exceeded groundwater protection or monitoring constituent standard (See Appendix A.) 
WELL HSB 86D (cont.)

Radioactive Constituents

Constituents

Americium-241

Carbon-14

Cobalt-60

Curium-242

Curium-243/244

Curium-245/246

Gross alpha

lodine-129

Nickel-63

Nonvolatile beta

Plutonium-238

Plutonium-239/240

Radium-226

Radium-228

Strontium-90

Technetium-99

Thorium-228

Thorium-230

Thorium-232

Sum of alphas

Sum of betas

Tritium

Uranium-233/234

Uranium-235

Uranium-238
3098

$9.0 \mathrm{E}+00 \quad / 1$

4.6E+02 //

$9.9 \mathrm{E}+02 \quad / 1$
STH DF Lab 4098

Mod

1

GP $1.2 \mathrm{E}+01 \quad /$

-

1 GP $5.3 E+02 \quad /$

GP $1.7 \mathrm{E}+03$
Filt.

ST $\underline{H}$ DF Lab Unit

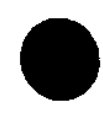

1 GE $\mathrm{pCi} / \mathrm{L}$

-

$1 \mathrm{GE} \quad \mathrm{pCi} / \mathrm{L}$

- 1 GE $\mathrm{pCi} / \mathrm{mL}$

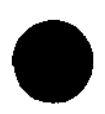

Notes:

- = exceeded holding time

- = exceeded groundwater protection or monitoring constituent standard (See Appendix A.) 
WELL HSB100C

\begin{tabular}{|c|c|}
\hline S Coord. & LatLo \\
\hline $\begin{array}{l}\text { N72077.2 } \\
\text { E58806.5 }\end{array}$ & 81.6 \\
\hline
\end{tabular}

SAMPLE DATE

$07 / 09 / 98$
Screen Zone Elevation

$163.0-153.0 \mathrm{ft} \mathrm{msl}$
Top of Casing

$260.2 \mathrm{ft} \mathrm{ms}$

10/07/98

FIELD DATA

Constituents

Water elevation

$\mathrm{pH}$

Sp. conductance

Water temperature

Alkalinity as $\mathrm{CaCO}_{3}$

Turbidity

Volumes purged

Sampling code

Synchronous water level

ANALYTICAL DATA

Inorganic Constituents

Constituents $\quad 3098$

Antimony, total recoverable $<2.0$

Arsenic, total recoverable $\quad<30$

Barium, total recoverable $\quad 4.8$

Cadmium, total recoverable $<10$

Chromium, total recoverable $<30$

Cobalt, total recoverable $<2.0$

Copper, total recoverable $\quad 2.1$

Cyanide

Lead, total recoverable $\quad 0.65$

Mercury, total recoverable $\quad<0.20$

Nickel, total recoverable $\quad 2.6$

Nitrate as nitrogen

Nitrate-nitrite as nitrogen $\quad 140$

Selenium, total recoverable 19

Silver, total recoverable $\quad<10$

Tin, total recoverable $<20$

Vanadium, total recoverable $<20$

Zinc, total recoverable

$<50$

Organic Constituents

Constituents $\quad 3098$

Benzene

Bis(2-ethythexyl) phthalate

Dichloromethane

Tetrachloroethylene

Trichloroethylene

Trichlorofluoromethane

\section{8}

$<1.0$

$<10$

$<1.0$

$<10$

$<1.0$

$<1.0$
3098

229.1

4.7

33

21.5

4

3.1

$229.1(09 / 17 / 98)$

\begin{tabular}{|c|c|}
\hline Mod & Filt. \\
\hline $\mathrm{U} / /$ & $<2.0$ \\
\hline $\begin{array}{l}\mathbf{u} / / \\
/ /\end{array}$ & $<30.0000$ \\
\hline $\mathrm{U} / /$ & $<10$ \\
\hline $\mathrm{U} / /$ & $<30.0000$ \\
\hline $\mathrm{U} / /$ & $<2.0$ \\
\hline "l & \\
\hline $\mathrm{U} / /$ & $<10$ \\
\hline $\mathrm{J} / \mathrm{E} /$ & NDD \\
\hline $\mathrm{U} / /$ & $<0.20$ \\
\hline NI & \\
\hline$N I$ & \\
\hline $\mathrm{J} / \mathrm{E} /$ & NDD \\
\hline $\mathrm{U} / /$ & $<10$ \\
\hline $\mathrm{U} / /$ & $<20.0000$ \\
\hline $\mathrm{U} / 1$ & $<20.0000$ \\
\hline $\mathrm{U} / /$ & $<50$ \\
\hline
\end{tabular}

ST브

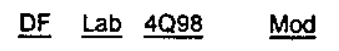

$\begin{array}{ll}10 & \mathrm{GE} \\ 10 & \mathrm{GE} \\ 10 & \mathrm{GE} \\ 10 & \mathrm{GE} \\ 10 & \mathrm{GE} \\ 10 & \mathrm{GE} \\ 10 & \mathrm{GE} \\ 1 & \mathrm{GE} \\ 10 & \mathrm{GE} \\ 1 & \mathrm{GE} \\ 10 & \mathrm{GE}\end{array}$

GE

GE

GE

GE

$\mathrm{GE}$

GE

$\begin{array}{lll}\text { Mod } & & \text { Filt. } \\ \text { U/I } & & <1.0 \\ U / I & & <10 \\ U / I & & <1.0 \\ U / I & & <1.0 \\ U / I & & <1.0 \\ U / I & & <1.0\end{array}$

STT

$\begin{array}{ll}\text { DF } & \text { Lab } \\ 1 & \text { GE } \\ 1 & \text { GE } \\ 1 & \text { GE } \\ 1 & \text { GE } \\ 1 & \text { GE } \\ 1 & \text { GE }\end{array}$

Casing Pump

4" PVC S

Screen Zone

Barnwell (IIB 1$)$ $\underline{4098}$

227.9

5.3

30

21.0

7

2.1

$226.4(12 / 21 / 98)$
Unit

$\mathrm{ft}$ msl

$\mathrm{pH}$

$\mu \mathrm{S} / \mathrm{cm}$

${ }^{\circ} \mathrm{C}$

$\mathrm{mg} / \mathrm{L}$

NTU

well vol

ft msl

Filt.

ST 브 DF Lab Unit

$1 \mathrm{GE}<0.20 \quad \mathrm{U} / / \quad<0.20$

$1 \mathrm{GE}$

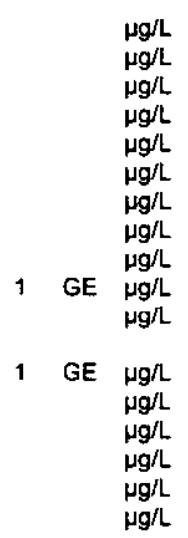

Mod

Filt.

ST 트 DF Lab Unit

$\mu \mathrm{g} / \mathrm{L}$

$\mu g / L$

$\mu g / L$

$\mu g / L$

$\mu g / L$

$\mu g / \mathrm{L}$

Notes:

- = exceeded holding time

- =xceeded groundwater protection or monitoring constituent standard (See Appendix A.) 
WELL HSB100C (cont.)

Radioactive Constituents

\begin{tabular}{|c|c|c|c|c|c|c|c|c|c|c|c|c|c|}
\hline Constituents & $\underline{3098}$ & Mod & Filt. & $\underline{\text { ST }} \underline{H}$ & $\underline{\mathrm{DF}}$ & $\underline{\mathrm{Lab}}$ & $4 Q 98$ & Mod & Filt. & $\underline{\mathrm{ST}} \underline{\mathrm{H}}$ & $\underline{D F}$ & $\underline{\mathrm{Lab}}$ & $\underline{\text { Unit }}$ \\
\hline Americium-241 & $<-1.7 \mathrm{E}-02$ & $\mathrm{UI} / I$ & $<0.1410$ & & 1 & GP & & & & & & & $\mathrm{pCi} / \mathrm{L}$ \\
\hline Carbon-14 & $<3.4 \mathrm{E}+00$ & $\mathrm{UI} / /$ & $<9.2300$ & & 1 & GP & & & & & & & $\mathrm{pCi} / \mathrm{L}$ \\
\hline Cobalt -60 & $<-1,4 E-01$ & $\mathrm{U} 1 / /$ & $<3.0400$ & & 1 & GP & & & & & & & $\mathrm{pCi} / \mathrm{L}$ \\
\hline Curium-242 & $<-3.5 E-03$ & $\mathrm{UI} / /$ & $<0.1420$ & & 1 & GP & & & & & & & $\mathrm{pCi} / \mathrm{L}$ \\
\hline Curium-243/244 & $<2.9 \mathrm{E}-02$ & $\mathrm{UI} / /$ & $<0.0870$ & & 1 & GP & & & & & & & $\mathrm{pCi} / \mathrm{L}$ \\
\hline Curium-245/246 & $<1.7 E-02$ & $\mathrm{UI} / /$ & $<0.0494$ & & 1 & GP & & & & & & & $\mathrm{pCi} / \mathrm{L}$ \\
\hline Gross alpha & $<-2.9 \mathrm{E}-02$ & $\mathrm{U} / /$ & $<0.7260$ & & 1 & GP & $<3.3 E-01$ & $\mathrm{U} / /$ & $<6.0$ E-01 & & 1 & GE & $\mathrm{pCi} / \mathrm{L}$ \\
\hline lodine-129 & $<9.8 \mathrm{E}-01$ & $\mathrm{U} / / /$ & $<1.3600$ & & 1 & GP & & & & & & & $\mathrm{pCi} / \mathrm{L}$ \\
\hline Nickel-63 & $<-1.4 \mathrm{E}-01$ & UI & $<3.0400$ & & & & & & & & & & $\mathrm{pCi} / \mathrm{L}$ \\
\hline Nonvolatije beta & $<6.1 E-01$ & $\mathrm{UI} / /$ & $<1.2100$ & & 1 & GP & $<9.7 \mathrm{E}-01$ & $\mathrm{U} / /$ & $<1.1 E+\infty 0$ & & 1 & GE & $\mathrm{pCi} / \mathrm{L}$ \\
\hline Plutonium-238 & $<1.7 E-02$ & $\mathrm{U} 1 / 1$ & $<0.0500$ & & 1 & GP & & & & & & & $\mathrm{pCi} / \mathrm{L}$ \\
\hline Plutonium-239/240 & $<1.7 \mathrm{E}-02$ & $\mathrm{UI} / \mathrm{I}$ & $<0.0499$ & & 1 & GP & & & & & & & $\mathrm{pCi} / \mathrm{L}$ \\
\hline Radium-226 & $<3.5 E-01$ & $\mathrm{UI} / /$ & $<0.6360$ & & 1 & GP & & & & & & & $\mathrm{pCi} / \mathrm{L}$ \\
\hline Radium-228 & $<1.3 \mathrm{E}-01$ & $\mathrm{UI} / /$ & $<0.9360$ & & 1 & GP & & & & & & & $\mathrm{pCi} / \mathrm{L}$ \\
\hline Strontium-90 & $<-5.8 \mathrm{E}-01$ & UIJ/L & & & 1 & $\mathrm{GP}$ & & & & & & & pCill \\
\hline Technetium-99 & $<-8.6 \mathrm{E}+00$ & $\mathrm{UI} / /$ & $<27.8000$ & & 1 & GP & & & & & & & $\mathrm{pCi} / \mathrm{L}$ \\
\hline Thorium-228 & $<1.3 E-01$ & UIII & $<0.5560$ & & 1 & GP & & & & & & & $\mathrm{pCi} / \mathrm{L}$ \\
\hline Thorium-230 & $<5.6 \mathrm{E}-02$ & $\mathrm{UI} / /$ & $<0.0841$ & & 1 & GP & & & & & & & $\mathrm{pCi} / \mathrm{L}$ \\
\hline Thorium-232 & $<-1.4 \mathrm{E}-02$ & $\mathrm{U1} / 1$ & $<0.1740$ & & 1 & GP & & & & & & & $\mathrm{pCi} / \mathrm{L}$ \\
\hline $\begin{array}{l}\text { Sum of alphas } \\
\text { Sum of betas }\end{array}$ & & & & & & & & & & & & & \\
\hline Tritium & $1.2 \mathrm{E}+00$ & 11 & & & 1 & GP & $1.8 E+00$ & $/ 1$ & & & 1 & GE & $\mathrm{pCi} / \mathrm{mL}$ \\
\hline Uranium-233/234 & $<2.6 \mathrm{E}-01$ & $\mathrm{UN} /$ & $<0.0647$ & & 1 & GP & & & & & & & $\mathrm{pCi} / \mathrm{L}$ \\
\hline Uranium-235 & $<2.2 \mathrm{E}-02$ & $\mathrm{UI} / /$ & $<0.0649$ & & 1 & GP & & & & & & & $\mathrm{pCill}$ \\
\hline Uranium-238 & $<3.8 E-02$ & UIII & $<0.1140$ & & 1 & GP & & & & & & & $\mathrm{pCi} / \mathrm{L}$ \\
\hline
\end{tabular}

Notes:

- =xceeded holding time

- = exceeded groundwater protection or monitoring constituent standard (See Appendix A.) 
WELL HSB100D

$\begin{array}{ll}\text { SRS Coord. } & \text { Lat/Longitude } \\ \text { N72073.8 } & 33.281113^{\circ} \mathrm{N} \\ \text { E58796.9 } & 81.651978^{\circ} \mathrm{W}\end{array}$

SAMPLE DATE

FIELD DATA

Constituents

Water elevation

$\mathrm{pH}$

Sp. conductance

Water temperature

Alkajinity as $\mathrm{CaCO}_{3}$

Turbidity

Volumes purged

Sampling code

Synchronous water level
$07 / 07 / 98$

3098

Screen Zone Elevation

236.9-216.9 ft msl

238.0

4.8

78

21.1

6

4.5

$237.2(09 / 17 / 98)$
Top of Casing

$260.1 \mathrm{ft} \mathrm{ms}$

$10 / 07 / 98$

$4 Q 98$
236.6
5.1
68
20.6
4
2
2.5
$234.4(12 / 21 / 98)$

ANALYTICAL DATA
Inorganic Constituents

\begin{tabular}{|c|c|c|c|c|c|c|c|c|c|c|c|c|c|}
\hline Constituents & 3098 & Mod & Filt. & $\underline{\text { ST }} \underline{H}$ & $\underline{\mathrm{DF}}$ & Lab & 4Q98 & Mod & Filt. & ST $\underline{H}$ & $\underline{\mathrm{DF}}$ & $\underline{\text { Lab }}$ & Unit \\
\hline Antimony, total recoverable & $<0.20$ & $\mathrm{U} / /$ & $<0.20$ & & 1 & GE & & & & & & & $\mu g / L$ \\
\hline Arsenic, total recoverable & $<3.0$ & $\mathbf{U} / /$ & $<3.0$ & & 1 & GE & & & & & & & $\mu g / L$ \\
\hline Barium, total recoverable & 15 & $\|$ & & & 1 & GE & & & & & & & $\mu g / L$ \\
\hline Cadmium, total recoverable & $<1.0$ & $\mathrm{U} / /$ & $<1.0$ & & 1 & GE & & & & & & & $\mu g / L$ \\
\hline Chromium, total recoverable & $<3.0$ & UII & $<3.0$ & & 1 & GE & & & & & & & $\mu g / L$ \\
\hline Cobalt, total recoverable & 0.17 & $\mathrm{~J} / \mathrm{E} /$ & NDD & & 1 & GE & & & & & & & $\mu g / L$ \\
\hline Copper, total recoverable & 21 & $\|$ & & & 1 & GE & & & & & & & د \\
\hline Cyaride & $<10$ & $\mathrm{U} / /$ & $<10$ & & 1 & GE & & & & & & & $\mu g / L$ \\
\hline Lead, total recoverable & 3.1 & $N I$ & & & 1 & GE & & & & & & & $\lg / \mathrm{L}$ \\
\hline Mercury, total recoverable & $<0.20$ & $\mathrm{U} / \mathrm{I}$ & $<0.20$ & & 1 & GE & $<0.20$ & $\mathrm{U} / /$ & $<0.20$ & & 1 & GE & $g / L$ \\
\hline Nickel, total recoverable & 0.77 & $/ /$ & & & 1 & GE & & & & & & & \\
\hline Nitrate as nitrogen & & & & & & & & & & & & & \\
\hline Nitrate-nitrite as nitrogen & 3,800 & $N i$ & & & 3 & GE & 3,600 & "l & & & 2 & GE & L \\
\hline Selenium, total recoverable & $<5.0$ & $\mathrm{U} / l$ & $<5.0$ & & 1 & GE & & & & & & & $g_{1}$ \\
\hline Silver, total recoverable & $<1.0$ & $\mathrm{U} / /$ & $<1.0$ & & 1 & GE & & & & & & & - \\
\hline Tin, total recoverable & $<2.0$ & $\mathrm{U} / /$ & $<2.0$ & & 1 & GE & & & & & & & \\
\hline Vanadium, total recoverable & $<2.0$ & $U / /$ & $<2.0$ & & 1 & GE & & & & & & & \\
\hline Zinc, total recoverable & 48 & $\|$ & & & 1 & GE & & & & & & & \\
\hline
\end{tabular}

Organic Constituemts

\begin{tabular}{|c|c|c|c|c|c|}
\hline Constituents & 3098 & Mod & Filt. & $\underline{\text { ST }} \underline{H}$ & DF \\
\hline Benzene & $<1.0$ & $\mathrm{U} / /$ & $<1.0$ & & 1 \\
\hline Bis(2-ethylhexyl) phthalate & $<9.9$ & $\mathrm{U} / /$ & $<9.9$ & & 1 \\
\hline Dichloromethane & $<0.79$ & UN8I & $<1.0$ & & 1 \\
\hline Tetrachloroethylene & $<1.0$ & $\mathrm{U} I I$ & $<1.0$ & & 1 \\
\hline Trichloroethylene & $<1.0$ & $\mathrm{U} / /$ & $<1.0$ & & 1 \\
\hline Trichlorofluoromethane & $<1.0$ & $\mathrm{U} / /$ & $<1.0$ & & 1 \\
\hline
\end{tabular}

Casing Pump

Screen Zone

Water Table (IIB2)
Unit

ft msl

$\mathrm{pH}$

$\mu \mathrm{S} / \mathrm{cm}$

${ }^{\circ} \mathrm{C}$

NTU

well vol

ft $\mathbf{m s l}$ 
WELL HSB100D (cont.)

Radioactive Constituents

\begin{tabular}{|c|c|c|c|c|c|c|c|c|c|c|c|c|c|}
\hline Constituents & 3Q98 & Mod & Filt. & $\underline{\mathbf{S T}} \underline{\mathrm{H}}$ & $\underline{D F}$ & $\underline{\text { Lab }}$ & $4 Q 98$ & Mod & Filt. & $\underline{\text { ST }} \underline{\mathrm{H}}$ & DF & $\underline{\text { Lab }}$ & $\underline{\text { Unit }}$ \\
\hline Americium-241 & $<1.6 E-02$ & $\mathrm{U} 1 / /$ & $<0.1020$ & & 1 & GP & & & & & & & $\mathrm{pCi} / \mathrm{L}$ \\
\hline Beta dose & 8.19 & & & - & & & & & & & & & $\mathrm{pCi} / \mathrm{L}$ \\
\hline Carbon-14 & $<8.1 E+00$ & $\mathrm{U} I / /$ & $<8.8000$ & & 1 & GP & & & & & & & $\mathrm{pCi} / \mathrm{L}$ \\
\hline Cobalt-60 & $<-9.0 E-01$ & $\mathrm{UI} / /$ & $<5.1300$ & & 1 & GP & & & & & & & $\mathrm{pCi} / \mathrm{L}$ \\
\hline Curium-242 & $<0.0 \mathrm{E}+00$ & $\mathrm{U} ! / /$ & $<0.0519$ & & 1 & GP & & & & & & & $\mathrm{pCi} / \mathrm{L}$ \\
\hline Curium-243/244 & $<0.0 E+00$ & $\mathrm{U}: / /$ & $<0.0491$ & & 1 & GP & & & & & & & $\mathrm{pCi} / \mathrm{L}$ \\
\hline Curium-245/246 & $<0.0 \mathrm{E}+00$ & $\mathrm{U} 1 / 1$ & $<0.0490$ & & 1 & GP & & & & & & & $\mathrm{pCi} / \mathrm{L}$ \\
\hline Gross alpha & $3.6 \mathrm{E}+00$ & $\|$ & & & 1 & GP & $<2.1 \mathrm{E}+00$ & $\mathrm{U} / /$ & $<8.9 E-01$ & & 1 & GE & $\mathrm{pC} i / \mathrm{L}$ \\
\hline lodine-129 & $<2.7 \mathrm{E}-01$ & $\mathrm{U} \mathrm{I} / \mathrm{I}$ & $<0.8360$ & & 1 & GP & & & & & & & $\mathrm{pCi} / \mathrm{L}$ \\
\hline Nickel-63 & $<-9.0 \mathrm{E}-01$ & U & $<5.1300$ & & & & & & & & & & $\mathrm{pCilL}$ \\
\hline Nonvolatile beta & $1.4 E+02$ & "l & & - & 1 & GP & $1.2 E+02$ & $/ 1$ & & - & 1 & GE & $\mathrm{pCi} / \mathrm{L}$ \\
\hline Plutonium-238 & $<1.9 \mathrm{E}-02$ & $\mathrm{UI} / I$ & $<0.2180$ & & 1 & GP & & & & & & & $\mathrm{pCi} / \mathrm{L}$ \\
\hline Plutonium-23\$/240 & $<4.3 E-03$ & $\mathrm{U} \mid / /$ & $<0.1070$ & & 1 & GP & & & & & & & $\mathrm{pCi} / \mathrm{L}$ \\
\hline Radium-226 & $<7.6 \mathrm{E}-01$ & UNI & $<0.6410$ & & 1 & GP & & & & & & & $\mathrm{pCi} / \mathrm{L}$ \\
\hline Radium-228 & $2.8 \mathrm{E}+00$ & "I & & & 1 & GP & & & & & & & $\mathrm{pCi} / \mathrm{L}$ \\
\hline Strontium-90 & $6.1 E+01$ & "I & & ש & 1 & GP & & & & & & & $\mathrm{pCi} / \mathrm{L}$ \\
\hline Technetium-99 & $<9.0 \mathrm{E}+00$ & $\mathrm{U} 11 / \mathrm{I}$ & $<20.7000$ & & 1 & GP & & & & & & & $\mathrm{pCi} / \mathrm{L}$ \\
\hline Thorium-228 & $<2.5 E-01$ & $\mathrm{UI} / /$ & $<0.6150$ & & 1 & GP & & & & & & & $\mathrm{pCi} / \mathrm{L}$ \\
\hline Thorium-230 & $<6.7 \mathrm{E}-02$ & $\mathrm{U} \mathrm{I} / \mathrm{I}$ & $<0.3630$ & & 1 & GP & & & & & & & pCill \\
\hline $\begin{array}{l}\text { Thorium-232 } \\
\text { Sum of atphas }\end{array}$ & $<4.0 \mathrm{E}-02$ & $U I / I$ & $<0.2760$ & & 1 & GP & & & & & & & $\mathrm{pCi} / \mathrm{L}$ \\
\hline Sum of betas & $6.4 E+01$ & & & $=$ & & & & & & & & & $\mathrm{pCi/L}$ \\
\hline Total radium & $2.8 E+00$ & & & & & & & & & & & & $\mathrm{pCi/L}$ \\
\hline Tritium & $8.2 E+01$ & "I & & - & 1 & GP & $8.9 E+01$ & /I & & - & 1 & GE & $\mathrm{pCi} / \mathrm{mL}$ \\
\hline Uranium-233/234 & $<1.8 \mathrm{E}-01$ & UN! & $<0.1040$ & & 1 & GP & & & & & & & pCill \\
\hline Uranium-235 & $<4.2 E-02$ & $\mathrm{UI} / 1$ & $<0.1040$ & & 1 & GP & & & & & & & $\mathrm{pCl} / \mathrm{L}$ \\
\hline Uranium-238 & $<1.3 \mathrm{E}-02$ & $\mathrm{UI} / /$ & $<0.0878$ & & 1 & GP & & & & & & & $\mathrm{pCi} / \mathrm{L}$ \\
\hline
\end{tabular}

Notes:

- exceeded holding time

- exceeded groundwater protection or monitoring constituent standard (See Appendix A.) 
WELL HSB101C

\begin{tabular}{ll} 
SRS Coord. & Latlongitude \\
\hline N72001.9 & $33.280640^{\circ} \mathrm{N}$ \\
E58604.4 & $81.652346^{\circ} \mathrm{W}$
\end{tabular}

SAMPLE DATE

07/07/98

\section{Screen Zone Elevation}

176.3-166.3 ft mst
Top of Casing

$258.5 \mathrm{ft} \mathrm{mst}$

FIELD DATA

Constituents

Water elevation

$\mathrm{pH}$

$\mathrm{Sp}$. conductance

Water temperature

Alkalinity as $\mathrm{CaCO}_{3}$

Turbidity

Volumes purged

Sampling code

Synchronous water level $\underline{3098}$

227.8

5.0

41

20.9

6

2.9

$233.4(09 / 17 / 98)$

\section{ANALYTICAL DATA}

Inorganic Constituents

\begin{tabular}{|c|c|c|c|}
\hline Constituents & 3098 & Mod & Filt. \\
\hline Antimony, total recoverable & $<0.085$ & UNI & $<0.20$ \\
\hline Arsenic, total recoverable & $<3.0$ & $U / I$ & $<3.0$ \\
\hline Barium, total recoverable & 12 & $\|$ & \\
\hline Cadmium, total recoverable & 0.16 & $\cdot \quad \mathrm{J} / \mathrm{E}$ & NDD \\
\hline Chromium, total recoverable & 3.5 & H & \\
\hline Cobalt, total recoverable & $<0.20$ & $\mathrm{U} / /$ & $<0.20$ \\
\hline Copper, total recoverable & 0.58 & $\|$ & \\
\hline Cyanide & 4.8 & $\mathrm{~J} / \mathrm{EI} / 2$ & NDD \\
\hline Lead, total recoverable & $<2.0$ & $\mathbf{U} / /$ & $<2.0$ \\
\hline Mercury, total recoverable & $<0.20$ & $\mathrm{U} / /$ & $<0.20$ \\
\hline $\begin{array}{l}\text { Nickel, total recoverabje } \\
\text { Nitrate as nitrogen }\end{array}$ & 1.2 & " & \\
\hline Nitrate-nitrite as nitrogen & 910 & $N !$ & \\
\hline Selenium, total recoverable & 0.96 & $\mathrm{~J} / \mathrm{E} /$ & NDD \\
\hline Silver, total recoverable & $<1.0$ & $\mathbf{U} / /$ & $<1.0$ \\
\hline Tin, total recoverable & $<2.0$ & $\mathrm{U} / I$ & $<2.0$ \\
\hline Vanadium, total recoverable & $<2.0$ & $\mathrm{U} / /$ & $<2.0$ \\
\hline Zinc, total recoverable & 7.8 & $/ /$ & \\
\hline
\end{tabular}

Organic Constituents

$\begin{array}{lllllll}\text { Constituents } & 3098 & \text { Mod } & \text { Filt. } & \text { ST H } & \text { DF } & \text { Lab } \\ \text { Benzene } & <1.0 & \mathrm{U} / \prime & <1.0 & & 1 & \text { GE } \\ \text { Bis(2-ethylhexyl) phthatate } & <9.9 & \mathrm{U} / \prime & <9.9 & & 1 & \text { GE } \\ \text { Dichloromethane } & <1.0 & \mathrm{U} / \prime & <1.0 & & 1 & \text { GE } \\ \text { Tetrachloroethylene } & <1.0 & \mathrm{U} / \prime & <1.0 & & 1 & \text { GE } \\ \text { Trichloroethylene } & <1.0 & \mathrm{U} / \prime & <1.0 & & 1 & \text { GE } \\ \text { Trichlorofluoromethane } & <1.0 & \mathrm{U} / / & <1.0 & & 1 & \text { GE }\end{array}$

Casing Pump

4" PVC S
Screen Zone

Barnwell (IIB $\left.B_{1}\right)$
10/30/98

40.98
232.4
6.2
340
19.4
42
39
0.023
$X N$
$230.9(12 / 21 / 98)$

(12/21/98)
Unit

ft msl

$\mathrm{pH}$

$\mu \mathrm{S} / \mathrm{cm}$

${ }^{\circ} \mathrm{C}$

mg/L

well vol

ftmsl

\begin{tabular}{|c|c|c|c|c|}
\hline \multirow{18}{*}{ SII브 } & $\underline{\mathrm{DF}}$ & Lab & 4Q98 & Mod \\
\hline & 1 & $\mathrm{GE}$ & & \\
\hline & 1 & $\mathrm{GE}$ & & \\
\hline & 1 & $\mathrm{GE}$ & & \\
\hline & 1 & $\overline{G E}$ & & \\
\hline & 1 & $\mathrm{GE}$ & & \\
\hline & 1 & $\mathrm{GE}$ & & \\
\hline & 1 & $\mathrm{GE}$ & & \\
\hline & 1 & $\mathrm{GE}$ & & \\
\hline & 1 & GE & & \\
\hline & 1 & $\mathrm{GE}$ & 4.8 & II \\
\hline & 1 & GE & & \\
\hline & 1 & GE & 29,000 & /I \\
\hline & $i$ & GE & & \\
\hline & 1 & GE & & \\
\hline & 1 & $\mathrm{GE}$ & & \\
\hline & 1 & GE & & \\
\hline & 1 & GE & & \\
\hline
\end{tabular}

Mod

Filt.

ST $\underline{H} \underline{\text { DF }} \underline{\text { Lab }} \underline{\text { Unit }}$

$\mu g / L$

$\mu g / L$

$\mu g / L$

$\mu g / L$

$\mu g h$

$\mu g / L$

$\mu g / L$

$\mu g / 2$

$\mu \mathrm{g} / \mathrm{L}$

GE $\mu g / L$

$\mu \mathrm{g} / \mathrm{L}$

$25 \mathrm{GE} \mu g / \mathrm{L}$

$\mu g / 2$

$\mu g / L$

$\mu g / L$

$\mu g / L$
$\mu g / L$

Trichlorofluoromethane

GE
Filt.

$\underline{\text { ST }} \underline{H}$ DF Lab Unit

$\mu g / L$

$\mu g / L$

$\mu g / L$

$\mu g / L$

$\mu g / L$

$\mu g / \mathrm{L}$

Notes:

- exceeded holding time

- exceeded groundwater protection or monitoring constituent standard (See Appendix A.) 
WELL HSB101C (cont.)

Radioactive Constituents

\begin{tabular}{|c|c|c|c|c|c|c|c|c|c|c|c|c|c|}
\hline Constituents & 3098 & Mod & Filt. & $\underline{\text { ST브 }}$ & $\underline{\text { DF }}$ & $\underline{\text { Lab }}$ & 4Q98 & Mod & Filt. & $\underline{\text { ST }} \underline{H}$ & $\underline{\mathrm{DF}}$ & $\underline{\text { Lab }}$ & Unit \\
\hline Americium-241 & $<1,6 \mathrm{E}-02$ & $\mathrm{UI} / /$ & $<0.0757$ & & 1 & GP & & & & & & & $\mathrm{pCi} / \mathrm{L}$ \\
\hline Carbon-14 & $<2.5 \mathrm{E}+00$ & $\mathbf{U} / /$ & $<8.9100$ & & 1 & GP & & & & & & & $\mathrm{pCi} / \mathrm{L}$ \\
\hline Cobalt -60 & $<-1.6 E+00$ & $\mathrm{UI} / /$ & $<3.3200$ & & 1 & GP & & & & & & & $\mathrm{pCi} / \mathrm{L}$ \\
\hline Curium-242 & $<-6.4 \mathrm{E}-03$ & $\mathrm{U} / /$ & $<0.1410$ & & 1 & GP & & & & & & & $\mathrm{pCi} / \mathrm{L}$ \\
\hline Curium-243/244 & $<7.0 \mathrm{E}-02$ & $\mathrm{Ul} / /$ & $<0.1330$ & & 1 & GP & & & & & & & $\mathrm{pCi} / \mathrm{L}$ \\
\hline Curium-245/246 & $<-6.1 E-03$ & $\mathrm{UI} / I$ & $<0.1330$ & & 1 & GP & & & & & & & $\mathrm{pCi} / \mathrm{L}$ \\
\hline Gross alpha & $<6.1 \mathrm{E}-01$ & $\mathrm{UI} / /$ & $<0.6300$ & & $\uparrow$ & GP & $<9.1 E+00$ & $\mathrm{U} / /$ & $<2.9 \mathrm{E}+00$ & & 1 & GE & $\mathrm{pCi} / \mathrm{L}$ \\
\hline lodine-129 & $<-3.0 E-01$ & $\mathrm{UI} / l$ & $<0.9360$ & & 1 & GP & & & & & & & $\mathrm{pCi} / \mathrm{L}$ \\
\hline Nickel-63 & $<-1.6 \mathrm{E}+00$ & UI & $<3.3200$ & & & & & & & & & & $\mathrm{pCi} / \mathrm{L}$ \\
\hline Nonvolatile beta & $<1.1 E+00$ & $\mathrm{U}: / /$ & $<1.7300$ & & 1 & GP & $7.6 \mathrm{E}+01$ & $/ 1$ & & $\mathbf{m}$ & 1 & GE & $\mathrm{pCi} / \mathrm{L}$ \\
\hline Plutonium-238 & $<6.1 E-04$ & $\mathrm{UH} / /$ & $<0.1150$ & & 1 & GP & & & & & & & $\mathrm{pCi} / \mathrm{L}$ \\
\hline Plutonium-239/240 & $<-1.8 \mathrm{E}-02$ & UI/I & $<0.1230$ & & 1 & GP & & & & & & & $\mathrm{pCi} / \mathrm{L}$ \\
\hline Radium-226 & $<4.9 E-01$ & UNI & $<0.1890$ & & 1 & GP & & & & & & & $\mathrm{pCi} / \mathrm{L}$ \\
\hline Radium-228 & $<5.9 E-01$ & $\mathrm{U} 1 / /$ & $<1.2100$ & & 1 & GP & & & & & & & $\mathrm{pCi} / \mathrm{L}$ \\
\hline Strontium-90 & $<9.4 E-01$ & $\mathrm{U} U / /$ & $<1.7600$ & & 1 & GP & & & & & & & $\mathrm{pCi} / \mathrm{L}$ \\
\hline Tectinetium-99 & $<2.9 E-01$ & $\mathrm{UI} / /$ & $<20.7000$ & & 1 & GP & & & & & & & $\mathrm{pCi} / \mathrm{L}$ \\
\hline Thorium-228 & $<1.8 \mathrm{E}-01$ & $\mathrm{UI} / l$ & $<0.8440$ & & 1 & GP & & & & & & & $\mathrm{pCi} / \mathrm{L}$ \\
\hline Thorium-230 & $<1.9 E-01$ & $\mathrm{UI} / / /$ & $<0.5180$ & & 1 & GP & & & & & & & pCint \\
\hline Thorium-232 & $<4.4 E-02$ & $U \mid / /$ & $<0.3030$ & & 1 & GP & & & & & & & pCill \\
\hline $\begin{array}{l}\text { Sum of alphas } \\
\text { Sum of betas }\end{array}$ & & & & & & & & & & & & & \\
\hline Tritium & $1.0 E+01$ & /l & & & 1 & GP & $2.9 E+03$ & $\|$ & & च & 1 & GE & $\mathrm{pCi} / \mathrm{mL}$ \\
\hline Uranium-233/234 & $<1.6 E-02$ & UIII & $<0.1120$ & & 1 & GP & & & & & & & pCir \\
\hline Uranium-235 & $<2.1 \mathrm{E}-02$ & $\mathrm{UI} / I$ & $<0.0640$ & & 1 & GP & & & & & & & $\mathrm{pCi} / \mathrm{L}$ \\
\hline Uranium-238 & $<1.6 \mathrm{E}-02$ & $\mathrm{UI} / /$ & $<0.1120$ & & 1 & GP & & & & & & & pCill \\
\hline
\end{tabular}

Notes: 
WELL HSB101D

$\begin{array}{lll}\text { SRS Coord. } & \text { Lat/Longitude } \\ \text { N71997.5 } & 33.280614^{\circ} \mathrm{N} \\ \text { E58594.8 } & 81.652362^{\circ} \mathrm{W}\end{array}$

SAMPLE DATE

FIELD DATA

Constituents

Water elevation

$\mathrm{pH}$

Sp. conductance

Water temperature

Alkalinity as $\mathrm{CaCO}_{3}$

Turbidity

Volumes purged

Sampling code

Synchronous water level

ANALYTICAL DATA
Screen Zone Elevation

236.1-216.1 ft msl

$07 / 27 / 98$

3Q98

257.7

21.5

7

280

124

0.0

$227.2(09 / 17 / 98)$
Top of Casing

$258.7 \mathrm{ft} \mathrm{msl}$

$11 / 05 / 98$

$\underline{4098}$

226.2

9.3
95

18.2

9

2

2.4

N

$225.5(12 / 21 / 98)$
Casing Pump

4" PVC V
Screen Zone

Water Table (IIB2)

Inorganic Constituents

\begin{tabular}{|c|c|c|c|c|c|c|c|c|c|c|c|c|c|}
\hline Constituents & $\underline{3098}$ & Mod & Filt. & $\underline{\mathrm{ST}} \underline{\mathrm{H}}$ & $\underline{\mathrm{DF}}$ & $\underline{\text { Lab }}$ & $\underline{4 Q 98}$ & Mod & Filt. & $\underline{\mathrm{ST}} \underline{\mathrm{H}}$ & $\underline{\mathrm{DF}}$ & Lab & Unit \\
\hline Antimony, total recoverable & 0.19 & $J / E J$ & NDD & & 1 & GE & & & & & & & 40/l \\
\hline Arsenic, total recoverable & 39 & NI & & & 1 & GE & & & & & & & $\mu g / L$ \\
\hline Barium, total recoverable & 3.1 & $/ 1$ & & & 1 & GE & & & & & & & $\mu g / L$ \\
\hline Cadmium, total recoverable & $<1,0$ & $\mathrm{U} / /$ & $<1.0$ & & 1 & GE & & & & & & & $\mu \mathrm{g} / \mathrm{L}$ \\
\hline Chromium, total recoverable & 2.0 & $\mathrm{~J} / \mathrm{E} /$ & NDD & & 1 & GE & & & & & & & HolL \\
\hline Cobalt, total recoverable & 0.13 & $\mathrm{~J} / \mathrm{E} /$ & NDD & & 1 & GE & & & & & & & $\mu g / L$ \\
\hline Copper, totai recoverable & 0.40 & $/ 1$ & & & 1 & GE & & & & & & & $\mu \mathrm{g} / \mathrm{L}$ \\
\hline Cyanide & $<10$ & $\mathrm{u} / /$ & $<10$ & & 1 & GE & & & & & & & $\mu \mathrm{g} / \mathrm{L}$ \\
\hline Lead, total recoverable & $<2.0$ & $\mathrm{U} / /$ & $<2.0$ & & 1 & GE & & & & & & & $\mu \mathrm{g} / \mathrm{L}$ \\
\hline Mercury, total recoverable & 2.4 & $" /$ & & - & 1 & $\mathrm{GE}$ & $<0.20$ & $\mathrm{U} / \prime$ & $<0.20$ & & 1 & GE & $\mu \mathrm{g} / \mathrm{L}$ \\
\hline $\begin{array}{l}\text { Nickel, total recoverable } \\
\text { Nitrate as nitrogen }\end{array}$ & $<0.20$ & $\mathrm{U} / /$ & $<0.20$ & & 1 & $\mathrm{GE}$ & & & & & & & $\mu \mathrm{g} / \mathrm{L}$ \\
\hline Nitrate-nitrite as nitrogen & 17,000 & NI & & a & 10 & $\mathrm{GE}$ & 600 & /l & & & 1 & GE & $\mu \mathrm{g} / \mathrm{L}$ \\
\hline Selenium, total recoverable & $<5.0$ & $\mathrm{U} / /$ & $<5.0$ & & 1 & $\mathrm{GE}$ & & & & & & & $\mu g / \mathrm{L}$ \\
\hline Silver, total recoverable & $<1.0$ & $\mathrm{U} / \mathrm{I}$ & $<1.0$ & & 1 & GE & & & & & & & $\mu g / \mathrm{h}$ \\
\hline Tin, total recoverable & $<2.0$ & $\mathrm{U} / /$ & $<2.0$ & & 1 & GE & & & & & & & $\mu / \mathrm{l}$ \\
\hline Vanadium, total recoverable & 140 & $\|$ & & - & 1 & GE & & & & & & & $\mu g / \mathrm{h}$ \\
\hline Zinc, total recoverable & $<5.0$ & $\mathrm{U} / /$ & $<5.0$ & & 1 & GE & & & & & & & $\mu g / L$ \\
\hline \multicolumn{14}{|l|}{ Organic Constituents } \\
\hline Constituents & $\underline{3098}$ & Mod & Filt. & $\underline{\text { ST} \underline{~}}$ & $\underline{D F}$ & $\underline{\text { Lab }}$ & 4098 & Mod & Filt. & $\underline{S T} \underline{H}$ & $\underline{\mathrm{DF}}$ & Lab & Unit \\
\hline Benzene & $<1.0$ & $\mathrm{UJ} / \mathrm{O} / 1$ & $<1.0$ & & 1 & GE & & & & & & & $\mu g / \mathrm{L}$ \\
\hline Bis(2-ethylhexyl) phthalate & $<10$ & $\mathrm{U} / I$ & $<10$ & & 1 & GE & & & & & & & $\mu g / \mathrm{L}$ \\
\hline Dichloromethane & $<16$ & UJ/OV/I & $<1.0$ & & 1 & GE & & & & & & & Hoh \\
\hline Tetrachloroethylene & $<1.0$ & $\mathrm{UJ} / \mathrm{O} / 1$ & $<1.0$ & & 1 & $\mathrm{GE}$ & & & & & & & $\mu \mathrm{g} / \mathrm{L}$ \\
\hline Trichloroethylene & $<1.0$ & $\mathrm{UJ} / \mathrm{O} / 1$ & $<1.0$ & & 1 & $\mathrm{GE}$ & & & & & & & \\
\hline Trichlorofluoromethane & $<1.0$ & UJ/O/1 & $<1.0$ & & 1 & $\mathrm{GE}$ & & & & & & & $\mu \mathrm{g} / \mathrm{L}$ \\
\hline
\end{tabular}

Notes:

- = exceeded holding time

- exceeded groundwater protection or monitoring constituent standard (See Appendix A.) 
WELL HSB101D (cont.)

Radioactive Constituents

\begin{tabular}{|c|c|c|c|c|c|c|c|c|c|c|c|c|c|}
\hline Constituents & 3098 & Mod & Filt. & $\underline{\text { ST }} \underline{H}$ & $\underline{\mathrm{DF}}$ & $\underline{\text { Lab }}$ & 4Q98 & Mod & Filt. & $\underline{\mathrm{ST}} \underline{\mathrm{H}}$ & $\underline{D F}$ & $\underline{\text { Lab }}$ & Unit \\
\hline Americium-241 & $<3.0 \mathrm{E}-02$ & $\mathrm{UI} / /$ & $<0.0297$ & & 1 & GP & & & & & & & $\mathrm{pCi} / \mathrm{L}$ \\
\hline Beta dose & 8.03 & & & $\mathbf{\square}$ & & & & & & & & & pCill \\
\hline Carbon-14 & $5.8 \mathrm{E}+02$ & $\|$ & & $\mathbf{\square}$ & 1 & GP & & & & & & & $\mathrm{pCi} / \mathrm{L}$ \\
\hline Cobalt-60 & $<-1.5 E+00$ & $\mathrm{UU} / /$ & $<3.4200$ & & 1 & GP & & & & & & & $\mathrm{pCi} / \mathrm{L}$ \\
\hline Curium-242 & $<1.1 E-02$ & $\mathrm{UI} / I$ & $<0.0316$ & & 1 & $G P$ & & & & & & & $\mathrm{pCi} / \mathrm{L}$ \\
\hline Curium-243/244 & $<1.5 E-02$ & $\mathrm{UI} / I$ & $<0.0626$ & & 1 & GP & & & & & & & $\mathrm{pCi} / \mathrm{L}$ \\
\hline Curium-245/246 & $<0.0 \mathrm{E}+00$ & $\mathrm{UI} / \mathrm{I}$ & $<0.0297$ & & 1 & GP & & & & & & & $\mathrm{pCi} / \mathrm{L}$ \\
\hline Gross alpha & $9.5 \mathrm{E}-01$ & $/ /$ & & & 1 & GP & $<3.4 \mathrm{E}-01$ & $\mathrm{U} / /$ & $<1.1 \mathrm{E}+00$ & & 1 & GE & $\mathrm{pCilL}$ \\
\hline lodine-129 & $5.2 \mathrm{E}+00$ & $/ /$ & & & 1 & GP & & & & & & & $\mathrm{pCi} / \mathrm{L}$ \\
\hline Nickel-63 & $<-1.5 E+00$ & U! & $<3.4200$ & & & & & & & & & & $\mathrm{pCi} / \mathrm{L}$ \\
\hline Nonvolatile beta & $1.0 E+01$ & $/ /$ & & & 1 & GP & $<1.5 \mathrm{E}+00$ & $\mathrm{U} / /$ & $<1.8 \mathrm{E}+00$ & & 1 & GE & $\mathrm{pCi} / \mathrm{L}$ \\
\hline Plutonium-238 & $<0.0 \mathrm{E}+00$ & $\mathrm{UI} / /$ & $<0.0246$ & & 1 & GP & & & & & & & $\mathrm{pCi} / \mathrm{L}$ \\
\hline Plutonium-239/240 & $<1.6 \mathrm{E}-02$ & $\mathrm{UI} / /$ & $<0.0245$ & & 1 & GP & & & & & & & $\mathrm{pCi} / \mathrm{L}$ \\
\hline Radium-226 & $1.6 E+00$ & $/ /$ & & & 1 & GP & & & & & & & $\mathrm{pCi} / \mathrm{L}$ \\
\hline Radium-228 & $<-2.6 \mathrm{E}-01$ & UIII & $<1.0900$ & & 1 & GP & & & & & & & $\mathrm{pCi} / \mathrm{L}$ \\
\hline Strontium-90 & $2.0 E+01$ & $/ /$ & & $\boldsymbol{\square}$ & 1 & GP & & & & & & & $\mathrm{pCi} / \mathrm{L}$ \\
\hline Technetium-99 & $3.9 E+01$ & /I & & & 1 & GP & & & & & & & $\mathrm{pCi} / \mathrm{L}$ \\
\hline Thorium-228 & $<1.2 \mathrm{E}-01$ & $\mathrm{UIJ} / \mathrm{Cl}$ & $<0.659$ & & 1 & GP & & & & & & & $\mathrm{pCi} / \mathrm{L}$ \\
\hline Thorium-230 & $<-6.8 E-02$ & $\mathrm{U} \mid \mathrm{J} / \mathrm{C} /$ & $<0.652$ & & 1 & GP & & & & & & & $\mathrm{pCuL}$ \\
\hline Thorium-232 & $<-4.5 E-02$ & $\mathrm{UIJ} / \mathrm{C} /$ & $<0.584$ & & 1 & GP & & & & & & & $\mathrm{pCill}$ \\
\hline Sum of alphas & $3.3 E+\infty 0$ & & & & & & & & & & & & pCill \\
\hline Sum of betas & $6.4 \mathrm{E}+02$ & & & $\mathbf{0}$ & & & & & & & & & pCi/L \\
\hline Total radium & $1.6 \mathrm{E}+00$ & & & & & & & & & & & & $\mathrm{pCilL}$ \\
\hline Tritium & $1.2 E+03$ & $/ /$ & & $\square$ & 1 & GP & $6.6 E+00$ & $/ /$ & & & 1 & GE & $\mathrm{pCi} / \mathrm{mL}$ \\
\hline Uranium-233/234 & $1.3 E+\infty 0$ & /I & & & 1 & GP & & & & & & & $\mathrm{pCill}$ \\
\hline Uranium-235 & 2.5E-01 & $\|$ & & & 1 & GP & & & & & & & pCill \\
\hline Uranium-238 & $1.8 E+00$ & $/ /$ & & & 1 & GP & & & & & & & $\mathrm{pCi} / \mathrm{L}$ \\
\hline
\end{tabular}

Notes:

- = exceeded holding time

- exceeded groundwater protection or monitoring constituent standard (See Appendix A.) 
WELL HSB102C

\begin{tabular}{|c|c|c|c|}
\hline SRS Coord. & Lat/Longitude & & Screen Zone Elevation \\
\hline $\begin{array}{l}\text { N71960.1 } \\
\text { E58399.7 }\end{array}$ & $\begin{array}{l}33.280213^{\circ} \mathrm{N} \\
81.652803^{\circ} \mathrm{W}\end{array}$ & & $176.7-166.7 \mathrm{ft} \mathrm{msl}$ \\
\hline SAMPLE DA & & $07 / 07 / 98$ & \\
\hline FIELD DATA & & & \\
\hline Constituents & & $\underline{3098}$ & \\
\hline Water elevat & & 226.7 & \\
\hline $\mathrm{pH}$ & & 5.6 & \\
\hline Sp. conducte & & 180 & \\
\hline Water tempe & & 21.5 & \\
\hline Alkalinity as & $\mathrm{O}_{3}$ & 34 & \\
\hline Turbidity & & 3 & \\
\hline $\begin{array}{l}\text { Volumes pur } \\
\text { Sampling cor }\end{array}$ & & 3.0 & \\
\hline Synchronous & ter level & 226.0105 & \\
\hline
\end{tabular}

\section{Top of Casing}

$259 \mathrm{ft} \mathrm{msl}$

$10 / 07 / 98$

4Q98

225.7

5.5

170

213

16

$224.3(12 / 21 / 98)$
Casing Pump

$4^{\text {" PVC } \quad S}$
Screen Zone

Bamwell $\left(\mathrm{IB}_{1}\right)$

ANALYTICAL DATA

Inorganic Constituents

Constituents

Antimony, total recoverable Arsenic, total recoverable Barium, total recoverable

Cadmium, total recoverable

Chromium, total recoverable Cobalt, total recoverable

Copper, total recoverable

Cyanide

Lead, total recoverable $<2.0$

Mercury, total recoverable $\quad 0.40$

Nickel, total recoverable $\quad 5.5$

Nitrate as nitrogen

Nitrate-nitrite as nitrogen $\quad 16,000$

Selenium, total recoverable

Silver, total recoverable $<1.0$

Tin, total recoverable $\quad 0.19$

Vanadium, total recoverable $<2.0$

Zinc, total recoverable

28

Organic Constituents

Constituents
Benzene
Bis(2-ethylhexyl) phthalate
Dichloromethane
Tetrachloroethylene
Trichloroethylene
Trichlorofluoromethane

3098
$<1.0$
$<10$
$<1.0$
$<1.0$
$<1.0$
$<1.0$

Unit

ft ms

$\mathrm{pH}$

$\mu \mathrm{S} / \mathrm{cm}$

${ }^{\circ} \mathrm{C}$

$\mathrm{mg} / \mathrm{L}$

NTU

well vol

ft msł

\begin{tabular}{|c|c|}
\hline Mod & Filt. \\
\hline UNI & $<0.20$ \\
\hline $\mathrm{U} / /$ & $<3.0$ \\
\hline ll & \\
\hline $\mathrm{J} / \mathrm{E} /$ & NDD \\
\hline $\mathrm{J} / \mathrm{E} /$ & NDD \\
\hline "I & \\
\hline$/ /$ & \\
\hline $\mathrm{U} / /$ & $<10$ \\
\hline $\mathrm{U} / /$ & $<2.0$ \\
\hline$/ 1$ & \\
\hline 11 & \\
\hline$N /$ & \\
\hline$J / E J$ & NDD \\
\hline $\mathrm{U} / /$ & $<1.0$ \\
\hline $\mathrm{J} / \mathrm{E} /$ & NDD \\
\hline $\mathrm{U} / \prime$ & $<2.0$ \\
\hline
\end{tabular}

STH

1
1
1
1
1
1

$\begin{array}{ll}\text { DF } & \text { La } \\ 1 & \text { GE } \\ 1 & \text { GE } \\ 1 & \text { GE } \\ 1 & \text { GE } \\ 1 & \text { GE } \\ 1 & \text { GE } \\ 1 & \text { GE } \\ 1 & \text { GE } \\ 1 & \text { GE }\end{array}$

Lab 4Q98 Mod

Filt.
- 25 GE 14,000 /l

$1.0 \quad 1$ GE

1 GE

1 GE

\begin{tabular}{|c|c|}
\hline ST브 & DF \\
\hline & 1 \\
\hline & 1 \\
\hline & 1 \\
\hline & 1 \\
\hline & 1 \\
\hline & 1 \\
\hline
\end{tabular}

ST H DF Lab Unit

$\mu g / L$

$\mu g / L$

$\mu g / L$

$\mu g / L$

$\mu g / L$

$\mu g / L$

$\mu g / L$

$\mu g / L$

$\mu g / L$

$1 \mathrm{GE} \mu g / \mathrm{L}$ $\mu g / L$

- 5 GE $\mu g / L$

$\mu g$ h

$\mu \mathrm{g} / \mathrm{L}$

$\mu \mathrm{g} / \mathrm{L}$

$\mu g / L$

$\mu g / L$

$\begin{array}{ll}\text { Mod } & \text { Filt. } \\ U / I & <1.0 \\ U / / & <10 \\ U / I & <1.0 \\ U / / & <1.0 \\ U / 1 & <1.0 \\ U / / & <1.0\end{array}$

GE

Filt.

ST $\underline{H}$ DF Lab Unit

$\mu g / L$

$\mu g / L$

$\mu g / L$

$\mu g / L$

$\mu g / L$

$\mu g / L$

Notes:

- =xceeded holding time

- exceeded groundwater protection or monitoring constituent standard (See Appendix A.) 
WELL HSB102C (cont.)

Radioactive Constituents

\begin{tabular}{|c|c|c|c|c|c|c|c|c|c|c|c|c|c|}
\hline Constituents & $3 Q 98$ & Mod & Filt. & $\underline{\text { ST }} \underline{\text { H }}$ & $\underline{\text { DF }}$ & $\underline{\text { Lab }}$ & 4Q98 & Mod & Filt. & $\underline{\mathrm{ST}} \underline{\mathrm{H}}$ & $\underline{\mathrm{DF}}$ & $\underline{L a b}$ & Unit \\
\hline Americium-241 & $<-9.3 E-03$ & $\mathrm{Ul} / /$ & $<0.0474$ & & 1 & GP & & & & & & & $\rho \mathrm{Ci} / \mathrm{L}$ \\
\hline Beta dose & 0.58 & & & & & & & & & & & & $\mathrm{pCi} / \mathrm{L}$ \\
\hline Carbon-14 & $<3.3 E+00$ & $\mathrm{UII} / /$ & $<9.0100$ & & 1 & GP & & & & & & & $p \mathrm{Ci} / \mathrm{L}$ \\
\hline Cobalt-60 & $<-1.1 E+00$ & UIII & $<4.0800$ & & 1 & GP & & & & & & & $\mathrm{pCi} / \mathrm{L}$ \\
\hline Curium-242 & $<4.7 E-03$ & $\mathrm{UI} / I$ & $<0.1160$ & & 1 & GP & & & & & & & $\mathrm{pCi} / \mathrm{L}$ \\
\hline Curium-243/244 & $<1.6 \mathrm{E}-02$ & UIII & $<0.0474$ & & 1 & GP & & & & & & & $\mathrm{pCi} / \mathrm{L}$ \\
\hline Curium-245/246 & $<0.0 E+00$ & Ut/I & $<0.0474$ & & 1 & GP & & & & & & & $\mathrm{pCi} / \mathrm{L}$ \\
\hline Gross alpha & $3.3 E+00$ & $/ /$ & & & 1 & GP & $1.9 E+00$ & $\|$ & & & 1 & GE & $\rho \mathrm{Ci} / \mathrm{L}$ \\
\hline lodine-129 & $<8.3 E-01$ & $\mathrm{UI} / /$ & $<0.9600$ & & 1 & GP & & & & & & & $\mathrm{pCi} / \mathrm{L}$ \\
\hline Nickel-63 & $<-1.1 E+00$ & UI & $<4.0800$ & & & & & & & & & & $\mathrm{pCi} / \mathrm{L}$ \\
\hline Nonvolatile beta & $5.6 \mathrm{E}+00$ & 11 & & & 1 & GP & $3.7 \mathrm{E}+00$ & $/ 1$ & & & 1 & GE & $\mathrm{pCi} / \mathrm{L}$ \\
\hline Plutonium-238 & $<8.0 \mathrm{E}-03$ & $\mathrm{UI} / /$ & $<0.1220$ & & 1 & GP & & & & & & & $\mathrm{pCi} / \mathrm{L}$ \\
\hline Plutonium-239/240 & $<-6.8 \mathrm{E}-03$ & $\mathrm{UI} / /$ & $<0.0886$ & & 1 & GP & & & & & & & $\mathrm{pCi} / \mathrm{L}$ \\
\hline Radium-226 & $2.1 E+00$ & /I & & & 1 & GP & & & & & & & $\mathrm{pCi} / \mathrm{L}$ \\
\hline Radium-228 & $1.4 E+00$ & $/ 1$ & & & 1 & GP & & & & & & & $\mathrm{pCi} / \mathrm{L}$ \\
\hline Strontium-90 & $2.4 E+\infty 0$ & $/ 1$ & & & 1 & GP & & & & & & & $\mathrm{pCi} / \mathrm{L}$ \\
\hline Technetium-99 & $<1.7 E+00$ & $\mathrm{U} \mid / /$ & $<19.8000$ & & 1 & GP & & & & & & & pCill \\
\hline Thorium-228 & $<-1.9 \mathrm{E}-01$ & $\mathrm{UI} / /$ & $<0.7590$ & & 1 & GP & & & & & & & $\mathrm{pCi} / \mathrm{L}$ \\
\hline Thorium-230 & $<8.3 E-02$ & UII/ & $<0.3730$ & & 1 & GP & & & & & & & $\mathrm{pCi} / \mathrm{L}$ \\
\hline Thorium-232 & $<-9.2 E-03$ & $\mathrm{U} / /$ & $<0.3730$ & & 1 & GP & & & & & & & $\mathrm{pCi} / \mathrm{L}$ \\
\hline Sum of alphas & & & & & & & & & & & & & \\
\hline $\begin{array}{l}\text { Sum of betas } \\
\text { Total radium }\end{array}$ & $\begin{array}{l}3.8 E+00 \\
3.5 E+00\end{array}$ & & & & & & & & & & & & $\begin{array}{l}\mathrm{pCi} / \mathrm{L} \\
\mathrm{pCi} / \mathrm{L}\end{array}$ \\
\hline Tritium & $1.5 E+02$ & $/ /$ & & - & 1 & GP & $1.7 E+02$ & $/ /$ & & $\mathbf{a}$ & 1 & GE & $\mathrm{pCi} / \mathrm{mL}$ \\
\hline Uranium-233/234 & $<4.2 \mathrm{E}-02$ & $\mathrm{UI} / /$ & $<0.1280$ & & 1 & GP & & & & & & & $\mathrm{pCi} / \mathrm{L}$ \\
\hline Uranium-235 & $<4.7 E-02$ & $\mathrm{UI} / /$ & $<0.1150$ & & 1 & GP & & & & & & & $\mathrm{pCi} / \mathrm{L}$ \\
\hline Uranium-238 & $<1,4 E-02$ & $\mathrm{UI} / /$ & $<0.0974$ & & 1 & GP & & & & & & & $\mathrm{pCi} / \mathrm{L}$ \\
\hline
\end{tabular}

Notes:

- = exceeded holding time

= exceeded groundwater protection or monitoring constituent standard (See Appendix A.) 
WELL HSB102D

\begin{tabular}{|c|c|}
\hline SRS Coord. & Lat/Longitude \\
\hline $\begin{array}{l}\text { N71952.4 } \\
\text { E58393.4 }\end{array}$ & 81.652805 o \\
\hline
\end{tabular}

SAMPLE DATE

$07 / 21 / 98$

FIELD DATA

Constituents

Water elevation

$\mathrm{pH}$

Sp. conductance

Water temperature

Alkalinity as $\mathrm{CaCO}_{3}$

Turbidity

Volumes purged

Sampling code

Synchronous water level

Screen Zone Elevation

236.3-216.3 ft msl

ANALYTICAL DATA

Inorganic Constituents

3098

Antimony total recoverable $<0.20$

Arsenic, total recoverable $\quad<3.0$

Barium, total recoverable 14

Cadmium, total recoverable 0.34

Chromium, total recoverable 1.1

Cobalt, total recoverable $\quad 1.5$

Copper, total recoverable $\quad 9.0$

Cyanide

Lead, total recoverable

Mercury, total recoverable

Nickel, total recoverable

Nitrate as nitrogen

Nitrate-nitrite as nitrogen $\quad 10,000$

Selenium, total recoverable $<5.0$

Silver, total recoverable $<1.0$

Tin, total recoverable $<2.0$

Vanadium, total recoverable $<2.0$

Zinc, total recoverable

38

Organic Constituents

Benzene

3098

Bis(2-ethylhexyl) phthalate $\quad<9.6$

Dichloromethane

Tetrachloroethylene

Trichloroethylene

Trichlorofluoromethane

\section{8}

231.2

3.6

120

20.0

5

0.10

$\mathrm{XN}$

$232.7(09 / 17 / 98)$
Top of Casing

$258.6 \mathrm{ft} \mathrm{ms}$

$10 / 13 / 98$

$4 Q 98$
230.1
3.3
170
18.2
0
7
0.0
$X N$
$228.3(12 / 21 / 98)$

Unit

ft ms!

pH

$\mu S / \mathrm{cm}$

${ }^{\circ} \mathrm{C}$

$\mathrm{mg} / \mathrm{L}$

well vol

ft msl 
WELL HSB102D (cont.)

Radioactive Constituents

\begin{tabular}{|c|c|c|c|c|c|c|c|c|c|c|c|c|c|}
\hline Constituents & $\underline{3 Q 98}$ & Mod & Filt. & ST브 & $\underline{D F}$ & $\underline{L a b}$ & 4098 & Mod & Filt. & $\underline{\mathrm{ST}} \underline{H}$ & $\underline{\mathrm{DF}}$ & $\underline{L a b}$ & Unit \\
\hline Americium-241 & $<7.4 \mathrm{E}-02$ & $\mathrm{UI} / /$ & $<0.138$ & & 1 & GP & & & & & & & $\mathrm{pCi} / \mathrm{L}$ \\
\hline Beta dose & 93.71 & & & 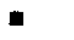 & & & & & & & & & $\mathrm{pCi} / \mathrm{L}$ \\
\hline Carbon-14 & $3.8 E+01$ & $\prime \prime$ & & & 1 & GP & & & & & & & $\mathrm{pCi} / \mathrm{L}$ \\
\hline Cobalt-60 & $1.3 E+01$ & $\|$ & & & 1 & GP & & & & & & & $\mathrm{pCi} / \mathrm{L}$ \\
\hline Curium-242 & $<0.0 \mathrm{E}+00$ & $\mathrm{UI} / \mathrm{I}$ & $<0.149$ & & 1 & GP & & & & & & & $\mathrm{pCi} / \mathrm{L}$ \\
\hline Curium-243/244 & $<4.6 \mathrm{E}-02$ & $\mathrm{U} / \mathrm{I}$ & $<0.139$ & & 1 & GP & & & & & & & $\mathrm{pCi} / \mathrm{L}$ \\
\hline Curium-245/246 & $<-1.1 E-02$ & $\mathrm{UI} / /$ & $<0.243$ & & 1 & $\mathrm{GP}$ & & & & & & & $\mathrm{pCi} / \mathrm{L}$ \\
\hline Gross alpha & $5.0 \mathrm{E}+01$ & "I & & - & 1 & GP & $5.8 \mathrm{E}+01$ & /l & & - & 1 & GE & $\mathrm{pCi} / \mathrm{L}$ \\
\hline lodine-129 & $2.0 \mathrm{E}+00$ & /I & & & 1 & $G P$ & & & & & & & $\mathrm{pCi/L}$ \\
\hline Nickel-63 & $4.5 \mathrm{E}+01$ & "I & & & 1 & GP & & & & & & & $\mathrm{pCil}$ \\
\hline Nonvolatile beta & $1.6 \mathrm{E}+03$ & $\prime \prime$ & & - & 1 & GP & $2.3 E+03$ & "I & & ש & 1 & GE & $\mathrm{pCil}$ \\
\hline Plutonium-238 & $<5.2 \mathrm{E}-02$ & $U 1 / /$ & $<0.156$ & & 1 & GP & & & & & & & $\mathrm{pCi} / \mathrm{L}$ \\
\hline Plutonium-239/240 & $<5.2 \mathrm{E}-02$ & $\mathrm{UI} / /$ & $<0.156$ & & 1 & GP & & & & & & & $\mathrm{pCi} / \mathrm{L}$ \\
\hline Radium-226 & $2.6 \mathrm{E}+00$ & $\|$ & & & 1 & GP & & & & & & & $\mathrm{pCi} / \mathrm{L}$ \\
\hline Radium-228 & $3.3 E+\infty 0$ & /I & & & 1 & GP & & & & & & & $\mathrm{pCi} / \mathrm{L}$ \\
\hline Strontium-90 & $7.2 \mathrm{E}+02$ & "I & & - & 1 & GP & & & & & & & $\mathrm{pCi} / \mathrm{L}$ \\
\hline Technetium-99 & $<1.8 E+01$ & $\mathrm{U} 1 / 1 /$ & $<22.4$ & & 1 & GP & & & & & & & $\mathrm{pCi} / \mathrm{L}$ \\
\hline Thorium-228 & $5.0 \mathrm{E}-01$ & R/4/ & Rej & & 1 & GP & & & & & & & $\mathrm{pCi} / \mathrm{L}$ \\
\hline Thorium-230 & $<1.5 \mathrm{E}-01$ & $\mathrm{UI} / /$ & $<0.224$ & & 1 & GP & & & & & & & pCill \\
\hline Thorium-232 & $<0.0 E+00$ & $\mathrm{UI} / /$ & $<0.224$ & & 1 & GP & & & & & & & $\mathrm{pCi} / \mathrm{L}$ \\
\hline Sum of alphas & $3.0 E+01$ & & & - & & & & & & & & & $\mathrm{pCi} / \mathrm{L}$ \\
\hline Sum of betas & $\begin{array}{l}8.2 E+02 \\
6.0 F+00\end{array}$ & & & - & & & & & & & & & PCilL \\
\hline $\begin{array}{l}\text { Total radium } \\
\text { Tritium }\end{array}$ & $\begin{array}{l}6.0 E+00 \\
2.6 E+02\end{array}$ & $/ /$ & & $\mathbf{n}$ & 1 & & & & & & & GF & PCill \\
\hline Uranium-233/234 & $1.6 E+01$ & /I & & $\approx$ & 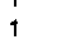 & GP & $1.4 E+03$ & "l & & 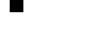 & 1 & GE & $\mathrm{pCi} / \mathrm{mL}$ \\
\hline Uranium-235 & $7.9 \mathrm{E}-01$ & $\|$ & & & 1 & GP & & & & & & & $\mathrm{pCil}$ \\
\hline Uranium-238 & $1.3 \mathrm{E}+01$ & // & & & .1 & GP & & & & & & & pCVL \\
\hline
\end{tabular}


WELL HSB103C

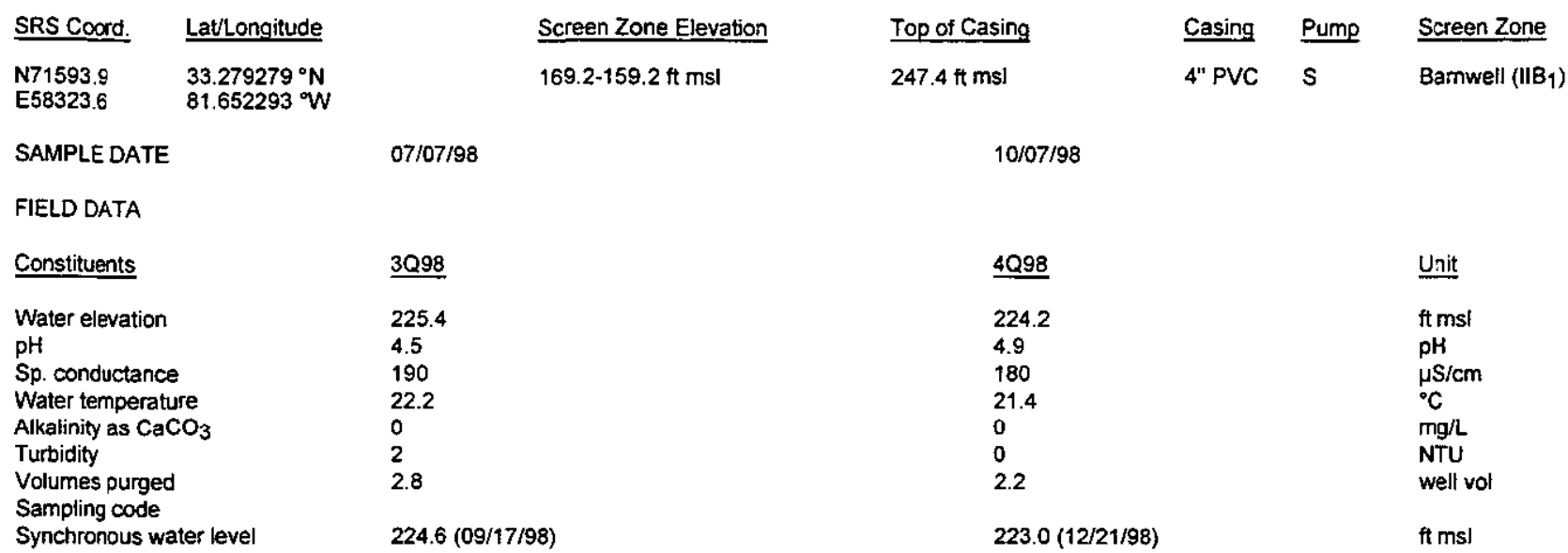

ANALYTICAL DATA

Inorganic Constituents

\begin{tabular}{|c|c|c|c|c|c|c|c|c|c|c|c|c|c|}
\hline Constituents & $\underline{3 Q 98}$ & Mod & Filt. & $\underline{\text { ST }} \underline{H}$ & $\underline{D F}$ & Lab & $\underline{4098}$ & Mod & Filt. & $\underline{\mathbf{S T}} \underline{\mathrm{H}}$ & $\underline{\mathrm{DF}}$ & Lab & $\underline{\text { Unit }}$ \\
\hline Antimony, total recoverable & $<0.077$ & UNI & $<0.20$ & & 1 & GE & & & & & & & $\mu \mathrm{g} / \mathrm{h}$ \\
\hline Arsenic, total recoverable & 0.62 & $3 / E J$ & NDD & & 1 & GE & & & & & & & $\mu g / L$ \\
\hline Barium, total recoverable & 75 & "I & & & 1 & GE & & & & & & & $\mu \mathrm{g} / \mathrm{L}$ \\
\hline Cadmium, total recoverable & 0.64 & J/EI & NDD & & 1 & $\mathrm{GE}$ & & & & & & & $\mu g / L$ \\
\hline Chromium, total recoverable & 0.64 & $J / E J$ & NDD & & 1 & $\mathrm{GE}$ & & & & & & & $\mu g / L$ \\
\hline Cobalt, total recoverable & 15 & $/ /$ & & m & 1 & $\mathrm{GE}$ & & & & & & & $\mu g / L$ \\
\hline Copper, total recoverable & 0.96 & /I & & & 1 & GE & & & & & & & $\mu g / L$ \\
\hline Cyanide & $<10$ & $\mathrm{U} / I$ & $<10$ & & 1 & GE & & & & & & & $\mu g / L$ \\
\hline Lead, total recoverable & $<2.0$ & $\mathrm{U} / /$ & $<2.0$ & & 1 & $\mathrm{GE}$ & & & & & & & $\mu g / L$ \\
\hline Mercury, total recoverable & 1.3 & $" 1$ & & & 1 & GE & 1.2 & $\|$ & & & 1 & GE & $\mu g / L$ \\
\hline $\begin{array}{l}\text { Nickel, total recoverable } \\
\text { Nitrate as nitrogen }\end{array}$ & 6.0 & $\|$ & & & 1 & $\mathrm{GE}$ & & & & & & & $\mu g / L$ \\
\hline Nitrate-nitrite as nitrogen & 20,000 & $N I$ & & m & 25 & GE & 21,000 & $/ 1$ & & - & 25 & GE & $\mu g / L$ \\
\hline Selenium, total recoverable & 0.95 & $\mathrm{~J} / \mathrm{E} /$ & NDD & & 1 & $\mathrm{GE}$ & & & & & & & $\mu g / 1$ \\
\hline Silver, total recoverable & $<1.0$ & $\mathrm{u} / 1$ & $<1.0$ & & 1 & $\mathrm{GE}$ & & & & & & & $\mu \mathrm{g} / \mathrm{L}$ \\
\hline Tin, total recoverable & $<2.0$ & $\mathrm{U} / /$ & $<2.0$ & & 1 & $\mathrm{GE}$ & & & & & & & $\mu g / L$ \\
\hline Vanadium, total recoverable & $<2.0$ & $\mathrm{U} / \prime$ & $<2.0$ & & 1 & GE & & & & & & & $\mu g / L$ \\
\hline Zinc, total recoverable & 21 & $/ 1$ & & & 1 & GE & & & & & & & $\mu g / 2$ \\
\hline \multicolumn{14}{|l|}{ Organic Constituents } \\
\hline Constituents & $\underline{3098}$ & Mod & Filt. & $\underline{\text { ST }} \underline{H}$ & $\underline{\mathrm{DF}}$ & Lab & $\underline{4 Q 98}$ & Mod & Filt. & $\underline{\text { ST }} \underline{H}$ & $\underline{D F}$ & $\underline{\text { Lab }}$ & $\underline{\text { Unit }}$ \\
\hline Benzene & $<1.0$ & $\mathbf{U} / 1$ & $<1.0$ & & 1 & GE & & & & & & & $\mu g / \mathrm{L}$ \\
\hline Bis(2ethylhexyl) phthalate & $<9.9$ & $\mathrm{U} / 1$ & $<9.9$ & & 1 & GE & & & & & & & $\mu g / L$ \\
\hline Dichloromethane & $<1.0$ & $\mathrm{U} / \mathrm{I}$ & $<1.0$ & & 1 & $\mathrm{GE}$ & & & & & & & $\mu g / L$ \\
\hline Tetrachloroethylene & $<1.0$ & $\mathrm{U} / \mathrm{I}$ & $<1.0$ & & 1 & GE & & & & & & & $\mu g / L$ \\
\hline Trichloroethylene & 3.8 & $/ 1$ & & & 1 & GE & & & & & & & $\mu g / L$ \\
\hline Trichlorofluoromethane & $<1.0$ & $\mathrm{U} / /$ & $<1.0$ & & 1 & GE & & & & & & & $\mu g / L$ \\
\hline
\end{tabular}

Notes:

- exceeded holding time

- = exceeded groundwater protection or monitoring constituent standard (See Appendix A.) 
WELL HSB103C (cont.)

Radioactive Constituents

\begin{tabular}{|c|c|c|c|c|c|c|c|c|c|c|c|c|c|}
\hline Constituents & $\underline{3 Q 98}$ & Mod & Filt. & $\underline{\text { ST }} \underline{H}$ & $\underline{\mathrm{DF}}$ & $\underline{\text { Lab }}$ & $\underline{4 Q 98}$ & Mod & Filt. & $\underline{\text { ST }} \underline{H}$ & DF & $\underline{\text { Lab }}$ & $\underline{\text { Unit }}$ \\
\hline Americium-241 & $<-9.3 \mathrm{E}-03$ & $\mathrm{UI} / \mathrm{I}$ & $<0.0637$ & & 1 & GP & & & & & & & $\mathrm{pCi} / \mathrm{L}$ \\
\hline Beta dose & 4.93 & & & $\square$ & & & & & & & & & $\mathrm{PCi} / \mathrm{L}$ \\
\hline Carbon-14 & $1.3 E+01$ & $" 1$ & & & 1 & GP & & & & & & & pCi/L \\
\hline Cobalt- 60 & $<-1.8 \mathrm{E}-01$ & $\mathrm{UII} / \mathrm{H}$ & $<4.3500$ & & 1 & GP & & & & & & & $\mathrm{pCi} / \mathrm{L}$ \\
\hline Curium-242 & $<0.0 E+00$ & $\mathrm{U} I / /$ & $<0.0673$ & & 1 & GP & & & & & & & $\mathrm{PCi} / \mathrm{L}$ \\
\hline Curium-243/244 & $<2.1 \mathrm{E}-02$ & $\mathrm{U} / / /$ & $<0.0637$ & & 1 & GP & & & & & & & $\mathrm{pCi} / \mathrm{L}$ \\
\hline Curium-245/246 & $<0.0 E+00$ & $\mathrm{UI} / /$ & $<0.0636$ & & 1 & GP & & & & & & & $\mathrm{pCi} / \mathrm{L}$ \\
\hline Gross alpha & $4.4 E+00$ & $1 /$ & & & 1 & GP & $4.6 E+\infty 0$ & $/ 1$ & & & 1 & GE & pCill \\
\hline lodine-129 & $4.9 E+00$ & $N l$ & & & 1 & GP & & & & & & & $\mathrm{PC} / / \mathrm{L}$ \\
\hline Nickel-63 & $<-1.8 \mathrm{E}-01$ & U! & $<4.3500$ & & & & & & & & & & $\mathrm{pCi} / \mathrm{L}$ \\
\hline Nonvolatile beta & $1.3 E+01$ & 11 & & & 1 & GP & $1.3 \mathrm{E}+01$ & /I & & & 1 & GE & $\mathrm{pC} / / \mathrm{L}$ \\
\hline Plutonium-238 & $<2.6 \mathrm{E}-02$ & $\mathrm{UI} / /$ & $<0.0771$ & & 1 & GP & & & & & & & $\mathrm{PCi} / \mathrm{L}$ \\
\hline Plutonium-239/240 & $<-3.5 \mathrm{E}-03$ & Uil/ & $<0.0771$ & & 1 & GP & & & & & & & $\mathrm{pCi} / \mathrm{L}$ \\
\hline Radium-226 & $2.2 \mathrm{E}+00$ & 11 & & & 1 & GP & & & & & & & $\mathrm{pCi} / \mathrm{L}$ \\
\hline Radium-228 & $<8.1 \mathrm{E}-01$ & $\mathrm{UI} / /$ & $<1.1200$ & & 1 & GP & & & & & & & $\mathrm{pCi} / \mathrm{L}$ \\
\hline Strontium-90 & $<1.2 E+00$ & $\mathrm{UW} / /$ & $<1.8800$ & & 1 & GP & & & & & & & $\mathrm{pCi} / \mathrm{L}$ \\
\hline Technetium-99 & $2.4 E+01$ & "I & & & 1 & GP & & & & & & & $\mathrm{pCi} / \mathrm{L}$ \\
\hline Thorium-228 & $<3.4 E-01$ & $\mathrm{UIII}$ & $<0.6350$ & & 1 & GP & & & & & & & $\mathrm{pCi} / \mathrm{L}$ \\
\hline Thorium-230 & $<4.9 \mathrm{E}-02$ & $\mathrm{UI} / 1$ & $<0.1470$ & & 1 & GP & & & & & & & $\mathrm{pCi} / \mathrm{L}$ \\
\hline Thorium-232 & $<-3.5 \mathrm{E}-02$ & $\mathrm{UI} / /$ & $<0.3400$ & & 1 & GP & & & & & & & $\mathrm{pCi/L}$ \\
\hline Sum of alphas & 9.7E-02 & & & & & & & & & & & & $\mathrm{pCi} / \mathrm{L}$ \\
\hline $\begin{array}{l}\text { Sum of betas } \\
\text { Total radium }\end{array}$ & $4.2 E+01$ & & & & & & & & & & & & $\mathrm{pCi} / \mathrm{L}$ \\
\hline $\begin{array}{l}\text { Total radium } \\
\text { Tritium }\end{array}$ & $2.2 \mathrm{E}+00$ & & & $\boldsymbol{\sigma}$ & & & & & & & & & $\mathrm{pCi} / \mathrm{L}$ \\
\hline $\begin{array}{l}\text { Tritium } \\
\text { Uranium-233/234 }\end{array}$ & $\begin{array}{l}4.4 E+02 \\
<7.7 E-02\end{array}$ & $\mathrm{U}: 1 / 1$ & & $\omega$ & $\begin{array}{l}1 \\
1\end{array}$ & GP & $5.5 E+02$ & $\|$ & & घ & 1 & GE & $\mathrm{pCi} / \mathrm{mL}$ \\
\hline $\begin{array}{l}\text { Uranium-233/234 } \\
\text { Uranium-235 }\end{array}$ & $\begin{array}{l}<7.7 \mathrm{E}-02 \\
<3.3 \mathrm{E}-02\end{array}$ & $\mathrm{U} 1 / 1$ & $<0.0487$ & & 1 & GP & & & & & & & $\mathrm{pCi} / \mathrm{L}$ \\
\hline Uranium-238 & $\begin{array}{l}<3.3 \mathrm{E}-02 \\
9.7 \mathrm{E}-02\end{array}$ & $\|$ & & & 1 & GP & & & & & & & $\mathrm{PCi} / \mathrm{L}$ \\
\hline & & & & & & & & & & & & & $\mathrm{pCi} / \mathrm{L}$ \\
\hline
\end{tabular}

Notes:

- exceeded holding time

= exceeded groundwater protection or monitoring constituent standard (See Appendix A.) 
WELL HSB103D

\begin{tabular}{|c|c|c|c|}
\hline SRS Coord. & \multicolumn{2}{|l|}{ Lat/Longitude } & Screen Zone Elevation \\
\hline $\begin{array}{l}\text { N71588.1 } \\
\text { E58315.6 }\end{array}$ & \multicolumn{2}{|l|}{$\begin{array}{l}33.279253^{\circ} \mathrm{N} \\
81.652302^{\circ} \mathrm{W}\end{array}$} & 233.7-213.7 ft msl \\
\hline \multicolumn{2}{|c|}{ SAMPLE DATE } & \multicolumn{2}{|l|}{$07 / 14 / 98$} \\
\hline \multicolumn{4}{|l|}{ FIELD DATA } \\
\hline \multicolumn{2}{|l|}{ Constituents } & \multicolumn{2}{|l|}{3098} \\
\hline \multicolumn{2}{|c|}{ Water elevation } & \multicolumn{2}{|l|}{226.6} \\
\hline & \multicolumn{2}{|l|}{4.4} \\
\hline \multirow{2}{*}{\multicolumn{2}{|c|}{$\begin{array}{l}\text { Sp. conductance } \\
\text { Water temperature }\end{array}$}} & \multicolumn{2}{|l|}{120} \\
\hline & & \multicolumn{2}{|l|}{20.3} \\
\hline \multicolumn{2}{|c|}{ Alkalinity as $\mathrm{CaCO}_{3}$} & \multicolumn{2}{|l|}{0} \\
\hline \multicolumn{2}{|c|}{ Turbidity } & \multicolumn{2}{|l|}{2} \\
\hline \multicolumn{2}{|c|}{ Volumes purged } & \multicolumn{2}{|l|}{4.4} \\
\hline \multicolumn{2}{|c|}{ Synchronous water level } & \multicolumn{2}{|c|}{$226.0(09 / 17 / 98)$} \\
\hline
\end{tabular}

$\begin{array}{llll}\text { Top of Casing } & \text { Casing } & \text { Pump } & \frac{\text { Screen Zone }}{247.6 \mathrm{ft} \mathrm{msl}} \\ \text { 4" PVC } & \text { V } & \text { Water Table (IIB2) }\end{array}$

$10 / 13 / 98$

4098

225.6

4.7

150

17.6

0

0.0

XN

$224.1(12 / 21 / 98)$

$\underline{\text { Unit: }}$

ft msi

$\mathrm{pH}$

$\mu \mathrm{S} / \mathrm{cm}$

${ }^{\circ} \mathrm{C}$

$\mathrm{mg} / \mathrm{L}$

NTU

well vol

$f t$ msl

\section{ANALYTICAL DATA}

Inorganic Constrituents

\begin{tabular}{|c|c|c|c|c|c|c|c|c|c|c|c|c|c|}
\hline Constituents & $3 Q 98$ & Mod & Filt. & ST브 & $\underline{\text { DF }}$ & Lab & 4Q98 & Mod & Filt. & $\underline{\text { ST }} \underline{H}$ & DF & Lab & $\underline{\text { Unit }}$ \\
\hline Antimony, total recoverable & $<0.20$ & $\mathbf{U} / /$ & $<0.20$ & & 1 & GE & & & & & & & $\mu g / L$ \\
\hline Arsenic, total recoverable & $<3.0$ & $\mathbf{U} / /$ & $<3.0$ & & 1 & GE & & & & & & & $\mu g / L$ \\
\hline Barium, total recoverable & 26 & $/ 1$ & & & 1 & GE & & & & & & & Hg/L \\
\hline Cadmilum, total recoverabie & 0.28 & $\mathrm{~J} / \mathrm{E} /$ & NDD & & 1 & GE & & & & & & & $\mu g / L$ \\
\hline Chromium, total recoverable & 1.3 & $\mathrm{~J} / \mathrm{E} /$ & NDD & & 1 & GE & & & & & & & $\mu \mathrm{g} / \mathrm{L}$ \\
\hline Cobalt, total recoverable & 1.3 & $/ /$ & & & 1 & GE & & & & & & & $\mu \mathrm{g} / \mathrm{L}$ \\
\hline Copper total recoverable & 2.3 & $/ /$ & & & 1 & $\overline{G E}$ & & & & & & & $\mu g / L$ \\
\hline Cyanide & $<10$ & $\mathrm{U} / /$ & $<10$ & & 1 & GE & & & & & & & $\mu g / L$ \\
\hline Lead, total recoverable & 0.64 & $\mathrm{~J} / \mathrm{E} /$ & NDD & & 1 & GE & & & & & & & $\mu g / L$ \\
\hline Mercury, total recoverable & 2.5 & $/ 1$ & & $\boldsymbol{\square}$ & 1 & GE & 7.7 & $\|$ & & $\mathbf{\square}$ & 1 & GE & $\mu g / L$ \\
\hline $\begin{array}{l}\text { Nickel, total recoverable } \\
\text { Nitrate as nitrogen }\end{array}$ & 3.2 & $/ /$ & & & 1 & GE & & & & & & & $\mu g / L$ \\
\hline Nitrate-nitrite as nitrogen & 14,000 & $N /$ & & $\mathbf{\square}$ & 10 & GE & 15,000 & $\|$ & & $\mathbf{E}$ & 10 & GE & $\mu g / L$ \\
\hline Selenium, total recoverable & $<5.0$ & $\mathrm{U} / /$ & $<5.0$ & & 1 & GE & & & & & & & Hg/L \\
\hline Silver, total recoverable & $<1.0$ & $\mathrm{U} / /$ & $<1.0$ & & 1 & GE & & & & & & & $\mu g / L$ \\
\hline Tin, total recoverable & $<2.0$ & $\mathrm{U} / l$ & $<2.0$ & & 1 & GE & & & & & & & $\mu g / L$ \\
\hline Vanadium, total recoverable & $<2.0$ & $\mathrm{U} / /$ & $<2.0$ & & 1 & GE & & & & & & & $\mu g / L$ \\
\hline Zinc, total recoverable & 9.1 & $/ /$ & & & 1 & GE & & & & & & & $\mu g / L$ \\
\hline \multicolumn{14}{|l|}{ Organic Constituents } \\
\hline Constituents & 3Q998 & Mod & Filt. & ST브 & $\underline{\text { DF }}$ & $\underline{\text { Lab }}$ & 4Q98 & Mod & Filt. & ST $\underline{\text { HI }}$ & DF & $\underline{\text { Lab }}$ & Unit \\
\hline Benzene & $<1.0$ & $\mathrm{UJ} / \mathrm{O} / 1$ & $<1.0$ & & 1 & GE & & & & & & & $\mu g / L$ \\
\hline Bis(2-ethylhexyl) phthalate & $<10$ & $\mathrm{UJ} / \mathrm{Q} /$ & $<10$ & - & 1 & GE & & & & & & & $\mu g / L$ \\
\hline Dichloromethane & $<1.9$ & UJNOB/1 & $<1.0$ & & 1 & GE & & & & & & & $\mu g / L$ \\
\hline Tetsachioroethylene & $<1.0$ & UJ/O/1 & $<1.0$ & & 1 & GE & & & & & & & $\mu g / L$ \\
\hline Trichloroethylene & $<1.0$ & $U \mathrm{~J} / \mathrm{O} / 1$ & $<1.0$ & & 1 & GE & & & & & & & $\mu g / L$ \\
\hline Trichlorofluoromethane & $<1.0$ & $\mathrm{UJ} / \mathrm{O} / 1$ & $<1.0$ & & 1 & GE & & & & & & & $\mu g / L$ \\
\hline
\end{tabular}

Notes:

- = exceeded holding time

- = exceeded groundwater protection or monitoring constituent standard (See Appendix A.) 
WELL HSB103D (cont.)

Radioactive Constituents

\begin{tabular}{|c|c|c|c|c|c|c|c|}
\hline Constituents & 3098 & Mod & Filt. & ST브 & $\underline{\mathrm{DF}}$ & $\underline{\text { Lab }}$ & 4Q98 \\
\hline Americium-241 & $<9.5 \mathrm{E}-02$ & $\mathrm{U} 1 / /$ & $<0.2810$ & & 1 & $G P$ & \\
\hline Beta dose & 81.43 & & & E & & & \\
\hline Carbon-14 & $7.2 \mathrm{E}+01$ & $/ 1$ & & $\mathbf{0}$ & 1 & GP & \\
\hline Cobalt- 60 & $<2.8 \mathrm{E}+00$ & UIII & $<5.9700$ & & 1 & GP & \\
\hline Curium-242 & $<0.0 E+00$ & $\mathrm{UI} / /$ & $<0.1500$ & & 1 & GP & \\
\hline Curium-243/244 & $<4.5 E-02$ & $\mathrm{UI} / /$ & $<0.1360$ & & 1 & GP & \\
\hline Curium-245/246 & $<0.0 \mathrm{E}+00$ & $\mathrm{UI} / /$ & $<0.1360$ & & 1 & GP & \\
\hline Gross alpha & $1.4 \mathrm{E}+01$ & $/ /$ & & & 1 & GP & $9.4 E+00$ \\
\hline lodine-129 & $7.6 \mathrm{E}+00$ & /I & & & 1 & GP & \\
\hline Nickel-63 & $<2.8 E+00$ & UI & $<5.9700$ & & & & \\
\hline Nonvolatile beta & $1.0 \mathrm{E}+03$ & $/ 1$ & & $\mathbf{v}$ & 1 & GP & $1.1 E+03$ \\
\hline Plutonium-238 & $<-3.4 \mathrm{E}-02$ & $\mathrm{UI} / /$ & $<0.3290$ & & 1 & GP & \\
\hline Plutonium-239/240 & $<1.9 \mathrm{E}-03$ & $\mathrm{UI} / l$ & $<0.3570$ & & 1 & GP & \\
\hline Radium-226 & $3.4 \mathrm{E}+00$ & $/ 1$ & & & 1 & GP & \\
\hline Radium-228 & $<8.6 \mathrm{E}-01$ & $\mathrm{U} \mid / /$ & $<1.1100$ & & 1 & GP & \\
\hline Strontium-90 & $5.9 E+02$ & $/ /$ & & ש & 1 & GP & \\
\hline Technetium-99 & $3.6 \mathrm{E}+01$ & $/ /$ & & & 1 & GP & \\
\hline Thorium-228 & $<4.9 E-01$ & $\mathrm{UI} / /$ & $<0.6380$ & & 1 & GP & \\
\hline Thorium-230 & $<-1.7 E-02$ & $\mathrm{UI} / \mathrm{I}$ & $<0.3800$ & & 1 & GP & \\
\hline Thorium-232 & $<0.0 E+00$ & $\mathrm{U} 1 / /$ & $<0.2730$ & & 1 & GP & \\
\hline Sum of alphas & $1.4 \mathrm{E}+00$ & & & & & & \\
\hline Sum of betas & $7.1 E+02$ & & & $\boldsymbol{\omega}$ & & & \\
\hline Total radium & $3.4 E+00$ & & & & & & \\
\hline Tritium & $5.5 E+02$ & "l & & - & 1 & & $1.1 E+03$ \\
\hline Uranium-233/234 & $1.1 E+\infty 0$ & $\|$ & & & 1 & GP & \\
\hline Uranium-235 & $<0.0 E+00$ & UII/ & $<0.1800$ & & 1 & GP & \\
\hline Uranium-238 & $3.0 E-01$ & $\|$ & & & 1 & GP & \\
\hline
\end{tabular}

Filt. $\quad$ ST $\underline{H}$ DF Lab Unit

pCi/L.

$\mathrm{pCi} / \mathrm{L}$

$\mathrm{pC} / \mathrm{i}$

pCill

$\mathrm{pCi} / \mathrm{L}$

pCi/L

pCi/l

$1 \mathrm{GE} \mathrm{pCi} / \mathrm{L}$

$\mathrm{pCi} / \mathrm{L}$

$\mathrm{pCi} / \mathrm{L}$

GE $\mathrm{pCi} / \mathrm{L}$

$\mathrm{pCi} / \mathrm{L}$

pCi/l

$\mathrm{pCi} / \mathrm{L}$

$\mathrm{pCi} / \mathrm{L}$

$\mathrm{PCi} /$

$\mathrm{pCi} / \mathrm{L}$

pCi/L

$\mathrm{pCi} / \mathrm{L}$

pCill

$\mathrm{pCi} / \mathrm{L}$

$\mathrm{pCi} / \mathrm{L}$

- 1 GE $\begin{aligned} & \mathrm{pCi} / \mathrm{L} \\ & \mathrm{pCi} / \mathrm{mL}\end{aligned}$

$\mathrm{pCi} / \mathrm{L}$

$\mathrm{pCi} / \mathrm{L}$

$\mathrm{pCi} / \mathrm{L}$

Notes:

- = exceeded holding time

- exceeded groundwater protection or monitoring constituent standard (See Appendix A.) 
WELL HSB104C

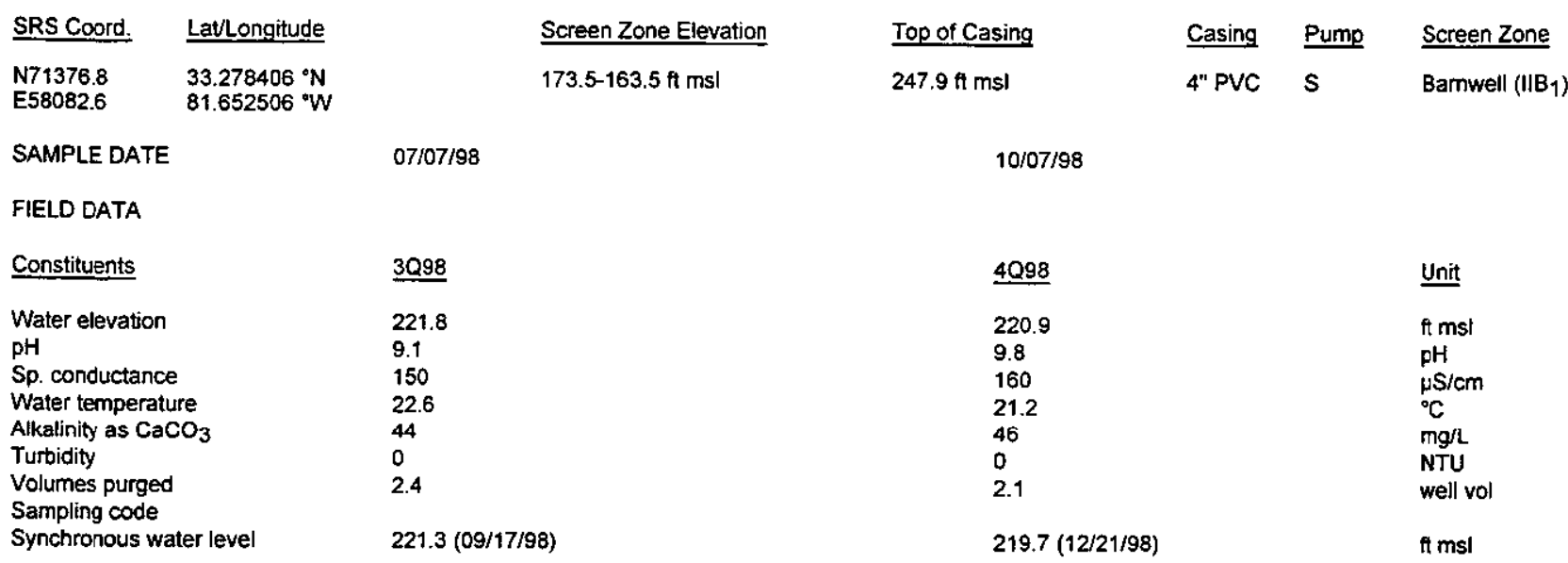

ANALYTICAL DATA

Inorganic Constituents

\begin{tabular}{|c|c|c|c|c|c|c|c|c|c|c|c|c|c|}
\hline Constituents & $\underline{3098}$ & Mod & Filt. & $\underline{\text { STI }} \underline{\mathrm{H}}$ & DF & $\underline{L a b}$ & $4 Q 98$ & Mod & Filt. & ST $\underline{H}$ & $\underline{D F}$ & Lab & Unit \\
\hline Antimony, total recoverable & $<0.070$ & UNI & $<0.20$ & & 1 & GE & & & & & & & $\mu g / L$ \\
\hline Arsenic, total recoverable & $<3.0$ & $\mathrm{U} / /$ & $<3.0$ & & 1 & GE & & & & & & & $\mu g / L$ \\
\hline Barium, total recoverable & 85 & "I & & & 1 & $\mathrm{GE}$ & & & & & & & $\mu g / L$ \\
\hline Cadmium, total recoverable & $<1.0$ & $\mathrm{u} / /$ & $<1.0$ & & 1 & $\mathrm{GE}$ & & & & & & & $\mu g / L$ \\
\hline Chromium, total recoverable & 1.7 & $\mathrm{~J} / \mathrm{E}$ & NDD & & $i$ & GE & & & & & & & $\mu \mathrm{g} / \mathrm{L}$ \\
\hline Cobait, total recoverable & 0.18 & $\mathrm{~J} / \mathrm{E} J$ & NDD & & 1 & $\mathrm{GE}$ & & & & & & & $\mu g / \mathrm{L}$ \\
\hline Copper, total recoverable & 0.48 & 11 & & & 1 & GE & & & & & & & $\mu g / \mathrm{h}$ \\
\hline Cyanide & $<10$ & $\mathrm{U} / / \mathrm{I}$ & $<10$ & & $\uparrow$ & GE & & & & & & & $\mu \mathrm{g} / \mathrm{L}$ \\
\hline Lead, total recoverable & $<2.0$ & $\mathrm{U} / /$ & $<2.0$ & & 1 & $\mathrm{GE}$ & & & & & & & $\mu g / L$ \\
\hline Mercury, total recoverable & $<0.20$ & $U / /$ & $<0.20$ & & $i$ & $\mathrm{GE}$ & $<0.20$ & $\mathrm{u} / /$ & $<0.20$ & & 1 & GE & $\mu g / 2$ \\
\hline $\begin{array}{l}\text { Nickel, total recoverable } \\
\text { Nitrate as nitrogen }\end{array}$ & 0.66 & $\|$ & & & 1 & GE & & & & & 1 & L & $\mu g / L$ \\
\hline Nitrate-nitrite as nitrogen & 7,200 & $N I$ & & & 5 & GE & 4,800 & $\|$ & & & 2 & GE & $\mu g / L$ \\
\hline Selenium, total recoverable & 1.5 & $\mathrm{~J} / \mathrm{E} /$ & NDD & & 1 & $\mathrm{GE}$ & & & & & & & $\mu g / \mathrm{L}$ \\
\hline Silver, total recoverable & $<1.0$ & $\mathrm{U} / 1$ & $<1.0$ & & 1 & GE & & & & & & & $\mu \mathrm{g} / \mathrm{L}$ \\
\hline Tin, total recoverable & $<2.0$ & $\mathrm{U} / \mathrm{I}$ & $<2.0$ & & 1 & GE & & & & & & & $\mu g / \mathrm{h}$ \\
\hline Vanadium, total recoverable & $<2.0$ & $\mathrm{U} / /$ & $<2.0$ & & 1 & GE & & & & & & & $\mu g / \mathrm{L}$ \\
\hline Zinc, total recoverable & 6.7 & $/ 1$ & & & 1 & GE & & & & & & & $\mu g / L$ \\
\hline \multicolumn{14}{|l|}{ Organic Constituents } \\
\hline Constituents & $3 Q 98$ & Mod & Filt. & ST브 & $\underline{\mathrm{DF}}$ & Lab & 4Q98 & $M \propto d$ & Fill. & $\underline{\text { ST }} \underline{H}$ & DF & Lab & Unit \\
\hline Benzene & $<1.0$ & $\mathrm{U} / /$ & $<1.0$ & & 1 & GE & & & & & & & $\mu \mathrm{g} / \mathrm{L}$ \\
\hline Bis(2-ethylhexyl) phthalate & $<9.8$ & $\mathrm{U} / /$ & $<9.8$ & & 1 & $\mathrm{GE}$ & & & & & & & $\mu / \mathrm{L}$ \\
\hline Dichtoromethane & $<0.79$ & UN8I & $<1.0$ & & 1 & GE & & & & & & & $\mu g / L$ \\
\hline Tetrachloroethylene & $<1.0$ & $v / I$ & $<1.0$ & & 1 & GE & & & & & & & $\mu g / \mathrm{L}$ \\
\hline Trichloroethylene & 0.67 & $\mathrm{~J} / \mathrm{E}$ & NDD & & 1 & $\mathrm{GE}$ & & & & & & & $\mu g / 2$ \\
\hline Trichlorofluoromethane & $<1.0$ & $\mathrm{U} / I$ & $<1.0$ & & 1 & $\mathrm{GE}$ & & & & & & & $g / L$ \\
\hline
\end{tabular}

Notes:

- = exceeded holding time

- exceeded groundwater protection or monitoring constituent standard (See Appendix A.) 
WELL HSB104C (cont.)

Radioactive Constituents

\begin{tabular}{|c|c|c|c|c|c|c|c|c|c|c|c|c|c|}
\hline Constituents & $\underline{3 Q 98}$ & Mod & Filt. & ST브 & $\underline{\mathrm{DF}}$ & Lab & 4Q98 & Mod & Filt. & $\underline{\mathrm{ST}} \underline{\mathrm{H}}$ & $\underline{\mathrm{DF}}$ & $\underline{L a b}$ & Unit \\
\hline Americium-241 & $<-1.4 E-02$ & UIII & $<0.1120$ & & 1 & GP & & & & & & & $\mathrm{pCi} / \mathrm{L}$ \\
\hline Beta dose & 0.69 & & & & & & & & & & & & $\mathrm{pCi} / \mathrm{L}$ \\
\hline Carbon-14 & $<8.0 \mathrm{E}+00$ & $\mathrm{UI} / /$ & $<8.7500$ & & 1 & GP & & & & & & & $\mathrm{pCi} / \mathrm{L}$ \\
\hline Cobalt-60 & $<4.0 \mathrm{E}-01$ & UI/I & $<2.6100$ & & 1 & GP & & & & & & & $\mathrm{pCi} / \mathrm{L}$ \\
\hline Curium-242 & $<4.5 \mathrm{E}-02$ & $\mathrm{UI} / /$ & $<0.0675$ & & 1 & GP & & & & & & & $\mathrm{pCi} / \mathrm{L}$ \\
\hline Curium-243/244 & $<1.6 \mathrm{E}-02$ & UU/ & $<0.1120$ & & 1 & GP & & & & & & & $\mathrm{pCi} / \mathrm{L}$ \\
\hline Curium-245/246 & $<1,6 \mathrm{E}-02$ & $\mathrm{Ul} / /$ & $<0.1120$ & & 1 & GP & & & & & & & $\mathrm{pCi} / \mathrm{L}$ \\
\hline Gross alpha & $2.2 E+00$ & 11 & & & 1 & GP & $<8.7 E-01$ & $\mathrm{U} / /$ & $<6.4 E-01$ & & 1 & GE & $\mathrm{pCi} / \mathrm{L}$ \\
\hline lodine-129 & $<2.5 \mathrm{E}-01$ & $\mathrm{U} \mid / /$ & $<0.7380$ & & 1 & GP & & & & & & & $\mathrm{pCi} / \mathrm{L}$ \\
\hline Nickel-63 & $<4,0 \mathrm{E}-01$ & UI & $<2.6100$ & & & & & & & & & & $\mathrm{pCi} / \mathrm{L}$ \\
\hline Nonvolatile beta & $1.1 E+01$ & $1 /$ & & & 1 & GP & $1.2 \mathrm{E}+01$ & /l & & & 1 & GE & $\mathrm{pCi} / \mathrm{L}$ \\
\hline Plutonium-238 & $<1.5 \mathrm{E}-02$ & $\mathrm{UI} / /$ & $<0.1340$ & & 1 & GP & & & & & & & $\mathrm{pCi} / \mathrm{L}$ \\
\hline Plutonium-239/240 & $<-1.4 \mathrm{E}-02$ & $\mathrm{UI} / /$ & $<0.1080$ & & 1 & GP & & & & & & & $\mathrm{pCi} / \mathrm{L}$ \\
\hline Radium-226 & $1.6 E+00$ & $/ 1$ & & & 1 & GP & & & & & & & $\mathrm{pCi} / \mathrm{L}$ \\
\hline Radium-228 & $1.3 E+00$ & /l & & & 1 & GP & & & & & & & $\mathrm{pCi} / \mathrm{L}$ \\
\hline Strontium-90 & $3.4 E+00$ & $/ 1$ & & & 1 & $\mathrm{GP}$ & & & & & & & $\mathrm{pCi} / \mathrm{L}$ \\
\hline Technetium-99 & $<1.1 \mathrm{E}+01$ & $\mathrm{U} \mathbf{l} / /$ & $<20.9000$ & & 1 & GP & & & & & & & $\mathrm{pCi} / \mathrm{L}$ \\
\hline Thorium-228 & $<9.9 \mathrm{E}-02$ & $\mathrm{UI} / /$ & $<0.4480$ & & 1 & GP & & & & & & & $\mathrm{pCi} / \mathrm{L}$ \\
\hline Thorium-230 & $<-4.5 E-02$ & $\mathrm{UI} / /$ & $<0.3500$ & & 1 & GP & & & & & & & $\mathrm{pCi} / \mathrm{L}$ \\
\hline Thorium-232 & $<0.0 \mathrm{E}+00$ & $\mathrm{UI} / /$ & $<0.1390$ & & 1 & GP & & & & & & & $\mathrm{pCi} / \mathrm{L}$ \\
\hline Sum of alphas & & & & & & & & & & & & & \\
\hline Sum of betas & $4.7 E+00$ & & & & & & & & & & & & pCill \\
\hline Total radium & $2.9 E+00$ & & & & & & & & & & & & $\mathrm{pCi} / \mathrm{L}$ \\
\hline Tritium & $1.9 E+02$ & $1 /$ & & $\boldsymbol{\omega}$ & 1 & GP & $2.3 E+02$ & $\|$ & & $\mathbf{n}$ & 1 & GE & pCi/mL \\
\hline Uranium-233/234 & $<2.8 \mathrm{E}-02$ & $\mathrm{UI} / /$ & $<0.1150$ & & 1 & GP & & & & & & & $\mathrm{pCi} / \mathrm{L}$ \\
\hline Uranium-235 & $<1.9 \mathrm{E}-02$ & $\mathrm{UI} / /$ & $<0.1400$ & & 1 & GP & & & & & & & $\mathrm{pCi} / \mathrm{L}$ \\
\hline Uranium-238 & $<1.4 \mathrm{E}-02$ & $\mathrm{UI} / /$ & $<0.0972$ & & 1 & GP & & & & & & & pCilL \\
\hline
\end{tabular}

Notes:

- = exceeded holding time

- exceeded grouncwater protection or monitoring constituent standard (See Appendix A.) 


\section{WELL HSB104D}

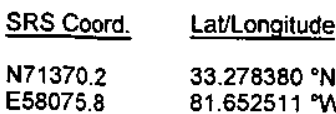

SAMPLE DATE
Screen Zone Elevation

230.6-210.6 ft msl

$07 / 08 / 98$
Top of Casing

$247.8 \mathrm{ft} \mathrm{msl}$

\section{Casing Pump}

4" PVC V
Screen Zone

Water Table (IIB2)

FIELD DATA

\section{Constituents}

Water elevation

pH

Sp. conductance

Water temperature

Alkalinity as $\mathrm{CaCO}_{3}$

Turbidity

Volumes purged

Sampling code

Synchronous water level
3098

225.3

4.4

64

20.2

0

3.7

$218.0(09 / 17 / 98)$
$10 / 12 / 98$

4Q98

224.7

4.4

60

21.7

3

3.6

$222.9(12 / 21 / 98)$
Unit

ft msi

$\mathrm{pH}$

$\mathrm{S} / \mathrm{cm}$

$\mathrm{mg} / \mathrm{L}$

NTU

well vol

Atms!

ANALYTICAL DATA

Inorganic Constituents

\begin{tabular}{|c|c|c|c|}
\hline Constituents & 3Q98 & Mod & Filt. \\
\hline Antimony, total recoverable & $<0.20$ & $\mathrm{U} / /$ & $<0.20$ \\
\hline Arsenic, total recoverable & $<3.0$ & $\mathrm{U} / /$ & $<3.0$ \\
\hline Barium, total recoverable & 16 & $/ 1$ & \\
\hline Cadmium, total recoverable & $<1.0$ & $\mathrm{U} / /$ & $<1.0$ \\
\hline Chromium, total recoverable & $<3.0$ & $\mathrm{U} / /$ & $<3.0$ \\
\hline Cobalt, total recoverable & 1.0 & /1 & \\
\hline Copper, total recoverable & $<2.7$ & UNI & $<0.20$ \\
\hline Cyanide & $<10$ & $\mathrm{U} / /$ & $<10$ \\
\hline Lead, total recoverable & 0.53 & $\mathrm{~J} / \mathrm{EV} /$ & NDD \\
\hline Mercury, total recoverable & 1.8 & $/ /$ & \\
\hline $\begin{array}{l}\text { Nickel, total recoverable } \\
\text { Nitrate as nitrogen }\end{array}$ & $<2.4$ & UNI & $<0.20$ \\
\hline Nitrate-nitrite as nitrogen & 3,100 & $N i$ & \\
\hline Selenium, total recoverable & $<5.0$ & $\mathbf{U} / 1$ & $<5.0$ \\
\hline Silver, total recoverable & $<1.0$ & $\mathrm{U} / /$ & $<1.0$ \\
\hline Tin, total recoverable & $<0.17$ & UNI & $<2.0$ \\
\hline Vanadium, total recoverable & $<2.0$ & $\mathrm{U} / /$ & $<2.0$ \\
\hline Zinc, total recoverable & $<11$ & $\mathrm{U} N /$ & $<5.0$ \\
\hline
\end{tabular}

Mod$$
\text { GE }
$$$$
\text { GE }
$$$$
\text { GE }
$$

GE

GE

GE

1 GE

1 G

GE

5.7

/l

3 GE 2,900 $/ 1$

1 GE

1 GE

1 GE

Organic Constituents

Constituents

3098

Benzene

Bis(2-ethylhexyl) phthalate

Dichloromethane

Tetrachloroethylene

Trichloroethylene

Trichlorofluoromethane
$<1.0$

$<9.9$

$<2.4$

1.6

$<1.0$

$<1.0$

$\begin{array}{ll}\text { Mod } & \text { Filt. } \\ \text { UJ/O/1 } & <1.0 \\ \text { U/I } & <9.9 \\ \text { UJ/OV/1 } & <1.0 \\ \text { J/O/1 } & \text { NDD } \\ \text { UJ/O/1 } & <1.0 \\ \text { UJ/O/1 } & <1.0\end{array}$

ST브

$\begin{array}{ll}\text { DF } & \text { Lab } \\ 1 & \text { GE } \\ 1 & \mathrm{GE} \\ 1 & \mathrm{GE} \\ 1 & \mathrm{GE} \\ 1 & \mathrm{GE}\end{array}$

Mod

Fijt.

ST 브 DF Lab Unit

$\mathrm{\mu g} / \mathrm{L}$

$\mu g / L$

$\mu g / L$

$\mu g / L$

$\mu g / L$

Notes:

- = exceeded holding time

a = exceeded groundwater protection or monitoring constituent standard (See Appendix A.) 
WELL HSB104D (cont.)

Radioactive Constituents

\begin{tabular}{|c|c|c|c|c|c|c|c|c|c|c|c|c|c|}
\hline Constituents & $\underline{3098}$ & Mod & Filt. & $\underline{\text { ST}} \underline{H}$ & DF & $\underline{\text { Lab }}$ & $4 Q 98$ & Mod & Filt. & ST $\underline{H}$ & DF & $\underline{\text { Lab }}$ & Unit \\
\hline $\begin{array}{l}\text { Americium-241 } \\
\text { Beta dose }\end{array}$ & $\begin{array}{l}<1.2 \mathrm{E}-01 \\
36.20\end{array}$ & $\mathrm{UI} / /$ & $<0.1460$ & घ & 1 & GP & & & & & & & $\begin{array}{l}\mathrm{pCi} / \mathrm{L} \\
\mathrm{pCi/L}\end{array}$ \\
\hline Carbon-14 & $<7.1 E+\infty 0$ & $\mathrm{UI} / /$ & $<8.8400$ & & 1 & GP & & & & & & & $\mathrm{pCi} / \mathrm{L}$ \\
\hline Cobalt-60 & $<2.7 \mathrm{E}+00$ & $\mathrm{U} / \mathrm{l}$ & $<3.7900$ & & 1 & GP & & & & & & & $\mathrm{pCi} / \mathrm{L}$ \\
\hline Curium-242 & $<-7.9 \mathrm{E}-03$ & $\mathrm{U} / /$ & $<0.1530$ & & 1 & GP & & & & & & & $\mathrm{pCi} / \mathrm{L}$ \\
\hline Curium-243/244 & $<4.3 \mathrm{E}-02$ & $U W I$ & $<0.1060$ & & 1 & GP & & & & & & & $\mathrm{pCi} / \mathrm{L}$ \\
\hline Curium-245/246 & $<0.0 E+00$ & $\mathrm{U} \| / I$ & $<0.0508$ & & 1 & GP & & & & & & & $\mathrm{pCi} / \mathrm{L}$ \\
\hline Gross alpha & $7.1 E+00$ & 11 & & & 1 & GP & $4.4 E+00$ & "I & & & 1 & GE & $\mathrm{pCi} / \mathrm{L}$ \\
\hline lodine-129 & $1.2 \mathrm{E}+00$ & $/ 1$ & & & 1 & GP & & & & & & & $\mathrm{pCi} / \mathrm{L}$ \\
\hline Nickel-63 & $<2.7 E+00$ & UI & $<3.7900$ & & & & & & & & & & PCilL \\
\hline Nonvolatile beta & $6.3 E+02$ & /I & & - & 1 & GP & $3.3 \mathrm{E}+02$ & "I & & - & 1 & GE & $\mathrm{pCi} / \mathrm{L}$ \\
\hline Plutonium-238 & $<-3.5 \mathrm{E}-02$ & $\mathrm{U} \mathbf{1} / /$ & $<0.2050$ & & 1 & GP & & & & & & & $\mathrm{pCi} / \mathrm{L}$ \\
\hline Plutonium-239/240 & $<2.4 E-02$ & $\mathrm{U} ! \prime \prime$ & $<0.0604$ & & 1 & GP & & & & & & & $\mathrm{pCi} / \mathrm{L}$ \\
\hline Radium-226 & $1.3 E+00$ & 11 & & & 1 & GP & & & & & & & $\mathrm{pCi} / \mathrm{L}$ \\
\hline Radium-228 & $<-3.4 \mathrm{E}-01$ & $\mathrm{UI} / /$ & $<1.1200$ & & 1 & GP & & & & & & & $\mathrm{pCi} / \mathrm{L}$ \\
\hline Strontium-90 & $2.8 E+02$ & 11 & & - & 1 & GP & & & & & & & $\mathrm{pCi} / \mathrm{L}$ \\
\hline Technetium-99 & $<1.3 E+01$ & $\mathrm{UI} / /$ & $<21.3000$ & & 1 & GP & & & & & & & PCi/L \\
\hline Thorium-228 & $<8.2 \mathrm{E}-02$ & UIII/ & $<0.8260$ & & 1 & GP & & & & & & & $\mathrm{pCill}$ \\
\hline Thorium-230 & $<-3.7 \mathrm{E}-02$ & $\mathrm{UI} / /$ & $<0.3580$ & & 1 & GP & & & & & & & $\mathrm{pCi} / \mathrm{L}$ \\
\hline Thorium-232 & $<-1.2 \mathrm{E}-02$ & $\mathrm{UI} / /$ & $<0.2720$ & & 1 & GP & & & & & & & $\mathrm{pCi} / \mathrm{L}$ \\
\hline Sum of alphas & $9.2 \mathrm{E}-01$ & & & & & & & & & & & & $\mathrm{pCi} / \mathrm{L}$ \\
\hline $\begin{array}{l}\text { Sum of betas } \\
\text { Total radium }\end{array}$ & $\begin{array}{l}2.8 E+02 \\
1.3 E+00\end{array}$ & & & - & & & & & & & & & $\begin{array}{l}\mathrm{pCi} / \mathrm{L} \\
\mathrm{pCi} / \mathrm{L}\end{array}$ \\
\hline Tritium & 2.1E+02 & /I & & 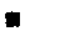 & 1 & GP & $2.8 \mathrm{E}+02$ & "I & & - & 1 & GE & $\mathrm{pCi} / \mathrm{mL}$ \\
\hline Uranjum-233/234 & 7.5E-01 & $" 1$ & & & 1 & GP & & & & & & & $\mathrm{pCi} / \mathrm{L}$ \\
\hline Uranium-235 & $<0.0 E+00$ & UII/ & $<0.0567$ & & 1 & GP & & & & & & & $\mathrm{pCi} / \mathrm{L}$ \\
\hline Uranium-238 & 1.7E-01 & " & & & 1 & GP & & & & & & & $\mathrm{pCi} / \mathrm{L}$ \\
\hline
\end{tabular}

Notes:

- = exceeded holding time

- exceeded groundwater protection or monitoring constituent standard (See Appendix A.) 
WELL HSB105C

\begin{tabular}{|c|c|c|c|c|c|c|c|}
\hline SRS Coord. & Lat/Longitude & & $\underline{\text { Screen Zone Elevation }}$ & Top of Casing & Casing & Pump & Screen Zone \\
\hline $\begin{array}{l}\text { N71447.3 } \\
\text { E57883.8 }\end{array}$ & $\begin{array}{l}33.278237^{\circ} \mathrm{N} \\
81.653166^{\circ} \mathrm{W}\end{array}$ & & $162.2-152.2 \mathrm{ft} \mathrm{msl}$ & $249.5 \mathrm{ft} \mathrm{msl}$ & 4" PVC & $s$ & Bamwell (IIB 1 ) \\
\hline \multicolumn{2}{|c|}{ SAMPLE DATE } & \multicolumn{2}{|l|}{$07 / 09 / 98$} & \multicolumn{3}{|l|}{$10 / 12 / 98$} & \\
\hline \multicolumn{8}{|l|}{ FIELD DATA } \\
\hline \multicolumn{2}{|l|}{ Constituents } & \multicolumn{2}{|l|}{$\underline{3098}$} & \multicolumn{3}{|l|}{$\underline{4098}$} & $\underline{\text { Unit }}$ \\
\hline \multicolumn{2}{|c|}{$\begin{array}{l}\text { Water elevation } \\
\text { pH }\end{array}$} & \multicolumn{2}{|l|}{$\begin{array}{l}220.7 \\
5.3\end{array}$} & \multicolumn{3}{|l|}{$\begin{array}{l}219.8 \\
5.5\end{array}$} & $\mathrm{ft} \mathrm{msl}$ \\
\hline \multicolumn{2}{|c|}{$\begin{array}{l}\mathrm{pH} \\
\mathrm{Sp} \text {. conductance }\end{array}$} & \multicolumn{2}{|l|}{100} & \multicolumn{3}{|l|}{91} & $\begin{array}{l}\mathrm{pH} \\
\mu \mathrm{S} / \mathrm{cm}\end{array}$ \\
\hline \multicolumn{2}{|c|}{ Water temperature } & \multicolumn{2}{|l|}{20.2} & \multicolumn{3}{|l|}{19.3} & ${ }^{\circ} \mathrm{C}$ \\
\hline \multicolumn{2}{|c|}{ Alkalinity as $\mathrm{CaCO}_{3}$} & 30 & & \multirow{2}{*}{\multicolumn{3}{|c|}{$\begin{array}{l}0 \\
0\end{array}$}} & $\mathrm{mg} / \mathrm{h}$ \\
\hline \multicolumn{2}{|c|}{$\begin{array}{l}\text { Turbidity } \\
\text { Volumes purged }\end{array}$} & 1 & & & & & NTU \\
\hline \multirow{2}{*}{\multicolumn{2}{|c|}{$\begin{array}{l}\text { Sampling code } \\
\text { Synchronous water level }\end{array}$}} & \multicolumn{2}{|l|}{2.8} & \multicolumn{3}{|l|}{0.90} & well vol \\
\hline & & \multicolumn{2}{|c|}{$220.2(09 / 17 / 98)$} & \multicolumn{3}{|l|}{$218.8(12 / 21 / 98)$} & $\mathrm{ft} \mathrm{msl}$ \\
\hline
\end{tabular}

\section{ANALYTICAL DATA}

Inorganic Constituents

\begin{tabular}{|c|c|c|c|c|c|c|c|c|c|c|c|c|c|}
\hline Constituents & 3098 & Mod & Filt. & ST브 & $\underline{\text { DF }}$ & $\underline{\text { Lab }}$ & 4Q98 & Mod & Filt. & $\underline{\text { ST }} \underline{H}$ & DF & $\underline{\text { Lab }}$ & Unit \\
\hline Antimony, total recoverable & $<0.20$ & $\mathrm{U} / /$ & $<0.20$ & & 1 & GE & & & & & & & $\mu g / L$ \\
\hline Arsenic, total recoverable & $<3.0$ & $\mathrm{U} / I$ & $<3.0$ & & 1 & $\overline{G E}$ & & & & & & & $\mu g / L$ \\
\hline Barium, total recoverable & 8.6 & $\|$ & & & 1 & GE & & & & & & & $\mu g / L$ \\
\hline Cadmium, total recoverable & $<1.0$ & $\mathrm{U} / \prime$ & $<1.0$ & & 1 & GE & & & & & & & $\mu g / L$ \\
\hline Chromium, total recoverable & $<3.7$ & $\mathrm{U} N /$ & $<3.0$ & & 1 & GE & & & & & & & $\mu g / L$ \\
\hline Cobalt, total recoverable & $<0.20$ & $\mathrm{U} / /$ & $<0.20$ & & 1 & $\overline{G E}$ & & & & & & & $\mu g / L$ \\
\hline Copper, total recoverable & 2.9 & $\|$ & & & 1 & $\overline{G E}$ & & & & & & & $\mu \mathrm{g} / \mathrm{L}$ \\
\hline Cyanide & 8.0 & $\mathrm{~J} / \mathrm{E} J$ & NDD & & 1 & GE & & & & & & & $\mu \mathrm{g} / \mathrm{L}$ \\
\hline Lead, total recoverable & 0.39 & $\mathrm{~J} / \mathrm{E} /$ & NDD & & 1 & GE & & & & & & & $\mu g / L$ \\
\hline Mercury, total recoverable & $<0.20$ & $\mathrm{U} / 1$ & $<0.20$ & & 1 & GE & 0.084 & $\mathrm{~J} / 1 /$ & NDD & & 1 & GE & $\mu g / L$ \\
\hline Nickel, total recoverable & 1.7 & $N I$ & & & 1 & GE & & & & & & & $\mu g / L$ \\
\hline $\begin{array}{l}\text { Nitrate as nitrogen } \\
\text { Nitrate-nitrite as nitrogen }\end{array}$ & 4,400 & $N /$ & & & & & & & & & & & \\
\hline & 0.59 & $\mathrm{~J} / \mathrm{E} /$ & NDD & & 5 & GE & 4,800 & /I & & & 3 & GE & $\mu g / L$ \\
\hline $\begin{array}{l}\text { Selenium, rotal recoverable } \\
\text { Silver, total recoverable }\end{array}$ & $<1.0$ & $\mathrm{U} / 1$ & $<1.0$ & & 1 & GE & & & & & & & $\mu g / L$ \\
\hline Tin, total recoverable & $<0.36$ & UNI & $<20$ & & 1 & GE & & & & & & & $\mu g / L$ \\
\hline Vanadium, total recoverable & $<20$ & UII & $<20$ & & 1 & GE & & & & & & & $\mu g / L$ \\
\hline Zinc, total recoverable & 16 & 11 & & & 1 & GE & & & & & & & $\mu g / L$ \\
\hline & & & & & & & & & & & & & g/L \\
\hline \multicolumn{14}{|l|}{ Organic Constituents } \\
\hline Constituents & 3Q98 & Mod & Filt. & ST$\underline{\text { H }}$ & $\underline{\mathrm{DF}}$ & $\underline{\text { Lab }}$ & 4098 & Mod & Filt. & $\underline{\text { ST }} \underline{H}$ & $\underline{\mathrm{DF}}$ & $\underline{\text { Lab }}$ & $\underline{\text { Unit }}$ \\
\hline Benzene & $<1.0$ & $\mathrm{U} / \prime$ & $<1.0$ & & 1 & GE & & & & & & & $\mu g / L$ \\
\hline Bis(2-ethylhexyl) phthalate & $<10$ & $\mathrm{U} / 1$ & $<10$ & & 1 & GE & & & & & & & $\mu g / L$ \\
\hline Dichloromethane & $<1.0$ & $\mathrm{U} / 1$ & $<1.0$ & & 1 & GE & & & & & & & $\mu g / L$ \\
\hline Tetrachioroethylene & $<1.0$ & $\mathrm{U} / 1$ & $<1.0$ & & 1 & GE & & & & & & & $\mu g / L$ \\
\hline Trichloroethylene & $<1.0$ & $\mathrm{U} / 1$ & $<1.0$ & & 1 & GE & & & & & & & $\mu g / L$ \\
\hline Trichlorofluoromethane & $<1.0$ & $\mathrm{U} / 1$ & $<1.0$ & & 1 & GE & & & & & & & $g / L$ \\
\hline
\end{tabular}

Notes:

- exceeded holding time

- exceeded groundwater protection or monitoring constituent standard (See Appendix A.) 
WELL HSB105C (cont.)

Radioactive Constituents

\begin{tabular}{|c|c|c|c|c|c|c|c|c|c|c|c|c|c|}
\hline Constifuents & 3098 & Mod & Filt. & $\underline{\text { ST }} \underline{\underline{H}}$ & DF & $\underline{\text { Lab }}$ & 4Q98 & Mod & Filt. & $\underline{\text { ST }} \underline{\mathrm{H}}$ & $\underline{\mathrm{DF}}$ & $\underline{\text { Lab }}$ & Unit \\
\hline Americium-241 & $<4.4 E-02$ & Uil/ & $<0.1330$ & & 1 & GP & & & & & & & $\mathrm{pCi} / \mathrm{L}$ \\
\hline Carbon-14 & $<6.8 \mathrm{E}+00$ & $\mathrm{UI} / /$ & $<9.0400$ & & 1 & GP & & & & & & & $\mathrm{pCi} / \mathrm{L}$ \\
\hline Cobalt- 60 & $<-6.2 E-02$ & $\mathrm{U} / / /$ & $<3.2900$ & & 1 & $\mathrm{GP}$ & & & & & & & $\mathrm{pCi} / \mathrm{L}$ \\
\hline Curium-242 & $<9.8 E-03$ & $\mathrm{U} I / /$ & $<0.1170$ & & 1 & GP & & & & & & & $\mathrm{pCi} / \mathrm{L}$ \\
\hline Curium-243/244 & $<-4.2 \mathrm{E}-03$ & $\mathrm{UH} / /$ & $<0.0928$ & & 1 & GP & & & & & & & $\mathrm{pCi} / \mathrm{L}$ \\
\hline Curium-245/246 & $<-4.2 E-03$ & $\mathrm{UI} / /$ & $<0.0927$ & & 1 & GP & & & & & & & $\mathrm{pCi} / \mathrm{L}$ \\
\hline Gross alpha & $9.2 E-01$ & $\|$ & & & 1 & GP & $<4.2 E-01$ & $\mathrm{u} / /$ & $<7.2 \mathrm{E}-01$ & & 1 & GE & $\mathrm{pCi} / \mathrm{L}$ \\
\hline lodine-129 & $<3.8 E-01$ & $\mathrm{UI} / /$ & $<0.6940$ & & 1 & GP & & & & & & & $\mathrm{pCi} / \mathrm{L}$ \\
\hline Nickel-63 & $<-6.2 \mathrm{E}-02$ & UI & $<3.2900$ & & & & & & & & & & $\mathrm{pCi} / \mathrm{L}$ \\
\hline Nonvolatile beta & $<8.0 \mathrm{E}-01$ & UI// & $<1.2100$ & & 1 & GP & $<2.6 \mathrm{E}+00$ & $\mathrm{U} / /$ & $<1.3 E+00$ & & 1 & GE & $\mathrm{pCi} / \mathrm{L}$ \\
\hline Plutonium-238 & $<8.6 E-03$ & $\mathrm{UI} / /$ & $<0.1020$ & & 1 & GP & & & & & & & $\mathrm{pCi} / \mathrm{L}$ \\
\hline Plutonium-239/240 & $<-4.0 E-03$ & $\mathrm{UI} / /$ & $<0.0868$ & & 1 & $\mathrm{GP}$ & & & & & & & $\mathrm{pCi} / \mathrm{L}$ \\
\hline Radium-226 & 5.2E-01 & $/ 1$ & & & 1 & GP & & & & & & & $\mathrm{pCi} / \mathrm{L}$ \\
\hline Radium-228 & $<6.3 \mathrm{E}-01$ & $\mathrm{UL} / \prime$ & $<1.1400$ & & 1 & GP & & & & & & & $\mathrm{pCi} / \mathrm{L}$ \\
\hline Strontium-90 & $<1,1 \mathrm{E}-02$ & UlJ/U & & & 1 & GP & & & & & & & $\mathrm{pCi} / \mathrm{L}$ \\
\hline Technetium-99 & $<-1.4 \mathrm{E}+01$ & $\mathrm{Ul} / /$ & $<31.2000$ & & 1 & GP & & & & & & & $\mathrm{pCi} / \mathrm{L}$ \\
\hline Thorium-228 & $<2.8 \mathrm{E}-01$ & $\mathrm{UI} / /$ & $<0.4190$ & & 1 & GP & & & & & & & $\mathrm{pCi} / \mathrm{h}$ \\
\hline Thorium-230 & $<1.1 \mathrm{E}-01$ & $\mathrm{U}: / /$ & $<0.1630$ & & 1 & GP & & & & & & & $\mathrm{pCi} / \mathrm{L}$ \\
\hline Thorium-232 & $<0.0 \mathrm{E}+00$ & U1 $/ /$ & $<0.0645$ & & 1 & GP & & & & & & & $\mathrm{pCi} / \mathrm{L}$ \\
\hline $\begin{array}{l}\text { Sum of alphas } \\
\text { Sum of betas }\end{array}$ & $4.9 E+00$ & & & & & & & & & & & & $\mathrm{pCi} / \mathrm{L}$ \\
\hline Total radium & $5.2 \mathrm{E}-01$ & & & & & & & & & & & & $p C i / L$ \\
\hline Tritium & $8.9 E+01$ & $/ /$ & & 口 & 1 & GP & $9.2 \mathrm{E}+01$ & $/ /$ & & $\mathbf{v}$ & 1 & GE & $\mathrm{pCi} / \mathrm{mL}$ \\
\hline Uranium-233/234 & $1.4 E+00$ & $/ 1$ & & & 1 & GP & & & & & & & $\mathrm{pCi} / \mathrm{L}$ \\
\hline Uranium-235 & 2.3E-01 & $/ /$ & & & 1 & GP & & & & & & & $\mathrm{pCi} / \mathrm{L}$ \\
\hline Uranium-238 & $3.3 E+00$ & $1 /$ & & & 1 & GP & & & & & & & $\mathrm{pCi} / \mathrm{L}$ \\
\hline
\end{tabular}

Notes:

- exceeded holding time

- = exceeded groundwater protection or monitoring constituent standard (See Appendix A.) 


\section{WELL HSB105D}

$\begin{array}{ll}\text { SRS Coord. } & \text { Lat/Longitude } \\ \text { N71454.8 } & \quad 33.278244^{\circ} \mathrm{N} \\ \text { E57877.4 } & 81.653197^{\circ} \mathrm{W}\end{array}$

SAMPLE DATE

07/08/98

Screen Zone Elevation

231.8-211.8 ft ms
Top of Casing

249.5 ft ms $\begin{array}{ll}\text { Casing } & \text { Pump } \\ \text { 4" PVC } & V\end{array}$

Screen Zone

Water Table (IIB2)

FIELD DATA

Constituents

Water elevation

pH

Sp. conductance

Water temperature

Alkalinity as $\mathrm{CaCO}_{3}$

Turbidity

Volumes purged

Sampling code

Synchronous water level
3Q98

222.9

4.0

100

21.8

0

2

$225.9(09 / 17 / 98)$
$10 / 12 / 98$

$4 Q 98$
225.3
3.9
130
21.6
0
1
4.3
$223.1(12 / 21 / 98)$

Unit

ft ms!

$\mathrm{pH}$

$\mu \mathrm{S} / \mathrm{cm}$

${ }^{\circ} \mathrm{C}$

$\mathrm{mg} / \mathrm{L}$

NTU

well vol

ft msl

ANALYTICAL DATA

Inorganic Constituents

\begin{tabular}{|c|c|c|c|c|c|c|c|c|c|c|c|c|c|}
\hline Constituents & 3098 & Mod & Filt. & $\underline{\text { STT }} \underline{H}$ & $\underline{\text { DF }}$ & Lab & 4Q98 & Mad & Filt. & $\underline{\text { ST }} \underline{H}$ & $\underline{\text { DF }}$ & Lab & Unit \\
\hline Antimony, total recoverable & $<0.20$ & $\mathrm{U} / /$ & $<0.20$ & & 1 & GE & & & & & & & $\mu g / L$ \\
\hline Arsenic, total recoverable & $<3.0$ & $\mathrm{U} / /$ & $<3.0$ & & 1 & GE & & & & & & & $\mu g / \mathrm{L}$ \\
\hline Barium, total recoverable & 25 & II & & & 1 & $\overline{G E}$ & & & & & & & $\mu g / L$ \\
\hline Cadmium, total recoverable & 0.22 & $\mathrm{~J} / \mathrm{E} /$ & NDD & & 1 & GE & & & & & & & $\mu g / L$ \\
\hline Chromium, total recoverable & 1.7 & $\mathrm{~J} / \mathbf{E}$ & NDD & & 1 & GE & & & & & & & $\mu g / L$ \\
\hline Cobalt, total recoverable & 5.3 & $/ 1$ & & $\boldsymbol{\square}$ & 1 & GE & & & & & & & $\mu g / L$ \\
\hline Copper, total recoverable & 12 & NI & & & 1 & GE & & & & & & & $\mu g / L$ \\
\hline Cyanide & $<10$ & $\mathrm{U} / /$ & $<10$ & & 1 & GE & & & & & & & $\mu g / L$ \\
\hline Lead, total recoverable & 3.0 & $N /$ & & & 1 & GE & & & & & & & $\mu g / L$ \\
\hline Mercury, total recoverable & 5.3 & $/ /$ & & च & 1 & GE & 3.5 & // & & ש & 1 & GE & $\mu g / 2$ \\
\hline $\begin{array}{l}\text { Nickel, total recoverable } \\
\text { Nitrate as nitrogen }\end{array}$ & $<6.3$ & UNI & $<0.20$ & & 1 & GE & & & & & & & $\mu g / L$ \\
\hline Nitrate-nitrite as nitrogen & 12,000 & $N /$ & & घ & 5 & GE & 13,000 & $/ /$ & & $\mathbf{E}$ & 5 & GE & $\mu g / L$ \\
\hline Selenium, total recoverable & $<5.0$ & $\mathrm{U} / 1$ & $<5.0$ & & 1 & GE & & & & & & & $\mu g / L$ \\
\hline Silver, total recoverable & $<1.0$ & $\mathrm{U} / I$ & $<1.0$ & & 1 & GE & & & & & & & $\mu g / L$ \\
\hline Tin, total recoverable & $<0.41$ & UNI & $<2.0$ & & 1 & GE & & & & & & & $\mu g / L$ \\
\hline Vanadium, total recoverable & $<2.0$ & $\mathrm{U} / /$ & $<2.0$ & & 1 & GE & & & & & & & $\mu g / L$ \\
\hline Zinc, total recoverable & 53 & $N /$ & & & 1 & $\overline{G E}$ & & & & & & & $\mu g / L$ \\
\hline \multicolumn{14}{|l|}{ Organic Constituemts } \\
\hline Constituents & $\underline{3 Q 98}$ & Mod & Filt. & ST브 & $\underline{\text { DF }}$ & $\underline{\mathrm{Lab}}$ & 4098 & Mod & Filt. & $\underline{\text { ST }} \underline{H}$ & $\underline{D F}$ & $\underline{\text { Lab }}$ & $\underline{\text { Unit }}$ \\
\hline Benzene & $<1.0$ & $\mathrm{U} J / O / 1$ & $<1.0$ & & 1 & GE & & & & & & & $\mu g^{\prime}$ \\
\hline Bis(2-ethylhexyl) phthalate & $<10$ & $\mathrm{U} / /$ & $<10$ & & 1 & GE & & & & & & & $\mu g / L$ \\
\hline Dichloromethane & $<2.5$ & UJ/OV/1 & $<1.0$ & & 1 & GE & & & & & & & $\mu g / L$ \\
\hline Tetrachloroethylene & 1.3 & $\mathrm{~J} / \mathrm{O} / 1$ & NDD & & 1 & GE & & & & & & & $\mu g / L$ \\
\hline Trichloroethylene & $<1.0$ & $\mathrm{UJ} / \mathrm{O} / 1$ & $<1.0$ & & 1 & $\overline{G E}$ & & & & & & & $\mu g / L$ \\
\hline Trichlorofluoromethane & $<1.0$ & $\mathrm{UJ} / \mathrm{O} / 1$ & $<1.0$ & & 1 & $\overline{G E}$ & & & & & & & L \\
\hline
\end{tabular}

Notes:

- exceeded holding time

- exceeded groundwater protection or monitoring constituent standard (See Appendix A.) 
WELL HSB105D (cont.)

Radioactive Constituents

\begin{tabular}{|c|c|c|c|c|c|c|c|c|c|c|c|c|c|}
\hline Constituents & $3 Q 98$ & Mod & Filt. & ST브 & DF & $\underline{\text { Lab }}$ & 4Q98 & Mod & Filt. & $\underline{\text { ST }} \underline{H}$ & DF & Lab & Unit \\
\hline Americium-241 & $<3.6 \mathrm{E}-02$ & $\mathrm{U} I / I$ & $<0.1520$ & & 1 & GP & & & & & & & $\mathrm{pCi} / \mathrm{L}$ \\
\hline Beta dose & 64.16 & & & ט & & & & & & & & & $\mathrm{pCi} / \mathrm{L}$ \\
\hline Carbon-14 & $3.7 E+01$ & $/ /$ & & & 1 & GP & & & & & & & $\mathrm{pCi} / \mathrm{L}$ \\
\hline Cobalt -60 & $1.3 E+01$ & $/ l$ & & & 1 & GP & & & & & & & $\mathrm{pCi} / \mathrm{L}$ \\
\hline Curium-242 & $<1.4 \mathrm{E}-02$ & $\mathrm{UI} / \mathrm{I}$ & $<0.0977$ & & 1 & GP & & & & & & & $\mathrm{pCi} / \mathrm{L}$ \\
\hline Curium-243/244 & $<2.7 \mathrm{E}-02$ & $\mathrm{UI} / /$ & $<0.1090$ & & 1 & GP & & & & & & & $\mathrm{pCi} / \mathrm{L}$ \\
\hline Curium-245/246 & $<0.0 E+00$ & $\mathrm{UI} / /$ & $<0.0533$ & & 1 & GP & & & & & & & $\mathrm{pCi} / \mathrm{L}$ \\
\hline Gross alpha & $1.7 E+01$ & $/ /$ & & 口 & 1 & GP & $1.2 \mathrm{E}+01$ & $\|$ & & & 1 & GE & $\mathrm{pCi} / \mathrm{L}$ \\
\hline lodine-129 & $1.0 \mathrm{E}+01$ & $\|$ & & & 1 & GP & & & & & & & $\mathrm{pCi} / \mathrm{L}$ \\
\hline Nickel-63 & $1.3 \mathrm{E}+01$ & 11 & & & & & & & & & & & $\mathrm{pCi} / \mathrm{L}$ \\
\hline Nonvolatile beta & $1.0 \mathrm{E}+03$ & $/ 1$ & & घ & 1 & GP & $1.0 E+03$ & $1 /$ & & a & 1 & GE & $\mathrm{pCi} / \mathrm{L}$ \\
\hline Plutonium-238 & $<-7.9 E-03$ & $\mathrm{UN} /$ & & & 1 & GP & & & & & & & $\mathrm{pCi} / \mathrm{L}$ \\
\hline Plutonium-239/240 & $<-4.0 E-03$ & $\mathrm{UI} / /$ & $<0.1730$ & & 1 & GP & & & & & & & $\mathrm{pCi} / \mathrm{L}$ \\
\hline Radium-226 & $2.5 \mathrm{E}+00$ & $/ /$ & & & 1 & GP & & & & & & & $\mathrm{pCi} / \mathrm{L}$ \\
\hline Radium-228 & $<4.6 \mathrm{E}-01$ & $\mathrm{UI} / \mathrm{I}$ & $<0.7290$ & & 1 & GP & & & & & & & $\mathrm{pCi} / \mathrm{L}$ \\
\hline Strontium-90 & $4.3 E+02$ & $/ /$ & & 口 & 1 & GP & & & & & & & $\mathrm{pCi} / \mathrm{L}$ \\
\hline Technetium-99 & $<1.9 E+01$ & $\mathrm{U} 1 / /$ & $<21.0$ & & 1 & GP & & & & & & & $\mathrm{pCi} / \mathrm{L}$ \\
\hline Thorium-228 & $<3.4 E-02$ & Uill & $<0.4910$ & & 1 & GP & & & & & & & $\rho \mathrm{Ci} / \mathrm{L}$ \\
\hline Thorium-230 & $<8.4 E-02$ & $\mathrm{U}: / /$ & $<0.2080$ & & 1 & GP & & & & & & & $\mathrm{pCi} / \mathrm{L}$ \\
\hline Thorium-232 & $<0.0 E+00$ & $\mathbf{U I} / /$ & $<0.1000$ & & 1 & GP & & & & & & & $\mathrm{pCi} / \mathrm{L}$ \\
\hline Sum of alphas & $1.5 \mathrm{E}+00$ & & & & & & & & & & & & $\mathrm{pCi} / \mathrm{L}$ \\
\hline $\begin{array}{l}\text { Sum of betas } \\
\text { Total radium }\end{array}$ & $\begin{array}{l}5.0 \mathrm{E}+02 \\
2.5 \mathrm{E}+00\end{array}$ & & & $\mathbf{v}$ & & & & & & & & & $\mathrm{pCi} / \mathrm{L}$ \\
\hline Tritium & $1.6 \mathrm{E}+03$ & $/ /$ & & $\boldsymbol{w}$ & 1 & GP & $13 E+03$ & /I & & 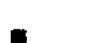 & 1 & GF & $\mathrm{pCi} / \mathrm{L}$ \\
\hline Uranium-233/234 & $1.3 E+00$ & $N /$ & & & 1 & GP & & & & & & & $\mathrm{pCi} / \mathrm{L}$ \\
\hline Uranium-235 & $<2.8 \mathrm{E}-02$ & $\mathrm{UI} / \mathrm{I}$ & $<0.1150$ & & 1 & GP & & & & & & & $\mathrm{pCi} / \mathrm{L}$ \\
\hline Uranium-238 & 2.1E-01 & $/ 1$ & & & 1 & GP & & & & & & & $\mathrm{pCi} / \mathrm{L}$ \\
\hline
\end{tabular}

Notes:

- = exceeded holding time

- exceeded groundwater protection or monitoring constituent standard (See Appendix A.) 


\section{WELL HSB106C}

\begin{tabular}{|c|c|c|c|}
\hline SRS Coord. & Lat/Longitude & & Screen Zone Elevation \\
\hline $\begin{array}{l}N 71720.9 \\
\text { E57651.5 }\end{array}$ & $\begin{array}{l}33.278464^{\circ} \mathrm{N} \\
81.654309^{\circ} \mathrm{W}\end{array}$ & & $168.7-158.7 \mathrm{ft} \mathrm{msl}$ \\
\hline \multicolumn{2}{|c|}{ SAMPLE DATE } & \multicolumn{2}{|l|}{$07 / 09 / 98$} \\
\hline \multicolumn{4}{|c|}{ FIELD DATA } \\
\hline \multicolumn{2}{|l|}{ Constituents } & \multicolumn{2}{|l|}{$\underline{3098}$} \\
\hline \multicolumn{2}{|c|}{ Water elevation } & \multicolumn{2}{|l|}{223.3} \\
\hline \multirow{2}{*}{\multicolumn{2}{|c|}{$\begin{array}{l}\text { pH } \\
\text { Sp. conductance }\end{array}$}} & \multicolumn{2}{|l|}{4.8} \\
\hline & & \multirow{2}{*}{\multicolumn{2}{|c|}{$\begin{array}{l}74 \\
23.9\end{array}$}} \\
\hline \multicolumn{2}{|c|}{ Water temperature } & & \\
\hline \multicolumn{2}{|c|}{ Alkalinity as $\mathrm{CaCO}_{3}$} & \multicolumn{2}{|l|}{6} \\
\hline \multicolumn{2}{|l|}{ Turbidity } & \multicolumn{2}{|l|}{1} \\
\hline \multicolumn{2}{|c|}{ Volumes purged } & \multicolumn{2}{|l|}{2.8} \\
\hline Synchronou & ter level & \multicolumn{2}{|c|}{$222.5(09 / 17 / 98)$} \\
\hline
\end{tabular}

\begin{tabular}{llll} 
Top of Casing & Casing & Pump & $\frac{\text { Screen Zone }}{252.9 \mathrm{ft} \mathrm{msl}}$ \\
\hline 4" PVC & S & Bamwell (IIB $\left.\mathrm{B}_{1}\right)$
\end{tabular}

$10 / 12 / 98$

ANALYTICAL DATA

Inorganic Constituemts

\begin{tabular}{|c|c|c|c|c|c|c|c|c|c|c|c|c|c|}
\hline Constituents & 3098 & Mod & Filt. & $\underline{\text { ST}} \underline{H}$ & $\underline{\text { DF }}$ & Lab & 4098 & Mod & Filt. & $\underline{\mathrm{ST}} \underline{\mathrm{H}}$ & $\underline{\mathrm{DF}}$ & $\underline{L a b}$ & Unit \\
\hline Antimony, total recoverable & $<0.20$ & $\mathrm{U} / /$ & $<0.20$ & & 1 & GE & & & & & & & $\mu g / L$ \\
\hline Arsenic, total recoverable & $<3.0$ & $\mathrm{U} / /$ & $<3.0$ & & 1 & GE & & & & & & & $\mu g / L$ \\
\hline Barium, total recoverable & 14 & $/ 1$ & & & 1 & GE & & & & & & & $\mu g / L$ \\
\hline Cadmium, total recoverable & $<1.0$ & $\mathrm{U} / \mathrm{I}$ & $<1.0$ & & 1 & GE & & & & & & & $\mu g / L$ \\
\hline Chromium, total recoverable & $<2.0$ & UNI & $<3.0$ & & 1 & GE & & & & & & & $\mu g / L$ \\
\hline Cobalt, total recoverable & 0.089 & $\mathrm{~J} / \mathrm{E} /$ & NDD & & 1 & GE & & & & & & & $\mu g / L$ \\
\hline Copper, total recoverable & 0.89 & /I & & & 1 & GE & & & & & & & $\mu g / L$ \\
\hline Cyanide & $<10$ & $\mathrm{U} / \mathrm{I}$ & $<10$ & & 1 & GE & & & & & & & $\mu g / L$ \\
\hline Lead, total recoverable & 0.19 & $\mathrm{~J} / \mathrm{E} /$ & NDD & & 1 & GE & & & & & & & $\mu g / L$ \\
\hline Mercury, total recoverable & 0.59 & $\|$ & & & 1 & GE & 0.57 & $\|$ & & & 1 & GE & $\mu g / L$ \\
\hline $\begin{array}{l}\text { Nickel, total recoverable } \\
\text { Nitrate as nitrogen }\end{array}$ & 1.8 & $N /$ & & & 1 & GE & & & & & & & $\mu g / L$ \\
\hline Nitrate-nitrite as nitrogen & 5,800 & $N /$ & & & 5 & GE & 6,400 & $\|$ & & & 3 & GE & $\mu g^{\prime} L$ \\
\hline Selenium, total recoverable & $<5.0$ & $\mathrm{U} / /$ & $<5.0$ & & 1 & GE & & & & & & & $\mu g / L$ \\
\hline Silver, total recoverable & $<1,0$ & $\mathrm{U} / /$ & $<1.0$ & & 1 & GE & & & & & & & $\mu g / L$ \\
\hline Tin, total recoverable & $<0.16$ & UNI & $<2.0$ & & 1 & GE & & & & & & & $\mu g / L$ \\
\hline Vanadium, total recoverable & $<2.0$ & $\mathrm{U} / /$ & $<2.0$ & & 1 & GE & & & & & & & 每 \\
\hline Zinc, total recoverable & 12 & $\|$ & & & 1 & $\mathrm{GE}$ & & & & & & & $\mu g / L$ \\
\hline
\end{tabular}

Organic Constituents

\begin{tabular}{|c|c|c|c|c|c|c|c|c|c|c|}
\hline Constituents & $\underline{3098}$ & Mod & Filt. & ST브 & $\underline{D F}$ & Lab 4098 & Mod & Filt. & $\underline{\text { ST }} \underline{H}$ DF Lab & Unit \\
\hline Benzene & $<1.0$ & $\mathrm{U} / /$ & $<1.0$ & & 1 & $\mathrm{GE}$ & & & & $\mu g / L$ \\
\hline Bis(2-ethylhexyl) phthalate & 59 & $/ /$ & & E & 1 & $\mathrm{GE}$ & & & & $\mu g /$ \\
\hline $\begin{array}{l}\text { Dichloromethane } \\
\text {. }\end{array}$ & $<1.0$ & $u / I$ & $<1.0$ & & 1 & GE & & & & $\mu \mathrm{g} / \mathrm{L}$ \\
\hline Tetrachloroethylene & $<1.0$ & $\mathrm{u} / /$ & $<1.0$ & & 1 & GE & & & & $\mu g / L$ \\
\hline Trichloroethylene & $<1.0$ & $\mathrm{u} / /$ & $<1.0$ & & 1 & $\mathrm{GE}$ & & & & $\mu g / L$ \\
\hline Trichlorofluoromethane & $<1.0$ & $\mathrm{u} / \prime$ & $<1.0$ & & $i$ & GE & & & & $\mu \alpha^{\prime} \mathrm{L}$ \\
\hline
\end{tabular}

Notes:

- = exceeded holding time

- exceeded groundwater protection or monitoring constituent standard (See Appendix A.) 
WELL HSB106C (cont.)

Radioactive Constituents

\begin{tabular}{|c|c|c|c|c|c|c|c|c|c|c|c|c|c|}
\hline Constituents & $\underline{3098}$ & Mod & Filt. & $\underline{\text { ST }} \underline{H}$ & DF & $\underline{\mathrm{Lab}}$ & $\underline{4 Q 98}$ & Mod & Filt. & $\underline{\text { ST }} \underline{H}$ & $\underline{D F}$ & Lab & Unit \\
\hline $\begin{array}{l}\text { Americium-241 } \\
\text { Beta dose }\end{array}$ & $\begin{array}{l}<3.1 E-02 \\
0.01\end{array}$ & $\mathrm{UI} / / \mathrm{I}$ & $<0.1500$ & & 1 & GP & & & & & & & $\mathrm{pCin}$ \\
\hline $\begin{array}{l}\text { Beta dose } \\
\text { Carbon-14 }\end{array}$ & $\begin{array}{l}0.01 \\
1.1 E+01\end{array}$ & /1 & & & 1 & GP & & & & & & & $\mathrm{pCi} / \mathrm{L}$ \\
\hline $\begin{array}{l}\text { Carbon-14 } \\
\text { Cobalt-60 }\end{array}$ & $<2.3 \mathrm{E}-01$ & $\mathrm{U} / /$ & $<3.7300$ & & 1 & $\begin{array}{l}\text { GP } \\
\text { GP }\end{array}$ & & & & & & & $\mathrm{pCi} / \mathrm{L}$ \\
\hline Curium-242 & $<-1.5 \mathrm{E}-02$ & $\mathrm{U} ! / /$ & $<0.1470$ & & 1 & $G P$ & & & & & & & $\begin{array}{l}\mathrm{PCi} / \mathrm{L} \\
\mathrm{OCi} / \mathrm{L}\end{array}$ \\
\hline Curium-243/244 & $<3.5 \mathrm{E}-02$ & $\mathrm{U}: / /$ & $<0.1050$ & & 1 & GP & & & & & & & $\mathrm{pCi} / \mathrm{L}$ \\
\hline Curium-245/246 & $<2.0 \mathrm{E}-02$ & $\mathrm{U} 1 / /$ & $<0.0594$ & & 1 & GP & & & & & & & pCi/L \\
\hline Gross alpha & $7.5 \mathrm{E}-01$ & "I & & & 1 & GP & 8.1E-01 & $\mathrm{J} / / \mathrm{K} / \mathrm{C}$ & NDD & & 1 & $\mathrm{TM}$ & $\mathrm{pCi} / \mathrm{L}$ \\
\hline lodine-129 & $<8.2 \mathrm{E}-01$ & UII/ & $<1.2200$ & & 1 & GP & & & & & & & $\mathrm{pCi} / \mathrm{L}$ \\
\hline Nickel-63 & $<2.3 E-01$ & UI & $<3.7300$ & & & & & & & & & & $\mathrm{pCi} / \mathrm{L}$ \\
\hline Nonvolatile beta & $2.0 E+00$ & "I & & & 1 & $G P$ & $3.6 \mathrm{E}+00$ & /I & & & 1 & $\mathrm{TM}$ & $\mathrm{pCi} / \mathrm{L}$ \\
\hline Plutonium-238 & $<7.4 \mathrm{E}-02$ & $\mathrm{UI} / /$ & $<0.1020$ & & 1 & GP & & & & & & & $\mathrm{pCilL}$ \\
\hline Plutonium-239/240 & $<2.9 \mathrm{E}-02$ & $\mathrm{U} \mid \mathrm{I} /$ & $<0.0863$ & & 1 & GP & & & & & & & $\mathrm{pCi} / \mathrm{L}$ \\
\hline Radium-226 & $<6.0 \mathrm{E}-01$ & UNI & $<0.5580$ & & 1 & GP & & & & & & & $\mathrm{pCi} / \mathrm{L}$ \\
\hline Radium-228 & $<4.2 \mathrm{E}-01$ & $\mathrm{U} ! / /$ & $<1.2700$ & & 1 & GP & & & & & & & $\mathrm{pCi} / \mathrm{L}$ \\
\hline Strontium-90 & $<-3.3 \mathrm{E}-02$ & $\mathrm{UIJ} / \mathrm{U}$ & & & 1 & GP & & & & & & & $\mathrm{pCi} / \mathrm{L}$ \\
\hline Technetium-99 & $<-1.0 \mathrm{E}+01$ & $\mathrm{UI} / 2$ & $<22.1000$ & & 1 & GP & & & & & & & $\mathrm{pCi} / \mathrm{L}$ \\
\hline Thorium-228 & $<9.7 \mathrm{E}-02$ & $\mathrm{U} ! / !$ & $<0.3990$ & & 1 & GP & & & & & & & $\mathrm{pCi} / \mathrm{L}$ \\
\hline Thorium-230 & $<9.8 \mathrm{E}-02$ & UII/ & $<0.1340$ & & 1 & GP & & & & & & & $\mathrm{pCi} / \mathrm{L}$ \\
\hline $\begin{array}{l}\text { Thorium-232 } \\
\text { Sum of alphas }\end{array}$ & $<2.2 \mathrm{E}-02$ & UIIII & $<0.0648$ & & 1 & GP & & & & & & & $\mathrm{pCi} / \mathrm{L}$ \\
\hline Sum of betas & $1.1 \mathrm{E}+01$ & & & & & & & & & & & & $\mathrm{pCi} / \mathrm{L}$ \\
\hline Tritium & 1.6E+02 & /I & & E & 1 & GP & $1.8 \mathrm{E}+02$ & "I & & - & 1 & GE & $\mathrm{pCi} / \mathrm{mL}$ \\
\hline Uranium-233/234 & $<1,3 \mathrm{E}-01$ & UNI & $<0.1160$ & & 1 & GP & & & & & & & PCi/L \\
\hline Uranium-235 & $<6.6 \mathrm{E}-02$ & UIII & $<0.0662$ & & 1 & GP & & & & & & & PCi $/ L$ \\
\hline Uranium-238 & $<3.9 \mathrm{E}-02$ & UII/ & $<0.1160$ & & 1 & GP & & & & & & & pCi/L \\
\hline
\end{tabular}

Notes:

- = exceeded holding time

= exceeded groundwater protection or monitoring constituent standard (See Appendix A.) 


\section{WELL HSB106D}

\begin{tabular}{|c|c|}
\hline SRS Coord. & Lat/Longitude \\
\hline $\begin{array}{l}\text { N71727.8 } \\
\text { E57644.8 }\end{array}$ & $\begin{array}{l}33.278468^{\circ} \mathrm{N} \\
81.654340^{\circ} \mathrm{W}\end{array}$ \\
\hline
\end{tabular}

SAMPLE DATE

Water elevation

pH

Sp. conductance

Water temperature

Alkalinity as $\mathrm{CaCO}_{3}$

Turbidity

Volumes purged

Sampling code

Synchronous water level

ANALYTICAL DATA
3Q98

228.6

\section{Screen Zone Elevation}

230.7-210.7 ft ms

$07 / 08 / 98$

4.2

88

19.5

0

1

$226.6(09 / 17 / 98)$
Top of Casing

$252.9 \mathrm{ft}$ mst

$10 / 14 / 98$

$4 Q 98$
226.0
3.9
81
18.2
0
1
3.7
$224.5(12 / 21 / 98)$

Unit

ft ms!

$\mathrm{pH}$

$\mu S / c m$

${ }^{\circ} \mathrm{C}$

$\mathrm{mg} / \mathrm{L}$

NTU

well vol

ft msl

Inorganic Constituents

\begin{tabular}{|c|c|c|c|c|c|c|c|c|c|c|c|c|c|}
\hline Constituents & $\underline{3098}$ & Mod & Filtt. & $\underline{\text { ST }} \underline{\mathrm{H}}$ & $\underline{\text { DF }}$ & $\underline{\text { Lab }}$ & 4Q98 & Mod & Filt. & $\underline{\mathrm{ST}} \underline{\mathrm{H}}$ & $\underline{\text { DF }}$ & $\underline{L a b}$ & Unit \\
\hline Antimony, total recoverable & $<0.20$ & $\mathrm{U} / /$ & $<0.20$ & & 1 & GE & & & & & & & $\mu g /$ \\
\hline Arsenic, total recoverable & $<3.0$ & $\mathrm{U} / /$ & $<3.0$ & & 1 & GE & & & & & & & $\mu g / L$ \\
\hline Barium, total recoverable & 20 & $/ 1$ & & & 1 & GE & & & & & & & $\mu \mathrm{g} / \mathrm{L}$ \\
\hline Cadmium, total recoverable & 0.19 & $\mathrm{~J} / \mathrm{E} /$ & NDD & & 1 & GE & & & & & & & $\mu g / L$ \\
\hline Chromium, total recoverable & 0.63 & $\mathrm{~J} / \mathrm{E} /$ & NDD & & 1 & GE & & & & & & & $\mu g / L$ \\
\hline Cobalt, total recoverable & 2.2 & $/ 1$ & & & 1 & $\overline{G E}$ & & & & & & & $\mu g / L$ \\
\hline Copper, total recoverable & 8.0 & $N \prime$ & & & 1 & GE & & & & & & & $\mu g / L$ \\
\hline Cyanide & $<10$ & $\mathrm{U} / /$ & $<10$ & & 1 & GE & & & & & & & $\mu g / L$ \\
\hline Lead, total recoverable & 1.5 & J/EV/ & NDD & & 1 & GE & & & & & & & $\mu g / L$ \\
\hline Mercury, total recoverable & 19 & $\|$ & & a & 2 & GE & 6.3 & $\|$ & & घ & 1 & GE & $\mu g / L$ \\
\hline $\begin{array}{l}\text { Nickel, total recoverable } \\
\text { Nitrate as nitrogen }\end{array}$ & $<5.3$ & $\mathrm{U} N I$ & $<0.20$ & & 1 & GE & & & & & & & $\mathrm{\mu g} / \mathrm{L}$ \\
\hline Nitrate-nitrite as nitrogen & 7,300 & $N \prime$ & & & 5 & GE & 10,000 & II & & v & 5 & $\mathrm{GE}$ & $\mu g / L$ \\
\hline Selenium, total recoverable & $<5.0$ & $\mathrm{U} / /$ & $<5.0$ & & 1 & GE & & & & & 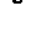 & كا & $\mu g / L$ \\
\hline Silver, total recoverable & $<1.0$ & $\mathrm{U} / l$ & $<1.0$ & & 1 & GE & & & & & & & $\mu g / L$ \\
\hline Tin, total recoverabie & $<0.54$ & UNI & $<2.0$ & & 1 & GE & & & & & & & $\mu g / L$ \\
\hline Vanadium, total recoverable & $<2.0$ & $\mathrm{U} / 1$ & $<2.0$ & & 1 & GE & & & & & & & $\mu g / L$ \\
\hline Zinc, total recoverable & $<24$ & UNI & $<5.0$ & & 1 & GE & & & & & & & $\mu g / L$ \\
\hline \multicolumn{14}{|l|}{ Organic Constituents } \\
\hline Constituents & $\underline{3098}$ & Mod & Filt. & $\underline{\text { ST브 }}$ & $\underline{\mathrm{DF}}$ & $\underline{\text { Lab }}$ & 4Q98 & Mod & Filt. & ST $H$ & $\underline{\mathrm{DF}}$ & Lab & $\underline{\text { Unit }}$ \\
\hline Benzene & $<1.0$ & $\mathrm{UJ} / \mathrm{O} / 1$ & $<1.0$ & & 1 & GE & & & & & & & $\mu g / L$ \\
\hline Bis(2-ethylhexyl) phthalate & $<10$ & UJ/Q/ & $<10$ & $\bullet$ & 1 & GE & & & & & & & $\mu g / L$ \\
\hline Dichloromethane & $<2.3$ & $\mathrm{UJ} / O \mathrm{OV} / 1$ & $<1.0$ & & 1 & GE & & & & & & & $\mu g / L$ \\
\hline Tetrachloroethylene & $<1.0$ & $\mathrm{UJ} / \mathrm{O} / 1$ & $<1.0$ & & 1 & GE & & & & & & & $\mu g / L$ \\
\hline Trichloroethylene & $<1.0$ & UJ/O/1 & $<1.0$ & & 1 & GE & & & & & & & $\mu g / L$ \\
\hline Trichlorofluoromethane & $<1.0$ & UJ/O/1 & $<1.0$ & & 1 & GE & & & & & & & \\
\hline
\end{tabular}

$\mu g / L$

$\mu g / L$

$\mu g / L$

w/L

Notes:

- exceeded holding time

- = exceeded groundwater protection or monitoring constituent standard (See Appendix A.) 
WELL HSB106D (cont.)

Radioactive Constituents

\begin{tabular}{|c|c|c|c|c|c|c|c|c|c|c|c|c|c|}
\hline Constituents & $\underline{3098}$ & Mod & Filt. & $\underline{\text { ST }} \underline{\mathrm{H}}$ & $\underline{D F}$ & $\underline{L a b}$ & $\underline{4 Q 98}$ & Mod & Filt. & $\underline{\mathrm{ST}} \underline{\mathrm{H}}$ & $\underline{\mathrm{DF}}$ & $\underline{L a b}$ & $\underline{\text { Unit }}$ \\
\hline Americium-241 & $2.2 \mathrm{E}-01$ & /1 & & & 1 & GP & & & & & & & $\mathrm{pCi} / \mathrm{L}$ \\
\hline Beta dose & 29.61 & & & - & & & & & & & & & $\mathrm{pCi} / \mathrm{L}$ \\
\hline Carbon-14 & $2.2 E+01$ & "I & & & 1 & GP & & & & & & & $\mathrm{pCi} / \mathrm{L}$ \\
\hline Cobalt-60 & $<1.4 E+\infty$ & $\mathrm{UI} / /$ & $<4.7000$ & & 1 & GP & & & & & & & $\mathrm{pCin}$ \\
\hline Curium-242 & $<-1.0 \mathrm{E}-02$ & $U 1 / I$ & $<0.1330$ & & 1 & GP & & & & & & & $\mathrm{pCi} / \mathrm{L}$ \\
\hline Curium-243/244 & $<1.4 \mathrm{E}-01$ & $U N /$ & $<0.1070$ & & 1 & GP & & & & & & & $\mathrm{pCi} / \mathrm{L}$ \\
\hline Curium-245/246 & $<-4.9 E-03$ & $\mathrm{UI} / /$ & $<0.1070$ & & 1 & GP & & & & & & & $\mathrm{pCi} / \mathrm{L}$ \\
\hline Gross alpha & $7.0 E+00$ & $1 /$ & & & 1 & GP & $4.7 E+00$ & $/ 1$ & & & 1 & GE & $\mathrm{pCi} / \mathrm{L}$ \\
\hline lodine- 129 & $7.1 \mathrm{E}+00$ & $/ /$ & & & 1 & GP & & & & & & & $\mathrm{pCi} / \mathrm{L}$ \\
\hline Nickel-63 & $<1.4 \mathrm{E}+00$ & Ut & $<4.7000$ & & & & & & & & & & $\mathrm{pCi} / \mathrm{L}$ \\
\hline Nonvolatile beta & $4.5 \mathrm{E}+02$ & "I & & घ & 1 & GP & $5.2 \mathrm{E}+02$ & $/ 1$ & & a & 1 & GE & $\mathrm{pCi} / \mathrm{L}$ \\
\hline Plutonium-238 & $<2.9 E-03$ & $\mathrm{U}] / /$ & $<0.1620$ & & 1 & $\mathrm{GP}$ & & & & & & & $\mathrm{pCill}$ \\
\hline Plutonium-239/240 & $<1.2 \mathrm{E}-02$ & $\mathrm{U} 1 / \mathrm{I}$ & $<0.0505$ & & 1 & GP & & & & & & & $\mathrm{pCin}$ \\
\hline Radium-226 & $2.1 \mathrm{E}+00$ & 11 & & & 1 & GP & & & & & & & $\mathrm{pCi} / \mathrm{L}$ \\
\hline Radium-228 & $<-1.7 \mathrm{E}-01$ & $\mathrm{UI} / /$ & $<1.3400$ & & 1 & GP & & & & & & & $\mathrm{pCi} / \mathrm{L}$ \\
\hline Strontium-90 & $1.8 \mathrm{E}+02$ & $/ 1$ & & - & 1 & GP & & & & & & & $\mathrm{pCi} / \mathrm{L}$ \\
\hline Technetium-99 & $<2.0 E+00$ & UII/ & $<20.7000$ & & 1 & GP & & & & & & & $\mathrm{pCi} / \mathrm{L}$ \\
\hline Thorium-228 & $<6.7 \mathrm{E}-01$ & $\mathrm{UI} / \mathrm{I}$ & $<0.8490$ & & 1 & GP & & & & & & & $\mathrm{pCi} / \mathrm{L}$ \\
\hline Thorium-230 & $<4.6 \mathrm{E}-02$ & $\mathrm{U} M / \mathrm{I}$ & $<0.3210$ & & 1 & GP & & & & & & & $\mathrm{pCi} / \mathrm{L}$ \\
\hline Thorium-232 & $<1.2 E-01$ & $\mathbf{U} \| /$ & $<0.1820$ & & 1 & GP & & & & & & & $\mathrm{pCi} / \mathrm{L}$ \\
\hline Sum of alphas & $1.4 E+00$ & & & & & & & & & & & & $\mathrm{pCi} / \mathrm{L}$ \\
\hline $\begin{array}{l}\text { Sum of betas } \\
\text { Total radium }\end{array}$ & $\begin{array}{l}2.0 E+02 \\
2.1 E+00\end{array}$ & & & - & & & & & & & & & $\begin{array}{l}\mathrm{pCi} / \mathrm{L} \\
\mathrm{pCi} / \mathrm{L}\end{array}$ \\
\hline Tritium & $2.9 E+02$ & "I & & घ & 1 & GP & $7.1 E+02$ & $/ /$ & & ט & 1 & GE & $\mathrm{pCi} / \mathrm{mL}$ \\
\hline Uranium-233/234 & $9.9 \mathrm{E}-01$ & $N /$ & & & 1 & GP & & & & & & & $\mathrm{pCi} / \mathrm{L}$ \\
\hline Uranium-235 & $<6.2 \mathrm{E}-02$ & $\mathrm{UI} / /$ & $<0.0618$ & & 1 & GP & & & & & & & $\mathrm{pCi} / \mathrm{L}$ \\
\hline Uranium-238 & $2.0 \mathrm{E}-01$ & $\|$ & & & 1 & GP & & & & & & & $\mathrm{pCi} / \mathrm{L}$ \\
\hline
\end{tabular}


WELL HSB107C

$\begin{array}{lll}\text { SRS Coord. } & \text { Lat/Longitude } \\ \text { N71698.5 } & 33.278056^{\circ} \mathrm{N} \\ \text { E57432.0 } & 81.654844^{\circ} \mathrm{W}\end{array}$

SAMPLE DATE

07/22/98

Screen Zone Elevation

169.3-159.3 ft ms

FIELD DATA

\section{Constituents}

Water elevation

$\mathrm{pH}$

$\mathrm{Sp}$. conductance

Water temperature

Alkalinity as $\mathrm{CaCO}_{3}$

Turbidity

Volumes purged

Sampling code

Synchronous water level

$\underline{3098}$

220.0

6.7

120

21.4

16

3

2.9

$219.6(09 / 17 / 98)$
Top of Casing

$261.6 \mathrm{ft} \mathrm{ms}$

$10 / 12 / 98$

$4 Q 98$
219.3
6.5
140
20.5
25
0
2.3
$218.3(12 / 21 / 98)$

ANALYTICAL DATA

Inorganic Constituents

Constituents $\quad \underline{3098}$

Antimony, total recoverable $<0.14$

Arsenic, total recoverable $<3.0$

Barium, total recoverable 38

Cadmium, total recoverable 0.36

Chromium, total recoverable 0.89

Cobalt, total recoverable $\quad<0.20$

Copper, total recoverable $\quad 0.89$

Cyanide

Lead, total recoverable $<2.0$

Mercury, total recoverable $\quad<0.20$

Nickel, total recoverable $\quad 2.9$

Nitrate as nitrogen

Nitrate-nitrite as nitrogen $\quad 7,900$

Selenium, total recoverable $<5.0$

Silver, total recoverable $\quad<1.0$

Tin, total recoverable $<2.0$

Vanadium, total recoverable $<20$

Zinc, total recoverable

12

Organic Constituents

Constituents

Benzene

Bis(2-ethylhexyl) phthalate

Dichloromethane

Tetrachloroethylene

Trichlorothylene

Trichlorofluoromethane $\underline{3098}$

$<1.0$

$<11$

$<1.0$

$<1.2$

$<1.4$

4.4

\begin{tabular}{|c|c|}
\hline Mod & Filt. \\
\hline $\begin{array}{l}\mathrm{U} N / \\
\mathrm{U} / / \\
/ /\end{array}$ & $\begin{array}{l}<0.20 \\
<3.0\end{array}$ \\
\hline $\mathrm{J} / \mathrm{E}$ & NDD \\
\hline $\mathrm{J} / \mathrm{E} /$ & NDD \\
\hline $\mathrm{U} / /$ & $<0.20$ \\
\hline "I & \\
\hline $\mathrm{U} / /$ & $<10$ \\
\hline $\mathrm{U} / 1$ & $<2.0$ \\
\hline $\mathrm{U} / /$ & $<0.20$ \\
\hline "l & \\
\hline$N /$ & \\
\hline $\mathrm{u} / /$ & $<5.0$ \\
\hline $\mathrm{U} / /$ & $<1.0$ \\
\hline $\mathrm{U} / /$ & $<2.0$ \\
\hline $\mathrm{U} / \prime$ & $<2.0$ \\
\hline
\end{tabular}

Mod

UJ/O/1

U/I

$\mathrm{U} / / \mathrm{O} / \mathrm{t}$

UJ/O/1

UJ/O/1

$\mathrm{J} / \mathrm{O} / 1$
Filt.

$<1.0$

$<11$

$<1.0$

$<1.0$

$<1.0$

NDD
$<1.0$

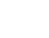

ST브

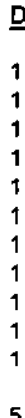



GE

$G E$

GE

$\mathrm{GE}$

GE

GE

GE

GE

$<0.20$

U/I

$\mathrm{GE}$
$\mathrm{GE}$
$\mathrm{GE}$
$\mathrm{GE}$
$\mathrm{GE}$
$\mathrm{GE}$

$\mathrm{GE}$
$\mathrm{GE}$
$\mathrm{GE}$
$\mathrm{GE}$
$\mathrm{GE}$
$\mathrm{GE}$

.

1.0
11
1.0
1.0
1.0
$D D$

$\begin{array}{ll}\text { DF } & \text { Lab } \\ 1 & \text { GE } \\ 1 & \text { GE } \\ 1 & \text { GE } \\ 1 & \text { GE } \\ 1 & \text { GE } \\ 1 & \text { GE }\end{array}$

$\begin{array}{lll}\text { Casing } & \text { Pump } & \text { Screen Zone } \\ 4^{\prime \prime} \text { PVC } & \mathrm{S} & \left.\text { Barnwell (IIB } \mathrm{B}_{1}\right)\end{array}$


WELL HSB107C (cont.)

Radioactive Constituents

\begin{tabular}{|c|c|c|c|c|c|c|c|c|c|c|c|c|c|}
\hline Constituents & 3098 & Mod & Filt. & ST브 & DF & $\underline{L a b}$ & $4 Q 98$ & Mod & Filt. & $\underline{\text { ST }} \underline{H}$ & $\underline{D F}$ & $\underline{\text { Lab }}$ & Unit \\
\hline Americium-241 & $<-1,3 \mathrm{E}-01$ & $\mathrm{UI} / /$ & $<0.2930$ & & 1 & $G P$ & & & & & & & $\mathrm{pCi} / \mathrm{L}$ \\
\hline Beta dose & 0.20 & & & & & & & & & & & & $\mathrm{pCi} / \mathrm{L}$ \\
\hline Carbon-14 & $<4.6 \mathrm{E}+00$ & $\mathrm{U} 1 / /$ & $<8.5100$ & & 1 & GP & & & & & & & $\mathrm{pCi} / \mathrm{L}$ \\
\hline Cobalt-60 & $<2.1 \mathrm{E}-01$ & $\mathrm{U} 1 / /$ & $<3.5100$ & & 1 & GP & & & & & & & $\mathrm{pCi} / \mathrm{L}$ \\
\hline Curium-242 & $<0.0 E+00$ & $\mathrm{UI} / /$ & $<0.0665$ & & 1 & GP & & & & & & & $\mathrm{pCi} / \mathrm{L}$ \\
\hline Curium-243/244 & $<4.2 \mathrm{E}-02$ & $\mathrm{UI} / /$ & $<0.0632$ & & 1 & GP & & & & & & & $\mathrm{pCi} / \mathrm{L}$ \\
\hline Curium-245/246 & $<-1.5 E-02$ & $\mathrm{U} \mathrm{l} / /$ & $<0.1460$ & & 1 & GP & & & & & & & $\mathrm{pCi} / \mathrm{L}$ \\
\hline Gross alpha & $9.4 \mathrm{E}-01$ & $/ 1$ & & & 1 & GP & $<8.1 E-01$ & $\mathrm{U} / /$ & $<5.2 E-01$ & & 1 & GE & $\mathrm{pCi} / \mathrm{L}$ \\
\hline lodine-129 & $<-1,5 E-01$ & $\mathrm{UI} / /$ & $<0.7400$ & & 1 & GP & & & & & & & $\mathrm{pCi} / \mathrm{L}$ \\
\hline Nickel-63 & $<2.1 E-01$ & UI & $<3.5100$ & & & & & & & & & & $\mathrm{pCi} / \mathrm{L}$ \\
\hline Nonvolatile beta & $5.1 E+00$ & $1 /$ & & & 1 & GP & $5.3 E+00$ & $/ /$ & & & 1 & GE & $\mathrm{pCi} / \mathrm{L}$ \\
\hline Plutonium-238 & $<-2.6 E-02$ & $\mathrm{UI} / /$ & $<0.1310$ & & 1 & GP & & & & & & & $\mathrm{pCi} / \mathrm{L}$ \\
\hline Plutonium-239/240 & $<1.7 \mathrm{E}-02$ & UII/ & $<0.0999$ & & 1 & GP & & & & & & & $\mathrm{pCi} / \mathrm{L}$ \\
\hline Radium-226 & $<3.4 E-01$ & UNI & $<0.1540$ & & 1 & GP & & & & & & & $\mathrm{pCi} / \mathrm{L}$ \\
\hline Radium-228 & $<1.0 \mathrm{E}+00$ & $\mathrm{UI} / /$ & $<1.2600$ & & 1 & GP & & & & & & & $\mathrm{pCi} / \mathrm{L}$ \\
\hline Strontium-90 & $1.6 \mathrm{E}+00$ & $1 /$ & & & 1 & GP & & & & & & & $\mathrm{pCi} / \mathrm{L}$ \\
\hline Technetium-99 & $<2.1 E+00$ & $\mathrm{UI} / /$ & $<24.2000$ & & 1 & GP & & & & & & & $\mathrm{pCi} / \mathrm{L}$ \\
\hline Thorium-228 & $<9.1 E-02$ & $\mathrm{UI} / /$ & $<0.4050$ & & 1 & GP & & & & & & & $\mathrm{pCi} / \mathrm{L}$ \\
\hline Thorium-230 & 3.7E-01 & 11 & & & 1 & GP & & & & & & & $\mathrm{pCi} / \mathrm{L}$ \\
\hline Thorium-232 & $<2.8 \mathrm{E}-02$ & $\mathrm{UI} / /$ & $<0.1970$ & & 1 & GP & & & & & & & $\mathrm{pCi} / \mathrm{L}$ \\
\hline Sum of alphas & 3.7E-01 & & & & & & & & & & & & $\mathrm{pCi} / \mathrm{L}$ \\
\hline Sum of betas & $1.6 \mathrm{E}+00$ & & & & & & & & & & & & $\mathrm{pCi} / \mathrm{L}$ \\
\hline Tritium & $3.2 E+02$ & $\|$ & & $\mathbf{\square}$ & 1 & GP & 4.1E+02 & $/ /$ & & $\mathbf{\square}$ & 1 & $\mathrm{GE}$ & $\mathrm{pCi} / \mathrm{mL}$ \\
\hline Uranium-233/234 & $<2.5 \mathrm{E}-01$ & UII/ & $<0.3480$ & & 1 & GP & & & & & & & pCill \\
\hline Uranium-235 & $<6.6 \mathrm{E}-02$ & $\mathrm{UI} / /$ & $<0.1980$ & & 1 & GP & & & & & & & $\mathrm{pCi} / \mathrm{L}$ \\
\hline Uranium-238 & $<1.3 E-01$ & $\mathrm{UI} / /$ & $<0.1980$ & & 1 & GP & & & & & & & $\mathrm{pCi} / \mathrm{L}$ \\
\hline
\end{tabular}

Notes:

- = exceeded holding time

= = exceeded groundwater protection or monitoring constituent standard (See Appendix A.) 
WELL HSB107D

\begin{tabular}{|c|c|c|c|}
\hline SRS Coord. & Lat/Longitude & & Screen Zone Elevation \\
\hline $\begin{array}{l}\text { N71696.6 } \\
\text { E57412.2 }\end{array}$ & $\begin{array}{l}33.278019^{\circ} \mathrm{N} \\
81.654892^{\circ} \mathrm{W}\end{array}$ & & $235.1-215.1 \mathrm{ft} \mathrm{msl}$ \\
\hline \multicolumn{2}{|c|}{ SAMPLE DATE } & \multicolumn{2}{|l|}{ 07/08/98 } \\
\hline \multicolumn{4}{|l|}{ FIELD DATA } \\
\hline \multicolumn{2}{|l|}{ Constituents } & $\underline{3098}$ & \\
\hline \multirow{2}{*}{\multicolumn{2}{|c|}{ Water elevation }} & 225.7 & \\
\hline & & 4.6 & \\
\hline & 120 & \\
\hline & & 20.0 & \\
\hline \multicolumn{2}{|c|}{ Water temperature } & 0 & \\
\hline \multicolumn{2}{|l|}{ Turbidity } & 1 & \\
\hline \multicolumn{2}{|c|}{$\begin{array}{l}\text { Volumes purged } \\
\text { Sampling code }\end{array}$} & 6.1 & \\
\hline \multicolumn{2}{|c|}{ Synchronous water level } & 224.7105 & \\
\hline
\end{tabular}

\begin{tabular}{|c|c|c|c|}
\hline Top of Casing & Casing & Pump & Screen Zone \\
\hline $262.3 \mathrm{ft} \mathrm{msl}$ & 4" PVC & v & Water Table (IIB2) \\
\hline $10 / 13 / 98$ & & & \\
\hline$\underline{4 Q 98}$ & & & $\underline{\text { Unit }}$ \\
\hline 224.3 & & & $\mathrm{ft} \mathrm{msl}$ \\
\hline 4.6 & & & $\mathrm{pH}$ \\
\hline 120 & & & $\mu \mathrm{S} / \mathrm{cm}$ \\
\hline 18.0 & & & \\
\hline 0 & & & $\mathrm{mg} / \mathrm{L}$ \\
\hline 1 & & & NTUU \\
\hline 7.2 & & & well vol \\
\hline $223.0(12 / 21 / 98)$ & & & $\mathrm{ft} \mathrm{msl}$ \\
\hline
\end{tabular}

ANALYTICAL DATA

Inorganic Constituents

\begin{tabular}{|c|c|c|c|c|c|c|c|c|c|c|c|c|c|}
\hline Constituents & $\underline{3 Q 98}$ & Mod & Filt. & $\underline{\text { STI }} \underline{H}$ & $\underline{\mathrm{DF}}$ & Lab & $\underline{4098}$ & Mod & Filt. & $\underline{\text { ST }} \underline{H}$ & DF & $\underline{\text { Lab }}$ & $\underline{\text { Unit }}$ \\
\hline Antimony, total recoverable & $<0.20$ & $\mathrm{U} / 1$ & $<0.20$ & & 1 & GE & & & & & & & $\mu \mathrm{g} / \mathrm{L}$ \\
\hline Arsenic, total recoverable & $<3.0$ & $\mathrm{U} / /$ & $<3.0$ & & 1 & GE & & & & & & & $\begin{array}{l}\beta g / L \\
\mu g / L\end{array}$ \\
\hline Barium, total recoverable & 34 & "I & & & 1 & GE & & & & & & & $\mu g / L$ \\
\hline Cadmium, total recoverable & $<1.0$ & U/I & $<1.0$ & & 1 & GE & & & & & & & $\mu \mathrm{g} / \mathrm{L}$ \\
\hline Chromium, total recoverable & 0.74 & $\mathrm{~J} / \mathrm{E} /$ & NDD & & 1 & GE & & & & & & & $\mu g / \mathrm{L}$ \\
\hline Cobalt, total recoverable & 1.7 & "I & & & 1 & $\mathrm{GE}$ & & & & & & & $\mu g / L$ \\
\hline Copper, total recoverable & $<3.3$ & $\mathrm{U} N I$ & $<0.20$ & & 1 & GE & & & & & & & $\mu g / L$ \\
\hline Cyanide & $<10$ & $\mathrm{U} / \mathrm{I}$ & $<10$ & & 1 & GE & & & & & & & $\mu g / \mathrm{L}$ \\
\hline Lead, total recoverable & $<0.42$ & UNI & $<2.0$ & & 1 & GE & & & & & & & $\mu g / L$ \\
\hline Mercury, total recoverable & 4.8 & $\prime \prime$ & & - & 1 & GE & 3.6 & $\|$ & & ๗ & 1 & GE & $\mu g / L$ \\
\hline $\begin{array}{l}\text { Nickel, total recoverable } \\
\text { Nitrate as nitrogen }\end{array}$ & $<3.1$ & UNI & $<0.20$ & & 1 & GE & & & & & & & $\mu \mathrm{g} / \mathrm{L}$ \\
\hline Nitrate-nitrite as nitrogen & 13,000 & $N /$ & & - & 25 & GE & 12,000 & $\|$ & & 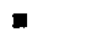 & 10 & GE & $\mu g / L$ \\
\hline Selenium, total recoverable & $<5.0$ & $\mathrm{U} / \mathrm{I}$ & $<5.0$ & & 1 & GE & & & & & & & $\mu g / L$ \\
\hline Silver, total recoverable & $<1.0$ & $\mathrm{U} / /$ & $<1.0$ & & 1 & GE & & & & & & & $\mu g / L$ \\
\hline Tin, total recoverable & $<0.27$ & $\mathrm{UN} /$ & $<2.0$ & & 1 & GE & & & & & & & $\mu g / L$ \\
\hline Vanadium, total recoverable & $<1.7$ & UNI & $<2.0$ & & 1 & GE & & & & & & & $\mu g / L$ \\
\hline Zinc, total recoverable & $<16$ & UNI & $<5.0$ & & 1 & GE & & & & & & & $\mu g / L$ \\
\hline \multicolumn{14}{|l|}{ Organic Constituents } \\
\hline Constituents & $\underline{3 Q 98}$ & Mod & Filt. & $\underline{\text { STI }} \underline{H}$ & $\underline{\mathrm{DF}}$ & $\underline{\text { Lab }}$ & $\underline{4 Q 98}$ & Mod & Filt. & $\underline{\text { ST }} \underline{H}$ & DF & $\underline{\text { Lab }}$ & $\underline{\text { Unit }}$ \\
\hline Benzene & $<1.0$ & $\mathrm{UJ} / \mathrm{O} / 1$ & $<1.0$ & & 1 & GE & & & & & & & $\mu g / L$ \\
\hline Bis(2-ethylhexyl) phthalate & $<10$ & $\mathrm{U} / \mathrm{I}$ & $<10$ & & 1 & $\mathrm{GE}$ & & & & & & & $\mu g / \mathrm{L}$ \\
\hline Dichloromethane & $<3.0$ & UJ/OV/1 & $<1.0$ & & 1 & GE & & & & & & & $\mu g / L$ \\
\hline Tetrachloroethylene & $<1.0$ & UJJ/O/1 & $<1.0$ & & 1 & GE & & & & & & & $\mu g / L$ \\
\hline Trichloroethylene & $<1.0$ & $\mathrm{UJ} / \mathrm{O} / 1$ & $<1.0$ & & 1 & GE & & & & & & & $\mu g / L$ \\
\hline Trichlorofluoromethane & $<1.0$ & $\mathrm{UJ} / \mathrm{O} / 1$ & $<1.0$ & & 1 & GE & & & & & & & $\mu g / L$ \\
\hline
\end{tabular}

Notes:

- = exceeded holding time

- = exceeded groundwater protection or monitoring constituent standard (See Appendix A.) 
WELL HSB107D (cont.)

Radioactive Constituents

\begin{tabular}{|c|c|c|c|c|c|c|c|c|c|c|c|c|c|}
\hline Constituents & 3098 & Mod & Filt. & ST브 & $\underline{D F}$ & $\underline{\mathrm{Lab}}$ & $4 Q 98$ & Mod & Fillt. & ST $\mathrm{H}$ & DF & Lab & Unit \\
\hline Americium-241 & $<2.9 E-02$ & $\mathrm{UH} / \mathrm{I}$ & $<0.1170$ & & 1 & GP & & & & & & & $p C i / L$ \\
\hline Beta dose & 65.73 & & & 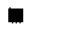 & & & & & & & & & $\mathrm{pCi} / \mathrm{L}$ \\
\hline Carbon-14 & $<2.0 \mathrm{E}+00$ & $\mathrm{U}: /$ & $<9.0100$ & & 1 & GP & & & & & & & $\mathrm{pCi} / \mathrm{L}$ \\
\hline Cobalt-60 & $1.0 \mathrm{E}+01$ & $\|$ & & & 1 & GP & & & & & & & $\mathrm{pCi} / \mathrm{L}$ \\
\hline Curium-242 & $<0.0 \mathrm{E}+00$ & $\mathrm{UI} / /$ & $<0.0540$ & & 1 & GP & & & & & & & $\mathrm{pCi} / \mathrm{L}$ \\
\hline Curium-243/244 & $<6.3 \mathrm{E}-02$ & $\mathrm{UI} / /$ & $<0.0889$ & & 1 & $G P$ & & & & & & & $\mathrm{pCi} / \mathrm{L}$ \\
\hline Curium-245/246 & $<-4.0 \mathrm{E}-03$ & $\mathrm{UI} / /$ & $<0.0887$ & & 1 & GP & & & & & & & $\mathrm{pCi} / \mathrm{L}$ \\
\hline Gross alpha & $1.0 \mathrm{E}+01$ & $/ /$ & & & 1 & GP & $1.0 \mathrm{E}+01$ & $/ /$ & & & 1 & GE & $\mathrm{pCi} / \mathrm{L}$ \\
\hline lodine-129 & $5.4 \mathrm{E}+00$ & $/ /$ & & & 1 & GP & & & & & & & $\mathrm{pCi} / \mathrm{L}$ \\
\hline Nickel-63 & $1.0 E+01$ & $\|$ & & & & & & & & & & & $\mathrm{pCi} / \mathrm{L}$ \\
\hline Nonvolatile beta & $1.2 E+03$ & $\|$ & & $\square$ & 1 & GP & $1.1 E+03$ & $\|$ & & ש & 1 & GE & $\mathrm{pCi} / \mathrm{L}$ \\
\hline Plutonium-238 & $<0.0 \mathrm{E}+00$ & $\mathrm{U}[\mathrm{J} / \mathrm{C} /$ & & & 1 & $G P$ & & & & & & & $\mathrm{pCj} / \mathrm{L}$ \\
\hline Plutonium-239/240 & $<9.1 E-03$ & $\mathrm{U}] \mathrm{J} / \mathrm{C} /$ & & & 1 & GP & & & & & & & $\mathrm{pCi} / \mathrm{L}$ \\
\hline Radium-226 & $1.8 E+00$ & $/ /$ & & & 1 & GP & & & & & & & $\mathrm{pCi} / \mathrm{L}$ \\
\hline Radium-228 & $<5.3 E-01$ & $\mathrm{UIII}$ & $<1.0800$ & & 1 & GP & & & & & & & $\mathrm{pCi} / \mathrm{L}$ \\
\hline Strontjum-90 & $4.8 \mathrm{E}+02$ & $\|$ & & D & 1 & GP & & & & & & & $p C i / L$ \\
\hline Technetium-99 & $3.0 E+01$ & $\|$ & & & 1 & GP & & & & & & & $\mathrm{pCi} / \mathrm{L}$ \\
\hline Thorium-228 & $<1.6 \mathrm{E}-01$ & $\mathbf{U 1} / /$ & $<0.5250$ & & 1 & GP & & & & & & & $\mathrm{pCi} / \mathrm{L}$ \\
\hline Thorium-230 & $<2.7 \mathrm{E}-02$ & $\mathbf{U I} / /$ & $<0.2410$ & & $t$ & GP & & & & & & & $\mathrm{pCi} / \mathrm{L}$ \\
\hline Thorium-232 & $<3,0 \mathrm{E}-02$ & $\mathrm{UI} / /$ & $<0.0911$ & & 1 & GP & & & & & & & $\mathrm{pCi} / \mathrm{L}$ \\
\hline Sum of alphas & $4.3 E+00$ & & & & & & & & & & & & $\mathrm{pCi} / \mathrm{L}$ \\
\hline Sum of betas & $5.3 E+02$ & & & 口 & & & & & & & & & $\mathrm{pCi} / \mathrm{L}$ \\
\hline Total radium & $1.8 \mathrm{E}+00$ & & & & & & & & & & & & $\mathrm{pCi} / \mathrm{L}$ \\
\hline Tritium & $3.6 \mathrm{E}+02$ & $/ 1$ & & $\mathbf{0}$ & 1 & GP & 4.5E+02 & $/ 1$ & & a & 1 & $\mathrm{GE}$ & $\mathrm{pCi} / \mathrm{mL}$ \\
\hline Uranium-233/234 & $3.0 \mathrm{E}+00$ & $N I$ & & & 1 & GP & & & & & & & $\mathrm{pCi} / \mathrm{L}$ \\
\hline Uranium-235 & 7.5E-01 & $N I$ & & & 1 & GP & & & & & & & $\mathrm{pCi} / \mathrm{L}$ \\
\hline Uranium-238 & 5.2E-01 & $/ /$ & & & 1 & GP & & & & & & & $\mathrm{pCi} / \mathrm{L}$ \\
\hline
\end{tabular}

Notes:

- =xceeded holding time

- exceeded groundwater protection or monitoring constituent standard (See Appendix A.) 


\section{WELL HSB108C}

\begin{tabular}{lll} 
SRS Coord. & & Lat/Longitude \\
\hline N71688.7 & & $33.277583^{\circ} \mathrm{N}$ \\
E57155.5 & $81.655553^{\circ} \mathrm{W}$
\end{tabular}

SAMPLE DATE

$07 / 22 / 98$

\section{Screen Zone Elevation}

196.0-186.0 ft msl
Top of Casing

$266.2 \mathrm{ft} \mathrm{msl}$
Casing Pump

4" PVC S
Screen Zone

Barnwell (IIB 1 )
$10 / 12 / 98$

$4 Q 98$
218.3
6.7
125
20.0
48
1
2.8
$217.4(12 / 21 / 98)$

$\underline{\text { Unit }}$

ft ms

$\mathrm{pH}$

$\mu \mathrm{S} / \mathrm{cm}$

${ }^{\circ} \mathrm{C}$

$\mathrm{mg} / \mathrm{L}$

NTU

well vol

ft msi

\section{ANALYTICAL DATA}

Inorganic Constituents

Constituents

Mod

UNI

$<0.20$

Arsenic, total recoverable $<3.0$

Barium, total recoverable $\quad 9.7$

Cadmium, total recoverable $<1$.

Chromium, total recoverable 3.8

Cobalt, total recoverable $\quad 0.047$

Copper, total recoverable $\quad 3.8$

Cyanide

Lead, total recoverable

Mercury, total recoverable

Nickel, total recoverable

Nitrate as nitrogen

Nitrate-nitrite as nitrogen $\quad 1,800$

Selenium, total recoverable $<5.0$

Silver, total recoverable $<1.0$

Tin, total recoverable $\quad<2.0$

Vanadium, total recoverable $<2.0$

Zinc, total recoverable

UII $<3.0$

$U / / \quad<1.0$

II

J/EJ NDD

UII $<10$

$\begin{array}{ll}\mathrm{U} / / & <10\end{array}$

$\mathrm{U} / / \quad<0.20$

/I

NI

$\mathrm{U} / 1$

UII $<1.0$

$\mathrm{U} / \mathrm{U}<20$

$\mathrm{U} / /<2.0$

Onganic Constituents

Constituents

Benzene

Bis(2-ethylhexyl) phthalate

Dichloromethane

Tetrachioroethylene

Trichloroethylene

Trichiorofluoromethane

3098
$<1.0$
$<10$
$<1.0$
$<1.0$
$<1.0$
$<1.0$

$\begin{array}{ll}\text { Mod } & \text { Filt. } \\ \text { UJ/O/1 } & <1.0 \\ U / / & <10 \\ U J / O / 1 & <1.0 \\ U J / O / 1 & <1.0 \\ U J / O / 1 & <1.0 \\ U J / O / 1 & <1.0\end{array}$

sT브

ab 4Q98 Mod

Filt.

ST 브 DF Lab Unit

GE
$G E$
$G E$
$G E$
$G E$
$G E$
$G E$
$G E$
$G E$
$G E$
$G E$
$G E$
$G E$
$G E$
$G E$
$G E$
$G E$

$<0.20$

U/I

ST브

$\begin{array}{ll}\text { DF } & \text { Lab } \\ 1 & \text { GE } \\ 1 & \text { GE } \\ 1 & \text { GE } \\ 1 & \text { GE } \\ 1 & \text { GE } \\ 1 & \text { GE }\end{array}$

$<0.20$

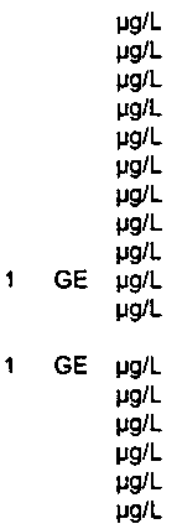

Filt.
ST H DF Lab Unit

$\mu g / L$

$\mu g / L$

$\mu g / L$

$\mu \mathrm{g} / \mathrm{L}$

$\mu \mathrm{g} / \mathrm{L}$

$\mu g / L$

$\begin{array}{ll}\text { DF } & \text { Lab } \\ 1 & \\ 1 & \\ 1 & \\ 1 & \\ 1 & \\ 1 & \\ 1 & \\ 1 & \\ 1 & \\ 1 & \\ 1 & \end{array}$

Notes:

- = exceeded holding time

= exceeded groundwater protection or monitoring constituent standard (See Appendix A.) 
WELL HSB108C (cont.)

Radioactive Constituents

\begin{tabular}{|c|c|c|c|c|c|c|c|c|c|c|c|c|c|}
\hline Constituents & 3098 & Mod & Filt. & $\underline{\text { ST }} \underline{H}$ & $\underline{\text { DF }}$ & $\underline{\mathrm{Lab}}$ & 4098 & Mod & Filt. & $\underline{\mathrm{ST}} \underline{\mathrm{H}}$ & $\underline{D F}$ & Lab & Unit \\
\hline Americium-241 & $<3.2 \mathrm{E}-02$ & $\mathrm{U} 1 / /$ & $<0.0621$ & & 1 & GP & & & & & & & $p C i / L$ \\
\hline Beta dose & 2.40 & & & $\mathbf{0}$ & & & & & & & & & $\mathrm{pCi} / \mathrm{L}$ \\
\hline Carbon-14 & $<3.1 E+\infty 0$ & $\mathrm{U} / / /$ & $<8.7700$ & & 1 & $G P$ & & & & & & & $\mathrm{pCi} / \mathrm{L}$ \\
\hline Cobalt-60 & $<-1.0 E+00$ & UI/I & $<3.1800$ & & 1 & GP & & & & & & & $\mathrm{pCi} / \mathrm{L}$ \\
\hline Curium-242 & $<0.0 \mathrm{E}+00$ & $\mathrm{UI} / /$ & $<0.0653$ & & 1 & GP & & & & & & & $\mathrm{pCi} / \mathrm{L}$ \\
\hline Curium-243/244 & $<3.6 \mathrm{E}-02$ & $\mathrm{UI} / /$ & $<0.1090$ & & $i$ & GP & & & & & & & $\mathrm{pCi} / \mathrm{L}$ \\
\hline Curium-245/246 & $<0.0 E+00$ & $\mathrm{UI} / /$ & $<0.0620$ & & 1 & GP & & & & & & & $\mathrm{pCi} / \mathrm{L}$ \\
\hline Gross alpha & 4.9E-01 & $/ 1$ & & & 1 & GP & $<5.8 \mathrm{E}-01$ & $\mathbf{U} / /$ & $<7.0 \mathrm{E}-01$ & & 1 & $\mathrm{GE}$ & $\mathrm{pCi} / \mathrm{L}$ \\
\hline lodine- 129 & $2.4 E+00$ & $\|$ & & & $i$ & $\mathrm{GP}$ & & & & & & & $\mathrm{pCi} / \mathrm{L}$ \\
\hline Nickel-63 & $<-1.0 \mathrm{E}+00$ & ال & $<3.1800$ & & & & & & & & & & $\mathrm{pCi} / \mathrm{L}$ \\
\hline Nonvolatile beta & $<8.1 E-01$ & $\mathrm{UI} / /$ & $<1.1400$ & & 1 & GP & $6.2 E+00$ & $/ /$ & & & 1 & GE & $\mathrm{pCi} / \mathrm{L}$ \\
\hline Plutonium-238 & 1.0E-01 & $/ 1$ & & & 1 & GP & & & & & & & pCill \\
\hline Plutonium-239/240 & $1.4 \mathrm{E}-01$ & $/ /$ & & & 1 & GP & & & & & & & $\mathrm{pCi} / \mathrm{L}$ \\
\hline Radium-226 & $<1.6 \mathrm{E}-01$ & $\mathrm{UI} / /$ & $<0.4880$ & & 1 & GP & & & & & & & $\mathrm{pCi} / \mathrm{L}$ \\
\hline Radium-228 & $<1.7 E+00$ & $\mathrm{UN} /$ & $<1.0600$ & & 1 & GP & & & & & & & $\mathrm{pCi} / \mathrm{L}$ \\
\hline Strontium-90 & $<-2.6 E-02$ & $\mathrm{UI} / I$ & $<2.3700$ & & 1 & GP & & & & & & & $\mathrm{pCi} / \mathrm{L}$ \\
\hline Technetium-99 & $<5.8 E+00$ & $\mathrm{UI} / /$ & $<23.9000$ & & 1 & GP & & & & & & & PCiL \\
\hline Thorium-228 & $<1.3 E-01$ & $\mathrm{UII} /$ & $<0.2810$ & & 1 & GP & & & & & & & pCill \\
\hline Thorium-230 & $<1.3 E-01$ & $\mathrm{UI} / /$ & $<0.1790$ & & 1 & GP & & & & & & & $\mathrm{pCi} / \mathrm{L}$ \\
\hline Thorium-232 & $<2.6 \mathrm{E}-02$ & $\mathrm{UI} / /$ & $<0.1790$ & & 1 & GP & & & & & & & pCint \\
\hline Sum of alphas & $2.4 E-01$ & & & & & & & & & & & & $\mathrm{pCi} / \mathrm{L}$ \\
\hline Sum of betas & $2,4 E+00$ & & & & & & & & & & & & $\mathrm{pCi} / \mathrm{L}$ \\
\hline Tritium & $1.3 E+02$ & $/ 1$ & & ש & 1 & GP & $1.5 E+02$ & $/ /$ & & 口 & 1 & GE & $\mathrm{pCi} / \mathrm{mL}$ \\
\hline Uranium-233/234 & $<4.3 E-02$ & $\mathrm{UI} / \mathrm{I}$ & $<0.3000$ & & 1 & GP & & & & & & & $\mathrm{pCi} / \mathrm{L}$ \\
\hline Uranium-235 & $<1.6 \mathrm{E}-01$ & $\mathrm{UI} / \mathrm{I}$ & $<0.3010$ & & 1 & GP & & & & & & & $\mathrm{pCi} / \mathrm{L}$ \\
\hline Uranium-238 & $<4.0 E-01$ & UNI & $<0.1710$ & & 1 & GP & & & & & & & $\mathrm{pCi} / \mathrm{L}$ \\
\hline
\end{tabular}

Notes:

- = exceeded holding time

- =xceeded groundwater protection or monitoring constituent standard (See Appendix A.) 


\section{WELL HSB108D}

\begin{tabular}{|c|c|}
\hline SRS Coord. & Lat/Longitude \\
\hline $\begin{array}{l}\text { N71688.0 } \\
\text { E57145.6 }\end{array}$ & $\begin{array}{l}33.2775 \\
81.6555\end{array}$ \\
\hline
\end{tabular}

SAMPLE DATE

FIELD DATA

Constituents

Water elevation

$\mathrm{pH}$

Sp. conductance

Water temperature

Alkalinity as $\mathrm{CaCO}_{3}$

Turbidity

Volumes ourged

Sampling code

Synchronous water level

\section{Screen Zone Elevation}

232.0-212.0 ft ms|

$07 / 14 / 98$

3Q98

224.2

4.8

76

20.0

0

$223.2(09 / 17 / 98)$
Top of Casing

$266.3 \mathrm{ft} \mathrm{msl}$

$10 / 13 / 98$

4098

222.8

4.9

19.4

0

5.8

$221.8(12 / 21 / 98)$
Casing Pump

4" PVC V

\section{Screen Zone}

Water Table (IIB2)

ANALYTICAL DATA

Inorganic Constituemts

Constituents

Antimony, total recoverable $<0.11$

Arsenic, total recoverable $<3.0$

Barium, total recoverable $\quad 15$

Cadmium, total recoverable 0.30

Chromium, total recoverable $<2.0$

Cobalt, total recoverable

Copper, total recoverable

Cyanide

1.8

Lead, total recoverable $\quad 3.7$

Mercury, total recoverable 2.8

Nickel, total recoverable $\quad 5.9$

Nitrate as nitrogen

Nitrate-nitrite as nitrogen $\quad 6,900$

Selenium, total recoverable $<5.0$

Silver, total recoverable $\quad<0.093$

Tin, total recoverable

$<0.43$

Vanadium, total recoverable $<2.0$

Zinc, total recoverable

48

\begin{tabular}{|c|c|}
\hline Mod & Filt. \\
\hline $\mathrm{U} N I$ & $<0.20$ \\
\hline $\mathrm{U} / /$ & $<3.0$ \\
\hline $\mathrm{J} / \mathrm{E}$ & NDD \\
\hline $\begin{array}{l}\mathrm{U} N / \\
\prime \prime\end{array}$ & $<3.0$ \\
\hline /I & \\
\hline $\begin{array}{l}\mathrm{U} / / \\
/ /\end{array}$ & $<10$ \\
\hline 11 & \\
\hline /I & \\
\hline /l & \\
\hline $\mathrm{U} / 1$ & $<5.0$ \\
\hline $\mathrm{US} / \mathrm{CV} /$ & $<1.0$ \\
\hline UNI & $<2.0$ \\
\hline $\begin{array}{l}\mathrm{U} / / \\
/ /\end{array}$ & $<2.0$ \\
\hline
\end{tabular}

ST

DF Lab 4Q98 Mod

Filt.

ST 브 DF Lab Unit

Organic Constituents

\begin{tabular}{ll} 
Constituents & 3098 \\
\hline & $<1.0$ \\
Benzene & $<9.9$ \\
Bis(2-ethylhexyl) phthalate & $<2.4$ \\
Dichloromethane & $<1.0$ \\
Tetrachloroethylene & $<1.0$ \\
Trichloroethylene & $<1.0$ \\
Trichlorofuoromethane &
\end{tabular}

$\begin{array}{ll}\text { Mod } & \text { Filt. } \\ \text { UJ/O/1 } & <1.0 \\ \text { U/I } & <9.9 \\ \text { UJNO8/1 } & <1.0 \\ \text { UJ/O/1 } & <1.0 \\ \text { UJ/O/1 } & <1.0 \\ \text { UJ/O/1 } & <1.0\end{array}$

Unit

ft msl

$\mathrm{pH}$

$\mu \mathrm{S} / \mathrm{cm}$

$\mathrm{mg} / \mathrm{L}$

NTU

well vol

ft msl
ST브 DF Lab $4 Q 98 \quad \underline{\text { Mod }}$

$\begin{array}{ll}\text { DF } & \text { Lab } \\ 1 & \text { GE } \\ 1 & \text { GE } \\ 1 & \text { GE } \\ 1 & \text { GE } \\ 1 & \text { GE }\end{array}$

GE
GE
GE
GE
GE
GE
GE
GE
GE
GE
GE
GE
GE
GE
GE
GE
GE

Lab
GE
GE
GE
GE
GE
GE

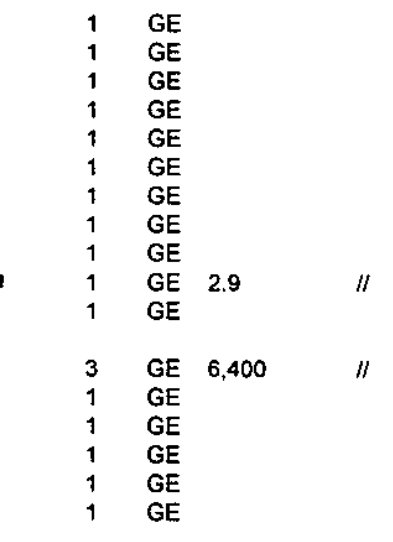

Filt.

ST 브 DF Lab Unit

$\mu g / L$

$\mu g / L$

$\mu g / L$

$\mu g / L$

HglL

$\mu \mathrm{g} / \mathrm{L}$

Notes:

- = exceeded holding time

- = exceeded groundwater protection or monitoring constituent standard (See Appendix A.) 
WELL HSB108D (cont.)

Radioactive Constituents

\begin{tabular}{|c|c|c|c|c|c|c|c|c|}
\hline Constituents & $\underline{3098}$ & Mod & Filt. & ST븝 & $\underline{D F}$ & $\underline{\text { Lab }}$ & 4Q98 & Mod \\
\hline Americium-241 & $<4.4 \mathrm{E}-03$ & $\mathrm{U} / 1 /$ & $<0.2950$ & & 1 & $G P$ & & \\
\hline Beta dose & 50.32 & & & - & & & & \\
\hline Carbon-14 & $2.5 E+01$ & $J / X I$ & NDD & & 1 & GP & & \\
\hline Cobalt-60 & $7.4 \mathrm{E}+00$ & "I & & & 1 & GP & & \\
\hline Curium-242 & $<0.0 \mathrm{E}+00$ & UI// & $<0.20$ & & 1 & GP & & \\
\hline Curium-243/244 & $<0.0 E+00$ & $\mathrm{U} 1 / /$ & $<0.1920$ & & 1 & GP & & \\
\hline Curium-245/246 & $<0.0 \mathrm{E}+00$ & $\mathrm{UI} / /$ & $<0.1920$ & & 1 & GP & & \\
\hline Gross alpha & $1.5 \mathrm{E}+01$ & "I & & ש & 1 & $G P$ & $1,2 \mathrm{E}+01$ & /I \\
\hline lodine-129 & $2.6 \mathrm{E}+00$ & /1 & & & 1 & $\mathrm{GP}$ & & \\
\hline Nickel-63 & $7.4 E+00$ & "I & & & & & & \\
\hline Nonvolatile beta & $9.2 E+02$ & /l & & - & 1 & GP & $9.9 \mathrm{E}+02$ & /I \\
\hline Plutonium-238 & $<0.0 E+00$ & $\mathrm{UI} / /$ & $<0.1850$ & & 1 & GP & & \\
\hline Plutonium-239/240 & $<-1.5 \mathrm{E}-02$ & $\mathrm{U} \mathbf{1} / 1$ & $<0.3250$ & & 1 & GP & & \\
\hline Radium-226 & $1.7 \mathrm{E}+01$ & 11 & & " & 1 & GP & & \\
\hline Radium-228 & $<-1.2 \mathrm{E}-02$ & $\mathrm{U} \mathrm{U} / /$ & $<1.3800$ & & 1 & GP & & \\
\hline Strontium-90 & $3.8 \mathrm{E}+02$ & /I & & च & 1 & GP & & \\
\hline Technetium-99 & $<-9.1 E+\infty 0$ & $\mathrm{U} \mid / /$ & $<22.3000$ & & 1 & GP & & \\
\hline Thorium-228 & $<5.2 \mathrm{E}-02$ & $U \mathbf{I} / /$ & $<0.1890$ & & 1 & GP & & \\
\hline Thorium-230 & $<5.3 \mathrm{E}-02$ & UII/ & $<0.0606$ & & 1 & GP & & \\
\hline Thorium-232 & $<-9.7 \mathrm{E}-03$ & UII/ & $<0.0737$ & & 1 & $G P$ & & \\
\hline Sum of alphas & $1.1 \mathrm{E}+00$ & & & & & & & \\
\hline Sum of betas & $4.2 E+02$ & & & - & & & & \\
\hline Total radium & $1.7 \mathrm{E}+01$ & & & & & & & \\
\hline Tritium & $2.3 E+02$ & "I & & - & 1 & GP & $3.7 E+02$ & "l \\
\hline Uranium-233/234 & $1.1 \mathrm{E}+00$ & 11 & & & 1 & $G P$ & & \\
\hline Uranium-235 & $<-4.5 \mathrm{E}-03$ & $\mathrm{UI} / / \mathrm{I}$ & $<0.0998$ & & 1 & GP & & \\
\hline Uranium-238 & $<3.8 \mathrm{E}-02$ & $\mathrm{UI} / /$ & $<0.0566$ & & 1 & GP & & \\
\hline
\end{tabular}

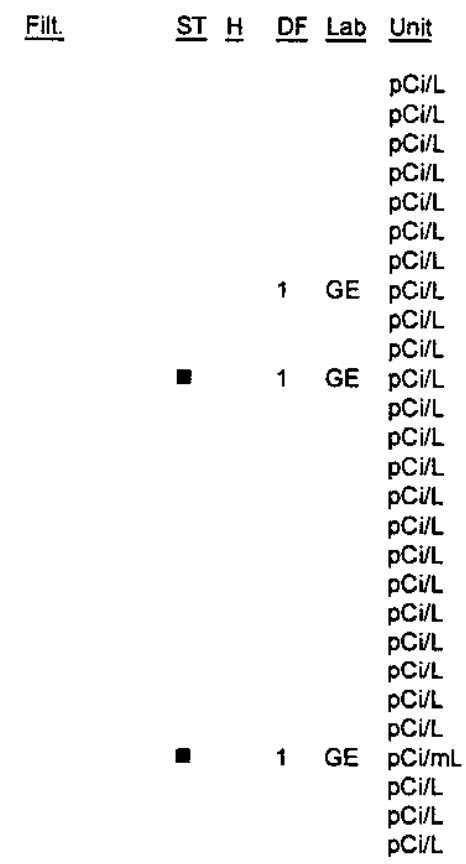

Notes:

- = exceeded holding time

- exceeded groundwater protection or monitoring constituent standard (See Appendix A.) 
WELL HSB109C

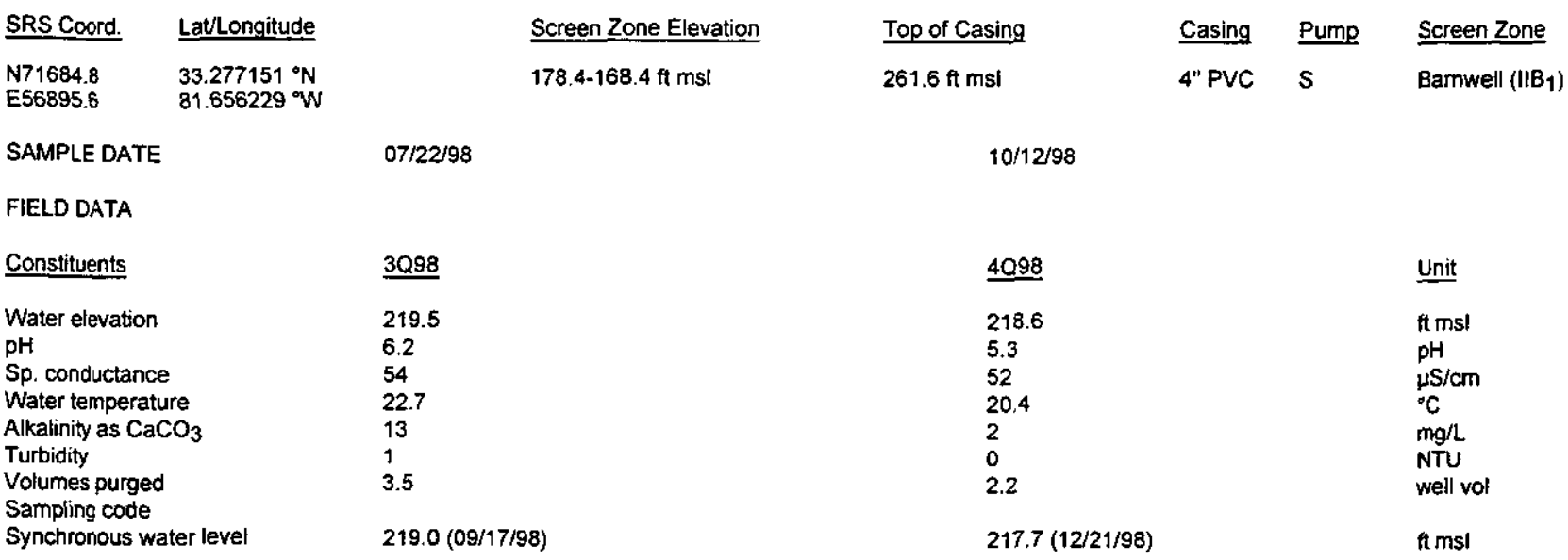

\section{ANALYTICAL DATA}

Inorganic Constituents

\begin{tabular}{|c|c|c|c|c|c|c|c|c|c|c|c|c|c|}
\hline Constituents & $\underline{3098}$ & Mod & Filt. & $\underline{\text { ST}} \underline{H}$ & $\underline{\mathrm{DF}}$ & $\underline{\text { Lab }}$ & 4098 & Mod & Filt. & $\underline{\text { ST }} \underline{H}$ & $\mathrm{DF}$ & Lab & $\underline{\text { Unit }}$ \\
\hline Antimony, total recoverable & $<0.20$ & $\mathrm{u} / /$ & $<0.20$ & & 1 & GE & & & & & & & ugh \\
\hline Arsenic, total recoverable & $<3.0$ & $\mathrm{U} / /$ & $<3.0$ & & 1 & GE & & & & & & & $\mu g / L$ \\
\hline Barium, total recoverable & 4.9 & "l & & & 1 & GE & & & & & & & $\mu g / L$ \\
\hline Cadmium, total recoverable & $<1.0$ & $\mathrm{u} / /$ & $<1.0$ & & 1 & GE & & & & & & & $\mu g / \mathrm{L}$ \\
\hline Chromium, total recoverable & 1.4 & $J / E J$ & NDD & & $i$ & GE & & & & & & & $\mathrm{\mu g} / \mathrm{L}$ \\
\hline Cobalt, total recoverable & $<0.20$ & $\mathrm{U} / 1$ & $<0.20$ & & 1 & $\mathrm{GE}$ & & & & & & & ug/L \\
\hline Copper, total recoverable & 2.4 & $\|$ & & & 1 & GE & & & & & & & 4o/L \\
\hline Cyanide & $<10$ & $\mathrm{U} / /$ & $<10$ & & 1 & GE & & & & & & & Hg/L \\
\hline Lead, total recoverable & 0.41 & $\mathrm{~J} / \mathrm{E} /$ & NDD & & $i$ & GE & & & & & & & $\mu g / L$ \\
\hline Mercury, total recoverable & $<0.20$ & $\mathrm{U} / 1 /$ & $<0.20$ & & $i$ & GE & $<0.20$ & $\mathrm{U} / /$ & $<0.20$ & & 1 & GE & $\mu g / \mathrm{h}$ \\
\hline $\begin{array}{l}\text { Nickel, total recoverable } \\
\text { Nitrate as nitrogen }\end{array}$ & 0.80 & "I & & & 1 & GE & & & & & & & $\mu g / 2$ \\
\hline Nitrate-nitrite as nitrogen & 1,800 & $N /$ & & & 1 & GE & 1900 & "l & & & 1 & GE & 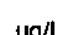 \\
\hline Selenium, total recoverable & $<5.0$ & $\mathrm{u} / /$ & $<5.0$ & & 1 & GE & & & & & & & $\mu g / \mathrm{L}$ \\
\hline Silver, total recoverable & $<1.0$ & $\mathrm{U} / 1$ & $<1.0$ & & 1 & GE & & & & & & & $\mu g / L$ \\
\hline Tin, total recoverable & $<2,0$ & $\mathrm{U} / 1$ & $<2.0$ & & 1 & GE & & & & & & & $\mu g / \mathrm{L}$ \\
\hline Vanadium, total recoverable & $<2.0$ & $\mathrm{U} / /$ & $<2.0$ & & 1 & $\mathrm{GE}$ & & & & & & & $\mathrm{Hg} / \mathrm{h}$ \\
\hline Zinc, total recoverable & $<5.0$ & $\mathrm{U} / /$ & $<5.0$ & & 1 & GE & & & & & & & $\mu g / \mathrm{L}$ \\
\hline \multicolumn{14}{|l|}{ Organic Constituents } \\
\hline Constituents & $\underline{3 Q 98}$ & $\underline{\text { Mod }}$ & Filt. & $\underline{\text { ST}} \underline{H}$ & $\underline{D F}$ & $\underline{\text { Lab }}$ & 4Q98 & Mod & Filt. & $\underline{S T} \underline{H}$ & DF & Lab & Unit \\
\hline Benzene & $<1.0$ & $U J / O / 1$ & $<1.0$ & & 1 & $\mathrm{GE}$ & & & & & & & Ho/ \\
\hline Bis(2-ethylhexyl) phthalate & $<11$ & $\mathrm{U} / /$ & $<11$ & & 1 & GE & & & & & & & $g / \mathrm{h}$ \\
\hline Dichloromethane & $<1.0$ & $\mathrm{UJ} / \mathrm{O} / 1$ & $<1.0$ & & 1 & GE & & & & & & & ug/L \\
\hline Tetrachloroethylene & $<1.0$ & UJ/O/1 & $<1.0$ & & 1 & GE & & & & & & & 4o/k \\
\hline Trichloroethylene & $<1.0$ & $\mathrm{UJ} / \mathrm{O} / 1$ & $<1.0$ & & 1 & GE & & & & & & & $\mu g / L$ \\
\hline Trichlorofluoromethane & $<1.0$ & $\mathrm{UJ} / \mathrm{O} / \mathrm{I}$ & $<1.0$ & & 1 & GE & & & & & & & L \\
\hline
\end{tabular}

Notes:

= exceeded holding time

= exceeded groundwater protection or monitoring constituent standard (See Appendix A.) 
WELL HSB109C (cont.)

Radioactive Constituents

\begin{tabular}{|c|c|c|c|c|c|c|c|c|c|c|c|c|c|}
\hline Constituents & $\underline{3 Q 98}$ & Mod & Filt. & $\underline{\text { ST }} \underline{H}$ & $\underline{\mathrm{DF}}$ & Lab & $\underline{4 Q 98}$ & Mod & Filt. & $\underline{\mathrm{ST}} \underline{\mathrm{H}}$ & $\underline{\mathrm{DF}}$ & $\underline{\text { Lab }}$ & Unit \\
\hline Americium-241 & $<3.8 E-03$ & $\mathrm{Ul} / /$ & $<0.1560$ & & 1 & GP & & & & & & & $\mathrm{pCi} / \mathrm{L}$ \\
\hline Carbon-14 & $<1.2 E-01$ & $\mathrm{U} \mid / I$ & $<8.6100$ & & 1 & GP & & & & & & & $\mathrm{pCi} / \mathrm{L}$ \\
\hline Cobalt-60 & $<-3.4 \mathrm{E}-02$ & $\mathrm{UI} / /$ & $<3.4800$ & & 1 & GP & & & & & & & $\mathrm{pCi} / \mathrm{L}$ \\
\hline Curium-242 & $<0.0 E+\infty 0$ & $\mathrm{U} 1 / /$ & $<0.0793$ & & 1 & GP & & & & & & & $\mathrm{pCi} / \mathrm{L}$ \\
\hline Curium-243/244 & $<5.0 \mathrm{E}-02$ & $\mathrm{U} \mid / /$ & $<0.0754$ & & 1 & GP & & & & & & & $\mathrm{pCi} / \mathrm{h}$ \\
\hline Curium-245/246 & $<5.0 \mathrm{E}-02$ & $\mathrm{UI} / /$ & $<0.0753$ & & 1 & GP & & & & & & & $\mathrm{pCi} / \mathrm{L}$ \\
\hline Gross alpha & $<2.2 \mathrm{E}-01$ & $\mathrm{UI} / /$ & $<0.3720$ & & 1 & GP & $<5.3 E-01$ & $\mathrm{U} / /$ & $<6.4 E-01$ & & 1 & GE & $\mathrm{pCi} / \mathrm{L}$ \\
\hline lodine-129 & $<8,1 E-01$ & $\mathrm{U} \mathrm{I} / /$ & $<0.8900$ & & 1 & GP & & & & & & & $\mathrm{pCi} / \mathrm{L}$ \\
\hline Nickel-63 & $<-3.4 \mathrm{E}-02$ & U1 & $<3.4800$ & & & & & & & & & & $\mathrm{pCi} / \mathrm{L}$ \\
\hline Nonvolatile beta & $<9.9 E-01$ & $\mathrm{UH} / /$ & $<1.0500$ & & 1 & GP & $6.2 E+00$ & // & & & 1 & GE & $\mathrm{pCi} / \mathrm{L}$ \\
\hline Plutonium-238 & $<2.3 \mathrm{E}-02$ & $\mathrm{U} \mathrm{l} / /$ & $<0.0706$ & & 1 & GP & & & & & & & $\mathrm{pCi} / \mathrm{L}$ \\
\hline Plutonium-239/240 & $<1.6 E-02$ & $\mathrm{U} \mathrm{H} / /$ & $<0.0934$ & & 1 & GP & & & & & & & $\mathrm{pCi} / \mathrm{L}$ \\
\hline Radium-226 & $<2.6 E-01$ & $\mathrm{UI} / /$ & $<0.3830$ & & 1 & GP & & & & & & & $\mathrm{pCi} / \mathrm{L}$ \\
\hline Radium-228 & $<4.7 E-01$ & $\mathrm{UI} / /$ & $<1.1100$ & & 1 & GP & & & & & & & $\mathrm{pCi} / \mathrm{L}$ \\
\hline Strontium-90 & $<-1.3 E-01$ & $\mathrm{UI} / /$ & $<1.6300$ & & 1 & GP & & & & & & & $\mathrm{pCi} / \mathrm{L}$ \\
\hline Technetium-99 & $<-1,5 E+01$ & $\mathrm{UI} / /$ & $<24.2000$ & & 1 & GP & & & & & & & $\mathrm{pCi} / \mathrm{L}$ \\
\hline Thorium-228 & $<1.7 E-01$ & $\mathrm{UI} / /$ & $<0.2320$ & & $\uparrow$ & GP & & & & & & & $\mathrm{pCi} / \mathrm{L}$ \\
\hline Thorium-230 & $<1.7 \mathrm{E}-01$ & $\mathrm{UI} / /$ & $<0.1920$ & & 1 & GP & & & & & & & $\mathrm{pCi} / \mathrm{L}$ \\
\hline Thorium-232 & $<-8.8 E-03$ & $\mathrm{UI} / /$ & $<0.1920$ & & 1 & GP & & & & & & & $\mathrm{pCi} / \mathrm{L}$ \\
\hline $\begin{array}{l}\text { Sum of alphas } \\
\text { Sum of betas }\end{array}$ & & & & & & & & & & & & & \\
\hline Tritium & 4.1E+01 & $\|$ & & $\mathbf{0}$ & 1 & GP & $4.9 E+01$ & $/ /$ & & $\mathbf{n}$ & 1 & GE & $\mathrm{pCi} / \mathrm{mL}$ \\
\hline Uranium-233/234 & $<2.3 E-01$ & $\mathrm{UI} / /$ & $<0.1690$ & & 1 & GP & & & & & & & $\mathrm{pCi} / \mathrm{L}$ \\
\hline Uranium-235 & $<4.3 E-02$ & $\mathrm{UI} / /$ & $<0.2980$ & & 1 & GP & & & & & & & $\mathrm{pCi} / \mathrm{L}$ \\
\hline Uranium-238 & $<1.7 E-01$ & $\mathrm{UU} / /$ & $<0.1690$ & & 1 & GP & & & & & & & $\mathrm{pCi} / \mathrm{L}$ \\
\hline
\end{tabular}

Notes:

- = exceeded holding time

- exceeded groundwater protection or monitoring constituent standard (See Appendix A.) 


\section{WELL HSB109D}

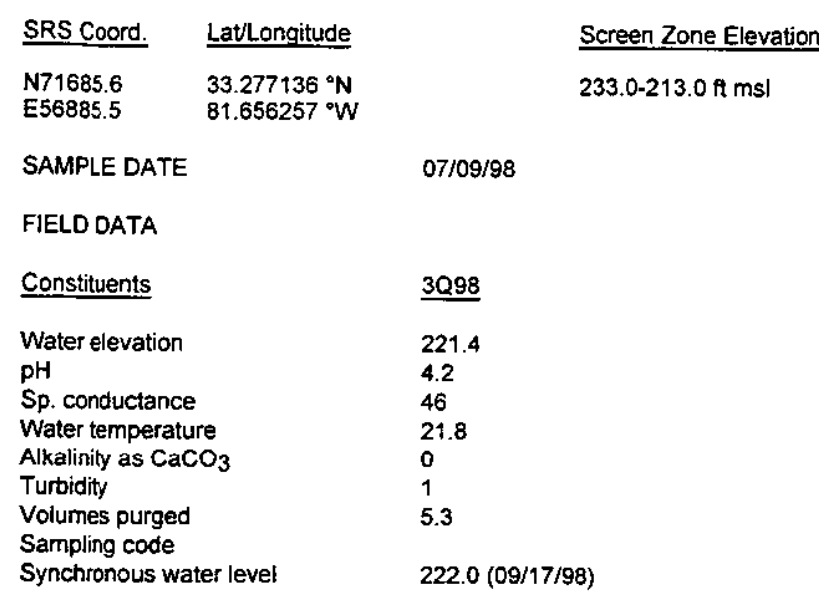

\begin{tabular}{|c|c|c|c|}
\hline Top of Casing & Casing & Pump & Screen Zone \\
\hline $261.2 \mathrm{ft} \mathrm{msl}$ & 4" PVC & $s$ & Water Table (IIB2) \\
\hline $10 / 12 / 98$ & & & \\
\hline 4098 & & & Unit \\
\hline 221.7 & & & $\mathrm{ft} \mathrm{ms}]$ \\
\hline 4.4 & & & $\mathrm{pH}$ \\
\hline 40 & & & $\mu \mathrm{S} / \mathrm{cm}$ \\
\hline 23.2 & & & ${ }^{\circ} \mathrm{C}$ \\
\hline 0 & & & $\mathrm{mg} / \mathrm{L}$ \\
\hline 0 & & & NTU \\
\hline 2.5 & & & well vol \\
\hline$(12 / 21 / 98)$ & & & $\mathrm{ft} \mathrm{msl}$ \\
\hline
\end{tabular}

ANALYTICAL DATA

Inorganic Constituents

\begin{tabular}{|c|c|c|c|c|c|c|c|c|c|c|c|c|c|}
\hline Constituents & $\underline{3098}$ & Mod & Filt. & $\underline{\text { ST }} \underline{\mathrm{H}}$ & $\underline{\mathrm{DF}}$ & Lab & $\underline{4 Q 98}$ & Mod & Filt. & $\underline{S T} \underline{H}$ & $\underline{\mathrm{DF}}$ & $\underline{\operatorname{Lab}}$ & Unit \\
\hline Antimony, total recoverable & $<0.20$ & $\mathrm{U} / /$ & $<0.20$ & & 1 & GE & & & & & & & $\mu g / L$ \\
\hline Arsenic, total recoverable & $<3.0$ & $\mathrm{U} / /$ & $<3.0$ & & 1 & GE & & & & & & & $\mu g / L$ \\
\hline Barium, total recoverable & 14 & $\|$ & & & 1 & GE & & & & & & & $\mu g / L$ \\
\hline Cadmium, total recoverable & $<1.0$ & $\mathrm{U} / /$ & $<1.0$ & & 1 & $G E$ & & & & & & & $\mu g / L$ \\
\hline Chromium, total recoverable & $<1.0$ & UNI & $<3.0$ & & 1 & GE & & & & & & & $\mu g / L$ \\
\hline Cobalt, total recoverable & 1.3 & 11 & & & 1 & GE & & & & & & & $\mu g / L$ \\
\hline Copper, total recoverable & 3.5 & $\|$ & & & 1 & $\overline{G E}$ & & & & & & & $\mu g / \mathrm{L}$ \\
\hline Cyanide & $<10$ & $\mathrm{U} / /$ & $<10$ & & 1 & GE & & & & & & & $\mu g / L$ \\
\hline Lead, total recoverable & 1.4 & $\mathrm{~J} / \mathrm{E} /$ & NDD & & 1 & $\overline{G E}$ & & & & & & & $\mu g / L$ \\
\hline Mercury, total recoverable & 0.64 & $\|$ & & & 1 & GE & 0.73 & $\|$ & & & 1 & GE & $\mu g / L$ \\
\hline $\begin{array}{l}\text { Nickel, total recoverable } \\
\text { Nitrate as nitrogen }\end{array}$ & 3.3 & $N /$ & & & 1 & GE & & & & & & & \\
\hline Nitrate-nitrite as nitrogen & 2,800 & $N /$ & & & 1 & GE & 2,800 & $\|$ & & & 2 & GE & طالورا \\
\hline Selenium, total recoverable & $<5.0$ & $\mathrm{U} / /$ & $<5.0$ & & 1 & GE & & & & & & & L \\
\hline Silver, total recoverable & $<1.0$ & $\mathrm{U} / \mathrm{I}$ & $<1.0$ & & 1 & GE & & & & & & & $g / L$ \\
\hline Tin, total recoverable & $<0.22$ & UNI & $<2.0$ & & 1 & GE & & & & & & & $\mu \mathrm{g} / \mathrm{L}$ \\
\hline Vanadium, total recoverable & $<2.0$ & $\mathrm{U} / /$ & $<2.0$ & & 1 & GE & & & & & & & $\mathrm{g} / \mathrm{L}$ \\
\hline Zinc, total recoverable & 13 & $/ 1$ & & & 1 & $\overline{G E}$ & & & & & & & \\
\hline \multicolumn{14}{|l|}{ Organic Constituents } \\
\hline Constituents & $\underline{3 Q 98}$ & Mod & Filt. & ST브 & $\underline{D F}$ & $\underline{\text { Lab }}$ & 4Q98 & Mod & Filt. & $\underline{\text { ST }} \underline{H}$ & $\underline{\mathrm{DF}}$ & $\underline{L a b}$ & Jnit \\
\hline Benzene & $<1.0$ & $\mathrm{U} / /$ & $<1.0$ & & 1 & GE & & & & & & & \\
\hline Bis(2-ethylhexyl) phthalate & $<9.8$ & $\mathrm{U} / /$ & $<9.8$ & & 1 & GE & & & & & & & $g / L$ \\
\hline Dichloromethane & $<1.0$ & $\mathrm{U} / /$ & $<1.0$ & & 1 & GE & & & & & & & $g / L$ \\
\hline Tetraciloroethylene & $<1.0$ & $\mathrm{U} / /$ & $<1.0$ & & 1 & GE & & & & & & & 几 \\
\hline Trichloroethylene & $<1.0$ & $\mathrm{U} / I$ & $<1.0$ & & 1 & $\mathrm{GE}$ & & & & & & & $g / L$ \\
\hline Trichlorofluoromethane & $<1.0$ & $\mathrm{U} / /$ & $<1.0$ & & 1 & GE & & & & & & & \\
\hline
\end{tabular}

Notes:

- exceeded holding time

- exceeded groundwater protection or monitoring constituent standard (See Appendix A.) 
WELL HSB109D (cont.)

Radioactive Constituents

Constituents
Americium-241
Carbon-14
Cobalt-60
Curium-242
Curium-243/244
Curium-245/246
Gross alpha
lodine-129
Nickel-63
Nonvolatile beta
Plutonium-238
Plutonium-239/240
Radium-226
Radium-228
Strontium-90
Technetium-99
Thorium-228
Thorium-230
Thorium-232
Sum of alphas
Sum of betas
Total radium
Tritium
Uranium-233/234
Uranium-235
Uranium-238

\begin{tabular}{|c|c|}
\hline 3098 & 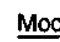 \\
\hline$<4.9 E-03$ & \\
\hline$<7.0 E+00$ & \\
\hline$<2.3 E+00$ & \\
\hline$<3.3 E-02$ & \\
\hline$<9.2 E-03$ & \\
\hline$<-4.2 \mathrm{E}-03$ & \\
\hline $5.1 E+00$ & \\
\hline$<4.7 \mathrm{E}-01$ & \\
\hline$<2.3 \mathrm{E}+00$ & \\
\hline $1.9 E+02$ & /I \\
\hline$<0.0 E+00$ & \\
\hline$<1.1 E-02$ & 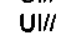 \\
\hline $2.9 E+00$ & \\
\hline$<1.0 \mathrm{E}+00$ & \\
\hline $9.5 E+01$ & \\
\hline$<-1.0 E+01$ & \\
\hline$<1.7 E-01$ & 0 \\
\hline$<3.6 \mathrm{E}-02$ & \\
\hline$<1.9 E-02$ & \\
\hline $1.4 E+00$ & \\
\hline $9.5 \mathrm{E}+01$ & \\
\hline $2.9 E+00$ & \\
\hline $3.1 E+01$ & $/ /$ \\
\hline $8.4 \mathrm{E}-01$ & $H$ \\
\hline 3.0 E-01 & /I \\
\hline 2.4E-01 & $\|$ \\
\hline
\end{tabular}

Filt.

$<0.1430$

$<9.4500$

$<2.7600$

$<0.0994$

$<0.1100$

$<0.0930$

$<1.2200$

$<2.7600$

$<0.0449$

$<0.0788$

$<1.1700$

NDD

$<20.9000$

$<0.4400$

$<0.1970$

$<0.0560$
STH DF Lab 4Q998 Mod

$G P$

GP

GP

$G P$

GP

$G P$

$\begin{array}{ll}1 & G P \\ 1 & G P\end{array}$

- 1 GP 1.2E+01 //

$<1.2 \mathrm{E}+00 \quad \mathrm{U} / 1$

GP

GP

GP

GP

GP

GP

GP

GP

GP

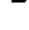

D

$\begin{array}{lll}1 & \text { GP } & 6.7 E+01 \quad / / \\ 1 & \text { GP } & \\ 1 & \text { GP } & \\ 1 & \text { GP } & \end{array}$

Filt.

ST $\underline{H}$ DF Lab Unit

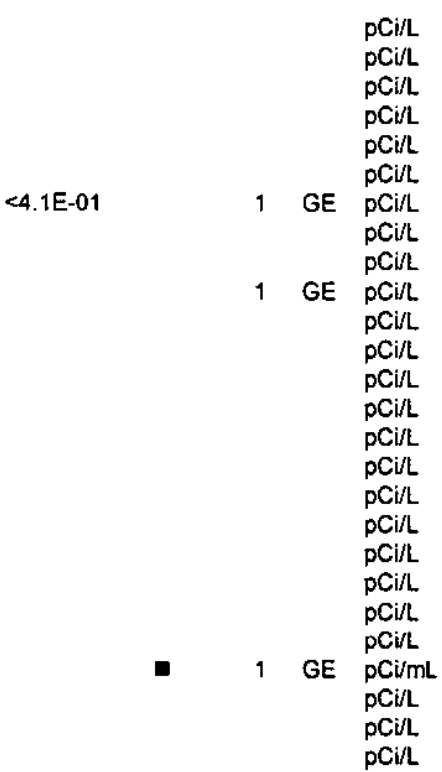

Notes:

- exceeded holding time

= exceeded groundwater protection or monitoring constituent standard (See Appendix A.) 


\section{WELL HSB110C}

\begin{tabular}{|c|c|}
\hline SRS Coord. & Lat/Longitude \\
\hline $\begin{array}{l}\text { N71779.3 } \\
\text { E56680.7 }\end{array}$ & $\begin{array}{l}33.277009^{\circ} \mathrm{N} \\
81.656979^{\circ} \mathrm{W}\end{array}$ \\
\hline
\end{tabular}

SAMPLE DATE

$07 / 23 / 98$

\section{Screen Zone Elevation}

$181.4-171.4 \mathrm{ft} \mathrm{msl}$

FIELD DATA

Constituents

Water elevation

$\mathrm{pH}$

Sp. conductance

Water temperature

Alkalinity as $\mathrm{CaCO}_{3}$

Turbidity

Volumes purged

Sampling code

Synchronous water level

ANALYTICAL DATA

Inorganic Constituents
Constituents

Antimony, total recoverable

Arsenic, total recoverable

Barium, total recoverable

Cadmium, total recoverable $<1.0$

Chromium, total recoverable

Cobalt, total recoverable $\quad 0.12$

Copper, total recoverable $\quad 5.6$

Cyanide

Lead, total recoverable

Mercury, total recoverable

Nickel, total recoverable

Nitrate as nitrogen

Nitrate-nitrite as nitrogen $\quad \mathbf{5 3 0}$

Selenium, total recoverable

Silver, total recoverable

Tin, total recoverable

Zinc, total recoverable

30.98
$<0.20$
$<3.0$
5.3
$<1.0$
2.3
0.12
5.6
$<10$
3.3
$<0.20$
1.2

530
$<5.0$
$<1.0$
$<2.0$
$<2.0$
8.8

Organic Constituents

Constituents

Benzene

Bis(2-ethylhexyl) phthalate

Dichloromethane

Tetrachloroethylene

Trichloroethylene

Trichlorofluoromethane
3098

$<1.0$

32

$<1.5$

$<10$

$<1.0$

$<1.0$
3098

219.8

5.6

26

20.8

2

$219.2(09 / 17 / 98)$
Top of Casing

255.7 ft msl

$10 / 12 / 98$

4Q98

218.8

6.8

20.4

1

2
0.064
$X N$

XN

$217.9(12 / 21 / 98)$
Casing Pump

4" PVC S
Screen Zone

Barnwell (IIB 1 )
Unit

ft msl

$\mathrm{pH}$

$\mu \mathrm{S} / \mathrm{cm}$

${ }^{\circ} \mathrm{C}$

$\mathrm{mg} / \mathrm{L}$

NTU

well vol

ft msl

\begin{tabular}{|c|c|c|c|c|c|c|c|c|c|c|c|}
\hline Mod & Filt. & $\underline{\text { ST}} \underline{\underline{H}}$ & DF & Lab & 4Q98 & Mod & Filt. & ST 브 & DF & $\underline{\text { Lab }}$ & Unit \\
\hline $\mathrm{U} / \mathrm{I}$ & $<0.20$ & & 1 & GE & & & & & & & $\mu g / L$ \\
\hline $\mathrm{U} / /$ & $<3.0$ & & 1 & $\overline{G E}$ & & & & & & & $\mu \mathrm{g} / \mathrm{L}$ \\
\hline /I & & & 1 & GE & & & & & & & $\mu g / L$ \\
\hline $\mathrm{U} / /$ & $<1.0$ & & 1 & GE & & & & & & & $\mu g / L$ \\
\hline $\mathrm{J} / \mathrm{E}$ & NDD & & 1 & GE & & & & & & & $\mu \mathrm{g} / \mathrm{L}$ \\
\hline $\mathrm{J} / \mathrm{E} /$ & NDD & & 1 & GE & & & & & & & $\mu g / L$ \\
\hline II & & & 1 & GE & & & & & & & L \\
\hline $\mathrm{U} / /$ & $<10$ & & 1 & GE & & & & & & & $\mathrm{\mu g} / \mathrm{L}$ \\
\hline$/ 1$ & & & 1 & GE & & & & & & & $\mu g / L$ \\
\hline $\mathrm{U} / \mathrm{I}$ & $<0.20$ & & 1 & GE & $<0.20$ & $\mathrm{U} / /$ & $<0.20$ & & 1 & GE & $\mu \mathrm{g} / \mathrm{h}$ \\
\hline$/ /$ & & & 1 & GE & & & & & & & $\mu \mathrm{g} / \mathrm{h}$ \\
\hline $\mathrm{J} / \mathrm{IV} / 1$ & NDD & & 1 & GE & 530 & $\|$ & & & 1 & GE & $\mu g / L$ \\
\hline $\mathbf{U} / /$ & $<5.0$ & & 1 & GE & & & & & & & $\mu g / L$ \\
\hline UII & $<1.0$ & & 1 & GE & & & & & & & $\mu g / L$ \\
\hline $\mathbf{U} / /$ & $<2.0$ & & 1 & GE & & & & & & & $\mu \mathrm{g} / \mathrm{h}$ \\
\hline $\mathbf{U} / /$ & $<2.0$ & & 1 & GE & & & & & & & $\mu \mathrm{g} / \mathrm{L}$ \\
\hline 11 & & & 1 & GE & & & & & & & Lالود \\
\hline
\end{tabular}

Notes:

- exceeded holding time

E = exceeded groundwater protection or monitoring constituent standard (See Appendix A.)

$\mu \mathrm{g} / \mathrm{L}$

$\mu g / L$

$\mu g / L$

$\mu g / L$

$\mu g / L$

$\mu g / L$ 
WELL HSB110C (cont.)

Radioactive Constituents

\begin{tabular}{|c|c|c|c|c|c|c|c|c|c|c|c|c|c|}
\hline Constituents & 3Q98 & Mod & Filit. & ST브 & $\mathrm{DF}$ & $\underline{\text { Lab }}$ & $4 Q 98$ & Mod & Filt. & $\underline{\text { ST }} \underline{H}$ & $\underline{\text { DF }}$ & Lap & Unit \\
\hline Americium-241 & $<5.9 \mathrm{E}-02$ & $\mathrm{UI} / /$ & $<0.0680$ & & 1 & GP & & & & & & & $\mathrm{pCi} / \mathrm{L}$ \\
\hline Carbon-14 & $<3.0 E+00$ & $\mathrm{UI} / /$ & $<8.4900$ & & 1 & GP & & & & & & & $\mathrm{pCi} / \mathrm{L}$ \\
\hline Cobalt -60 & $<7.8 \mathrm{E}-01$ & $U ! / /$ & $<3.6000$ & & 1 & GP & & & & & & & $\mathrm{pCi} / \mathrm{L}$ \\
\hline Curium-242 & $<0.0 \mathrm{E}+00$ & $\mathrm{UI} / /$ & $<0.0713$ & & 1 & $\mathrm{GP}$ & & & & & & & $\mathrm{pCi} / \mathrm{L}$ \\
\hline Curium-243/244 & $<5.7$ E-02 & $\mathrm{UI} / /$ & $<0.1410$ & & 1 & GP & & & & & & & $\mathrm{pCi} / \mathrm{L}$ \\
\hline Curium-245/246 & $<0.0 E+00$ & $\mathrm{UI} / I$ & $<0.0680$ & & 1 & $G P$ & & & & & & & $\mathrm{pCi} / \mathrm{L}$ \\
\hline Gross alpha & $<3.3 E-01$ & UI/I & $<0.4320$ & & 1 & GP & $<1.5 E-01$ & $\mathrm{U} / /$ & $<5.3 E-01$ & & 1 & GE & $\mathrm{pCi} / \mathrm{L}$ \\
\hline lodine-129 & $<1.9 E-01$ & $\mathrm{UH} / /$ & $<0.5930$ & & 1 & GP & & & & & & & $\mathrm{pCi} / \mathrm{L}$ \\
\hline Nickel-63 & $<7.8 E-01$ & UI & $<3.6000$ & & & & & & & & & & $\mathrm{pCi} / \mathrm{L}$ \\
\hline Nonvolatile beta & $<4.4 E-01$ & U1/I & $<0.9690$ & & 1 & GP & $<1.2 \mathrm{E}+00$ & $\mathbf{U} / \prime$ & $<1.0 E+00$ & & 1 & $\mathrm{GE}$ & $\mathrm{pCi} / \mathrm{L}$ \\
\hline Plutonium-238 & $<-4.5 \mathrm{E}-02$ & $\mathrm{UI} / /$ & $<0.2170$ & & 1 & GP & & & & & & & $\mathrm{pCi} / \mathrm{L}$ \\
\hline Plutonium-239/240 & $<8.9 E-02$ & $\mathrm{UI} / /$ & $<0.3250$ & & 1 & GP & & & & & & & $\mathrm{pCi} / \mathrm{L}$ \\
\hline Radium-226 & $<7.8 \mathrm{E}-01$ & $\mathrm{U} H / /$ & $<0.8050$ & & 1 & GP & & & & & & & $\mathrm{pCi} / \mathrm{L}$ \\
\hline Radium-228 & $<6.9 E-01$ & $\mathrm{U} \mid / /$ & $<1.3000$ & & 1 & GP & & & & & & & $\mathrm{pCi} / \mathrm{L}$ \\
\hline Strontium-90 & $<3.5 E-02$ & $\mathrm{U} / \mathrm{J} / \mathrm{C} /$ & & & 1 & GP & & & & & & & $\mathrm{pCi} / \mathrm{L}$ \\
\hline Technetium-99 & $<-2.1 E+\infty 0$ & $\mathrm{UI} / /$ & $<22.0$ & & 1 & $\mathrm{GP}$ & & & & & & & $\mathrm{pCi} / \mathrm{L}$ \\
\hline Thorium-228 & $<1.6 \mathrm{E}-02$ & $\mathrm{UI} / /$ & $<0.1860$ & & 1 & GP & & & & & & & $\mathrm{pCi} / \mathrm{L}$ \\
\hline Thorium-230 & $<1.6 \mathrm{E}-01$ & $\mathrm{UI} / /$ & $<0.2510$ & & 1 & GP & & & & & & & $\mathrm{pCi} / \mathrm{L}$ \\
\hline Thorium-232 & $<-2.8 E-02$ & $\mathrm{UU} / /$ & $<0.2210$ & & 1 & GP & & & & & & & $\mathrm{pCil}$ \\
\hline $\begin{array}{l}\text { Sum of alphas } \\
\text { Sum of betas }\end{array}$ & & & & & & & & & & & & & \\
\hline Tritium & $7.1 \mathrm{E}+00$ & $/ /$ & & & 1 & GP & 1.1E+01 & $/ /$ & & & $t$ & $\mathrm{GE}$ & $\mathrm{pCi} / \mathrm{mL}$ \\
\hline Uranium-233/234 & $<2.0 E-03$ & $\mathrm{UI} / /$ & $<0.3760$ & & 1 & GP & & & & & & & $\mathrm{pCi} / \mathrm{L}$ \\
\hline Uranium-235 & $<-2.4 \mathrm{E}-02$ & $\mathrm{UH} / \mathrm{I}$ & $<0.3100$ & & 1 & GP & & & & & & & $\mathrm{pCi} / \mathrm{h}$ \\
\hline Uranium-238 & $<5.0 \mathrm{E}-02$ & $\mathrm{U} / /$ & $<0.1490$ & & 1 & GP & & & & & & & $\mathrm{pCi} / \mathrm{L}$ \\
\hline
\end{tabular}

Notes: 
WELL HSB110D

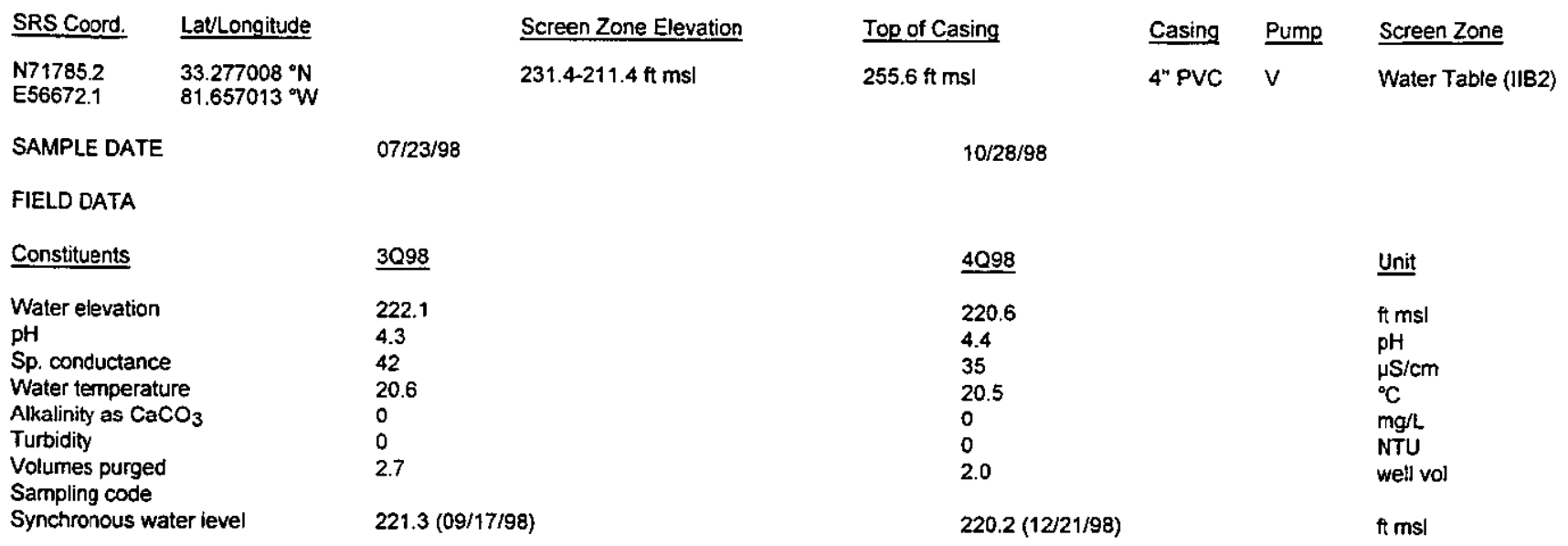

\section{ANALYTICAL DATA}

Inorganic Constituents

\begin{tabular}{|c|c|c|c|c|c|c|c|c|c|c|c|c|c|}
\hline Constituents & 3098 & Mod & Filt. & SIㅗㅂ & $\underline{D F}$ & $\underline{\text { Lab }}$ & $\underline{4 Q 98}$ & Mod & Filt. & $\underline{\mathrm{ST}} \underline{\mathrm{H}}$ & $\underline{\mathrm{DF}}$ & $\underline{L a b}$ & $\underline{\text { Unit }}$ \\
\hline Antimony, total recoverable & $<0.20$ & $\mathrm{U} / \prime$ & $<0.20$ & & 1 & GE & & & & & & & $\mu g / L$ \\
\hline Arsenic, total recoverable & $<3.0$ & $\mathrm{U} / /$ & $<3.0$ & & 1 & GE & & & & & & & $\mu g / L$ \\
\hline Barium, total recoverable & 3.9 & $\|$ & & & 1 & GE & & & & & & & $\mu g / L$ \\
\hline Cadmium, total recoverable & $<1.0$ & $\mathrm{U} / /$ & $<1.0$ & & 1 & GE & & & & & & & $\mu g / L$ \\
\hline Chromium, total recoverable & 1.2 & $\mathrm{~J} / \mathrm{E}$ & NDD & & 1 & GE & & & & & & & $\mu g / \mathrm{L}$ \\
\hline Cobalt, total recoverable & 2.9 & $\|$ & & & 1 & GE & & & & & & & $\mu g / L$ \\
\hline Copper, total recoverable & 2.0 & /I & & & 1 & GE & & & & & & & $\mu g / L$ \\
\hline Cyanide & $<10$ & $\mathrm{U} / /$ & $<10$ & & 1 & GE & & & & & & & $\mu g / L$ \\
\hline Lead, total recoverable & $<2.0$ & $\mathrm{U} / /$ & $<2.0$ & & 1 & GE & & & & & & & $\mu g / L$ \\
\hline Mercury, total recoverable & $<0.20$ & $U / I$ & $<0.20$ & & 1 & $\mathrm{GE}$ & $<0.20$ & $\mathrm{U} / /$ & $<0.20$ & & 1 & GE & $\mu g / L$ \\
\hline $\begin{array}{l}\text { Nickel, total recoverable } \\
\text { Nitrate as nitrogen }\end{array}$ & 1.6 & $/ /$ & & & 1 & GE & & & & & & & $\mu g / L$ \\
\hline Nitrate-nitrite as nitrogen & 1,500 & $\mathrm{~J} / \mathrm{V} / 1$ & NDD & & 1 & GE & 1,400 & $/ /$ & & & 1 & GE & $\mu g / L$ \\
\hline Selenium, total recoverable & $<5.0$ & $\mathrm{U} / /$ & $<5.0$ & & 1 & GE & & & & & & & $\mu \mathrm{g} / \mathrm{L}$ \\
\hline Silver, total recoverable & $<1.0$ & $\mathrm{U} / /$ & $<1.0$ & & 1 & GE & & & & & & & $\mu g / L$ \\
\hline Tin, total recoverable & $<2.0$ & $\mathrm{U} / /$ & $<2.0$ & & 1 & GE & & & & & & & $\mu g / L$ \\
\hline Vanadium, total recoverable & $<2.0$ & $\mathrm{U} / /$ & $<2.0$ & & 1 & GE & & & & & & & $\mu g / L$ \\
\hline Zinc, total recoverable & $<5.0$ & $\mathrm{U} / /$ & $<5.0$ & & 1 & GE & & & & & & & $\mu$ L \\
\hline \multicolumn{14}{|l|}{ Organic Constituents } \\
\hline Constituents & 3Q98 & Mod & Filt. & ST브 & $\underline{D F}$ & $\underline{\text { Lab }}$ & 4098 & Mod & Filt. & $\underline{\text { ST }} \underline{\underline{H}}$ & $\underline{\text { DF }}$ & $\underline{\text { Lab }}$ & $\underline{\text { Unit }}$ \\
\hline Benzene & $<1.0$ & $\mathrm{UJ} / \mathrm{O} / 1$ & $<1.0$ & & 1 & GE & & & & & & & $\mu \mathrm{g} / \mathrm{L}$ \\
\hline Bis (2-ethylhexyl) phthaiate & $<10$ & $\mathrm{U} / l$ & $<10$ & & 1 & GE & & & & & & & $\mu \mathrm{g} / \mathrm{L}$ \\
\hline Dichloromethane & $<1.6$ & UJNO8/1 & $<1.0$ & & 1 & GE & & & & & & & $\mu g / \mathrm{L}$ \\
\hline Tetrachloroethylene & $<1.0$ & $\mathrm{UJ} / \mathrm{O} / \mathrm{1}$ & $<1.0$ & & 1 & GE & & & & & & & L \\
\hline Trichloroethylene & $<1.0$ & $\mathrm{UJ} / \mathrm{O} / \mathrm{A}$ & $<1.0$ & & 1 & GE & & & & & & & $\mu g / L$ \\
\hline Trichlorofluoromethane & $<1.0$ & $\mathrm{UJ} / \mathrm{O} / 1$ & $<1.0$ & & 1 & GE & & & & & & & II \\
\hline
\end{tabular}

Notes:

- = exceeded holding time

w = exceeded groundwater protection or monitoring constituent standard (See Appendix A.) 
WELL HSB110D (cont.)

Radioactive Constituents

\begin{tabular}{|c|c|c|c|c|c|c|c|c|c|c|c|c|c|}
\hline Constituents & $3 Q 98$ & Mod & Filt. & ST브 & DF & $\underline{\text { Lab }}$ & $4 Q 98$ & Mod & Filt. & $\underline{\mathrm{ST}} \underline{\mathrm{H}}$ & DF & Lab & Unit \\
\hline Americium-241 & $<-3.1 E-03$ & $\mathrm{UI} / /$ & $<0.4200$ & & 1 & GP & & & & & & & $p C i / L$ \\
\hline Carbon-14 & $<1.4 E+00$ & $\mathrm{U} \mathrm{l} / /$ & $<8.7600$ & & 1 & GP & & & & & & & $\mathrm{pCi} / \mathrm{L}$ \\
\hline Cobatt-60 & $<6.8 \mathrm{E}-01$ & $\mathrm{UI} / 1$ & $<2.8700$ & & 1 & GP & & & & & & & $\mathrm{pCi} / \mathrm{L}$ \\
\hline Curium-242 & $<1.9 \mathrm{E}-01$ & $\mathrm{UV} / /$ & $<0.3590$ & & 1 & GP & & & & & & & $\mathrm{pCi} / \mathrm{L}$ \\
\hline Curium-243/244 & $<4.6 \mathrm{E}-01$ & $\mathbf{U I} / 1$ & $<0.6190$ & & 1 & GP & & & & & & & $\mathrm{pCi} / \mathrm{L}$ \\
\hline Curium-245/246 & $<6.0 \mathrm{E}-02$ & $\mathrm{UI} / /$ & $<0.1800$ & & 1 & GP & & & & & & & $\mathrm{pCi} / \mathrm{L}$ \\
\hline Gross alpha & $1.5 \mathrm{E}+00$ & $\mathrm{~J} / \mathrm{C} /$ & NDD & & 1 & GP & $<2.2 \mathrm{E}+00$ & $\mathrm{U} / 1$ & $<8.0 E-01$ & & 1 & GE & $\mathrm{pCi} / \mathrm{L}$ \\
\hline lodine-129 & $<5.1 \mathrm{E}-01$ & $\mathrm{UI} / /$ & $<0.8240$ & & 1 & GP & & & & & & & $\mathrm{pCi} / \mathrm{L}$ \\
\hline Nickel-63 & $<0.0 \mathrm{E}+00$ & $\mathrm{UI} / I$ & $<24.0000$ & & 1 & GP & & & & & & & $\mathrm{pCi} / \mathrm{L}$ \\
\hline Nonvolatile beta & $6.4 E+01$ & $/ 1$ & & $\mathbf{\square}$ & 1 & GP & $5.8 E+01$ & $\|$ & & 日 & 1 & GE & $\mathrm{pCi} / \mathrm{L}$ \\
\hline Plutonium-238 & $<-4.1 E-03$ & $\mathrm{UI} / 1$ & $<0.0861$ & & 1 & GP & & & & & & & $\mathrm{pCi} / \mathrm{L}$ \\
\hline Plutonium-239/240 & $<2,0 \mathrm{E}-02$ & $\mathrm{UI} / /$ & $<0.1140$ & & 1 & GP & & & & & & & $\mathrm{pCi} / \mathrm{L}$ \\
\hline Radium-226 & $8.1 E-01$ & $/ 1$ & & & 1 & GP & & & & & & & $\mathrm{pC} / \mathrm{L}$ \\
\hline Radium-228 & $<1.4 \mathrm{E}+00$ & UNI & $<0.8250$ & & 1 & GP & & & & & & & $\mathrm{pCi} / \mathrm{L}$ \\
\hline Strontium-90 & $3.0 \mathrm{E}+01$ & $\mathrm{~J} / \mathrm{C} /$ & NDD & & 1 & GP & & & & & & & $\mathrm{pCi} / \mathrm{L}$ \\
\hline Technetium-99 & $<1.4 \mathrm{E}+00$ & $\mathrm{UI} / /$ & $<20.3000$ & & 1 & GP & & & & & & & $\mathrm{pCi} / \mathrm{L}$ \\
\hline Thorium-228 & $<1.7 \mathrm{E}-02$ & $U 1 / /$ & $<0.2080$ & & 1 & GP & & & & & & & $\mathrm{pCi} / \mathrm{L}$ \\
\hline Thorium-230 & $<1.3 E-01$ & U1/I & $<0.0978$ & & 1 & GP & & & & & & & $\mathrm{pCi} / \mathrm{L}$ \\
\hline Thorium-232 & $<3.3 E-02$ & $\mathrm{UI} / /$ & $<0.0978$ & & 1 & GP & & & & & & & $\mathrm{pCi} / \mathrm{L}$ \\
\hline Sum of alphas & 4.6E-01 & & & & & & & & & & & & $\mathrm{pCi} / \mathrm{L}$ \\
\hline Sum of betas & $3.0 E+01$ & & & & & & & & & & & & $\mathrm{pCi} / \mathrm{L}$ \\
\hline Total radium & $8.1 E-01$ & & & & & & & & & & & & $\mathrm{pCi} / \mathrm{L}$ \\
\hline Tritium & $1.5 E+01$ & $/ /$ & & & 1 & GP & $1.8 E+01$ & $/ /$ & & & 1 & GE & $\mathrm{pCi} / \mathrm{mL}$ \\
\hline Uranium-233/234 & 4.6E-01 & $/ /$ & & & 1 & GP & & & & & & & $\mathrm{pCi} / \mathrm{L}$ \\
\hline Uranium-235 & $<0.0 \mathrm{E}+00$ & $\mathrm{UI} / /$ & $<0.2050$ & & 1 & GP & & & & & & & $\mathrm{pCi} / \mathrm{L}$ \\
\hline Uranium-238 & $<5.2 \mathrm{E}-02$ & $\mathrm{Ut} / /$ & $<0.3600$ & & 1 & GP & & & & & & & $\mathrm{pCi} / \mathrm{L}$ \\
\hline
\end{tabular}

Notes:

- exceeded holding time

- = exceeded groundwater protection or monitoring constituent standard (See Appendix A.) 
WELL HSB111C

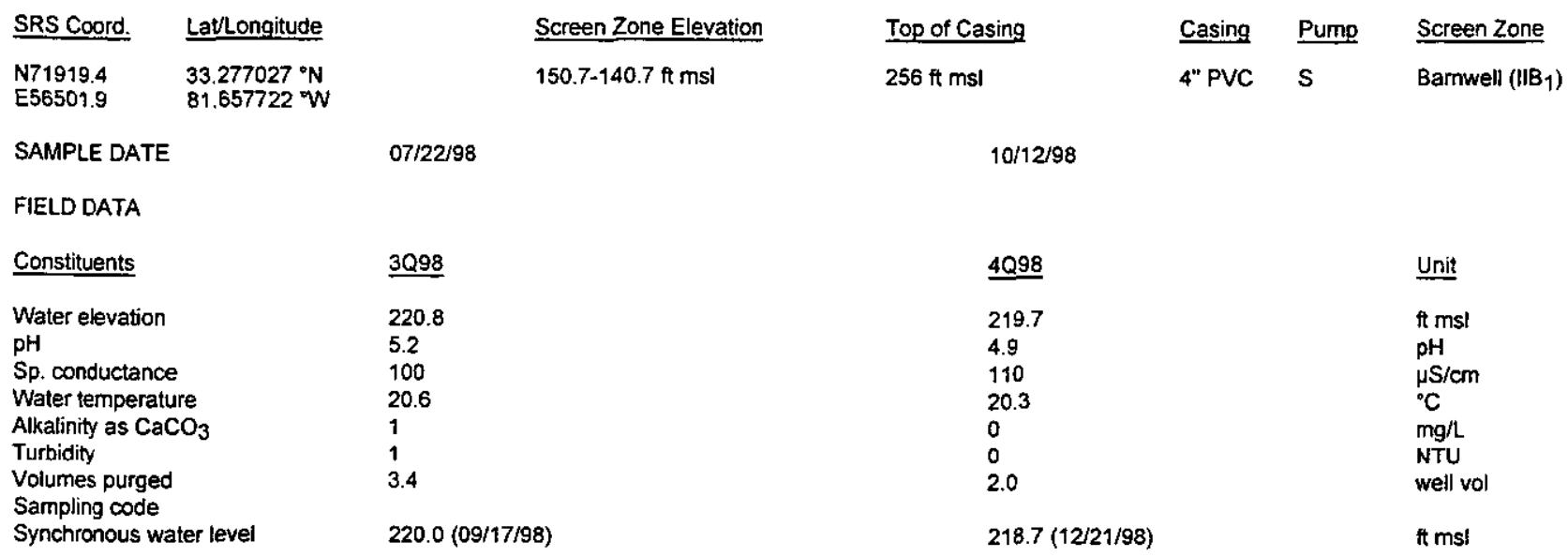

\section{ANALYTICAL DATA}

Inorganic Constituents

\begin{tabular}{|c|c|c|c|c|c|c|c|c|c|c|c|c|c|}
\hline Constituents & $\underline{3098}$ & Mod & Filt. & $\underline{\text { ST}} \underline{H}$ & $\underline{\text { DF }}$ & $\underline{\text { Lab }}$ & $\underline{4098}$ & Mod & Filt. & $\underline{\text { ST }} \underline{H}$ & $\underline{\mathrm{DF}}$ & $\underline{\text { Lab }}$ & Unit \\
\hline Antimony, total recoverable & $<0.20$ & $\mathrm{U} / \mathrm{I}$ & $<0.20$ & & 1 & GE & & & & & & & $\mu \mathrm{g} / \mathrm{L}$ \\
\hline Arsenic, total recoverable & $<3.0$ & $\mathrm{U} / /$ & $<3.0$ & & 1 & GE & & & & & & & $\mu \mathrm{g} / \mathrm{L}$ \\
\hline Barium, total recoverable & 9.9 & /I & & & 1 & GE & & & & & & & Hgl \\
\hline Cadmium, total recoverable & 0.35 & $\mathrm{~J} / \mathrm{E} /$ & NDD & & 1 & GE & & & & & & & $\mu \mathrm{g} / \mathrm{L}$ \\
\hline Chromium, total recoverable & 1.9 & $J / E /$ & NDD & - & 1 & GE & & & & & & & $\mu g / \mathrm{h}$ \\
\hline Cobalt, total recoverable & 0.75 & $1 /$ & & & 1 & $\mathrm{GE}$ & & & & & & & $\mu \mathrm{g} / \mathrm{L}$ \\
\hline Copper, total recoverable & 2.7 & $\|$ & & & $i$ & $\mathrm{GE}$ & & & & & & & $\mu g / L$. \\
\hline Cyanide & $<10$ & $\mathrm{u} / /$ & $<10$ & & 1 & $\mathrm{GE}$ & & & & & & & $\mu \mathrm{g} / \mathrm{L}$ \\
\hline Lead, total recoverable & 0.36 & $\mathrm{~J} / \mathrm{E} /$ & NOD & & 1 & GE & & & & & & & $\mathrm{g} / \mathrm{L}$ \\
\hline Mercury, total recoverable & $<0.20$ & $\mathrm{U} / 1 /$ & $<0.20$ & & 1 & GE & $<0.20$ & $\mathrm{U} / 1$ & $<0.20$ & & 1 & $\mathrm{GE}$ & $\mu \mathrm{g} / \mathrm{L}$ \\
\hline Nickel, total recoverable & 3.4 & "l & & & 1 & $G E$ & & & & & & & $\mu g / L$ \\
\hline Nitrate-nitrite as nitrogen & 12,000 & NI & & a & 5 & GE & 11000 & $u$ & & $=$ & 6 & GE & ton \\
\hline Selenium, total recoverable & $<5.0$ & $\mathrm{U} / /$ & $<5.0$ & & 1 & GE & & & & & & & $\begin{array}{ll}\mu g / \mathrm{L} \\
\mu \mathrm{g} / \mathrm{L}\end{array}$ \\
\hline Silver, total recoverable & $<1.0$ & $\mathrm{U} / /$ & $<1.0$ & & 1 & $\mathrm{GE}$ & & & & & & & $\mu g / \mathrm{L}$ \\
\hline Tin, total recoverable & $<2.0$ & $\mathrm{U} / \prime$ & $<2.0$ & & 1 & GE & & & & & & & $\mu g / L$ \\
\hline Vanadium, total recoverable & $<2.0$ & $\mathrm{U} / /$ & $<2.0$ & & 1 & GE & & & & & & & $\mu \mathrm{g} / \mathrm{L}$ \\
\hline Zinc, total recoverable & 16 & "I & & & 1 & $\mathrm{GE}$ & & & & & & & $\mu g / L$ \\
\hline \multicolumn{14}{|l|}{ Organic Constituents } \\
\hline Constituents & 3098 & Mod & Filit. & $\underline{\text { ST}} \underline{H}$ & DF & Lab & $\underline{4 Q 98}$ & $\underline{\text { Mod }}$ & Filt. & $\underline{\text { ST }}$ 브 & $\underline{\mathrm{DF}}$ & $\underline{\text { Lab }}$ & Unit \\
\hline Benzene & $<1.0$ & $\mathrm{UJ} / \mathrm{O} / 1$ & $<1.0$ & & 1 & GE & & & & & & & \\
\hline Bis(2-ethylhexyl) phthalate & $<10$ & $\mathrm{u} / 1$ & $<10$ & & 1 & $\mathrm{GE}$ & & & & & & & 1 \\
\hline Dichloromethane & $<1.0$ & $\mathrm{UJ} / \mathrm{O} / 1$ & $<1.0$ & & 1 & $\mathrm{GE}$ & & & & & & & \\
\hline Tetrachloroethylene & $<1.0$ & UJ/O/1 & $<1.0$ & & 1 & $\mathrm{GE}$ & & & & & & & ig/L \\
\hline Trichloroethylene & $<1.0$ & UJ/O/1 & $<1.0$ & & 1 & GE & & & & & & & \\
\hline Trichlorofluoromethane & $<1.0$ & UJ/O/1 & $<1.0$ & & 1 & $\mathrm{GE}$ & & & & & & & \\
\hline
\end{tabular}

Notes:

- exceeded holding time

- exceeded groundwater protection or monitoring constituent standard (See Appendix A.) 
WELL HSB111C (cont.)

Radioactive Constituents

Constituents
Americium-241
Beta dose
Carbon-14
Cobalt-60
Curium-242
Curium-243/244
Curium-245/246
Gross alpha
lodine-129
Nickel-63
Nonvolatile beta
Plutonium-238
Plutonium-239/240
Radium-226
Radium-228
Strontium-90
Technetium-99
Thorium-228
Thorium-230
Thorium-232
Sum of alphas
Sum of betas
Tritium
Uranium-233/234
Uranium-235
Uranium-238

\begin{tabular}{|c|c|c|c|c|c|c|c|c|}
\hline 3Q98 & Mod & Filt. & ST브 & $\underline{\mathrm{DF}}$ & Lab & $4 Q 98$ & Mod & Filt. \\
\hline $\begin{array}{l}<9.6 \mathrm{E}-03 \\
0.06\end{array}$ & $\mathrm{UI} / /$ & $<0.0566$ & & 1 & GP & & & \\
\hline$<5.0 E+00$ & UII/ & $<8.6700$ & & 1 & GP & & & \\
\hline$<3.0 E-01$ & $\mathrm{UI} / /$ & $<3.3500$ & & 1 & GP & & & \\
\hline$<-4,8 \mathrm{E}-03$ & UIII & $<0.1050$ & & 1 & $G P$ & & & \\
\hline$<1.9 E-02$ & $\mathrm{UI} / /$ & $<0.0566$ & & 1 & GP & & & \\
\hline$<0.0 \mathrm{E}+00$ & UIII & $<0.0566$ & & 1 & GP & & & \\
\hline 8.8E-01 & /I & & & 1 & $\mathrm{GP}$ & $1.2 E+00$ & $\mathrm{~J} / \mathrm{IK} / \mathrm{C}$ & NDD \\
\hline$<1.8 E-01$ & $\mathrm{UI} / /$ & $<1.1800$ & & 1 & GP & & & \\
\hline$<3.0 E-01$ & Ui & $<3.3500$ & & & & & & \\
\hline $1.8 E+01$ & $/ 1$ & & & 1 & GP & $2.9 E+01$ & /I & \\
\hline$<5.7 E-02$ & $\mathrm{U} \mathrm{K} / \mathrm{I}$ & $<0.082 t$ & & 1 & GP & & & \\
\hline$<1.5 E-02$ & $\mathrm{U} \mid / /$ & $<0.0458$ & & 1 & GP & & & \\
\hline$<3.1 E-01$ & Ul/I & $<0.3780$ & & 1 & $G P$ & & & \\
\hline$<5.3 E-01$ & $\mathrm{UI} / \mathrm{I}$ & $<0.9320$ & & 1 & GP & & & \\
\hline$<2.3 E+00$ & $\mathrm{U} / / /$ & $<4.6300$ & & 1 & $G P$ & & & \\
\hline $5.5 E+01$ & /I & & 口 & 1 & GP & & & \\
\hline$<1.9 \mathrm{E}-02$ & $\mathrm{U} \mid / /$ & $<0.3160$ & & 1 & GP & & & \\
\hline$<1.4 \mathrm{E}-01$ & $\mathrm{UI} / /$ & $<0.1600$ & & 1 & GP & & & \\
\hline$<0.0 \mathrm{E}+00$ & $\mathrm{UI} / /$ & $<0.0907$ & & 1 & GP & & & \\
\hline $5.5 E+01$ & & & - & & & & & \\
\hline $1.2 E+03$ & /I & & 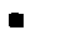 & 1 & GP & $1.4 E+03$ & /I & \\
\hline$<5.5 \mathrm{E}-02$ & $\mathrm{UI} / /$ & $<0.1640$ & & 1 & $\mathrm{GP}$ & & & \\
\hline$<4.2 \mathrm{E}-02$ & UIII & $<0.2890$ & & 1 & GP & & & \\
\hline$<-1.3 E-02$ & $\mathrm{UI} / /$ & $<0.2880$ & & 1 & GP & & & \\
\hline
\end{tabular}

Filt. $\quad \underline{S T} \underline{H}$ DF Lab Unit

$\mathrm{pCi} / \mathrm{L}$

pCi/L

pCi/L

$\mathrm{pCi} / \mathrm{L}$

PCilL

pCinl

$\mathrm{pCi} / \mathrm{L}$

$1 \mathrm{TM} \mathrm{pCi}$

$\mathrm{pCi} / \mathrm{L}$

pCill

1 TM $\mathrm{pCi} / \mathrm{L}$

pCill

pCill

pCi/L

pCin

pCill

pCill

pCill

pCilL

pCirl.

pCirL

- 1 GE $\stackrel{\mathrm{pCi} / \mathrm{mL}}{\mathrm{pCi}}$

pCi/L

$\mathrm{pCi} / \mathrm{L}$

$\mathrm{pCi} / \mathrm{L}$

Notes:

- = exceeded holding time

E = exceeded groundwater protection or monitoring constituent standard (See Appendix A.) 
WELL HSB111D

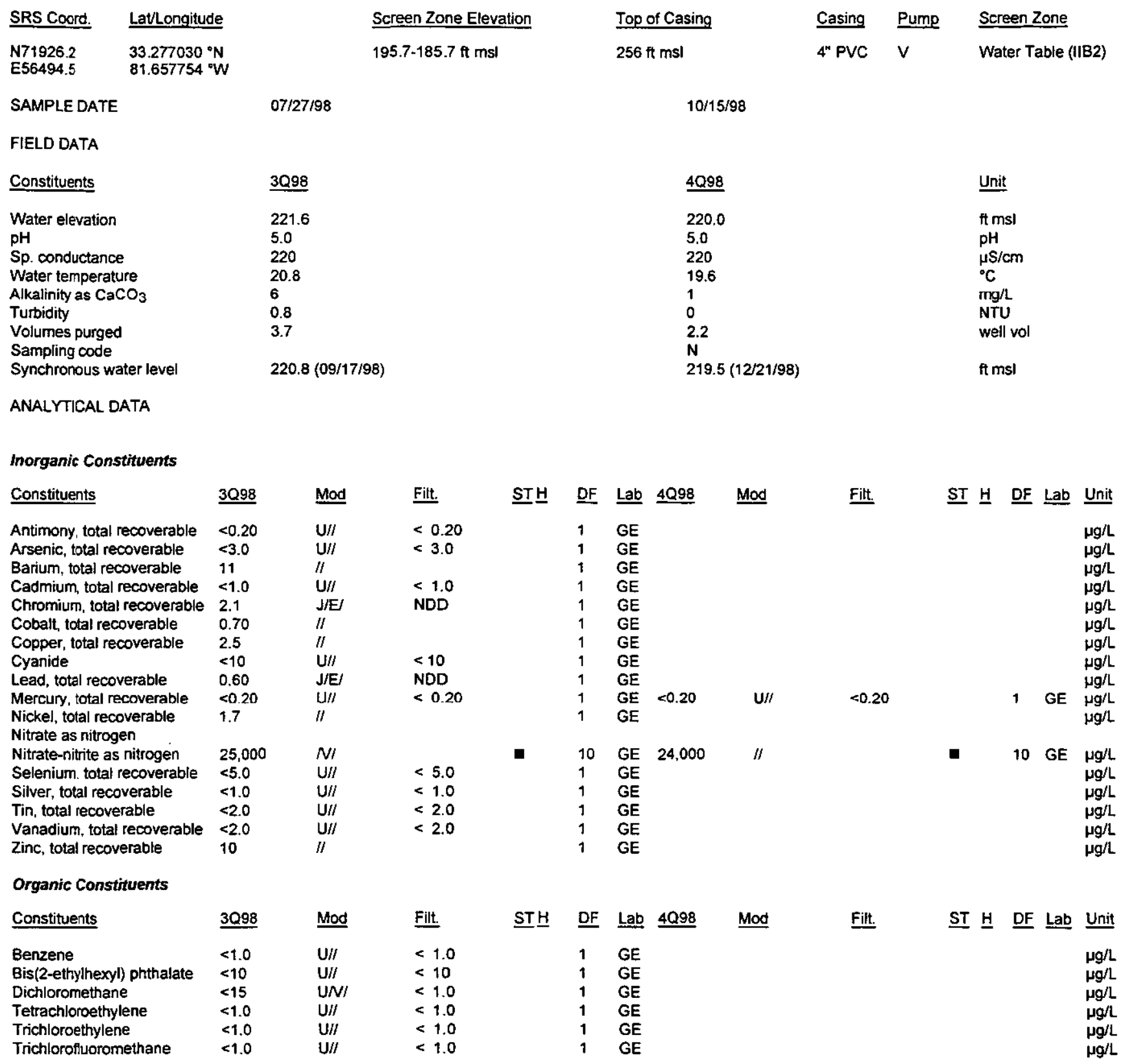

Notes:

- exceeded holding time

- = exceeded groundwater protection or monitoring constituent standard (See Appendix A.) 
WELL HSB111D (cont.)

Radioactive Constituents

\begin{tabular}{|c|c|c|c|c|c|c|c|c|c|c|c|c|c|}
\hline Constituents & 3098 & Mod & Filt. & $\underline{\mathrm{ST}} \underline{\mathrm{H}}$ & $\underline{D F}$ & $\underline{\text { Lab }}$ & $\underline{4 Q 98}$ & Mod & Filt. & $\underline{\mathrm{ST}} \underline{\mathrm{H}}$ & $\underline{\mathrm{DF}}$ & $\underline{L a b}$ & $\underline{\text { Unit }}$ \\
\hline Americium-24i & $<2.5 \mathrm{E}-02$ & $U W / I$ & $<0.0625$ & & 1 & GP & & & & & & & $\mathrm{pCi} / \mathrm{L}$ \\
\hline Beta dose & 4.06 & & & - & & & & & & & & & PCiL \\
\hline Carbon-14 & $4.8 E+01$ & $\|$ & & & 1 & GP & & & & & & & $\mathrm{pCi} / \mathrm{L}$ \\
\hline Cobalt-60 & $<4.7 \mathrm{E}-01$ & $\mathrm{UI} / 1$ & $<3.3900$ & & 1 & GP & & & & & & & $\mathrm{pCi} / \mathrm{L}$ \\
\hline Curium-242 & $<0.0 \mathrm{E}+00$ & $\mathrm{UI} / \prime$ & $<0.0317$ & & 1 & GP & & & & & & & $\mathrm{pCi} / \mathrm{L}$ \\
\hline Curium-243/244 & $<9.9 E-03$ & $\mathrm{UI} / /$ & $<0.0298$ & & 1 & GP & & & & & & & $\mathrm{pCi} / \mathrm{L}$ \\
\hline Curium-245/246 & $<0.0 \mathrm{E}+\infty 0$ & $\mathrm{U} \mid / /$ & $<0.0297$ & & 1 & GP & & & & & & & $\mathrm{pCi} / \mathrm{L}$ \\
\hline Gross alpha & $4.2 \mathrm{E}+00$ & $\|$ & & & 1 & GP & $<1.6 \mathrm{E}+00$ & $\mathrm{U} / /$ & $<1.7 \mathrm{E}+00$ & & 1 & GE & $\mathrm{pCi} / \mathrm{L}$ \\
\hline lodine-129 & $3.9 \mathrm{E}+00$ & /I & & & 1 & GP & & & & & & & $\mathrm{pCi} / \mathrm{L}$ \\
\hline Nickel-63 & $<4.7 \mathrm{E}-01$ & U1 & $<3.3900$ & & & & & & & & & & $\mathrm{pCi} / \mathrm{L}$ \\
\hline Nonvolatile beta & $4.8 \mathrm{E}+01$ & "I & & & 1 & GP & $2.0 E+01$ & 11 & & & 1 & GE & $\mathrm{pCi} / \mathrm{L}$ \\
\hline Plutonium-238 & $<0.0 E+00$ & $U M /$ & $<0.0317$ & & 1 & GP & & & & & & & $\mathrm{pCi} / \mathrm{L}$ \\
\hline Plutonium-239/240 & $<1.1 \mathrm{E}-02$ & $\mathrm{UI} / \mathrm{I}$ & $<0.0317$ & & 1 & GP & & & & & & & $\mathrm{pCi} / \mathrm{L}$ \\
\hline Radium-226 & $2.8 E+00$ & "I & & & 1 & GP & & & & & & & pCill \\
\hline Radium-228 & $<-2.5 \mathrm{E}-01$ & UIIII & $<1.3300$ & & 1 & GP & & & & & & & $\mathrm{pCilL}$ \\
\hline Strontium-90 & $<3.1 \mathrm{E}-01$ & $\mathrm{UI} / /$ & $<1.2300$ & & 1 & GP & & & & & & & $\mathrm{pCi} / \mathrm{L}$ \\
\hline Technetium-99 & $1.2 \mathrm{E}+02$ & 11 & & - & 1 & GP & & & & & & & $\mathrm{pCill}$ \\
\hline Thorium-228 & $<3.7$ E-03 & UIJ $/ \mathrm{C} /$ & & & 4 & GP & & & & & & & $\mathrm{pCill}$ \\
\hline Thorium-230 & $<-2.2 \mathrm{E}-02$ & $\mathrm{U}[\mathrm{J} / \mathrm{C} / \mathrm{I}$ & & & 1 & GP & & & & & & & $\mathrm{pCi} / \mathrm{L}$ \\
\hline Thorium-232 & $<0.0 \mathrm{E}+00$ & $\mathrm{UIJ} / \mathrm{C} /$ & & & 1 & GP & & & & & & & $\mathrm{pCi} / \mathrm{L}$ \\
\hline Sum of alphas & 4.2E-01 & & & & & & & & & & & & $\mathrm{pCi} / \mathrm{L}$ \\
\hline Sum of betas & $1.7 \mathrm{E}+02$ & & & a & & & & & & & & & $\mathrm{pCi} / \mathrm{L}$ \\
\hline Total radium & $2.8 \mathrm{E}+00$ & & & & & & & & & & & & $\mathrm{pCi/L}$ \\
\hline Tritium & $3.0 E+03$ & "I & & - & 1 & GP & $3.2 E+03$ & $\|$ & & - & 1 & GE & $\mathrm{pCi} / \mathrm{mL}$ \\
\hline Uranium-233/234 & $2.6 \mathrm{E}-01$ & "l & & & 1 & GP & & & & & & & $\mathrm{pCi} / \mathrm{L}$ \\
\hline Uranium-235 & $<8.0 \mathrm{E}-02$ & $\mathrm{U} 1 / /$ & $<0.1840$ & & 1 & GP & & & & & & & $\mathrm{pCi} / \mathrm{L}$ \\
\hline Uranium-238 & $1.5 \mathrm{E}-01$ & /I & & & 1 & GP & & & & & & & $\mathrm{pCi} / \mathrm{L}$ \\
\hline
\end{tabular}

Notes: 
WELL HSB111E

\begin{tabular}{|c|c|}
\hline SRS Coord. & Lat/Longitude \\
\hline $\begin{array}{l}\text { N71932.8 } \\
\text { E56487.2 }\end{array}$ & $81.657786^{\circ}$ \\
\hline
\end{tabular}

SAMPLE DATE

FIELD DATA

Constituents

Water elevation

$\mathrm{pH}$

Sp. conductance

Water temperature

Alkalinity as $\mathrm{CaCO}_{3}$

Turbidity

Volumes purged

Sampling code

Synchronous water level

\section{ANALYTICAL DATA}

Inorganic Constrituents

Constituents 309

Antimony, total recoverable $<0.20$

Arsenic, total recoverable $<3.0$

Barium, total recoverable $\quad 5.1$

Cadmium, total recoverable $<1.0$

Chromium, total recoverable $<1$,

Cobalt, total recoverable $\quad 0.41$

Copper, total recoverable

Cyanide

Lead, total recoverable $\quad 0.40$

Mercury, lotal recoverable $\quad<0.20$

Nickel, total recoverable $\quad 1.1$

Nitrate as nitrogen

Nitrate-nitrite as nitrogen $\quad 2,300$

Selenium, total recoverable 0.60

Silver, total recoverable $\quad<1.0$

Tin, total recoverable $<0.23$

Vanadium, total recoverable $<2.0$

Zinc, total recoverable $\quad 4.7$

Organic Constituents

\begin{tabular}{ll} 
Constituents & 3098 \\
\hline & $<1.0$ \\
Benzene & $<10$ \\
Bis(2-ethylhexyl) phthalate & $<1.0$ \\
Dichloromethane & $<1.0$ \\
Tetrachloroethylene & $<1.0$ \\
Trichloroethylene & $<1.0$ \\
Trichlorofluoromethane &
\end{tabular}

Trichlorofluoromethane $<1.0$
Screen Zone Elevation

231.7-211.7 ft ms!

$07 / 09 / 98$

3Q98

217.9

4.4

42

20.5

0

1

8.8

$220.9(09 / 17 / 98)$
Top of Casing

$255.9 \mathrm{ft} \mathrm{msl}$

$10 / 14 / 98$

4Q98

220.3

4.3

40

8.7

0

5.0

N

$219.5(12 / 21 / 98)$

Casing Pump

4" PVC V
Screen Zone

Water Table (IIB2)

\begin{tabular}{|c|c|c|}
\hline Q98 & Mod & Filt. \\
\hline .20 & $\mathrm{U} / /$ & $<0.20$ \\
\hline 3.0 & $\begin{array}{l}\mathrm{U} / / \\
/ /\end{array}$ & $<3.0$ \\
\hline 1.0 & $\mathrm{U} / \mathrm{I}$ & $<1.0$ \\
\hline $\begin{array}{l}1.9 \\
41\end{array}$ & $\begin{array}{l}\text { UNI } \\
/ /\end{array}$ & $<3.0$ \\
\hline 5 & $/ /$ & \\
\hline & $\mathrm{U} / I$ & $<10$ \\
\hline 4 & $\mathrm{~J} / \mathrm{E} /$ & NDD \\
\hline 20 & $\mathrm{U} / \mathrm{I}$ & $<0.20$ \\
\hline 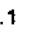 & $N I$ & \\
\hline 00 & $N /$ & \\
\hline 60 & $\mathrm{~J} / \mathrm{E} /$ & NDD \\
\hline 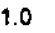 & $U / I$ & $<1.0$ \\
\hline & UNI & $<2.0$ \\
\hline 2. & $\mathrm{U} / /$ & $<2.0$ \\
\hline 7 & $\mathrm{~J} / \mathrm{E} /$ & NDD \\
\hline
\end{tabular}

ST보
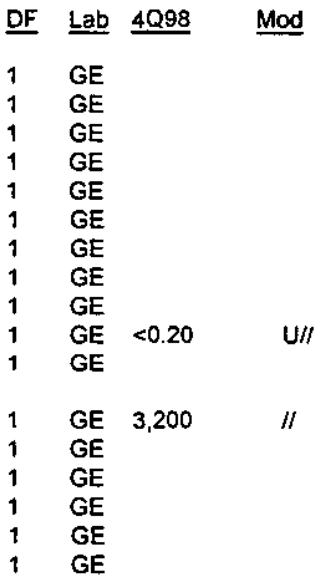

Filt.

ST $\underline{H}$ DF Lab Unit

At ms

pH

$\mu \mathrm{S} / \mathrm{cm}$

${ }^{\circ} \mathrm{C}$

mg/t

well vol

ft mst

$\begin{array}{ll}\text { Mod } & \text { Filt. } \\ U / / & <1.0 \\ U / I & <10 \\ U / I & <1.0 \\ U / I & <1.0 \\ U / / & <1.0 \\ U / I & <1.0\end{array}$

ST브

$\begin{array}{ll}\text { DF } & \text { Lab } \\ 1 & \text { GE } \\ 1 & \text { GE } \\ 1 & \text { GE } \\ 1 & \text { GE } \\ 1 & \text { GE } \\ 1 & \text { GE }\end{array}$

Notes:

- = exceeded holding time

- exceeded groundwater protection or monitoring constituent standard (See Appendix A.) 
WELL HSB111E (cont.)

Radioactive Constituents

\begin{tabular}{|c|c|c|c|c|c|c|c|c|c|c|c|c|c|}
\hline Constituents & $\underline{3 Q 98}$ & Mod & Filt: & $\underline{\mathrm{ST}} \underline{\mathrm{H}}$ & $\underline{\text { DF }}$ & $\underline{\text { Lab }}$ & 4Q98 & Mod & Filt. & $\underline{\text { ST }} \underline{H}$ & $\underline{D F}$ & Lab & Unit \\
\hline $\begin{array}{l}\text { Americium-241 } \\
\text { Beta dose }\end{array}$ & $\begin{array}{l}<-2.1 E-02 \\
0.28\end{array}$ & $\mathrm{UI} / I$ & $<0.1500$ & & 1 & GP & & & & & & & $\mathrm{pCi} / \mathrm{L}$ \\
\hline $\begin{array}{l}\text { Beta dose } \\
\text { Carbon-14 }\end{array}$ & $\begin{array}{l}0.28 \\
<-1.2 E+\infty 00\end{array}$ & $\mathrm{UI} / /$ & $<8.9300$ & & 1 & GP & & & & & & & $\mathrm{pCi} / \mathrm{L}$ \\
\hline $\begin{array}{l}\text { Carbon-14 } \\
\text { Cobalt-60 }\end{array}$ & $<1.4 \mathrm{E}+00$ & $\mathrm{UI} / /$ & $<4.9100$ & & 1 & GP & & & & & & & $\mathrm{pCi} / \mathrm{L}$ \\
\hline Curium-242 & $<-6.2 E-03$ & $\mathrm{UI} / / \mathrm{I}$ & $<0.1360$ & & 1 & GP & & & & & & & $\mathrm{PCi} / \mathrm{L}$ \\
\hline Curium-243/244 & $<1.4 \mathrm{E}-02$ & $\mathrm{UI} / \mathrm{I}$ & $<0.1660$ & & 1 & GP & & & & & & & pCi/L \\
\hline Curium-245/246 & $<0.0 \mathrm{E}+00$ & $\mathrm{UI} / /$ & $<0.0725$ & & 1 & GP & & & & & & & pCilL \\
\hline Gross alpha & $4.2 \mathrm{E}+00$ & "l & & & 1 & GP & $3.4 \mathrm{E}+00$ & $\mathrm{~J} / \mathrm{l} /$ & NDD & & 1 & GE & $\mathrm{PCi} / \mathrm{L}$ \\
\hline lodine-129 & $<2.1 E-01$ & $\mathrm{UI} / 1$ & $<0.7810$ & & 1 & GP & & & & & & & $\mathrm{PCi} / \mathrm{L}$ \\
\hline Nickel-63 & $<1.4 \mathrm{E}+00$ & UI & $<4.9100$ & & & & & & & & & & $\mathrm{pCi} / \mathrm{L}$ \\
\hline Nonvolatile beta & $1.6 \mathrm{E}+02$ & /I & & - & 1 & GP & $1.2 E+02$ & $\|$ & & - & 1 & GE & $\mathrm{pCi} / \mathrm{L}$ \\
\hline Plutonjum-238 & $<0.0 \mathrm{E}+00$ & $\mathrm{UI} / / /$ & $<0.0527$ & & 1 & GP & & & & & & & $\mathrm{pCi} / \mathrm{L}$ \\
\hline Plutonium-239/240 & $<0.0 \mathrm{E}+00$ & $\mathrm{U} \mid / /$ & $<0.0527$ & & 1 & $G P$ & & & & & & & $\mathrm{pCi} / \mathrm{L}$ \\
\hline Radium-226 & $5.7 \mathrm{E}+00$ & "I & & " & 1 & GP & & & & & & & $\mathrm{pCi} / \mathrm{L}$ \\
\hline Radium-228 & $1.4 \mathrm{E}+00$ & "I & & & 1 & GP & & & & & & & $\mathrm{pCi} / \mathrm{L}$ \\
\hline Strontium-90 & $8.3 E+01$ & $\mathrm{~J} / \mathrm{U}$ & NDD & & 1 & GP & & & & & & & $\mathrm{pCi} / \mathrm{L}$ \\
\hline Technetium-99 & $<-5.5 \mathrm{E}+00$ & $\mathrm{UIII}$ & $<21.60$ & & 1 & GP & & & & & & & $\mathrm{pCi} / \mathrm{L}$ \\
\hline Thorium-228 & $<2.6 \mathrm{E}-02$ & $\mathrm{UI} / /$ & $<0.495$ & & 1 & GP & & & & & & & $\mathrm{pCi} / \mathrm{L}$ \\
\hline Thorium-230 & $<1.1 \mathrm{E}-01$ & $\mathrm{UI} / /$ & $<0.199$ & & 1 & GP & & & & & & & $\mathrm{pCi} / \mathrm{L}$ \\
\hline Thorium-232 & $<1.2 E-02$ & $\mathrm{U} / /$ & $<0.144$ & & 1 & $G P$ & & & & & & & $\mathrm{pCi} / \mathrm{L}$ \\
\hline Sum of alphas & 7.0E-01 & & & & & & & & & & & & $\mathrm{pCi} / \mathrm{L}$ \\
\hline Sum of betas & $8.4 E+01$ & & & . & & & & & & & & & $\mathrm{pCi} / \mathrm{L}$ \\
\hline Tritium & $4.1 \mathrm{E}+03$ & 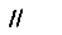 & & - & 1 & GP & $7.9 E+02$ & $\|$ & & - & 1 & GE & $\mathrm{pCi} / \mathrm{mL}$ \\
\hline Uranium-233/234 & $5.3 E-01$ & "I & & & 1 & GP & & & & & & & $\mathrm{pCi} / \mathrm{L}$ \\
\hline Uranium-235 & $1.6 \mathrm{E}-01$ & "I & & & 1 & GP & & & & & & & $\mathrm{pCi} / \mathrm{L}$ \\
\hline Uranium-238 & $<7.7 \mathrm{E}-02$ & $\mathrm{UI} / /$ & $<0.1080$ & & 1 & GP & & & & & & & $\mathrm{pCi} / \mathrm{L}$ \\
\hline
\end{tabular}

Notes:

- = exceeded holding time

= exceeded groundwater protection or monitoring constituent standard (See Appendix A.) 
WELL HSB112C

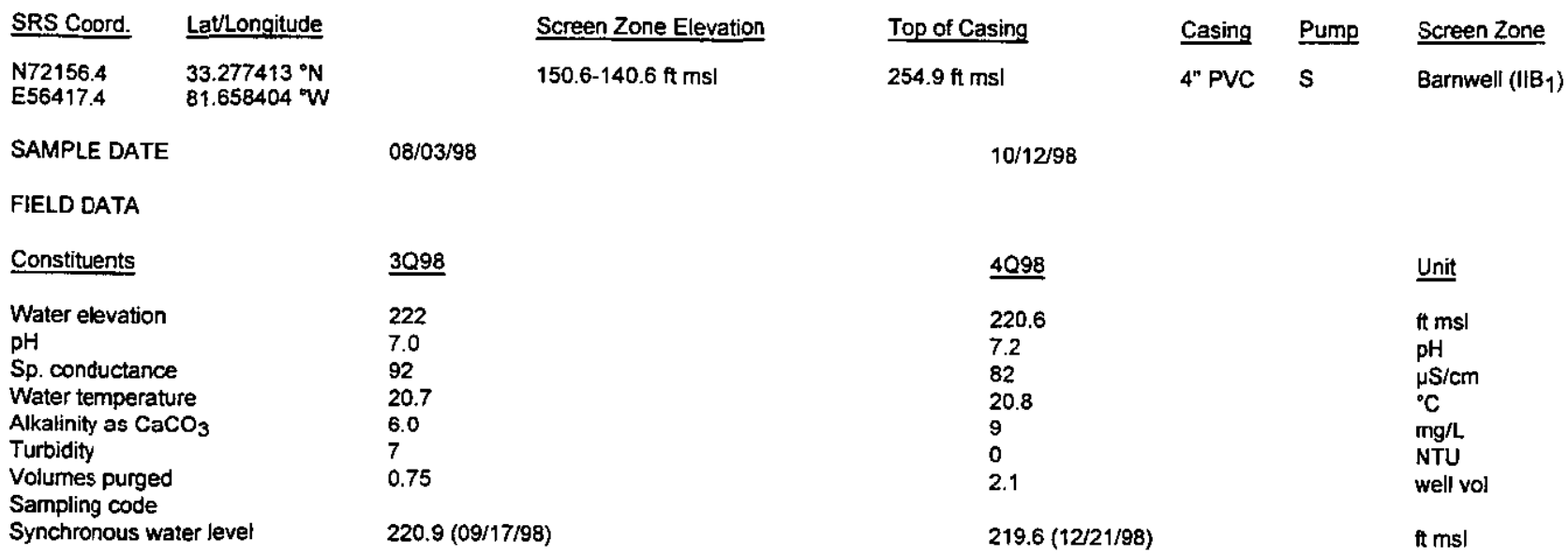

\section{ANALYTICAL DATA}

Inorganic Constifuents

\begin{tabular}{|c|c|c|c|c|c|c|c|c|c|c|c|c|c|}
\hline Constituents & 3098 & Mod & Filt. & SIT $\underline{H}$ & $\underline{\mathrm{DF}}$ & $\underline{\mathrm{Lab}}$ & $\underline{4 Q 98}$ & Mod & Filt. & $\underline{\text { ST }} \underline{H}$ & $\underline{\mathrm{DF}}$ & $\underline{\text { Lab }}$ & Unit \\
\hline Antimony, total recoverable & 0.24 & /I & & & 1 & GE & & & & & & & $\mu g / L$ \\
\hline Arsenic, total recoverable & $<3.0$ & $\mathrm{u} / !$ & $<3.0$ & & 1 & GE & & & & & & & $\mu g / L$ \\
\hline Barium, total recoverable & 14 & $/ /$ & & & 1 & GE & & & & & & & $\mu \mathrm{g} / \mathrm{L}$ \\
\hline Cadmium, total recoverable & 0.37 & $\mathrm{~J} / \mathrm{E} /$ & NDD & & 1 & $\overline{G E}$ & & & & & & & 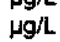 \\
\hline Chromium, total recoverable & 1.6 & $\mathrm{~J} / \mathrm{E} /$ & NDD & & 1 & GE & & & & & & & $\mu{ }^{\prime}$ \\
\hline Cobalt, total recoverable & 0.54 & $\|$ & & & 1 & GE & & & & & & & $\mu \mathrm{g} / \mathrm{L}$ \\
\hline Copper, total recoverable & 1.5 & $\|$ & & & 1 & GE & & & & & & & $\mu g / L$ \\
\hline Cyanide & $<10$ & $\mathrm{U} / /$ & $<10$ & & 1 & $\overline{G E}$ & & & & & & & $\mu g / L$ \\
\hline Lead, total recoverable & 0.40 & $\mathrm{~J} / \mathrm{E} /$ & NDD & & 1 & $\overrightarrow{G E}$ & & & & & & & $\mu g / L$ \\
\hline Mercury, total recoverable & 0.070 & $\mathrm{~J} / \mathrm{E} /$ & NDD & & 1 & GE & $<0.20$ & $\mathrm{U} / /$ & $<0.20$ & & 1 & GE & $\mathrm{Hg} / \mathrm{L}$ \\
\hline $\begin{array}{l}\text { Nickel, total recoverable } \\
\text { Nitrate as nitrogen }\end{array}$ & 2.7 & $/ 1$ & & & 1 & GE & & 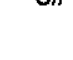 & -0.20 & & 1 & SL & $\mu g / L$ \\
\hline Nitrate-nitrite as nitrogen & 5,900 & $N \prime$ & & & 5 & GE & 4,300 & /I & & & 3 & GE & $\mu g / L$ \\
\hline Selenium, total recoverable & $<5.0$ & $\mathrm{U} / /$ & $<5.0$ & & 1 & GE & & & & & & & pg/L \\
\hline Silver, total recoverable & 0.74 & $\mathrm{~J} / \mathrm{E} /$ & NDD & & 1 & $\overrightarrow{G E}$ & & & & & & & H \\
\hline Tin, total recoverable & 4.0 & $/ /$ & & $\mathbf{E}$ & 1 & GE & & & & & & & با \\
\hline Vanadium, total recoverable & $<2.0$ & $\mathrm{U} / /$ & $<2.0$ & & 1 & GE & & & & & & & $\mu g / L$ \\
\hline Zinc, total recoverable & 32 & $\|$ & & & 1 & GE & & & & & & & $\mu g / L$ \\
\hline \multicolumn{14}{|l|}{ Organic Constituents } \\
\hline Constituents & $\underline{3098}$ & Mod & Filt. & ST & $\underline{D F}$ & Lab & 4Q98 & Mod & Filt. & $\underline{\text { ST }} \underline{H}$ & $\underline{\mathrm{DF}}$ & $\underline{\mathrm{Lab}}$ & Unit \\
\hline Benzene & $<1.0$ & $\mathrm{UJ} / \mathrm{O} / 1$ & $<1.0$ & & 1 & GE & & & & & & & $19 / \mathrm{L}$ \\
\hline Bis(2-ethylhexyl) phthalate & $<10$ & $\mathrm{UJ} / \mathrm{Q} /$ & $<10$ & $\bullet$ & 1 & GE & & & & & & & $g / h$ \\
\hline Dichloromethane & $<1.0$ & UJ/O/1 & $<1.0$ & & 1 & $\overline{G E}$ & & & & & & & $g / L$ \\
\hline Tetrachloroethylene & $<1.0$ & $\mathrm{UJ} / \mathrm{O} / 1$ & $<1.0$ & & 1 & GE & & & & & & & $g / L$ \\
\hline Trichloroethylene & $<1.0$ & $\mathrm{UJ} / \mathrm{O} / 1$ & $<1.0$ & & 1 & $\overline{G E}$ & & & & & & & 912 \\
\hline Trichioroftuoromethane & $<1.0$ & $\mathrm{U} J / O / 1$ & $<1.0$ & & 1 & $G E$ & & & & & & & \\
\hline
\end{tabular}

Notes:

- exceeded holding time

- exceeded groundwater protection or monitoring constituent standard (See Appendix A.) 
WELL HSB112C (cont.)

Radjoactive Constituents

\section{Constituents}

Americium-241

Beta dose

Carbon-14

Cobalt-60

Curium-242

Curium-243/244

Curium-245/246

Gross alpha

lodine-129

Nickel-63

Nonvolatile beta

Plutonium-238

Plutonium-239/240

Radium-226

Radium-228

Strontium-90

Technetium-99

Thorium-228

Thorium-230

Thorium-232

Sum of alphas

Sum of betas

Total radium

Total radium

Tritium

Uranium-233/234

Uranium-235

Uranium-238

\begin{tabular}{|c|c|c|c|c|c|c|c|}
\hline 3098 & Mod & Filt. & ST $\underline{H}$ & DF & $\underline{\text { Lab }}$ & 4Q98 & Mod \\
\hline $\begin{array}{l}<6.2 E-02 \\
0.34\end{array}$ & UI/I & $<0.0681$ & & 1 & GP & & \\
\hline $2.6 \mathrm{E}+01$ & $/ 1$ & & & 1 & GP & & \\
\hline$<-3.4 E-02$ & $\mathrm{UV} / /$ & $<3.2500$ & & 1 & GP & & \\
\hline$<2.4 E-02$ & $\mathrm{UI} / /$ & $<0.0733$ & & 1 & GP & & \\
\hline$<4.7 \mathrm{E}-03$ & $\mathrm{UI} / /$ & $<0.1620$ & & 1 & GP & & \\
\hline$<2.3 E-02$ & $\mathrm{UI} / /$ & $<0.0681$ & & 1 & GP & & \\
\hline$<6.2 E-01$ & $\mathrm{UI} / /$ & $<1.2100$ & & 1 & GP & $<2.5 \mathrm{E}-01$ & $U / /$ \\
\hline$<3.9 E-02$ & $\mathrm{UI} / /$ & $<1.3000$ & & 1 & GP & & \\
\hline$<-3.4 E-02$ & UI & $<3.2500$ & & & & & \\
\hline $1.1 E+01$ & 11 & & & 1 & GP & $<6.7 \mathrm{E}-01$ & $\mathrm{U} / /$ \\
\hline$<3.8 \mathrm{E}-02$ & $\mathrm{UI} / I$ & $<0.1140$ & & 1 & GP & & \\
\hline$<6.7 \mathrm{E}-02$ & $\mathrm{UI} / /$ & $<0.20$ & & 1 & GP & & \\
\hline$<4.5 \mathrm{E}-01$ & UNI & $<0.4140$ & & 1 & GP & & \\
\hline $1.4 \mathrm{E}+00$ & /I & & & 1 & GP & & \\
\hline$<1.2 E+00$ & $\mathrm{UIJ} / \mathrm{Cl}$ & & & 1 & GP & & \\
\hline $4.2 \mathrm{E}+01$ & 11 & & & 1 & $\mathrm{GP}$ & & \\
\hline$<8.7$ E-02 & $\mathrm{UI} / /$ & $<0.2930$ & & 1 & GP & & \\
\hline$<1,0 E-01$ & $\mathrm{UI} / /$ & $<0.1000$ & & 1 & GP & & \\
\hline $\begin{array}{l}<-8.0 E-03 \\
1.4 E+01\end{array}$ & UU/I & $<0.1760$ & & 1 & GP & & \\
\hline $\begin{array}{l}6.9 E+01 \\
1.4 E+00 \\
7.1 E+00\end{array}$ & & & D & & & & \\
\hline $8.5 E+02$ & $/ /$ & & $\square$ & 1 & $\mathrm{GP}$ & $4.8 E+02$ & $/ 1$ \\
\hline $8.4 E+00$ & /I & & & 1 & GP & & \\
\hline 8.6E-01 & /I & & & 1 & GP & & \\
\hline $4.4 E+00$ & $1 /$ & & & 1 & GP & & \\
\hline
\end{tabular}

Fitt.

ST 브 DF Lab Unit

$\mathrm{pCi} / \mathrm{L}$

$\mathrm{pCi} / \mathrm{L}$

pCi/L

pCill

pCi/L

$\mathrm{pCi} / \mathrm{L}$

$<5.1 E-01$

1 GE

pCi/L

$\mathrm{pCi} / \mathrm{L}$

$<1.1 E+00$

$\mathrm{pCi} / \mathrm{L}$

1 GE $\mathrm{pCi} / \mathrm{L}$

pCill

pCill

$\mathrm{PCil}$

pCill

$\mathrm{pCi/L}$

$\mathrm{pCil}$

pCi/L

$\mathrm{pCi} / \mathrm{L}$

$\mathrm{pCi} / \mathrm{L}$

pCi/L

pCi/l,

$\mathrm{pCi} / \mathrm{L}$

$\mathrm{pCi} / \mathrm{L}$

- 1 GE $\mathrm{pCi} / \mathrm{mL}$

$\mathrm{PCi} / \mathrm{L}$

$\mathrm{pCi} / \mathrm{L}$

PCi/L 
WELL HSB112D

$\begin{array}{lll}\text { SRS Coord. } & \text { Lat/Longitude } \\ \text { N72161.6 } & & 33.277410^{\circ} \mathrm{N} \\ \text { E56408.1 } & 81.658439^{\circ} \mathrm{W}\end{array}$

SAMPLE DATE

FIELD DATA

\section{Constituents}

Water elevation

$\mathrm{pH}$

Sp. conductance

Water temperature

Alkalinity as $\mathrm{CaCO}_{3}$

Turbidity

Volumes purged

Sampling code

Synchronous water level

ANALYTICAL DATA

Inorganic Constituents

\begin{tabular}{ll} 
Constituents & $\underline{3098}$ \\
\cline { 2 - 2 } Antimony, total recoverable & $<0.20$ \\
Arsenic, total recoverable & $<3.0$ \\
Barium, total recoverable & 1.7 \\
Cadmium, total recoverable & $<1.0$ \\
Chromium, total recoverable & 1.4 \\
Cobalt, total recoverable & 0.21 \\
Copper, total recoverable & 0.74 \\
Cyanide & $<10$ \\
Lead, total recoverable & $<2.0$ \\
Mercury, total recoverable & $<0.20$ \\
Nickel, total recoverable & $<0.20$ \\
Nitrate as nitrogen & \\
Nitrate-nitrite as nitrogen & 5,800 \\
Selenium, total recoverable & $<5.0$ \\
Silver, total recoverable & 0.33 \\
Tin, total recoverable & 4.4 \\
Vanadium, total recoverable & $<2.0$ \\
Zinc, total recoverable & $<5.0$
\end{tabular}

\begin{tabular}{|c|c|}
\hline Mod & Filt. \\
\hline $\mathrm{U} / \mathrm{I}$ & $<0.20$ \\
\hline $\mathrm{U}_{/ /} / \mathrm{I}$ & $<3.0$ \\
\hline U:I & $<1.0$ \\
\hline $\begin{array}{l}\mathrm{J} / \mathrm{E} / \\
/ /\end{array}$ & NOD \\
\hline "l & \\
\hline $\mathrm{U} / /$ & $<10$ \\
\hline $\mathrm{U} / /$ & $<2.0$ \\
\hline $\mathrm{U} / /$ & $<0.20$ \\
\hline $\mathrm{U} / \prime$ & $<0.20$ \\
\hline$N I$ & \\
\hline $\mathrm{U} / 1$ & $<5.0$ \\
\hline$J / E /$ & NDD \\
\hline $\mathrm{u} / /$ & $<2.0$ \\
\hline $\mathrm{U} / /$ & $<5.0$ \\
\hline
\end{tabular}

Onganic Constituents

\begin{tabular}{llll} 
Constituents & 3098 & Mod & Filt. \\
\hline Benzene & $<1.0$ & UJ/O/1 & $<1.0$ \\
Bis(2-thylhexyl) phthalate & $<10$ & U/I & $<10$ \\
Dichloromethane & $<1.0$ & UJ/O/1 & $<1.0$ \\
Tetrachloroethylene & $<1.0$ & UJ/O/1 & $<1.0$ \\
Trichloroethylene & $<1.0$ & UJ/O/1 & $<1.0$ \\
Trichlorofluoromethane & $<1.0$ & UJ/O/1 & $<1.0$
\end{tabular}

Screen Zone Elevation

198.3-188.3 ft ms

08/03/98

$\underline{3 Q 98}$

222.6

80

19.6

0.3

$221.7(09 / 17 / 98)$
Top of Casing

255.1 ft ms

$10 / 28 / 98$

4Q98

220.9

5.5

80

19.6

2.2

$220.2(12 / 21 / 98)$
Casing Pump Screen Zone

4" PVC V Water Table (IIB2)
Unit

$\mathrm{ft} \mathrm{msl}$

$\mathrm{pH}$

$\mu \mathrm{S} / \mathrm{cm}$

${ }^{\circ} \mathrm{C}$

$\mathrm{mg} / \mathrm{L}$

NTU

well vol

$f \mathrm{msl}$

\begin{tabular}{|c|c|c|c|c|c|c|c|c|c|}
\hline$\underline{\text { ST }} \underline{\underline{H}}$ & DF & $\underline{\text { Lab }}$ & 4Q988 & Mod & Filt. & ST $\underline{H}$ & $\underline{\mathrm{DF}}$ & Lab & Unit \\
\hline & 1 & $\mathrm{GE}$ & & & & & & & $\mu \sigma^{\prime}$ \\
\hline & 1 & $\mathrm{GE}$ & & & & & & & $\mu g / L$ \\
\hline & $\uparrow$ & GE & & & & & & & $\mu g / L$ \\
\hline & 1 & $\mathrm{GE}$ & & & & & & & $\mu g^{\prime} / \mathrm{L}$ \\
\hline & 1 & $\mathrm{GE}$ & & & & & & & $\mu g / L$ \\
\hline & 1 & GE & & & & & & & $\mu g / L$ \\
\hline & 1 & GE & & & & & & & $\mu g / L$ \\
\hline & 1 & GE & & & & & & & $\mu g / L$ \\
\hline & 1 & $\mathrm{GE}$ & & & & & & & $\mu g^{\prime} \mathrm{L}$ \\
\hline & 1 & GE & $<0.20$ & $\mathrm{U} / 1$ & $<0.20$ & & 1 & GE & $\mu g^{\prime} / \mathrm{L}$ \\
\hline & 1 & GE & & & & & & & (1) \\
\hline & 5 & GE & 6,300 & II & & & 5 & GE & \\
\hline & 1 & $\mathrm{GE}$ & & & & & & & $g / \mathrm{L}$ \\
\hline & 1 & GE & & & & & & & $g / L$ \\
\hline ש & 1 & GE & & & & & & & $g / L$ \\
\hline & 1 & GE & & & & & & & /L \\
\hline & 1 & $\mathrm{G}$ & & & & & & & \\
\hline
\end{tabular}

ST브

$\begin{array}{ll}\text { DF } & \text { Lab } \\ 1 & \text { GE } \\ 1 & \text { GE } \\ 1 & \text { GE } \\ 1 & \text { GE } \\ 1 & \text { GE } \\ 1 & \text { GE }\end{array}$

.

4Q98 Mod

Filt.

Notes:

- = exceeded holding time

= exceeded groundwater protection or monitoring constituent standard (See Appendix A.) 
WELL HSB112D (cont.)

Radioactive Constituents

\begin{tabular}{|c|c|c|c|c|c|c|c|c|c|c|c|c|c|}
\hline Constituents & $\underline{3 Q 98}$ & Mod & Filt. & $\underline{\text { ST }} \underline{H}$ & $\underline{\mathrm{DF}}$ & $\underline{\text { Lab }}$ & 4098 & Mod & Filt. & $\underline{S T} \underline{H}$ & $\underline{\mathrm{DF}}$ & $\underline{\text { Lab }}$ & Unit \\
\hline $\begin{array}{l}\text { Americium-241 } \\
\text { Beta dose }\end{array}$ & $\begin{array}{l}<6.9 \mathrm{E}-02 \\
4.72\end{array}$ & $\mathrm{UI} / I$ & $<0.1590$ & च & 1 & $\mathrm{GP}$ & & & & & & & $\mathrm{PCi} / \mathrm{L}$ \\
\hline Carbon-14 & $<8.9 E+00$ & $\mathrm{UII}$ & $<9.1200$ & & 1 & GP & & & & & & & $\mathrm{pCi} / \mathrm{L}$ \\
\hline Cobalt-60 & $<-8.3 E-02$ & $\mathrm{U} \mid / /$ & $<3.3700$ & & 1 & GP & & & & & & & $\mathrm{pCi} / \mathrm{L}$ \\
\hline Curium-242 & $<5.0 E-02$ & $\mathrm{UI} / \mathrm{I}$ & $<0.1870$ & & 1 & GP & & & & & & & $\mathrm{pCi} / \mathrm{L}$ \\
\hline Curium-243/244 & $<3.1 E-02$ & $\mathrm{UI} / /$ & $<0.2420$ & & 1 & GP & & & & & & & $\mathrm{PC} / \mathrm{L} / \mathrm{L}$ \\
\hline Curium-245/246 & $<0.0 E+00$ & $\mathrm{UI} / \mathrm{I}$ & $<0.0686$ & & 1 & GP & & & & & & & $\mathrm{pCi} / \mathrm{L}$ \\
\hline Gross alpha & $<5.3 E-02$ & $U \mathrm{I} / /$ & $<0.6600$ & & 1 & GP & $3.1 E+00$ & $/ 1$ & & & 1 & GE & $\mathrm{pCi} / \mathrm{L}$ \\
\hline lodine-129 & $4.7 \mathrm{E}+00$ & NI & & & 1 & GP & & & & & & & $\mathrm{pCi} / \mathrm{L}$ \\
\hline Nickel-63 & $<-8.3 E-02$ & UI & $<3.3700$ & & & & & & & & & & $\mathrm{pCi} / \mathrm{L}$ \\
\hline Nonvolatile beta & $2.1 E+00$ & $\|$ & & & 1 & GP & $4.0 E+00$ & $\|$ & & & 1 & GE & $\mathrm{pCi} / \mathrm{L}$ \\
\hline Plutonium-238 & $<0.0 E+00$ & $\mathrm{U} \mathrm{I} / \mathrm{I}$ & $<0.1240$ & & 1 & GP & & & & & & & $\mathrm{pCi} / \mathrm{L}$ \\
\hline Plutonium-239/240 & $<8.3 E-02$ & $\mathrm{U} \mathbf{I} / \mathrm{I}$ & $<0.1240$ & & 1 & $G P$ & & & & & & & $\mathrm{pCi} / \mathrm{L}$ \\
\hline Radium-226 & 7.6E-01 & $/ /$ & & & 1 & GP & & & & & & & $\mathrm{pCi} / \mathrm{L}$ \\
\hline Radium-228 & $<1.2 \mathrm{E}+00$ & $\mathrm{U} \mathbf{I} / /$ & $<1.2900$ & & 1 & GP & & & & & & & $\mathrm{pCi} / \mathrm{L}$ \\
\hline Strontium-90 & $<9.7 \mathrm{E}-02$ & $\mathrm{UIJ} / \mathrm{C} / \mathrm{I}$ & & & $i$ & GP & & & & & & & $\mathrm{pCi} / \mathrm{L}$ \\
\hline Technetium-99 & 1. $6 \mathrm{E}+01$ & $\|$ & & & 1 & GP & & & & & & & $\mathrm{pCi} / \mathrm{L}$ \\
\hline Thorium-228 & $<-6.7 \mathrm{E}-03$ & $\mathrm{U} \mathrm{I} / /$ & $<0.4120$ & & 1 & GP & & & & & & & $\mathrm{pCi} / \mathrm{L}$ \\
\hline Thorium-230 & $<6.2 \mathrm{E}-02$ & $\mathrm{UI} / /$ & $<0.2550$ & & 1 & GP & & & & & & & $\mathrm{pCi} / \mathrm{L}$ \\
\hline Thorium-232 & $<0.0 E+00$ & UIII & $<0.1230$ & & 1 & GP & & & & & & & $\mathrm{pCi} / \mathrm{L}$ \\
\hline Sum of alphas & 1.7E-01 & & & & & & & & & & & & $\mathrm{pCi} / \mathrm{L}$ \\
\hline Sum of betas & $2.1 E+01$ & & & & & & & & & & & & $\mathrm{PCi} / \mathrm{L}$ \\
\hline Total radjum & 7.6E-01 & & & & & & & & & & & & $\mathrm{pCi} / \mathrm{L}$ \\
\hline Tritium & $7.9 \mathrm{E}+02$ & $\|$ & & a & 1 & GP & $7.9 E+02$ & $\|$ & & - & 1 & GE & $\mathrm{pCi} / \mathrm{mL}$ \\
\hline Uranium-233/234 & $<1.5 \mathrm{E}-01$ & UNI & $<0.1260$ & & 1 & GP & & & & & & & $\mathrm{pCi} / \mathrm{L}$ \\
\hline Uranium-235 & $<4.0 E-02$ & $U \mathrm{UI} / \mathrm{I}$ & $<0.0596$ & & 1 & GP & & & & & & & $\mathrm{pCi} / \mathrm{L}$ \\
\hline Uranium-238 & $1.7 \mathrm{E}-01$ & $\|$ & & & 1 & GP & & & & & & & $\mathrm{pCi} / \mathrm{L}$ \\
\hline
\end{tabular}

Notes: 
WELL HSB112E

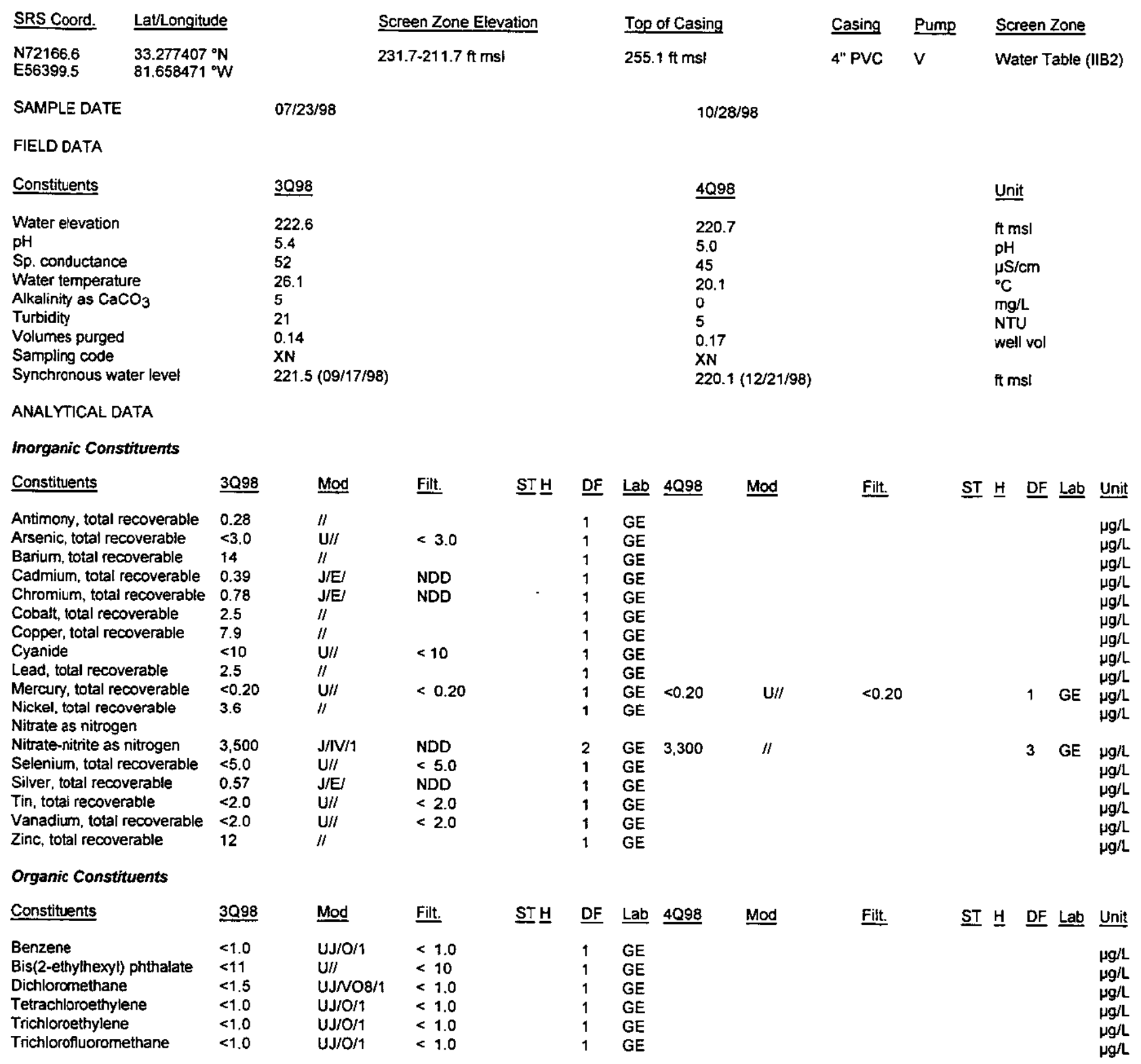

Notes:

$\bullet=$ exceeded holding time

- = exceeded groundwater protection or monitoring constituent standard (See Appendix A.) 
WELL HSB112E (cont.)

Radioactive Constituents

\begin{tabular}{|c|c|c|c|c|c|c|c|c|c|c|c|c|c|}
\hline Constituents & $\underline{3 Q 98}$ & Mod & Filt. & $\underline{\text { ST}} \underline{H}$ & $\underline{\mathrm{DF}}$ & $\underline{L a b}$ & $\underline{4 Q 98}$ & Mod & Filt. & $\underline{S T} \underline{H}$ & DF & $\underline{\text { Lab }}$ & Unit \\
\hline Americium-241 & $<5.2 \mathrm{E}-02$ & $\mathrm{UI} / /$ & $<0.1510$ & & 1 & GP & & & & & & & $\mathrm{pCi} / \mathrm{L}$ \\
\hline Beta dose & 1.60 & & & घ & & & & & & & & & $\mathrm{pCi} / \mathrm{L}$ \\
\hline Carbon-14 & $<2.8 \mathrm{E}+01$ & UNI & $<9.4300$ & & 1 & GP & & & & & & & $\mathrm{pCi} / \mathrm{L}$ \\
\hline Cobalt-60 & $<9.9 \mathrm{E}-01$ & $\mathrm{UI} / /$ & $<2.9300$ & & 1 & GP & & & & & & & $\mathrm{pCi} / \mathrm{L}$ \\
\hline Curium-242 & $<0.0 \mathrm{E}+00$ & $\mathrm{UI} / /$ & $<0.0765$ & & 1 & GP & & & & & & & $\mathrm{pCi} / \mathrm{L}$ \\
\hline Curium-243/244 & $<-5.8 \mathrm{E}-03$ & UIII & $<0.1290$ & & 1 & GP & & & & & & & $\mathrm{pCi} / \mathrm{L}$ \\
\hline Curium-245/246 & $<0.0 E+00$ & $\mathrm{U} 1 / /$ & $<0.0730$ & & 1 & GP & & & & & & & $\mathrm{pCi} / \mathrm{L}$ \\
\hline Gross alpha & $2.1 E+00$ & $\mathrm{~J} / \mathrm{Cl}$ & NDD & & 1 & GP & $<2.7 \mathrm{E}+00$ & $\mathrm{U} / /$ & $<1.2 \mathrm{E}+00$ & & 1 & GE & $\mathrm{pCi} / \mathrm{L}$ \\
\hline lodine-129 & $1.6 E+00$ & II & & & 1 & $\mathrm{GP}$ & & & & & & & $\mathrm{PCi} / \mathrm{L}$ \\
\hline Nickel-63 & $<9.9 \mathrm{E}-01$ & UI & $<2.9300$ & & & & & & & & & & $\mathrm{pCi} / \mathrm{L}$ \\
\hline Nonvolatile beta & $8.4 E+01$ & $\|$ & & - & 1 & GP & $9.7 E+01$ & $1 /$ & & - & 1 & GE & $\mathrm{pCi} / \mathrm{L}$ \\
\hline Plutonium-238 & $<-1.5 \mathrm{E}-02$ & $\mathrm{UI} / / \mathrm{I}$ & $<0.1350$ & & 1 & GP & & & & & & & pCirt \\
\hline Plutonium-239/240 & $<-1.1 \mathrm{E}-04$ & $\mathrm{UI} / /$ & $<0.1470$ & & 1 & GP & & & & & & & $\mathrm{pCi} / \mathrm{L}$ \\
\hline Radium-226 & $1.6 \mathrm{E}+00$ & "I & & & $t$ & $\mathrm{GP}$ & & & & & & & $\mathrm{pCi} / \mathrm{L}$ \\
\hline Radium-228 & $<2.3 E+00$ & UNI & $<1.1900$ & & 1 & GP & & & & & & & $\mathrm{pCi} / \mathrm{L}$. \\
\hline Strontium-90 & $3.6 E+01$ & $\mathrm{~J} / \mathrm{C} /$ & NDD & & 1 & GP & & & & & & & $\mathrm{pCi} / \mathrm{L}$ \\
\hline Technetium-99 & $<3.8 E+00$ & $\mathrm{U} \mathrm{I} / /$ & $<21.2000$ & & 1 & GP & & & & & & & $\mathrm{pCi} / \mathrm{h}$ \\
\hline Thorium-228 & $1.9 E+00$ & R/4/ & Rej & & 1 & GP & & & & & & & $\mathrm{pCi} / \mathrm{L}$ \\
\hline Thorium-230 & $\angle 4.0 \mathrm{E}-02$ & $\mathrm{UI} / /$ & $<0.2910$ & & 1 & GP & & & & & & & $\mathrm{pCi} / \mathrm{h}$ \\
\hline Thorium-232 & $<1.2 E-01$ & $\mathrm{UI} / /$ & $<0.1150$ & & 1 & GP & & & & & & & $\mathrm{pCi} / \mathrm{L}$ \\
\hline Sum of betas & $3.7 E+01$ & & & & & & & & & & & & $\mathrm{pCi} / \mathrm{L}$ \\
\hline Total radium & $1.6 \mathrm{E}+00$ & & & & & & & & & & & & $\mathrm{pCi} / \mathrm{L}$ \\
\hline Tritium & $3.5 E+01$ & /I & & घ & 1 & GP & $3.9 \mathrm{E}+01$ & $\|$ & & घ & 1 & GE & $\mathrm{pCi} / \mathrm{mL}$ \\
\hline Uranium-233/234 & $<1.0 \mathrm{E}-01$ & $\mathrm{UI} / /$ & $<0.1510$ & & 1 & GP & & & & & & & $\mathrm{pCi} / \mathrm{L}$ \\
\hline Uranium-235 & $<1.0 \mathrm{E}-01$ & $\mathrm{UI} / /$ & $<0.1520$ & & 1 & GP & & & & & & & $\mathrm{pCi} / \mathrm{L}$ \\
\hline Uranium-238 & $<1.0 \mathrm{E}-0$ & $\mathrm{UI} / /$ & $<0.1510$ & & 1 & GP & & & & & & & $\mathrm{pCi} / \mathrm{L}$ \\
\hline
\end{tabular}

Notes:

- = exceeded holding time

- = exceeded groundwater protection or monitoring constituent standard (See Appendix A.) 
WELL HSB113C

\begin{tabular}{|c|c|}
\hline RS Coord. & Lat/Longitude \\
\hline $\begin{array}{l}72312 . \\
56160 .\end{array}$ & W \\
\hline
\end{tabular}

SAMPLE DATE

\section{Screen Zone Elevation}

161.7-151.7 ft msl
Top of Casing

$261 \mathrm{ft}$ msl $\begin{array}{ll}\text { Casing Pump } & \text { P"PVC }\end{array}$

Barnwell (IIB 1$)$
Screen Zone

FJELD DATA
Water elevation

$\mathrm{pH}$

Sp. conductance

Water temperature

Alkalinity as $\mathrm{CaCO}_{3}$

Turbidity

Volumes purged

Sampling code

Synchronous water level
Constituents
$07 / 28 / 98$

3098

222.0

5.2

100

22,4

2.8

$220.9(09 / 17 / 98)$
$10 / 12 / 98$

4Q98
220.6
4.6
100
20.9
0
0
2.2
$219.4(12 / 21 / 98)$

Unit

ft msi

$\mathrm{pH}$ $\mu \mathrm{S} / \mathrm{cm}$ ${ }^{\circ} \mathrm{C}$

$\mathrm{mg} / \mathrm{L}$

NTU

well vol

$\mathrm{ft}$ msl
ANALYTICAL DATA

Inorganic Constituents

Constituents

Antimony, total recoverable

Arsenic, total recoverable

Barium, total recoverable

Cadmium, total recoverable

Chromium, total recoverable 0.68

Cobalt, total recoverable $\quad 1.3$

Copper, total recoverable 1.1

Cyanide

Lead, total recoverable $\quad 0.42$

Mercury, total recoverable $\quad 0.13$

Nickel, total recoverable $\quad 2.7$

Nitrate as nitrogen

Nitrate-nitrite as nitrogen $\quad 12,000$

Selenium, total recoverable $<5.0$

Silver, total recoverable $\quad<1.0$

Tin, total recoverable $\quad<2.0$

Vanadium, total recoverable $<2.0$

Zinc, total recoverable

22

\begin{tabular}{|c|c|}
\hline Mod & Filt. \\
\hline$U / I$ & $<0.20$ \\
\hline $\mathrm{U} / 1$ & $<3.0$ \\
\hline$/ 1$ & \\
\hline$J / E /$ & NDD \\
\hline $\mathrm{J} / \mathrm{E} /$ & NDD \\
\hline 11 & \\
\hline$\| 1$ & \\
\hline U/I & $<10$ \\
\hline J/EV/ & NDD \\
\hline $\begin{array}{l}3 / E / \\
/ /\end{array}$ & NDD \\
\hline$N /$ & \\
\hline $\mathrm{U} / /$ & $<5.0$ \\
\hline U/I & $<1.0$ \\
\hline $\mathrm{U} / 1$ & $<2.0$ \\
\hline $\mathrm{U} / /$ & $<2.0$ \\
\hline
\end{tabular}

ST본

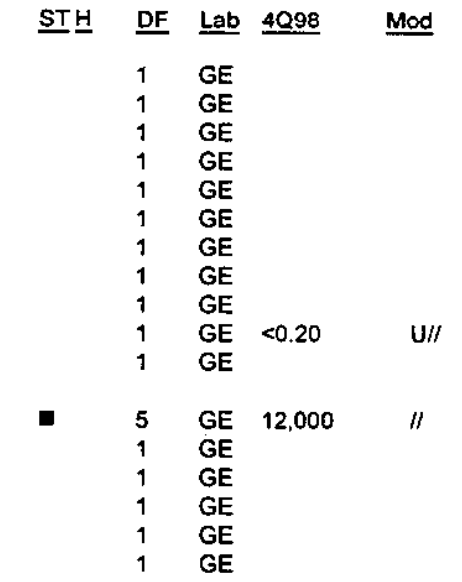

Filt.

ST 브 DF Lab Unit

Organic Constituents
Constituents

Benzene

Bis(2-ethylhexyl) phthalate

Dichloromethane

Tetrachloroethylene

Trichloroethylene

Trichtorofluoromethane
3Q98

$<i .0$

$<10$

$<5.1$

$<1.0$

$<1.0$

$<1.0$
Mo

$\mathrm{UJ} / \mathrm{O} / 1$

$\mathrm{U} / I$

UJNO8/1

$\mathrm{UJ} / \mathrm{O} / 1$

U $/ / O / 1$
Filt.

$<1.0$

$<10$

$<1.0$

$<1.0$

$<1.0$
ST브

$\begin{array}{ll}\text { DF } & \text { Lab } \\ 1 & \text { GE } \\ 1 & \text { GE } \\ 1 & \text { GE } \\ 1 & \text { GE } \\ 1 & \text { GE } \\ 1 & \text { GE }\end{array}$$$
4 Q 98
$$

Mod

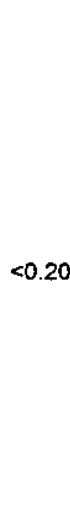

Filt.

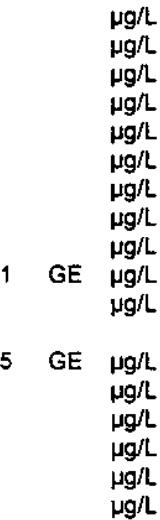

$\mathrm{g} / \mathrm{L}$

$\mu g /$

$\mu g / \mathrm{k}$

$\mu g /$

$\mu g / L$

$\mu g / L$

$\mu g / L$

$\mu g / k$

$\mathrm{\mu g} / \mathrm{L}$

$\mu g / L$ $\mu g / L$

$\mu g / \mathrm{L}$

$\mu g / L$

$\mu g / L$

$\mu g / L$

$\mu g / L$

Notes:

- exceeded holding time

- exceeded groundwater protection or monitoring constituent standard (See Appencix A.) 
WELL HSB113C (cont.)

Radioactive Constituents

\begin{tabular}{|c|c|c|c|c|c|c|c|}
\hline Constituents & 3098 & Mod & Filt. & ST보 & $\mathrm{DF}$ & Lab & 4Q98 \\
\hline Americium-241 & $<-2.5 E-01$ & UII/ & $<0.5460$ & & 1 & GP & \\
\hline Beta dose & 0.10 & & & & & & \\
\hline Carbon-14 & $1.0 E+02$ & $\|$ & & $\mathbf{\square}$ & 1 & GP & \\
\hline Cobalt-60 & $<-7.3 \mathrm{E}-01$ & $\mathrm{UI} / /$ & $<3.5600$ & & 1 & GP & \\
\hline Curium-242 & $<-2.7 \mathrm{E}-02$ & $\mathrm{UW} / /$ & $<0.2620$ & & 1 & GP & \\
\hline Curium-243/244 & $<-7.8 \mathrm{E}-02$ & $\mathrm{UI} / /$ & $<0.4120$ & & 1 & $\mathrm{GP}$ & \\
\hline Curium-245/246 & $<1.9 E-02$ & $\mathrm{U} 1 / /$ & $<0.2290$ & & 1 & GP & \\
\hline Gross alpha & 9.5E-01 & $\|$ & & & 1 & GP & $2.5 E+00$ \\
\hline lodine-129 & $<1.0 \mathrm{E}+00$ & $\mathrm{UI} / /$ & $<1.3000$ & & 1 & GP & \\
\hline Nickel-63 & $<-7.3 E-01$ & UI & $<3.5600$ & & & & \\
\hline Nonvolatile beta & $2.1 E+01$ & $\|$ & & & 1 & GP & $2.2 E+02$ \\
\hline Plutonium-238 & $<0.0 \mathrm{E}+00$ & UI/I & $<0.0537$ & & 1 & GP & \\
\hline Plutonium-239/240 & $<-4.3 E-03$ & $\mathrm{Ut} / I$ & $<0.0945$ & & 1 & GP & \\
\hline Radium-226 & 6.7E-01 & $/ /$ & & & 1 & GP & \\
\hline Radium-228 & $<6.9 E-01$ & $\mathrm{U} \mid / /$ & $<0.9960$ & & 1 & $\mathrm{GP}$ & \\
\hline Strontium-90 & $<2.3 E-01$ & UIJ/C/ & & & 1 & GP & \\
\hline Technetium-99 & $4.9 E+01$ & $/ /$ & & & 1 & GP & \\
\hline Thorium-228 & 2.1E-01 & $R / 4 /$ & Rej & & 1 & $\mathrm{GP}$ & \\
\hline Thorium-230 & $1.7 E-01$ & /I & & & $i$ & GP & \\
\hline Thorium-232 & $<0.0 \mathrm{E}+00$ & $\mathrm{UI} / /$ & $<0.1020$ & & 1 & GP & \\
\hline Sum of alphas & 1.7E-01 & & & & & & \\
\hline Sum of betas & $1.5 E+02$ & & & $\mathbf{\square}$ & & & \\
\hline Total radium & $6.7 E-01$ & & & & & & \\
\hline Tritium & $9.9 E+02$ & /I & & m & 1 & GP & $1.3 E+03$ \\
\hline Uranium-233/234 & $<3.5 \mathrm{E}-02$ & $\mathrm{U} / /$ & $<0.4240$ & & 1 & GP & \\
\hline Uranium-235 & $<0.0 E+00$ & UIII & $<0.2050$ & & 1 & GP & \\
\hline Uranium-238 & $<0.0 E+00$ & UI/I & $<0.1690$ & & 1 & GP & \\
\hline
\end{tabular}

Filt. $\quad \underline{\text { ST }} \underline{\mathrm{H}}$ DF Lab Unit

$\mathrm{pCin}$

pCin

pCi/L

$\mathrm{pCi} / \mathrm{L}$

pCi/l

$\mathrm{pCi/L}$

pCinL

$1 \mathrm{GE} \mathrm{pCin}$

$\mathrm{pCi} / \mathrm{L}$

$\mathrm{pCi} / \mathrm{L}$

- $1 \mathrm{GE}$ pCi/L

$\mathrm{pCi} / 2$

$\mathrm{pCi} / \mathrm{L}$

pCi/L

$\mathrm{pCi} / \mathrm{L}$

$\mathrm{pCi} / \mathrm{L}$

$\mathrm{pCi} / \mathrm{L}$

$\mathrm{pCi} / \mathrm{L}$

$\mathrm{pCi} / \mathrm{L}$

$\mathrm{pCi} / \mathrm{L}$

$\mathrm{pCi} / \mathrm{L}$

$\mathrm{pCi} / \mathrm{L}$

PCi/L

$<0.1690$ 


\section{WELL HSB113D}

\begin{tabular}{|c|c|}
\hline SRS Coord. & Lat/Longitude \\
\hline $\begin{array}{l}\text { N72302.7 } \\
\text { E56164.3 }\end{array}$ & $81.659355 \%$ \\
\hline
\end{tabular}

SAMPLE DATE

$07 / 14 / 98$

Screen Zone Elevation

$236.2-216.2 \mathrm{ft} \mathrm{msl}$

FIELD DATA

Constituents

Water elevation

pH

Sp. conductance

Water temperature

Alkalinity as $\mathrm{CaCO}_{3}$

Turbidity

Volumes purged

Sampling code

Synchronous water level

ANALYTICAL DATA

Inorganic Constituents

Constituents

0.21

Arsenic, total recoverable $\quad<3.0$

Barium, total recoverabie 50

Cadmium, total recoverable 0.30

Chromium, total recoverable 2.3

Cobalt, total recoverable $\quad 5.5$

Copper, total recoverable 12

Cyanide

Lead, total recoverable

Mercury, total recoverable

Nickel, total recoverable

Nitrate as nitrogen

Nitrate-nitrite as nitrogen $\quad 26.000$

Selenium, total recoverable $<5.0$

Silver, total recoverable $\quad<1.0$

Tin, total recoverable $<2.0$

Vanadium, total recoverable $<2.0$

Zinc, total recoverable

77

3Q98

221.7

3.8

240

22.3

0

1

3.6

$220.9(09 / 17 / 98)$

Filt

/I

UII $<3.0$

J/E

J/E I NDD

$\mathrm{U} / 1$

$\mathrm{J} / \mathrm{E} /$ NDD

Ni

$\mathrm{U} / 1<5.0$

$\mathrm{U} / 1<1.0$

$\mathrm{U} / 1<2.0$

UII $<2.0$

$<2.0$
Top of Cașing

$260.9 \mathrm{ft} \mathrm{ms}$

$11 / 10 / 98$

4Q98

220.7

3.7

250

19.1

0

20

$\mathrm{XN}$

$219.7(12 / 21 / 98)$
Casing Pump Screen Zone

4" PVC V Water Table (IIB2)

Organic Constrituents

Constituents
Benzene
Bis(2-ethylhexyl) phthatate
Dichloromethane
Tetrachloroethylene
Trichloroethylene
Trichlorofluoromethane

3098
$<1.0$
$<10$
$<1.9$
$<1.0$
$<1.0$
$<1.0$

ST브

Trichlorofluoromethane

$\begin{array}{ll}\text { Mod } & \text { Filt. } \\ \text { UJ/O/1 } & <1.0 \\ \text { UJ/Q/ } & <10 \\ \text { UJNO/O8/1 } & <1.0 \\ \text { UJ/O/1 } & <1.0 \\ \text { UJ/O/1 } & <1.0 \\ \text { UJ/O/1 } & <1.0\end{array}$

ST브

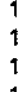

$\mathrm{L}$
$\mathrm{G}$
$\mathrm{G}$
$\mathrm{G}$
$\mathrm{G}$
$\mathrm{G}$
$\mathrm{G}$
$\mathrm{G}$
$\mathrm{G}$

Lab 4Q98 Mod

0.38

- 25 GE 25,000 ll
DF

Lab
GE
GE
GE
GE
GE
GE
Unit

ft mst

$\mathrm{pH}$

$\mu \mathrm{S} / \mathrm{cm}$

${ }^{\circ} \mathrm{C}$

$\mathrm{mg} / \mathrm{L}$

NTU

well vol

$\mathrm{ft} \mathrm{msl}$

Filt.

ST $\mathrm{H}$ DF Lab Unit

$\mu g / L$

$\mu g /$

$\mu g / L$

$\mu g / L$

Hg/L.

Hg/L.

$\mu g / L$

$\mu g / L$

$\mu g$ L

1 GE $\mu g / L$

$\mu g / L$

- $25 \mathrm{GE} \mu \mathrm{g} / \mathrm{L}$

$\mu g / L$

$\mu g /$.

$\mu g / L$

$\mu$ 이.

$\mu g / L$

1 GE

- 1 GE

4Q98 Mod

Filt.

$\underline{\text { ST }} \underline{H}$ DF Lab Unit

HglL

$\mu g / L$

$\mu g / L$

$\mu g / L$

$\mu g / L$

$\mu g / L$

Notes:

- = exceeded holding time

- exceeded groundwater protection or monitoring constituent standard (See Appendix A.) 


\section{WELL. HSB113D (cont.)}

Radioactive Constituents

\begin{tabular}{|c|c|c|c|c|c|c|c|c|c|c|c|c|c|}
\hline Constituents & 3098 & Mod & Filt. & $\underline{\text { ST }} \underline{H}$ & $\underline{\mathrm{DF}}$ & $\underline{\text { Lab }}$ & $4 Q 98$ & Mod & Fill. & $\underline{\mathrm{ST}} \underline{\mathrm{H}}$ & $\underline{\mathrm{DF}}$ & $\underline{\text { Lab }}$ & $\underline{\text { Unit }}$ \\
\hline Americium-241 & $3.4 \mathrm{E}-01$ & /l & & & 1 & GP & & & & & & & $\mathrm{pCi} / \mathrm{L}$ \\
\hline Beta dose & 111.74 & & & a & & & & & & & & & $\mathrm{pCi} / \mathrm{L}$ \\
\hline Carbon-14 & $1.3 E+02$ & "I & & a & 1 & GP & & & & & & & $\mathrm{pCi} / \mathrm{L}$ \\
\hline Cobalt-60 & $3.3 E+01$ & 11 & & & 1 & GP & & & & & & & $\mathrm{pCi} / \mathrm{L}$ \\
\hline Curium-242 & $<-1,2 \mathrm{E}-02$ & $\mathrm{Ut} / /$ & $<0.261$ & & 1 & GP & & & & & & & $\mathrm{pCi} / \mathrm{L}$ \\
\hline Curium-243/244 & $1.3 E+00$ & $/ /$ & & & 1 & GP & & & & & & & $\mathrm{pCi/L}$ \\
\hline Curium-245/246 & $<4.5 \mathrm{E}-02$ & $\mathrm{UI} / /$ & $<0.134$ & & 1 & GP & & & & & & & $\mathrm{pCi} / \mathrm{L}$ \\
\hline Gross alpha & $2.1 \mathrm{E}+01$ & 11 & & ш & 1 & GP & $2.9 \mathrm{E}+01$ & $\|$ & & - & 1 & GE & $\mathrm{pCi} / \mathrm{L}$ \\
\hline lodine-129 & $4.5 \mathrm{E}+01$ & 11 & & & 1 & $G P$ & & & & & & & $\mathrm{pCi} / \mathrm{L}$ \\
\hline Nickel-63 & $3.3 E+01$ & "I & & & & & & & & & & & $\mathrm{pCi} / \mathrm{L}$ \\
\hline Nonvolatile beta & $9.2 E+02$ & "I & & - & 1 & GP & $9.3 E+02$ & $\|$ & & - & 1 & GE & $\mathrm{pCi} / \mathrm{L}$ \\
\hline Plutonium-238 & $<-1.2 \mathrm{E}-02$ & $\mathrm{U} 1 / /$ & $<0.273$ & & 1 & $G P$ & & & & & & & $\mathrm{pCi} / \mathrm{L}$ \\
\hline Plutonium-239/240 & $<3.9 \mathrm{E}-02$ & $\mathrm{U}: / /$ & $<0.273$ & & 1 & GP & & & & & & & $\mathrm{pCilL}$ \\
\hline Radium-226 & $9.7 E+\infty 0$ & 11 & & - & 1 & GP & & & & & & & $\mathrm{pCin}$ \\
\hline Radium-228 & $3.0 \mathrm{E}+00$ & 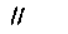 & & & 1 & GP & & & & & & & $\mathrm{pCi} / \mathrm{L}$ \\
\hline Strontium-90 & $5.2 \mathrm{E}+02$ & "I & & - & 1 & GP & & & & & & & $\mathrm{pCi} / \mathrm{L}$ \\
\hline Technetium-99 & $7.7 \mathrm{E}+01$ & "I & & - & 1 & GP & & & & & & & $\mathrm{pCi} / \mathrm{L}$ \\
\hline Thorium-228 & $<2.0 \mathrm{E}-01$ & $U \mathrm{U} / /$ & $<0.799$ & & 1 & GP & & & & & & & $\mathrm{pCi} / \mathrm{L}$ \\
\hline Thorium-230 & $<3.7 \mathrm{E}-01$ & $U: / /$ & $<0.374$ & & 1 & GP & & & & & & & $\mathrm{pCi} / \mathrm{L}$ \\
\hline Thorium-232 & $<1.3 \mathrm{E}-01$ & $\mathrm{U} / / \mathrm{I}$ & $<0.374$ & & 1 & GP & & & & & & & $\mathrm{pCi} / \mathrm{L}$ \\
\hline Sum of alphas & $4.0 \mathrm{E}+00$ & & & & & & & & & & & & $\mathrm{pCi} / \mathrm{L}$ \\
\hline Sum of betas & $8.4 E+02$ & & & - & & & & & & & & & $\mathrm{pCi} / \mathrm{L}$ \\
\hline Total radium & 1.3E+01 & & & & & & & & & & & & $\mathrm{pCi} / \mathrm{L}$ \\
\hline Tritium & $2.2 \mathrm{E}+03$ & "I & & - & 1 & GP & $1.1 \mathrm{E}+03$ & $\|$ & & - & 1 & GE & $\mathrm{pCi} / \mathrm{mL}$ \\
\hline Uranium-233/234 & $1.3 \mathrm{E}+00$ & "I & & & 1 & GP & & & & & & & $\mathrm{pCi} / \mathrm{L}$ \\
\hline Uranium-235 & $2.8 E-01$ & "I & & & 1 & GP & & & & & & & $\mathrm{pCi} / \mathrm{h}$ \\
\hline Uranium-238 & $8.1 E-01$ & "l & & & 1 & GP & & & & & & & $\mathrm{pCl} / \mathrm{L}$ \\
\hline
\end{tabular}

Notes:

- = exceeded holding time

- exceeded groundwater protection or monitoring constituent standard (See Appendix A.) 
WELL HSB114C

$\begin{array}{lll}\text { SRS Coord. } & \text { Lat/Longitude } \\ \text { N72464.6 } & & 33.277589^{\circ} \mathrm{N} \\ \text { E56107.0 } & 81.659820^{\circ} \mathrm{W}\end{array}$

SAMPLE DATE

\section{FIELD DATA}

Constituents

Water elevation

$\mathrm{pH}$

Sp. conductance

Water temperature

Alkalinity as $\mathrm{CaCO}_{3}$

Turbidity

Volumes purged

Sampling code

Synchronous water level
Screen Zone Elevation

$195.6-185.6 \mathrm{ft} \mathrm{ms}$

07/29/98
Top of Casing

$263.8 \mathrm{ft} \mathrm{ms}$

$11 / 05 / 98$

\section{ANALYTICAL DATA}

Inorganic Constituents

\begin{tabular}{|c|c|c|c|c|c|c|c|c|c|c|c|c|c|}
\hline Constituents & $\underline{3098}$ & Mod & Filt. & $\underline{\text { ST }} \underline{H}$ & DF & Lab & 4Q98 & Mod & Filt. & $\underline{\mathrm{ST}} \underline{\mathrm{H}}$ & $\underline{\mathrm{DF}}$ & $\underline{\text { Lab }}$ & Unit \\
\hline Antimony, total recoverable & $<0.20$ & $u / \prime$ & $<0.20$ & & 1 & GE & & & & & & & $\mu g / L$ \\
\hline Arsenic, total recoverable & $<3.0$ & $\mathrm{U} / /$ & $<3.0$ & & 1 & GE & & & & & & & $\mu g / L$ \\
\hline Barium, total recoverable & 25 & "l & & & 1 & GE & & & & & & & $\mu g / L$ \\
\hline Cadmium, total recoverable & 0.52 & $\mathrm{~J} / \mathrm{E} /$ & NDD & & 1 & GE & & & & & & & $\mu g / L$ \\
\hline Chromium, total recoverable & 0.87 & $\mathrm{~J} / \mathrm{E} /$ & NDD & & 1 & GE & & & & & & & $\mu g / L$ \\
\hline Cobalt, total recoverable & 2.0 & "I & & & 1 & GE & & & & & & & $\mu g / L$ \\
\hline Copper, total recoverable & 2.4 & "1 & & & 1 & $\mathrm{GE}$ & & & & & & & $\mu g / L$ \\
\hline Cyanide & $<10$ & $\mathrm{U} / I$ & $<10$ & & 1 & GE & & & & & & & $\mu g / L$ \\
\hline Lead, total recoverable & $<2.0$ & $\mathrm{U} / \mathrm{I}$ & $<2.0$ & & 1 & GE & & & & & & & $\mu g / h$ \\
\hline Mercury, total recoverable & 0.73 & "l & & & 1 & GE & $<0.20$ & $u / I$ & $<0.20$ & & 1 & GE & $\mu g / L$ \\
\hline $\begin{array}{l}\text { Nickel, total recoverable } \\
\text { Nitrate as nitrogen }\end{array}$ & 4.0 & "I & & & 1 & $G E$ & & & & & & & \\
\hline Nitrate-nitrite as nitrogen & 22,000 & NI & & - & 25 & GE & 20,000 & 11 & & ! & 10 & GE & إ \\
\hline Selenium, total recoverable & $<5.0$ & $\mathrm{u} / 1$ & $<5.0$ & & 1 & GE & & & & & & & $g / \mathrm{L}$ \\
\hline Silver, total recoverable & $<1.0$ & $\mathrm{v} / \prime$ & $<1.0$ & & 1 & $\mathrm{GE}$ & & & & & & & $\mu g / L$ \\
\hline Tin, total recoverable & $<2.0$ & $\mathrm{U} / 1$ & $<2.0$ & & 1 & GE & & & & & & & $\mu g / L$ \\
\hline Vanadium, total recoverable & $<2.0$ & $\mathrm{u} / I$ & $<2.0$ & & 1 & GE & & & & & & & $\mu g / L$ \\
\hline Zinc, total recoverable & 20 & $\|$ & & & 1 & $\mathrm{GE}$ & & & & & & & 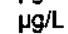 \\
\hline
\end{tabular}

Organic Constituents

$\begin{array}{lllllll}\text { Constituents } & 3098 & \text { Mod } & \text { Filt. } & \text { STH } & \text { DF } & \text { Lab } \\ \text { Benzene } & <1.0 & \text { UJ/O/1 } & <1.0 & & 1 & \text { GE } \\ \text { Bis(2-ethylhexyl) phthalate } & <10 & \text { U/I } & <10 & & 1 & \text { GE } \\ \text { Dichloromethane } & 5.5 & \mathrm{~J} / O / 1 & \text { NDD } & & 1 & \text { GE } \\ \text { Tetrachloroethylene } & <1.0 & \text { UJ/O/1 } & <1.0 & & 1 & \mathrm{GE} \\ \text { Trichloroethylene } & <1.0 & \text { UJ/O/1 } & <1.0 & & 1 & \mathrm{GE} \\ \text { Trichlorofluoromethane } & <1.0 & \mathrm{UJ} / 0 / 1 & <1.0 & & 1 & \mathrm{GE}\end{array}$

$\begin{array}{ll}\text { 4Q98 } & \text { Unit } \\ 221.1 & \mathrm{ftmsl} \\ 4.1 & \mathrm{pH} \\ 200 & \mathrm{HS} / \mathrm{cm} \\ 17.8 & { }^{\circ} \mathrm{C} \\ 0 & \mathrm{mg} / \mathrm{L} \\ 1 & \mathrm{NTU} \\ 3.0 & \text { well vol } \\ \mathrm{N} & \\ (12 / 21 / 98) & \mathrm{ft} \mathrm{msl}\end{array}$

222.8
4.6
200
19.9
0
0.9
2.0
$221.61(9 / 17 / 98)$

Casing Pump

4" PVC V
Barnwell (l1B 1 )

ms

Filt. $\quad \underline{\mathrm{ST}} \underline{\mathrm{H}}$ DF Lab $\underline{\text { Unit }}$

$\mu g / L$

$\mu g / L$

$\mu \mathrm{g} / \mathrm{L}$

$\mu g / \mathrm{L}$

$\mu g / \mathrm{L}$

$\mu g / 2$

Notes:

- = exceeded holding time

- exceeded groundwater protection or monitoring constituent standard (See Appendix A.) 
WELL HSB114C (cont.)

Radioactive Constituents

\begin{tabular}{|c|c|c|c|c|c|c|c|c|c|c|c|c|c|}
\hline Constituents & 3Q98 & Mod & Filt. & $\underline{\text { ST브 }}$ & $\underline{\mathrm{DF}}$ & $\underline{\text { Lab }}$ & $\underline{4 Q 98}$ & Mod & Filt. & $\underline{\mathrm{ST}} \underline{\mathrm{H}}$ & DF & Lab & Unit \\
\hline Americium-241 & $<9.3 E-02$ & $\mathrm{U} \mathrm{W} / \mathrm{I}$ & $<0.2370$ & & 1 & GP & & & & & & & $\mathrm{pCi} / \mathrm{L}$ \\
\hline Beta dose & 3.52 & & & 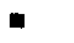 & & & & & & & & & $\mathrm{pCi/L}$ \\
\hline Carbon-14 & $8.6 \mathrm{E}+01$ & $" 1$ & & - & 1 & GP & & & & & & & $\mathrm{pCi} / \mathrm{L}$ \\
\hline Cobalt-60 & $<-5.6 \mathrm{E}-01$ & $U ! I /$ & $<3.7500$ & & 1 & GP & & & & & & & $\mathrm{pCi} / \mathrm{L}$ \\
\hline Curium-242 & $<-1.4 E-02$ & $\mathrm{UI} / /$ & $<0.2660$ & & 1 & $\mathrm{GP}$ & & & & & & & $\mathrm{pCi} / \mathrm{L}$ \\
\hline Curium-243/244 & $<8.2 \mathrm{E}-02$ & $\mathrm{U}: / /$ & $<0.2370$ & & 1 & $G P$ & & & & & & & $\mathrm{pCi} / \mathrm{L}$ \\
\hline Curium-245/246 & $<0.0 E+00$ & $\mathrm{U} \mathrm{I} / \mathrm{I}$ & $<0.0876$ & & 1 & GP & & & & & & & $\mathrm{pCi} / \mathrm{L}$ \\
\hline Gross alpha & $4.4 E+00$ & /I & & & 1 & GP & $<4.4 E+00$ & $\mathrm{U} / /$ & $<1.4 \mathrm{E}+00$ & & 1 & GE & $\mathrm{pCi} / \mathrm{L}$ \\
\hline lodine-129 & $3.4 \mathrm{E}+00$ & "I & & & 1 & GP & & & & & & & $\mathrm{pCi} / \mathrm{L}$ \\
\hline Nickel-63 & $<-5.6 \mathrm{E}-01$ & UH & $<3.7500$ & & & & & & & & & & $\mathrm{pCi} / \mathrm{L}$ \\
\hline Nonvolatile beta & $2.8 \mathrm{E}+01$ & 11 & & & 1 & GP & $1.6 \mathrm{E}+01$ & // & & & 1 & GE & $\mathrm{pCi} / \mathrm{L}$ \\
\hline Plutonium-238 & $<8.7 E-02$ & UIIII & $<0.3220$ & & 1 & GP & & & & & & & $\mathrm{pCi} / \mathrm{L}$ \\
\hline Plutonium-239/240 & $<6.5 E-02$ & $\mathrm{UI} / 1$ & $<0.2650$ & & 1 & GP & & & & & & & $\mathrm{pCi} / \mathrm{L}$ \\
\hline Radium-226 & $1.0 E+01$ & 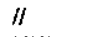 & & - & 1 & GP & & & & & & & $\mathrm{pCi} i \mathrm{~L}$ \\
\hline Radium-228 & $<-5.8 \mathrm{E}-01$ & UIII & $<1.2700$ & & 1 & GP & & & & & & & $\mathrm{pCi} / \mathrm{L}$ \\
\hline Strontium-90 & $<2.4 \mathrm{E}-01$ & UIJ/U & & & 1 & GP & & & & & & & $\mathrm{pCi} / \mathrm{L}$ \\
\hline Technetium-99 & $7.1 E+01$ & "I & & a & 1 & GP & & & & & & & $\mathrm{pCi} / \mathrm{L}$ \\
\hline Thorium-228 & $<8.3 \mathrm{E}-02$ & $\mathrm{U} \mathrm{UJ} / \mathrm{C} /$ & & & 1 & GP & & & & & & & $\mathrm{\rho Ci} / \mathrm{L}$ \\
\hline Thorium-230 & $5.4 \mathrm{E}-01$ & $\mathrm{~J} / \mathrm{C} /$ & NDD & & 1 & GP & & & & & & & $\mathrm{pCi} / \mathrm{L}$ \\
\hline Thorium-232 & $<3.6 \mathrm{E}-02$ & $\mathrm{UIJ} / \mathrm{C} /$ & & & 1 & GP & & & & & & & $\mathrm{pCi} / \mathrm{L}$ \\
\hline Sum of alphas & 5.4E-01 & & & & & & & & & & & & $\mathrm{pCi} / \mathrm{L}$ \\
\hline $\begin{array}{l}\text { Sum of betas } \\
\text { Total radium }\end{array}$ & $\begin{array}{l}1.6 E+02 \\
1.0 E+01\end{array}$ & & & m & & & & & & & & & $\mathrm{PCi} / \mathrm{L}$ \\
\hline Tritium & $2.2 \mathrm{E}+03$ & "l & & - & 1 & GP & $2.6 E+03$ & $\|$ & & n & 1 & $G F$ & $\mathrm{pCi} / \mathrm{L}$ \\
\hline Uranium-233/234 & $<8.2 \mathrm{E}-02$ & $\mathrm{UI} / /$ & $<0.2360$ & & 1 & GP & & & & & 1 & GE & $\begin{array}{l}\mathrm{pCi} / \mathrm{mL} \\
\mathrm{pCi} / \mathrm{L}\end{array}$ \\
\hline Uranium-235 & $<8.1 \mathrm{E}-02$ & $\mathrm{UI} / /$ & $<0.1540$ & & 1 & GP & & & & & & & $\mathrm{pCi} / \mathrm{L}$ \\
\hline Uranium-238 & $<1.4 \mathrm{E}-01$ & $\mathrm{UI} / /$ & $<0.1540$ & & 1 & GP & & & & & & & $\mathrm{pCi} / \mathrm{L}$ \\
\hline
\end{tabular}

Notes:

- = exceeded holding time

- = exceeded groundwater protection or monitoring constituent standard (See Appendix A.) 
WELL HSB114D

\begin{tabular}{|c|c|}
\hline SRS Coord. & Lat/Longitude \\
\hline $\begin{array}{l}N 72474.2 \\
\text { E56104.6 }\end{array}$ & $\begin{array}{l}33.277606^{\circ} \mathrm{N} \\
81.659845^{\circ} \mathrm{V}\end{array}$ \\
\hline
\end{tabular}

SAMPLE DATE

Screen Zone Elevation

232.8-212.8 $f$ msl

FIELD DATA

Constituents

Water elevation

$\mathrm{pH}$

Sp. conductance

Water temperature

Alkalinity as $\mathrm{CaCO}_{3}$

Turbidity

Volumes purged

Sampling code

Synchronous water level

260

20.6

0

10

$221.5(09 / 47 / 98)$
Top of Casing

$264 \mathrm{ft} m s \mathrm{~s}$

$10 / 14 / 98$

220.9

40

320

19.5

2

2.3

$220.4(12 / 21 / 98)$
Casing Pump

4" PVC V
Screen Zone

Water Table (IIB2)
ANALYTICAL DATA

Inorganic Constituents

\begin{tabular}{|c|c|c|c|c|c|c|c|c|c|c|c|c|c|}
\hline Constituents & 3098 & Mod & Filt. & $\underline{\mathrm{ST}} \underline{\mathrm{H}}$ & $\underline{\mathrm{DF}}$ & Lab & 4Q98 & Mod & Filt. & $\underline{\mathrm{ST}} \underline{\mathrm{H}}$ & DF & $\underline{L a b}$ & Unit \\
\hline Antimony, total recoverable & 0.19 & $J / E /$ & NDD & & 1 & GE & & & & & & & $\mu g / L$ \\
\hline Arsenic, total recoverable & $<3.0$ & $\mathrm{U} / /$ & $<3.0$ & & 1 & GE & & & & & & & $\mu g / L$ \\
\hline Barium, total recoverable & 77 & $\|$ & & & 1 & $\overline{G E}$ & & & & & & & $\mu g^{\prime} L$ \\
\hline Cadmium, total recoverable & 0.65 & J/E $/$ & NDD & & 1 & GE & & & & & & & $\mu g / L$ \\
\hline Chromium, total recoverable & 0.95 & $\mathrm{~J} / \mathrm{E} /$ & NDD & & 1 & GE & & & & & & & $\mu g / L$ \\
\hline Cobalt, total recoverable & 16 & $\|$ & & $\mathbf{c}$ & 1 & GE & & & & & & & $\mu g / L$ \\
\hline Copper, total recoverable & 11 & $/ 1$ & & & 1 & GE & & & & & & & $\mu g / \mathrm{L}$ \\
\hline Cyanide & $<10$ & $\mathrm{U} / 1$ & $<10$ & & 1 & $\overline{G E}$ & & & & & & & $\mu g / \mathrm{h}$ \\
\hline Lead, total recoverable & 1.6 & $\mathrm{~J} / \mathrm{E} /$ & NDD & & 1 & GE & & & & & & & Hgil. \\
\hline Mercury, total recoverable & 0.64 & $/ 1$ & & & 1 & GE & 0.050 & $\mathrm{~J} / \mathrm{N}$ & NDD & & 1 & GE & $\mu g / L$ \\
\hline $\begin{array}{l}\text { Nickel, total recoverable } \\
\text { Nitrate as nitrogen }\end{array}$ & 25 & $\|$ & & & 1 & GE & & & & & & & $\mu g / L$ \\
\hline Nitrate-nitrite as nitrogen & 28,000 & $N I$ & & • & 25 & GE & 41,000 & $\|$ & & $\mathbf{w}$ & 25 & GE & $\mu g / L$ \\
\hline Selenium, total recoverable & $<5.0$ & $\mathrm{U} / /$ & $<5.0$ & & 1 & GE & & & & & & & $\mu g / L$ \\
\hline Silver, total recoverable & $<1.0$ & $\mathrm{U} / 1$ & $<1.0$ & & 1 & GE & & & & & & & $\mu g / L$ \\
\hline Tin, total recoverable & $<2.0$ & $\mathrm{U} / 1$ & $<2.0$ & & 1 & GE & & & & & & & $\mu g / L$ \\
\hline Vanadium, total recoverable & $<2.0$ & $\mathrm{U} / /$ & $<2.0$ & & 1 & GE & & & & & & & $\mu g / L$ \\
\hline Zinc, total recoverable & 77 & $/ 1$ & & & 1 & GE & & & & & & & $\mu g / L$ \\
\hline
\end{tabular}

Organic Constituemts

Constituents
Benzene
Bis(2-ethyihexyl) phthalate
Dichloromethane
Tetrachloroethylene
Trichloroethylene
Trichlorofluoromethane

3098
$<1.0$
$<10$
$<2.5$
$<1.0$
$<1.0$
$<1.0$

$\begin{array}{ll}\text { Mod } & \text { Filt. } \\ \text { UJ/O/1 } & <1.0 \\ \text { UJ/Q/ } & <10 \\ U J / N O 8 / 1 & <1.0 \\ U J / O / 1 & <1.0 \\ U J / O / 1 & <1.0 \\ U J / O / 1 & <1.0\end{array}$

ST브

$\begin{array}{ll}\text { OF } & \text { Lab } \\ 1 & \text { GE } \\ 1 & \text { GE } \\ 1 & \text { GE } \\ 1 & \text { GE } \\ 1 & \text { GE } \\ 1 & \text { GE }\end{array}$

Mod

Filt.

ST $\mathrm{H}$ DF Lab Unit

$\mu g / L$

$\mu g /$.

$\mu g / L$

$\mu g / L$

ught

$\mu \mathrm{g} / \mathrm{h}$

Notes:

- exceeded holding time

- exceeded groundwater protection or monitoring constituent standard (See Appendix A.) 
WELL HSB114D (cont.)

Radioactive Constituents

\begin{tabular}{|c|c|c|c|c|c|c|c|}
\hline Constituents & 3Q98 & Mod & Filt. & $\underline{\mathrm{ST}} \underline{\mathrm{H}}$ & DF & Lab & 4Q98 \\
\hline Americium-241 & $<1.0 E-01$ & $\mathrm{Ul} / /$ & $<0.2340$ & & 1 & GP & \\
\hline Beta dose & 208.64 & & & - & & & \\
\hline Carbon-14 & $1.3 E+02$ & $\|$ & & - & 1 & GP & \\
\hline Cobalt-60 & $4.5 E+01$ & $/ /$ & & & 1 & GP & \\
\hline Curium-242 & $<0.0 \mathrm{E}+00$ & $\mathrm{U} 1 / /$ & $<0.1470$ & & 1 & GP & \\
\hline Curium-243/244 & 2.2E-01 & /I & & & 1 & GP & \\
\hline Currium-245/246 & $<0.0 \mathrm{E}+00$ & $\mathrm{UI} / /$ & $<0.1330$ & & 1 & GP & \\
\hline Gross alpha & $3.5 E+01$ & $/ 1$ & & $\mathbf{\square}$ & 1 & GP & $2.5 E+01$ \\
\hline lodine-129 & 1.1E+02 & $/ /$ & & $\mathbf{D}$ & 1 & GP & \\
\hline Nickel-63 & $4.5 E+01$ & $/ 1$ & & & & & \\
\hline Nonvolatile beta & $1.6 E+03$ & $\| /$ & & $\mathbf{\square}$ & 1 & GP & $1.2 E+03$ \\
\hline Plutonium-238 & $<0.0 E+00$ & $\mathrm{UI} / /$ & $<0.1590$ & & 1 & GP & \\
\hline Plutonium-239/240 & $<1.1 \mathrm{E}-01$ & UU/ & $<0.1590$ & & 1 & GP & \\
\hline Radium-226 & $1.6 E+01$ & $/ /$ & & $\mathbf{B}$ & 1 & GP & \\
\hline Radium-228 & $4.5 E+\infty 0$ & $\|$ & & & 1 & GP & \\
\hline Strontium-90 & $7.7 \mathrm{E}+02$ & $\|$ & & a & $i$ & GP & \\
\hline Technetium-99 & $9.7 \mathrm{E}+01$ & $/ 1$ & & $\mathbf{n}$ & 1 & GP & \\
\hline Thorium-228 & $<4.3 \mathrm{E}-01$ & $\mathrm{Ul} / /$ & $<0.8000$ & & 1 & GP & \\
\hline Thorium-230 & $6.6 \mathrm{E}-01$ & 11 & & & $\uparrow$ & GP & \\
\hline Thorium-232 & $<-2.0 E-02$ & $\mathrm{UI} / /$ & $<0.4340$ & & 1 & GP & \\
\hline Sum of alphas & $4.5 E+00$ & & & & & & \\
\hline Sum of betas & $1.2 \mathrm{E}+03$ & & & a & & & \\
\hline Total radium & $2.1 E+01$ & & & & & & \\
\hline Tritium & $4.1 E+03$ & /l & & a & 1 & GP & $6.1 E+03$ \\
\hline Uranium-233/234 & $2.4 \mathrm{E}+00$ & $1 /$ & & & 1 & GP & \\
\hline Uranium-235 & $<5.1 \mathrm{E}-02$ & $\mathrm{UI} / /$ & $<0.1520$ & & 1 & GP & \\
\hline Uranium-238 & $1.3 E+00$ & $/ 1$ & & & 1 & $\mathrm{GP}$ & \\
\hline
\end{tabular}

Filt. $\quad \underline{\mathrm{ST}} \underline{H}$ DF Lab Unit

pCill

$\mathrm{pCi} / \mathrm{L}$

pCi/L

oCi/L

$\mathrm{pCi} / \mathrm{L}$

pCi/l

$p \mathrm{Ci} / \mathrm{L}$

- 1 GE $\mathrm{pCi} / \mathrm{L}$

$\mathrm{pCi} / \mathrm{L}$

$\mathrm{pCi} / \mathrm{L}$

- 1 gE pCirl

pCi/L

$p \mathrm{Ci} / \mathrm{L}$

$\mathrm{pCi} / \mathrm{L}$

pCi/L

$\mathrm{pCi} / \mathrm{L}$

$\mathrm{pCi} / \mathrm{L}$

$\mathrm{pCi} / \mathrm{L}$

pCi/L

pCi/L

$\mathrm{pCi} / \mathrm{L}$

$\mathrm{pCill}$

$\mathrm{pCi} / \mathrm{L}$

$\mathrm{pCi} / \mathrm{mL}$

pCill.

pCilL

$\mathrm{PCi} / \mathrm{L}$

Notes:

- = exceeded holding time

- = exceeded groundwater protection or monitoring constituent standard (See Appendix A.) 
WELL HSB115C

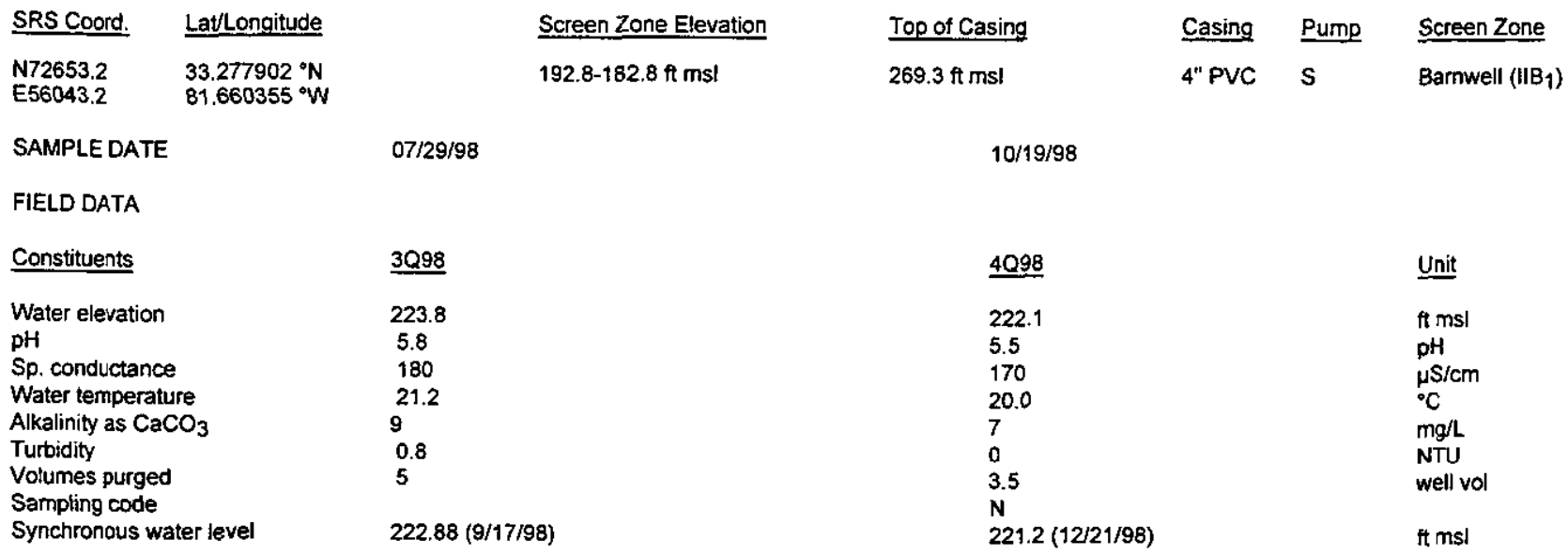

ANALYTICAL DATA

Inorganic Constituemts

\begin{tabular}{|c|c|c|c|c|c|c|c|c|c|c|c|c|c|}
\hline Constituents & $\underline{3098}$ & Mod & Filt. & $\underline{\text { ST}} \underline{\underline{H}}$ & DF & $\underline{\text { Lab }}$ & 4Q98 & Mod & Filt. & $\underline{S T} \underline{H}$ & DF & $\underline{L a b}$ & Unit \\
\hline Antimony, total recoverable & $<0.20$ & $\mathrm{U} / /$ & $<0.20$ & & 1 & $\mathrm{GE}$ & & & & & & & \\
\hline Arsenic, total recoverable & $<3.0$ & $\mathrm{U} / /$ & $<3.0$ & & 1 & GE & & & & & & & \\
\hline Barium, total recoverable & 21 & /I & & & 1 & GE & & & & & & & \\
\hline Cadmium, total recoverable & 0.62 & $\mathrm{~J} / \mathrm{E} /$ & NDD & & 1 & $\mathrm{GE}$ & & & & & & & \\
\hline Chromium, total recoverable & 0.61 & $J / E I$ & NDD & & 1 & $\mathrm{GE}$ & & & & & & & \\
\hline Cobalt, total recoverable & 1.7 & /I & & & 1 & GE & & & & & & & \\
\hline Copper, total recoverable & 1.7 & "ll & & & $i$ & GE & & & & & & & \\
\hline Cyanide & $<10$ & $\mathrm{U} / \mathrm{I}$ & $<10$ & & 1 & $\mathrm{GE}$ & & & & & & & \\
\hline Lead, total recoverable & 0.76 & J/EV/ & NDD & & 1 & GE & & & & & & & \\
\hline Mercury, total recoverable & $<0.20$ & $\mathrm{u} / \prime$ & $<0.20$ & & 1 & GE & $<0.20$ & $\mathrm{U} / /$ & $<0.20$ & & 1 & GE & \\
\hline $\begin{array}{l}\text { Nickel, total recoverable } \\
\text { Nitrate as nitrogen }\end{array}$ & 2.0 & /l & & & 1 & GE & & & & & & & \\
\hline Nitrate-nitrite as nitrogen & 17,000 & $N /$ & & $=$ & 25 & GE & 17000 & $1 / 6$ & & n & 10 & GE & \\
\hline Selenium, total recoverable & $<5.0$ & $\mathrm{U} / /$ & $<5.0$ & & 1 & GE & & & & & & & \\
\hline Silver, total recoverable & $<1.0$ & $\mathrm{U} / \mathrm{I}$ & $<1.0$ & & $i$ & $\mathrm{GE}$ & & & & & & & \\
\hline Tin, total recoverable & $<2.0$ & $\mathrm{U} / / \mathrm{I}$ & $<2.0$ & & 1 & GE & & & & & & & \\
\hline Vanadium, total recoverable & $<2.0$ & $\mathrm{U} / / \mathrm{I}$ & $<2.0$ & & 1 & GE & & & & & & & \\
\hline Zinc, total recoverable & $<5.0$ & $\mathrm{U} / /$ & $<5.0$ & & 1 & $\mathrm{GE}$ & & & & & & & \\
\hline \multicolumn{14}{|l|}{ Organic Constituemts } \\
\hline Constituents & 3098 & Mod & Filt. & $\underline{\text { ST }} \underline{\underline{H}}$ & $\underline{\text { DF }}$ & $\underline{\underline{L a b}}$ & $4 Q 98$ & Mod & Filt. & $\underline{\mathrm{ST}} \underline{\mathrm{H}}$ & DF & Lab & \\
\hline Benzene & $<1.0$ & $\mathrm{UJ} / \mathrm{O} / 1$ & $<1.0$ & & 1 & GE & & & & & & & \\
\hline Bis(2-ethylhexyl) phthalate & 31 & $\|$ & & $=$ & 1 & GE & & & & & & & \\
\hline Dichloromethane & 5.9 & $\mathrm{~J} / \mathrm{O} / 1$ & NDD & & 1 & GE & & & & & & & \\
\hline Tetrachioroethylene & $<1.0$ & $\mathrm{UJ} / \mathrm{O} / 1$ & $<1.0$ & & 1 & GE & & & & & & & \\
\hline Trichloroethylene & $<1.0$ & UJ/O/1 & $<1.0$ & & 1 & $\mathrm{GE}$ & & & & & & & \\
\hline Trichlorofluoromethane & $<1.0$ & UJ/O/1 & $<1.0$ & & $i$ & $\mathrm{GE}$ & & & & & & & \\
\hline
\end{tabular}

Notes:

- exceeded holding time

- exceeded groundwater protection or monitoring constituent standard (See Appendix A.) 
WELL HSB115C (cont.)

Radioactive Constituents

\begin{tabular}{|c|c|c|c|c|c|c|c|}
\hline Constituents & 3Q98 & Mod & Filt. & $\underline{\text { ST }} \underline{\text { H }}$ & $\underline{\mathrm{DF}}$ & $\underline{\text { Lab }}$ & 4Q98 \\
\hline Americium-241 & $<4.9 \mathrm{E}-02$ & $\mathrm{U} 1 / l$ & $<0.2480$ & & 1 & GP & \\
\hline Beta dose & 8.77 & & & $\mathbf{\square}$ & & & \\
\hline Carbon-14 & $5.7 E+01$ & $/ 1$ & & घ & 1 & GP & \\
\hline Cobalt -60 & $<5.5 \mathrm{E}-01$ & $\mathrm{UI} / /$ & $<3.8200$ & & 1 & GP & \\
\hline Curium-242 & $<9.7 E-03$ & $\mathrm{UI} / /$ & $<0.2420$ & & $t$ & GP & \\
\hline Curium-243/244 & $<1.6 \mathrm{E}-01$ & $\mathrm{UI} / /$ & $<0.1730$ & & 1 & $\mathrm{GP}$ & \\
\hline Curium-245/246 & $<3.3 E-02$ & $\mathrm{UI} / I$ & $<0.0984$ & & 1 & GP & \\
\hline Gross alpha & $5.1 E+00$ & $/ 1$ & & & $\uparrow$ & GP & $5.9 E+00$ \\
\hline lodine-129 & $8.7 E+00$ & $/ /$ & & & 1 & GP & \\
\hline Nickel-63 & $<5.5 E-01$ & UI & $<3.8200$ & & & & \\
\hline Nonvolatile beta & $3.1 E+01$ & $/ 1$ & & & 1 & GP & $5.3 E+01$ \\
\hline Plutonium-238 & $<1.2 E-01$ & $\mathrm{UI} / /$ & $<0.3590$ & & 1 & GP & \\
\hline Plutonium-239/240 & $<-8.7 E-02$ & $\mathrm{UI} / /$ & $<0.4660$ & & 1 & GP & \\
\hline Radium-226 & $3.8 E+00$ & $/ /$ & & & 1 & GP & \\
\hline Radium-228 & $<-1.2 E+00$ & $\mathrm{UI} / /$ & $<1.2000$ & & 1 & GP & \\
\hline Strontium-90 & $5.5 E+00$ & $\mathrm{~J} / \mathrm{L} /$ & NDD & & 1 & GP & \\
\hline Technetium-99 & $3.9 E+01$ & $/ /$ & & & 1 & GP & \\
\hline Thorium-228 & $<-5.6 \mathrm{E}-03$ & Uls/Cl & & & 1 & GP & \\
\hline Thorium-230 & $2.3 E-01$ & $\mathrm{~J} / \mathrm{C} /$ & NDD & & 1 & GP & \\
\hline Thorium-232 & $<8.7 E-02$ & $\mathrm{UIJ} / \mathrm{Cl}$ & & & 1 & $G P$ & \\
\hline Sum of alphas & 2.3E-01 & & & & & & \\
\hline Sum of betas & $1.1 E+02$ & & & $=$ & & & \\
\hline Total radium & $3.8 E+00$ & & & & & & \\
\hline Tritium & $2.4 E+03$ & $\|$ & & E & 1 & $G P$ & $2.7 E+03$ \\
\hline Uranium-233/234 & $<1.4 \mathrm{E}-01$ & UI/I & $<0.2760$ & & 1 & GP & \\
\hline Uranium-235 & $<-2.0 E-02$ & UIII & $<0.1960$ & & 1 & $\mathrm{GP}$ & \\
\hline Uranium-238 & $<-3.4 \mathrm{E}-02$ & UIII & $<0.2270$ & & 1 & GP & \\
\hline
\end{tabular}

Filt. $\quad \underline{\text { ST }} \underline{\mathrm{H}}$ DF Lab Unit

$\mathrm{pCi} / \mathrm{L}$

PCi/L

pCi/L

$\mathrm{PCi} / \mathrm{L}$

PCill

PCil

$\mathrm{pCi/L}$

$1 \mathrm{TM} \mathrm{PCi} / \mathrm{h}$

pCill

$\mathrm{pCilL}$

TM PCi/L

$\mathrm{pCi}$.

pCinl

pCi/L

$\mathrm{pCi} / \mathrm{L}$

$\mathrm{pCi} / \mathrm{L}$

pCi/L

$\mathrm{PCi} / \mathrm{L}$

pCi/L

pCi/L

$\mathrm{pCi} / \mathrm{L}$

pCi/L

$\mathrm{pCi} / \mathrm{L}$

$<0.2270$

$\mathrm{pCi} / \mathrm{mL}$

$\mathrm{pCi} / \mathrm{L}$

PCi/L

pCi/L

\section{Notes:}

- exceeded holding time

- = exceeded groundwater protection or monitoring constituent standard (See Appendix A.) 
WELL HSB115D

\begin{tabular}{ll} 
SRS Coord. & Lat/Longitude \\
\hline N72662.3 & $33.277916^{\circ} \mathrm{N}$ \\
E56039.8 & $81.660381^{\circ} \mathrm{W}$
\end{tabular}

Screen Zone Elevation

233.9-213.9 ft msl
SAMPLE DATE

$09 / 29 / 98$

Top of Casing

$269.1 \mathrm{ft} \mathrm{ms!}$

10/14/98

FIELD DATA

Constituents

Water elevation

$\mathrm{pH}$

Water temperature

Alkalinity as $\mathrm{CaCO}_{3}$

Turbidity

Volumes purged

Sampling code

Synchronous water level

ANALYTICAL DATA

Inorganic Constituents
3098

222.4

3.9

280

23.3

0

2.9

$\mathrm{XN}$

$222.6(09 / 17 / 98)$
$\mathrm{Sp}$. conductance
Casing Pump
4" PVC S
Screen Zone

Water Table (IIB2)

\begin{tabular}{|c|c|c|c|c|c|c|c|c|c|c|c|c|c|}
\hline Constituents & $\underline{3098}$ & $\underline{\text { Mod }}$ & Filt. & $\underline{\text { ST }} \underline{H}$ & $\underline{\mathrm{DF}}$ & Lab & 4098 & Mod & Filt. & $\underline{\text { ST }} \underline{H}$ & $\underline{D F}$ & $\underline{\text { Lab }}$ & Unit \\
\hline Antimony, total recoverable & 0.61 & /I & & & 1 & GE & & & & & & & $\mu g / L$ \\
\hline Arsenic, total recoverable & $<3.0$ & $\mathrm{U} / / \mathrm{I}$ & $<3.0$ & & 1 & $\mathrm{GE}$ & & & & & & & $\mu g / L$ \\
\hline Barium, total recoverable & 63 & $/ 1$ & & & $i$ & GE & & & & & & & $\mu g / 1$ \\
\hline Cadmium, total recoverable & 0.77 & $\mathrm{~J} / \mathrm{E} /$ & NDD & & 1 & $\mathbf{G E}$ & & & & & & & $\mu g / L$ \\
\hline Chromium, total recoverable & 3.5 & 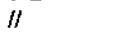 & & & 1 & GE & & & & & & & $\mu g / L$ \\
\hline Cobalt, total recoverable & 7.3 & "I & & - & 1 & $\mathrm{GE}$ & & & & & & & $\mu g / L$ \\
\hline Copper, total recoverable & 27 & /l & & & 1 & GE & & & & & & & $\mu g / L$ \\
\hline Cyanide & 2.3 & $\mathrm{~J} / \mathrm{E} /$ & NDD & & 1 & $\mathrm{GE}$ & & & & & & & $\mu g / L$ \\
\hline Lead, total recoverable & 21 & 11 & & - & $i$ & $\mathrm{GE}$ & & & & & & & $\mu \mathrm{g} / \mathrm{L}$ \\
\hline Mercury, total recoverable & $<0.20$ & $\mathrm{U} / \prime$ & $<0.20$ & & 1 & GE & $<0.20$ & $\mathrm{U} / /$ & $<0.20$ & & 1 & GE & $\mu g / L$ \\
\hline $\begin{array}{l}\text { Nickel, total recoverable } \\
\text { Nitrate as nitrogen }\end{array}$ & 77 & $\|$ & & & 1 & GE & & & & & & & $\mu g h$ \\
\hline Nitrate-nitrite as nitrogen & 9,100 & $N /$ & & & 5 & GE & 31,000 & $\|$ & & - & 25 & GE & $\mu g / L$ \\
\hline Selenium, total recoverable & $<5.0$ & $\mathrm{U} / \mathrm{I}$ & $<5.0$ & & 1 & $\mathrm{GE}$ & & & & & & & $\mu g / \mathrm{L}$ \\
\hline Silver, total recoverable & $<1.0$ & $\mathrm{U} / /$ & $<1.0$ & & 1 & GE & & & & & & & $\mu \mathrm{g} / \mathrm{L}$ \\
\hline Tin, total recoverable & $<2.0$ & $\mathrm{U} / /$ & $<2.0$ & & $i$ & GE & & & & & & & Ho/L \\
\hline Vanadium, total recoverable & 3.9 & $\|$ & & & 1 & GE & & & & & & & $\mu g / L$ \\
\hline Zinc, total recoverable & 45 & $\|$ & & & 1 & $\mathrm{GE}$ & & & & & & & $\mu \mathrm{g} / \mathrm{L}$ \\
\hline \multicolumn{14}{|l|}{ Organic Constituents } \\
\hline Constituents & $\underline{3098}$ & Mod & Filt. & $\underline{\text { ST }} \underline{H}$ & $\underline{D F}$ & $\underline{\text { Lab }}$ & 4098 & Mod & Filt. & $\underline{\mathrm{ST}} \underline{\mathrm{H}}$ & DF & $\underline{\text { Lab }}$ & Unit \\
\hline Benzene & $<1.0$ & $\mathrm{UJ} / \mathrm{O} / 1$ & $<1.0$ & & 1 & GE & & & & & & & \\
\hline Bis(2-ethylhexyl) phthalate & $<10$ & $\mathrm{UJ} / \mathrm{Q} /$ & $<10$ & - & 1 & $\mathrm{GE}$ & & & & & & & $\mu \mathrm{g} / \mathrm{L}$ \\
\hline Dictiloromethane & $<2.6$ & UJNO8/1 & $<1.0$ & & 1 & GE & & & & & & & ug/h \\
\hline Tetrachloroethylene & $<1.0$ & $\mathrm{UJ} / \mathrm{O} / \mathrm{A}$ & $<1.0$ & & 1 & GE & & & & & & & $\mu g / L$ \\
\hline Trichloroethylene & $<1.0$ & UJ/O/1 & $<1.0$ & & 1 & GE & & & & & & & $\mu \mathrm{g} / \mathrm{L}$ \\
\hline Trichlorofluoromethane & $<1.0$ & UJ/O/1 & 1.0 & & 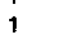 & $=$ & & & & & & & \\
\hline
\end{tabular}

\section{Lead, to}

Nitrate-nitnite as nitroger

(t)

Zinc, total recoverable
$<1.0$

$4 Q 98$
222.1
4.0
250
26.0
0
12
1.3
$X N$
$221.2(12 / 21 / 98)$

$\underline{\text { Unit }}$

$\mathrm{ft} \mathrm{ms}$

$\mathrm{pH}$

$\mu S / \mathrm{cm}$

${ }^{\circ} \mathrm{C}$

NTU

well vol

ft ms! 
WELL HSB115D (cont.)

Radioactive Constituents

\begin{tabular}{|c|c|c|c|c|c|c|c|c|c|c|c|c|c|}
\hline Constituents & $\underline{3 Q 98}$ & Mod & Filt. & $\underline{\text { ST }} \underline{\mathrm{H}}$ & $\underline{\mathrm{DF}}$ & Lab & 4Q98 & Mod & Filt. & $\underline{\mathrm{ST}} \underline{\mathrm{H}}$ & DF & $\underline{L a b}$ & Unit \\
\hline Americium-241 & $<-1.9 E-02$ & $\mathrm{UI} / /$ & $<0.1380$ & & 1 & GP & & & & & & & $\mathrm{pCi} / \mathrm{L}$ \\
\hline Beta dose & 123.88 & & & - & & & & & & & & & $\mathrm{pCi} / \mathrm{L}$ \\
\hline Carbon-14 & $3.2 E+01$ & $/ /$ & & & 1 & GP & & & & & & & $\mathrm{pCi} / \mathrm{L}$ \\
\hline Cobalt-60 & $2.8 E+01$ & /I & & & 1 & GP & & & & & & & $\mathrm{pCi} / \mathrm{L}$ \\
\hline Curium-242 & $<0.0 \mathrm{E}+00$ & $\mathrm{UI} / /$ & $<0.1530$ & & 1 & GP & & & & & & & $\mathrm{pCi} / \mathrm{L}$ \\
\hline Curium-243/244 & $<-1.1 E-02$ & $\mathrm{U} / / /$ & $<0.2440$ & & 1 & GP & & & & & & & $\mathrm{pCi} / \mathrm{L}$ \\
\hline Curium-245/246 & $<0.0 E+00$ & $\mathrm{UI} / /$ & $<0.1380$ & & 1 & GP & & & & & & & pCilL \\
\hline Gross alpha & $1.4 E+01$ & $\|$ & & & 1 & GP & $3.5 E+01$ & $/ /$ & & - & 1 & GE & $\mathrm{pCi} / \mathrm{L}$ \\
\hline lodine-129 & $5.8 E+00$ & /I & & & 1 & GP & & & & & & & $\mathrm{pCill}$ \\
\hline Nickel-63 & $2.8 E+01$ & // & & & & & & & & & & & $\mathrm{PCi} / \mathrm{L}$ \\
\hline Nonvolatile beta & $1.7 \mathrm{E}+03$ & $/ /$ & & a & 1 & GP & $2.7 E+03$ & $/ /$ & & ש & 1 & GE & $\mathrm{pCi} / \mathrm{L}$ \\
\hline Plutonium-238 & $<3.3 E-02$ & $\mathrm{UI} / /$ & $<0.2290$ & & 1 & GP & & & & & & & pCill \\
\hline Plutonium-239/240 & $<-3.1 E-02$ & $\mathrm{UI} / /$ & $<0.3010$ & & $i$ & GP & & & & & & & $\mathrm{pCi} / \mathrm{L}$ \\
\hline Radium-226 & $6.0 E+00$ & $\|$ & & $\mathbf{a}$ & 1 & GP & & & & & & & $\mathrm{pCi} / \mathrm{L}$ \\
\hline Radium-228 & $4.6 E+00$ & $/ 1$ & & & 1 & GP & & & & & & & pCill \\
\hline Strontium-90 & $9.3 \mathrm{E}+02$ & $\|$ & & $\mathbf{\square}$ & 1 & GP & & & & & & & $\mathrm{pCi} / \mathrm{L}$ \\
\hline Technetium-99 & $4.5 E+01$ & $/ /$ & & & 1 & GP & & & & & & & $\mathrm{pCi} / \mathrm{L}$ \\
\hline Thorium-228 & $7.4 E-01$ & $R / 4 /$ & Rej & & 1 & GP & & & & & & & $\mathrm{pCi} / \mathrm{L}$ \\
\hline Thorium-230 & $<0.0 E+00$ & U!ll & $<0.3060$ & & 1 & GP & & & & & & & $\mathrm{pCi} / \mathrm{L}$ \\
\hline Thorium-232 & $<0.0 E+00$ & $\mathrm{UI} / /$ & $<0.3060$ & & 1 & GP & & & & & & & $\mathrm{pCi} / \mathrm{L}$ \\
\hline Sum of alphas & $1.8 E+00$ & & & & & & & & & & & & $\mathrm{pCi} / \mathrm{L}$ \\
\hline Sum of betas & $1.1 E+03$ & & & $\boldsymbol{\square}$ & & & & & & & & & $\mathrm{pCi} / \mathrm{L}$ \\
\hline Total radium & $1.1 E \div 01$ & & & & & & & & & & & & $\mathrm{pCi} / \mathrm{L}$ \\
\hline Tritium & $2.8 \mathrm{E}+02$ & $/ 1$ & & $\mathbf{0}$ & 1 & GP & $4.2 \mathrm{E}+03$ & $\|$ & & a & 1 & GE & $\mathrm{pCi} / \mathrm{mL}$ \\
\hline Uranium-233/234 & $1.3 E+00$ & $\|$ & & & 1 & GP & & & & & & & $\mathrm{pCi} / \mathrm{L}$ \\
\hline Uranium-235 & $<1,2 \mathrm{E}-01$ & $\mathrm{U}] / /$ & $<0.1730$ & & 1 & GP & & & & & & & $\mathrm{pCi} / \mathrm{L}$ \\
\hline Uranium-238 & $5.0 \mathrm{E}-01$ & $\|$ & & & 1 & GP & & & & & & & $\mathrm{pCi} / \mathrm{L}$ \\
\hline
\end{tabular}

Notes:

- exceeded holding time

- exceeded groundwater protection or monitoring constituent standard (See Appendix A.) 


\section{WELL HSB116C}

\begin{tabular}{|c|c|}
\hline SRS Coord. & Lat/Longitude \\
\hline $\begin{array}{l}\text { N72888.1 } \\
\text { E55989.1 }\end{array}$ & $81.660953^{\circ}$ \\
\hline
\end{tabular}

SAMPLE DATE

07/23/98

$190.5-180.5 \mathrm{ft} \mathrm{ms}$

FIELD DATA

Constituents

$\underline{3098}$

225.2

pH

Sp. conductance

Water temperature

Alkalinity as $\mathrm{CaCO}_{3}$

Turbidity

Volumes purged

Sampling code

Synchronous water level

ANALYTICAL DATA

Inorganic Constituents

Constituents

Antimony, total recoverable

Arsenic, total recoverable

Barium, total recoverable

Cadmium, total recoverable $<1.0$

Chromium, total recoverable $<3.0$

Cobalt, total recoverable $\quad 3.8$

Copper, total recoverable $\quad 0.65$

Cyanide

Lead, total recoverable

Mercury, total recoverable

Nickel, total recoverable

Nitrate as nitrogen

Nitrate-nitrite as nitrogen $\quad 8,800$

Selenium, total recoverabie $<5.0$

Silver, total recoverable

Tin, total recoverable

$<1.0$

$<2.0$

Zinc, total recoverable

$<2.0$

Organic Constituents

Constituents

Benzene

Bis(2-ethylhexyl) phthalate

Dichloromethane

Tetrachloroethylene

Trichloroethylene

Trichlorofluoromethane
3098

$<1.0$

$<10$

$<1.5$

$<1.0$

$<1.0$

$<1.0$
5.0

92

20.2

$224.5(09 / 17 / 98)$

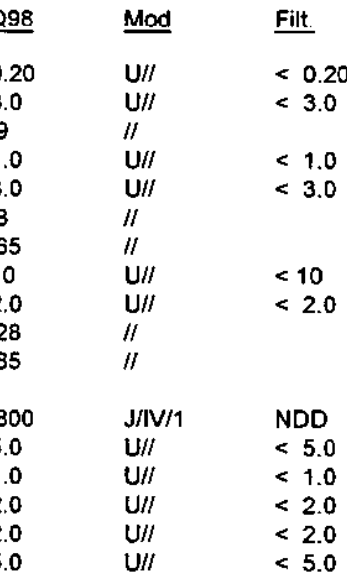

$\begin{array}{ll}\text { Mod } & \text { Filt. } \\ \text { UJ/O/1 } & <1.0 \\ \text { UII } & <10 \\ \text { UJNOO8/1 } & <1.0 \\ \text { UJ/O/1 } & <1.0 \\ \text { UJ/O/1 } & <1.0 \\ \text { UJ/O/1 } & <1.0\end{array}$

\section{Top of Casing}

$257.5 \mathrm{ft} \mathrm{msi}$

10/28/98

4098
223.5
5.2
85
19.5
0
0
2.6
$222.7(12 / 21 / 98)$

Casing Pump

4" PVC V

Screen Zone

Bamwell ( $\left(\mathrm{IB}_{1}\right)$
Unit

$\mathrm{ftms}$

$\mathrm{pH}$

$\mu \mathrm{S} / \mathrm{cm}$

${ }^{\circ} \mathrm{C}$

$\mathrm{mg} / \mathrm{L}$

well vol

ft msl

ST브

DF Lab 4Q98 Mod

Filt.

ST 브 DF Lab Unit

$\begin{array}{ccccc} & 1 & \mathrm{GE} & & \\ & 1 & \mathrm{GE} & & \\ & 1 & \mathrm{GE} & & \\ & 1 & \mathrm{GE} & & \\ - & 1 & \mathrm{GE} & & \\ 1 & \mathrm{GE} & & \\ 1 & \mathrm{GE} & & \\ 1 & \mathrm{GE} & & \\ 1 & \mathrm{GE} & & \\ 1 & \mathrm{GE} & 0.24 & & \\ 1 & \mathrm{GE} & & \\ & & & \\ & & & \\ 1 & \mathrm{GE} & 8.900 & & \end{array}$

GE $\mu g / L$

$\mu g / L$

3 GE $\mu g / L$

$\mu g / L$

$\mu g / 2$

$\mu g / L$

$\mu g / L$

$\underline{\text { ST }} \underline{H}$

$\begin{array}{ll}\text { DF } & \text { Lab } \\ 1 & \mathrm{GE} \\ 1 & \mathrm{GE} \\ 1 & \mathrm{GE} \\ 1 & \mathrm{GE} \\ 1 & \mathrm{GE} \\ 1 & \mathrm{GE}\end{array}$

Mod

Filt.

$\underline{\text { ST }}$ H DF Lab Unit

$\mu g / L$

$\mu g / L$

$\mu g / L$

$\mu g / L$

$\mu g / 2$

$\mu g / L$

Notes:

- = exceeded holding time

a = exceeded groundwater protection or monitoring constituent standard (See Appendix A.) 
WELL HSB116C (cont.)

Radioactive Constituents

\begin{tabular}{|c|c|c|c|c|c|c|c|c|c|c|c|c|c|}
\hline Constituents & $\underline{3 Q 98}$ & Mod & Filt. & STI보 & $\underline{\mathrm{DF}}$ & $\underline{\text { Lab }}$ & $4 Q 98$ & Mod & Filt. & $\underline{\mathrm{ST}} \underline{\mathrm{H}}$ & DF & $\underline{\text { Lab }}$ & Unit \\
\hline $\begin{array}{l}\text { Americium-241 } \\
\text { Beta dose }\end{array}$ & $\begin{array}{l}<3.4 E-02 \\
2.44\end{array}$ & $\mathrm{UI} / /$ & $<0.0642$ & - & 1 & GP & & & & & & & $\mathrm{pCi} / \mathrm{L}$ \\
\hline Carbon-14 & $<5.8 E+00$ & $\mathrm{UI} / /$ & $<8.4100$ & & 1 & GP & & & & & & & $\mathrm{pCi} / \mathrm{L}$ \\
\hline Cobalt-60 & $<7.2 \mathrm{E}-01$ & $\mathrm{U} \mathrm{l} / /$ & $<4.0500$ & & 1 & GP & & & & & & & $\mathrm{pCi} / \mathrm{L}$ \\
\hline Curium-242 & $<-1.1 E-02$ & $\mathrm{U} I / /$ & $<0.1400$ & & 1 & GP & & & & & & & $\mathrm{pCi/h}$ \\
\hline Curium-243/244 & $<0.0 \mathrm{E}+00$ & UIII & $<0.0642$ & & 1 & GP & & & & & & & $\mathrm{PCV}$ \\
\hline Curium-245/246 & $<0.0 \mathrm{E}+00$ & $U 1 / /$ & $<0.0642$ & & 1 & GP & & & & & & & $\begin{array}{l}\mathrm{pCi} / \mathrm{L} \\
\mathrm{pCi} / \mathrm{L}\end{array}$ \\
\hline Gross alpha & $1.1 \mathrm{E}+00$ & $\mathrm{~J} / \mathrm{C} /$ & NDD & & 1 & GP & $<1.4 \mathrm{E}+00$ & $U / I$ & $<1.0 \mathrm{E}+00$ & & 1 & GE & $\mathrm{pCi} / \mathrm{L}$ \\
\hline lodine- 129 & $2.4 E+00$ & /1 & & & 1 & GP & & & & & & & $\mathrm{pCi} / \mathrm{L}$ \\
\hline Nicke!-63 & $<7.2 \mathrm{E}-01$ & UI & $<4.0500$ & & & & & & & & & & $\mathrm{pCi} / \mathrm{L}$ \\
\hline Nonvolatile beta & $7.1 E+\infty$ & "I & & & 1 & GP & $<2.4 \mathrm{E}+\infty 0$ & $\mathrm{U} / /$ & $<1.6 \mathrm{E}+00$ & & 1 & GE & $\mathrm{pCi} / \mathrm{L}$ \\
\hline Plutonium-238 & $<0.0 \mathrm{E}+00$ & $\mathrm{U} 1 / /$ & $<0.0521$ & & 1 & GP & & & & & & & $\mathrm{pCi} / \mathrm{L}$ \\
\hline Plutonium-239/240 & $<1.7 E-02$ & $U \mathrm{II} / \mathrm{I}$ & $<0.0520$ & & 1 & GP & & & & & & & $\mathrm{pC} / 2$ \\
\hline Radium-226 & $1.5 \mathrm{E}+00$ & /I & & & 1 & GP & & & & & & & $\mathrm{pCi} / \mathrm{L}$ \\
\hline Radium-228 & $<7.9 \mathrm{E}-01$ & UNI & $<0.7220$ & & 1 & GP & & & & & & & $\mathrm{PCi} / \mathrm{L}$ \\
\hline Strontium-90 & $<-1.3 \mathrm{E}-01$ & $\mathrm{UIJ} / \mathrm{C} /$ & & & 1 & GP & & & & & & & $\mathrm{pCi} / \mathrm{L}$ \\
\hline Technetium-99 & $3.2 E+01$ & 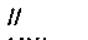 & & & 1 & GP & & & & & & & $\mathrm{pCi} / \mathrm{L}$ \\
\hline Thorium-228 & $<-2.6 E-02$ & $\mathrm{U} / / /$ & $<0.2500$ & & 1 & GP & & & & & & & $\mathrm{pCi} / /$ \\
\hline Thorium-230 & $1.8 \mathrm{E}-01$ & "I & & & 1 & GP & & & & & & & $\mathrm{pCi} / \mathrm{L}$ \\
\hline Thorium-232 & $<0.0 E+00$ & $\mathrm{U}: / /$ & $<0.1050$ & & 1 & GP & & & & & & & $\mathrm{pC} / \mathrm{L}$ \\
\hline Sum of alphas & $1.8 \mathrm{E}-0 \mathrm{t}$ & & & & & & & & & & & & $\mathrm{pCi} / \mathrm{L}$ \\
\hline $\begin{array}{l}\text { Sum of betas } \\
\text { Total radium }\end{array}$ & $\begin{array}{l}3.4 \mathrm{E}+01 \\
1.5 \mathrm{E}+00\end{array}$ & & & & & & & & & & & & $\mathrm{PCi} / \mathrm{L}$ \\
\hline Tritium & $1.1 E+03$ & /l & & - & 1 & GP & $1.0 \mathrm{E}+03$ & /1 & & n & 1 & GE & $\begin{array}{l}\mathrm{pCV} / \mathrm{L} \\
\mathrm{DC} / \mathrm{mL}\end{array}$ \\
\hline Uranium-233/234 & $<2.0 E-01$ & $\mathrm{U}: / /$ & $<0.5990$ & & $i$ & GP & & & & & & & $\mathrm{PC}_{\mathrm{i} / \mathrm{L}}$ \\
\hline Uranium-235 & $<1.1 \mathrm{E}-01$ & UIII & $<0.5150$ & & 1 & GP & & & & & & & $\mathrm{PCi} / \mathrm{L}$ \\
\hline Uranium-238 & $<6.8 \mathrm{E}-02$ & $U I / /$ & $<0.5990$ & & 1 & GP & & & & & & & $\mathrm{pCi} / \mathrm{L}$ \\
\hline
\end{tabular}




\section{WELL HSB116D}

SRS Coord. LatlLongitude

N72898.1 $33.278354^{\circ} \mathrm{N}$

E55988.2 81.660975 oW

SAMPLE DATE
$07 / 22 / 98$

Screen Zone Elevation

234.5-214.5 ft msl

3Q98

225.5

4.2

94

20.2

0

1

5.0
$224.5(09 / 17 / 98)$
Top of Casing

$256.8 \mathrm{ft} \mathrm{msl}$

$10 / 14 / 98$

$\underline{4 Q 98}$

223.8

4.0

93

178

0

2.9

$222.6(12 / 21 / 98)$

\section{Casing Pump Screen Zone}

4" PVC V Water Table (IIB2)
Volumes purged

Sampling code

Synchronous water level

ANALYTICAL DATA

Inorganic Constituents
Antimony, total recoverable

Arsenic total recoverable

Barium, total recoverable

Cadmium, total recoverable $<1.0$

Chromium, total recoverable 3.6

Cobalt, total recoverable $\quad 1.4$

Copper, total recoverable $\quad 4.4$

Cyanide

Mercury, total recoverable $\quad 0.047$

Nickel, total recoverable $\quad 4.2$

Nitrate as nitrogen

Nitrate-nitrite as nitrogen $\quad 8,500$

Selenium, total recoverable $<50$

Silver, total recoverable $<1.0$

Tin, total recoverable

Vanadium total recoverable

Zinc, total recoverable
Lead, total recoverable 2.2

$\begin{array}{ll}\text { Mod } & \text { Filt. } \\ \mathrm{U} / / & <0.20 \\ \mathrm{U} / / & <3.0 \\ / I & <1.0 \\ \mathrm{U} / / & \\ / I & \\ / I & \\ / I & <10 \\ \mathrm{U} / / & \mathrm{NDD} \\ / I & \\ \mathrm{~J} / \mathrm{E} / & \\ / I & \\ \mathrm{~N} / & <5.0 \\ \mathrm{U} / / & <2.0 \\ \mathrm{U} / / & 2.0 \\ \mathrm{U} / / & \\ \mathrm{U} / / & \\ I & \end{array}$

Organic Constituents

\section{Constituents}

Benzene

Bis(2-ethylhexyl) phthalate

Dichloromethane

Tetrachloroethylene

Trichloroethylene

Trichlorofluoromethane

\section{$\underline{3098}$}

$<10$

$<9.9$

$<1.0$

$<10$

$<10$

$<1.0$
Mod

$\mathrm{UJ} / \mathrm{O} / 1$

$\mathrm{UJ} / \mathrm{Q} / \mathrm{Q}$

$\mathrm{UJ} / \mathrm{O} / \mathrm{I}$

$\mathrm{UJ} / \mathrm{O} / 1$

$\mathrm{UJ} / \mathrm{O} / 1$

UJ/O/1
Filt.

$<1.0$

$<90$

$<1.0$

$<10$

$<1.0$

$<1.0$
ST브

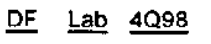

Mod

$\mathrm{GE}$
$\mathrm{GE}$
$\mathrm{GE}$
$\mathrm{GE}$
$\mathrm{GE}$
$\mathrm{GE}$
$\mathrm{G}$
$\mathrm{G}$
$\mathrm{G}$

GE

GE

$E$

0.18

$\mathrm{J} / \mathrm{I}$

NDD

ST브

$\begin{array}{ll}\text { DF } & \text { Lab } \\ 1 & \text { GE } \\ 1 & \text { GE } \\ 1 & \text { GE } \\ 1 & \text { GE } \\ 1 & \text { GE } \\ 1 & \text { GE }\end{array}$

Filt.

Mod

40

$\begin{array}{ll}5 & \mathrm{GE} \\ 1 & \mathrm{GE} \\ 1 & \mathrm{GE} \\ 1 & \mathrm{GE} \\ 1 & \mathrm{GE} \\ 1 & \mathrm{GE}\end{array}$

Unit

ft msl

$\mathrm{pH}$

$\mu \mathrm{S} / \mathrm{cm}$

${ }^{\circ} \mathrm{C}$

$\mathrm{mg} / \mathrm{L}$

well vol

ft msl $\mu g / L$

$\mu g / L$

$\mu g / L$

$\mu g / L$

$\mu \mathrm{g} / \mathrm{L}$

$\mu \mathrm{g} / \mathrm{L}$

$\mu g / L$

$\mu g / L$

$\mu g / L$

1 GE $\mu g / h$

$\mu g / L$

$5 \mathrm{GE} \mu \mathrm{g} / \mathrm{L}$

H

$\mu g / L$

$\mu g / L$

$\mu g / L$

$\mu g / L$
Filt.

$\underline{\text { ST }} \underline{H}$ DF Lab Unit

$\mu g / L$

$\mu \mathrm{gl}$

$\mu g / L$

$\mu g / L$

$\mu g / L$

$\mu g / L$
ST $\underline{H}$ DF Lab Unit

Notes:

- = exceeded holding time

- exceeded groundwater protection or monitoring constituent standard (See Appendix A.) 
WELL HSB116D (cont.)

Radioactive Constituents

\begin{tabular}{|c|c|c|c|c|c|c|c|c|c|c|c|c|c|}
\hline Constituents & 3Q98 & Mod & Filt. & ST브 & $\underline{\mathrm{DF}}$ & Lab & 4098 & Mod & Filt. & $\underline{S T} \underline{H}$ & $\underline{D F}$ & $L a b$ & Unit \\
\hline Americium-241 & $<-1.4 E-02$ & $\mathrm{Ui} / /$ & $<0.1090$ & & 1 & GP & & & & & & & $\mathrm{pCi} / \mathrm{L}$ \\
\hline Beta dose & 85.48 & & & a & & & & & & & & & $\mathrm{pCill}$ \\
\hline Carbon-14 & $<1.2 \mathrm{E}+01$ & $\mathrm{U} N /$ & $<8.5000$ & & 1 & GP & & & & & & & $\mathrm{pCilL}$ \\
\hline Cobalt-60 & $1.6 \mathrm{E}+01$ & $/ /$ & & & 1 & GP & & & & & & & $\mathrm{pCi} / \mathrm{L}$ \\
\hline Curium-242 & $<0.0 E+00$ & $\mathrm{UI} / /$ & $<0.0651$ & & 1 & GP & & & & & & & $\mathrm{pCi} / \mathrm{L}$ \\
\hline Curium-243/244 & $<2.1 \mathrm{E}-02$ & $\mathrm{UI} / /$ & $<0.0618$ & & 1 & GP & & & & & & & $\mathrm{pCi} / \mathrm{L}$ \\
\hline Curium-245/246 & $<0,0 \mathrm{E}+00$ & $\mathrm{UI} / /$ & $<0.0617$ & & 1 & GP & & & & & & & $\mathrm{pCi} / \mathrm{L}$ \\
\hline Gross alpha & $1.2 E+01$ & $/ 1$ & & & 1 & GP & $1.0 E+01$ & $/ 1$ & & & 1 & GE & $\mathrm{pCi} / \mathrm{L}$ \\
\hline lodine-129 & $1.2 E+00$ & $\mathrm{R} / 4 /$ & Rej & & 1 & GP & & & & & & & $\mathrm{pCi} / \mathrm{L}$ \\
\hline Nickel-63 & $1.6 E+01$ & $\|$ & & & & & & & & & & & $\mathrm{pCill}$ \\
\hline Nonvolatile beta & $7.7 \mathrm{E}+02$ & $/ /$ & & $\mathbf{a}$ & 1 & GP & $1.0 \mathrm{E}+03$ & $/ /$ & & ט & 1 & GE & $\mathrm{pCi} / \mathrm{L}$ \\
\hline Plutonium-238 & $<-1.2 \mathrm{E}-02$ & $\mathrm{UI} / /$ & $<0.1100$ & & 1 & GP & & & & & & & $\mathrm{pCi} / \mathrm{L}$ \\
\hline Plutonium-239/240 & $<1.2 \mathrm{E}-02$ & $\mathrm{UI} / /$ & $<0.0832$ & & 1 & GP & & & & & & & $\mathrm{pCi} / \mathrm{L}$ \\
\hline Radium-226 & $3.0 \mathrm{E}+00$ & $/ /$ & & & 1 & GP & & & & & & & $\mathrm{pCi} / \mathrm{L}$ \\
\hline Radium-228 & $<1.7 \mathrm{E}+00$ & $\mathrm{U} N /$ & $<0.8390$ & & 1 & GP & & & & & & & $\mathrm{pCi} / \mathrm{L}$ \\
\hline Strontium-90 & $6.8 E+02$ & $\|$ & & $\mathbf{n}$ & $\hat{\imath}$ & GP & & & & & & & $\mathrm{pCi} / \mathrm{L}$ \\
\hline Technetium-99 & $<2.9 \mathrm{E}+00$ & $\mathrm{U} / /$ & $<24.6000$ & & 1 & GP & & & & & & & $\mathrm{pCi} / \mathrm{L}$ \\
\hline Thoritum-228 & $<8,1 \mathrm{E}-02$ & $\mathrm{U} \mid / /$ & $<0.2800$ & & 1 & GP & & & & & & & $\mathrm{pCi} / \mathrm{L}$ \\
\hline Thorium-230 & $<6.3 \mathrm{E}-02$ & $\mathrm{UI} / /$ & $<0.1930$ & & 1 & GP & & & & & & & $\mathrm{pCi} / \mathrm{L}$ \\
\hline Thorium-232 & $<0.0 \mathrm{E}+00$ & $\mathrm{UI} / /$ & $<0.0833$ & & 1 & GP & & & & & & & $\mathrm{pCi} / \mathrm{L}$ \\
\hline Sum of alphas & $1.2 E+00$ & & & & & & & & & & & & $\mathrm{pCi} / \mathrm{L}$ \\
\hline Sum of betas & $7.1 E+02$ & & & $\mathbf{\square}$ & & & & & & & & & $\mathrm{pCi} / \mathrm{L}$ \\
\hline Total radium & $3.0 E+00$ & & & & & & & & & & & & pCill \\
\hline Tritium & 1.1E+02 & $/ /$ & & m & 1 & GP & $8.3 E+01$ & $/ /$ & & $\mathbf{a}$ & 1 & GE & $\mathrm{pCi} / \mathrm{mL}$ \\
\hline Uranium-233/234 & $1.2 E+00$ & /I & & & 1 & GP & & & & & & & $\mathrm{pCi} / \mathrm{L}$ \\
\hline Uranium-235 & $<-2.8 \mathrm{E}-02$ & $\mathrm{UI} / /$ & $<0.3660$ & & 1 & GP & & & & & & & $\mathrm{pCi} / \mathrm{L}$ \\
\hline Uranium-238 & $<\uparrow .8 \mathrm{E}-01$ & $\mathrm{UI} / /$ & $<0.1760$ & & 1 & GP & & & & & & & $\mathrm{pCi} / \mathrm{L}$ \\
\hline
\end{tabular}

Notes:

- exceeded holding time

a = exceeded groundwater protection or monitoring constituent standard (See Appendix A.) 
WELL HSB117A

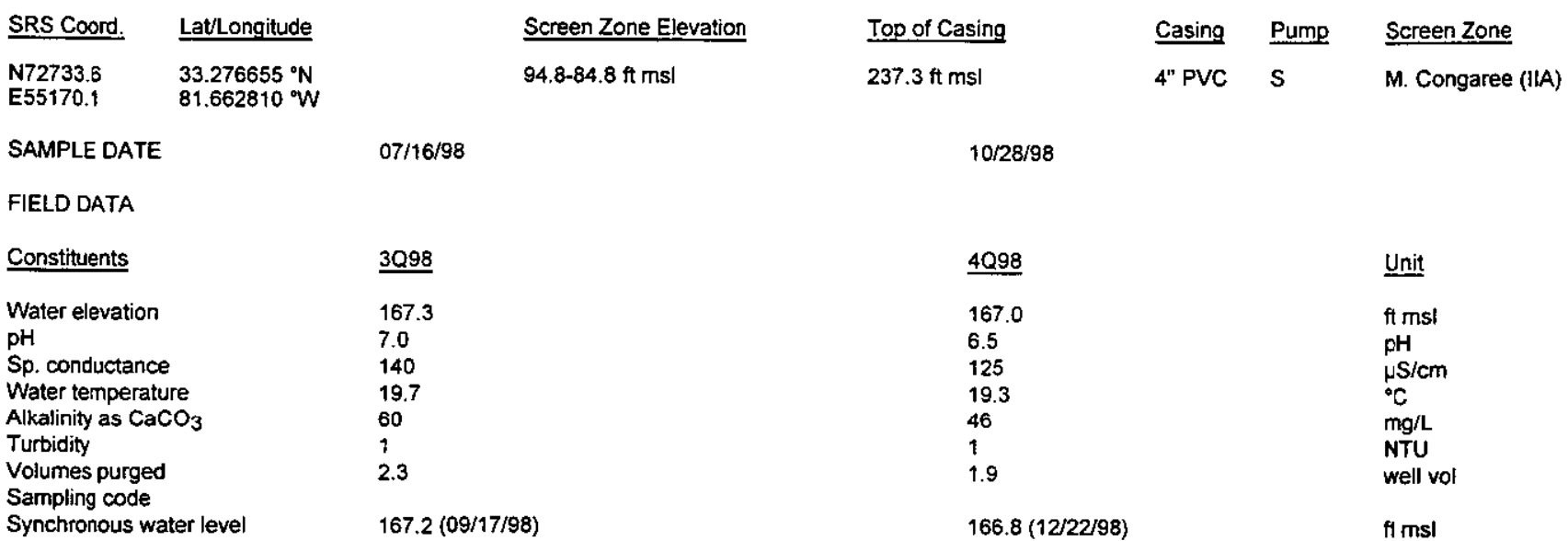

ANALYTICAL DATA

Inorganic Constituents

Constituents

Antimony, total recoverable

Arsenic, total recoverable

Barium, total recoverable

Cadmium, total recoverable

Chromium, total recoverable

Cobalt, total recoverable

Copper, total recoverable

Cyanide

Lead, total recoverable

Mercury, total recoverable

Nickel, total recoverable

Nitrate as nitrogen

Nitrate-nitrite as nitrogen

Selenium, total recoverable

Silver, total recoverable

Tin, total recoverable

Vanadium, total recoverable

Zinc, total recoverable

Organic Constituents

Constituents

0.11

10
Mod

Filt.

NDD

$\mathrm{J} / \mathrm{E} /$

NDD

1

$\mathrm{U} / 1$

1 GE 20

$\mathrm{J} / \mathrm{I}$

NDD

Benzene

Bis(2-ethylhexyl) phthalate

Dichloromethane

Tetrachloroethylene

Trichlorøethylene

Trichlorofluoromethane

Notes:

$\bullet=$ exceeded holding time

- = exceeded groundwater protection or monitoring constituent standard (See Appendix A.) 
WELL HSB117A (cont.)

Radioactive Constituents

Constituents

Americium-241

Carbon-14

Cobalt-60

Curium-242

Curium-243/244

Curium-245/246

Gross alpha

lodine-129

Nickel-63

Nonvolatile beta

Plutonium-238

Plutonium-239/240

Radium-226

Radium-228

Strontium-90

Technetium-99

Thorium-228

Thorium-230

Thorium-232

Sum of alphas

Sum of betas

Tritium

Uranium-233/234

Uranium-235

Uranium-238
3098

Mod

Filt.

ST브 DF Lab 4Q98

1

GP $<5.5 \mathrm{E}-01$

U/I

1

$\mathrm{GP}<2.6 \mathrm{E}+00 \quad \mathrm{U} / /$

$2.4 \mathrm{E}+00 \quad / /$

$<-6.4 \mathrm{E}-01 \quad \mathrm{U} 1 / 1$

$<6.9 \mathrm{E}-01$

1

GP <-3.7E-01 U//

$<6.3 E-01$

$1 \mathrm{GE} \quad \mathrm{pCi} / \mathrm{mL}$

Notes:

- exceeded holding time

- = exceeded grouncwater protection or monitoring constituent standard (See Appendix A.) 
WELL HSB117C

\begin{tabular}{|c|c|}
\hline SRS Coord. & Lat/Longitude \\
\hline $\begin{array}{l}\text { N72 } 2740.7 \\
\text { E55162.9 }\end{array}$ & $\begin{array}{l}33.2766 \\
81.6628\end{array}$ \\
\hline
\end{tabular}

SAMPLE DATE

$07 / 27 / 98$

Screen Zone Elevation

$175.1-165.1 \mathrm{ft} \mathrm{ms|}$
Top of Casing

$237.4 \mathrm{ft} \mathrm{msl}$
Casing Pump

4" PVC V
Screen Zone

Barnwell (IIB 1$)$

FIELD DATA

Constituents

Water elevation

$\mathrm{pH}$

Sp. conductance

Water temperature

Alkalinity as $\mathrm{CaCO}_{3}$

Turbidity

Volumes purged

Sampling code

Synchronous water level

ANALYTICAL DATA

inorganic Constituents
3Q98

237.1

18.5

5

300

16

0.0

$220.7(09 / 17 / 98)$

$10 / 19 / 98$

4Q98

219.3

4.3

300

19.5

0

3.4

$N$

$218.0(12 / 22 / 98)$
Unit

ft msl

$\mathrm{pH}$

$\mu \mathrm{S} / \mathrm{cm}$

$\mathrm{mg} / \mathrm{L}$

NTU

well vol

ft msi

\begin{tabular}{|c|c|c|c|c|c|c|c|c|c|c|c|c|c|}
\hline Constituents & $\underline{3 Q 98}$ & Mod & Filt. & $\underline{\text { ST}} \underline{H}$ & $\underline{\mathrm{DF}}$ & Lab & $\underline{4098}$ & $\underline{\text { Mod }}$ & Filt. & $\underline{\mathrm{ST}} \underline{\mathrm{H}}$ & $\underline{\text { DF }}$ & $\underline{\text { Lab }}$ & Unit \\
\hline Antimony, total recoverable & $<0.20$ & $u \prime \prime$ & $<0.20$ & & 1 & GE & & & & & & & $\mu g / L$ \\
\hline Arsenic, total recoverable & $<3.0$ & $\mathrm{u} / /$ & $<3.0$ & & 1 & GE & & & & & & & $\mu g / \mathrm{L}$ \\
\hline Barium, total recoverable & 62 & "I & & & 1 & GE & & & & & & & $\mu g / L$ \\
\hline Cadmium, total recoverable & $<1.0$ & $\mathrm{U} / /$ & $<1.0$ & & 1 & GE & & & & & & & $\mu g / L$ \\
\hline Chromium, total recoverable & $<3.0$ & $\mathrm{U} / / \mathrm{I}$ & $<3.0$ & & 1 & GE & & & & & & & $\mu g / L$ \\
\hline Cobalt, total recoverable & 3.9 & $\|$ & & ש & 1 & GE & & & & & & & $\mu g / L$ \\
\hline Copper, total recoverable & 0.94 & $" 1$ & & & 1 & GE & & & & & & & $\mu g / L$ \\
\hline Cyanide & $<10$ & $\mathrm{U} / \mathrm{l}$ & $<10$ & & 1 & $\mathrm{GE}$ & & & & & & & $\mu g / L$ \\
\hline Lead, total recoverable & $<2.0$ & $\mathrm{U} / /$ & $<2.0$ & & 1 & $\mathrm{GE}$ & & & & & & & $\mu g / L$ \\
\hline Mercury, total recoverable & 0.20 & /I & & & 1 & GE & 0.12 & $\mathrm{~J} / / /$ & NDD & & 1 & GE & $\mu$ L \\
\hline $\begin{array}{l}\text { Nickel, total recoverable } \\
\text { Nitrate as nitrogen }\end{array}$ & 3.0 & $" 1$ & & & 1 & GE & & & & & & & $\mu g / L$ \\
\hline Nitrate-nitrite as nitrogen & 37,000 & NI & & = & 25 & GE & 36,000 & "l & & - & 25 & GE & $\mu g / L$ \\
\hline Selenium, total recoverable & $<5.0$ & $\mathrm{U} / /$ & $<5.0$ & & 1 & GE & & & & & & & $\mu$ L \\
\hline Silver, total recoverable & $<1.0$ & $\mathrm{U} / /$ & $<1.0$ & & 1 & GE & & & & & & & $\mu g / L$ \\
\hline Tin, total recoverable & $<2.0$ & $\mathrm{u} / /$ & $<2.0$ & & 1 & GE & & & & & & & $\mu g / L$ \\
\hline Vanadium, total recoverable & $<2.0$ & Uil & $<2.0$ & & 1 & $\mathrm{GE}$ & & & & & & & $\mu \mathrm{g} / \mathrm{L}$ \\
\hline Zinc, total recoverable & 10 & /l & & & 1 & GE & & & & & & & $\mu g / L$ \\
\hline \multicolumn{14}{|l|}{ Organic Constituents } \\
\hline Constituents & $\underline{3 Q 98}$ & Mod & Filt. & $\underline{\text { ST }} \underline{H}$ & DF & $\underline{\text { Lab }}$ & $\underline{4098}$ & $\underline{\text { Mod }}$ & Filt. & ST $\underline{H}$ & $\underline{\mathrm{DF}}$ & $\underline{\text { Lab }}$ & $\underline{\text { Unit }}$ \\
\hline Benzene & $<1.0$ & $\mathrm{U} / 1$ & $<1.0$ & & 1 & GE & & & & & & & $\mu g / L$ \\
\hline Bis (2-ethylhexyl) phthalate & $<10$ & $\mathrm{U} / /$ & $<10$ & & 1 & GE & & & & & & & $\mu g / L$ \\
\hline Dichloromethane & $<17$ & UNI & $<1.0$ & & 1 & GE & & & & & & & $\mu g / L$ \\
\hline Tetrachloroethylene & $<1.0$ & $\mathrm{u} / /$ & $<1.0$ & & 1 & GE & & & & & & & $\mu \mathrm{g} / \mathrm{L}$ \\
\hline Trichioroethylene & $<1.0$ & $\mathrm{U} / /$ & $<1.0$ & & 1 & GE & & & & & & & $\mu g / 2$ \\
\hline Trichlorofluoromethane & $<\uparrow .0$ & $\mathrm{U} / /$ & $<1.0$ & & 1 & GE & & & & & & & $\mu g / \mathrm{L}$ \\
\hline
\end{tabular}

Notes:

- = exceeded holding time

- exceeded groundwater protection or monitoring constituent standard (See Appendix A.) 
WELL HSB117C (cont.)

Radioactive Constituents

\begin{tabular}{|c|c|c|c|c|c|c|c|c|c|c|c|c|c|}
\hline Constituents & $\underline{3 Q 98}$ & Mod & Filt. & $\underline{\text { ST }} \underline{H}$ & $\underline{\mathrm{DF}}$ & $\underline{L a b}$ & $\underline{4 Q 98}$ & Mod & Filt. & $\underline{\mathrm{ST}} \underline{\mathrm{H}}$ & $\mathrm{DF}$ & Lab & $\underline{\text { Unit }}$ \\
\hline Americium-241 & 1.1E-01 & $/ 1$ & & & 1 & GP & & & & & & & $\mathrm{pCi} / \mathrm{L}$ \\
\hline Beta dose & 4.72 & & & " & & & & & & & & & $\mathrm{pCi} / \mathrm{h}$ \\
\hline Carbon-14 & $5.2 E+01$ & "I & & - & 1 & GP & & & & & & & $\mathrm{pCi} / \mathrm{L}$ \\
\hline Cobali- 60 & $<-5.0 E-01$ & $\mathrm{UI} / /$ & $<2.4400$ & & 1 & GP & & & & & & & $\mathrm{pCi} / \mathrm{L}$ \\
\hline Currium-242 & $<0.0 \mathrm{E}+00$ & $\mathrm{U}: / /$ & $<0.0526$ & & 1 & GP & & & & & & & $\mathrm{pCi} / \mathrm{L}$ \\
\hline Curium-243/244 & $<0.0 E+00$ & $\mathrm{UI} / /$ & $<0.0495$ & & 1 & GP & & & & & & & PCi $/ L$ \\
\hline Curium-245/246 & 1.5E-01 & "l & & & 1 & GP & & & & & & & $\mathrm{pCi} / \mathrm{L}$ \\
\hline Gross alpha & $8.8 E+00$ & $/ 1$ & & & 1 & GP & $<7.5 E+00$ & $\mathrm{U} / /$ & $<4,2 \mathrm{E}+00$ & & 1 & GE & $\mathrm{pC} / / \mathrm{L}$ \\
\hline lodine- 129 & $4.1 \mathrm{E}+00$ & "l & & & 1 & GP & & & & & & & $\mathrm{pCi} / \mathrm{L}$ \\
\hline Nickel-63 & $1.9 \mathrm{E}+01$ & "l & & & 1 & GP & & & & & & & $\mathrm{PCi} / \mathrm{L}$ \\
\hline Nonvolatile beta & $2.9 E+01$ & "l & & & $i$ & GP & $3.2 E+01$ & $/ /$ & & & 1 & GE & $\mathrm{pCi} / \mathrm{L}$ \\
\hline Pfutonium-238 & $<0.0 E+00$ & $\mathrm{U} \mid / /$ & $<0.1500$ & & 1 & GP & & & & & & & $\mathrm{pCi} / \mathrm{L}$ \\
\hline Plutonium-239/240 & $<0.0 \mathrm{E}+00$ & $\mathrm{U}] / /$ & $<0.1500$ & & 1 & GP & & & & & & & $\mathrm{pCi} / \mathrm{L}$ \\
\hline Radjum-226 & $6.4 E+\infty 0$ & $\|$ & & m & 1 & GP & & & & & & & $\mathrm{pCi} / \mathrm{L}$ \\
\hline Radium-228 & $<-1.9 \mathrm{E}-01$ & $\mathrm{U} \mathbf{I} / \mathrm{I}$ & $<1.0900$ & & 1 & GP & & & & & & & $\mathrm{pCi} / \mathrm{L}$ \\
\hline Strontium-90 & $<1.9 E-01$ & $\mathrm{UI} / \mathrm{I}$ & $<1.5300$ & & 1 & GP & & & & & & & $\mathrm{pCi} / \mathrm{L}$ \\
\hline Technetium-99 & $1.9 \mathrm{E}+02$ & 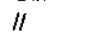 & & - & 1 & GP & & & & & & & $\mathrm{pCi} / \mathrm{L}$ \\
\hline Thorium-228 & $<-7.3 E-02$ & $\mathrm{UIJ} / \mathrm{C} /$ & & & 1 & GP & & & & & & & $\mathrm{pCi} / \mathrm{L}$ \\
\hline Thorium-230 & $<1,1 \mathrm{E}-01$ & $\mathrm{UIJ} / \mathrm{C} / \mathrm{I}$ & & & 1 & GP & & & & & & & $\mathrm{pCi} / \mathrm{L}$ \\
\hline Thorium-232 & $<0.0 \mathrm{E}+00$ & $\mathrm{ULJ} / \mathrm{C} /$ & & & 1 & GP & & & & & & & $\mathrm{pCi} / \mathrm{L}$ \\
\hline Sum of alphas & $1.2 \mathrm{E}+00$ & & & & & & & & & & & & $\mathrm{pCi} / \mathrm{L}$ \\
\hline $\begin{array}{l}\text { Sum of betas } \\
\text { Total radium }\end{array}$ & $\begin{array}{l}2.7 E+02 \\
6.4 E+00\end{array}$ & & & - & & & & & & & & & $\begin{array}{l}\mathrm{PCi} / \mathrm{L} \\
\mathrm{PCi} / \mathrm{L}\end{array}$ \\
\hline Tritium & $4.6 \mathrm{E}+03$ & $" 1$ & & - & 1 & GP & $5.0 \mathrm{E}+03$ & $1 /$ & & a & 1 & GE & $\mathrm{pCi} / \mathrm{mL}$ \\
\hline Uranium-233/234 & $3.7 \mathrm{E}-01$ & 11 & & & 1 & GP & & & & & & & $\mathrm{pCi} / \mathrm{L}$ \\
\hline Uranium-235 & $2.2 \mathrm{E}-01$ & 11 & & & 1 & GP & & & & & & & $\mathrm{pCi} / \mathrm{L}$ \\
\hline Uranium-238 & $3.5 \mathrm{E}-01$ & "l & & & 1 & GP & & & & & & & $\mathrm{pCi} / \mathrm{L}$ \\
\hline
\end{tabular}

Notes:

- = exceeded holding time

- exceeded groundwater protection or monitoring constituent standard (See Appendix A.) 
WELL HSB117D

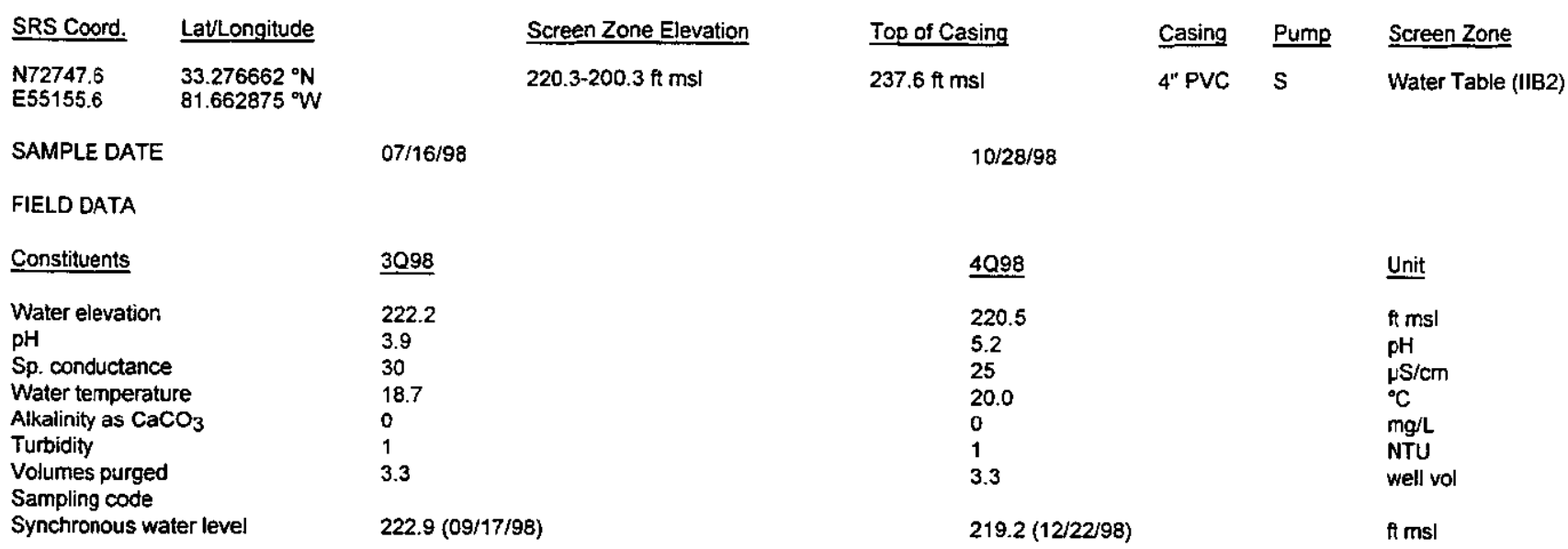

ANALMICAL DATA

Inorganic Constituents

\section{Constituents}

Antimony, total recoverable

Arsenic, total recoverable

Barium, total recoverable

Cadmium, total recoverable

Chromium, total recoverable

Cobalt, total recoverable

Copper, total recoverable

Cyanide

Lead, total recoverable

Mercury, total recoverable

Nickel, total recoverable

Nitrate as nitrogen

Nitrate-nitrite as nitrogen

Selenium, total recoverable

Silver, total recoverable

Tin, total recoverable

Vanadium, total recoverable

Zinc, total recoverable

Organic Constituents

Constituents

Benzene

Bis(2-ethy|hexyl) phthalate

Dichloromethane

Tetrachloroethylene

Trichloroethylene

Trichlorofiuoromethane
Mod

Filt.

ST브

DF Lab 4098 Mod

NDD

1

GE $<0.20$

$\mathrm{U} / /$

1 GE 720

/

Mod

Filt.

ST브

DF ab $4098 \quad$ Mod
Filt.

ST $\underline{H} \underline{\text { DF Lab Unit }}$
1 GE $\mu g / L$

$1 \mathrm{GE} \mu g / L$

Notes:

= exceeded holding time

- = exceeded groundwater protection or monitoring constituent standard (See Appendix A.) 


\section{WELL HSB117D (cont.)}

\section{Radioactive Constituents}

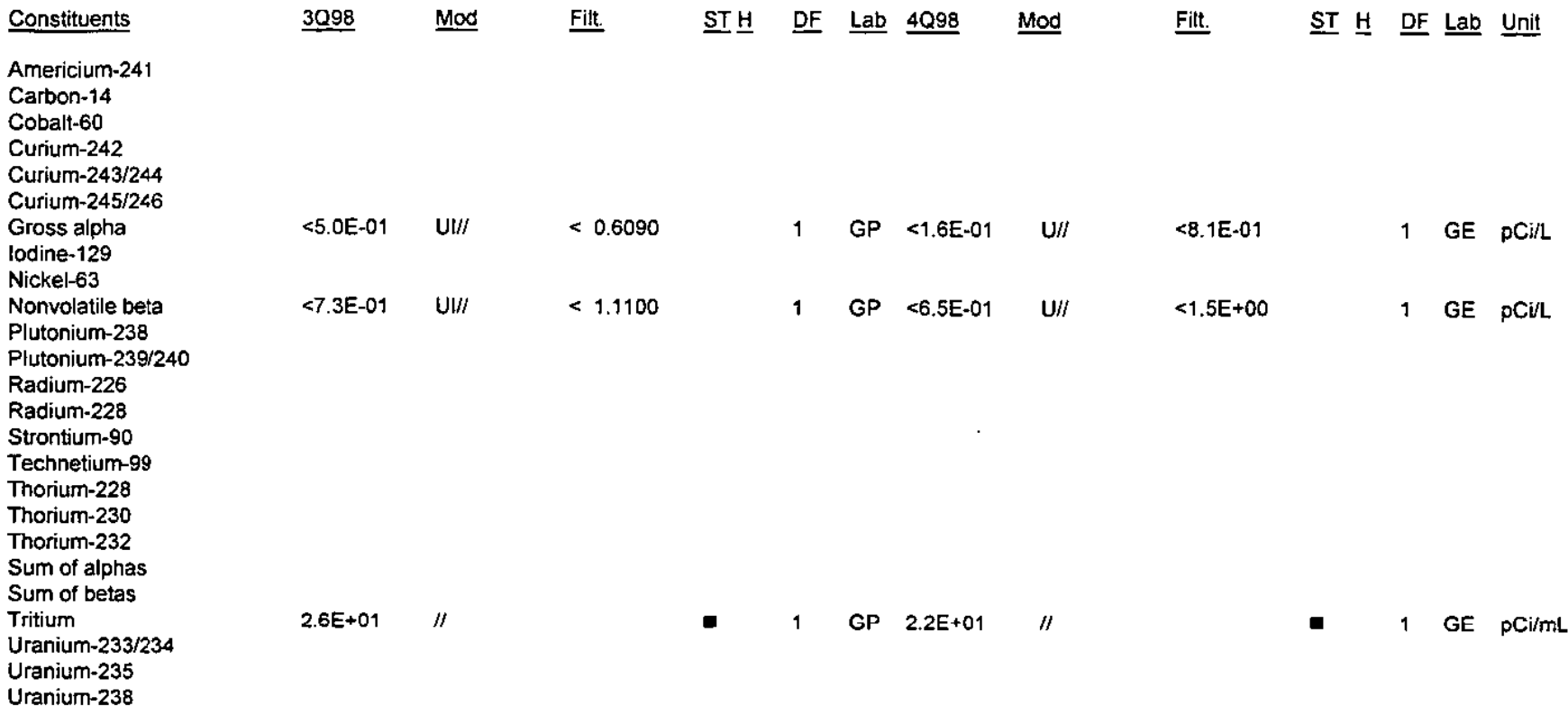

Notes: 
WELL HSB118A

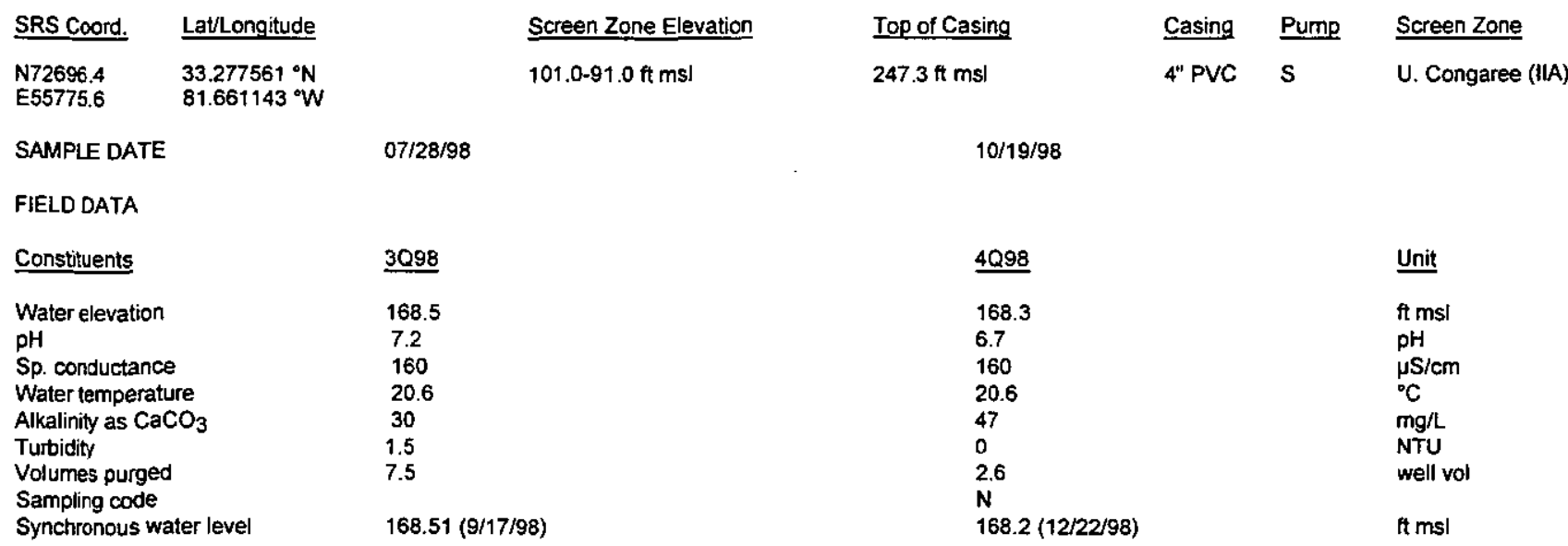

ANALYTICAL DATA

Inorganic Constituents

\begin{tabular}{|c|c|c|c|c|c|c|c|c|c|c|c|c|c|}
\hline Constituents & $\underline{3098}$ & Mod & Fut. & ST브 & DF & Lab & $\underline{4 Q 98}$ & Mod & Filt. & $\underline{\text { ST }} \underline{H}$ & $\underline{\mathrm{DF}}$ & $\underline{L a b}$ & Unit \\
\hline Antimony, total recoverable & $<0.20$ & $\mathrm{U} / /$ & $<0.20$ & & 1 & GE & & & & & & & $\mu g / L$ \\
\hline Arsenic, total recoverable & 1.7 & S/E/ & NDD & & 1 & GE & & & & & & & $\mu g / L$ \\
\hline Barium, total recoverable & 27 & $/ /$ & & & 1 & GE & & & & & & & $\mu g / L$ \\
\hline Cadmium, total recoverable & $<1.0$ & U/I & $<1.0$ & & 1 & GE & & & & & & & $\mu g / L$ \\
\hline Chromium, total recoverable & $<3.0$ & $\mathrm{U} / /$ & $<3.0$ & & 1 & GE & & & & & & & $\mu g / L$ \\
\hline Cobalt, total recoverable & 0.11 & $\mathrm{~J} / \mathrm{E} /$ & NDD & & 1 & GE & & & & & & & $\mu g / L$ \\
\hline Coppes, total recoverable & 0.50 & $/ 1$ & & & 1 & GE & & & & & & & $\mu g / L$ \\
\hline Cyanide & $<10$ & $\mathrm{U} / /$ & $<10$ & & 1 & GE & & & & & & & $\mu \mathrm{g} / \mathrm{L}$ \\
\hline Lead, total recoverable & 0.66 & J/EV/ & NDD & & 1 & GE & & & & & & & $\mu g / L$ \\
\hline Mercury, total recoverable & $<0.20$ & $\mathrm{U} / /$ & $<0.20$ & & 1 & $\overline{G E}$ & $<0.20$ & $\mathrm{U} / /$ & $<0.20$ & & 1 & GE & $\mu g / L$ \\
\hline $\begin{array}{l}\text { Nickel, total recoverable } \\
\text { Nitrate as nitrogen }\end{array}$ & $<0.20$ & $\mathrm{U} / /$ & $<0.20$ & & 1 & GE & & & & & & & \\
\hline Nitrate-nitrite as nitrogen & 3,500 & $N /$ & & & 3 & GE & 3,500 & $/ /$ & & & 2 & GE & $\mu g / L$ \\
\hline Selenium, total recoverable & $<5.0$ & $U / I$ & $<5.0$ & & 1 & GE & & & & & & & \\
\hline Silver, total recoverable & $<1.0$ & $\mathrm{U} / /$ & $<1.0$ & & 1 & GE & & & & & & & $g / L$ \\
\hline Tin, total recoverable & $<2.0$ & $\mathrm{U} / /$ & $<2.0$ & & 1 & GE & & & & & & & $\mathrm{g} / \mathrm{L}$ \\
\hline Vanadium, total recoverable & $<2.0$ & $\mathrm{U} / /$ & $<2.0$ & & 1 & $\overline{G E}$ & & & & & & & g/L \\
\hline Zinc, total recoverable & $<5.0$ & $\mathrm{U} / /$ & $<5.0$ & & 1 & GE & & & & & & & \\
\hline
\end{tabular}

Organic Constituents

\begin{tabular}{|c|c|c|c|c|c|}
\hline Constituents & 3Q98 & Mod & Filt. & $\underline{\text { ST }} \underline{H}$ & DF \\
\hline Benzene & $<1.0$ & $\mathrm{UJ} / \mathrm{O} / 1$ & $<1.0$ & & 1 \\
\hline Bis $(2$-ethylhexyl) phthalate & $<9.9$ & UII & $<9.9$ & & 1 \\
\hline Dichloromethane & 6.1 & $\mathrm{~J} / \mathrm{O} / 1$ & NDD & & 1 \\
\hline Tetrachioroethylene & $<1.0$ & $\mathrm{UJ} / \mathrm{O} / 1$ & $<1.0$ & & 1 \\
\hline Trichloroethylene & $<1.0$ & $\mathrm{UJ} / \mathrm{O} / 1$ & $<1.0$ & & 1 \\
\hline Trichlorofluoromethane & $<1.0$ & UJ/O/1 & $<1.0$ & & 1 \\
\hline
\end{tabular}

Mod

Filt.

$\underline{\text { ST }} \underline{H} \underline{\text { DF Lab }} \underline{\text { Unit }}$

$\mu g / L$

$\mu g / L$

$\mu \mathrm{gl}$

$\mu g / L$

$\mu g / L$

$\mu g / L$

Notes:

- exceeded holding time

- exceeded groundwater protection or monitoring constituent standard (See Appendix A.) 
WELL HSB118A (cont.)

Radioactive Constituents

Constituents
Americium-241
Beta dose
Carbon-14
Cobalt-60
Curium-242
Curium-243/244
Curium-245/246
Gross atpha
lodine-129
Nickel-63
Nonvolatile beta
Plutonium-238
Plutonium-239/240
Radium-226
Radium-228
Strontium-90
Technetium-99
Thorium-228
Thorium-230
Thorium-232
Sum of alphas
Sum of betas
Tritium
Uranium-233/234
Uranium-235
Uranium-238

\begin{tabular}{|c|c|c|c|c|c|c|c|}
\hline 3098 & Mod & Filt. & ST $\underline{H}$ & $\underline{D F}$ & $\underline{L a b}$ & 4Q98 & Mod \\
\hline $\begin{array}{l}<7.4 E-02 \\
0.01\end{array}$ & $\mathrm{UI} / /$ & $<0.2660$ & & 1 & GP & & \\
\hline $1.0 \mathrm{E}+01$ & /I & & & 1 & GP & & \\
\hline$<-2.0 E-03$ & UII/ & $<3.7700$ & & 1 & $G P$ & & \\
\hline$<5.3 E-02$ & $\mathrm{UI} / \mathrm{I}$ & $<0.2170$ & & 1 & GP & & \\
\hline$<2.1 E-01$ & $\mathrm{UI} / 1$ & $<0.2290$ & & 1 & GP & & \\
\hline$<0.0 E+00$ & $\mathrm{UI} / I$ & $<0.0986$ & & 1 & $\mathrm{GP}$ & & \\
\hline$<6.8 \mathrm{E}-01$ & $\mathrm{UI} / /$ & $<0.6960$ & & 1 & $\mathrm{GP}$ & $<2.2 \mathrm{E}+00$ & $\mathrm{U} / /$ \\
\hline$<4.5 E-01$ & $U I I I$ & $<0.8190$ & & 1 & $\mathrm{GP}$ & & \\
\hline$<-2.0 \mathrm{E}-03$ & UI & $<3.7700$ & & & & & \\
\hline $3,6 E+00$ & /I & & & 1 & GP & 1.1E+01 & $\|$ \\
\hline$<2.2 \mathrm{E}-03$ & U: $/ I$ & $<0.4060$ & & 1 & $\mathrm{GP}$ & & \\
\hline$<-3.9 E-02$ & $\mathrm{UI} / 1$ & $<0.3720$ & & 1 & GP & & \\
\hline$<5.7 E-01$ & UIII & $<0.7510$ & & 1 & GP & & \\
\hline$<-6.5 E-01$ & $\mathrm{UI} / /$ & $<1.3100$ & & 1 & GP & & \\
\hline$<-3.6 \mathrm{E}-01$ & UIJ/U & & & 1 & GP & & \\
\hline$<-8.3 E+00$ & $\mathrm{UI} / /$ & $<15.8000$ & & 1 & GP & & \\
\hline$<1.8 E-02$ & $\mathrm{UIJ} / \mathrm{Cl}$ & & & 1 & $\mathrm{GP}$ & & \\
\hline 3.3E-01 & $\mathrm{J} / \mathrm{C} /$ & NDD & & 1 & GP & & \\
\hline$<0.0 \mathrm{E}+00$ & $\mathrm{U} / \mathrm{J} / \mathrm{C} /$ & & & 1 & $G P$ & & \\
\hline 3.3E-01 & & & & & & & \\
\hline $1.0 E+01$ & & & & & & & \\
\hline $1.1 E+03$ & /I & & $\mathbf{0}$ & 1 & GP & 1.1E+03 & // \\
\hline$<5.0 \mathrm{E}-02$ & $\mathrm{UII} /$ & $<0.2250$ & & 1 & $\mathrm{GP}$ & & \\
\hline$<4.9 \mathrm{E}-02$ & $\mathrm{UI} / /$ & $<0.1470$ & & 1 & GP & & \\
\hline$<-6.7 E-03$ & $\mathrm{U} / /$ & $<0.1470$ & & 1 & GP & & \\
\hline
\end{tabular}

Filt. $\quad \underline{\text { ST }} \underline{H}$ DF Lab Unit

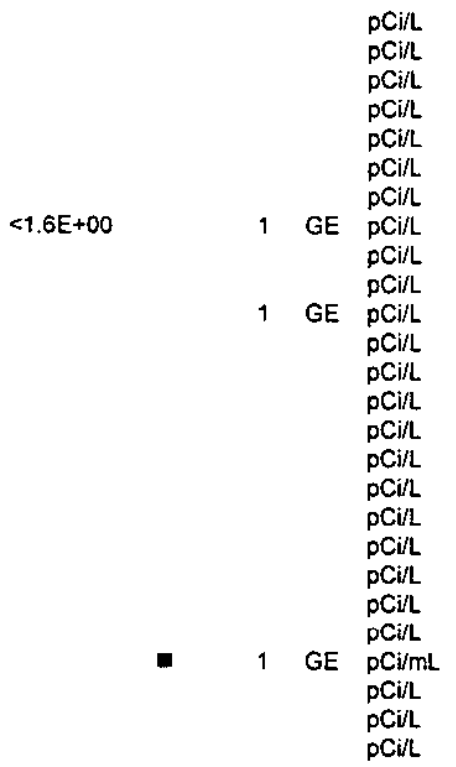

Notes:

- = exceeded holding time

- = exceeded groundwater protection or monitoring constituent standard (See Appendix A.) 


\section{WELL HSB119A}

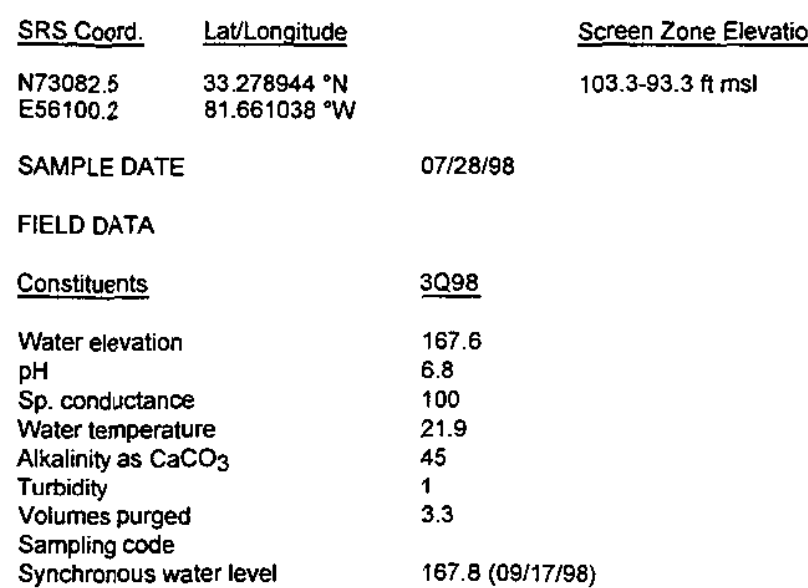

ANALYTICAL DATA

Inorganic Constituents

\begin{tabular}{|c|c|c|c|c|c|c|c|c|c|c|c|c|c|}
\hline Constituents & 3Q98 & Mod & Filt. & $\underline{\text { ST브 }}$ & DF & $\underline{L a b}$ & $\underline{4098}$ & Mod & Filt. & $\underline{\mathrm{ST}} \underline{\mathrm{H}}$ & $\underline{D F}$ & $\underline{\text { Lab }}$ & Unit \\
\hline Antimony, total recoverable & $<0.20$ & $\mathrm{U} / 1$ & $<0.20$ & & 1 & GE & & & & & & & $\mu g / L$ \\
\hline Arsenic, total recoverable & 2.2 & $\mathrm{~J} / \mathrm{E}$ & NDD & & 1 & GE & & & & & & & $\mu g / L$ \\
\hline Barium, total recoverable & 13 & $/ /$ & & & 1 & GE & & & & & & & $\mu g / L$ \\
\hline Cadmium, total recoverabie & $<1.0$ & $\mathbf{U} / /$ & $<1.0$ & & 1 & GE & & & & & & & $\mu g / L$ \\
\hline Chromium, total recoverable & 0.86 & JiE & NDD & & 1 & GE & & & & & & & $\mu g / L$ \\
\hline Cobalt, total recoverable & 0.072 & $\mathrm{~J} / \mathrm{E} /$ & NDD & & 1 & GE & & & & & & & $\mu g / L$ \\
\hline Copper, total recoverable & 0.72 & $/ 1$ & & & 1 & GE & & & & & & & $\mu g / L$ \\
\hline Cyanide & $<10$ & $\mathrm{U} / /$ & $<10$ & & 1 & GE & & & & & & & $\mu g / L$ \\
\hline Lead, total recoverable & $<2.0$ & $\mathrm{U} / /$ & $<2.0$ & & 1 & GE & & & & & & & $\mu g /$ \\
\hline Mercury, total recoverable & $<0.20$ & $\mathrm{U} / /$ & $<0.20$ & & 1 & GE & $<0.20$ & $\mathrm{u} / /$ & $<0.20$ & & 1 & GE & $\mu g / L$ \\
\hline $\begin{array}{l}\text { Nickel, total recoverable } \\
\text { Nitrate as nitrogen }\end{array}$ & 2.1 & $\|$ & & & 1 & GE & & & & & & & $\mu g / L$ \\
\hline Nitrate-nitrite as nitrogen & 5,400 & $N !$ & & & 3 & GE & 5,800 & $/ / 6$ & & & 3 & GE & $\mu g / L$ \\
\hline Selenium, total recoverable & $<5.0$ & $\mathrm{U} / /$ & $<5.0$ & & 1 & $\mathrm{GE}$ & & & & & & & $\mu g / L$ \\
\hline Silver, total recoverable & $<1.0$ & $\mathrm{U} / /$ & $<1.0$ & & 1 & $\overline{G E}$ & & & & & & & $\mu g / L$ \\
\hline Tin, total recoverable & $<2.0$ & $\mathrm{U} / /$ & $<2.0$ & & 1 & GE & & & & & & & $\mu \mathrm{g} / \mathrm{L}$ \\
\hline Vanadium, total recoverable & $<2.0$ & $\mathbf{U} / /$ & $<2.0$ & & 1 & GE & & & & & & & $\mu g / L$ \\
\hline Zinc, total recoverable & 16 & $\|$ & & & 1 & GE & & & & & & & $\mu g / L$ \\
\hline \multicolumn{14}{|l|}{ Organic Constituents } \\
\hline Constituents & 3Q98 & Mod & Filt. & ST브 & DF & $\underline{\text { Lab }}$ & $\underline{4 Q 98}$ & Mod & Filt. & ST $\underline{ }$ & $\underline{D F}$ & Lab & Unit \\
\hline Benzene & $<1.0$ & UJ/O/1 & $<1.0$ & & 1 & GE & & & & & & & $g / L$ \\
\hline Bis(2-ethylhexyl) phthalate & $<10$ & $\mathrm{U} / /$ & $<10$ & & 1 & $\mathrm{GE}$ & & & & & & & L \\
\hline Dichloromethane & $<4.8$ & UJ/NO8/1 & $<1.0$ & & 1 & GE & & & & & & & $g / L$ \\
\hline Tetrachloroethylene & $<1.0$ & $\mathrm{UJ} / \mathrm{O} / 1$ & $<1,0$ & & 1 & GE & & & & & & & L \\
\hline Trichloroethylene & $<1.0$ & UJ/O/1 & $<1.0$ & & 1 & GE & & & & & & & $\mathrm{g} / \mathrm{L}$ \\
\hline Trichlorofluoromethane & $<1.0$ & UJ/O/1 & $<1.0$ & & 1 & GE & & & & & & & \\
\hline
\end{tabular}

$\underline{4098}$

167.3

6.4

125

20.6

23

3

$\mathrm{XN}$

$167.5(12 / 21 / 98)$
Casing Pump

4" PVC S

Screen Zone

U. Congaree (IIA)
Unit

ft msl

$\mathrm{pH}$

$\mu \mathrm{S} / \mathrm{cm}$

mg/t

well vol

$\mathrm{ft}$ msl $\mu g / L$

g/L

$\mu g / L$

$\mu g / L$

Notes:

- exceeded holding time

a exceeded groundwater protection or monitoring constituent standard (See Appendix A.) 
WELL HSB119A (cont.)

Radioactive Constituents

\begin{tabular}{|c|c|c|c|c|c|c|c|c|}
\hline Constituents & 3Q98 & Mod & Filt. & ST브 & $\underline{D F}$ & Lab & 4Q98 & Mod \\
\hline $\begin{array}{l}\text { Americium-241 } \\
\text { Beta dose }\end{array}$ & $\begin{array}{l}<5.2 E-03 \\
0.08\end{array}$ & $\mathrm{UI} / /$ & $<0.1000$ & & 1 & GP & & \\
\hline Carbon-14 & $8.3 E+01$ & $\|$ & & $\mathbf{v}$ & 1 & GP & & \\
\hline Cobalt -60 & $<-1.6 E+00$ & $\mathrm{UI} / /$ & $<2.9500$ & & 1 & GP & & \\
\hline Curium-242 & $<0.0 E+00$ & $\mathrm{UI} / /$ & $<0.0584$ & & 1 & GP & & \\
\hline Curium-243/244 & $<1.4$ E.02 & $\mathrm{U}: / /$ & $<0.1000$ & & 1 & GP & & \\
\hline Curium-245/246 & $<0.0 \mathrm{E}+\infty 0$ & $\mathrm{UI} / /$ & $<0.0568$ & & 1 & GP & & \\
\hline Gross alpha & $1.1 E+00$ & $/ 1$ & & & 1 & GP & $2.6 E+00$ & $1 /$ \\
\hline lodine-129 & $<-2.4 \mathrm{E}-01$ & $\mathrm{UI} / /$ & $<0.9910$ & & 1 & GP & & \\
\hline Nickel-63 & $<-1.6 E+00$ & UI & $<2.9500$ & & & & & \\
\hline Nonvolatile beta & $8.3 E+00$ & /I & & & 1 & GP & $2.3 E+01$ & /I \\
\hline Plutonium-238 & $<0.0 E+00$ & $\mathrm{UI} / /$ & $<0.0529$ & & 1 & GP & & \\
\hline Plutonium-239/240 & $<1.8 E-02$ & $\mathrm{U} 1 / /$ & $<0.0528$ & & 1 & GP & & \\
\hline Radium-226 & $1.7 E+00$ & $/ /$ & & & 1 & GP & & \\
\hline Radium-228 & $<2.8 E-01$ & $\mathrm{U} 1 / /$ & $<0.9450$ & & 1 & GP & & \\
\hline Strontium-90 & $<4.8 \mathrm{E}-02$ & $\mathrm{U} ! \mathrm{J} / \mathrm{C} /$ & & & 1 & GP & & \\
\hline Technetium-99 & $3.5 E+01$ & $/ /$ & & & 1 & GP & & \\
\hline Thorium-228 & $<-1.9 E-02$ & U1// & $<0.2470$ & & 1 & GP & & \\
\hline Thorium-230 & $<3.9 E-02$ & $\mathrm{UI} / /$ & $<0.1170$ & & 1 & GP & & \\
\hline Thorium-232 & $<3.9 E-02$ & Uil/ & $<0.1170$ & & 1 & GP & & \\
\hline \multicolumn{9}{|l|}{ Sum of atphas } \\
\hline Sum of betas & $1.2 \mathrm{E}+02$ & & & $\mathbf{v}$ & & & & \\
\hline Total radium & $1.7 E+00$ & & & & & & & \\
\hline Tritium & $3.7 E+02$ & $/ /$ & & घ & 1 & GP & $5.0 \mathrm{E}+02$ & $/ 1$ \\
\hline Uranium-233/234 & $<0.0 \mathrm{E}+00$ & $\mathrm{UI} / /$ & $<0.2180$ & & 1 & GP & & \\
\hline Uranium-235 & $<0.0 \mathrm{E}+00$ & UIII & $<0.2180$ & & 1 & GP & & \\
\hline Uranium-238 & $<3.8 \mathrm{E}-02$ & UIII & $<0.4510$ & & 1 & GP & & \\
\hline
\end{tabular}

Filt. $\quad \underline{\text { ST }} \underline{H}$ DF Lab Unit

$\mathrm{pCi} / \mathrm{L}$

PCi/L

$\mathrm{PCi} / \mathrm{L}$

oCi/L

$\mathrm{PCi} / \mathrm{L}$

PCilL

$\mathrm{pCi} / \mathrm{L}$

$1 \mathrm{GE} \mathrm{pCi} / \mathrm{L}$

PCi/L

PCill

$1 \mathrm{TM} \mathrm{PCi/L}$

pCill

PCilL

PCi/L

pCi/L

pCill

$\mathrm{PCilL}$

$\mathrm{PCi} / \mathrm{L}$

$\mathrm{PCi}$

pCi/l

PCi/L

$\mathrm{pCi} / \mathrm{L}$

$\mathrm{PCi} / \mathrm{mL}$

$\mathrm{PCi} / \mathrm{L}$

PCi/L

$\mathrm{PCi} / \mathrm{L}$

Notes:

- = exceeded holding time

$\boldsymbol{w}=$ exceeded groundwater protection or monitoring constituent standard (See Appendix A.) 
WELL HSB120A

$\begin{array}{ll}\text { SRS Coord. } & \text { Lat/Longitude } \\ \text { N73395.1 } & 33.280177 \mathrm{~N} \\ \text { E56431.9 } & 81.660772 \mathrm{~W}\end{array}$

SAMPLE DATE

Screen Zone Elevation

$101.0-91.0 \mathrm{ft} \mathrm{ms}$
Top of Casing

$268.2 \mathrm{ft} \mathrm{ms}$
Casing Pump

4" PVC S
Screen Zone

U. Congaree (IIA)
$10 / 28 / 98$

4Q98

166.9

7.2

200

19.2

86

2.2

$166.7(12 / 21 / 98)$ $\underline{\text { Unit }}$

ft msl

$\mathrm{pH}$

$\mu S / \mathrm{cm}$

${ }^{\circ} \mathrm{C}$

$\mathrm{mg} / \mathrm{L}$

NTU

well vol

ft ms!

Synchronous water level

$167.0(09 / 17 / 98)$

ANALYTICAL DATA

Inorganic Constituents

\begin{tabular}{|c|c|c|c|}
\hline Constituents & 3098 & Mod & Filt. \\
\hline Antimony, total recoverable & $<0.20$ & $\mathrm{U} / /$ & $<0.20$ \\
\hline Arsenic, total recoverable & 1.2 & J/E & NDD \\
\hline Barium, total recoverable & 31 & /I & \\
\hline Cadmium, total recoverable & 0.47 & $\mathrm{~J} / \mathrm{E} /$ & NDD \\
\hline Chromium, total recoverable & 0.77 & $\mathrm{~J} / \mathrm{E} /$ & NDD \\
\hline Cobalt, total recoverable & 0.042 & J/E & NDD \\
\hline Copper, total recoverable & 1.1 & /I & \\
\hline Cyanide & $<10$ & $\mathrm{U} / 1$ & $<10$ \\
\hline Lead, total recoverable & $<2.0$ & $\mathrm{U} / \mathrm{I}$ & $<2.0$ \\
\hline Mercury, total recoverable & $<0.20$ & $\mathrm{U} / /$ & $<0.20$ \\
\hline $\begin{array}{l}\text { Nickel, total recoverable } \\
\text { Nitrate as nitrogen }\end{array}$ & $<0.20$ & $\mathrm{U} / I$ & $<0.20$ \\
\hline Nitrate-nitrite as nitrogen & $<40$ & UJ/VII & $<50$ \\
\hline Selenium, total recoverable & $<5.0$ & $\mathbf{U} / /$ & $<5.0$ \\
\hline Silver, total recoverable & $<1.0$ & $\mathrm{U} / \mathrm{I}$ & $<1.0$ \\
\hline Tin, total recoverable & $<2.0$ & $\mathrm{U} / I$ & $<2.0$ \\
\hline Vanadium, total recoverable & $<2.0$ & $\mathrm{U} / /$ & $<2,0$ \\
\hline Zinc, tota! recoverable & $<5.0$ & $\mathrm{U} / /$ & $<5.0$ \\
\hline
\end{tabular}

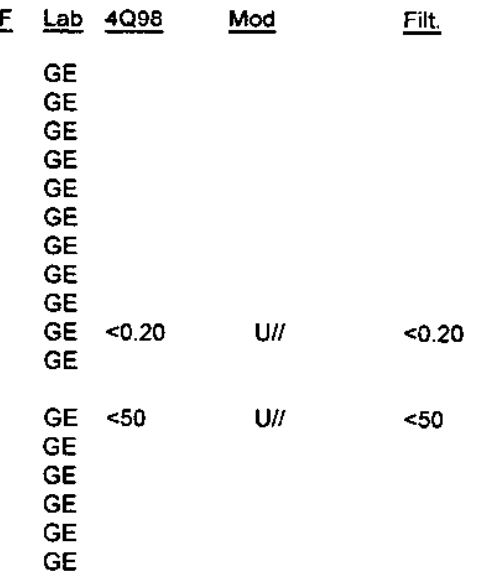

ST H DF Lab Unit

Organic Constituents

Constituents
Benzene
Bis(2-ethylhexyl) phthalate
Dichloromethane
Tetrachloroethylene
Trichloroethylene
Trichlorofluoromethane

3098
$<1.0$
$<10$
$<1.6$
$<1.0$
$<1.0$
$<1.0$

$\begin{array}{ll}\text { Mod } & \text { Filt. } \\ \text { UJ/O/1 } & <1.0 \\ U / / & <10 \\ U J / N O 8 / 1 & <1.0 \\ U J / O / 1 & <1.0 \\ U J / O / 1 & <1.0 \\ U J / O / 1 & <1.0\end{array}$

ST브 $\begin{array}{cc}\text { DF } & \text { Lab } \\ 1 & \text { GE } \\ 1 & \text { GE } \\ 1 & \text { GE } \\ 1 & \text { GE } \\ 1 & \text { GE } \\ 1 & \text { GE }\end{array}$

Mod

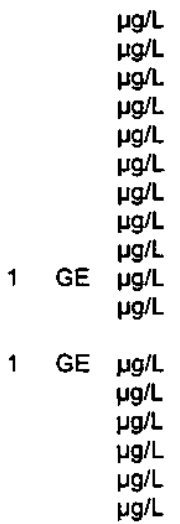

Filt.

ST $\underline{\text { DF Lab Unit }}$

$\mu \mathrm{g} / \mathrm{L}$

$\mu g / L$

$\mu g / L$

$\mu g / L$

$\mu g / L$

$\mu g / L$

Notes:

- exceeded holding time

- = exceeded groundwater protection or monitoring constituent standard (See Appendix A.) 
WELL HSB120A (cont.)

Radioactive Constituents

\begin{tabular}{|c|c|c|c|c|c|c|c|c|c|c|c|c|c|}
\hline Constituents & 3098 & Mod & Filt. & ST브 & DF & $\underline{L a b}$ & $4 Q 98$ & Mod & Filt. & $\underline{\text { ST }} \underline{H}$ & $\underline{D F}$ & $\underline{\text { Lab }}$ & Unit \\
\hline Americium-241 & $<4.2 \mathrm{E}-02$ & $\mathrm{UH} / \mathrm{I}$ & $<0.0515$ & & 1 & GP & & & & & & & $p C i / L$ \\
\hline Carbon-14 & $<-1.6 \mathrm{E}+00$ & $\mathrm{UI} / /$ & $<8.7000$ & & 1 & GP & & & & & & & $\mathrm{pCi} / \mathrm{L}$ \\
\hline Cobalt-60 & $<-1.1 E-01$ & $\mathrm{UI} / /$ & $<3.2600$ & & 1 & GP & & & & & & & $\mathrm{pCi} / \mathrm{L}$ \\
\hline Curium-242 & $<0.0 E+00$ & $\mathrm{UI} / /$ & $<0.0540$ & & 1 & GP & & & & & & & $\mathrm{pCi} / \mathrm{L}$ \\
\hline Curium-243/244 & $<1.7 E-02$ & $\mathrm{UI} / /$ & $<0.0515$ & & 1 & GP & & & & & & & $\mathrm{pCl} / \mathrm{L}$ \\
\hline Curium-245/246 & $<-8.3 \mathrm{E}-03$ & $\mathrm{UI} / /$ & $<0.1610$ & & 1 & GP & & & & & & & $\mathrm{pCi} / \mathrm{L}$ \\
\hline Gross alpha & $1.5 E+\infty 0$ & $\mathrm{~J} / \mathrm{C} /$ & NDD & & 1 & $\mathrm{GP}$ & $<1.9 E+\infty 0$ & $\mathrm{U} / /$ & $<8.3 E-01$ & & 1 & GE & $\mathrm{pCi} / \mathrm{L}$ \\
\hline lodine-129 & $<3.8 \mathrm{E}-01$ & $\mathrm{U} \backslash / !$ & $<0.5330$ & & 1 & GP & & & & & & & pCin \\
\hline Nickel-63 & $<-1,1 E-01$ & UH & $<3.2600$ & & & & & & & & & & $\mathrm{pCi} / \mathrm{L}$ \\
\hline Nonvolatile beta & $1.6 E+00$ & $\|$ & & & 1 & GP & $2.9 E+\infty 0$ & $/ /$ & & & 1 & GE & $\mathrm{pC} U \mathrm{~L}$ \\
\hline Plutonium-238 & $<0.0 E+\infty 0$ & $\mathrm{UI} / /$ & $<0.0799$ & & 1 & GP & & & & & & & $\mathrm{pCi} / \mathrm{L}$ \\
\hline Plutonium-239/240 & $<-6.5 E-03$ & $\mathrm{U} ! / /$ & $<0.2300$ & & 1 & GP & & & & & & & $\mathrm{pCi} / \mathrm{L}$ \\
\hline Radium-226 & $<9.0 E-01$ & $\mathrm{UI} / /$ & $<0.9670$ & & 1 & GP & & & & & & & $\mathrm{pCi} / \mathrm{L}$ \\
\hline Radium-228 & $<6.1 E-02$ & $U(/ /$ & $<0.6960$ & & 1 & GP & & & & & & & $\mathrm{pCi} / \mathrm{L}$ \\
\hline Strontium-90 & $<3.0 \mathrm{E}-01$ & $\mathrm{UIJ} / \mathrm{Cl}$ & & & 1 & GP & & & & & & & $\mathrm{pCi} / \mathrm{L}$ \\
\hline Technetium-99 & $<3.5 \mathrm{E}+00$ & $\mathrm{UI} / /$ & $<22.6000$ & & 1 & GP & & & & & & & $\mathrm{pCi} / \mathrm{L}$ \\
\hline Thorium-228 & $<3,1 \mathrm{E}-02$ & $\mathrm{U} / / /$ & $<0.3580$ & & 1 & GP & & & & & & & $\mathrm{pCi} / \mathrm{L}$ \\
\hline Thorium-230 & 5.3E-01 & $/ 1$ & & & 1 & GP & & & & & & & $\mathrm{pCi} / \mathrm{L}$ \\
\hline Thorium-232 & $<-1.7 E-02$ & $\mathrm{U} 1 / /$ & $<0.2210$ & & 1 & GP & & & & & & & $\mathrm{pCi} / \mathrm{L}$ \\
\hline $\begin{array}{l}\text { Sum of alphas } \\
\text { Sum of betas }\end{array}$ & 5.3E-01 & & & & & & & & & & & & $\mathrm{pCi} / \mathrm{L}$ \\
\hline Tritium & $<-3.2 E-01$ & $\mathrm{UH} / \mathrm{I}$ & $<6.3 E-01$ & & 1 & GP & $<-2.4 E-01$ & $\mathrm{U} / /$ & $<6.3 E-01$ & & 1 & GE & $\mathrm{pCi} / \mathrm{ml}$ \\
\hline Uranium-233/234 & $<6.2 E-02$ & UU/ & $<0.1870$ & & 1 & GP & & & & & & & $\mathrm{pCi} / \mathrm{L}$ \\
\hline Uranium-235 & $<4.8 E-02$ & $\mathrm{UW} / /$ & $<0.3310$ & & 1 & GP & & & & & & & $\mathrm{pCi} / \mathrm{L}$ \\
\hline Uranium-238 & $<1.9 E-01$ & $\mathrm{U} / / /$ & $<0.1870$ & & 1 & GP & & & & & & & $\mathrm{pCi} / \mathrm{L}$ \\
\hline
\end{tabular}

Notes:

- = exceeded holding time

= exceeded groundwater protection or monitoring constituent standard (See Appendix A.) 
WELL HSB121A

$\begin{array}{lll}\text { SRS Coord. } & \text { Lat/Longitude } \\ \text { N72024.8 } & & 33.278709^{\circ} \mathrm{N} \\ \text { E57389.6 } & 81.655589^{\circ} \mathrm{W}\end{array}$

SAMPLE DATE

Constituents

Water elevation

$\mathrm{pH}$

Sp. conductance

Water temperature

Alkalinity as $\mathrm{CaCO}_{3}$

Turbidity

Volumes purged

Sampling code

Synchronous water level
3098

Screen Zone Elevation

98.3-88.3 ft msl

08/03/98

172.5

7.9

220

20.2

90

0.3

2.1

$172.49(9 / 17 / 98)$
Top of Casing

$274.6 \mathrm{ft} \mathrm{msl}$

$10 / 05 / 98$

4Q998

172.0

7.2

200

20.3

93

$172.1(12 / 22 / 98)$
Casing Pump

4" PVC S
Screen Zone

U. Congaree (IIA)

ANALYTICAL DATA

Inorganic Constituents

\section{Constituents}

Antimony, total recoverable

Arsenic, total recoverable

Barium, total recoverable

Cadmium total recoverable $<1.0$

Chromium, total recoverable $<3.0$

Cobalt, total recoverable $\quad 0.63$

Copper, total recoverable $\quad<0.43$

Cyanide

Lead, total recoverable $\quad<2.0$

Mercury, total recoverable $<0.20$

Nickel, total recoverable $\quad 0.84$

Nitrate as nitrogen

Nitrate-nitrite as nitrogen $\quad<22$

Selenium, total recoverable $<5.0$

Silver, total recoverable $<1.0$

Tin, total recoverable $\quad<2.0$

Vanadium, total recoverable $<2.0$

Zinc, total recoverable

10

Organic Constituents

Constituents

Benzene

Bis(2-ethylhexyl) phthalate

Dichloromethane

Tetrachloroethylene

Trichloroethylene

Trichlorofluoromethane
3098

$<1.0$

$<3.6$

$<1.0$

$<1.0$

$<1.0$

$<1.0$

\begin{tabular}{|c|c|}
\hline Mod & Filt. \\
\hline $\begin{array}{l}\mathrm{u} / / \\
\mathrm{J} / \mathrm{E}^{\prime} \\
/ /\end{array}$ & $\begin{array}{l}<0.20 \\
\text { NDD }\end{array}$ \\
\hline $\mathrm{U} / 1$ & $<1.0$ \\
\hline $\mathbf{U} / /$ & $<3.0$ \\
\hline $\mathrm{J} / \mathrm{E} /$ & NDD \\
\hline U/6/ & $<0.20$ \\
\hline $\mathrm{U} / /$ & $<10$ \\
\hline U/I & $<2.0$ \\
\hline $\mathrm{U} / /$ & $<0.20$ \\
\hline$/ 1$ & \\
\hline $\mathrm{U} N /$ & $<20.0000$ \\
\hline $\mathrm{u} / /$ & $<5.0$ \\
\hline $\mathrm{v} / I$ & $<1.0$ \\
\hline $\mathrm{U} / /$ & $<2.0$ \\
\hline $\mathrm{U} / /$ & $<2.0$ \\
\hline $\mathrm{J} / \mathrm{E} /$ & NDD \\
\hline
\end{tabular}

ST브

$$
\begin{aligned}
& \mathrm{D} \\
& 1 \\
& 1 \\
& 1 \\
& 1 \\
& 1 \\
& 1 \\
& 1 \\
& 1 \\
& 1 \\
& 1 \\
& 1
\end{aligned}
$$

DF Lab

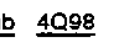

Mod

Filt.

ST $\underline{H}$ DF Lab Unit

GE
GE
GE
GE
GE
WA
GE
GE
GE
GE
GE
WA
GE
GE
$G E$
$G E$
$W A$

Unit

ft msl

$\mathrm{pH}$

$\mu S / c m$

${ }^{\circ} \mathrm{C}$

$\mathrm{mg} / \mathrm{L}$

NTU

well vol

tิ msl

$\mu g / L$

$\mu g / L$

$\mu g h$

$\mu g / L$

$\mu g / L$

$\mu g l$

$\mu g / L$

$\mu g / L$

$<0.20 \quad \mathrm{U} / / \quad<0.20$

$\mu g / L$

1 GE $\mu g / L$

$\mu g / L$

1 GE $\mu g / L$

$\mu \mathrm{g} / \mathrm{L}$

$\mu \mathrm{g} L$

$\mu g / L$

$\mu / L$
$\mu g / L$

\begin{tabular}{|c|c|}
\hline Mod & Filt. \\
\hline $\mathrm{U} / I$ & $<1.0$ \\
\hline UN/ & $<10$ \\
\hline $\mathrm{U} / /$ & $<1.0$ \\
\hline $\mathrm{U} / /$ & $<1.0$ \\
\hline $\mathrm{U} / \prime$ & $<1.0$ \\
\hline $\mathrm{U} / /$ & $<1.0$ \\
\hline
\end{tabular}

ST브

$\begin{array}{ll}\text { DF } & \text { Lab } \\ 1 & \text { GE } \\ 1 & \text { WA } \\ 1 & \text { GE } \\ 1 & \text { GE } \\ 1 & \text { GE } \\ 1 & \text { GE }\end{array}$

Mod

Filt.

ST 브 DF Lab Unit

$\mu g / h$

$\mu g / L$

$\mu g / L$

$\mu g / 2$

$\mu g / L$

$\mu g /$

Notes:

- exceeded holding time

- exceeded groundwater protection or monitoring constituent standard (See Appendix A.) 
WELL HSB121A (cont.)

Radioactive Constituents

\begin{tabular}{|c|c|c|c|c|c|c|c|c|c|c|c|c|c|}
\hline Constituents & 3098 & Mod & Filt. & $\underline{\text { ST }} \underline{\underline{H}}$ & $\underline{\mathrm{DF}}$ & Lab & 4Q998 & Mod & Filt. & $\underline{S T} \underline{H}$ & $\underline{\text { DF }}$ & $\underline{\text { Lab }}$ & Unit \\
\hline Americium-241 & $<1.6 \mathrm{E}-02$ & $\mathrm{UI} / /$ & $<0.1900$ & & 1 & GP & & & & & & & $\mathrm{pCi} / \mathrm{L}$ \\
\hline Carbon-14 & $<1.7 \mathrm{E}+00$ & $\mathrm{UI} / /$ & $<9.4200$ & & 1 & GP & & & & & & & $\mathrm{pCi} / \mathrm{L}$ \\
\hline Cobalt-60 & $<1.7 \mathrm{E}-01$ & $\mathrm{UI} / /$ & $<6.9600$ & & 1 & $\mathrm{TM}$ & & & & & & & $\mathrm{pCi} / \mathrm{L}$ \\
\hline Curium-242 & $<-2.8 E-02$ & $\mathrm{UI} / /$ & $<0.2360$ & & 1 & GP & & & & & & & $\mathrm{pCi} / \mathrm{L}$ \\
\hline Curium-243/244 & $<2.4 \mathrm{E}-02$ & $\mathrm{U} ! / /$ & $<0.2100$ & & 1 & GP & & & & & & & $\mathrm{pC}: \mathrm{L}$ \\
\hline Curium-245/246 & $<0.0 E+00$ & $\mathrm{UI} / /$ & $<0.0687$ & & 1 & GP & & & & & & & $\mathrm{pCi} / \mathrm{h}$ \\
\hline Gross alpha & $2.0 E+00$ & $/ 1$ & & & 1 & TM & $<9.8 \mathrm{E}-01$ & $\mathrm{U} / \mathrm{I}$ & $<7.9 E-01$ & & 1 & GE & $\mathrm{pCi} / \mathrm{L}$ \\
\hline Jodine-129 & $<1.2 \mathrm{E}-01$ & $\mathrm{UI} / /$ & $<0.9960$ & & 1 & GP & & & & & & & $\mathrm{pCi} / \mathrm{L}$ \\
\hline Nickel-63 & $<1.7 \mathrm{E}-01$ & Ul & $<6.9600$ & & & & & & & & & & PCill \\
\hline Nonvolatile beta & $8.4 E+00$ & $J / X \mid$ & NDD & & 1 & $\mathrm{TM}$ & $<1.1 E+\infty 0$ & $\mathrm{U} / /$ & $<1.1 \mathrm{E}+00$ & & 1 & GE & $\mathrm{pCi} / \mathrm{L}$ \\
\hline Plutonium-238 & $<-9.6 \mathrm{E}-03$ & UIII & $<0.2100$ & & 1 & GP & & & & & & & $\mathrm{pCi} / \mathrm{L}$ \\
\hline Plutonium-239/240 & $<0.0 \mathrm{E}+00$ & $\mathrm{UI} / /$ & $<0.1190$ & & 1 & GP & & & & & & & $\mathrm{pCi} / \mathrm{L}$ \\
\hline Radium-226 & $1.2 \mathrm{E}+00$ & $/ 1$ & & & 1 & GP & & & & & & & $\mathrm{pCi} / \mathrm{L}$ \\
\hline Radium-228 & $<-5.7 E-01$ & $\mathrm{UIII}$ & $<2.9200$ & & 1 & $T M$ & & & & & & & $\mathrm{pCi} / \mathrm{L}$ \\
\hline Strontium-90 & $<0.0 E+00$ & $\mathrm{UI} / /$ & $<2.0300$ & & 1 & $\mathrm{TM}$ & & & & & & & $\mathrm{pCi} / \mathrm{L}$ \\
\hline Technetium-99 & $<-3.9 E-01$ & $\mathrm{U} ! / /$ & $<6.5300$ & & 1 & GP & & & & & & & $\mathrm{pCi} / \mathrm{L}$ \\
\hline Thorium-228 & $2.9 \mathrm{E}-01$ & $/ 1$ & & & 1 & GP & & & & & & & $\mathrm{pCi} / \mathrm{L}$ \\
\hline Thorium-230 & $1.1 E+00$ & /I & & & 1 & $T M$ & & & & & & & $\mathrm{pCi} / \mathrm{L}$ \\
\hline Thorium-232 & $<0.0 \mathrm{E}+\infty 0$ & UIII & $<0.0900$ & & 1 & GP & & & & & & & $\mathrm{pCi} / \mathrm{L}$ \\
\hline $\begin{array}{l}\text { Sum of atphas } \\
\text { Sum of betas }\end{array}$ & $1.4 \mathrm{E}+00$ & & & & & & & & & & & & $\rho \mathrm{Ci} / \mathrm{L}$ \\
\hline Total radium & $1.2 \mathrm{E}+00$ & & & & & & & & & & & & $\mathrm{pCi} / \mathrm{L}$ \\
\hline Tritium & $<1.7 \mathrm{E}-01$ & $\mathrm{U} 1 / /$ & $<1.2000$ & & 1 & $T M$ & $<2.5 E-01$ & $\mathrm{U} / /$ & $<5.3 E-01$ & & 1 & GE & $\mathrm{pCi} / \mathrm{mL}$ \\
\hline Uranium-233/234 & $<1.3 E-01$ & $\mathrm{UNI}$ & $<0.0895$ & & 1 & GP & & & & & & & $\mathrm{pCi} / \mathrm{L}$ \\
\hline Uranium-235 & $<1.7 E-02$ & $\mathrm{UI} / /$ & $<0.0499$ & & 1 & GP & & & & & & & $\mathrm{pCi} / \mathrm{L}$ \\
\hline Uranium-238 & $<3.6 \mathrm{E}-02$ & UIII & $<0.0533$ & & 1 & GP & & & & & & & $\mathrm{pCill}$ \\
\hline
\end{tabular}

Notes:

- = exceeded holding time

- = exceeded groundwater protection or monitoring constituent standard (See Appendix A.) 
WELL HSB122A

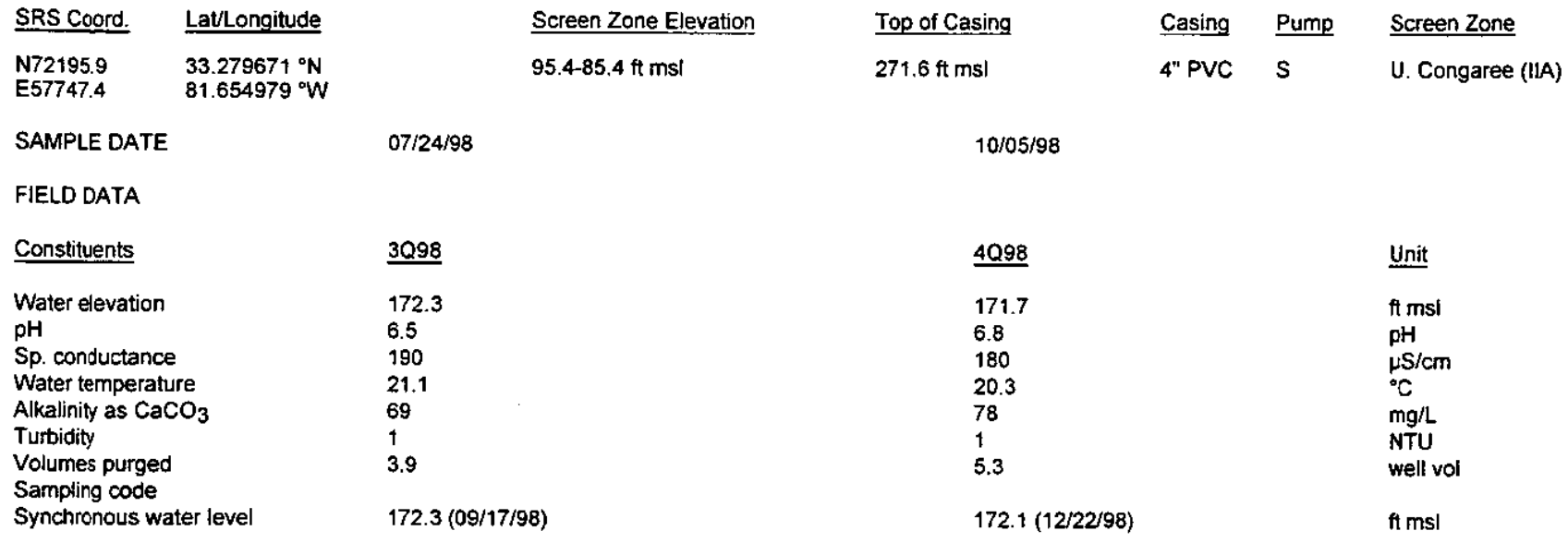

ANALYTICAL DATA

Inorganic Constituents

\begin{tabular}{|c|c|c|c|c|c|c|c|c|c|}
\hline Constituents & $\underline{3 Q 98}$ & Mod & Filt. & $\underline{\text { ST }} \underline{H}$ & $\underline{\mathrm{DF}}$ & $\underline{\underline{L a b}}$ & $\underline{4 Q 98}$ & Mod & Fill. \\
\hline Antimony, total recoverable & $<0.20$ & $\mathrm{U} / /$ & $<0.20$ & & 1 & GE & & & \\
\hline Arsenic, total recoverable & $<3.0$ & U/I & $<3.0$ & & 1 & GE & & & \\
\hline Barium, total recoverable & 24 & /I & & & 1 & $\mathrm{GE}$ & & & \\
\hline Cadmitm, total recoverable & $<1.0$ & U/I & $<1.0$ & & 1 & $\mathrm{GE}$ & & & \\
\hline Chromium, total recoverable & $<3.0$ & $\mathrm{U} / 1$ & $<3.0$ & & 1 & GE & & & \\
\hline Cobalt, total recoverable & $<0.20$ & $\mathrm{U} / 1$ & $<0.20$ & & 1 & $\mathrm{GE}$ & & & \\
\hline Copper, total recoverable & 0.36 & "I & & & 1 & GE & & & \\
\hline Cyanide & $<10$ & $\mathrm{U} / /$ & $<10$ & & 1 & $\mathrm{GE}$ & & & \\
\hline Lead, total recoverable & $<2.0$ & $\mathrm{U} / /$ & $<2.0$ & & 1 & $\mathrm{GE}$ & & & \\
\hline Mercury, total recoverable & 0.039 & $\mathrm{~J} / \mathrm{E}$ & NDD & & 1 & $\mathrm{GE}$ & $<0.20$ & $\mathrm{U} / \mathrm{l}$ & $<0.20$ \\
\hline Nickel, total recoverable & 1.3 & "ll & & & 1 & GE & & & \\
\hline Nitrate-nitrite as nitrogen & $<20$ & UJ/V/1 & $<50$ & & 1 & $\mathrm{GE}$ & $<10$ & uNI & $<$ \\
\hline Selenium, total recoverable & $<5.0$ & & $<5.0$ & & 1 & GE & & & \\
\hline Silver, total recoverable & $<1.0$ & $\mathrm{u} / /$ & $<1.0$ & & 1 & GE & & & \\
\hline Tin, total recoverable & $<2.0$ & $\mathrm{u} / 1$ & $<2.0$ & & 1 & $\mathrm{GE}$ & & & \\
\hline Vanadium, total recoverable & $<2.0$ & $\mathrm{U} / 1$ & $<2.0$ & & 1 & GE & & & \\
\hline & & & & & & & & & \\
\hline
\end{tabular}

\section{Organic Constituents}

\begin{tabular}{ll} 
Constituents & 3098 \\
\hline Benzene & $<1.0$ \\
Bis(2-ethylhexyl) phthalate & $<9.9$ \\
Dichloromethane & $<1.0$ \\
Tetrachloroethylene & $<1.0$ \\
Trichloroethylene & $<1.0$ \\
Trichlorofluoromethane & $<1.0$
\end{tabular}

$\begin{array}{lllll}\text { Mod } & \text { Filt. } & \text { ST브 } & \underline{\text { DF }} & \text { Lab } \\ U J / O / 1 & <1.0 & & 1 & \mathrm{GE} \\ \mathrm{U} / / & <9.9 & & 1 & \mathrm{GE} \\ \mathrm{UJ} / \mathrm{O} / 1 & <1.0 & & 1 & \mathrm{GE} \\ \mathrm{UJ} / \mathrm{O} / 1 & <1.0 & & 1 & \mathrm{GE} \\ \mathrm{UJ} / \mathrm{O} / 1 & <1.0 & & 1 & \mathrm{GE} \\ \mathrm{UJ} / \mathrm{O} / 1 & <1.0 & & 1 & \mathrm{GE}\end{array}$

\section{$4098 \quad$ Mod}

Notes:

- = exceeded holding time

- exceeded groundwater protection or monitoring constituent standard (See Appendix A.) 
WELL HSB122A (cont.)

Radioactive Constituents

\begin{tabular}{|c|c|c|c|c|c|c|c|c|c|c|c|c|c|}
\hline Constituents & 3098 & Mod & Filt. & ST & $\underline{D F}$ & $\underline{\text { Lab }}$ & $\underline{4 Q 98}$ & Mod & Filt. & $\underline{\text { ST }} \underline{H}$ & $\underline{\mathrm{DF}}$ & $\underline{L a b}$ & Unit \\
\hline Americium-241 & $<1.5 E-02$ & $\mathrm{UI} / /$ & $<0.0724$ & & 1 & GP & & & & & & & $\mathrm{pCi} / \mathrm{L}$ \\
\hline Carbon-14 & $<-2.0 E+00$ & $\mathrm{UI} / /$ & $<9.8500$ & & 1 & GP & & & & & & & $\mathrm{pCi} / \mathrm{L}$ \\
\hline Cobalt-60 & $<-1.3 E-01$ & $\mathrm{UI} / I$ & $<3.5100$ & & 1 & GP & & & & & & & $\mathrm{pCilt}$ \\
\hline Curium-242 & $<0.0 E+\infty 0$ & $\mathrm{UI} / I$ & $<0.0756$ & & 1 & GP & & & & & & & $\mathrm{pCi} / \mathrm{L}$ \\
\hline Curium-243/244 & $<4.3 E-02$ & $\mathrm{UI} / I$ & $<0.1270$ & & 1 & GP & & & & & & & $\mathrm{pCi} / \mathrm{L}$ \\
\hline Curium-245/246 & $<2.4 \mathrm{E}-02$ & $\mathrm{U} 1 / /$ & $<0.0723$ & & 1 & GP & & & & & & & $\mathrm{pCi} / \mathrm{L}$ \\
\hline Gross alpha & $<3.8 \mathrm{E}-01$ & UH $/ /$ & $<0.8560$ & & 1 & $\mathrm{GP}$ & $<4.3 \mathrm{E}-01$ & $\mathrm{U} / 1$ & $<7.8 E-01$ & & 1 & GE & $\mathrm{pCi} / \mathrm{L}$ \\
\hline lodine-129 & $<4,2 E-01$ & UI/I & $<1.1000$ & & 1 & $\mathrm{GP}$ & & & & & & & $\mathrm{pCi} / \mathrm{L}$ \\
\hline Nickel-63 & $<-1.3 \mathrm{E}-01$ & UI & $<3.5100$ & & & & & & & & & & $\mathrm{pCi} / \mathrm{L}$ \\
\hline Nonvolatile beta & $<9.9 E-01$ & $\mathrm{UI} / /$ & $<1.1700$ & & 1 & GP & $<1.5 E+00$ & $\mathrm{U} / 1$ & $<1.2 \mathrm{E}+00$ & & 1 & GE & $\mathrm{pCi} / \mathrm{L}$ \\
\hline Plutonium-238 & $<0.0 \mathrm{E}+00$ & $\mathrm{UI} / /$ & $<0.0589$ & & 1 & $G P$ & & & & & & & $\mathrm{pCi} / \mathrm{L}$ \\
\hline Plutonium-239/240 & $<2.2 E-02$ & $\mathrm{UI} / /$ & $<0.0666$ & & 1 & GP & & & & & & & $\mathrm{pCi} / \mathrm{L}$ \\
\hline Radium-226 & $<8.0 E-01$ & UI/ & $<0.9600$ & & 1 & GP & & & & & & & $\mathrm{pCi} / \mathrm{L}$ \\
\hline Radium-228 & $<6.4 \mathrm{E}-01$ & $\mathrm{UH} / \mathrm{I}$ & $<0.8810$ & & 1 & GP & & & & & & & $\mathrm{pCi} / \mathrm{L}$ \\
\hline Strontium-90 & $<6.5 E-02$ & UlJ/Cl & & & 1 & GP & & & & & & & $\mathrm{pCi} / \mathrm{L}$ \\
\hline Technetium-99 & $<5.0 E+00$ & $\mathrm{U!} / /$ & $<21.2000$ & & 1 & GP & & & & & & & pCilL \\
\hline Thorium-228 & $<1.6 \mathrm{E}-01$ & $\mathrm{U}: / /$ & $<0.2130$ & & 1 & $\mathrm{GP}$ & & & & & & & $\mathrm{pCi} / \mathrm{L}$ \\
\hline Thorium-230 & 1.7E-01 & $/ 1$ & & & 1 & $\mathrm{GP}$ & & & & & & & $\mathrm{pCi} / \mathrm{L}$ \\
\hline $\begin{array}{l}\text { Thorium- } 232 \\
\text { Sum of alphas } \\
\text { Sum of betas }\end{array}$ & $\begin{array}{l}<0.0 E+00 \\
1.7 E-01\end{array}$ & $\mathrm{UI} / /$ & $<0.1000$ & & 1 & GP & & & & & & & $\begin{array}{l}\mathrm{pCi} / \mathrm{L} \\
\mathrm{pCi} / \mathrm{L}\end{array}$ \\
\hline Tritium & $<-9.3 E-02$ & $\mathrm{UI} / /$ & $<5.9 E-01$ & & $t$ & GP & $<1.0 \mathrm{E}-01$ & $\mathrm{w} / \mathrm{I}$ & $<5.3 \mathrm{E}-01$ & & 1 & $\mathrm{GE}$ & \\
\hline Uranium-233/234 & $<9.0 \mathrm{E}-02$ & UII/ & $<0.4900$ & & $t$ & GP & & & & & & & $\mathrm{pCi} / \mathrm{L}$ \\
\hline Uranium-235 & $<-1,7 \mathrm{E}-02$ & U1// & $<0.3730$ & & 1 & GP & & & & & & & $\mathrm{pCi} / \mathrm{h}$ \\
\hline Uranium-238 & $<7.1 \mathrm{E}-02$ & $\mathrm{U} \mathrm{I} / \mathrm{I}$ & $<0.2120$ & & 1 & GP & • & & & & & & $\mathrm{Ci} / \mathrm{L}$ \\
\hline
\end{tabular}

Notes:

- exceeded holding time

- exceeded groundwater protection or monitoring constituent standard (See Appendix A.) 
WELL HSB123A

\begin{tabular}{lll} 
SRS Coord. & Lat/Longitude \\
\cline { 1 - 1 } N72189.8 & & $33.280273^{\circ} \mathrm{N}$ \\
E58124.8 & & $81.653973^{\circ} \mathrm{W}$
\end{tabular}

SAMPLE DATE

$07 / 24 / 98$

\section{Screen Zone Elevation}

103.6-93.6 ft ms

FIELD DATA

Constituents

Water elevation

$\mathrm{pH}$

Sp. conductance

Water temperature

Alkalinity as $\mathrm{CaCO}_{3}$

Turbidity

Volumes purged

Sampling code

Synchronous water level

\section{$\underline{3 Q 98}$}

173.4

10.9

1000

22.2

151

0.0

$\mathrm{XN}$

$174.0(09 / 17 / 98)$
Top of Casing

$265.7 \mathrm{ft} \mathrm{msl}$

$10 / 05 / 98$

4Q98

174.3

10.9

700

27.3

92

0.019

XN

$173.0(12 / 22 / 98)$
Casing Pump Screen Zone

$4^{n}$ PVC S $\quad$ U. Congaree (IIA)

ANALYTICAL DATA

Inorganic Constituents

Constituents

30.98

Mod

Filt

$<0.20$

Arsenic, total recoverable $<3.0$

Barium, total recoverable $\quad 310$

Cadmium, total recoverable $<10$

Chromium, total recoverable $<3.0$

Cobalt, total recoverable $\quad 0.060$

Copper, total recoverable $\quad 0.43$

Cyanide

$<10$

Lead, total recoverable

2.7

Mercury, total recoverable

Nickel, total recoverable

Nitrate as nitrogen

Nitrate-nitrite as nitrogen $\quad<40$

Selenium, total recoverable $<5.0$

Sitver, total recoverable $<1.0$

Tin, total recoverable $<2.0$

Vanadium, total recoverable $<2.0$

Zinc, total recoverable

25

\begin{tabular}{|c|c|}
\hline U/I & $<0.20$ \\
\hline $\mathrm{U} / I$ & $<3.0$ \\
\hline $\mathrm{u} / 1$ & $<1.0$ \\
\hline $\mathrm{U} / /$ & $<3.0$ \\
\hline $\begin{array}{l}\mathrm{J} / \mathrm{E} / \\
/ /\end{array}$ & NDD \\
\hline U/I & $<10$ \\
\hline $\begin{array}{l}\mathrm{U} / / \\
/ /\end{array}$ & $<0.20$ \\
\hline$U J / V / 1$ & $<50$ \\
\hline $\mathrm{U} / \mathrm{I}$ & $<5.0$ \\
\hline $\mathrm{U} / /$ & $<1.0$ \\
\hline $\mathrm{U} / I$ & $<2.0$ \\
\hline & $<2.0$ \\
\hline
\end{tabular}

Organic Constituents

Constituents

Benzene

Bis(2-ethylhexyl) phthalate

Dichloromethane

Tetrachloroethylene

Trichloroethylene

Trichlorofluoromethane
3098

$<1.0$

$<9.8$

$<1.0$

$<1.0$

$<1.0$

$<1.0$
Mod

$\mathrm{UJ} / \mathrm{O} / 1$

U/I

$\mathrm{UJ} / \mathrm{O} / 1$

UJ/O/1

$\mathrm{UJ} / \mathrm{O} / 1$
Filt.

$<1.0$

$<9.8$

$<1.0$

$<1.0$

$<1.0$ $\underline{\text { ST}} \underline{H}$

$\mathrm{La}$
$\mathrm{GE}$
$\mathrm{GE}$
$\mathrm{GE}$
$\mathrm{GE}$
$\mathrm{GE}$
$\mathrm{GE}$
$\mathrm{GE}$
$\mathrm{GE}$
$\mathrm{GE}$
$\mathrm{GE}$
$\mathrm{GE}$
$\mathrm{GE}$
$\mathrm{GE}$
$\mathrm{GE}$
$\mathrm{GE}$
$\mathrm{GE}$

$<0.20$

$\mathrm{U} /$

120

EE

GE

GE

$\underline{\text { ST}} \underline{H}$

$\begin{array}{ll}\text { DF } & \text { Lab } \\ 1 & \text { GE } \\ 1 & \text { GE } \\ 1 & \text { GE } \\ 1 & \text { GE } \\ 1 & \text { GE } \\ 1 & \text { GE }\end{array}$

$\underline{\text { Unit }}$

ft msl

$\mathrm{pH}$

$\mu \mathrm{S} / \mathrm{cm}$

${ }^{\circ} \mathrm{C}$

$\mathrm{mg} / \mathrm{L}$

NTU

well vol

$\mathrm{ft}$ ms:

Filt.

ST 브 LF Lab Unit

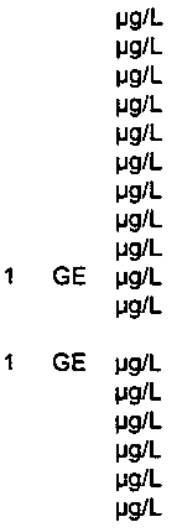

Filt.

ST 브 DF Lab Unit

$\mu g / L$

$\mu g / L$

$\mu g / L$

$\mu g /$

$\mu g / L$

Notes:

- = exceeded holding time

- exceeded groundwater protection or monitoring constituent standard (See Appendix A.) 


\section{WELL HSB123A (cont.)}

\section{Radioactive Constituents}

\section{Constituents}

Americium-241

Beta dose

Carbon-14

Cobait-60

Curium-242

Curium-243/244

Curium-245/246

Gross alpha

lodine-129

Nickel-63

Nonvolatile beta

Plutonium-238

Plutonium-239/240

Radium-226

Radium-228

Strontium-90

Technetium-99

Thorium-228

Thorium-230

Thorium-232

Sum of alphas

Sum of betas

Total radium

Tritium

Uranium-233/234

Uranium-235

Uranium-238

\begin{tabular}{|c|c|c|c|c|c|c|c|}
\hline 3Q98 & Mod & Filt. & ST브 & $\underline{\mathrm{DF}}$ & Lab & $4 Q 98$ & Mod \\
\hline $\begin{array}{l}<2.5 E-01 \\
0.20\end{array}$ & $\mathrm{U} N /$ & $<0.1630$ & & 1 & $G P$ & & \\
\hline$<-2.7 E+00$ & UII/ & $<9.2200$ & & 1 & $G P$ & & \\
\hline$<1.0 \mathrm{E}+00$ & $\mathrm{UI} / /$ & $<3.0400$ & & 1 & GP & & \\
\hline$<0.0 \mathrm{E}+00$ & Ull/ & $<0.1830$ & & 1 & GP & & \\
\hline$<-5.3 E-02$ & UU/I & $<0.4140$ & & 1 & GP & & \\
\hline$<5.4 E-02$ & $\mathrm{UI} / /$ & $<0.1630$ & & 1 & GP & & \\
\hline $2.4 \mathrm{E}+00$ & $1 /$ & & & 1 & $G P$ & $<2.9 \mathrm{E}+00$ & $\mathrm{U} / /$ \\
\hline$<3.3 E-01$ & $\mathrm{UI} / /$ & $<0.6190$ & & 1 & GP & & \\
\hline$<1.0 \mathrm{E}+00$ & UI & $<3.0400$ & & & & & \\
\hline $3.3 E+00$ & /I & & & 1 & GP & $5.1 E+00$ & $\|$ \\
\hline$<0.0 E+00$ & UI// & $<0.0600$ & & 1 & $G P$ & & \\
\hline$<0.0 E+00$ & $\mathrm{U} 1 / /$ & $<0.0599$ & & 1 & GP & & \\
\hline $6.4 E+00$ & /I & & ט & 1 & GP & & \\
\hline $1.0 \mathrm{E}+00$ & /I & & & 1 & GP & & \\
\hline$<5,1 \mathrm{E}-02$ & $\mathrm{U} \mid \mathrm{J} / \mathrm{C} /$ & & & 1 & GP & & \\
\hline$<1.2 \mathrm{E}+\infty 0$ & $\mathrm{U} 1 / /$ & $<21.2000$ & & 1 & GP & & \\
\hline$<9.8 E-02$ & $\mathrm{UI} / /$ & $<0.2430$ & & 1 & GP & & \\
\hline 1.6E-01 & 11 & & & 1 & $G P$ & & \\
\hline$<3.1 E-02$ & U:II & $<0.0942$ & & 1 & GP & & \\
\hline $\begin{array}{l}1.6 \mathrm{E}-01 \\
1.0 \mathrm{E}+00\end{array}$ & & & & & & & \\
\hline $7.5 E+00$ & & & & & & & \\
\hline$<-7.7 E-03$ & UI// & $<6.3 E-01$ & & 1 & GP & $<9.3 \mathrm{E}-02$ & $\mathrm{U} / 1$ \\
\hline$<5.1 \mathrm{E}+02$ & $\mathrm{UI} / /$ & $<0.3520$ & & 1 & GP & & \\
\hline$<5.1 E-02$ & UIII & $<0.3530$ & & 1 & GP & & \\
\hline$<5.1 E-02$ & $\mathrm{UI} / /$ & $<0.3520$ & & 1 & GP & & \\
\hline
\end{tabular}

Fist.

ST $\underline{H}$ DF Lab Un

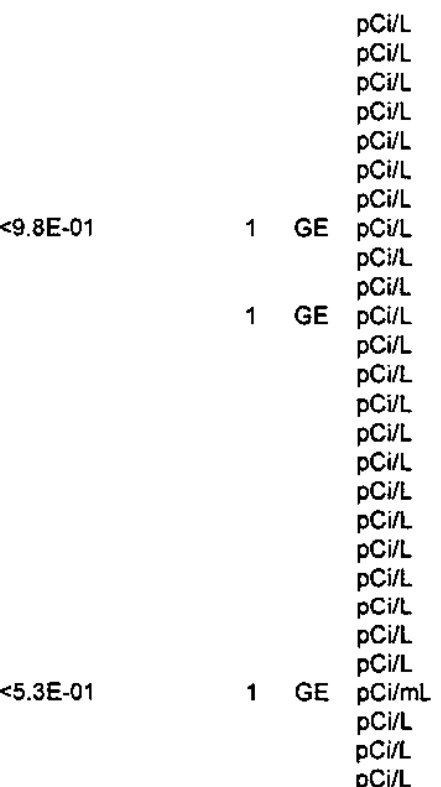

Notes:

- exceeded holding time

- exceeded groundwater protection or monitoring constituent standard (See Appendix A.) 
WELL HSB124AR

\begin{tabular}{|c|c|}
\hline SRS Coord. & Lat/Longitude \\
\hline $\begin{array}{l}N 72202.7 \\
E 58531.7\end{array}$ & 81.652927 \\
\hline
\end{tabular}

SAMPLE DATE

FIELD DATA

Constituents

Water elevation

$\mathrm{pH}$

Sp. conductance

Water temperature

Alkalinity as $\mathrm{CaCO}_{3}$

Turbidity

Volumes purged

Sampling code

Synchronous water leved
$08 / 03 / 98$

Screen Zone Elevation

$104.6-94.6 \mathrm{ft} \mathrm{ms|}$

Top of Casing

$266.8 \mathrm{ft} \mathrm{ms}$

$10 / 05 / 98$

3Q98

172.6

7.4

230

20.9

75

23

$172.73(9 / 17 / 98)$
4Q98

172.4

6.8

180

20.9

1

4.6

$172.4(12 / 22 / 98)$
Casing Pump Screen Zone

4" PVC S U. Congaree (IIA)

\section{ANALYTICAL DATA}

Inorganic Constituents

Constituents

Mod

Antimony, total recoverable $<0.20$

Arsenic, total recoverable $\mathbf{1 . 1}$

Barium, total recoverable 33

Cadmium, total recoverable $<1.0$

Chromium, total recoverable $<3.0$

Cobalt, total recoverable $\quad 0.16$

Copper, total recoverable $\quad 0.42$

Cyanide

Lead, total recoverable

Mercury, total recoverable $\quad 0.77$

Nickel, total recoverable $\quad 1.1$

Nitrate as nitrogen

Nitrate-nitrite as nitrogen $\quad<30$

Selenium, total recoverable $<5.0$

Silver, total recoverable $<1.0$

Tin, total recoverable $\quad<2.0$

Vanadium, total recoverable $<2.0$

Zinc, total recoverable

$<5.0$

Organic Constituents

Constituents

\section{Q98}

Benzene

$<1.0$

Bis(2-ethylhexyl) phthalate

Dichloromethane

Tetrachloroethylene

Trichloroethylene

$<9.9$

$<1.4$

$<1.0$

Trichlorofluoromethane
Filt.

\begin{tabular}{|c|c|}
\hline $\begin{array}{l}\mathrm{U} / / \\
\mathrm{J} / \mathrm{E} / \\
/ /\end{array}$ & $\begin{array}{l}<0.20 \\
\text { NDD }\end{array}$ \\
\hline $\mathrm{U} / /$ & $<1.0$ \\
\hline $\mathrm{U} / /$ & $<3.0$ \\
\hline $\begin{array}{l}\mathrm{J} / \mathrm{E} / \\
/ /\end{array}$ & NDD \\
\hline$U / I$ & $<10$ \\
\hline $\mathrm{J} / \mathrm{E} /$ & NDD \\
\hline
\end{tabular}

$\begin{array}{ll}\mathrm{U} N / & <50 \\ \mathrm{U} / & <5.0\end{array}$

$\mathrm{U} / 1 \quad<5.0$

$\mathrm{U} / 1 \quad<1.0$

$<2.0$

$<5.0$

$\begin{array}{ll}\text { Mod } & \text { Filt. } \\ \text { U/I } & <1.0 \\ U / \prime & <9.9 \\ \text { UNI } & <1.0 \\ U / I & <1.0 \\ U \prime \prime & <1.0 \\ U / I & <1.0\end{array}$

$<1.0$

ST브

$\begin{array}{ll}\text { DF } & \text { Lab } \\ 1 & \text { GE } \\ 1 & \text { GE } \\ 1 & \text { GE } \\ 1 & \text { GE } \\ 1 & \text { GE } \\ 1 & \text { GE }\end{array}$

DF Lab 4 QQ98

GE

GE

GE

1 GE

1 GE

GE

1 GE

1 GE

$\mathrm{GE}$

1 GE

1 GE

$<0.20$

Mod

$\mathrm{U} / 1$

$<20$

UNI

$\mathrm{GE}$

GE

GE

GE

1 GE
Unit

ft $\mathrm{ms}$

pH

$\mu \mathrm{S} / \mathrm{cm}$

${ }^{\circ} \mathrm{C}$

$\mathrm{mg} / \mathrm{L}$

NTU

well vol

ft mst
Mod

Filt.

ST $\underline{H}$ DF Lab Unit

$\mu g / L$

$\mu g / L$

$\mu g / L$

$\mu g / L$

$\mu \mathrm{g} / \mathrm{L}$

$\mu g / L$

Notes:

- = exceeded holding time

- exceeded groundwater protection or monitoring constituent standard (See Appendix A.) 
WELL HSB124AR (cont.)

Radioactive Constituents

\begin{tabular}{|c|c|c|c|c|c|c|c|c|c|c|c|c|c|}
\hline Constituents & 3Q98 & Mod & Filt. & $\underline{\text { ST }} \underline{H}$ & $\underline{\mathrm{DF}}$ & $\underline{\mathrm{Lab}}$ & 4098 & Mod & Filt. & ST $\underline{H}$ & DF & $\underline{\text { Lab }}$ & Unit \\
\hline $\begin{array}{l}\text { Americium-241 } \\
\text { Beta dose }\end{array}$ & $\begin{array}{l}<1.8 \mathrm{E}-02 \\
0.19\end{array}$ & $\mathrm{UI} / /$ & $<0.2180$ & & 1 & GP & & & & & & & $\mathrm{pCil}$ \\
\hline Carbon-14 & $<1.5 E+00$ & $\mathrm{UI} / /$ & $<9.2700$ & & 1 & GP & & & & & & & $P C i / L$ \\
\hline Cobalt-60 & $<9.9 E-01$ & $\mathrm{U} W /$ & $<3.7400$ & & 1 & GP & & & & & & & $\begin{array}{l}\mathrm{pCi} / \mathrm{L} \\
\mathrm{pCi} / \mathrm{L}\end{array}$ \\
\hline Curium-242 & $<-1.2 \mathrm{E}-02$ & $\mathrm{UI} / I$ & $<0.1550$ & & 1 & GP & & & & & & & $\mathrm{pCi} / \mathrm{L}$ \\
\hline Curium-243/244 & $<-1.1 \mathrm{E}-02$ & $\mathrm{UI} / /$ & $<0.1440$ & & 1 & GP & & & & & & & $\mathrm{pCi} / \mathrm{L}$ \\
\hline Curium-245/246 & $<0.0 E+00$ & $U I / /$ & $<0.0693$ & & 1 & GP & & & & & & & $\mathrm{pCi} / \mathrm{L}$ \\
\hline Gross alpha & $1.2 \mathrm{E}+00$ & $/ /$ & & & 1 & GP & $<1.1 E+00$ & $\mathrm{U} / /$ & $<8.2 \mathrm{E}-01$ & & 1 & GE & $\mathrm{pCi} / \mathrm{L}$ \\
\hline lodine-129 & $<4.4 \mathrm{E}-01$ & $\mathrm{UI} / /$ & $<0.5740$ & & 1 & GP & & & & & & & $\mathrm{pCi} / \mathrm{L}$ \\
\hline Nickel-63 & $<9.9 E-01$ & UI & $<3.7400$ & & & & & & & & & & $\mathrm{pCi} / \mathrm{L}$ \\
\hline Nonvolatile beta & $<1.8 \mathrm{E}+00$ & $\mathrm{UI} / /$ & $<1.9000$ & & 1 & GP & $<1.1 \mathrm{E}+00$ & $\mathrm{U} / /$ & $<1.3 E+00$ & & 1 & GE & $\mathrm{pCi} / \mathrm{L}$ \\
\hline Plutonium-238 & $<-8.9 E-03$ & $\mathrm{UI} / /$ & $<0.1960$ & & 1 & GP & & & & & & & pCill \\
\hline Plutonium-239/240 & $<3.7 E-02$ & $\mathrm{UI} / /$ & $<0.1110$ & & 1 & GP & & & & & & & pCi/L \\
\hline Radium-226 & 5.3E-01 & $/ /$ & & & 1 & GP & & & & & & & $\mathrm{pCi} / \mathrm{L}$ \\
\hline Radium-228 & 9.3E-01 & $/ /$ & & & 1 & GP & & & & & & & $\mathrm{pCi} / \mathrm{L}$ \\
\hline Strontium-90 & $<4.2 \mathrm{E}-01$ & $\mathrm{U}[\mathrm{J} / \mathrm{C} /$ & & & 1 & GP & & & & & & & $\mathrm{pCi} / \mathrm{L}$ \\
\hline Technetium-99 & $<-9.8 E-01$ & $\mathrm{U} 1 / /$ & $<6.9800$ & & 1 & GP & & & & & & & $\mathrm{pCi} / \mathrm{L}$ \\
\hline Thorium-228 & $<9.1 E-03$ & $\mathrm{UI} / /$ & $<0.2250$ & & 1 & GP & & & & & & & $\mathrm{pCi} / \mathrm{L}$ \\
\hline Thorium-230 & $<5.6 \mathrm{E}-02$ & $\mathrm{UI} / /$ & $<0.1670$ & & 1 & GP & & & & & & & $\mathrm{pCi} / \mathrm{L}$ \\
\hline $\begin{array}{l}\text { Thorium-232 } \\
\text { Sum of alphas }\end{array}$ & $<-7.6 \mathrm{E}-03$ & $\mathrm{UI} / /$ & $<0.1670$ & & 1 & GP & & & & & & & $\mathrm{pCi} / \mathrm{L}$ \\
\hline $\begin{array}{l}\text { Sum of betas } \\
\text { Total radium }\end{array}$ & $\begin{array}{l}9.3 \mathrm{E}-01 \\
1.5 \mathrm{E}+00\end{array}$ & & & & & & & & & & & & $\begin{array}{l}\mathrm{pCi} / \mathrm{L} \\
\mathrm{pCi} / \mathrm{L}\end{array}$ \\
\hline Tritium & $<-2.2 \mathrm{E}-01$ & $\mathrm{UI} / /$ & $<5.6 \mathrm{E}-01$ & & 1 & GP & $<-7.6 \mathrm{E}-02$ & $\mathrm{U} / /$ & $<5.3 E-01$ & & 1 & GE & $\mathrm{pCi} / \mathrm{mL}$ \\
\hline Uranium-233/234 & $<1,4 \mathrm{E}-0$ ? & UNI & $<0.0970$ & & 1 & GP & & & & & & & $\mathrm{pCi} / \mathrm{L}$ \\
\hline Uranium-235 & $<8.5 E-03$ & $\mathrm{UH} / /$ & $<0.1150$ & & 1 & GP & & & & & & & $\mathrm{pCi} / \mathrm{L}$ \\
\hline Uranium-238 & $<6.7 E-02$ & $\mathrm{UH} / /$ & $<0.0970$ & & 1 & GP & & & & & & & $\mathrm{pCi} / \mathrm{L}$ \\
\hline
\end{tabular}

Notes: 
WELL HSB125C

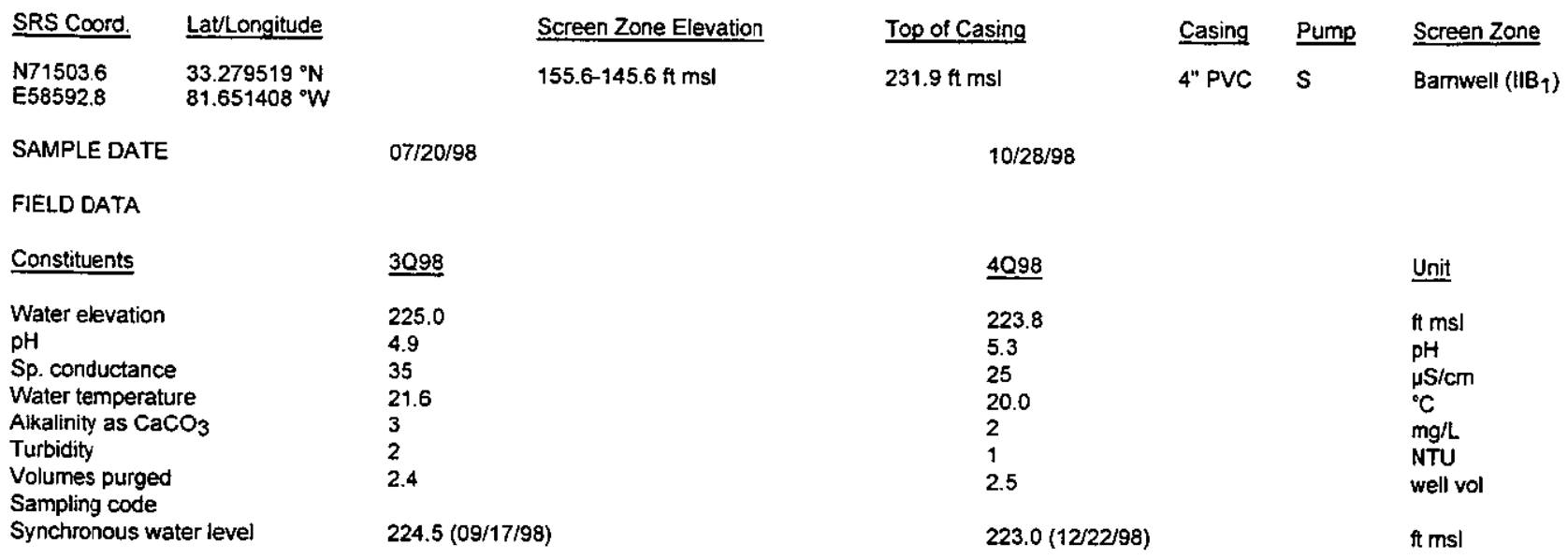

\section{ANALYTICAL DATA}

Inorganic Constituents

Constituents

Antimony, total recoverable Arsenic, total recoverable Barium, total recoverable

Cadmium, total recoverable

Chromium, total recoverable

Cobalt, total recoverable

Copper, total recoverable

Cyanide

Lead, total recoverable

Mercury, total recoverable

Nickel, total recoverable

Nitrate as nitrogen

Nitrate-nitrite as nitrogen

Selenium, total recoverable

Silver, total recoverable

Tin, total recoverable

Vanadium, total recoverable

Zinc, total recoverable

Organic Constituents

Constituents.

Benzene

Bis(2-ethylhexyl) phthalate

Dichloromethane

Tetrachloroethylene

Trichloroethylene

Trichlorofluoromethane

Mod

Filt.

ST

DF Lab 4 Q998 Mod

1 GE $<0.20$

UII

1 GE $<90$

U//6

NI

Mod
Filt.
Mod
$<0.20$

$<50$

Filt.
$1 \mathrm{GE} \mu \mathrm{g} / \mathrm{h}$

$1 \mathrm{GE} \mu \mathrm{g} / \mathrm{L}$

Notes:

- exceeded holding time

- exceeded groundwater protection or monitoring constituent standard (See Appendix A.) 
WELL HSB125C (cont.)

Radioactive Constituents

Constituents

Americium-241

Carbon-14

Cobalt-60

Curium-242

Curium-243/244

Curium-245/246

Gross alpha

lodine-129

Nickel-63

Nonvolatile beta

Plutonium-238

Plutonium-239/240

Radium-226

Radium-228

Strontium-90

Technetium-99

Thorium-228

Thorium-230

Thorium-232

Sum of alphas

Sum of betas

Tritium

Uranium-233/234

Uranium-235

Uranium-238

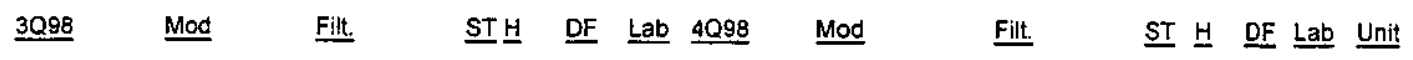

$<2.6 \mathrm{E}-0 \mathrm{U} \quad \mathrm{UI} / \quad \mathbf{0.6130} \quad 1$

1

GP $<2.2 \mathrm{E}-01 \quad \mathrm{U} / /$

$<8.7 \mathrm{E}-01$

$1 \mathrm{TM} \mathrm{PCi/L}$

$<1.9 E-02$

$\mathrm{UI} / 1$

$<1.3600$

$1 \quad$ GP $<-2,6 \mathrm{E}-01 \quad \mathrm{U} / /$

$<1.8 \mathrm{E}+00$

$1 \mathrm{TM} \quad \mathrm{pCi} / \mathrm{L}$
$2.3 \mathrm{E}+00 \quad / 1$

$1 \quad$ GP $\quad 2.4 \mathrm{E}+00$

ll

$1 \mathrm{GE} \quad \mathrm{pCi} / \mathrm{mL}$

Notes:

- exceeded holding time

- exceeded groundwater protection or monitoring constituent standard (See Appendix A.) 
WELL HSB125D

\begin{tabular}{|c|c|}
\hline SRS Coord. & Lat/Longitude \\
\hline $\begin{array}{l}\text { N71498.2 } \\
\text { E58584.1 }\end{array}$ & $\begin{array}{l}33.279492{ }^{\circ} \mathrm{N} \\
81.651421{ }^{\circ} \mathrm{W}\end{array}$ \\
\hline
\end{tabular}

SAMPLE DATE

$\underline{3 Q 98}$

220.7

6.4

150

19.2

9

0.4

2.4

$220.67(9 / 17 / 98)$

\section{Screen Zone Elevation}

219.4-199.4 ft msl

Top of Casing

$231.7 \mathrm{ft} \mathrm{msl}$

10/28/98

40.98
220.4
5.6
145
19.5
9
0
2.5
$220.0(12 / 22 / 98)$

$\underline{\text { Unit }}$

ft msl

$\mathrm{pH}$

$\mu \mathrm{S} / \mathrm{cm}$

${ }^{\circ} \mathrm{C}$

$\mathrm{mg} / \mathrm{L}$

NTU

well vol

ft ms!

\section{ANALYTICAL DATA}

Inorganic Constituents

Constifuents

Antimony, total recoverable $<0.20$

Arsenic, total recoverable $<3.0$

Barium, total recoverable 13

Cadmium, total recoverable $<1.0$

Chromium, total recoverable 2.4

Cobalt, total recoverable $\quad 2.8$
Copse

Copper, total recoverable $\quad 0.84$

Cyanide

Lead, total recoverable $\quad<2.0$

Mercury, total recoverable 1.2

Nickel, total recoverable $\quad 2.8$

Nitrate as nitrogen

Nitrate-nitrite as nitrogen $\quad 8,800$

Selenium, total recoverable $<5.0$

Silver, total recoverable $\quad<1.0$

Tin, total recoverable $<2.0$

Vanadium, total recoverable $<2.0$

Zinc, total recoverable $<5.0$

Organic Constituents

Benzene

Bis(2-ethylhexyl) phthalate

Dichloromethane

Tetrachloroethylene

Trichloroethylene

Trichlorofluoromethane
3Q98

$<1.0$

$<9.9$

$<1.0$

$<1.0$

$<1.0$

\begin{tabular}{|c|c|}
\hline Mod & Filt. \\
\hline $\mathrm{U} / 1$ & $<0.20$ \\
\hline $\begin{array}{l}\mathrm{U} / 1 \\
/ /\end{array}$ & $<3.0$ \\
\hline $\mathrm{U} / \prime$ & $<1.0$ \\
\hline $\begin{array}{l}\mathrm{J} / \mathrm{E} J \\
\prime \prime\end{array}$ & NDD \\
\hline $\mathrm{U} / 1$ & $<10$ \\
\hline $\begin{array}{l}\mathrm{U} / / \\
\|\end{array}$ & $<2.0$ \\
\hline
\end{tabular}

ST브

DF Lab 4Q98 Mod

$<5.0$

$<1.0$

$<2.0$

$<2.0$

$<5.0$

$\begin{array}{ll}N / & \\ U \prime \prime & <5.0 \\ U \prime \prime \prime & <1.0 \\ U \prime \prime \prime & <2.0 \\ U / I & <2.0 \\ U / I & <5.0\end{array}$

$\mathrm{GE}$

GE

GE

GE

GE

GE

GE

$G E$

GE 0.46

GE $\quad 11,000 \quad 1 / 6$

GE

GE

GE

1 GE

1 GE
4Q98 Mod

GE
GE
GE
GE
GE creen Zone

Water Table (IIB2) 
WELL HSB125D (cont.)

Radioactive Constituents

\begin{tabular}{|c|c|c|c|c|c|c|c|c|c|c|c|c|c|}
\hline Constituents & 3Q98 & Mod & Filt. & ST & $\underline{\text { DF }}$ & $\underline{\text { Lab }}$ & 4098 & Mod & Filt. & $\underline{\text { ST }} \underline{H}$ & $\underline{D F}$ & $\underline{L a b}$ & Unit \\
\hline Americium-241 & $<-2.0 \mathrm{E}-02$ & UIII & $<0.1740$ & & 1 & GP & & & & & & & pCill \\
\hline Beta dose & 0.64 & & & & & & & & & & & & $\mathrm{pCil}$ \\
\hline Carbon-14 & $4.8 \mathrm{E}+01$ & $/ 1$ & & & 1 & GP & & & & & & & $\mathrm{pCi} / \mathrm{L}$ \\
\hline Cobalt-60 & $<-9.5 E-01$ & $\mathrm{Ul} / /$ & $<3.2200$ & & 1 & GP & & & & & & & $\mathrm{pCi} / \mathrm{L}$ \\
\hline Curium-242 & $<-5.8 E-02$ & $\mathrm{UI} / /$ & $<0.2860$ & & 1 & GP & & & & & & & $\mathrm{pCi} / \mathrm{L}$ \\
\hline Curium-243/244 & $<9.9 E-02$ & $\mathrm{UI} / I$ & $<0.1750$ & & 1 & GP & & & & & & & $\mathrm{pCi} / \mathrm{L}$ \\
\hline Curium-245/246 & $<-6.7 E-03$ & $\mathrm{UI} / l$ & $<0.1480$ & & 1 & GP & & & & & & & $\mathrm{pCi} / \mathrm{L}$ \\
\hline Gross alpha & $<1.5 \mathrm{E}-01$ & $\mathbf{U} \mid / /$ & $<0.6660$ & & 1 & $\mathrm{GP}$ & $<1.6 E+00$ & $\mathrm{U} / /$ & $<7.3 E-01$ & & 1 & GE & $\mathrm{pCi} / \mathrm{L}$ \\
\hline lodine-129 & $<2.5 E+00$ & $\mathrm{UN} /$ & $<0.6110$ & & 1 & GP & & & & & & SL & $\mathrm{pCi} / \mathrm{L}$ \\
\hline Nickel-63 & $<-1,9 \mathrm{E}-01$ & $\mathrm{Ul} / /$ & $<19.0000$ & & 1 & GP & & & & & & & $\mathrm{pCi} / \mathrm{L}$ \\
\hline Nonvolatile beta & $1.9 \mathrm{E}+00$ & $/ 1$ & & & 1 & GP & $8.1 E+00$ & // & & & 1 & GE & $\mathrm{pCi} / \mathrm{L}$ \\
\hline Plutonium-238 & $<3.0 \mathrm{E}-02$ & $\mathrm{U} 1 / /$ & $<0.2050$ & & 1 & $\mathrm{GP}$ & & & & & & & pCi/L \\
\hline Plutonium-239/240 & $<-1.9 E-02$ & $\mathrm{UI} / /$ & $<0.2420$ & & 1 & GP & & & & & & & $\mathrm{pCi} / \mathrm{L}$ \\
\hline Radium-226 & $1.2 \mathrm{E}+\infty 0$ & $/ /$ & & & 1 & GP & & & & & & & $\mathrm{pCi} / \mathrm{L}$ \\
\hline Radium-228 & $3.0 \mathrm{E}+00$ & $\|$ & & & 1 & GP & & & & & & & $\mathrm{pCi} / \mathrm{L}$ \\
\hline Strontium-90 & $1.2 E+00$ & $\mathrm{~J} / \mathrm{C} /$ & NDD & & 1 & GP & & & & & & & pCill \\
\hline Technetium-99 & $1.3 E+01$ & $\|$ & & & 1 & GP & & & & & & & pCill. \\
\hline Thorium-228 & $<1.3 \mathrm{E}-02$ & $\mathrm{UI} / /$ & $<0.3300$ & & 1 & $G P$ & & & & & & & pCill \\
\hline Thorium-230 & $<1.3 E-01$ & $\mathrm{UI} / /$ & $<0.2450$ & & 1 & GP & & & & & & & pCill. \\
\hline Thorium-232 & $<2.4 \mathrm{E}-02$ & $\mathrm{UI} / /$ & $<0.2890$ & & 1 & GP & & & & & & & $\mathrm{pCi} / \mathrm{L}$ \\
\hline Sum of alphas & 2.6E-01 & & & & & & & & & & & & $\mathrm{pCi} / \mathrm{L}$ \\
\hline Sum of betas & $6.5 E+01$ & & & - & & & & & & & & & $\mathrm{pCi} / \mathrm{L}$ \\
\hline Total radium & $4.2 E+00$ & & & & & & & & & & & & $\mathrm{pCiLL}$ \\
\hline Tritium & $7.1 E+02$ & $\|$ & & $\mathbf{\square}$ & 1 & GP & $9.9 E+02$ & $\|$ & & च & 1 & GE & $\mathrm{pCi} / \mathrm{mL}$ \\
\hline Uranium-233/234 & $<2.8 E-01$ & $\mathrm{U} N I$ & $<0.0594$ & & 1 & GP & & & & & & & $\mathrm{pCi} / \mathrm{L}$ \\
\hline Uranium-235 & $<4.9 E-02$ & $\mathrm{Ut} / /$ & $<0.1270$ & & 1 & GP & & & & & & & $\mathrm{pCi} / \mathrm{L}$ \\
\hline Uranium-238 & $2.6 \mathrm{E}-01$ & $/ 1$ & & & 1 & GP & & & & & & & $\mathrm{pCi} / \mathrm{L}$ \\
\hline
\end{tabular}

Notes:

- = exceeded holding time

- = exceeded groundwater protection or monitoring constituent standard (See Appendix A.) 


\section{WELL HSB126C}

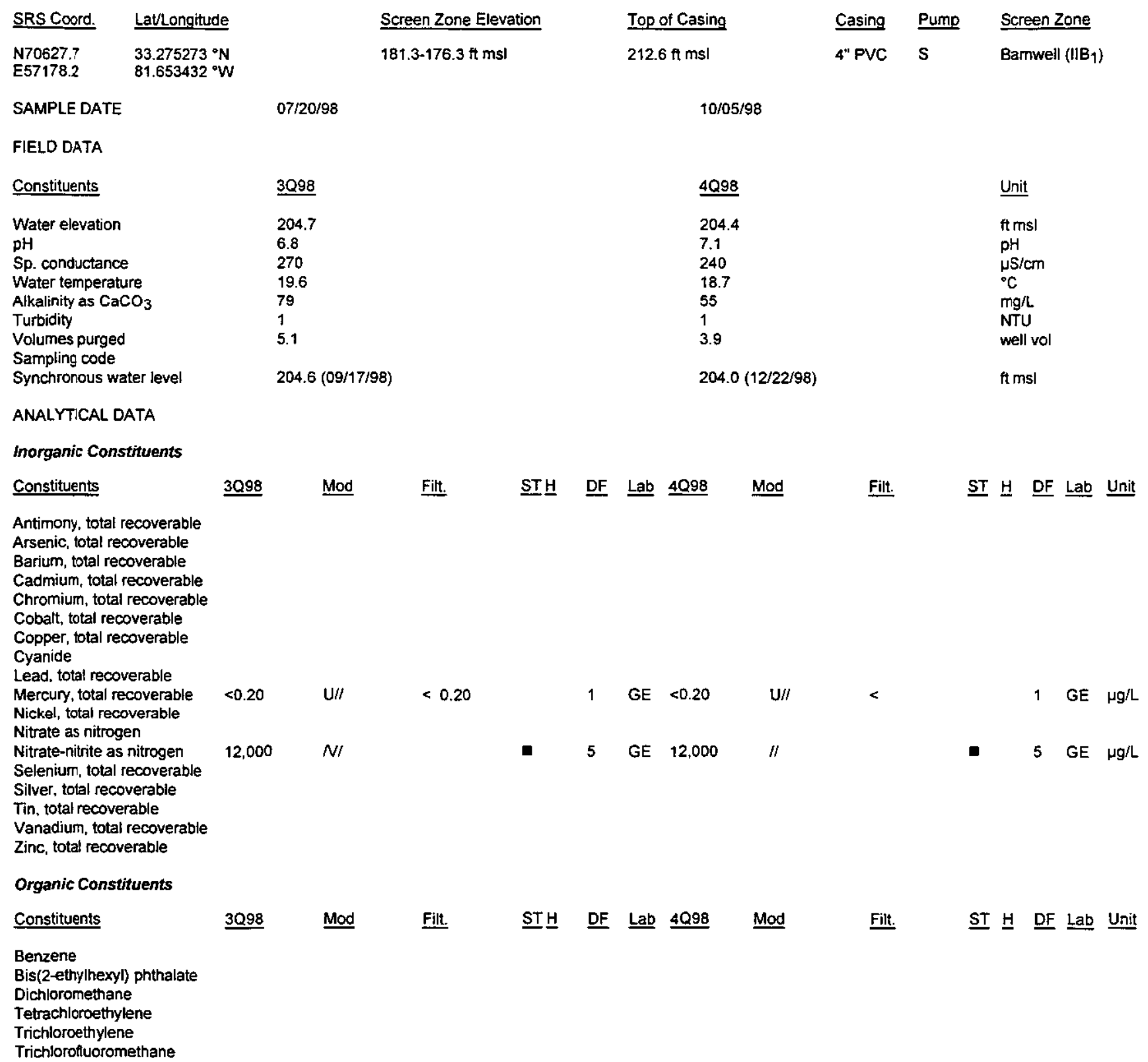

Notes:

- exceeded holding time

E = exceeded groundwater protection or monitoring constituent standard (See Appendix A.) 
WSRC-TR-99-00013

Unclassified

WELL HSB126C (cont.)

Radioactive Constituents

\section{Constituents}

Americium-241

Carbon-14

Cobalt-60

Curium-242

Curium-243/244

Curium-245/246

Gross alpha

lodine-129

Nickel-63

Nonvolatile beta

Plutonium-238

Plutonium-239/240

Radium-226

Radium-228

Strontium-90

Technetium-99

Thorium-228

Thorium-230

Thorium-232

Sum of alphas

Sum of betas

Tritium

Uranium-233/234

Uranium-235

Uranium-238 $\underline{3098}$

Mod

Filt.

ST브

DF Lab $4 Q 98$

Mod

$<7.8 \mathrm{E}-01$

$\mathrm{U} \mathrm{I} / \mathrm{I}$

$<1.0500$

1

GP $<1.5 \mathrm{E}-01$

U/I

1 GP $6.4 \mathrm{E}+00 \quad / /$

$<9.6 \mathrm{E}-01$

1 GE $\mathrm{pCi} / \mathrm{L}$

$3.5 \mathrm{E}+00 \quad / 1$

1 GE PCiL

$4.4 \mathrm{E}+02 \quad / /$
1 GP $5.3 E+02 \quad / /$ $\underline{\text { ST }} \underline{H}$ DF Lab $\underline{\text { Unit }}$

Filt.

$\underline{\underline{S T}} \underline{\mathrm{D}} \underline{\mathrm{Lab}} \underline{\mathrm{Uni}}$

- 1 GE $\mathrm{pCi} / \mathrm{mL}$

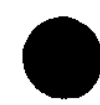

\section{Notes:}

- = exceeded hoiding time

= exceeded groundwater protection or monitoring constituent standard (See Appendix A.) 
WELL HSB126D

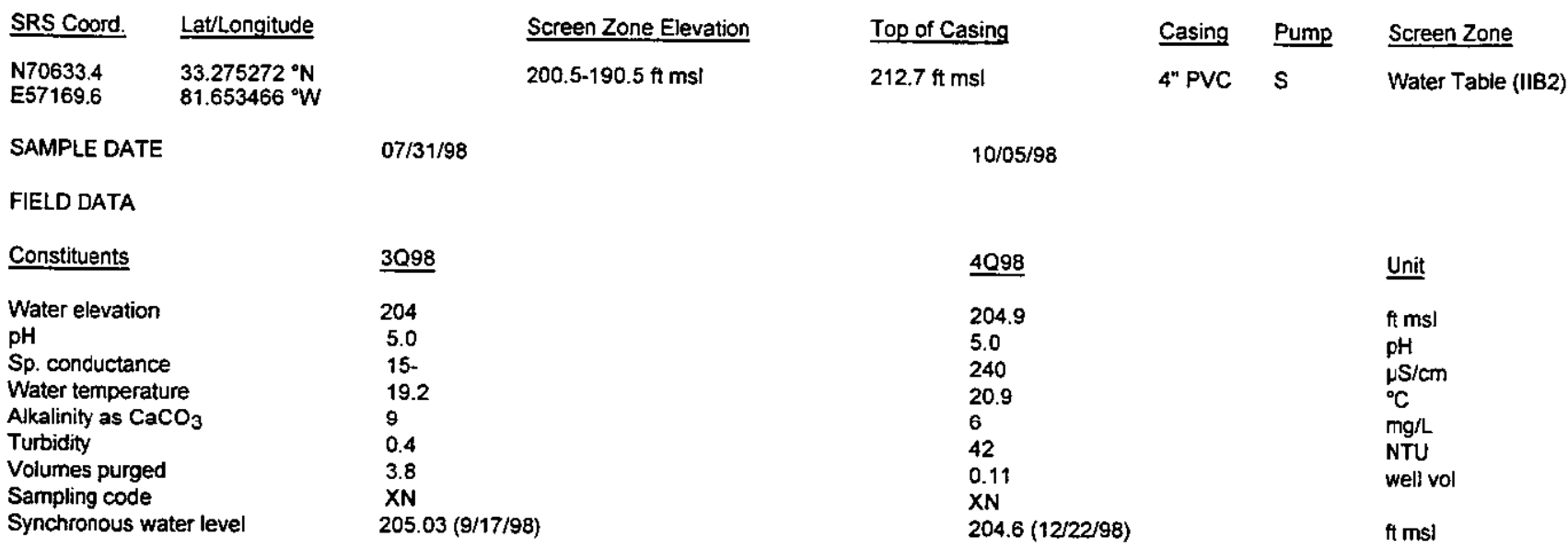

ANALYTICAL DATA

Inorganic Constituemts

\begin{tabular}{|c|c|c|c|c|c|c|c|c|c|c|c|c|c|}
\hline Constituents & $\underline{3 Q 98}$ & Mod & Filt. & STT 브 & $\underline{\mathrm{DF}}$ & Lab & 4Q98 & Mod & Filt. & $\underline{\text { ST }} \underline{\mathrm{H}}$ & DF & $\underline{\text { Lab }}$ & $\underline{\text { Unit }}$ \\
\hline Antimony, total recoverable & $<0.20$ & $\mathrm{U} / /$ & $<0.20$ & & 1 & GE & & & & & & & $\mu g / L$ \\
\hline Arsenic, total recoverable & $<3.0$ & $\mathrm{U} / /$ & $<3.0$ & & 1 & GE & & & & & & & $\begin{array}{ll}\mu g / L \\
\mu / L\end{array}$ \\
\hline Barium, total recoverable & 56 & $\|$ & & & 1 & GE & & & & & & & $\mu g / L$ \\
\hline Cadmium, total recoverable & $<1.0$ & $\mathrm{U} / /$ & $<1.0$ & & 1 & GE & & & & & & & $\mu g / L$ \\
\hline Chromium, total recoverable & 1.1 & $\mathrm{~J} / \mathrm{E}$ & NDD & & 1 & $\overrightarrow{G E}$ & & & & & & & $\mu g / L$ \\
\hline Cobalt, total recoverable & 2.0 & $/ 1$ & & & 1 & $\overline{G E}$ & & & & & & & $\mu g / L$ \\
\hline Copper, total recoverable & 3.4 & $\|$ & & & 1 & GE & & & & & & & ug/L \\
\hline Cyanide & $<10$ & $\mathrm{U} / /$ & $<10$ & & 1 & $G E$ & & & & & & & L \\
\hline Lead, total recoverable & 2.6 & NI & & & 1 & GE & & & & & & & $\begin{array}{l}\mu g h \text { L } \\
\mu g / L\end{array}$ \\
\hline Mercury, total recoverable & 0.090 & $\mathrm{~J} / \mathrm{E}$ & NDD & & 1 & GE & 0.61 & $/ 1$ & & & 1 & GE & $\mu g /$ \\
\hline $\begin{array}{l}\text { Nickel. total recoverable } \\
\text { Nitrate as nitrogen }\end{array}$ & 3.0 & 11 & & & 1 & GE & & & & & & & $\mu g / L$ \\
\hline Nitrate-nitrite as nitrogen & 29,000 & $N I$ & & $\boldsymbol{\omega}$ & 25 & GE & 32,000 & /1 & & $\mathbf{m}$ & 25 & GE & $\mu g / L$ \\
\hline Selenium, total recoverable & $<5.0$ & $\mathrm{U} / /$ & $<5.0$ & & 1 & GE & & & & & & & $\mu g / L$ \\
\hline Silver, total recoverable & $<1.0$ & $\mathrm{U} / /$ & $<1.0$ & & 1 & GE & & & & & & & $\mu g / L$ \\
\hline Tin, total recoverable & $<2.0$ & $\mathrm{U} / /$ & $<2.0$ & & 1 & GE & & & & & & & $\mu g / L$ \\
\hline Vanadium, total recoverable & $<2.0$ & $\mathrm{U} / /$ & $<2.0$ & & 1 & GE & & & & & & & $\mu g / L$ \\
\hline Zinc, total recoverable & 10 & /I & & & 1 & GE & & & & & & & \\
\hline
\end{tabular}

Organic Constituents

$\begin{array}{lllllll}\text { Constituents } & 3098 & \text { Mod } & \text { Filt. } & \text { ST } \underline{\text { S }} & \text { DF } & \text { Lab } \\ \text { Benzene } & <1.0 & \mathrm{U} / / & <1.0 & & 1 & \mathrm{GE} \\ \text { Bis(2-ethylhexyl) phthalate } & 140 & \mathrm{~J} / \mathrm{Q} / & \mathrm{NDD} & & 4 & \mathrm{GE} \\ \text { Dichloromethane } & <1.0 & \mathrm{U} / / & <1.0 & & 1 & \mathrm{GE} \\ \text { Tetrachloroethylene } & 0.74 & \mathrm{~J} / \mathrm{E} / & \mathrm{NDD} & & 1 & \mathrm{GE} \\ \text { Trichloroethylene } & <1.0 & \mathrm{U} / / & <1.0 & & 1 & \mathrm{GE} \\ \text { Trichlorofluoromethane } & <1.0 & \mathrm{U} / / & <1.0 & & 1 & \mathrm{GE}\end{array}$

Mod Filt.

ST H DF Lab Unit

$\mu g / L$

$\mu g$ L

$\mu g / L$

$\mu g / L$

$\mu \mathrm{g} L$

$\mu g / L$

Notes:

- = exceeded holding time

- =xceeded groundwater protection or monitoring constituent standard (See Appendix A.) 
WELL HSB126D (cont.)

Radioactive Constituents

\begin{tabular}{|c|c|c|c|c|c|c|c|c|c|c|c|c|c|}
\hline Constituents & 30998 & Mod & Filt. & $\underline{\text { ST }} \underline{H}$ & DF & $\underline{\text { Lab }}$ & 4Q98 & Mod & Filt. & $\underline{\text { ST }} \underline{H}$ & $\underline{D F}$ & $\underline{L a b}$ & Unit \\
\hline Americium-241 & $<1.5 E-01$ & $\mathrm{U} I I !$ & $<0.1720$ & & 1 & GP & & & & & & & $\mathrm{pCi} / \mathrm{L}$ \\
\hline Beta dose & 10.55 & & & a & & & & & & & & & $\mathrm{pCi} / \mathrm{L}$ \\
\hline Carbon-14 & $3.5 E+01$ & 11 & & & 1 & GP & & & & & & & $\mathrm{pCi} / \mathrm{L}$ \\
\hline Cobalt-60 & $<2.4 \mathrm{E}+00$ & UII/ & $<4.9000$ & & 1 & GP & & & & & & & $\mathrm{pCi} / \mathrm{L}$ \\
\hline Curium-242 & $<1.3 E-02$ & $\mathrm{UI} / /$ & $<0.2050$ & & 1 & GP & & & & & & & $\mathrm{pCi} / \mathrm{L}$ \\
\hline Curium-243/244 & $<8.2 \mathrm{E}-02$ & $\mathrm{UI} / /$ & $<0.2320$ & & 1 & GP & & & & & & & $\mathrm{pCi} / \mathrm{L}$ \\
\hline Curium-245/246 & $<0.0 \mathrm{E}+00$ & $\mathrm{UI} / /$ & $<0.0681$ & & 1 & GP & & & & & & & $\mathrm{pCi} / \mathrm{L}$ \\
\hline Gross aipha & $1.3 \mathrm{E}+00$ & $/ 1$ & & & 1 & $\mathrm{GP}$ & $4.0 E+00$ & $\| \prime$ & & & 1 & GE & $\mathrm{pCi} / \mathrm{L}$ \\
\hline lodine-129 & $1.0 \mathrm{E}+01$ & $N I$ & & & 1 & GP & & & & & & & $\mathrm{pCi} / \mathrm{L}$ \\
\hline Nickel-63 & $<2.4 E+00$ & UI & $<4.9000$ & & & & & & & & & & $\mathrm{pCi} / \mathrm{L}$ \\
\hline Nonvolatile beta & $9.5 E+00$ & 11 & & & 1 & GP & $1.2 \mathrm{E}+01$ & /l & & & 1 & GE & $\mathrm{pCi} / \mathrm{L}$ \\
\hline Plutonium-238 & $<-7.6 \mathrm{E}-03$ & $\mathrm{UI} / /$ & $<0.1670$ & & 1 & GP & & & & & & & $\mathrm{pCi} / \mathrm{L}$ \\
\hline Plutonium-239/240 & $<0.0 \mathrm{E}+00$ & $\mathrm{UI} / I$ & $<0.1790$ & & 1 & GP & & & & & & & $\mathrm{pCi} / \mathrm{L}$ \\
\hline Radium-226 & $1,2 \mathrm{E}+\infty$ & $/ 1$ & & & 1 & GP & & & & & & & $\mathrm{pCi} / \mathrm{L}$ \\
\hline Radium-228 & $2.6 \mathrm{E}+00$ & $\|$ & & & 1 & GP & & & & & & & $\mathrm{pCi} / \mathrm{L}$ \\
\hline Strontium-90 & $<3.7 \mathrm{E}-01$ & UIJ/CL/ & & & 1 & GP & & & & & & & $\mathrm{pCi} / \mathrm{L}$ \\
\hline Technetium-99 & $3.1 E+01$ & $/ /$ & & & 1 & GP & & & & & & & $\mathrm{pCi} / \mathrm{L}$ \\
\hline Thorium-228 & $<-1.1 E-02$ & UU/I & $<0.2200$ & & 1 & GP & & & & & & & $\mathrm{pCi} / \mathrm{L}$ \\
\hline Thorium-230 & $<4.2 E-01$ & UNI & $<0.1330$ & & 1 & $\mathrm{GP}$ & & & & & & & $\mathrm{pCi} / \mathrm{L}$ \\
\hline Thorium-232 & $<-6.1 \mathrm{E}-03$ & $\mathrm{UI} / /$ & $<0.1330$ & & 1 & GP & & & & & & & $\mathrm{pCi} / \mathrm{L}$ \\
\hline $\begin{array}{l}\text { Sum of aiphas } \\
\text { Sum of betas }\end{array}$ & $78 F+01$ & & & $=$ & & & & & & & & & \\
\hline $\begin{array}{l}\text { Sum of betas } \\
\text { Total radium }\end{array}$ & $\begin{array}{l}7.8 E+01 \\
3.8 E+00\end{array}$ & & & $\mathbf{E}$ & & & & & & & & & $\mathrm{pCi} / \mathrm{L}$ \\
\hline Tritium & $1.6 E+03$ & $\|$ & & - & 1 & GP & $1.8 E+03$ & /I & & E & 1 & FF & $\mathrm{pCi} / \mathrm{L}$ \\
\hline Uranium-233/234 & $<1.0 \mathrm{E}-01$ & $\mathrm{UU} / /$ & $<0.1110$ & & 1 & GP & & & & & & GE & $\begin{array}{l}\mathrm{pCl} / \mathrm{mL} \\
\mathrm{pC} / / \mathrm{L}\end{array}$ \\
\hline Uranium-235 & $<0.0 E+00$ & $\mathrm{UII}$ & $<0.0632$ & & 1 & GP & & & & & & & $\mathrm{pCi} / \mathrm{L}$ \\
\hline Uranium-238 & $<1.6 \mathrm{E}-02$ & $\mathrm{UI} / \mathrm{I}$ & $<0.1110$ & & 1 & GP & & & & & & & $\mathrm{pCi} / \mathrm{L}$ \\
\hline
\end{tabular}

Notes:

- = exceeded holding time

Q = exceeded groundwater protection or monitoring constituent standard (See Appendix A.) 


\section{WELL HSB127C}

\begin{tabular}{ll} 
SRS Coord. & Lat/Longitude \\
\hline N71210.1 & $33.275932^{\circ} \mathrm{N}$ \\
E56792.1 & $81.655580^{\circ} \mathrm{W}$
\end{tabular}

SAMPLE DATE

Turbidity

Volumes purged

Sampling code

Synchronous water level

ANALYTICAL DATA

Inorganic Constituents

Constituents

Antimony, total recoverable

Arsenic, total recoverable

Barium, total recoverable

Cadmium, total recoverable

Chromium, total recoverable

Cobalt, total recoverable

Copper, total recoverable

Cyanide

Lead, total recoverable

Mercury total recoverable

Nickel, total recoverable

Nitrate as nitrogen

Nitrate-nitrite as nitrogen

Selenium, total recoverable

Silver, total recoverable

Tin, total recoverable

Vanadium, total recoverable

Zinc, total recoverable

Organic Constituents

Constituents

Mod

Filt.

ST브

Filt.

STH

$N$
$07 / 21 / 98$

3098

210.8

6.9

250

20.2

78

3.

Top of Casing

$225.7 \mathrm{ft} \mathrm{msl}$

$10 / 05 / 98$

$\underline{4098}$

210.1

7.2

240

199

74

$210.3(09 / 17 / 98)$
Casing Pump

4" PVC S

Screen Zone

Bamwell $\left(\mathrm{HB}_{1}\right)$

Benzene

Bis(2-ethylhexyl) phthalate

Dichloromethane

Tetrachloroethylene

Trichloroethylene

Trichlorofluoromethane

DF Lab $\quad \underline{4 Q 98}$

Mod

Filt.

$\underline{\text { ST }} \underline{H}$ DF Lab Unit

$\underline{\text { Unit }}$

ft ms

$\mathrm{pH}$

$\mu \mathrm{S} / \mathrm{cm}$

${ }^{\circ} \mathrm{C}$

$\mathrm{mg} / \mathrm{L}$

NTU

well vol

fi msl

$209.7(12 / 22 / 98)$

GE $<0.20$

$\mathrm{U} / /$

$<0.20$

$1 \mathrm{GE} \mu \mathrm{g} / \mathrm{L}$

5 GE 9,700

5 GE $\mu g / L$

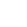

(n)

\section{Notes:}

= exceeded holding time

- = exceeded groundwater protection or monitoring constituent standard (See Appendix A.) 
WELL HSB127C (cont.)

Radioactive Constituents

Constituents

Americium-241

Carbon-14

Cobalt-60

Curium-242

Curium-243/244

Curium-245/246

Gross alpha

lodine-129

Nickel-63

Nonvolatile beta

Plutonium-238

Plutonium-239/240

Radium-226

Radium-228

Strontium-90

Technetium-99

Thorium-228

Thorium-230

Thorium-232

Sum of alphas

Sum of betas

Tritium

Uranium-233/234

Uranium-235

Uranium-238 $\underline{3 Q 98} \quad \underline{M 0 d} \quad \underline{F i l t .} \quad \underline{\text { ST }} \underline{\mathrm{H}} \quad \underline{\mathrm{DF}}$ Lab $\underline{4 Q 98} \quad \underline{M o d} \quad \underline{\text { Filt. }} \quad \underline{\text { ST }} \underline{\mathrm{H}}$ DF $\underline{\text { Lab }} \underline{\text { Unit }}$

9.3E-01 /I

1

GP $1.6 \mathrm{E}+00 \quad / 1$

$3.4 \mathrm{E}+00 \quad / /$

1 GP $\quad 9.9 E+00 \quad / /$

$1 \mathrm{GP} \mathrm{pCi} / \mathrm{L}$

1 GE $\mathrm{pCi} / \mathrm{L}$

$8.8 \mathrm{E}+02 \quad / 1$

GP $\quad 1.0 E+03 \quad / 1$

- 1 GE pCi/mL 
WELL HSB127D

SRS Coord. Lat/Longitude

E56788.0 $\quad 81.655608^{\circ} \mathrm{W}$

SAMPLE DATE

07/30/98

FIELD DATA

Constituents

Water elevation

$\mathrm{pH}$

Sp. conductance

Water temperature

Alkalinity as $\mathrm{CaCO}_{3}$

Turbidity

Volumes purged

Sampling code

Synchronous water level

3Q98

217.4

6.4

150

19.2

9

0.4

8.0

$215.68(9 / 17 / 98)$
Screen Zone Elevation

217.8-197.8 ft ms!

Top of Casing

$226.1 \mathrm{ft} \mathrm{msl}$

10/14/98

4098

216.2

4.7

100

19.7

0

7.0

$216.4(12 / 22 / 98)$

\section{Casing Pump}

4" PVC S
Screen Zone

Water Table (IIB2)

\section{ANALYTICAL DATA}

Inorganic Constituents

\begin{tabular}{ll} 
Constituents & 3098 \\
\hline & \\
Antimony, total recoverable & $<0.20$ \\
Arsenic, total recoverable & $<3.0$ \\
Barium, total recoverable & 10 \\
Cadmium, total recoverable & 0.81 \\
Chromium, total recoverable & 0.93 \\
Cobalt, total recoverable & 4.4 \\
Copper, total recoverable & 6.1 \\
Cyanide & $<10$ \\
Lead, total recoverable & 0.59 \\
Mercury, total recoverable & 4.5 \\
Nickel, total recoverable & 4.6 \\
Nitrate as nitrogen & \\
Nitrate-nitrite as nitrogen & 12,000 \\
Selenium, total recoverable & $<5.0$ \\
Silver, total recoverable & $<1.0$ \\
Tin, total recoverable & $<2.0$ \\
Vanadium, total recoverable & $<2.0$ \\
Zinc, total recoverable & $<5.0$
\end{tabular}

\begin{tabular}{|c|c|}
\hline Mod & Filt. \\
\hline $\mathrm{U} / /$ & $<0.20$ \\
\hline $\mathrm{U} / \prime$ & $<3.0$ \\
\hline /I & \\
\hline $\mathrm{J} / \mathrm{E}$ & NDD \\
\hline $\mathrm{J} / \mathrm{E} /$ & NDD \\
\hline$/ 1$ & \\
\hline II & \\
\hline $\mathrm{U} / /$ & $<10$ \\
\hline $\mathrm{J} / \mathrm{E}$ & NDD \\
\hline$/ /$ & \\
\hline /I & \\
\hline$N !$ & \\
\hline $\mathrm{U} / /$ & $<5.0$ \\
\hline $\mathrm{U} / /$ & $<1.0$ \\
\hline $\mathrm{U} / /$ & $<2.0$ \\
\hline $\mathrm{u} / /$ & $<2.0$ \\
\hline $\mathbf{U} / /$ & $<5.0$ \\
\hline
\end{tabular}

\begin{tabular}{|c|c|c|c|c|}
\hline \multirow[t]{6}{*}{ ST브 } & $\underline{D F}$ & Lab & 4Q98 & Mod \\
\hline & 1 & GE & & \\
\hline & 1 & GE & & \\
\hline & 1 & GE & & \\
\hline & 1 & GE & & \\
\hline & 1 & GE & & \\
\hline \multirow[t]{4}{*}{ 口 } & 1 & $\mathrm{GE}$ & & \\
\hline & 1 & GE & & \\
\hline & 1 & GE & & \\
\hline & 1 & GE & & \\
\hline \multirow[t]{2}{*}{$\boldsymbol{\omega}$} & 1 & GE & 2.6 & // \\
\hline & 1 & GE & & \\
\hline \multirow[t]{6}{*}{ - } & 5 & GE & 8,400 & $\|$ \\
\hline & 1 & $\mathrm{GE}$ & & \\
\hline & 1 & GE & & \\
\hline & 1 & GE & & \\
\hline & 1 & $\overline{G E}$ & & \\
\hline & 1 & GE & & \\
\hline
\end{tabular}

Filt.

Unit

ft msl

$\mathrm{pH}$

$\mu \mathrm{S} / \mathrm{cm}$

${ }^{\circ} \mathrm{C}$

$\mathrm{mg} / \mathrm{L}$

well vol

ft msl

Organic Constituents

3098

\begin{tabular}{|c|c|}
\hline Mod & Filt. \\
\hline $\mathrm{U} / 1$ & $<1.0$ \\
\hline $\mathrm{U} / \prime$ & $<10$ \\
\hline $\mathrm{U} / /$ & $<1.0$ \\
\hline $\mathrm{U} / /$ & $<1.0$ \\
\hline $\mathrm{U} / 1$ & $<1.0$ \\
\hline $\mathrm{U} / /$ & $<1.0$ \\
\hline
\end{tabular}

$\underline{\text { STI }}$

$\begin{array}{ll}\text { DF } & \text { Lab } \\ 1 & \text { GE } \\ 1 & \text { GE } \\ 1 & \text { GE } \\ 1 & \text { GE } \\ 1 & \text { GE } \\ 1 & \text { GE }\end{array}$

Mod

Filt.

ST $\underline{H} \underline{\text { DF Lab Unit }}$

$\begin{array}{ll}\text { Benzene } & <1.0 \\ \text { Bis(2-ethylhexyl) phthalate } & <10 \\ \text { Dichloromethane } & <1.0 \\ \text { Tetrachloroethylene } & <1.0 \\ \text { Trichloroethylene } & <1.0 \\ \text { Trichlorofluoromethane } & <1.0\end{array}$

1.0

$<1.0$

$<1.0$

$<1.0$

$\mu g / L$
$\mu g / L$
$\mu g / L$
$\mu g / L$
$\mu g / L$
$\mu g / L$
$\mu g / L$
$\mu g / L$
$\mu g / L$
$\mu g / L$
$\mu g / L$
$\mu g / L$
$\mu g / L$
$\mu g / L$
$\mu g / L$
$\mu g / L$
$\mu g / L$

$\mu g / L$
$\mu g / L$
$\mu g / L$
$\mu g / L$
$\mu g / L$
$\mu g / L$
$\mu g / L$
$\mu g / L$
$\mu g / L$
$\mu g / L$
$\mu g / L$
$\mu g / L$
$\mu g / L$
$\mu g / L$
$\mu g / L$
$\mu g / L$
$\mu g / L$

$\mu g / L$
$\mu g / L$
$\mu g / L$
$\mu g / L$
$\mu g / L$
$\mu g / L$
$\mu g / L$
$\mu g / L$
$\mu g / L$
$\mu g / L$
$\mu g / L$
$\mu g / L$
$\mu g / L$
$\mu g / L$
$\mu g / L$
$\mu g / L$
$\mu g / L$

$\mu g / L$
$\mu g / L$
$\mu g / L$
$\mu g / L$
$\mu g / L$
$\mu g / L$
$\mu g / L$
$\mu g / L$
$\mu g / L$
$\mu g / L$
$\mu g / L$
$\mu g / L$
$\mu g / L$
$\mu g / L$
$\mu g / L$
$\mu g / L$
$\mu g / L$

$\mu g / L$
$\mu g / L$
$\mu g / L$
$\mu g / L$
$\mu g / L$
$\mu g / L$
$\mu g / L$
$\mu g / L$
$\mu g / L$
$\mu g / L$
$\mu g / L$
$\mu g / L$
$\mu g / L$
$\mu g / L$
$\mu g / L$
$\mu g / L$
$\mu g / L$

Notes:

= exceeded holding time

- exceeded groundwater protection or monitoring constituent standard (See Appendix A.) 
WELL HSB127D (cont.)

Radioactive Constituents

\begin{tabular}{|c|c|c|c|c|c|c|c|c|c|c|c|c|c|}
\hline Constituents & 3098 & Mod & Filt. & $\underline{\text { ST }} \underline{\mathrm{H}}$ & $\underline{\mathrm{DF}}$ & $\underline{L a b}$ & $\underline{4 Q 98}$ & Mod & Filt. & $\underline{\text { ST }} \underline{H}$ & $\underline{\mathrm{DF}}$ & $\underline{L a b}$ & Unit \\
\hline $\begin{array}{l}\text { Americium-241 } \\
\text { Beta dose }\end{array}$ & $\begin{array}{l}<2.6 E-02 \\
5.46\end{array}$ & $\mathrm{U} \mid \mathrm{J} / \mathrm{C} /$ & & n & 1 & GP & & & & & & & $\mathrm{PCi} / \mathrm{L}$ \\
\hline $\begin{array}{l}\text { Beta dose } \\
\text { Carbon-14 }\end{array}$ & $4.1 \mathrm{E}+01$ & // & & & 1 & GP & & & & & & & $\mathrm{PCi} / \mathrm{L}$ \\
\hline Cobalt-60 & $<2.5 \mathrm{E}+00$ & UIII & $<3.2700$ & & 1 & GP & & & & & & & $\mathrm{pCi} / \mathrm{L}$ \\
\hline Curium-242 & $<0.0 E+00$ & $\mathrm{U}: 1 /$ & $<0.0621$ & & 1 & GP & & & & & & & $\mathrm{pCi} / \mathrm{L}$ \\
\hline Curium-243/244 & $<2.0 \mathrm{E}-02$ & $\mathrm{UI} / /$ & $<0.0593$ & & 1 & GP & & & & & & & $\mathrm{pCi} / \mathrm{L}$ \\
\hline Curium-245/246 & $<2.0 \mathrm{E}-02$ & UIII & $<0.0592$ & & 1 & GP & & & & & & & $\mathrm{pCi} / \mathrm{L}$ \\
\hline Gross alpha & $1.7 E+00$ & II & & & 1 & GP & $<2.0 \mathrm{E}+00$ & $\mathrm{U} / /$ & $<1.5 \mathrm{E}+00$ & & 1 & GE & $\mathrm{pCi} / \mathrm{L}$ \\
\hline lodine-129 & $5.0 \mathrm{E}+00$ & /l & & & 1 & GP & & & & & & & $\mathrm{pCi} / \mathrm{L}$ \\
\hline Nickel-63 & $<2.5 E+00$ & UI & $<3.2700$ & & & & & & & & & & $\mathrm{PCi} / \mathrm{L}$ \\
\hline Nonvolatile beta & $1.0 \mathrm{E}+01$ & ll & & & 1 & GP & 1.1E+02 & /I & & ש & 1 & GE & $\mathrm{pCi} / \mathrm{L}$ \\
\hline Plutonium-238 & $<2.0 \mathrm{E}-02$ & $\mathrm{U} / \mathrm{l} /$ & $<0.0593$ & & 1 & GP & & & & & & & $\mathrm{pCi} / \mathrm{L}$ \\
\hline Plutonium-239/240 & $<0.0 E+00$ & $\mathrm{U} \mid / /$ & $<0.0592$ & & 1 & GP & & & & & & & $\mathrm{pCi} / \mathrm{L}$ \\
\hline Radium-226 & $3.7 E+\infty 0$ & 11 & & & 1 & GP & & & & & & & $\mathrm{pCi} / \mathrm{L}$ \\
\hline Radium-228 & $2.1 \mathrm{E}+00$ & "I & & & 1 & GP & & & & & & & $\mathrm{pCil}$ \\
\hline Strontium-90 & $6.7 \mathrm{E}+00$ & $\mathrm{~J} / \mathrm{C} /$ & NDD & & 1 & GP & & & & & & & pCill \\
\hline Technetiutm-99 & $2.2 \mathrm{E}+01$ & II & & & 1 & GP & & & & & & & $\mathrm{pCi} / \mathrm{L}$ \\
\hline Thorium-228 & $<2.0 \mathrm{E}-01$ & $\mathrm{UI} / /$ & $<0.1980$ & & 1 & GP & & & & & & & pCill \\
\hline Thorium-230 & $<0.0 E+00$ & UIIII & $<0.2020$ & & 1 & GP & & & & & & & $\mathrm{pCi} / \mathrm{L}$ \\
\hline Thorium-232 & $<0.0 \mathrm{E}+00$ & $\mathrm{UI} / /$ & $<0.2020$ & & 1 & GP & & & & & & & $\mathrm{pCi} / \mathrm{L}$ \\
\hline Sum of alphas & $1.0 E+00$ & & & & & & & & & & & & $\mathrm{pCi} / \mathrm{L}$ \\
\hline $\begin{array}{l}\text { Sum of betas } \\
\text { Total radium }\end{array}$ & $\begin{array}{l}7.7 E+01 \\
5.7 E+00\end{array}$ & & & m & & & & & & & & & $\begin{array}{l}\mathrm{pCi} / \mathrm{L} \\
\mathrm{OCi} / \mathrm{L}\end{array}$ \\
\hline Tritium & $5.6 \mathrm{E}+02$ & "I & & - & 1 & GP & $2.9 E+02$ & 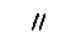 & & - & 1 & GE & $\mathrm{pCi} / \mathrm{mL}$ \\
\hline Uranium-233/234 & $1.0 \mathrm{E}+00$ & $1 /$ & & & 1 & GP & ate or & & & & 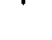 & SL & $\mathrm{pCi} / \mathrm{L}$ \\
\hline Uranium-235 & $<2.1 \mathrm{E}-02$ & 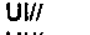 & $<0.0643$ & & 1 & GP & & & & & & & PCill \\
\hline Uranium-238 & $<4.3 \mathrm{E}-02$ & $\mathrm{UI} / /$ & $<0.0641$ & & 1 & GP & & & & & & & pCi/L \\
\hline
\end{tabular}

Notes:

- = exceeded hoiding time

- =xceeded groundwater protection or monitoring constituent standard (See Appendix A.) 


\section{WELL HSB129C}

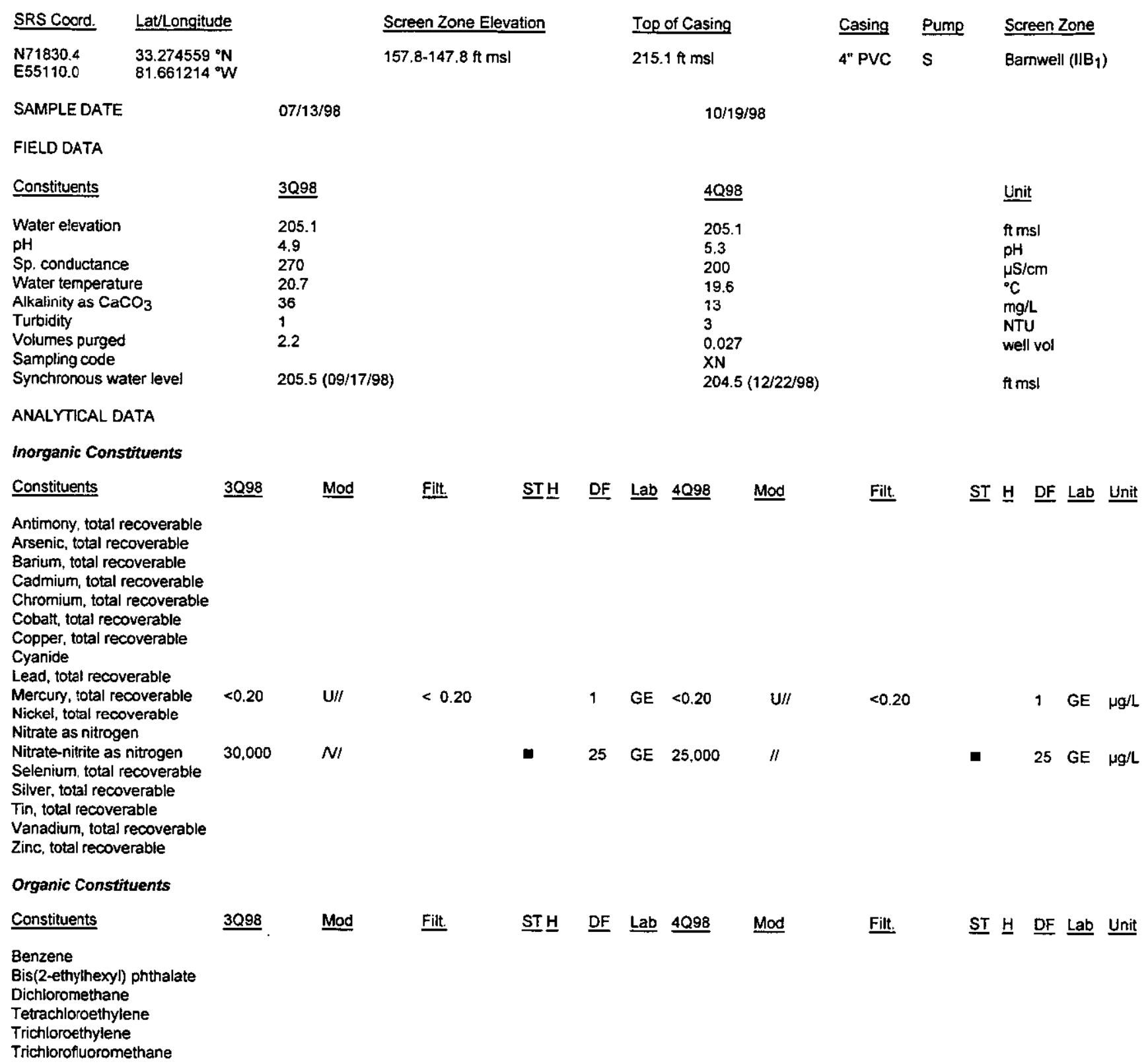

Notes:

= exceeded holding time

- exceeded groundwater protection or monitoring constituent standard (See Appendix A.) 
WELL HSB129C (cont.)

Radioactive Constituents

\begin{tabular}{|c|c|c|c|c|c|c|c|c|c|c|c|c|c|}
\hline Constituents & $\underline{3098}$ & Mod & Filt. & $\underline{\text { ST}} \underline{H}$ & $\underline{D F}$ & $\underline{\text { Lab }}$ & 4098 & Mod & Filt. & $\underline{\text { ST }} \underline{H}$ & $\underline{D F}$ & $\underline{L a b}$ & Unit \\
\hline $\begin{array}{l}\text { Americium-241 } \\
\text { Carbon-14 } \\
\text { Cobalt-60 } \\
\text { Curium-242 } \\
\text { Curium-243/244 } \\
\text { Curium-245/246 }\end{array}$ & & & & & & & & & & & & & \\
\hline $\begin{array}{l}\text { Gross alpha } \\
\text { lodine-129 } \\
\text { Nickel-63 }\end{array}$ & $5.0 \mathrm{E}+00$ & /I & & & 1 & GP & $<4.2 \mathrm{E}+00$ & $\mathrm{U} / I$ & $<2.1 \mathrm{E}+00$ & & 1 & $\mathrm{GE}$ & $\mathrm{pCi} / \mathrm{L}$ \\
\hline $\begin{array}{l}\text { Nonvolatile beta } \\
\text { Plutonium-238 } \\
\text { Plutonium-239/240 } \\
\text { Radium-226 } \\
\text { Radium-228 } \\
\text { Strontium-90 } \\
\text { Technetium-99 } \\
\text { Thorium-228 } \\
\text { Thorium-230 } \\
\text { Thorium-232 } \\
\text { Sum of alphas } \\
\text { Sum of betas }\end{array}$ & $5.3 E+01$ & $\|$ & & - & 1 & GP & $5.5 E+01$ & $/ 1$ & & - & 1 & GE & $\mathrm{pCi} / \mathrm{L}$ \\
\hline $\begin{array}{l}\text { Tritium } \\
\text { Uranium-233/234 } \\
\text { Uranium-235 } \\
\text { Uranium-238 }\end{array}$ & $2.5 E+03$ & " & & - & 1 & GP & $2.2 \mathrm{E}+03$ & /I & & - & 1 & GE & $\mathrm{pCi} / \mathrm{mL}$ \\
\hline
\end{tabular}

Notes:

- = exceeded holding time

- = exceeded groundwater protection or monitoring constituent standard (See Appendix A.) 
WELL HSB129D

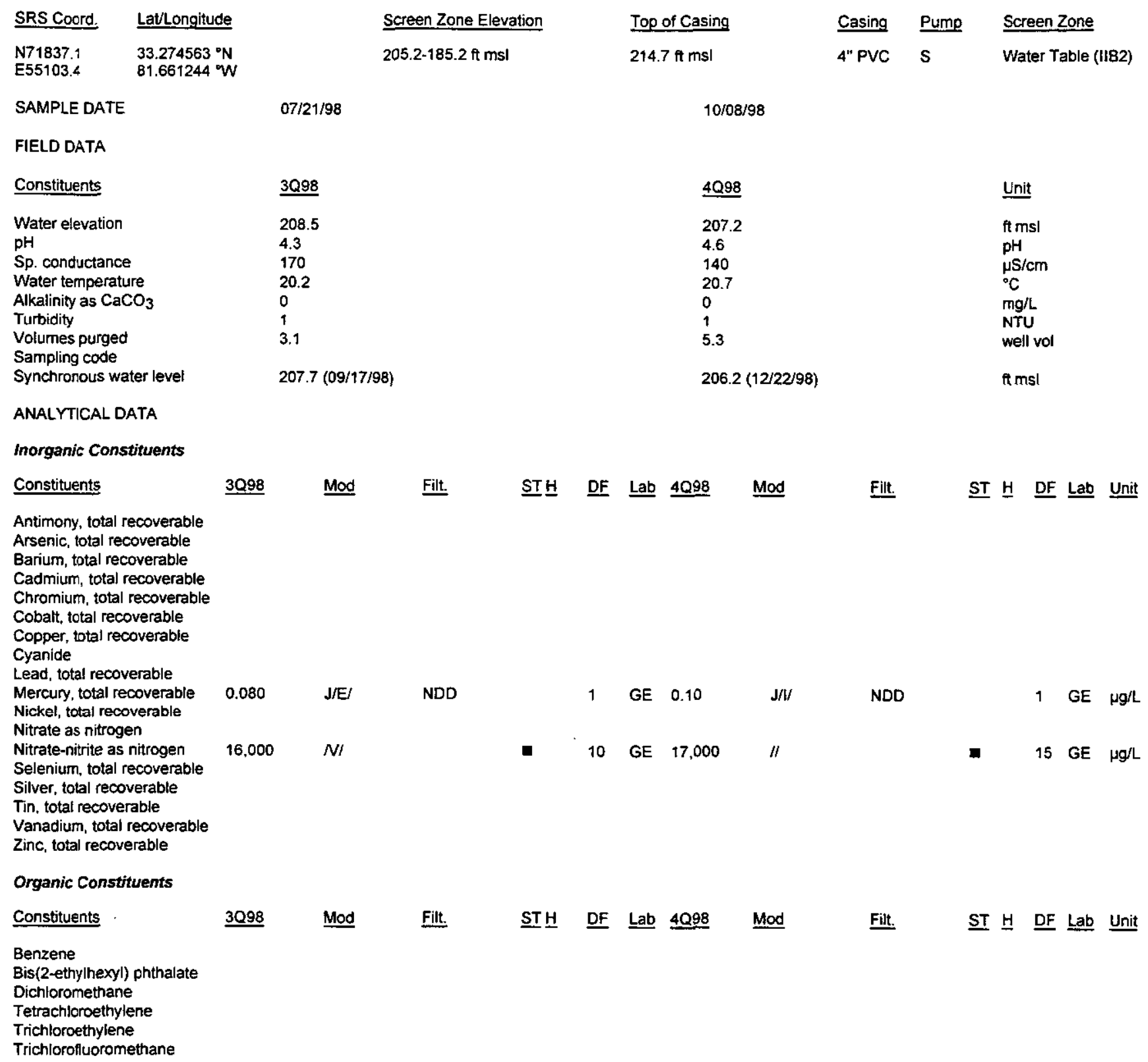

Notes:

- = exceeded holding time

- exceeded grouncwwater protection or monitoring constituent standard (See Appendix A.) 


\section{WELL HSB129D (cont.)}

Radioactive Constituents

Constituents

Americium-241

Carbon-14

Cobalt-60

Curium-242

Curium-243/244

Curium-245/246

Gross alpha

lodine-129

Nickel-63

Nonvolatile beta

Plutonium-238

Plutonium-239/240

Radium-226

Radium-228

Strontium-90

Technetium-99

Thorium-228

Thorium-230

Thorium-232

Sum of alphas

Sum of betas

Tritium

Uranium-233/234

Uranium-235

Uranium-238

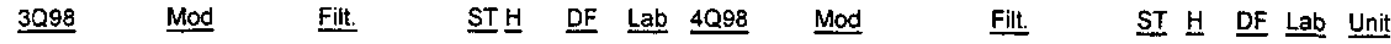

1

GP $<1.6 E+00 \quad \mathrm{JU} / \mathrm{L}$

1 GP $1.6 E+01 \quad J / L /$

$1.4 E+01 \quad / l$

$1.5 \mathrm{E}+03 \quad / /$

GP $\quad 1.5 E+03$
1 GE $\mathrm{pCi} / \mathrm{L}$

1 GE PCill
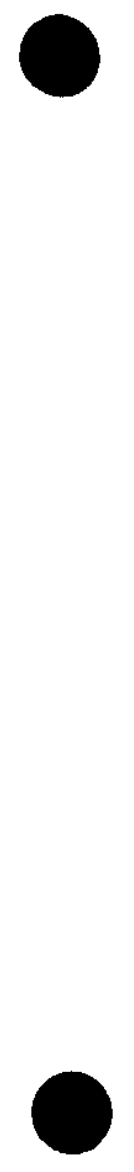

1 GE $\mathrm{pCi} / \mathrm{mL}$

Notes:

- = exceeded holding time

- = exceeded grounowater protection or monitoring constituent standard (See Appendix A.) 
WELL HSB130C

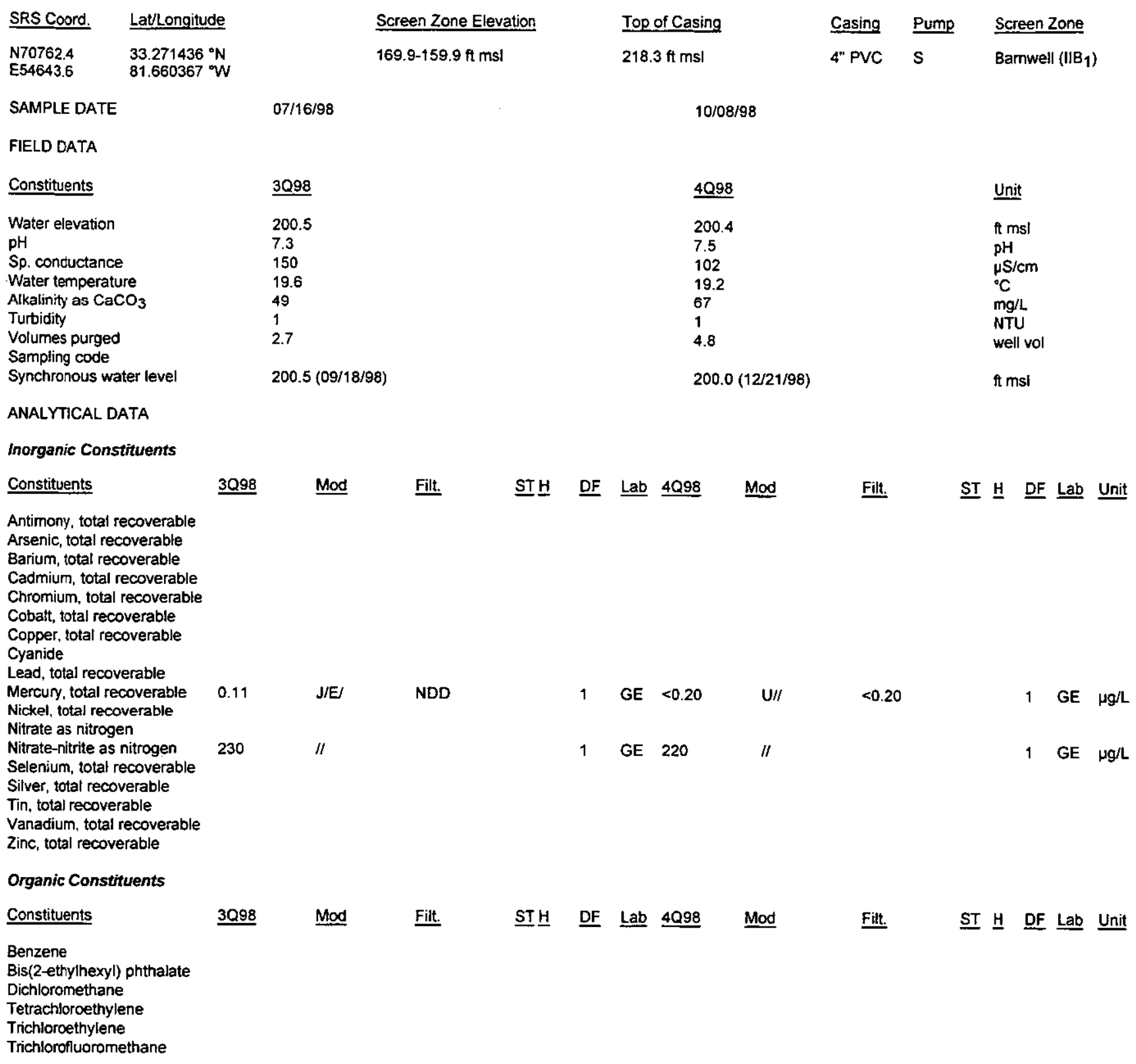

Notes:

- = exceeded holding time

- exceeded groundwater protection or monitoring constituent standard (See Appendix A.) 


\section{WELL HSB130C (cont.)}

\section{Radioactive Constituents}

\section{Constituents}

Americium-241

Carbon-14

Cobalt-60

Curium-242

Curium-243/244

Curium-245/246

Gross alpha

lodine-129

Nickel-63

Nonvolatile beta

Plutonium-238

Plutonium-239/240

Radium-226

Radium-228

Strontium-90

Technetium-99

Thorium-228

Thorium-230

Thorium-232

Sum of alphas

Sum of betas

Tritium

Uranium-233/234

Uranium-235

Uranium-238 $\underline{3 Q 98} \quad \underline{M a d} \quad \underline{F i t .} \quad \underline{\text { ST }} \underline{H}$ DF Lab $\underline{4098} \underline{M o d}$

1.1E+00 /I

$<9.7 \mathrm{E}-01 \quad$ UIII

$<1.3000$

1 GP $<1.4 E-02$ JU/LI

1 GP $<6.0 \mathrm{E}-01 \quad \mathrm{JU} / \mathrm{I}$

$<3.8 \mathrm{E}-01 \quad \mathrm{UI} / / \quad<6.9 \mathrm{E}-01$
Filt.

$\underline{\text { ST }} \underline{H}$ DF Lab Unit

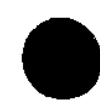

1 GE $\mathrm{pCi} / \mathrm{L}$

1 GE $\mathrm{pCi} / \mathrm{L}$

$1 \mathrm{GE} \quad \mathrm{pCi} / \mathrm{mL}$

Notes:

- = exceeded hoiding time

- = exceeded groundwater protection or monitoring constituent standard (See Appendix A.) 
WSRC-TR-99-00013

Unclassified

WELL HSB130D

SRS Coord. Lat/Longitude

N70757.2 $\quad 33.271438^{\circ} \mathrm{N}$

E54651.7 $81.660336^{\circ} \mathrm{W}$

SAMPLE DATE

$07 / 16 / 98$

FIELD DATA

Constituents

Water elevation

$\mathrm{pH}$

Sp. conductance

Water temperature

Alkalinity as $\mathrm{CaCO}_{3}$

Turbidity

Volumes purged

Sampling code

Synchronous water leve

$\underline{3098}$

200.9

5.5

61

18.9

32

$200.5(09 / 18 / 98)$
Screen Zone Elevation

202.1-182.1 ft ms

Top of Casing

$218.6 \mathrm{ft} \mathrm{ms}$

Casing

4" PVC

Pump

$\mathrm{s}$

Screen Zone

Water Table (I1B2)

ANALYTICAL DATA

Inorganic Constituents

Constituents

Antimony, total recoverable

Arsenic, total recoverable

Barium, total recoverable

Cadmium, total recoverable

Chromium, total recoverable

Cobalt, total recoverable

Copper, total recoverable

Cyanide

Lead, total recoverable

Mercury, total recoverable

Nickel, total recoverable

Nitrate as nitrogen

Nitrate-nitrite as nitrogen

Selenium, total recoverable

Silver, total recoverable

Tin, total recoverable

Vanadium, total recoverable

Zinc, total recoverable

Organic Constituents

Constituents

Benzene

Bis(2-ethylhexyl) phthalate

Dichloromethane

Tetrachloroethylene

Trichloroethyiene

Trichlorofluoromethane
Mod

Filt.

$\underline{\text { ST }} \underline{H}$

DF Lab 4 QQ98

Mod

1 GE $<0.20$

$\mathrm{U} / \prime$

1 GE 460

3098

$\underline{\operatorname{Mod}}$

Filt.

$\underline{\text { ST }}$

DF Lab $4 Q 99$

Mod $\underline{\text { Unit }}$

ft msi

$\mathrm{pH}$

$\mu \mathrm{S} / \mathrm{cm}$

${ }^{\circ} \mathrm{C}$

$\mathrm{mg} / \mathrm{L}$

NTU

well vol

ft ms!

Filt.

$\underline{\text { ST }} \underline{H}$ DF Lab Unit

$<0.20$

1 GE $\mu g / L$

1 GE $\mu g / L$

Filt.

$\underline{\text { ST }} \underline{H}$ DF Lab Unit

Notes:

- = exceeded holding time

- = exceeded groundwater protection or monitoring constituent standard (See Appendix A.) 
WELL HSB130D (cont.)

Radjoactive Constituents

\section{Constituents}

Americium-241

Carbon-14

Cobalt-60

Curium-242

Curium-243/244

Curium-245/246

Gross alpha

lodine-129

Nickel-63

Nonvolatile beta

Plutonium-238

Plutonium-239/240

Radium-226

Radium-228

Strontium-90

Technetium-99

Thorium-228

Thorium-230

Thorium-232

Sum of alphas

Sum of betas

Tritium

Uranium-233/234

Uranium-235

Uranium-238 $\underline{3098}$

Mod

Filt.

ST브 DF Lab $\underline{4 Q 98} \quad$ Mod

$<4.6 \mathrm{E}-01$

UiII

$<8.3 E-02$

UIII

$<0.4750$

1 GP $<6.0 \mathrm{E}-01$ JU/UI

$\angle N D D$

1 GE $\mathrm{pCi} / \mathrm{L}$

$<1.1700$

JU/LI

$<N D D$

$1 \mathrm{GE} \mathrm{pCi/L}$

\section{Notes:}

- exceeded holding time

- exceeded groundwater protection or monitoring constituent standard (See Appendix A.) 
WELL HSB131C

$\begin{array}{lll}\text { SRS Coord. } & \text { Lat/Longitude } \\ \text { N70374.7 } & 33.274252^{\circ} \mathrm{N} \\ \text { E56894.9 } & 81.653687^{\circ} \mathrm{W}\end{array}$

SAMPLE DATE

$07 / 16 / 98$

Screen Zone Elevation

$158.5-148.5 \mathrm{ft} \mathrm{msl}$

FIELD DATA

Constituents

Water elevation

$\mathrm{pH}$

Sp. conductance

Water temperature

Alkalinity as $\mathrm{CaCO}_{3}$

Turbidity

Volumes purged

Sampling code

Synchronous water level

ANALYTICAL DATA

Inorganic Constituents

\section{Constituents}

Antimony, total recoverable

Arsenic, total recoverable

Barium, total recoverable

Cadmium, total recoverable

Chromium, total recoverable

Cobalt, total recoverable

Copper, total recoverable

Cyanide

Lead, total recoverable

Mercury, total recoverable

Nickel, total recoverable

Nitrate as nitrogen

Nitrate-nitrite as nitrogen

Selenium, total recoverable

Silver, total recoverable

Tin, total recoverable

Vanadium, total recoverable

Zinc, total recoverable

Organic Constituents

Constituents

$204.3(09 / 18 / 98)$

\section{Top of Casing}

$211.7 \mathrm{ft} \mathrm{ms}$

10/12/98

4Q98

204.2

7.5

160

18.2

77

9.4

$203.7(12 / 21 / 98)$

\section{Casing Pump Screen Zone \\ $4^{n}$ PVC S \\ Barnwell (IIB 1 )}

$\underline{3098}$ Mod $\quad \underline{\text { Filt. }} \quad \underline{\text { ST }} \underline{H}$ DF Lab $\underline{4098}$ Mod $\quad \underline{\text { Filt. }} \underline{\text { ST }} \underline{\mathrm{H}} \underline{\text { DF }} \underline{\underline{\text { Lab }}} \underline{\text { Unit }}$

$J / E$

NDD

$1 \mathrm{GE}<0.20$

U/I

2 GE 3,200

/l

/

$\underline{\text { Mod }} \underline{\text { Filt. }}$

$\underline{\text { ST }} \underline{\mathrm{H}}$

DF Lab $\underline{\text { LQ98 }} \quad \underline{\text { Mod }}$

$<0.20$

$\begin{array}{lll}1 & \text { GE } & \mu g / L \\ & & \text { GE } \mu g / L\end{array}$

Benzene

Bis(2-ethylhexyl) phthalate

Dichloromethane

Tetrachloroethylene

Trichloroethylene

Trichlorofluoromethane

\section{Notes:}

- exceeded holding time

= exceeded groundwater protection or monitoring constituent standard (See Appendix A.) 
WELL HSB131C (cont.)

Radioactive Constituents

Constituents

Americium-241

Carbon-14

Cobalt-60

Curium-242

Curium-243/244

Curium-245/246

Gross alpha

lodine-129

Nickel-63

Nonvolatile beta

Plutonium-238

Plutonium-239/240

Radium-226

Radium-228

Strontium-90

Technetium-99

Thorium-228

Thorium-230

Thorium-232

Sum of alphas

Sum of betas

Tritium

Uranium-233/234

Uranium-235

Uranium-238
3098

$1,3 E+00 \quad / /$

$<1.1 \mathrm{E}+00 \quad$ UII $/$

$<1.2300$

1 GP $<3.1 \mathrm{E}-01$

$\mathrm{u} / 1$

$<9.5 E-01$

$1 \mathrm{GE} \mathrm{PCi/L}$

$1 \quad G P<6.4 E-01 \quad U / 1$

$<1,4 \mathrm{E}+00$

$\uparrow$ GE PCi/L
Filt.

$\underline{\text { ST H }}$ DF Lab Unit

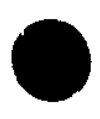




\section{WELL HSB131D}

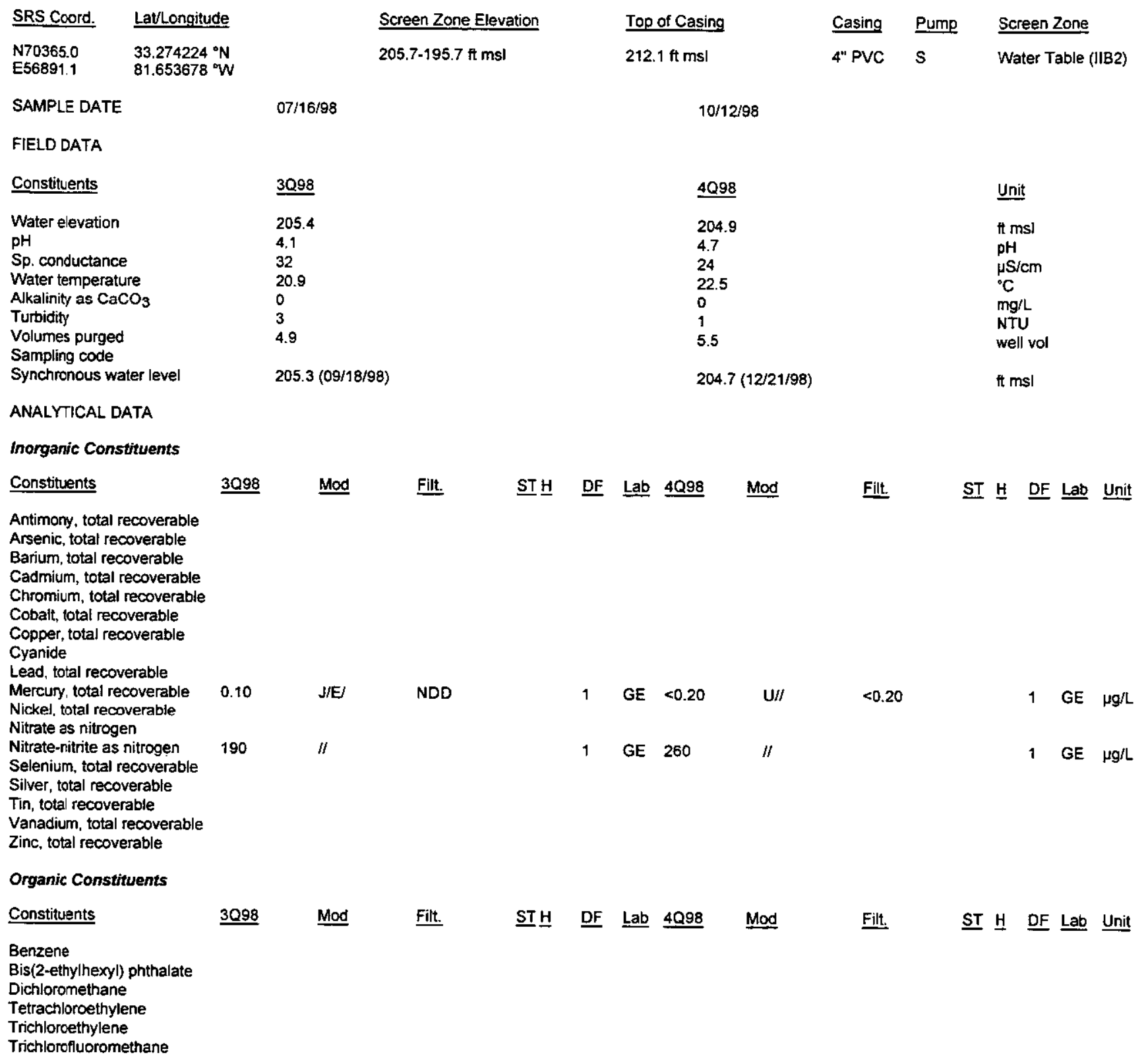

Notes:

- =xceeded holding time

- exceeded groundwater protection or monitoring constituent standard (See Appendix A.) 
WELL HSB131D (cont.)

Radioactive Constituents

Constituents

309

Mod

Filt.

ST브 DF Lab 4098 Mod

Filt.

ST 브 DF Lab Unit

Americium-241

Carbon-14

Cobalt-60

Curium-242

Curium-243/244

Curium-245/246

Gross alpha

lodine-129

Nickel-63

Nonvolatile beta

Plutonium-238

Plutonium-239/240

Radium-226

Radium-228

Strontium-90

Technetium-99

Thorium-228

Thorium-230

Thorium-232

Sum of alphas

Sum of betas

Tritium

Uranium-233/234

Uranium-235

Uranium-238

$1.4 \mathrm{E}+00 \quad /$

$<1.0 \mathrm{E}+00 \quad \mathrm{UI} / /$

$<1.3600$

8.4E+00 /
1

GP $<1.1 E+00 \quad U / /$

1

GP $<2.0 E+00 \quad U / 1$

$<5.6 \mathrm{E}-01$

$<1.1 \mathrm{E}+00$

GP $8.0 E+00 \quad / /$
$1 \mathrm{GE} \mathrm{pCil}$

$1 \mathrm{GE} p \mathrm{Cil}$

Notes:

- = exceeded holding time

- exceeded groundwater protection or monitoring constituent standard (See Appendix A.) 
WELL HSB132C

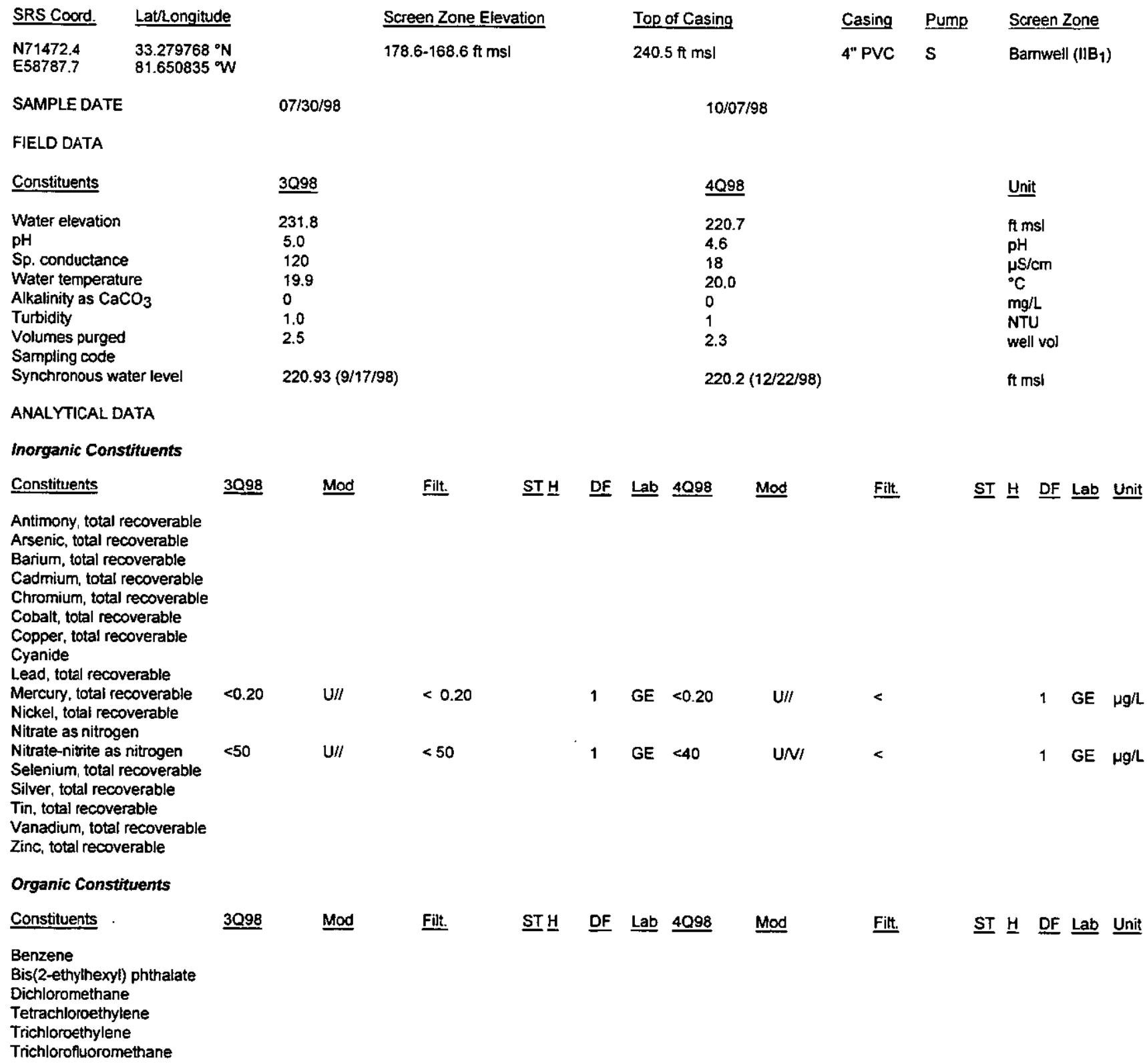

Notes:

- = exceeded holding time

- exceeded groundwater protection or monitoring constituent standard (See Appendix A.) 


\section{WELL HSB132C (cont.)}

\section{Radioactive Constituents}

Constituents

Americium-241

Carbon-14

Cobalt-60

Curium-242

Curium-243/244

Curium-245/246

Gross alpha

lodine-129

Nickel-63

Nonvolatile beta

Plutonium-238

Plutonium-239/240

Radium-226

Radium-228

Strontium-90

Technetium-99

Thorium-228

Thorium-230

Thorium-232

Sum of alphas

Sum of betas

Tritium

Uranium-233/234

Uranium-235

Uranium-238 $\underline{3098}$

Mod

Filt.

ST브 DF Llab 4Q98 $\underline{\text { Mod }}$

Filt.

ST $\underline{H}$ DF Lab Unit

$\begin{array}{lllllllllll}<1.3 \mathrm{E}-01 & \mathrm{UI} / / & <0.5710 & 1 & \mathrm{GP} & <4.0 \mathrm{E}-02 & \mathrm{JU} / \mathrm{LI} & <\mathrm{NDD} & & \mathrm{GE} & \mathrm{pCi} / \mathrm{L} \\ <5.7 \mathrm{E}-01 & \mathrm{UI} / / & <1.2900 & 1 & \mathrm{GP} & <-2.1 \mathrm{E}-01 \quad \mathrm{JU} / \mathrm{LI} & <\mathrm{NDD} & & \end{array}$

1

\begin{abstract}
GP $\quad<5.2 \mathrm{E}-02$
\end{abstract}
U/I

$<6.2 \mathrm{E}-01$

$\uparrow$ GE $\mathrm{pCi} / \mathrm{mL}$

Notes:

- =xceeded holding time

- exceeded groundwater protection or monitoring constituent standard (See Appendix A.) 


\section{WELL HSB132D}

$\begin{array}{lll}\text { SRS Coord. } & & \text { Lat/Longitude } \\ \text { N71469.5 } & & 33.279780^{\circ} \mathrm{N} \\ \text { E58799.3 } & & 81.650798^{\circ} \mathrm{W}\end{array}$

SAMPLE DATE

$07 / 30 / 98$

Screen Zone Elevation

226.5-206.5 ft ms]

FIELD DATA

Constituents

Water elevation

$\mathrm{pH}$

Sp. conductance

Water temperature

Alkalinity as $\mathrm{CaCO}_{3}$

Turbidity

Volumes purged

Sampling code

Synchronous water level

ANALYTICAL DATA

Inorganic Constituents

Constituents

Antimony, total recoverable

Arsenic, total recoverable

Barium, total recoverable

Cadmium, total recoverable

Chromium, total recoverable

Cobalt, total recoverable

Copper, total recoverable

Cyanide

Lead, total recoverable

Mercury, total recoverable

Nickel, total recoverable

Nitrate as nitrogen

Nitrate-nitrite as nitrogen

Selenium, total recoverable

Silver, total recoverable

Tin, total recoverable

Vanadium, total recoverable

Zinc, total recoverable

Organic Constituents

Constituents

Benzene

Bis(2-ethylhexyl) phthalate

Tetrachloroethylene

Trichloroethylene

Trichlorofluoromethane
3Q98

220.5

5.0

20

23.2

2

2.9

$220.26(9 / 17 / 98)$
Mod

Fitt.

$<0.20$

$\mathrm{U} / 1$

$<0.20$

$630 \quad N /$

Mod Filt

ST
Dichloromethane

\section{Top of Casing}

$240.7 \mathrm{ft} \mathrm{ms}$

$10 / 07 / 98$

4Q98

218.0

4.5

20.0

0

3.3

$219.7(12 / 22 / 98)$

Casing Pump

4" PVC S

Water Tabie (IIB2)
Unit

ft msl

pH

$\mathrm{\mu S} / \mathrm{cm}$

${ }^{\circ} \mathrm{C}$

$\mathrm{mg} / \mathrm{L}$

well vol

ft ms!
Screen Zone

ST브

DF Lab $4 Q 98$

Mod

Filt.

ST 브 DF Lab Unit

$1 \quad$ GE $<0.20$

$\mathrm{U} / \mathrm{I}$

$<0.20$

$1 \mathrm{GE} \mu g / \mathrm{L}$

1 GE 650

/I

1 GE $\mu g / L$

DF Lab $\underline{4 Q 98}$

$\underline{M o d}$

Filt.

$\underline{\text { ST }} \underline{H} \underline{\text { DF }} \underline{\text { Lab }} \underline{\text { Unit }}$

Notes:

- = exceeded holding time

= exceeded groundwater protection or monitoring constituent standard (See Appendix A.) 
WELL HSB132D (cont.)

Radioactive Constituents

Constituents

Americium-241

Carbon-14

Cobalt-60

Curium-242

Curium-243/244

Curium-245/246

Gross alpha

lodine-129

Nickel-63

Nonvolatile beta

Plutonium-238

Plutonium-239/240

Radium-226

Radium-228

Strontium-90

Technetium-99

Thorium-228

Thorium-230

Thorium-232

Sum of alphas

Sum of betas

Tritium

Uranium-233/234

Uranium-235

Uranium-238 $\underline{3098}$

Mod

Filt.

ST브 DF Lab $\underline{4 Q 98}$ Mod

Fịlt.

ST $\underline{H}$ DF Lab Unit

$<4.3 E-01 \quad U I / 4$

$<0.6840$

1 GP $\quad<7.5 \mathrm{E}-01$

JU/LI

$\angle N D D$

1 GE $\mathrm{pCi} / \mathrm{L}$

$<9.0 \mathrm{E}-01 \quad \mathrm{UI} / /$

$<1.1300$

$1 \quad G P \quad<8.2 E-01$

JU/U

<NDD

$1 \mathrm{GE} \mathrm{PCi/L}$

$1.1 E+01 \quad / /$

1 GP $1.3 E+01$

"I

1 GE $\mathrm{pCi} / \mathrm{mL}$ 
WELL HSB133C

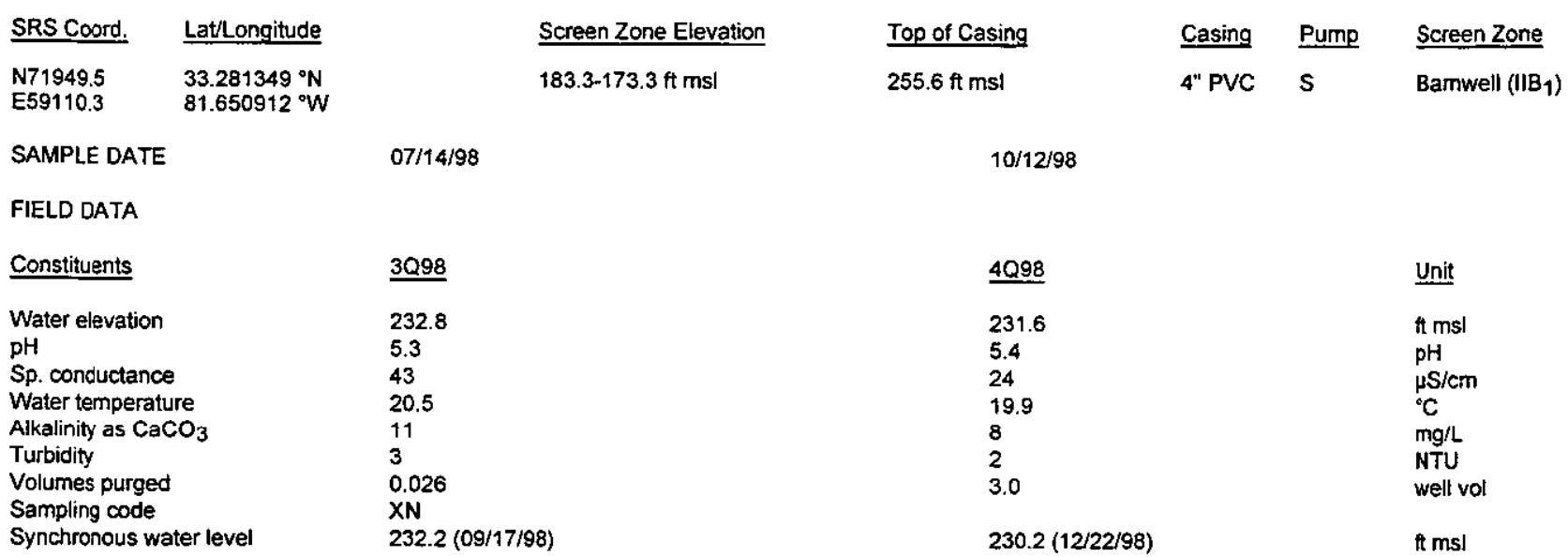

ANALYTICAL DATA

Inorganic Constituents

Constituents

Antimony, total recoverable

Arsenic, total recoverable

Barium, total recoverable

Cadmium, total recoverable

Chromium, total recoverable

Cobalt, total recoverable

Copper, total recoverable

Cyanide

Lead, total recoverable

Mercury, total recoverable

Nickel, total recoverable

Nitrate as nitrogen

Nitrate-nitrite as nitrogen

Selenium, total recoverable

Silver, total recoverable

Tin, total recoverable

Vanadium, total recoverable

Zinc, total recoverable

Organic Constituents

Constituents

Benzene

Bis(2-ethylhexyl) phthatate

Dichloromethane

Tetrachlorcethylene

Trichloroethylene

Trichlorofluoromethane

$<0.20$
Filt.

ST

DF Lab 4 Q998

Mod

$<0.20$
$\mathrm{U} / /$

1 GE $<0.20$

$\mathrm{U} / /$

NDD

GE 40

ST브

DF

$\underline{4098}$

Mod

$<0.20$

NDD

Mod Filt.

Filt.

Notes:

- = exceeded holding time

- = exceeded groundwater protection or monitoring constituent standard (See Appendix A.) 
WELL HSB133C (cont.)

Radioactive Constituents

\section{Constituents}

Americium-241

Carbon-14

Cobalt-60

Curium-242

Curium-243/244

Curium-245/246

Gross alpha

lodine-129

Nickel-63

Nonvolatile beta

Plutonium-238

Plutonium-239/240

Radium-226

Radium-228

Strontium-90

Technetium-99

Thorium-228

Thorium-230

Thorium-232

Sum of alphas

Sum of betas

Tritium

Uranium-233/234

Uranium-235

Uranium-238
3098

Mod

Filt.

STH DF Lab 4098 Mod

$<2.6 E-01$

Ut $/ I$

$1.5 E+\infty 0$

/I

$<-2.3 \mathrm{E}-01 \quad \mathrm{U} / \mathrm{l}$

$<6.2 E-01$

1 GP $<1.9 E-02$

UII

$<6.5 E-01$

$1 \mathrm{GE} \quad \mathrm{pCi} / \mathrm{mL}$

Notes: 
WELL HSB133D

$\begin{array}{ll}\text { SRS Coord. } & \text { LatlLongitude } \\ \text { N71943.5 } & \quad 33.281323^{\circ} \mathrm{N} \\ \text { E59102.3 } & 81.650921^{\circ} \mathrm{W}\end{array}$

Screen Zone Elevation

228.5-208.5 ft msl

$07 / 14 / 98$

SAMPLE DATE

FIELD DATA

Constituents

Water elevation

$\mathrm{pH}$

Sp. conductance

Water temperature

Alkalinity as $\mathrm{CaCO}_{3}$

Turbidity

Voiumes purged

Sampling code

Synchronous water level

3098

237.8

5.1

51

19.8

4

1
3.3

$237.4(09 / 17 / 98)$
Top of Casing

$255.3 \mathrm{ft} \mathrm{ms|}$

$10 / 12 / 98$

4Q98

236.8

4.9

44

0

4.0

$235.0(12 / 22 / 98)$
Casing Pump

4" PVC S
Screen Zone

Water Table (IIB2)

ANALYTICAL DATA

Inorganic Constituents

Constituents

Mod

Filt.

DF Lab $\underline{4098}$

Mod

1

GE $<0.20$

1 GE 530

U/I

"l

Nitrate-nitrite as nitrogen

Selenium, total recoverable

660

Silver, total recoverable

Tin, total recoverable

Vanadium, total recoverable

Zinc, total recoverable

Organic Constituents

Constituents

Benzene

Bis(2-ethylhexyl) phthalate

Dichloromethane

Tetrachloroethylene

Trichloroethylene

Trichlorofluoromethane

Notes:

- = exceeded holding time

- = exceeded groundwater protection or monitoring constituent standard (See Appendix A.) 


\section{WELL HSB133D (cont.)}

Radioactive Constituents

Constituents

Americium-241

Carbon-14

Cobalt-60

Curium-242

Curium-243/244

Curium-245/246

Gross alpha

lodine-129

Nickel-63

Nonvolatile beta

Plutonium-238

Plutonium-239/240

Radium-226

Radium-228

Strontium-90

Technetium-99

Thorium-228

Thorium-230

Thorium-232

Sum of alphas

Sum of betas

Tritium

Uranium-233/234

Uranium-235

Uranium-238
309

Mod

Filt.

$\underline{\text { ST }} \underline{\mathrm{H}}$

DF Lab $\underline{4 Q 98}$

Mod

Filt.

ST $\underline{H}$ DF Lab Unit

\author{
$<7.2 E-01$
}

UIII

$<0.8840$

1

GP $<2.5 E-01$

$\mathrm{u} / \mathrm{I}$

$<7.7 \mathrm{E}-01$

1

GP $<3.3 E-01$

unI

$<1.1 \mathrm{E}+00$

$1 \mathrm{GE}$ pCi/l

$<9.3 E-01$

$\mathrm{U} / /$

$<1.1800$

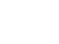

(2)

$1.7 \mathrm{E}+01$

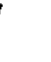

1 GP $2.1 E+01 \quad$ "

1 GP $2.1 \mathrm{E}+01 \quad$ / 
WELL HSB134C

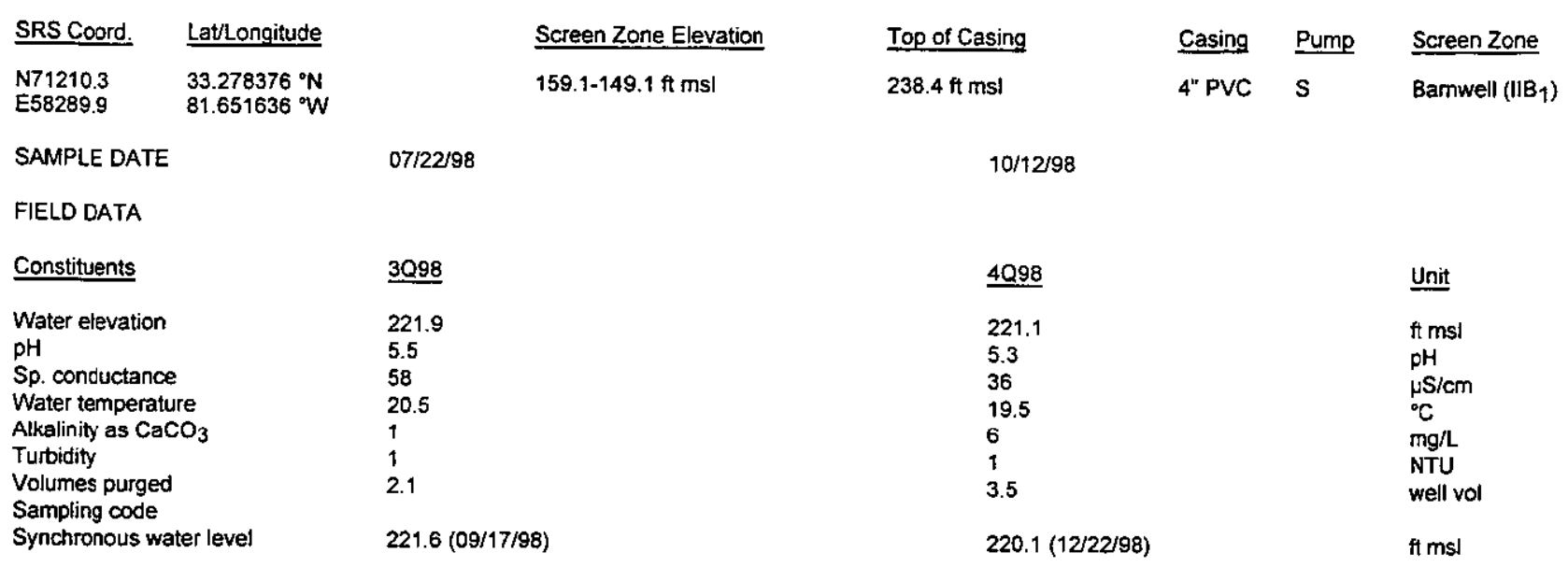

ANALYTICAL DATA

Inorganic Constituents

\section{Constituents}

Antimony, total recoverable Arsenic, total recoverable Barium, total recoverable Cadmium, total recoverable Chromium, total recoverable Cobalt, total recoverable Copper, total recoverable

Cyanide

Lead, total recoverable Mercury, total recoverable

Nickel, total recoverable

Nitrate as nitrogen

Nitrate-nitrite as nitrogen

Selenium, totai recoverable

Silver, total recoverable

Tin, total recoverable

Vanadium, total recoverable

Zinc, total recoverable

Organic Constituents

Constituents

Benzene

Bis(2-ethylhexyl) phthalate

Dichloromethane

Tetrachloroethylene

Trichloroethylene

Trichlorofluoromethane
Mod

Filt.

$\underline{\text { ST }} \underline{\underline{H}}$

DF Lab 4Q98 Mod

1 GE $<0.20$

$\mathrm{U} / /$

$<0.20$

1 GE $\mu g / L$

$1,200 \quad J / V 6 / 1 \quad$ NDD

1 GE $\quad 1,200$

II

$\underline{3098}$

Mod

Filt.

$\underline{\text { ST} ㅡ ㅡ ~}$

DF
Lab 409
Mod
Filt.

ST 브 DF Lab Unit

\section{Notes:}

- exceeded holding time

- exceeded groundwater protection or monitoring constituent standard (See Appendix A.) 
WELL HSB134C (cont.)

Radioactive Constituents

\begin{tabular}{|c|c|c|c|c|c|c|c|c|c|c|c|c|c|}
\hline Constituents & $\underline{3098}$ & Mod & Fitt. & $\underline{\text { ST }} \underline{H}$ & $\underline{\mathrm{DF}}$ & Lab & $\underline{4 Q 98}$ & Mod & Filt. & $\underline{\mathrm{ST}} \underline{H}$ & $\underline{D F}$ & $\underline{L a b}$ & $\underline{\text { Unit }}$ \\
\hline $\begin{array}{l}\text { Americium-241 } \\
\text { Carbon-14 } \\
\text { Cobalt-60 } \\
\text { Curium-242 } \\
\text { Curium-243/244 } \\
\text { Curium-245/246 }\end{array}$ & & & & & & & & & & & & & \\
\hline $\begin{array}{l}\text { Gross alpha } \\
\text { lodine-129 } \\
\text { Nickel-63 }\end{array}$ & $<3.8 \mathrm{E}-01$ & UI// & $<0.5110$ & & 1 & GP & $<1.2 E-01$ & $\mathrm{U} / /$ & $<6.1 E-01$ & & 1 & GE & $\mathrm{pCi} / \mathrm{L}$ \\
\hline $\begin{array}{l}\text { Nonvolatile beta } \\
\text { Plutonium-238 } \\
\text { Plutonium-239/240 } \\
\text { Radium-226 } \\
\text { Radium-228 } \\
\text { Strontium-90 } \\
\text { Technetium-99 } \\
\text { Thorium-228 } \\
\text { Thorium-230 } \\
\text { Thorium-232 } \\
\text { Sum of alphas } \\
\text { Sum of betas }\end{array}$ & $1.7 \mathrm{E}+00$ & "I & & & 1 & GP & $<7.5 \mathrm{E}-01$ & $\mathrm{u} / /$ & $<1.2 \mathrm{E}+00$ & & 1 & $G E$ & $\mathrm{pCi} / \mathrm{L}$ \\
\hline $\begin{array}{l}\text { Tritium } \\
\text { Uranium-233/234 } \\
\text { Uranium-235 } \\
\text { Uranium-238 }\end{array}$ & $2.4 \mathrm{E}+09$ & $/ 1$ & & - & 1 & $\mathrm{TM}$ & $2.5 E+01$ & $/ 1$ & & च & 1 & GE & $\mathrm{pCi} / \mathrm{mL}$ \\
\hline
\end{tabular}


WELL HSB134D

\begin{tabular}{|c|c|}
\hline SRS Coord. & Latho \\
\hline $\begin{array}{l}\text { N712 } \\
\text { E582 }\end{array}$ & 81.6 \\
\hline
\end{tabular}

SAMPLE DATE

FIELD DATA

Constituents

Water elevation

$\mathrm{pH}$

$\mathrm{Sp}$. conductance

Water temperature

Alkalinity as $\mathrm{CaCO}_{3}$

Turbidity

Volumes purged

Sampling code

Synchronous water level
Screen Zone Elevation

$225.8-205.8 \mathrm{ft} \mathrm{msl}$

$07 / 09 / 98$

$\underline{3 Q 98}$

212.8

4.0

88

19.7

0

10

$221.3(09 / 17 / 98)$
Top of Casing

$238.1 \mathrm{ft} \mathrm{ms}$

$10 / 14 / 98$

4Q98

220.8

3.9

120

18.8

0

3.

$220.1(12 / 22 / 98)$
Casing Pump Screen Zone

4" PVC S $\quad$ Water Table (IIB2)

\section{ANALYTICAL DATA}

Inorganic Constituents

\begin{tabular}{|c|c|c|c|c|c|c|c|c|}
\hline Constituents & $\underline{3098}$ & Mod & Filt. & $\underline{\text { ST }} \underline{H}$ & $\underline{D F}$ & Lab & 4098 & Mod \\
\hline Antimony, total recoverable & $<0.20$ & U/I & $<0.20$ & & 1 & GE & & \\
\hline Arsenic, total recoverable & $<3.0$ & $\mathrm{U} / 1$ & $<3.0$ & & 1 & $\mathrm{GE}$ & & \\
\hline Barium, total recoverable & 50 & ll & & & $i$ & GE & & \\
\hline Cadmium, total recoverable & 0.20 & $J / E /$ & NDD & & $i$ & GE & & \\
\hline Chromium, total recoverable & $<2.1$ & UNI & $<3.0$ & & 1 & GE & & \\
\hline Cobalt, total recoverable & 2.5 & $\|$ & & & 1 & GE & & \\
\hline Copper, total recoverable & 5.7 & $\|$ & & & 1 & $\mathrm{GE}$ & & \\
\hline Cyanide & 8.4 & $\mathrm{~J} / \mathrm{E} / \mathbf{2}$ & NDD & & 1 & $\mathrm{GE}$ & & \\
\hline Lead, total recoverable & 3.1 & $1 /$ & & & 1 & GE & & \\
\hline Mercury, total recoverable & & & & & & & 0.65 & $\mathrm{~J} / \mathrm{K} / \mathrm{C}$ \\
\hline $\begin{array}{l}\text { Nickel, total recoverable } \\
\text { Nitrate as nitrogen }\end{array}$ & 3.6 & NI & & & 1 & GE & & \\
\hline Nitrate-nitrite as nitrogen & 6.900 & NI & & & 5 & GF & 11000 & 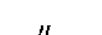 \\
\hline Selenium, total recoverable & $<5.0$ & $\mathrm{u} / /$ & $<5.0$ & & 1 & GE & 11,000 & $n$ \\
\hline Silver, total recoverable & $<1.0$ & $\mathrm{U} / /$ & $<1.0$ & & 1 & GE & & \\
\hline Tin, total recoverable & $<1.2$ & $U N /$ & $<2.0$ & & 1 & GE & & \\
\hline Vanadium, total recoverable & $<2.0$ & $\mathrm{U} / /$ & $<2.0$ & & 1 & GE & & \\
\hline Zinc, total recoverable & 22 & $/ 1$ & & & 1 & GE & & \\
\hline
\end{tabular}

Organic Constituents

\begin{tabular}{|c|c|c|c|c|c|}
\hline Constituents & $\underline{3098}$ & Mod & Filt. & $\underline{\text { ST }} \underline{H}$ & DF \\
\hline Benzene & $<1.0$ & $\mathrm{U} / /$ & $<1.0$ & & 1 \\
\hline Bis(2-ethylhexyl) phthalate & $<9.8$ & $\mathrm{U} / /$ & $<9.8$ & & 1 \\
\hline Dichloromethane & $<1.0$ & $\mathrm{U} / \mathrm{I}$ & $<1.0$ & & 1 \\
\hline Tetrachloroethylene & $<1.0$ & $\mathrm{U} / / \mathrm{I}$ & $<1.0$ & & 1 \\
\hline Trichloroethylene & $<1.0$ & $\mathrm{U} / /$ & $<1.0$ & & 1 \\
\hline Trichlorofluoromethane & $<1.0$ & $\mathrm{U} / /$ & $<1.0$ & & 1 \\
\hline
\end{tabular}

$\underline{\text { Unit }}$

ft msl

$\mathrm{pH}$

$\mu \mathrm{S} / \mathrm{cm}$

${ }^{\circ} \mathrm{C}$

$\mathrm{mg} / \mathrm{L}$

NTU

well vol

ft msl
ST $\underline{H}$ DF Lab Unit

$\mu \mathrm{g} / \mathrm{L}$

$\mu \mathrm{g} / \mathrm{L}$

$\mu g / L$

$\mu g / L$

$\mu g / L$

$\mu g / L$

$\mu \mathrm{g} / \mathrm{L}$

$\mu g / L$

GE $\mu g / L / L$

$\mu g / L$

- 5 GE $\mu g / L$

$\mu g / L$

$\mu g / L$

$\mu g / L$

$\mu g / L$

$\mu \mathrm{g} / \mathrm{L}$

4 Mod Filt. ST H DF Lab $\frac{\text { Unit }}{\mu g / L}$

Notes:

- = exceeded holding time

= exceeded groundwater protection or monitoring constituent standard (See Appendix A.) 
WELL HSB134D (cont.)

Radioactive Constituents

\begin{tabular}{|c|c|c|c|c|c|c|c|c|c|c|c|c|c|}
\hline Constituents & $\underline{3 Q 98}$ & Mod & Filt. & $\underline{\text { ST }} \underline{H}$ & $\underline{\mathrm{DF}}$ & $\underline{\text { Lab }}$ & $\underline{4 Q 98}$ & Mod & Filt. & $\underline{\text { ST }} \underline{H}$ & $\underline{\mathrm{DF}}$ & $\underline{L a b}$ & $\underline{\text { Unit }}$ \\
\hline Americium-241 & $<1.0 \mathrm{E}-01$ & $\mathrm{UI} / /$ & $<0.1430$ & & 1 & GP & & & & & & & $\mathrm{pCil}$ \\
\hline Beta dose & 5.50 & & & घ & & & & & & & & & $\mathrm{pCi} / \mathrm{L}$ \\
\hline Carbon-14 & $<3.3 E+00$ & $\mathrm{UI} / /$ & $<14.8000$ & & 1 & GP & & & & & & & $\mathrm{pCi} / \mathrm{L}$ \\
\hline Cobalt-60 & $<-6.5 \mathrm{E}-01$ & $U: / /$ & $<3.5600$ & & 1 & GP & & & & & & & $\mathrm{pCi} / \mathrm{L}$ \\
\hline Curium-242 & $<-2.0 \mathrm{E}-02$ & $\mathrm{U}[/ /$ & $<0.2090$ & & 1 & GP & & . & & & & & $\mathrm{pCi} / \mathrm{L}$ \\
\hline Curium-243/244 & $<6.3 \mathrm{E}-02$ & $\mathrm{U} / \mathrm{II}$ & $<0.1560$ & & 1 & GP & & & & & & & $\mathrm{pCi} / \mathrm{L}$ \\
\hline Curium-245/246 & $<0.0 \mathrm{E}+00$ & $\mathrm{U} \mid \prime \prime$ & $<0.0618$ & & 1 & $G P$ & & & & & & & $\mathrm{pCi} / \mathrm{L}$ \\
\hline Gross alpha & $1.1 \mathrm{E}+01$ & $\|$ & & & 1 & GP & $<2.2 \mathrm{E}+00$ & $\mathrm{U} / \mathrm{h}$ & $<7.5 \mathrm{E}-01$ & & 1 & GE & $\mathrm{pCi} / \mathrm{L}$ \\
\hline Iodine-129 & $5.5 E+\infty 0$ & 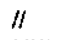 & & & 1 & GP & & & & & & & $\mathrm{pCi} / \mathrm{L}$ \\
\hline Nickel-63 & $<0.0 E+00$ & $\mathrm{U} \mid / /$ & $<220.0000$ & & 1 & GP & & & & & & & pCin \\
\hline Nonvolatile beta & $7.3 \mathrm{E}+02$ & $\prime \prime$ & & - & 1 & GP & $1.8 \mathrm{E}+02$ & "l & & घ & 1 & GE & $\mathrm{pCi} / \mathrm{L}$ \\
\hline Plutonium-238 & $<0.0 \mathrm{E}+00$ & $\mathbf{U} \mid / /$ & $<0.0525$ & & 1 & GP & & & & & & & $\mathrm{pCi} / \mathrm{L}$ \\
\hline Plutonium-239/240 & $<-8.4 \mathrm{E}-03$ & $\mathrm{U} \mathrm{1} / /$ & $<0.1090$ & & 1 & GP & & & & & & & $\mathrm{pCi} / \mathrm{L}$ \\
\hline Radium-226 & $2.8 \mathrm{E}+00$ & "I & & & 1 & GP & & & & & & & $\mathrm{pCil}$ \\
\hline Radium-228 & $<-3.6 \mathrm{E}-02$ & U:// & $<0.8800$ & & 1 & GP & & & & & & & $\mathrm{pCi} / \mathrm{L}$ \\
\hline Strontium-90 & $2.6 \mathrm{E}+02$ & $\mathrm{~J} / \mathrm{U}$ & NDD & & 1 & $G P$ & & & & & & & $\mathrm{pCill}$ \\
\hline Technetium-99 & $<-9.7 E+00$ & $U \mathrm{UI} /$ & $<23.1000$ & & 1 & GP & & & & & & & $\mathrm{PCilL}$ \\
\hline Thorium-228 & $<5.3 E-02$ & $\mathrm{UI} / /$ & $<0.3780$ & & 1 & GP & & & & & & & $\mathrm{pCi} / \mathrm{L}$ \\
\hline Thorium-230 & $<0.0 \mathrm{E}+00$ & UIII & $<0.0665$ & & 1 & GP & & & & & & & $\mathrm{pCi} / \mathrm{L}$ \\
\hline Thorium-232 & $<2.2 \mathrm{E}-02$ & $\mathrm{UIII}$ & $<0.0665$ & & 1 & GP & & & & & & & $\mathrm{pCi} \mathrm{L}$ \\
\hline Sum of alphas & $1.3 E+00$ & & & & & & & & & & & & $\mathrm{pCi} / \mathrm{h}$ \\
\hline $\begin{array}{l}\text { Sum of betas } \\
\text { Total radium }\end{array}$ & $\begin{array}{l}2.6 \mathrm{E}+02 \\
2.8 \mathrm{E}+00\end{array}$ & & & - & & & & & & & & & $\mathrm{pCi} / \mathrm{h}$ \\
\hline Tritium & $3.5 \mathrm{E}+02$ & /1 & & - & 1 & GP & $8.3 \mathrm{E}+02$ & $\|$ & & - & 1 & GE & $\mathrm{pCi} / \mathrm{mL}$ \\
\hline Uranium-233/234 & $1.0 E+00$ & "I & & & 1 & GP & & & & & & & $\mathrm{pCi} / \mathrm{h}$ \\
\hline Uranium-235 & $<4.4 \mathrm{E}-02$ & $\mathbf{U} \mathbf{1} / /$ & $<0.0662$ & & 1 & GP & & & & & & & $\mathrm{pCill}$ \\
\hline Uranium-238 & $2.9 \mathrm{E}-01$ & $/ 1$ & & & 1 & GP & & & & & & & $\mathrm{pCin}$ \\
\hline
\end{tabular}




\section{WELL HSB135C}

$\begin{array}{lll}\text { SRS Coord. } & \text { LatLongitude } \\ \text { N71390.2 } & & 33.275953^{\circ} \mathrm{N} \\ \text { E56560.8 } & 81.656539^{\circ} \mathrm{W}\end{array}$

Screen Zone Elevation

$157.3-147.3 \mathrm{ft} \mathrm{ms}$

$07 / 14 / 98$

3Q98

Constituents

Water elevation

pH

Sp. conductance

Water temperature

Alkalinity as $\mathrm{CaCO}_{3}$

Turbidity

Volumes purged

Sampling code

Synchronous water level

ANALYTICAL DATA

Inorganic Constituents

\section{Constituents}

Antimony, total recoverable

Arsenic, total recoverable

Barium, total recoverable

Cadmium, total recoverable

Chromium, total recoverable

Cobalt, total recoverable

Copper, total recoverable

Cyanide

Lead, total recoverable

Mercury, total recoverable

Nickel, total recoverable

Nitrate as nitrogen

Nitrate-nitrite as nitrogen

Selenium, total recoverable

Silver, total recoverable

Tin, total recoverable

Vanadium, total recoverable

Zinc, total recoverable

Organic Constituents

Constituents

Benzene

Bis(2-ethylhexyl) phthalate

Dichloromethane

Tetrachloroethylene

Trichloroethylene

Trichlorofluoromethane

Mod

Filt.

$\underline{3 Q 98}$

Mod

Filt.
STI

DF

Lab 4 Q98

Mod

1

GE $<0.20$

$\mathrm{u} / /$

1 GE 540

II

$430 \quad$ /I

STI

DF Lab $\underline{4 Q 98}$

Mod

Filt.

$<0.20$

1 GE $\mu g / L$

1 GE $\mu g / L$

ft ms

pH

$\mu \mathrm{S} / \mathrm{cm}$

${ }^{\circ} \mathrm{C}$

$\mathrm{mg} / \mathrm{L}$

NTU

well vol

ft msl

I

creen Zone

Bamwell ( $\mathrm{IB}_{1}$ )
Unit 
WELL HSB135C (cont.)

Radioactive Constituents

Constituents

$\underline{3098}$

Mod

Filt.

STH $\underline{H}$ Lab $\underline{4098}$ Mod

Filt.

$\underline{\text { ST }} \underline{H}$ DF Lab Unit

Americium-241

Carbon-14

Cobalt-60

Curium-242

Curium-243/244

Curium-245/246

Gross alpha

6.9E-01 /

$1 \quad$ GP $\quad<1.1 \mathrm{E}-01$

UII

$<7.6 \mathrm{E}-01$

1 GE $\mathrm{pCi} / \mathrm{L}$

$1.4 \mathrm{E}+\infty \quad / 1$

$1 \quad G P<1.1 E+\infty 0$

$\mathrm{U} / \prime$

$<1.2 E+00$

1 GE pCi/L

Plutonium be

Plutonium-239/240

Radium-226

Radium-228

Strontium-90

Technetium-99

Thorium-228

Thorium-230

Thorium-232

Sum of alphas

Sum of betas

Tritium

Uranium-233/234

$2.0 \mathrm{E}+01$

1 GP $\quad 3.3 E+01$

-

$1 \mathrm{GE} \mathrm{pCi} / \mathrm{mL}$

Uranium-238

Notes:

- exceeded holding time

= exceeded groundwater protection or monitoring constituent standard (See Appendix A.) 
WELL HSB135D

\begin{tabular}{|c|c|c|c|c|c|c|c|}
\hline SRS Coord. & Latllongitude & & Screen Zone Elevation & Top of Casing & Casing & Pump & Screen Zone \\
\hline $\begin{array}{l}\text { N71396.7 } \\
\text { E56552.8 }\end{array}$ & $\begin{array}{l}33.275954^{\circ} \mathrm{N} \\
81.656572^{\circ} \mathrm{W}\end{array}$ & & $219.9-199.9 \mathrm{ft} \mathrm{msl}$ & $232.3 \mathrm{ft} \mathrm{msl}$ & 4" PVC & $\mathbf{S}$ & Water Table (IIB2) \\
\hline \multicolumn{2}{|c|}{ SAMPLE DATE } & \multicolumn{2}{|l|}{$07 / 14 / 98$} & $10 / 12$ & & & \\
\hline \multicolumn{8}{|l|}{ FIELD DATA } \\
\hline \multicolumn{2}{|l|}{ Constituents } & \multicolumn{2}{|l|}{$\underline{3098}$} & $4 Q 98$ & & & $\underline{\text { Unit }}$ \\
\hline \multicolumn{2}{|c|}{ Water elevation } & \multicolumn{2}{|l|}{217.6} & 216.8 & & & $\mathrm{ft} \mathrm{msl}$ \\
\hline \multirow{2}{*}{\multicolumn{2}{|c|}{$\begin{array}{l}\text { pH } \\
\text { Sp. conductance }\end{array}$}} & \multicolumn{2}{|l|}{4.3} & 4.7 & & & $\mathrm{pH}$ \\
\hline & & \multicolumn{2}{|l|}{26} & 26 & & & $\mu \mathrm{S} / \mathrm{cm}$ \\
\hline \multicolumn{2}{|c|}{ Water temperature } & & 19.6 & & & ${ }^{\circ} \mathrm{C}$ \\
\hline \multicolumn{2}{|c|}{$\begin{array}{l}\text { Water temperature } \\
\text { Alkalinity as } \mathrm{CaCO}_{3}\end{array}$} & \multirow{2}{*}{\multicolumn{2}{|c|}{$\begin{array}{l}1 \\
1\end{array}$}} & 0 & & & $\mathrm{mg} / \mathrm{L}$ \\
\hline \multicolumn{2}{|l|}{ Turbidity } & & & 1 & & & NTU \\
\hline \multicolumn{2}{|c|}{ Volumes purged } & \multicolumn{2}{|l|}{4.3} & 7.2 & & & well vol \\
\hline \multicolumn{2}{|c|}{ Sampling code } & $217.2(09$ & 98) & 216.6 & & & $\mathrm{ft} \mathrm{msl}$ \\
\hline
\end{tabular}

ANALYMICAL DATA

Inorganic Constituents

Constituents

Antimony, total recoverable Arsenic, total recoverable Barium, total recoverable

Cadmium, total recoverable Chromium, total recoverable Cobalt, total recoverable Copper, total recoverable Cyanide

Lead, total recoverable Mercury, total recoverable

Nickel, total recoverable

Nitrate as nitrogen

Nitrate-nitrite as nitrogen

Selenium, total recoverable

Silver, total recoverable

Tin, total recoverable

Vanadium, total recoverable

Zinc, total recoverable

Organic Constituents

Constituents

$\underline{3098}$

Benzene

Bis(2-ethylhexyl) phthalate

Dichloromethane

Tetrachloroethylene

Trichloroethylene

Trichlorofluoromethane

309
Mod

Filt

ST $\underline{H}$

DF LLab 4098

Mod

1

GE $\quad 0.10$

$\mathrm{J} / / \mathrm{I}$

$1 \quad$ GE $\quad 2,100$

$\|$

NDD

1 GE $\mu g / L$

/l

2,100

II

Mod Fitt.

ST브

DF Lab 4 Q998

Mod

Filt.

ST $\underline{H}$ DF Lab Unit

\section{Notes:}

- = exceeded holding time

- exceeded groundwater protection or monitoring constituent standard (See Appendix A.) 
WSRC-TR-99-00013

Unclassified

\section{WELL HSB135D (cont.)}

Radioactive Constituents

Constituents

Americium-241

Carbon-14

Cobalt -60

Curium-242

Curium-243/244

Curium-245/246

Gross alpha

lodine-129

Nickel-63

Nonvolatile beta

Plutonium-238

Plutonium-239/240

Radium-226

Radium-228

Strontium-90

Technetium-99

Thorium-228

Thorium-230

Thorium-232

Sum of alphas

Sum of betas

Tritium

Uranium-233/234

Uranium-235

Uranium-238
3098

Mod

Filt.

$\underline{\text { ST }} \underline{\mathrm{H}}$

DF Lab 4 QQ98

Mod

6.2E-01 $/ /$

$3.2 \mathrm{E}+00 \quad / /$

1

GP $<3.9 \mathrm{E}-01 \quad \mathrm{U} / /$

$<6.3 \mathrm{E}-01$

1 GE $\mathrm{pCi} / \mathrm{L}$

GP 4.1E+00 $/ /$

1 GE $\mathrm{pCi} / \mathrm{L}$

2.4E+01 //

- 1 GP $3.3 E+01 \quad / /$

-

$1 \mathrm{GE} \mathrm{pCi} / \mathrm{mL}$

Notes:

- exceeded holding time

- exceeded groundwater protection or monitoring constituent standard (See Appendix A.) 


\section{WELL HSB136C}

\begin{tabular}{ll} 
SRS Coord. & Lat/Longitude \\
\hline N71900.3 & $33.276084^{\circ} \mathrm{N}$ \\
E55949.6 & $81.659139^{\circ} \mathrm{W}$
\end{tabular}

SAMPLE DATE

$07 / 28 / 98$

Screen Zone Elevation

$170.5-160.5 \mathrm{ft} \mathrm{msl}$

Top of Casing

$227.9 \mathrm{ft} \mathrm{msl}$

$10 / 12 / 98$

FIELD DATA

Constituents

3098

Water elevation

$\mathrm{pH}$

Sp. conductance

Water temperature

Alkalinity as $\mathrm{CaCO}_{3}$

Turbidity

Volumes purged

Sampling code

Synchronous water level

ANALYTICAL DATA

Inorganic Constituents

Constituents

Antimony, total recoverable Arsenic, total recoverable $<3.0$ Barium, total recoverable $\quad 87$

Cadmium, total recoverable $<1.0$

Chromium, total recoverable 1.0

Cobalt, total recoverable $\quad 0.55$

Copper, total recoverable $\quad 0.62$

Cyanide

Lead, total recoverable $\quad 0.55$

Mercury, total recoverable $\quad<0.20$

Nickel, total recoverable $\quad 1.5$

Nitrate as nitrogen

Nitrate-nitrite as nitrogen $\quad 30,000$

Selenium, total recoverable $<5.0$

Silver, total recoverable $<1.0$

Tin, total recoverable $<20$

Vanadium, total recoverable $<2.0$

Zinc, total recoverable

9.2

Organic Constituents

Benzene

Bis(2-ethylhexyl) phthalate

Dichioromethane

Tetrachloroethylene

Trichioroethylene

Trichloroftuoromethane
3Q98

$<1.0$

$<10$

5.4

$<1.0$

$<1.0$

$<1.0$

\begin{tabular}{|c|c|}
\hline Mod & Filt. \\
\hline $\mathrm{U} / 7$ & $<0.20$ \\
\hline $\begin{array}{l}\mathrm{U} / / \\
/ /\end{array}$ & $<3.0$ \\
\hline $\mathrm{U} / \mathrm{I}$ & $<1.0$ \\
\hline $\begin{array}{l}\mathrm{J} / \mathrm{E} / \\
/ /\end{array}$ & NDD \\
\hline "I & \\
\hline $\mathrm{U} / \mathrm{I}$ & $<10$ \\
\hline J/EV/ & NDD \\
\hline $\mathrm{U} / /$ & $<0.20$ \\
\hline "I & \\
\hline$N I$ & \\
\hline $\mathrm{U} / /$ & $<5.0$ \\
\hline $\mathrm{U} / /$ & $<1.0$ \\
\hline $\mathrm{U} / \mathrm{I}$ & $<2.0$ \\
\hline $\mathrm{U} / /$ & $<2.0$ \\
\hline
\end{tabular}

Mod

Fitt.

$<10$

$\mathrm{U} \mathrm{J} / \mathrm{O} / \mathrm{I}$

$\mathrm{U} / \mathrm{I}$

$\mathrm{J} / \mathrm{O} / 1$

$\mathrm{UJ} / \mathrm{O} / 1$

$\mathrm{U} J / \mathrm{O} / \mathrm{A}$

$<10$

NDD

$<1.0$

$<1.0$

$<1.0$

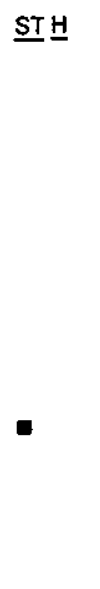

$\begin{array}{ll}\text { DFF } & \text { Lab } \\ 1 & \text { GE } \\ 1 & \text { GE } \\ 1 & \text { GE } \\ 1 & \text { GE } \\ 1 & \text { GE } \\ 1 & \text { GE } \\ 1 & \text { GE } \\ 1 & \text { GE } \\ 1 & \text { GE } \\ 1 & \text { GE } \\ 1 & \text { GE } \\ & \\ 25 & \text { GE } \\ 1 & \text { GE } \\ 1 & \text { GE } \\ 1 & \text { GE } \\ 1 & \text { GE } \\ 1 & \text { GE }\end{array}$

4098
214.6
9.8
200
22.7
35
6
$X N$
$214.7(12 / 22 / 98)$

Unit

ft ms!

$\mathrm{pH}$

$\mu S / c m$

${ }^{\circ} \mathrm{C}$

$\mathrm{mg} / \mathrm{L}$

NTU

ft ms! creen Zone

arnwell (IIB 1 ) 
WELL HSB136C (cont.)

Radioactive Constituents

\begin{tabular}{|c|c|c|c|c|c|c|c|c|c|c|c|c|c|}
\hline Constituents & 3Q98 & Mod & Filt. & ST $\underline{\text { H }}$ & DF & $\underline{\text { Lab }}$ & $4 Q 98$ & Mod & Filt. & $\underline{\text { ST }} \underline{H}$ & $\underline{\mathrm{DF}}$ & Lab & Unit \\
\hline Americium-241 & $<5.2 E-02$ & $\mathrm{UI} / /$ & $<0.1750$ & & 1 & GP & & & & & & & $\mathrm{pCi} / \mathrm{L}$ \\
\hline Beta dose & 38.23 & & & ш & & & & & & & & & $\mathrm{pCi} / \mathrm{L}$ \\
\hline Carbon-14 & $1.5 E+02$ & $/ /$ & & - & 1 & GP & & & & & & & $\mathrm{pCi} / \mathrm{L}$ \\
\hline Cobalt-60 & $<-1.3 E+00$ & $\mathrm{U} / /$ & $<2.9200$ & & 1 & GP & & & & & & & $\mathrm{pCi} / \mathrm{L}$ \\
\hline Curium-242 & $<8.4 \mathrm{E}-03$ & $\mathrm{UW} /$ & $<0.2070$ & & 1 & GP & & & & & & & $\mathrm{pCi} / \mathrm{L}$ \\
\hline Curium-243/244 & 1.7E-01 & $/ /$ & & & 1 & GP & & & & & & & $\mathrm{pCi} / \mathrm{L}$ \\
\hline Curium-245/246 & $<0.0 E+00$ & $\mathrm{U} M$ & $<0.1100$ & & 1 & GP & & & & & & & $\mathrm{pCi} / \mathrm{L}$ \\
\hline Gross alpha & $5.0 \mathrm{E}+00$ & $/ 1$ & & & 1 & GP & $<3.3 E+00$ & $\mathrm{U} / /$ & $<1.5 E+00$ & & 1 & GE & $\mathrm{pCi} / \mathrm{L}$ \\
\hline lodine-129 & $3.8 E+01$ & $/ 1$ & & & 1 & GP & & & & & & & $\mathrm{pCi} / \mathrm{L}$ \\
\hline Nickel-63 & $<-1.3 E+00$ & Ut & $<2.9200$ & & & & & & & & & & $\mathrm{pCi} / \mathrm{L}$ \\
\hline Nonvolatile beta & $7.3 E+01$ & $/ 1$ & & - & 1 & GP & 4.3E+01 & $\|$ & & & 1 & GE & $\mathrm{pCi} / \mathrm{L}$ \\
\hline Plutonium-238 & $<7.3 E-02$ & $\mathrm{UW} / /$ & $<0.2200$ & & 1 & GP & & & & & & & pCil \\
\hline Plutonium-239/240 & $<0.0 \mathrm{E}+00$ & $\mathrm{UW} / \mathrm{I}$ & $<0.1250$ & & 1 & GP & & & & & & & $\mathrm{pCi} / \mathrm{L}$ \\
\hline Radium-226 & $2.5 \mathrm{E}+00$ & $\|$ & & & 1 & GP & & & & & & & pCill \\
\hline Radium-228 & $<5.5 \mathrm{E}-01$ & $\mathrm{Ut} / I$ & $<1.1400$ & & 1 & GP & & & & & & & $\mathrm{pCi} L$ \\
\hline Strontium-90 & $<9.5 E-01$ & UIS/U & & & 1 & GP & & & & & & & pCill \\
\hline Technetium-99 & $1.4 E+02$ & /I & & - & 1 & GP & & & & & & & pCill \\
\hline Thorium-228 & $<7.6 \mathrm{E}-02$ & UIJ/C) & & & 1 & GP & & & & & & & $\mathrm{pCi} / \mathrm{L}$ \\
\hline Thorium-230 & $4.0 \mathrm{E}-01$ & $\mathrm{~J} / \mathrm{C} /$ & NDD & & 1 & GP & & & & & & & pCill \\
\hline Thorium-232 & $<0.0 \mathrm{E}+00$ & $\mathrm{U} \mathrm{H} / \mathrm{C} / \mathrm{C}$ & & & 1 & GP & & & & & & & pCill \\
\hline Sum of alphas & 5.7E-01 & & & & & & & & & & & & $\mathrm{pCi} / \mathrm{L}$ \\
\hline $\begin{array}{l}\text { Sum of betas } \\
\text { Total radium }\end{array}$ & $\begin{array}{l}3.2 E+02 \\
2.5 E+00\end{array}$ & · & & - & & & & & & & & & $\begin{array}{l}\mathrm{pCi} / \mathrm{L} \\
\mathrm{pCi} / \mathrm{L}\end{array}$ \\
\hline Tritium & $4.6 \mathrm{E}+03$ & $/ 1$ & & - & 1 & GP & $3.8 E+03$ & $\|$ & & $\mathbf{0}$ & 1 & GE & $\mathrm{pCl} / \mathrm{ml}$ \\
\hline Uranium-233/234 & $<1.7 E-02$ & $\mathrm{UU} / \mathrm{I}$ & $<0.3040$ & & 1 & GP & & & & & & & $\mathrm{pCi} / \mathrm{L}$ \\
\hline Uranium-235 & $<5.5 E-02$ & UIII & $<0.0830$ & & 1 & GP & & & & & & & $\mathrm{pCi} / \mathrm{L}$ \\
\hline Uranium-238 & $<-3.7 \mathrm{E}-02$ & UIII & $<0.2700$ & & 1 & GP & & & & & & & $\mathrm{pCi} / \mathrm{L}$ \\
\hline
\end{tabular}

Notes:

- exceeded holding time

- = exceeded groundwater protection or monitoring constituent standard (See Appendix A.) 


\section{WELL HSB136D}

\begin{tabular}{|c|c|}
\hline SRS Coord. & Lat/Longitude \\
\hline $\begin{array}{l}\text { N71906.0 } \\
\text { E55941.7 }\end{array}$ & $81.659171 \mathrm{~W}$ \\
\hline
\end{tabular}

07/13/98

Screen Zone Elevation

220.2-200.2 ft msl

FIELD DATA

Constituents

Water elevation

$\mathrm{pH}$

Sp. conductance

Water temperature

Alkalinity as $\mathrm{CaCO}_{3}$

Turbidity

Sampling code

Synchronous water level
SAMPLE DATE

Volumes purged
3Q988

219.8

3.8

220

21.0

10

$219.1(09 / 17 / 98)$
Top of Casing

$228 \mathrm{ft} \mathrm{msl}$

$10 / 14 / 98$

$4 Q 98$
218.8
4.2
200
19.8
0
0
3.7
$N$
$218.3(12 / 22 / 98)$

ANALYTICAL DATA

Inorganic Constituents

\begin{tabular}{|c|c|c|c|c|c|c|c|c|c|c|c|c|c|}
\hline Constituents & $\underline{3 Q 98}$ & Mod & $\underline{\text { Filt. }}$ & ST브 & DF & $\underline{\text { Lab }}$ & $4 Q 98$ & Mod & Filt. & $\underline{\text { ST }} \underline{H}$ & $\underline{\text { DF }}$ & $\underline{\mathrm{Lab}}$ & Unit \\
\hline Antimony, total recoverable & $<0.20$ & $\mathrm{U} / 1$ & $<0.20$ & & 1 & GE & & & & & & & $\mu g / L$ \\
\hline Arsenic, total recoverable & $<3.0$ & $\mathrm{U} / /$ & $<3.0$ & & 1 & GE & & & & & & & $\mu g / L$ \\
\hline Barium, total recoverable & 61 & $/ 1$ & & & 1 & GE & & & & & & & $\mu g / L$ \\
\hline Cadmium, total recoverable & 0.39 & $\mathrm{~J} / \mathrm{E} /$ & NDD & & 1 & GE & & & & & & & $\mu g / L$ \\
\hline Chromium, total recoverable & $<3.0$ & $\mathrm{U} / 1$ & $<3.0$ & & 1 & GE & & & & & & & $\mu g / L$ \\
\hline Cobalt, total recoverable & 5.0 & $\|$ & & $\mathbf{\square}$ & 1 & GE & & & & & & & $\mu g / L$ \\
\hline Copper, total recoverable & 2.7 & $\|$ & & & 1 & GE & & & & & & & $\mu g / L$ \\
\hline Cyanide & $<10$ & $\mathrm{U} / 1$ & $<10$ & & 1 & GE & & & & & & & $\mu g / L$ \\
\hline Lead, total recoverable & 0.37 & $J / E I$ & NDD & & 1 & GE & & & & & & & $\mu g / L$ \\
\hline Mercury, total recoverable & 0.29 & $\|$ & & & 1 & GE & 0.12 & $\mathrm{~J} / / /$ & NDD & & 1 & GE & $\mu g / L$ \\
\hline $\begin{array}{l}\text { Nickel, total recoverable } \\
\text { Nitrate as nitrogen }\end{array}$ & 7.5 & $\| /$ & & & 1 & GE & & & & & & & $\mu \mathrm{g} / \mathrm{L}$ \\
\hline Nitrate-nitrite as nitrogen & 28,000 & $N /$ & & $\mathbf{0}$ & 25 & GE & 30,000 & $/ 1$ & & $\mathbf{D}$ & 25 & GE & $\mu g / L$ \\
\hline Selenium, total recoverable & $<0.42$ & $\mathrm{UN} /$ & $<5.0$ & & 1 & GE & & & & & & & $\mu g / L$ \\
\hline Silver, total recoverable & $<1.0$ & $\mathrm{U} / /$ & $<1.0$ & & 1 & GE & & & & & & & $\mathrm{\mu g} / \mathrm{L}$ \\
\hline Tin, total recoverable & 0.20 & $\mathrm{~J} / \mathrm{E} /$ & NDD & & 1 & GE & & & & & & & $\mu g / L$ \\
\hline Vanadium, total recoverable & $<2.0$ & $\mathrm{U} / /$ & $<2.0$ & & 1 & GE & & & & & & & $\mu g / L$ \\
\hline Zinc, total recoverable & 36 & $/ /$ & & & 1 & GE & & & & & & & $\mu g / L$ \\
\hline \multicolumn{14}{|l|}{ Organic Constituents } \\
\hline Constituents & $\underline{3098}$ & Mod & Filt. & ST브 & $\underline{D F}$ & $\underline{\text { Lab }}$ & 4Q98 & Mod & Filt. & $\underline{\text { ST }} \underline{H}$ & $\underline{\mathrm{DF}}$ & $\underline{\text { Lab }}$ & $\underline{\text { Unit }}$ \\
\hline Benzene & $<1.0$ & $\mathrm{UJ} / \mathrm{O} / 1$ & $<1.0$ & & $\uparrow$ & GE & & & & & & & $\mu g / L$ \\
\hline Bis (2-ethylhexyl) phthalate & $<9.8$ & $\mathrm{UJ} / \mathrm{Q} /$ & $<9.8$ & $\bullet$ & 1 & GE & & & & & & & $\mu g / L$ \\
\hline Dichloromethane & $<2.2$ & UJNO8/1 & $<1.0$ & & 1 & GE & & & & & & & $\mu g / L$ \\
\hline Tetrachloroethylene & $<1.0$ & $\mathrm{UJ} / \mathrm{O} / 1$ & $<1,0$ & & 1 & GE & & & & & & & $\mu g / L$ \\
\hline Trichloroethylene & $<1.0$ & $\mathrm{UJ} / \mathrm{O} / 1$ & $<1.0$ & & 1 & GE & & & & & & & $\mu g / L$ \\
\hline Trichlorofluoromethane & $<1.0$ & $\mathrm{UJ} / \mathrm{O} / \mathrm{s}$ & $<1.0$ & & 1 & GE & & & & & & & \\
\hline
\end{tabular}

$\begin{array}{ll}\text { Casing } & \text { Pump } \\ \text { 4" PVC } & V\end{array}$

Screen Zone

Water Table (IIB2) $\underline{\text { Unit }}$

tims!

$\mathrm{pH}$

$\mu S / \mathrm{cm}$

${ }^{\circ} \mathrm{C}$

$\mathrm{mg} / \mathrm{L}$

well vol

ft ms! 
WELL HSB136D (cont.)

Radioactive Constituents

\section{Constituents}

Americium-241

Beta dose

Carbon-14

Cobalt-60

Curium-242

Curium-243/244

Curium-245/246

Gross alpha

lodine-129

Nickel-63

Nonvolatile beta

Plutonium-238

Plutonium-239/240

Radium-226

Radium-228

Strontium-90

Technetium-99

Thorium-228

Thorium-230

Thorium-232

Sum of alphas

Sum of betas

Total radium

Tritium

Uranium-233/234

Uraniurn-235

Uranium-238

\begin{tabular}{|c|c|c|c|c|c|c|c|}
\hline 3098 & Mod & Filt. & $\underline{\text { ST }} \underline{H}$ & $\underline{\mathrm{DF}}$ & $\underline{\text { Lab }}$ & $4 Q 98$ & Mod \\
\hline $\begin{array}{l}<3.2 \mathrm{E}-02 \\
110.28\end{array}$ & $\mathrm{UI} / \mathrm{I}$ & $<0.1520$ & - & 1 & GP & & \\
\hline $1.5 \mathrm{E}+02$ & "l & & m & 1 & GP & & \\
\hline $3.0 \mathrm{E}+01$ & "I & & & 1 & GP & & \\
\hline$<0.0 \mathrm{E}+00$ & $\mathrm{U} 3 / /$ & $<0.1690$ & & 1 & GP & & \\
\hline$<3.9 \mathrm{E}-02$ & $\mathrm{U}] / /$ & $<0.2680$ & & 1 & GP & & \\
\hline$<0.0 \mathrm{E}+00$ & $\mathrm{U} \mid / /$ & $<0.1520$ & & 1 & GP & & \\
\hline $4.1 E+01$ & /1 & & - & 1 & GP & $3.6 \mathrm{E}+01$ & "I \\
\hline $3.7 \mathrm{E}+01$ & "I & & & 1 & GP & & \\
\hline $3.0 E+01$ & II & & & & & & \\
\hline $1.1 E+03$ & "I & & $=$ & 1 & GP & $1.2 \mathrm{E}+03$ & /l \\
\hline$<-1.1 \mathrm{E}-02$ & $U 1 / I$ & $<0.2330$ & & 1 & GP & & \\
\hline$<-3.2 \mathrm{E}-02$ & $\mathrm{UI} / \mathrm{I}$ & $<0.3070$ & & 1 & GP & & \\
\hline $2.2 \mathrm{E}+01$ & "I & & - & 1 & GP & & \\
\hline $4.8 \mathrm{E}+00$ & $\|$ & & & 1 & GP & & \\
\hline $5.7 \mathrm{E}+02$ & "I & & ㅁ & 1 & GP & & \\
\hline $8.2 E+01$ & "I & & च & 1 & GP & & \\
\hline$<5.5 \mathrm{E}-01$ & U:// & $<0.6830$ & & 1 & GP & & \\
\hline$<1.8 \mathrm{E}-01$ & $\mathrm{U} \mid 1 / \prime$ & $<0.2630$ & & 1 & GP & & \\
\hline$<0.0 \mathrm{E}+00$ & $\mathrm{UI} / I$ & $<0.2630$ & & 1 & $\mathrm{GP}$ & & \\
\hline $2.4 \mathrm{E}+00$ & & & & & & & \\
\hline $9.0 \mathrm{E}+02$ & & & $\bullet$ & & & & \\
\hline $2.7 \mathrm{E}+01$ & & & $=$ & & & & $u$ \\
\hline 4.3E+03 & $" 1$ & & 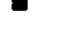 & $?$ & GP & $5.3 E+03$ & $n$ \\
\hline $1.5 E+00$ & $\mathrm{U} \mid /$ & $<0.3380$ & & $i$ & GP & & \\
\hline $\begin{array}{l}<8.3 E-02 \\
9.1 E-01\end{array}$ & $\|$ & 0.800 & & $i$ & GP & & \\
\hline $9.1 \mathrm{E}-01$ & & & & & & & \\
\hline
\end{tabular}

Filt.

$\underline{\text { ST }} \underline{H}$ DF Lab Unit

$\mathrm{pCi} / \mathrm{L}$

pCill

pCill

pCill

pCilL

$\mathrm{PCilL}$

$\mathrm{pCi} / \mathrm{L}$

- 1 GE $\mathrm{pCi} / \mathrm{L}$

$\mathrm{PCi} / \mathrm{L}$

- 1 GE $\mathrm{pCi} / \mathrm{L}$

pCi/L

pCill

$\mathrm{pCi} / \mathrm{L}$

pCill

$\mathrm{pCi} / \mathrm{L}$

$\mathrm{pCi} / \mathrm{L}$

$\mathrm{pCi} / \mathrm{L}$

$\mathrm{pCi} / \mathrm{L}$

pCi/L

pCi/l

$\mathrm{pCi} / \mathrm{L}$

pCilh

- 1 GE pCi/mL

$\mathrm{pCi} / \mathrm{L}$

pCilL

pCi/L

Notes:

- = exceeded holding time

= exceeded grounowater protection or monitoring constituent standard (See Appendix A.) 
WELL HSB137C

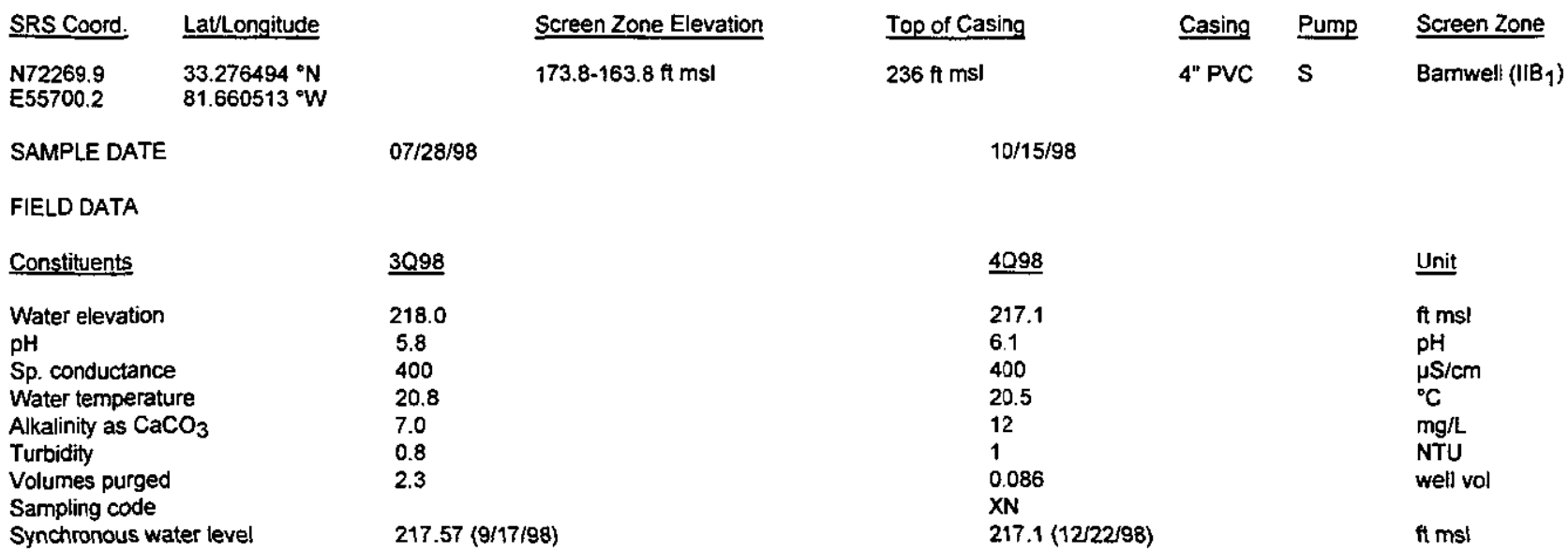

ANALYTICAL DATA

Inorganic Constituents

\begin{tabular}{|c|c|c|c|c|c|c|c|c|c|c|c|c|c|}
\hline Constituents & 3098 & Mod & Filt. & $\underline{\mathrm{ST}} \underline{\mathrm{H}}$ & $\underline{\mathrm{DF}}$ & Lab & $\underline{4 Q 98}$ & Mod & Filt. & $\underline{\text { ST }} \underline{H}$ & $\underline{\mathrm{DF}}$ & $\underline{\text { Lab }}$ & Unit \\
\hline Antimony, total recoverable & $<0.20$ & $\mathrm{U} / /$ & $<0.20$ & & 1 & GE & & & & & & & $\mu g / L$ \\
\hline Arsenic, total recoverable & $<3.0$ & $U / /$ & $<3.0$ & & 1 & GE & & & & & & & $\mu g / L$ \\
\hline Barium, total recoverable & 40 & $/ 1$ & & & 1 & GE & & & & & & & $\mu \mathrm{g} / \mathrm{L}$ \\
\hline Cadmium, total recoverable & 0.53 & $\mathrm{~J} / \mathrm{E} J$ & NDD & & 1 & GE & & & & & & & $\mu g / L$ \\
\hline Chromium, total recoverable & 1.2 & $\mathrm{~J} / \mathrm{E}$ & NDD & & 1 & GE & & & & & & & $\mu \mathrm{g} / \mathrm{L}$ \\
\hline Cobalt, total recoverable & 0.70 & "I & & & 1 & GE & & & & & & & $\mu g / L$ \\
\hline Copper, total recoverable & 1.1 & "l & & & 1 & GE & & & & & & & $\mu \mathrm{g} / \mathrm{L}$ \\
\hline Cyanide & $<10$ & $\mathrm{U} / /$ & $<10$ & & 1 & GE & & & & & & & $\mu g / L$ \\
\hline Lead, total recoverable & 0.50 & J/EVI & NOD & & 1 & GE & & & & & & & $\mu g / \mathrm{L}$ \\
\hline Mercury, total recoverable & 0.30 & /I & & & 1 & GE & $<0.20$ & $\mathrm{U} / /$ & $<0.20$ & & 1 & $\mathrm{GE}$ & $\mu g / L$ \\
\hline $\begin{array}{l}\text { Nickel, total recoverable } \\
\text { Nitrate as nitrogen }\end{array}$ & 3.5 & "I & & & 1 & GE & & & & & & & $\mu g / L$ \\
\hline Nitrate-nitrite as nitrogen & 42,000 & NI & & m & 25 & $\mathrm{GE}$ & 44,000 & $/ 1$ & & - & 25 & $\mathrm{GE}$ & $\mu g / L$ \\
\hline Selenium, total recoverable & $<5.0$ & $\mathrm{u} / I$ & $<5.0$ & & 1 & GE & & & & & & & $\mu g / L$ \\
\hline Silver, total recoverable & $<1.0$ & $\mathrm{U} / \mathrm{I}$ & $<1.0$ & & 1 & GE & & & & & & & $\mu g / \mathrm{L}$ \\
\hline Tin, total recoverable & $<2.0$ & $\mathrm{U} / I$ & $<2.0$ & & 1 & GE & & & & & & & $\mu g / L$ \\
\hline Vanadium, total recoverable & $<2.0$ & $\mathrm{U} / /$ & $<2.0$ & & 1 & $\mathrm{GE}$ & & & & & & & $\mu g / L$ \\
\hline Zinc, total recoverable & 24 & /I & & & 1 & $\mathrm{GE}$ & & & & & & & $\mu g / L$ \\
\hline
\end{tabular}

Organic Constituemts

\begin{tabular}{|c|c|c|c|c|c|c|c|c|c|c|}
\hline Constituents & $\underline{3 Q 98}$ & Mod & Filt. & $\underline{\text { ST}} \underline{H}$ & $\underline{\mathrm{DF}}$ & Lab 4098 & Mod & Filt. & ST $\underline{H}$ DF Lab & Unit \\
\hline Benzene & $<1.0$ & UJ/O/1 & $<1.0$ & & 1 & GE & & & & $\mu g / L$ \\
\hline Bis (2-ethylhexyl) phthalate & $<10$ & $\mathrm{U} / \mathrm{I}$ & $<10$ & & 1 & GE & & & & $\mu g / L$ \\
\hline Dichloromethane & 5.8 & $\mathrm{~J} / \mathrm{O} / 1$ & NDD & & 1 & $\mathrm{GE}$ & & & & $\mu g / \mathrm{L}$ \\
\hline Tetrachloroethylene & $<1.0$ & UJ/O/I & $<1.0$ & & 1 & GE & & & & $\mu g / L$ \\
\hline Trichloroethylene & $<1.0$ & $\mathrm{UJ} / \mathrm{O} / 1$ & $<1.0$ & & 1 & GE & & & & $\mu g / L$ \\
\hline Trichloroffuoromethane & $<1.0$ & $\mathrm{UJ} / \mathrm{O} / 1$ & $<1.0$ & & 1 & $\mathrm{GE}$ & & & & $g / L$ \\
\hline
\end{tabular}

Notes:

- = exceeded hoiding time

v = exceeded groundwater protection or monitoring constituent standard (See Appendix A.) 
WELL HSB137C (cont.)

Radioactive Constituents

Constituents

Americium-241

Beta dose

Carbon-14

Cobalt-60

Curium-242

Curium-243/244

Curium-245/246

Gross atpha

lodine-129

Nickel-63

Nonvolatile beta

Plutonium-238

Plutonium-239/240

Radium-226

Radium-228

Strontium-90

Technetium-99

Thorium-228

Thorium-230

Thorium-232

Sum of alphas

Sum of betas

Total radium

Tritium

Uranium-233/234

Uranium-235

Uranium-238

\begin{tabular}{|c|c|c|c|c|c|c|c|}
\hline 3098 & Mod & Filt. & $\underline{\text { ST }} \underline{H}$ & $\underline{D F}$ & $\underline{\text { Lab }}$ & 4Q98 & Mod \\
\hline$<-1.1 \mathrm{E}-01$ & $\mathrm{UI} / /$ & $<0.3680$ & & 1 & GP & & \\
\hline 9.43 & & & a & & & & \\
\hline $1.3 E+02$ & /I & & n & 1 & GP & & \\
\hline$<-6.6 \mathrm{E}-01$ & $\mathrm{UI} / \mathrm{H}$ & $<3.0300$ & & 1 & GP & & \\
\hline$<-4.6 \mathrm{E}-02$ & $\mathrm{U} \mid / /$ & $<0.3100$ & & 1 & GP & & \\
\hline$<1.8 \mathrm{E}-01$ & $\mathrm{UH} / \mathrm{I}$ & $<0.2730$ & & 1 & GP & & \\
\hline$<1.1 \mathrm{E}-01$ & $\mathrm{U} \mathrm{U} / \mathrm{I}$ & $<0.1080$ & & $i$ & GP & & \\
\hline $7.4 \mathrm{E}+00$ & $\|$ & & & 1 & $G P$ & $<4.4 \mathrm{E}+00$ & $u / \prime$ \\
\hline $9.2 E+00$ & /I & & & 1 & $G P$ & & \\
\hline$<-2,4 E-01$ & $\mathrm{UI} / /$ & $<17.9000$ & & 1 & GP & & \\
\hline $7.3 E+01$ & $\|$ & & $=$ & 1 & $G P$ & $3.4 \mathrm{E}+01$ & 11 \\
\hline$<1.4 \mathrm{E}-02$ & $\mathrm{UI} / /$ & $<0.3800$ & & 1 & $\mathrm{GP}$ & & \\
\hline$<3.4 \mathrm{E}-02$ & $\mathrm{U} 1 / /$ & $<0.3400$ & & 1 & $G P$ & & \\
\hline $1.8 E+00$ & 11 & & & 1 & GP & & \\
\hline$<1.8 \mathrm{E}-01$ & $\mathrm{UI} / \mathrm{I}$ & $<1.1600$ & & 1 & $G P$ & & \\
\hline$<2.4 \mathrm{E}-01$ & UIJ/L & & & 1 & GP & & \\
\hline $1.5 \mathrm{E}+02$ & $\|$ & & 口 & 1 & GP & & \\
\hline$<1,1 \mathrm{E}-01$ & $\mathrm{U} \mid \mathrm{J} / \mathrm{C} /$ & & & 1 & GP & & \\
\hline $3.8 \mathrm{E}-01$ & $\mathrm{~J} / \mathrm{C} /$ & NDD & & $\dagger$ & GP & & \\
\hline$<0.0 E+00$ & $\mathrm{UIJ} / \mathrm{Cl} /$ & & & $i$ & $G P$ & & \\
\hline 3.8E-01 & & & & & & & \\
\hline $\begin{array}{l}2.9 \mathrm{E}+02 \\
18 \mathrm{E}+00\end{array}$ & & & - & & & & \\
\hline $\begin{array}{l}1.8 \mathrm{E}+00 \\
7.9 \mathrm{E}+03\end{array}$ & $/ 1$ & & $\mathbf{v}$ & 1 & & $85 \mathrm{~F}+03$ & $\|$ \\
\hline $\begin{array}{l}7.9 E+03 \\
<1.5 E-01\end{array}$ & $\mathrm{U}: / /$ & $<0.1950$ & & 1 & $G P$ & $8.5 \mathrm{t}+03$ & $n$ \\
\hline$<-4.7 \mathrm{E}-02$ & $\mathrm{U} \mathbf{1} / /$ & $<0.2540$ & & 1 & GP & & \\
\hline$<3.8 \mathrm{E}-02$ & $\mathrm{U} \mid / /$ & $<0.2960$ & & $i$ & GP & & \\
\hline
\end{tabular}

Filt.

$\underline{\text { ST }}$ H $\underline{\text { DF Lab }} \underline{\text { Unit }}$

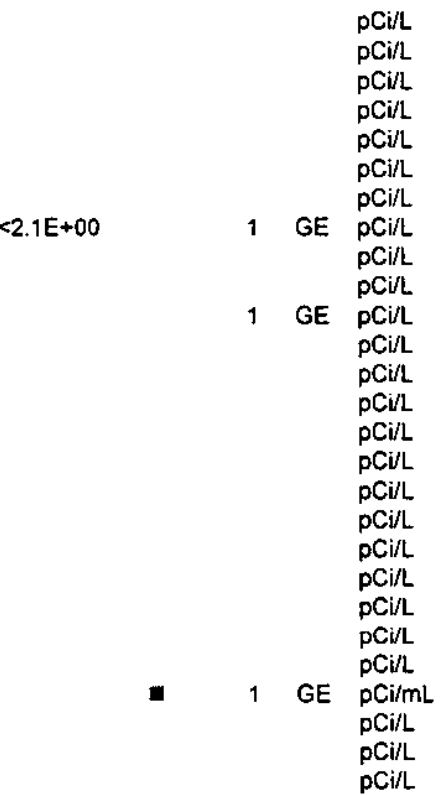

Notes:

$\bullet=$ exceeded holding time

- =xceeded groundwater protection or monitoring constituent standard (See Appendix A.) 


\section{WELL HSB137D}

\begin{tabular}{|c|c|}
\hline SRS Coord. & Lat/Longitude \\
\hline $\begin{array}{l}\text { N72278.9 } \\
\text { E55696.1 }\end{array}$ & $\begin{array}{l}33.276508 \\
81.660542\end{array}$ \\
\hline
\end{tabular}

SAMPLE DATE

$07 / 13 / 98$

Screen Zone Elevation

225.3-205.3 ft ms!

FIELD DATA

Constituents

Water elevation

$\mathrm{pH}$

Sp. conductance

Water temperature

Alkalinity as $\mathrm{CaCO}_{3}$

Turbidity

Volumes purged

Sampling code

Synchronous water level

ANALYTICAL DATA

Inorganic Constituents

Constituents

Antimony, total recoverable

Arsenic, total recoverable

Barium, total recoverable

Cadmium, total recoverable

Chromium, total recoverable

Cobalt, total recoverable

Copper, total recoverable

Cyanide

Lead, total recoverable

Mercury, total recoverable

Nickel, total recoverable

Nitrate as nitrogen

Nitrate-nitrite as nitrogen

Selenium, total recoverable

Silver, total recoverable

Tin, total recoverable

Vanadium, total recoverable

Zinc, total recoverable

Organic Constituents

Constituents

Benzene

Bis(2-ethylhexyl) phthalate

Dichloromethane

Tetrachioroethylene

Trichloroethylene

Trichlorofluoromethane
3Q98

220.8

4.5

150

19.1

0

1.

4.7

$220.3(09 / 17 / 98)$
$14,000 \quad N$
Top of Casing

$236.6 \mathrm{ft} \mathrm{msl}$

$10 / 15 / 98$
Casing Pump Screen Zone

4" PVC S Water Table (IIB2)
4Q98

219.6

5.1

150

18.9

0

3.3

$\mathrm{N}$

$218.9(12 / 22 / 98)$
Unit

$\mathrm{ft} \mathrm{ms}$

$\mathrm{pH}$

$\mu S / \mathrm{cm}$

${ }^{\circ} \mathrm{C}$

$\mathrm{mg} / \mathrm{L}$

NTU

well vol

ft msl

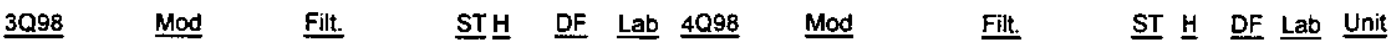

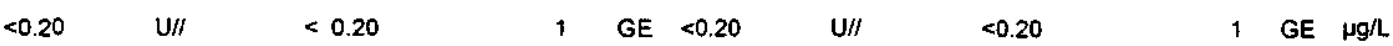

- 5 GE $14,000 \quad$ II

- $10 \mathrm{GE} \mu \mathrm{g} / \mathrm{L}$

ST브 DF Lab $\underline{4 Q 98} \quad \underline{\text { Mod }}$

Filt.

$\underline{\text { ST }} \underline{H} \underline{\text { DF }} \underline{\text { Lab }} \underline{\text { Unit }}$

Notes:

- exceeded holding time

- exceeded groundwater protection or monitoring constituent standard (See Appendix A.) 


\section{WELL HSB137D (cont.)}

Radioactive Constituents

Constituents

Americium-241

Carbon-14

Cobalt-60

Curium-242

Curium-243/244

Curium-245/246

Gross alpha

lodine-129

Nickel-63

Nonvolatile beta

Plutonium-238

Plutonium-239/240

Radium-226

Radium-228

Strontium-90

Technetium-99

Thorium-228

Thorium-230

Thorium-232

Sum of alphas

Sum of betas

Tritium

Uranium-233/234

Uranium-235

Uranium-238
$2.5 \mathrm{E}+00 \quad / /$

4.4E+01 //

$2.4 \mathrm{E}+03 \quad / 1$
STH DF Lab $\underline{4098}$ Mod

1

GP $<3.8 \mathrm{E}+00 \quad \mathrm{U} / \prime$

1 GP $4.6 \mathrm{E}+01 \quad h$

$<1,4 \mathrm{E}+00$

1 GE PCi/L

ST $\underline{H} \underline{\text { DF Lab Unit }}$

Filk

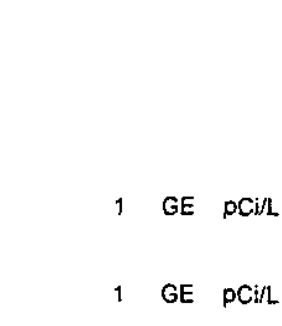

1 GP $2.5 \mathrm{E}+03 \quad / /$

- 1 GE $\mathrm{PCi} / \mathrm{mL}$

Notes:

- = exceeded holding time

I = exceeded groundwater protection or monitoring constituent standard (See Appendix A.) 


\section{WELL HSB138D}

\begin{tabular}{|c|c|}
\hline SRS Coord. & Lat/Longitude \\
\hline $\begin{array}{l}N 73160 \\
\text { E55260 }\end{array}$ & $\begin{array}{l}33.277746^{\circ} \mathrm{N} \\
81.663400^{\circ} \mathrm{W}\end{array}$ \\
\hline
\end{tabular}

SAMPLE DATE

Water temperature

Alkalinity as $\mathrm{CaCO}_{3}$

Turbidity

Volumes purged

Sampling code

Synchronous water level

ANALYTICAL DATA

Inorganic Constituents

Constituents

Antimony, total recoverable

Arsenic, total recoverable

Barium, total recoverable

Cadmium, total recoverable

Chromium, total recoverable

Cobalt, total recoverable

Copper, totai recoverable

Cyanide

Lead, total recoverable

Mercury, total recoverable

Nickel, total recoverable

Nitrate as nitrogen

Nitrate-nitrite as nitrogen

Selenium, total recoverable

Silver, total recoverable

Tin, total recoverable

Vanadium, total recoverable

Zinc, total recoverable

Organic Constituemts

Constituents

Benzene

Bis(2-ethylhexyl) phthalate

Dichloromethane

Tetrachloroethylene

Trichloroethylene

Trichlorofluoromethane

Mod

Filt.

STI

Mod

Filt.
Screen Zone Elevation

228.1-208.1 ft msl

$07 / 21 / 98$

3098

224.7

4.9

41

19.5

Top of Casing

$252.4 \mathrm{ft} \mathrm{ms \}}$

$10 / 08 / 98$

4Q98

223.

4.7

26

0

6

$2.9-0.10$

$223.8(09 / 17 / 98)$
Casing Pump

4" PVC S
Screen Zone

Water Table (UB2)

$\mathrm{XN}$
$221.5(12 / 22 / 98)$

Unit

ft msl

$\mathrm{pH}$

$\mu \mathrm{S} / \mathrm{cm}$

$\mathrm{mg} / \mathrm{L}$

NTU

well vol

ft msl

DF $\underline{\text { Lab }} \underline{4098}$

Mod

Filt.

$\underline{\text { ST }} \underline{H} \underline{\text { DF }} \underline{\text { Lab }} \underline{\text { Unit }}$

\begin{tabular}{|c|c|c|c|c|c|c|c|c|c|}
\hline$<0.20$ & $\mathrm{U} / /$ & $<0.20$ & 1 & $\mathrm{GE}$ & $<0.20$ & U/I & $<0.20$ & 1 & GE \\
\hline 1,500 & $N /$ & & 1 & GE & 420 & $/ /$ & & 1 & GE \\
\hline
\end{tabular}

ST브

DF L

4098

Mod

Filt.

ST $\underline{\text { H }} \underline{\text { DF Lab Unit }}$

Notes:

= exceeded holding time

$\mathbf{r}=$ exceeded groundwater protection or monitoring constituent standard (See Appendix A.) 


\section{WELL HSB138D (cont.)}

Radioactive Constituents

Constituents

Americium-241

Carbon-14

Cobalt-60

Curium-242

Curium-243/244

Curium-245/246

Gross alpha

lodine-129

Nickel-63

Nonvolatile beta

Plutonium-238

Plutonium-239/240

Radium-226

Radium-228

Strontium-90

Technetium-99

Thorium-228

Thorium-230

Thorium-232

Sum of alphas

Sum of betas

Tritium

Uranium-233/234

Uranium-235

Uranium-238 $\underline{3 Q 98} \quad \underline{M a d} \quad \underline{F i l t} . \quad \underline{S T} \underline{H} \quad \underline{D F} \underline{L a b} \underline{4 Q 98} \quad \underline{M o d} \quad \underline{\text { Filt. }} \quad \underline{\text { ST }} \underline{H} \quad \underline{\text { DF }} \underline{\underline{\text { Lab }}} \underline{\text { Unit }}$

$<4.5 E-01$

$<0.5980$

1

GP $\quad<7.1 E-01$

JU/L/I

$<$ NDD

$1 \mathrm{GE} \mathrm{pCi} / \mathrm{L}$

$<6.4 \mathrm{E}-01 \quad$ UI/

$<1.3600$

$1 \quad \mathrm{GP}<1.1 \mathrm{E}+00 \quad \mathrm{JU} / \mathrm{L} /$

<NDD

1 GE $\mathrm{pCi} / \mathrm{L}$
$2.4 \mathrm{E}+01$
GP 2.0E+01 //

1 GE PCímL

Notes:

- = exceeded holding time

- = exceeded groundwater protection or monitoring constituent standard (See Appendix A.) 


\section{WELL HSB139A}

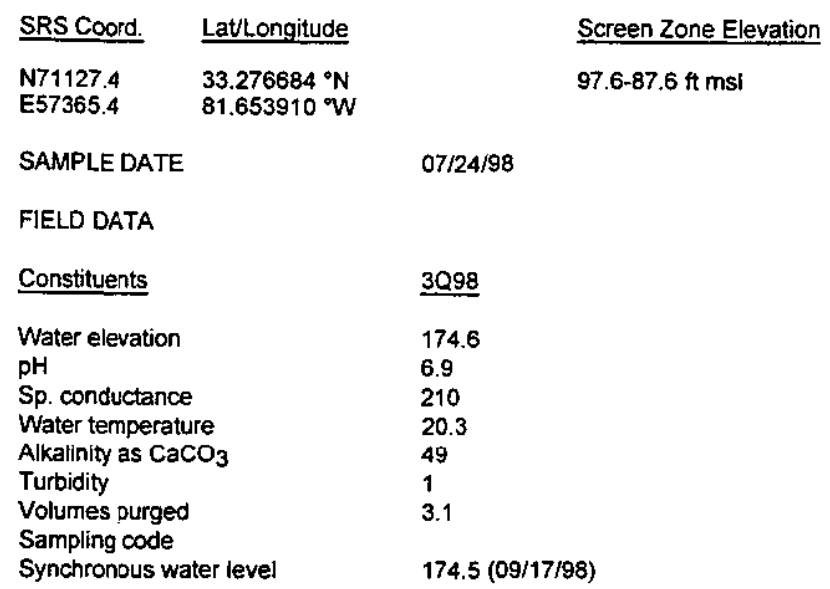

$\begin{array}{llll}\text { Top of Casing } & \text { Casing } & \text { Pump } & \text { Screen Zone } \\ 233.7 \mathrm{ft} \mathrm{ms} & \text { 4" PVC } & \text { S } & \text { U. Congaree (IIA) }\end{array}$

$10 / 12 / 98$

ANALYTICAL DATA

Inorganic Constituents

Constituents

Antimony. total recoverable Arsenic, total recoverable Barium, total recoverable

Cadmium, total recoverable

Chromium, total recoverable

Cobalt, total recoverable

Copper, total recoverable

Cyanide

Lead, total recoverable

Mercury, total recoverable

Nickel, total recoverable

Nitrate as nitrogen

Nitrate-nitrite as nitrogen

Selenium, total recoverable

Silver, total recoverable

Tin, total recoverable

Vanadium, total recoverable

Zinc, total recoverable

Organic Constituents

Constituents

$\underline{3 Q 98}$

Benzene

Bis(2-ethylhexyl) phthalate

Dichloromethane

Tetrachloroethylene

Trichioroethylene

Trichiorofuoromethane

$3 \mathrm{Q}$

64
U/I

$<0.20$

1 GE $<0.20$

$\mathrm{U} / \mathrm{I}$

SIVY/2

NDD

$\mathrm{J} / \mathrm{I} /$

NDD

Filt.

$\underline{\text { ST }} \underline{H} \quad \underline{\text { DF Lab Unit }}$

ST $\underline{H}$ DF Lab Unit

1 GE $\mu g / L$

1 GE $\mu g / L$

ST브 DF Lab $\quad \underline{4098} \quad$ Mot
Unit

$\mathrm{ftms}$

$\mathrm{pH}$

$\mu \mathrm{S} / \mathrm{cm}$

${ }^{\circ} \mathrm{C}$

mg/L

well vol

ft msl 


\section{WELL HSB139A (cont.)}

Radioactive Constituents

Constituents

Americium-241

Carbon-14

Cobalt-60

Curium-242

Curium-243/244

Curium-245/246

Gross alpha

lodine-129

Nickel-63

Nonvolatile beta

Plutonium-238

Plutonium-239/240

Radium-226

Radium-228

Strontium-90

Technetium-99

Thorium-228

Thorium-230

Thorium-232

Sum of alphas

Sum of betas

Tritium

Uranium-233/234

Uranium-235

Uranium-238
3098

$\underline{\text { Mod }}$

Filt.

STH

Mod

Filt.

$\underline{\text { ST }} \underline{H}$ DF Lab Unit

$<3.2 \mathrm{E}-01$

UIII

$<1.3900$

1

TM <2.9E-01 U $/ /$

$<6.7 \mathrm{E}-01$

1 GP $<8.6 E-01$

$\mathrm{u} / 1$

$<1.2 E+00$

1 GE $p \mathrm{Ci} /$

$1.5 \mathrm{E}+00 \quad / /$

$<5,1 \mathrm{E}-02$

UIII

$<6.0 \mathrm{E}-01$

1 GP $<4.8 E-01$

$\mathrm{U} / 1$

$<6.5 E-01$

1 GE $\mathrm{pCi} / \mathrm{mL}$

1 GE $\mathrm{pCi} / \mathrm{L}$

Notes:

- exceeded holding time

- =xceeded groundwater protection or monitoring constituent standard (See Appendix A.) 


\section{WELL HSB139C}

$\begin{array}{ll}\text { SRS Coord. } & \text { Lat/Longitude } \\ \text { N71129.8 } & 33.276704^{\circ} \mathrm{N} \\ \text { E57374.5 } & 81.653890^{\circ}\end{array}$

SAMPLE DATE

3098

214.6

4.5

330

18.8

Sp. conductance

Water temperature

Alkalinity as $\mathrm{CaCO}_{3}$

Turbidity

Volumes purged

Sampling code

Synchronous water level
Screen Zone Elevation

158.5-148.5 ft ms

$7 / 13 / 98$
Top of Casing

$233.8 \mathrm{ft} \mathrm{msi}$

$11 / 05 / 98$

4098

214.0

5.1

330

18.3

4

0.023

$\mathrm{XN}$

$213.3(12 / 22 / 98)$

Casing Pump

4" PVC S
Screen Zone

Bamwell (IIB 1 )

ANALYTICAL DATA

Inorganic Constituemts

\section{Constituents}

Antimony, total recoverable

Arsenic, total recoverable

Barium, total recoverable

Cadmium, total recoverable 0.69

Chromium, total recoverable $\mathbf{5 . 0}$

Cobalt, total recoverable $\quad 7.3$

Copper, total recoverable $\quad 2.9$

Cyanide

Lead, total recoverable $\quad 1.1$

Mercury, total recoverable $\quad 0.97$

Nickel, total recoverabl

Nitrate as nitrogen

Nitrate-nitrite as nitrogen

Selenium, total recoverable

Silver, total recoverable

Tin, total recoverable

Zinc, total recoverable

120

Organic Constituents

Constituents

3098

Mod

J/E

Filt.

NDD

$\mathrm{U} / /<15.0$

J/E/ NDD

$\mathrm{J} / \mathrm{E} /$ NDD

II

$\mathrm{J} / \mathrm{E}$

/I

/I

NI

J/EV/

U/I

$\mathrm{J} / \mathrm{E}$

U/I

Mod

NDD

NDD

NDD

$<5.0$

NDD

$<10$

Filt.
STI브

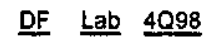

Mod

5 GE

GE

GE

GE

Mod

Filt.

Unit

ft $\mathrm{msl}$

pH

$\mu \mathrm{S} / \mathrm{cm}$

${ }^{\circ} \mathrm{C}$

$\mathrm{mg} / \mathrm{L}$

NTU

well vol

ft msl

Benzene

Bis(2-ethylhexyl) phthalate

Dichloromethane

Tetrachloroethylene

Trichtoroethylene

Trichlorofluoromethane

Notes:

= exceeded holding time

a = exceeded groundwater protection or monitoring constituent standard (See Appendix A.) 


\section{WELL HSB139C (cont.)}

Radioactive Constituents

Constituents

Americium-241

Carbon-14

Cobalt-60

Curium-242

Curium-243/244

Curium-245/246

Gross alpha

lodine-129

Nickel-63

Nonvolatile beta

Plutonium-238

Plutonium-239/240

Radium-226

Radium-228

Strontium-90

Technetium-99

Thorium-228

Thorium-230

Thorium-232

Sum of alphas

Sum of betas

Tritium

Uranium-233/234

Uranium-235

Uranium-238
3098

Mod

Fill.

ST

DF Lab $\underline{4 Q 98}$

$\underline{\text { Mod }}$

Filt.

$\underline{\text { ST }} \underline{H}$ DF Lab Unit

$3.0 E+00$

$1.7 E+01 \quad / 1$

1

GP $<4.4 E+00 \quad U / /$

1 GP $1.2 E+01 \quad / /$

$2.3 \mathrm{E}+03$

-

1

GP $2.3 E+03 \quad \|$
$1 \mathrm{GE} \mathrm{PCi/L}$

1 GE $\mathrm{pCi} / \mathrm{L}$

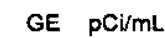

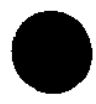

Notes

- = exceeded holding time

a = exceeded groundwater protection or monitoring constituent standard (See Appendix A.) 


\section{WELL HSB139D}

\begin{tabular}{|c|c|}
\hline SRS Coord. & Lat/Longitude \\
\hline $\begin{array}{l}\text { N711133.2 } \\
\text { E57384.4 }\end{array}$ & $\begin{array}{l}33.27672 \\
81.65387\end{array}$ \\
\hline
\end{tabular}

SAMPLE DATE

FIELD DATA

\section{Constituents}

Water elevation

$\mathrm{pH}$

Sp. conductance

Water temperature

Alkalinity as $\mathrm{CaCO}_{3}$

Turbidity

Volumes purged

Sampling code

Synchronous water level

ANALYTICAL DATA

Inorganic Constituents

Constituents

Antimony, total recoverable

Arsenic, total recoverable

Barium, total recoverable

Cadmium, total recoverable

Chromium, total recoverable

Cobalt, total recoverable

Copper, total recoverable

Cyanide

Lead, total recoverable

Mercury, total recoverable

Nickel, total recoverable

Nitrate as nitrogen

Nitrate-nitrite as nitrogen

Selenium. total recoverable

Silver, total recoverable

Tin, total recoverable

Vanadium, total recoverable

Zinc, total recoverable

Organic Constituents

Constituents

Benzene

Bis(2-ethylhexyl) phthalate

Dichloromethane

Tetrachloroethylene

Trichloroethylene

Trichlorofluoromethane
Screen Zone Elevation

226.7-206.7 ft ms

Top of Casing

$233.8 \mathrm{ft} \mathrm{ms}$

Casing Pump

4" PVC S

Screen Zone

$07 / 20 / 98$

$10 / 12 / 98$

3Q98

221.9

4.3

66

18.6

0

3

6.3

$221.4(09 / 17 / 98)$
4Q98

220.5

4.5

32

19.8

0

5.7

$219.6(12 / 22 / 98)$
Unit

ft msl

$\mathrm{pH}$

$\mu \mathrm{S} / \mathrm{cm}$

${ }^{\circ} \mathrm{C}$

$\mathrm{mg} / \mathrm{L}$

NTU

well vol

ft $\mathrm{msl}$

\section{.}

$\mathrm{cm}$

vol

Water Table (IIB2)
$3098 \quad$ Mod Filt.

$<0.20$

3,200

U/I

NI

Mod
ST브

Mod

1 GE $<0.20$

$\mathrm{U} / \mathrm{I}$

3 GE 2,400

II

Mod 


\section{WELL HSB139D (cont.)}

Radioactive Constituents

\section{Constituents}

Americium-241

Carbon-14

Cobalt- 60

Curium-242

Curium-243/244

Curium-245/246

Gross alpha

lodine-129

Nickel-63

Nonvolatile beta

Plutonium-238

Plutonium-239/240

Radium-226

Radium-228

Strontium-90

Technetium-99

Thorium-228

Thorium-230

Thorium-232

Sum of alphas

Sum of betas

Tritium

Uranium-233/234

Uranium-235

Uranium-238
309

$\underline{\text { Mod }}$

Filt.
$2.6 \mathrm{E}+00 \quad / 1$

$5.2 \mathrm{E}+01 \quad \|$

$1.2 \mathrm{E}+02$
1

GP $2.2 E+\infty 0 \quad / /$

- 1 GP $9.2 \mathrm{E}+01 \quad / /$

- 1 GP $7.4 \mathrm{E}+01 \quad / /$
1 GE pCin

- 1 GE pCi/L

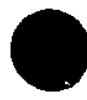


WELL HSB140A

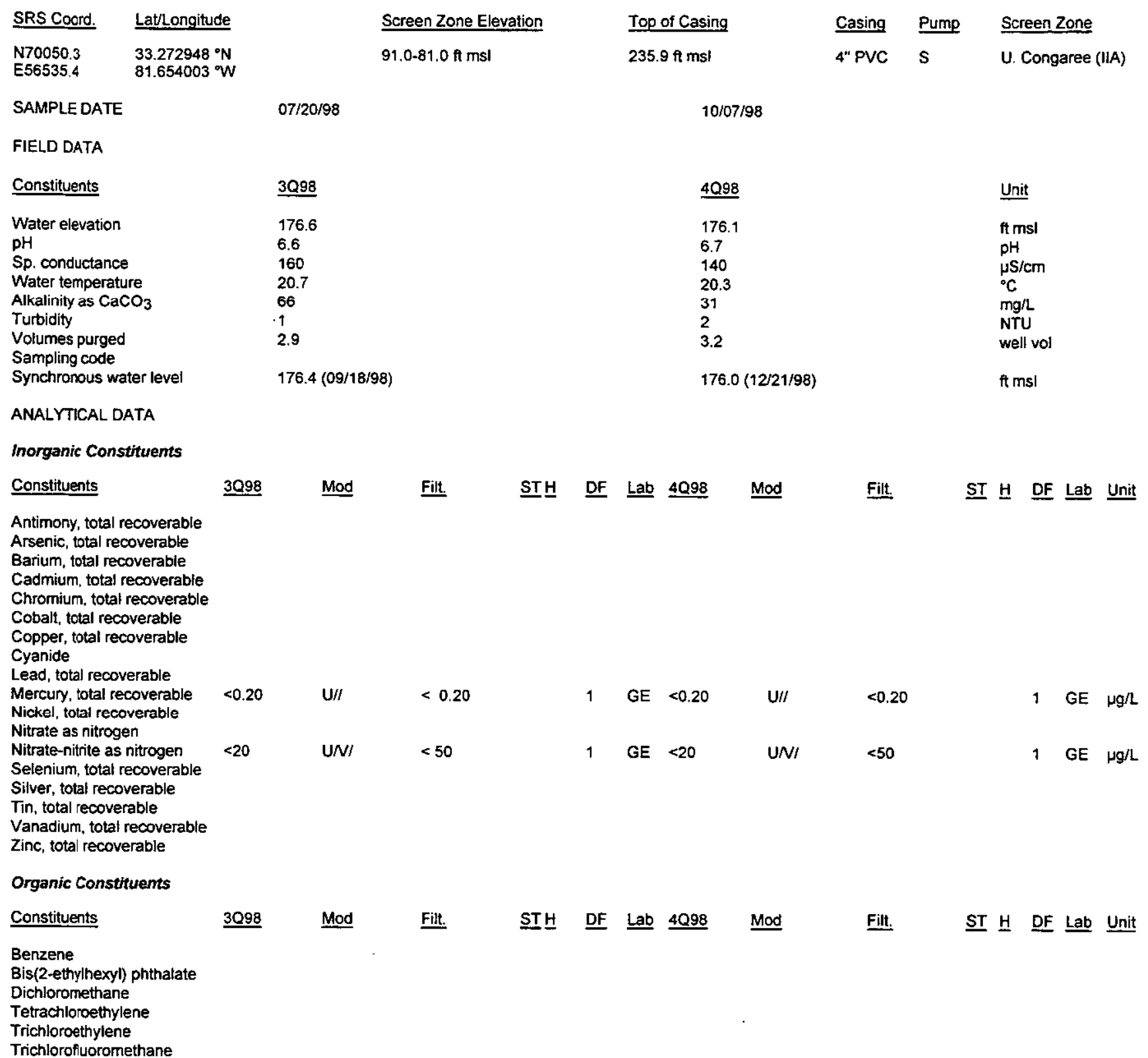

Notes:

- = exceeded holding time

- = exceeded groundwater protection or monitoring constituent standard (See Appendix A.) 


\section{WELL HSB140A (cont.)}

Radioactive Constituents

Constituents

Americium-241

Carbon-14

Cobalt-60

Curium-242

Curium-243/244

Curium-245/246

Gross alpha

lodine-129

Nickel-63

Nonvolatile beta

Plutonium-238

Plutonium-239/240

Radium-226

Radium-228

Strontium-90

Technetium-99

Thorium-228

Thorium-230

Thorium-232

Sum of alphas

Sum of betas

Tritium

Uranium-233/234

Uranium-235

Uranium-238

$\underline{3 Q 98} \quad$ Mod $\quad \underline{\text { Filt. }} \quad \underline{\text { ST }} \underline{H} \quad \underline{\text { DF }} \underline{\text { Lab }} \underline{4 Q 98} \quad \underline{\text { Mod }} \quad \underline{\text { Filt. }} \quad \underline{\text { ST }} \underline{H}$ DF Lab Unit

1

GP $\quad<1.9 E-01 \quad$ JU/UI

1 GP $\quad<6.9 \mathrm{E}-01 \quad \mathrm{JU} / \mathrm{L} /$
$<1.1100$

$<-7.4 \mathrm{E}-02 \quad \mathrm{UI} /$

$<5.9 E-01$
1 GP $<1.8 E-01$

U/I

$<6.3 E-01$
1 GE pCill

1 GE pCill 
WELL HSB140C

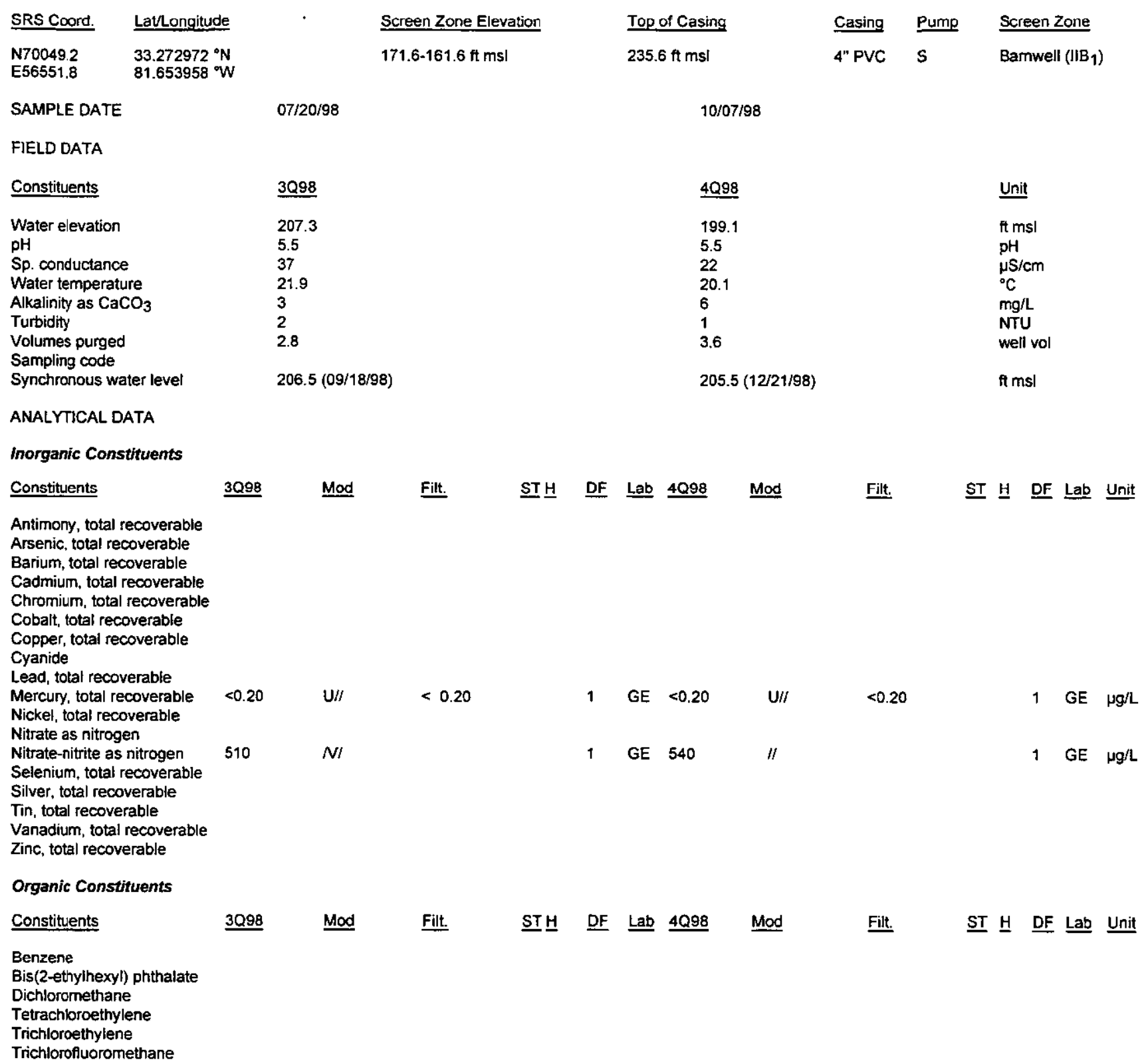

Notes:

- exceeded holding time

- exceeded groundwater protection or monitoring constituent standard (See Appendix A.) 


\section{WELL HSB140C (cont.)}

Radioactive Constituents

Constituents

Americium-241

Carbon-14

Cobalt-60

Curium-242

Curium-243/244

Curium-245/246

Gross atpha

lodine-129

Nickel-63

Nonvolatile beta

Plutonium-238

Plutonium-239/240

Radium-226

Radium-228

Strontium-90

Technetium-99

Thorium-228

Thorium-230

Thorium-232

Sum of alphas

Sum of betas

Tritium

Uranium-233/234

Uranium-235

Uranium-238

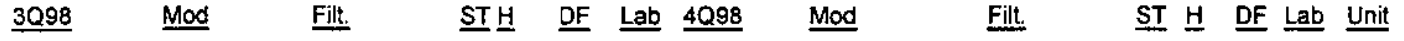

\begin{tabular}{|c|c|c|c|c|c|c|c|c|c|}
\hline$<4.5 E-01$ & $\mathrm{U} / \mathrm{I}$ & $<0.6420$ & 1 & GP & $<3.4 \mathrm{E}-02$ & JU/LI & $<N D D$ & 1 & GE \\
\hline$=7.9 E-01$ & UIII & $<1.0600$ & 1 & GP & $<t .1 E+00$ & JU/し/ & $<N D D$ & 1 & $\mathrm{GE}$ \\
\hline
\end{tabular}

$3.0 E+00 \quad / /$

1 GP $3.3 E+00$

ll

$1 \mathrm{GE} \quad \mathrm{pCi} / \mathrm{mL}$

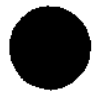

Notes:

= exceeded holding time

- exceeded groundwater protection or monitoring constituent standard (See Appendix A.) 


\section{WELL HSB140D}

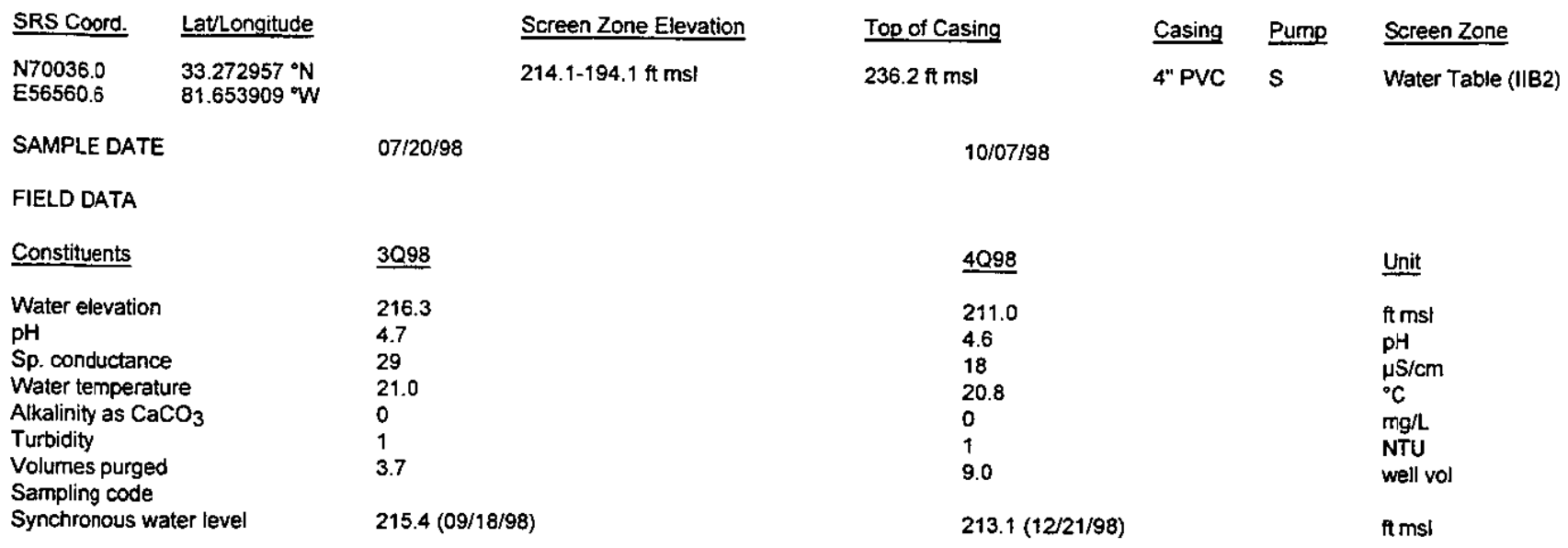

ANALYTICAL DATA

Inorganic Constituents

Constituents

Antimony, total recoverable Arsenic, total recoverable Barium, total recoverable

Cadmium, total recoverable

Chromium, total recoverable Cobalt, total recoverable

Copper, total recoverable

Cyanide

Lead, total recoverable

Mercury, total recoverable

Nickel, total recoverable

Nitrate as nitrogen

Nitrate-nitrite as nitrogen

Selenium, total recoverable

Silver, total recoverable

Tin, total recoverable

Vanadium, total recoverable

Zinc, total recoverable

Organic Constituents

Constituents

Benzene

Bis(2-ethylhexyl) phthalate

Dichloromethane

Tetrachloroethylene

Trichloroethylene

Trichlorofluoromethane

3Q98

400
Mod

Filt.

ST브

DF Lab $4 Q 98$

Mod

1 GE $<0.20$

U/I

1 GE 450

/I

NI

$<0.20$

(n)

\begin{tabular}{|c|c|c|c|c|c|c|c|c|c|c|}
\hline$<0.20$ & $\mathrm{U} / /$ & $<0.20$ & 1 & GE & $<0.20$ & $\mathrm{u} / /$ & $<0.20$ & 1 & GE & $\mu g / L$ \\
\hline 400 & $N /$ & & 1 & GE & 450 & $/ /$ & & 1 & GE & $\mathrm{g} / \mathrm{g}$ \\
\hline
\end{tabular}

\section{Notes:}

- exceeded ho!ding time

- = exceeded groundwater protection or monitoring constituent standard (See Appendix A.) 


\section{WELL HSB140D (cont.)}

Radioactive Constituents

Constituents

Americium-241

Carbon-14

Cobalt-60

Curium-242

Curium-243/244

Curium-245/246

Gross alpha

lodine-129

Nickel-63

Nonvolatile beta

Plutonium-238

Plutonium-239/240

Radium-226

Radium-228

Strontium-90

Technetium-99

Thorium-228

Thorium-230

Thorium-232

Sum of alphas

Sum of betas

Tritium

Uranium-233/234

Uranium-235

Uranium-238 $\underline{3098}$

Mod

Filt.

$\underline{\text { ST }} \underline{H}$

DF Lab 4098

Mod

Filt.

$\underline{\text { ST }} \underline{H}$ DF Lab Unit
1

GP $<8.6 E-01 \quad$ JU/LI

1

GP $<9.5 E-01 \quad J U / L /$
GP 1.2E+01 // $\uparrow$ GE $\mathrm{pCil}$

1 GE PCi/L

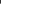

1 GE $\mathrm{pCi} / \mathrm{mL}$

Notes:

- = exceeded holding time

= exceeded groundwater protection or monitoring constituent standard (See Appendix A.) 
WELL HSB141A

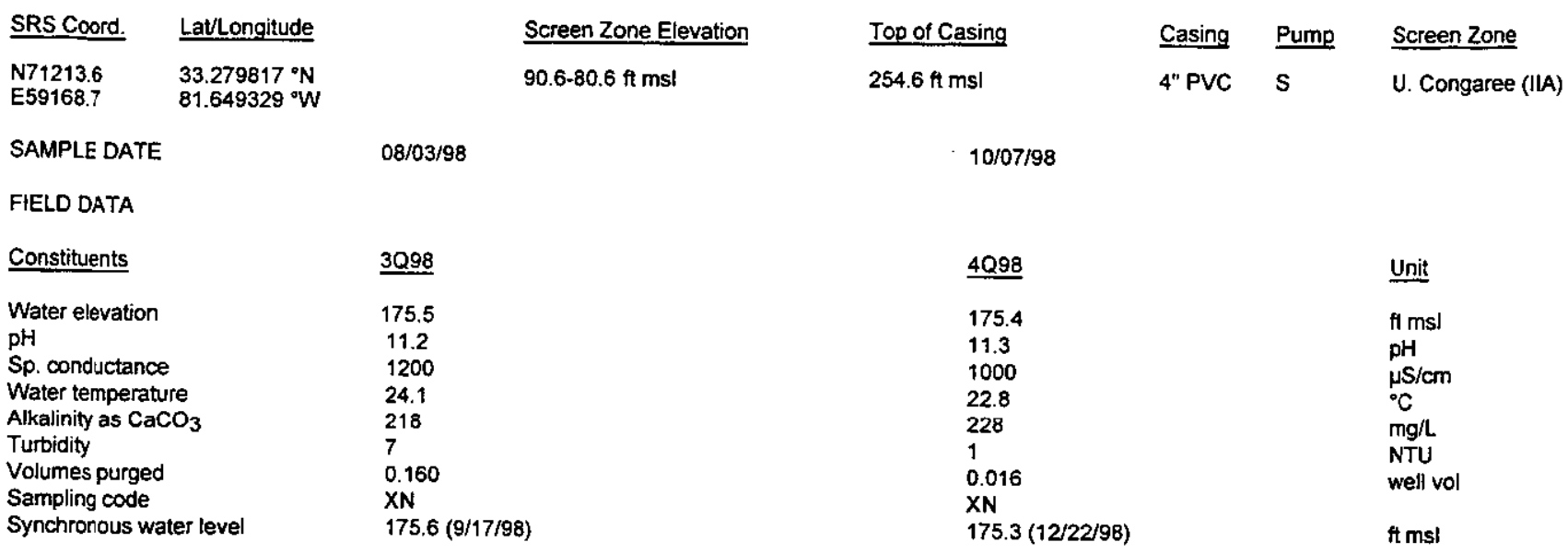

ANALYTICAL DATA

Inorganic Constituents

Constituents

Antimony, total recoverable

Arsenic, lotal recoverable

Barium, total recoverable

Cadmium, total recoverable

Chromium, total recoverable

Cobalt, total recoverable

Copper, total recoverable

Cyanide

Lead, total recoverable

Mercury, total recoverable

Nickel, total recoverable

J/E

NDD

GE $<0.20$

U/I

$<0.20$

1 GE $\mu g / L$

Nitrate as nitrogen

Nitrate-nitrite as nitrogen

$110 \quad$ NI

Selenium, total recoverable

Silver, total recoverable

Tin, total recoverable

Vanadium, total recoverable

Zinc, total recoverable

Organic Constituents

Constituents

Benzene

Bis(2-ethylhexyl) phthalate

Dichloromethane

Tetrachloroethylene

Trichloroethylene

Trichlorofluoromethane

Notes:

- = exceeded holding time

- exceeded groundwater protection or monitoring constituent standard (See Appendix A.) 
WELL HSB141A (cont.)

Radioactive Constituents

Constituents

Americium-241

Carbon-14

Cobalt-60

Curium-242

Curium-243/244

Curium-245/246

Gross alpha

lodine-129

Nickel-63

Nonvolatile beta

Plutonium-238

Plutonium-239/240

Radium-226

Radium-228

Strontium-90

Technetium-99

Thorium-228

Thorium-230

Thorium-232

Sum of alphas

Sum of betas

Tritium

Uranium-233/234

Uranium-235

Uranium-238 $\underline{3 Q 98}$

Mod

Filt.

STH

DF Lab 4 Q998

$\underline{\text { Mod }}$

Filt.

ST H DF Lab Unit

$<1.4 \mathrm{E}-$

UIII

$1.6 \mathrm{E}+\infty$

/I

$1.6 \mathrm{E}+00$

II
1

GP $\quad 1.7 E+\infty \quad J / / /$

NDD

1

GP $\quad 2.9 E+00$

$\mathrm{J} / \mathrm{I} /$

NDD

1

GP $3.3 E+00 \quad / /$
1 TM pCi/h

$1 \mathrm{TM} \mathrm{PCin}$
$1 \mathrm{TM} \quad \mathrm{pCi} / \mathrm{mL}$ 
WELL HSB141CR

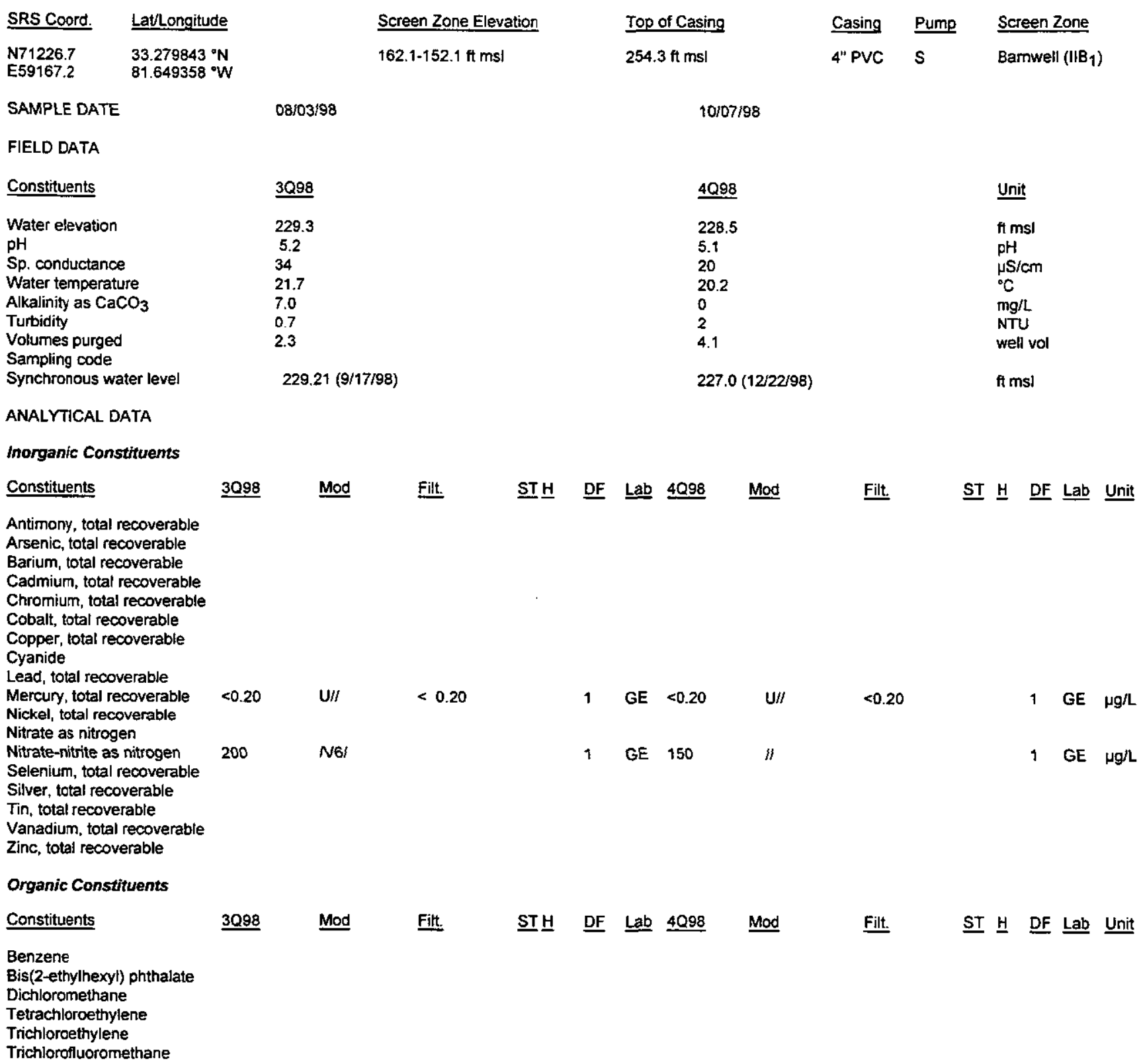

Notes:

= exceeded holding time

- = exceeded groundwater protection or monitoring constituent standard (See Appendix A.) 


\section{WELL HSB141CR (cont.)}

Radioactive Constituents

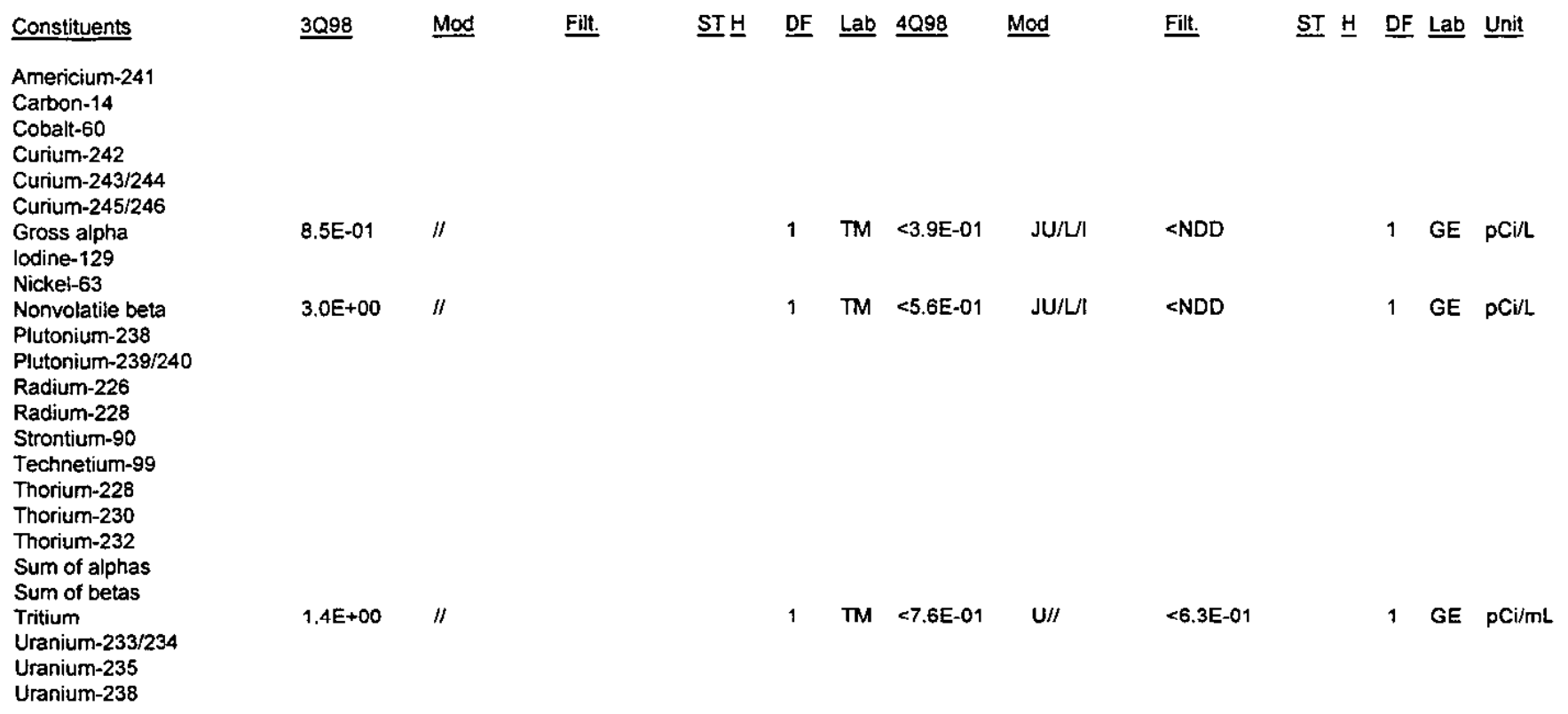

Notes:

- exceeded holding time

= exceeded groundwater protection or monitoring constituent standard (See Appendix A.) 
WELL HSB141D

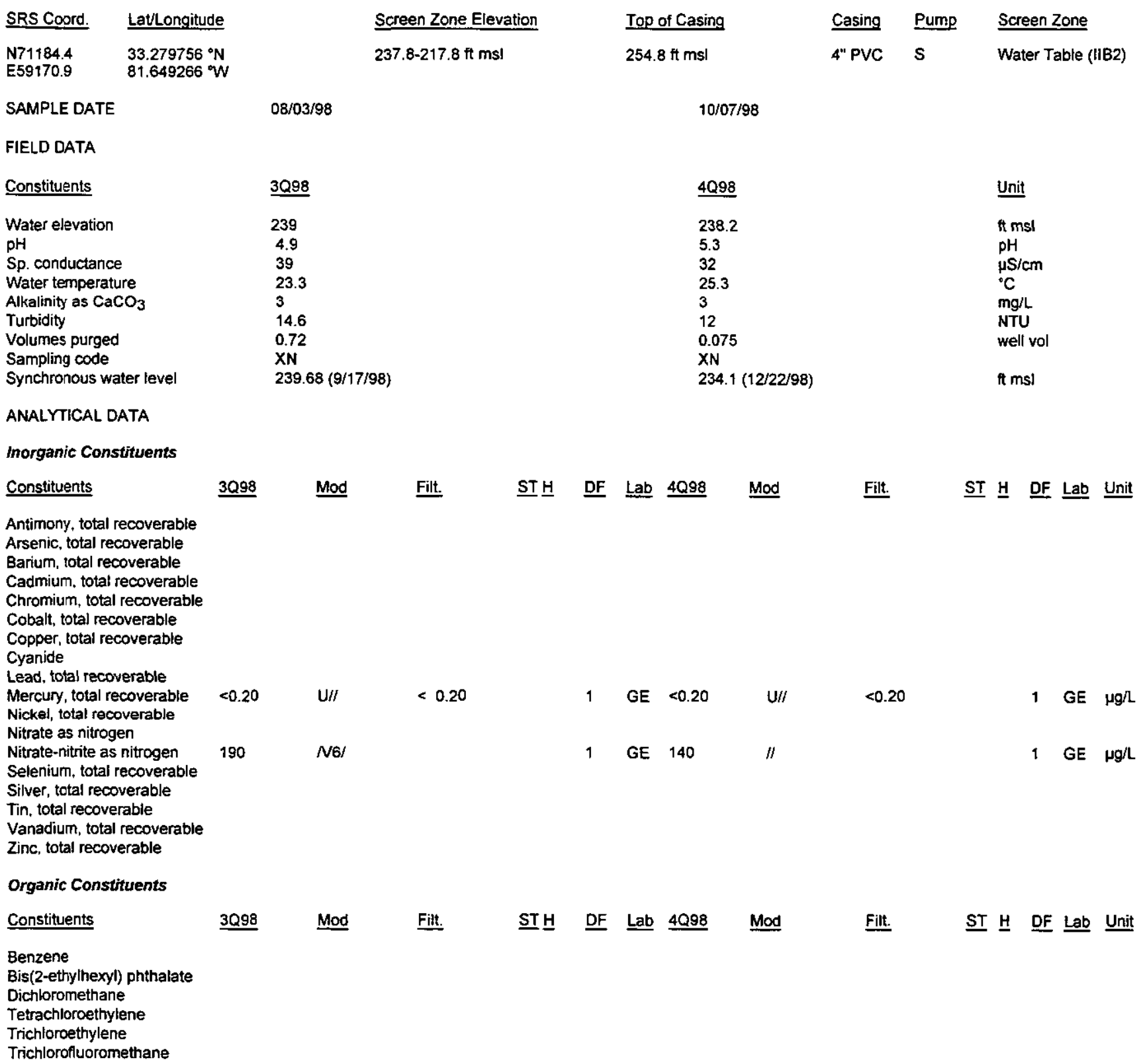

Notes:

- exceeded holding time

- = exceeded groundwater protection or monitoring constituent standard (See Appendix A.) 


\section{WELL HSB141D (cont.)}

Radioactive Constituents

\section{Constituents}

Americium-241

Carbon-14

Cobalt-60

Curium-242

Curium-243/244

Curium-245/246

Gross alpha

lodine-129

Nickel-63

Nonvolatile beta

Plutonium-238

Plutonium-239/240

Radium-226

Radium-228

Strontium-90

Technetium-99

Thorium-228

Thorium-230

Thorium-232

Sum of alphas

Sum of betas

Tritium

Uranium-233/234

Uranium-235

Uranium-238

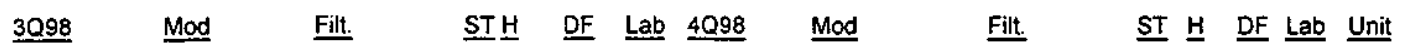

$1.7 \mathrm{E}+00$

$<8.6 \mathrm{E}-01 \quad \mathrm{UI} / \textrm{ } \quad<1.8800$

1

GP $<2,6 \mathrm{E}+00 \quad \mathrm{JU} / \mathrm{LI} \quad<\mathrm{NDD}$

1 GE $\mathrm{pCi} / \mathrm{L}$

$1 \quad G P<9.5 E-01 \quad J U / L / 1 \quad<N D D$

$1 \mathrm{GE} \mathrm{pCi} / \mathrm{L}$
$1.2 E+01 \quad / 1$
1

GP $\quad 1.3 E+01$

1 GE $\mathrm{pCi} / \mathrm{mL}$

Notes:

- =xceeded hoiding time

- exceeded groundwater protection or monitoring constituent standard (See Appendix A.) 


\section{WELL HSB142C}

\begin{tabular}{|c|c|}
\hline SRS Coord. & Latlongitude \\
\hline $\begin{array}{l}N 73119.0 \\
\text { E53505.3 }\end{array}$ & $\begin{array}{l}33.274791 \\
81.667942\end{array}$ \\
\hline
\end{tabular}

SAMPLE DATE

FIELD DATA

Constituents

Water elevation

pH

Sp. conductance

Water temperature

Alkalinity as $\mathrm{CaCO}_{3}$

Turbidity

Volumes purged

Sampling code

Synchronous water level
Screen Zone Elevation

$171.6-161.6 \mathrm{ft} \mathrm{msl}$

$07 / 14 / 98$

3098

197.4

4.8

21

17.9

1

3.2

$144.9(09 / 18 / 98)$
Top of Casing

$204 \mathrm{ft} \mathrm{msl}$

$10 / 06 / 98$
ANALYTICAL DATA

Inorganic Constituents

Constituents

Mod

Filt.

DF Lab 4Q98

Mod

Casing Pump

Screen Zone

4" PVC S

Barnwell (IIB 1$)$

Antimony, total recoverable

Arsenic, total recoverable

Barium, total recoverable

Cadmium, total recoverable

Chromium, total recoverable

Cobalt, total recoverable

Copper, lotal recoverable

Cyanide

Lead, total recoverable

Mercury, total recoverable

Nickel, total recoverable

$<0.20$

U/I

$<0.20$

1

GE $<0.20$

JU/LC

$<N D D$

1 GE $\mu g / L$

Nitrate as nitrogen

Nitrate-nitrite as nitrogen

310

ll

1 GE 910

II

Selenium, total recoverable

Silver, total recoverable

Tin, total recoverable

Vanadium, total recoverable

Zinc, total recoverable

Organic Constituents

Constituents

Filt.

DF

198.1
4.9
20
18.2
0
2
6.3
$195.9(12 / 22 / 98)$

Unit

ft msi

$\mathrm{pH}$

$\mu \mathrm{S} / \mathrm{cm}$

${ }^{\circ} \mathrm{C}$

$\mathrm{mg} / \mathrm{L}$

NTU

well vol

ft msl

Benzene

Bis(2-ethylhexyl) phthalate

Dichloromethane

Tetrachloroethylene

Trichloroethylene

Trichlorofluoromethane

Notes:

- = exceeded holding time

= exceeded groundwater protection or monitoring constituent standard (See Appendix A.) 
WELL HSB142C (cont.)

Radioactive Constituents

Constituents

Americium-241

Carbon-14

Cobalt- 60

Curium-242

Curium-243/244

Curium-245/246

Gross alpha

lodine-129

Nickel-63

Nonvolatile beta

Plutonium-238

Plutonium-239/240

Radium-226

Radium-228

Strontium-90

Technetium-99

Thorium-228

Thorium-230

Thorium-232

Sum of alphas

Sum of betas

Tritium

Uranium-233/234

Uranium-235

Uranium-238

$\begin{array}{lllllllll}<1.9 \mathrm{E}-02 \quad \mathrm{U} I / & <0.6150 & 1 & \mathrm{GP} & <1.0 \mathrm{E}+00 & \mathrm{U} / l & <5.3 \mathrm{E}-01 & & \\ <8.7 \mathrm{E}-01 & \mathrm{UI} / 1 & <1.1400 & 1 & \mathrm{GP} & 4.1 \mathrm{E}+00 & \| & \mathrm{PCi} / \mathrm{L}\end{array}$

$6.9 \mathrm{E}+00$

1 GP $3.1 E+02 \quad$ //

- 1 ge pCi/mL

\section{Mobs}

- = exceeded holding time

= exceeded groundwater protection or monitoring constituent standard (See Appendix A.) 


\section{WELL HSB142D}

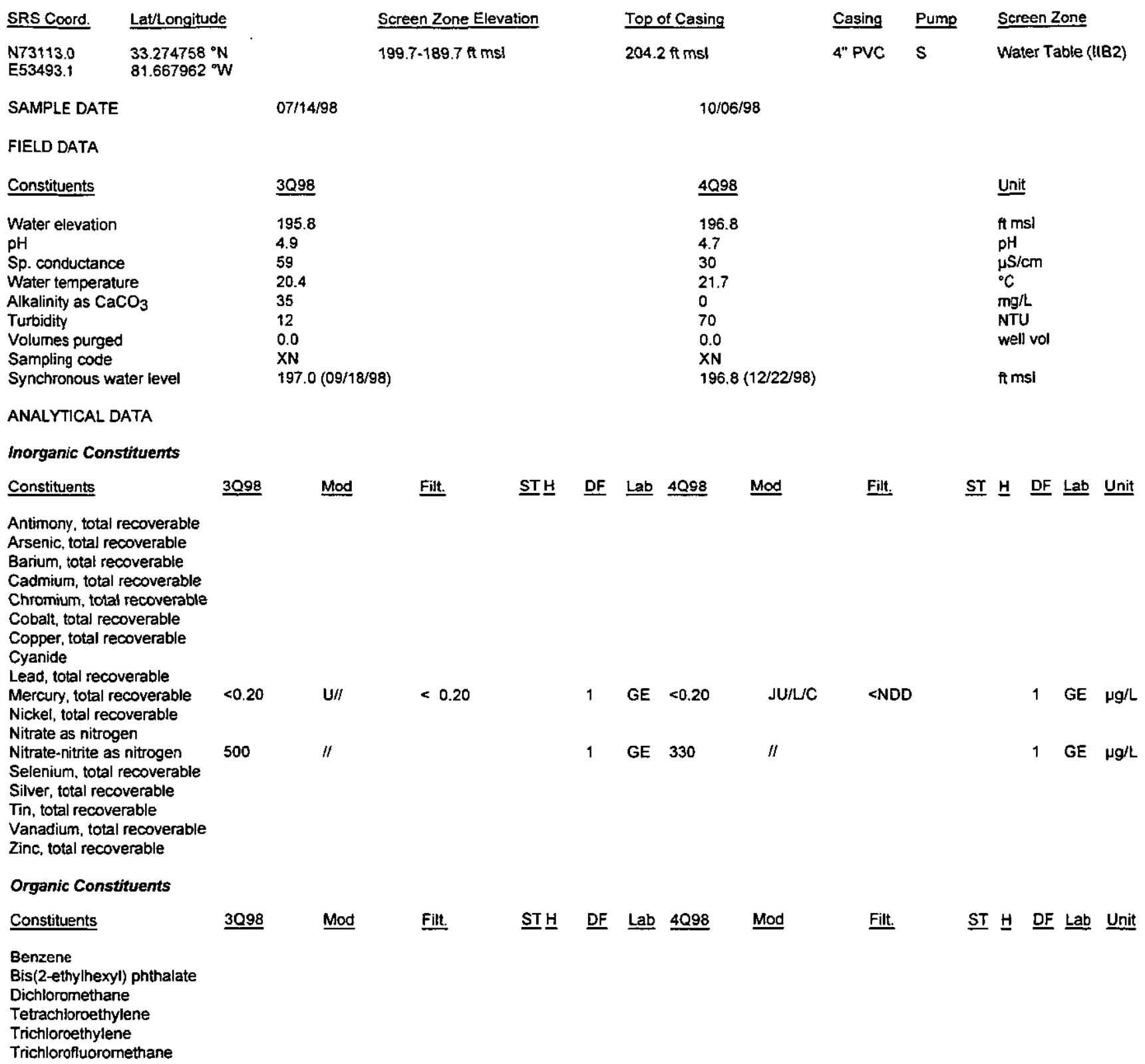

Notes:

- = exceeded holding time

w = exceeded groundwater protection or monitoring constituent standard (See Appendix A.) 


\section{WELL HSB142D (cont.)}

\section{Radioactive Constituents}

Constituents

Americium-241

Carbon-14

Cobalt-60

Curium-242

Curium-243/244

Curium-245/246

Gross alpha

lodine-129

Nickel-63

Nonvolatile beta

Plutonium-238

Plutonium-239/240

Radium-226

Radium-228

Strontium-90

Technetium-99

Thorium-228

Thorium-230

Thorium-232

Sum of alphas

Sum of betas

Tritium

Uranium-233/234

Uranium-235

Uranium-238 $\underline{3098}$

Mod

Filt.

ST브 DF Lab 4 Q998

Mod

Filt.

ST $\underline{H}$ DF Lab Unit

$<3.8 \mathrm{E}-$

UIII

$2.4 \mathrm{E}+00$

$3.4 \mathrm{E}+02 \quad 11$

"l

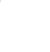

1

GP $<-3.9 E-03 \quad U / /$

1

GP $<7.4 \mathrm{E}-01$

$\mathrm{U} / /$

$<6.8 \mathrm{E}-01$

$<1.1 E+00$

GP $8.3 \mathrm{E}+00$
1 GE PCViL

1 GE pCi/L
$1 \mathrm{GE} \mathrm{pCi} / \mathrm{mL}$ 


\section{WELL HSB143C}

\begin{tabular}{|c|c|}
\hline SRS Coord. & Lat/Longitude \\
\hline $\begin{array}{l}\text { N737388.2 } \\
\text { E52773.2 }\end{array}$ & $\begin{array}{l}33.274966^{\circ} \mathrm{N} \\
81.6710722^{\circ} \mathrm{W}\end{array}$ \\
\hline
\end{tabular}

SAMPLE DATE

$07 / 14 / 98$

\section{Screen Zone Elevation}

179.1-169.1 ft ms!

FIELD DATA

Constituents

Water elevation

pH

Sp. conductance

Water temperature

Alkalinity as $\mathrm{CaCO}_{3}$

Turbidity

Volumes purged

Sampling code

Synchronous water level

ANALYTICAL DATA

Inorganic Constituemts

Constituents

Antimony, total recoverable

Arsenic, total recoverable

Barium, total recoverable

Cadmium, total recoverable

Chromium, total recoverable

Cobalt, total recoverable

Copper, total recoverable

Cyanide

Lead, total recoverable

Mercury, total recoverable

Nickel, total recoverable

Nitrate as nitrogen

Nitrate-nitrite as nitrogen

Selenium, total recoverable

Silver, total recoverable

Tin, total recoverable

Vanadium, total recoverable

Zinc, total recoverable

Organic Constituents

Constituents

Benzene

Bis(2-ethylhexyl) phthalate

Dichloromethane

Tetrachloroethylene

Trichloroethylene

Trichlorofluoromethane

\section{8}

209.4

4.4

45

20.2

2

3.4

$209.4(09 / 18 / 98)$
0.1

$\mathrm{J} / \mathrm{E} /$

NDD
Top of Casing

$222.2 \mathrm{ft} \mathrm{ms}$

$10 / 05 / 98$

4Q98

209.0

4.7

18.9

0

9.7

$208.5(12 / 21 / 98)$
Casing Pump

4" PVC S
Screen Zone

Bamwell (IIB 1 )

Unit

ft msl

$\mathrm{pH}$

$\mu \mathrm{S} / \mathrm{cm}$

${ }^{\circ} \mathrm{C}$

mgll

NTU

well vol

ft msl

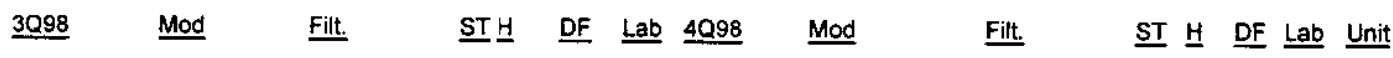

1 GE $<0.20$

$\mathrm{U} / 1$

1 GE 540

II

$\underline{\text { ST브 }}$

Mod

Filt.

ST $\underline{H}$ DF Lab Unit

Notes:

- exceeded holding time

- = exceeded groundwater protection or monitoring constituent standard (See Appendix A.) 


\section{WELL HSB143C (cont.)}

Radioactive Constituents

Constituents

Americium-241

Carbon-14

Cobalt-60

Curium-242

Curium-243/244

Curium-245/246

Gross alpha

lodine-129

Nickel-63

Nonvolatile beta

Plutonium-238

Plutonium-239/240

Radium-226

Radium-228

Strontium-90

Technetium-99

Thorium-228

Thorium-230

Thorium-232

Sum of alphas

Sum of betas

Tritium

Uranium-233/234

Uranium-235

Uranium-238
3098

Mod

Filt.

ST보

DF LLab 4 QQ98

Mod

Filt.

$\underline{\text { ST }} \underline{H}$ DF Lab Unit

$<1.5 E-01$

UI/I

$<0.7460$

1

GP $<7.5 E-01 \quad U / /$

$<5.9 E-01$

1

GP $\quad<5.3 \mathrm{E}-01 \quad \mathrm{U} / \prime$

$<1.2 E+00$

$<1.3000$

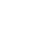

$<1.1 \mathrm{E}+00 \quad \mathrm{U} / /$

(n)

1 GE pCill

1 GE $\mathrm{pCi} / \mathrm{L}$
1

GP $8.1 E+00$

/l

1 GE pCimL
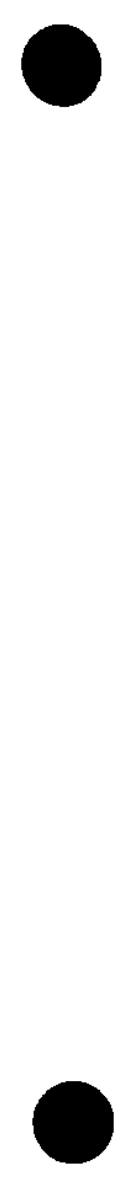

\section{Notes:}

- exceeded holding time

= exceeded groundwater protection or monitoring constituent standard (See Appendix A.) 


\section{WELL HSB143D}

\begin{tabular}{|c|c|}
\hline SRS Coord. & Lat/Longitude \\
\hline $\begin{array}{l}\text { N73754.0 } \\
\text { E52774.5 }\end{array}$ & $\begin{array}{l}33.2750 \\
81.6710\end{array}$ \\
\hline
\end{tabular}

SAMPLE DATE

$07 / 14 / 98$

Screen Zone Elevation

216.9-196.9 ft msl

Top of Casing

$222.9 \mathrm{ft} \mathrm{ms}$

$10 / 05 / 98$

FIELD DATA

Constituents

Water elevation

$\mathrm{pH}$

Sp. conductance

Water temperature

Alkalinity as $\mathrm{CaCO}_{3}$

Turbidity

Volumes purged

Sampling code

Synchronous water level

ANALYTICAL DATA

Inorganic Constituents

Constituents

Antimony, total recoverable

Arsenic, total recoverable

Barium, total recoverable

Cadmium, total recoverable

Chromium, total recoverable

Cobalt, total recoverable

Copper, total recoverable

Cyanide

Lead, total recoverable

Mercury, total recoverable

Nickel, total recoverable

Nitrate as nitrogen

Nitrate-nitrite as nitrogen

Selenium, total recoverable

Silver, total recoverable

Tin, total recoverable

Vanadium, total recoverable

Zinc, total recoverable

Organic Constituents

Constituents

22

20.4

0

3

3.8

$213.4(09 / 18 / 98)$

$390 \quad$ II

1 GE 560 /I
4Q98

213.4

4.4

19

19.5

0

9.2

$210.0(12 / 21 / 98)$
Casing Pump Screen Zone

4" PVC S Water Table (IIB2)

$\underline{3098}$ Mod $\underline{\text { Filt. }} \underline{\text { ST브 DF Lab }} \underline{4 Q 98} \quad \underline{\text { Mod }} \quad \underline{\text { Filt. }} \underline{\text { ST }} \underline{\text { DF }} \underline{\text { Lab }}$ Unit

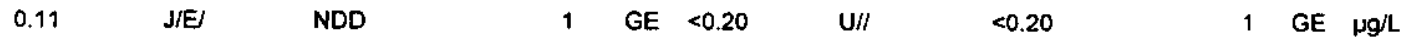

1 GE $\mu g / L$

Benzene

Bis(2-ethylhexyl) phthalate

Dichloromethane

Tetrachloroethylene

Trichloroethylene

Trichlorofluoromethane

Notes:

- = exceeded holding time

$\mathbf{a}=$ exceeded groundwater protection or monitoring constituent standard (See Appendix A.) 


\section{WELL HSB143D (cont.)}

\section{Radioactive Constituents}

Constituents

Americium-241

Carbon-14

Cobalt-60

Curium-242

Curium-243/244

Curium-245/246

Gross alpha

lodine-129

Nickel-63

Nonvolatile beta

Plutonium-238

Plutonium-239/240

Radium-226

Radium-228

Strontium-90

Technetium-99

Thorium-228

Thorium-230

Thorium-232

Sum of alphas

Sum of betas

Tritium

Uranium-233/234

Uranium-235

Uranium-238
3098

$1.4 E+00 \quad / /$

$<6.1 \mathrm{E}-01 \quad \mathrm{UI} /$

$<1.2000$

1

GP $<1.1 E+\infty \quad U / /$

1

GP <8.1E-01

U/I

$<1.1 E+\infty 0$

1 GP $9.1 E+00$ /I
Fist.

ST 브 DF Lab Unit
1 GE pCi/L

1 GE pCi/L

$1 \mathrm{GE} \rho \mathrm{Ci} / \mathrm{mL}$ 


\section{WELL HSB144A}

\begin{tabular}{|c|c|}
\hline SRS Coord. & Lat/Longitude \\
\hline $\begin{array}{l}\text { N7 1892.1 } \\
\text { E56200.5 }\end{array}$ & 81.658462 o \\
\hline
\end{tabular}

Screen Zone Elevation

88.6.78.6 ft ms

07/20/98

3098

Constituents

Water elevation

$\mathrm{pH}$

Sp. conductance

Water temperature

Alkalinity as $\mathrm{CaCO}_{3}$

Turbidity

Volumes purged

Sampling code

Synchronous water level

ANALYTICAL DATA

Inorganic Constituents

\section{Constituents}

Antimony, total recoverable

Arsenic, total recoverable

Barium, total recoverable

Cadmium, total recoverable

Chromium, total recoverable

Cobalt, total recoverable

Copper, total recoverable

Cyanide

Lead, total recoverable

Mercury, total recoverable

Nickel, total recoverable

Nitrate as nitrogen

Nitrate-nitrite as nitrogen

Selenium, total recoverable

Silver, total recoverable

Tin, total recoverable

Vanadium, total recoverable

Zinc, total recoverable

Organic Constituents

Constituents

Benzene

Bis(2-ethylhexyl) phthalate

Dichloromethane

Tetrachloroethylene

Trichloroethylene

Trichlorolluoromethane

Filit.

ST보
Top of Casing

$235.6 \mathrm{ft} \mathrm{msl}$

$10 / 06 / 98$

4Q98

171.2

6.3

120

41

1

4.3

$171.0(12 / 22 / 98)$
Casing Pump Screen Zone

4" PVC S
Unit

ft msl

$\mathrm{pH}$

HS/cm

${ }^{\circ} \mathrm{C}$

$\mathrm{mg} / \mathrm{L}$

NTU

well vol

ft ms!

$\underline{3098} \quad \underline{M o d} \quad$ Filt. $\quad \underline{\text { TTH }}$ DF Lab $\underline{4 Q 98} \quad \underline{M o d} \quad$ Filt. $\quad \underline{\text { ST }} \underline{H}$ DF Lab Unit

\begin{tabular}{|c|c|c|c|c|c|c|c|c|c|}
\hline$<0.20$ & $\mathrm{U} / /$ & $<0.20$ & 1 & GE & $<0.20$ & JU/UC & $\angle N D D$ & 1 & $\mathrm{GE}$ \\
\hline 180 & $N /$ & & 1 & GE & 80 & $/ 1$ & & 1 & GE \\
\hline
\end{tabular}

DF

DF 늠

4098

Mod

Filt.

ST $\underline{H}$ DF L Lab Unit

Notes:

- exceeded holding time

= exceeded groundwater protection or monitoring constituent standard (See Appendix A.) 


\section{WELL HSB144A (cont.)}

\section{Radioactive Constituents}

Constituents

Americium-241

Carbon-14

Cobalt-60

Curium-242

Curium-243/244

Curium-245/246

Gross alpha

lodine-129

Nickel-63

Nonvolatile beta

Plutonium-238

Plutonium-239/240

Radium-226

Radium-228

Strontium-90

Technetium-99

Thorium-228

Thorium-230

Thorium-232

Sum of alphas

Sum of betas

Tritium

Uranium-233/234

Uranium-235

Uranium-238
3Q98

Mod

Filt.

ST브 DF Lab $\underline{4 Q 98} \quad$ Mod

$<5.0$ E-01

UIII

$<0.6190$

$3.7 \mathrm{E}+00 \quad / /$

$1.6 \mathrm{E}+03 \quad / /$
1 GP $<7.1 E-01 \quad U / 1$

$1 \quad$ GP $<9.6 \mathrm{E}-01$

U/I

$<1.3 E+00$

GP $2.8 \mathrm{E}+01 \quad / /$
Filt.

$\underline{\text { ST }} \underline{H}$ DF Lab Unit

- 1 GE $\mathrm{pCi} / \mathrm{mL}$
1 GE pCi/L

$\uparrow$ GE $\mathrm{pCi} / \mathrm{L}$
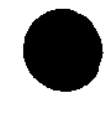
WELL HSB145C

\begin{tabular}{|c|c|}
\hline SRS Coord. & Lat/Longitude \\
\hline $\begin{array}{l}\text { N71 } \\
\text { E57 }\end{array}$ & 8 \\
\hline
\end{tabular}

SAMPLE DATE

$07 / 20 / 98$

Screen Zone Elevation

$174.7-164.7 \mathrm{ft} \mathrm{ms}$

Top of Casing

$235.7 \mathrm{ft} \mathrm{msl}$

$10 / 06 / 98$

FIELD DATA

Constituents

Water elevation

$\mathrm{pH}$

$\mathrm{Sp}$. conductance

Water temperature

Alkalinity as $\mathrm{CaCO}_{3}$

Turbidity

Volumes purged

Sampling code

Synchronous water level

3098

214.0

5.4

340

21.2

34

0

2.5

$213.7(09 / 17 / 98)$ $\underline{4 Q 98}$

213.3

5.5

280

20.2

9

3.2

$212.6(12 / 22 / 98)$
Casing Pump Screen Zone

4" PVC S

\section{ANALYTICAL DATA}

Inorganic Constituents

\section{Constituents}

Antimony, total recoverable

Arsenic, total recoverable

Barium, total recoverable

Cadmium, total recoverable

Chromium, total recoverable

Cobalt, total recoverable

Copper, total recoverable

Cyanide

Lead, total recoverable

Mercury, total recoverable

Nickel, total recoverable

Nitrate as nitrogen

Nitrate-nitrite as nitrogen

Selenium, total recoverable

Silver, total recoverable

Tin, total recoverable

Vanadium, total recoverable

Zinc, total recoverable

Organic Constituents

Constituents

Benzene

Bis(2-ethylhexyl) phthalate

Dichloromethane

Tetrachloroethylene

Trichloroethylene

Trichlorofluoromethane
3Q98

Mod

Filt.

ST브

DF 느

Lab $\underline{4 Q 98}$

Mod

1 GE 1.4

J/LC

NDD

- 25 GE 36,000

/l

3Q98 Mod

Filt

ST브

DF Lab 4 Q998

Mod

Filt.

Filt.

ST H DF Lab Unit

$\underline{\text { Unit }}$

ft msl

$\mathrm{pH}$

$\mu \mathrm{S} / \mathrm{cm}$

mgll

NTU

well vol

$\mathrm{ftms}$ 
WELL HSB145C (cont.)

Radioactive Constituents

Constituents

3098

Mod

Filt.

ST브 DF Lab $\underline{4098}$ Mod

Filt.

$\underline{\text { ST }}$ H DF Lab Unit

Americium-241

Carbon-14

Cobalt-60

Curium-242

Curium-243/244

Curium-245/246

Gross alpha

lodine-129

$3.6 \mathrm{E}+00 \quad / 1$

$1 \quad G P<1.4 E+00$

$\mathrm{U} / I$

$<1.1 \mathrm{E}+00$

$1 \mathrm{GE} \mathrm{PCil}$

Nickel-63

Nonvolatile beta

Piutonium-238

$3.0 \mathrm{E}+01 \quad / /$

1 GP $3.0 E+01 \quad / 1$

1 GE PCill

Plutonium-239/240

Radium-226

Radium-228

Strontium-90

Technetium-99

Thorium-228

Thorium-230

Thorium-232

Sum of alphas

Sum of betas

Tritium

Uranium-233/234

$3.7 \mathrm{E}+01 \quad \|$

- 1 GP $1.6 \mathrm{E}+03 \quad / /$

- 1 GE pCi/mL

Uranium-238

Notes:

$-=$ exceeded holding time

- = exceeded groundwater protection or monitoring constituent standard (See Appendix A.) 
WELL HSB145D

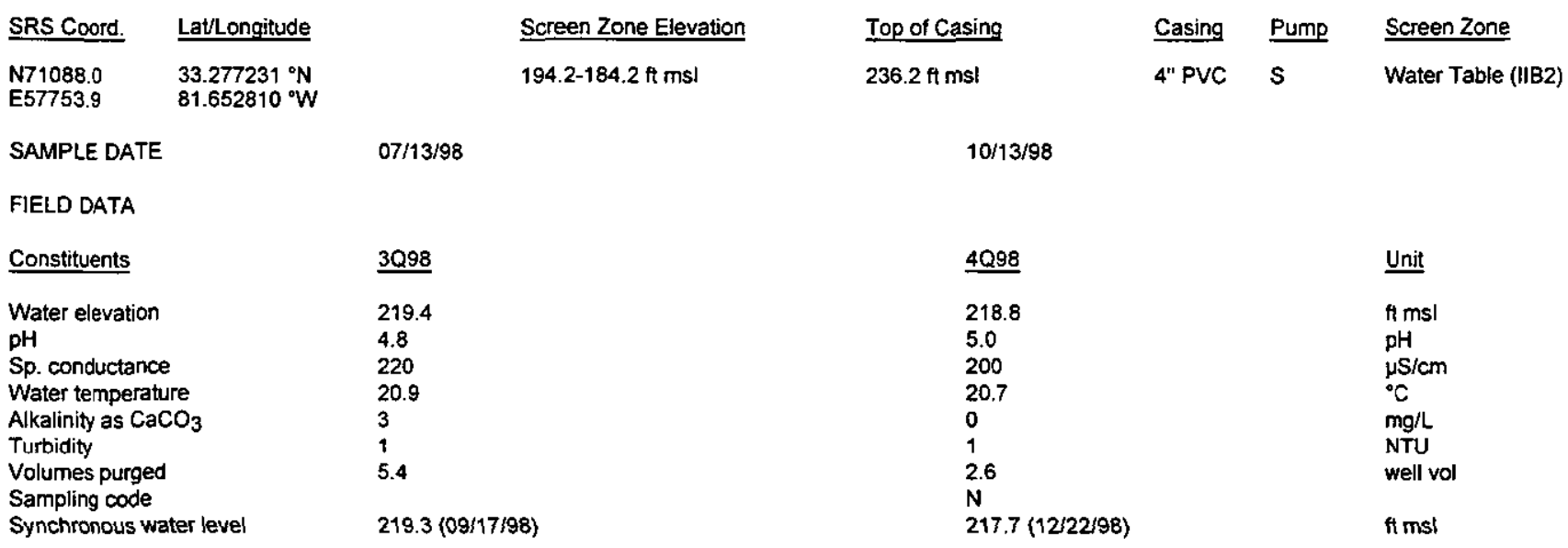

ANALYTICAL DATA

Inorganic Constituents

\section{Constituents}

Antimony, total recoverable

Arsenic, total recoverable

Barium, total recoverable

Cadmium, total recoverable

Chromium, total recoverable

Cobalt, total recoverable

Copper, total recoverable

Cyanide

Lead, total recoverable

Mercury, total recoverable

Nickel, total recoverable

Nitrate as nitrogen

Nitrate-ritrite as nitrogen

Selenium, total recoverable

$2.1 \quad$ II

$25,000 \quad N$

Silver, total recoverable

Tin, total recoverable

Vanadium, total recoverable

Zinc, total recoverable

Organic Constituemts

Constituents
Mod Filt.

Filt.

Mod

-

GE 2.7

$\mathrm{J} / \mathrm{K} / \mathrm{C}$

NDD

- 25 GE 29,000

/I

Benzene

Bis(2-ethylhexyl) phthalate

Dichloromethane

Tetrachloroethylene

Trichloroethylene

Trichlorofluoromethane

Notes:

- = exceeded holding time

a = exceeded groundwater protection or monitoring constituent standard (See Appendix A.) 
WELL HSB145D (cont.)

Radioactive Constituents

Constituents

Americium-241

Carbon-14

Cobalt-60

Curium-242

Curium-243/244

Curium-245/246

Gross a!pha

lodine-129

Nickel-63

Nonvolatile beta

Plutonium-238

Plutonium-239/240

Radium-226

Radium-228

Strontium-90

Technetium-99

Thorium-228

Thorium-230

Thorium-232

Sum of alphas

Sum of betas

Tritium

Uranium-233/234

4.8E+01 $\quad 1$

$5.8 \mathrm{E}+02$

1.7E+03 //

Mod

Filt.

ST브 DF Lab 4098

Mod

Filt.

ST 브 DF Lab Unit

Uranium-235

Uranium-238

Notes:

- = exceeded holding time

- exceeded groundwater protection or monitoring constituent standard (See Appendix A.)
- $\quad 1$ GE pCill

- $\quad 1$ GE pCill

- 1 GE pCi/mL 
WELL HSB146A

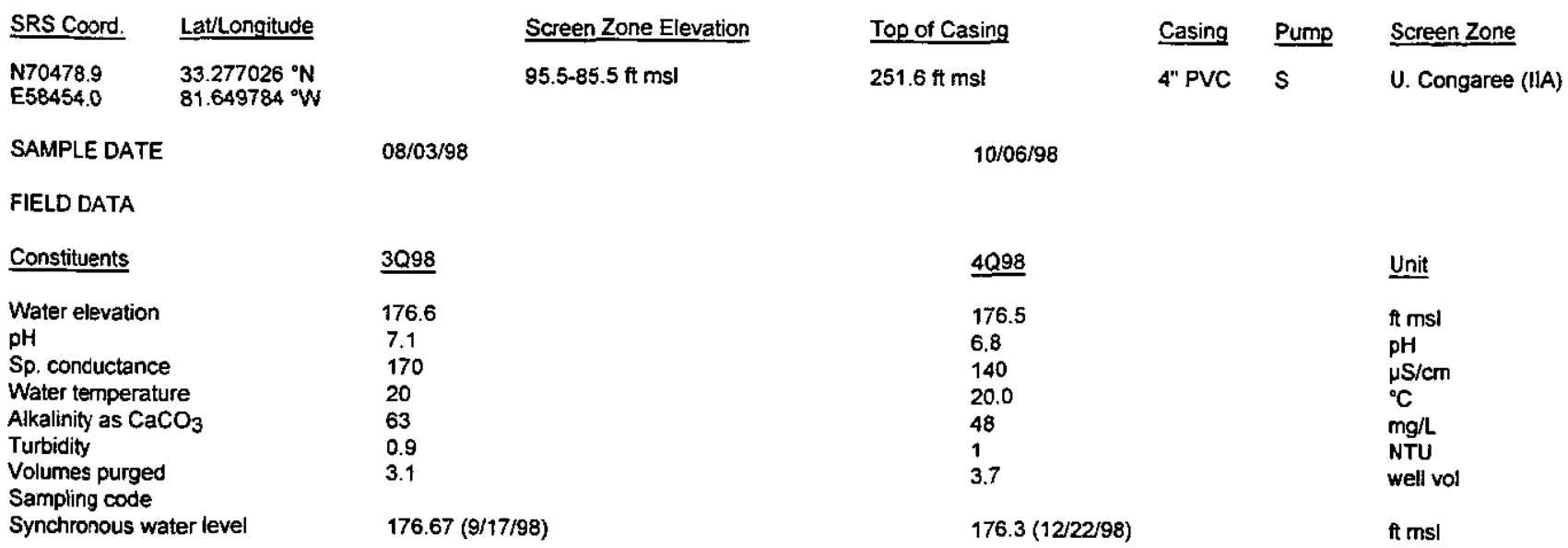

ANALYTICAL DATA

Inorganic Constituents

\section{Constituents}

Antimony, total recoverable Arsenic, total recoverable Barium, total recoverable Cadmium, total recoverable Chromium, total recoverable Cobalt, total recoverable Copper, total recoverable Cyanide

Lead, total recoverable Mercury, total recoverable Nickel, total recoverable

Nitrate as nitrogen

Nitrate-nitrite as nitrogen Selenium, total recoverable

Silver, total recoverable

Tin, total recoverable

Vanadium, total recoverable

Zinc, total recoverable

Organic Constituents

Constituents

Benzene

Bis(2-ethylhexyl) phthalate

Dichloromethane

Tetrachloroethylene

Trichloroethylene

Trichiorofluoromethane
3098

Mod

Filt.

$\underline{\text { STY }}$

DF

$\underline{L a b} \underline{4 Q 98}$

Mod

$<0.20$

1 GE $<0.20$

JU/LC

1 WA $<20$

UNI

$<50$

Filt.

ST 브 DF Lab Unit

NYGI
1 GE $\mu g / L$

1 GE $\mu g / L$

Mod

Filt.

$\underline{\text { ST }} \underline{H}$

DF 늠

4098

Mod

Filt.

Notes:

- = exceeded holding time

- exceeded groundwater protection or monitoring constituent standard (See Appendix A.) 


\section{WELL HSB146A (cont.)}

Radioactive Constituents

\section{Constituents}

Americium-241

Carbon-14

Cobalt-60

Curium-242

Curium-243/244

Curium-245/246

Gross alpha

lodine-129

Nickel-63

Nonvolatile beta

Plutonium-238

Plutonium-239/240

Radium-226

Radium-228

Strontium-90

Technetium-99

Thorium-228

Thorium-230

Thorium-232

Sum of alphas

Sum of betas

Tritium

Uranium-233/234

Uranium-235

Uranium-238
309

$1.2 E+00$

/l

$1.4 E+00$

/

$<-1.2 \mathrm{E}-01 \quad \mathrm{UI} / \mathrm{\prime}$

$<5,8 \mathrm{E}-01$

$1 \quad \mathrm{TM}<5.2 \mathrm{E}-01$

U/I

1 GP $<5.5 \mathrm{E}-01$

U/I

$<6.5 \mathrm{E}-01$

1 GP $<1.3 E-01$

$\mathrm{U} / \mathrm{I}$

GP $<1.3 E-01$
$<1.3 E+00$

Filt.

$\underline{\text { ST }}$ H DF Lab Unit
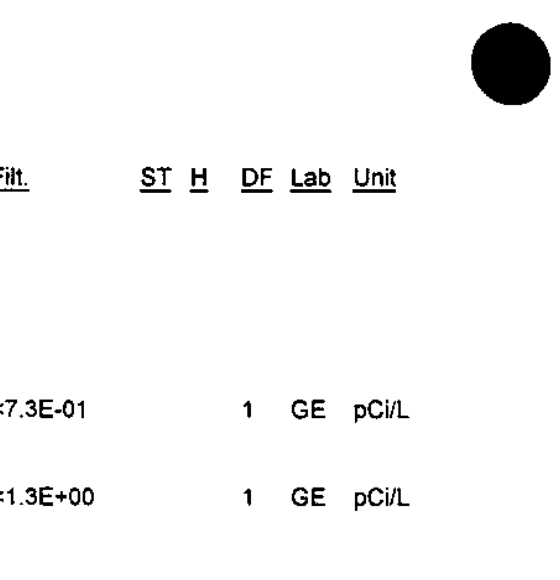

$1 \mathrm{GE} \quad \mathrm{PCi} / \mathrm{L}$

1 GE $\mathrm{pCi} / \mathrm{L}$

Notes:

- exceeded holding time

- exceeded groundwater protection or monitoring constituent standard (See Appendix A.) 
WELL HSB146C

\begin{tabular}{|c|c|c|c|c|c|c|c|}
\hline SRS Coord. & \multicolumn{2}{|l|}{ LatLongitude } & Screen Zone Elevation & Top of Casing & Casing & Pump & Screen Zone \\
\hline $\begin{array}{l}\text { N70471.6 } \\
\text { E58473.1 }\end{array}$ & \multicolumn{2}{|l|}{$\begin{array}{l}33.277041^{\circ} \mathrm{N} \\
81.649719^{\circ} \mathrm{W}\end{array}$} & $162.3-152.3 \mathrm{ft} \mathrm{msl}$ & $252.3 \mathrm{ft} \mathrm{msl}$ & 4" PVC & $s$ & Bamwell $\left(\|_{1} B_{1}\right)$ \\
\hline \multicolumn{2}{|c|}{ SAMPLE DATE } & \multicolumn{2}{|l|}{ 08/03/98 } & \multicolumn{2}{|c|}{$10 / 06 / 98$} & & \\
\hline \multicolumn{8}{|l|}{ FIELD DATA } \\
\hline \multicolumn{2}{|l|}{ Constituents } & \multicolumn{2}{|l|}{3098} & \multicolumn{2}{|l|}{$\underline{4098}$} & & Unit \\
\hline \multicolumn{2}{|c|}{ Water elevation } & \multicolumn{2}{|l|}{210.0} & \multicolumn{2}{|l|}{209.7} & & $\mathrm{ft} \mathrm{msl}$ \\
\hline & \multicolumn{2}{|l|}{8.1} & \multicolumn{2}{|l|}{6.9} & & $\mathrm{pH}$ \\
\hline \multirow{2}{*}{\multicolumn{2}{|c|}{ Water temperature }} & \multicolumn{2}{|l|}{77} & \multirow{2}{*}{\multicolumn{2}{|c|}{$\begin{array}{l}66 \\
200\end{array}$}} & & $\mu \mathrm{S} / \mathrm{cm}$ \\
\hline & & \multirow{2}{*}{\multicolumn{2}{|c|}{$\begin{array}{l}19.5 \\
21\end{array}$}} & \multirow{2}{*}{\multicolumn{2}{|c|}{$\begin{array}{l}20.0 \\
23\end{array}$}} & & ${ }^{\circ} \mathrm{C}$ \\
\hline \multicolumn{2}{|c|}{ Alkalinity as $\mathrm{CaCO}_{3}$} & & & & & & $\mathrm{mg} / \mathrm{L}$ \\
\hline \multicolumn{2}{|c|}{ Turbidity } & \multicolumn{2}{|l|}{0.4} & \multicolumn{2}{|l|}{2} & & NTU \\
\hline \multicolumn{2}{|c|}{ Volumes purged } & \multicolumn{2}{|l|}{2.6} & \multicolumn{2}{|l|}{3.2} & & well vol \\
\hline \multicolumn{2}{|c|}{$\begin{array}{l}\text { Sampling code } \\
\text { Synchronous water level }\end{array}$} & \multicolumn{2}{|c|}{$209.97(9 / 17 / 98)$} & \multicolumn{2}{|c|}{$209.3(12 / 22 / 98)$} & & $\mathrm{ftmsl}$ \\
\hline
\end{tabular}

ANALYTICAL DATA

Inorganic Constituents

Constituents

Mod

Antimony, total recoverable

Arsenic, total recoverable

Barium, total recoverable

Cadmium, total recoverable

Chromium, total recoverable

Cobalt, total recoverable

Copper, total recoverable

Cyanide

Lead, total recoverable

Mercury, total recoverable

Nickel, total recoverable

$<0.20$

U/I

$<0.20$

1 GE $<0.20$

JU/LC

$<N D D$

1 GE $\mu g / L$

Nitrate as nitrogen

Nitrate-nitrite as nitrogen

Selenium, total recoverable

$<610 \quad$ UNG/ $<50$

/I

1 GE $\mu g / L$

Silver, total recoverable

Tin, total recoverable

Vanadium, total recoverable

Zinc, total recoverable

Organic Constituents

Constituents

3098

Mod

Filt.

ST브

DF Lab $4 Q 99 \quad$ Mod

Filt.

ST 브 DF Lab Unit

Bis(2-ethylhexyl) phthalate

Dichloromethane

Tetrachloroethylene

Trichloroethylene

Trichlorofuoromethane

Notes:

- = exceeded holding time

- exceeded groundwater protection or monitoring constituent standard (See Appendix A.) 
WELL HSB146C (cont.)

Radioactive Constituents

Constituents

Americium-241

Carbon-14

Cobalt-60

Curium-242

Curium-243/244

Curium-245/246

Gross alpha

lodine-129

Nickel-63

Nonvolatile beta

Plutonium-238

Plutonium-239/240

Radium-226

Radium-228

Strontium-90

Technetium-99

Thorium-228

Thorium-230

Thorium-232

Sum of alphas

Sum of betas

Tritium

Uranium-233/234

Uranium-235

Uranium-238
$1.2 \mathrm{E}+00 \quad /$

$9.3 E+\infty \quad / /$
Filt.

ST브 DF Lab $\underline{4 Q 98} \quad \underline{\text { Mod }}$

1

GP $<1.1 E+00 \quad U / \prime$

$<5.1 E-01$

GP $<1.1 E+00 \quad U / /$

$<1.1 E+00$

GP $1.1 E+01 \quad /$
Filt.

ST 브 DF Lab Unit

$1 \mathrm{GE} \mathrm{pCi} / \mathrm{mL}$

Notes:

- exceeded holding time

- exceeded groundwater protection or monitoring constituent standard (See Appendix A.) 
WELL HSB146D

\begin{tabular}{|c|c|}
\hline SRS Coord. & Lat/Longitude \\
\hline $\begin{array}{l}\text { N70469.7 } \\
\text { E58493.0 }\end{array}$ & $\begin{array}{l}33.277069^{\circ} \mathrm{N} \\
81.649663^{\circ} \mathrm{W}\end{array}$ \\
\hline
\end{tabular}

Screen Zone Elevation

$224.1-204.0 \mathrm{ft} \mathrm{msl}$

08/03/98

$\underline{3 Q 98}$

Constituents

Water elevation

$\mathrm{pH}$

Sp. conductance

Water temperature

Alkalinity as $\mathrm{CaCO}_{3}$

Turbidity

Volumes purged

Sampling code

Synchronous water level

\section{ANALYTICAL DATA}

Inorganic Constituents

Constituents

Antimony, total recoverable

Arsenic, total recoverable

Barium, total recoverable

Cadmium, total recoverable

Chromium, total recoverable

Cobalt, total recoverable

Copper, total recoverable

Cyanide

Lead, total recoverable

Mercury, total recoverable

Nickel, total recoverable

Nitrate as nitrogen

Nitrate-nitrite as nitrogen

Selenium, total recoverable

Silver, total recoverable

Tin, total recoverable

Vanadium, total recoverable

Zinc, total recoverable

Organic Constituents

Constituents

Benzene

Bis(2-ethylhexyl) phthalate

Dichloromethane

Tetrachloroethylene

Trichloroethylene

Trichlorofluoromethane $\underline{3098}$

Mod

Filt.

ST

$<0.20$

$\mathrm{u} / /$

UN6/

$<0.20$

1 GE $<0.20$

JU/LC

$<N D D$

1 GE 260

"l

ST $\underline{H}$

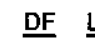

Lab

Mod

Fill.

Filt.
4Q98

222.2

5.1

24

$220.6(12 / 22 / 98)$

Casing Pump

4" PVC S

Screen Zone

Water Table (IIB2)

$\underline{\text { Unit }}$

ft msl

$\mathrm{pH}$

$\mu \mathrm{S} / \mathrm{cm}$

${ }^{\circ} \mathrm{C}$

$\mathrm{mg} / \mathrm{L}$

NTU

well vol

th msl

ST H DF Lab Unit

1 GE $\mu g / L$

Notes:

- exceeded holding time

= exceeded groundwater protection or monitoring constituent standard (See Appendix A.) 
WELL HSB146D (cont.)

Radioactive Constituents

Constituents

Americium-241

Carbon-14

Cobalt-60

Curium-242

Curium-243/244

Curium-245/246

Gross alpha

lodine-129

Nickel-63

Nonvolatile beta

Plutonium-238

Plutonium-239/240

Radium-226

Radium-228

Strontium-90

Technetium-99

Thorium-228

Thorium-230

Thorium-232

Sum of alphas

Sum of betas

Tritium

Uranium-233/234

Uranium-235

Uranium-238

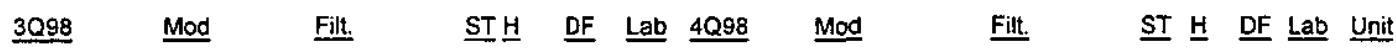

$<4.4 \mathrm{E}-01 \quad \mathrm{U} \mathrm{W} / \mathrm{<} \quad<.4950$

$1 \quad \mathrm{GP} \quad<2.7 \mathrm{E}+00 \quad \mathrm{U} / /$

$<9.7 E-01$

$1 \quad \mathrm{GP}<1.4 \mathrm{E}+\infty \quad \mathrm{U} / /$

$<2.2 E+00$

$<7.5 \mathrm{E}-01 \quad$ UII/

$<1.1200$

$\mathrm{U} / /$

$\uparrow$ GE pCir
1.1E+01 /

1 GP $\quad 1.2 \mathrm{E}+01$

$1 \mathrm{GE} \mathrm{pCi} / \mathrm{mL}$

Notes:

- exceeded holding time

U = exceeded groundwater protection or monitoring constituent standard (See Appendix A.) 
WELL HSB147D

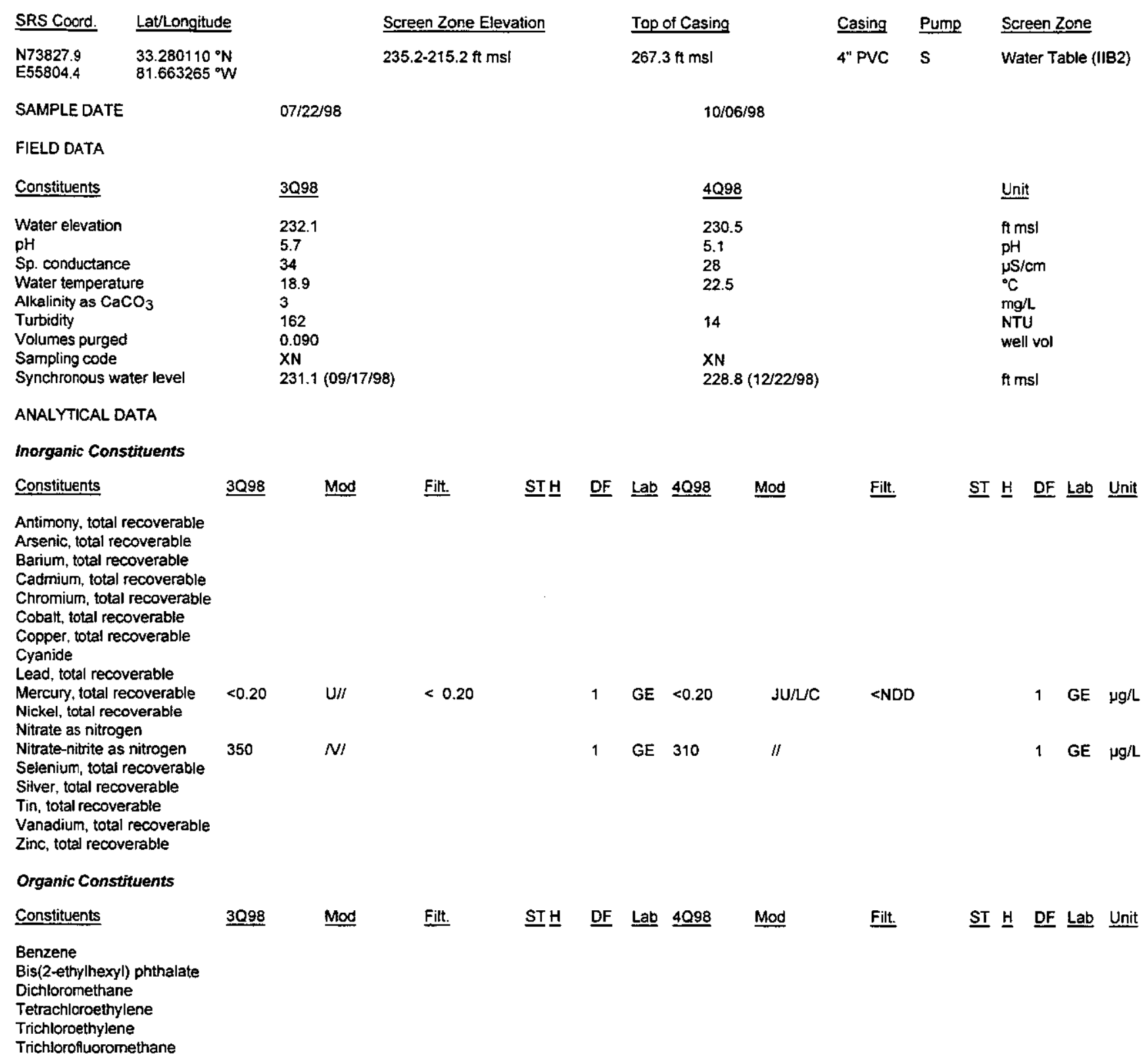

Notes:

- = exceeded holding time

= exceeded groundwater protection or monitoring constituent standard (See Appendix A.) 
WELL HSB147D (cont.)

Radioactive Constituents

Constituents

Americium-241

Carbon-14

Cobalt-60

Curium-242

Curium-243/244

Curium-245/246

Gross alpha

lodine-129

Nickel-63

Nonvoiatile beta

Plutonium-238

Plutonium-239/240

Radium-226

Radium-228

Strontium-90

Technetium-99

Thorium-228

Thorium-230

Thorium-232

Sum of alphas

Sum of betas

Tritium

Uranium-233/234

Uranium-235

Uranium-238 $\underline{3098}$

Mod

Filt.

ST브 DF Lab $\underline{4 Q 98} \quad \underline{\text { Mod }}$

$2.0 \mathrm{E}+00$

1

GP $<1.3 E+00 \quad U / 1$

GP

$<1.1 E+00$

$1 \mathrm{GE} \mathrm{PCi/L}$
$1.9 E+00 \quad / /$

1

UII

(n)

1 GE pCir

$9.0 \mathrm{E}+00 \quad / 1$

1 GP $1.1 \mathrm{E}+01 \quad / /$

$1 \mathrm{GE} \quad \mathrm{pCi} / \mathrm{mL}$

Notes:

- =xceeded holding time

- exceeded groundwater protection or monitoring constituent standard (See Appendix A.) 
WELL HSB148C

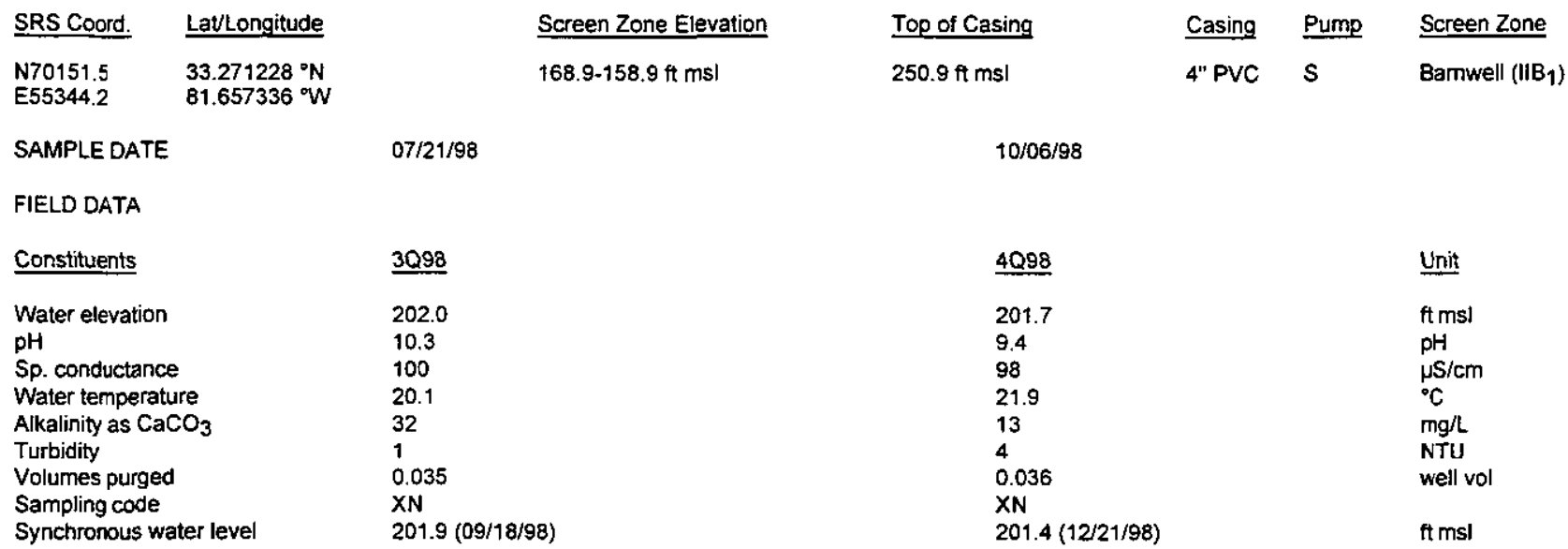

ANALYTICAL DATA

Inorganic Constituents

Constituents

Antimony, total recoverable Arsenic, total recoverable Barium, total recoverable Cadmium, total recoverable Chromium, total recoverable Cobalt, total recoverable Copper, total recoverable Cyanide

Lead, total recoverable Mercury, total recoverable Nickel, total recoverable

Nitrate as nitrogen

Nitrate-nitrite as nitrogen

Selenium, total recoverable

Silver, total recoverable

Tin, total recoverable

Vanadium, total recoverable

Zinc, total recoverable

Organic Constituents

Constituents

JU/UC

1 GE 540

"l

Benzene

Bis(2-ethylhexyl) phthalate

Dichloromethane

Tetrachloroethylene

Trichloroethylene

Trichlorofiuoromethane 


\section{WELL HSB148C (cont.)}

Radioactive Constituents

Constituents

Americium-241

Carbon-14

Cobalt-60

Curium-242

Curium-243/244

Curium-245/246

Gross alpha

lodine-129

Nickel-63

Nonvolatile beta

Plutonium-238

Plutonium-239/240

Radium-226

Radium-228

Strontium-90

Technetium-99

Thorium-228

Thorium-230

Thorium-232

Sum of alphas

Sum of betas

Tritium

Uranium-233/234

Uranium-235

Uranium-238
3Q98

Mod

Filt.

ST $\underline{H}$
Filt.

ST 브 DF Lab Unit
1

GP $<1.2 E+00 \quad U / /$

1 GP $2.6 \mathrm{E}+00 \quad / 1$

$3.0 \mathrm{E}+00 \quad / 1$

$1.8 \mathrm{E}+00 \quad / 1$
GP $1.8 E+00$
1 GE $\mathrm{pCi} / \mathrm{L}$

1 GE $\mathrm{pCi} / \mathrm{L}$

$1 \mathrm{GE} \mathrm{pCi} / \mathrm{mL}$

Notes:

- exceeded holding time

E = exceeded groundwater protection or monitoring constituent standard (See Appendix A.) 
WELL HSB148D

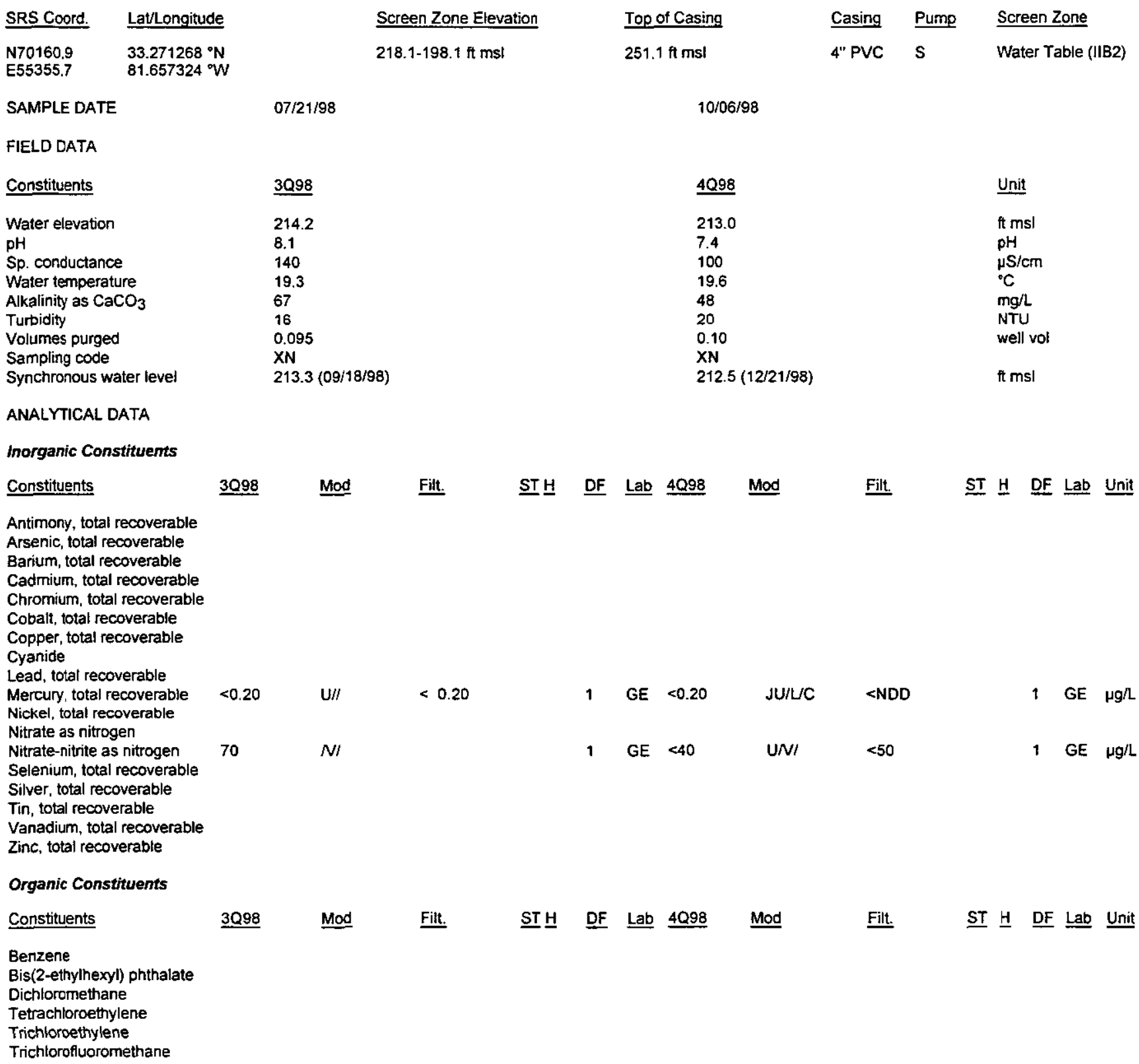

Notes:

- = exceeded holding time

- exceeded groundwater protection or monitoring constituent standard (See Appendix A.) 


\section{WELL HSB148D (cont.)}

Radioactive Constituents

\section{Constituents}

3098

Mod

Filt.

ST브 DF Lab $\underline{4098}$ Mod

Filt.

ST 브 DF Lab Unit

Americium-241

Carbon-14

Cobalt -60

Curium-242

Curium-243/244

Curium-245/246

Gross alpha

lodine-129

$1.6 E+01 \quad / /$

- 1 GP $8.9 E+00 \quad / /$

1 GE $\mathrm{pCi} / \mathrm{L}$

Nickel-63

Nonvolatile beta

Plutonium-238

Plutonium-239/240

Radium-226

Radium-228

Strontium-90

Technetium-99

Thorium-228

Thorium-230

Thorium-232

Sum of alphas

Sum of betas

Tritium

Uranium-233/234

$1.7 E+01 \quad / /$

1 GP $8.5 E+00$

1 GE $\mathrm{pCi} / \mathrm{L}$

Uranium-235

Uranium-238 
WELL HSB149D

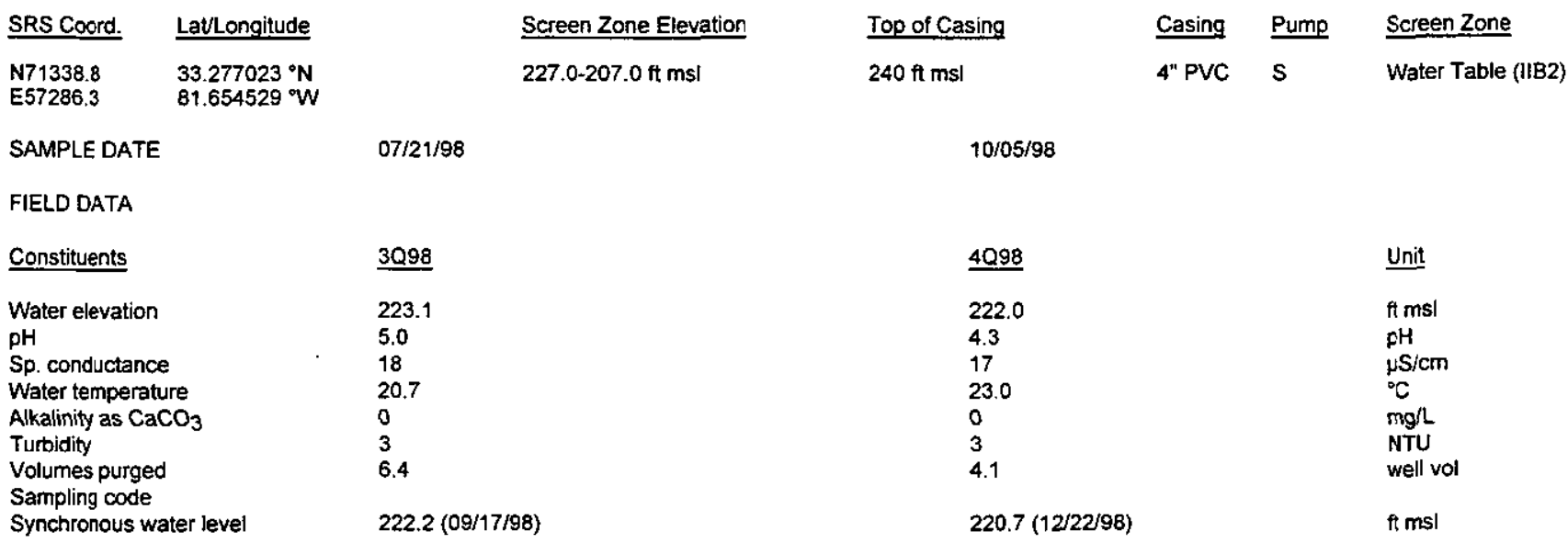

ANALYTICAL DATA

Inorganic Constituents

Constituents

Antimony, total recoverable

Arsenic, total recoverable

Barium, total recoverable

Cadmium, total recoverable

Chromium, total recoverable

Cobalt, total recoverable

Copper, total recoverable

Cyanide

Lead, total recoverable

Mercury, total recoverable

Nickel, total recoverable

Nitrate as nitrogen

Nitrate-nitrite as nitrogen

Selenium, totai recoverable

Silver, total recoverable

Tin, total recoverabie

Vanadium, total recoverable

Zinc, total recoverable

Organic Constituents

Constituents
Mod

Filt.

$\underline{\text { ST }}$

1

GE

$<0.20$

UII

1 GE 280

II

Benzene

Bis(2-ethylhexyl) phthalate

Dichloromethane

Tetrachloroethylene

Trichlorcethylene

Trichlorofluoromethane
NI

220

Mod
Filt.

ST브
DF Lab
4098
Mod
$<0.20$

Filt.
Filt.

ST 브 DF Lab Unit

Notes:

- exceeded holding time

= exceeded groundwater protection or monitoring constituent standard (See Appendix A.) 
WELL HSB149D (cont.)

Radioactive Constituents

Constituents

Americium-241

Carbon-14

Cobalt-60

Curium-242

Curium-243/244

Curium-245/246

Gross alpha

lodine-129

Nickel-63

Nonvolatile beta

Plutonium-238

Plutonium-239/240

Radium-226

Radium-228

Strontium-90

Technetium-99

Thorium-228

Thorium-230

Thorium-232

Sum of alphas

Sum of betas

Tritium

Uranium-233/234

Uranium-235

Uranium-238
$3 Q 98$

7.1E-01

ll

$<6.6 \mathrm{E}-01$

UII/

$<1.1500$

1 GP $<5.9 E-01$

U/I

1 GP $<7.7 E-01$

$\mathrm{U} / /$

1 GE $\rho \mathrm{Ci} / \mathrm{L}$

1 GE $p C i / L$

1.9E+01 $/ /$

1 GP 2.4E+01 $/$

- 1 GE $p \mathrm{Ci} / \mathrm{mL}$

Notes: 
WELL HSB150D

$\begin{array}{ll}\text { SRS Coord. } & \text { Latllongitude } \\ \text { N71692.6 } & 33.280100^{\circ} \mathrm{N}\end{array}$

SAMPLE DATE
Screen Zone Elevation

226.9-206.9 ft ms

$08 / 03 / 98$
Top of Casing

$239 \mathrm{ft} \mathrm{ms}$
Casing Pump

4" PVC S
Screen Zone

Water Table (IIB2)

FIELD DATA

\begin{tabular}{ll} 
Constituents & $\underline{3098}$ \\
\hline Water elevation & 224.6 \\
pH & 5.1 \\
Sp. conductance & 49 \\
Water temperature & 21.1 \\
Alkalinity as $\mathrm{CaCO}_{3}$ & 0 \\
Turbidity & 8.2 \\
Volumes purged & 0.086 \\
Sampling code & $\mathrm{XN}$ \\
Synchronous water level & $225.9(9 / 17 / 98)$
\end{tabular}

10/12/98

ANALYTICAL DATA

Inorganic Constituents

\begin{tabular}{|c|c|c|c|}
\hline Constituents & $\underline{3098}$ & Mod & Filt. \\
\hline Antimony, total recoverable & $<0.20$ & $\mathrm{U} / 1$ & $<0.20$ \\
\hline Arsenic, total recoverable & $<3.0$ & $\mathrm{U} / /$ & \\
\hline Barium, total recoverable & 7.5 & "I & \\
\hline Cadmium, total recoverable & $<1.0$ & $\mathrm{u} / \mathrm{I}$ & $<1.0$ \\
\hline Chromium, total recoverable & 6.9 & $\|$ & \\
\hline Cobalt, total recoverable & 0.30 & $\|$ & \\
\hline Copper, total recoverable & 13 & "I & \\
\hline Cyanide & $<10$ & $\mathrm{U} / /$ & $<10$ \\
\hline Lead, total recoverable & 1.7 & $\mathrm{~J} / \mathrm{E} /$ & NDD \\
\hline Mercury, total recoverable & 0.060 & $\mathrm{~J} / \mathrm{E} /$ & NDD \\
\hline Nickel, total recoverable & 5.0 & "I & \\
\hline Nitrate as nitrogen & & & \\
\hline Nitrate-nitrite as nitrogen & 2,500 & $N /$ & \\
\hline Selenium, total recoverable & $<5.0$ & $\mathrm{U} / /$ & $<5.0$ \\
\hline Silver, total recoverable & $<1.0$ & $\mathrm{U} / /$ & $<1.0$ \\
\hline Tin, total recoverable & $<2.0$ & $\mathrm{U} / /$ & 2.0 \\
\hline Vanadium, total recoverable & $<2.0$ & $\mathrm{U} / /$ & 2.0 \\
\hline & & $\mathrm{U} / /$ & \\
\hline
\end{tabular}

ST브

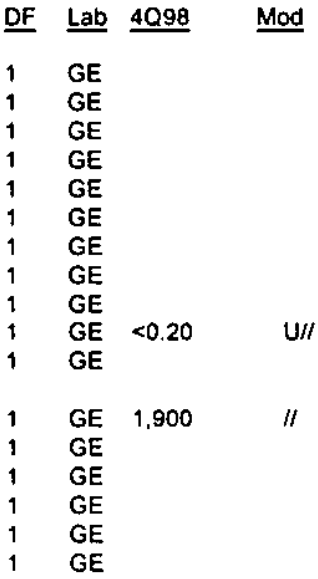

Organic Constituents

Constituents

3098

$\begin{array}{ll}\text { Mod } & \text { Filt. } \\ U / I & <1.0 \\ U / \prime & <9.9 \\ U \prime \prime & <1.0 \\ U / \prime & <1.0 \\ U \prime \prime & <1.0 \\ U \prime \prime & <1.0\end{array}$

ST브

$\begin{array}{ll}\text { DF } & \text { Lab } \\ 1 & \text { GE } \\ 1 & \text { GE } \\ 1 & \text { GE } \\ 1 & \text { GE } \\ 1 & \text { GE } \\ 1 & \text { GE }\end{array}$

4098
225.4
4.7
38
22.3
0
63
0.082
$X N$
$224.6(12 / 22 / 98)$

Unit

ft ms

$\mathrm{pH}$

$\mathrm{S} / \mathrm{cm}$

${ }^{\circ} \mathrm{C}$

$\mathrm{mg} / \mathrm{l}$

NTU

well vol

$\mathrm{ft} \mathrm{msi}$

Benzene $<10$

Bis(2-ethylhexyl) phthalate $<9.9$

Dichloromethane $<1.0$

Tetrachloroethylene $<10$

Trichloroethylene $\quad<1.0$

Trichlorofluoromethane

$<1.0$

$<1.0$

4Q98 Mod

Filt.

ST $\underline{H} \underline{\text { DF }} \underline{\text { Lab }} \underline{\text { Unit }}$

$\mu g / L$

$\mu \mathrm{g} / \mathrm{L}$

$\mu g / L$

$\mu g / L$

$\mu g / L$

$\mu g / L$

Notes:

- = exceeded holding time

- = exceeded groundwater protection or monitoring constituent standard (See Appendix A.) 
WELL HSB150D (cont.)

Radioactive Constituents

\begin{tabular}{|c|c|c|c|c|c|c|c|c|c|c|c|c|c|}
\hline Constituents & $\underline{3098}$ & Mod & Fitt. & STY $\underline{H}$ & $\underline{\text { DF }}$ & $\underline{\text { Lab }}$ & 4Q98 & Mod & Filt. & $\underline{\mathrm{ST}} \underline{\mathrm{H}}$ & $\underline{\mathrm{DF}}$ & $\underline{L a b}$ & Unit \\
\hline Americium-241 & $<-6.5 \mathrm{E}-03$ & $\mathrm{UI} / l$ & $<0.0977$ & & 1 & GP & & & & & & & $\mathrm{PCi} / \mathrm{L}$ \\
\hline Beta dose & 0.30 & & & & & & & & & & & & $\mathrm{pCi} / \mathrm{L}$ \\
\hline Carbon-14 & $<1.2 \mathrm{E}-01$ & $U 1 / /$ & $<8.9700$ & & 1 & GP & & & & & & & DCilL \\
\hline Cobalt-60 & $<4.7 E-01$ & $\mathrm{U} 1 / 1$ & $<4.0700$ & & 1 & GP & & & & & & & $\mathrm{pCi} / \mathrm{L}$ \\
\hline Curium-242 & $<0.0 \mathrm{E}+00$ & $\mathrm{UI} / \mathrm{I}$ & $<0.1060$ & & 1 & GP & & & & & & & $\mathrm{pCi} / \mathrm{L}$ \\
\hline Curium-243/244 & $<-5.9 \mathrm{E}-03$ & UIIII & $<0.1620$ & & 1 & GP & & & & & & & $\mathrm{pCi} / \mathrm{L}$ \\
\hline Curium-245/246 & $<0.0 E+00$ & $\mathrm{UI} / \mathrm{I}$ & $<0.0976$ & & 1 & GP & & & & & & & $\mathrm{pCi} / \mathrm{L}$ \\
\hline Gross alpha & $<5.2 \mathrm{E}-01$ & $\mathrm{UI} / /$ & $<0.7920$ & & 1 & GP & $<9.8 \mathrm{E}-01$ & $\mathrm{U} / \mathrm{I}$ & $<1.0 \mathrm{E}+00$ & & 1 & GE & $\mathrm{pCi} / \mathrm{L}$ \\
\hline lodine-129 & $<1.1 E+00$ & UN & $<0.7300$ & & 1 & GP & & & & & & & $\mathrm{pC} / \mathrm{L}$ \\
\hline Nickel-63 & $<4.7 E-01$ & UI & $<4.0700$ & & & & & & & & & & $\mathrm{PCill}$ \\
\hline Nonvolatile beta & $2.5 \mathrm{E}+00$ & "I & & & 1 & GP & $<2.4 E+00$ & $\mathrm{U} / /$ & $<1.5 E+\infty 0$ & & 1 & GE & $\mathrm{pCilL}$ \\
\hline Plutonium-238 & $<-1.7 \mathrm{E}-02$ & $\mathrm{U} 1 / /$ & $<0.2230$ & & 1 & GP & & & & & & & pCill \\
\hline Plutonium-239/240 & $<2.7 \mathrm{E}-02$ & $\mathrm{U} 1 / /$ & $<0.1890$ & & 1 & GP & & & & & & & $\mathrm{pCill}$ \\
\hline Radium-226 & $<4.7 \mathrm{E}-01$ & $\mathrm{UI} / / \mathrm{I}$ & $<0.6340$ & & 1 & GP & & & & & & & $\mathrm{pCi} / \mathrm{L}$ \\
\hline Radium-228 & $1.5 \mathrm{E}+00$ & "I & & & 1 & GP & & & & & & & $\mathrm{pCill}$ \\
\hline Strontium-90 & $<6.5 \mathrm{E}-02$ & UIJ/CI & & & 1 & GP & & & & & & & $\mathrm{pCi} / \mathrm{L}$ \\
\hline Technetium-99 & $<5.8 E+00$ & $\mathrm{Ut} / \mathrm{I}$ & $<6.6000$ & & 1 & GP & & & & & - & & $\mathrm{pCil}$ \\
\hline Thorium-228 & $<1,1 \mathrm{E}-02$ & $\mathrm{U} / / /$ & $<0.2810$ & & 1 & GP & & & & & & & $\mathrm{pCV} / \mathrm{L}$ \\
\hline Thorium-230 & $<1.9 E-01$ & $\mathrm{UI} / /$ & $<0.2090$ & & 1 & GP & & & & & & & $\mathrm{pCill}$ \\
\hline Thorium-232 & $<-1.9 \mathrm{E}-02$ & $\mathrm{UI} / \mathrm{I}$ & $<0.2470$ & & 1 & GP & & & & & & & pCill \\
\hline Sum of alphas & & & & & & & & & & & & & \\
\hline Sum of betas & $\begin{array}{l}1.5 \mathrm{E}+00 \\
1.5 \mathrm{E}+00\end{array}$ & & & & & & & & & & & & DCi/L \\
\hline Total radium & $1.5 \mathrm{E}+00$ & $1 /$ & & n & & & $6.4 F+01$ & & & & & & pCirl \\
\hline Tstium & $5.8 E+01$ & $\mathrm{U} \mid 1 /$ & $<0.1360$ & 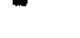 & 9 & GP & $6.4 E+01$ & 11 & & 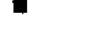 & 1 & GE & $\mathrm{pCi} / \mathrm{mL}$ \\
\hline Uranium-233/234 & $<3.4 \mathrm{E}-02$ & UIII & $<0.1360$ & & 1 & GP & & & & & & & $\mathrm{pCi} / \mathrm{L}$ \\
\hline Uranium-235 & $<0.0 E+00$ & UIII & $<0.0546$ & & 1 & GP & & & & & & & $\mathrm{pCil}$ \\
\hline Uranium-238 & $<2.7 \mathrm{E}-02$ & $\mathrm{UV} / /$ & $<0.1160$ & & 1 & GP & & & & & & & $\mathrm{pCi} / \mathrm{L}$ \\
\hline
\end{tabular}




\section{WELL HSB151C}

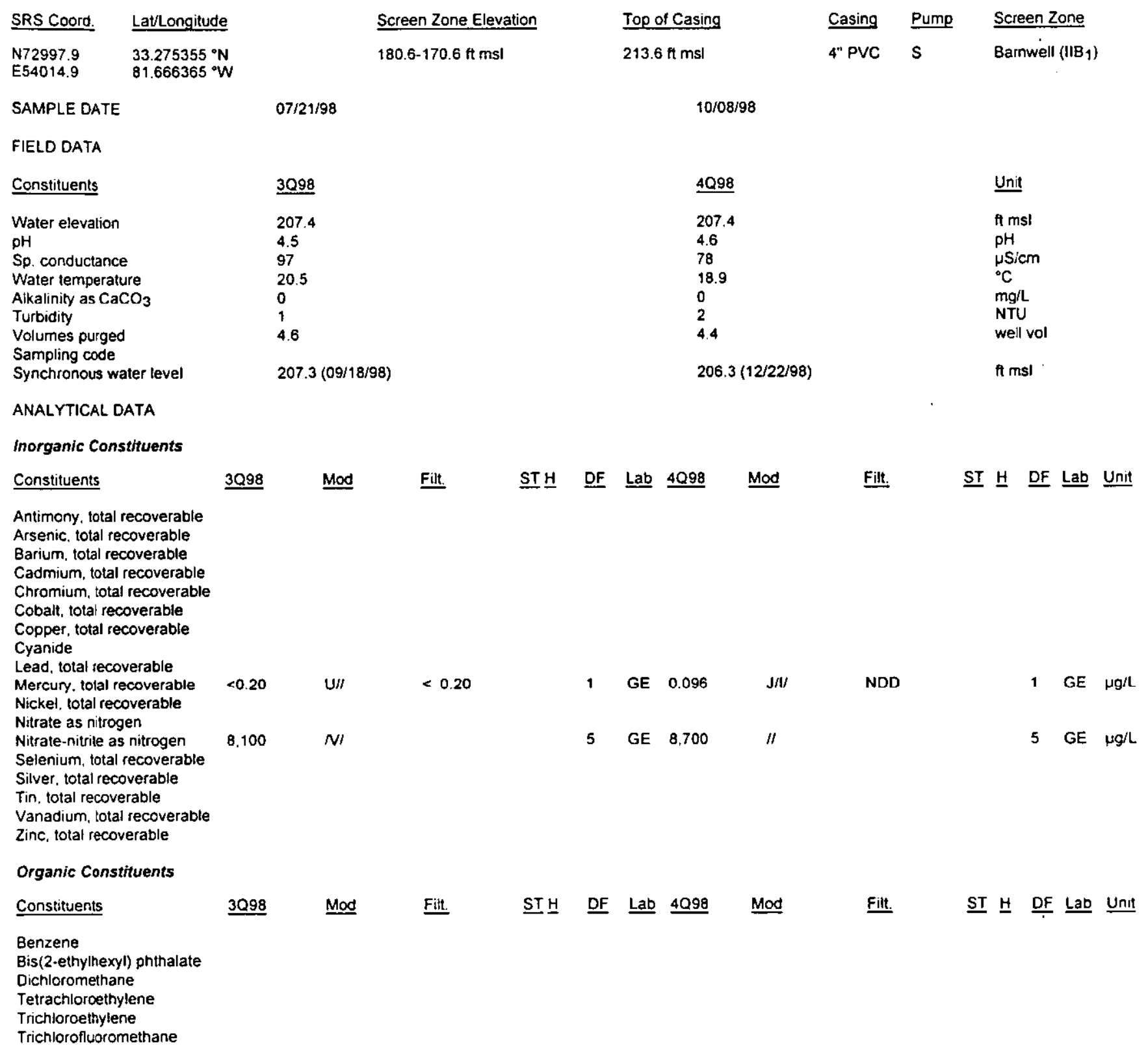

\section{Notes:}

- = exceeded holding time

- exceeded groundwater protection or monitoring constituent standard (See Appendix A.) 


\section{WELL HSB151C (cont.)}

\section{Radloactive Constituents}

Constituents

Americium-241

Carbon-14

Cobalt-60

Curium-242

Curium-243/244

Curium-245/246

Gross alpha

lodine-129

Nickel-63

Nonvolatile beta

Plutonium-238

Plutonium-239/240

Radium-226

Radium-228

Strontium-90

Technetium-99

Thorium-228

Thorium-230

Thorium-232

Sum of alphas

Sum of betas

Tritium

Uranium-233/234

Uranium-235

Uranium-238
3098

$1.9 E+00$

1

GP 9.6E-01

$\mathrm{J} / \mathrm{L} / \mathrm{I}$

NDD

1 GP $6.8 E+00$

Jル/

NDD

$9.1 E+00 / /$

$9.8 \mathrm{E}+02$

/I

GP $1.1 \mathrm{E}+03 \quad / /$
Filt.

ST $\underline{H}$ OF Lab Unit

$1 \mathrm{GE} \mathrm{pCi} / \mathrm{L}$

- 1 GE pCi/mL

Notes:

- = exceeded holding time

- exceeded groundwater protection or monitoring constituent standard (See Appendix A.) 


\section{WELL HSB151D}

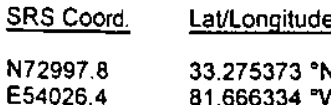

SAMPLE DATE

FIELD DATA

Constituents

Water elevation

$\mathrm{pH}$

$\mathrm{Sp}$. conductance

Water temperature

Alkalinity as $\mathrm{CaCO}_{3}$

Turbidity

Volumes purged

Sampling code

Synchronous water level

ANALYTICAL OATA

Inorganic Consthuents

Constituents

Antimony, total recoverable

Arsenic. total recoverable

Barium, total recoverable

Cadmium, total recoverable

Chromium, total recoverable

Cobalt, total recoverable

Copper, tolal recoverable

Cyanide

Lead, total recoverable

Mercury, Iotal recoverable

Nickel, total recoverable

Nitrate as nitrogen

Nitrate-nitrite as nitrogen

Selenium, total recoverable

Silver, total recoverable

Tin, total recoverable

Vanadium, total recoverable

Zinc totad recoverable

Organic Constituents

Constituents

\section{Screen Zone Elevation}

$207.6-197.6 \mathrm{ft} \mathrm{ms}$

Top of Casing

$213.6 \mathrm{ft} \mathrm{ms}$

$10 / 08 / 98$

3098

206.6

4.3

41

21.0

8.9

$206.6(09 / 18 / 98)$
4Q98

206.0

24

21.5

7.1

$205.4(12 / 22 / 98)$
Casing Pump

4" PVC S

\section{Screen Zone}

Water Table (IB2)

$\underline{3098} \quad \underline{M a d} \quad \underline{\text { Filt. }} \quad \underline{\mathrm{ST}} \underline{\mathrm{H}} \quad \underline{\mathrm{DF}} \underline{\text { Lab }} \underline{4098} \quad \underline{\text { Mod }} \quad \underline{\text { Filt. }} \underline{\text { ST }} \underline{\mathrm{H}} \underline{\mathrm{DF}} \underline{\underline{\text { Lab}}} \underline{\text { Unit }}$

$1,700 \quad N$

1 GE $<0.20$

$\mathrm{U} / I$

1 GE 1,800

$<0.20$

1 GE $\mu g / L$

1 GE $\mu g / L$

Benzene

Bis(2-ethylhexyl) phthalate

Dichloromethane

Tetrachioroethylene

Trichloroethylene

Trichlorofluoromethane

Notes

- = exceeded holding time

- exceeded groundwater protection or monitoring constituent standard (See Appendix A.) 


\section{WELL HSB151D (cont.)}

Radioactive Constituents

Constituents

Americium-241

Carbon-14

Cobalt-60

Curium-242

Curium-243/244

Curium-245/246

Gross alpha

lodine-129

Nickel-63

Nonvolatile beta

Plutonium-238

Plutonium-239/240

Radium-226

Radium-228

Strontium-90

Technetium-99

Thorium-228

Thorium-230

Thorium-232

Sum of alphas

Sum of betas

Tritium

Uranium-233/234

Uranium-235

Uranium-238
3098

Mod

Filt.

STH

DF Lab $\underline{4 Q 98}$

Mod

$1.0 E+\infty 0$

$1.8 E+00 \quad / 1$

1

GP $<1.5 E-01$

JU/LI

1 GP $<1.0 E+00 \quad J U / U 1$

$$
4.4 E+01 \quad / /
$$

GP $4.3 E+01 \quad / 1$

$<N D D$

Filt. $\quad \underline{\text { ST }} \underline{\mathrm{D}}$ DE Lab Unit

1 GE $p C i / L$

1 GE $p C i / L$

Notes:

- = exceeded holding time

- exceeded groundwater protection or monitoring constituent standard (See Appendix A.) 


\section{WELL HSB152C}

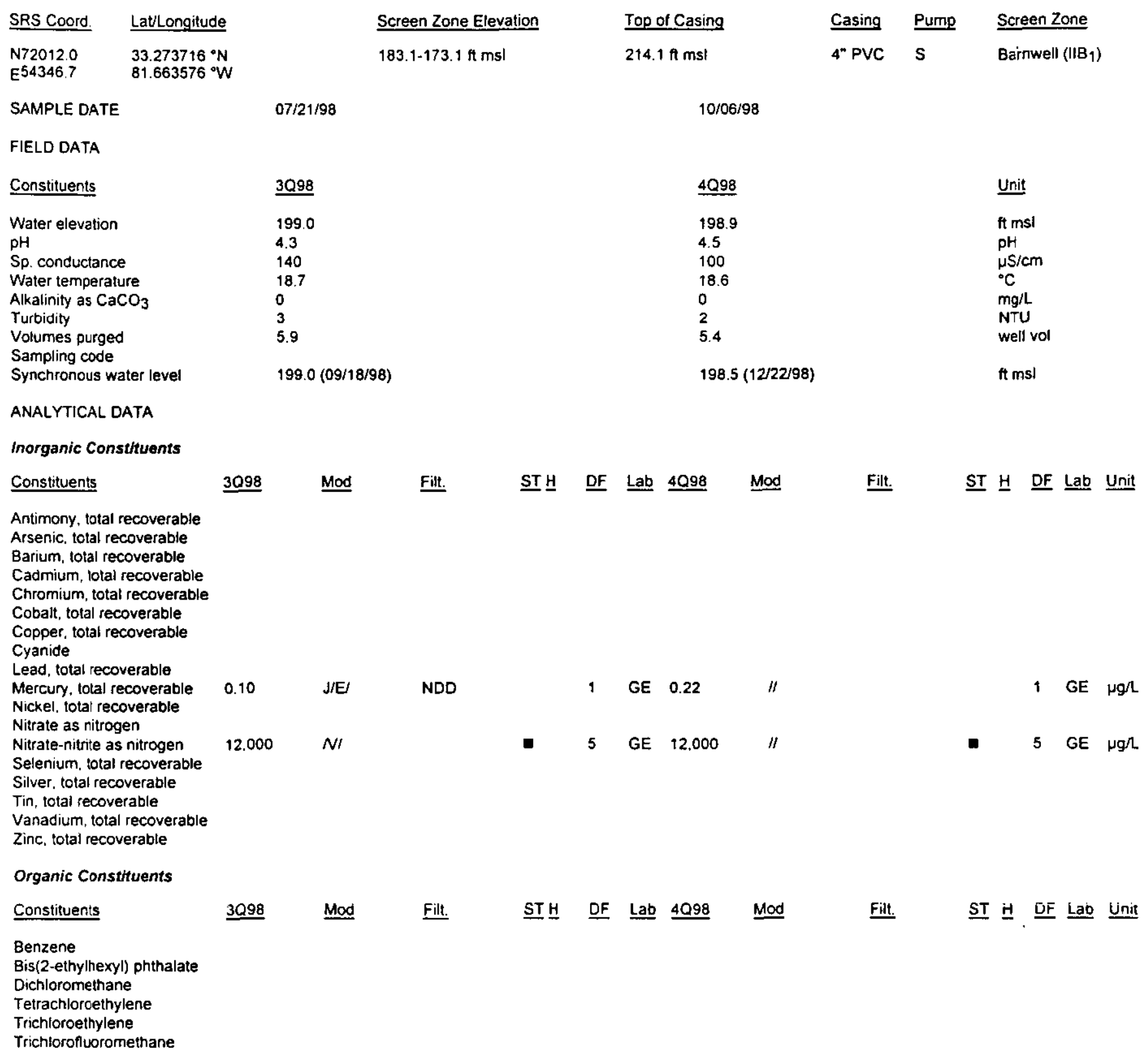

Notes:

- = exceeded holding time

W = exceeded grouncwater protection or monitoring constituent standard (See Appendix A.) 
WELL HSB152C (cont.)

Radioactive Constituents

Constituents

3098

Mod

Filt.

STH 므 Lab $\underline{4 Q 98}$ Mod

Filt.

ST H DF Lab Unit

Americium-241

Carbon-14

Cobalt-60

Curium-242

Curium-243/244

Curium-245/246

Gross alpha

$1.6 \mathrm{E}+\infty \quad / 1$

lodine-129

Nickel-63

Nonvolatile beta

2.6E+01

1

GP $\quad<1.8 \mathrm{EE}+00 \quad$ UII

1

GP $\quad 1.7 \mathrm{E}+01 \quad / /$

Plutonium-239/240

Radium-226

Radium-228

Strontium-90

Technetium-99

Thorium-228

Thorium-230

Thorium-232

Sum of alphas

Sum of betas

Tritium

Uranium-233/234

Uranium-235

Uranium-238

Notes:

- = exceeded holding time

- exceeded groundwater protection or monitoring constituent standard (See Appendix A.)

H-Area HWMF. 
WELL HSB152D

$\begin{array}{ll}\text { SRS Coord. } & \text { Latllongitude } \\ \text { N72011.7 } & 33.273740^{\circ} \mathrm{N} \\ \text { E54362.1 } & 81.663535^{\circ} \mathrm{W}\end{array}$

SAMPLE DATE

FIELD DATA

Constituents

Water elevation

$\mathrm{pH}$

Sp. conductance

Water temperature

Alkalinity as $\mathrm{CaCO}_{3}$

Jurbidity

Volumes purged

Sampling code

Synchronous water level

ANALYTICAL DATA

inorganic Constituents

Constituents

Antimony, total recoverable Arsenic, total recoverable

Barium, total recoverable

Cadmium, total recoverable

Chromium, total recoverable

Cobalt, total recoverable

Copper, total recoverable

Cyanide

Lead, total recoverable

Mercury, total recoverable

Nickel, total recoverable

Nitrate as nitrogen

Nitrate-nitrite as nitrogen

Selerium, total recoverable

Silver, total recoverable

Tin, total recoverable

Vanadium, total recoverable

Zinc, total recoverable

Organic Constituents

Constituents

309

$2,100 \quad N I$

\section{Screen Zone Elevation \\ 207.0-197.0 ft msI}

Top of Casing

$214.1 \mathrm{ft} \mathrm{ms}$

10/06/98

$\underline{4 Q 98}$

5.0

36

22.3

2

13

NSX

(09/18/98)
Casing Pump

4" PVC S

\section{Screen Zone}

Water Table (IIB 2$)$

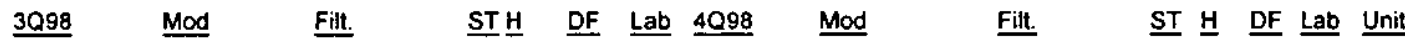

U/I

$<0.20$

$1 \quad$ GE $<0.20$

U/I

$<0.20$

1 GE $\mu g / L$

1 GE 700

$1 \mathrm{GE} \mu g / \mathrm{L}$

Benzene

Bis(2-ethylhexyl) phthalate

Dichloromethane

Tetrachloroethylene

Trichloroethylene

Trichlorofluoromethane

Notes:

- exceeded holding time

- exceeded groundwater protection or monitoring constituent standard (See Appendix A.) 
WELL HSB152D (cont.)

Radioactive Constituents

Constituents

Americium-241

Carbon-14

Cobalt-60

Curium-242

Curium-243/244

Curium-245/246

Gross atpha

locine-129

Nickel-63

Nonvolatile beta

Plutonium-238

Plutonium-239/240

Radium-226

Radium-228

Strontium-90

Technetium-99

Thorium-228

Thorium-230

Thorium-232

Sum of alphas

Sum of betas

Tritium

Uranium-233/234

Uranium-235

Uranium-238

3098 Mod Filt. $\underline{\text { STH}}$ DF Lab $\underline{4098}$ Mod $\quad \underline{\text { Filt. }} \underline{\text { ST }} \underline{H}$ DF Lab Unit

1 GP $3.1 E+01 \quad / /$

$1 \mathrm{GP}<1.6 \mathrm{E}+01 \quad \mathrm{U} / 1$

$4.2 \mathrm{E}+00 \quad / 1$

$1.6 \mathrm{E}+02 \quad / 1$

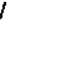

$<9.3 E+00$

- 1 GE $\mathrm{pCi} / \mathrm{L}$

1 GE $\mathrm{pCi} / \mathrm{L}$

Notes:

- = exceeded holding time

- = exceeded grouncwater protection or monitoring constituent standard (See Appendix A.) 
WELL HSL 1D

\begin{tabular}{|c|c|}
\hline SRS coord. & LatLongitude \\
\hline $\begin{array}{l}\text { N72179.6 } \\
\text { E58925.0 }\end{array}$ & 8165184 \\
\hline
\end{tabular}

SAMPLE DATE

FIELD DATA

Constituents

Water elevation

pH

Sp. conductance

Water temperature

Alkalinity as $\mathrm{CaCO}_{3}$

Turbidity

Volumes purged

Sampling code

Synchronous water level

ANALYTICAL DATA

Inorganic Constituents

Constituents

Mod

Filt.

$<1.0$

Arsenic, total recoverable

Barium, total recoverable

Cadmium, total recoverable Chromium, total recoverable

Cobalt, total recoverable

Copper, total recoverable

Cyanide

Lead, total recoverable

Mercury, total recoverable

0.48

$<0.20$

J/E/

NDD

Nitrate as nitrogen

Nitrate-nitrite as nitrogen

2.900

U/I

$<0.20$

NI

Selenium, total recoverable

Silver, total recoverable

Tin, total recoverable

Vanadium, total recoverable

Zinc, total recoverable

Organic Constituents

Constituents
Screen Zone Elevation

239.8-219.8 $\mathrm{ft} \mathrm{msl}$

Top of Casing

$264 \mathrm{ft} \mathrm{msl}$

10/19/98

4Q98

237.3

3.8

50

21.5

3.1

$235.4(12 / 21 / 98)$
Casing Pump

2" PVC V

\section{Screen Zone}

Water Table ( $\left.\mathrm{IIB}_{2}\right)$

Benzene

Bis(2-ethylhexyl) phthalate

Dichloromethane

Tetrachloroethylene

Trichloroethylene

Trichlorofluoromethane

Notes:

- =xceeded holding time

= exceeded groundwater protection or monitoring constituent standard (See Appendix A.) 
WELL HSL 1D (cont.)

Radioactive Constituents

Constituents

Filt. ST브 DF Lab $\underline{4098}$ Mod

Filt.

$\underline{\text { ST }} \underline{\mathrm{H}}$ DF Lab Unit

Americium-241

Carbon-14

Cobalt-60

Curium-242

Curium-243/244

Curium-245/246

Gross alpha

1

GP $\quad 4.8 \mathrm{E}+00 \quad / /$

1 GE $\mathrm{pCi} / \mathrm{L}$

lodine-129

Nickel-63

Nonvolatile beta

$1.6 \mathrm{E}+01 / /$

1 GP $1.8 \mathrm{E}+01 \quad / /$

$1 \mathrm{GE} \mathrm{pCi/L}$

Plutonium-238

Plutonium-239/240

Radium-226

Radium-228

Strontium-90

Technetium-99

Thorium-228

Thorium-230

Thorium-232

Sum of alphas

Sum of betas

Tritium

$7.7 \mathrm{E}+01 \quad / 1$

GP $1.2 \mathrm{E}+02 \quad / 1$

- 1 GE $\mathrm{pCi} / \mathrm{mL}$

Uranium-233/234

Uranium-235

Uranium-238

Notes:

- = exceeded holding time

a = exceeded groundwater protection or monitoring constituent standard (See Appendix A.) 


\section{WELL HSL 2D}

$\begin{array}{ll}\text { SRS Coord. } & \text { Latllongitude } \\ \text { N72190.8 } & \\ \text { E59423.5 } & 33.282394^{\circ} \mathrm{N} \\ 81.650556^{\circ} \mathrm{W}\end{array}$

SAMPLE DATE

FIELD DATA

Constituents

Water elevation

$\mathrm{pH}$

Sp. conductance

Water temperature

Alkalinity as $\mathrm{CaCO}_{3}$

Turbidity

Volumes purged

Sampling code

Synchronous water level

ANALYTICAL DATA

Inorganic Constituents

Constituents

Mod

Filt.

$\underline{\text { ST }} \underline{H}$

DF Lab 4 QQ98

Mod

Antimony, total recoverable

Arsenic, total recoverable

Barium, total recoverable

Cadmium, total recoverable

Chromium, total recoverable

$<1.0$

Cobalt, total recoverable

Copper, total recoverable

Cyanide

Lead, total recoverable

Mercury, total recoverable

Nickel, total recoverable

Nitrate as nitrogen

Nitrate-nitrite as nitrogen

Selenium, total recoverable

Silver, total recoverable

Tin, total recoverable

Vanadium, total recoverable

Zinc. total recoverable

Organic Constituents

Constituents

0.046

$J / E$

NDD

1 GE $<0.20$

U/I

1 GE 890

II

$980 \quad N I$

Benzene

Bis(2-ethylhexyl) phthalate

Dichloromethane

Tetrachloroethylene

Trichloroethylene

Trichlorofluoromethane
$265.5 \mathrm{ft} \mathrm{ms!}$

$10 / 19 / 98$

4Q98

242.6

4.4

0

3.9

$240.6(12 / 22 / 98)$
Casing Pump

2" PVC V
Screen Zone

Water Table $\left(\mathrm{IB}_{2}\right)$
Unit

ft msl

$\mathrm{pH}$

$\mathrm{S} / \mathrm{cm}$

${ }^{\circ} \mathrm{C}$

$\mathrm{mg} / \mathrm{h}$

NTU

well vol

ft msi

$\mu g / L$

$<0.20$

1 GE $\mu g / L$

1 GE $\mu g / L$

Notes:

- $=$ exceeded holding time

- = exceeded groundwater protection or monitoring constituent standard (See Appendix A.) 


\section{WELL HSL 2D (cont.)}

Radioactive Constituents

Constituents

Americium-241

Carbon-14

Cobalt-60

Curium-242

Curium-243/244

Curium-245/246

Gross alpha

lodine-129

Nickel-63

Nonvolatile beta

Plutonium-238

Plutonium-239/240

Radium-226

Radium-228

Strontium-90

Technetium-99

Thorium-228

Thorium-230

Thorium-232

Sum of alphas

Sum of betas

Tritium

Uranium-233/234

Uranium-235

Uranium-238
3098

$<5.6 E-01$

UIII

$<0.6100$

$<9.0 E \cdot 01$

$<9.0 E-01 \quad$ UIII

$<1.1900$

1

GP $<1.0 E+00 \quad U / 1$

$<6.4 E \cdot 01$

1 GE PCi/L

1

$G P<1.8 E+\infty \quad U / /$

$<1.2 E+00$

$1 \mathrm{GE} \mathrm{pCi} / \mathrm{L}$

Notes:

- = exceeded holding time

- exceeded groundwater protection or monitoring constituent standard (See Appendix A.) 
WELL HSL 3D

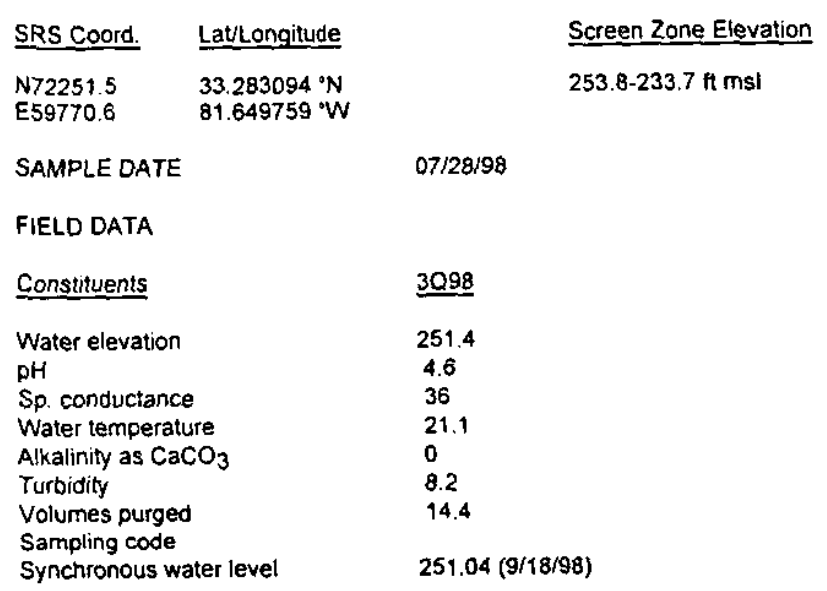

$\begin{array}{llll}\text { Top of Casing } & \text { Casing } & \text { Pump } & \text { Screen Zone } \\ 267.6 \mathrm{ft} \mathrm{msl} & \text { 2" PVC } & \text { V } & \text { Water Table (IIB } 2)\end{array}$

ANALYTICAL DATA

inorganic Constituents

Constituents

Antimony, total recoverable

Arsenic total recoverable

Barium, total recoverable

Cadmium, lotal recoverable $<1.0$

Chromium, total recoverable $<3.0$

Cobalt, total recoverable

Copper, total recoverable

Cyanide

Lead total recoverable

Mercury, total recoverable

Nickel, total recoverable

Nitrate as nitrogen

Nitrate-nitrite as nitrogen

Selenium, total recoverable

Silver, total recoverable

Tin, total recoverable

Vanadium, total recoverable

Zinc, total recoverabie

Organic Constituents

\section{Constituents}

Benzene

Bis(2-ethylhexyl) phthalate

Dichloromethane

Tetrachloroethylene

Trichioroethylene

Trichiorofluoromethane

\section{8}

Mod

Filt.

UII

$\mathrm{U} / \prime$

UII

J/I

1,500

$=1.0$

NI

v $/ /$

$<3.0$

$<1.0$

$<3.0$
0.92 J/EVI NDD

$<0.20$

$<1.0$

\section{SIH}

DF Lab 4Q98

Mod

1 GE

1 GE

1 GE

10/19/98

4098

249.8

4.2

26

23.0

4.9

$247.6(12 / 22 / 98)$

$1 \quad G E$

$<0.20 \quad u / I$

1 GE 1,700

1 GE
Water Table (llB 2$)$

Unit

ft msi

$\mathrm{pH}$

$\mu \mathrm{S} / \mathrm{cm}$

${ }^{\circ} \mathrm{C}$

$\mathrm{mg} / \mathrm{h}$

NTU

well vol

ft ms!

ST $\underline{H}$ DF Lab Unit

$\mu g / L$

$\mu g / L$

$\mu g / L$

$<0.20$

1 GE $\mu g /$

$1 \mathrm{GE} \mu g / \mathrm{L}$

$\mu \mathrm{g} / \mathrm{L}$

ST브 DF L Lab $\underline{\text { 4Q98 }}$ Mod

Fiit.

ST $\underline{H}$ DF Lab Unit

$\mu g / l$

$\mu g / \mathrm{L}$ $\mu g / L$

Notes:

- exceeded holding time

- exceeded groundwater protection or monitoring constituent standard (See Appendix A.) 
WELL HSL 3D (cont.)

Radioactive Constituents

Constituents

Americium-241

Carbon-14

Cobalt -60

Curium-242

Curium-243/244

Curium-245/246

Gross alpha

lodine-129

Nickel-63

Nonvolatile beta

Plutorium-238

Plutonium-239/240

Radium-226

Radium-228

Strontium -90

Technetium-99

Thorium-228

Thorium-230

Thorium-232

Sum of alphas

Sum of betas

Tritium

Uranium-233/234

Uranium-235

Uranium-238
3098

$1.8 \mathrm{E}+00 \quad / /$

2. $1 E+00 \quad / /$

$9.9 E+09 \quad$ /I
STH DF Lab $4 Q 998$ Mod

1

GP $<1.1 E+\infty \quad U / 1$

$1 \quad \mathrm{GP}<1.1 \mathrm{E}+00 \quad \mathrm{U} / /$

$<1.2 E+00$
Filt.

ST $\underline{H}$ DF Lab Unit 


\section{WELL HSL 4D}

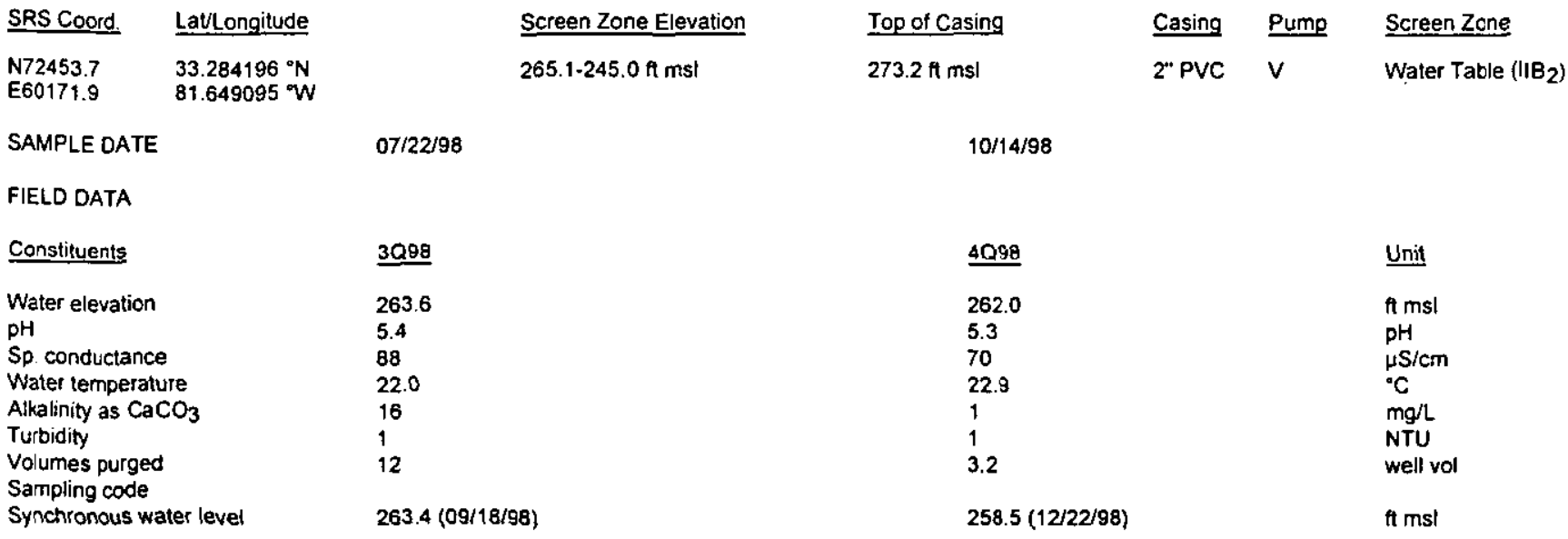

\section{ANALYTICAL DATA}

Inorganic Constituents

\begin{tabular}{|c|c|c|c|c|c|c|c|c|c|c|c|c|c|}
\hline Constituents & 3098 & Mod & Filt. & $\underline{\text { STI }} \underline{H}$ & $\underline{\mathrm{DF}}$ & Lab & $\underline{4098}$ & Mod & Fill. & $\underline{\mathrm{ST}} \underline{\mathrm{H}}$ & $\underline{\text { DF }}$ & Lab & Unit \\
\hline $\begin{array}{l}\text { Antimony, total recoverable } \\
\text { Arsenic. total recoverable } \\
\text { Barium, total recoverable }\end{array}$ & $<3.0$ & $\mathrm{U} / /$ & $<3.0$ & & 1 & $\mathrm{GE}$ & & & & & & & $\mu g / L$ \\
\hline $\begin{array}{l}\text { Cadmium, total recoverable } \\
\text { Chromium, total recoverable } \\
\text { Cobalt, total recoverable } \\
\text { Copper, total recoverable } \\
\text { Cyanide }\end{array}$ & $\begin{array}{l}<1.0 \\
<3.0\end{array}$ & $\begin{array}{l}\mathrm{U} / \prime \\
\mathrm{U} / \prime\end{array}$ & $\begin{array}{l}<1.0 \\
<3.0\end{array}$ & & $\begin{array}{l}1 \\
1\end{array}$ & $\begin{array}{l}\mathrm{GE} \\
\mathrm{GE}\end{array}$ & & & & & & & $\begin{array}{l}\mu g / L \\
\mu g / L\end{array}$ \\
\hline $\begin{array}{l}\text { Lead, total recoverable } \\
\text { Mercury, total recoverable } \\
\text { Nickel, total recoverable } \\
\text { Nitrate as nitrogen }\end{array}$ & $\begin{array}{l}0.72 \\
0.083\end{array}$ & $\begin{array}{l}J / E / \\
J / E J\end{array}$ & $\begin{array}{l}\text { NDD } \\
\text { NDD }\end{array}$ & & $\begin{array}{l}1 \\
1\end{array}$ & $\begin{array}{l}\text { GE } \\
\text { GE }\end{array}$ & 0.080 & $\mathrm{~J} / \mathrm{IK} / \mathrm{C}$ & NDD & & 1 & GE & $\begin{array}{l}\mu g / L \\
\mu g / L\end{array}$ \\
\hline $\begin{array}{l}\text { Nitrate-nitrite as nitrogen } \\
\text { Selenium, total fecoverable }\end{array}$ & 710 & $N /$ & & & 1 & GE & 430 & $n$ & & & 1 & GE & $\mu g / L$ \\
\hline $\begin{array}{l}\text { Silver, total recoverable } \\
\text { Tin, total recoverable } \\
\text { Vanadium, total recoverable } \\
\text { Zinc, total recoverable }\end{array}$ & $<1.0$ & $\mathrm{U} / 1$ & $<1.0$ & & 1 & GE & & & & & & & $\mu g / \mathrm{L}$ \\
\hline \multicolumn{14}{|l|}{ Organic Consthuemts } \\
\hline Constituents & 3098 & Mod & Filt. & $\underline{S T} \underline{H}$ & $\underline{D F}$ & $\underline{\text { Lab }}$ & 4099 & Mod & Fill. & $\underline{\text { ST }} \underline{H}$ & DF & $\underline{\text { Lab }}$ & $\underline{\text { Unit }}$ \\
\hline $\begin{array}{l}\text { Benzene } \\
\text { Bis (2-ethylhexyl) phthalate } \\
\text { Dichloromethane }\end{array}$ & $<1.0$ & $\mathrm{UJ} / \mathrm{O} / \mathrm{I}$ & $<1.0$ & & 1 & GE & & & & . & & & $\mu g / L$ \\
\hline $\begin{array}{l}\text { Tetrachloroethylene } \\
\text { Trichloroethylene }\end{array}$ & $\begin{array}{l}<1.0 \\
<1.0\end{array}$ & $\begin{array}{l}\mathrm{UJ} / \mathrm{O} / 1 \\
\mathrm{U} / \mathrm{O} / 1\end{array}$ & $\begin{array}{l}<1.0 \\
<1.0\end{array}$ & & 1 & GE & & & & & & & $\mu g / L$ \\
\hline $\begin{array}{l}\text { Micnlordeinylene } \\
\text { Trichloroflueromethane }\end{array}$ & $<1.0$ & UST 11 & $<1.0$ & & 1 & GL & & & & & & & $\mu g / L$ \\
\hline
\end{tabular}

Notes:

- = exceeded holding time

- = exceeded groundwater protection or monitoring constituent standard (See Appendix A.) 
WELL HSL 4D (cont.)

Radloactive Constituents

Constituents

Americium-241

Carbon-14

Cobalt- 60

Curium-242

Curium-243/244

Curium-245/246

Gross alpha

lodine-129

Nickel-63

Nonvolatile beta

Plutonium-238

Plutonium-239/240

Radium-226

Radium-228

Strontium-90

Technetium-99

Thorium-228

Thorium-230

Thorium-232

Sum of alphas

Sum of betas

Tritium

Uranium-233/234

Uranium-235

Uranium-238
3 Q98

Mod

Filt.

STH $\underline{\text { DF }}$ Lab 4 QQ98 $\quad$ Mod

$1.3 \mathrm{E}+00 \quad / /$

$6.9 E+01 \quad / 1$

$1 \quad \mathrm{GP}<1.5 \mathrm{E}+00 \quad \mathrm{U} / 1$

$<6.1 E-01$

- 1 GP $6.4 \mathrm{E}+01 \quad / 1$

3.3E+01 $/ 1$

- 1 GP $3.6 E+01$ "

- 1 ge pCi/mL

Notes: 


\section{WELL HSL 5D}

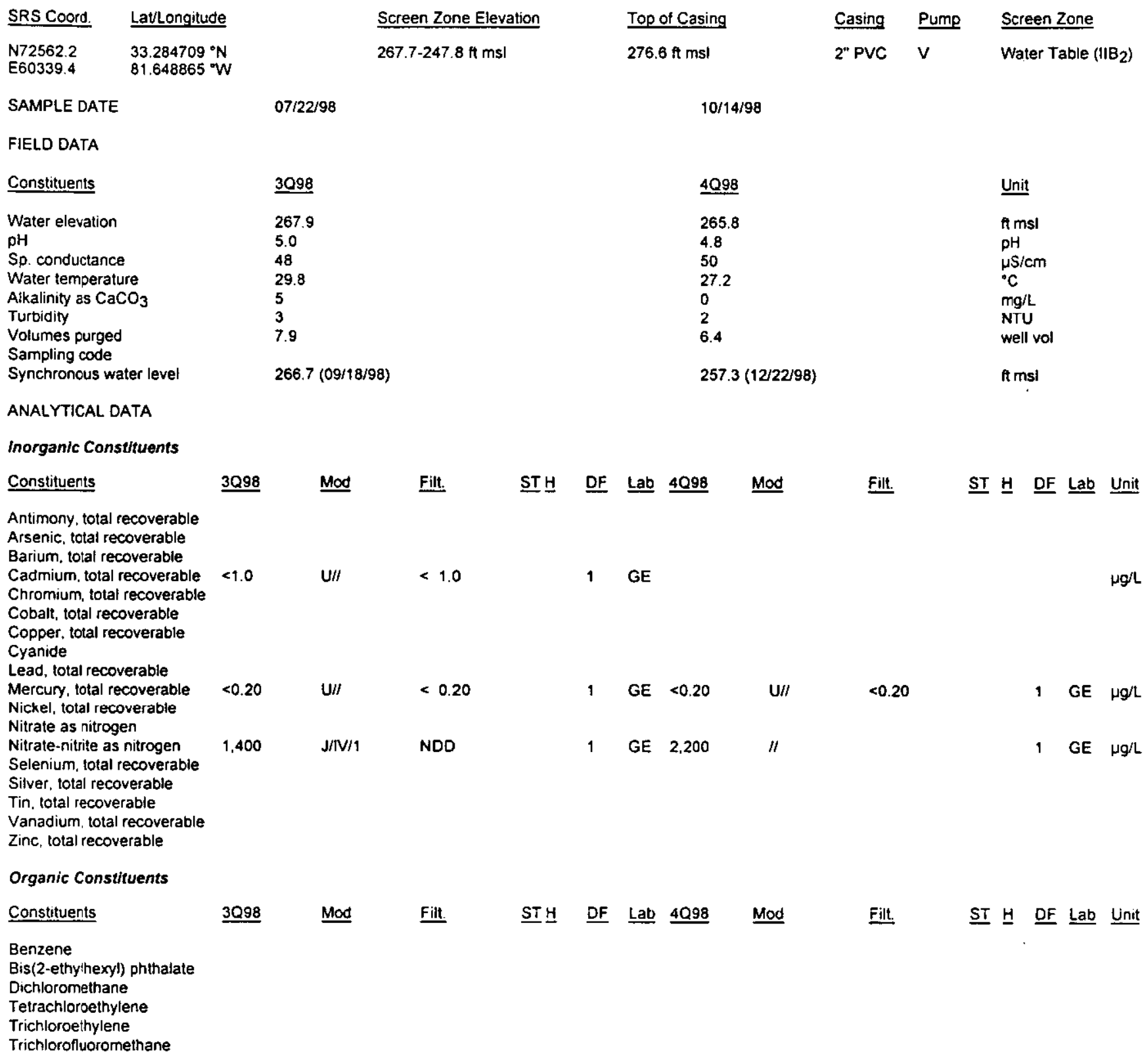

Notes:

- = exceeded holding time

= exceeded groundwater protection or monitoring constituent standard (See Appendix A.) 
WELL HSL 5D (cont.)

Radioactive Constituents

Constituents

3Q98

Mod

Filt.

STH $\underline{\text { DF Lab }} \underline{\text { 4Q98 }} \underline{\text { Mod }}$

Filt.

ST $\underline{H}$ OF Lab Unit

Americium-241

Carbon-14

Cobalt-60

Curium-242

Curium-243/244

Curium-245/246

Gross alpha

lodine-129

Nickel-63

Nonvolatile beta

Plutonium-238

Plutonium-239/240

Radium-226

Radium-228

Strontium-90

Technetium-99

Thorium-228

Thorium-230

Thorium-232

Sum of alphas

Sum of betas

Tritium

$1.5 E+01 \quad \|$

1

GP $<6.3 \mathrm{E} \cdot 01 \quad \mathrm{U} / 1$

$<6.7 \mathrm{E}-01$

1 GE pCill

$1.3 E+01$

1

$1 \mathrm{GE} \mathrm{pCi} / \mathrm{L}$

Uranium-233/234

Uranium-235

Uranium-238

Notes:

- = exceeded holding time

- = exceeded groundwater protection or monitoring constituent standard (See Appendix A.) 
WELL HSL 6D

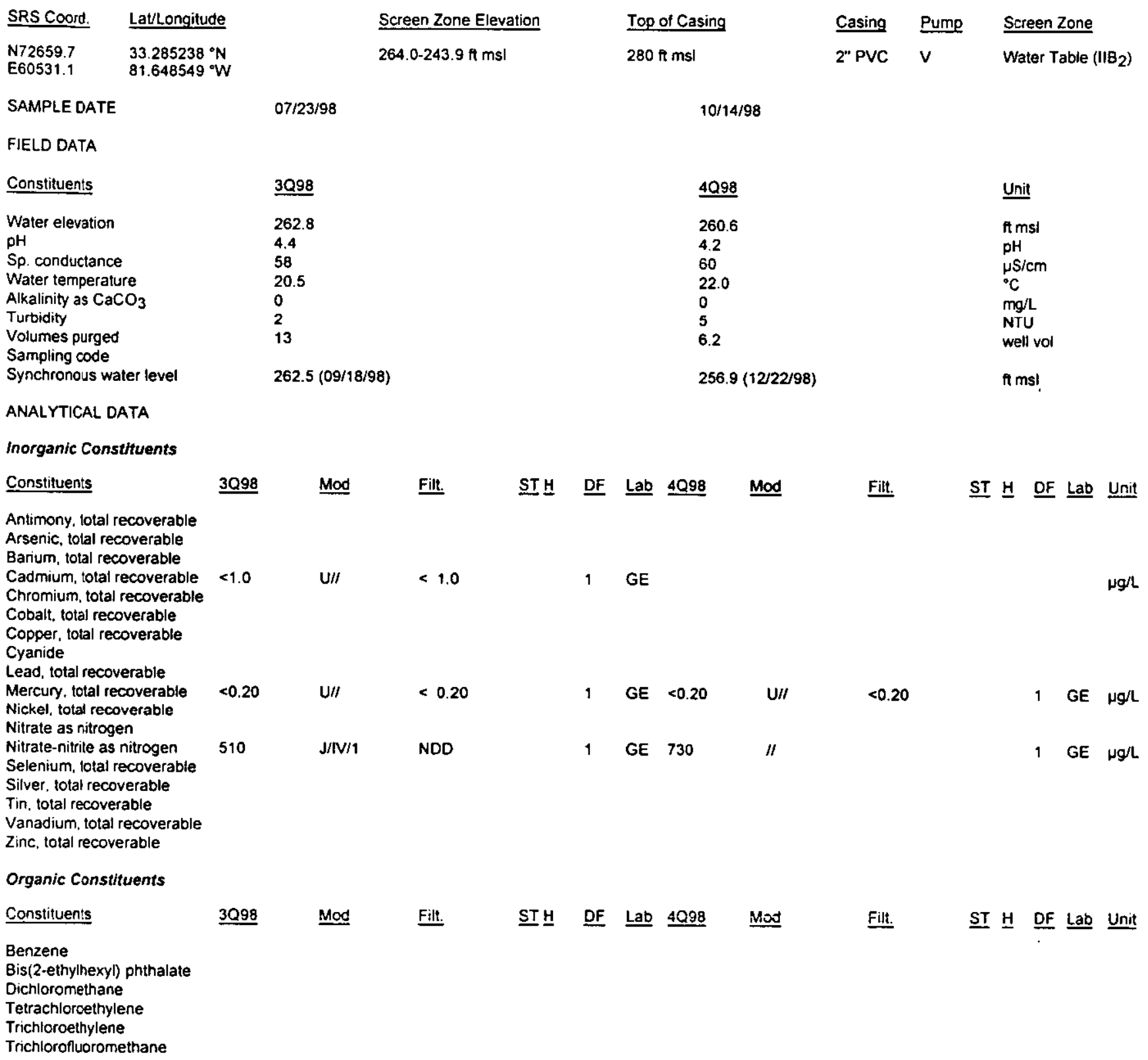

\section{Notes:}

- exceeded holding time

- exceeded groundwater protection or monitoring constituent standard (See Appendix A.) 
WELL HSL 6D (cont.)

Radloactive Constituents

Constituents

Americium-241

Carbon-14

Cobalt-60

Curium-242

Curium-243/244

Curium-245/246

Gross alpha

lodine-129

Nickel-63

Nonvolatile beta

Plutonium-238

Plutonium-239/240

Radium-226

Radium-22B

Strontium-90

Technetium-99

Thorium-228

Thorium-230

Thorium-232

Sum of alphas

Sum of betas

Tritium

Uranitum-233/234

Uranium-235

Uranium-238
309

Mod

Filt.

STㅂ DF Lab 4098 Mod

$3.3 E+00 \quad \mathrm{~J} / \mathrm{Cl} \quad \mathrm{NDD}$

$3.9 E+01 \quad / /$

4.1E+01 /

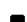

Filt.

$\underline{\text { ST }} \underline{H}$ ' $\underline{\text { LF }}$ Lab Unit
1 GE pCill

1 GE $p C i / L$
1 GP 4.3E+01 //

- 1 GE pCiml.

Notes:

- = exceeded holding time

n = exceeded groundwater protection or monitoring constituent standard (See Appendix A.) 
WELL HSL 7D

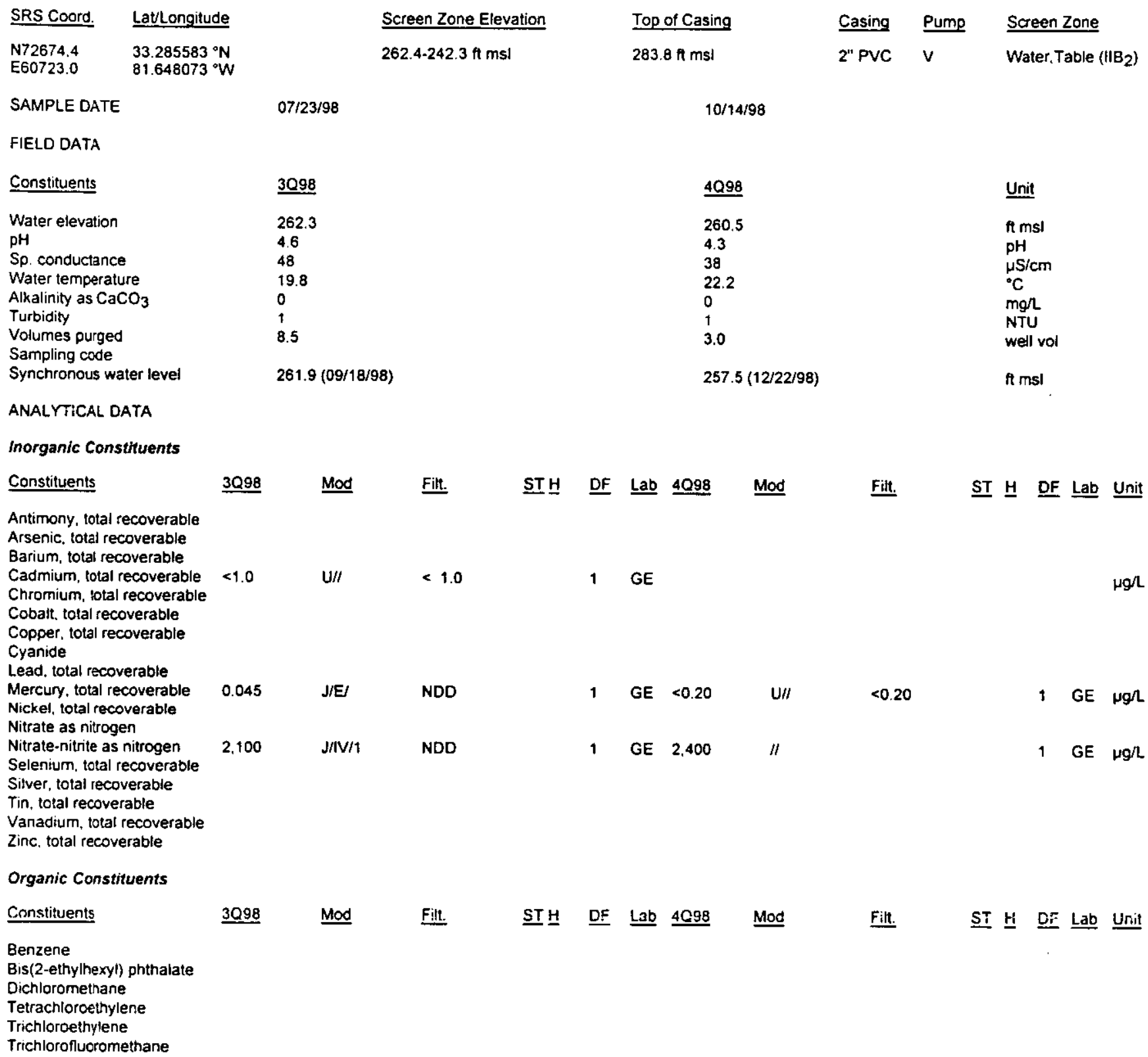

Notes:

- exceeded holding time

- exceeded groundwater protection or monitoring constituent standard (See Appendix A.) 
WELL HSL 7D (cont.)

Radioactive Constituents

Constituents

Americium-241

Carbon-14

Cobalt-60

Curium-242

Curium-243/244

Curium-245/246

Gross alpha

lodine-129

Nickel-63

Nonvolatile beta

Plutonium-238

Plutonium-239/240

Radium-226

Radium-228

Strontium-90

Technetium-99

Thorium-228

Thorium-230

Thorium-232

Sum of alphas

Sum of betas

Tritium

$\underline{3098}$

Mod

Filt.

STH

$1.4 \mathrm{E}+00 \quad \mathrm{~J} / \mathrm{C} /$

NDD

$1.4 \mathrm{E}+00 \quad / 1$

$3.4 \mathrm{E}+01 \quad / 1$

1 GP $3.8 \mathrm{E}+01$

Uranium-235

Uranium-238
GP $1.6 E+00$

GP $<1.9 \mathrm{E}+00 \quad \mathrm{U} / /$

$<9.8 \mathrm{E}-01$

$1 \mathrm{GE} \quad \mathrm{pCi} / \mathrm{L}$

1 GE $\mathrm{pCi} / \mathrm{L}$ 
WELL HSL 8D

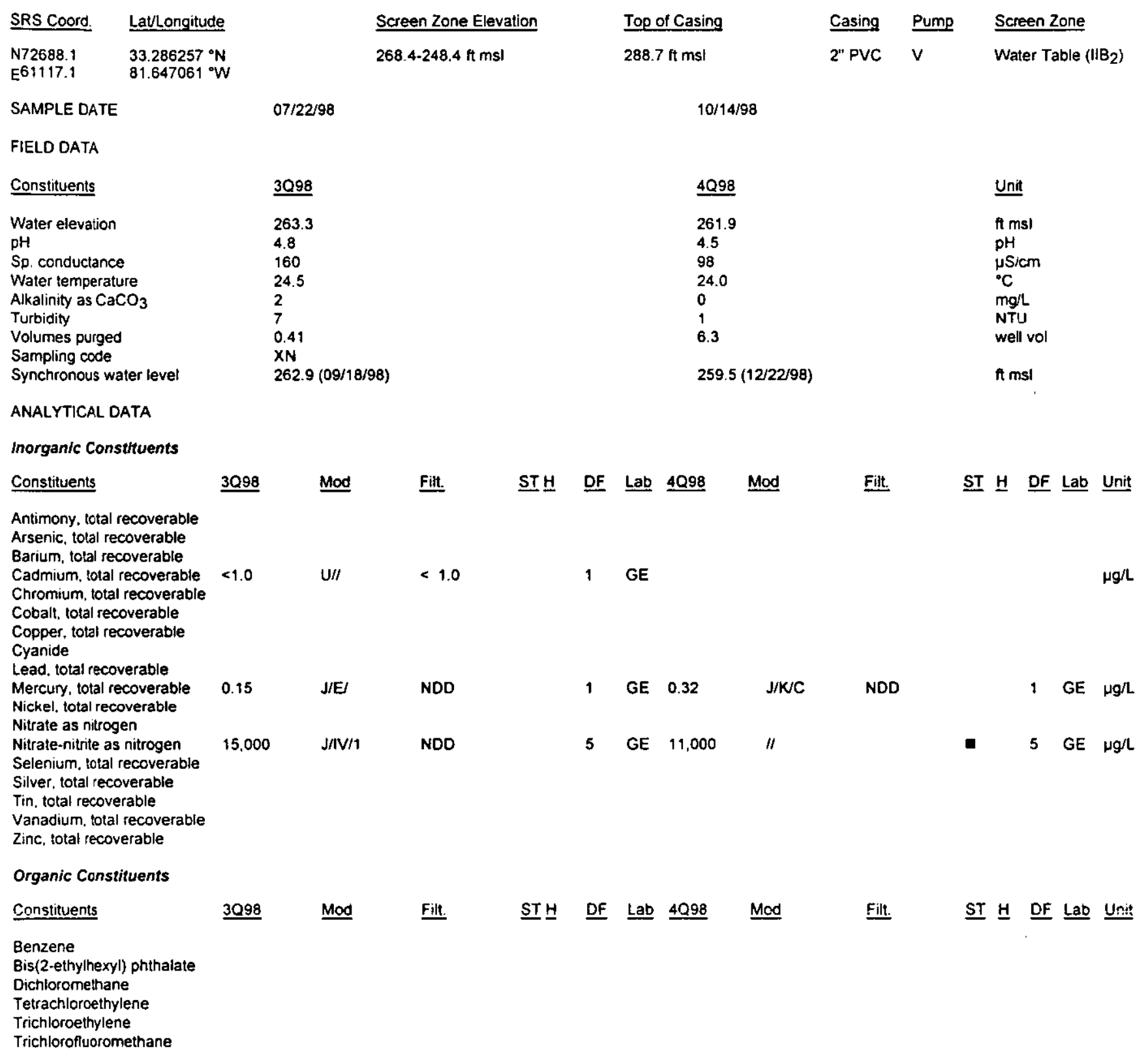

Notes:

- exceeded holding time

= exceeded groundwater protection or monitoring constituent standard (See Appendix A.) 


\section{WELL HSL 80 (cont.)}

\section{Radioactive Constituents}

\section{Constituents}

Americium-241

Carbon-14

Cobalt -60

Curium-242

Curium-243/244

Curium-245/246

Gross alpha

lodine-129

Nickel-63

Nonvolatile beta

Plutonium-238

Plutonium-239/240

Radium-226

Radium-228

Strontium-90

Technetium-99

Thorium-228

Thorium-230

Thorium-232

Sum of alphas

Sum of betas

Tritium

Uranium-233/234

Uranium-235

Uranium-238
3. $6 E+0$

$2.8 \varepsilon+01 \quad / 1$

GP $<1.1 E+00 \quad$ UII

2.1E+02 //
1 GP $1.4 \mathrm{E}+01$

Filt. $\quad$ STH $\underline{H}$ DF Lab $\underline{4098}$ Mod

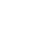

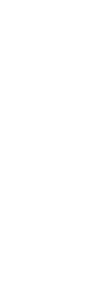

1 GP $1.4 E+02 \quad$ /
Filt.

ST $\underline{H}$ DF Lab $\cdot \underline{\text { Unit }}$

Notes:

- exceeded holding time

- exceeded groundwater protection or monitoring constituent standard (See Appendix A.) 


\begin{tabular}{|c|c|c|c|c|c|c|c|c|}
\hline \multicolumn{2}{|c|}{ UIC Permitted Constituents } & \multirow{2}{*}{$\begin{array}{l}\text { Reg } \\
\text { Limit }\end{array}$} & \multirow{2}{*}{$\begin{array}{l}\text { Jul-98 } \\
\text { Result }\end{array}$} & \multirow{2}{*}{$\frac{\text { Aug-98 }}{\text { Result }}$} & \multirow{2}{*}{$\frac{\text { Sep-98 }}{\text { Result }}$} & \multirow{2}{*}{$\begin{array}{l}\text { Oct-98 } \\
\text { Result }\end{array}$} & \multirow{2}{*}{$-\frac{\text { Nov-98 }}{\text { Result }}$} & \multirow{2}{*}{$\begin{array}{c}\text { Dec-98 } \\
\text { Result }\end{array}$} \\
\hline Constituent & Unit & & & & & & & \\
\hline Section I, INORGANICS & 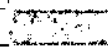 & $\because 7$ & 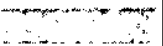 & $x_{0}+\cdots$ & $n^{2}$ & $-7 \mathrm{~m}$ & $7 \%$ & -7DSW \\
\hline Arsenic & $u g / \mathrm{L}$ & 50 & $<3$ & $<3$ & $<3$ & $<3$ & $<3$ & $<3$ \\
\hline Barium & $u \mathrm{~g} / \mathrm{L}$ & 2000 & $2.6 \overline{6}$ & 3.63 & 2.69 & 2.83 & 32.90 & 2.07 \\
\hline Cadmium & $u g / L$ & 5 & $<1$ & $<1$ & $<1$ & $<1$ & $<1$ & $<1$ \\
\hline Chromium & $\mu g / L$ & $100^{\circ}$ & $<3$ & $<3$ & $<3$ & $<3$ & $<3$ & $<3$ \\
\hline Lead & $\mu \mathrm{g} / \mathrm{L}$ & 50 & $<2$ & $<2$ & $<2$ & $<2$ & $<2$ & $<2$ \\
\hline Mercury & $\mu \mathrm{g} / \mathrm{L}$ & 2 & 0.65 & 0.74 & 1.00 & $<2$ & 0.82 & 0.98 \\
\hline Selenium & $\mu g / \mathrm{L}$ & 50 & $\overline{<5}$ & $<5$ & $<5$ & $<5$ & $<5$ & $<5$ \\
\hline Silver & $\mu g / L$ & 50 & $<1$ & $<1$ & $<1$ & $<1$ & $<1$ & $<1$ \\
\hline \multicolumn{9}{|l|}{ Soction II, ORGANICS } \\
\hline Antimony & $\mu g / L$ & 6 & $<0.2$ & $<0.2$ & $<0.2$ & $<0.2$ & 2.04 & $<0.2$ \\
\hline Cobalt & $\mu g / L$ & 140 & $<0.2$ & $0.63^{-}$ & 0.34 & 0.49 & 5.15 & $<0.2$ \\
\hline Copper & $\mu \mathrm{g} / \mathrm{L}$ & $130 \overrightarrow{0}$ & $<0.2$ & 1.47 & $<0.2$ & 0.84 & 1.76 & 4.08 \\
\hline Cyanide & $\mu g / L$ & 200000 & $<10$ & $<10$ & $<10$ & NR & $<10$ & $<10$ \\
\hline Benzene & $\mu g / L$ & 5 & $<1$ & $<1$ & $<1$ & $<1$ & $<1$ & $<1$ \\
\hline BEHP & $\mu \mathrm{g} / \mathrm{L}$ & 140 & $<10.1$ & $<9.9$ & $<10.2$ & $<10.1$ & $<10$ & $<10$ \\
\hline Methylene Chloride & $\mu g / L$ & 5 & $<1$ & $<1$ & $<5$ & $<5$ & $<5$ & $<5$ \\
\hline Nickel & $\mu g / L$ & 100 & $<\overline{0.2}$ & 1.68 & 2.82 & 1.23 & 5.65 & $<0.2$ \\
\hline Tetrachloroethylene & $\mu g / L$ & 5 & $<1$ & $<1$ & $<1$ & $<1$ & $<1$ & $<1$ \\
\hline & $\mu g / L$ & 50 & $<2$ & $<2$ & $<2$ & $<2$ & $<2$ & $<2$ \\
\hline Trichloroethylene & $\mu g / L$ & 5 & $<1$ & $<1$ & $<1$ & $<1$ & $<1$ & $<1$ \\
\hline Trichlorofluoromethane & $\mu g / L$ & 100 & $<1$ & $<1$ & $<5$ & $<5$ & $<5$ & $<5$ \\
\hline Vanadium & $\mu g / L$ & 49 & $<2$ & $<2$ & $<2$ & $<2$ & $<2$ & $<2$ \\
\hline Zinc & $\mu g / L$ & 5000 & $<5$ & $<5$ & $<5$ & $<5$ & 64.80 & $<5$ \\
\hline & & & $3 \sqrt{1} \times 3$ & $x_{0}+x^{2}$ & Wh & 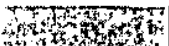 & Mroment & 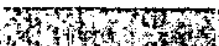 \\
\hline \multicolumn{2}{|l|}{ Section III, RADIONUCLIOES } & tov & W & 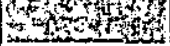 & Hint & 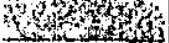 & 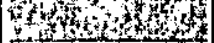 & $x+3, n$ \\
\hline Gross Alpha & $\mathrm{pCi} / \mathrm{L}$ & 15 & $<0.781$ & $<0.709$ & 1.24 & $<29.18$ & $<4.77$ & $<2.46$ \\
\hline Gross Beta & $\mathrm{pCi} / \mathrm{L}$ & 50 & 9.56 & 12.80 & 13.80 & $<52.8$ & 16.90 & 6.12 \\
\hline Total Radium $(226+228)$ & $\mathrm{pCi} / \mathrm{L}$ & 5 & BDL & 3.19 & BDL & BDL & BDL & BDL \\
\hline Soction IV, RADIONUCLID & ES & & 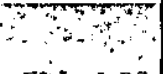 & Pat? & $7+4 x^{3}$ & 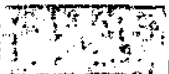 & (5) & (7P, \\
\hline Americium-241 & $\mathrm{pCi} / \mathrm{L}$ & SOA & $<0.0873$ & $<0.653$ & $<0.237$ & $<0.2098$ & $<1.858$ & $<0.708$ \\
\hline Cesium-137 & $\mathrm{pCi} / \mathrm{L}$ & SOB & $<3.5$ & $<3.77$ & $<3.49$ & $\overrightarrow{\mathbf{R}}$ & $\dot{\mathbf{R}}-$ & $<7.77$ \\
\hline Curium-242 & $\mathrm{pCi} / \mathrm{L}$ & SOA & $<0.0325$ & $<0.891$ & $<0.334$ & $<0.1369$ & $<0.327$ & $<0.219$ \\
\hline Curium-243/244 & $\mathrm{pCi} / \mathrm{L}$ & SOA & $<0.0632$ & $<1.19$ & $<0.146$ & $<0.3696$ & $<0.892$ & $<0.652$ \\
\hline Curium-246 & $\mathrm{pCi} / \mathrm{L}$ & SOA & $<0.03$ & $<0.504$ & $<0.107$ & $<0.1242$ & $<0.308$ & $<0.208$ \\
\hline Carbon-14 & $\mathrm{pCi} / \mathrm{L}$ & SOB & 24.30 & $<16.1$ & 48.50 & 42.90 & $<33.1$ & 24.10 \\
\hline Cobalt -60 & $\mathrm{pCi} / \mathrm{L}$ & SOB & $<3.81$ & $<4.07$ & $<4.36$ & $<7 . \overline{41}$ & $<<14.87$ & $<8.11$ \\
\hline lodine-129 & $\mathrm{pCi} / \mathrm{L}$ & SOB & 12.40 & 5.25 & $11.40^{\circ}$ & 12.20 & $<3.5$ & 6.20 \\
\hline Plutonium-238 & $\mathrm{pCi} / \mathrm{L}$ & SOA & $<0.122$ & $\mathbf{R}$ & $<0.251$ & $<0.4546$ & $<0.806$ & $<0.116$ \\
\hline Plutonium-239/240 & $\mathrm{pCi} / \mathrm{L}$ & SOAA & $<0.0419$ & $\bar{R}$ & $<0.203$ & $<0.5662$ & $<0.671$ & $<0.116$ \\
\hline Nickel-63 & $\mathrm{pCi} / \mathrm{L}$ & $\mathrm{SOB}$ & $<24.2$ & $<18.4$ & $<14.7$ & $<32.38$ & $<30.24$ & $<29.42$ \\
\hline Radium-226 & $\mathrm{pCi} / \mathrm{L}$ & SOR & $<0.17$ & 3.19 & $<0.782$ & $<1.029$ & $<1.295$ & $<1.502$ \\
\hline Radium-228 & $\mathrm{pCi} / \mathrm{L}$ & SOR & $<1.19$ & $<1.6$ & $<1.2$ & $<2.738$ & $<2.876$ & $<2.494$ \\
\hline Strontium-90 & pCill & $\mathrm{SOB}$ & 10.10 & 5.79 & 9.16 & 6.39 & $<5.81$ & $<1.891$ \\
\hline Technetium-99 & $\mathrm{pCi} / \mathrm{L}$ & $\mathrm{SOB}$ & $<7.42$ & $<8.16$ & $<20.7$ & $<1 \overline{7} .27$ & $<41.34$ & $<12.38$ \\
\hline Thorium-228 & $\mathrm{pCi} / \mathrm{L}$ & SOA & $<0.8 \overline{1}^{-}$ & $<\overline{11}$ & $<0.571$ & $<0.6068$ & $<\overline{0.525}$ & $<0.487$ \\
\hline Thorium-230 & $\mathrm{pC} \mathrm{i} / \bar{L}$ & $\mathrm{SOA}$ & $<0.399$ & $<0.369$ & $<\overline{0.497}$ & $<1.037$ & $<0.387$ & $<0.2464$ \\
\hline Thorium-232 & $\mathrm{pC \textrm {i } / \mathrm { L }}$ & SÖA- & $<0.227$ & $<0.435$ & $<0.31$ & $<0.197$ & $<0.272$ & $<0.048$ \\
\hline Uranium-233/234 & $\mathrm{pCi} / \mathrm{L}$ & SOA & 0.76 & $<0.717$ & $<0.309$ & $<0.1566$ & $<2.87$ & $<0.231$ \\
\hline Uranium-235 & $\mathrm{pCi} / \mathrm{L}$ & SOA & $<0 . \overline{192}$ & $<0.719$ & $<0.189$ & $<0.1061$ & $<2.618$ & $<0.1736$ \\
\hline Uranium-238 & $\mathrm{pCi} / \mathrm{L}$ & $\triangle O A$ & 0.19 & $<0.717$ & $<0.16$ & $<0.1265$ & $<1.13$ & $<0.1732$ \\
\hline $\begin{array}{l}\text { Sum of Alphas } \\
\text { Sum of Betas }\end{array}$ & & & $\begin{array}{r}0.95 \\
46.80\end{array}$ & $\frac{3.19}{11.04}$ & $\frac{\mathrm{BDL}}{20.56}$ & $\begin{array}{l}\mathrm{BDL} \\
61.49\end{array}$ & $\begin{array}{c}\mathrm{BDL} \\
\mathrm{BDL}\end{array}$ & $\frac{\mathrm{BDL}}{30.30}$ \\
\hline
\end{tabular}




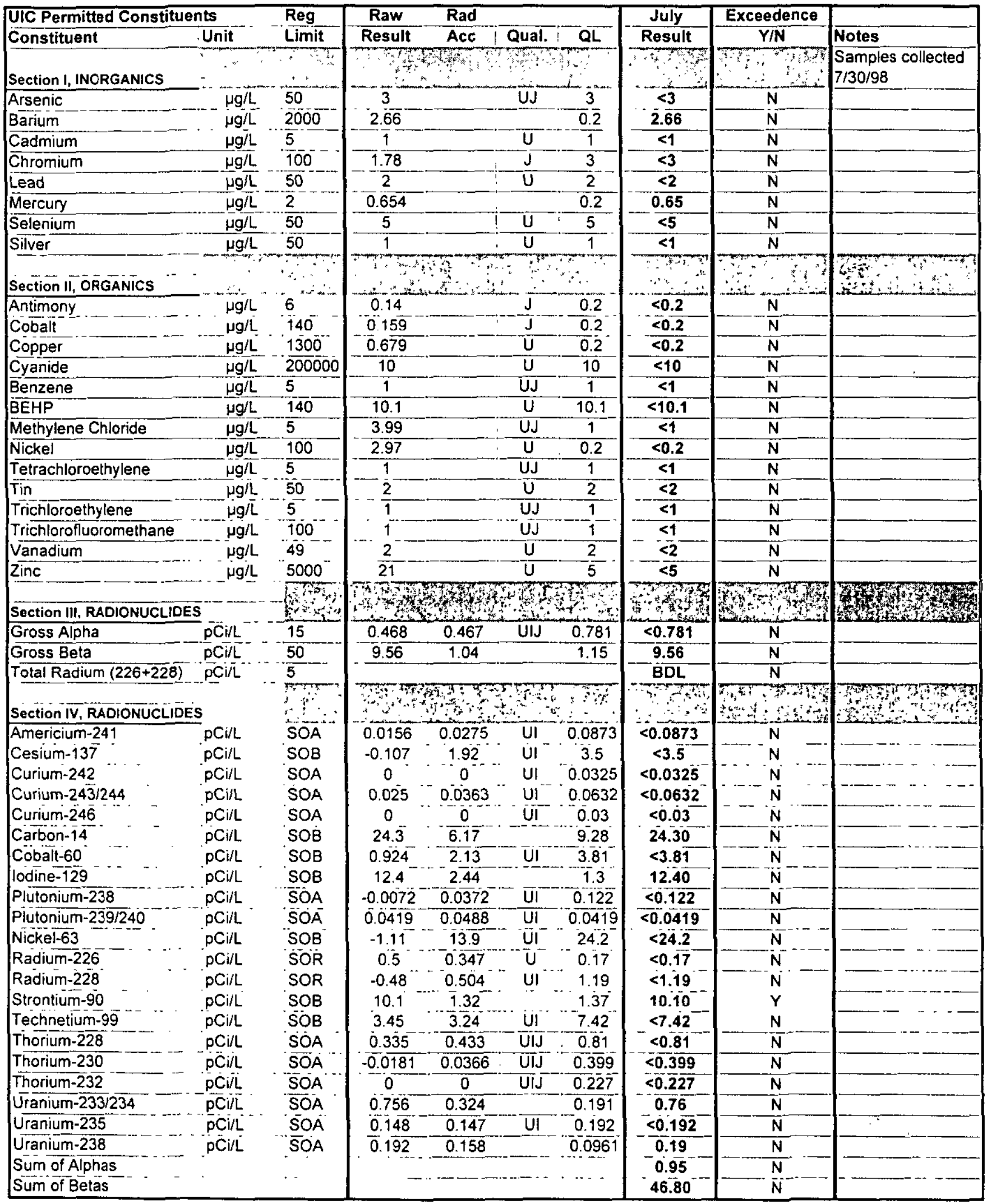




\begin{tabular}{|c|c|c|c|c|c|c|c|c|}
\hline \multirow{2}{*}{\begin{tabular}{|l|} 
UIC Permitted Constituents \\
Constituent \\
\end{tabular}} & \multirow{2}{*}{ Reg } & \multirow{2}{*}{$\frac{\operatorname{Raw}}{\text { Result }}$} & \multicolumn{3}{|l|}{ Rad } & \multirow{2}{*}{$\begin{array}{l}\text { August } \\
\text { Result }\end{array}$} & \multirow{2}{*}{$\frac{\text { Exceedence }}{\mathrm{Y} / \mathrm{N}}$} & \multirow[b]{2}{*}{ Notes } \\
\hline & & & Acc & Qual. & QL & & & \\
\hline Section l, INORGANICS & & then & WP & Whis & :14 & कर & 34 & $\begin{array}{l}\text { Samples collected } \\
8 / 20 / 98\end{array}$ \\
\hline Arsenic & $50^{\circ}$ & -3 & & $\mathrm{UJ}$ & 3 & $<3$ & $N$ & \\
\hline Barium & 2000 & $-\overline{3} .63$ & & & 0.2 & 3.63 & $\mathbf{N}$ & \\
\hline Cadmium & $\breve{5}$ & 1 & & $U$ & 1 & $<1$ & $\mathbf{N}$ & \\
\hline Chromium & 100 & 1.09 & & $\mathrm{~J}$ & 3 & $<3$ & $\mathrm{~N}$ & \\
\hline Lead & 50 & 2 & & $\mathrm{U}$ & 2 & $<2$ & $\mathbf{N}$ & \\
\hline Mercury & 2 & 0.741 & & & 0.2 & 0.74 & $\mathrm{~N}$ & \\
\hline Selenium & 50 & 5 & & $\mathrm{U}$ & 5 & $<5$ & $\mathbf{N}$ & \\
\hline Silver & 50 & 1 & & $U$ & 1 & $<1$ & $N$ & \\
\hline Section II, ORGANICS & & 13 & & 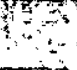 & $\because$ & 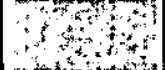 & $\because w^{2}$ & \\
\hline Antimony & 6 & 0.195 & & $\vec{u}$ & $0.2^{-1}$ & $<0.2$ & $\mathrm{~N}$ & \\
\hline Cobalt & 140 & 0.632 & & & 0.2 & 0.63 & $\mathbf{N}$ & \\
\hline Copper & 1300 & 1.47 & & & 0.2 & 1.47 & $\mathbf{N}$ & \\
\hline Cyanide & 200000 & 10 & & UJ & 10 & $<10$ & $\mathbf{N}$ & \\
\hline Benzene & 5 & 1 & & UJ & 1 & $<1$ & $\mathbf{N}$ & \\
\hline BEHP & 140 & 9.9 & & $\mathrm{U}$ & 9.9 & $<9.9$ & $\mathbf{N}$ & \\
\hline Methylene Chloride & 5 & 1 & & $\bar{U}$ & 1 & $<1$ & $\mathbf{N}$ & \\
\hline Nickel & 100 & 1.68 & & & 0.2 & 1.68 & $\mathbf{N}$ & \\
\hline Tetrachloroethylene & 5 & 1 & & $\bar{U}$ & $T$ & $<1$ & $\mathbf{N}$ & \\
\hline$\mu g / \mathrm{L}$ & 50 & 2 & & U & 2 & $<2$ & $\mathrm{~N}$ & \\
\hline Trichloroethylene & 5 & 1 & & $\bar{U}$ & 1 & $<1$ & $\mathbf{N}$ & \\
\hline Trichlorofluoromethane & 100 & 1 & & $\mathrm{U}$ & 1 & $<1$ & $\mathbf{N}$ & \\
\hline Vañadium & $49^{-}$ & 2 & & $u$ & 2 & $<2$ & $\mathbf{N}$ & \\
\hline$\mu g / \mathrm{t}$ & $5000^{--}$ & 5 & & $\bar{U}$ & 5 & $<5$ & $\mathrm{~N}$ & \\
\hline ONUCLIDES & Whas & 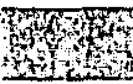 & to & 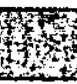 & 4he & my & 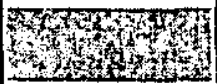 & W \\
\hline Gross Alpha & 15 & $\frac{11}{1.11}$ & 0.577 & $\mathrm{~J}$ & 0.709 & $<0.709$ & $\frac{N}{N}$ & \\
\hline Gross Beta & $50^{-}$ & 12.8 & 1.13 & & 1.05 & 12.80 & $\mathrm{~N}$ & \\
\hline Total Radium $(226+228) \quad \mathrm{pCi} / \mathrm{L}$ & 5 & & & & & 3.19 & $\mathbf{N}$ & \\
\hline Section IV, RADIONUCUIDES & & F & & & & wot & 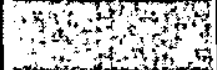 & 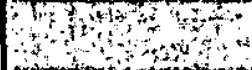 \\
\hline Americium-241 & $S O A$ & 0.107 & 0.218 & UI & 0.653 & $<0.653$ & $N$ & \\
\hline Cesium-137 & SOB & 0.413 & 2.07 & Üi & 3.77 & $<3.77$ & $\bar{N}$ & \\
\hline Curium-242 & SOA & -0.0344 & 0.239 & UI & 0.891 & $<\overline{0.891}$ & $\mathrm{~N}$ & \\
\hline Curium-243/244 & SOA & 0.645 & 0.665 & uii & 1.19 & $<1.19$ & $\mathrm{~N}$ & \\
\hline Curium $-2 \overline{46}$ & SOA & 0.182 & $0.288^{\circ}$ & Uा & 0.504 & $<0.504$ & $\mathbf{N}$ & \\
\hline Carbon-14 & SOB & 3.63 & 9.44 & Ūi & 16.1 & $<16.1$ & $\mathrm{~N}$ & \\
\hline Cobalt- 60 & SOB & $2 . \overline{2} 2$ & $2.09^{-}$ & uI & 4.07 & $<\overline{4.07}$ & N & \\
\hline $\mathrm{pCi} / \mathrm{L}$ & SOB & 5.25 & $2 . \overline{4} \overline{2}$ & & 1.21 & 5.25 & $\mathbf{N}$ & \\
\hline Plutonium-238 & SOA & 2.04 & 0.408 & $R$ & 0.0405 & $\overline{\mathbf{R}}$ & $\bar{N}$ & \\
\hline Plutonium-239/240 & SOA & 0.324 & $0.14 \overline{2}$ & $\bar{R}^{-}$ & 0.103 & $\mathbf{R}$ & $\bar{N}$ & \\
\hline Nickel-63 & $S O B$ & 7.75 & 10.9 & ii & 18.4 & $<18.4$ & $N$ & \\
\hline Radium-226 & SOR & 3.19 & $0.834^{\circ}$ & & 3.19 & $3 . \overline{19}$ & $N$ & \\
\hline Radium-228 & SOR & -0.243 & 0.678 & ÜاJ & 1.6 & $<1.6$ & $\mathrm{~N}$ & \\
\hline Strontium-90 & $S O B$ & 5.79 & 1.75 & & $2.52^{-}$ & $5 . \overline{79}$ & $\mathrm{~N}$ & \\
\hline Technetium-99 & SOB & -1.41 & 3.2 & पाँ & $8 . \overline{16}-$ & $<8.16$ & $\mathrm{~N}$ & \\
\hline Thorium-228 & S̄OA & -0.172 & 0.37 & पा & 1.11 & $<1.11$ & $\mathbf{N}$ & \\
\hline Thorium-230 & SOA & 0.193 & 0.247 & $\mathrm{UI}^{-}$ & 0.369 & $<0.369$ & $\mathrm{~N}$ & \\
\hline Thorium-232 & $S O A$ & -0.0335 & 0.0479 & UI & $0 . \overline{435}$ & $<0.435$ & $\mathrm{~N}$ & \\
\hline Uranium-233/234 & SOA & 0.0875 & 0.262 & UI & 0.717 & $<0.717$ & $\mathbf{N}$ & \\
\hline Uranium-235 & $\overline{S O A}$ & -0.0403 & 0.0573 & UI & 0.719 & $<0.719$ & $\bar{N}$ & \\
\hline Uranium-238 & SOA & 0.0875 & 0.262 & Uit & 0.717 & $<0.717$ & $\mathbf{N}$ & \\
\hline Sum of Alphas & & & & & & 3.19 & $N$ & \\
\hline Sum of Betas & & & & & & 11.04 & $\bar{N}$ & \\
\hline
\end{tabular}




\begin{tabular}{|c|c|c|c|c|c|c|c|c|c|}
\hline UIC Permitted Constitue & ents & Reg & Raw & Rad & & & September & Exceedence & \\
\hline Constituent & Unit & Limit & Result & Acc & Qual. & QL & Result & $\mathbf{Y} / \mathbf{N}$ & Notes \\
\hline Section I, INORGANICS & s & $\because$ & 3 & $\therefore$ & W: & 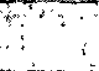 & $\because 4$ & $\therefore ?$ & $\begin{array}{l}\text { Samples collected } \\
\text { 9/22/98 }\end{array}$ \\
\hline Arsenic & $\mu \mathrm{g} / \mathrm{L}$ & 50 & 3 & & $U$ & 3 & $<3$ & $\mathrm{~N}$ & \\
\hline Barium & $\mu g / L$ & 2000 & 2.69 & & & $0 . \overline{2}$ & 2.69 & $\bar{N}$ & \\
\hline Cadmium & $\mu g / L$ & 5 & 1 & & U & 1 & $<1$ & $N$ & \\
\hline Chromium & $\mu g / L$ & 100 & 1.87 & & $\mathrm{~J}$ & 3 & $<3$ & $N$ & \\
\hline Lead & $\mu g / L$ & 50 & 2 & & $\mathrm{U}$ & 2 & $<2$ & $\bar{N}$ & \\
\hline Mercury & $\mu g / L$ & 2 & 1 & & & 0.2 & 1.00 & $\mathbf{N}$ & \\
\hline Selenium & $\mu g / L$ & 50 & 5 & & $\mathrm{U}$ & 5 & $<5$ & $\mathbf{N}$ & \\
\hline Silver & $\mu g / L$ & 50 & 1 & & $u$ & 1 & $<1$ & $\mathbf{N}$ & \\
\hline Section II, ORGANICS & & & $\because$ & & 3 & & 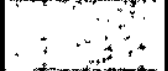 & 28 & $\therefore$ \\
\hline Antimony & $\mu g / L$ & 6 & 0.2 & & $u$ & 0.2 & $<0.2$ & $\overline{\mathrm{N}}$ & \\
\hline Cobalt & $\mu g / L$ & 140 & 0.335 & & & 0.2 & 0.34 & $\mathbf{N}$ & \\
\hline Copper & $\mu g / L$ & $\overline{1300}$ & $0.5 \overline{16}$ & & $\mathrm{U}$ & 0.2 & $<0.2$ & $\bar{N}$ & \\
\hline Cyanide & $\mu g / L$ & $2 \overline{00000}$ & 10 & & $u$ & 10 & $<10$ & $\mathrm{~N}$ & \\
\hline Benzene & $\mu g / \mathrm{L}$ & 5 & 1 & & U & 1 & $<1$ & $\bar{N}$ & \\
\hline BEHP & $\mu g / L$ & 140 & 10.2 & & $\mathrm{U}$ & 10.2 & $<10.2$ & $\mathrm{~N}$ & \\
\hline Methylene Chloride & $\mu g / L$ & 5 & 5 & & $\mathrm{U}$ & 5 & $<5$ & $\mathrm{~N}$ & \\
\hline Nickel & $\mu g / L$ & 100 & 2.82 & & & 0.2 & 2.82 & $\mathrm{~N}$ & \\
\hline Tetrachloroethylene & $\mu g / L$ & 5 & 1 & & $u$ & 1 & $<1$ & $\mathrm{~N}$ & \\
\hline Tin & $\mu g / L$ & 50 & 2 & & $\mathrm{U}$ & 2 & $<2$ & $N$ & \\
\hline Trichloroethylene & $\mu g / L$ & 5 & 1 & & U & 1 & $<1$ & $\bar{N}$ & \\
\hline Trichlorofluoromethane & $\mu g / L$ & 100 & 5 & & $\bar{u}$ & 5 & $<5$ & $\mathrm{~N}$ & \\
\hline Vanadium & $\mu g / L$ & 49 & 2 & & $\bar{u}$ & 2 & $<2$ & $\mathrm{~N}$ & \\
\hline Zinc & $\mu g / L$ & 5000 & 5 & & $\mathrm{U}$ & 5 & $<5$ & $\mathrm{~N}$ & \\
\hline RADIONUCLIO & & wis & 20 & $w^{2}$ & 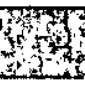 & The & का & Whand & why \\
\hline Gross Alpha & $\mathrm{pCi} / L$ & 15 & 1.24 & 0.648 & & 0.878 & 1.24 & $\mathrm{~N}$ & \\
\hline Gross Beta & $p C i / L$ & 50 & 13.8 & 1.22 & & 1.28 & 13.80 & $\mathrm{~N}$ & \\
\hline Total Radium $(226+228)$ & $\mathrm{pCi} / \mathrm{L}$ & 5 & & & & & $\mathrm{BDL}$ & $\mathbf{N}$ & \\
\hline RADIONUCLIDI & & & 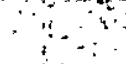 & & & $\because$ & $9+\cdots$ & 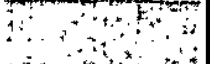 & $2+3 x$ \\
\hline Americ & $\mathrm{pCi} / \mathrm{L}$ & $\mathrm{SOA}$ & $-0.079^{-1}$ & 0.098 & $\mathrm{ui}^{12}$ & 0.237 & $<0.237$ & N & \\
\hline $\operatorname{siun}$ & $\mathrm{pCi} / \mathrm{L}$ & SOB & -1.04 & 2.04 & ui & 3.49 & $<3.49$ & $\bar{N}$ & \\
\hline ium-242 & $p \mathrm{Ci} / \mathrm{L}$ & SOA & -0.128 & 0.113 & UI & 0.334 & $<0.334$ & $\mathrm{~N}$ & \\
\hline $3 / 244$ & $\mathrm{pCi} / \mathrm{L}$ & SOA & -0.14 & 0.167 & $\mathrm{Ui}^{-}$ & 0.416 & $<0 . \overline{46}$ & $N$ & \\
\hline Curium-246 & $\mathrm{pCi} / \mathrm{L}$ & SOA & 0.0594 & 0.0704 & Uiা & 0.107 & $<0.107$ & $\overline{\mathrm{N}}$ & \\
\hline Carbon-14 & $\mathrm{pCi} / \mathrm{L}$ & $S O B$ & $48.5^{-}$ & 7.52 & & $10 . \overline{3}$ & $48.50^{\circ}$ & $\mathrm{N}$ & \\
\hline Co & $\mathrm{pCi} / \mathrm{L}$ & SOB & 2.35 & 1.58 & บĩ & 4.36 & $<4.36$ & $\mathrm{~N}$ & \\
\hline lodine-129 & $\mathrm{pCi} / \mathrm{L}$ & $\mathrm{SOB}$ & 111.4 & 2.2 & & 1.01 & 11.40 & $\hat{N}$ & \\
\hline Plutonium-238 & $\mathrm{pCi} / \mathrm{L}$ & $\mathrm{SOA}^{-}$ & 0.0457 & 0.107 & $\mathrm{Ui}$ & 0.251 & $<0.251$ & $\mathrm{~N}$ & \\
\hline Plutonium-239/240 & $\mathrm{pCi} / \mathrm{L}$ & SOA & -0.0211 & 0.0245 & UIJ & 0.203 & $<0.203$ & - & \\
\hline kel-63 & $\mathrm{pCi} / \mathrm{L}$ & SOB & 9.82 & $8 . \overline{8} 4$ & Ui & 14.7 & $<14.7$ & - & - \\
\hline dium-226 & $\mathrm{pCi} / \mathrm{L}$ & SOR & 0.504 & 0.521 & UI & 0.782 & $<0.782$ & $\mathrm{~N}$ & \\
\hline Radic & $\mathrm{pCi} / \mathrm{L}$ & SOR & -1.56 & 0.521 & UIJ. & 1.2 & $<1.2$ & - & \\
\hline Strontium-90 & $\mathrm{pCi} / \mathrm{L}$ & $\mathrm{SOB}$ & 9.16 & 0.958 & & 1.03 & 9.16 & $Y$ & \\
\hline Technetium-99 & $p C i / L$ & SOB & -2.76 & 8.17 & $\mathrm{Ui}^{-}$ & $20.7^{-}$ & $<20.7$ & - & \\
\hline Thorium-228 & $\mathrm{pCi} / \mathrm{L}$ & $\overline{S O A}$ & $-0.009 \overline{4}$ & 0.229 & $\mathrm{Ui}^{-1}$ & $0 . \overline{571}$ & $<0.571$ & $N$ & \\
\hline Thorium-230 & $\mathrm{PCi} / \mathrm{L}$ & $S O A$ & 0.42 & 0.334 & UI & 0.497 & $<0.497$ & $\bar{N}$ & \\
\hline Thorium-232 & $\mathrm{pCi} / \mathrm{L}$ & $S O A$ & 0.00198 & 0.0993 & $\overline{U i}-$ & 0.31 & $<0.31$ & $N$ & \\
\hline Uranium-233/234 & $\mathrm{pCi} / \mathrm{L}$ & SŌA & 0.2 & 0.189 & UI & 0.309 & $<0.309$ & $\mathrm{~N}$ & \\
\hline Uranium-235 & $\mathrm{pCi} / \mathrm{L}$ & SOA- & 0.0158 & 0.0643 & Ui & 0.189 & $<0.189$ & $\mathrm{~N}^{-}$ & \\
\hline Urañium-238 & $\mathrm{pCi} / \mathrm{L}$ & SOA & 0.023 & 0.0624 & Ui. & 0.16 & $<0.16$ & $N$ & \\
\hline Sum of Alphas & & & & & & & $B D L$ & $\bar{N}$ & \\
\hline Sum of Betas & & & & & & & 20.56 & $\mathrm{~N}$ & \\
\hline
\end{tabular}




\begin{tabular}{|c|c|c|c|c|c|c|c|c|c|}
\hline UIC Permitted Constitue & & $\operatorname{Reg}$ & Raw & Rad & & & October & Exceedence & \\
\hline Constituent & Unit & Limit & Result & Acc & Qual. & QL & Result & YIN & Notes \\
\hline Section I, INORGANICS & 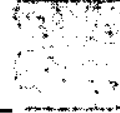 & $\therefore 6$ & We & 6 & $\begin{array}{l}x^{2} \\
4=3\end{array}$ & 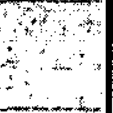 & 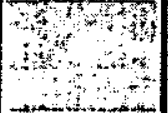 & 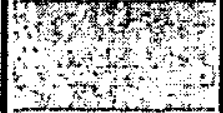 & $\begin{array}{l}\text { Samples } \\
\text { collected } \\
10 / 13 / 98\end{array}$ \\
\hline Arsenic & $\mu g / L$ & $50^{\circ}$ & 3 & & u & 3 & $<3$ & $\mathrm{~N}$ & \\
\hline Barium & $\mu g / L$ & $\overline{2000}$ & $\overline{2.83}$ & & & $0 . \overline{2}$ & 2.83 & $\mathbf{N}$ & \\
\hline Cadmium & $\mu \mathrm{g} / \mathrm{L}$ & 5 & 1 & & $\mathrm{U}$ & 1 & $<1$ & $\mathbf{N}$ & \\
\hline Chromium & $\mu \mathrm{g} / \mathrm{L}$ & 100 & $0.78 \overline{9}$ & & $\mathrm{~J}$ & 3 & $<3$ & $N$ & \\
\hline Lead & $\mu \mathrm{g} / \mathrm{L}$ & 50 & 2 & & $\bar{U}$ & 2 & $<\overline{2}$ & $\mathbf{N}$ & \\
\hline Mercury & $\mu g / L$ & 2 & 1.66 & & $J$ & 0.2 & $<2$ & $\mathbf{N}$ & \\
\hline Selenium & $\mu \mathrm{g} / \mathrm{L}$ & $50^{-7}$ & 5 & & $u$ & 5 & $<5$ & $\mathbf{N}$ & \\
\hline silver. & $\mu g / L$ & 50 & 1 & & $U$ & 1 & $<1$ & $\bar{N}$ & \\
\hline Section II, ORGANNICS & 8 & & 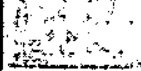 & 6 & 8 & & $2 x+4$ & a & $6 \mathrm{mos}$ \\
\hline Antimony & $\mu g / \mathrm{L}$ & 6 & 0.2 & & $\mathrm{U}$ & 0.2 & $<0.2$ & $\mathbf{N}$ & \\
\hline Cobalt & $\mu g h$ & 140 & 0.494 & & & 0.2 & 0.49 & $\mathbf{N}$ & \\
\hline Copper & $\mu g / L$ & 1300 & 0.841 & & & 0.2 & 0.84 & $\mathbf{N}$ & \\
\hline Cyanide & $\mu \mathrm{g} / \mathrm{L}$ & 200000 & & & & & & $\mathrm{~N}$ & \\
\hline Benzene & $\mu g / L$ & 5 & 1 & & U & 1 & $<1$ & $\mathbf{N}$ & \\
\hline BEHP & $\mu g / L$ & 140 & 10.1 & & UJ & 10.1 & $<10.1$ & $N$ & \\
\hline Methylene Chloride & $\mu g / h$ & 5 & 5 & & $u$ & 5 & $<5$ & $\mathbf{N}$ & \\
\hline Nickel & $\mu g / L$ & 100 & 1.23 & & & 0.2 & 1.23 & $\mathbf{N}$ & \\
\hline Tetrachloroethylene & $\mu g / L^{-}$ & 5 & 1 & & $u$ & 1 & $<1$ & $\mathbf{N}$ & \\
\hline & $\mu g / L$ & 50 & 2 & & u & 2 & $<2$ & $\mathbf{N}$ & \\
\hline Trichloroethylene & $\mu g / L$ & 5 & 1 & & $\bar{U}$ & 1 & $<1$ & $\bar{N}$ & \\
\hline Trichlorofluoromethane & $\mu g /$ & 100 & 5 & & $\underline{U}$ & 5 & $<5$ & $\mathbf{N}$ & \\
\hline Vanadium & $\mu g / L$ & 49 & 2 & & $\bar{u}$ & 2 & $<2$ & $\mathbf{N}$ & \\
\hline Zinc & $\mu g / L$ & 5000 & 5 & & $\dot{U}$ & 5 & $<5$ & $\mathrm{~N}$ & \\
\hline Section III, RADIONUCLIDE & & 7y & mots & tyst & the & pits & Mtron & Wxy & Whomp \\
\hline Gross Alpha & $\mathrm{pCi/L}$ & 15 & 6.03 & 7.94 & U & 29.18 & $<29.18$ & $\mathrm{~N}$ & \\
\hline Gross Beta & $\mathrm{pC} / \mathrm{I}$ & 50 & 15.9 & 14.4 & $\mathrm{u}$ & 52.8 & $<52.8$ & & $\mathrm{DL}$ too high \\
\hline Total Radium $(226+228)$ & $\mathrm{pCi} / \mathrm{L}$ & 5 & & & & & $\mathrm{BDL}$ & $\mathbf{N}$ & \\
\hline Section IV, RADIONUCLIO & & & & & & 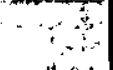 & 10 & ond & 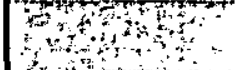 \\
\hline Americium-241 & $\mathrm{pCi} / \mathrm{L}$ & SOA & 0.0292 & 0.0469 & $\bar{U}$ & 0.2098 & $<0.2098$ & $\mathbf{N}$ & \\
\hline Cesium-137 & $\mathrm{pCi} / \mathrm{L}$ & $S O B$ & $6.9^{-1}+x-1$ & 2.38 & $R$ & 9.76 & $\mathbf{R}$ & $\bar{N}$ & Rejected Analysi \\
\hline Curium-242 & $\mathrm{pCi} / \mathrm{L}$ & SOA & 0.00805 & 0.0288 & $\bar{u}$ & 0.1369 & $<0.1369^{\circ}$ & $\mathrm{N}$ & \\
\hline Curium-243/244 & $\mathrm{pCill}$ & SOA & 0.0467 & 0.0908 & $u$ & 0.3696 & $<0.3696$ & $N$ & \\
\hline Curium-246. & $\mathrm{pCi} / \mathrm{L}$ & SOA & 0.00731 & 0.0261 & $u$ & 0.1242 & $<0.1242$ & $\bar{N}$ & \\
\hline Carbon-14 & pCi/L & SOB & $42 . \overline{9}$ & 6.76 & & $22.89^{-}$ & 42.90 & $N$ & \\
\hline Cobalt-60 & $\mathrm{PCi} / \mathrm{L}$ & SOB & 0.822 & 1.84 & $\bar{U}$ & 7.41 & $<7.41$ & $\bar{N}$ & \\
\hline lodine-129 & $\mathrm{p}$ & SOB & 12.2 & 2.71 & & 6.7 & 12.20 & $N$ & \\
\hline Plutonium-238 & $\mathrm{pCi} / \mathrm{L}$ & SOAA & $-0.0 \overline{302}$ & 0.0313 & i & 0.4546 & $<0.4546$ & $\mathrm{~N}$ & \\
\hline Plutonium-239/240 & $\mathrm{pCi} / \mathrm{L}$ & SOA & -0.0604 & 0.0456 & u & 0.5662 & $<0.5662$ & $N$ & \\
\hline Nickel-63 & $\mathrm{pCi} / \mathrm{L}$ & SOB & -6.3 & 8.59 & $\mathrm{U}$ & 32.38 & $<32.38$ & $N$ & \\
\hline Radium-226 & $\mathrm{pCi} / \mathrm{L}$ & SOR & $0.463^{-}$ & $0.319^{\circ}$ & II & 1.029 & $<1.029$ & $\bar{N}$ & \\
\hline Radium-228 & $\mathrm{pC} \mathrm{i} / \mathrm{L}$ & SOR & -0.123 & 0.644 & Uj & $2.738^{\circ}$ & $<2.738$ & $\mathbf{N}$ & \\
\hline Strontium-90 & $\mathrm{pCi} / \mathrm{L}$ & SOB & 6.39 & 1.61 & & 584 & 6.39 & $\mathrm{~N}$ & \\
\hline Technetium-99 & $\mathrm{p} C \mathrm{C} i{ }_{\mathrm{L}}$ & $S O B$ & 8.37 & 4.23 & $\bar{u}$ & $1 \overline{7} .27$ & $<17.27$ & $\mathrm{~N}$ & \\
\hline Thorium-228 & $\mathrm{pCi} / \mathrm{L}$ & $S O \bar{A}$ & -0.064 & 0.0514 & $u$ & 0.6068 & $<0.6068$ & $\bar{N}$ & \\
\hline Thorium-230 & $\mathrm{pCi} / \mathrm{L}$ & $\overline{S O A}$ & 0.524 & 0.42 & $\mathrm{U}$ & $1.03 \overline{7}$ & $<1.037$ & $\mathbf{N}$ & \\
\hline Thorium-232 & $p C i / L$ & SOA & 0 & $\overline{0}$ & $\bar{U}$ & $0 . \overline{197}$ & $<0.197$ & $N$ & \\
\hline Uranium-233/234 & $p \bar{C} \bar{i} / L$ & SOA & 0.0216 & 0.0386 & $\bar{U}$ & 0.1566 & $<0.1566$ & $\mathbf{N}$ & \\
\hline Uranium-235 & $\mathrm{pCi} / \mathrm{L}$ & SOA & 0.0144 & 0.0264 & $u$ & 0.1061 & $<0.1061$ & $\mathrm{~N}$ & \\
\hline Uranium-238 & $\mathrm{pCi} / \mathrm{L}$ & $S O A$ & 0.0323 & 0.0367 & U & 0.1265 & $<0.1265$ & $\mathrm{~N}$ & \\
\hline Sum of Alphas & & & & & & & $\overline{B D L}$ & $\mathrm{~N}$ & \\
\hline Sum of Betas & & & & & & & 61.49 & $Y$ & \\
\hline
\end{tabular}




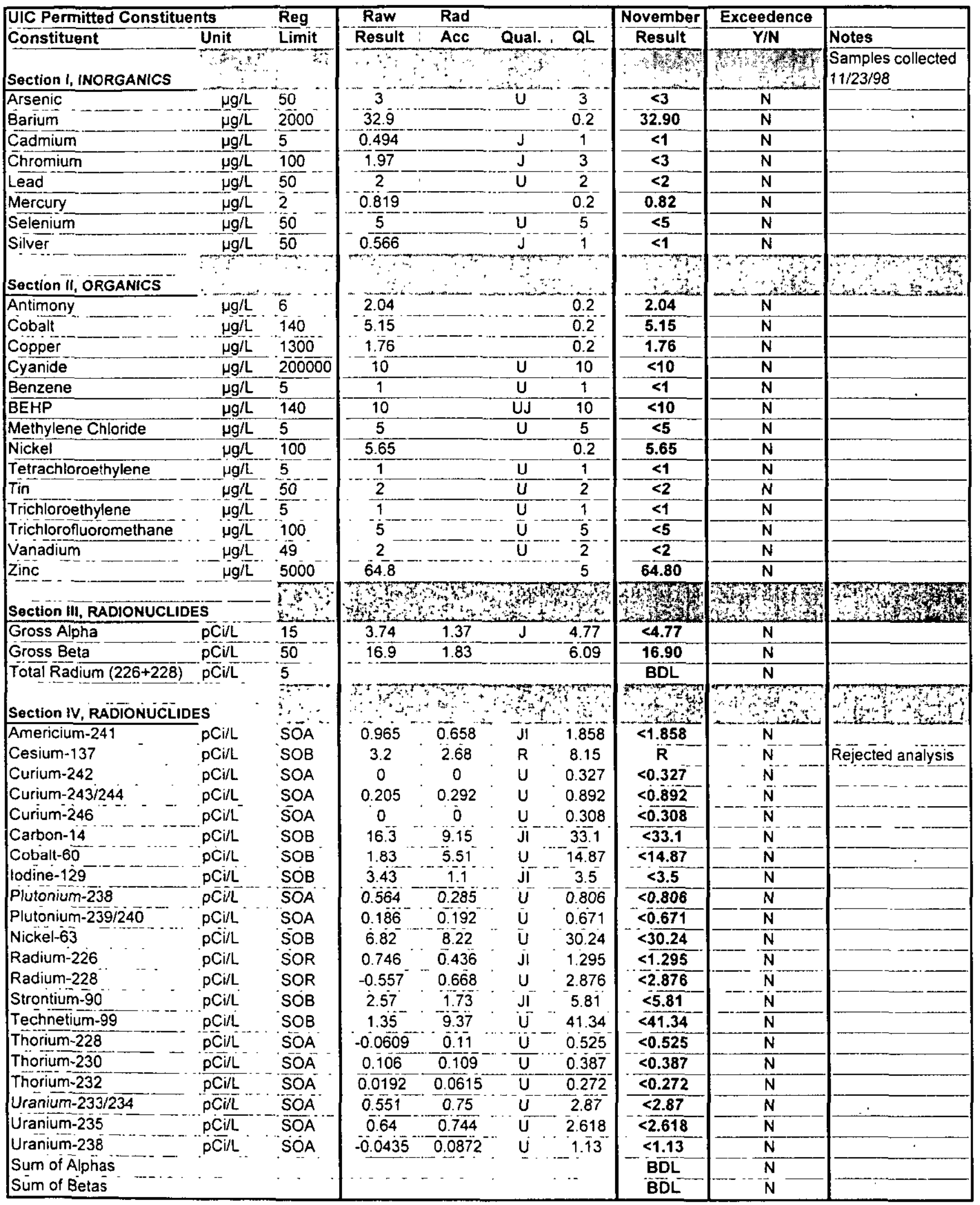




\begin{tabular}{|c|c|c|c|c|c|c|c|c|}
\hline UIC Permitted Constituents & $! \operatorname{Reg}$ & Raw & Rad & & & December & Exceedence & \\
\hline Constituent & Limit & Result & ACC & Qual. & QL & Result & Y/N & Notes \\
\hline Section I, INORGANICS & 78 & ming & PW & & $a^{2}$ & Wen & 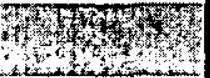 & $\begin{array}{l}\text { Samples collected } \\
12 / 09 / 98\end{array}$ \\
\hline Arsenic & 50 & 3 & & $\mathrm{U}$ & 3 & $<3$ & $\mathrm{~N}$ & \\
\hline Barium & 2000 & 2.07 & & & 0.2 & 2.07 & $\mathbf{N}$ & \\
\hline Cadmium & 5 & 1 & & $\bar{U}$ & 1 & $<1$ & $\mathbf{N}$ & \\
\hline Chromium & 100 & 1.13 & & $\mathrm{~J}$ & 3 & $<3$ & $\bar{N}$ & \\
\hline$\mu g / L$ & 50 & 2 & & $\bar{u}$ & 2 & $<2$ & $N$ & \\
\hline Mercuny & 2 & 0.977 & & & $0 . \overline{2}$ & 0.98 & $\mathbf{N}$ & \\
\hline Selenium & 50 & 5 & & $\bar{u}$ & 5 & $<5$ & $\mathrm{~N}$ & \\
\hline Silver & 50 & 1 & & U & 1 & $<1$ & $\mathbf{N}$ & \\
\hline Section II, ORGANICS & 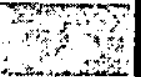 & $2+$ & in & & & $\therefore$ & 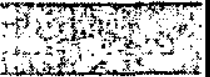 & Wh, \\
\hline Antimony & 6 & 0.2 & & $\mathrm{U}$ & 0.2 & $<0.2$ & $\mathrm{~N}$ & \\
\hline Cobait & 140 & 0.15 & & $\mathrm{~J}$ & 0.2 & $<0.2$ & $\mathbf{N}$ & \\
\hline Copper & 1300 & 4.08 & & & 0.2 & 4.08 & $\mathbf{N}$ & \\
\hline Cyanide & 200000 & $\overline{2} . \overline{67}$ & & $U$ & 10 & $<10$ & $\mathbf{N}$ & \\
\hline Benzene & 5 & 1 & & $u$ & 1 & $<1$ & $\bar{N}$ & \\
\hline$B E H P$ & 140 & 10 & & UJ & 10 & $<10$ & $\mathbf{N}$ & \\
\hline Methylene Chloride & 5 & 5 & & $\bar{U}$ & 5 & $<5$ & $\mathbf{N}$ & \\
\hline Nickel & 100 & $0 . \overline{2}$ & 1 & $U$ & 0.2 & $<0.2$ & $\mathbf{N}$ & \\
\hline Tietrachloroethylene & 5 & 1 & & $\bar{U}$ & 1 & $<1$ & $\mathbf{N}$ & \\
\hline$\mu g / L$ & 50 & 2 & & $\mathrm{U}$ & 2 & $<2$ & $\mathbf{N}$ & \\
\hline Trichloroethylene & 5 & 1 & & U & 1 & $<1$ & $\mathbf{N}$ & \\
\hline Trichlorofluoromethane & 100 & 5 & & $\bar{U}$ & 5 & $<5$ & $\mathbf{N}$ & \\
\hline Vanadium & 49 & 2 & & $\bar{u}$ & 2 & $<2$ & $\mathbf{N}$ & \\
\hline Zinc & 5000 & 5 & & u & 5 & $<5$ & $N$ & \\
\hline Section III, RADIONUCLIDES & (x) & 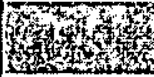 & 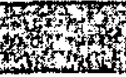 & 㷊 & the & \% & 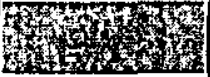 & 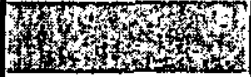 \\
\hline Gross Alpha & 15 & 0.834 & 0.65 & $U$ & 2.46 & $<2.46$ & $\mathrm{~N}$ & \\
\hline Gross Beta & 50 & 6.12 & 0.91 & & 3.04 & 6.12 & $\mathbf{N}$ & \\
\hline Total Radium $(226+228)$, pCi/L & 5 & & & & & BDL & $\mathbf{N}$ & \\
\hline ONUCLIDES & Wh & Pot & Why & & & Why & 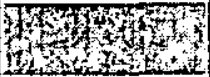 & W \\
\hline Americium-241 & SOA & 0.104 & 0.171 & U & 0.708 & $<0.708$ & $\mathrm{~N}$ & \\
\hline Cesium-137 & SOB & 1.02 & 1.98 & u & 7.77 & $<7.77$ & $N$ & Rejected analysis \\
\hline urium-242 & SOA & 0 & 0 & $u$ & 0.219 & $<0.219$ & $\mathbf{N}$ & \\
\hline urium-243/244 & SOA & 0.0528 & 0.143 & u & 0.652 & $<0.652$ & $N$ & \\
\hline rium-246 & $S O A^{-}$ & 0 & 0 & u & 0.208 & $<0.208$ & $\bar{N}$ & \\
\hline $\mathrm{PC}$ ill & SÖB & 24.1 & 4.29 & & 14.98 & 24.10 & $\mathrm{~N}$ & \\
\hline $\mathrm{pCi} / \mathrm{L}$ & SOB & $-0 . \overline{149}$ & 2.09 & $u$ & 8.11 & $<8.11$ & $\bar{N}$ & \\
\hline lodine-129 & SOB & 6.2 & 1.79 & & 4.87 & 6.20 & $N$ & \\
\hline Plutonium-238 & SOA & 0 & 0 & $\vec{u}$ & 0.116 & 16 & $\mathrm{~N}$ & \\
\hline Plutonium-239/240 & $\overline{S O A}$ & 0 & 0 & u & $0 . \overline{116}$ & $<0.116$ & $\mathrm{~N}$ & \\
\hline Nickel-63 & SŌB ${ }^{-}$ & -1.91 & 7.86 & $\underline{u}$ & 29.42 & $<29.42$ & $\mathrm{~N}$ & \\
\hline Radium-226 & SOR & 0.901 & 0.481 & $\mathrm{Ji}$ & 1.502 & $<1 . \overline{502}$ & $N$ & \\
\hline Radium- $2 \overline{28}$ & SOR & -0.908 & 0.557 & $u$ & $2.4 \overline{9} 4$ & $<2.494$ & $\mathbf{N}$ & \\
\hline Strontium-90 & SOB & 0.957 & 0.521 & Jٓ & $1.891^{\circ}$ & $3 \overline{91}$ & $\mathrm{~N}$ & \\
\hline$p \overline{C i l}$ & SOB & -0.269 & 2.77 & U & 12.38 & $<12.38$ & $N$ & \\
\hline Thorium-228 & $S O A$ & -0.0257 & 0.109 & Ü & $0 . \overline{487}$ & $<0.487$ & $N$ & \\
\hline Thorium-230 & SOA & 0.00776 & 0.0532 & $u$ & 0.2464 & $<0.2464$ & $\bar{N}$ & \\
\hline Thorium & $S \overline{O A}$ & 0 & 0 & $\bar{u}$ & 0.048 & $<0.048$ & $N$ & \\
\hline Uranium-233/234 & SOA & 0.022 & 0.0565 & u & 0.231 & $<0.231$ & $N$ & \\
\hline Uranium-235 & SOA & $0 . \overline{0274}$ & 0.0451 & $\bar{U}$ & 0.1736 & $<0.1736$ & $N$ & \\
\hline $\begin{array}{l}\text { uranium-238 } \\
\text { Sum of Alphas }\end{array}$ & SOA. & 0.0273 & 0.045 & $\bar{U}$ & 0.1732 & $\begin{array}{c}<0.1732 \\
B D L\end{array}$ & $\frac{N}{N}$ & \\
\hline Sum of Betas & & & & & & $30 . \overline{30}$ & $\bar{N}$ & \\
\hline
\end{tabular}


This page was left blank intentionally. 


\section{Appendix D}

Time Series Plots 
WSRC-TR-99-00013

Unclassified

THIS PAGE LEFT BLANK INTENTIONALLY. 


\section{Mercury Concentrations}

Well Cluster HSB 68

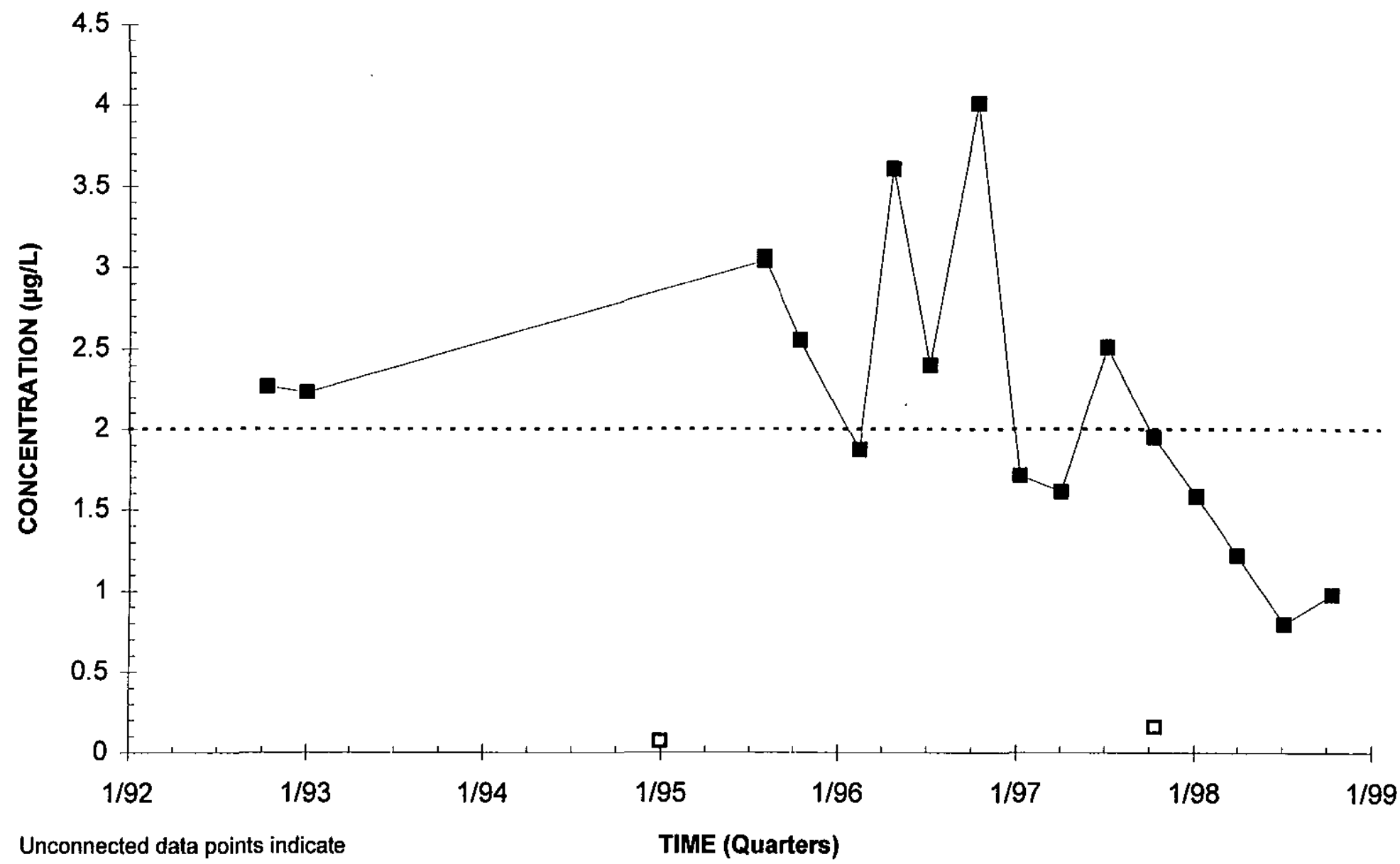

intervals of dry wells or values

below the detection limit.

GWPS $2 \mu \mathrm{g} / \mathrm{L}$

HSB $68(W)-$ - - HSB $68 A(L C) \cdots . .$. GWPS

Note: W=Water Table (IIB2); B=Barnwell (IIB1); M=McBean (IIB1); UC=Upper Congaree (IIA); MC=Middle Congaree (IIA); LC=Lower Congaree (IIA) 


\section{Mercury Concentrations \\ Well HSB 83D}

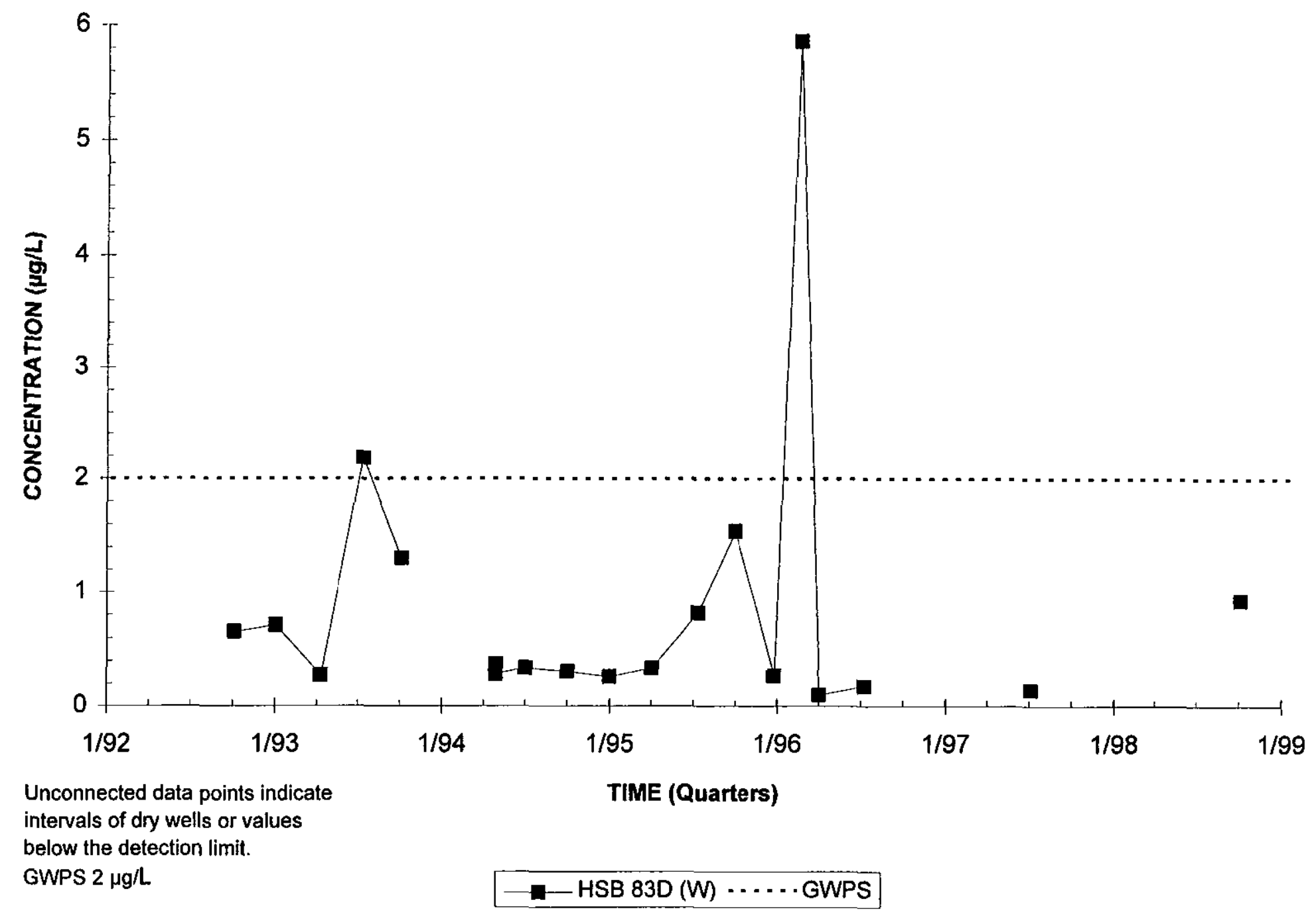

Note: W=Water Table (IIB2); B=Barnwell (IIB1); M=McBean (IIB1); UC=Upper Congaree (IIA); MC=Middle Congaree (IIA); LC=Lower Congaree (IIA) 


\section{Mercury Concentrations}

Well HSB101D

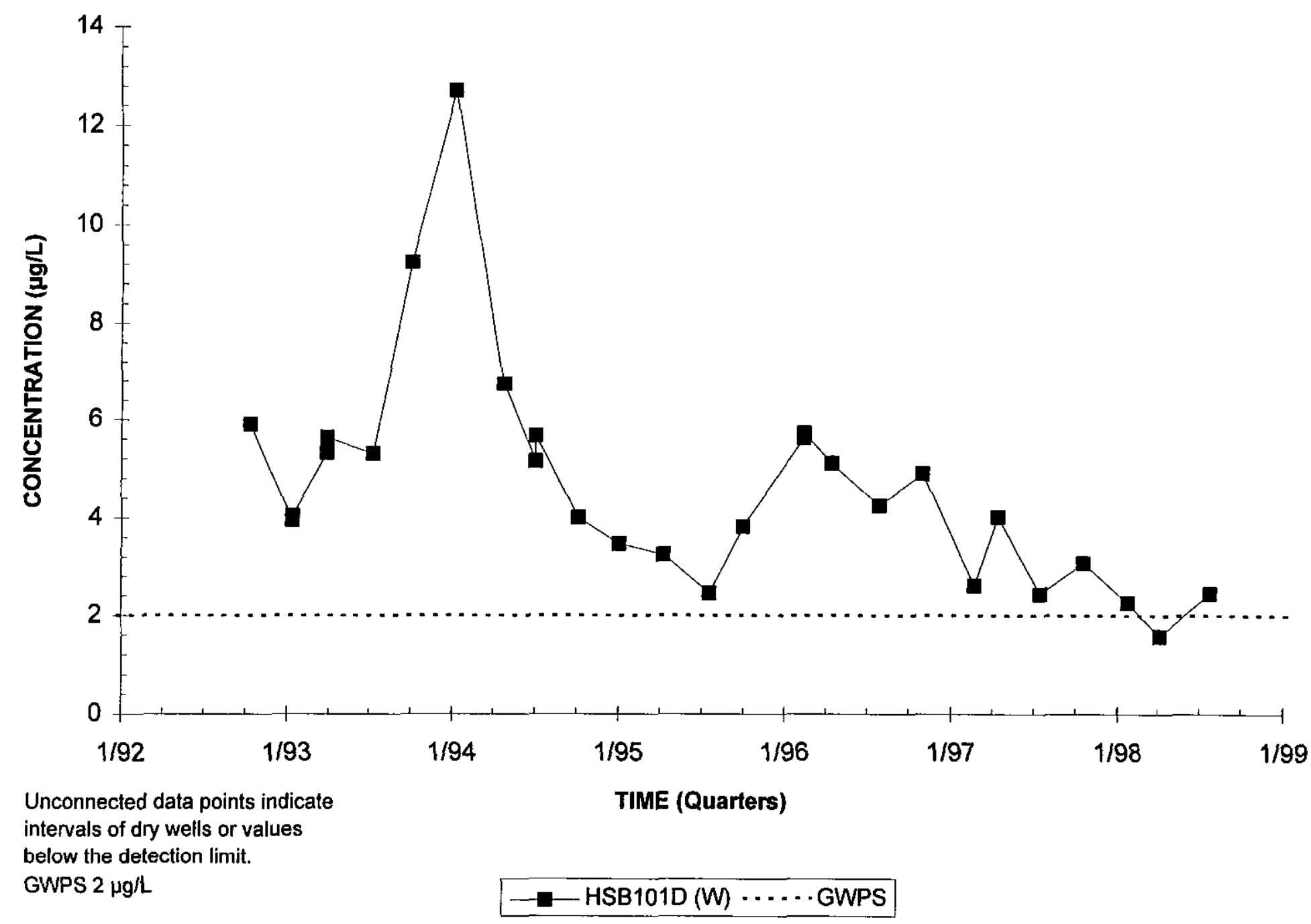

Note: $W=$ Water Table (IIB2); B=Barnwell (IBB1); M=McBean (IIB1); UC=Upper Congaree (IIA); MC=Middle Congaree (IIA); LC=Lower Congaree (IIA) 


\section{Mercury Concentrations \\ Well HSB102D}

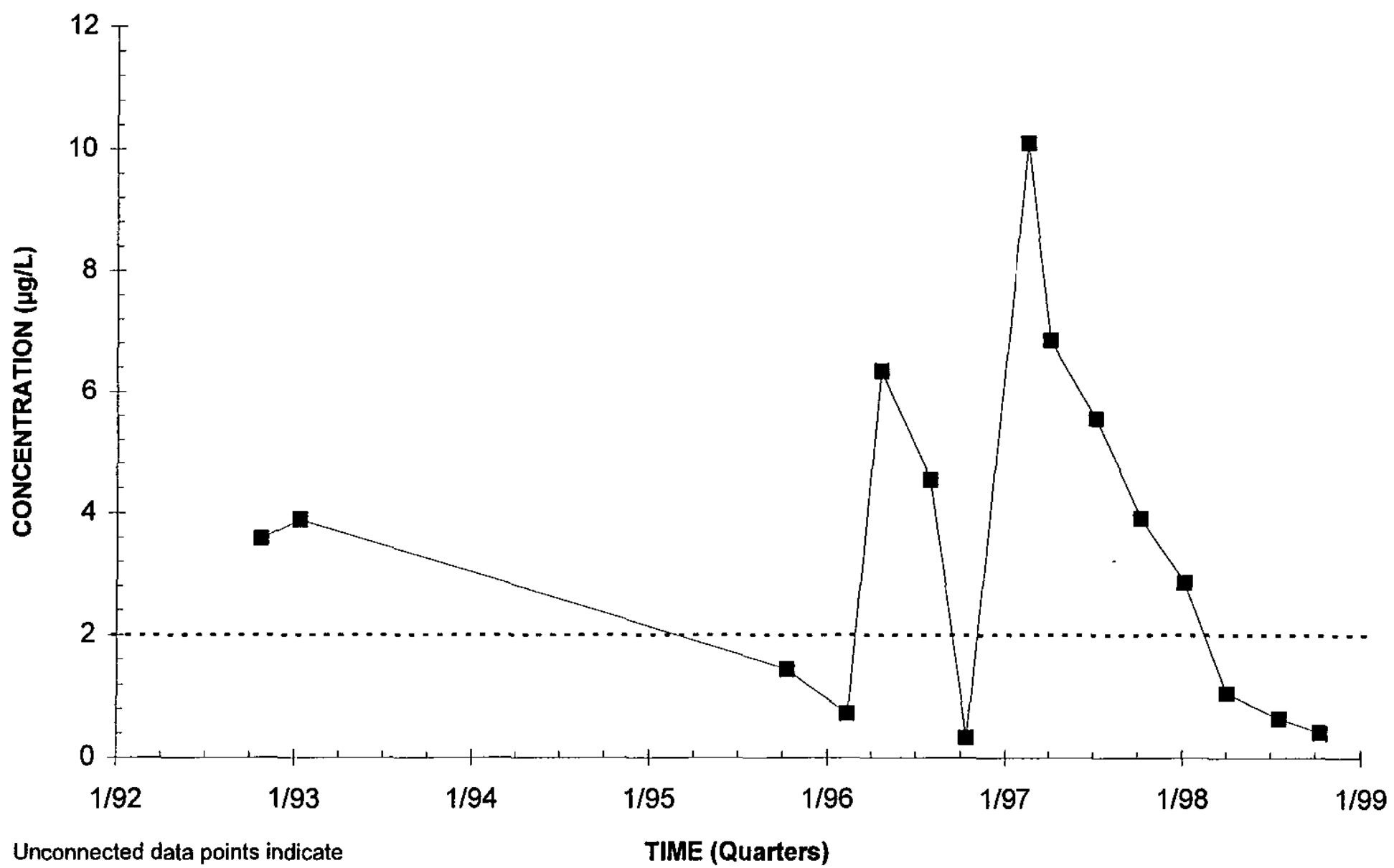

intervals of dry wells or values

below the detection limit.

GWPS $2 \mu \mathrm{g} / \mathrm{L}$

m-HSB102D (W) $\cdots \ldots$ GWPS

Note: $W=$ Water Table (IIB2); B=Barnwell (IIB1); M=McBean (IIB1); UC=Upper Congaree (IIA); MC=Middle Congaree (IIA); LC=Lower Congaree (IIA) 


\section{Mercury Concentrations}

Well HSB103D

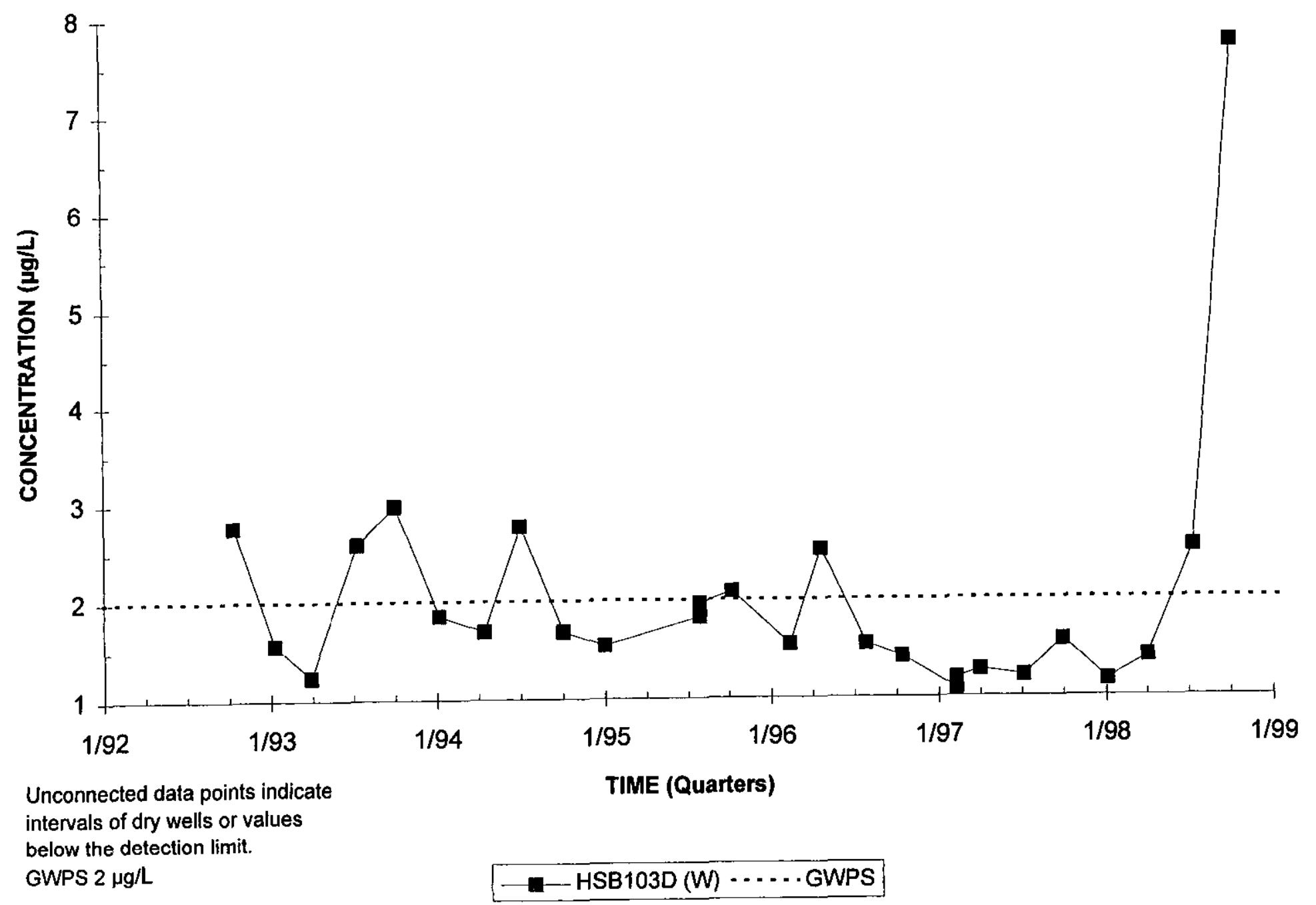

Note: W=Water Table (IIB2); B=Barnwell (IIB1); M=McBean (IIB1); UC=Upper Congaree (IIA); MC=Middle Congaree (IIA); LC=Lower Congaree (IIA) 


\section{Mercury Concentrations \\ Well HSB104D}

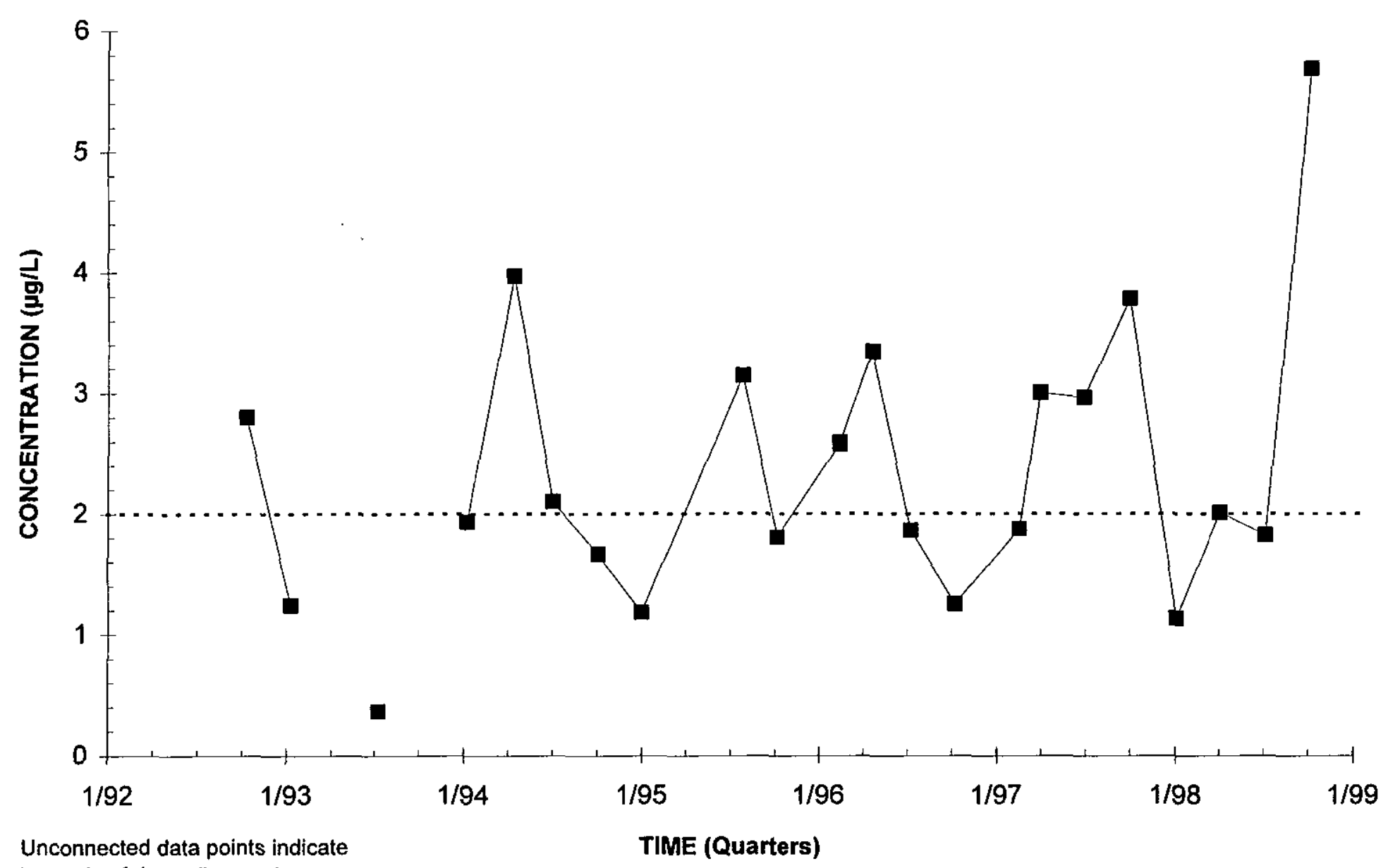

intervals of dry wells or values

below the detection limit.

GWPS $2 \mu \mathrm{g} / \mathrm{L}$

HSB104D (W) $\ldots \ldots$. GWPS

Note: $W=$ Water Table (IIB2); $B=B a r n w e l l$ (IIB1); $M=M c B e a n$ (IIB1); UC=Upper Congaree (IIA); $M C=$ Middle Congaree (IIA); LC=Lower Congaree (IIA) 


\section{Mercury Concentrations Well Cluster HSB105}

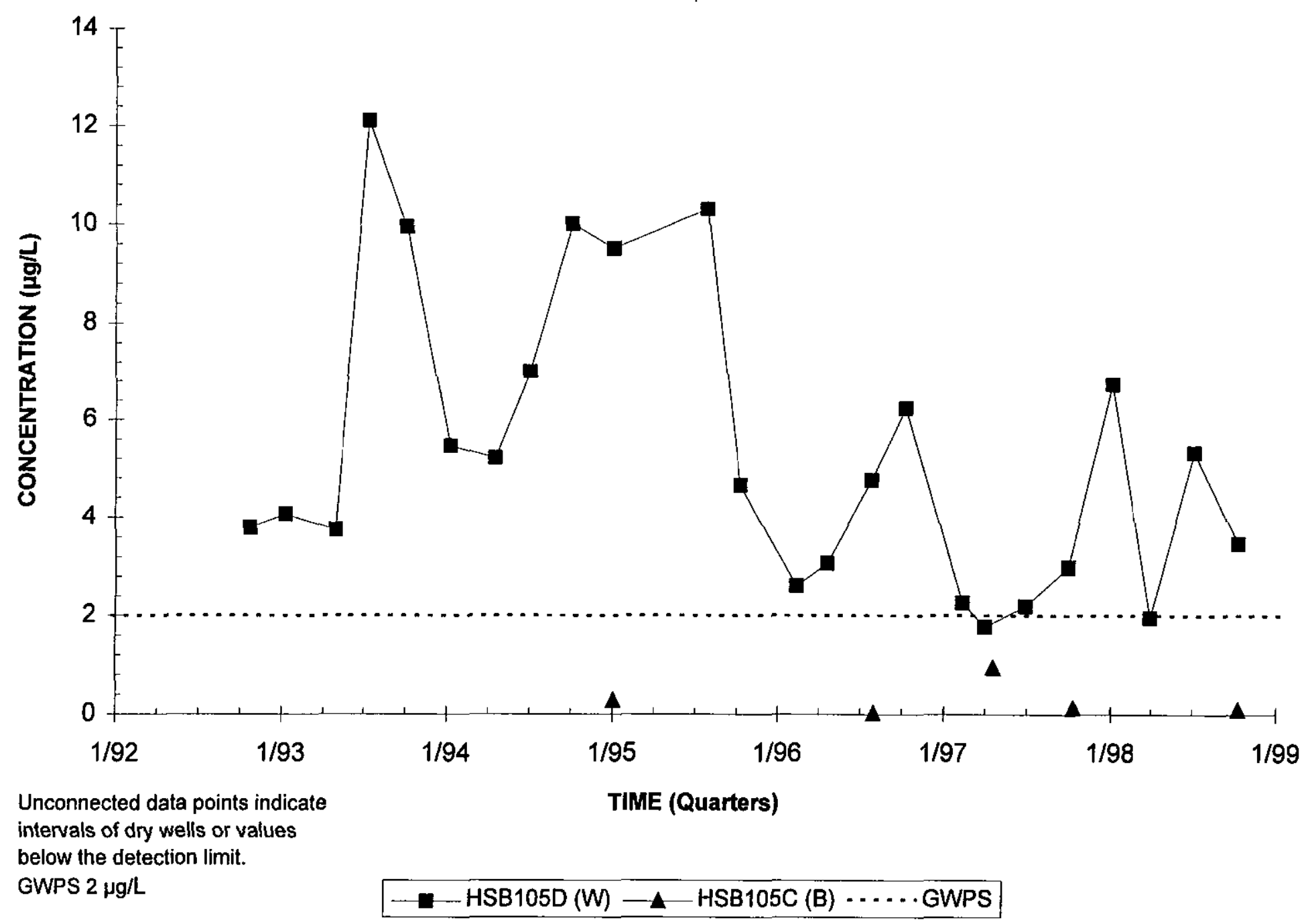

Note: $W=$ Water Table (IIB2); B=Barnwell (IIB1); M=MCBean (IIB1); UC=Upper Congaree (IIA); MC=Middle Congaree (IIA); LC=Lower Congaree (IAA) 


\section{Mercury Concentrations \\ Well HSB106D}

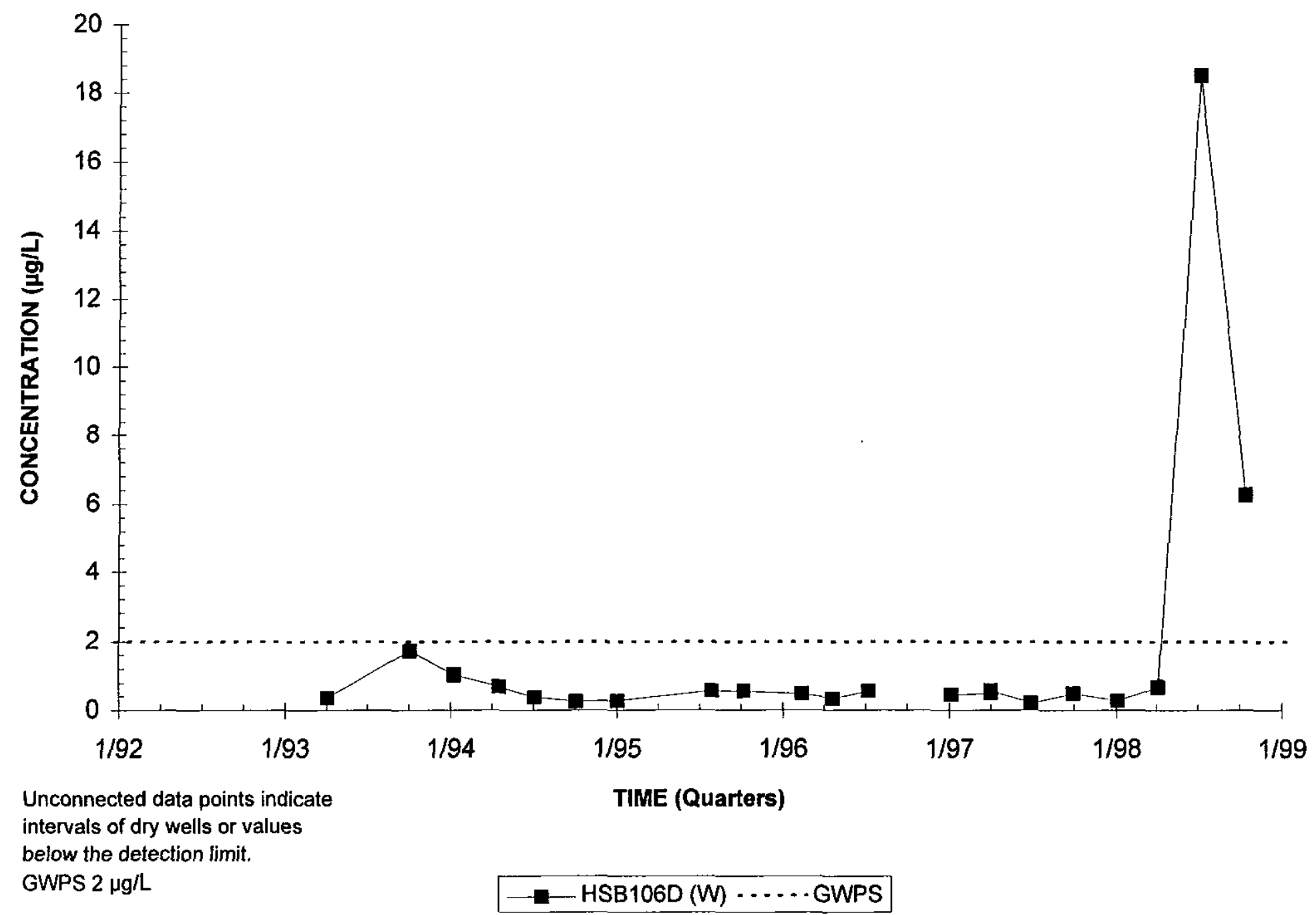

Note: $W=$ Water Table (IIB2); B=Barnwell (IIB1); M=McBean (IIB1); UC=Upper Congaree (IIA); MC=Middle Congaree (IIA); LC=Lower Congaree (IIA) 


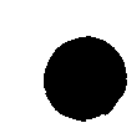

WSRC-TR-99-00013

Unclassified

\section{Mercury Concentrations \\ Well HSB108D}

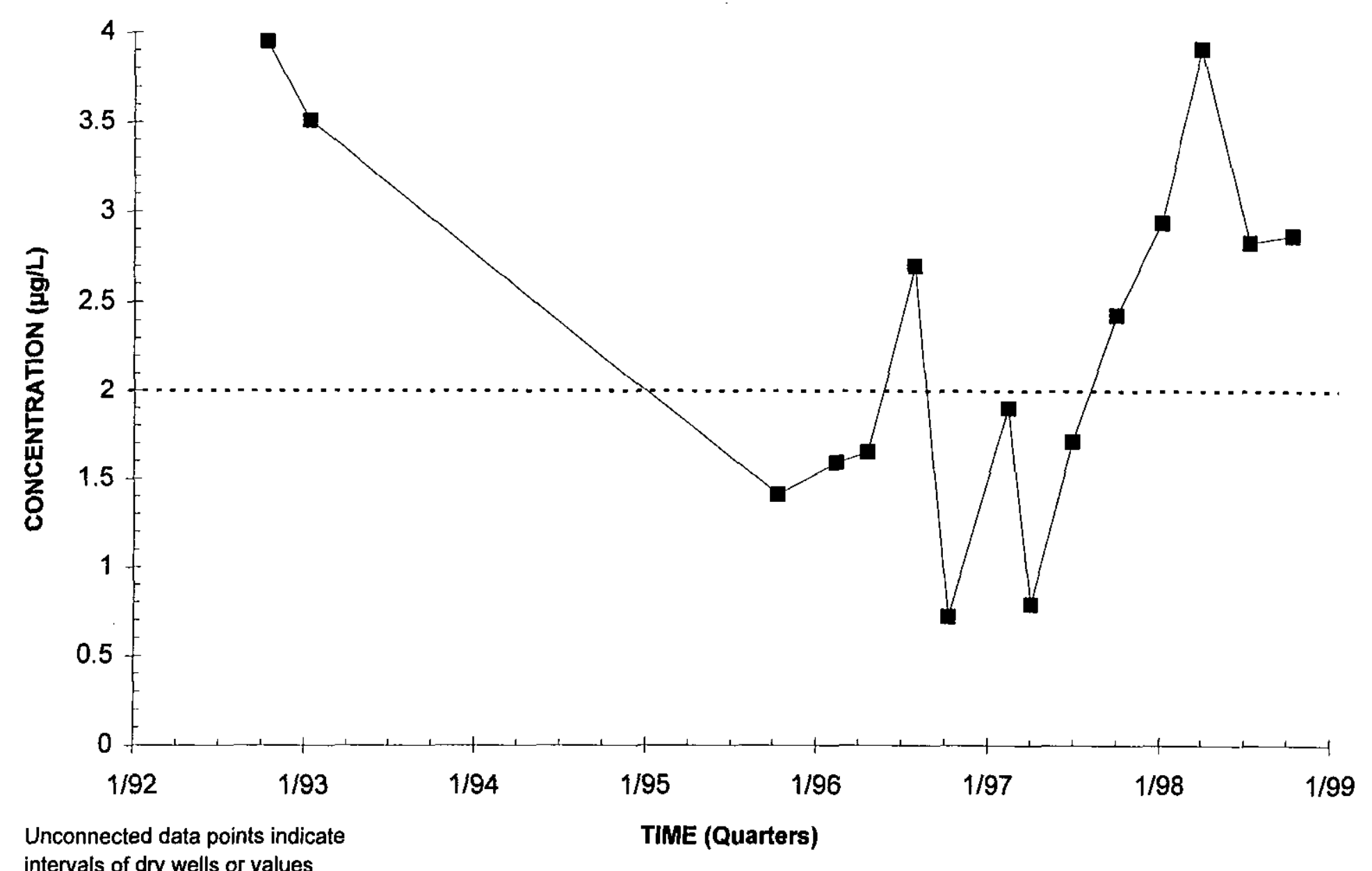

intervals of dry wells or values

below the detection limit.

GWPS $2 \mu \mathrm{g} / \mathrm{L}$

HSB108D $(\bar{W}) \cdots . . . \mathrm{GWPS}$

Note: W=Water Table (IIB2); B=Barnwell (IIB1); M=McBean (IIB1); UC=Upper Congaree (IIA); MC=Middle Congaree (IIA); LC=Lower Congaree (IIA) 


\section{Mercury Concentrations \\ Well HSB116C}

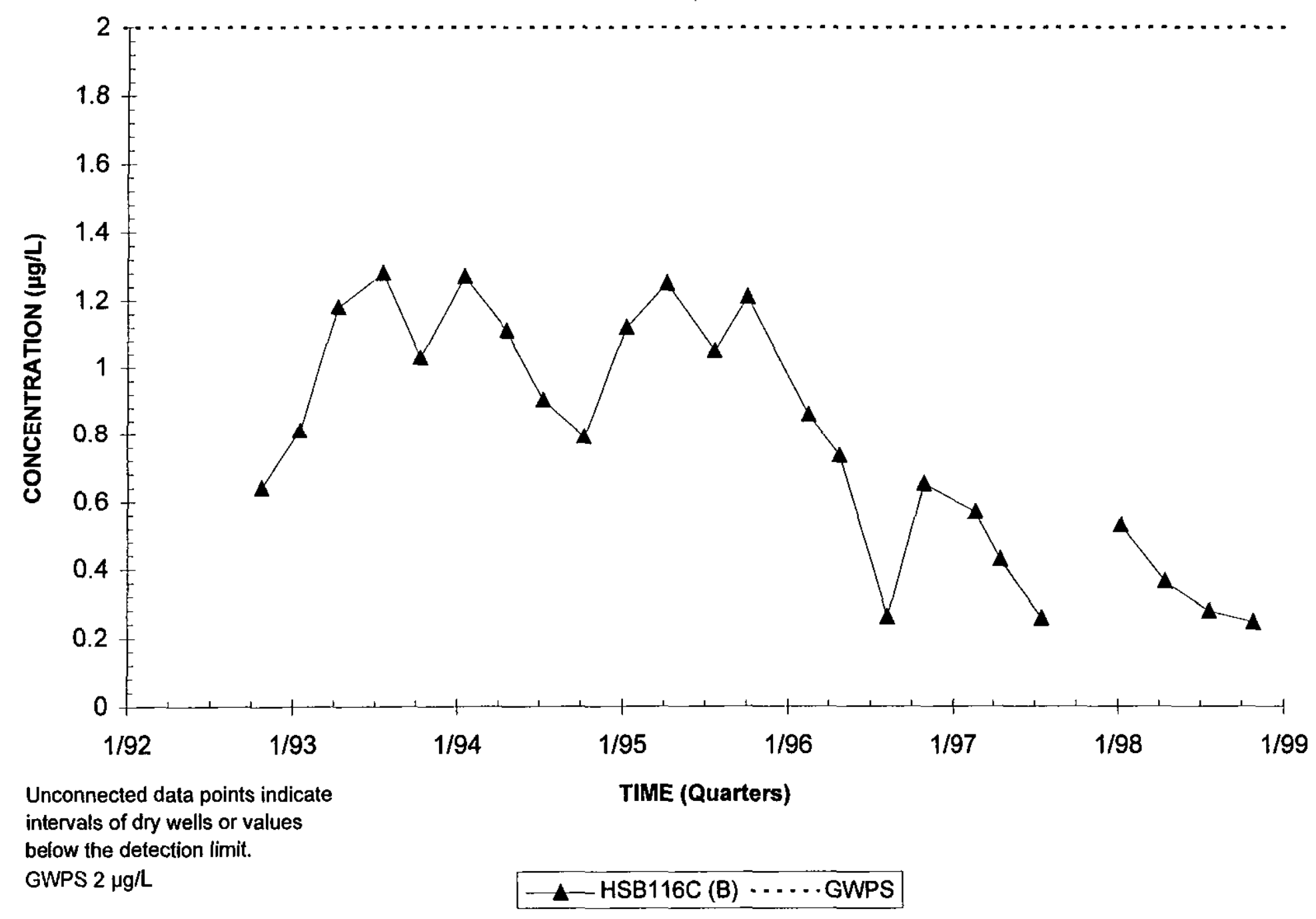

Note: W=Water Table (IIB2); B=Barnwell (IIB1); M=McBean (IIB1); UC=Upper Congaree (IIA); MC=Middle Congaree (IIA); LC=L.ower Congaree (IIA) 


\section{Mercury Concentrations \\ Well HSB125D}

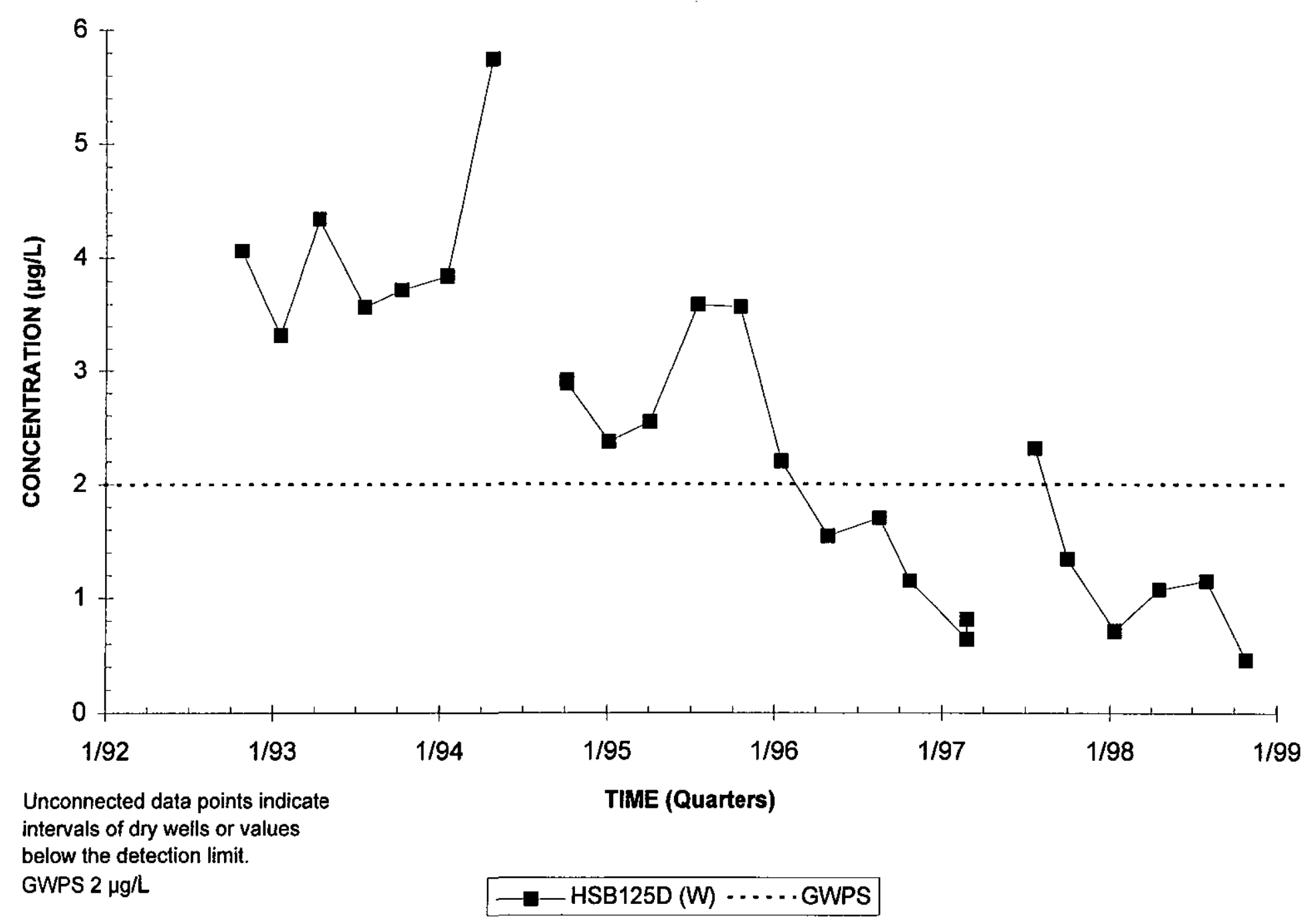

Note: $W=$ Water Table (IIB2); B=Barnwell (IIB1); M=McBean (IIB1); UC=Upper Congaree (IIA); MC=Middle Congaree (IIA); LC=Lower Congaree (IIA) 


\section{Mercury Concentrations \\ Well Cluster HSB127}

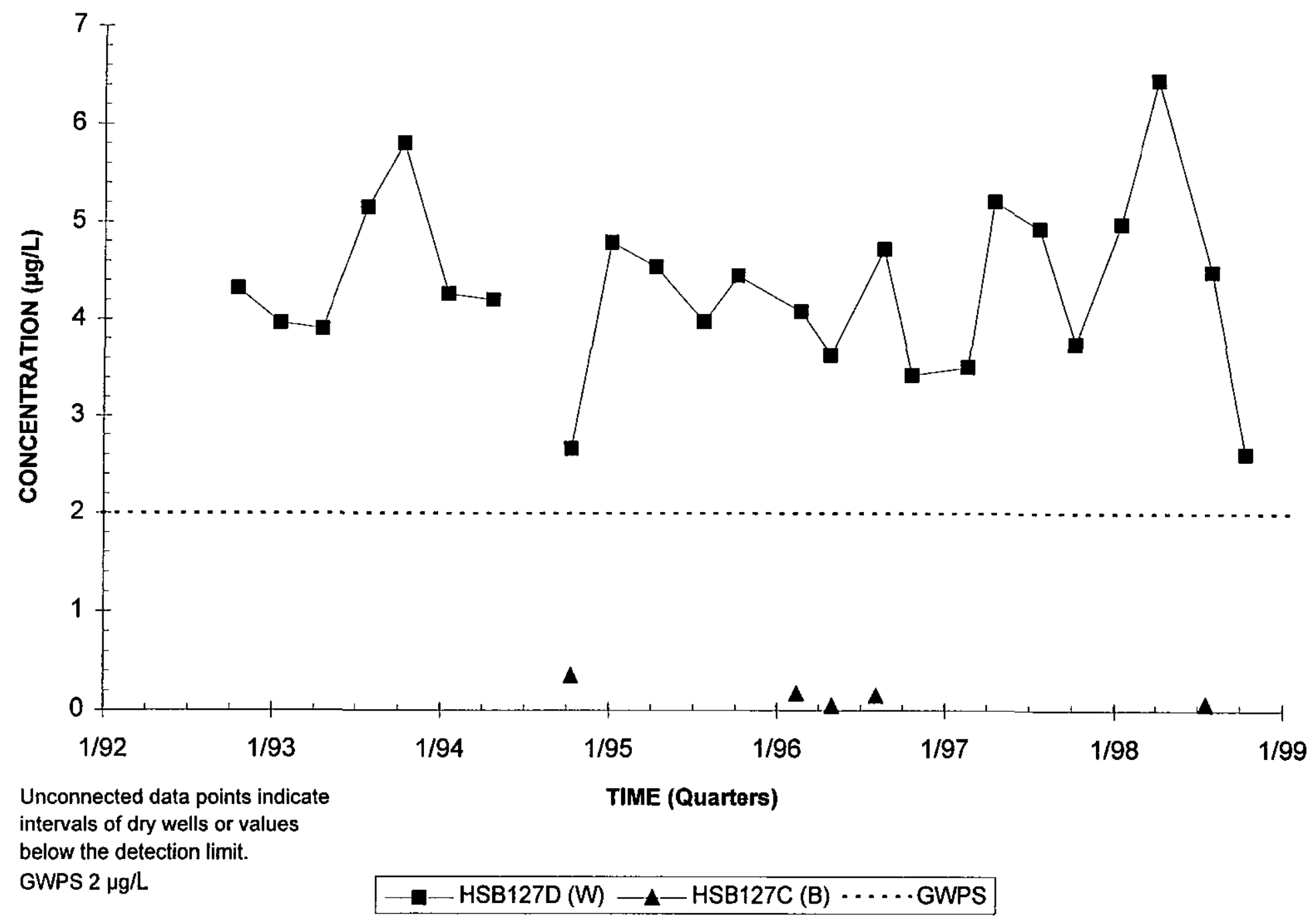

Note: W=Water Table (IIB2); B=Barnwell (IIB1); M=MCBean (IIB1); UC=Upper Congaree (IIA); MC=Middle Congaree (IIA); LC=Lower Congaree (IIA) 


\section{Mercury Concentrations \\ Well HSB145D}

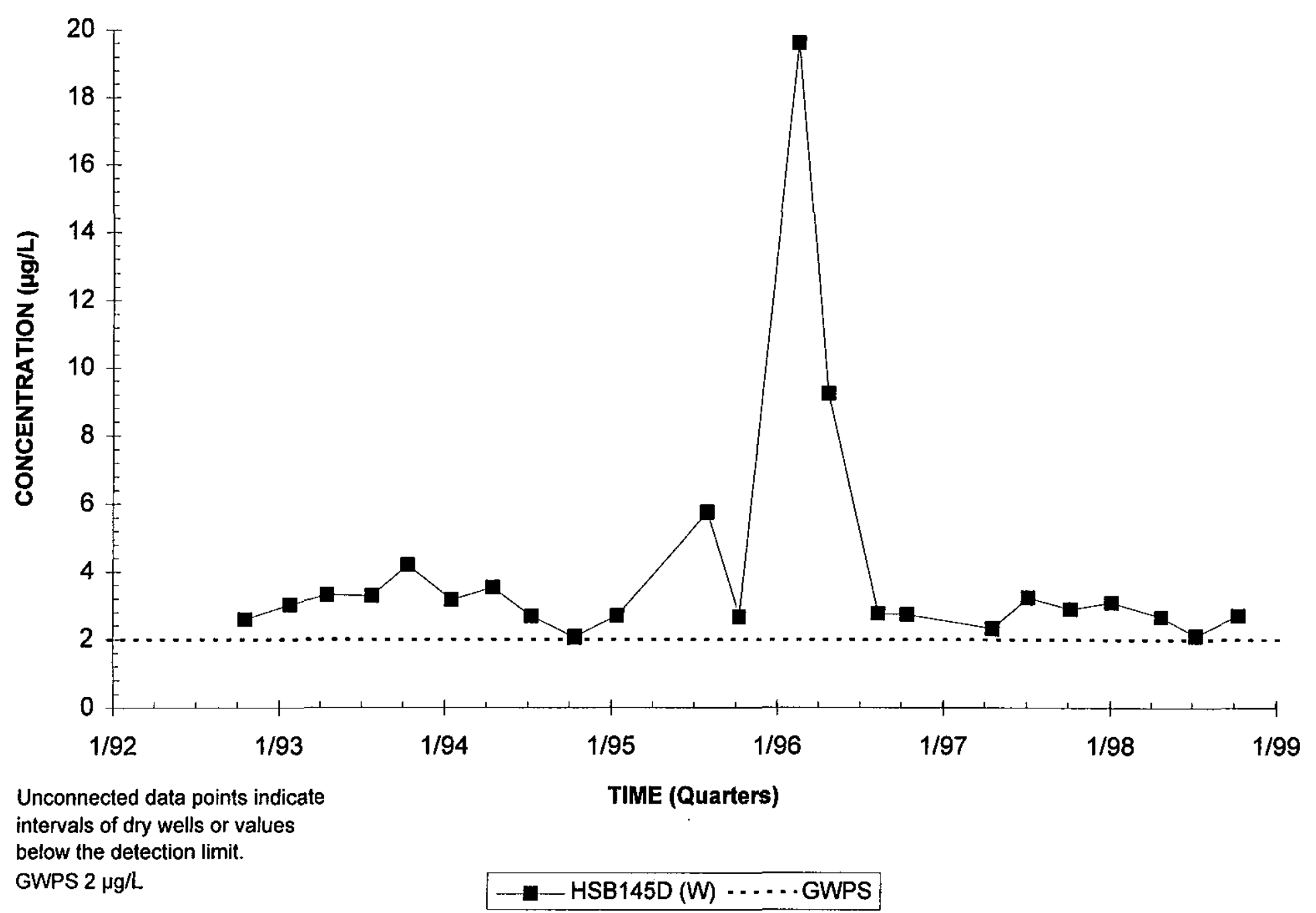

Note: W=Water Table (IIB2); B=Barnwell (IIB1); M=McBean (IIB1); UC=Upper Congaree (IIA); MC=Middle Congaree (IIA); LC=Lower Congaree (IIA) 


\section{Tritium Activities}

Well Cluster HSB 84

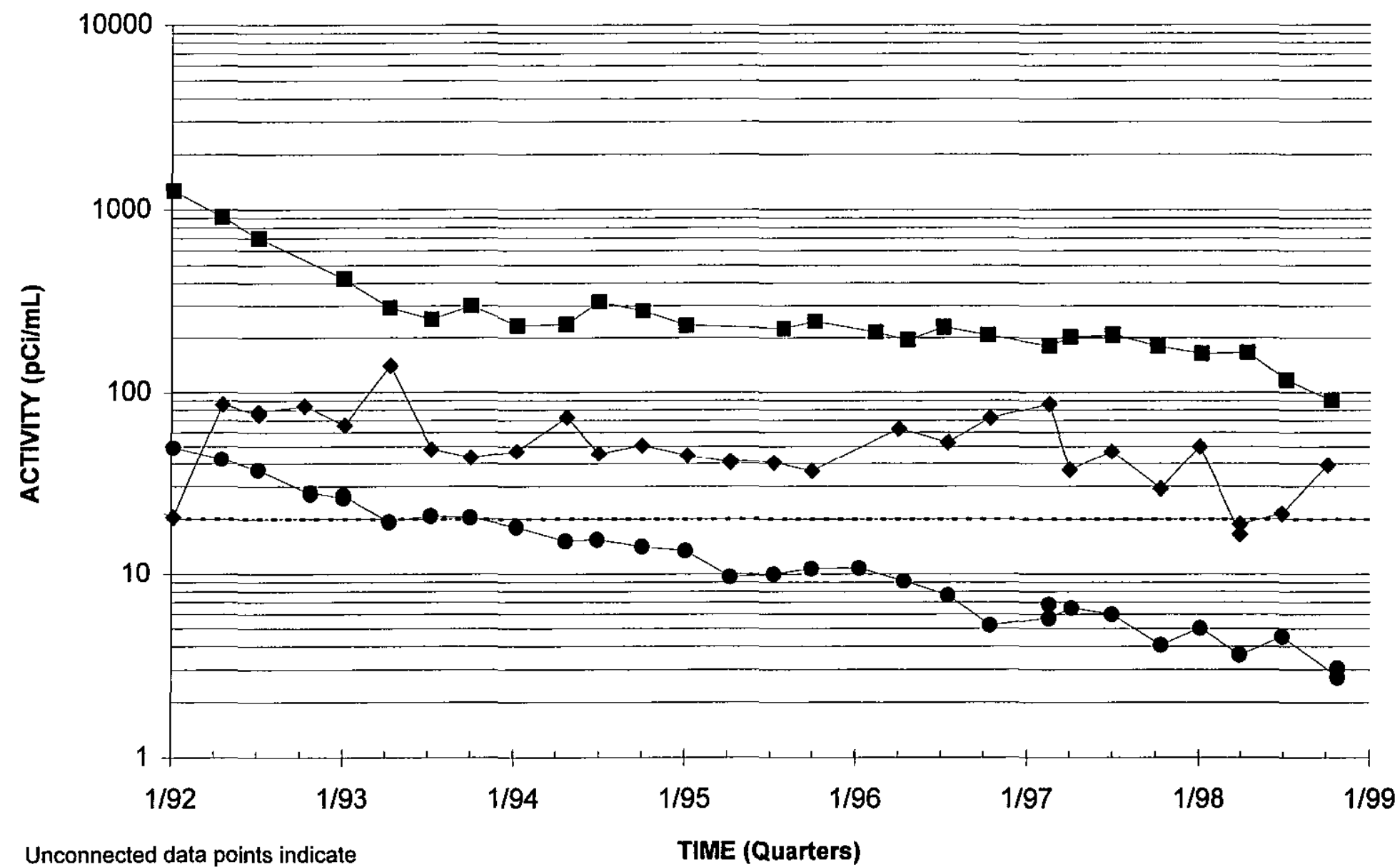

Unconnected data points indicate intervals of dry wells or values

below the detection limit.

GWPS $20 \mathrm{pCi} / \mathrm{mL}$

$$
-\mathrm{HSB} 84 \mathrm{D}(\mathrm{W})-\mathrm{HSB} 84 \mathrm{~B}(\mathrm{M}) \multimap \mathrm{HSB} 84 \mathrm{~A}(\mathrm{LC}) \ldots \ldots \text { GWPS }
$$

Note: $W=$ Water Table (IIB2); B=Barnwell (IIB1); M=McBean (IIB1); UC=Upper Congaree (IIA); MC=Middle Congaree (IIA); LC=Lower Congaree (IIA) 


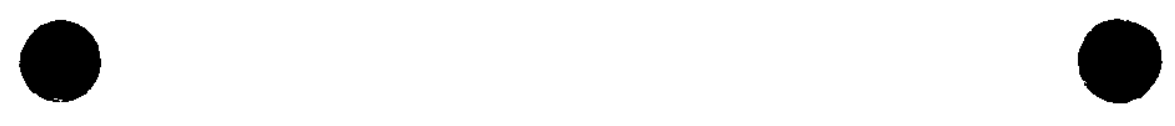

WSRC-TR-99-00013 Unclassified

\section{Tritium Activities} Well Cluster HSB102

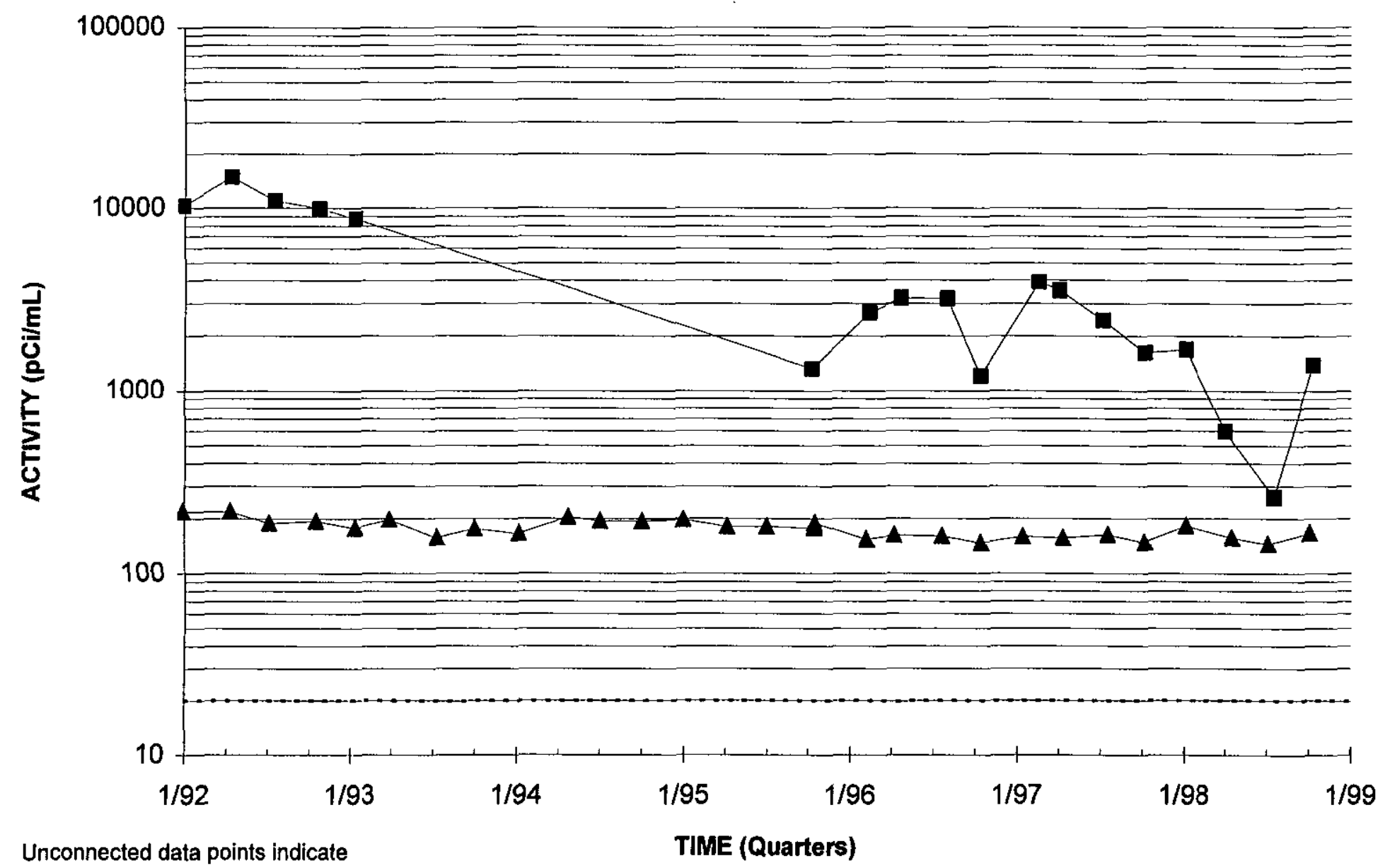
intervals of dry wells or values below the detection limit. GWPS $20 \mathrm{pCi} / \mathrm{mL}$

$$
\text { - }
$$

Note: $W=$ Water Table (IIB2); $B=B$ arnwell (IIB1); $M=M C B e a n$ (IIB1); UC=Upper Congaree (IIA); $M C=$ Mlddle Congaree (IIA); LC=Lower Congaree (IIA) 


\section{Tritium Activities \\ Well HSB104D}

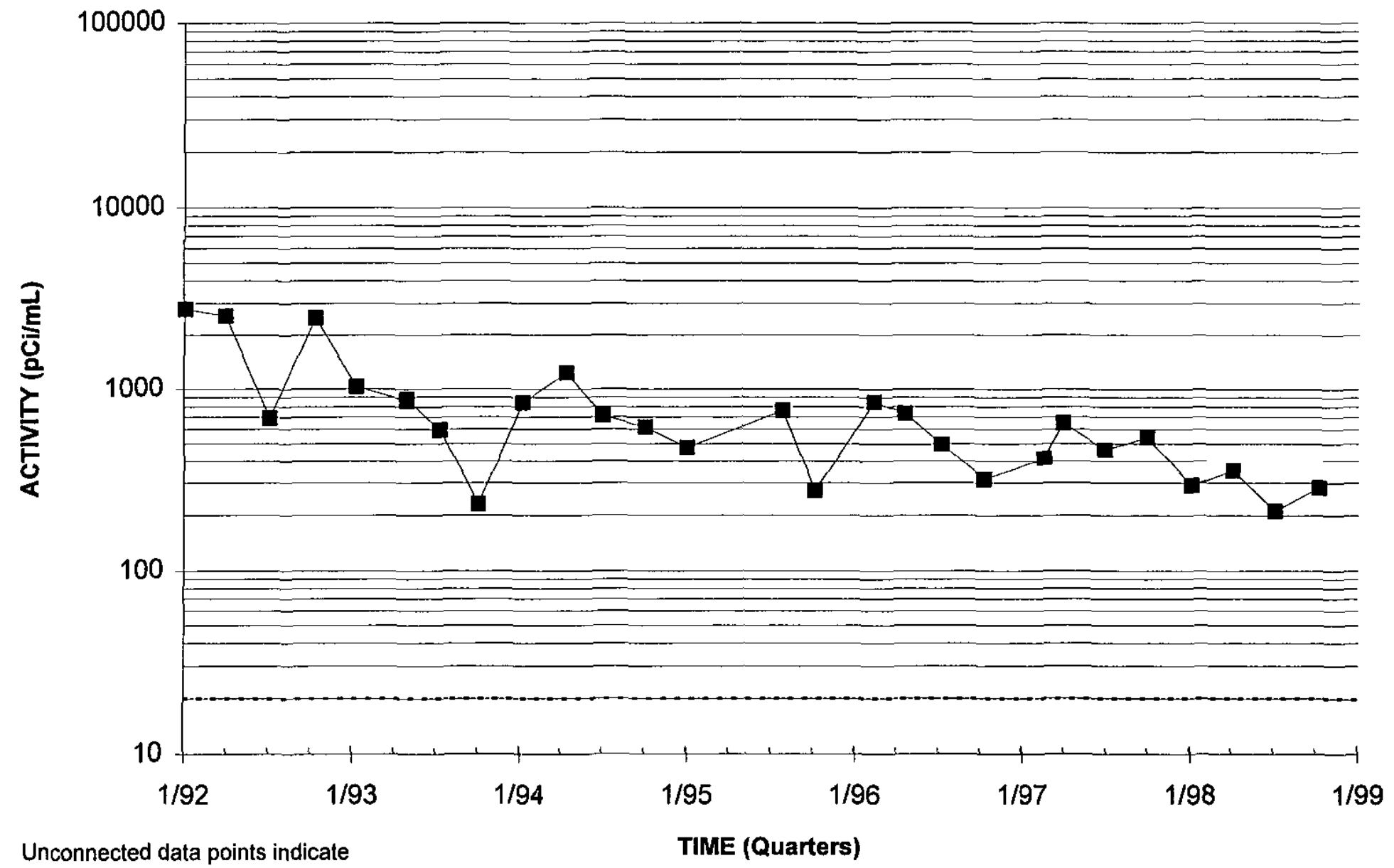

intervals of dry wells or values

below the detection limit.

GWPS $20 \mathrm{pCi} / \mathrm{mL}$

$$
\text { —-HSB104D (W) } \cdots . . . \text { GWPS }
$$

Note: W=Water Table (IIB2); B=Barnwell (IIB1); M=McBean (IIB1); UC=Upper Congaree (IIA); MC=Middle Congaree (IIA); LC=Lower Congaree (IIA) 


\section{Tritium Activities}

Well HSB108D

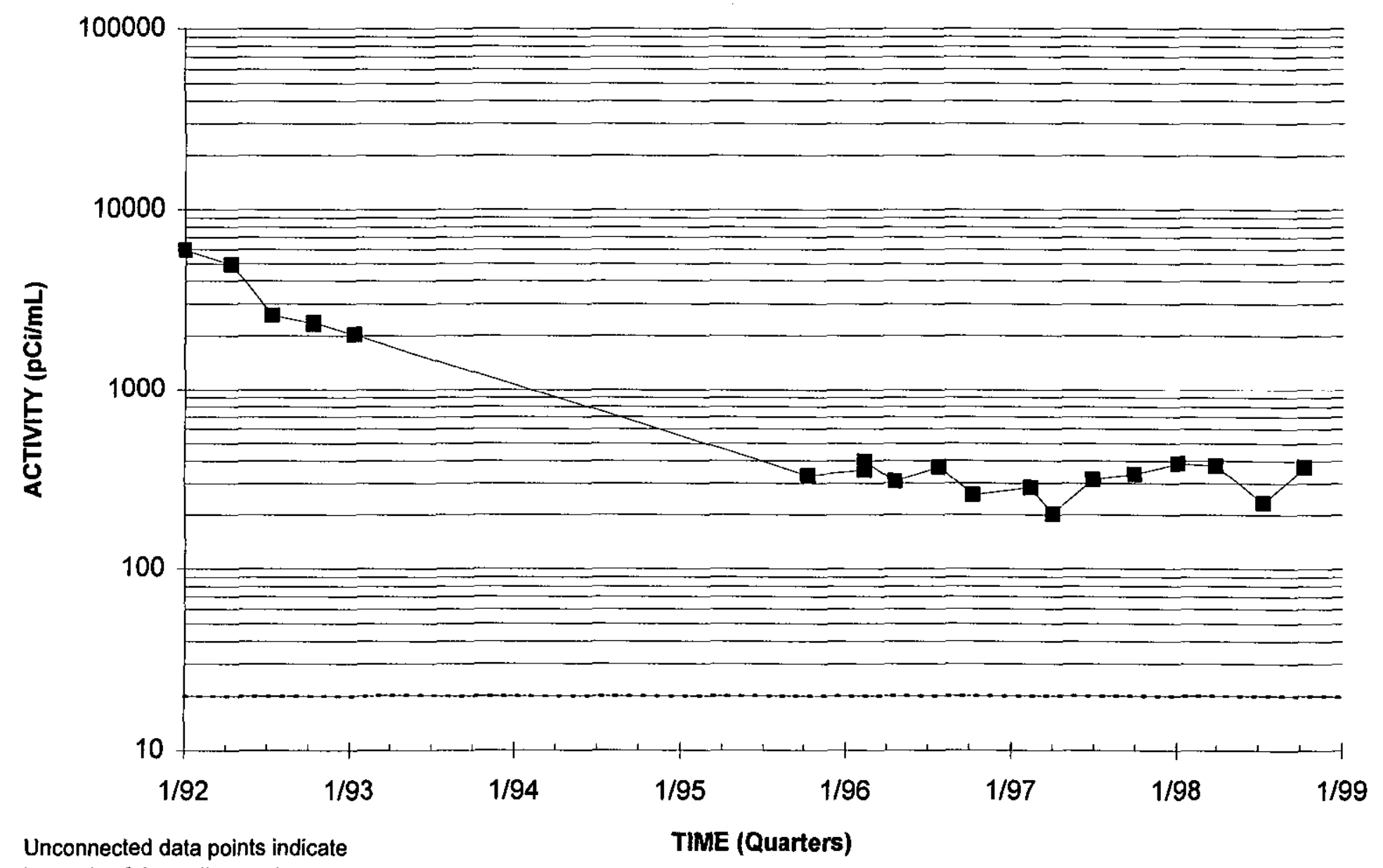

Uncorve of dry wells

below the detection limit.

GWPS $20 \mathrm{pCi} / \mathrm{mL}$

a-HSB108D (W) ...... GWPS

Note: W=Water Table (IIB2); B=Barnwell (IIB1); M=McBean (IIB1); UC=Upper Congaree (IIA); MC=Middle Congaree (IIA); LC=Lower Congaree (IIA) 


\section{Tritium Activities \\ Well Cluster HSB111}

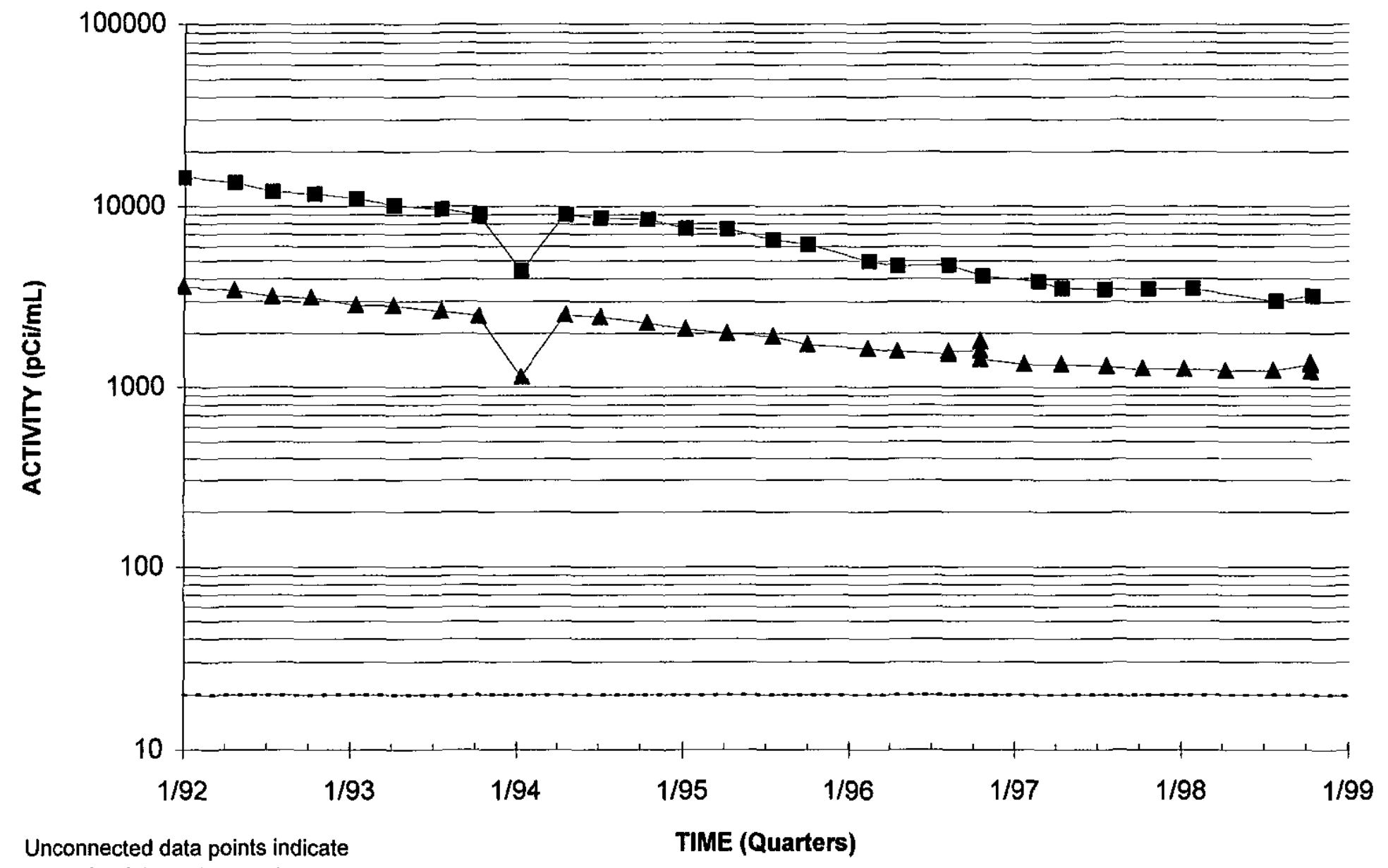

intervals of dry wells or values

below the detection limit.

GWPS $20 \mathrm{pCi} / \mathrm{mL}$

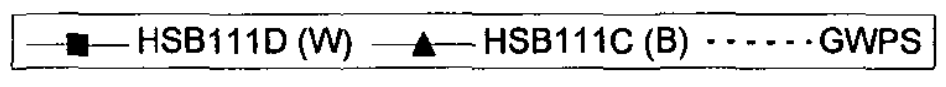

Note: W=Water Table (IIB2); 8=Barnwell (IIB1); M=McBean (IIB1); UC=Upper Congaree (IIA); MC=Middle Congaree (IIA); LC=Lower Congaree (IIA) 


\section{Tritium Activities}

\section{Well Cluster HSB117}

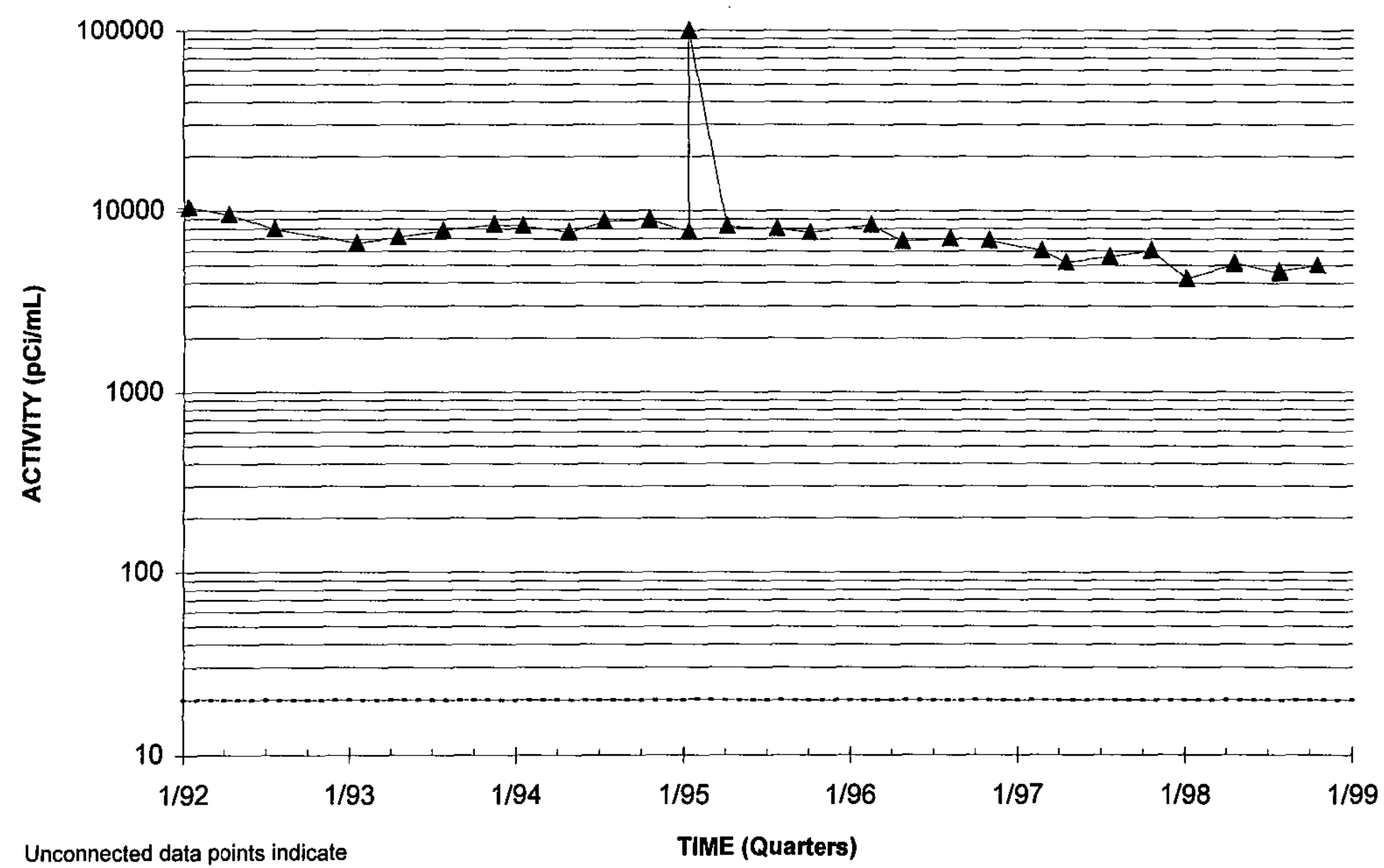

intervals of dry wells or values

below the detection limit.

GWPS $20 \mathrm{pCi} / \mathrm{mL}$

$-4-\mathrm{HSB117C}(\mathrm{B})-x-$ HSB117A (MC) $\cdots \cdots$ GWPS

Note: $W=$ Water Table (IIB2); B=Barnwell (IIB1); M=McBean (IIB1); UC=Upper Congaree (IIA); MC=Middle Congaree (IIA); LC=Lower Congaree (IIA) 


\section{Tritium Activities}

Well HSB118A

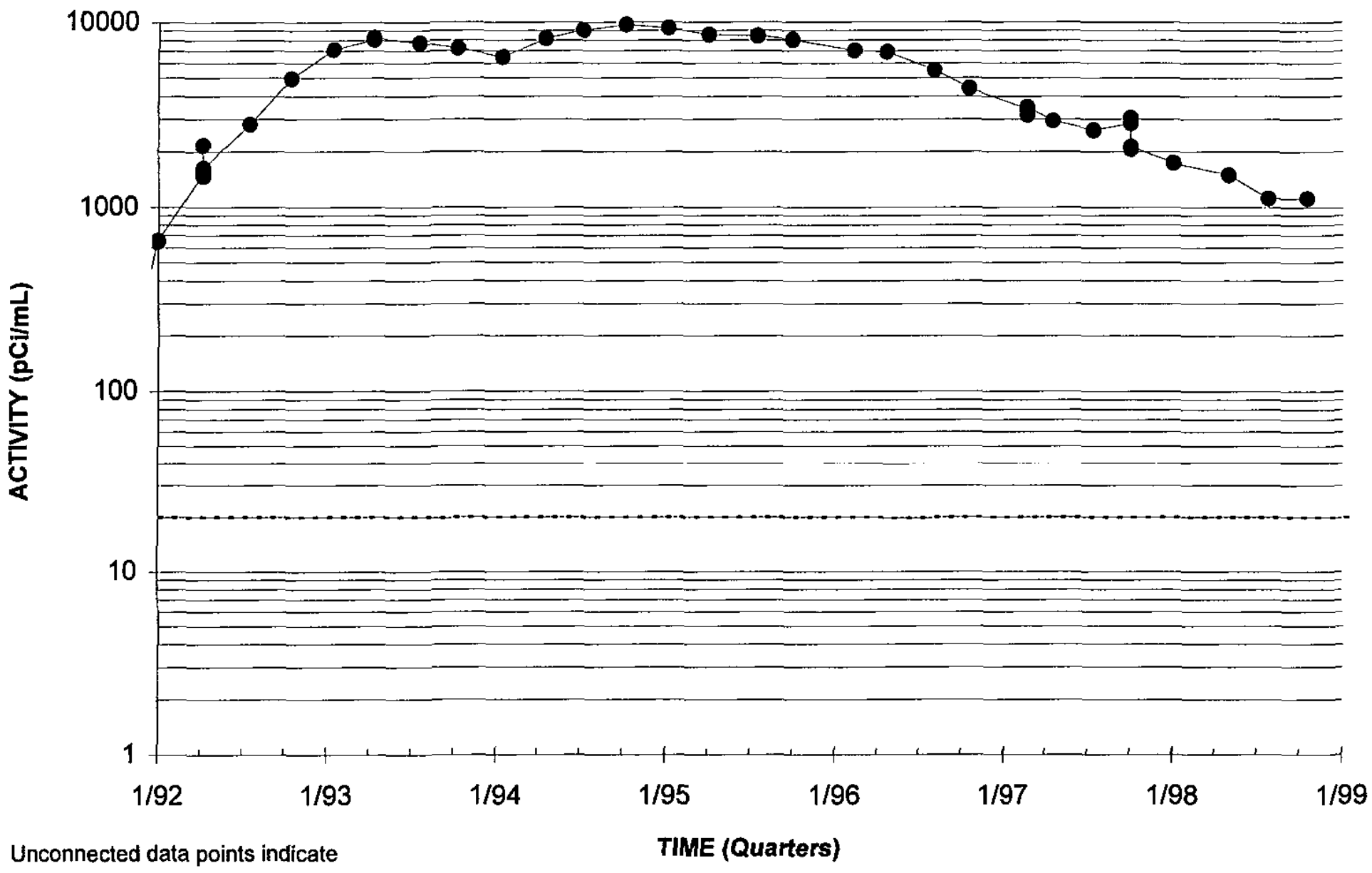

intervals of dry wells or values

below the detection limit.

GWPS $20 \mathrm{pCi} / \mathrm{mL}$

$\ldots$ HSB118A (UC) …...GWPS

Note: $W=$ Water Table (IIB2); B=Barnwell (IIB1); M=McBean (IIB1); UC=Upper Congaree (IIA); MC=Middle Congaree (IIA); LC=Lower Congaree (IIA) 


\section{Tritium Activities \\ Well HSB127D}

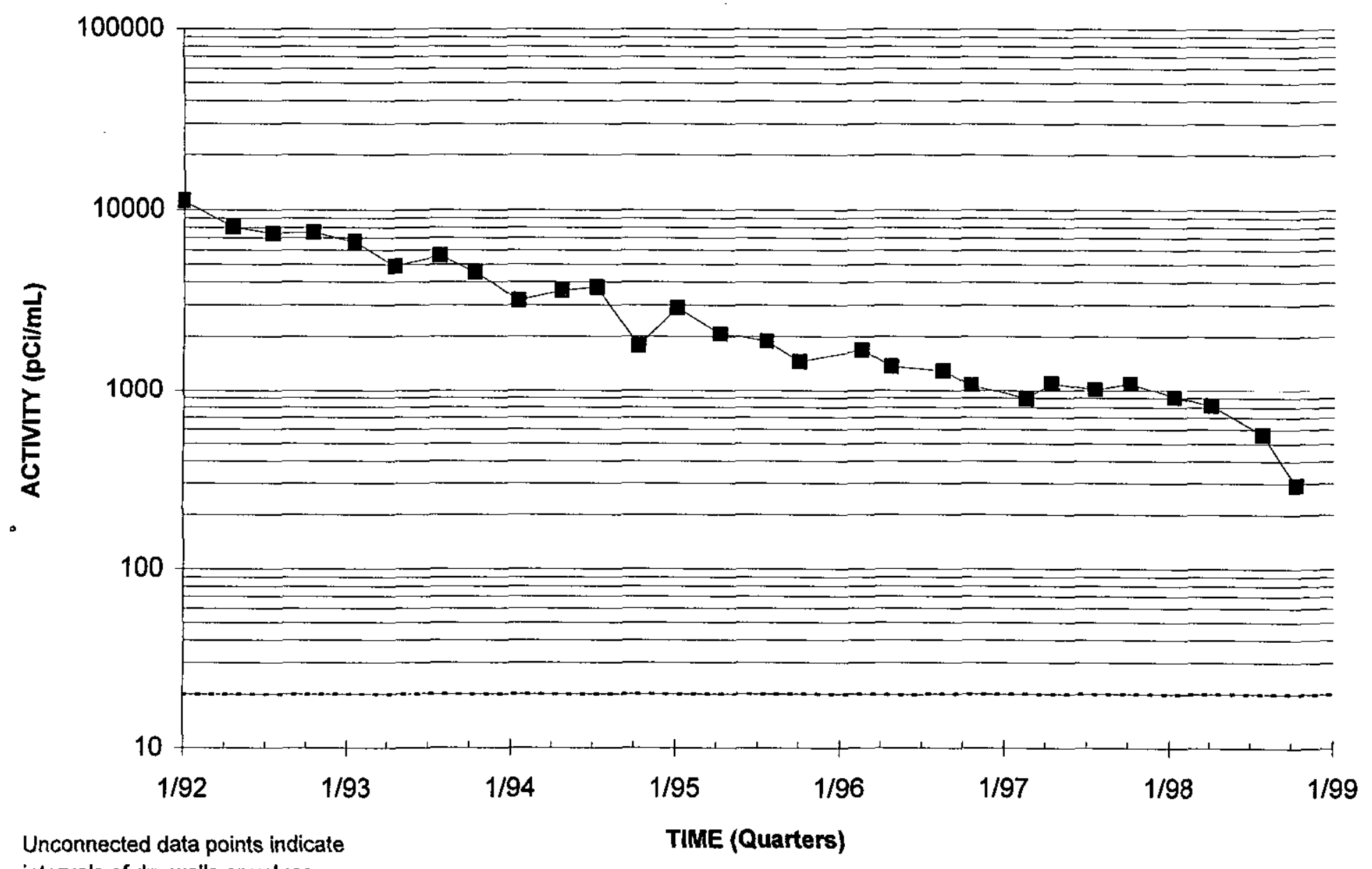

intervals of dry wells or values

below the detection limit.

GWPS $20 \mathrm{pCi} / \mathrm{mL}$

$-\mathrm{HSB} 127 \mathrm{D}(\mathrm{W}) \cdots \ldots$..... GWPS

Note: W=Water Table (IIB2); B=Barnwell (IIB1); M=McBean (IIB1); UC=Upper Congaree (IIA); MC=Middle Congaree (IIA); LC=Lower Congaree (IIA) 


\section{Tritium Activities}

Well Cluster HSB129

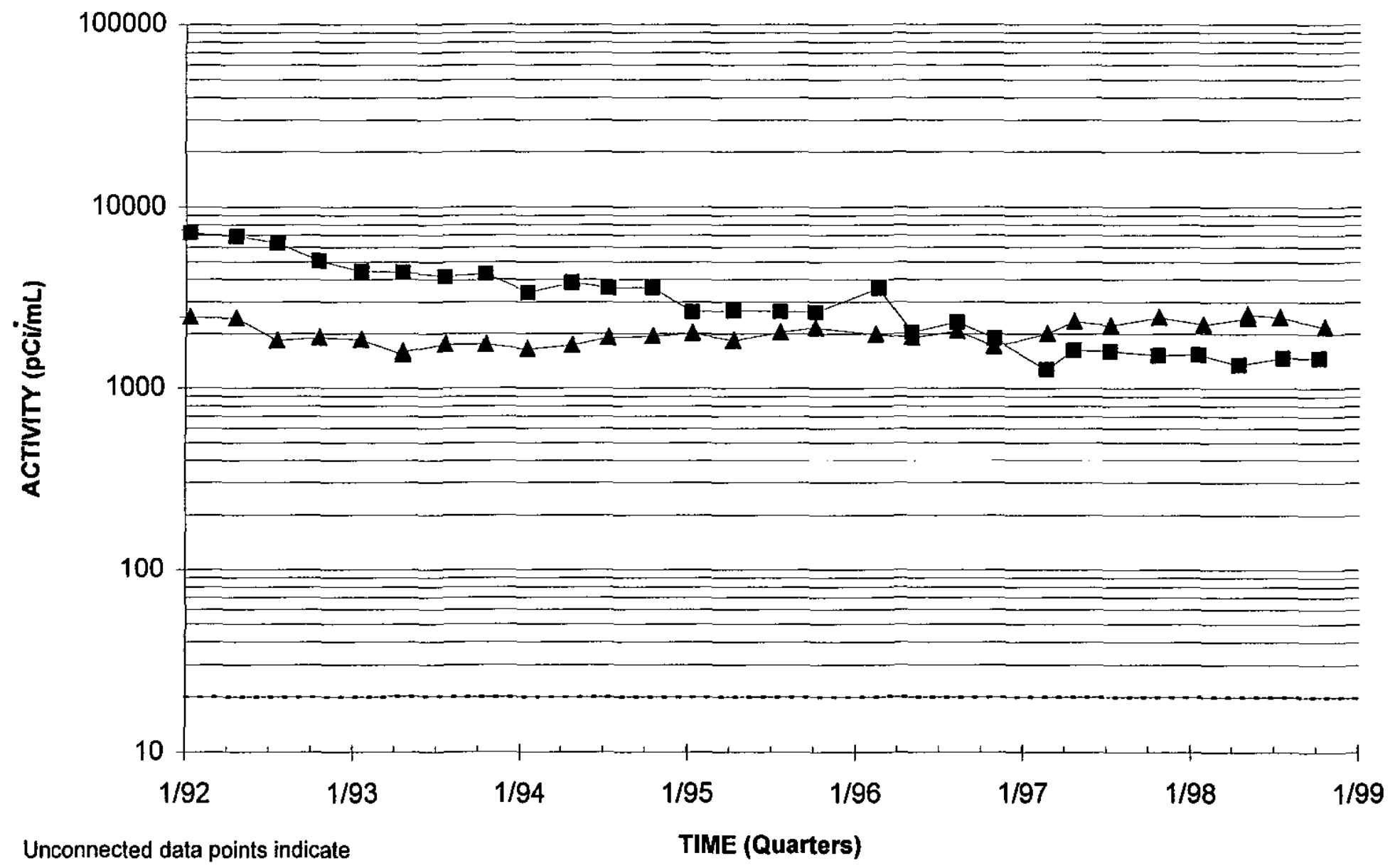

intervals of dry wells or values

below the detection limit.

GWPS $20 \mathrm{pCi} / \mathrm{mL}$

$$
\rightarrow-H S B 129 D(W)-A-H S B 129 C(B) \cdots \cdots \text { GWPS }
$$

Note: W=Water Table (IIB2); B=Barnwell (IIB1); M=McBean (IIB1); UC=Upper Congaree (IIA); MC=Middle Congaree (IIA); LC=Lower Congaree (IIA)

\section{H-Area HWMF}

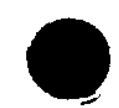




\section{Tritium Activities \\ Well HSB138D}

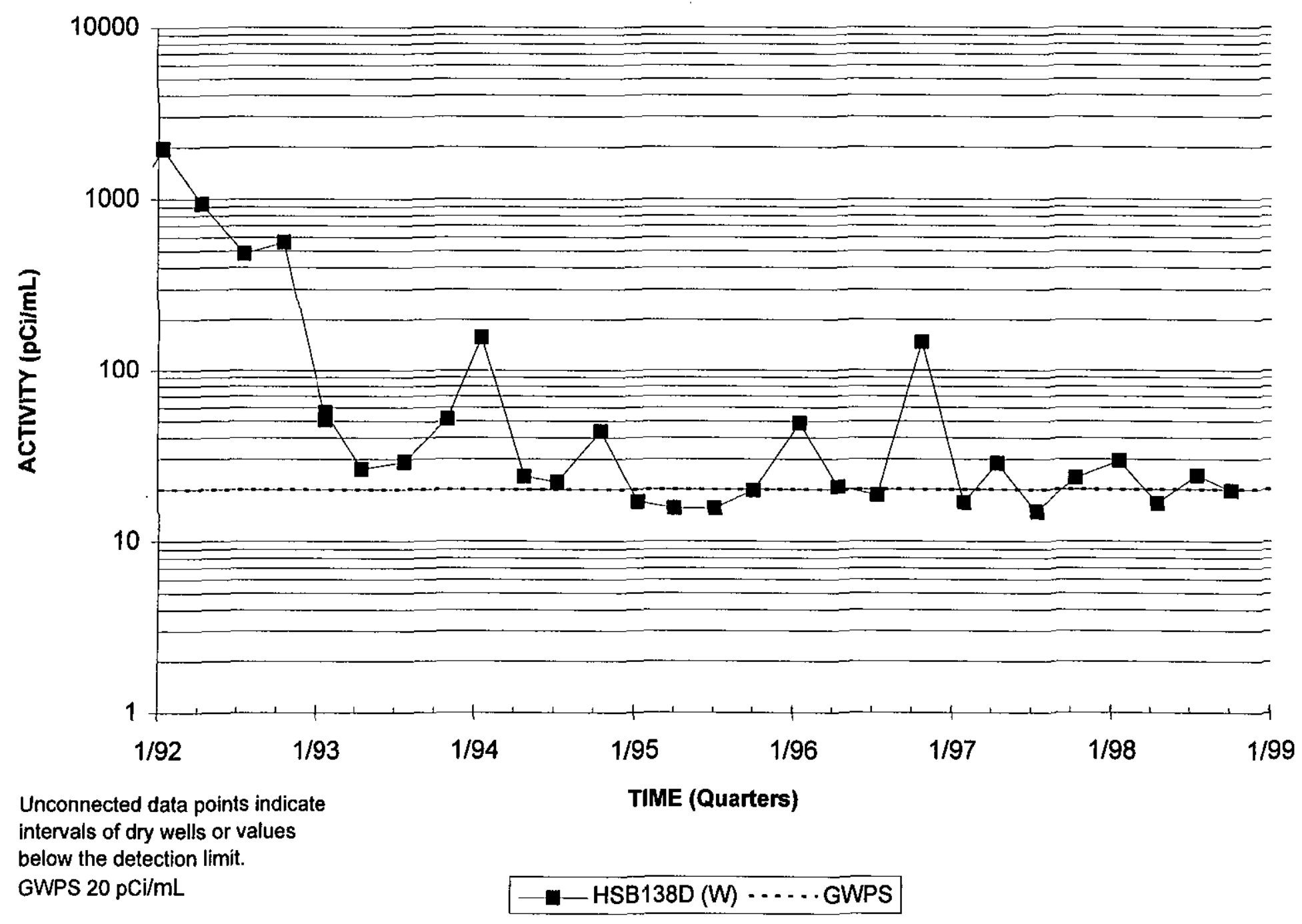

Note: $W=$ Water Table (IIB2); B=Barnwell (IIB1); M=McBean (IIB1); UC=Upper Congaree (IIA); MC=Middle Congaree (IIA); LC=Lower Congaree (IIA) 


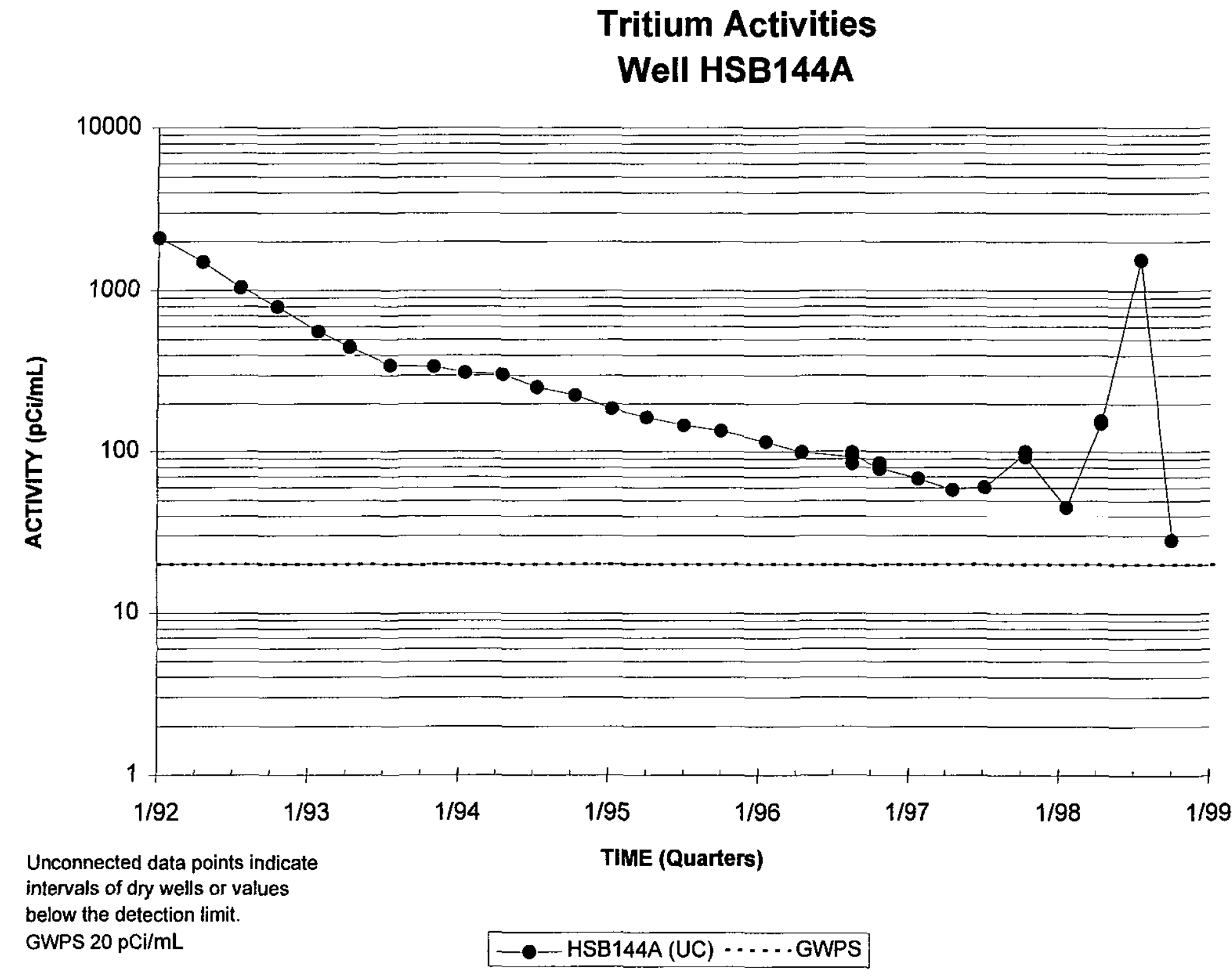

Note: $W=$ Water Table (IIB2); B=Barnwell (IIB1); M=McBean (IIB1); UC=Upper Congaree (IIA); MC=Middle Congaree (lIA); LC=Lower Congaree (IIA) 


\section{Tritium Activities}

Well HSB145D

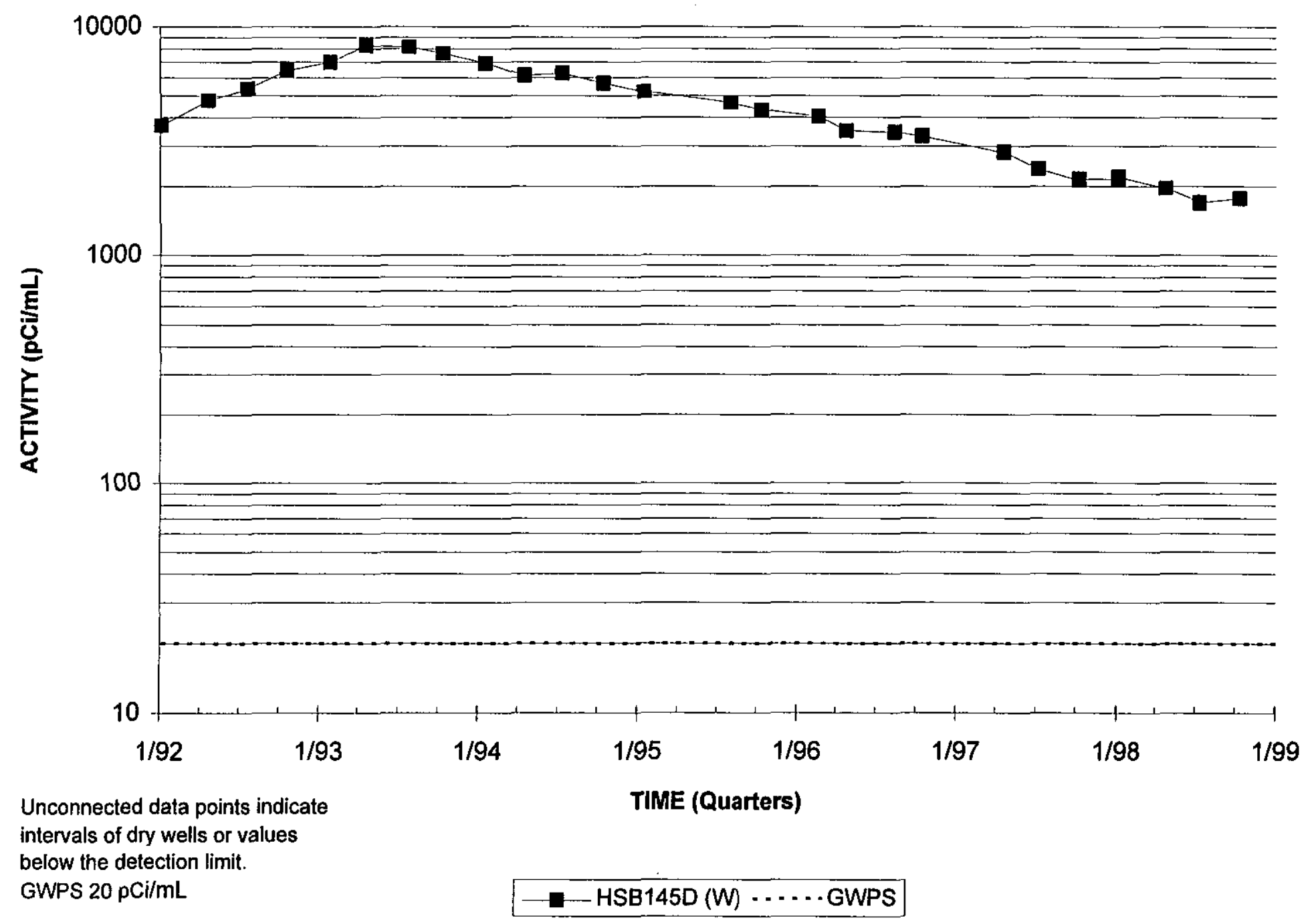

Note: W=Water Table (IIB2); B=Barnwell (IIB1); M=McBean (IIB1); UC=Upper Congaree (IIA); MC=Middle Congaree (IIA); LC=Lower Congaree (IIA) 


\section{Tritium Activities \\ Well HSB151C}

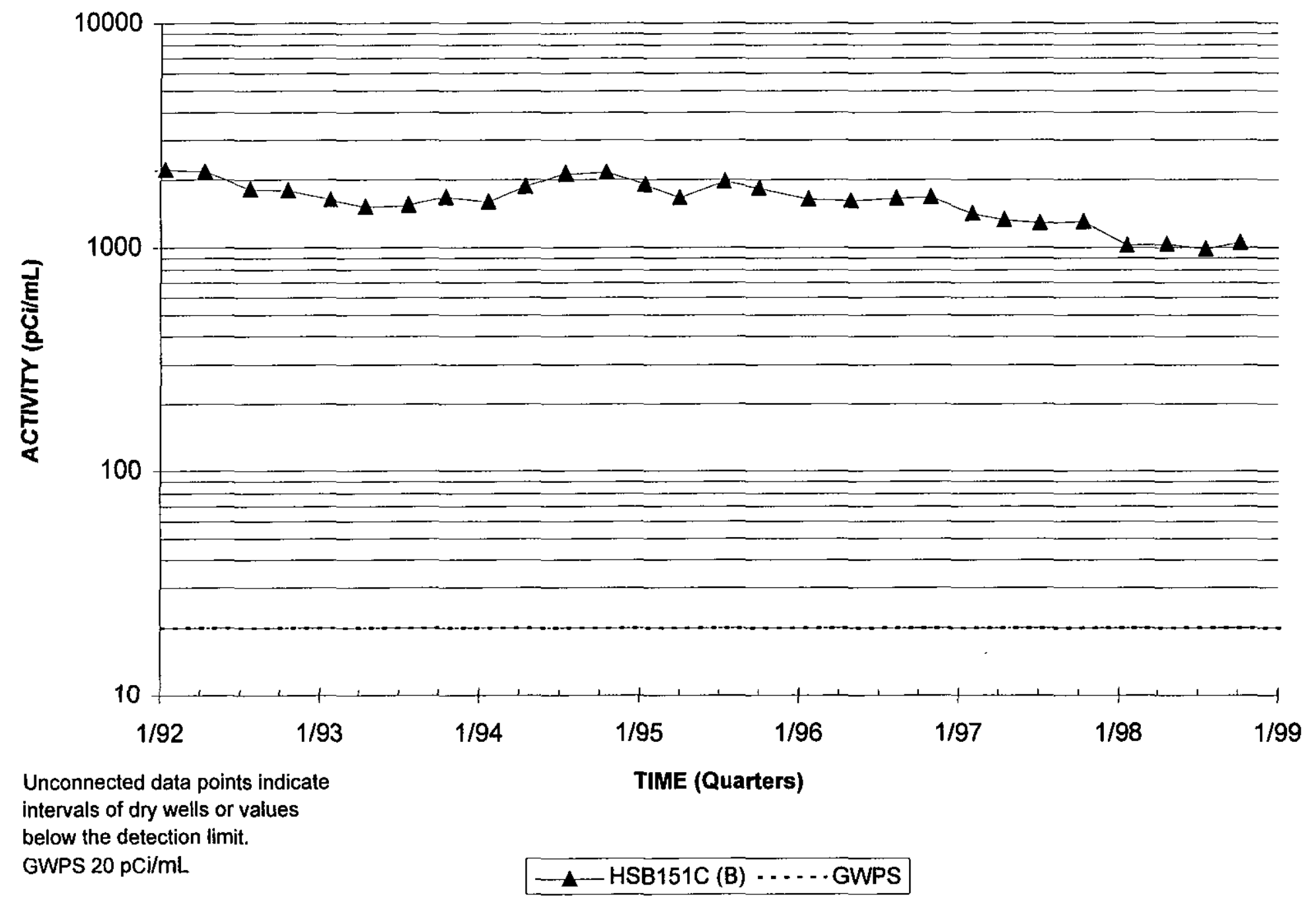

Note: W=Water Table (IIB2); B=Barnwell (IIB1); M=McBean (IIB1); UC=Upper Congaree (IIA); MC=Middle Congaree (IIA); LC=Lower Congaree (IIA) 
WSRC-TR-99-00013

Unclassified

Nitrate Concentrations

Well Cluster HSB 68

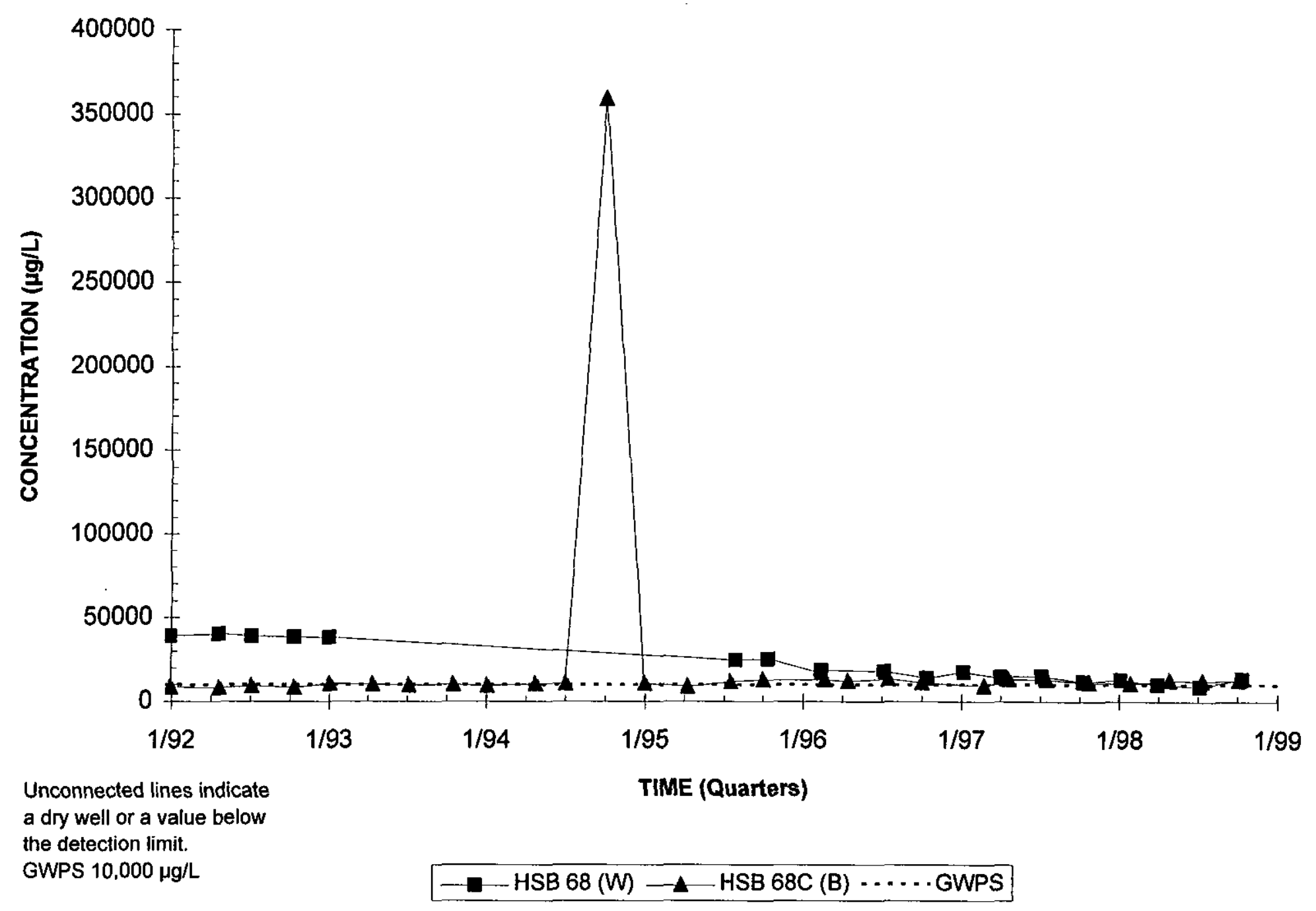

Note: W=Water Table (IIB2); B=Barnwell (IIB1); M=McBean (IIB1); UC=Upper Congaree (IIA); MC=Middle Congaree (IIA); LC=Lower Congaree (IIA) 


\section{Nitrate Concentrations}

Well Cluster HSB 70

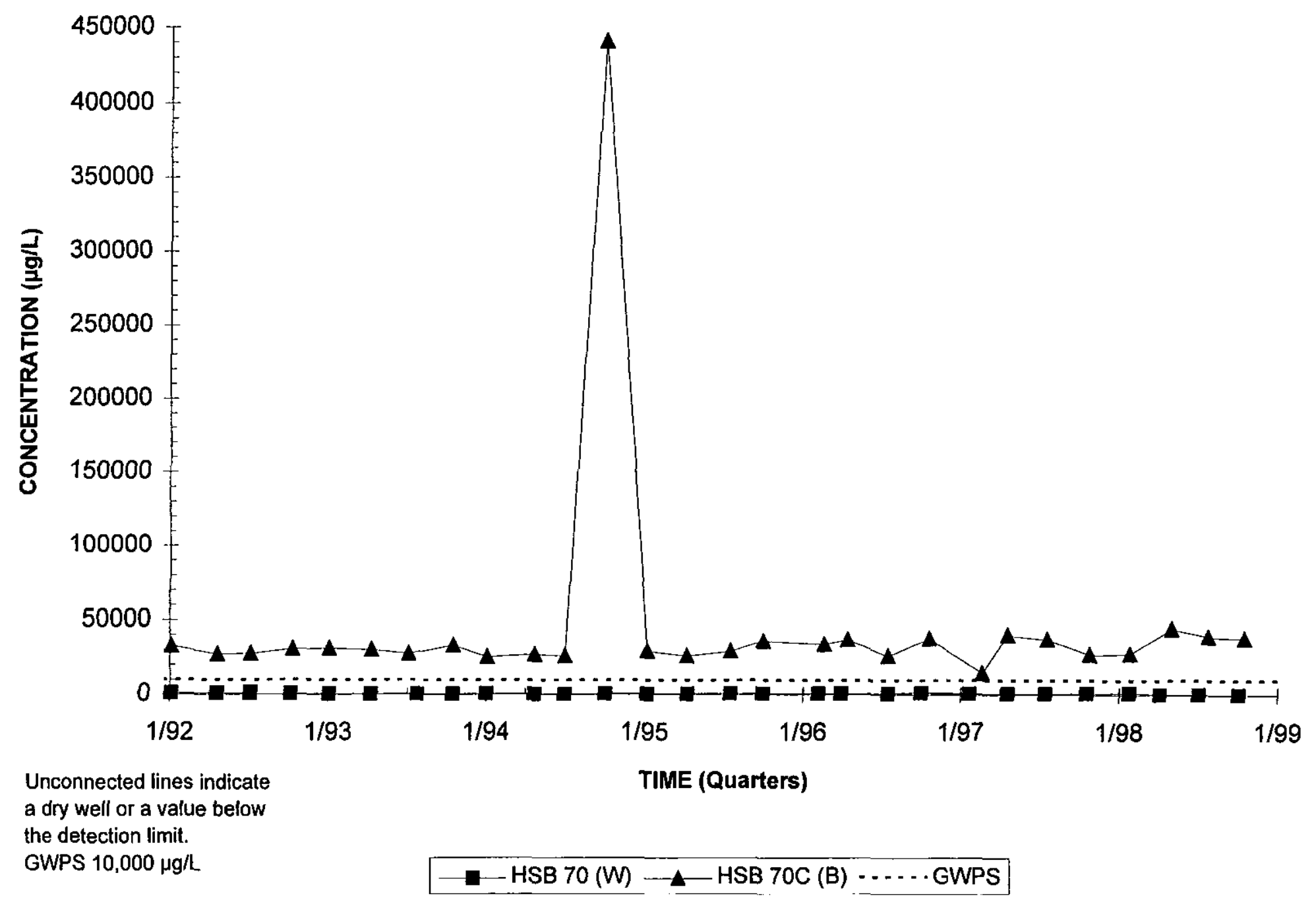

Note: $W=$ Water Table (IIB2); B=Barnwell (IIB1); M=MCBean (IIB1); UC=Upper Congaree (IIA); MC=Middle Congaree (IIA); LC=Lower Congaree (IIA) 


\section{Nitrate Concentrations \\ Well HSB101D}

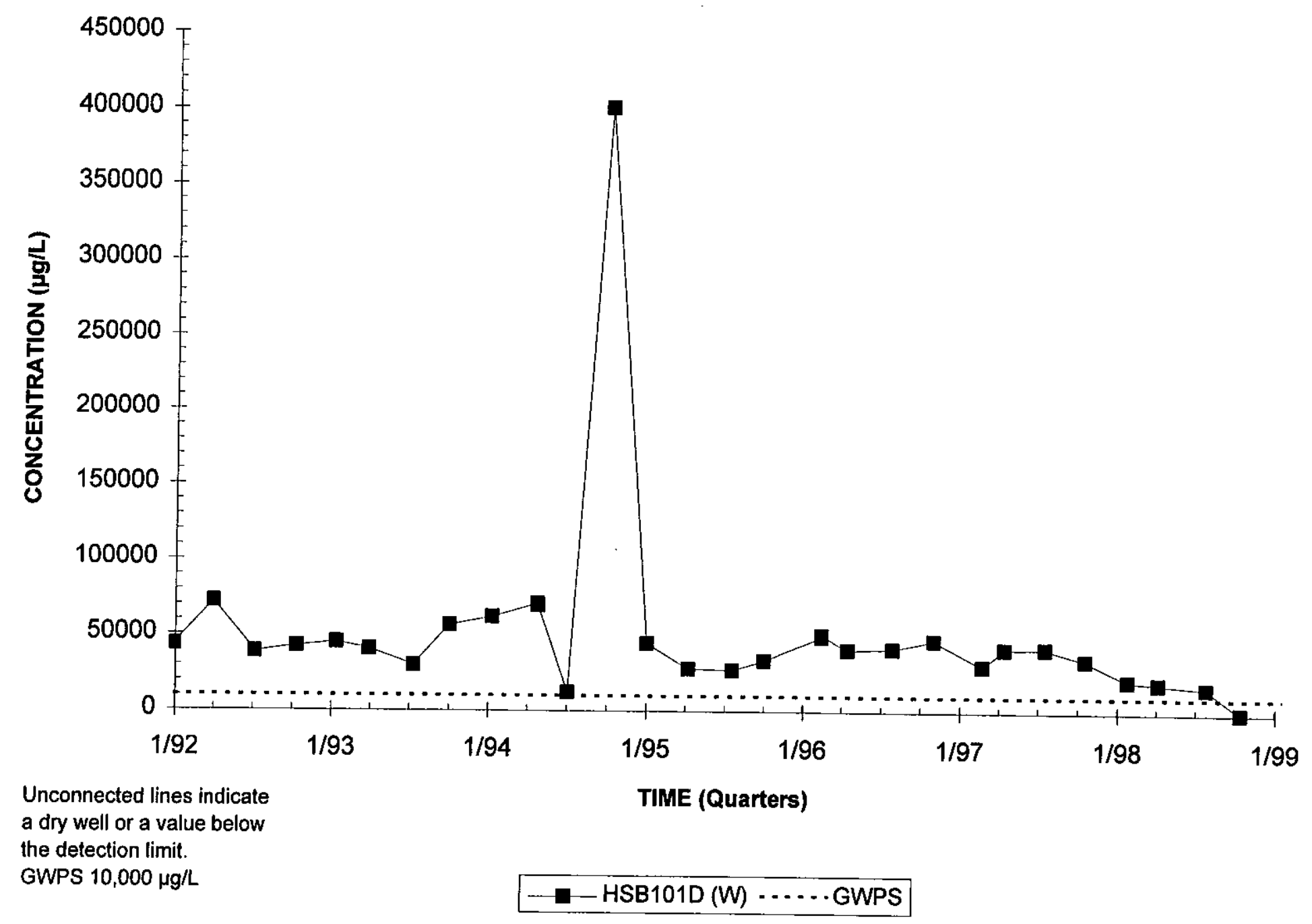

Note: W=Water Table (IIB2); B=Barnwell (IIB1); M=McBean (IIB1); UC=Upper Congaree (IIA); MC=Middle Congaree (IIA); LC=Lower Congaree (IIA) 


\section{Nitrate Concentrations}

Well HSB106D

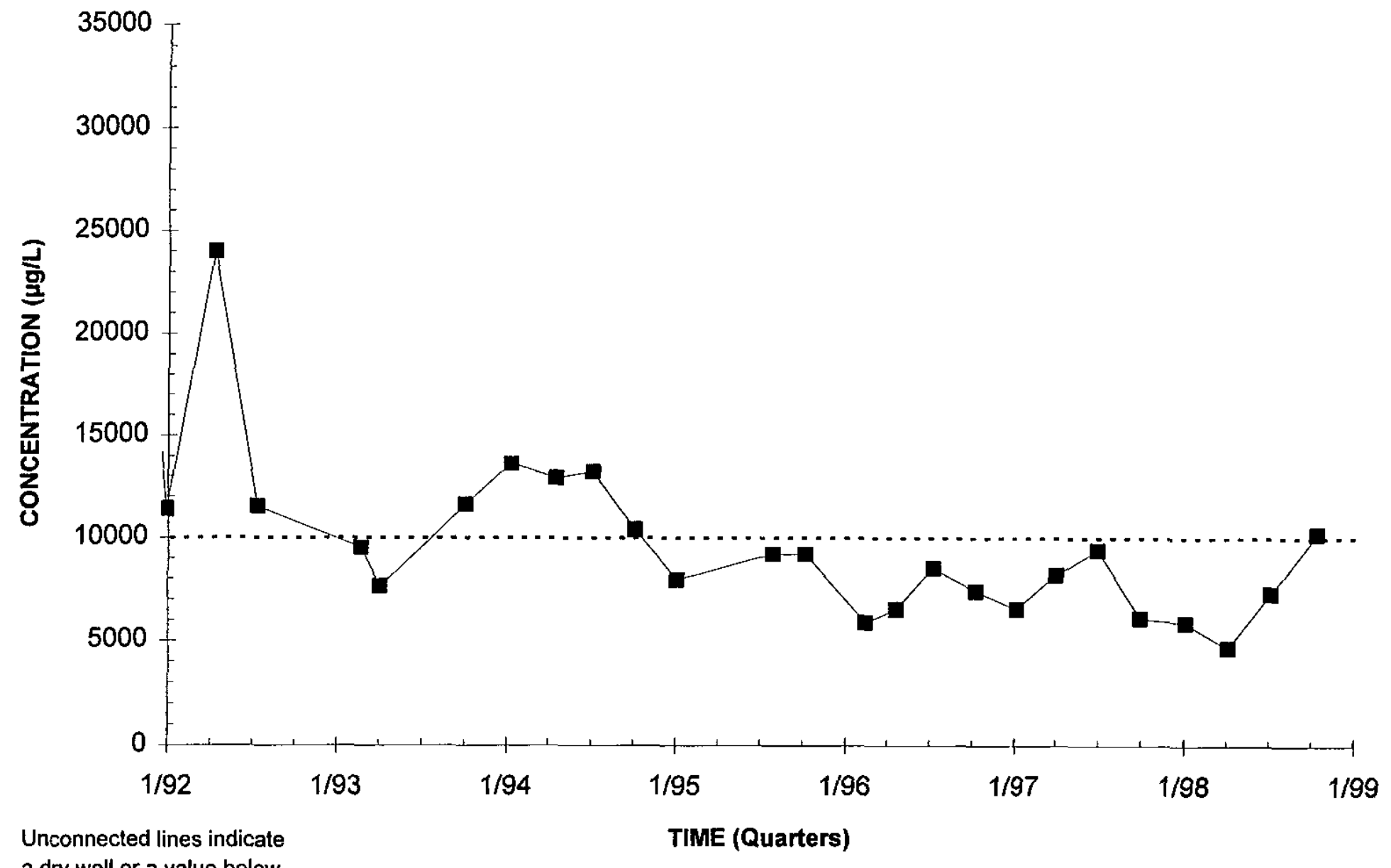

a dry well or a value below

the detection limit.

GWPS $10,000 \mu \mathrm{g} / \mathrm{L}$

- HSB106D (W) -......GWPS

Note: $W=$ Water Table (IIB2); B=Barnwell (IIB1); $M=M c B e a n$ (IIB1); UC=Upper Congaree (IIA); MC=Middle Congaree (IIA); LC=Lower Congaree (IIA) 


\section{Nitrate Concentrations}

Well Cluster HSB107

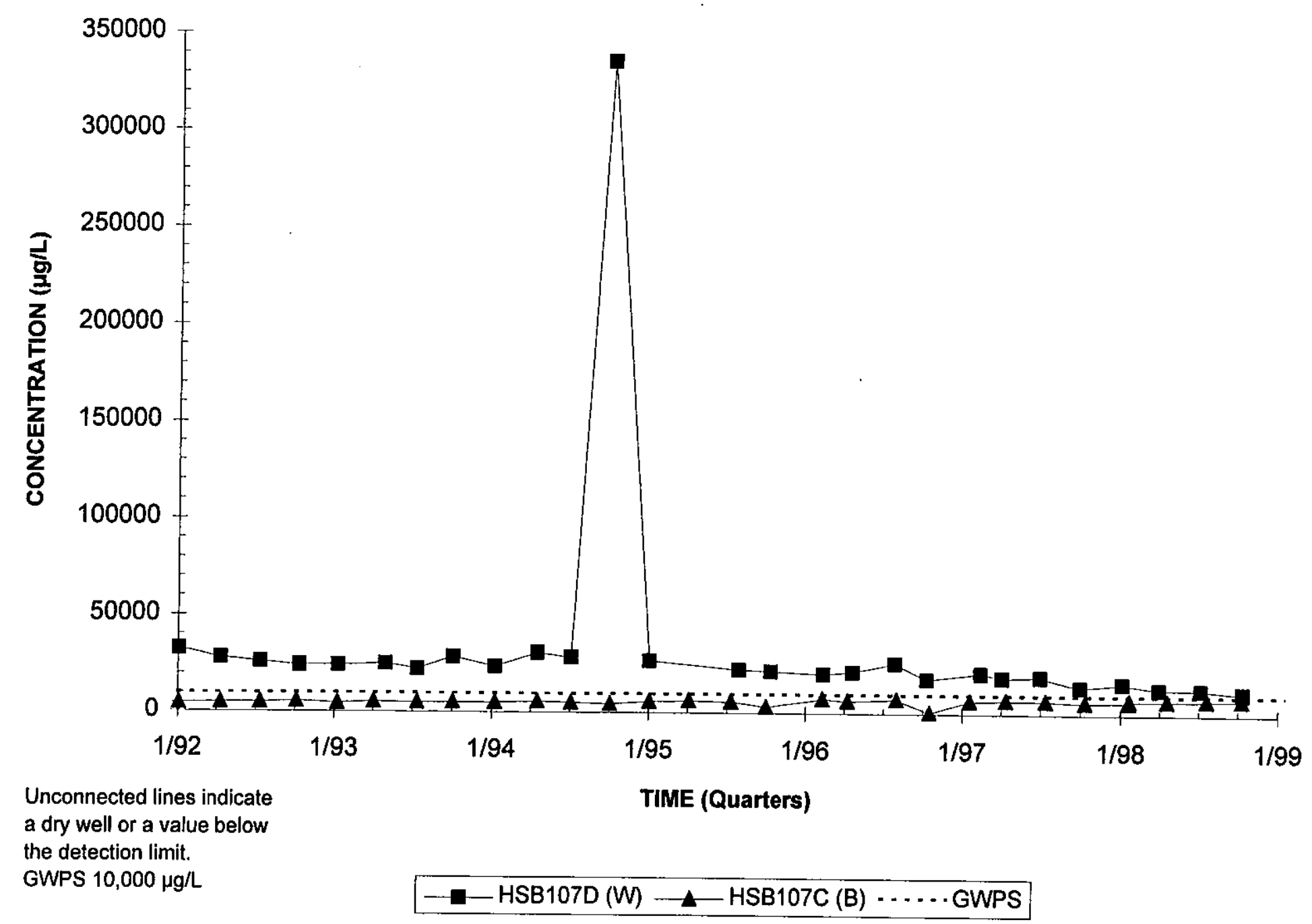

Note: $W=$ Water Table (IIB2); B=Barnwell (IIB1); M=MCBean (IIB1); UC=Upper Congaree (IIA); MC=Middle Congaree (IIA); LC=Lower Congaree (IIA) 


\section{Nitrate Concentrations}

Well Cluster HSB108

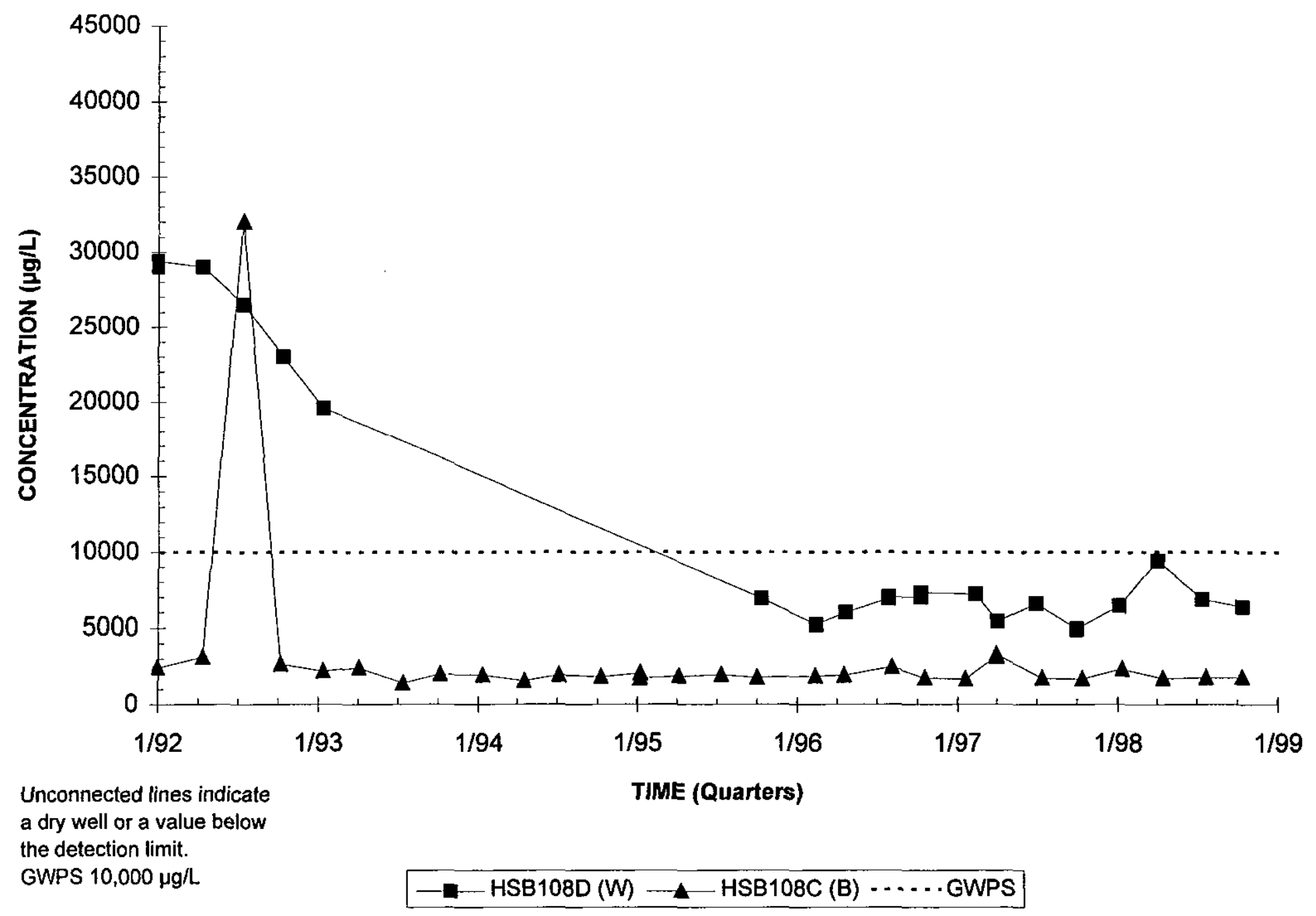

Note: W=Water Table (IIB2); B=Barnwell (IIB1); M=McBean (IIB1); UC=Upper Congaree (IIA); MC=Middle Congaree (IIA); LC=Lower Congaree (IIA) 


\section{Nitrate Concentrations \\ Well Cluster HSB111}

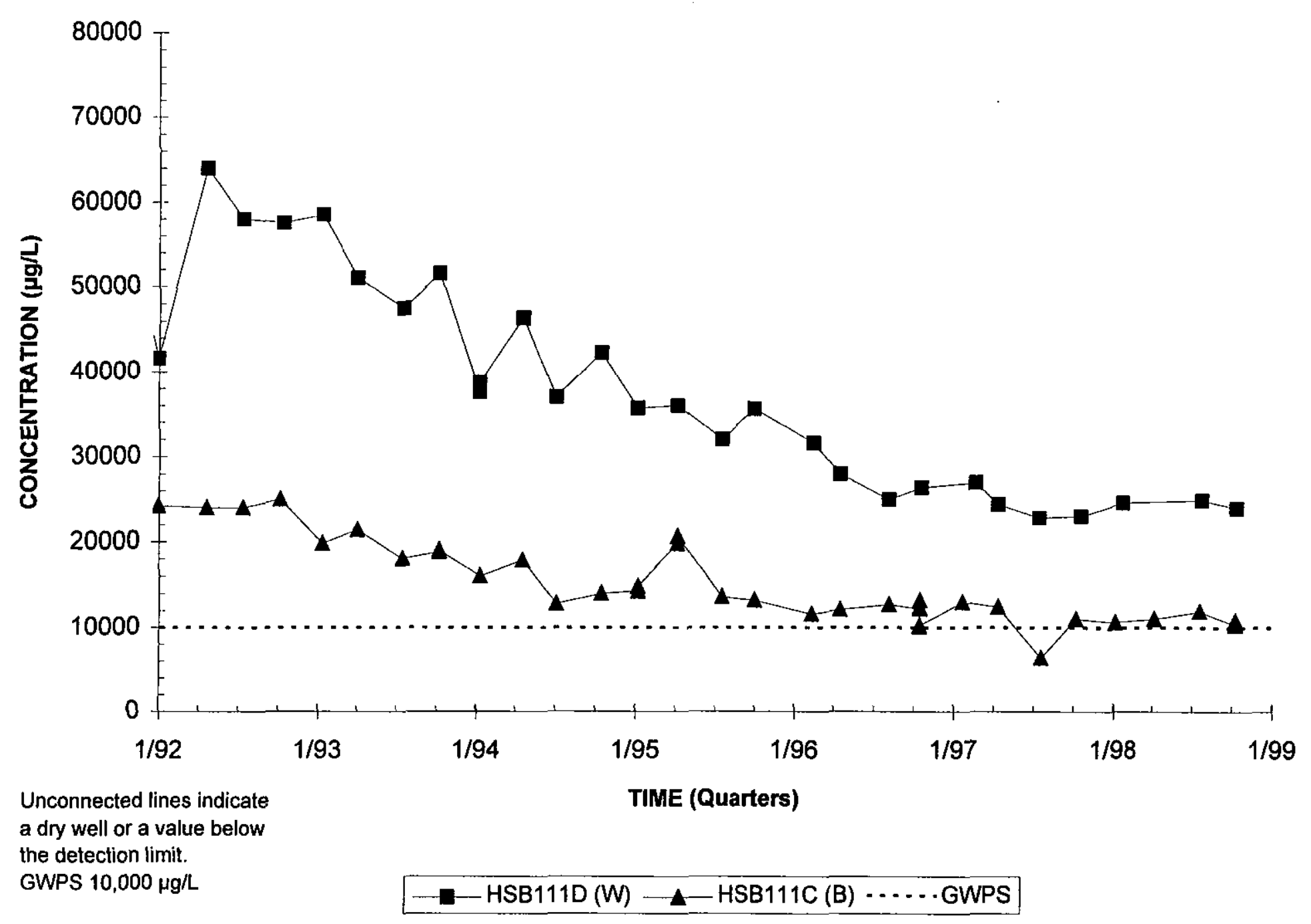

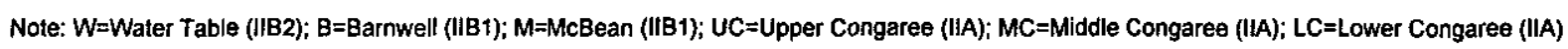




\section{Nitrate Concentrations \\ Well Cluster HSB114}

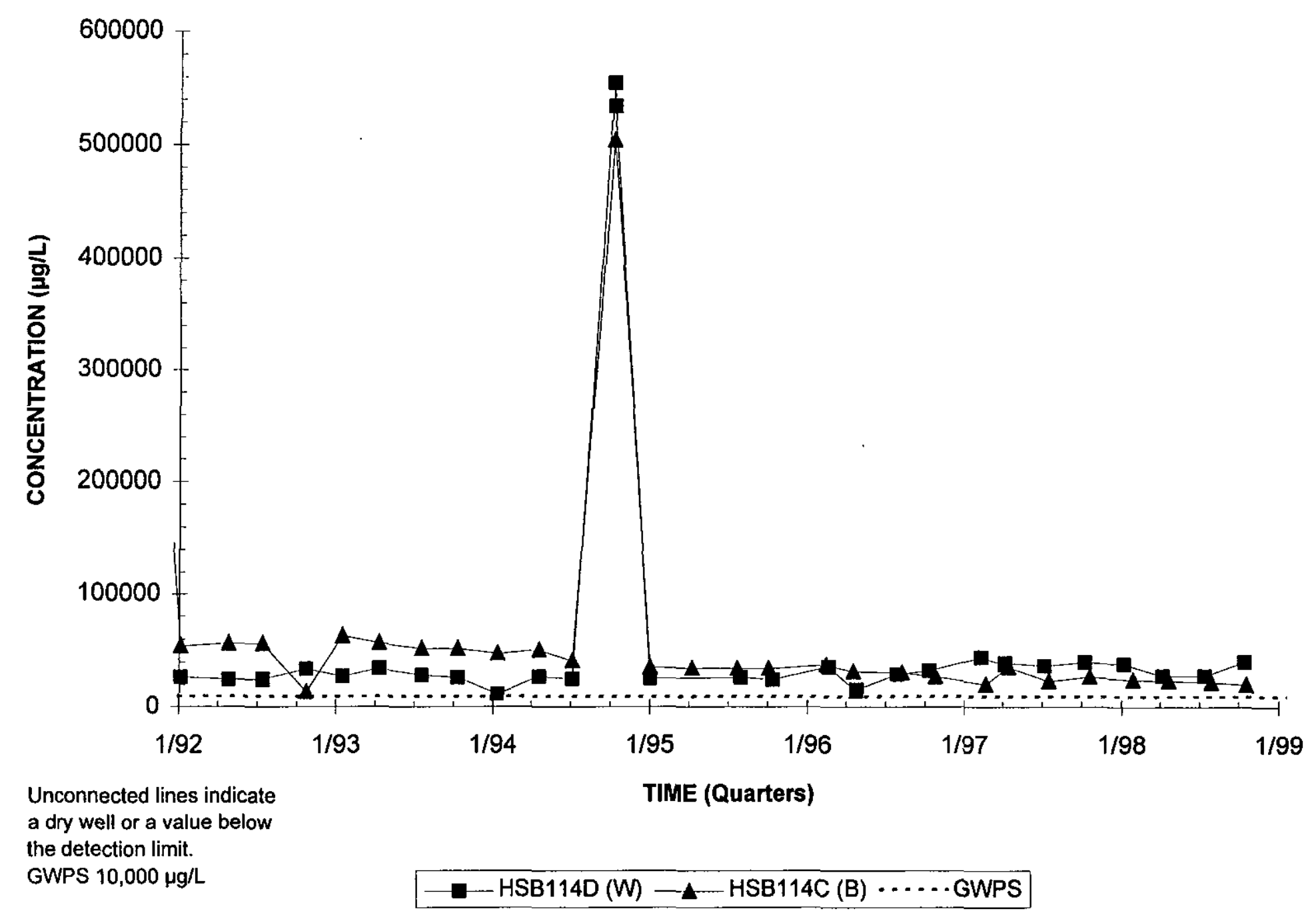

Note: $W=$ Water Table (IIB2); B=Barnwell (IIB1); M=MCBean (IIB1); UC=Upper Congaree (IIA); MC=Middle Congaree (IIA); LC=Lower Congaree (IIA) 


\section{Nitrate Concentrations}

Well HSB118A

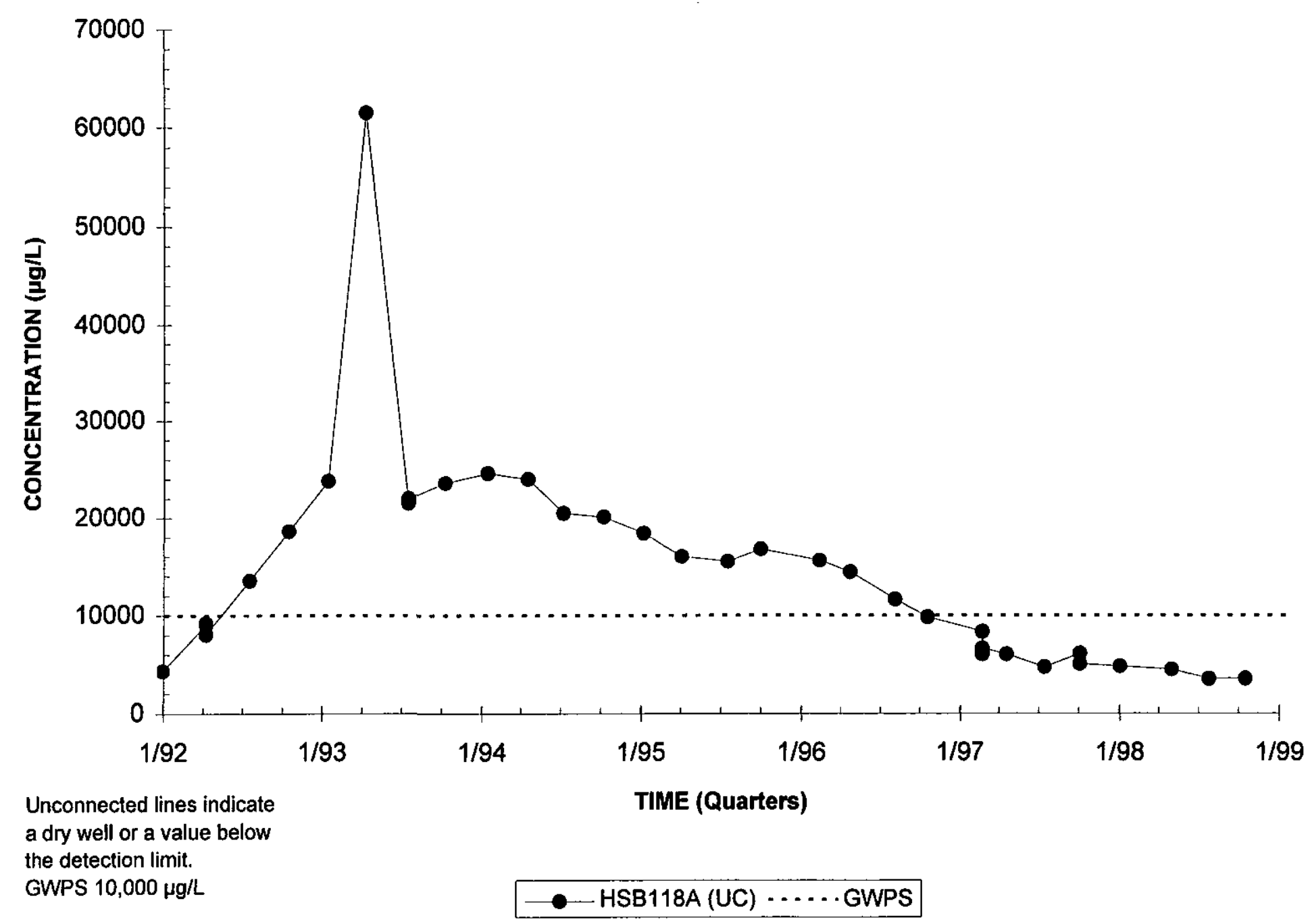

Note: $W=$ Water Table (IIB2); B=Barnwell (IIB1); M=McBean (IIB1); UC=Upper Congaree (IIA); MC=Middle Congaree (IIA); LC=Lower Congaree (IIA) 


\section{Nitrate Concentrations}

\section{Well HSB135D}

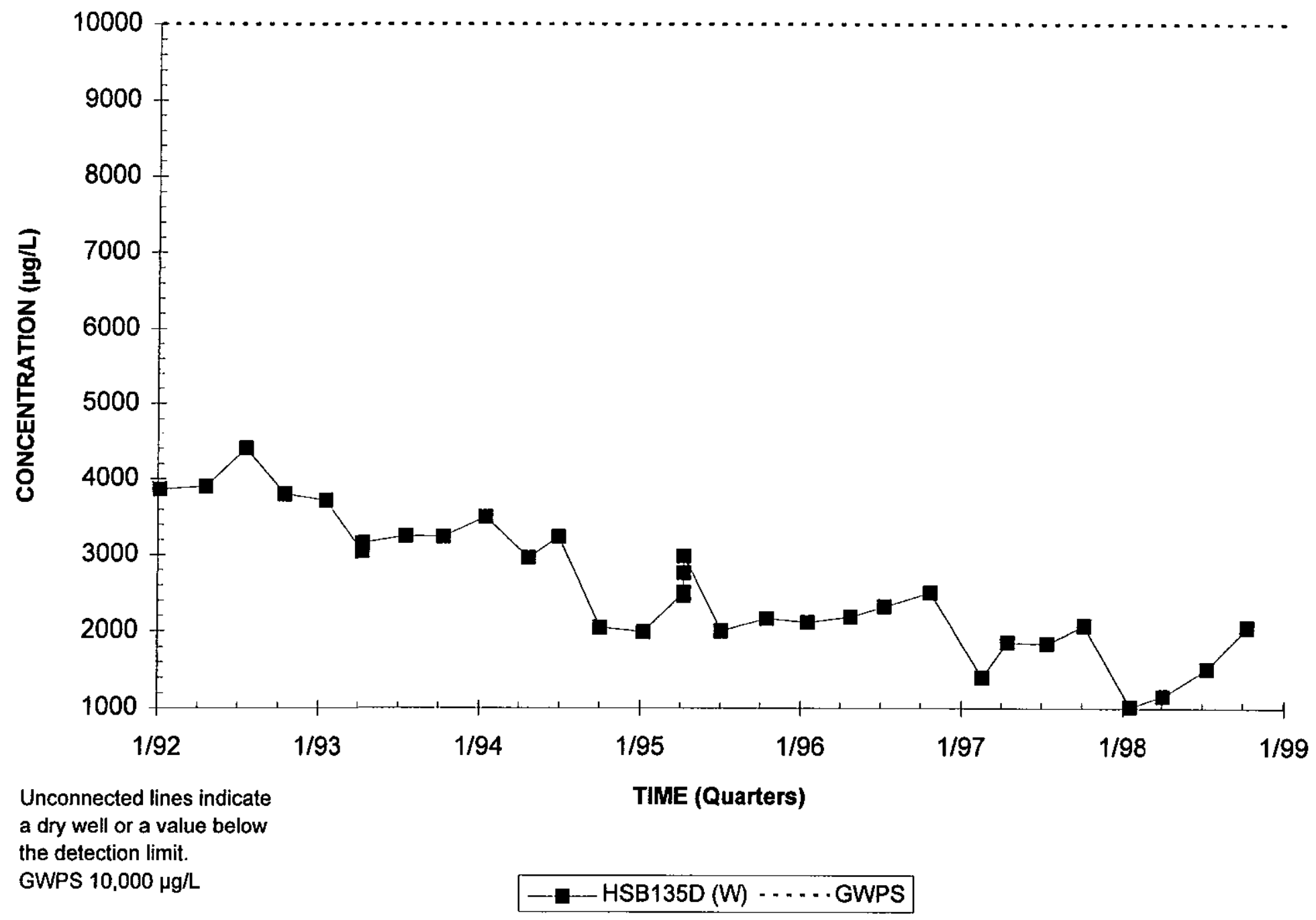

Note: W=Water Table (IIB2); B=Barnwell (IIB1); M=McBean (IIB1); UC=Upper Congaree (IIA); MC=Middle Congaree (IIA); LC=Lower Congaree (IIA) 


\section{Nitrate Concentrations \\ Well Cluster HSB139}

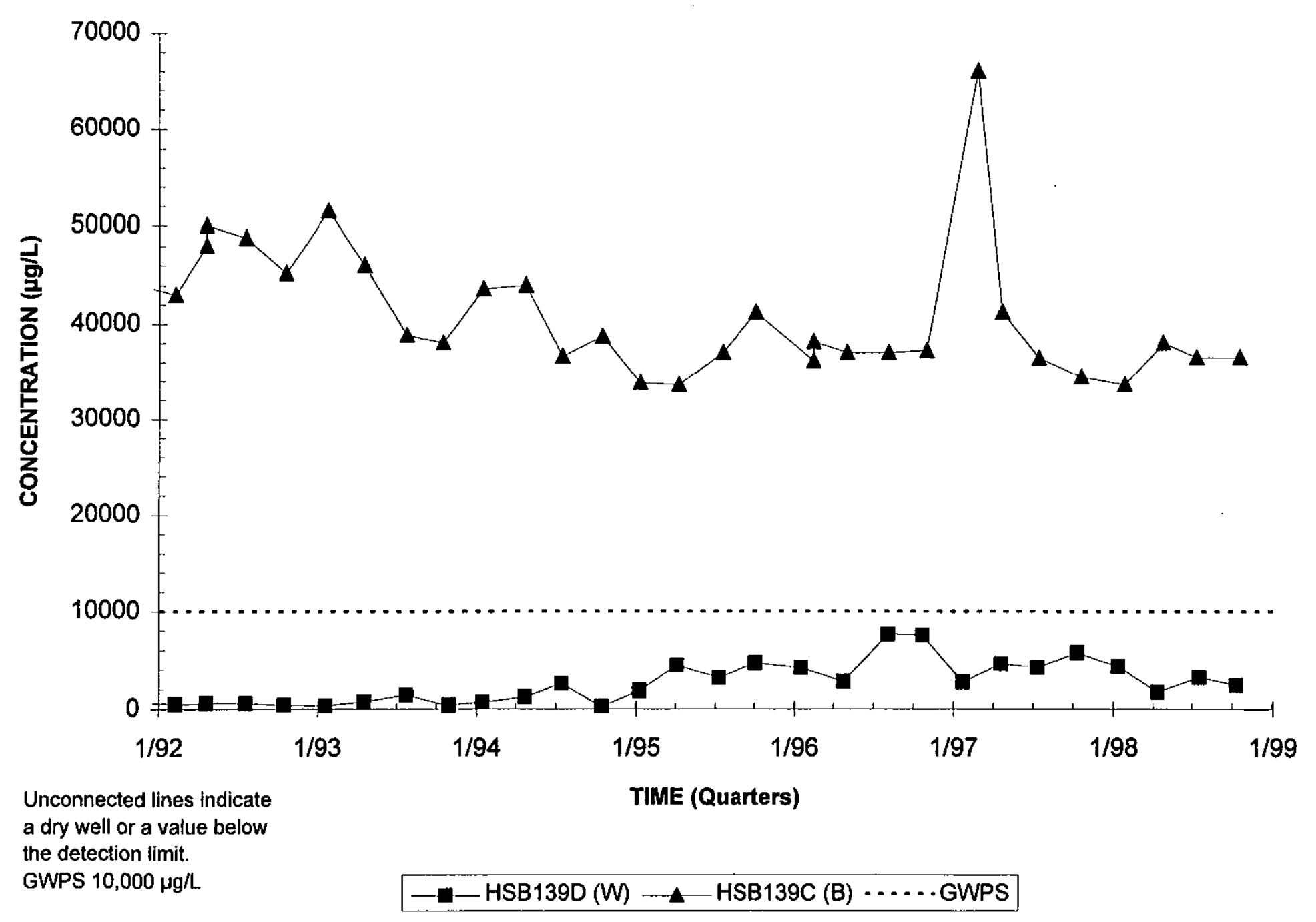

Note: W=Water Table (IIB2); B=Barnwell (IIB1); M=MCBean (IIB1); UC=Upper Congaree (IIA); MC=Middle Congaree (IIA); LC=Lower Congaree (IIA) 


\section{Nitrate Concentrations \\ Well Cluster HSB145}

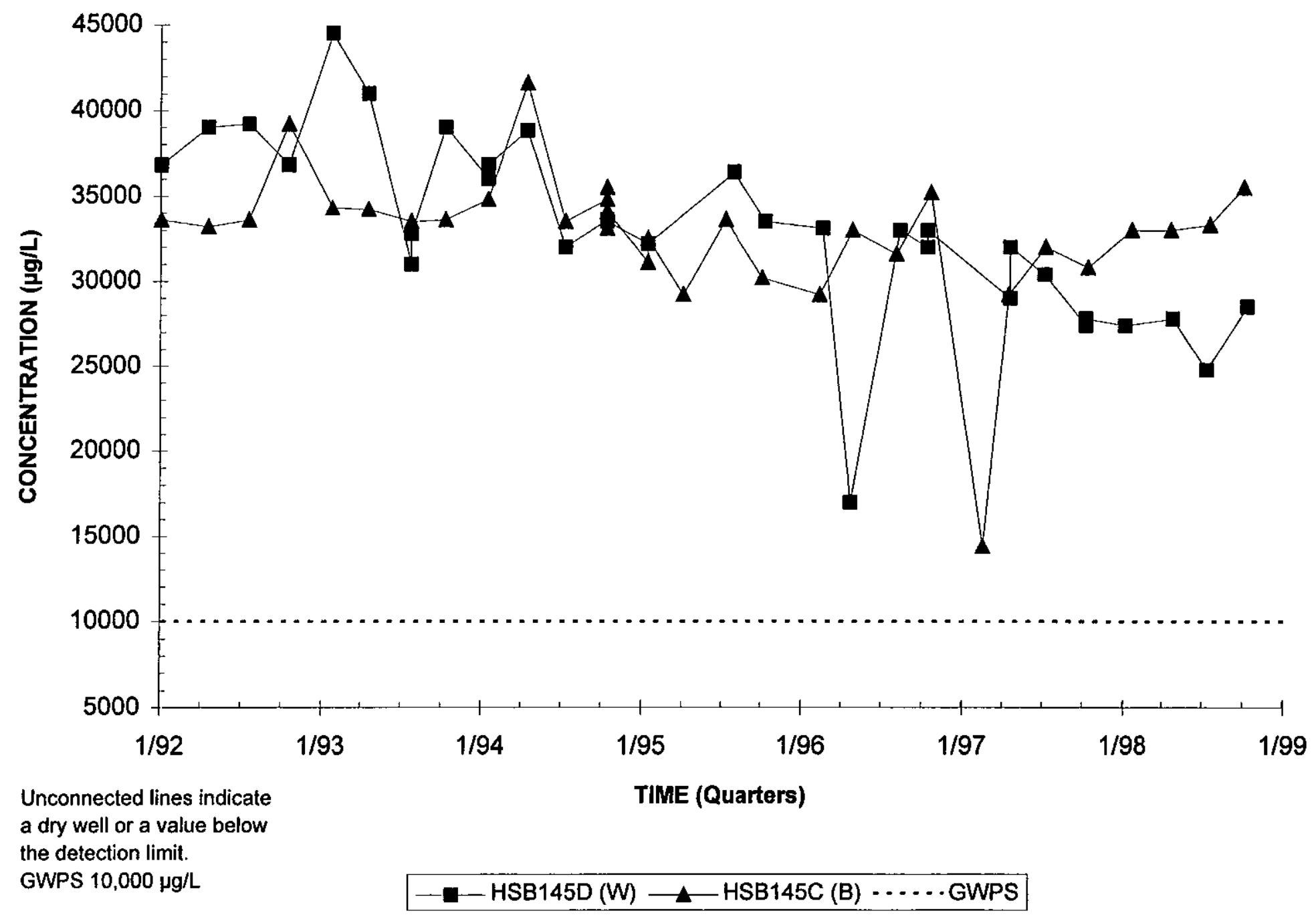

Note: W=Water Table (IIB2); B=Barnwell (IIB1); M=McBean (IIB1); UC=Upper Congaree (IIA); MC=Middle Congaree (IIA); LC=Lower Congaree (IIA) 


\section{Nitrate Concentrations}

Well HSB151C

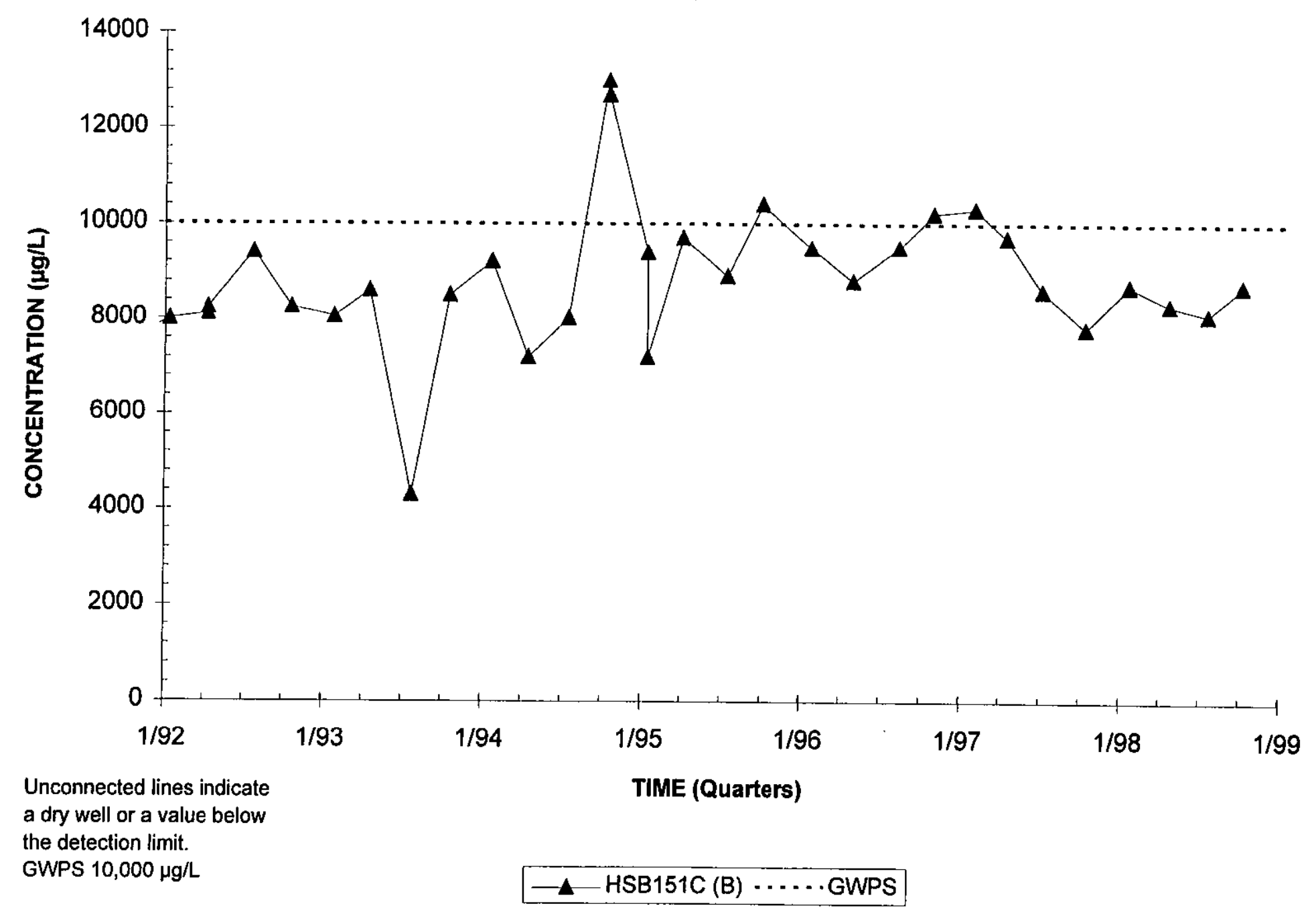

Note: $W=$ Water Table (IIB2); B=Barnwell (IIB1); $M=M C B e a n$ (IIB1); UC=Upper Congaree (IIA); MC=Middle Congaree (IIA); LC=Lower Congaree (IIA) 


\section{lodine-129 Activities}

Well HSB 69

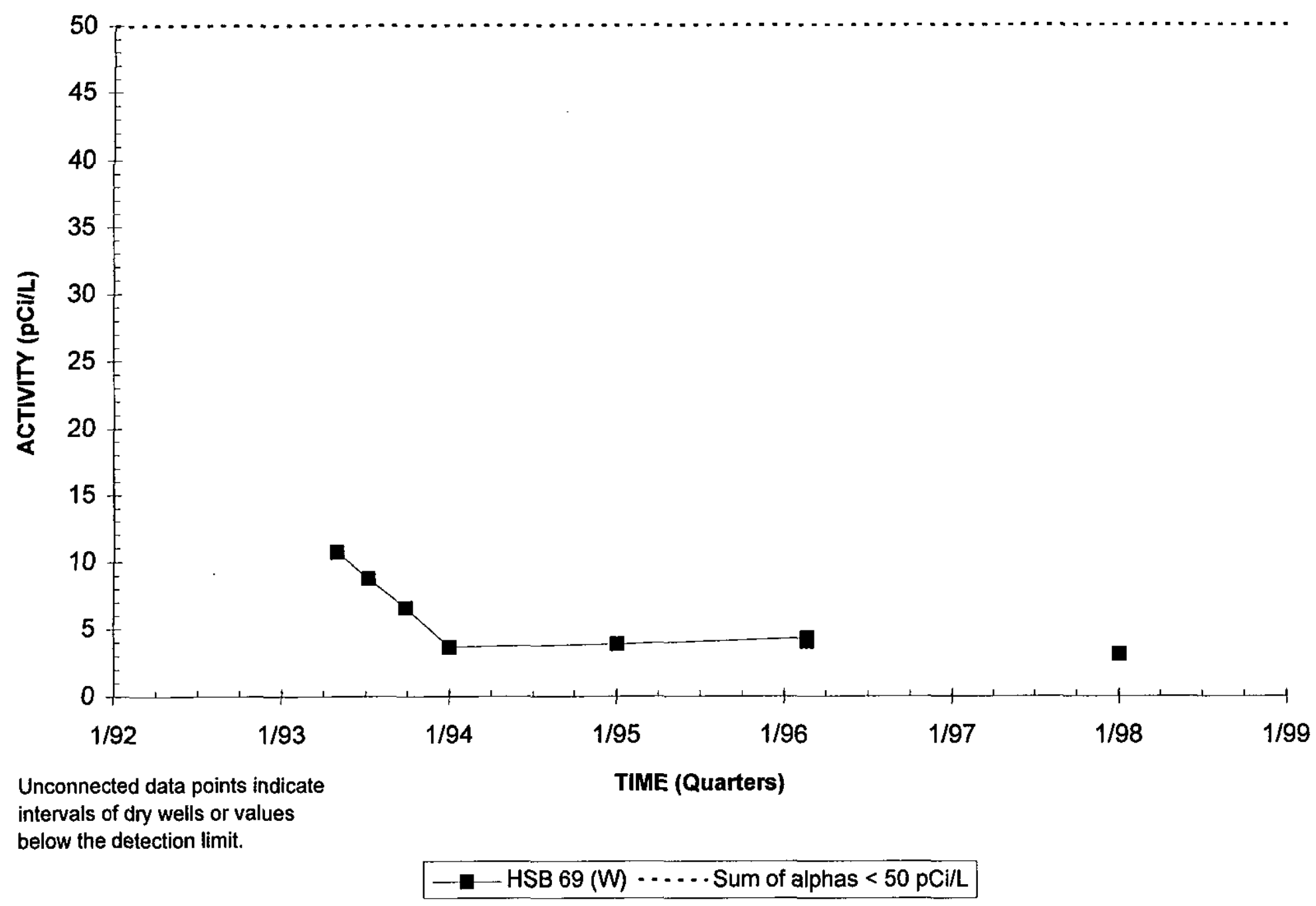

Note: W=Water Table (IIB2); B=Barnwell (IIB1); M=McBean (IIB1); UC=Upper Congaree (IIA); MC=Middle Congaree (IIA); LC=Lower Congaree (IIA) 


\section{lodine-129 Activities}

Well Cluster HSB 84

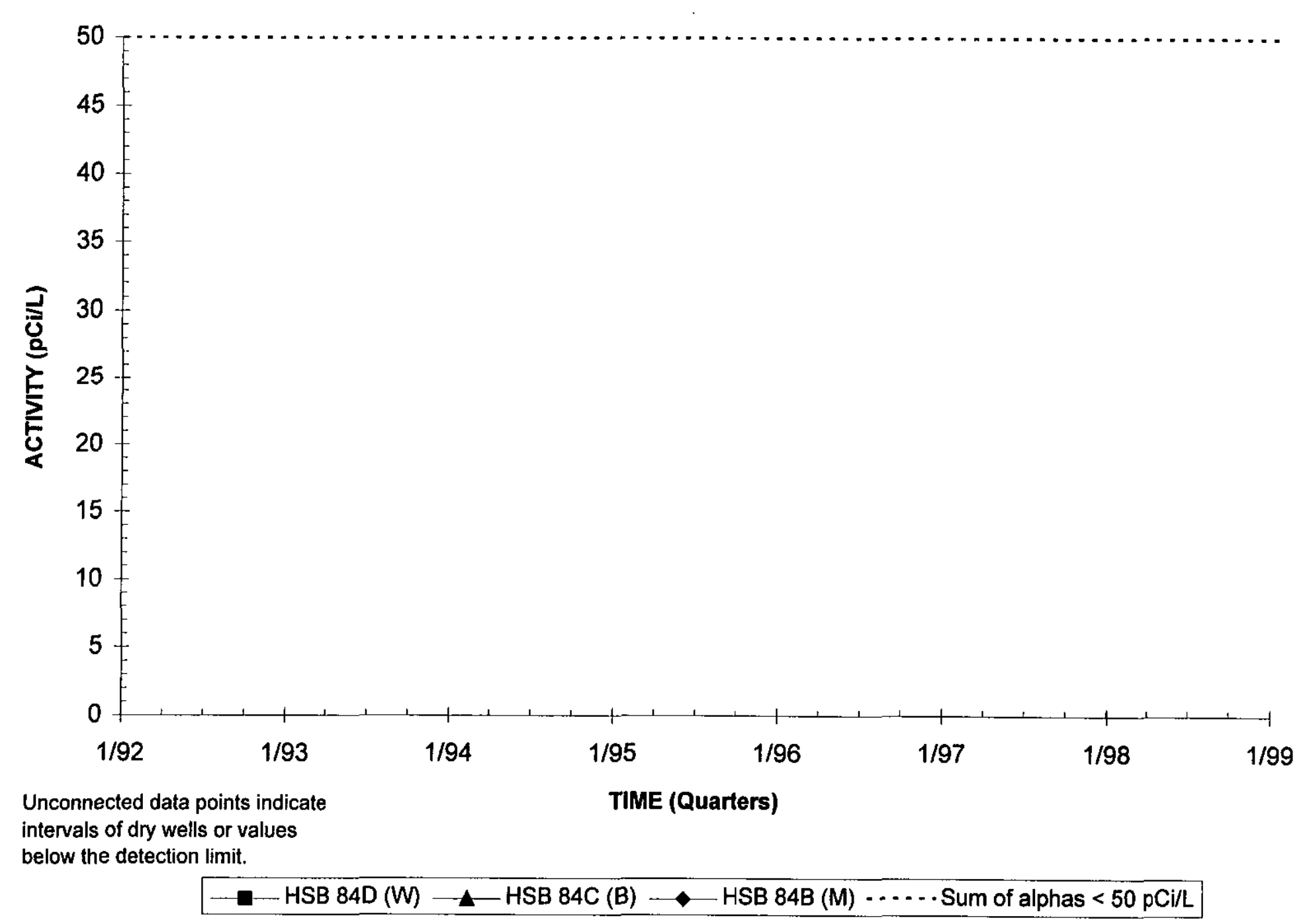

Note: W=Water Table (IIB2); B=Barnwell (IIB1); M=McBean (IIB1); UC=Upper Congaree (IIA); MC=Middle Congaree (IIA); LC=Lower Congaree (IIA) 


\section{lodine-129 Activities \\ Well Cluster HSB 86}

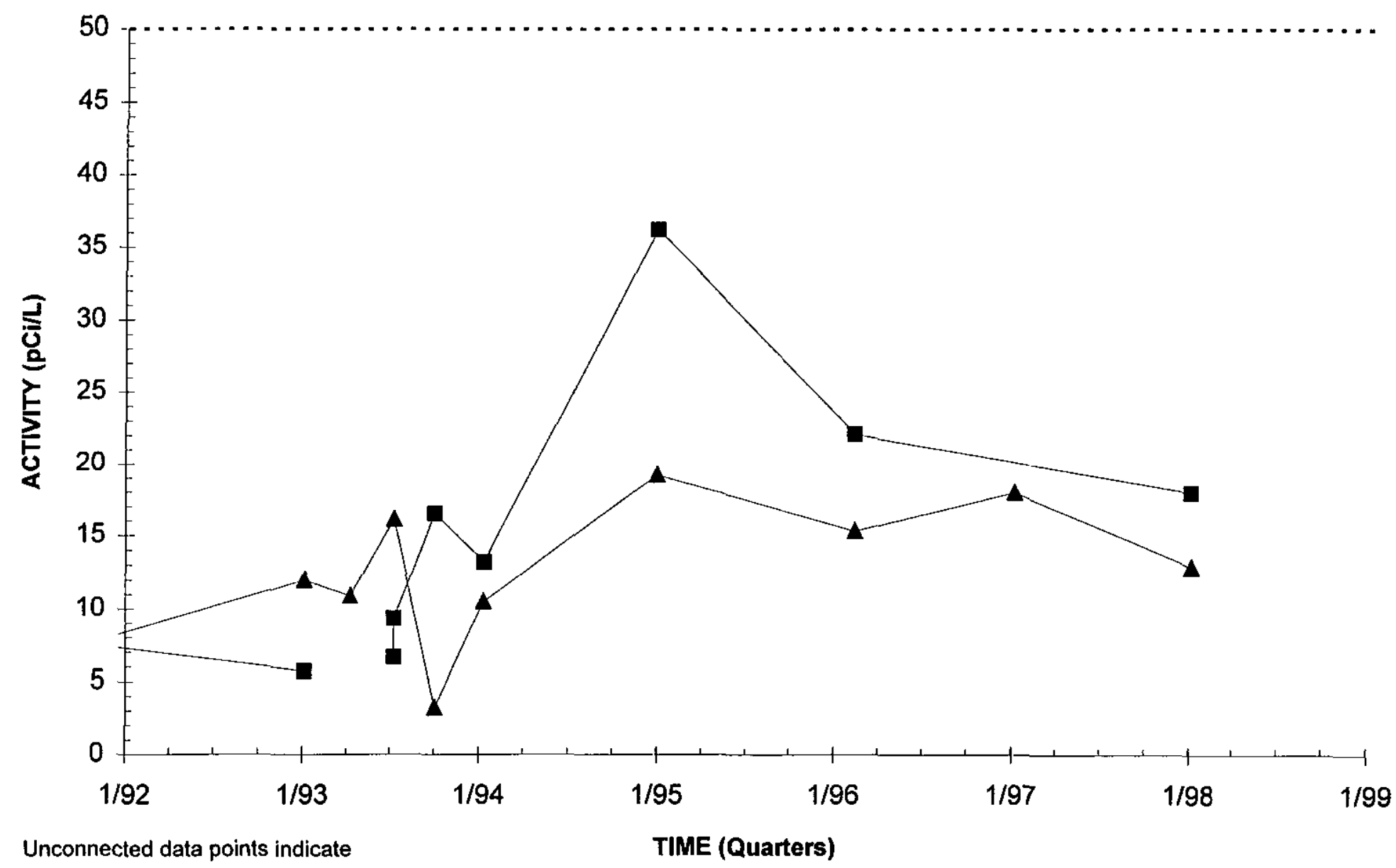

intervals of dry wells or values

below the detection limit.

$$
-\mathrm{HSB} 86 \mathrm{D}(\mathrm{W}) \_ \text {HSB 86C }(\mathrm{B}) \_ \text {HSB } 86 \mathrm{~B}(\mathrm{M}) \cdots \cdots \text {. } \ldots \text { Sum of alphas }<50 \mathrm{pCi} / \mathrm{L}
$$

Note: W=Water Table (IIB2); B=Barnwell (IIB1); M=MCBean (IIB1); UC=Upper Congaree (IIA); MC=Middle Congaree (IIA); LC=Lower Congaree (IIA) 


\section{lodine-129 Activities}

\section{Well Cluster HSB100}

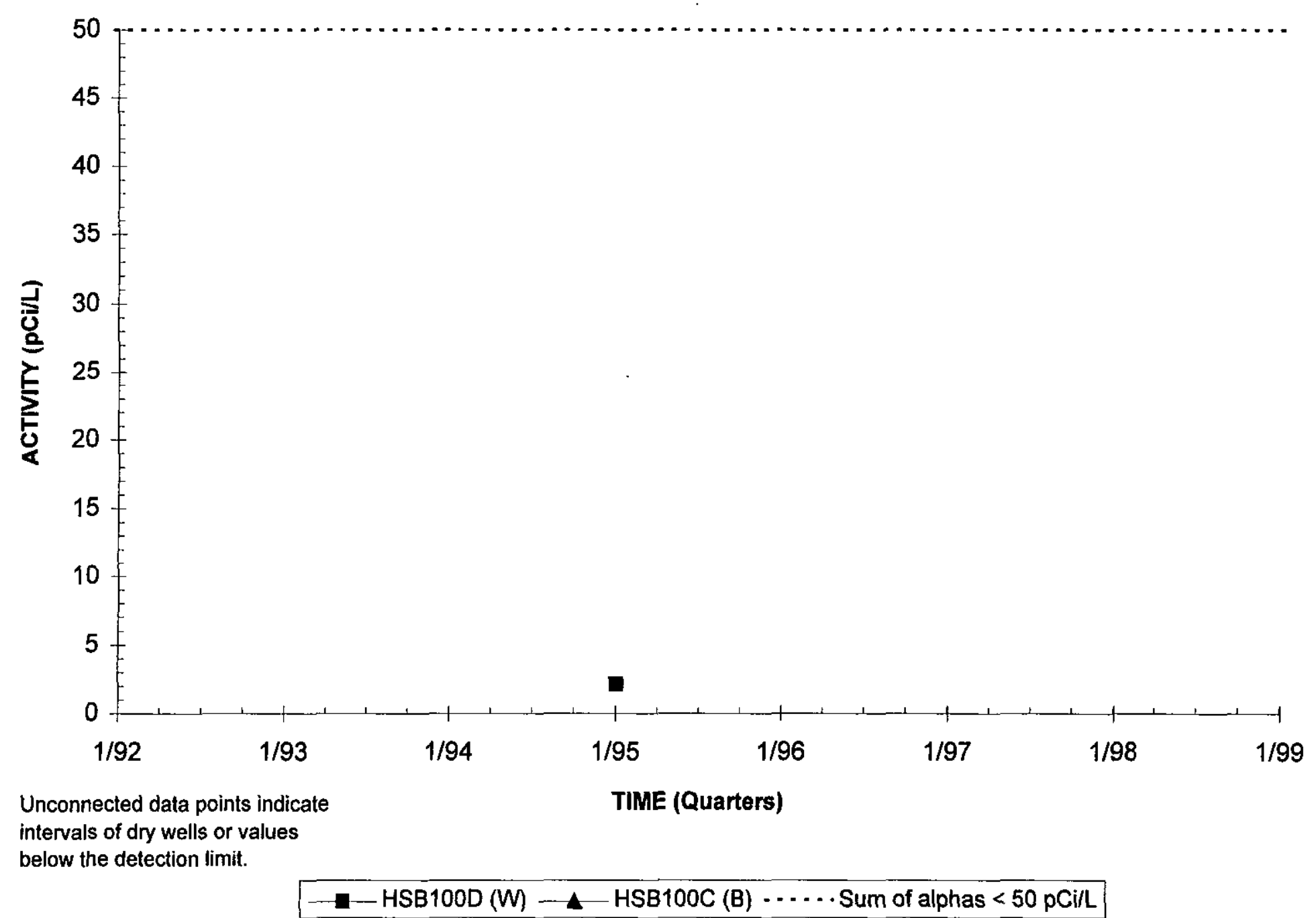

Note: W=Water Table (IIB2); B=Barnwell (IIB1); M=MCBean (IIB1); UC=Upper Congaree (IIA); MC=Middle Congaree (IIA); LC=Lower Congaree (IIA) 


\section{lodine-129 Activities}

\section{Well Cluster HSB104}

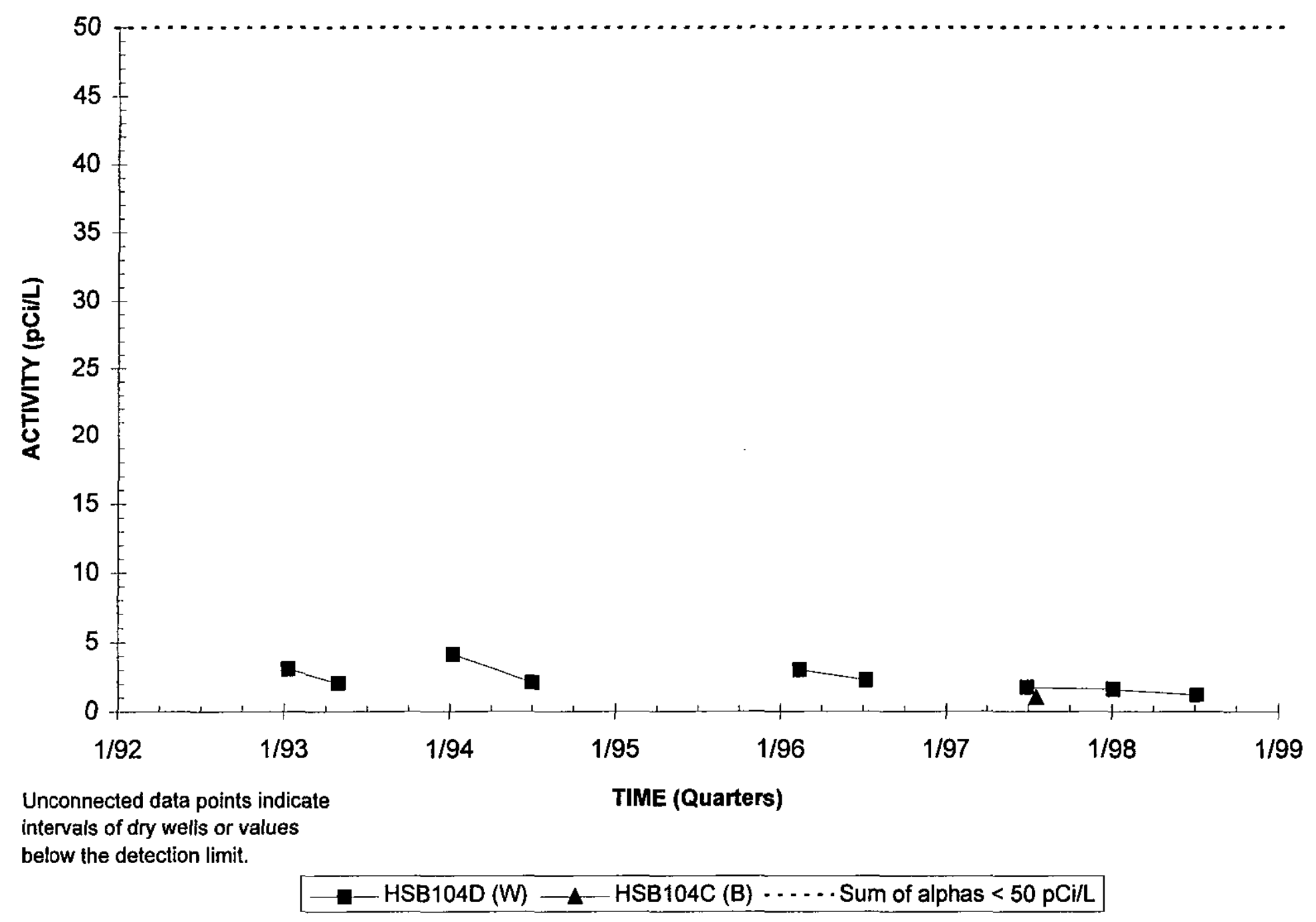

Note: W=Water Table (IIB2); B=Barnwell (IIB1); M=McBean (IIB1); UC=Upper Congaree (IAA); MC=Middle Congaree (IIA); LC=Lower Congaree (IIA) 
WSRC-TR-99-00013

Unclassified

\section{lodine-129 Activities \\ Well Cluster HSB105}

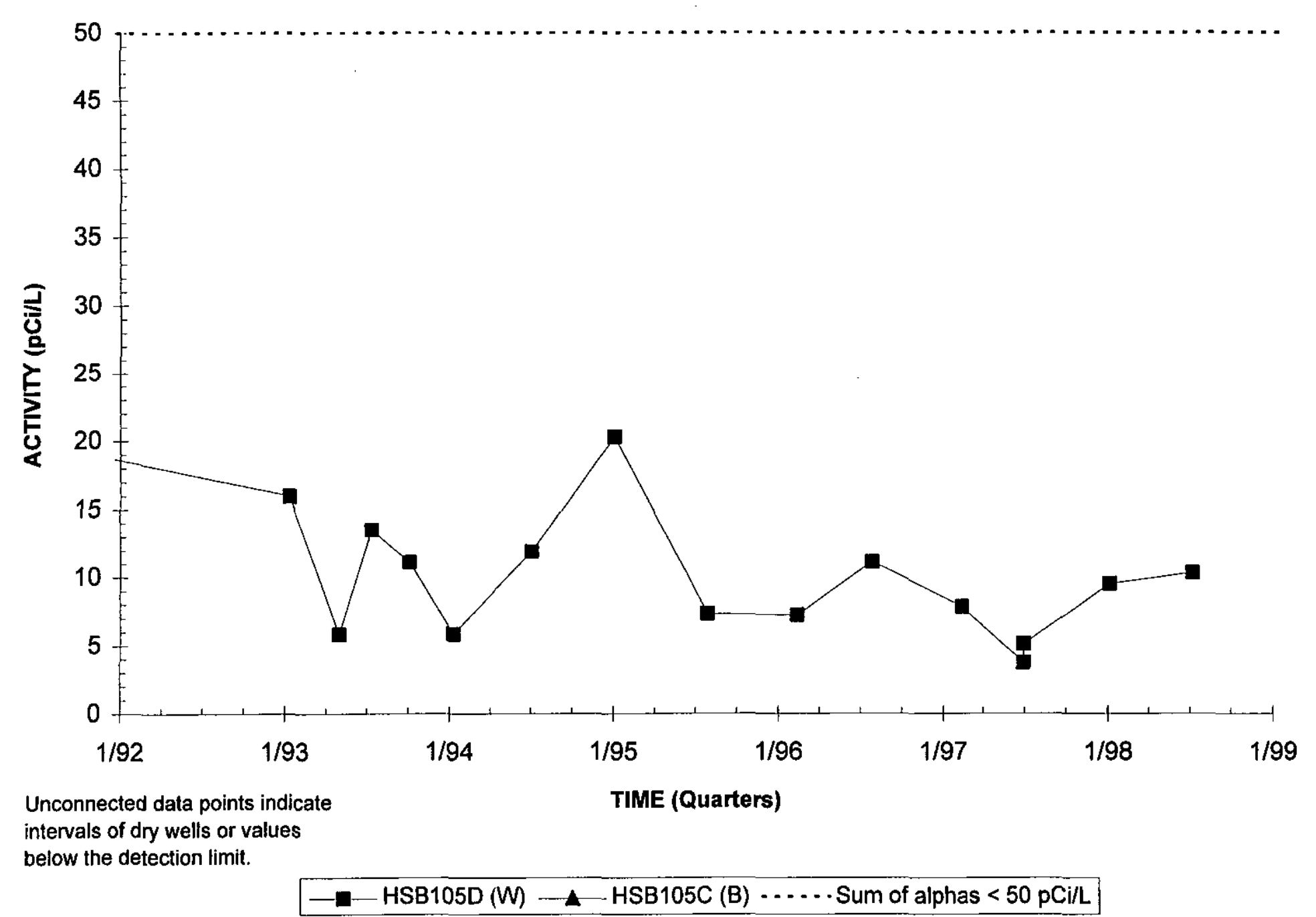

Note: W=Water Table (IIB2); B=Barnwell (IIB1); M=McBean (IIB1); UC=Upper Congaree (IIA); MC=Middle Congaree (IIA); LC=Lower Congaree (IIA) 
lodine-129 Activities

Well Cluster HSB106

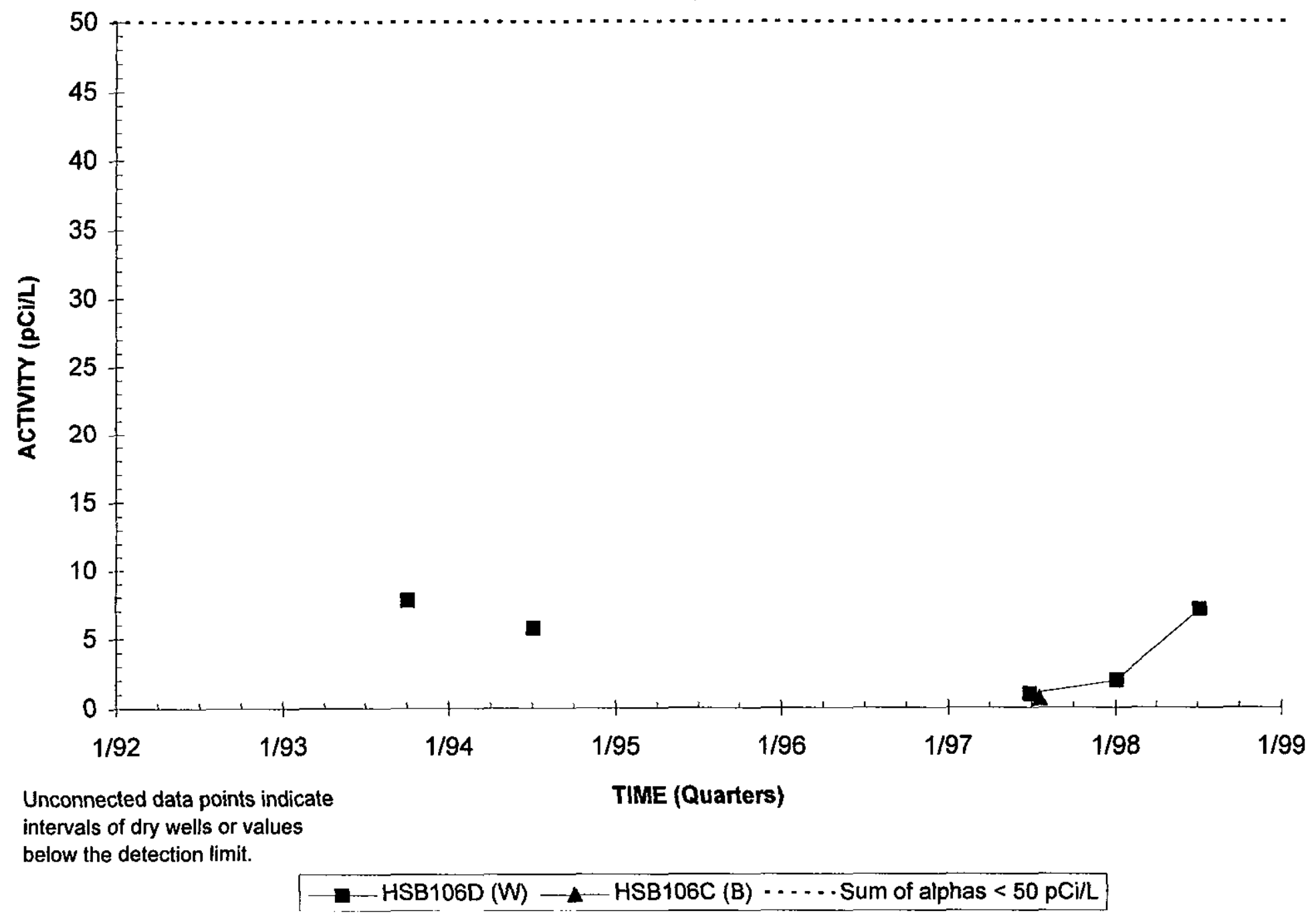

Note: W=Water Table (IIB2); B=Barnwell (IIB1); $M=$ MCBean (IIB1); UC=Upper Congaree (IIA); MC=Middle Congaree (IIA); LC=Lower Congaree (IIA) 


\section{lodine-129 Activities \\ Well Cluster HSB107}

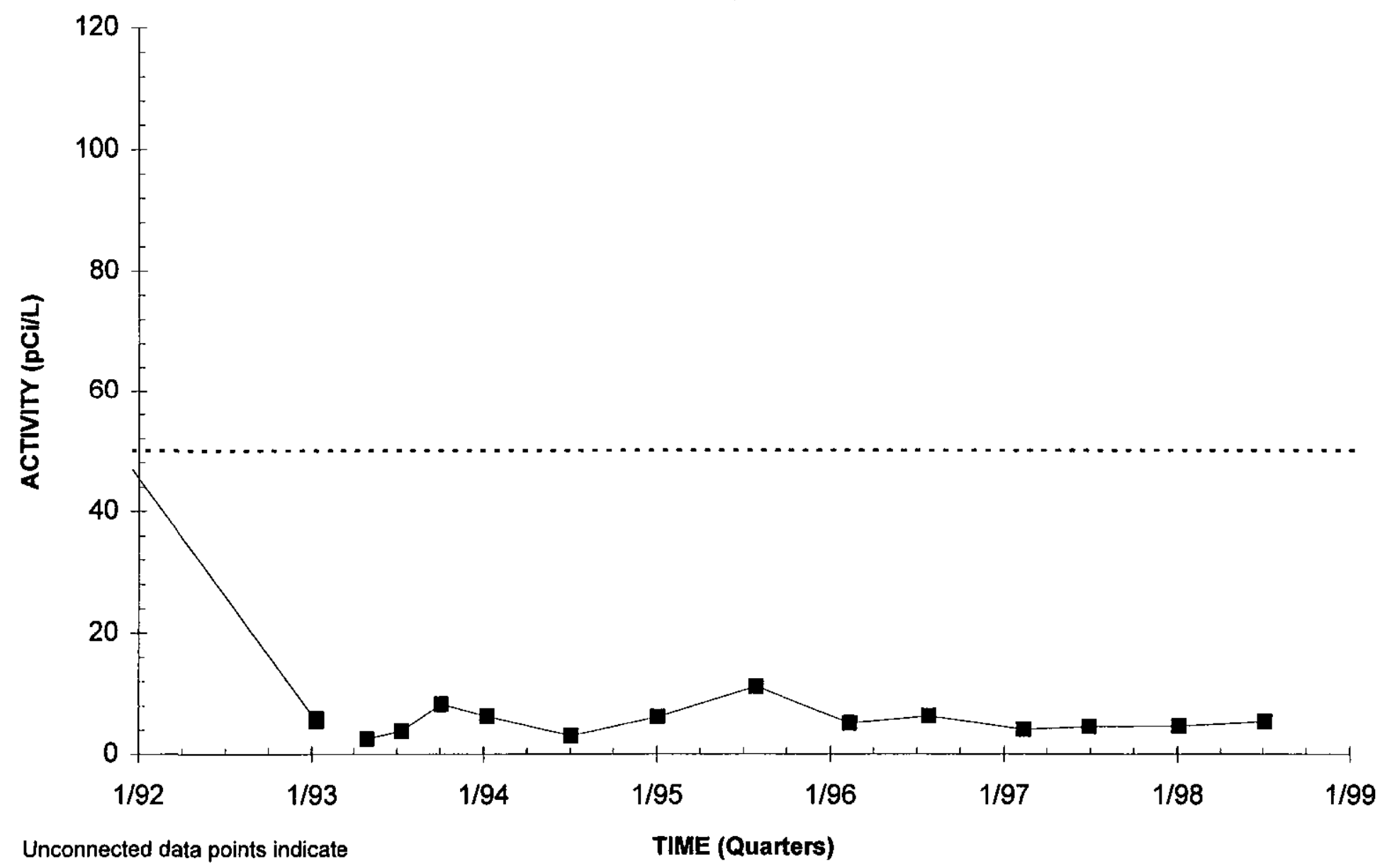

Unconnected data points indicat intervals of dry wells or values below the detection limit.

Note: W=Water Table (IIB2); B=Barnwell (IIB1); M=McBean (IIB1); UC=Upper Congaree (IIA); MC=Middle Congaree (IIA); LC=Lower Congaree (IIA) 


\section{lodine-129 Activities}

Well Cluster HSB113

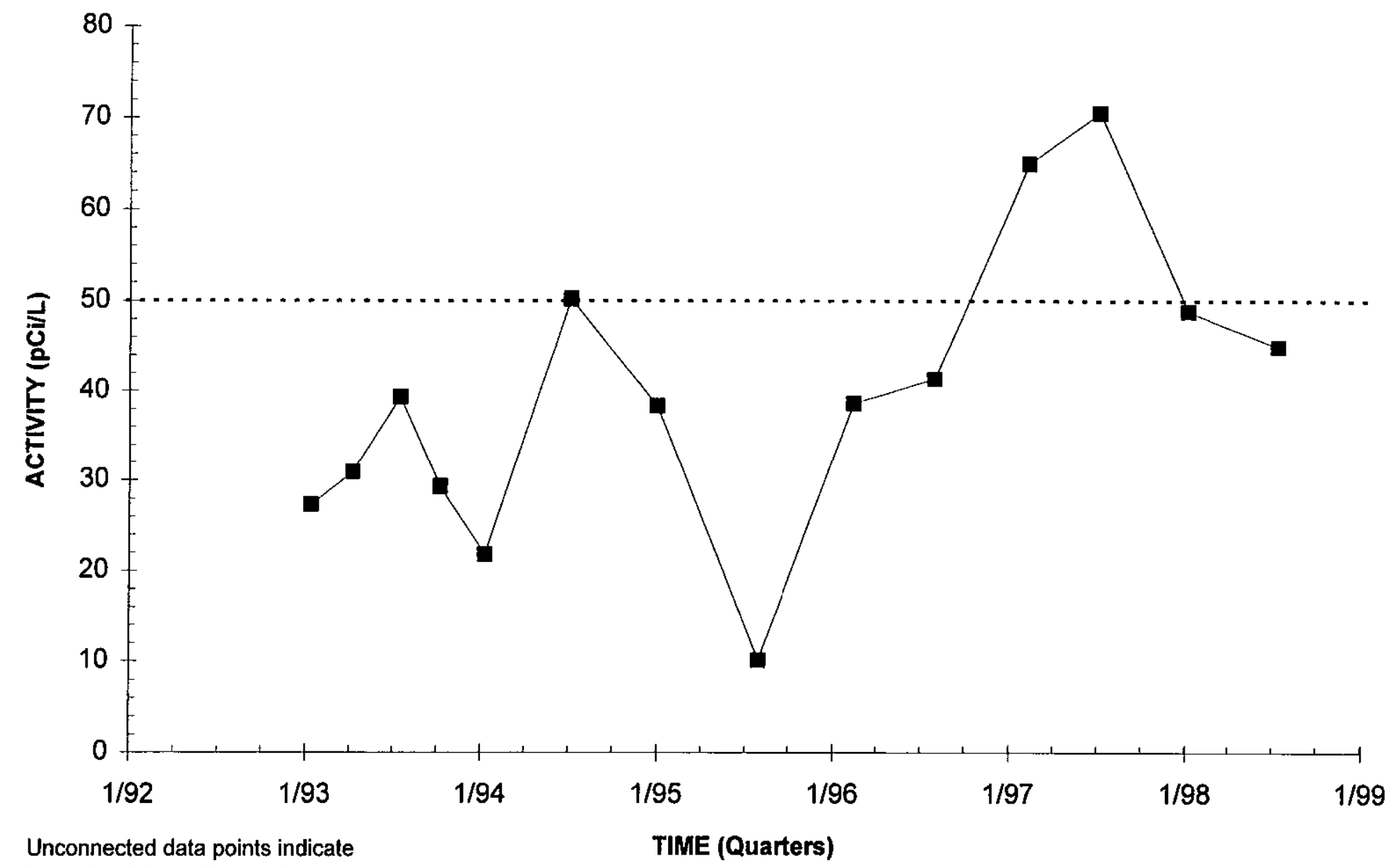

intervals of dry wells or values

below the detection limit.

Sum of alphas $<50 \mathrm{pCi} / \mathrm{L}$

Note: W=Water Table (IIB2); B=Barnwell (IIB1); M=MCBean (IIB1); UC=Upper Congaree (IIA); MC=Middle Congaree (IIA); LC=Lower Congaree (IIA)

H-Area HWMF

D -50

Third and Fourth Quarter 1998

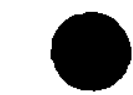




\section{lodine-129 Activities \\ Well Cluster HSB114}

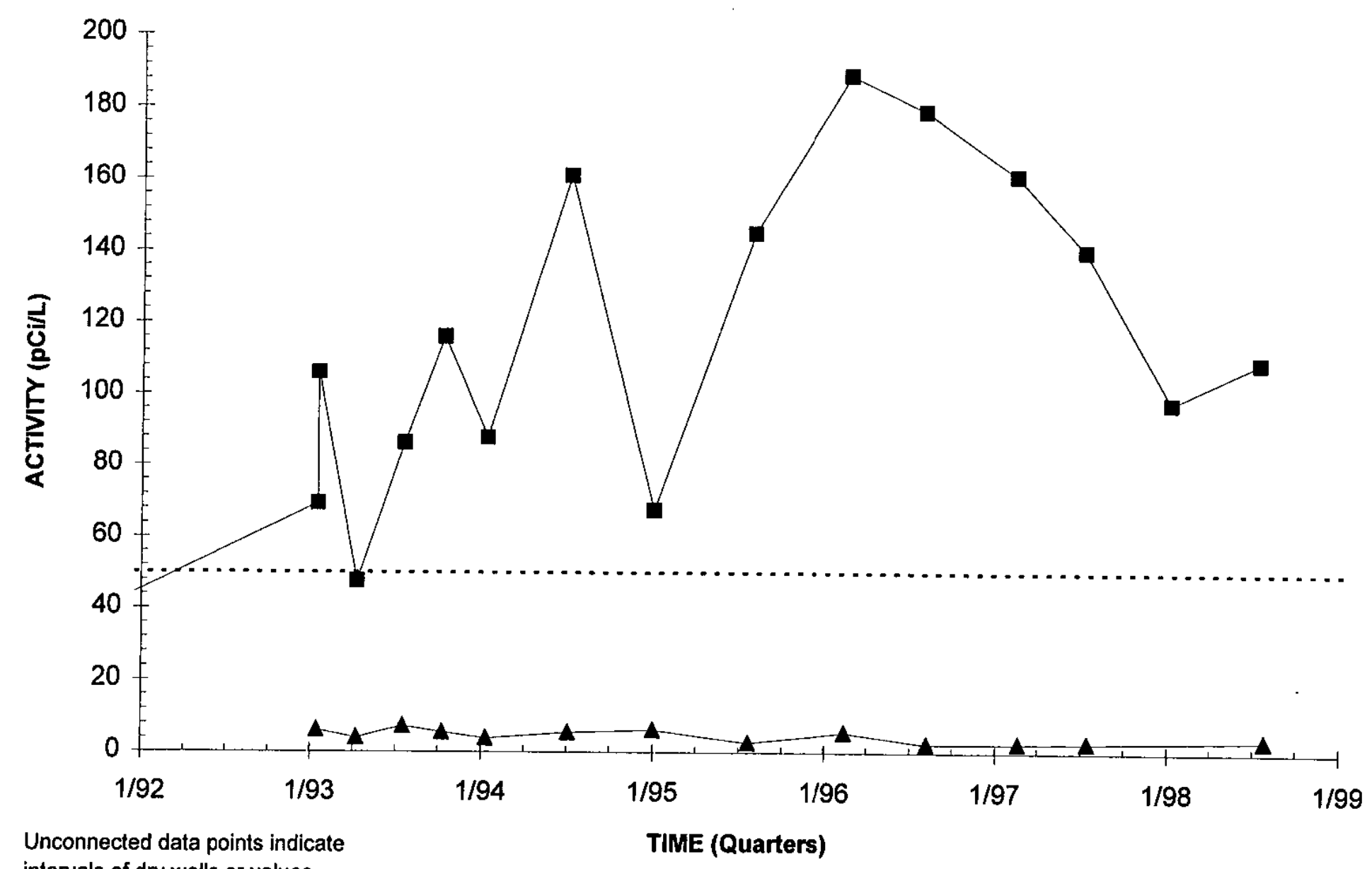

intervals of dry wells or values

below the detection limit.

$$
\text { HSB114D (W) - HSB114C (B) } \cdots \ldots \text { Sum of alphas }<50 \mathrm{pCi} / \mathrm{L}
$$

Note: $W=$ Water Table (IIB2); B=Barnwell (IIB1); $M=M C B$ ean (IIB1); UC=Upper Congaree (IAA); MC=Middle Congaree (IIA); LC=Lower Congaree (IIA) 


\section{lodine-129 Activities}

Well Cluster HSB115

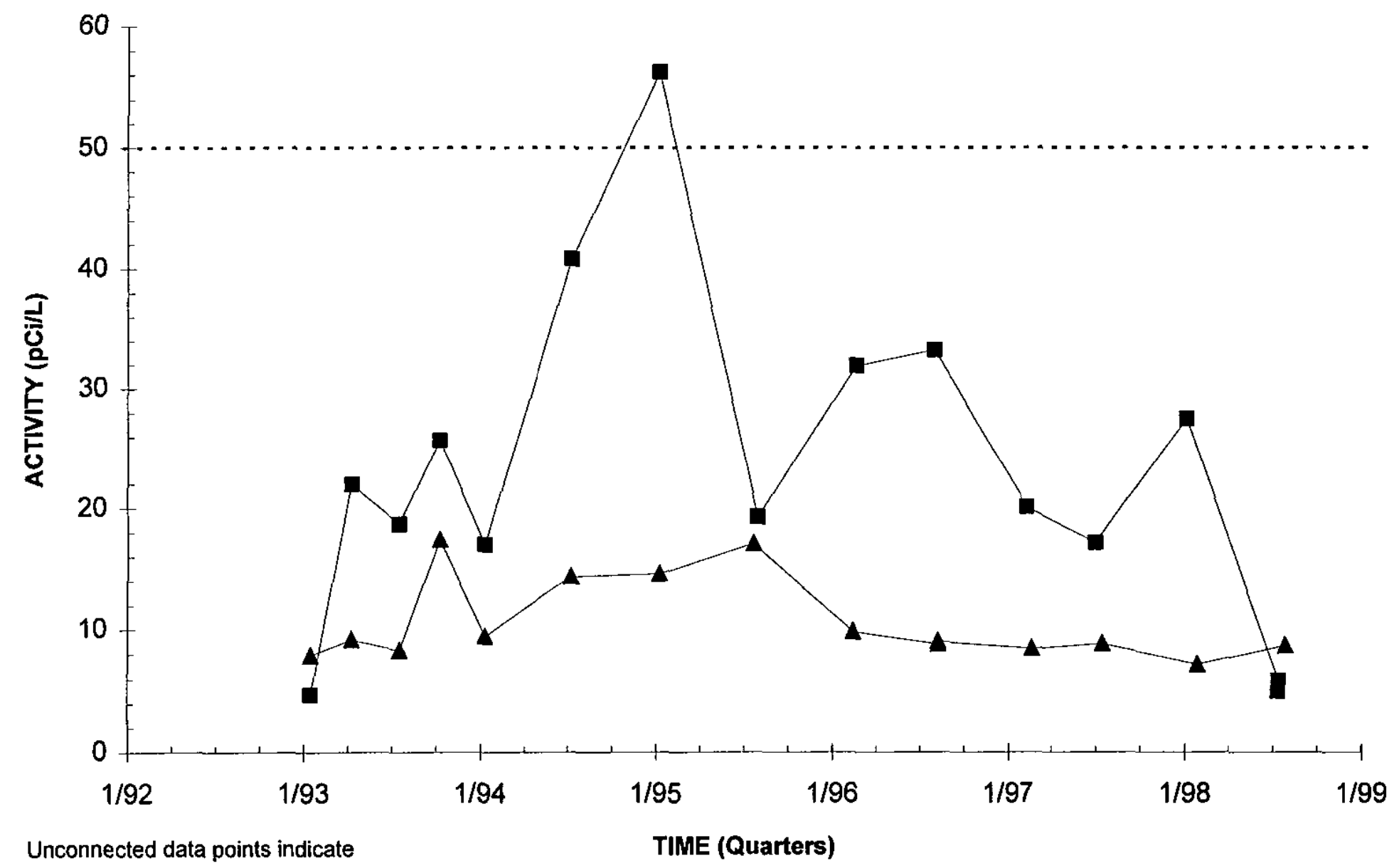

intervals of dry wells or values

below the detection limit.

HSB115D (W) $-\mathrm{HSB} 115 \mathrm{C}(\mathrm{B}) \cdots \cdots$. $\cdots$ Sum of alphas $<50 \mathrm{pCi} / \mathrm{L}$

Note: $W=$ Water Table (IIB2); $B=B a r n w e l l$ (IIB1); $M=M C B e a n$ (IIB1); UC=Upper Congaree (IIA); MC=Middle Congaree (IIA); LC=Lower Congaree (IIA)

H-Area HWMF

D - 52

Third and Fourth Quarter 1998
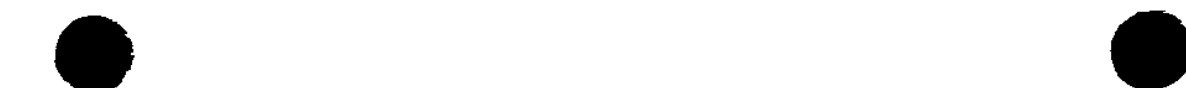


\section{lodine-129 Activities}

Well Cluster HSB136

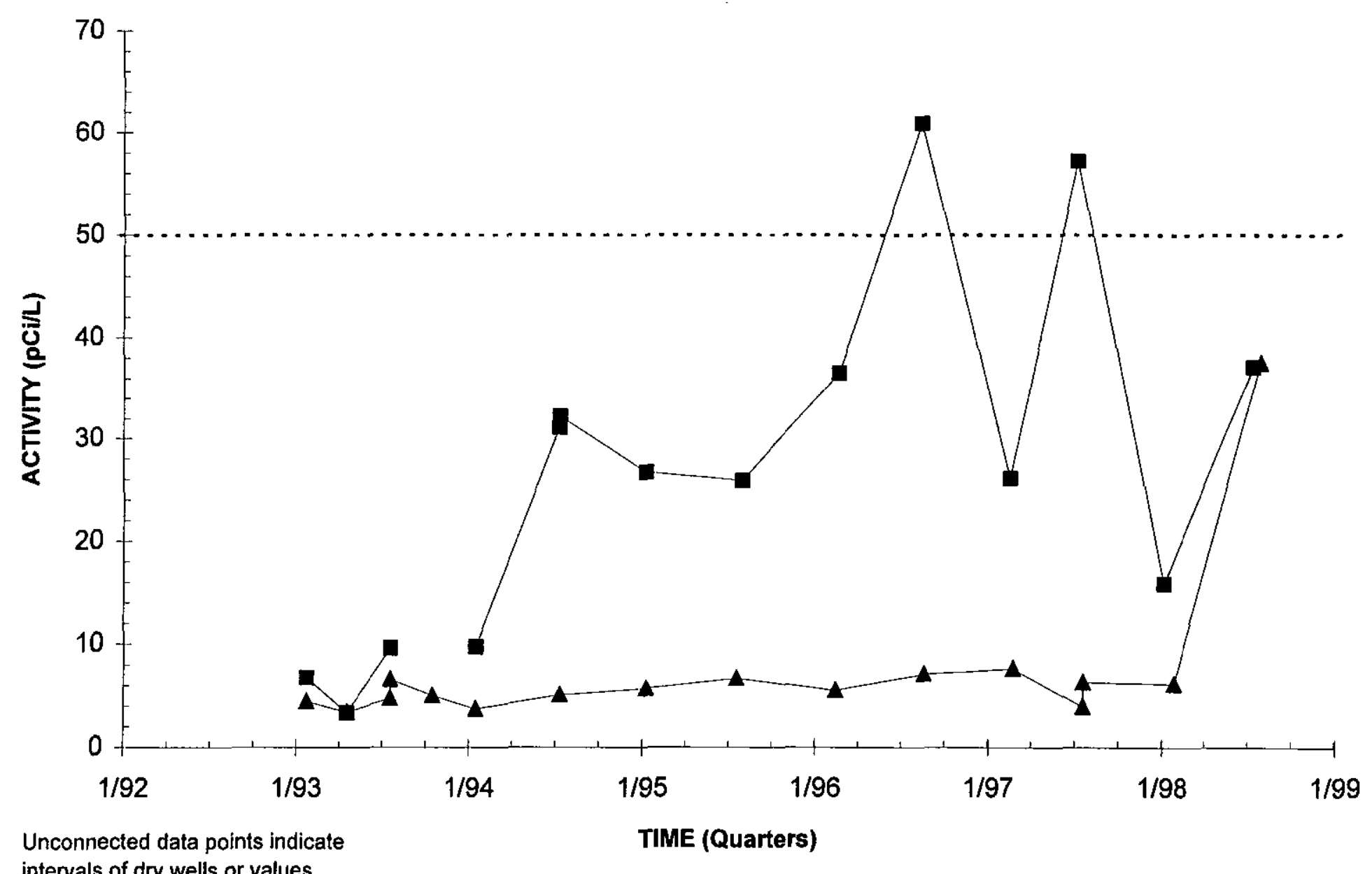

intervals of dry wells or values

below the detection limit.

m-HSB136D (W) - HSB136C (B) $\cdots$. . Sum of alphas $<50 \mathrm{pCi} / \mathrm{L}$

Note: W=Water Table (IIB2); B=Barnwell (IIB1); M=McBean (IIB1); UC=Upper Congaree (IIA); MC=Middle Congaree (IIA); LC=Lower Congaree (IIA) 


\section{lodine-129 Activities}

Well Cluster HSB145

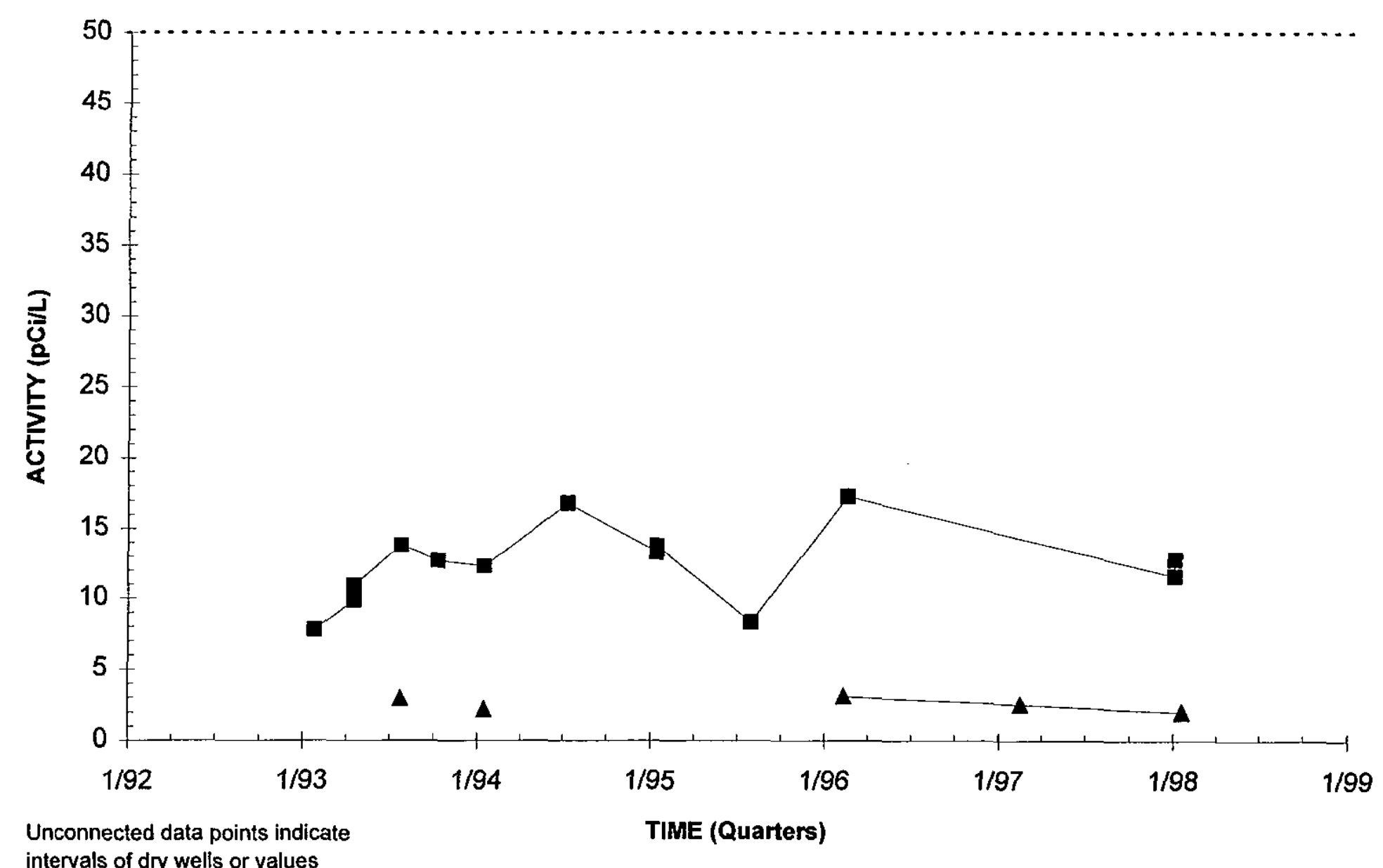

intervals of dry wells or values

below the detection limit.

- HSB145D (W) - HSB145C (B) $\ldots . .$. Sum of alphas $<50 \mathrm{pCi} / \mathrm{L}$

Note: $W=$ Water Table (II82); B=Barnwell (IIB1); M=McBean (IIB1); UC=Upper Congaree (IIA); MC=Middie Congaree (IIA); LC=Lower Congaree (IIA) 


\section{Strontium-90 Activities \\ Well HSB 69}

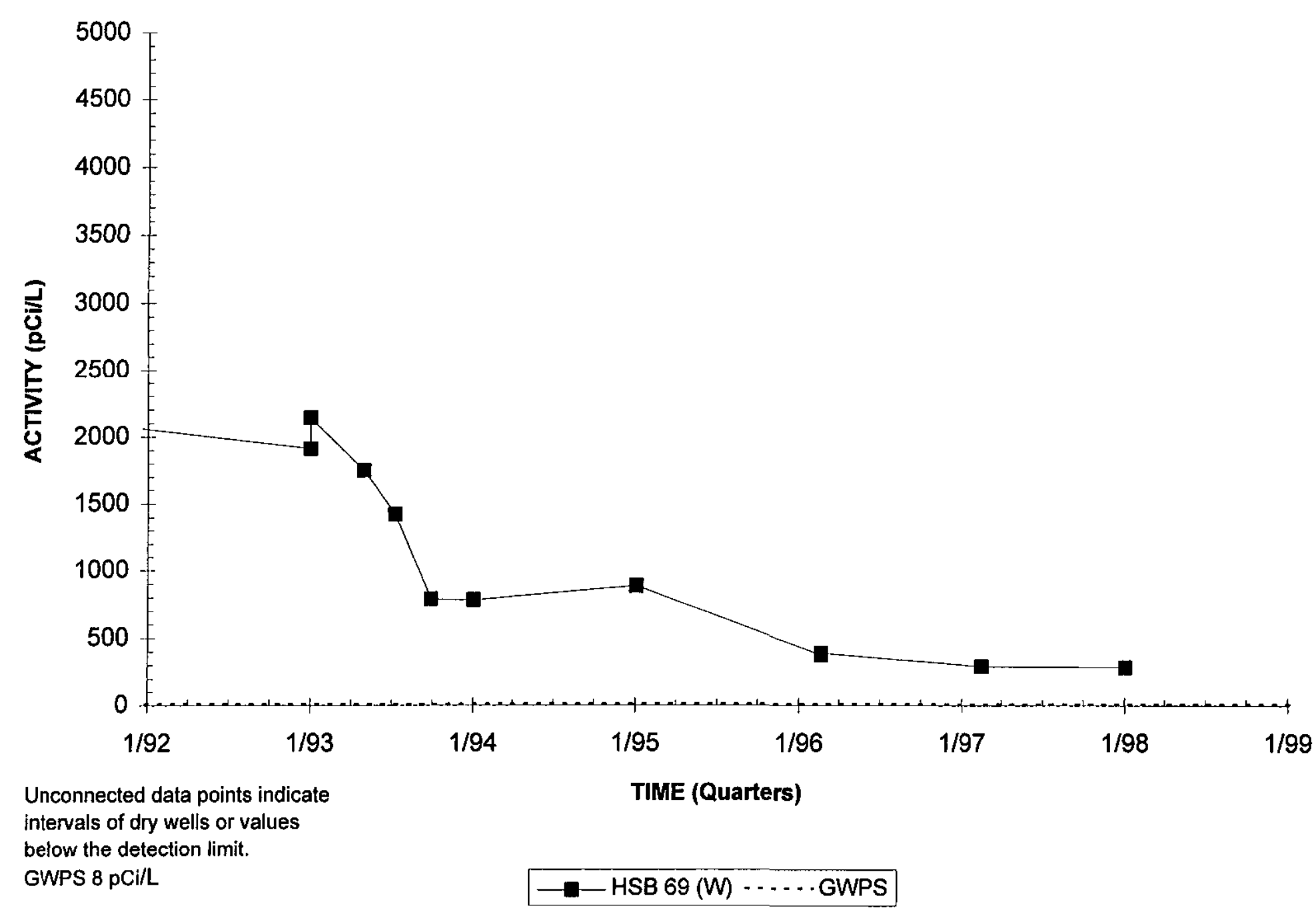

Note: $W=$ Water Table (IIB2); B=Barnwell (IIB1); M=McBean (IIB1); UC=Upper Congaree (IIA); MC=Middle Congaree (IIA); LC=Lower Congaree (IIA) 


\section{Strontium-90 Activities \\ Well Cluster HSB 84}

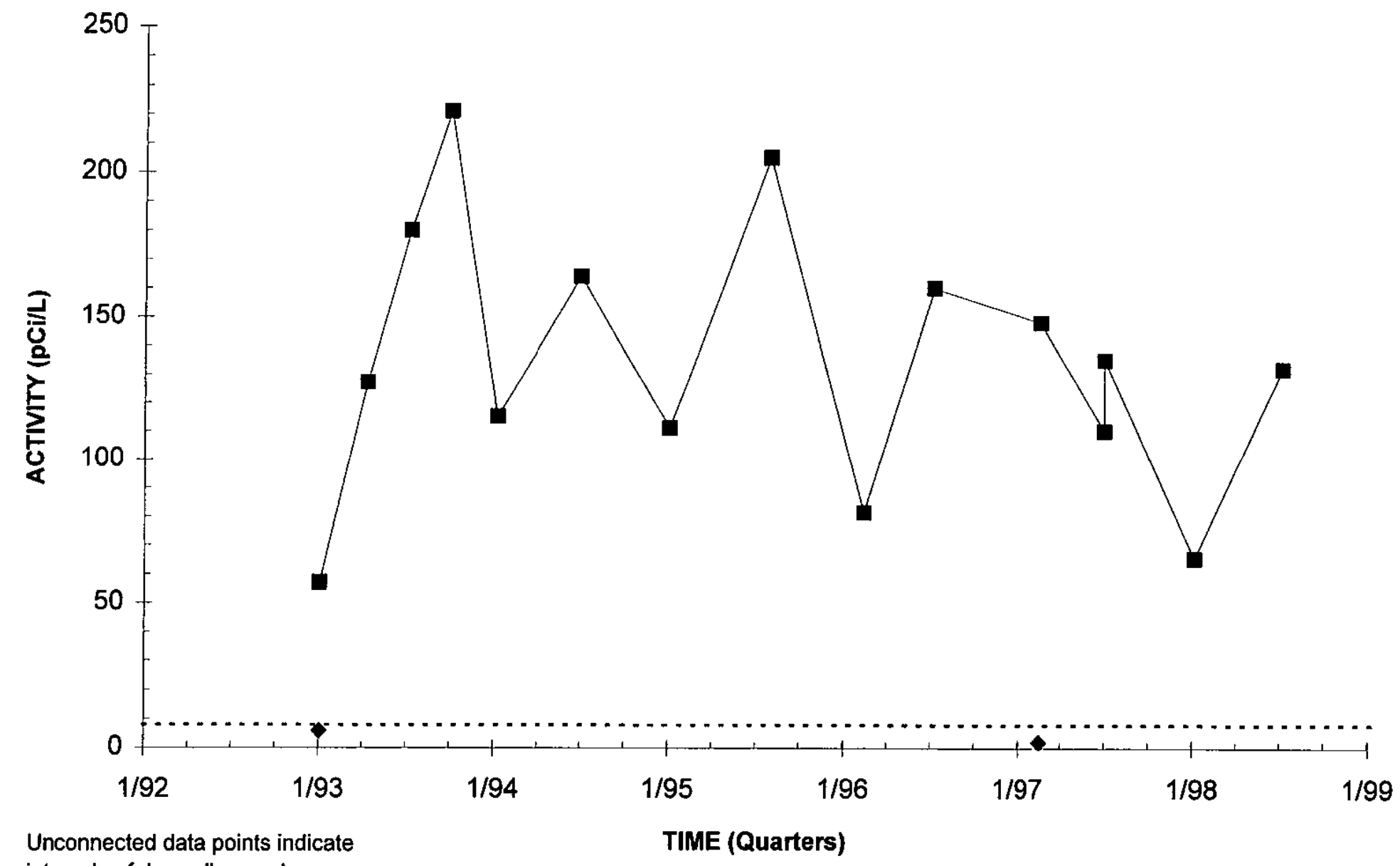

intervals of dry wells or values

below the detection limit.

GWPS $8 \mathrm{pCi} / \mathrm{L}$

- HSB 84D (W) - $\mathrm{HSB} 84 \mathrm{C}(\mathrm{B})$

HSB 84B (M) $\cdots \ldots$ GWPS

Note: $W=$ Water Table (IIB2); B=Barnwell (IIB1); M=McBean (IIB1); UC=Upper Congaree (IIA); MC=Middle Congaree (IIA); LC=Lower Congaree (IIA) 


\section{Strontium-90 Activities}

Well Cluster HSB 86

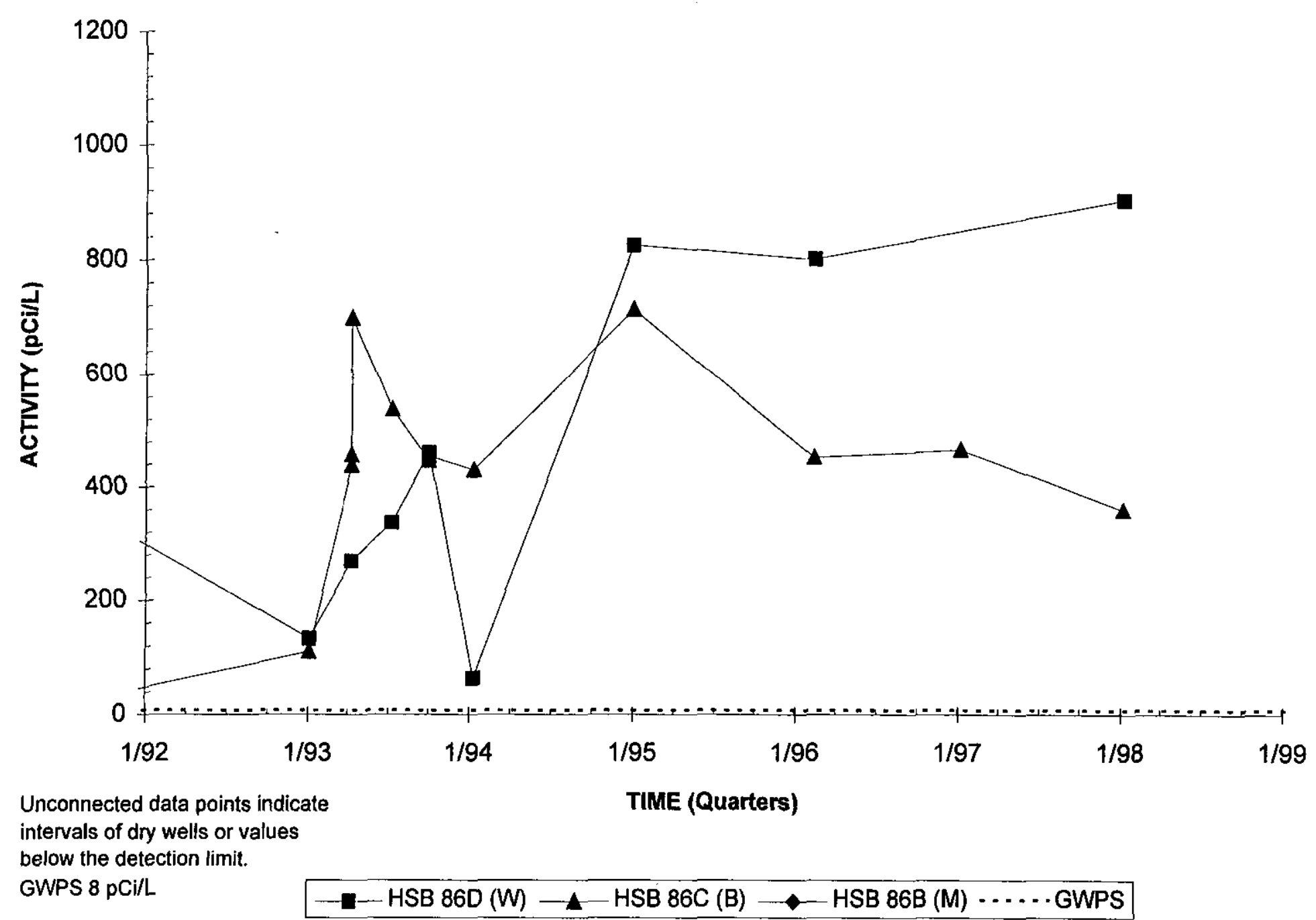

Note: W=Water Table (IIB2); B=Barnwell (IIB1); M=McBean (IIB1); UC=Upper Congaree (IIA); MC=Middle Congaree (IIA); LC=Lower Congaree (IIA) 


\section{Strontium-90 Activities \\ Well Cluster HSB100}

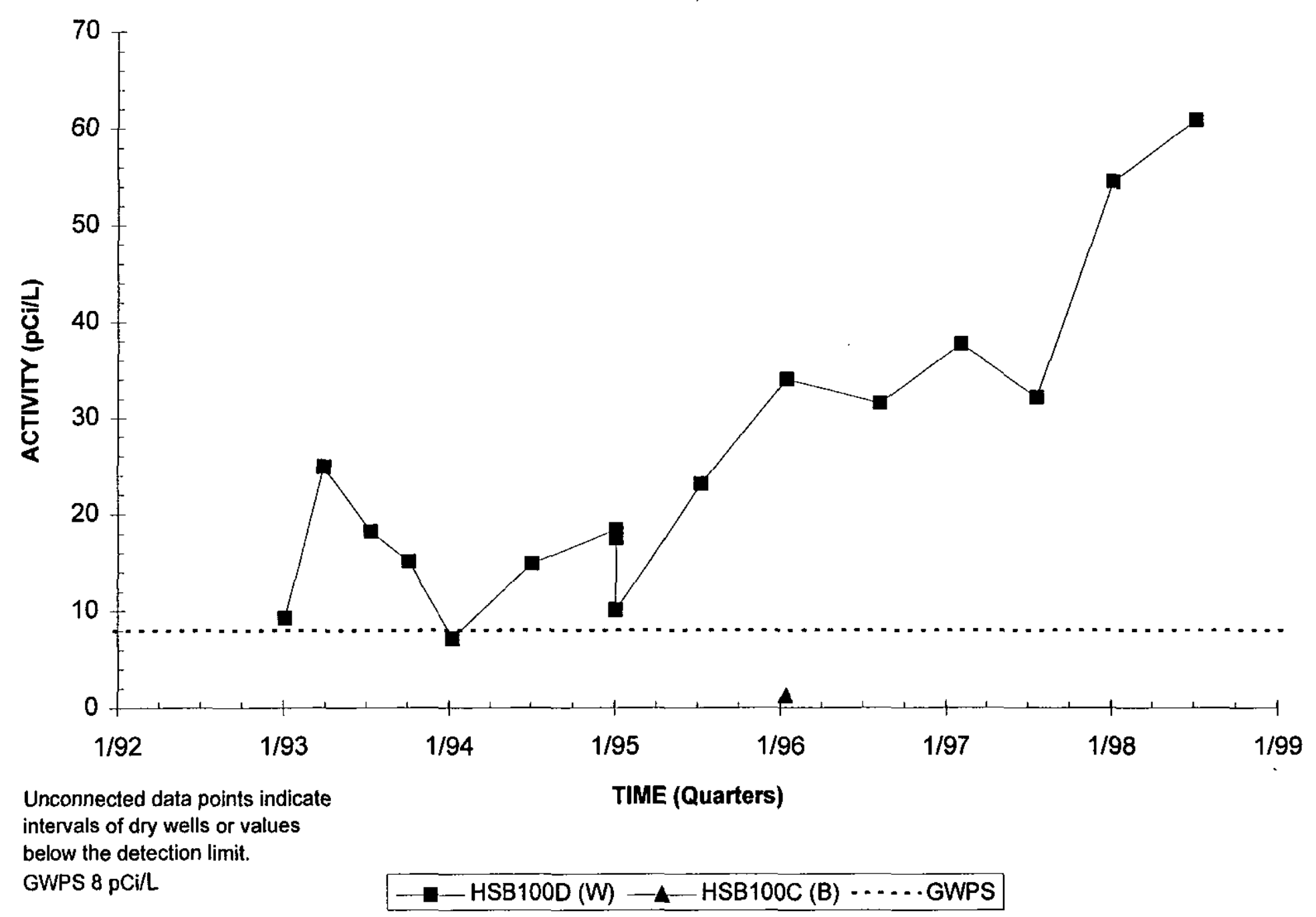

Note: W=Water Table (IIB2); B=Barnwell (IIB1); M=McBean (IIB1); UC=Upper Congaree (IIA); MC=Middle Congaree (IIA); LC=Lower Congaree (IIA) 


\section{Strontium-90 Activities \\ Well Cluster HSB104}

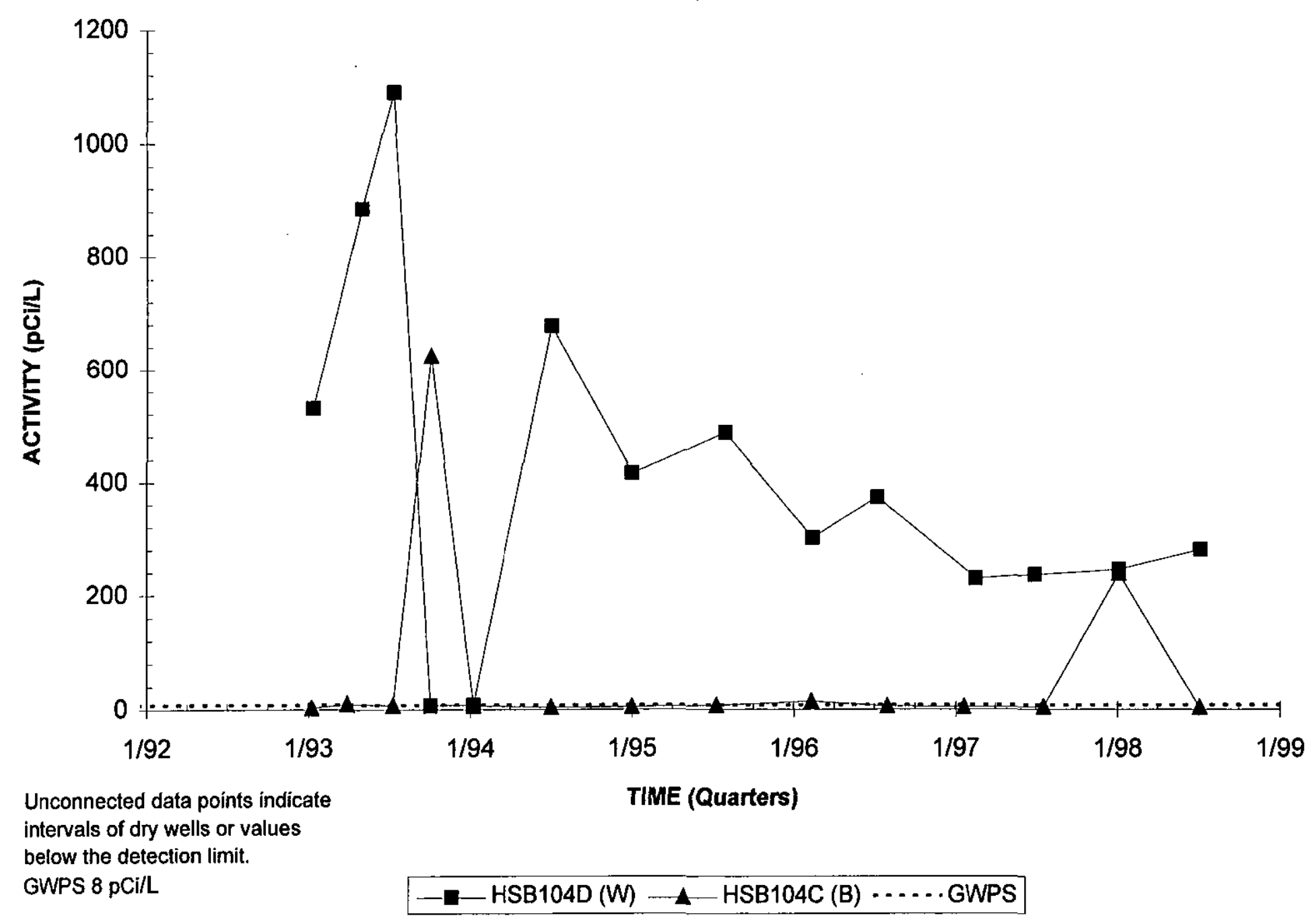

Note: $W=$ Water Table (IIB2); B=Barnwell (IIB1); M=McBean (IIB1); UC=Upper Congaree (IIA); MC=Middle Congaree (IIA); LC=Lower Congaree (IIA) 


\section{Strontium-90 Activities \\ Well Cluster HSB105}

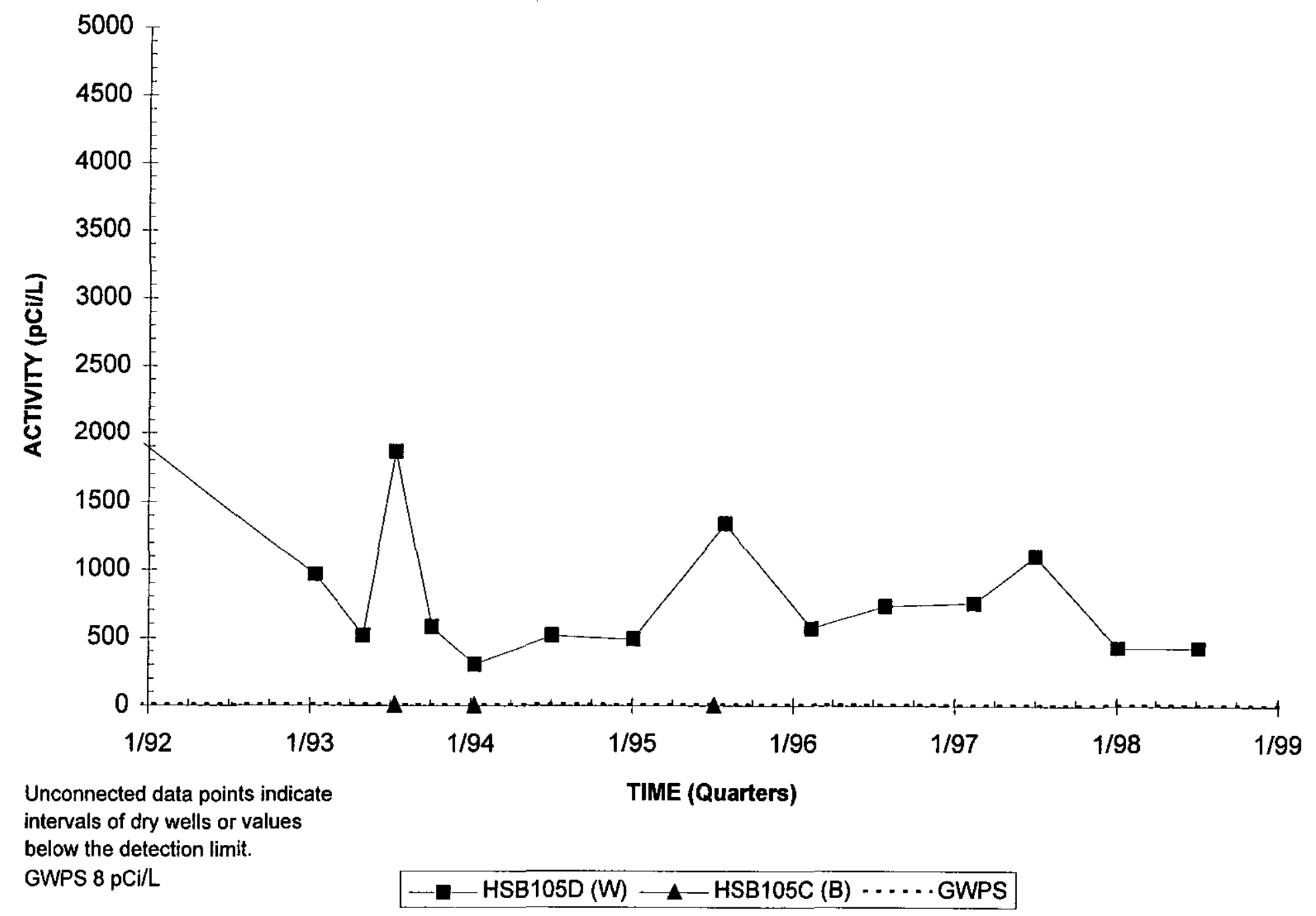

Note: W=Water Table (IIB2); B=Barnwell (IIB1); M=McBean (IIB1); UC=Upper Congaree (IIA); MC=Middle Congaree (IIA); LC=Lower Congaree (IIA) 


\section{Strontium-90 Activities}

Well Cluster HSB106

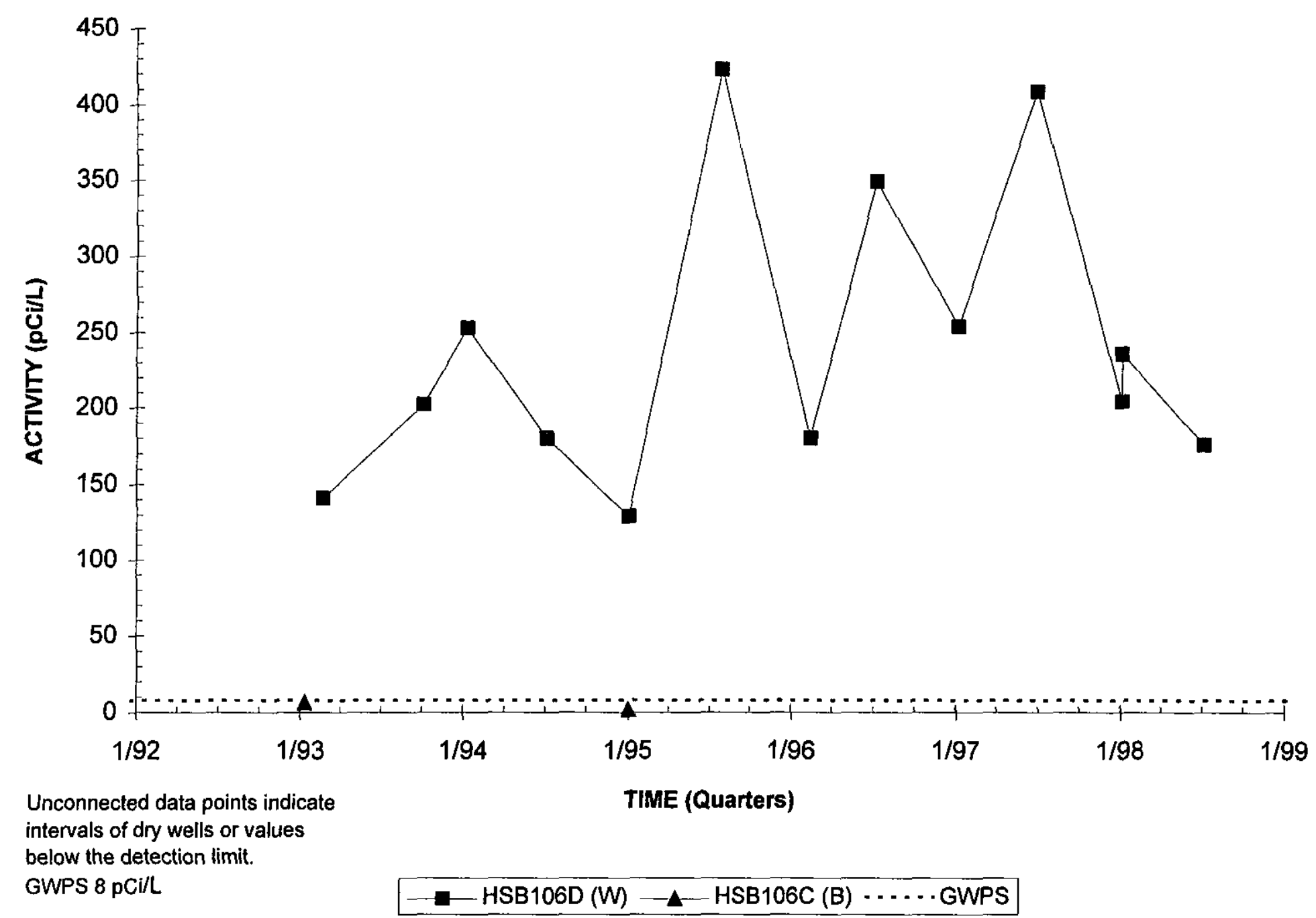

Note: W=Water Table (IIB2); B=Barnwell (IIB1); M=McBean (IIB1); UC=Upper Congaree (IIA); MC=Middle Congaree (IIA); LC=Lower Congaree (IIA) 


\section{Strontium-90 Activities \\ Well Cluster HSB107}

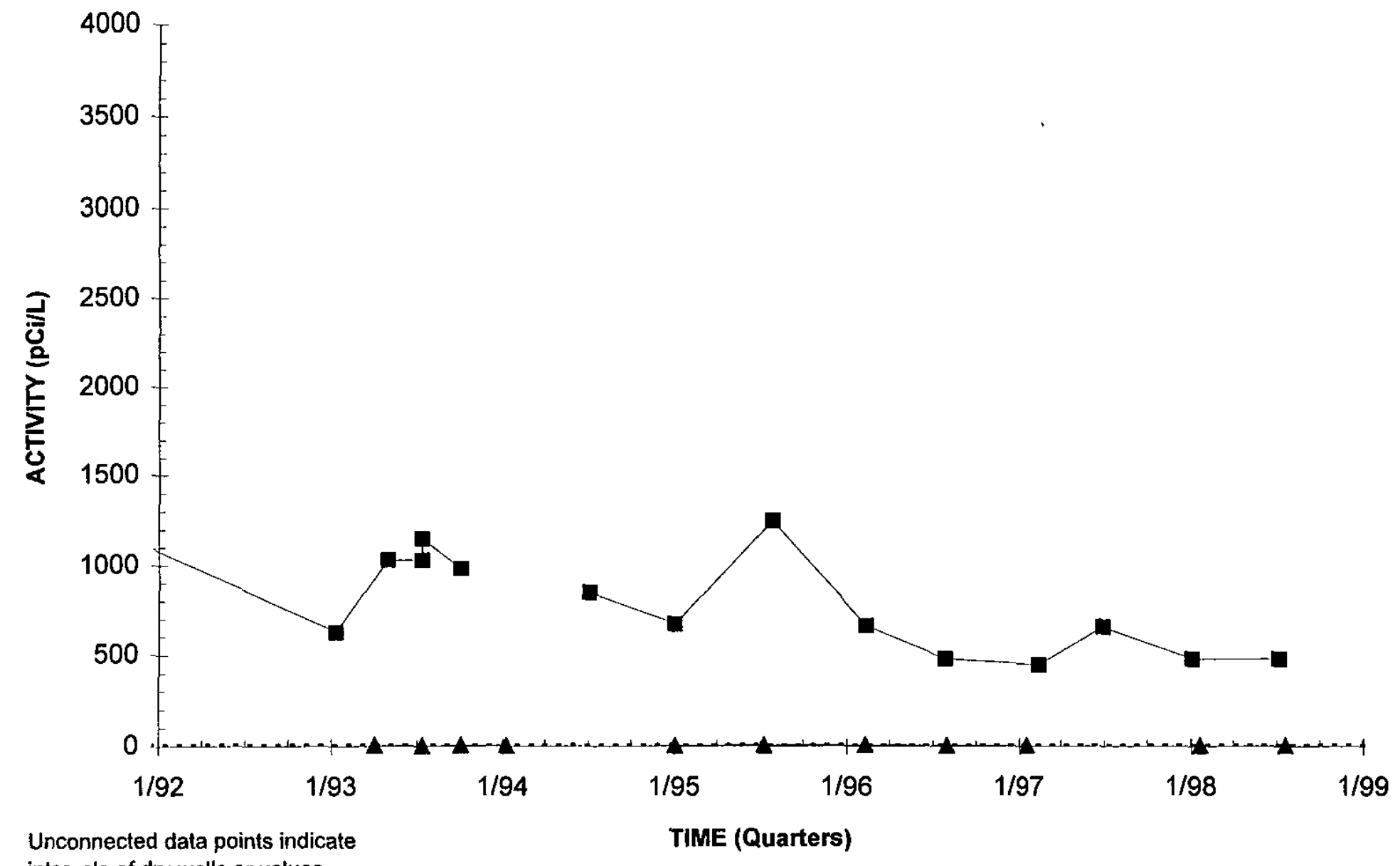

intervals of dry wells or values

below the detection limit.

GWPS 8 pCi/L

$$
\text { a-HSB107D }(\mathrm{W})-\mathrm{A} \text {-HSB107C (B) ......GWPS }
$$

Note: $W=$ Water Table (IIB2); B=Barnwell (IIB1); M=McBean (IIB1); UC=Upper Congaree (IIA); MC=Middle Congaree (IIA); LC=Lower Congaree (IIA) 


\section{Strontium-90 Activities}

Well Cluster HSB113

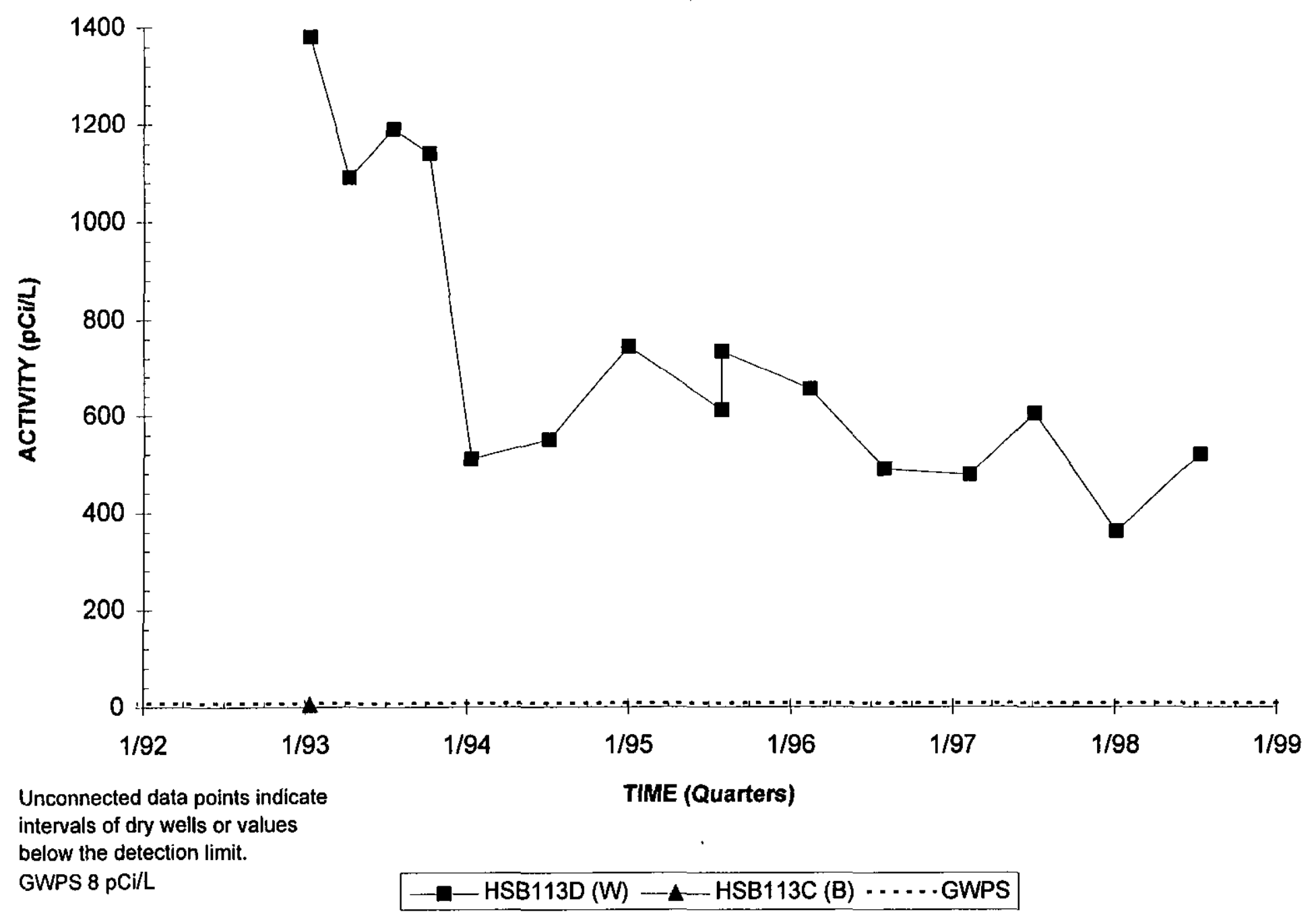

Note: $W=$ Water Table (IIB2); B=Barnwell (IIB1); M=McBean (IIB1); UC=Upper Congaree (IIA); MC=Middle Congaree (IIA); LC=Lower Congaree (IIA) 


\section{Strontium-90 Activities \\ Well Cluster HSB114}

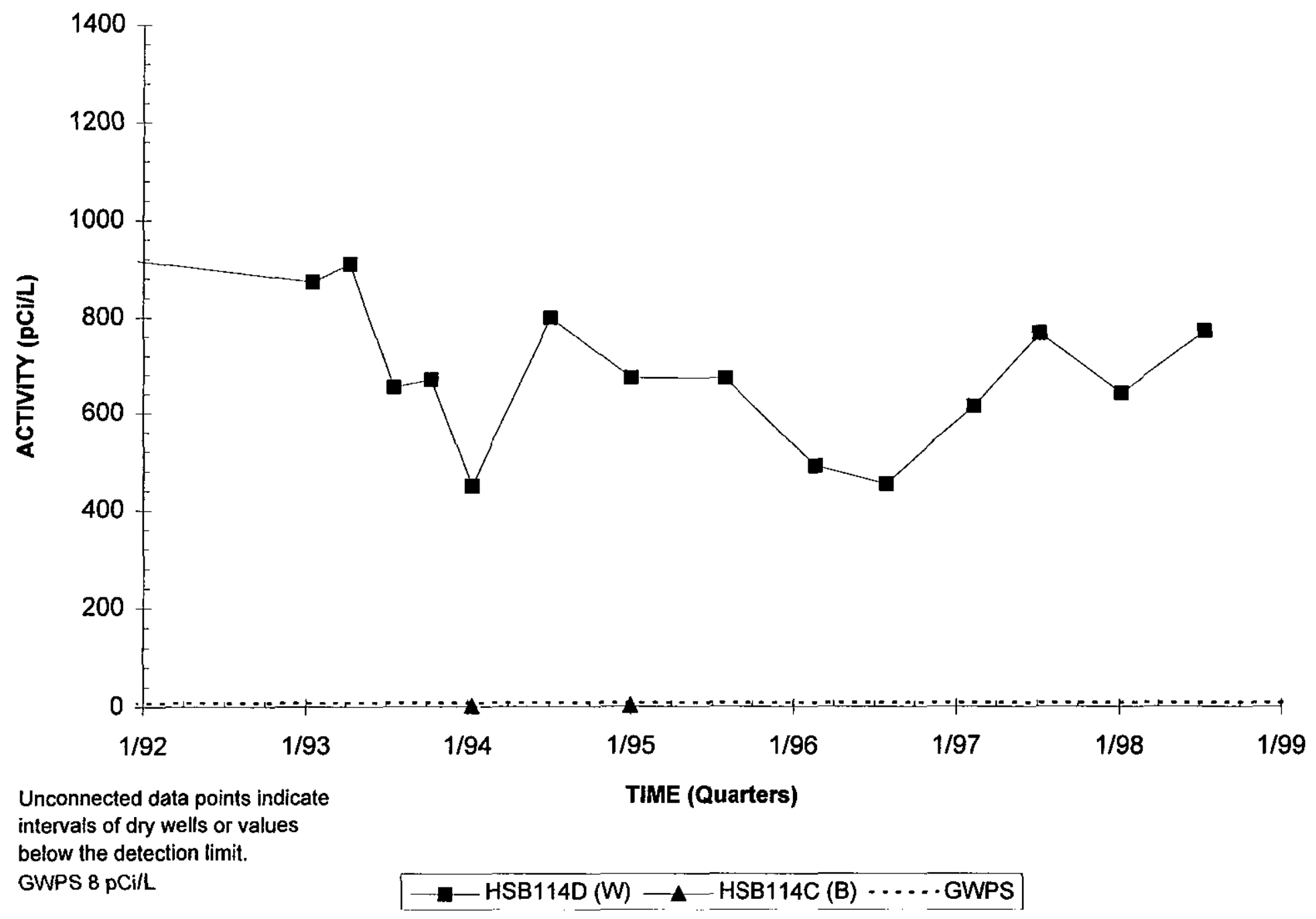

Note: $W=$ Water Table (IIB2); B=Barnwell (IIB1); M=McBean (IIB1); UC=Upper Congaree (IIA); MC=Middle Congaree (IIA); LC=Lower Congaree (IIA) 


\section{Strontium-90 Activities \\ Well Cluster HSB115}

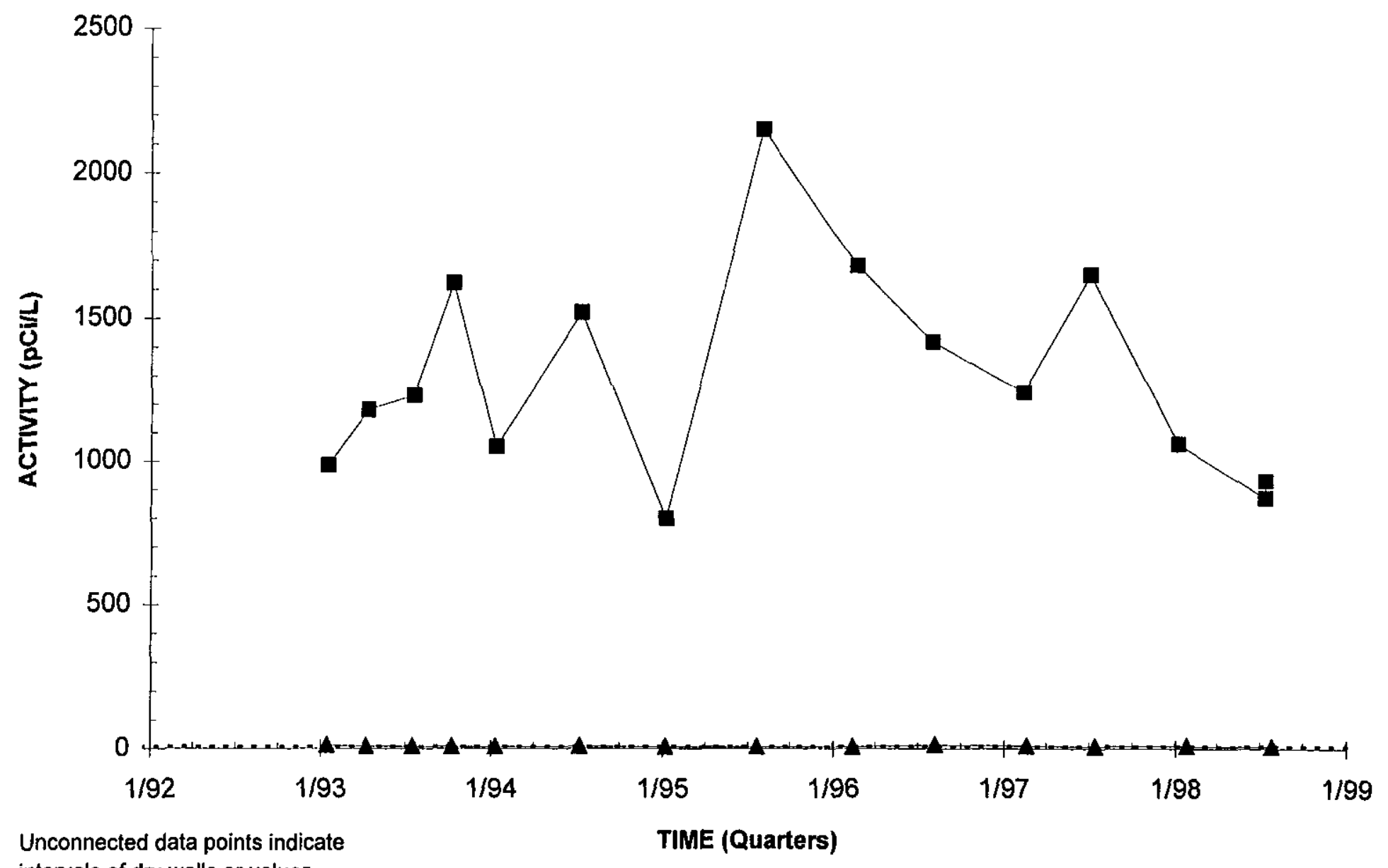

intervals of dry wells or values

below the detection limit.

GWPS $8 \mathrm{pCi} / \mathrm{L}$

$\rightarrow$ HSB115D (W) -A-HSB115C (B) …...GWPS

Note: $W=$ Water Table (IIB2); B=Barnwell (IIB1); M=McBean (IIB1); UC=Upper Congaree (IIA); MC=Middle Congaree (IIA); LC=Lower Congaree (IIA) 


\section{Strontium-90 Activities}

\section{Well Cluster HSB136}

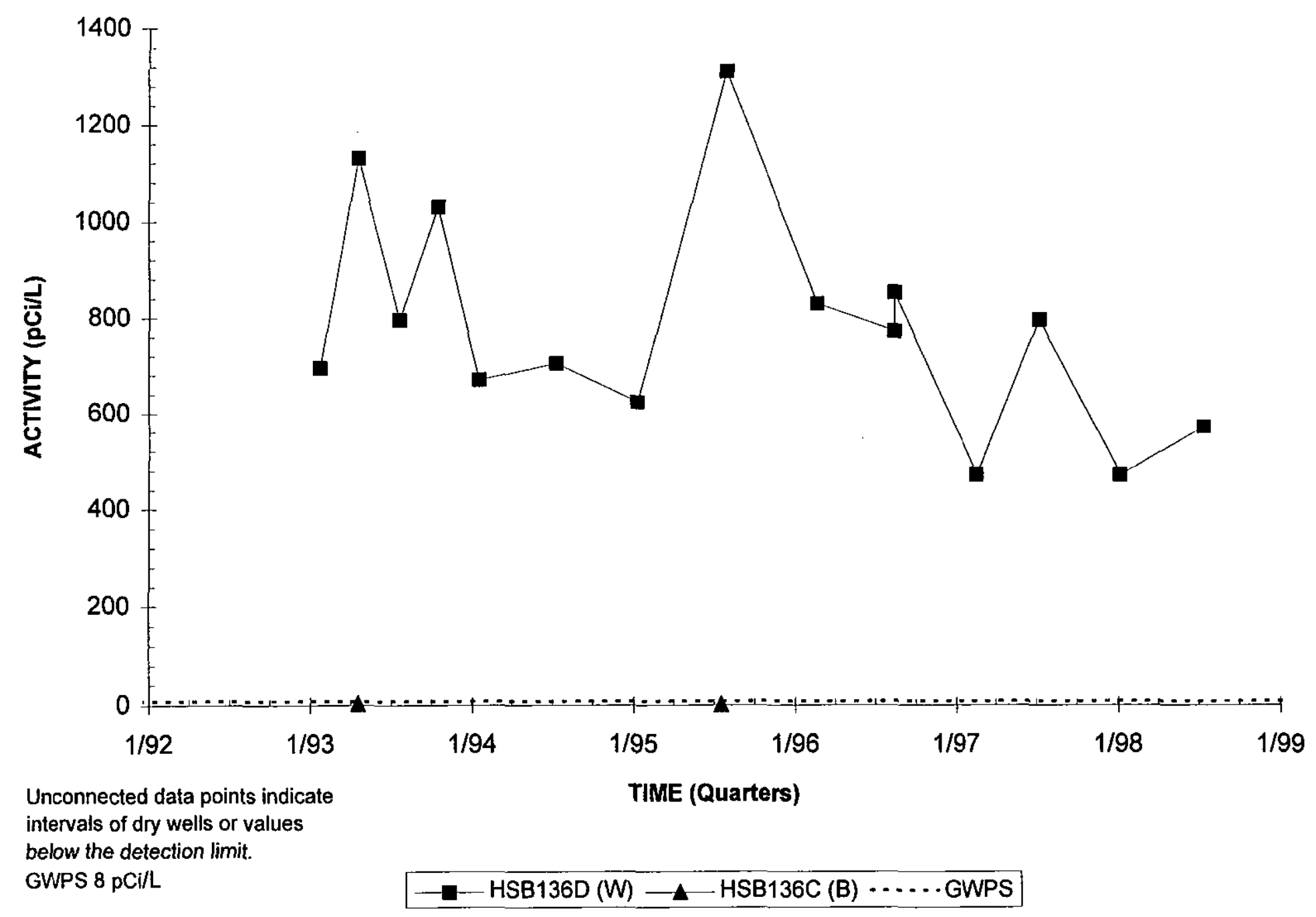

Note: $W=$ Water Table (IIB2); B=8arnwell (IIB1); $M=M c B e a n$ (IIB1); UC=Upper Congaree (IIA); MC=Middle Congaree (IIA); LC=Lower Congaree (IIA) 


\section{Strontium-90 Activities}

\section{Well Cluster HSB145}

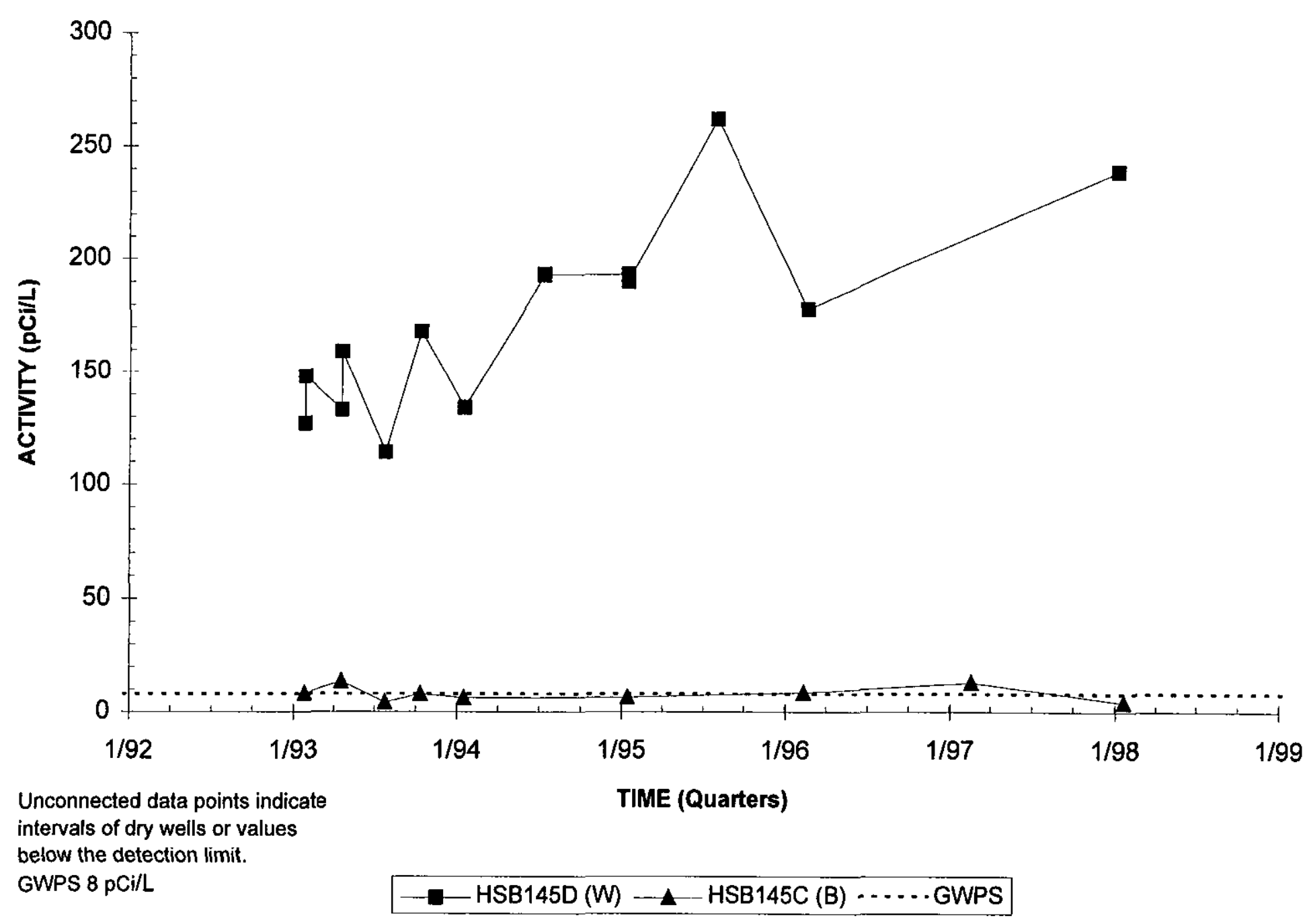

Note: W=Water Table (IIB2); B=Barnwell (IIB1); M=MCBean (IIB1); UC=Upper Congaree (IIA); MC=Middle Congaree (IIA); LC=Lower Congaree (IIA) 


\section{Gross Alpha Activities \\ Well Cluster HSB 71}

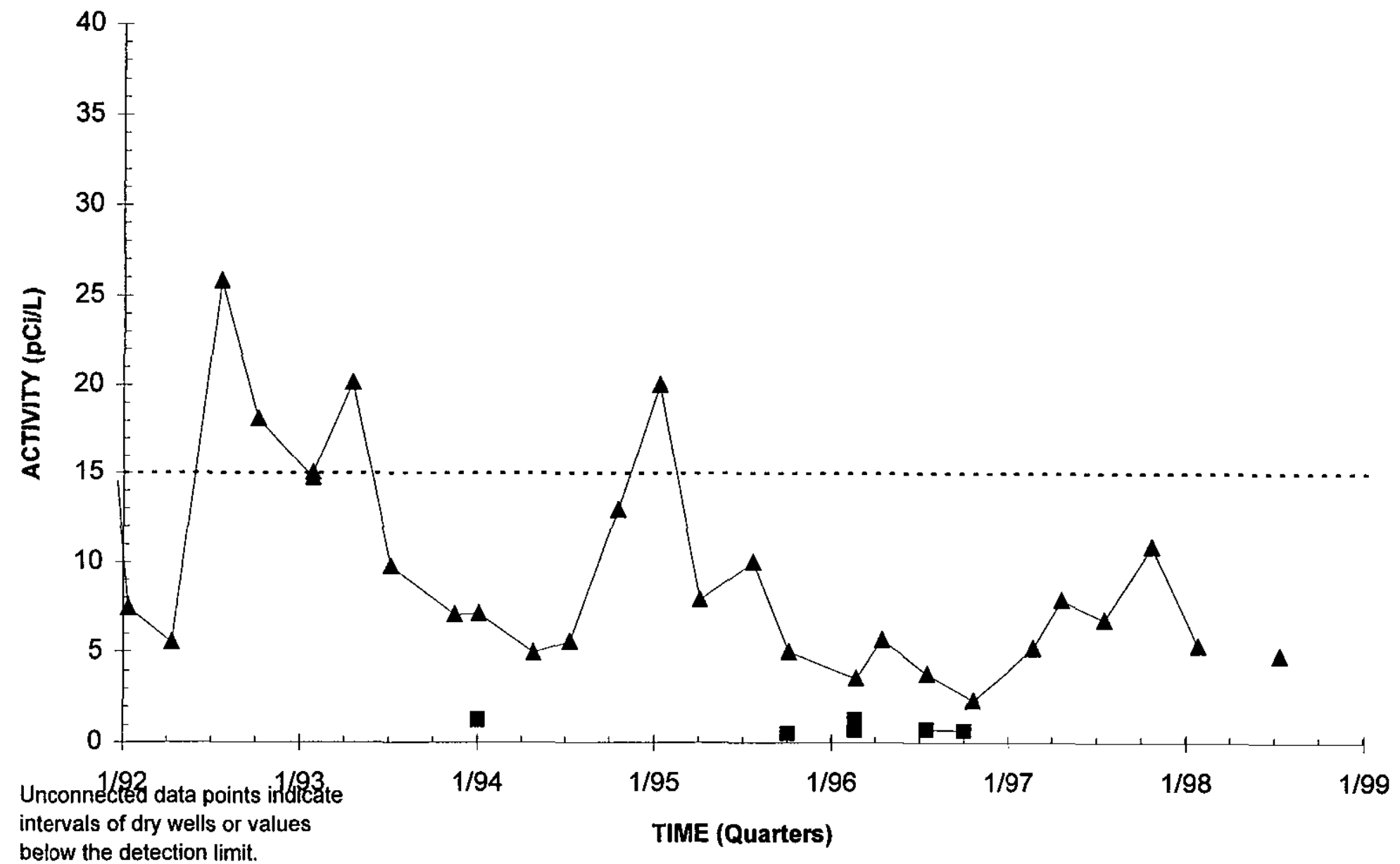

below the detection limit.

TIME (Quarters)

GWPS $15 \mathrm{pCi} / \mathrm{L}$

- HSB $71(\mathrm{~W})-\mathrm{A}$ HSB 71C (B) ...... GWPS

Note: W=Water Table (IIB2); B=Barnwell (IIB1); M=MCBean (IIB1); UC=Upper Congaree (IIA); MC=Middle Congaree (IIA); LC=Lower Congaree (IIA) 


\section{Gross Alpha Activities}

Well HSB 83D

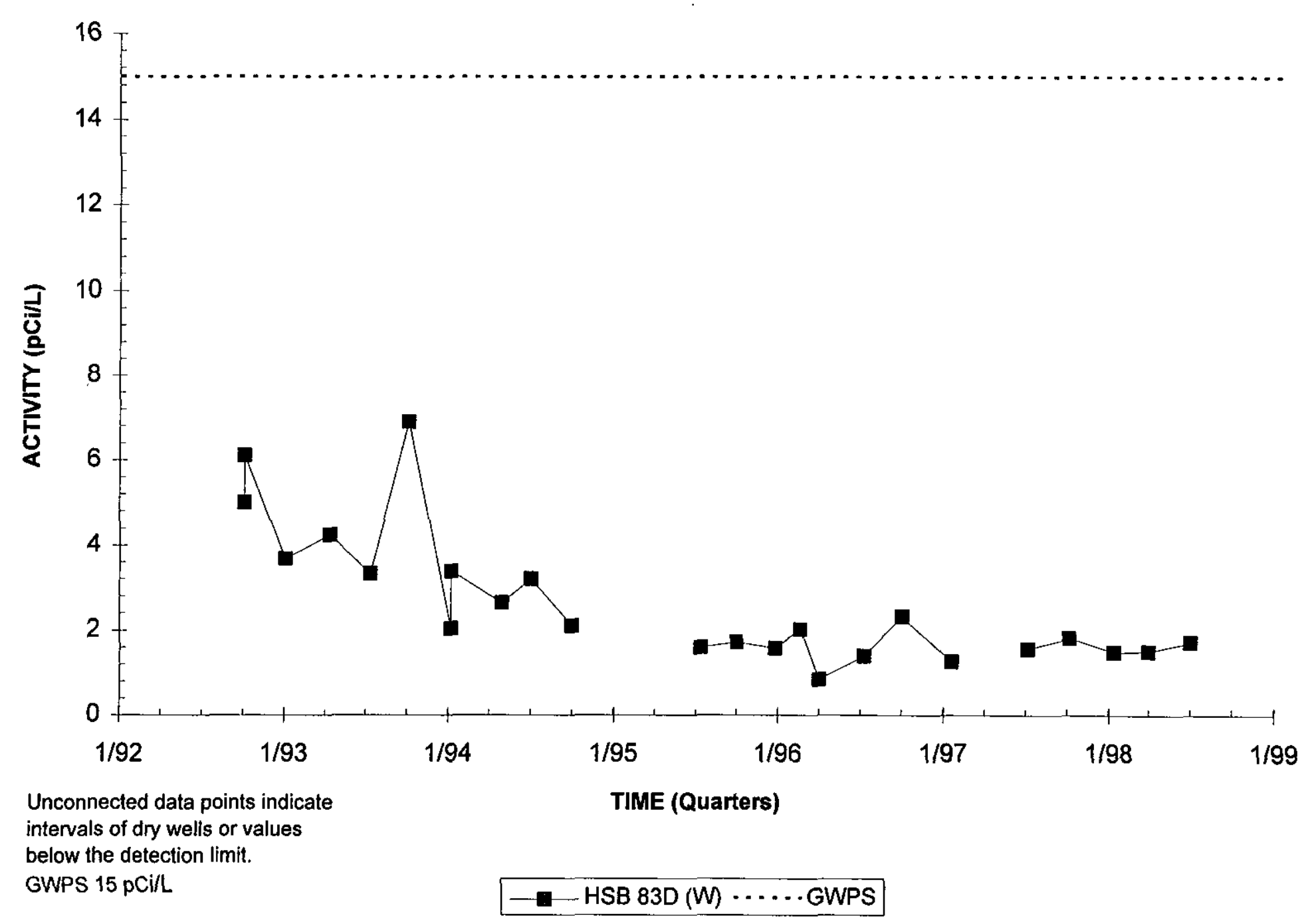

Note: W=Water Table (IIB2); B=Barnwell (IIB1); M=McBean (IIB1); UC=Upper Congaree (IIA); MC=Middle Congaree (IIA); LC=Lower Congaree (IIA) 


\section{Gross Alpha Activities \\ Well Cluster HSB 84}

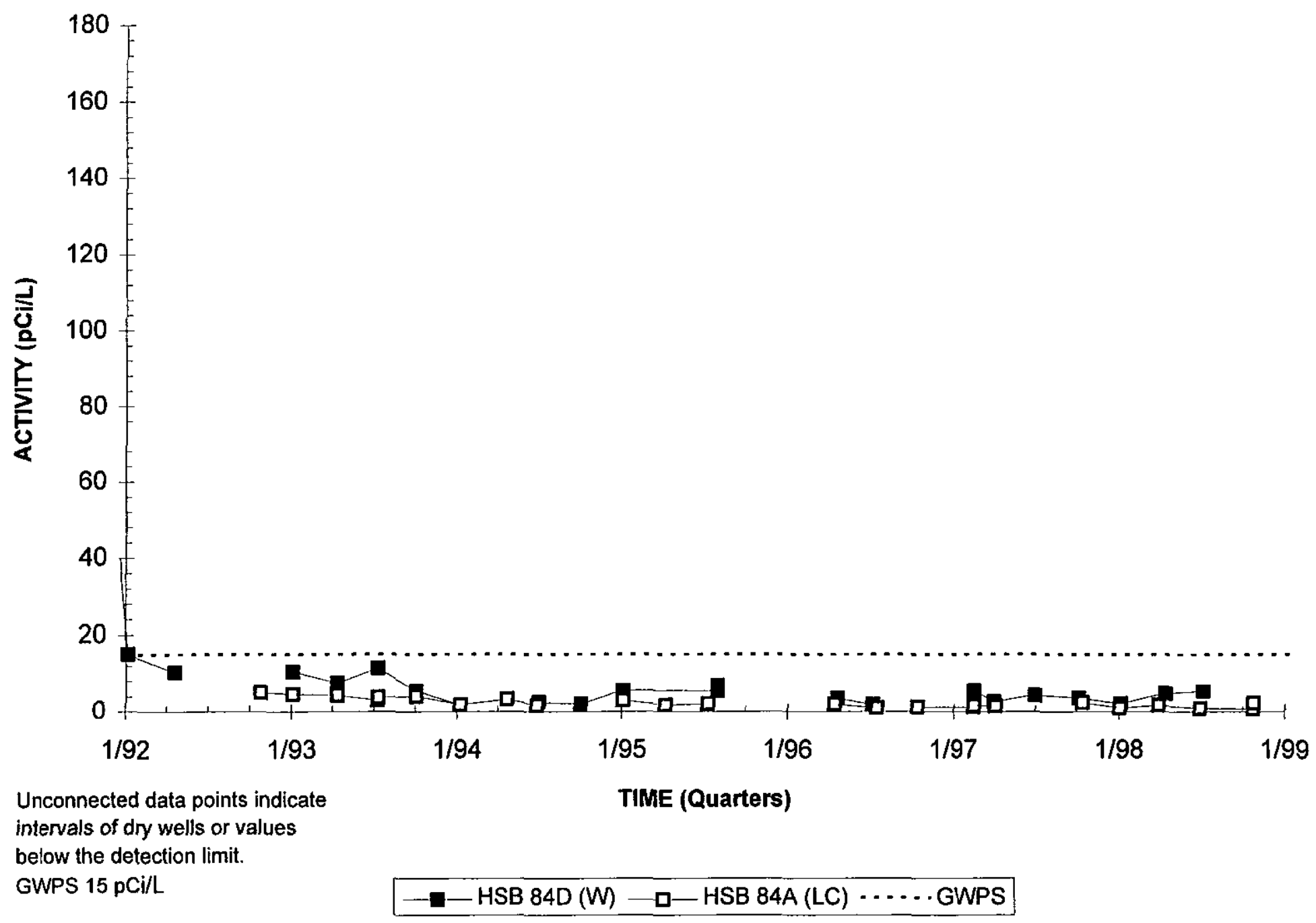

Note: W=Water Table (IIB2); B=Barnwell (IIB1); M=MCBean (IBB1); UC=Upper Congaree (IIA); MC=Middle Congaree (IIA); LC=Lower Congaree (IIA) 


\section{Gross Alpha Activities}

Well Cluster HSB 86

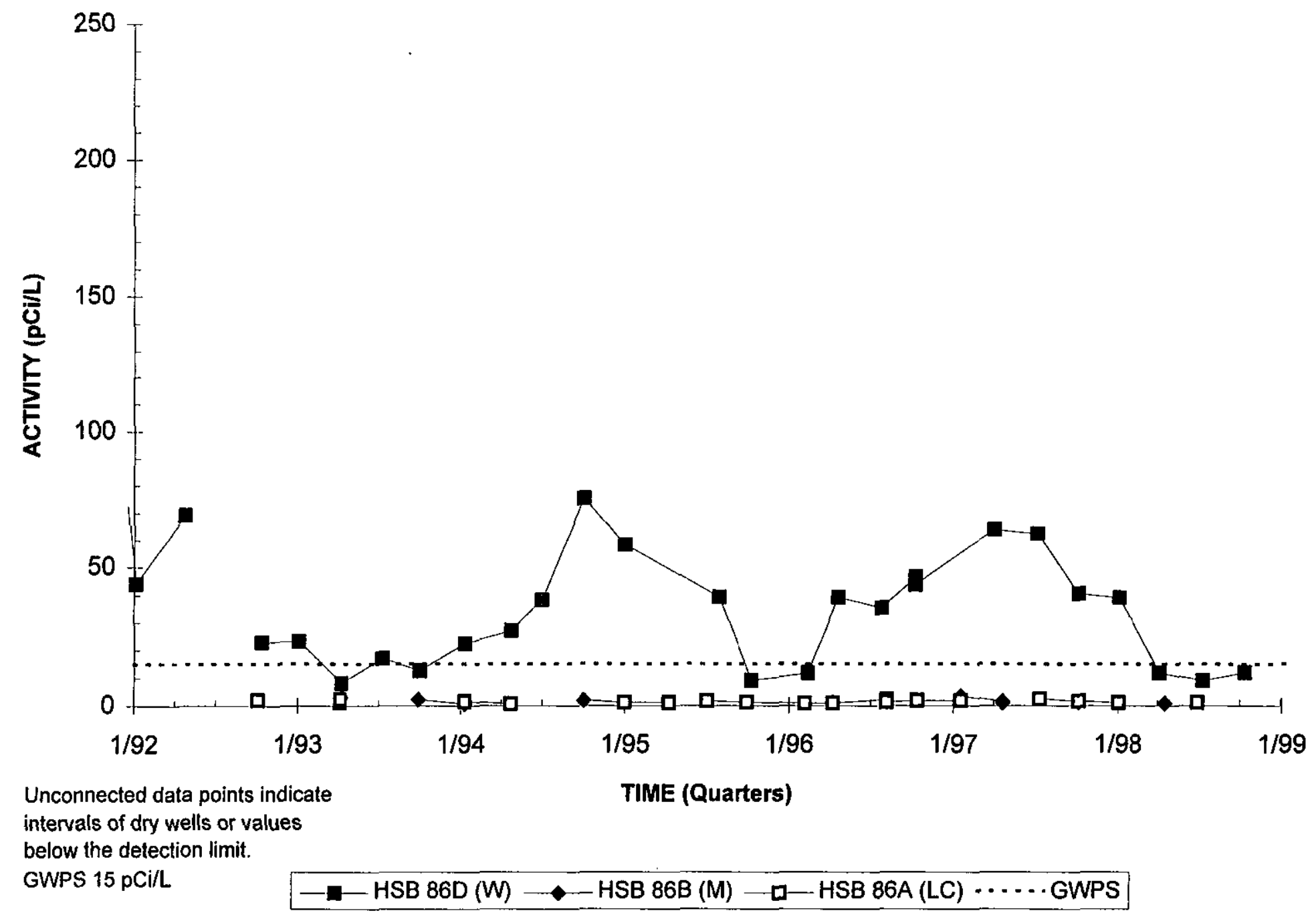

Note: W=Water Table (IIB2); B=Barnwell (IIB1); M=McBean (IIB1); UC=Upper Congaree (IIA); MC=Middle Congaree (IIA); LC=Lower Congaree (IIA) 


\section{Gross Alpha Activities \\ Well HSB101D}

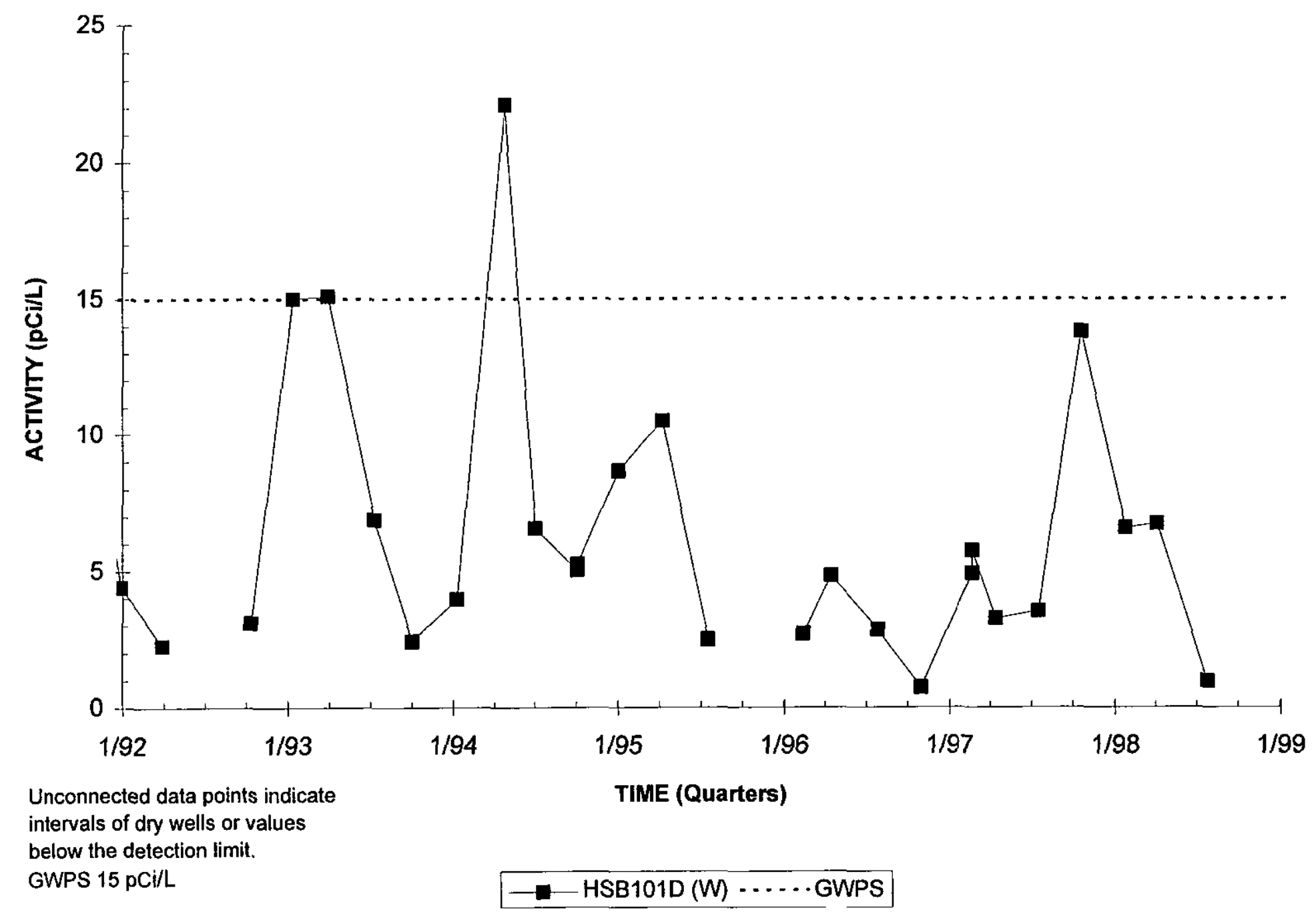

Note: W=Water Table (IIB2); B=Barnwell (IIB1); M=McBean (IIB1); UC=Upper Congaree (IIA); MC=Middle Congaree (IIA); LC=Lower Congaree (IIA) 


\section{Gross Alpha Activities \\ Well HSB104D}

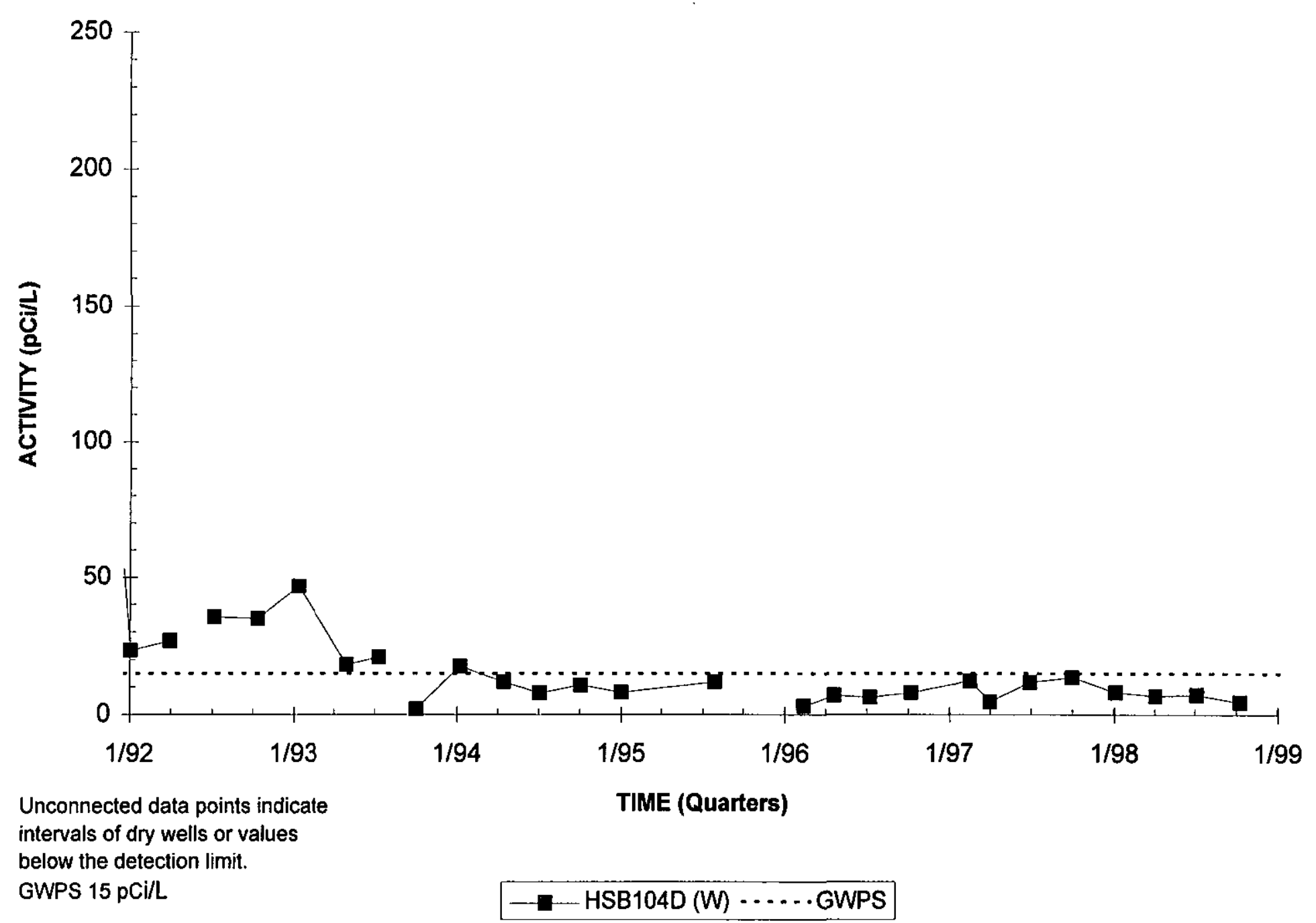

Note: W=Water Table (IIB2); B=Barnwell (IIB1); M=McBean (IIB1); UC=Upper Congaree (IIA); MC=Middle Congaree (IIA); LC=Lower Congaree (IIA) 


\section{Gross Alpha Activities \\ Well HSB106D}

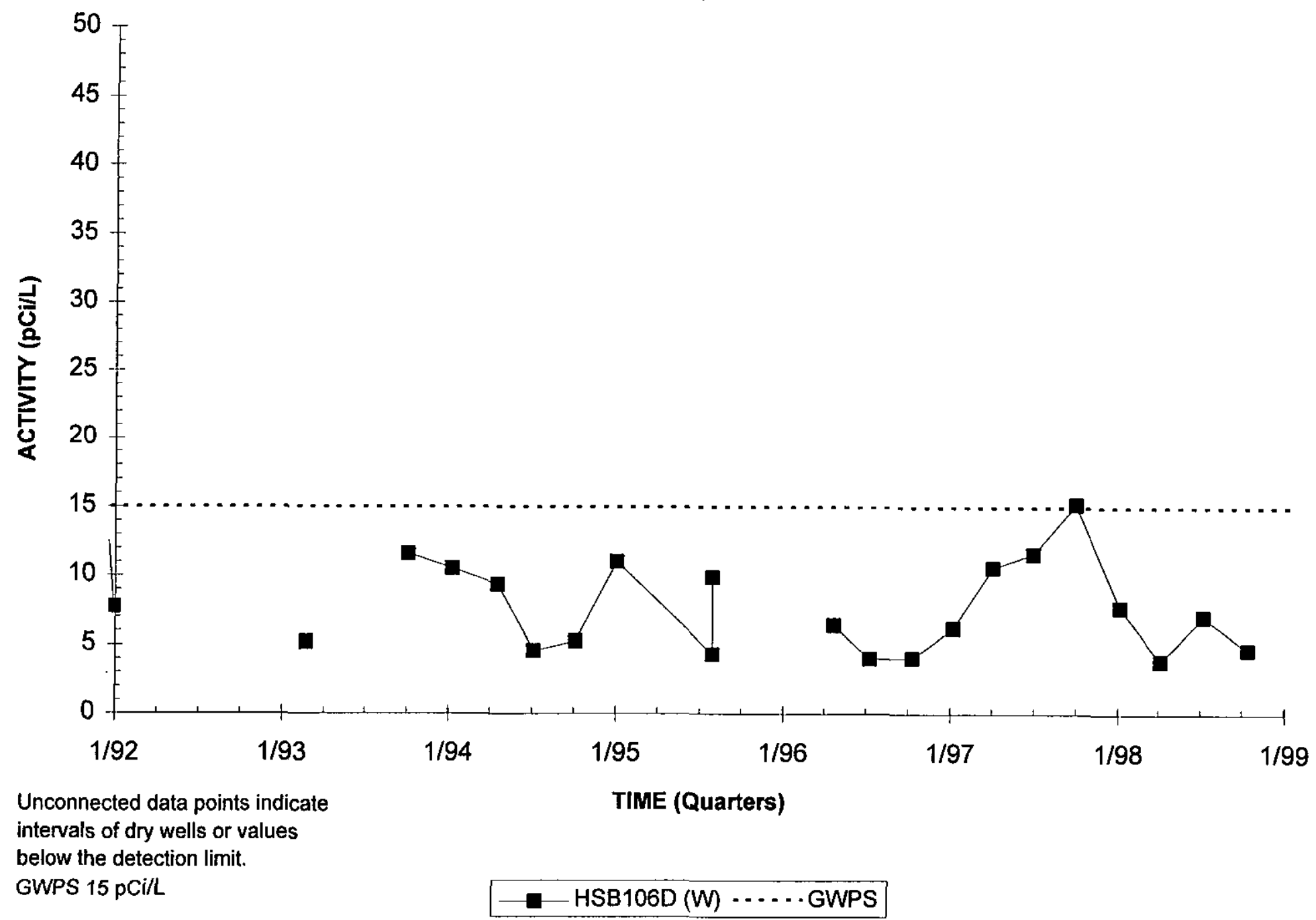

Note: W=Water Table (IIB2); B=Barnwell (IIB1); M=MCBean (IIB1); UC=Upper Congaree (IIA); MC=Middle Congaree (IIA); LC=Lower Congaree (IIA) 


\section{Gross Alpha Activities \\ Well HSB111D}

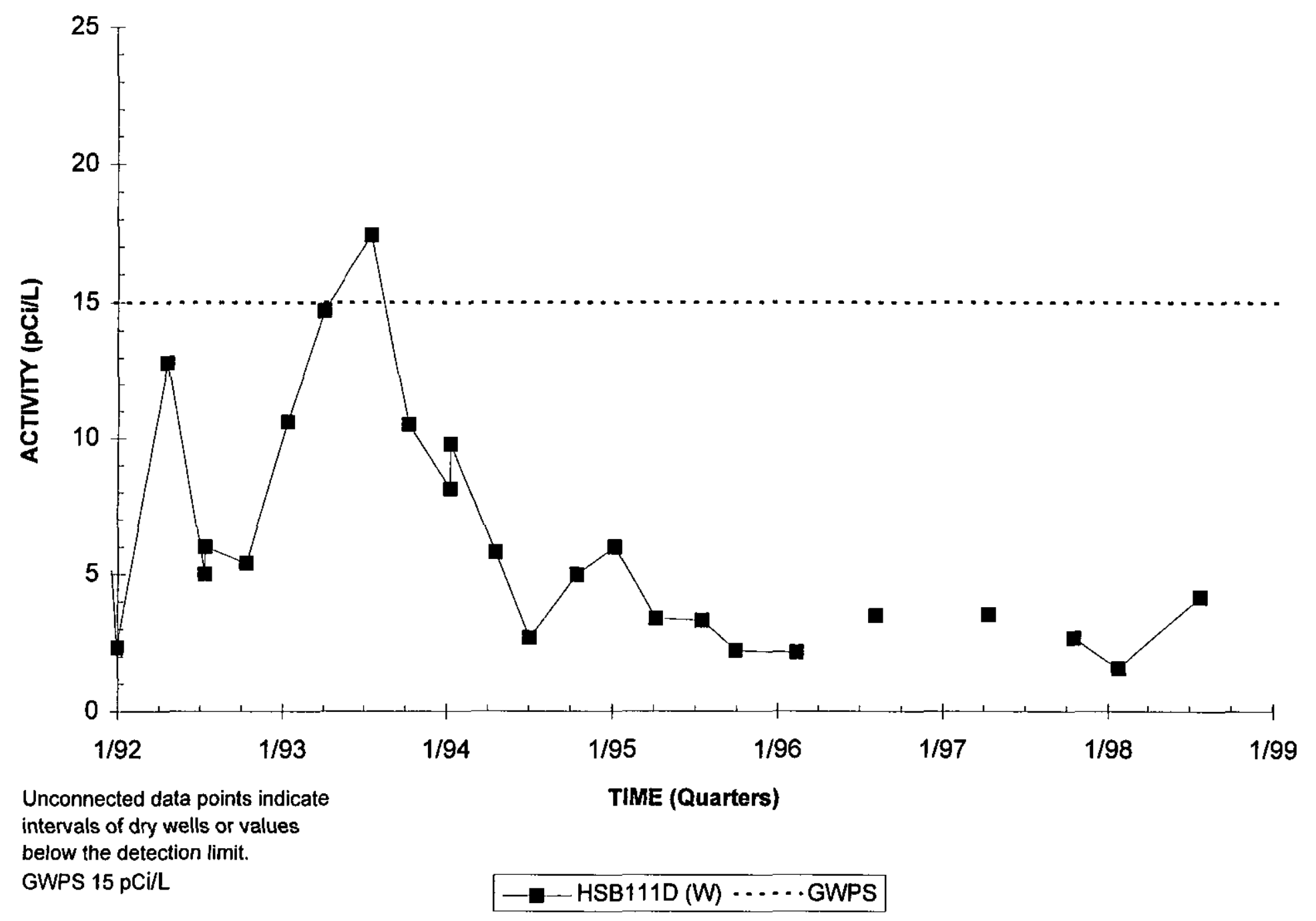

Note: $W=$ Water Table (IIB2); B=Barnwell (IIB1); $M=M c B e a n$ (IIB1); UC=Upper Congaree (IIA); MC=Middle Congaree (IIA); LC=Lower Congaree (IIA) 


\section{Gross Alpha Activities}

Well HSB112D

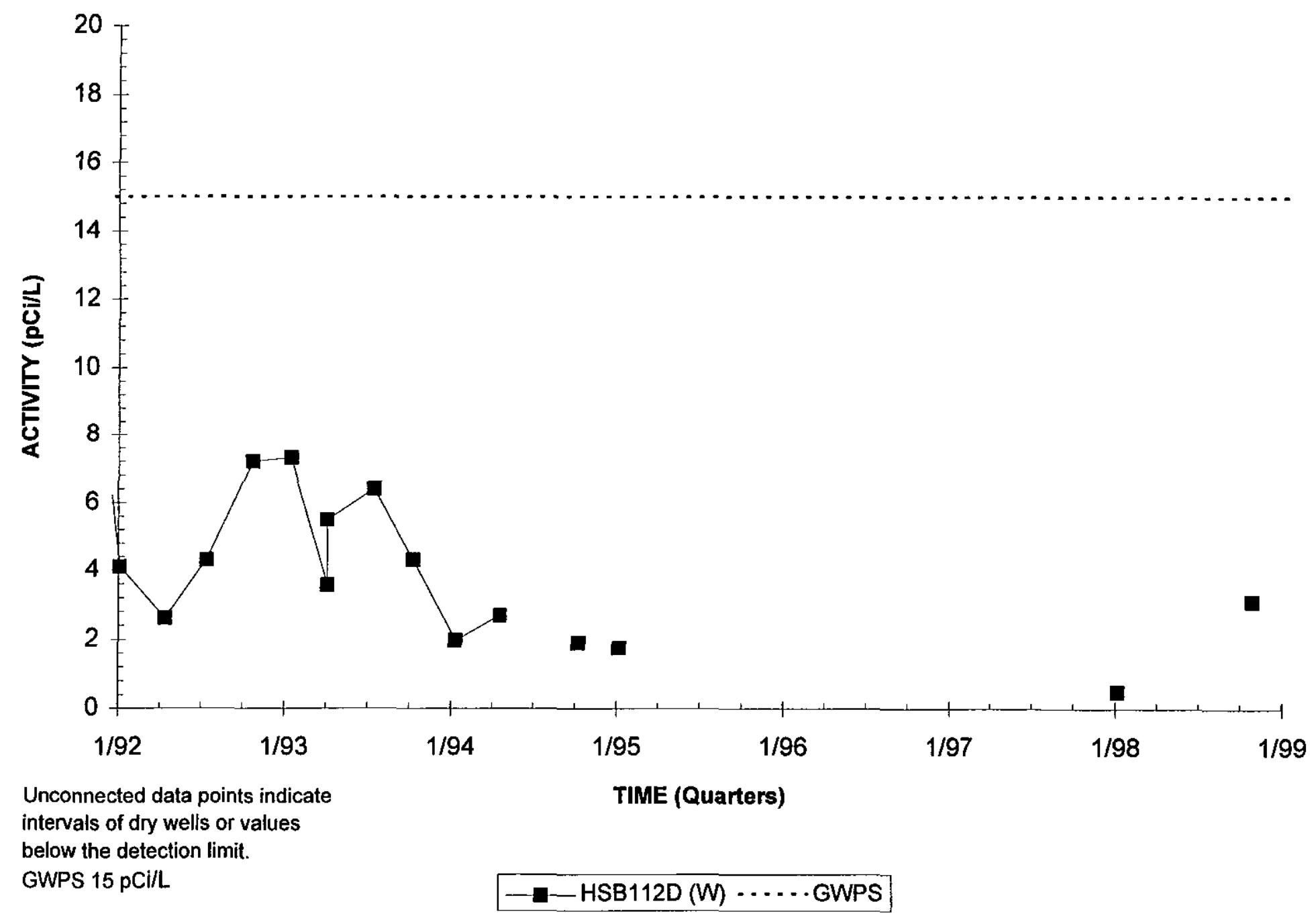

Note: W=Water Table (IIB2); B=Barnwell (IIB1); M=McBean (IIB1); UC=Upper Congaree (IIA); MC=Middle Congaree (IIA); LC=Lower Congaree (IIA) 


\section{Gross Alpha Activities}

\section{Well Cluster HSB114}

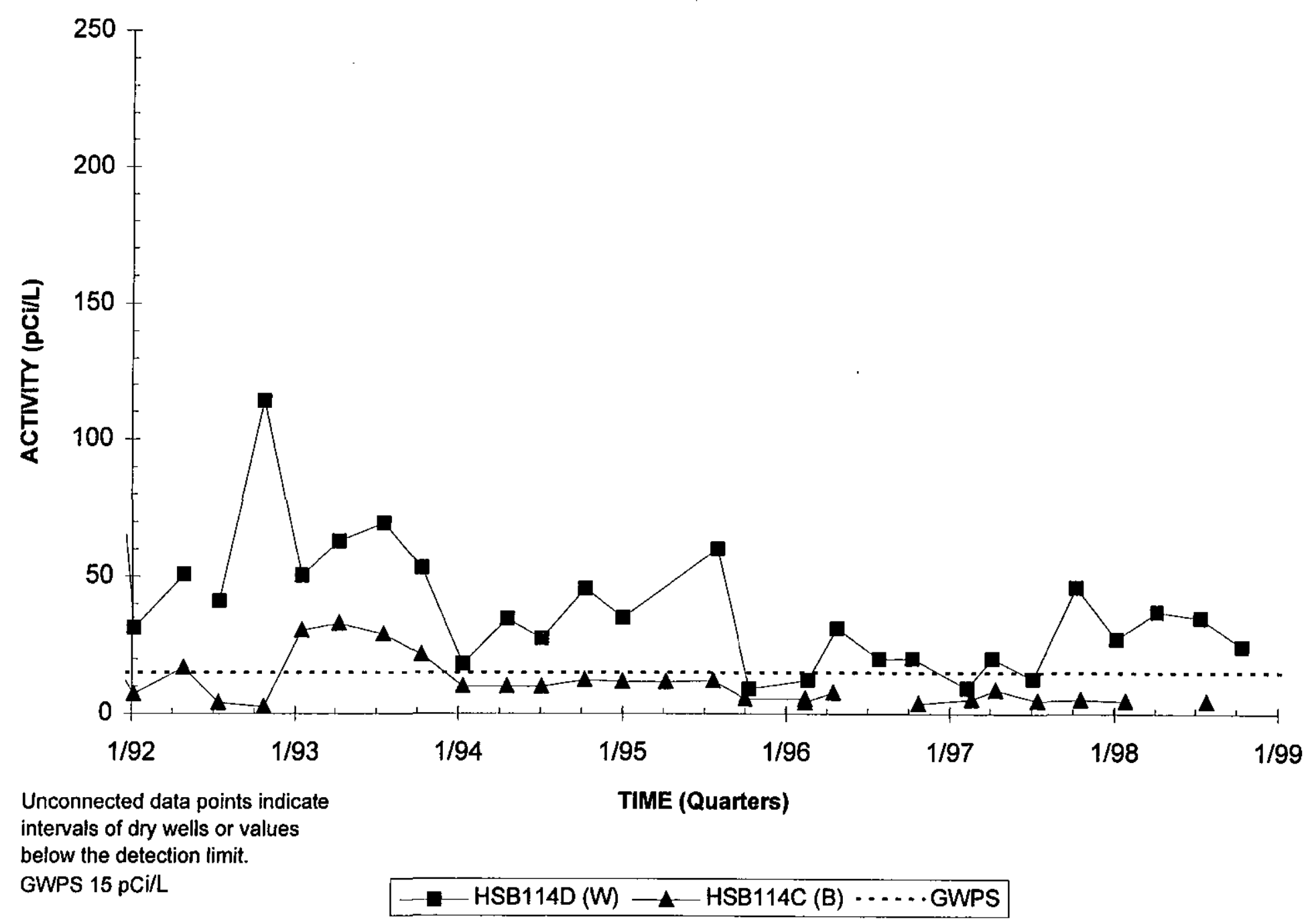

Note: $W=$ Water Table (IIB2); B=Barnwell (IIB1); M=McBean (IIB1); UC=Upper Congaree (IIA); MC=Middle Congaree (IIA); LC=Lower Congaree (IIA) 


\section{Gross Alpha Activities \\ Well Cluster HSB116}

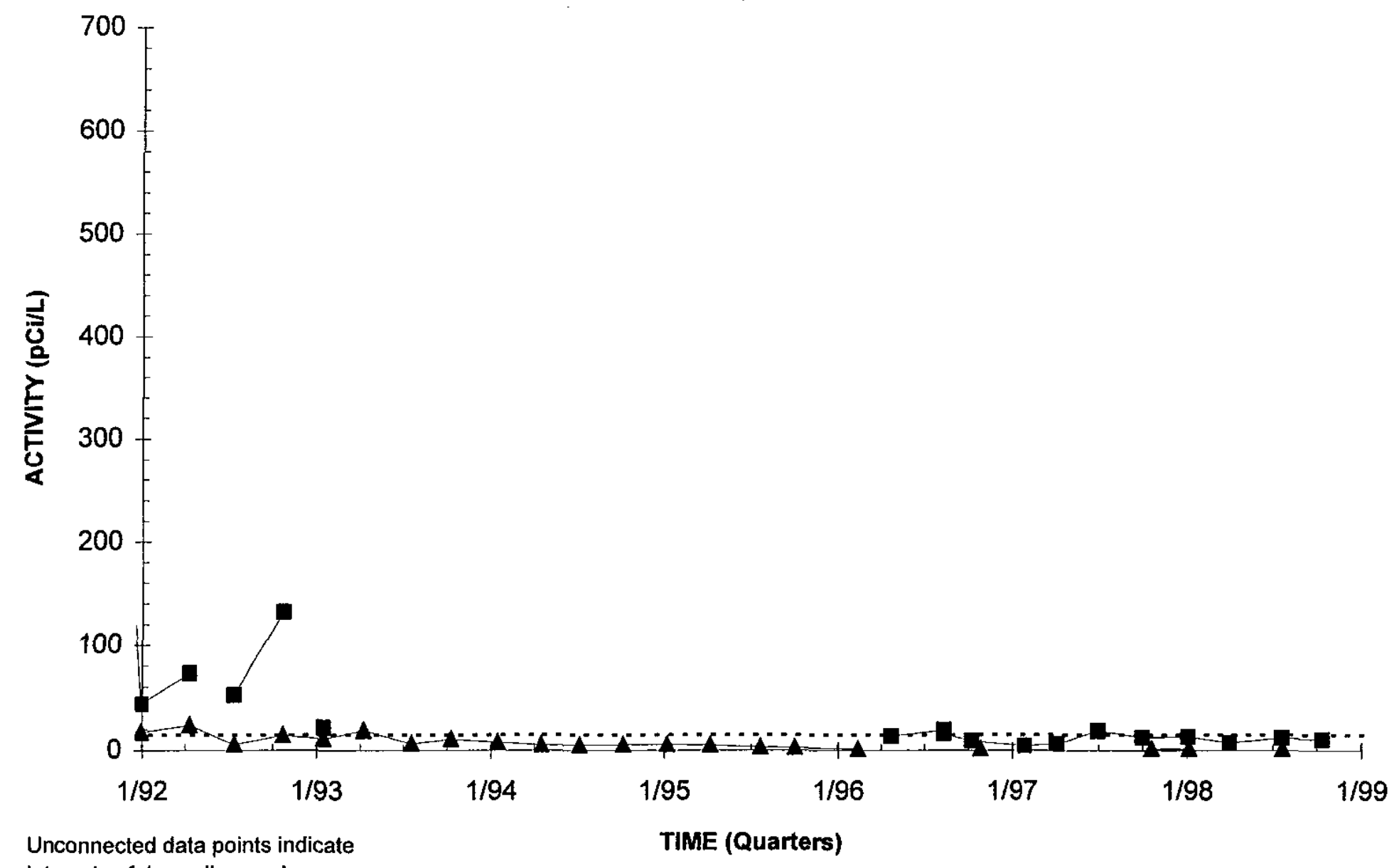

intervals of dry wells or values

below the detection limit.

GWPS $15 \mathrm{pCi} / \mathrm{L}$

m-HSB116D (W) - - HSB116C (B) $\ldots .$. GWPS

Note: W=Water Table (IIB2); B=Barnwell (IIB1); M=McBean (IIB1); UC=Upper Congaree (IIA); MC=Middle Congaree (IIA); LC=Lower Congaree (IIA) 


\section{Gross Alpha Activities}

Well Cluster HSB117

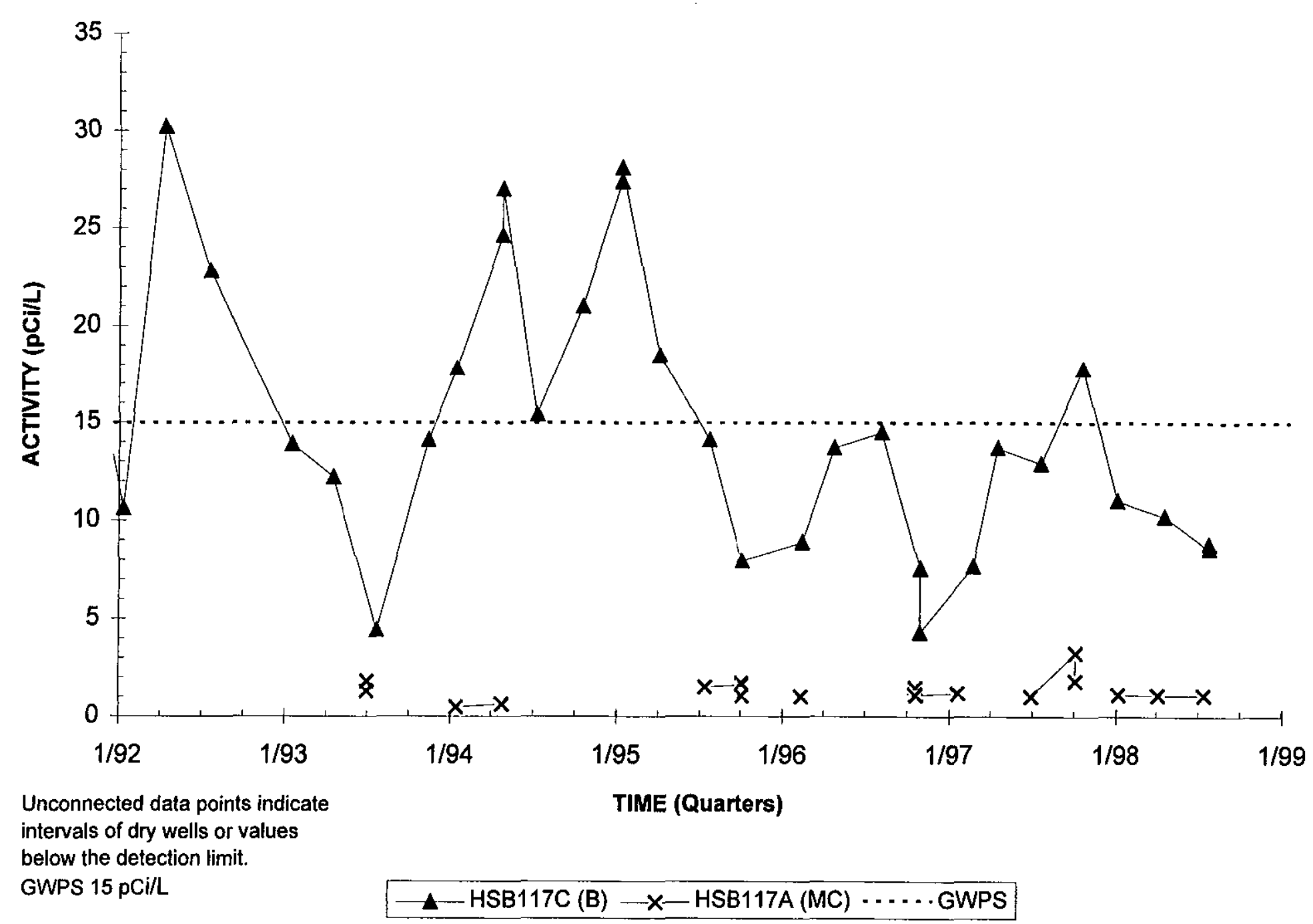

Note: W=Water Table (IIB2); B=Barnwell (IIB1); M=McBean (IIB1); UC=Upper Congaree (IIA); MC=Middle Congaree (IIA); LC=Lower Congaree (IIA) 


\section{Gross Alpha Activities \\ Well HSB125D}

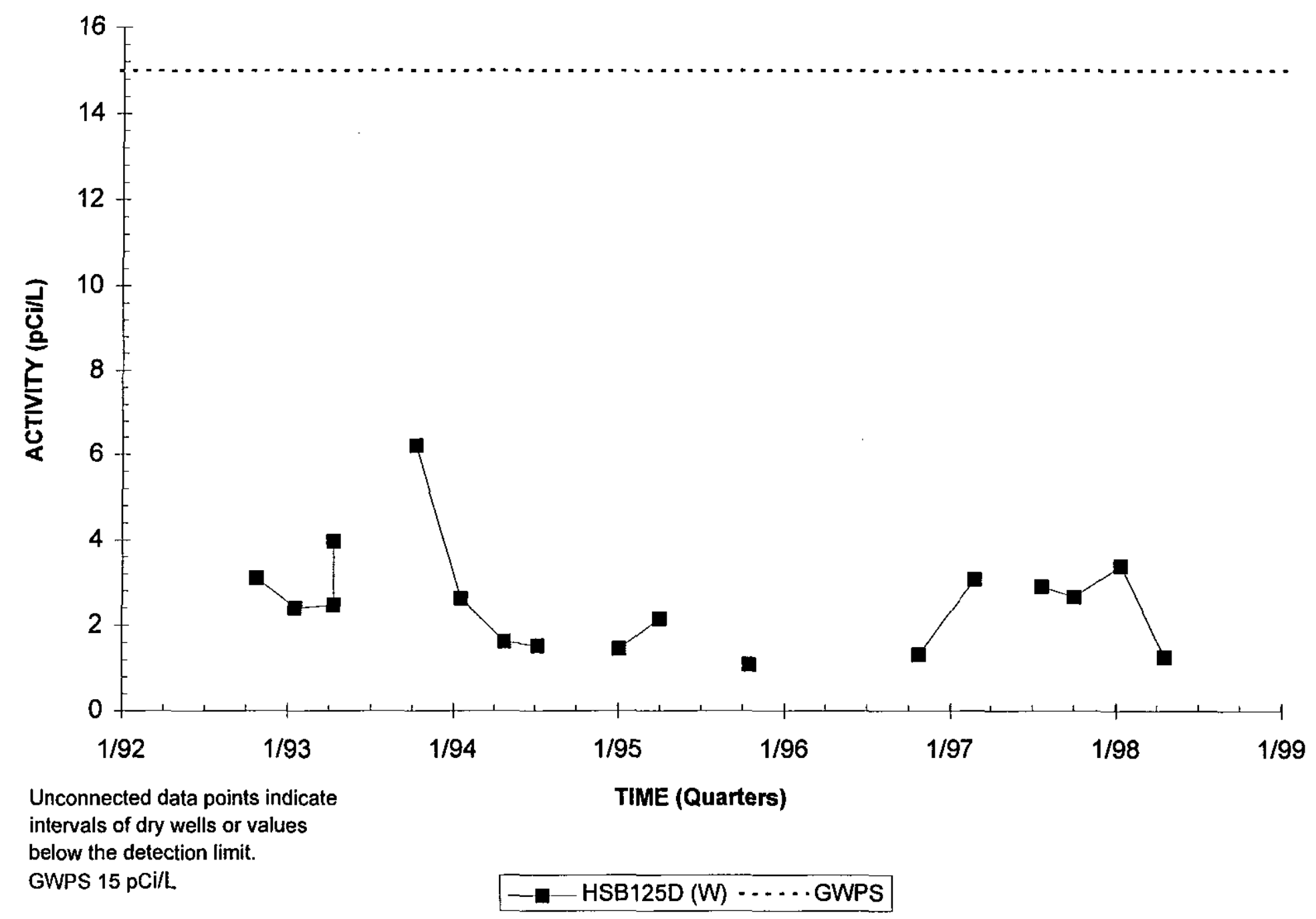

Note: $W=$ Water Table (IIB2); B=Barnwell (IIB1); M=McBean (IIB1); UC=Upper Congaree (IIA); MC=Middle Congaree (IIA); LC=Lower Congaree (IIA) 


\section{Gross Alpha Activities}

Well Cluster HSB136

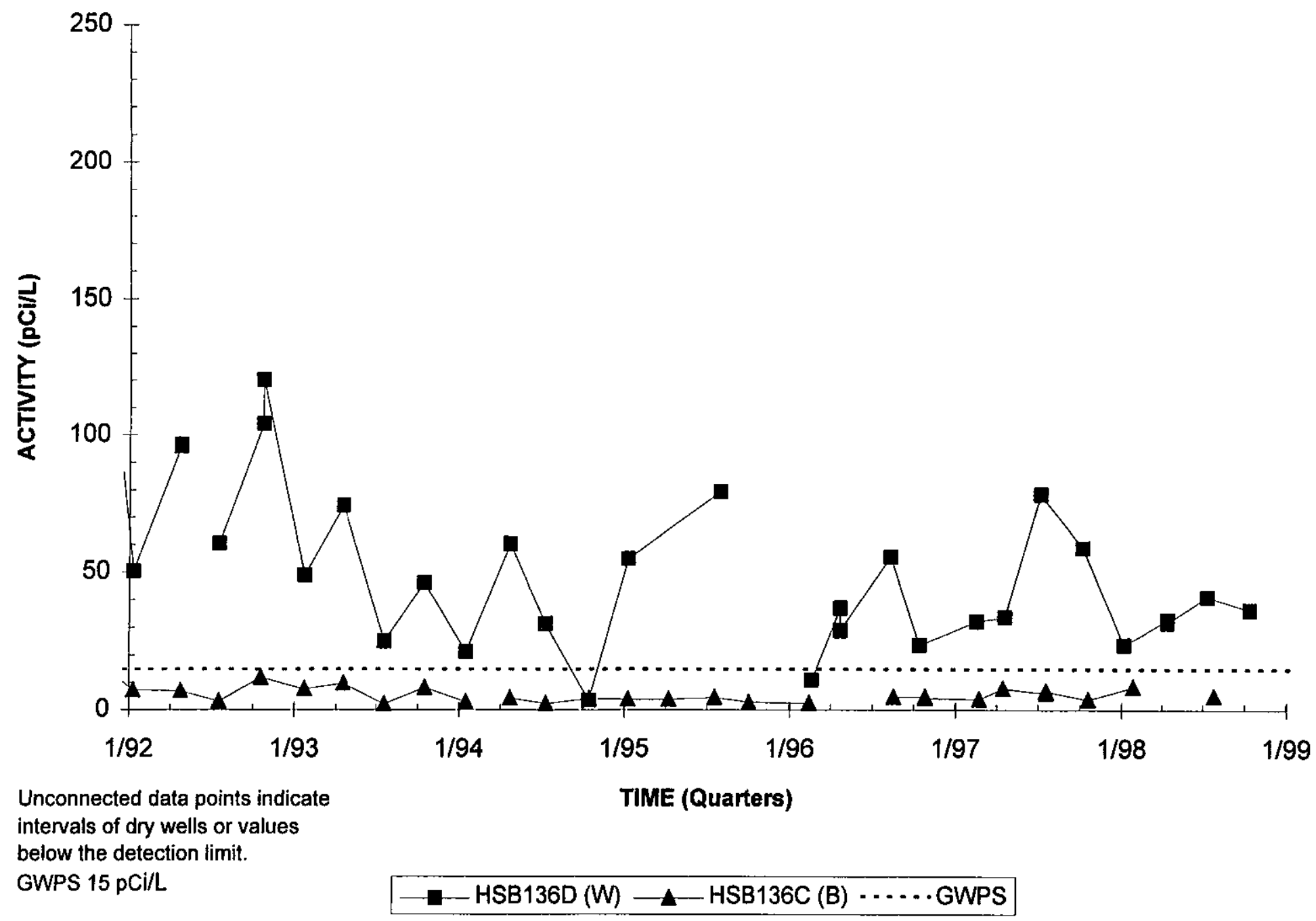

Note: $W=$ Water Table (IIB2); B=Barnwell (IIB1); M=McBean (IIB1); UC=Upper Congaree (IIA); MC=Middle Congaree (IIA); LC=Lower Congaree (IIA) 


\section{Nonvolatile Beta Activities \\ Well HSB 69}

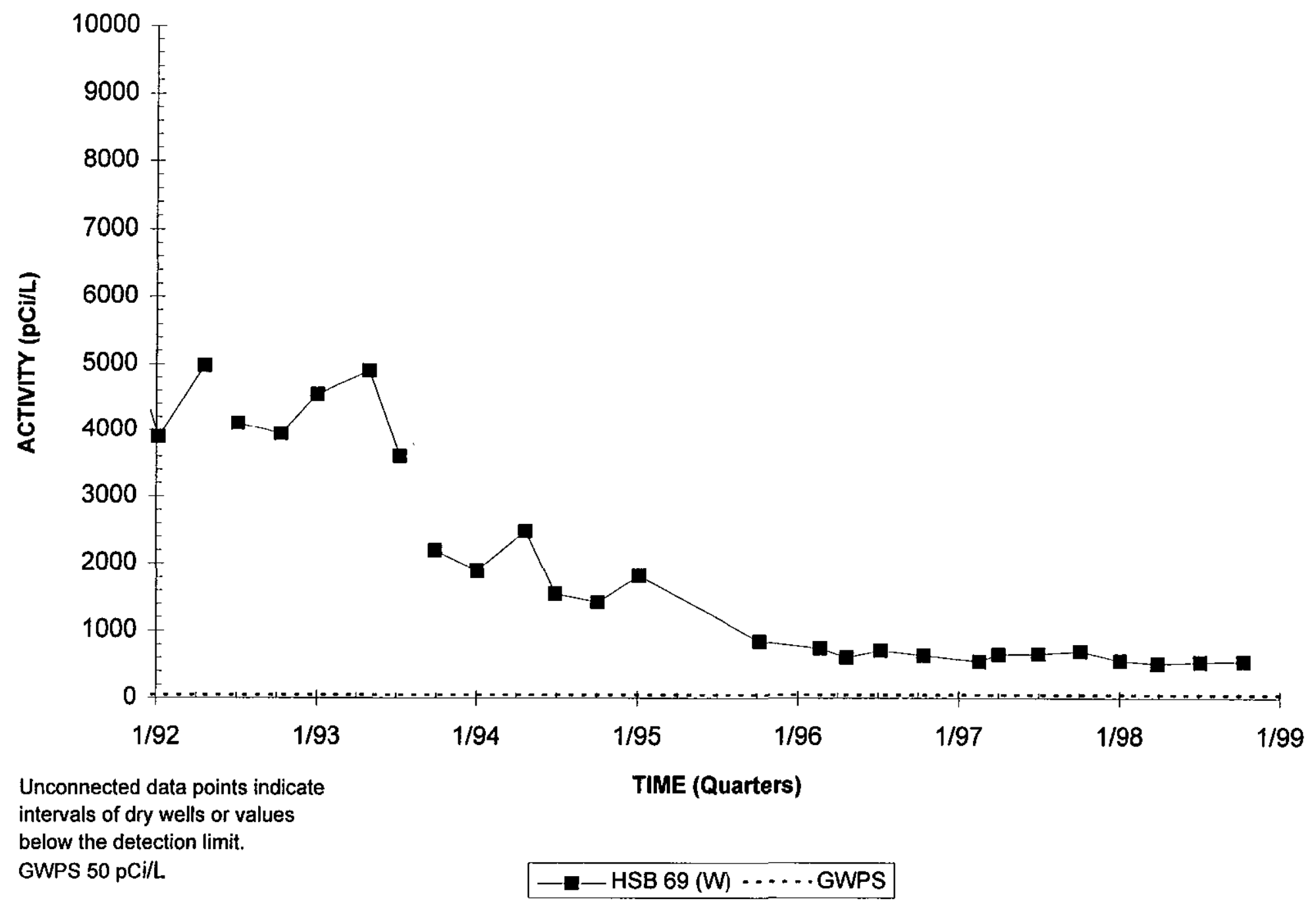

Note: W=Water Table (IIB2); B=Barnwell (IIB1); M=McBean (IIB1); UC=Upper Congaree (IIA); MC=Middle Congaree (IIA); LC=Lower Congaree (IIA)

H-Area HWMF

D -82

Third and Fourth Quarter 1998

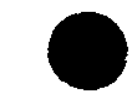




\section{Nonvolatile Beta Activities \\ Well Cluster HSB 84}

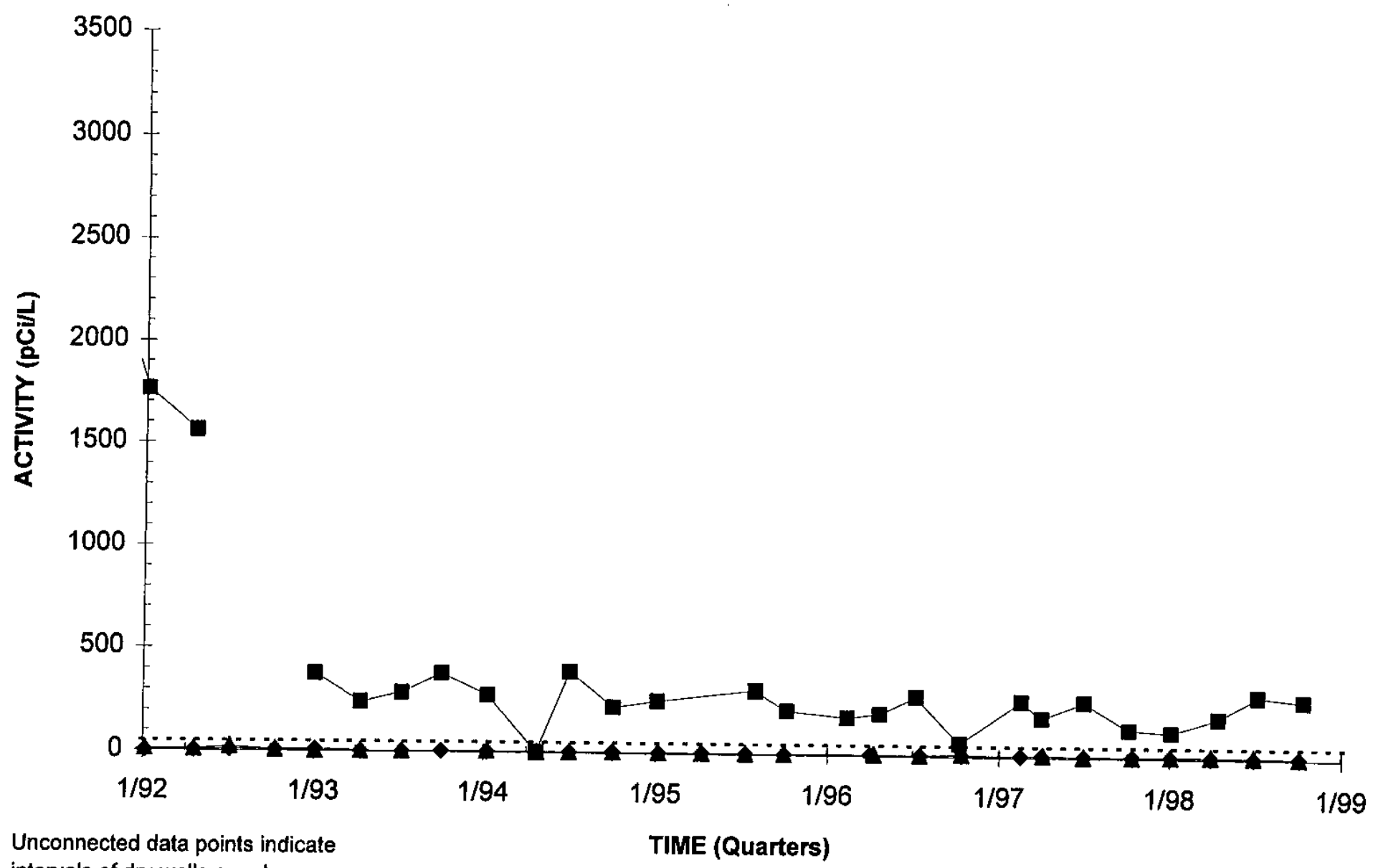

intervals of dry wells or values

below the detection limit.

GWPS $50 \mathrm{pCi} / \mathrm{L}$

$$
\square-\mathrm{HSB} 84 \mathrm{D}(\mathrm{W}) \multimap \mathrm{HSB} 84 \mathrm{C}(\mathrm{B}) \multimap \mathrm{HSB} 84 \mathrm{~B}(\mathrm{M}) \cdots \cdots \text { GWPS }
$$

Note: W=Water Table (IIB2); B=Barnweil (IIB1); $M=M c B e a n$ (IIB1); UC=Upper Congaree (IIA); MC=Middle Congaree (IIA); LC=Lower Congaree (IIA) 


\section{Nonvolatile Beta Activities \\ Well Cluster HSB 86}

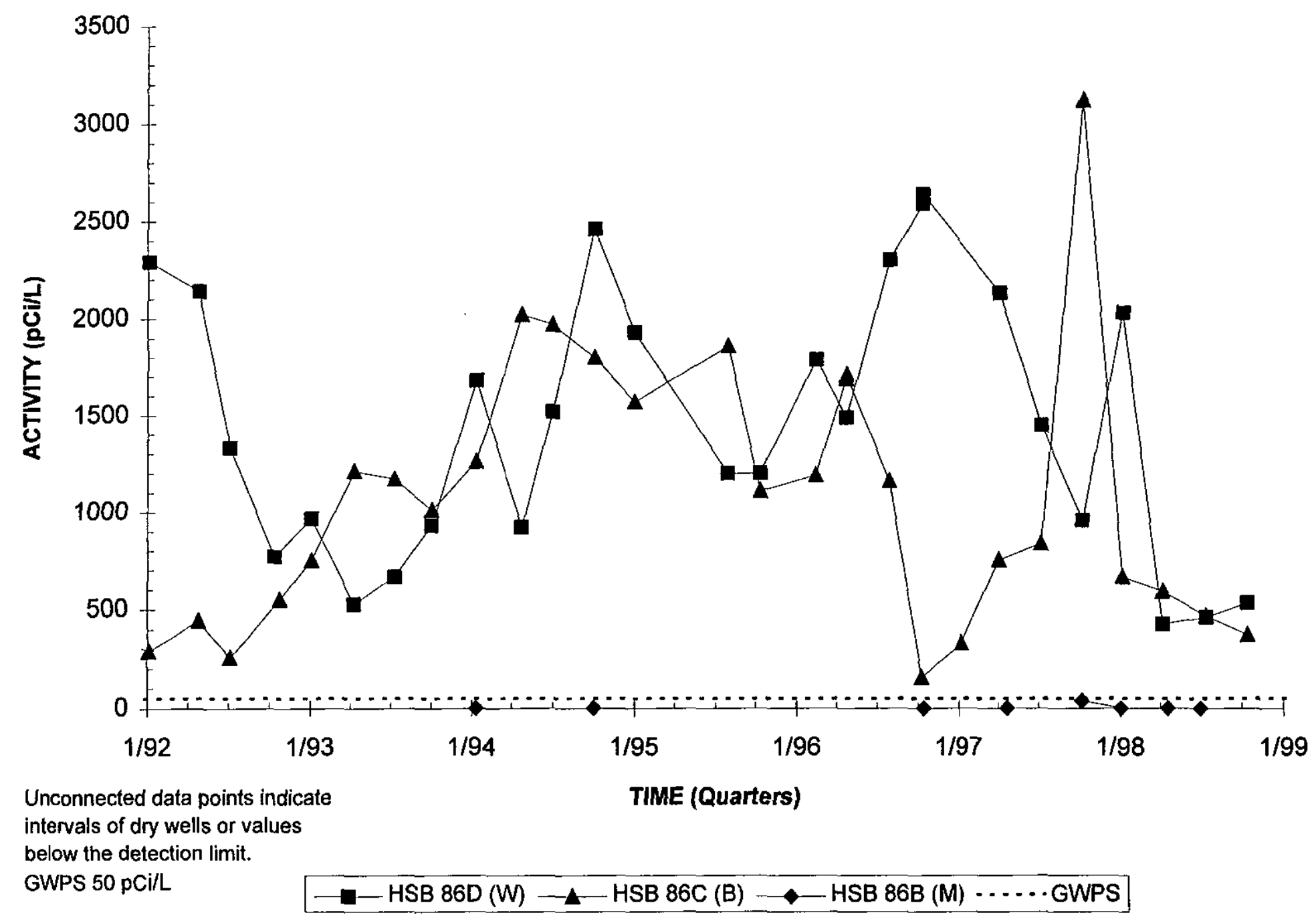

Note: $W=$ Water Table (IIB2); B=Barnwell (IIB1); $M=M c B e a n$ (IIB1); UC=Upper Congaree (IIA); MC=Middle Congaree (IIA); LC=Lower Congaree (IIA) 


\section{Nonvolatile Beta Activities \\ Well Cluster HSB100}

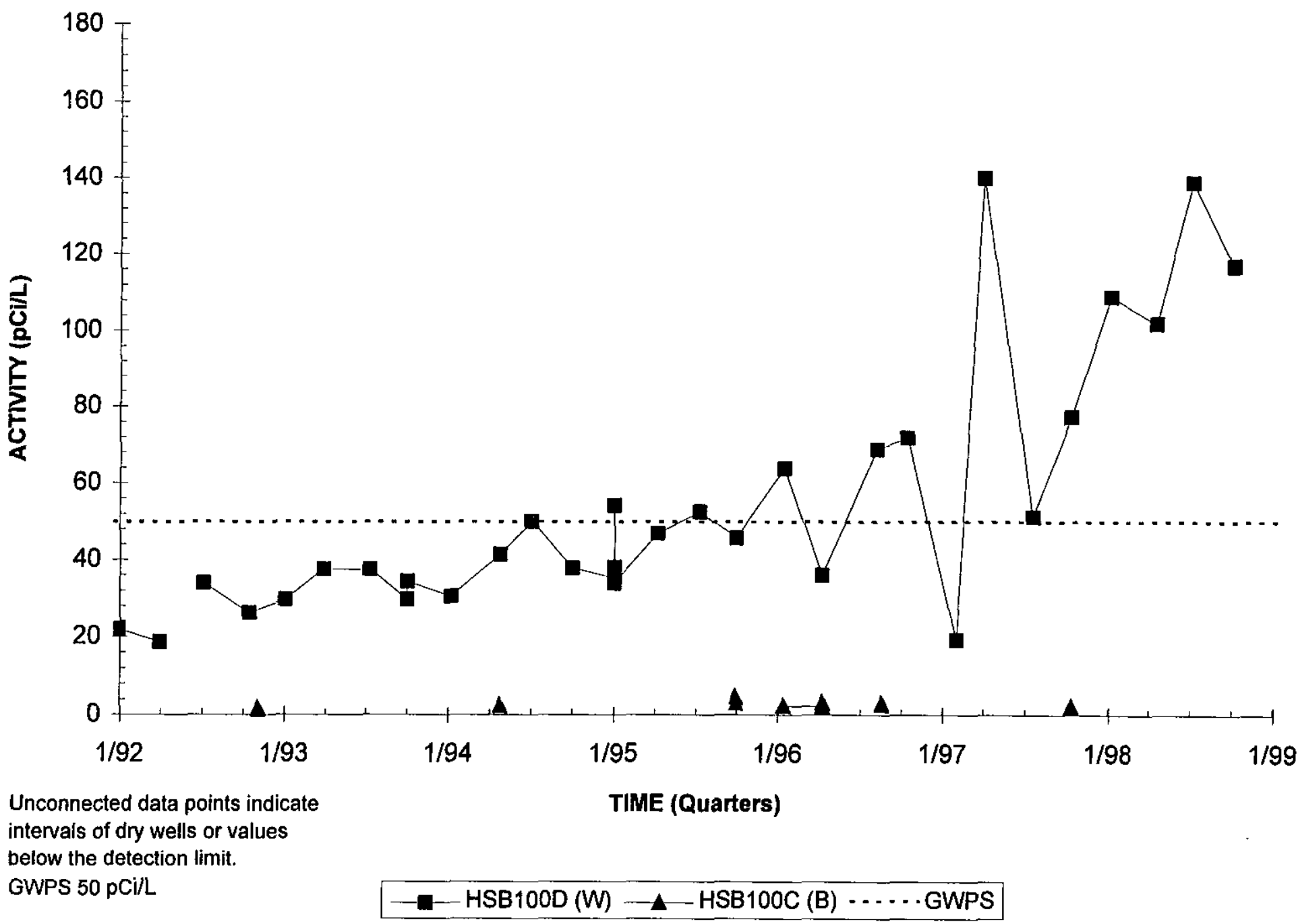

Note: W=Water Table (IIB2); B=Barnwell (IIB1); $M=$ MCBean (IIB1); UC=Upper Congaree (IIA); MC=Middle Congaree (IIA); LC=Lower Congaree (IIA) 


\section{Nonvolatile Beta Activities \\ Well Cluster HSB104}

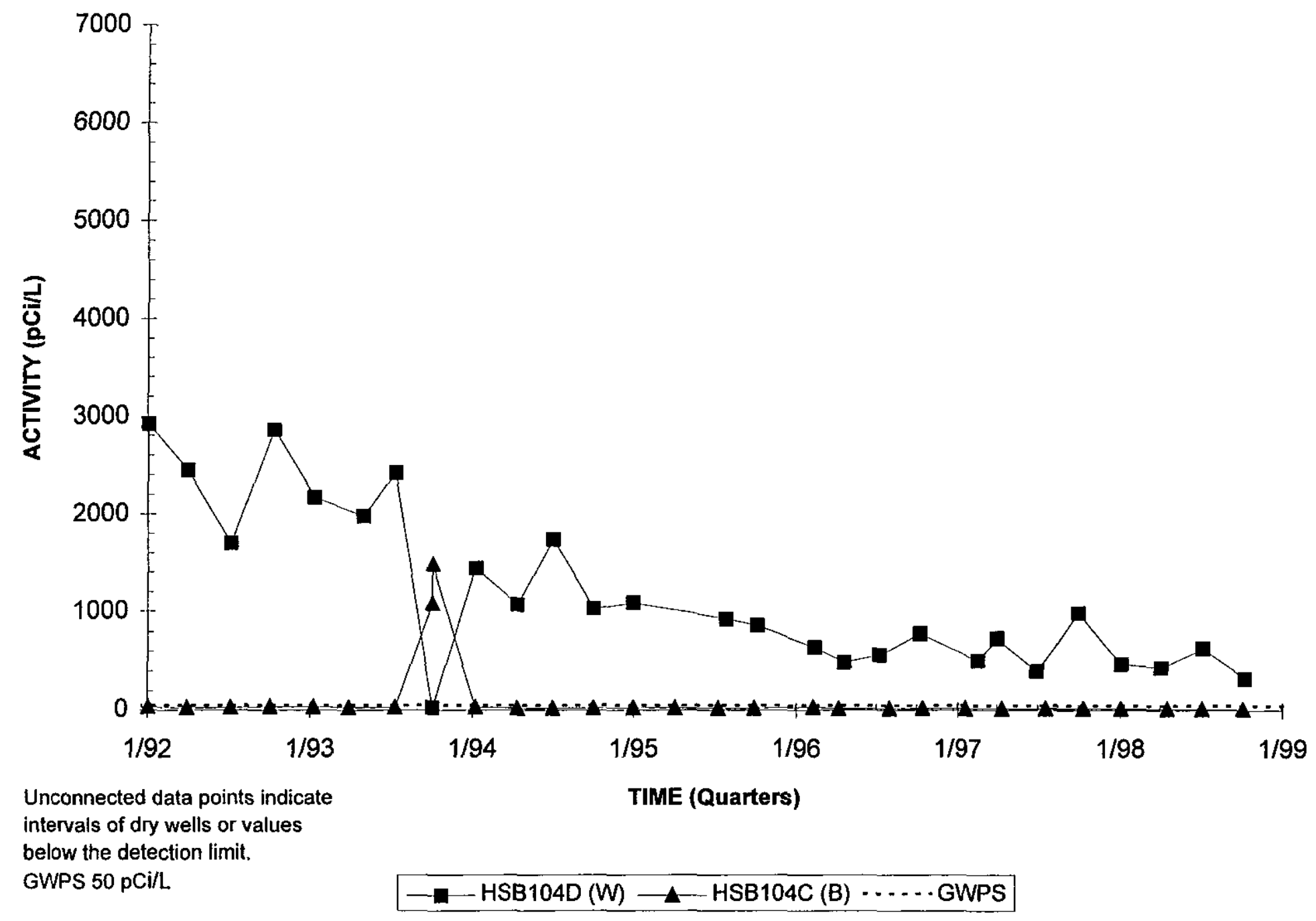

Note: $W=$ Water Table (IIB2); B=Barnwell (IIB1); M=McBean (IIB1); UC=Upper Congaree (IIA); MC=Middle Congaree (IIA); LC=Lower Congaree (IIA) 


\section{Nonvolatile Beta Activities \\ Well Cluster HSB105}

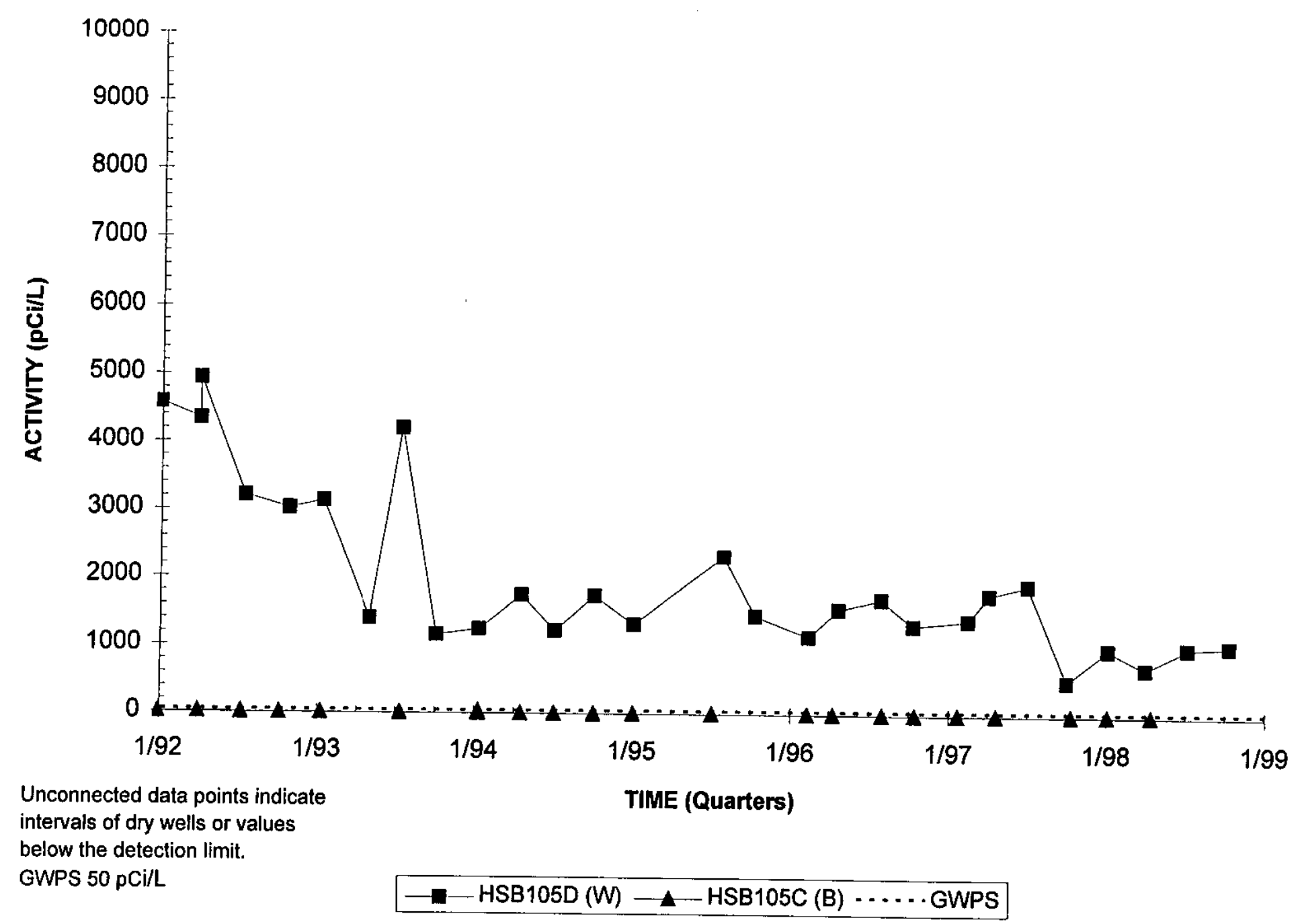

Note: $W=$ Water Table (IIB2); B=Barnwell (IIB1); $M=M C B e a n$ (IIB1); UC=Upper Congaree (IIA); MC=Middle Congaree (IIA); LC=Lower Congaree (IIA) 


\section{Nonvolatile Beta Activities \\ Well Cluster HSB106}

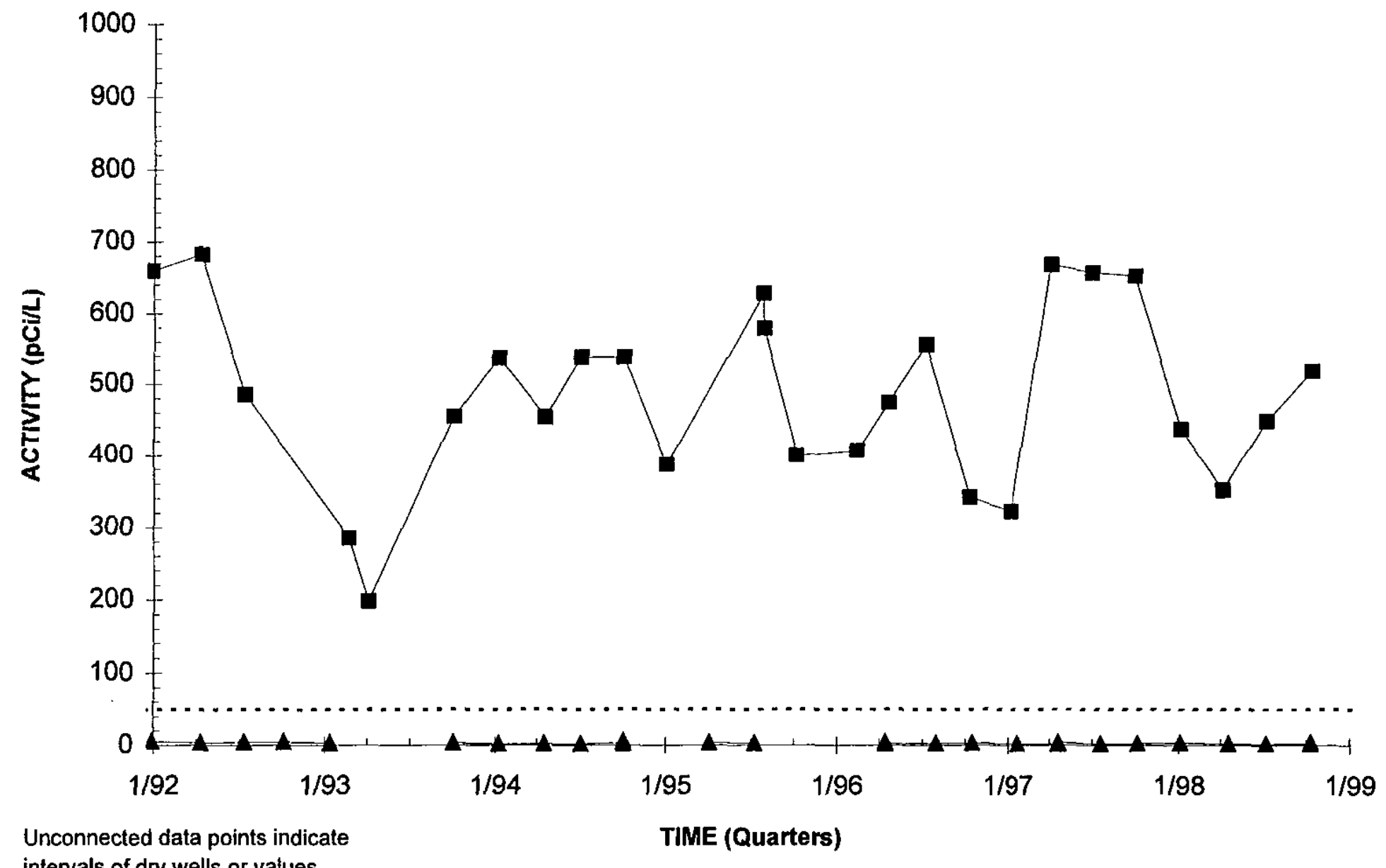

intervals of dry wells or values

below the detection limit.

GWPS $50 \mathrm{pCi} / \mathrm{L}$

$$
\text { -HSB106D (W) - HSB106C (B) -......GWPS }
$$

Note: $W=$ Water Table (IIB2); B=Barnwell (IIB1); $M=M c B e a n$ (IIB1); UC=Upper Congaree (IIA); MC=Middle Congaree (IIA); LC=Lower Congaree (IIA) 


\section{Nonvolatile Beta Activities \\ Well Cluster HSB113}

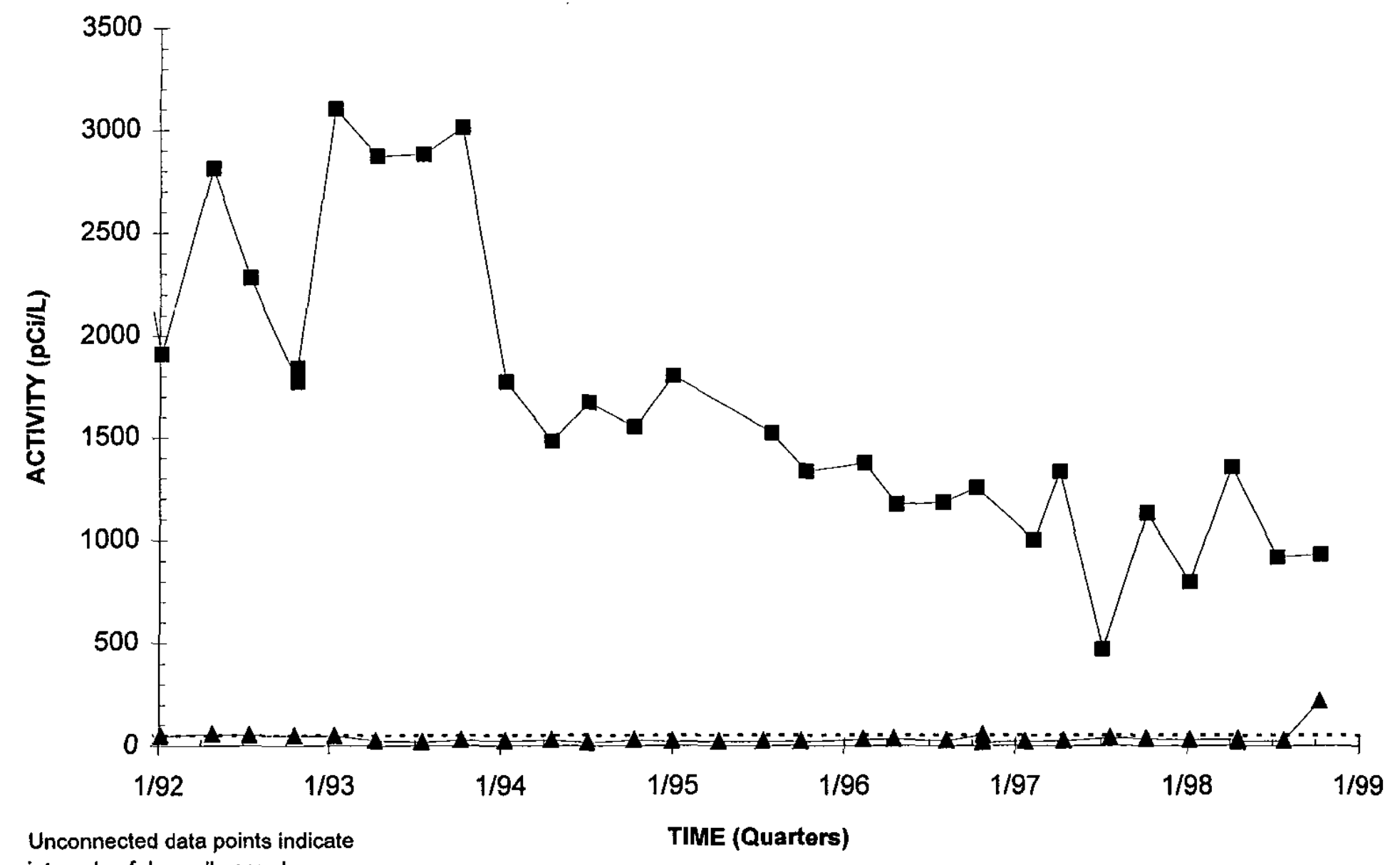

intervals of dry wells or values

below the detection limit.

GWPS $50 \mathrm{pCi} / \mathrm{L}$

$$
\text { - m-HSB113D (W) }-\triangle \text { HSB113C (B) } \cdots \cdots \text {. GWPS }
$$

Note: $W=$ Water Table (IIB2); B=Barnwell (IIB1); $M=M C B e a n$ (IIB1); UC=Upper Congaree (IIA); MC=Middle Congaree (IIA); LC=Lower Congaree (IIA) 


\section{Nonvolatile Beta Activities}

Well Cluster HSB114

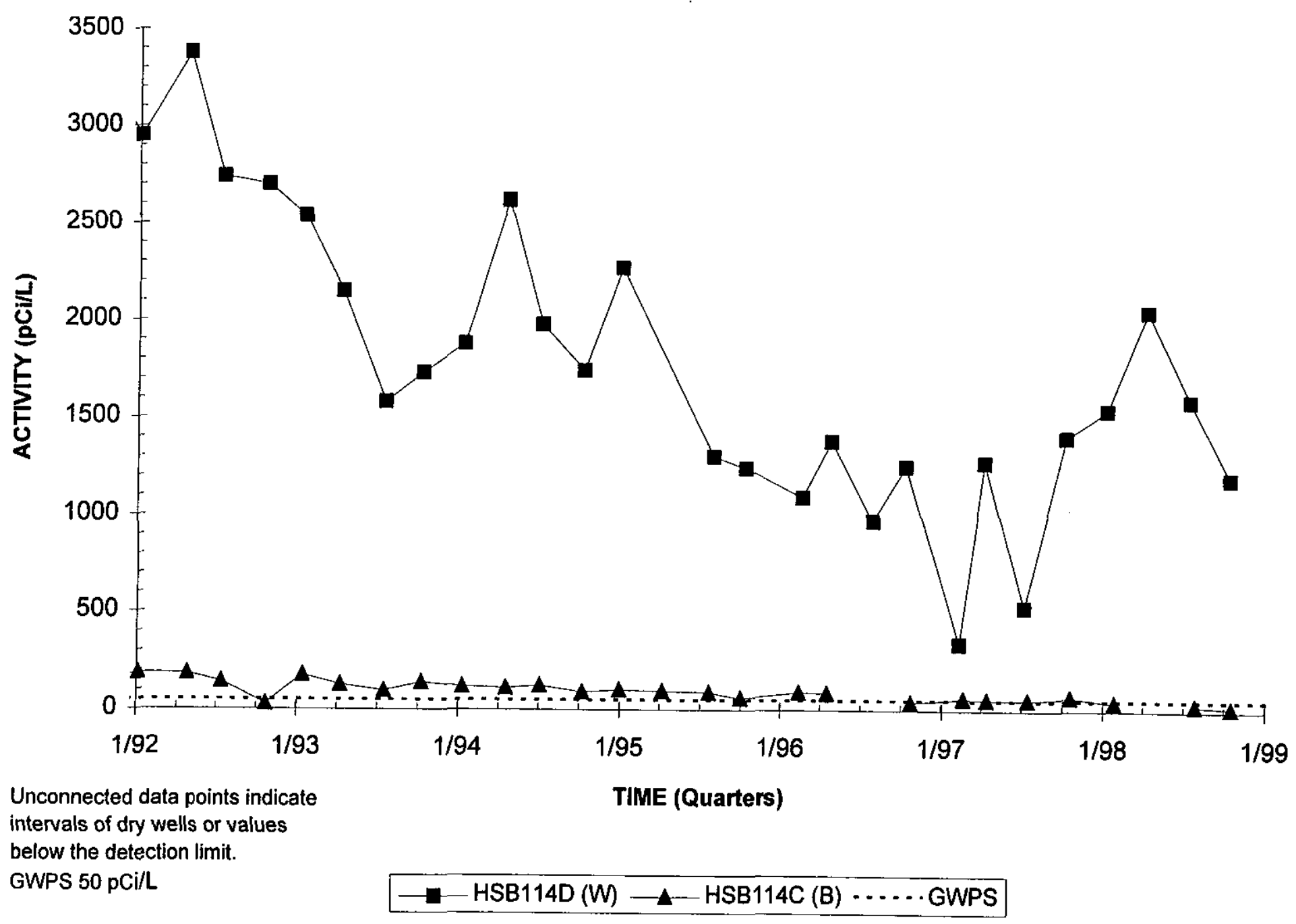

Note: W=Water Table (IIB2); B=Barnwell (IIB1); M=McBean (IIB1); UC=Upper Congaree (IIA); MC=Middle Congaree (IIA); LC=Lower Congaree (IIA) 


\section{Nonvolatile Beta Activities}

Well Cluster HSB115

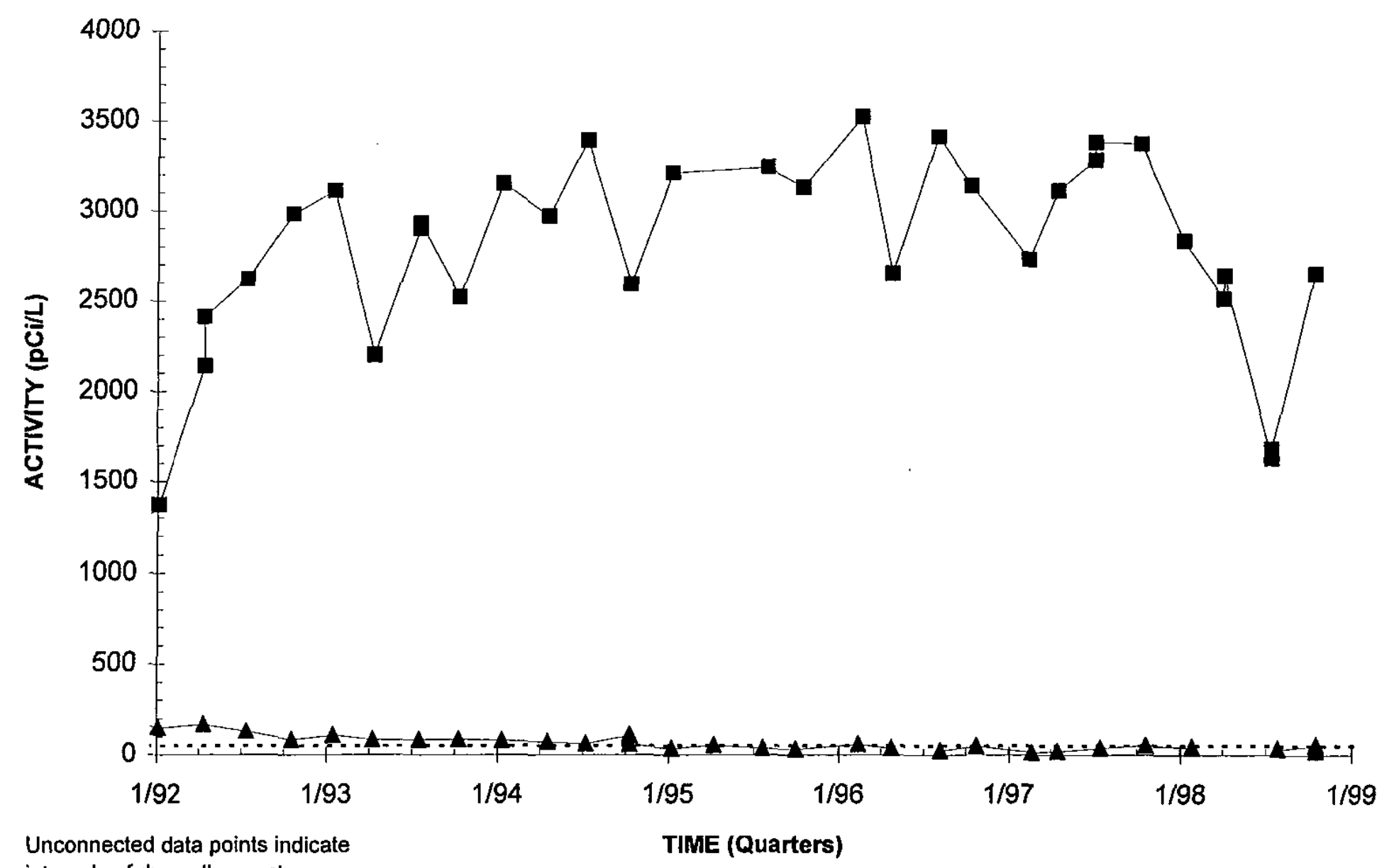

intervals of dry wells or values

below the detection limit.

GWPS $50 \mathrm{pCi} / \mathrm{L}$

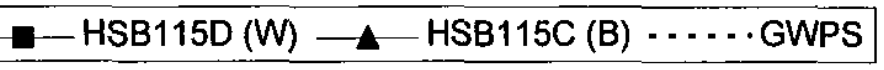

Note: W=Water Table (IIB2); B=Barnwell (IIB1); M=MCBean (IIB1); UC=Upper Congaree (IIA); MC=Middle Congaree (IIA); LC=Lower Congaree (IIA) 


\section{Nonvolatile Beta Activities \\ Well Cluster HSB136}

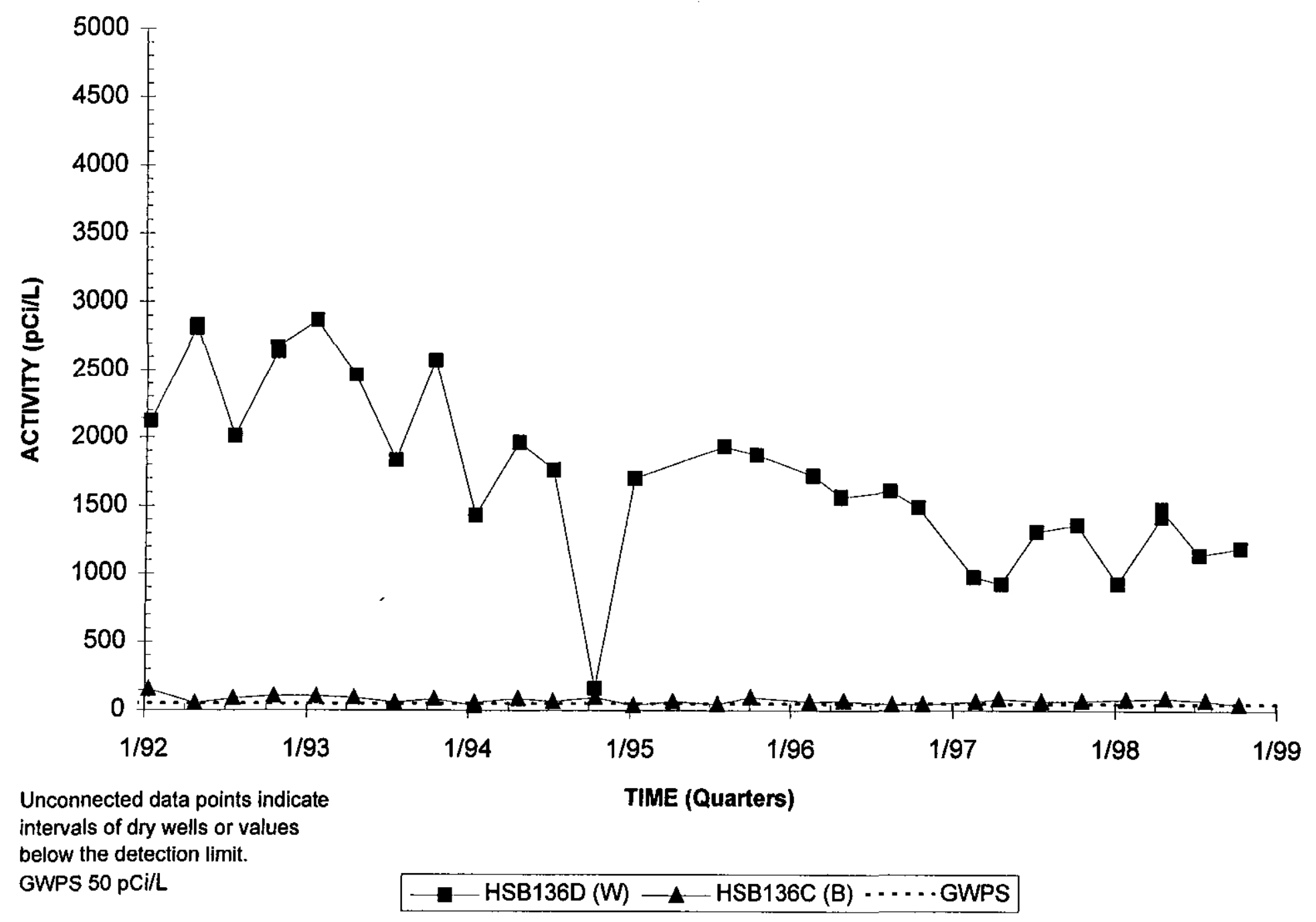

Note: W=Water Table (IIB2); B=Barnwell (IIB1); M=MCBean (IIB1); UC=Upper Congaree (IIA); MC=Middie Congaree (IIA); LC=Lower Congaree (IIA) 


\section{Nonvolatile Beta Activities \\ Well Cluster HSB145}

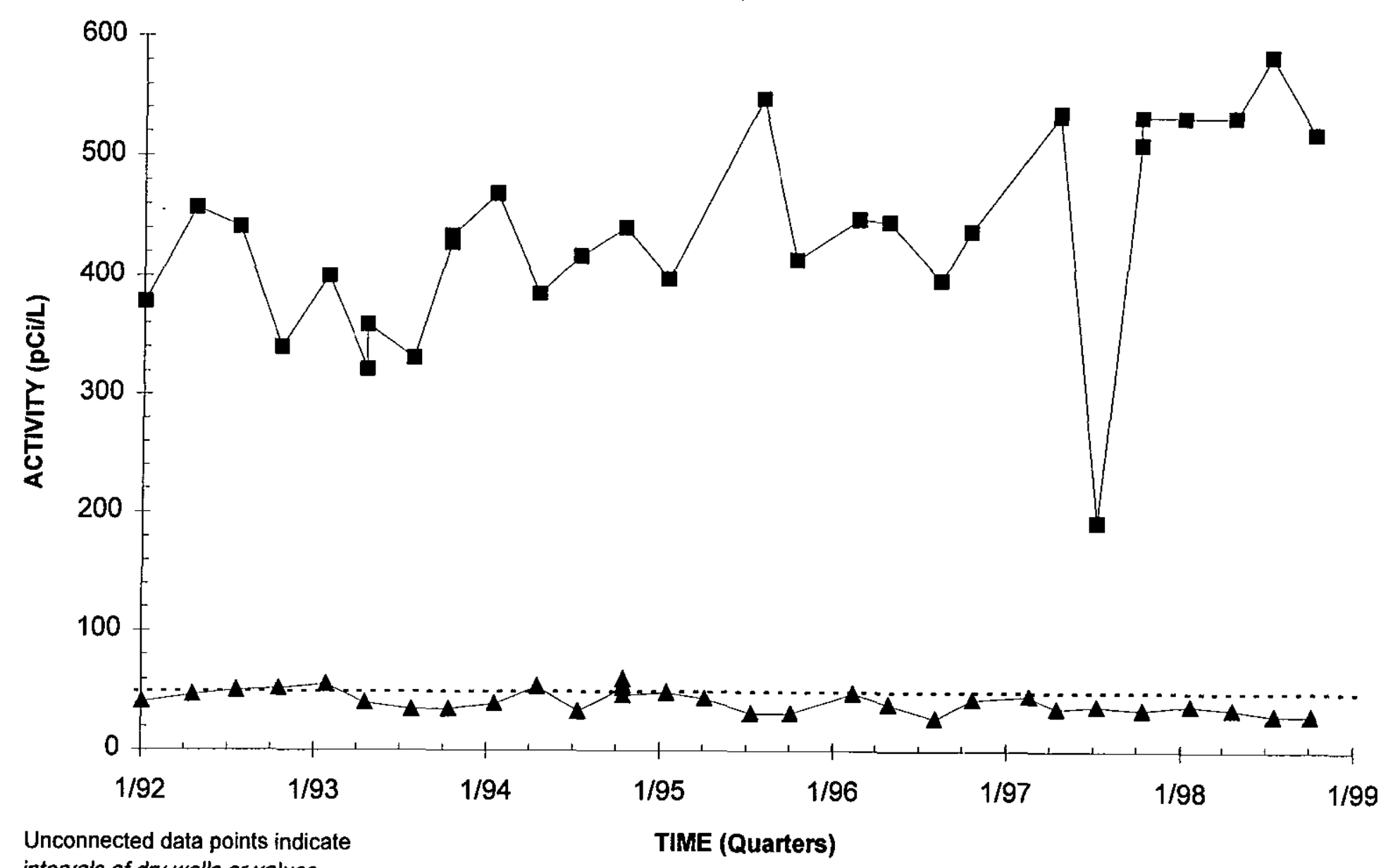

intervals of dry wells or values

below the detection limit.

GWPS $50 \mathrm{pCi} / \mathrm{L}$

HSB145D (W) $\longleftarrow$ HSB145C (B) ......GWPS

Note: W=Water Table (IIB2); B=Barnwell (IIB1); M=McBean (IIB1); UC=Upper Congaree (IIA); MC=Middle Congaree (IIA); LC=Lower Congaree (IIA) 
WSRC-TR-99-00013 Unclassified

Part II

\section{Appendix E}

Hydrographs

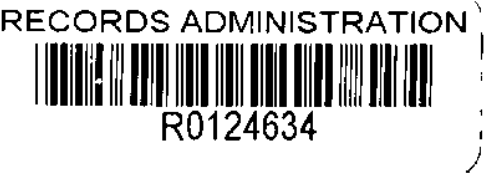




\section{Hydrograph \\ Well Cluster HSB 65}

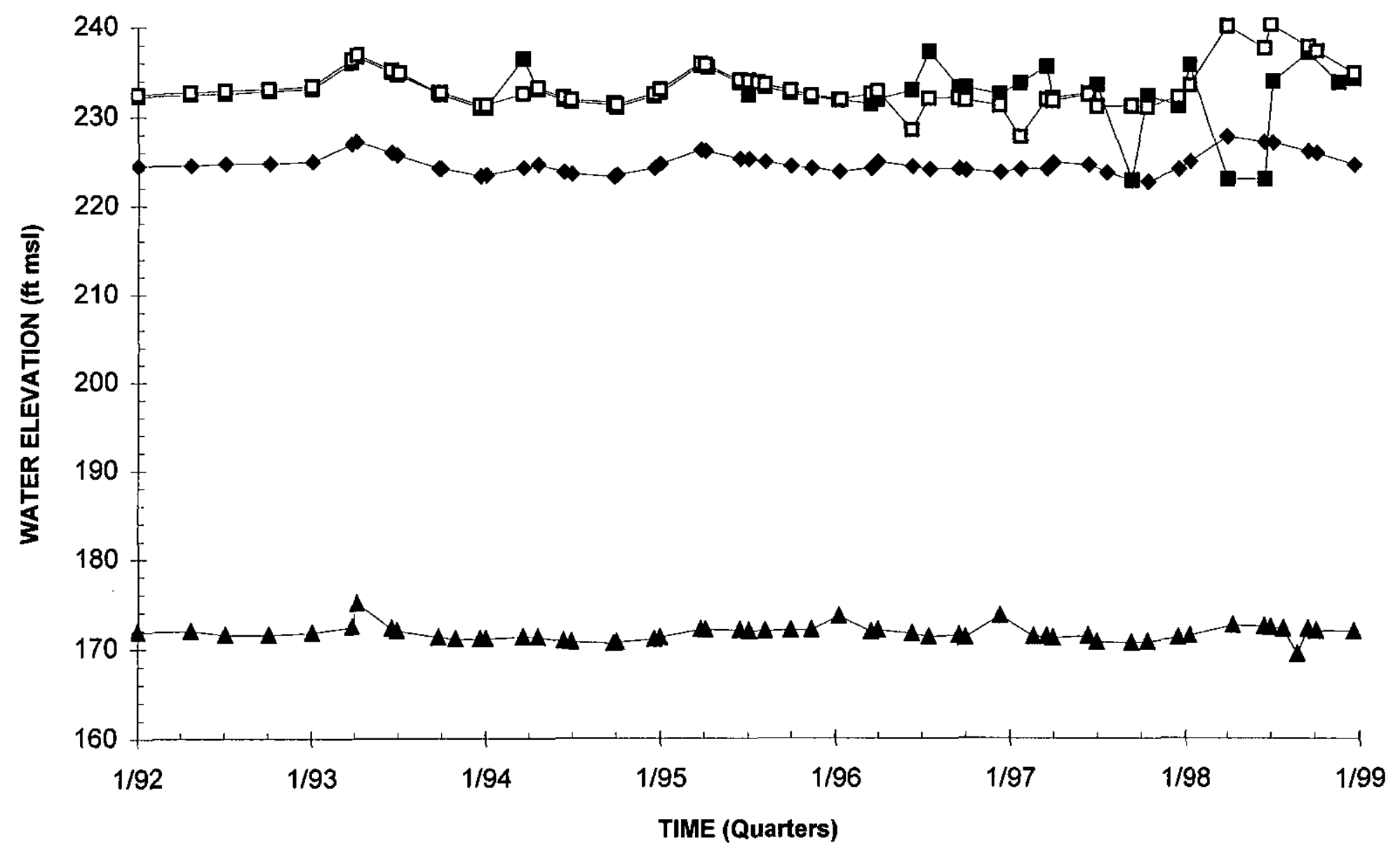

$-\mathrm{HSB} 65(\mathrm{~W})-\mathrm{-HSB} 65 \mathrm{C}(\mathrm{W}) \multimap \mathrm{HSB} 65 \mathrm{~B}(\mathrm{M})-\mathrm{HSB} 65 \mathrm{~A}(\mathrm{LC})$

Note: $W=$ Water Table (IIB2); B=Barnwell (IIB1); M=McBean (IIB1); UC=Upper Congaree (IIA); MC=Middle Congaree (IIA); LC=Lower Congaree (IIA) 


\section{Hydrograph \\ Well HSB 66}

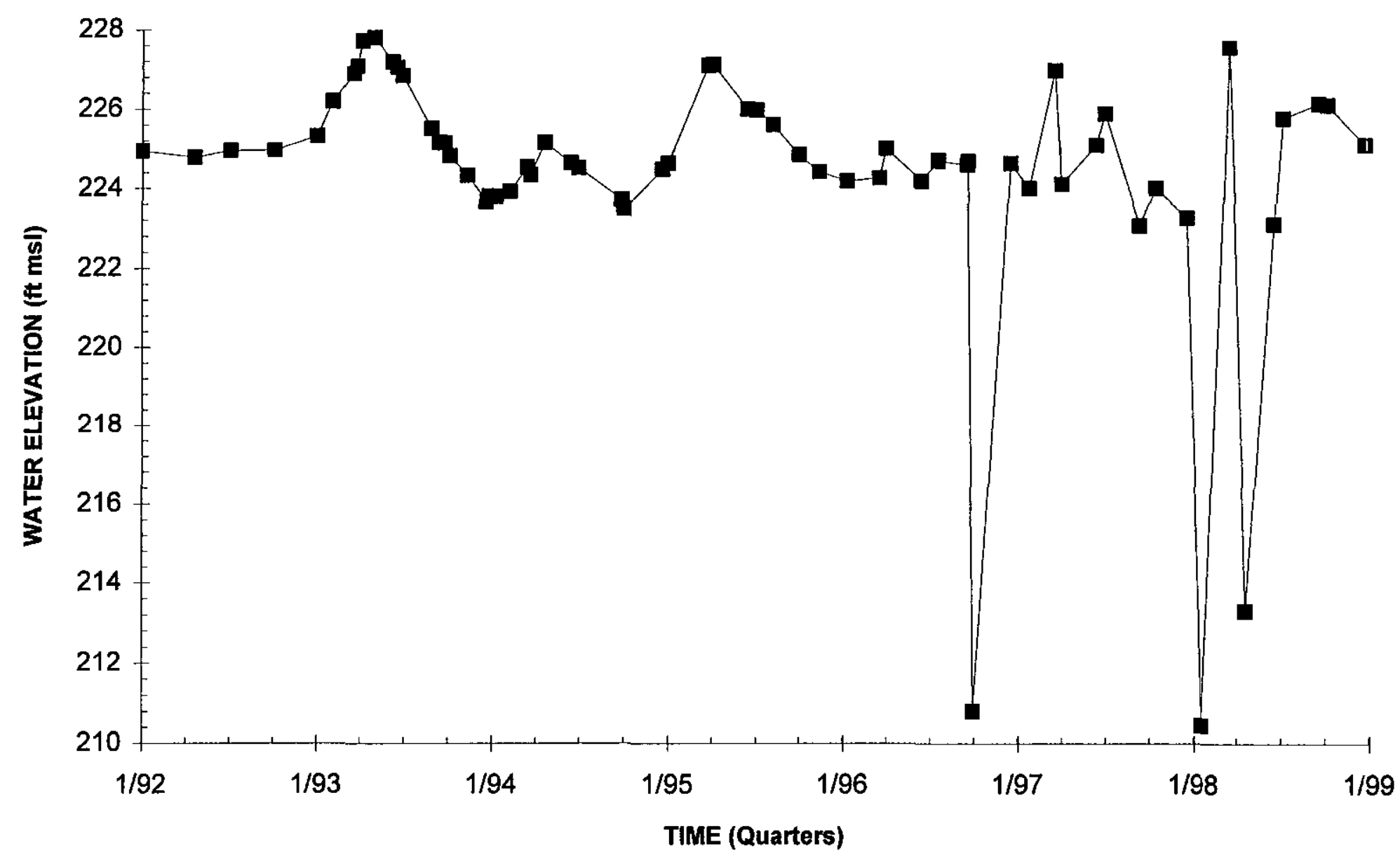

HSB 66 (W)

Note: W=Water Table (IIB2); B=Barnwell (IIB1); M=McBean (IIB1); UC=Upper Congaree (IIA); MC=Middle Congaree (IIA); LC=Lower Congaree (IIA) 


\section{Hydrograph}

\section{Well HSB 67}

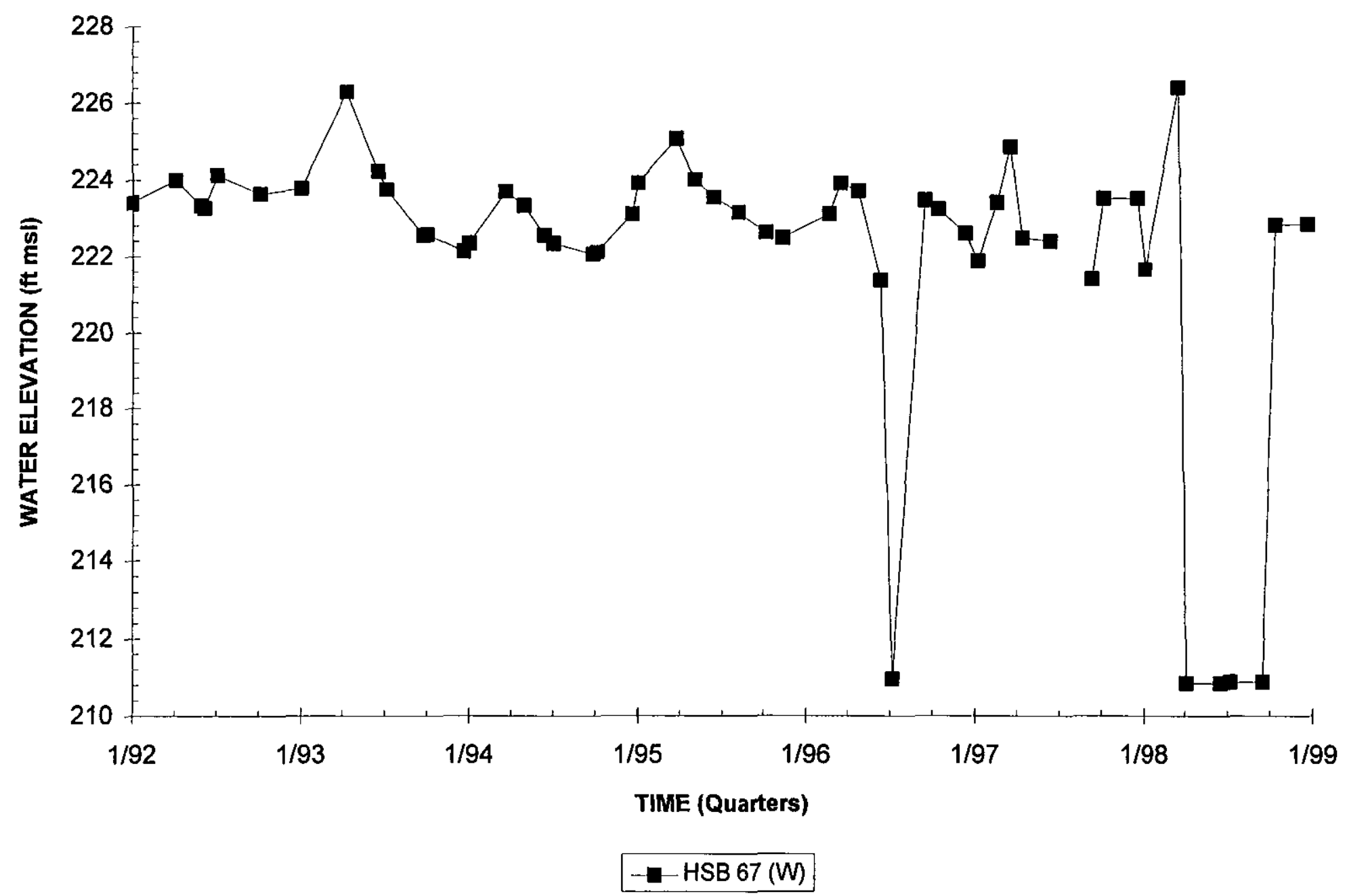

Note: W=Water Table (IIB2); B=Barnwell (IBB1); M=McBean (IIB1); UC=Upper Congaree (IIA); MC=Middle Congaree (IIA); LC=Lower Congaree (IIA) 


\section{Hydrograph \\ Well Cluster HSB 68}

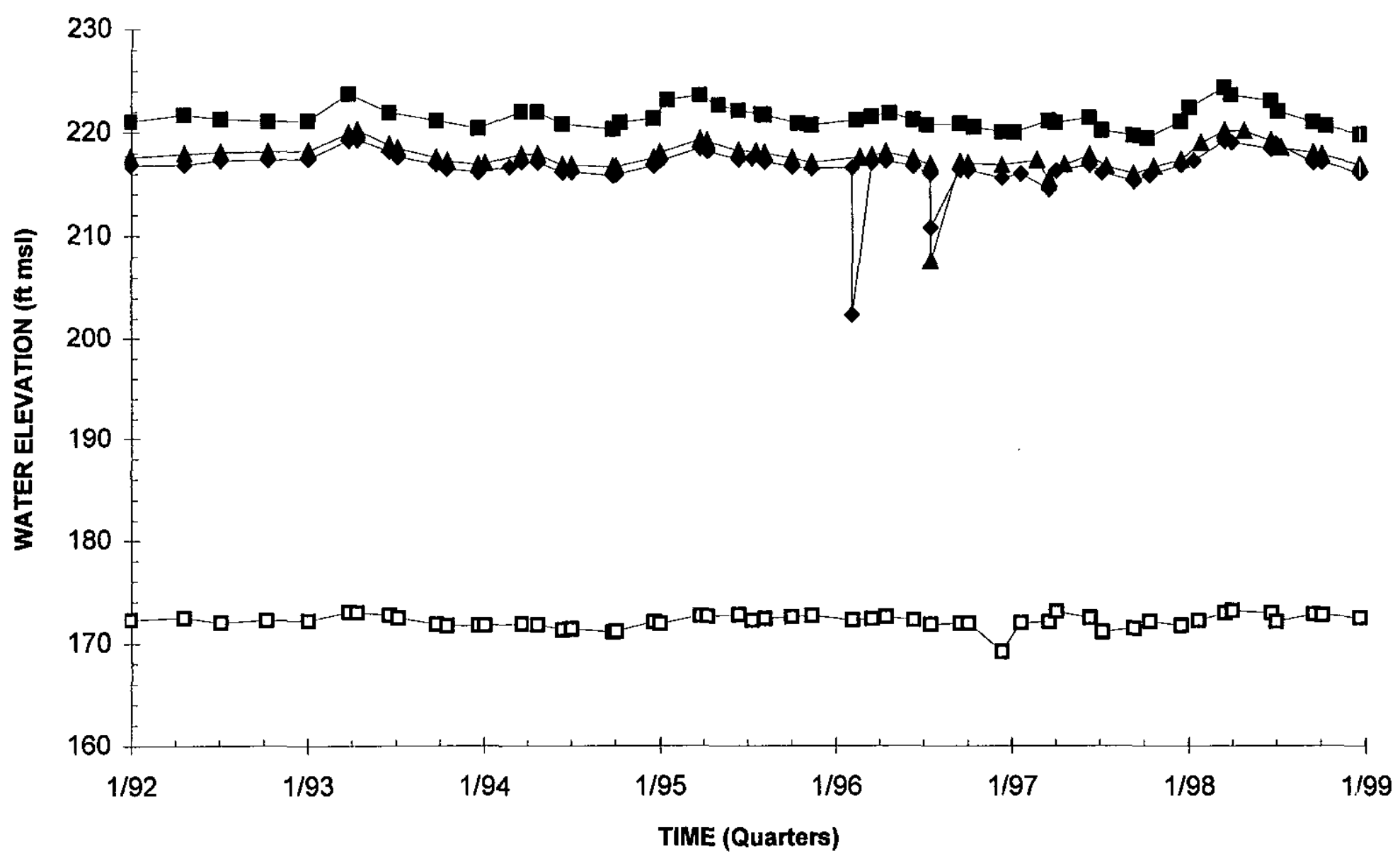

$$
\rightarrow-\mathrm{HSB} 68(\mathrm{~W})-\mathrm{ASB} 68 \mathrm{C}(\mathrm{B})-\mathrm{HSB} 68 \mathrm{~B}(\mathrm{M})-\mathrm{D}-\mathrm{HSB} 68 \mathrm{~A}(\mathrm{LC})
$$

Note: $W=$ Water Table (IIB2); $B=B a r n w e l l$ (IIB1); $M=M C B e a n$ (IIB1); UC=Upper Congaree (IIA); MC=Middle Congaree (IIA); LC=Lower Congaree (IIA) 


\section{Hydrograph \\ Well Cluster HSB 69}

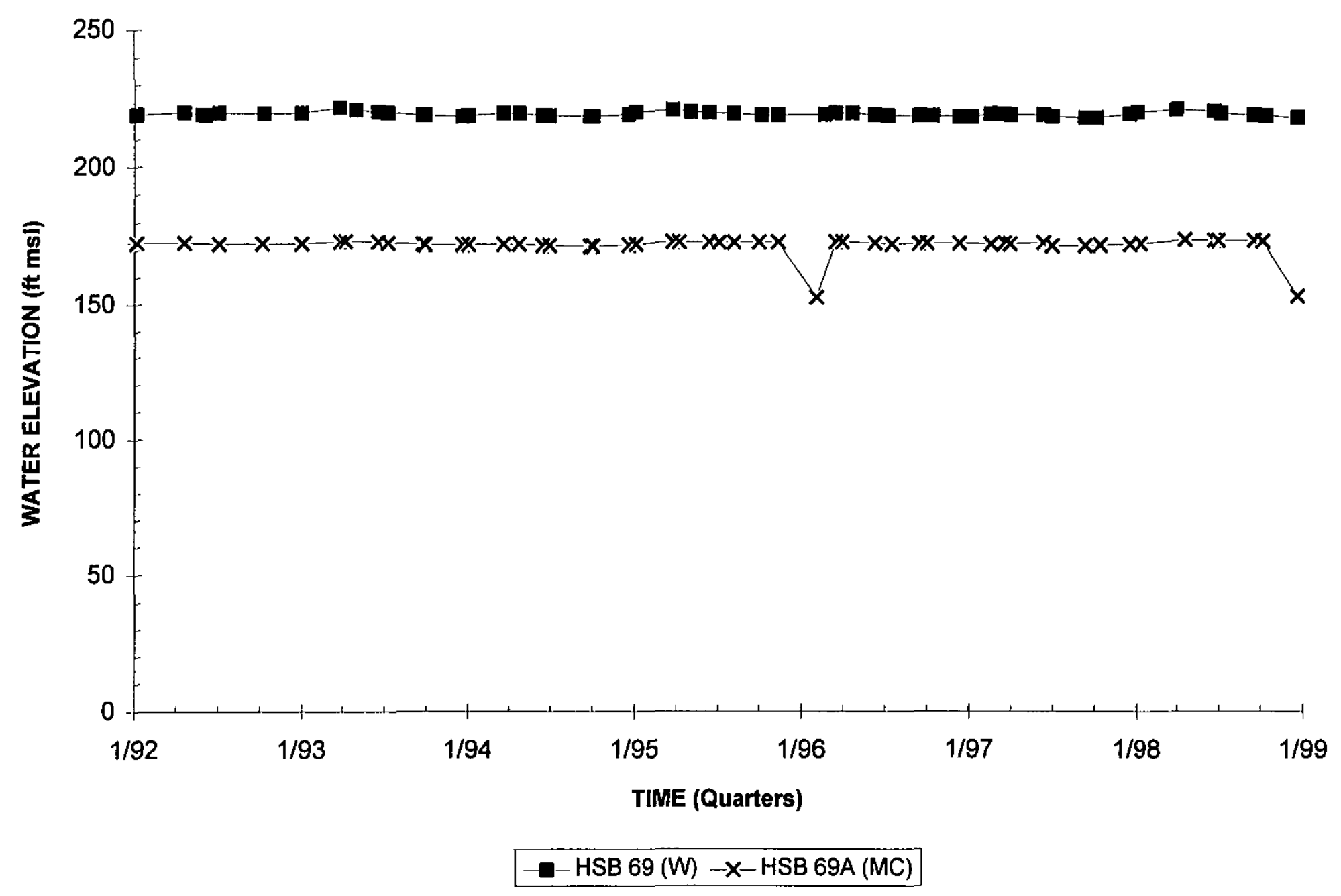

Note: W=Water Table (IIB2); B=Barnwell (IIB1); M=McBean (IIB1); UC=Upper Congaree (IIA); MC=Middle Congaree (IIA); LC=Lower Congaree (IIA) 


\section{Hydrograph \\ Well Cluster HSB 70}

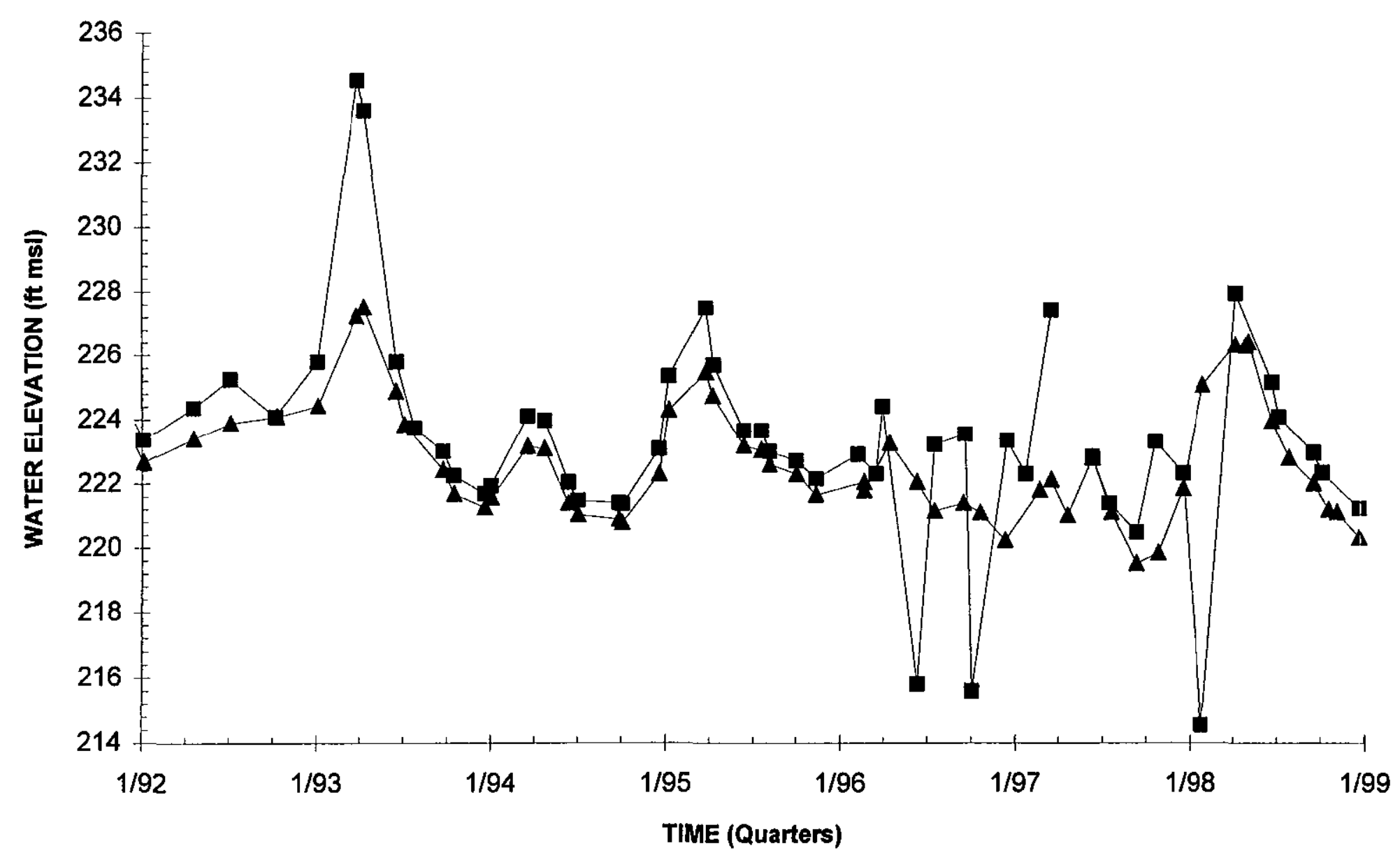

- HSB $70(W)-$ HSB $70 C(B)$

Note: W=Water Table (IIB2); B=Barnwell (IIB1); M=McBean (IIB1); UC=Upper Congaree (IIA); MC=Middle Congaree (IIA); LC=Lower Congaree (IIA) 


\section{Hydrograph}

\section{Well Cluster HSB 71}

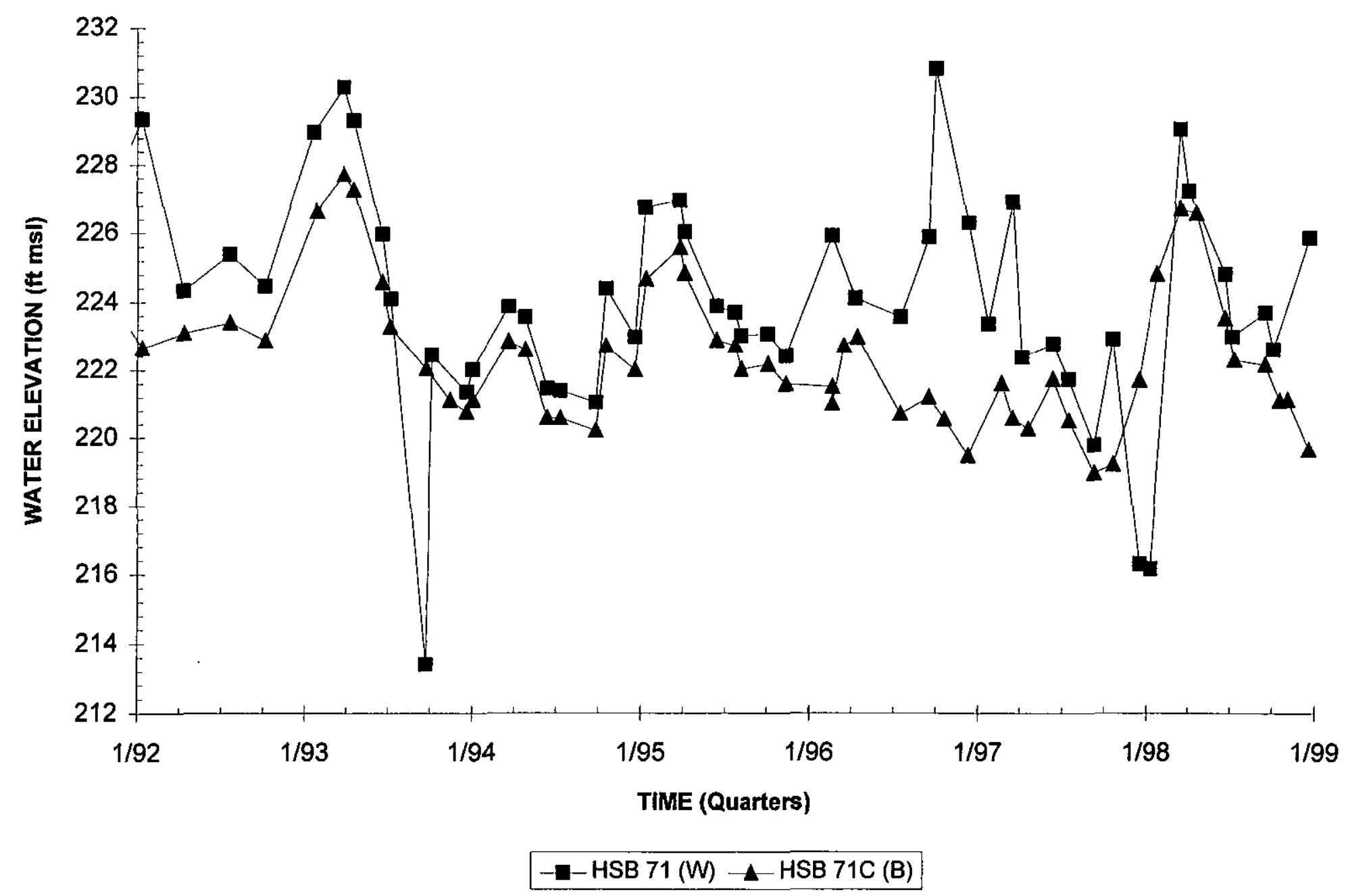

Note: $W=$ Water Table (IIB2); B=Barnwell (IIB1); M=McBean (IIB1); UC=Upper Congaree (IIA); MC=Middle Congaree (IAA); LC=Lower Congaree (IIA) 


\section{Hydrograph \\ Well Cluster HSB 83}

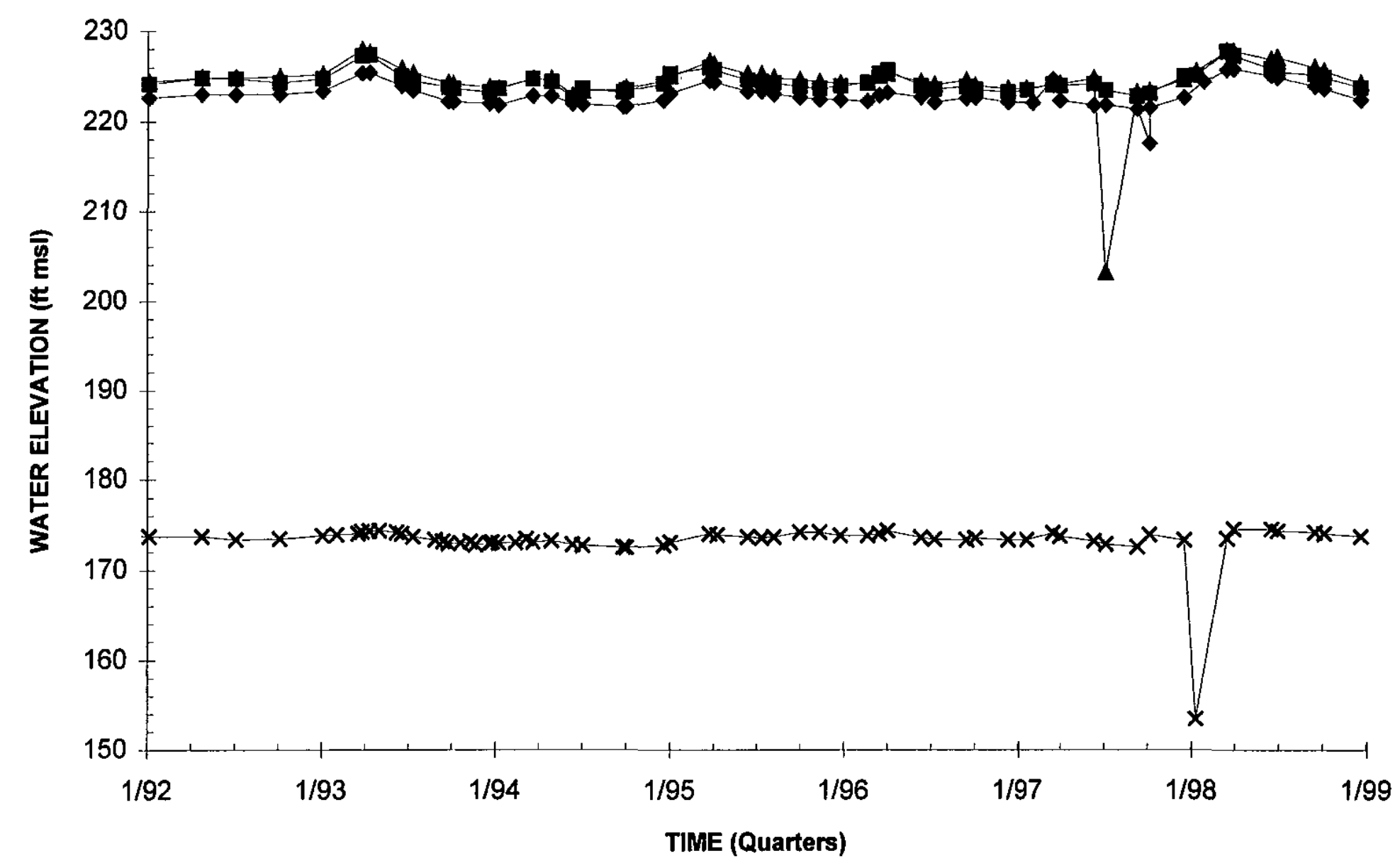

$-\operatorname{HSB} 83 \mathrm{D}(\mathrm{W})-\mathrm{HSB} 83 \mathrm{C}(\mathrm{B})-\mathrm{HSB} 83 \mathrm{~B}(\mathrm{M}) \rightarrow \mathrm{HSB} 83 \mathrm{~A}(\mathrm{MC})$

Note: W=Water Table (IIB2); B=Barnwell (IIB1); M=McBean (IIB1); UC=Upper Congaree (IIA); MC=Middle Congaree (IIA); LC=Lower Congaree (IIA) 


\section{Hydrograph}

Well Cluster HSB 84

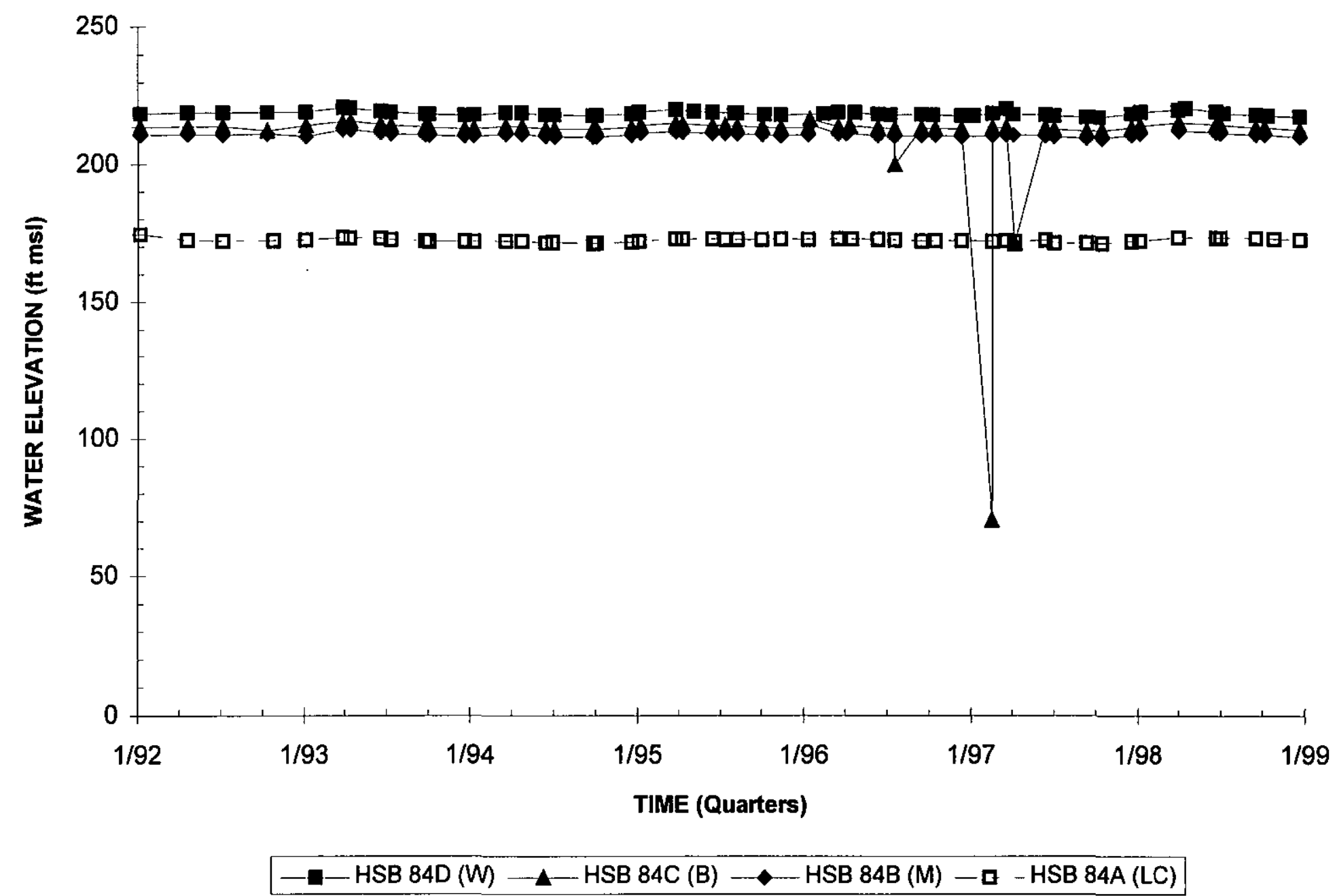

Note: $W=$ Water Table (IIB2); B=Barnwell (IIB1); M=MCBean (IIB1); UC=Upper Congaree (IIA); MC=Middle Congaree (IIA); LC=Lower Congaree (IIA) 


\section{Hydrograph \\ Well Cluster HSB 85}

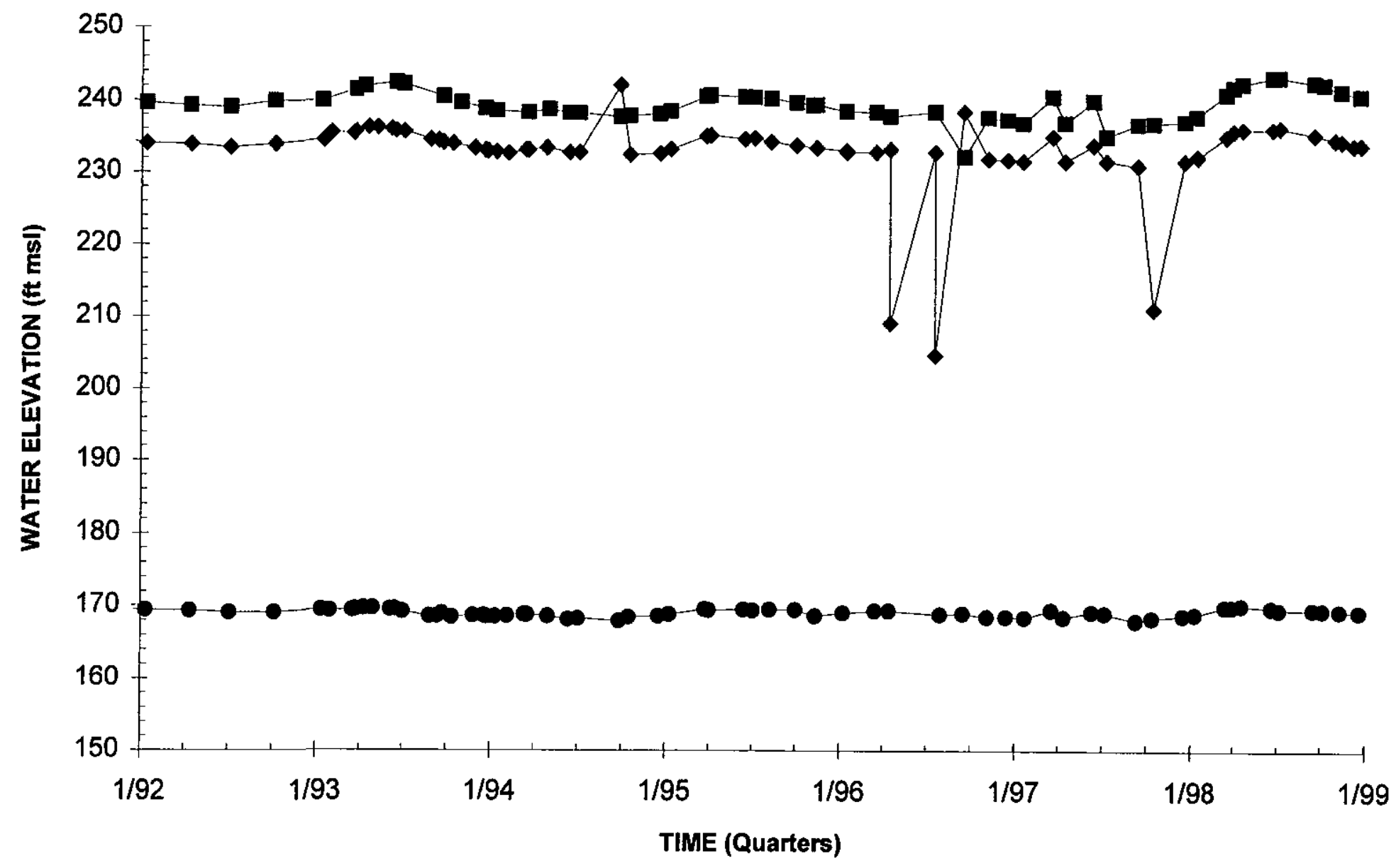

- HSB 85C (W) $\bullet$ HSB 85B (M) - HSB 85A (UC)

Note: W=Water Table (IIB2); B=Barnwell (IIB1); M=MCBean (IIB1); UC=Upper Congaree (IIA); MC=Middle Congaree (IIA); LC=Lower Congaree (IIA) 


\section{Hydrograph}

Well Cluster HSB 86

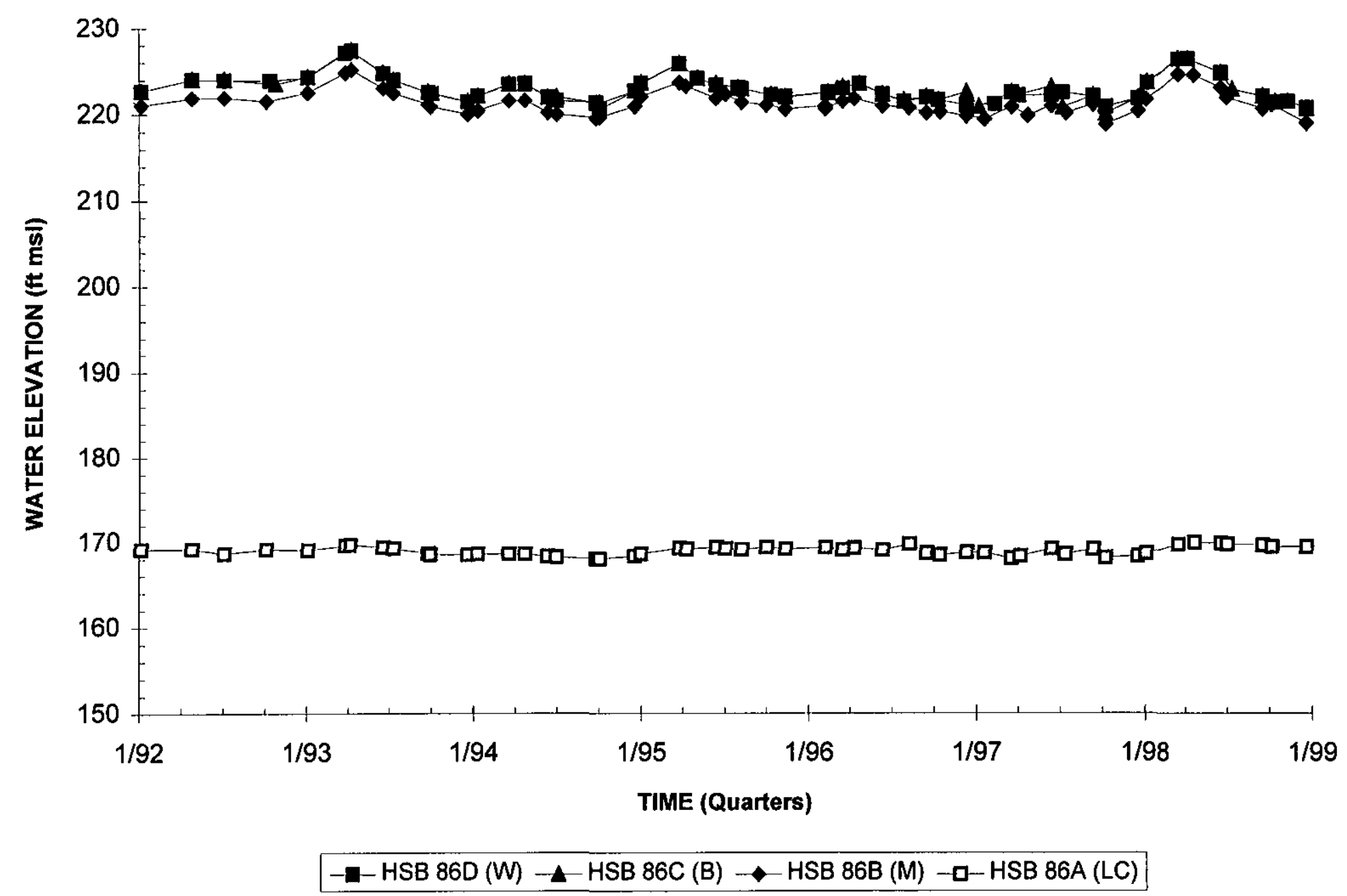

Note: W=Water Table (IIB2); B=Barnwell (IIB1); M=McBean (IIB1); UC=Upper Congaree (IIA); MC=Middle Congaree (IIA); LC=Lower Congaree (IIA) 


\section{Hydrograph}

\section{Well Cluster HSB100}

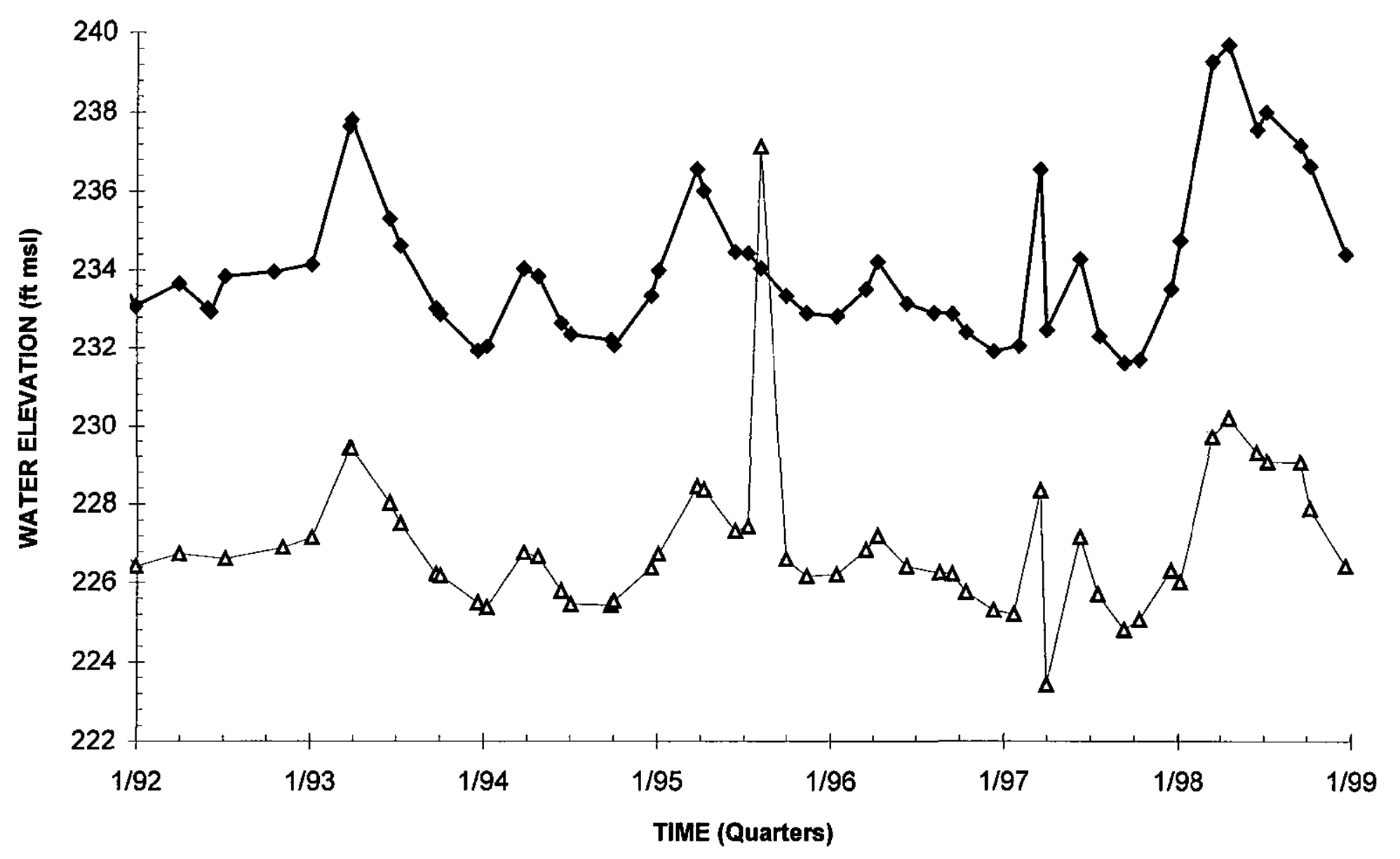

$$
\triangle-\mathrm{HSB} 100 \mathrm{C}(\mathrm{B}) \multimap \mathrm{HSB} 100 \mathrm{D}(\mathrm{W})
$$

Note: W=Water Table (IIB2); B=Barnwell (IIB1); M=McBean (IIB1); UC=Upper Congaree (IIA); MC=Middle Congaree (IIA); LC=Lower Congaree (IIA) 
Hydrograph

\section{Well Cluster HSB101}

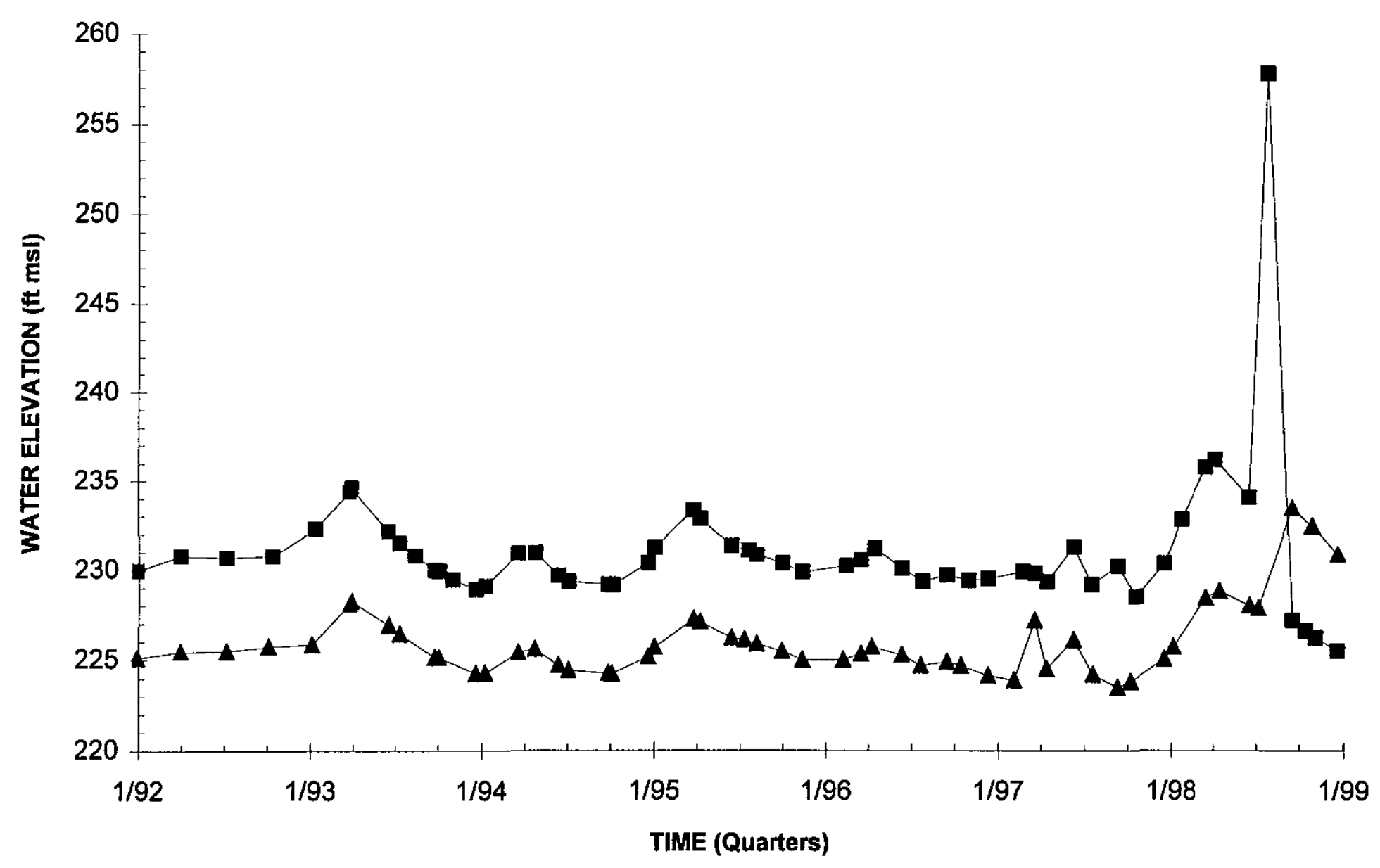

-HSB101D (W) - HSB101C (B)

Note: W=Water Table (IIB2); B=Barnwell (IIB1); M=McBean (IIB1); UC=Upper Congaree (IIA); MC=Middle Congaree (IIA); LC=Lower Congaree (IIA) 


\section{Hydrograph \\ Well Cluster HSB102}

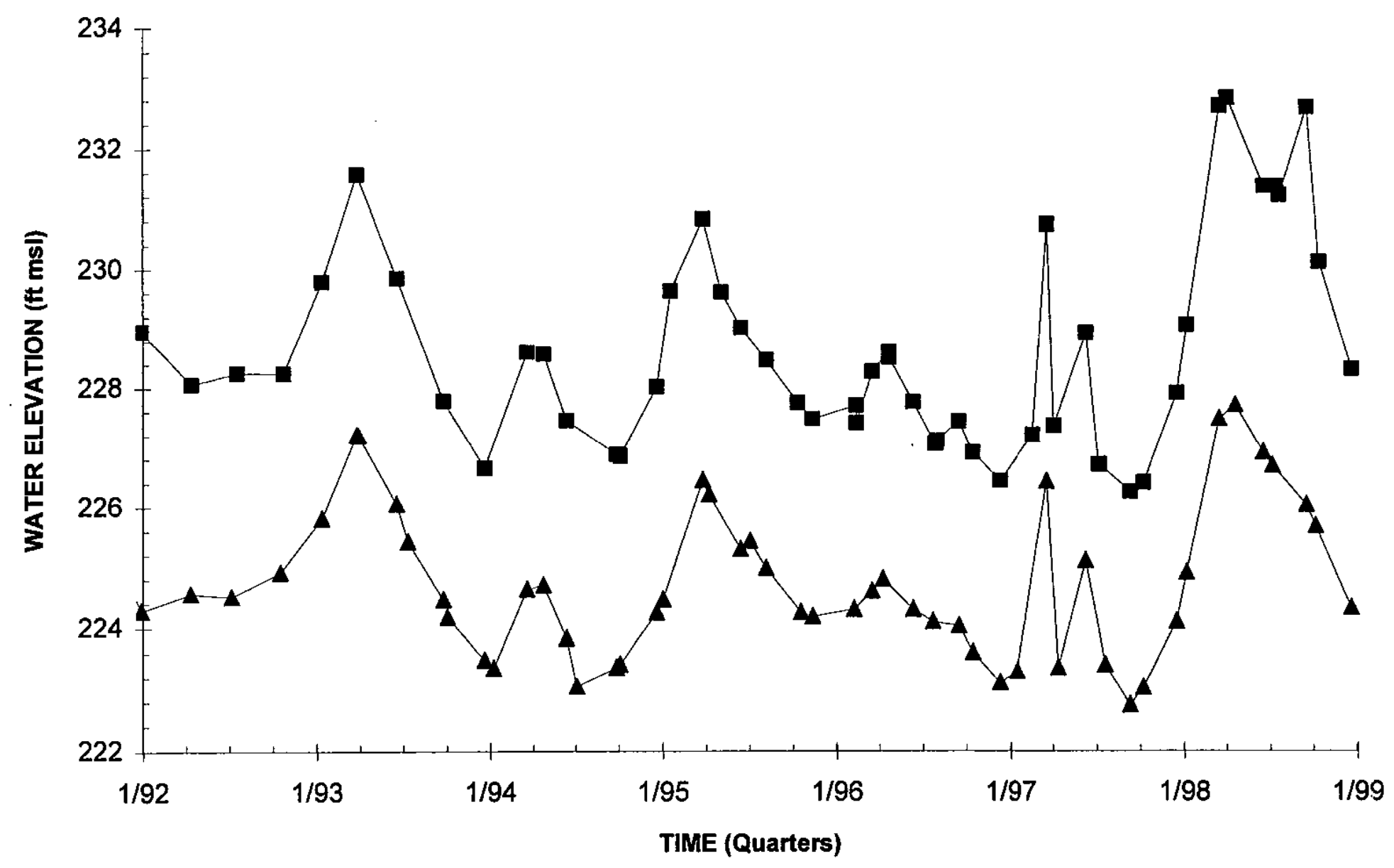

- HSB102D (W) - HSB102C (B)

Note: W=Water Table (IIB2); B=Barnwell (IIB1); M=McBean (IIB1); UC=Upper Congaree (IIA); MC=Middle Congaree (IIA); LC=Lower Congaree (IIA) 
Hydrograph

\section{Well Cluster HSB103}

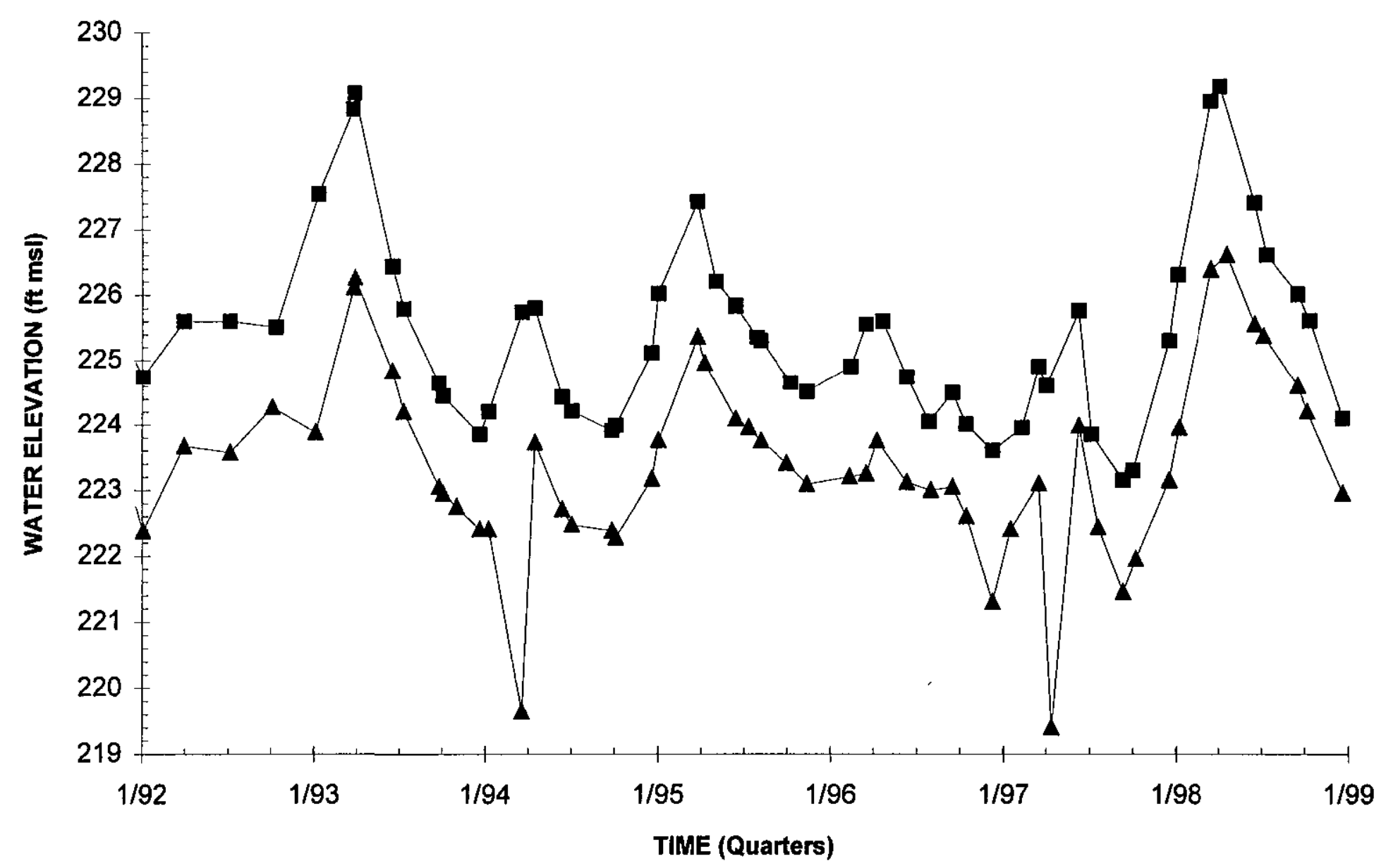

HSB103D (W) -A-HSB103C (B)

Note: $W=$ Water Table (IIB2); B=Barnwell (IIB1); M=MCBean (HB1); UC=Upper Congaree (IIA); MC=Middle Congaree (IIA); LC=Lower Congaree (IIA) 
Hydrograph

Well Cluster HSB104

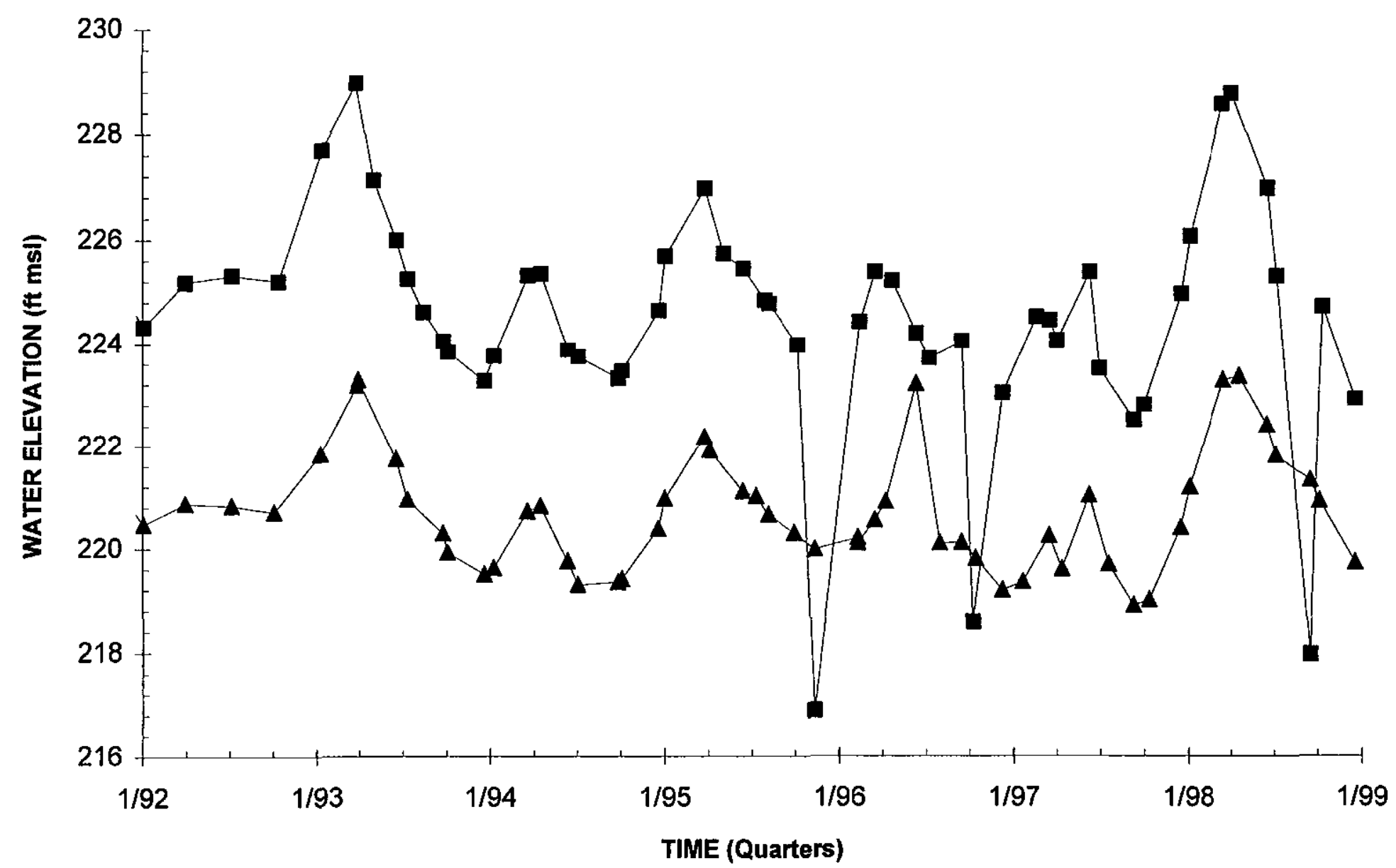

-HSB104D (W) - - HSB104C (B)

Note: W=Water Table (IIB2); B=Barnwell (IIB1); M=McBean (IIB1); UC=Upper Congaree (IIA); MC=Middle Congaree (IIA); LC=Lower Congaree (IIA) 


\section{Hydrograph}

\section{Well Cluster HSB105}

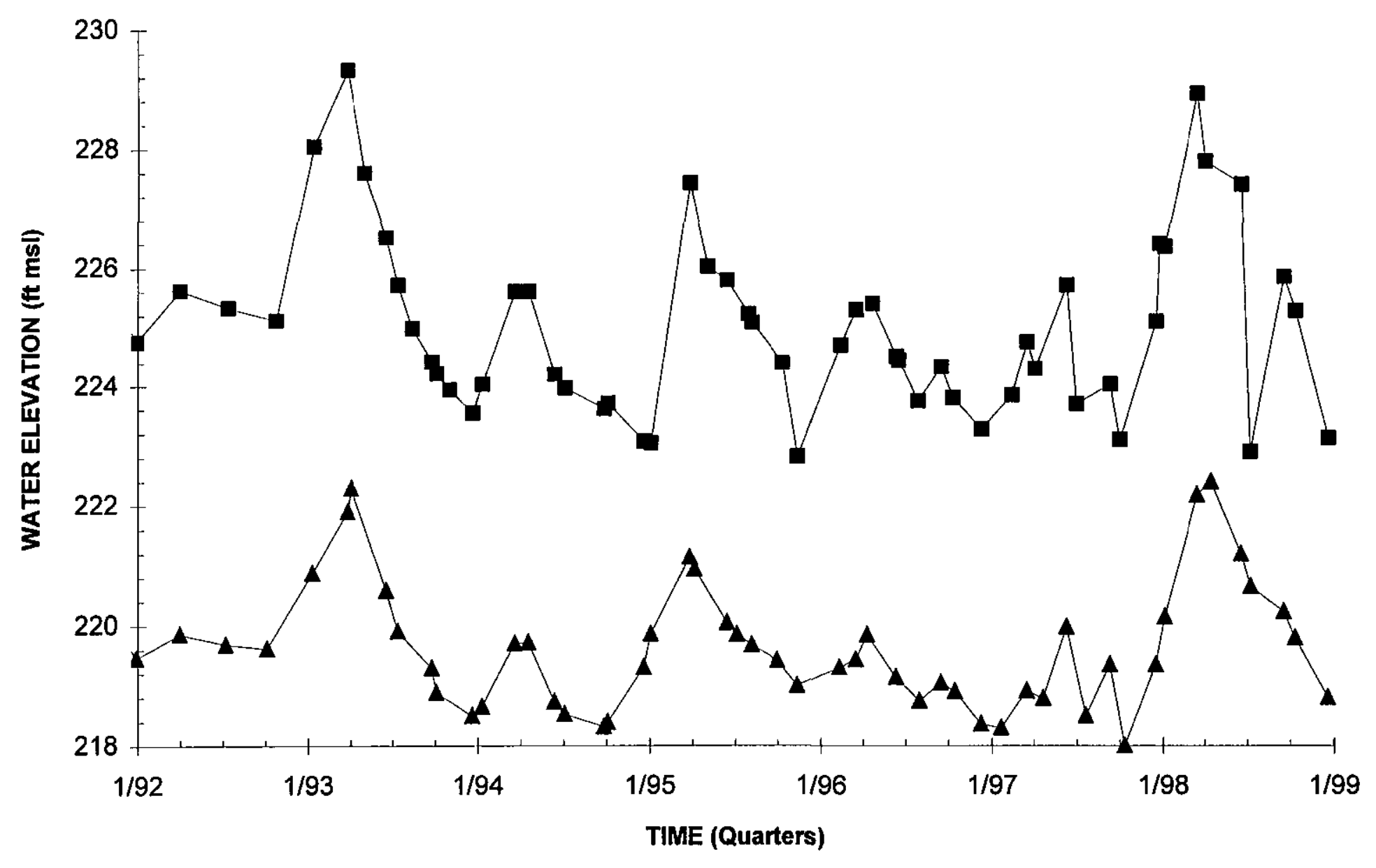

-HSB105D (W) $\_$HSB105C (B)

Note: W=Water Table (IIB2); B=Barnwell (IIB1); M=MCBean (IIB1); UC=Upper Congaree (IIA); MC=Middle Congaree (IIA); LC=Lower Congaree (IIA) 


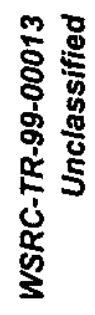
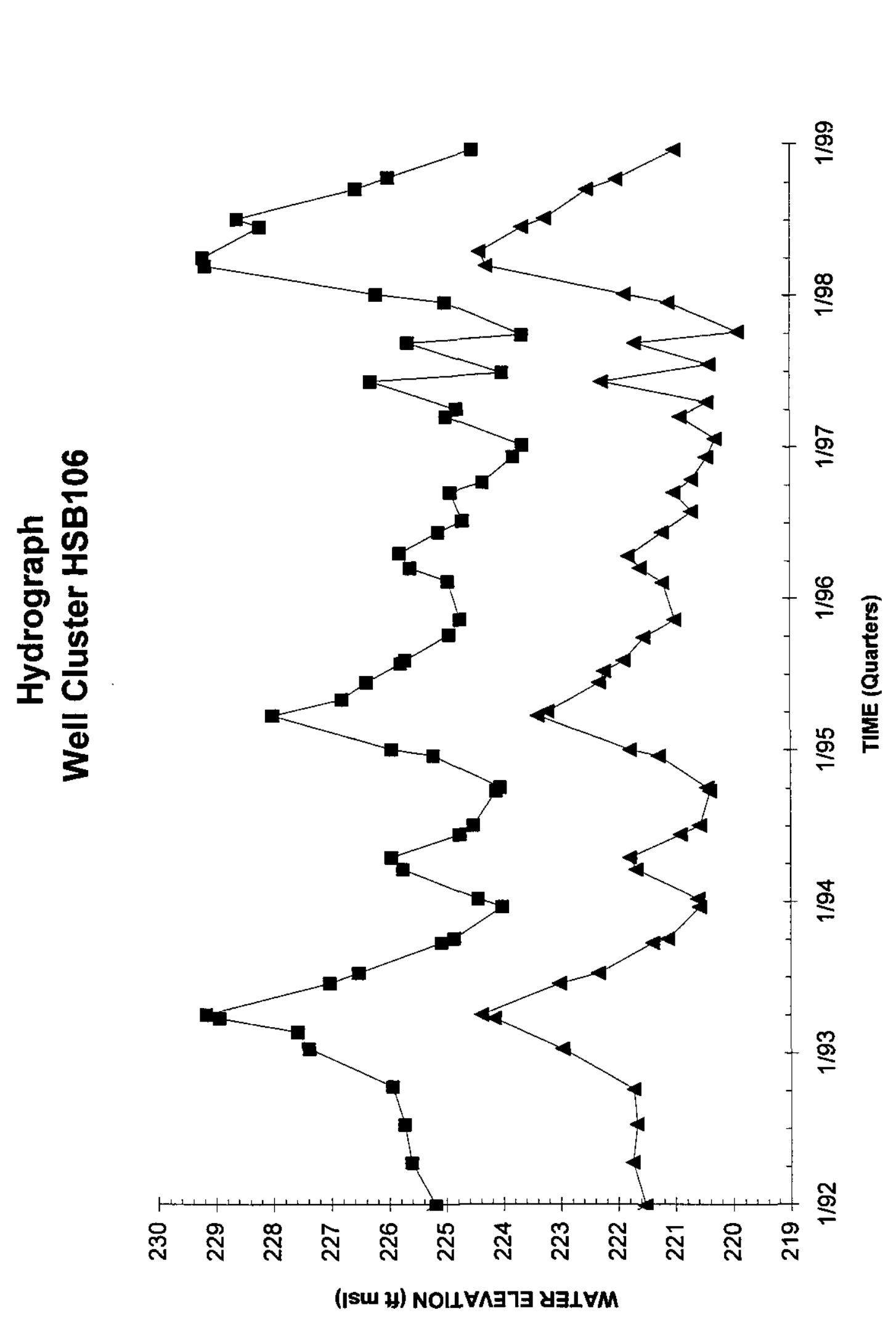

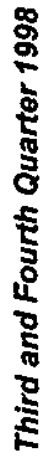

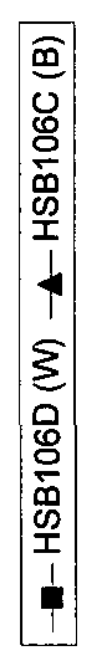

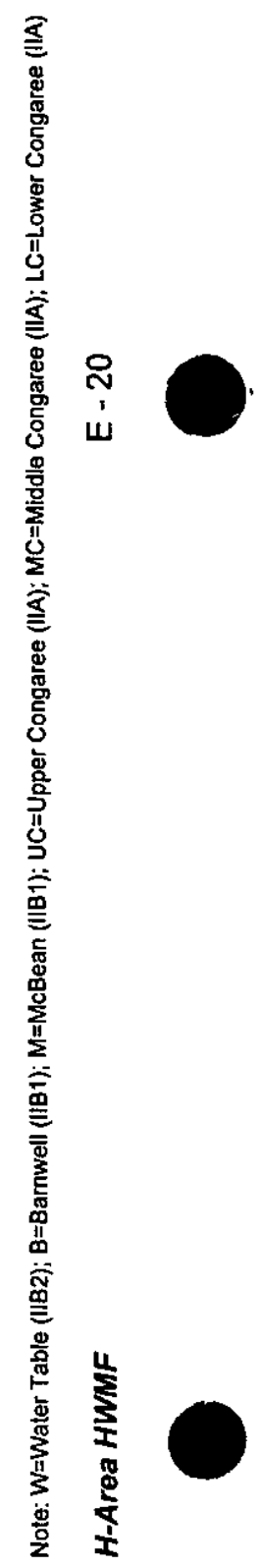


Hydrograph

\section{Well Cluster HSB107}

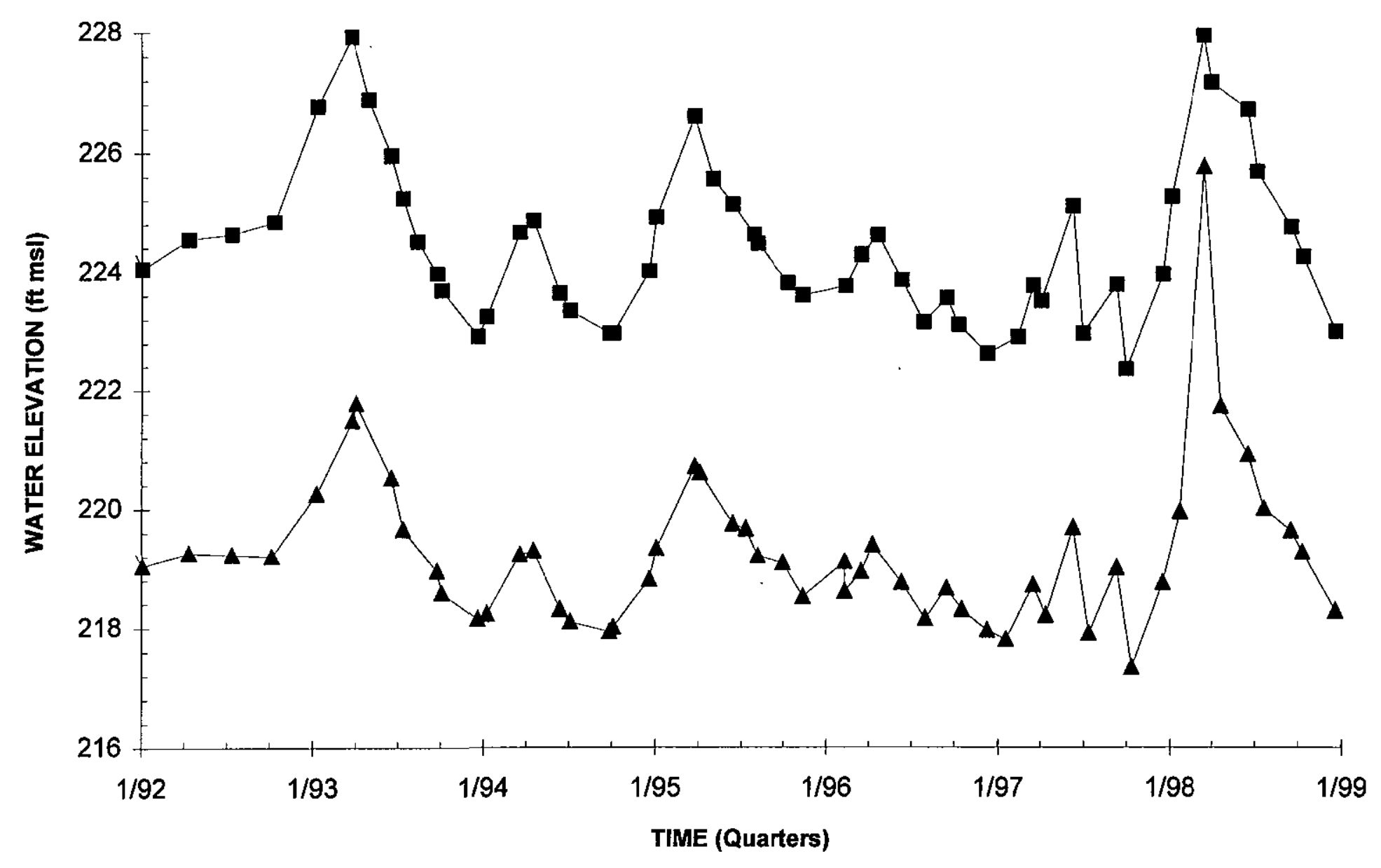

- HSB107D (W) - - HSB107C (B)

Note: W=Water Table (IIB2); B=8arnwell (IIB1); M=MCBean (IIB1); UC=Upper Congaree (IIA); MC=Middle Congaree (IIA); LC=Lower Congaree (IIA) 


\section{Hydrograph \\ Well Cluster HSB108}

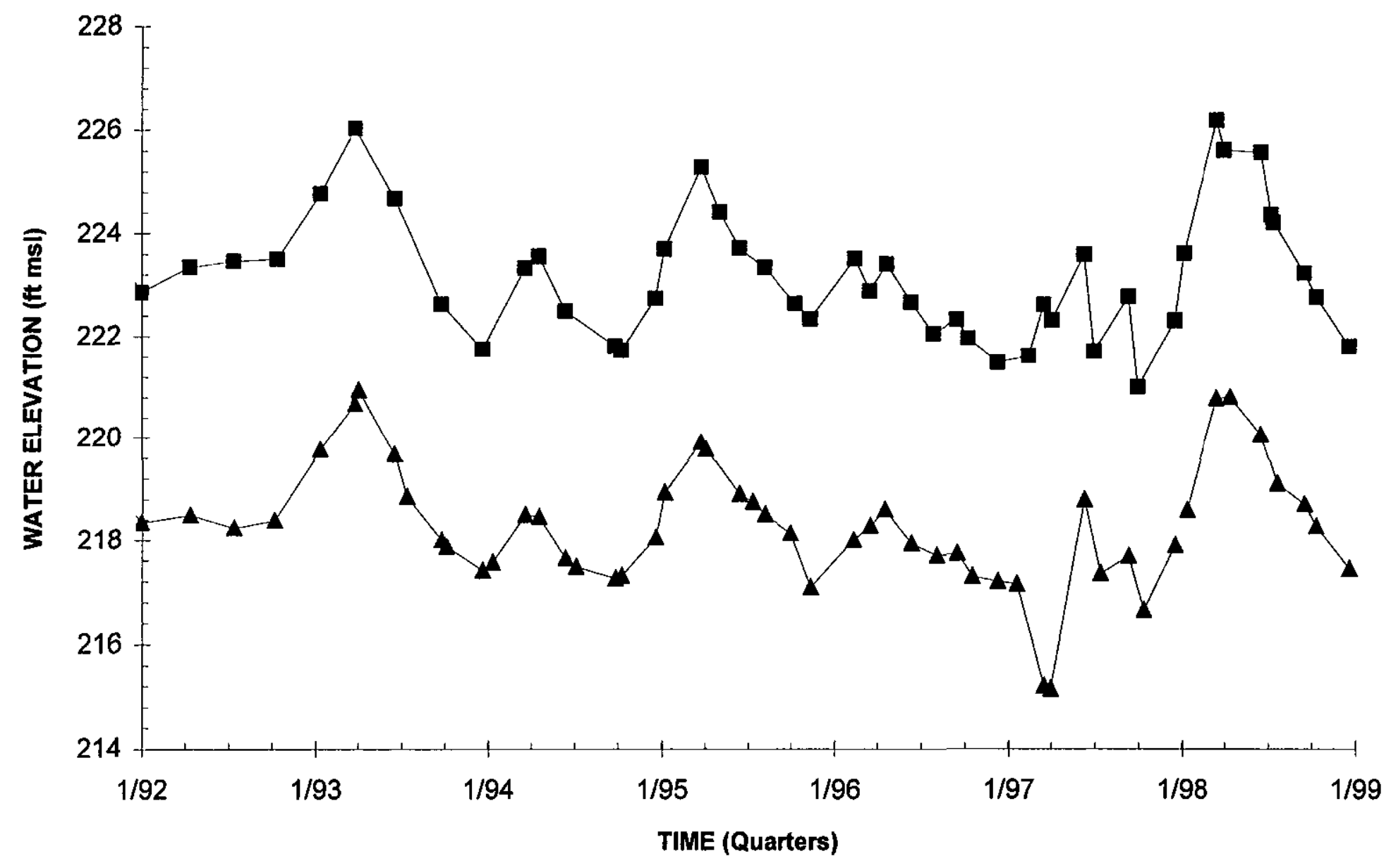

- $-\mathrm{HSB108D}(\mathrm{W})-\mathrm{A}-\mathrm{HSB108C}(\mathrm{B})$

Note: W=Water Table (IIB2); B=Barnwell (IIB1); M=McBean (IIB1); UC=Upper Congaree (IIA); MC=Middle Congaree (IIA); LC=Lower Congaree (IIA) 
Hydrograph

Well Cluster HSB109

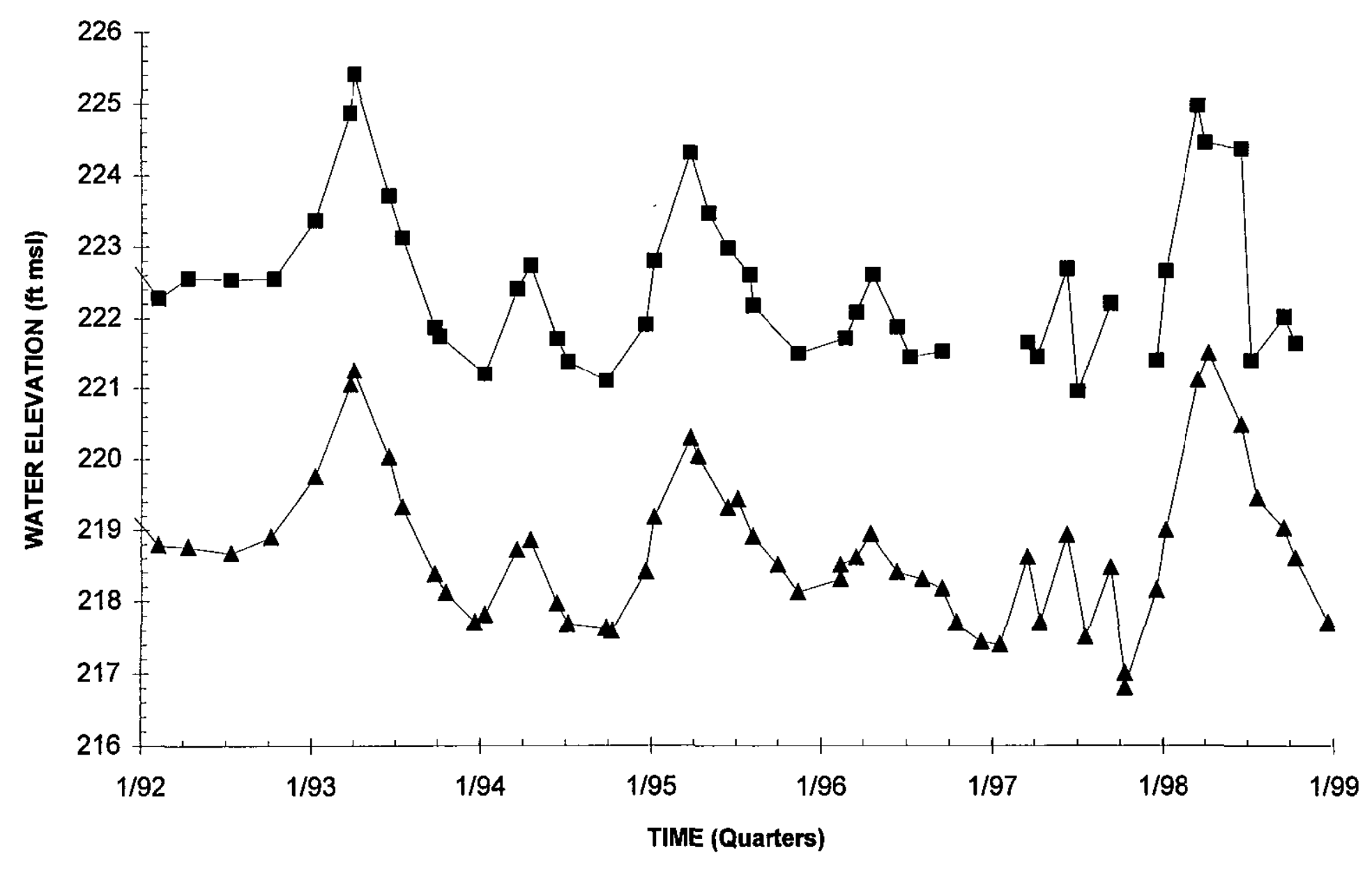

HSB109D (W) - HSB109C (B)

Note: W=Water Table (IIB2); B=Barnwell (IIB1); M=MCBean (IIB1); UC=Upper Congaree (IIA); MC=Middle Congaree (IIA); LC=Lower Congaree (IIA) 


\section{Hydrograph}

\section{Well Cluster HSB110}

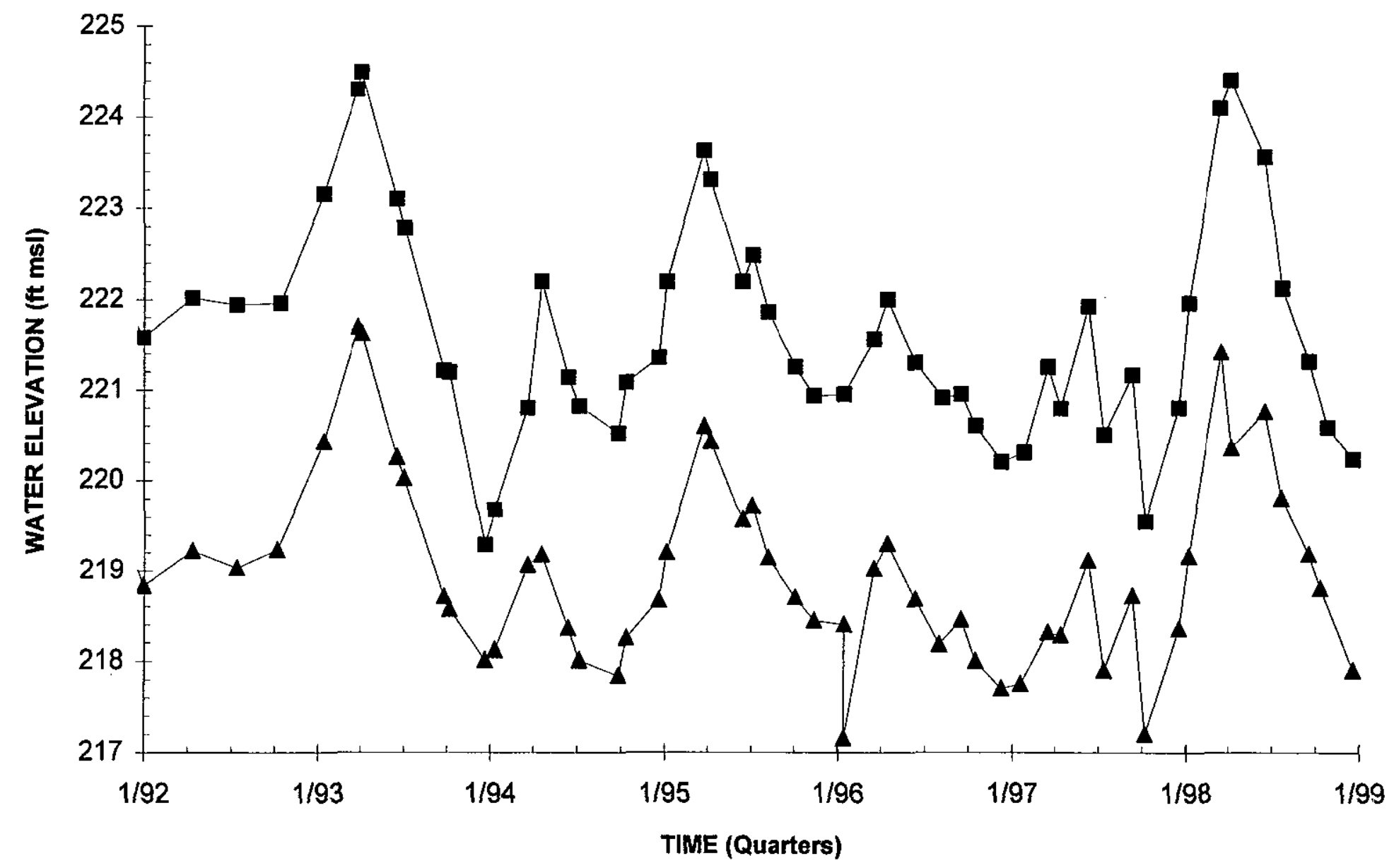

$-\mathrm{HSB110D}(\mathrm{W})-\mathrm{A}-\mathrm{HSB110C}(\mathrm{B})$

Note: W=Water Table (IIB2); B=Barnwell (IIB1); M=MCBean (IIB1); UC=Upper Congaree (IIA); MC=Middle Congaree (IIA); LC=Lower Congaree (IIA) 


\section{Hydrograph}

Well Cluster HSB111

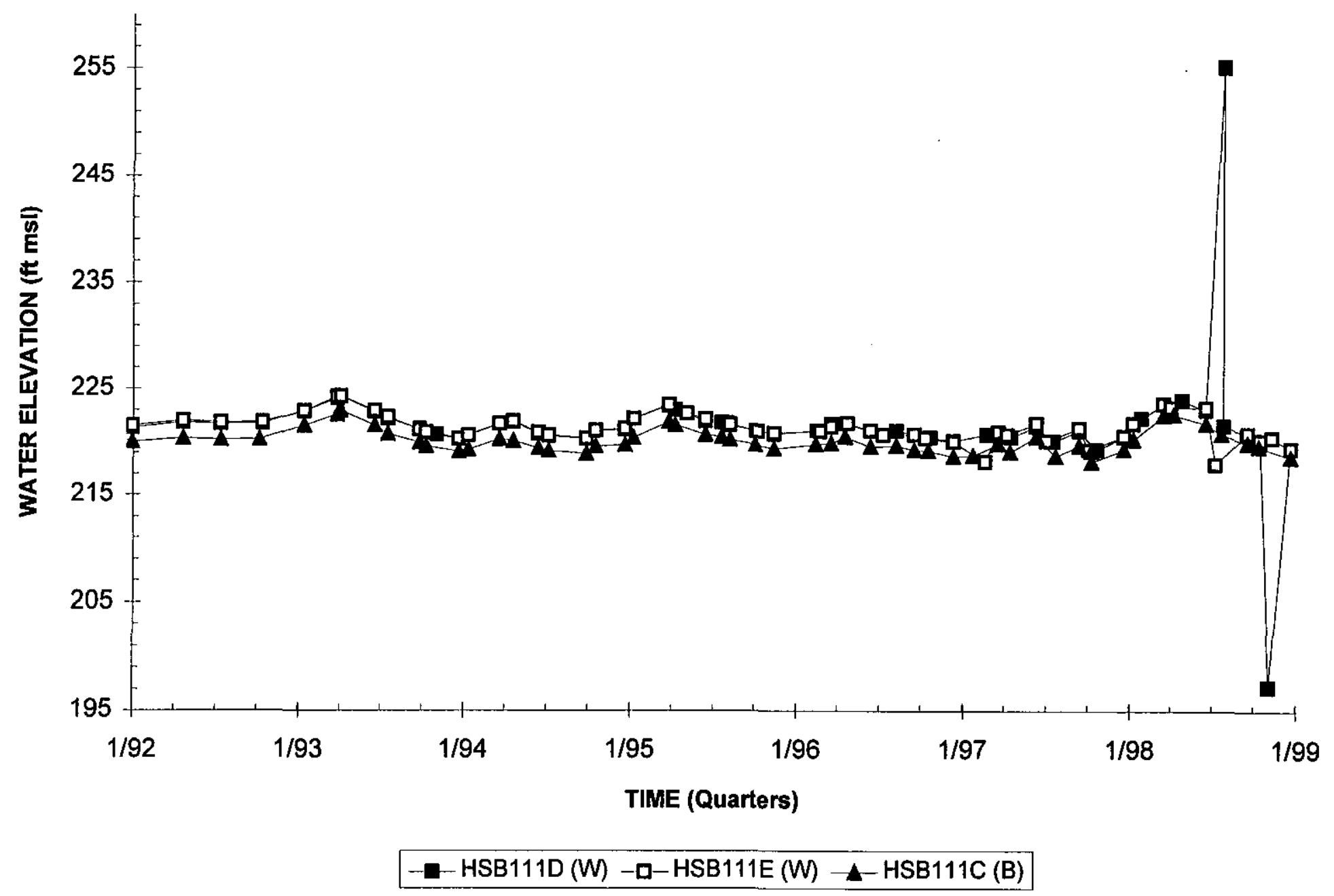

Note: W=Water Table (IIB2); B=Barnwell (IIB1); $M=M C B$ Bean (IIB1); UC=Upper Congaree (IIA); MC=Middle Congaree (IIA); LC=Lower Congaree (IIA) 


\section{Hydrograph \\ Well Cluster HSB112}

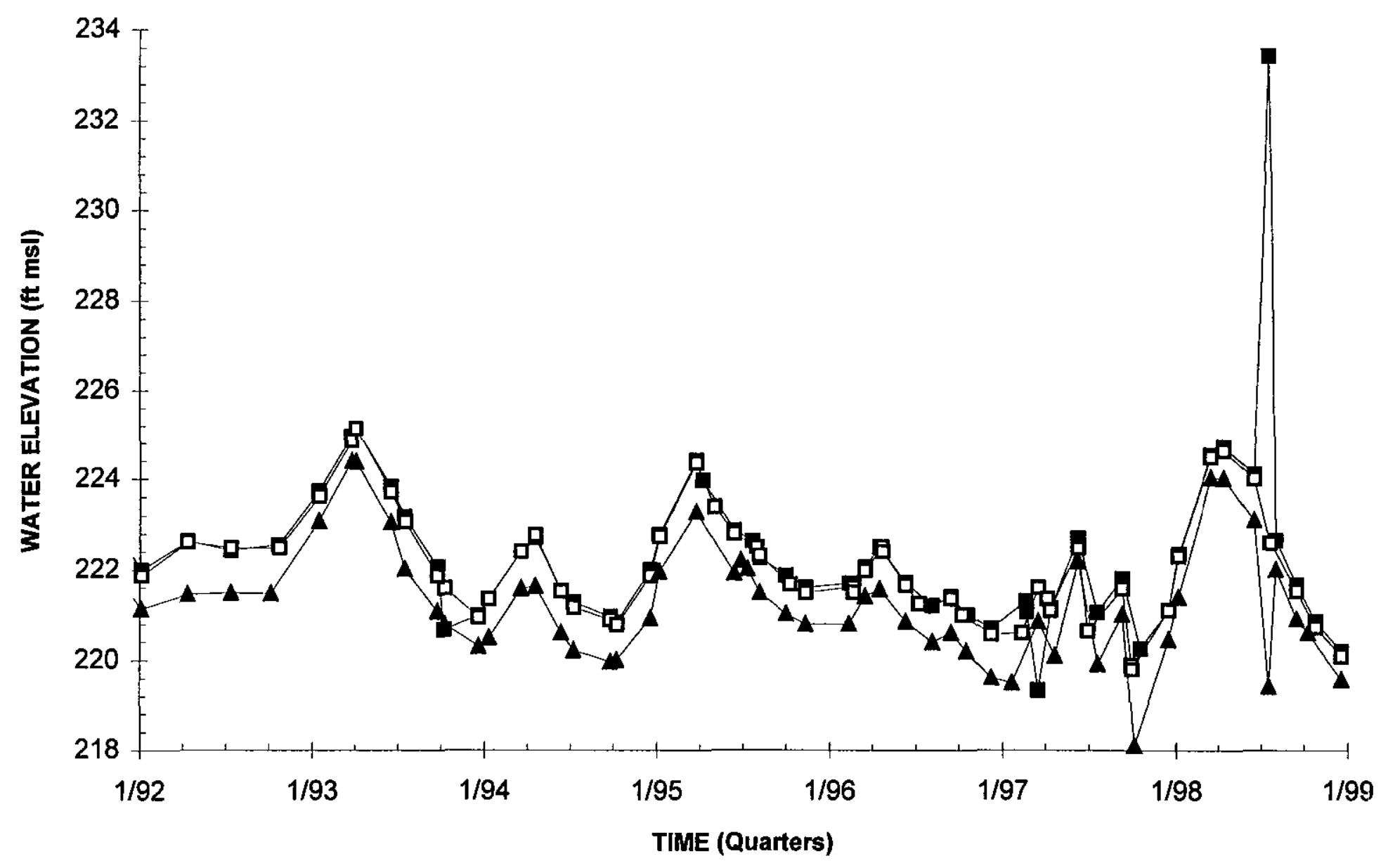

$-\mathrm{HSB} 112 \mathrm{D}(\mathrm{W})-\mathrm{HSB} 112 \mathrm{E}(\mathrm{W})-\mathrm{ASB} 112 \mathrm{C}(\mathrm{B})$

Note: $W=$ Water Table (IIB2); B=Barnwell (IIB1); M=McBean (IIB1); UC=Upper Congaree (IIA); MC=Middle Congaree (IIA); LC=Lower Congaree (IIA) 


\section{Hydrograph \\ Well Cluster HSB113}

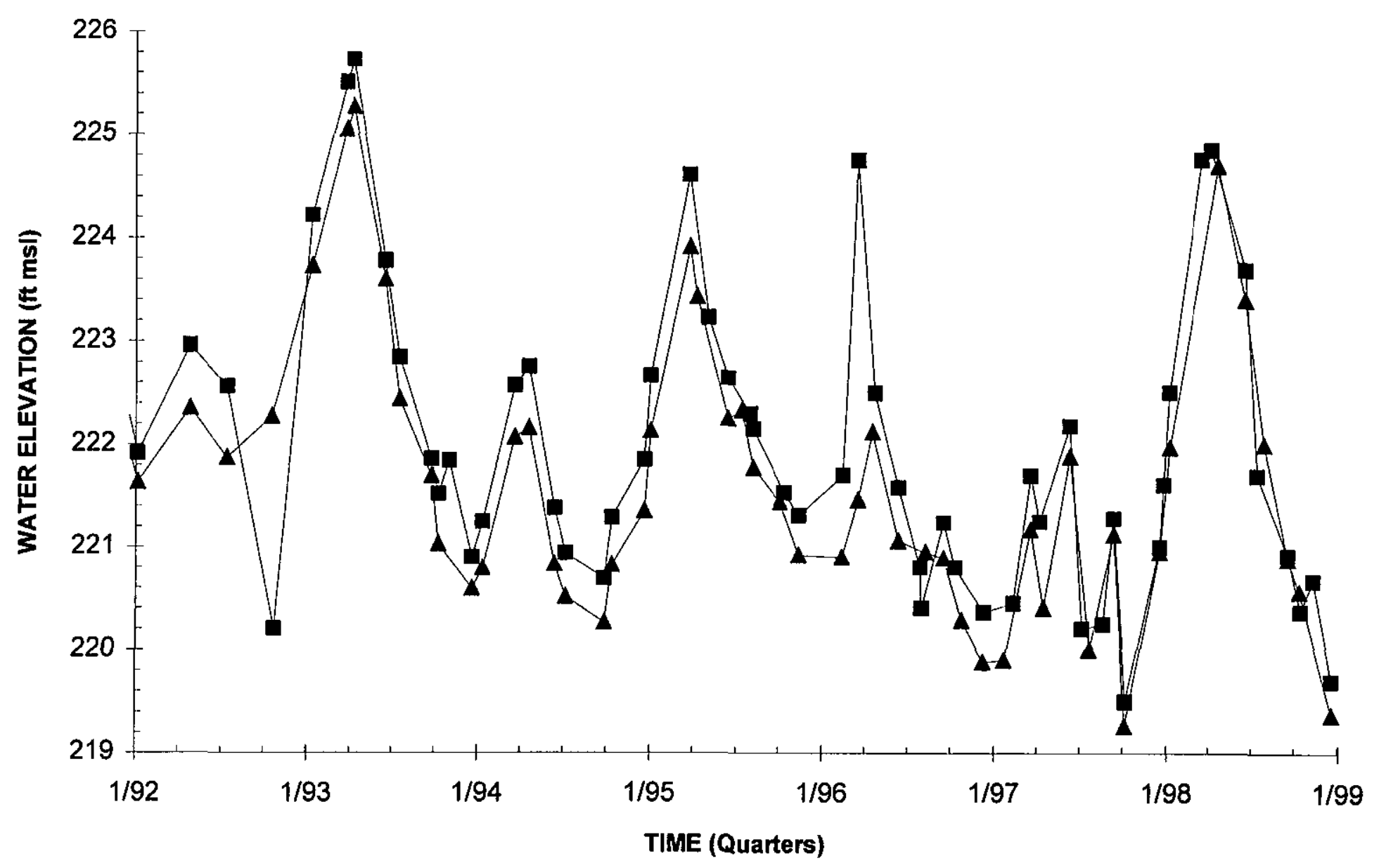

- HSB113D (W) -A-HSB113C (B)

Note: $W=$ Water Table (IIB2); B=Barnwell (IIB1); $M=M C B$ ean (IIB1); UC=Upper Congaree (IIA); MC=Middle Congaree (IIA); $L C=L$ tower Congaree (IIA) 


\section{Hydrograph \\ Well Cluster HSB114}

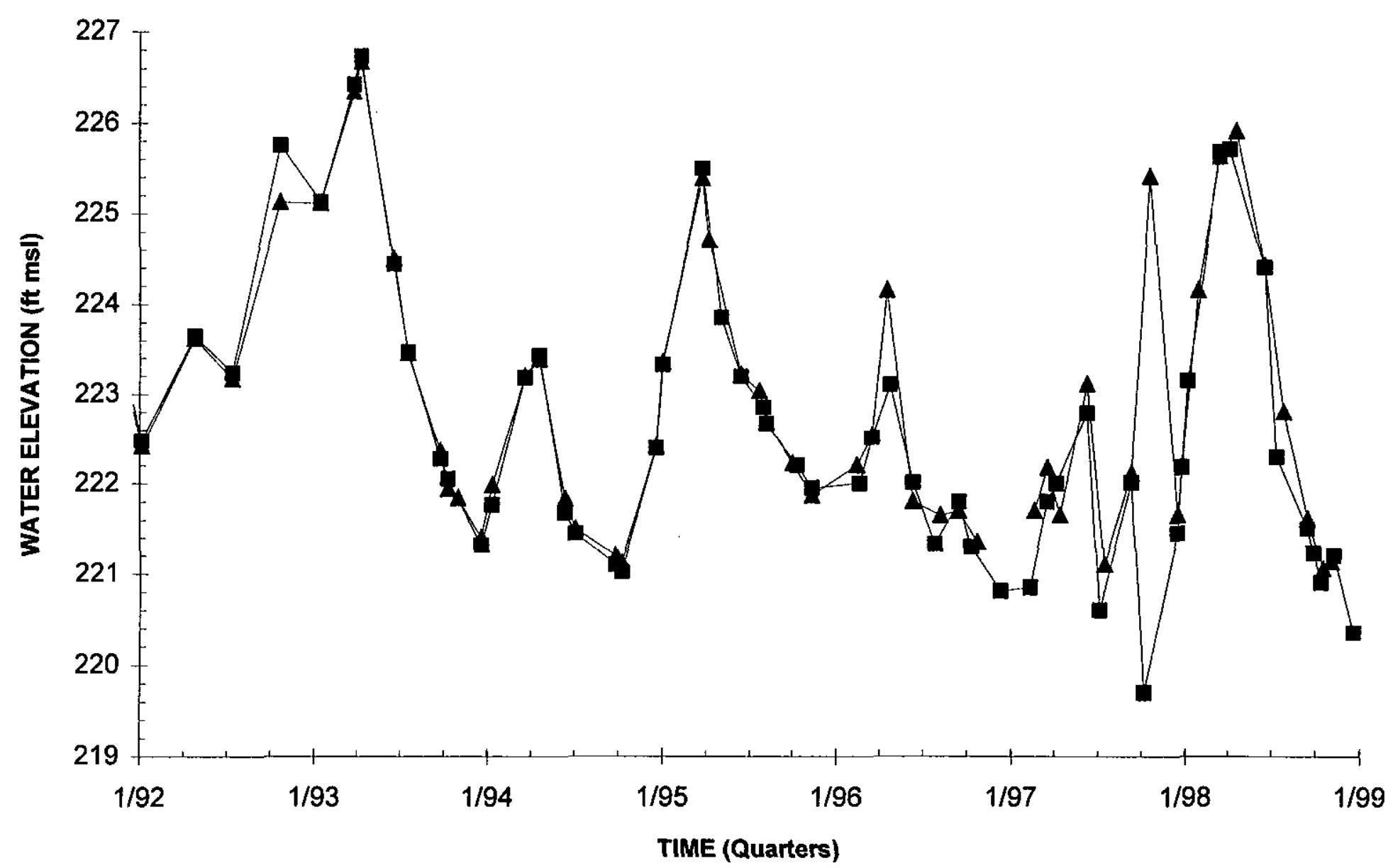

HSB114D (W) - - HSB114C (B)

Note: W=Water Table (IIB2); B=Barnwell (IIB1); M=MCBean (IIB1); UC=Upper Congaree (IIA); MC=Middle Congaree (IIA); LC=Lower Congaree (IIA) 


\section{Hydrograph \\ Well Cluster HSB115}

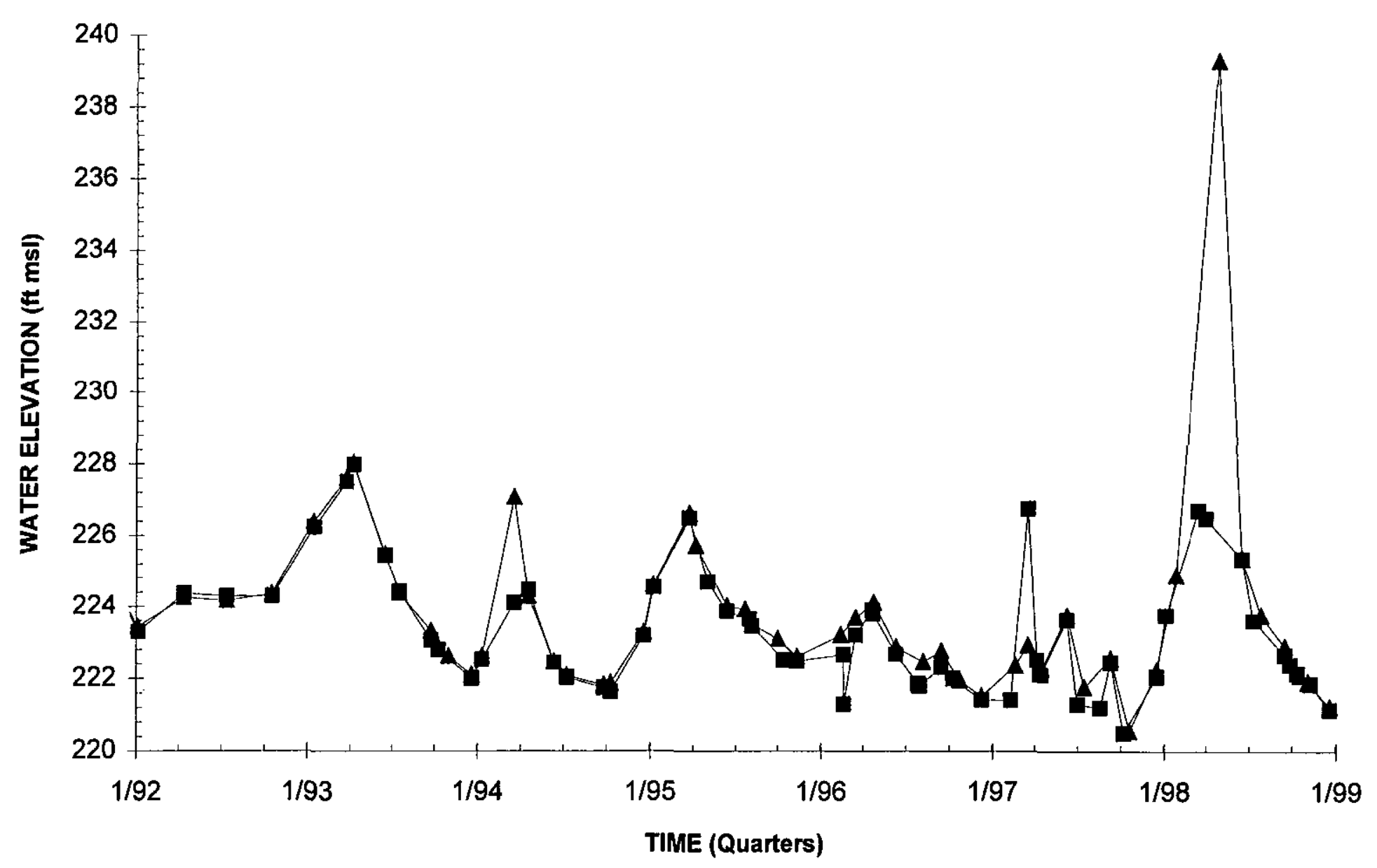

$-\mathrm{HSB} 115 \mathrm{D}(\mathrm{W})-\mathrm{HSB115C}(\mathrm{B})$

Note: $W=$ Water Table (IIB2); B=Barnwell (IIB1); M=McBean (IIB1); UC=Upper Congaree (IIA); MC=Middle Congaree (IIA); LC=Lower Congaree (IIA) 


\section{Hydrograph \\ Well Cluster HSB116}

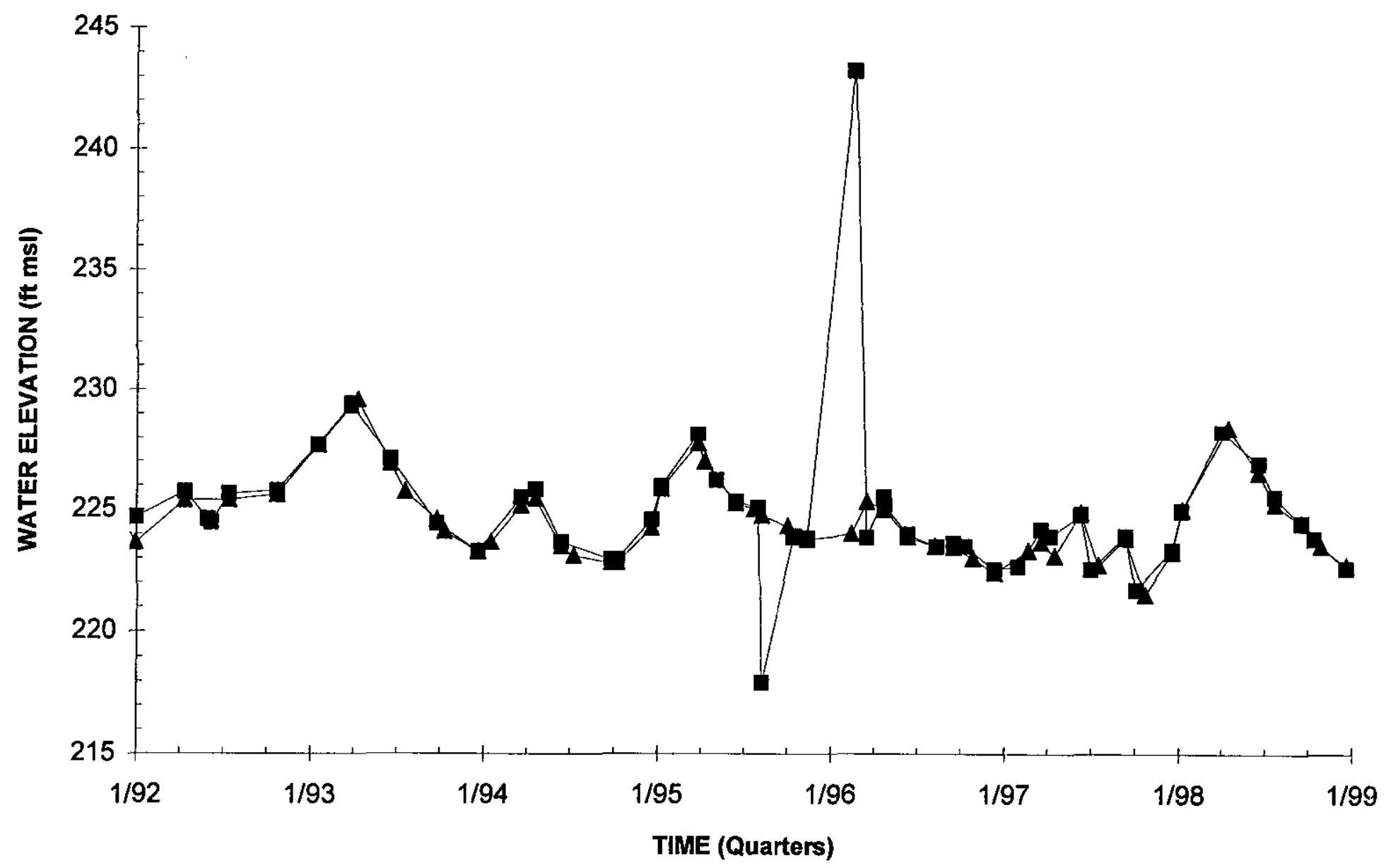

HSB116D (W) - HSB116C (B)

Note: $W=$ Water Table (IIB2); B=Barnwell (IIB1); M=McBean (IIB1); UC=Upper Congaree (IIA); MC=Middle Congaree (IIA); LC=Lower Congaree (IIA) 


\section{Hydrograph \\ Well Cluster HSB117}

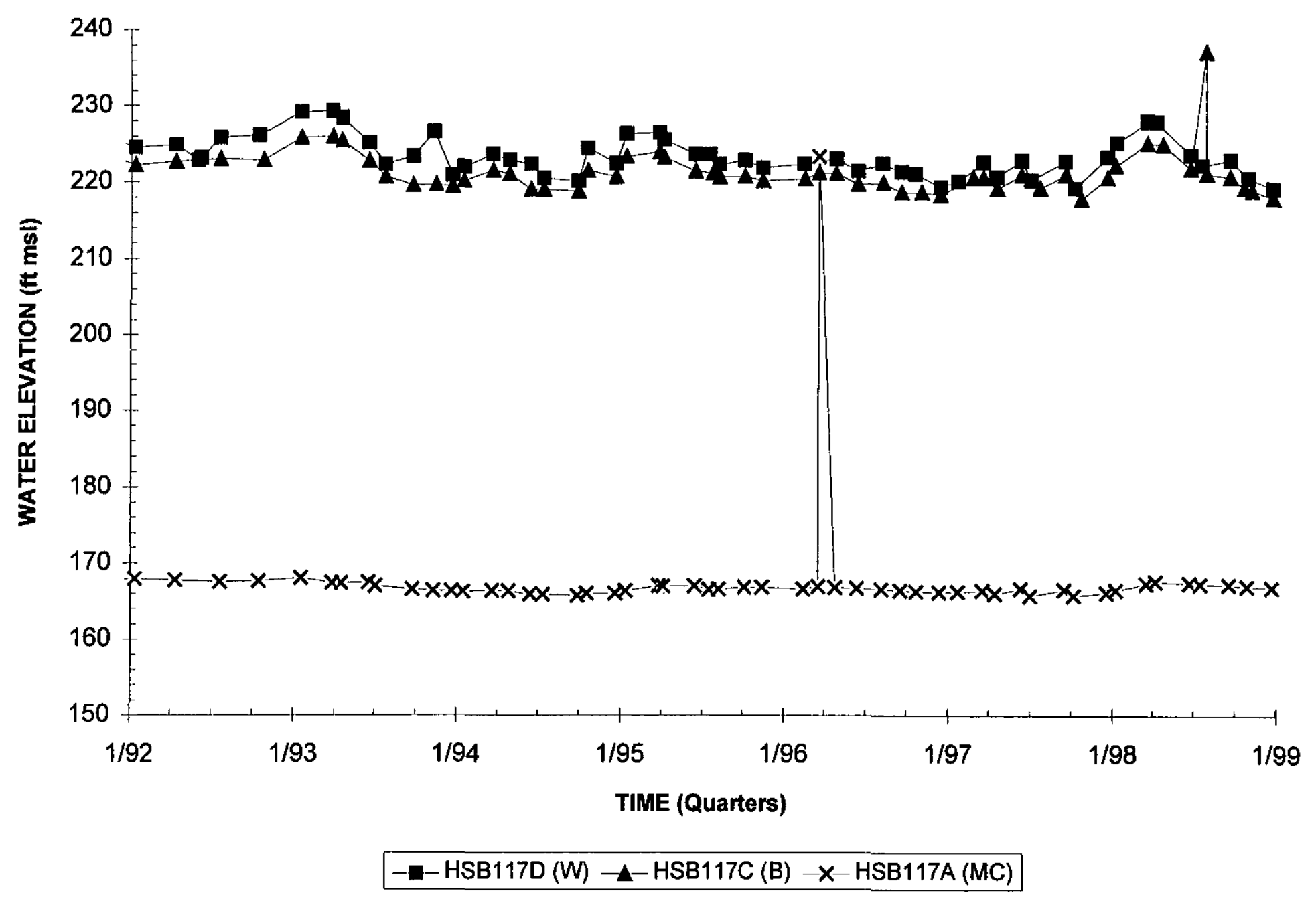

Note: W=Water Table (IIB2); B=Barnwell (IIB1); M=MCBean (IIB1); UC=Upper Congaree (IIA); MC=Middle Congaree (IIA); LC=Lower Congaree (IIA) 


\section{Hydrograph}

\section{Well HSB118A}

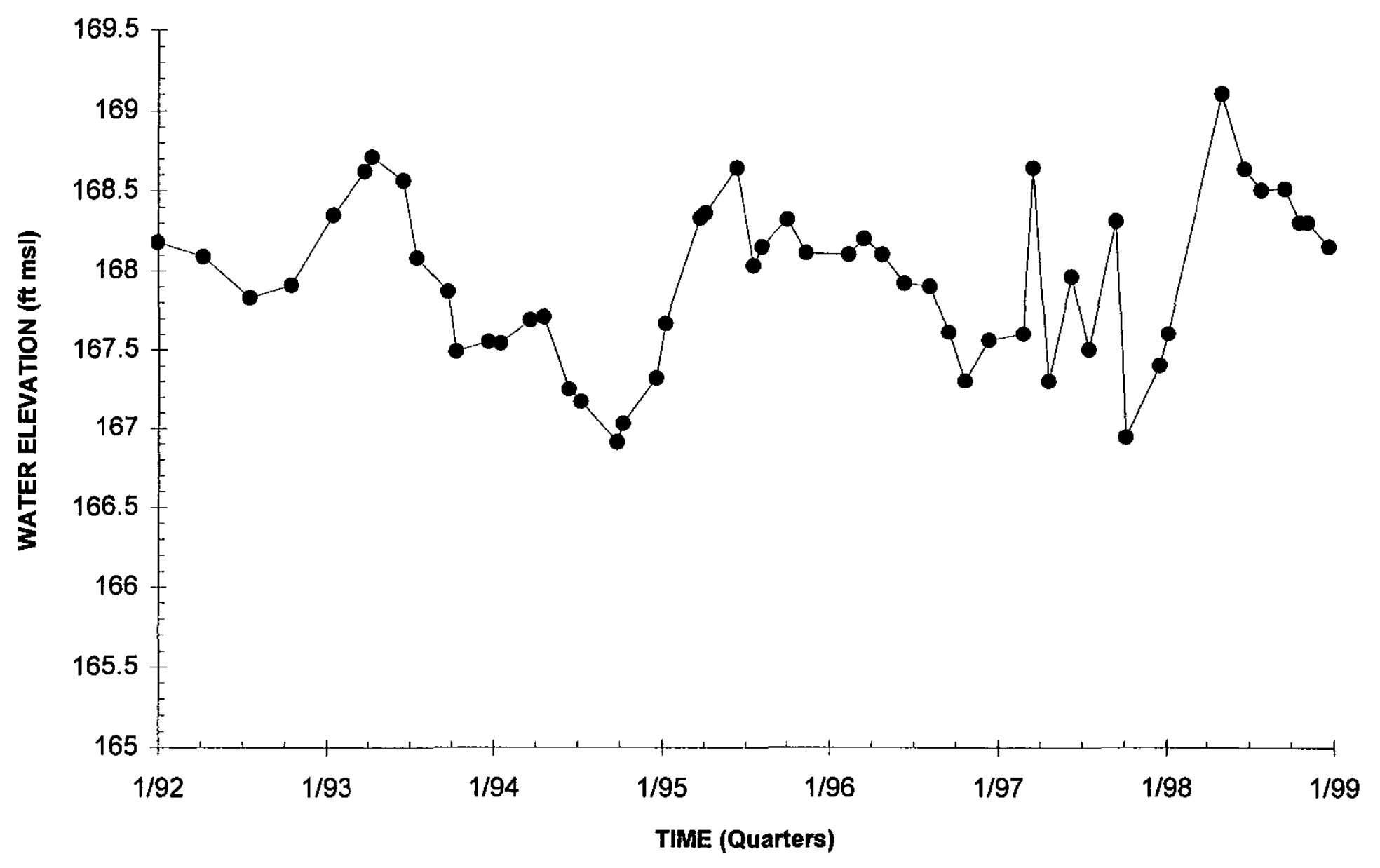

HSB118A (UC)

Note: W=Water Table (IIB2); B=Barnwell (IIB1); M=MCBean (IIB1); UC=Upper Congaree (IIA); MC=Middle Congaree (IIA); LC=Lower Congaree (IIA) 


\section{Hydrograph}

\section{Well HSB119A}

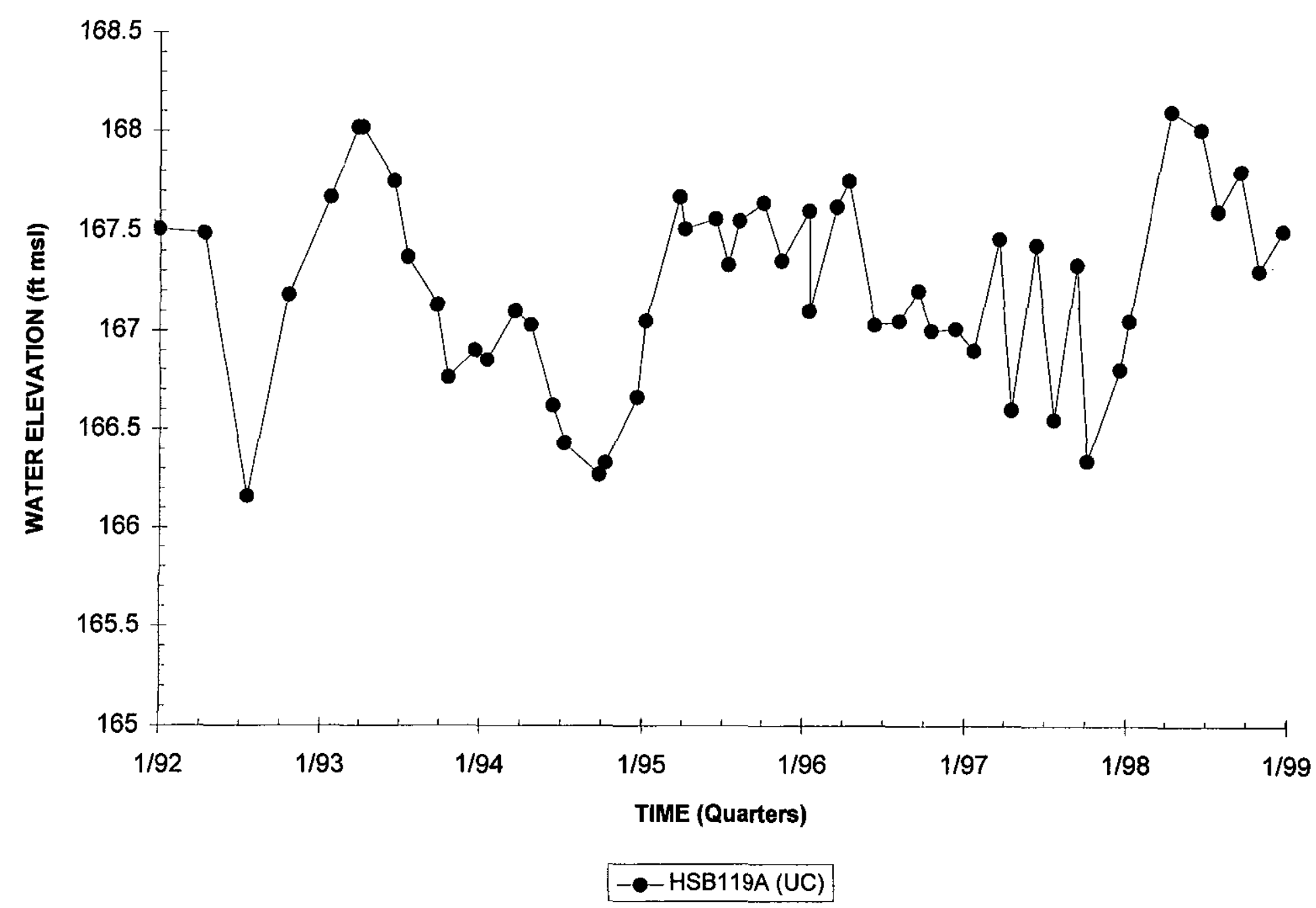

Note: $W=$ Water Table (IIB2); B=Barnwell (IIB1); M=McBean (IIB1); UC=Upper Congaree (IIA); MC=Middle Congaree (IIA); LC=Lower Congaree (IIA) 


\section{Hydrograph \\ Well HSB120A}

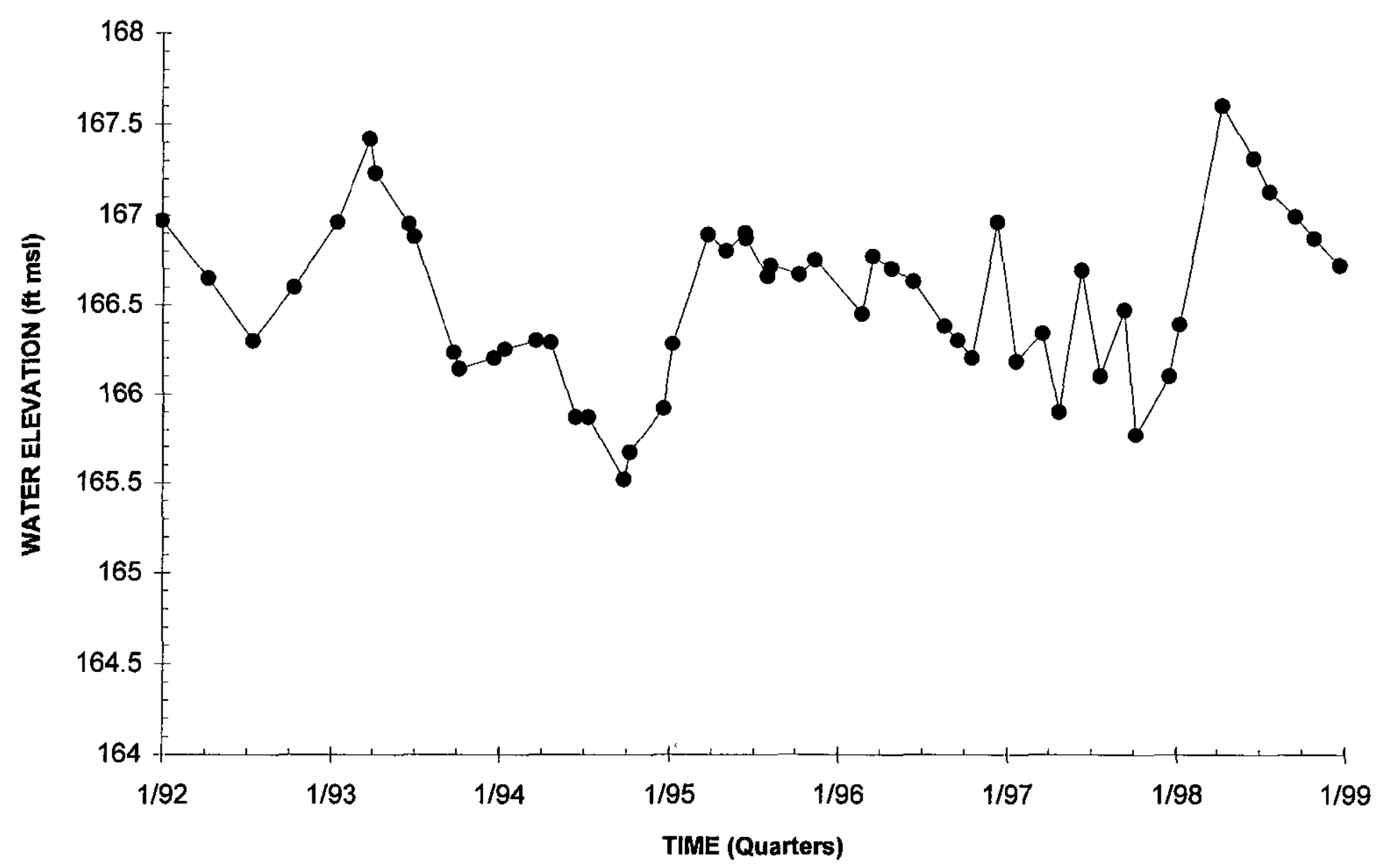

HSB120A (UC)

Note: $W=$ Water Table (IIB2); B=Barnwell (IIB1); M=MCBean (IIB1); UC=Upper Congaree (IIA); MC=Middle Congaree (IIA); LC=Lower Congaree (IIA)

H-Area HWMF

E - 34

Third and Fourth Quarter 1998
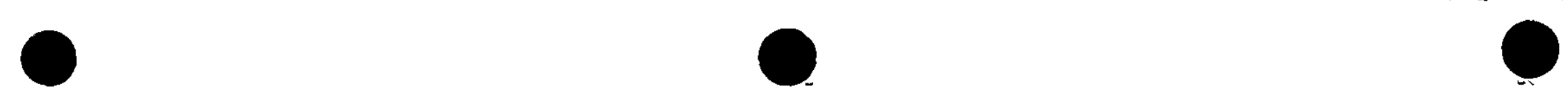


\section{Hydrograph}

\section{Well HSB121A}

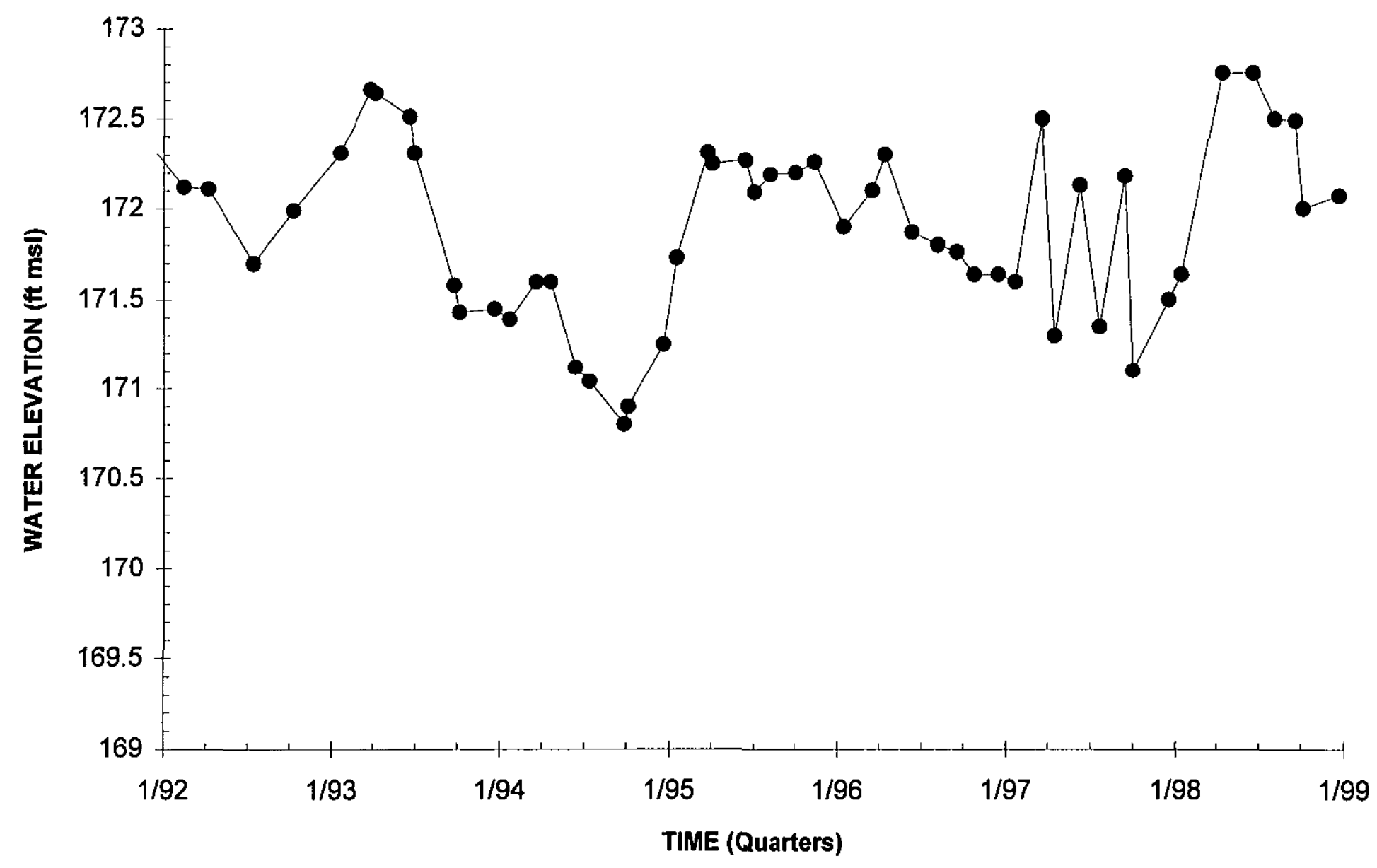

- HSB121A (UC)

Note: W=Water Table (IIB2); B=Barnwell (IIB1); M=McBean (IIB1); UC=Upper Congaree (IIA); MC=Middle Congaree (IIA); LC=Lower Congaree (IIA) 


\section{Hydrograph \\ Well HSB122A}

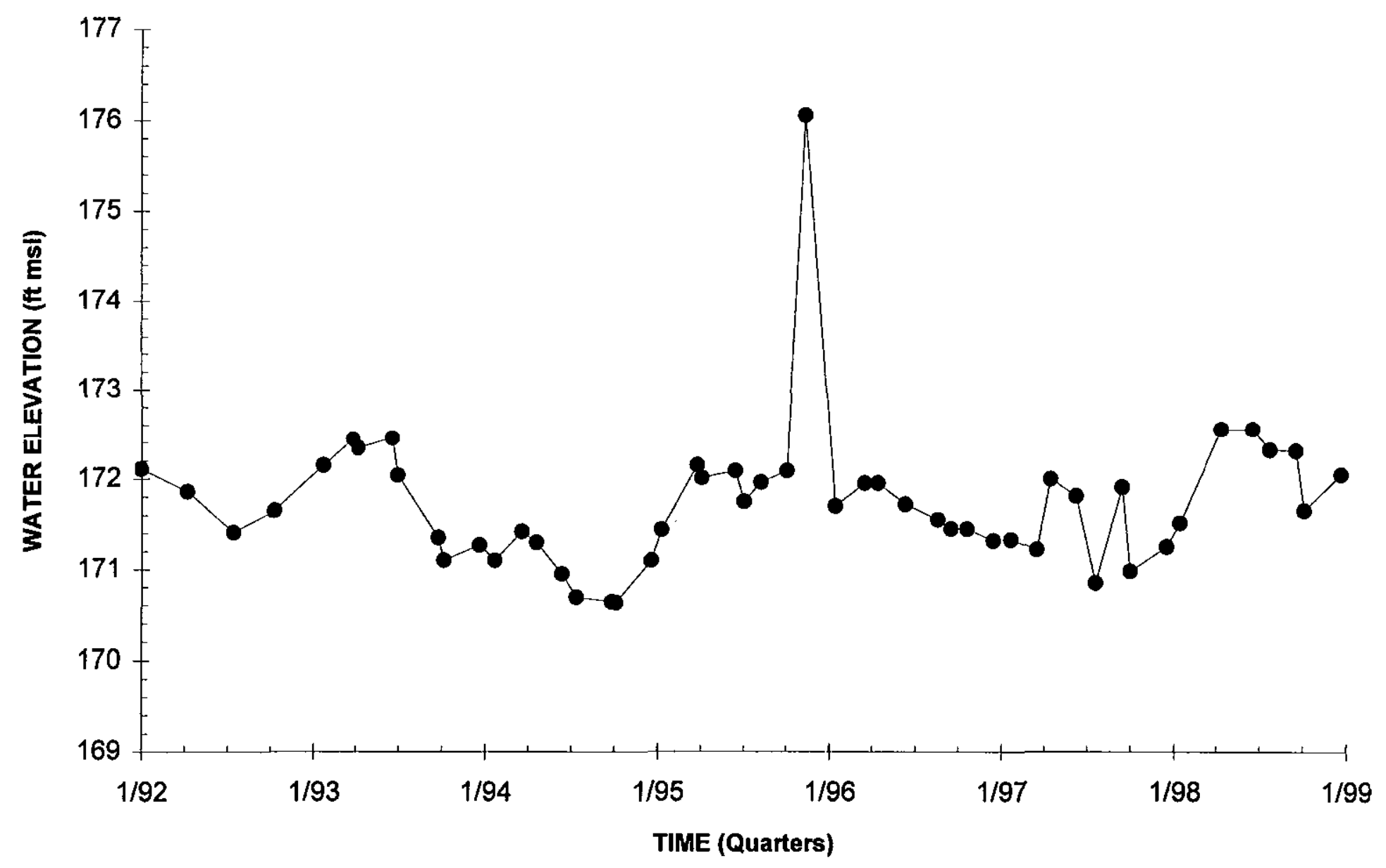

$$
\text { - HSB122A (UC) }
$$

Note: $W=$ Water Table (IIB2); B=Barnwell (IIB1); M=McBean (IIB1); UC=Upper Congaree (IIA); MC=Middle Congaree (IIA); LC=Lower Congaree (IIA) 


\section{Hydrograph}

Well HSB123A

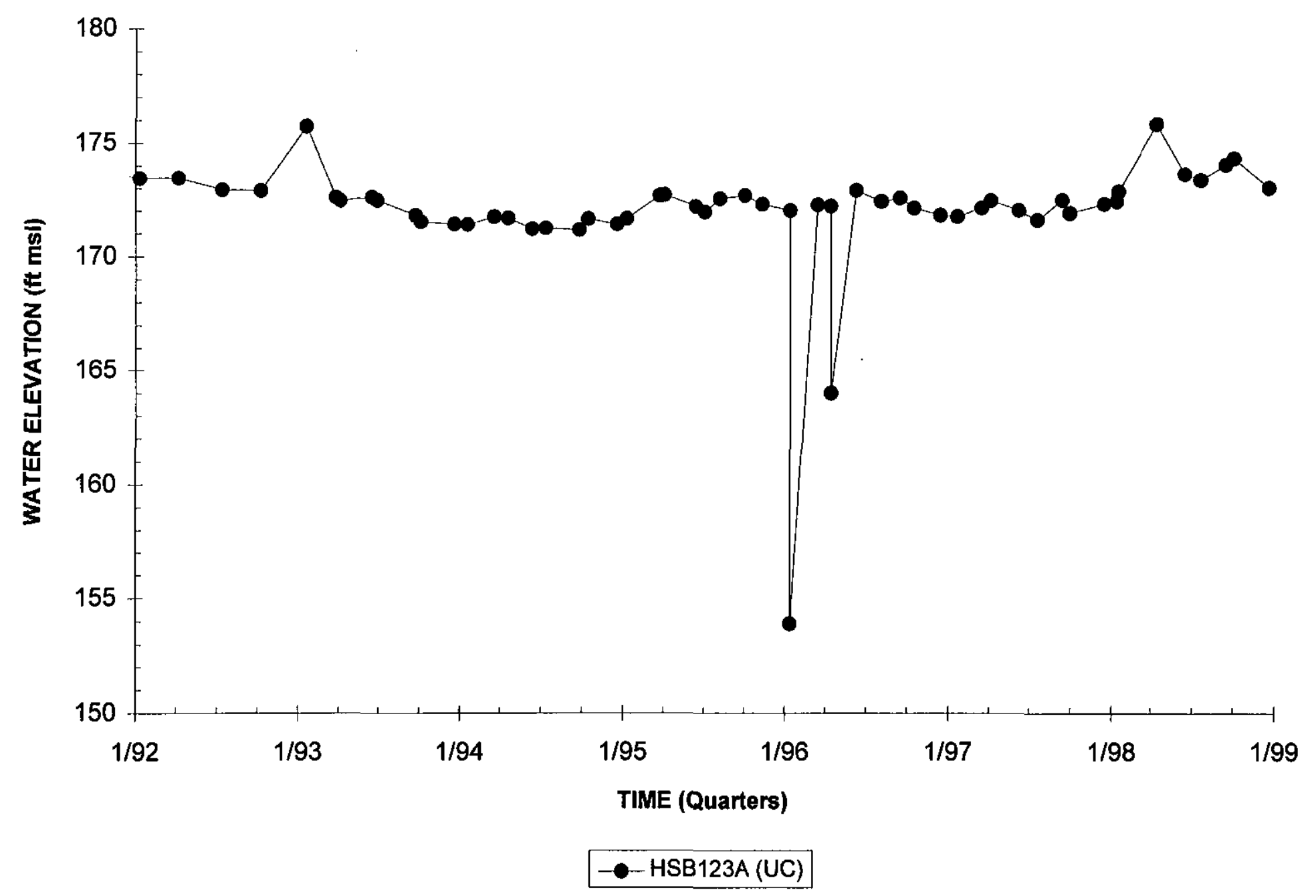

Note: W=Water Table (IIB2); B=Barnwell (IIB1); M=McBean (IIB1); UC=Upper Congaree (IIA); MC=Middle Congaree (IIA); LC=Lower Congaree (IIA) 


\section{Hydrograph \\ Well HSB124AR}

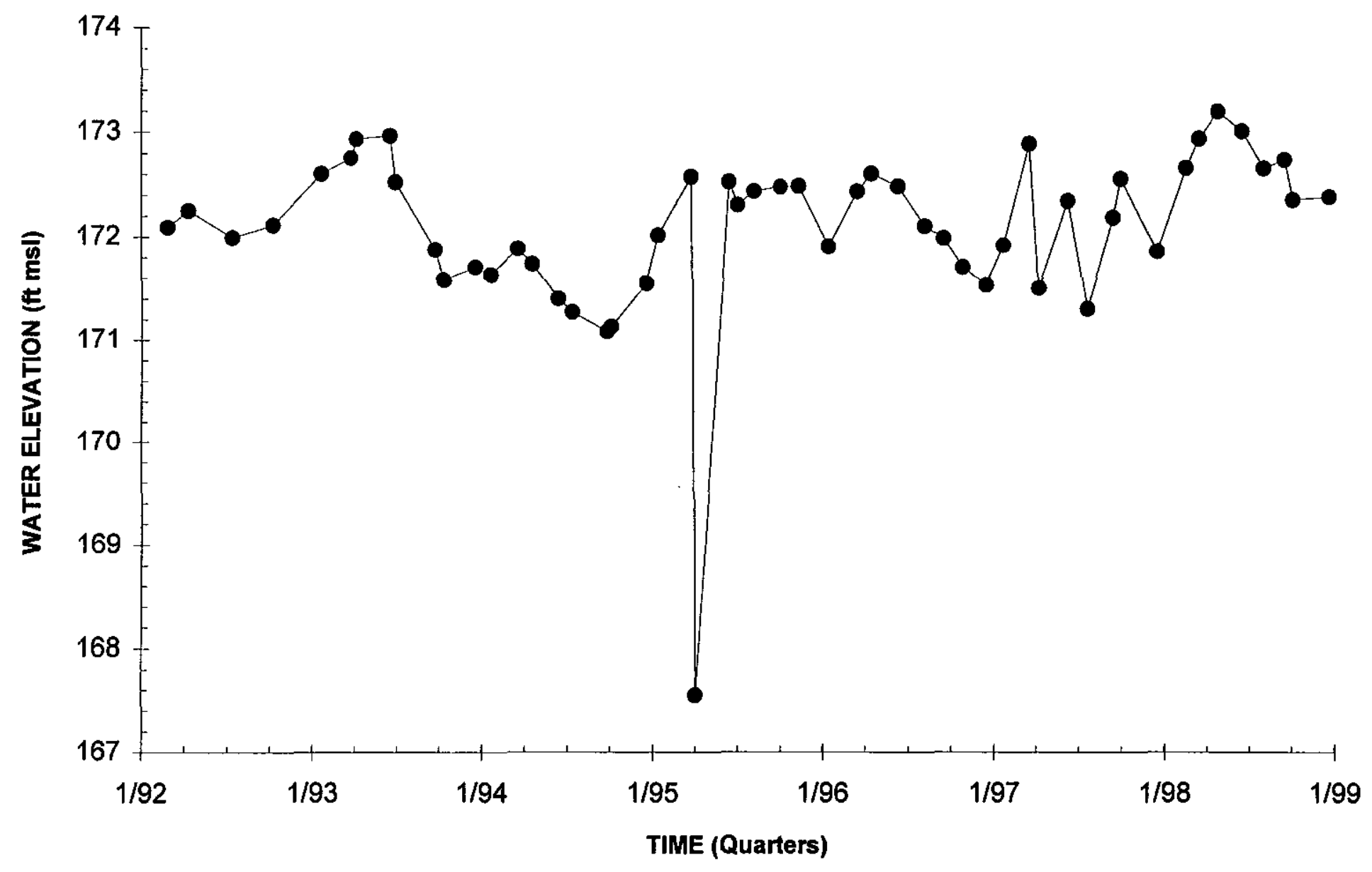

- HSB124AR (UC)

Note: W=Water Table (IIB2); B=Barnwell (IIB1); M=McBean (IIB1); UC=Upper Congaree (IIA); MC=Middle Congaree (IIA); LC=Lower Congaree (IIA) 


\section{Hydrograph \\ Well Cluster HSB125}

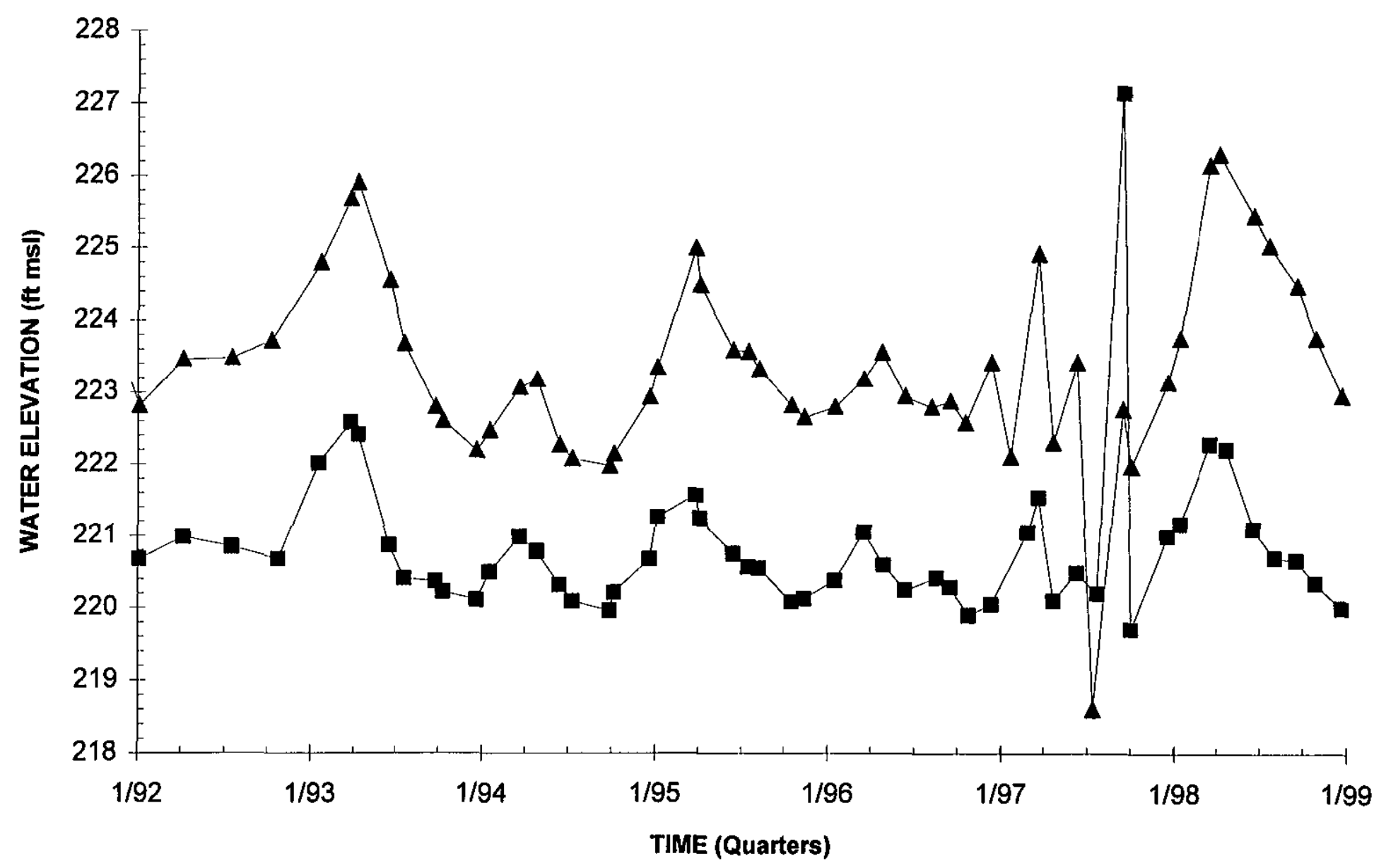

$$
-\mathrm{HSB125D}(\mathrm{W}) \_\mathrm{HSB125C}(\mathrm{B})
$$

Note: W=Water Table (IIB2); B=Barnwell (IIB1); M=McBean (IIB1); UC=Upper Congaree (IIA); MC=Middle Congaree (IIA); LC=Lower Congaree (IIA) 


\section{Hydrograph \\ Well Cluster HSB126}

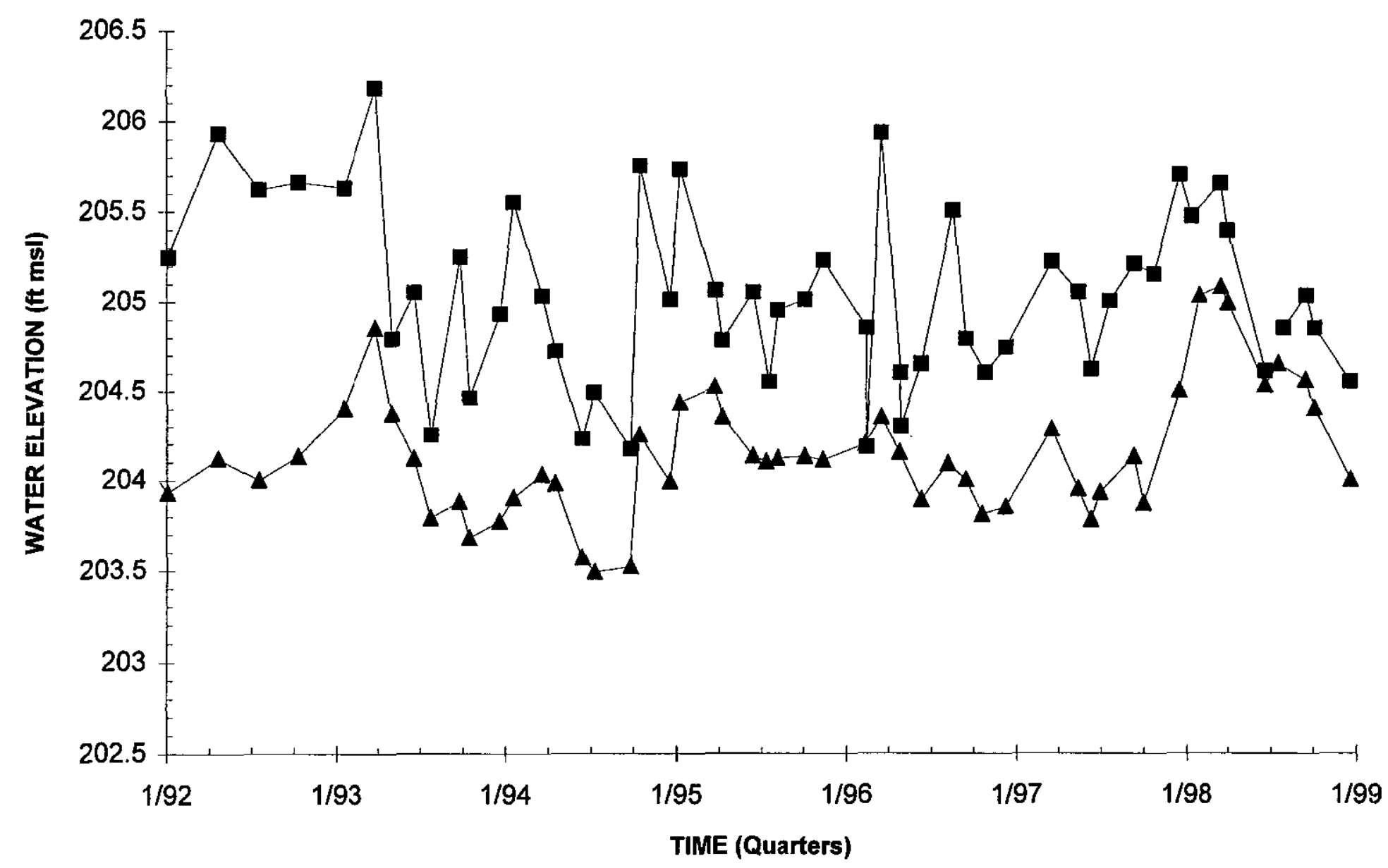

$\mathrm{HSB126D}(\mathrm{W})-\mathrm{ASB126C}(\mathrm{B})$

Note: W=Water Table (IIB2); B=Barnwell (IIB1); M=McBean (IIB1); UC=Upper Congaree (IIA); MC=Middle Congaree (IIA); LC=Lower Congaree (IIA)

H-Area HWMF

$E-40$

Third and Fourth Quarter 1998

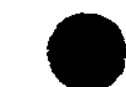




\section{Hydrograph \\ Well Cluster HSB127}

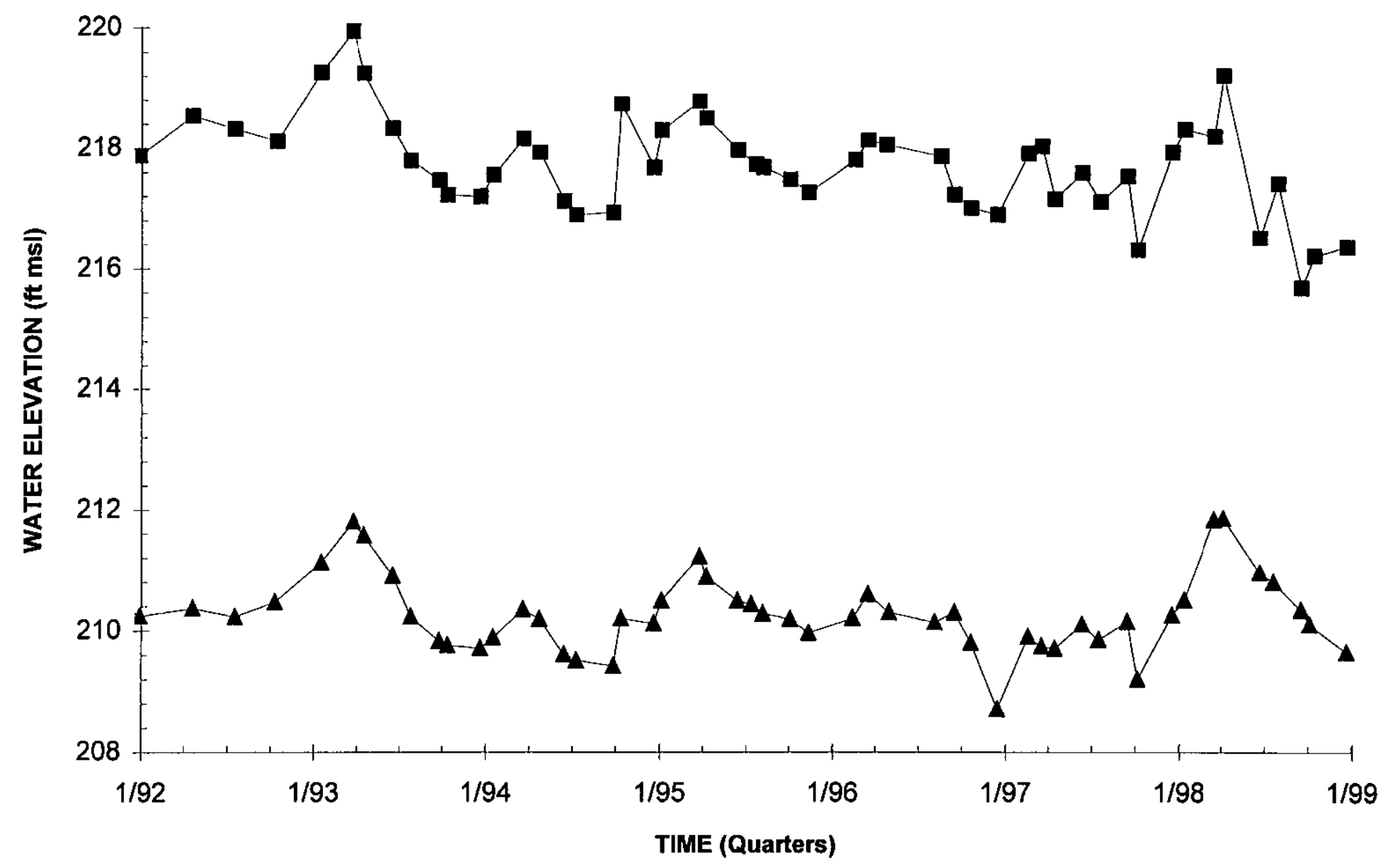

- HSB127D (W) -A-HSB127C (B)

Note: $W=$ Water Table (IIB2); B=Barnwell (IIB1); $M=M C B e a n$ (IIB1); UC=Upper Congaree (IIA); MC=Middle Congaree (IIA); LC=Lower Congaree (IIA) 


\section{Hydrograph \\ Well Cluster HSB129}

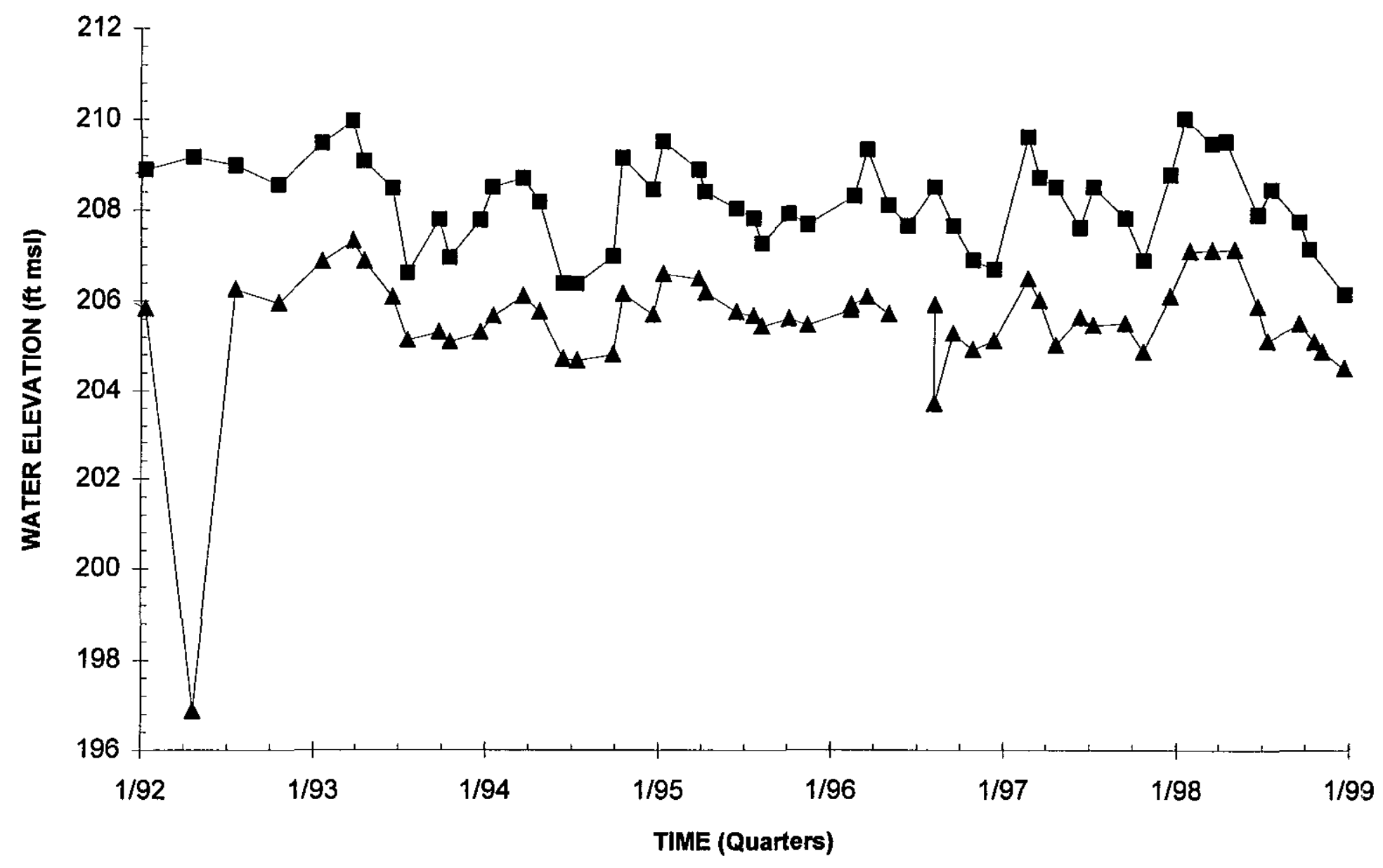

$-H S B 129 D(W)-H S B 129 C(B)$

Note: W=Water Table (IIB2); B=8arnwell (IIB1); M=McBean (IIB1); UC=Upper Congaree (IIA); MC=Middle Congaree (IIA); LC=Lower Congaree (IIA)

H-Area HWMF 
Hydrograph

Well Cluster HSB130

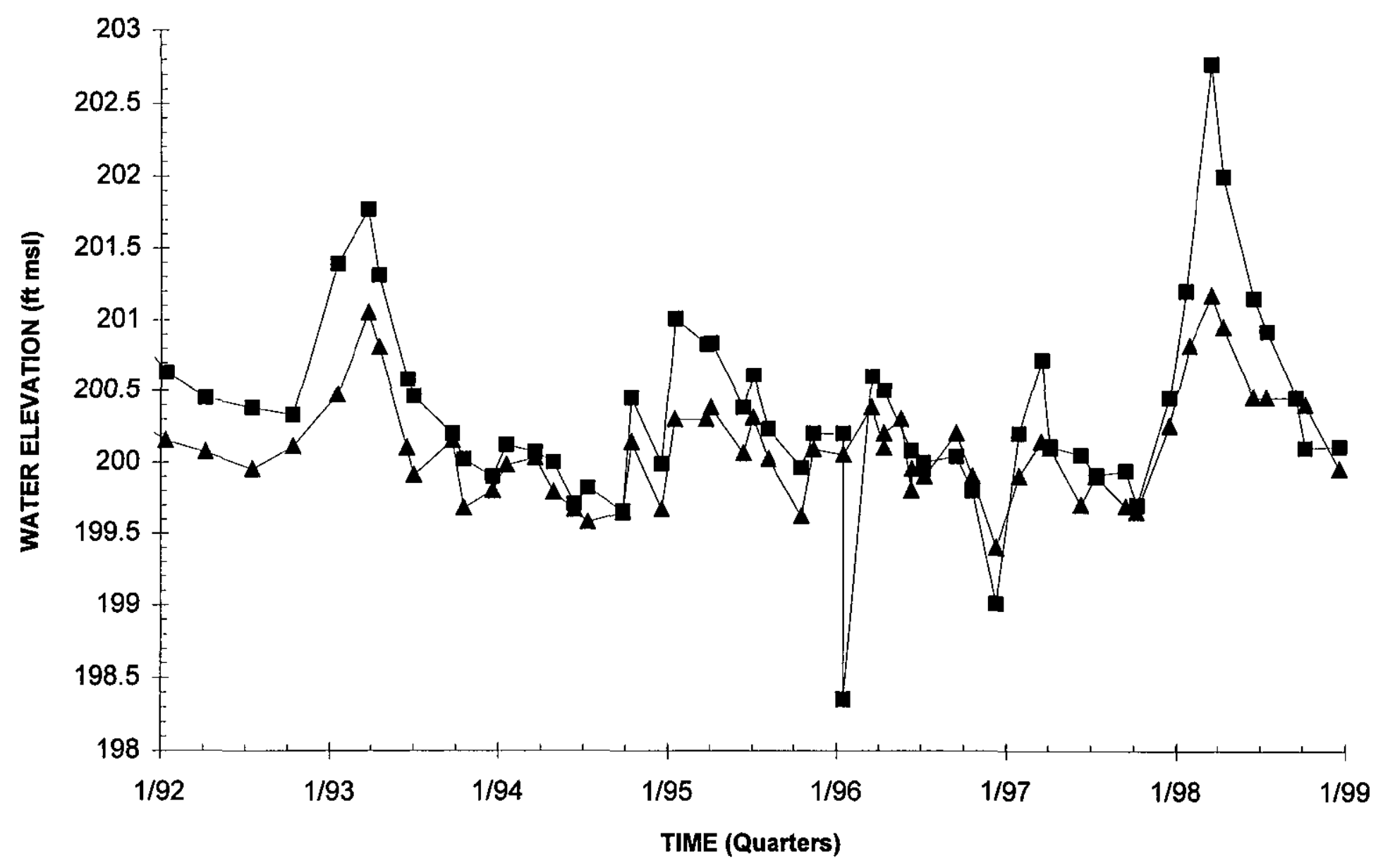

$-\operatorname{HSB130D}(\mathrm{W})-\mathbf{A}-\mathrm{HSB} 130 \mathrm{C}(\mathrm{B})$

Note: W=Water Table (IIB2); B=Barnwell (IIB1); M=McBean (IIB1); UC=Upper Congaree (IIA); MC=Middle Congaree (IIA); LC=Lower Congaree (IIA) 


\section{Hydrograph \\ Well Cluster HSB131}

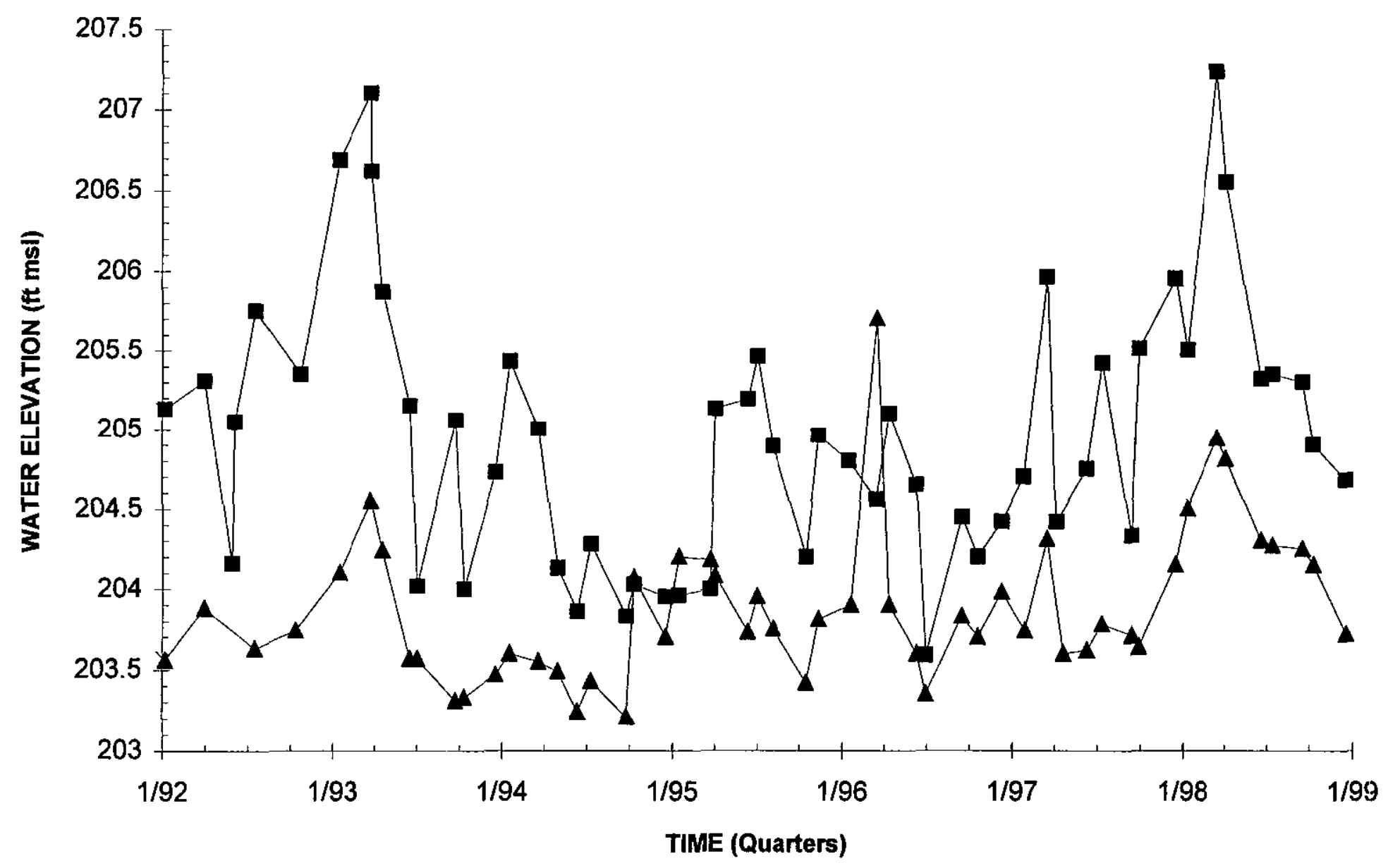

- - HSB131D (W)-HSB131C (B)

Note: $W=$ Water Table (IIB2); B=Barnwell (IIB1); M=McBean (IIB1); UC=Upper Congaree (IIA); MC=Middle Congaree (IIA); LC=Lower Congaree (IIA) 


\section{Hydrograph}

\section{Well Cluster HSB132}

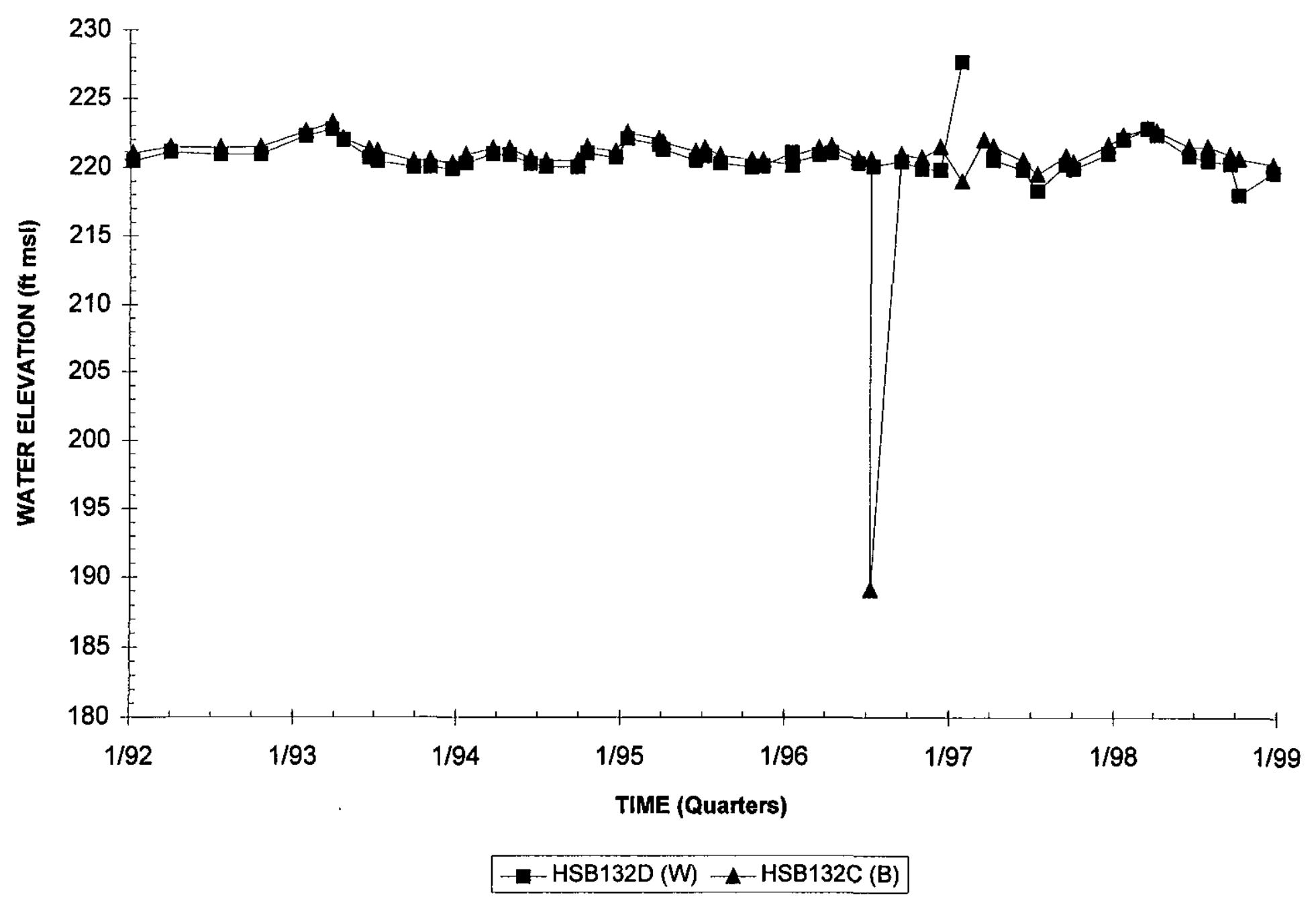

Note: $W=$ Water Table (IIB2); B=Barnwell (IBB1); M=McBean (IIB1); UC=Upper Congaree (IIA); MC=Middle Congaree (IIA); LC=Lower Congaree (IIA) 


\section{Hydrograph \\ Well Cluster HSB133}

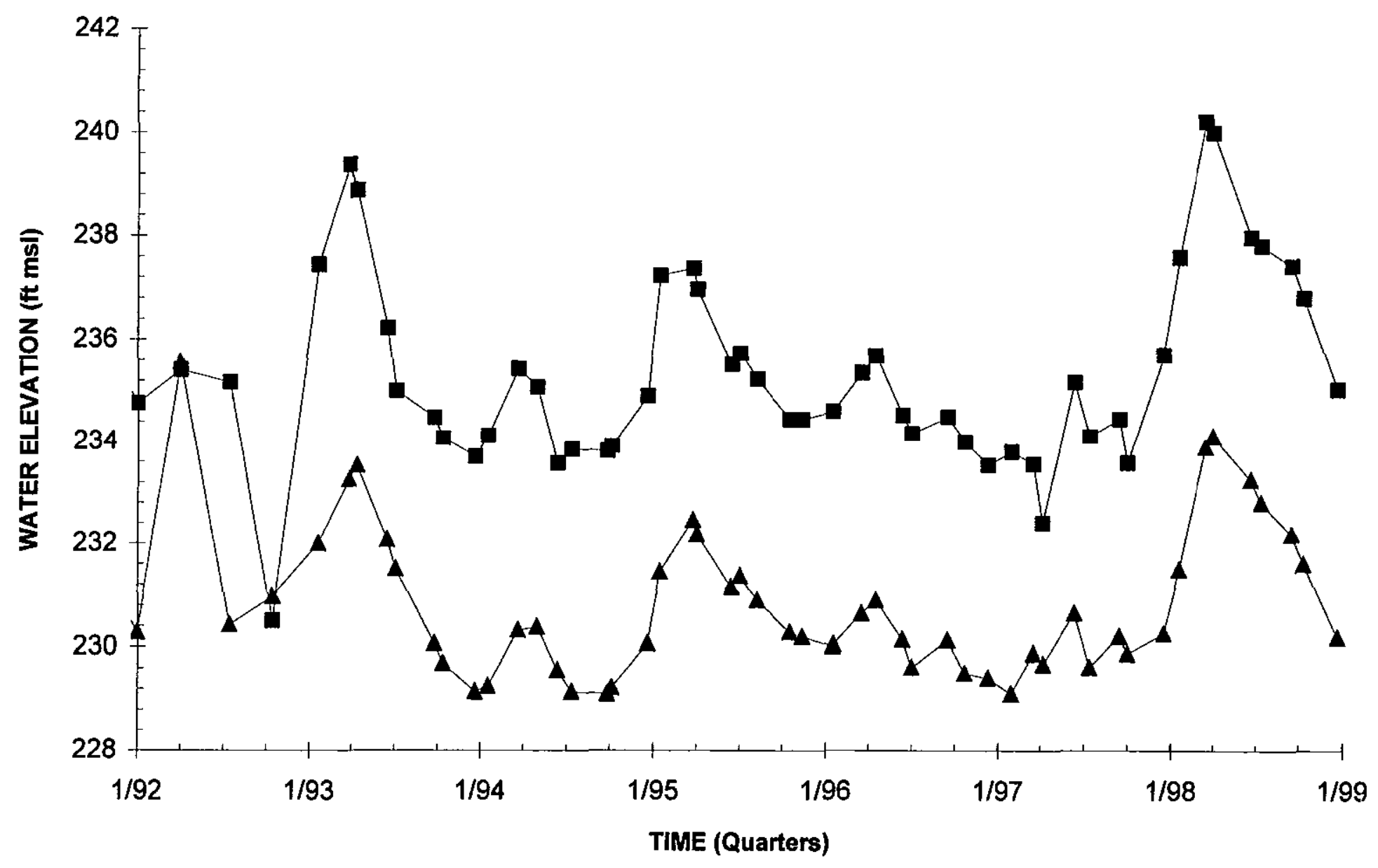

- HSB133D (W) - HSB133C (B)

Note: W=Water Table (IIB2); B=Barnwell (IIB1); M=McBean (IIB1); UC=Upper Congaree (IIA); MC=Middle Congaree (IIA); LC=Lower Congaree (IIA) 


\section{Hydrograph}

Well Cluster HSB134

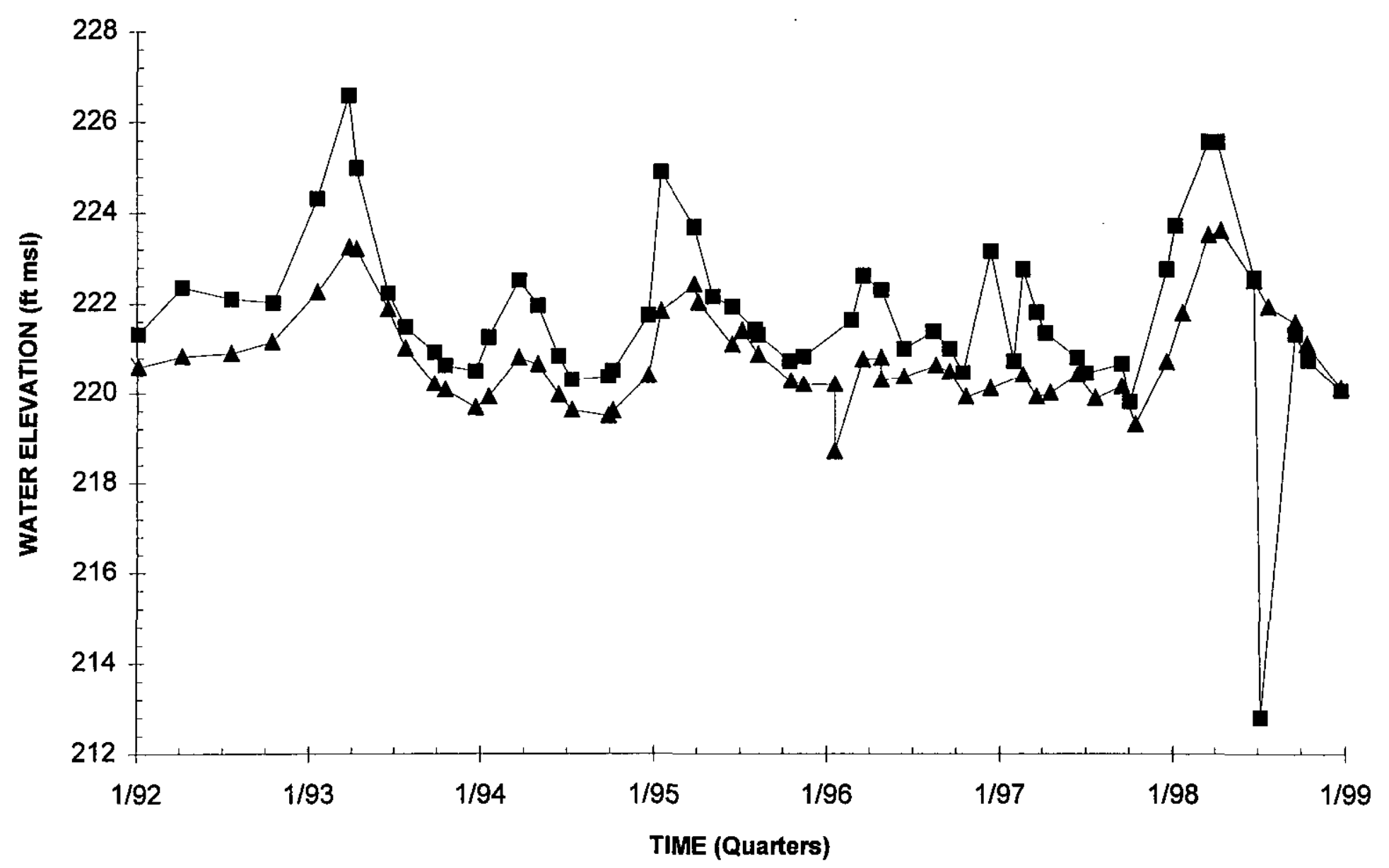

- HSB134D (W) - A-HSB134C (B)

Note: $W=$ Water Table (IIB2); B=Barnwell (IIB1); M=McBean (IIB1); UC=Upper Congaree (IIA); MC=Middle Congaree (IIA); LC=Lower Congaree (IIA) 


\section{Hydrograph \\ Well Cluster HSB135}

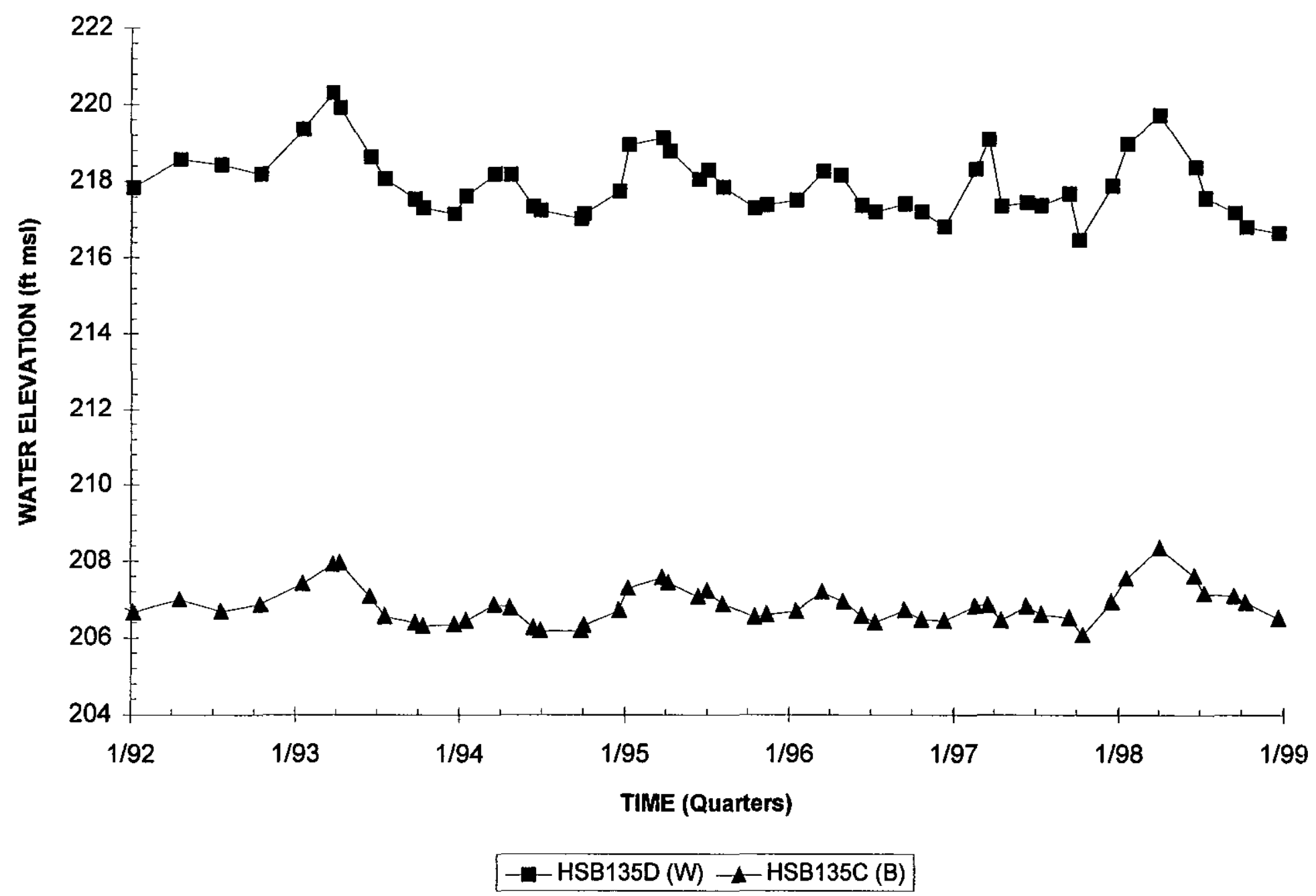

Note: $W=$ Water Table (IIB2); B=Barnwell (IIB1); M=McBean (IIB1); UC=Upper Congaree (IIA); MC=Middle Congaree (IIA); LC=Lower Congaree (IIA) 


\section{Hydrograph}

\section{Well Cluster HSB136}

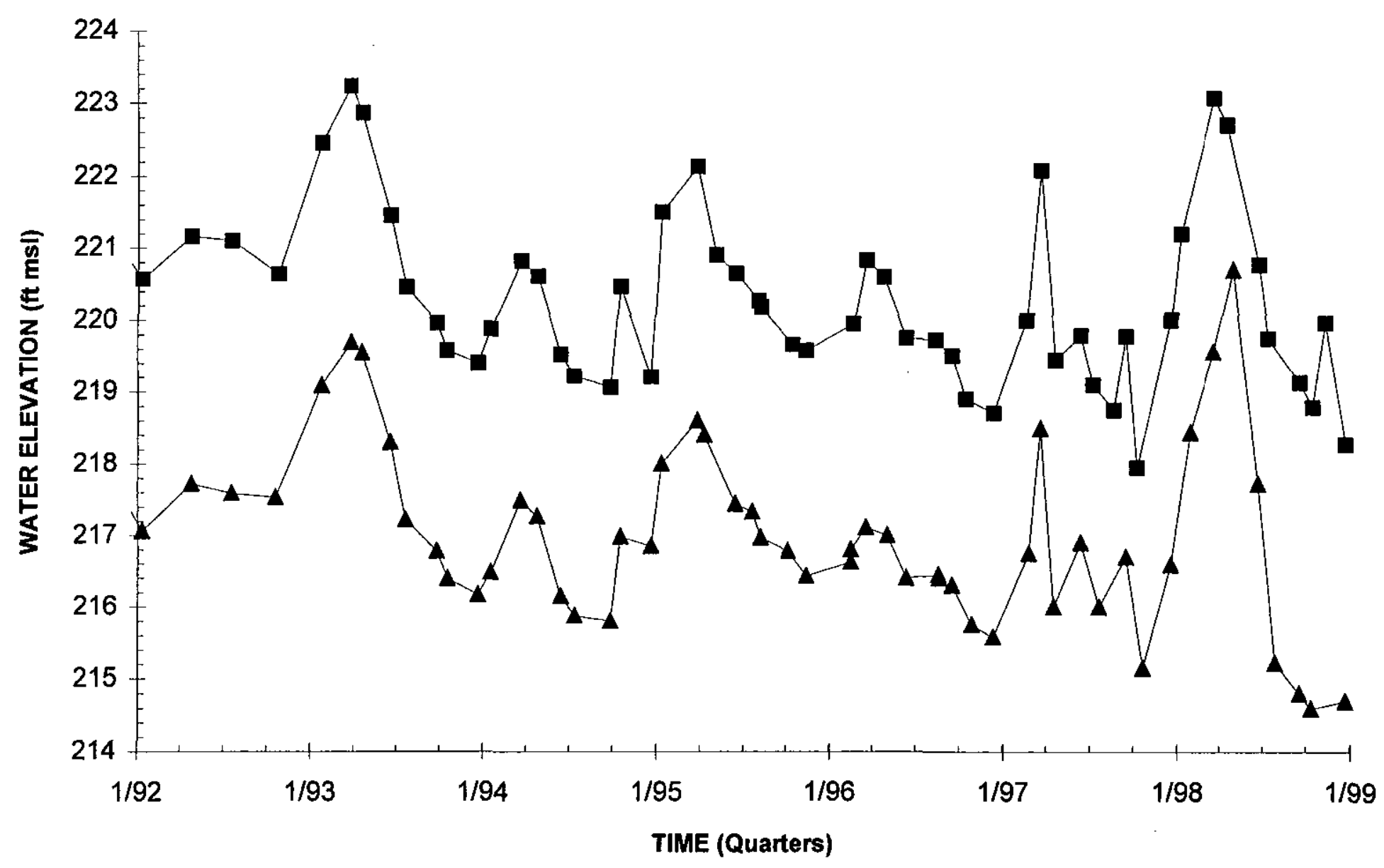

$-\mathrm{HSB136D}(\mathrm{W})-\mathrm{HSB136C}(\mathrm{B})$

Note: W=Water Table (IIB2); B=Barnwell (IIB1); M=McBean (IBB1); UC=Upper Congaree (IIA); MC=Middle Congaree (IIA); LC=Lower Congaree (IIA) 


\section{Hydrograph \\ Well Cluster HSB137}

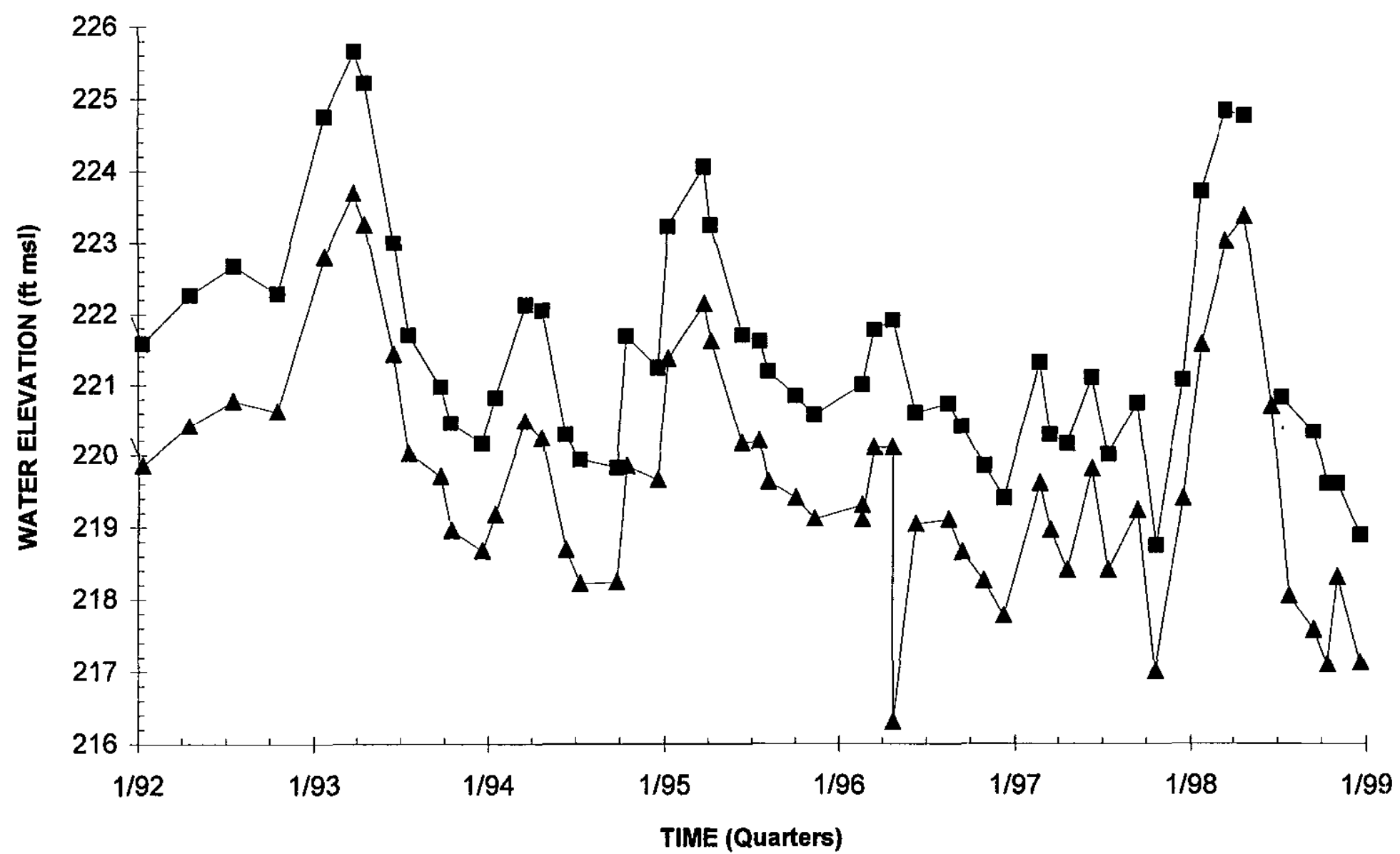

HSB137D (W) $\triangle$ HSB137C (B)

Note: $W=$ Water Table (IIB2); B=Barnwell (IIB1); M=McBean (IIB1); UC=Upper Congaree (IIA); MC=Middle Congaree (IIA); LC=Lower Congaree (IIA) 


\section{Hydrograph \\ Well HSB138}

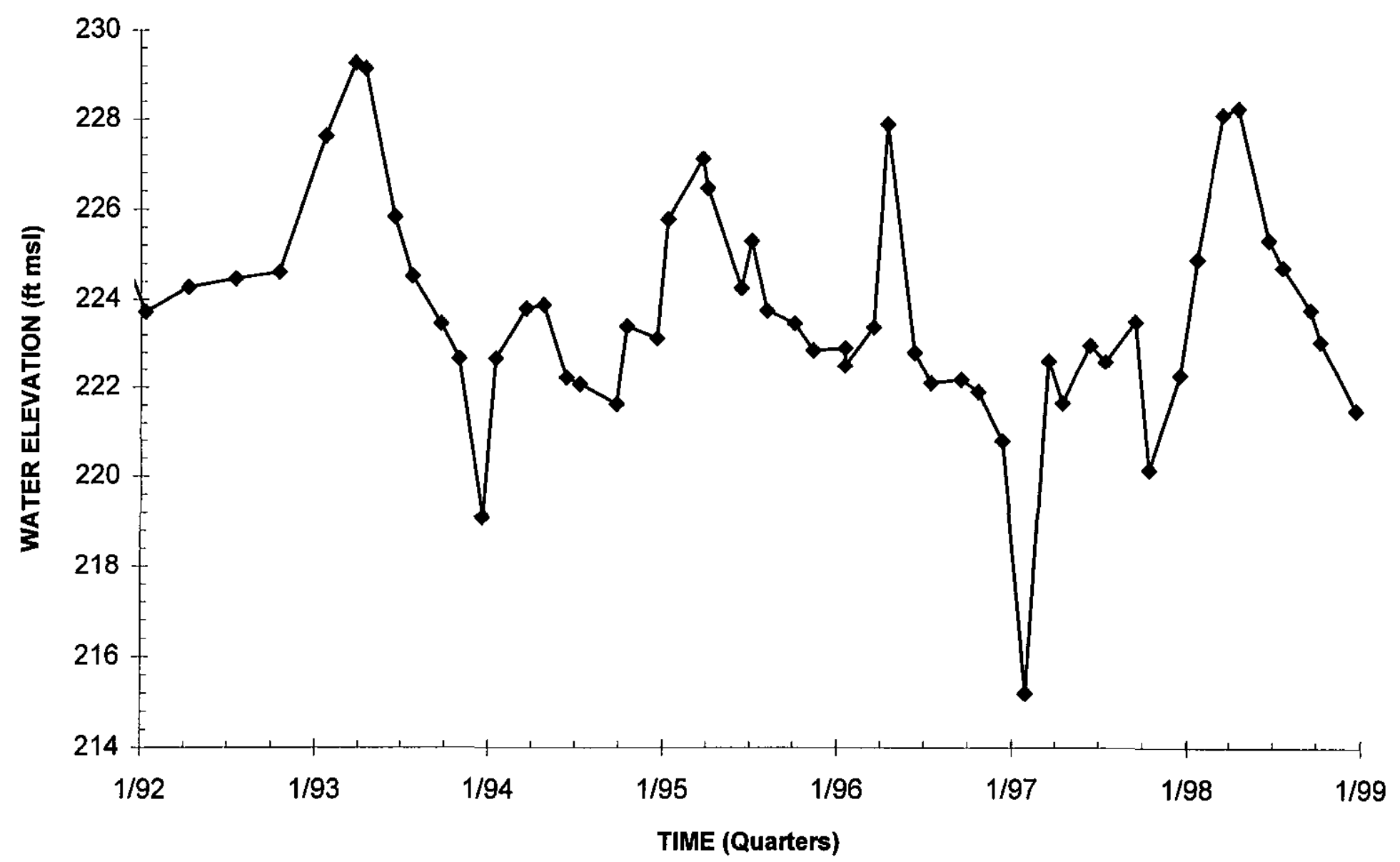

$\leadsto-\mathrm{HSB} 138 \mathrm{D}(\mathrm{W})$

Note: W=Water Table (IIB2); B=Barnwell (IIB1); M=McBean (IIB1); UC=Upper Congaree (IIA); MC=Middle Congaree (IIA); LC=Lower Congaree (IIA) 


\section{Hydrograph \\ Well Cluster HSB139}

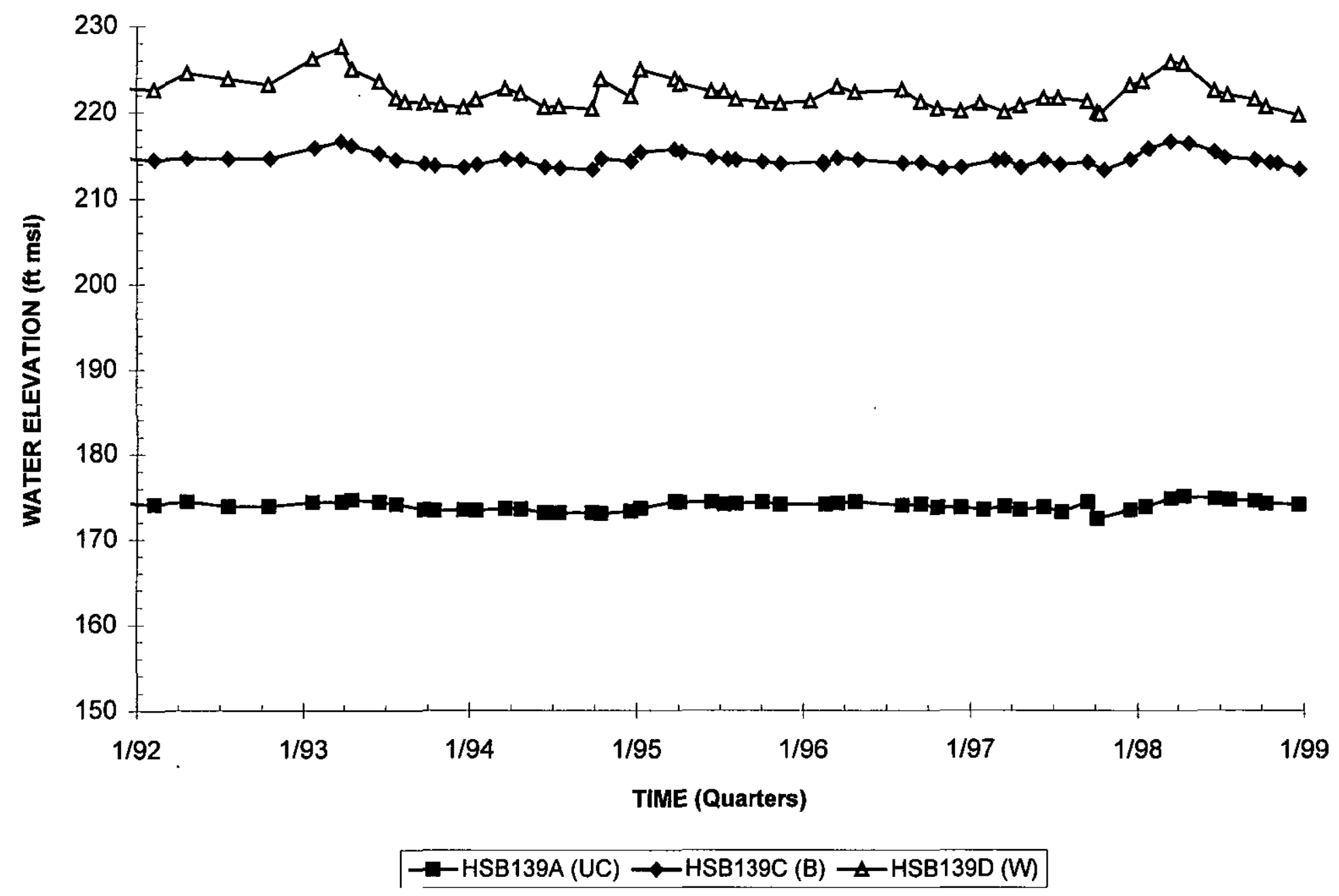

Note: W=Water Table (IIB2); B=Barnwell (IIB1); M=McBean (IIB1); UC=Upper Congaree (IIA); MC=Middle Congaree (IIA); LC=Lower Congaree (IIA)

H-Area HWMF

E - 52

Third and Fourth Quarter 1998

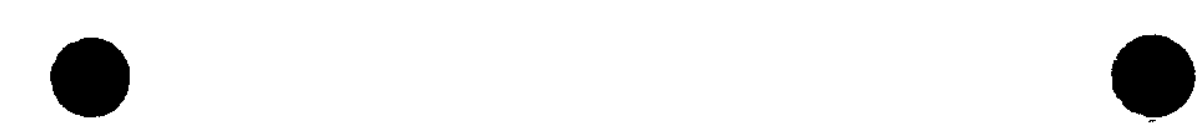




\section{Hydrograph}

\section{Well Cluster HSB140}

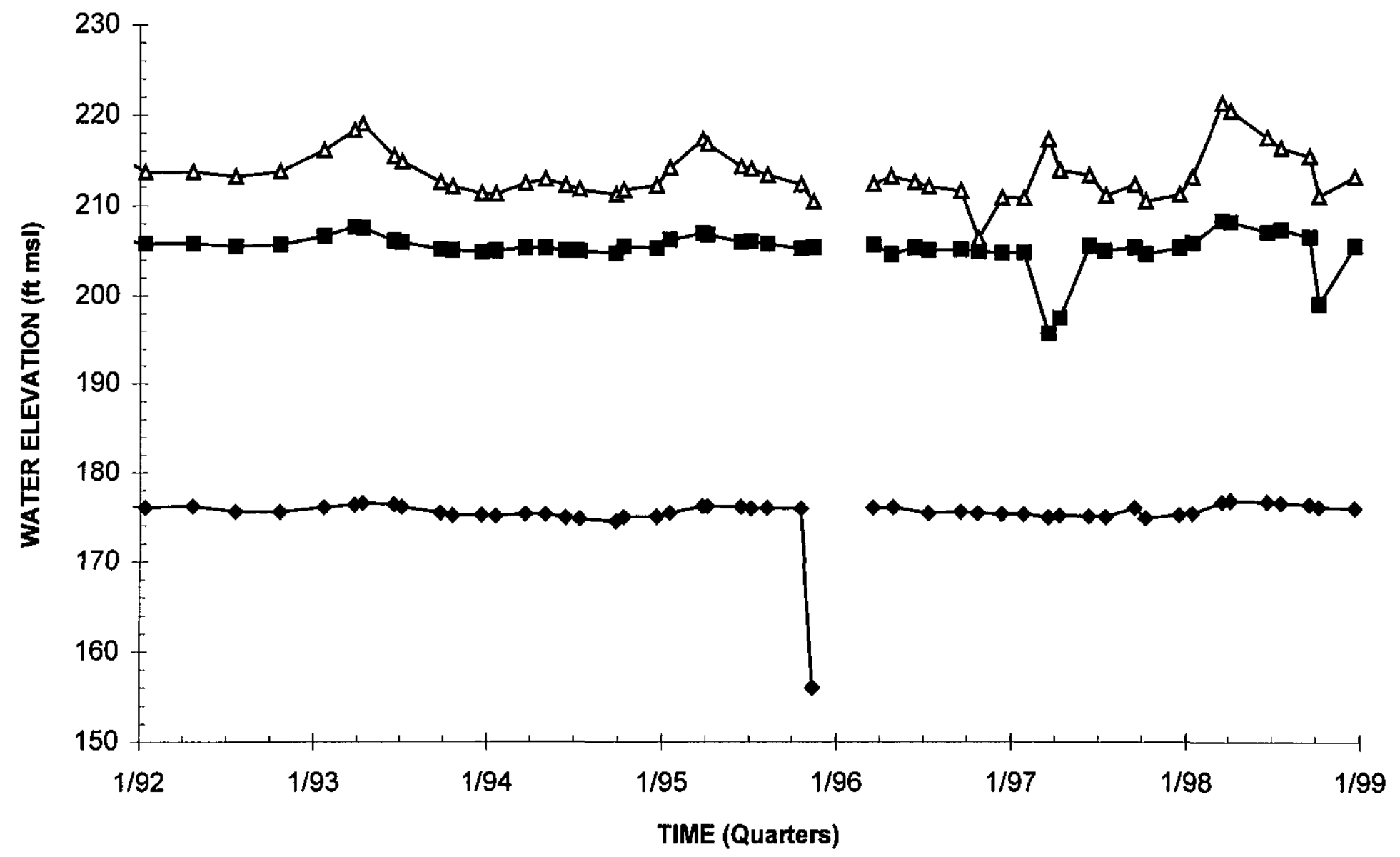

$\rightarrow-$ HSB140A (UC) $\rightarrow-H S B 140 C$ (B) $₫$ HSB140D (W)

Note: W=Water Table (IIB2); B=Barnwell (IIB1); M=McBean (IIB1); UC=Upper Congaree (IIA); MC=Middle Congaree (IIA); LC=Lower Congaree (IIA) 


\section{Hydrograph}

\section{Well Cluster HSB141}

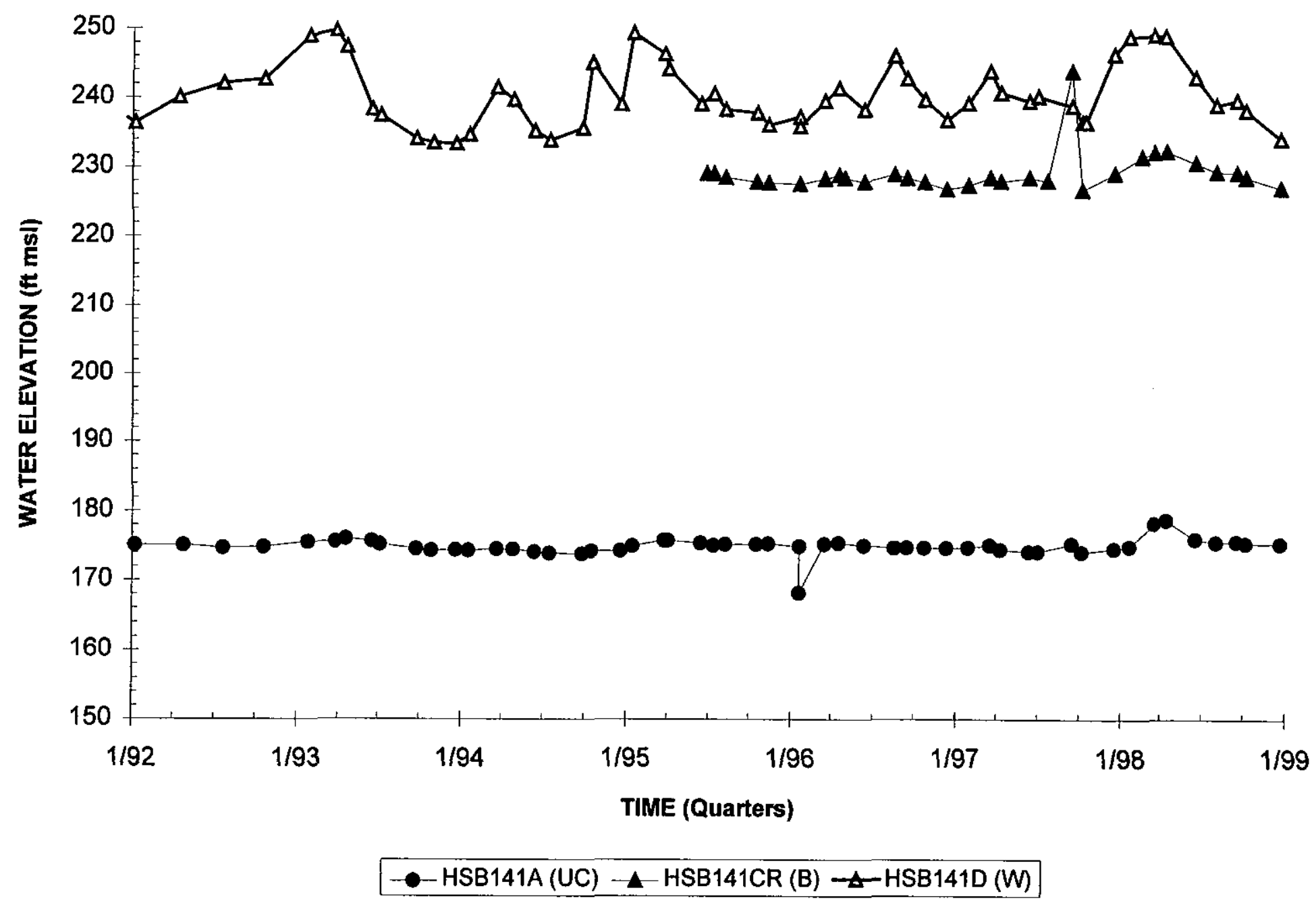

Note: W=Water Table (IIB2); B=Barnwell (IIB1); M=McBean (IIB1); UC=Upper Congaree (IIA); MC=Middle Congaree (IIA); LC=Lower Congaree (IIA) 


\section{Hydrograph}

Well Cluster HSB142

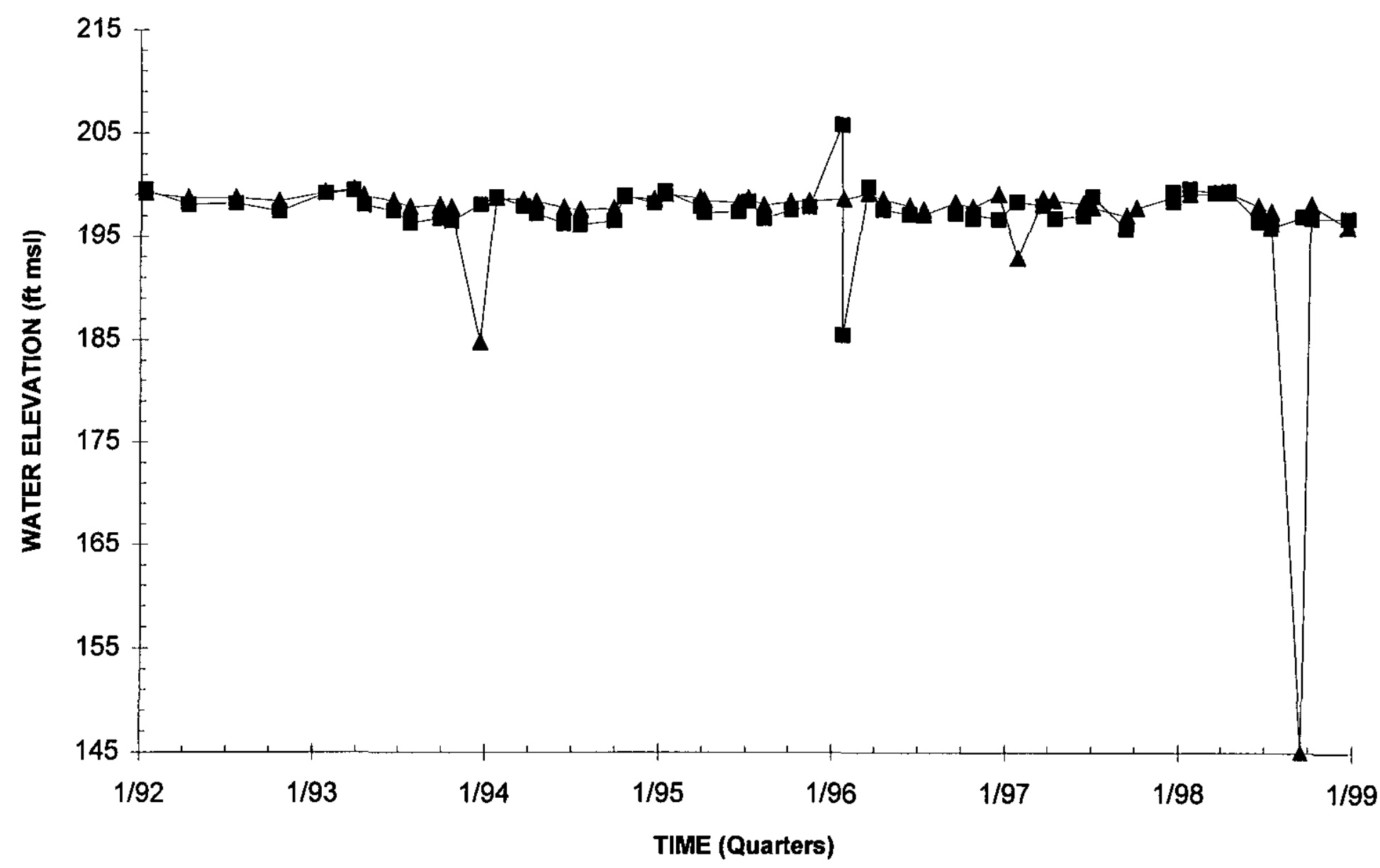

HSB142D (W) - HSB142C (B)

Note: W=Water Table (IIB2); B=Barnwell (IIB1); M=MCBean (IIB1); UC=Upper Congaree (IIA); MC=Middle Congaree (IIA); LC=Lower Congaree (IIA) 


\section{Hydrograph}

\section{Well Cluster HSB143}

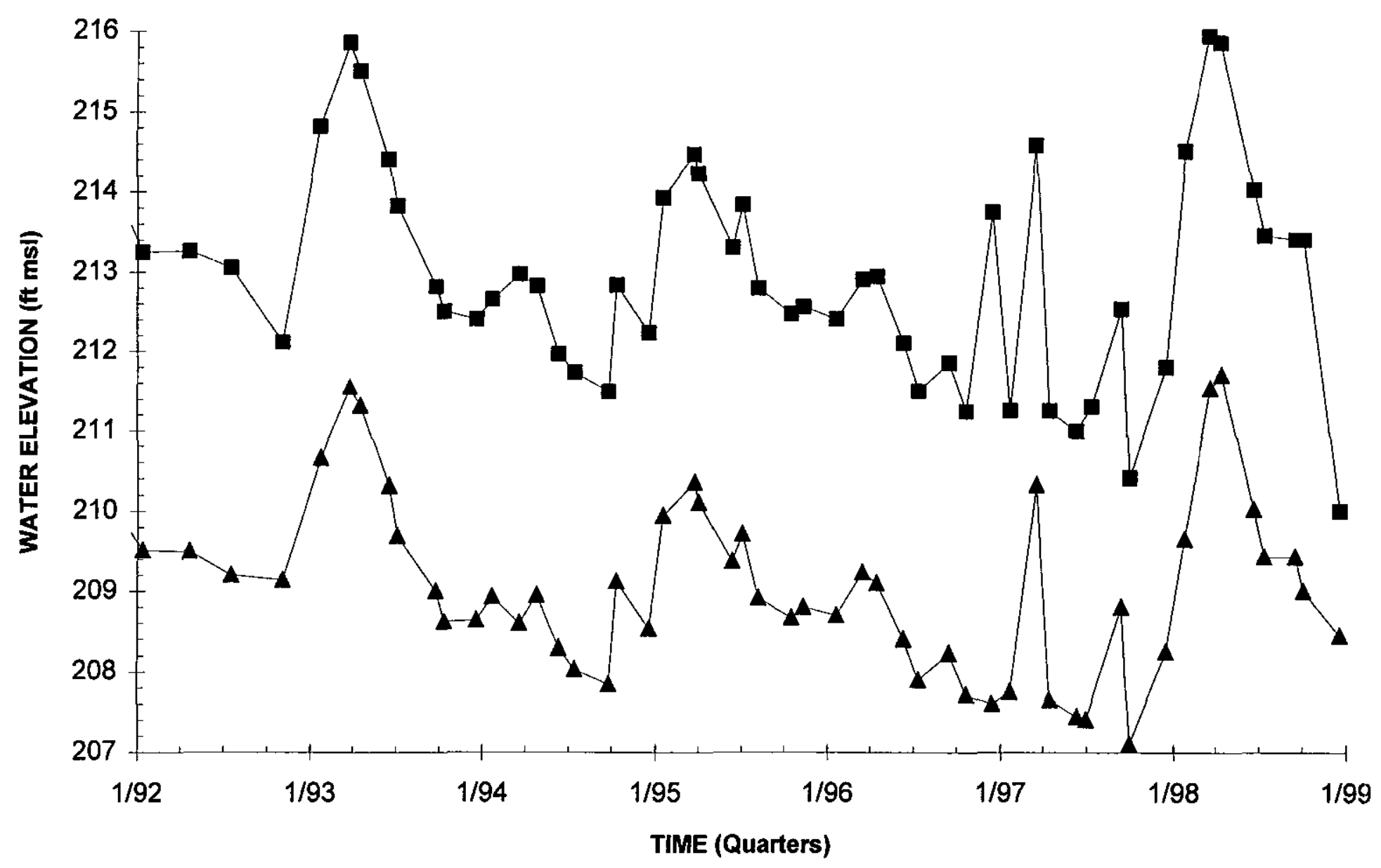

- HSB143D (W) -A-HSB143C (B)

Note: $W=$ Water Table (IIB2); $B=B a r n w e l l($ (IIB1); $M=M C B e a n$ (IIB1); UC=Upper Congaree (IIA); MC=Middle Congaree (IIA); LC=Lower Congaree (IIA) 


\section{Hydrograph}

Well HSB144A

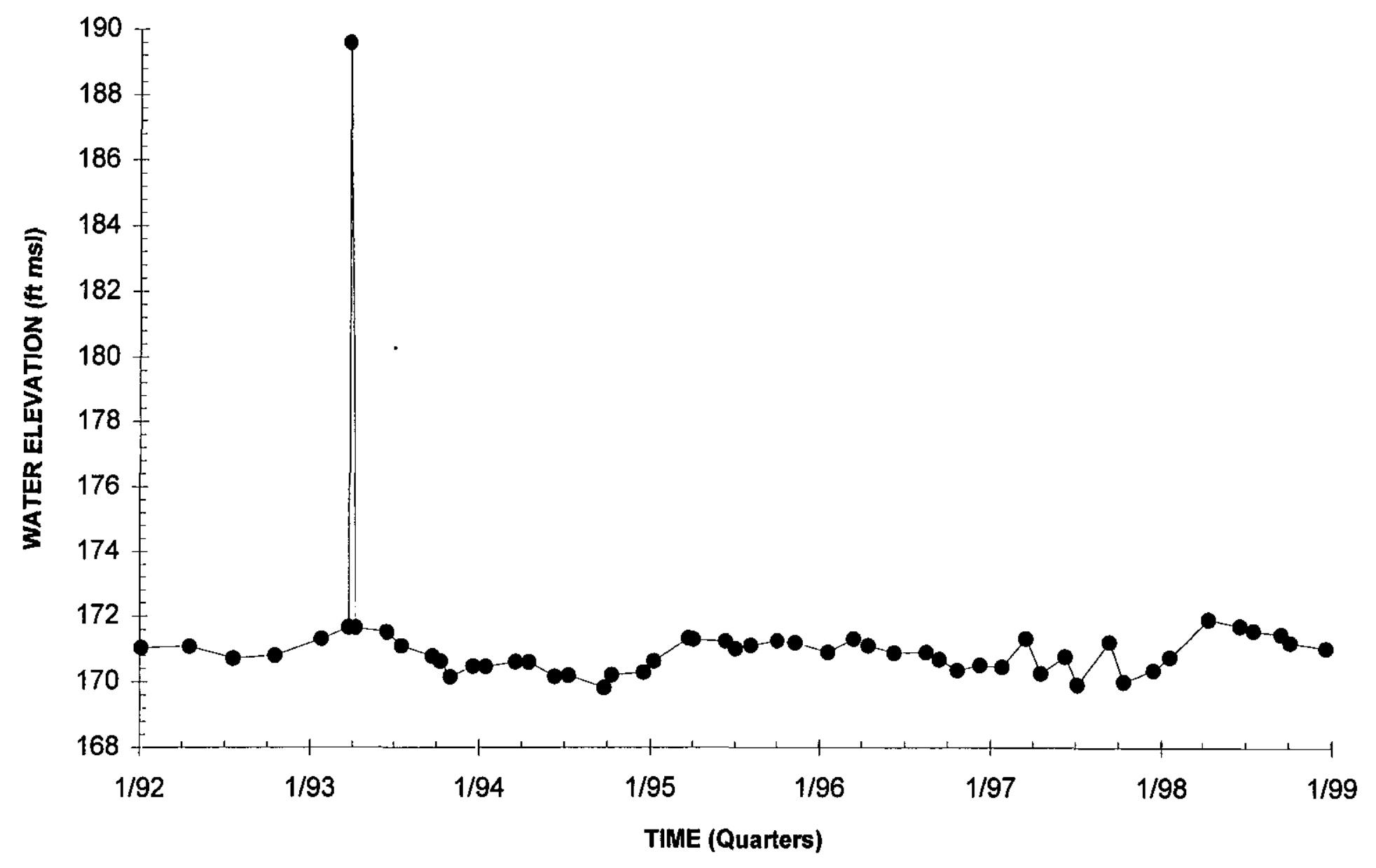

$-\mathrm{HSB} 144 \mathrm{~A}(\mathrm{UC})$

Note: $W=$ Water Table (IIB2); B=Barnwell (IBB1); M=MCBean (IIB1); UC=Upper Congaree (IIA); MC=Middle Congaree (IIA); LC=Lower Congaree (IIA) 


\section{Hydrograph \\ Well Cluster HSB145}

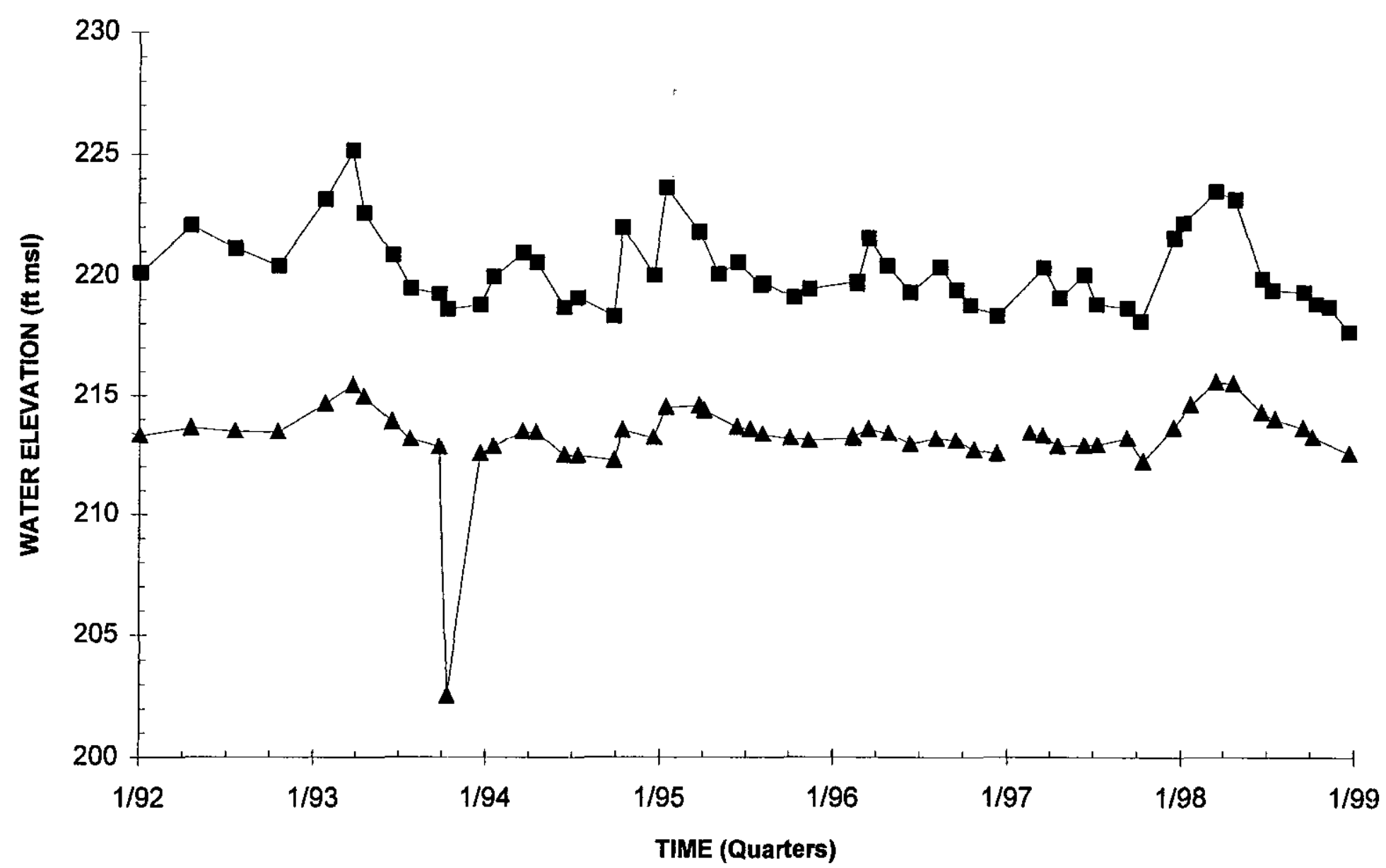

$-\mathrm{HSB145D}(\mathrm{W})-\leftarrow-\mathrm{HSB145C}(\mathrm{B})$

Note: W=Water Table (IIB2); B=8arnwell (IIB1); M=MCBean (IIB1); UC=Upper Congaree (IIA); MC=Middle Congaree (IIA); LC=Lower Congaree (IIA) 


\section{Hydrograph}

\section{Well Cluster HSB146}

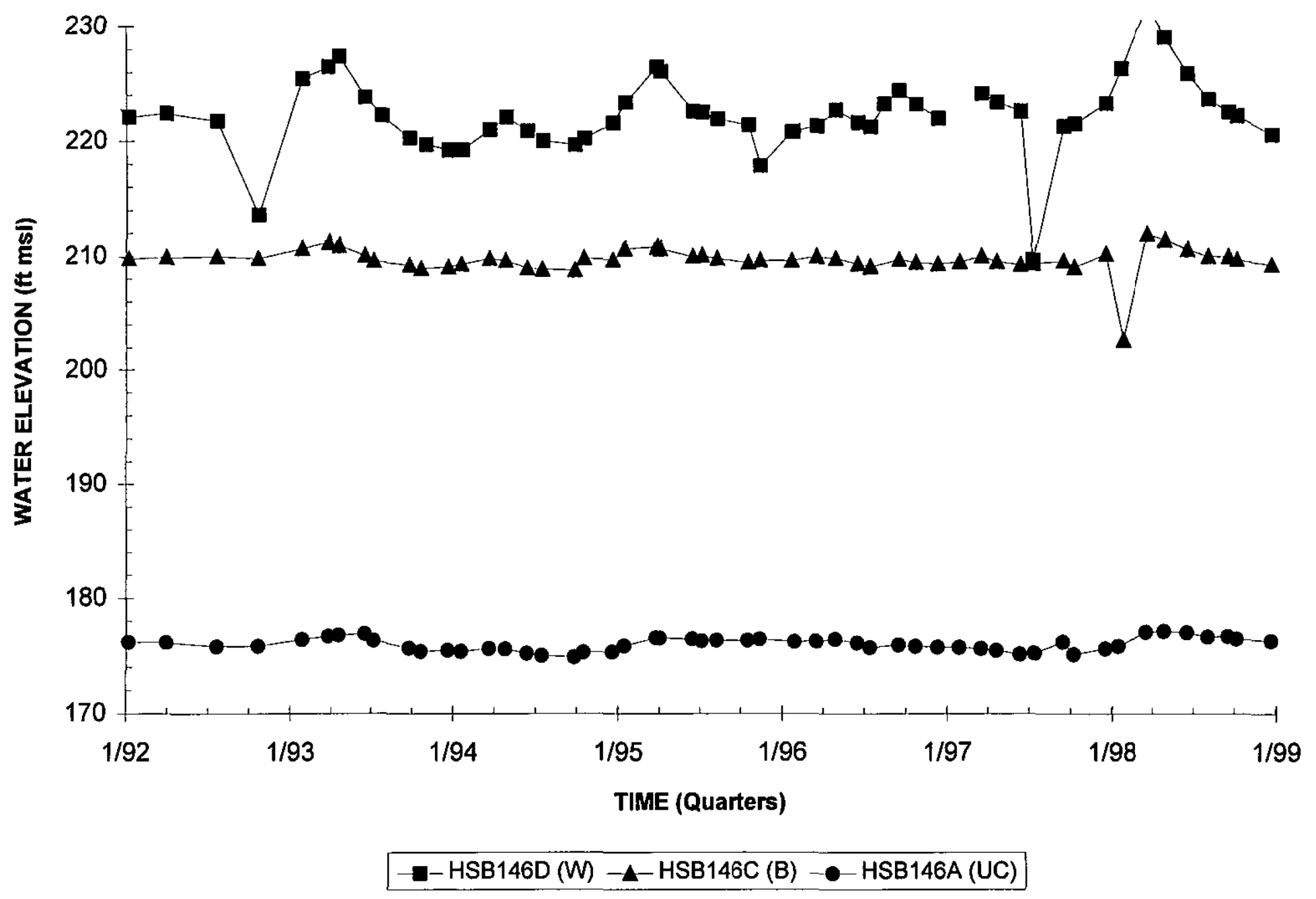

Note: W=Water Table (IIB2); B=Barnwell (IIB1); M=McBean (IIB1); UC=Upper Congaree (IIA); MC=Middle Congaree (IIA); LC=Lower Congaree (IAA) 


\section{Hydrograph}

\section{Well HSB147D}

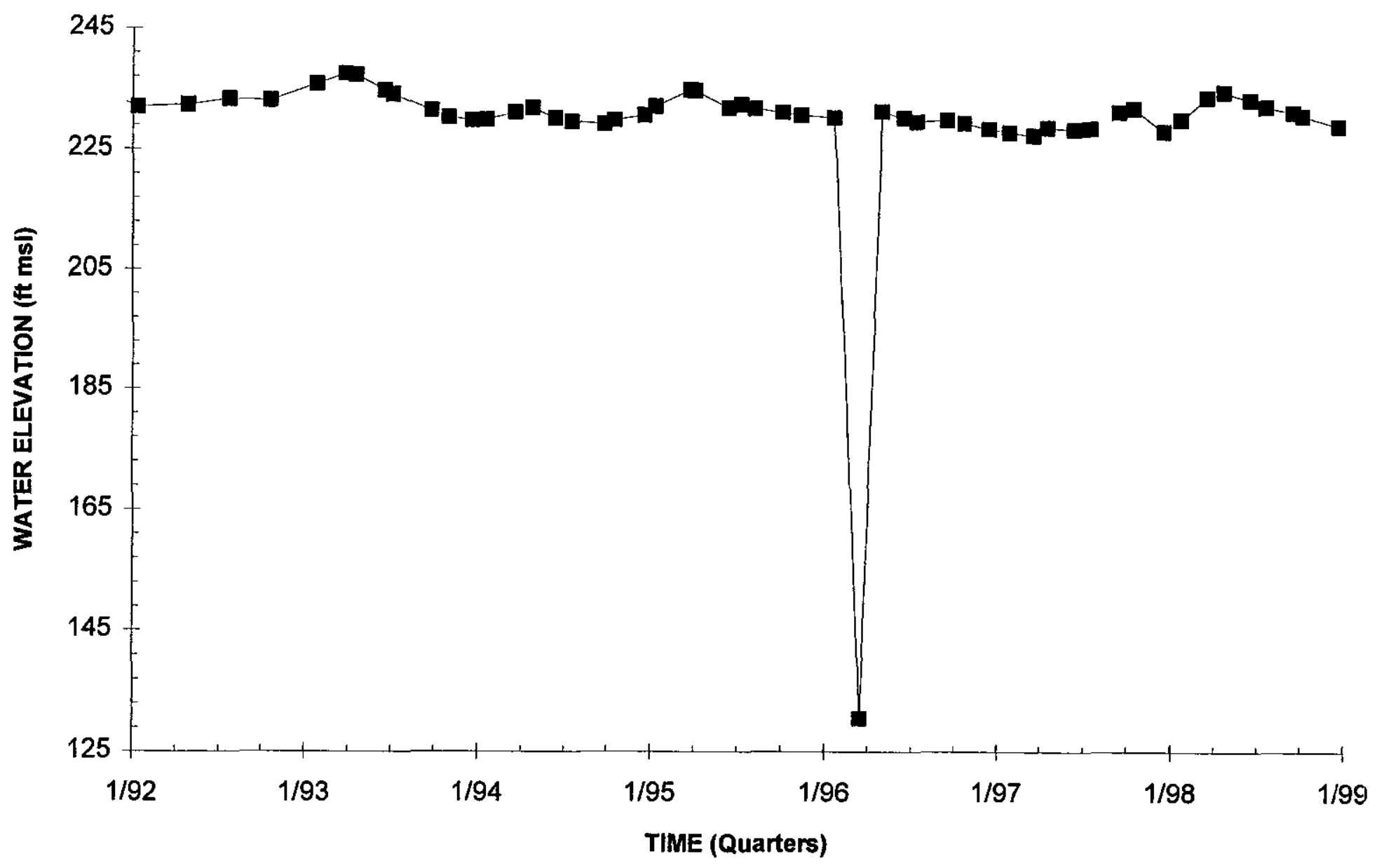

$$
\text { HSB147D (W) }
$$

Note: W=Water Table (IIB2); B=Barnwell (IIB1); M=MCBean (IIB1); UC=Upper Congaree (IIA); MC=Middle Congaree (IIA); LC=Lower Congaree (IIA) 


\section{Hydrograph}

\section{Well Cluster HSB148}

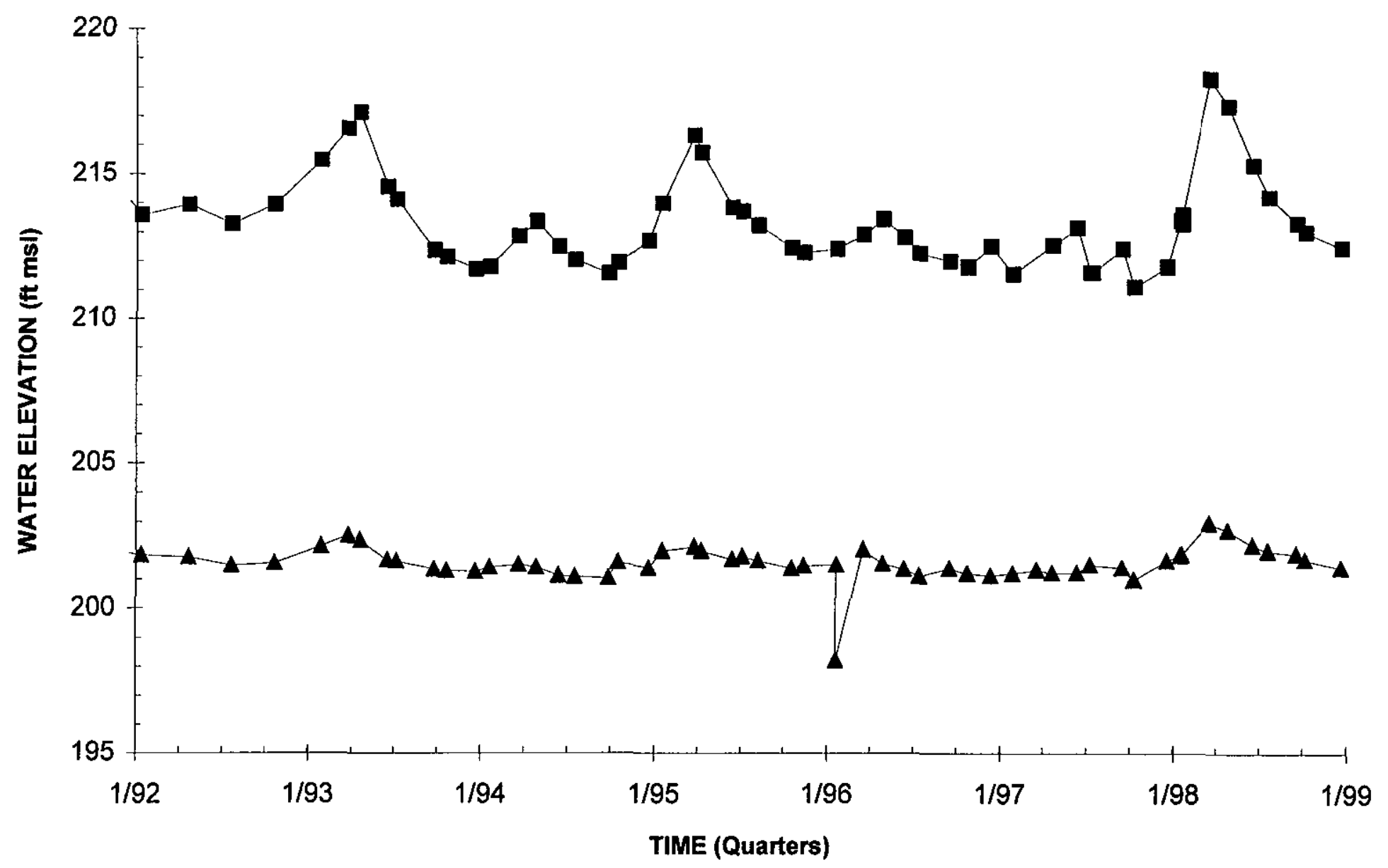

$-\mathrm{HSB} 148 \mathrm{D}(\mathrm{W})-\mathbf{A}-\mathrm{HSB148C}(\mathrm{B})$

Note: W=Water Table (IIB2); B=Barnwell (IIB1); M=McBean (IIB1); UC=Upper Congaree (IIA); MC=Middle Congaree (IIA); LC=Lower Congaree (IIA) 


\section{Hydrograph \\ Well HSB149D}

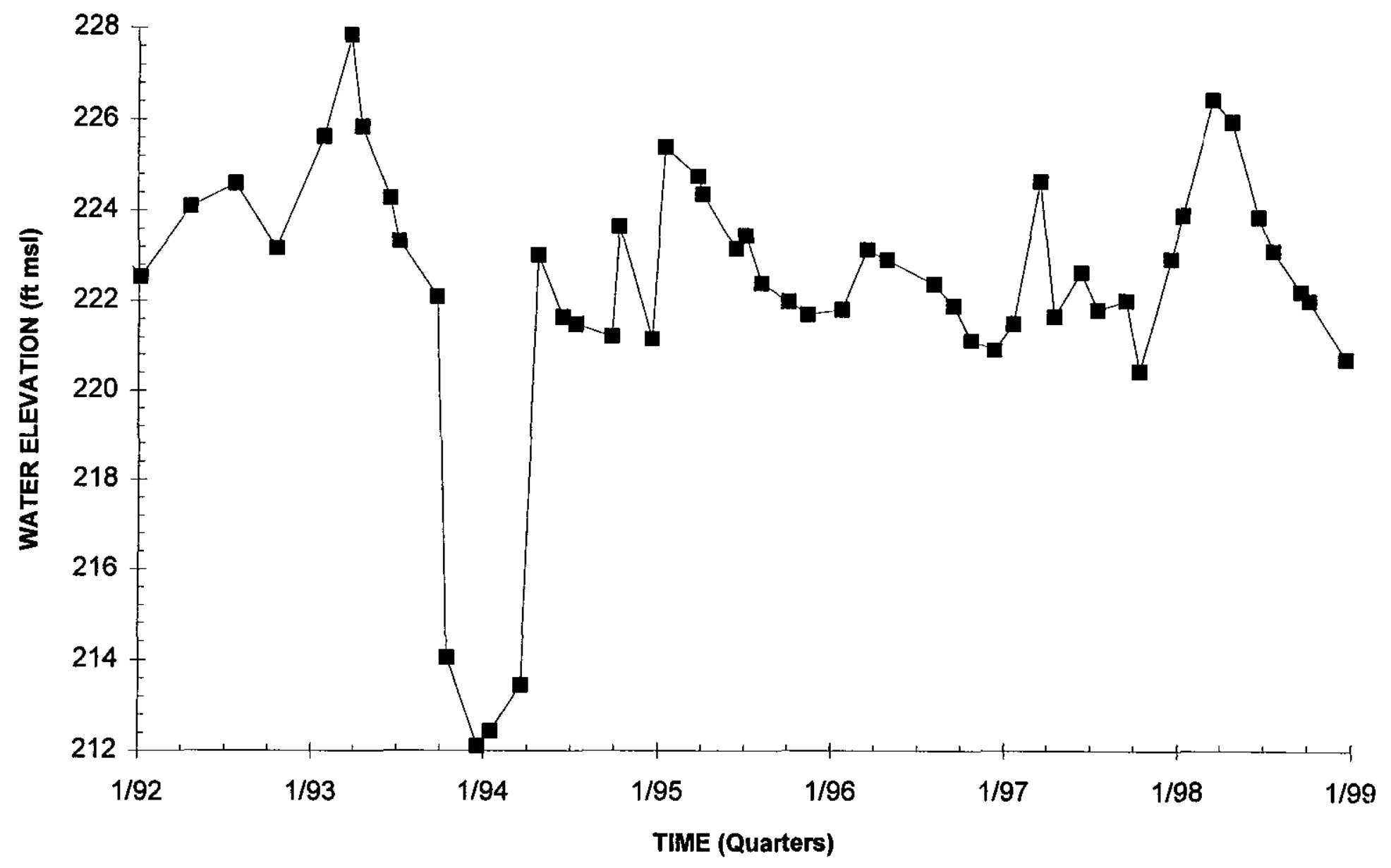

$-\mathrm{HSB149D}(\mathrm{W})$

Note: $W=$ Water Table (IIB2); B=Barnwell (IIB1); M=MCBean (IIB1); UC=Upper Congaree (IIA); MC=Middle Congaree (IIA); LC=Lower Congaree (IIA) 


\section{Hydrograph}

Well HSB150D

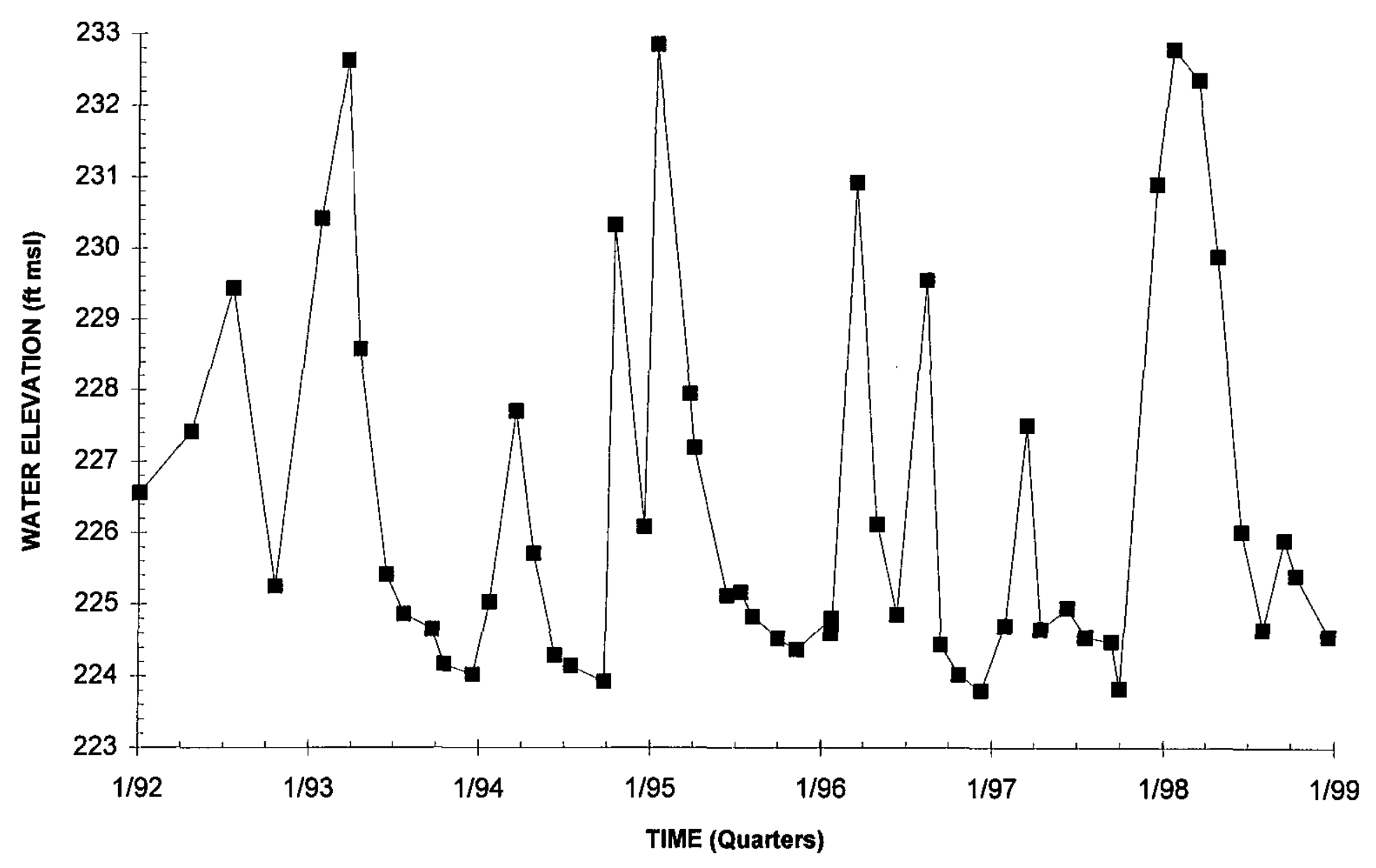

$-\operatorname{HSB150D}(\mathrm{W})$

Note: $W=$ Water Table (IIB2); B=Barnwell (IIB1); M=McBean (IIB1); UC=Upper Congaree (IIA); MC=Middle Congaree (IIA); LC=Lower Congaree (IIA) 


\section{Hydrograph \\ Well Cluster HSB151}

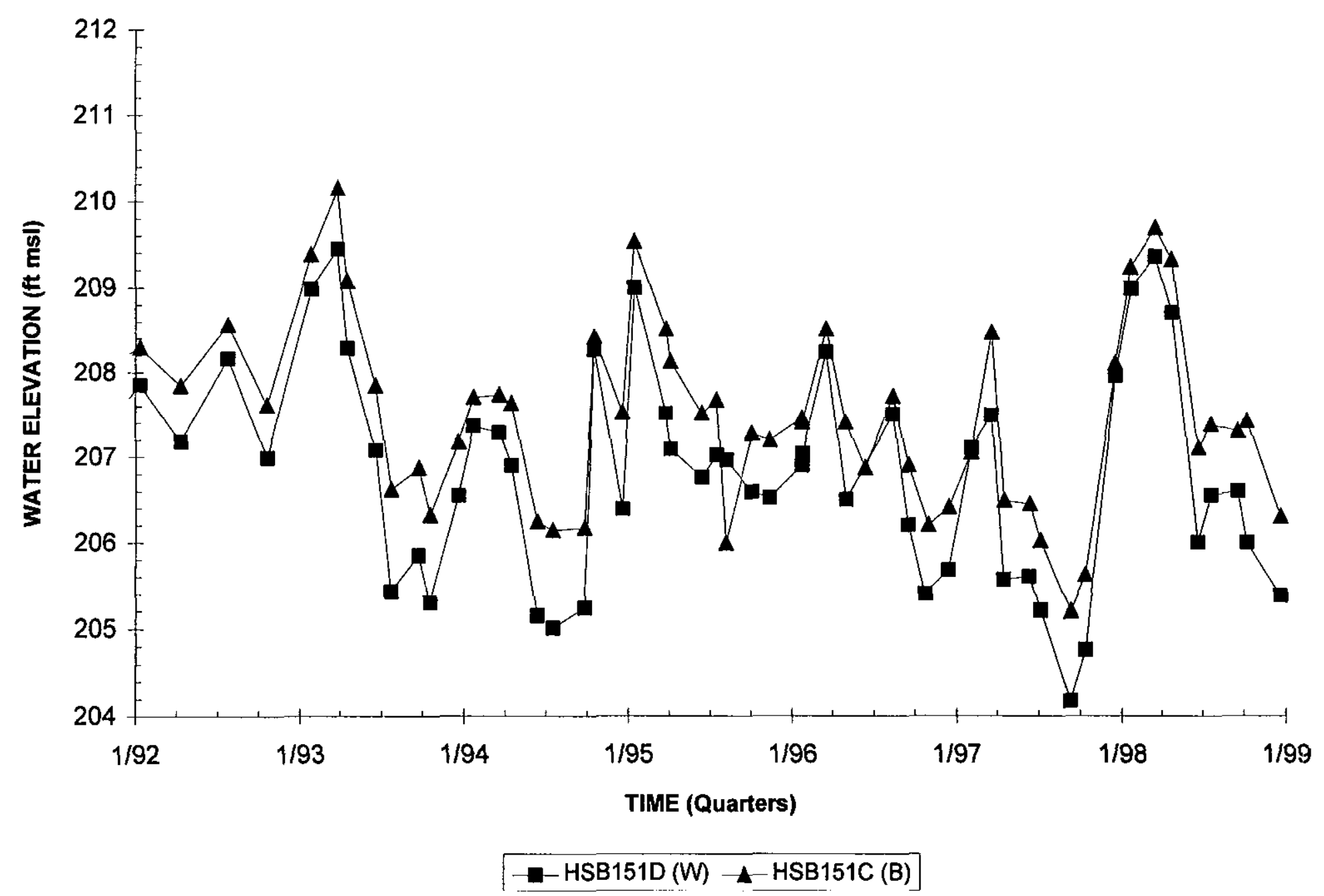

Note: $W=$ Water Table (IIB2); B=Barnwell (IIB1); $M=$ McBean (IIB1); UC=Upper Congaree (IIA); MC=Middle Congaree (IIA); LC=Lower Congaree (IIA) 


\section{Hydrograph}

\section{Well Cluster HSB152}

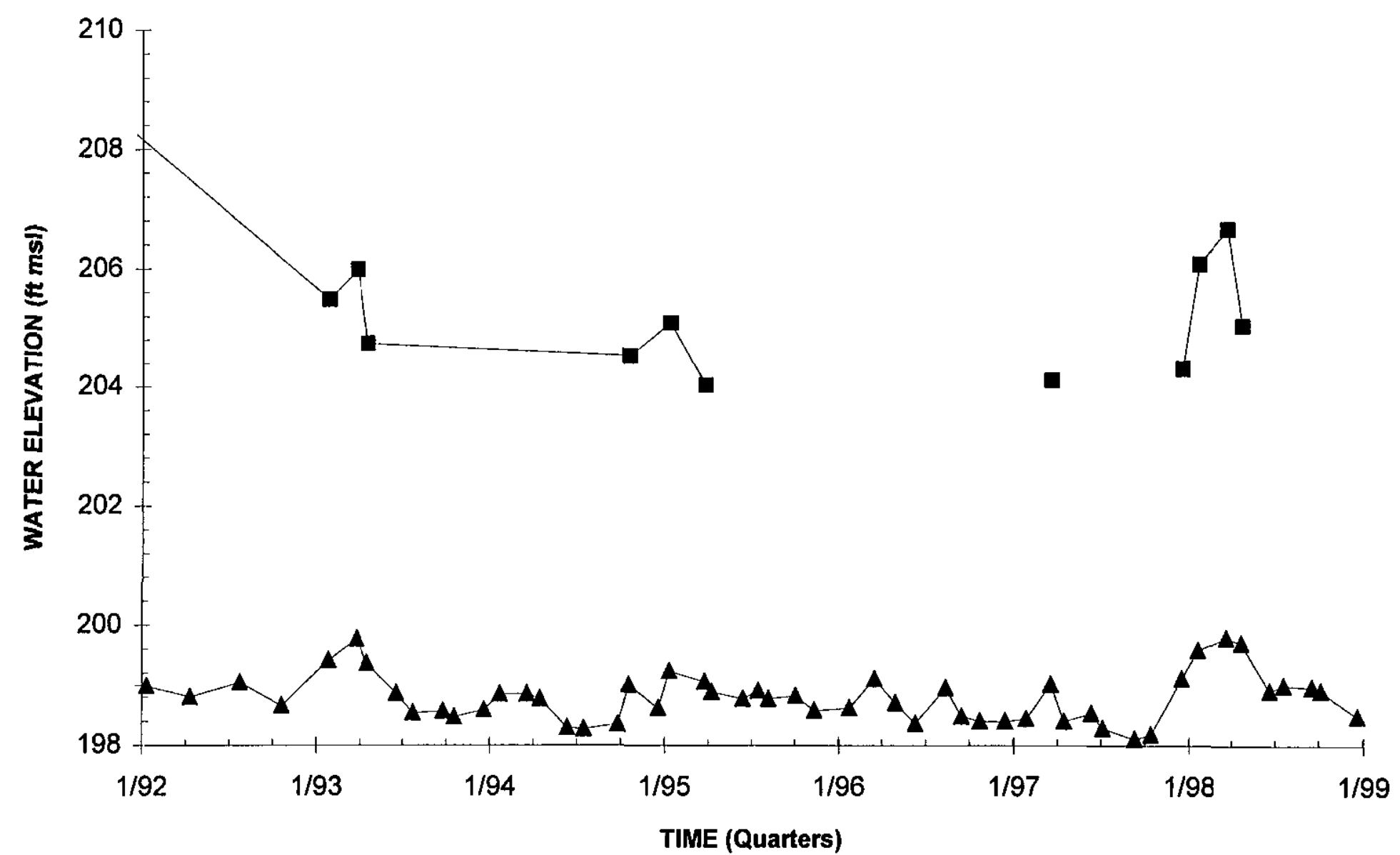

- HSB152D (W) - HSB152C (B)

Note: $W=$ Water Table (IIB2); B=Barnwell (IIB1); M=McBean (IIB1); UC=Upper Congaree (IIA); MC=Middle Congaree (IIA); LC=Lower Congaree (IIA) 


\section{Hydrograph}

Well HSL 1D

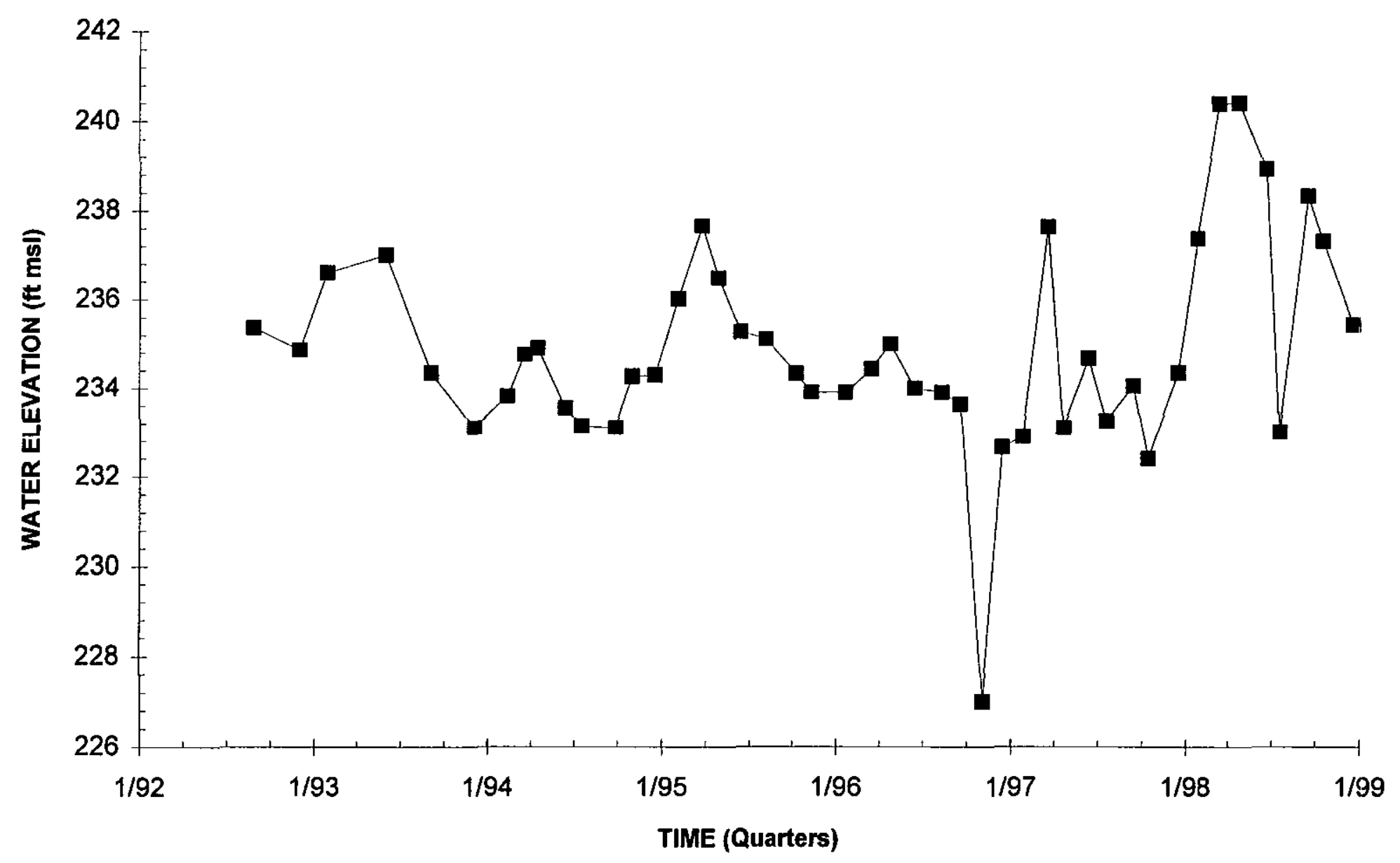

$-\mathrm{HSL} 1 \mathrm{D}(\mathrm{W})$

Note: W=Water Table (IIB2); B=Bamwell (IIB1); M=McBean (IIB1); UC=Upper Congaree (IIA); MC=Middle Congaree (IIA); LC=Lower Congaree (IIA) 


\section{Hydrograph}

\section{Well HSL 2D}

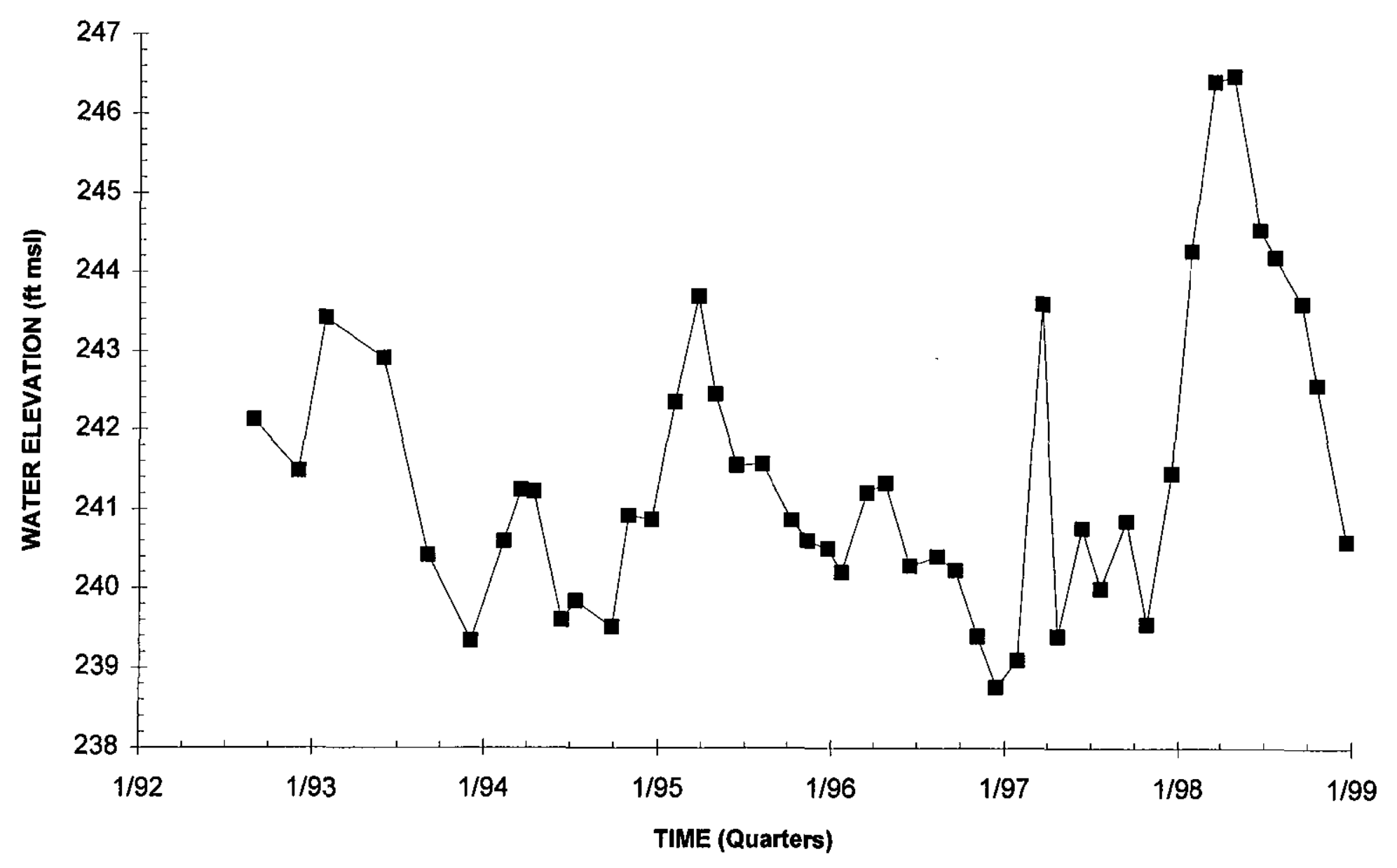

$-\mathrm{HSL} 2 \mathrm{D}(\mathrm{W})$

Note: W=Water Table (IIB2); B=Barnwell (IIB1); M=MCBean (IIB1); UC=Upper Congaree (IIA); MC=Middle Congaree (IIA); LC=Lower Congaree (IIA) 


\section{Hydrograph \\ Well HSL 3D}

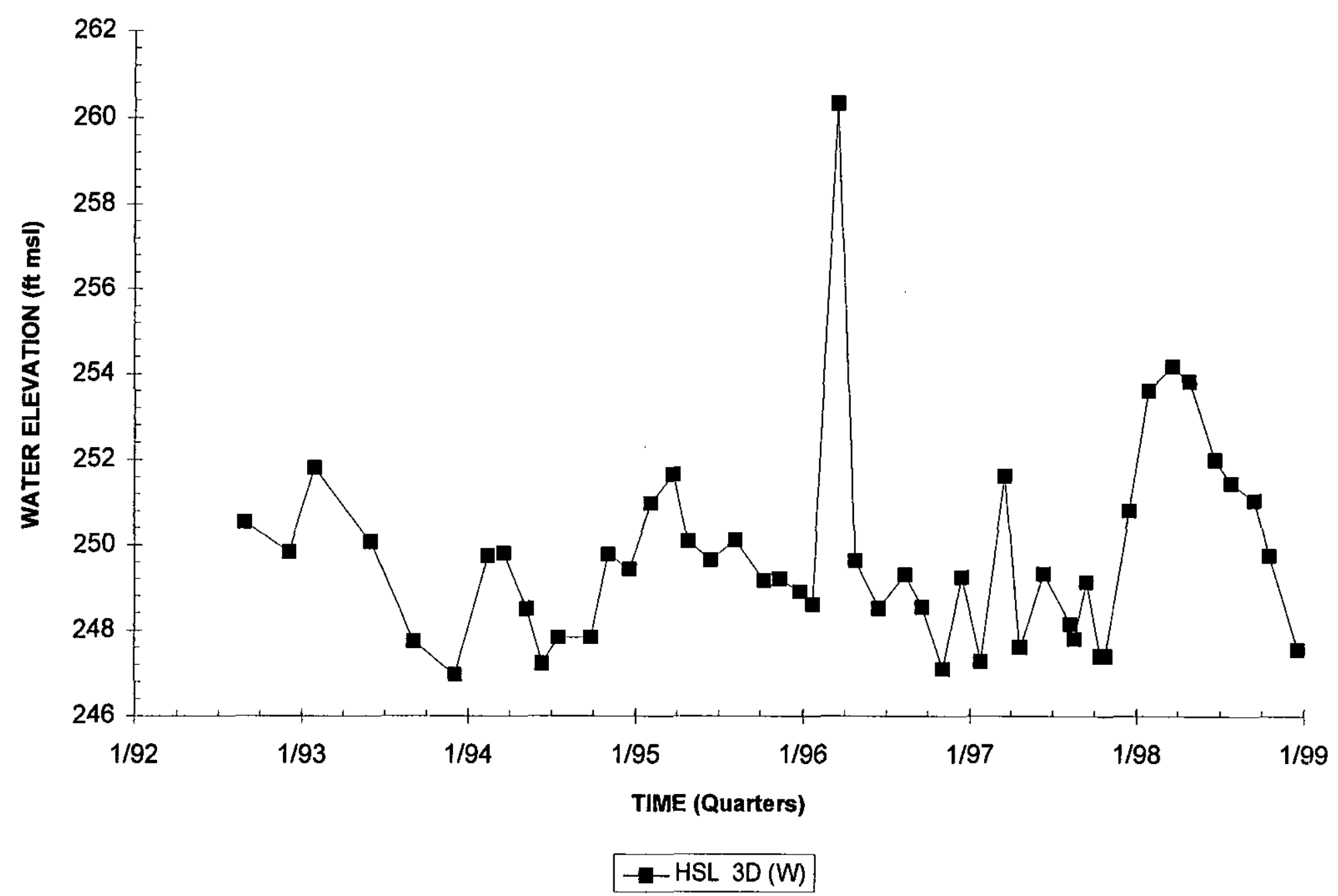

Note: W=Water Table (IIB2); B=Barnwell (IIB1); M=McBean (IIB1); UC=Upper Congaree (IIA); MC=Middle Congaree (IIA); LC=Lower Congaree (IIA) 
WSRC-TR-99-00013 Unclassified

\section{Hydrograph}

Well HSL 4D

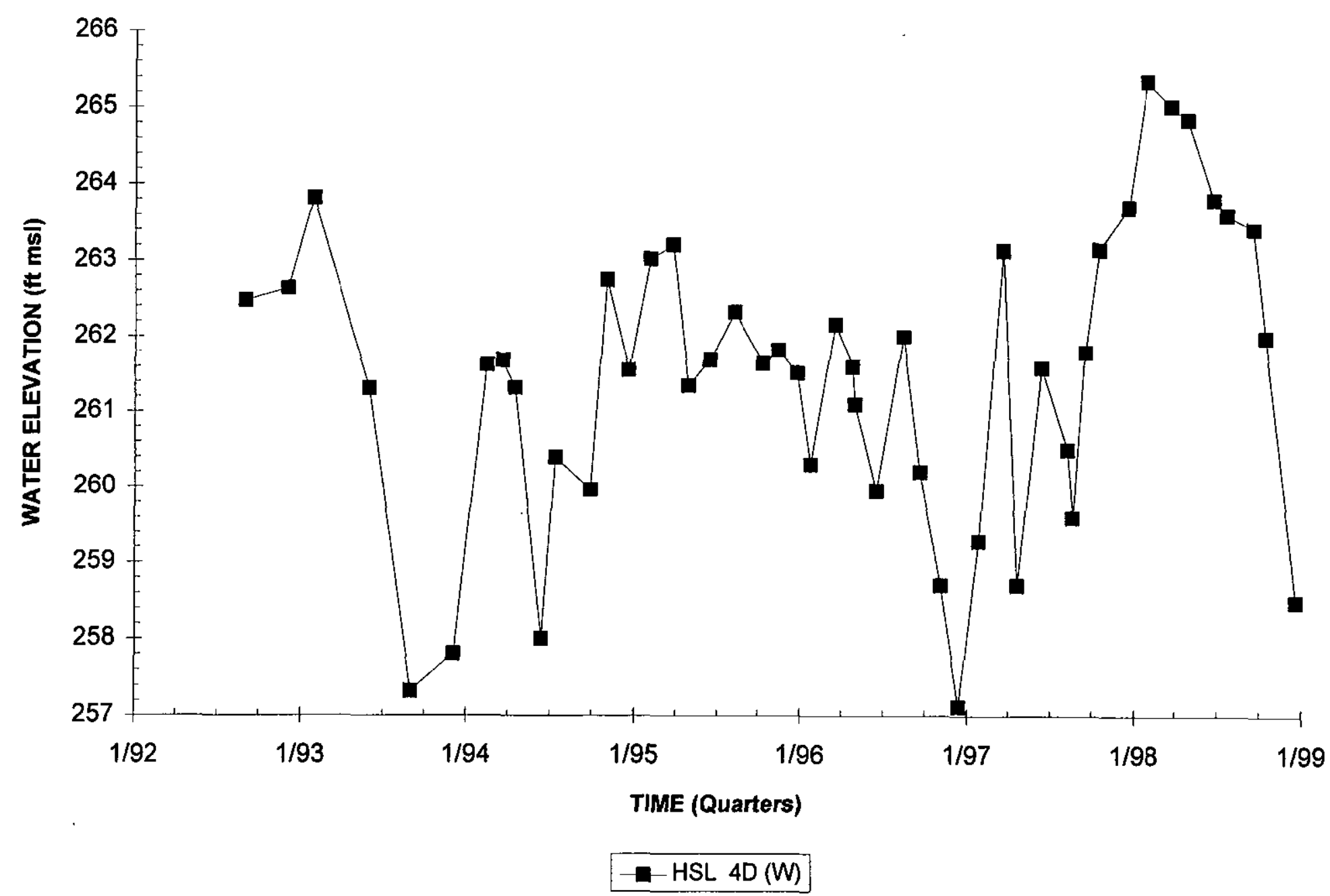

Note: $W=$ Water Table (IIB2); B=Barnwell (IBB1); M=McBean (IIB1); UC=Upper Congaree (IIA); MC=Middle Congaree (IIA); LC=Lower Congaree (IIA) 


\section{Hydrograph}

Well HSL 5D

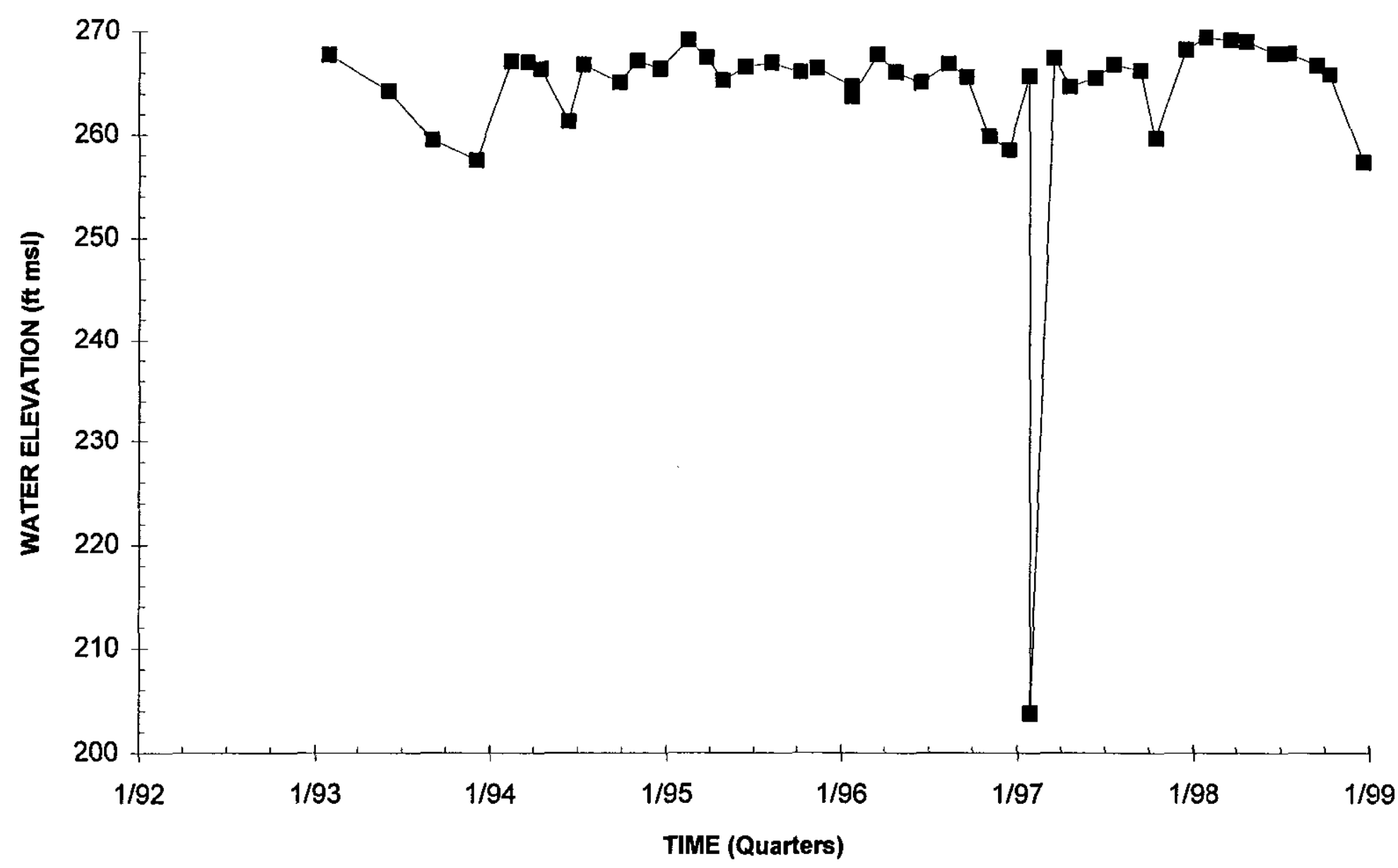

- HSL $5 \mathrm{D}(\mathrm{W})$

Note: $W=$ Water Table (IIB2); B=Barnwell (IIB1); $M=M C B e a n$ (IIB1); UC=Upper Congaree (IIA); MC=Middle Congaree (IIA); LC=Lower Congaree (IIA) 


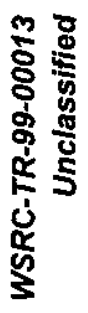
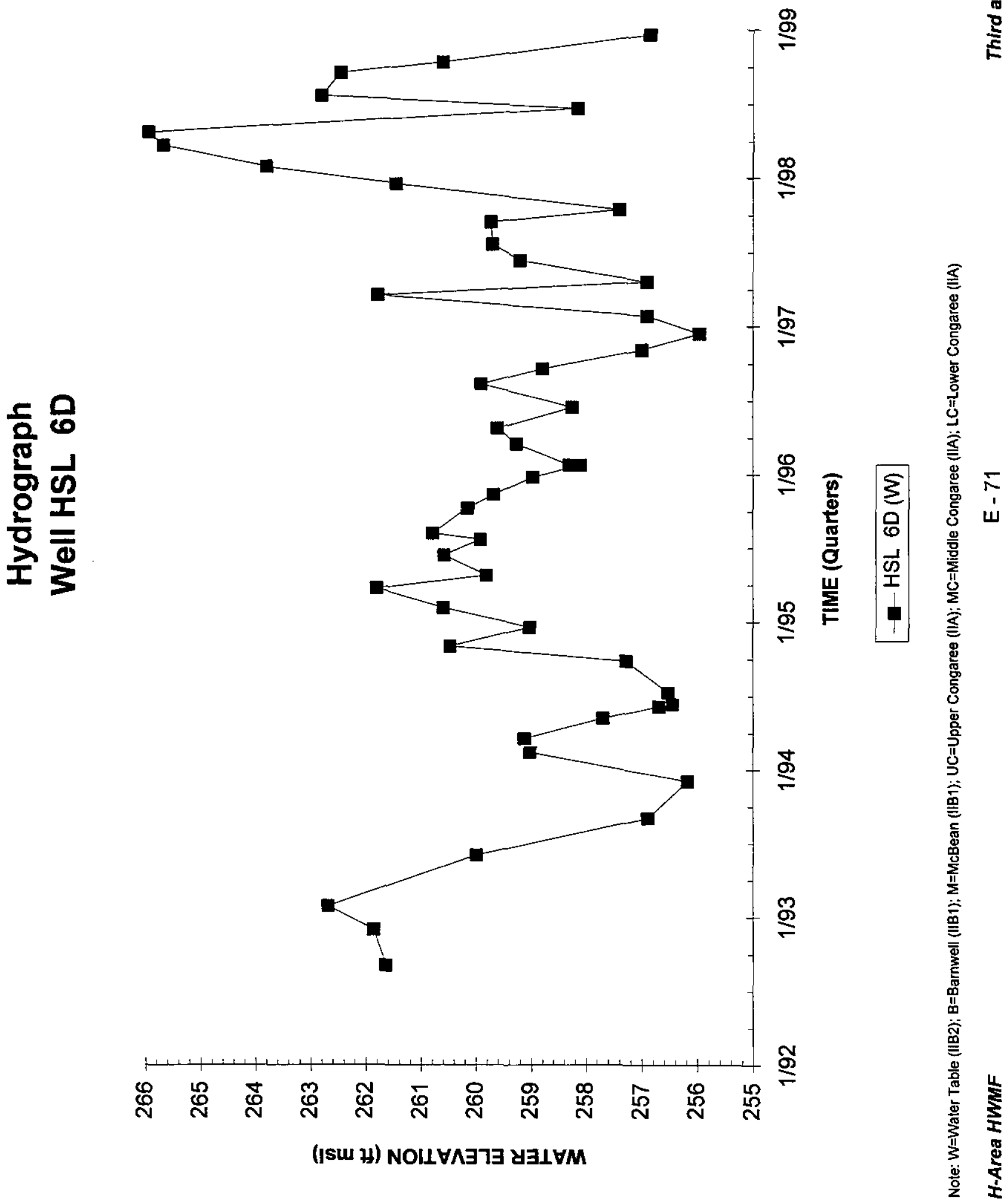


\section{Hydrograph}

Well HSL 7D

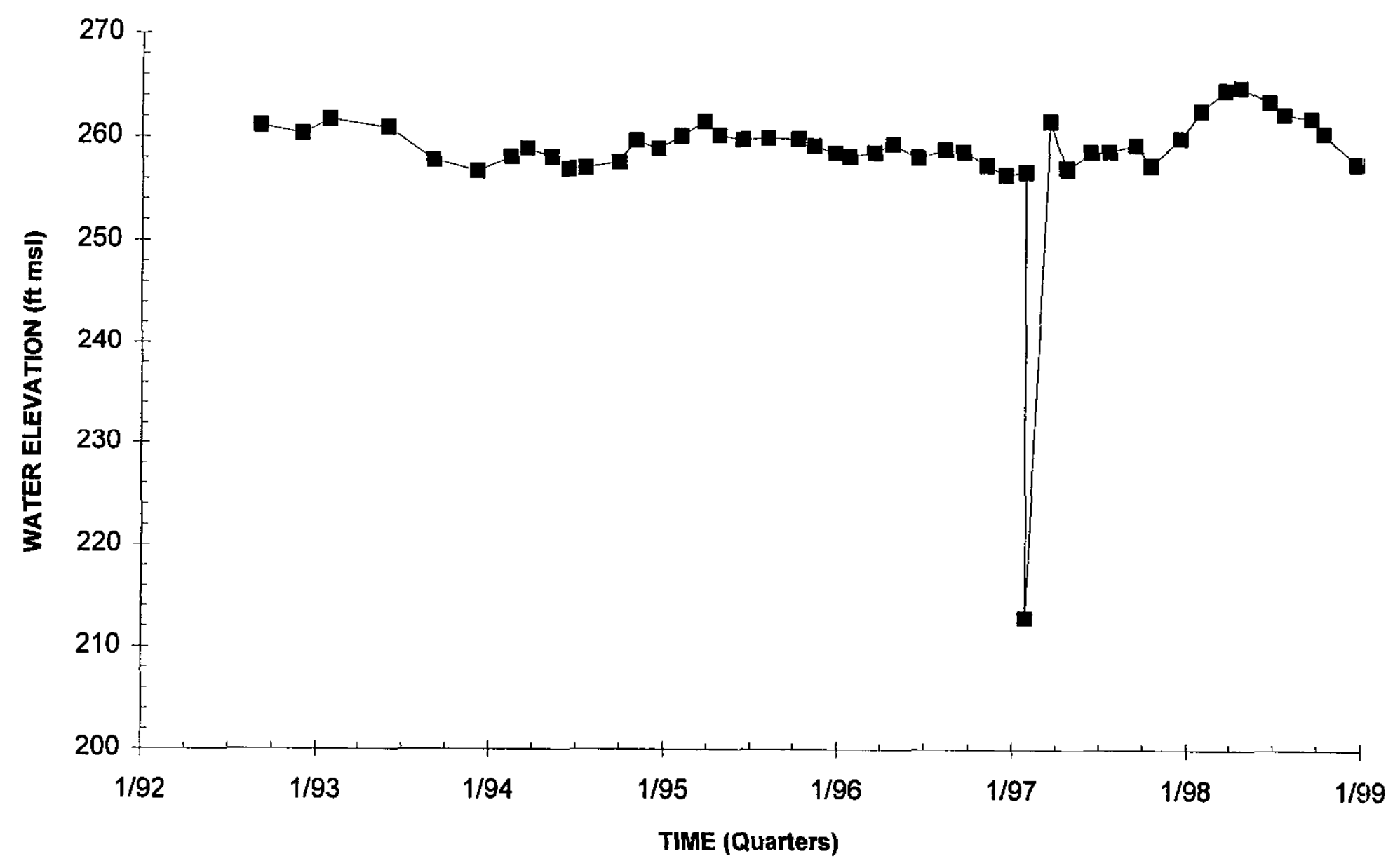

- HSL $7 \mathrm{D}(\mathrm{W})$

Note: $W=$ Water Table (IIB2); B=Barnwell (IIB1); M=McBean (IIB1); UC=Upper Congaree (IIA); MC=Middle Congaree (IIA); LC=Lower Congaree (IIA) 


\section{Hydrograph}

Well HSL 8D

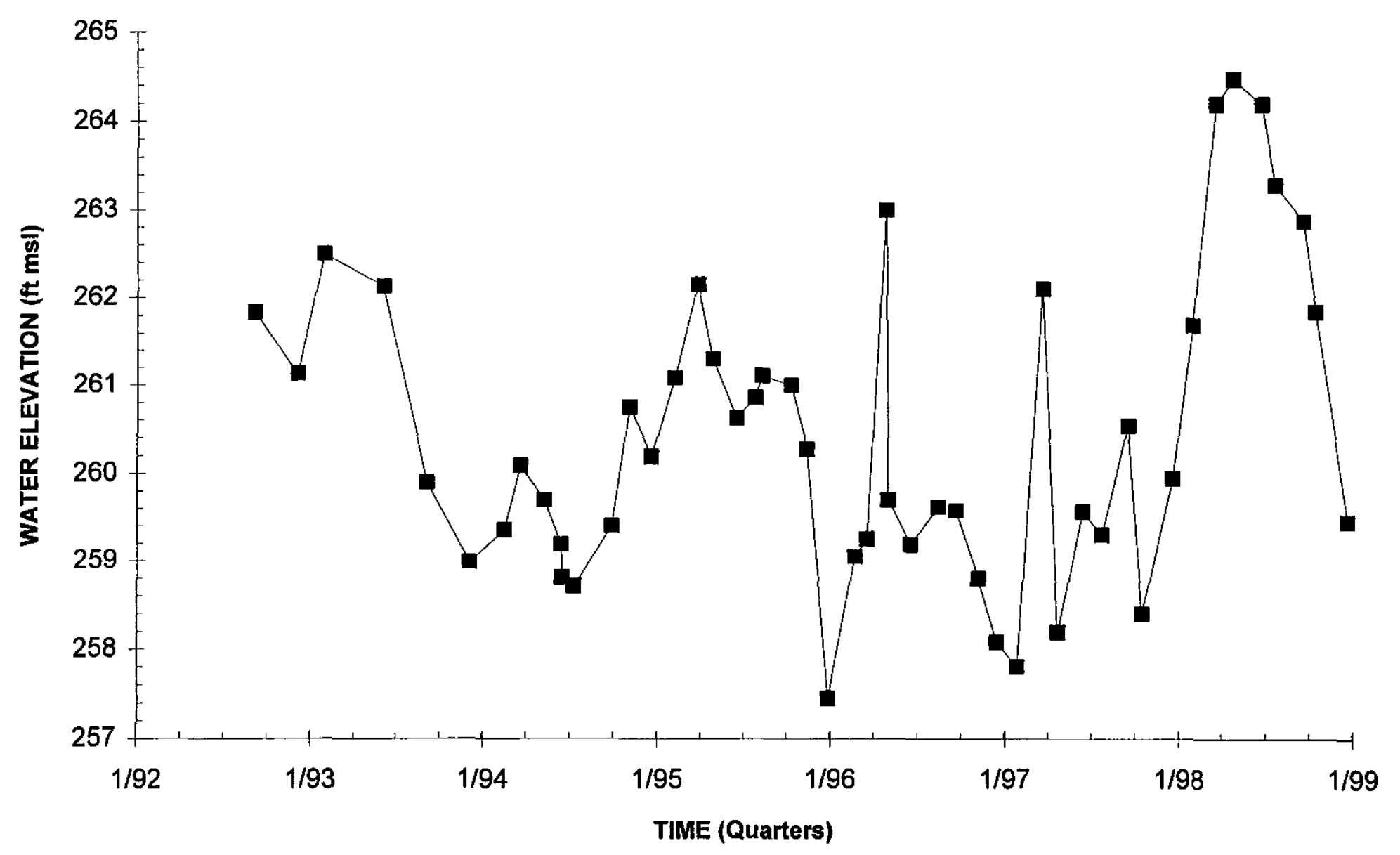

$-\mathrm{HSL} 8 \mathrm{D}(\mathrm{W})$

Note: $W=$ Water Table (IBB2); B=Barnwell (IIB1); $M=M C B$ ean (IIB1); UC=Upper Congaree (IIA); MC=Middle Congaree (IIA); LC=Lower Congaree (IIA) 
WSRC-TR-99-00013

Unclassified

THIS PAGE LEFT BLANK INTENTIONALLY. 


\section{Appendix F}

\section{Statistical Evaluations}


WSRC-TR-99-00013

Unclassified

This page was left blank intentionally. 


\section{Introduction}

The $1995 \mathrm{H}$-Area RCRA Part B Permit requires annual reports on the effectiveness of the corrective action program that are to include a "statistical evaluation of water quality data and water elevation data for significant changes." The permit application proposed that "SRS will use the combined Shewhart-CUSUM charts for detecting both increasing and decreasing trends, and to identify either sudden incursions (a contaminant slug) or steady drift (a change in plume concentration or size)." The text below reviews the appropriate statistical evaluation techniques for monitoring the effectiveness of remediation systems and outlines the technically correct procedures for the statistical evaluations.

\section{Purpose of the Statistical Evaluation}

The purpose of the statistical evaluation is to identify remediation system impacts to the groundwater quality in various wells. Identifying impacts requires a statistical evaluation since normal concentration fluctuations and trends make a non-statistical approach ineffective. The F\&HASB Remediation Projects (F\&H) permit application proposed the use of combined Shewhart-CUSUM control charts for the statistical evaluation of monitoring well data.

Typically, a control chart is the statistical method used identify intrawell changes, with the combined ShewhartCUSUM control charts being most useful. Combined Shewhart-CUSUM control charts are designed to provide a visual tool to detecting both trends and abrupt changes in concentration levels, and is the method suggested by the EPA for intrawell comparisons. However, because F\&H data show existing contamination, often with exponential trend decreases, the original proposal to use control charts is at odds with EPA recommendations and guidance. Specifically:

"The control chart method is recommended for uncontaminated wells only..."

"CAUTIONARY NOTE: Control charts are a useful supplement to other statistical techniques because they are graphical and simple to use. However, it is inappropriate to construct a control chart on wells that have shown evidence of contamination or an increasing trend (see $\$ 264.97(a)(1)(i))$...."

It should be noted, however, that the EPA is referring to only the textbook combined Shewhart-CUSUM control chart, which is constructed to identify increasing levels of concentration at a single uncontaminated well over time. At $\mathrm{F} \& \mathrm{H}$, we wish to monitor both increasing and decreasing effects in already contaminated monitoring wells over time. Thus, the "textbook" combined Shewhart-CUSUM control chart method can be slightly modified from the EPA procedure and the ASTM Provisional Standard Guide for use at F\&H to statistically monitor both increasing and decreasing changes to our groundwater quality data. The following procedure details the modified combined Shewhart-CUSUM control chart method to be used for $\mathrm{F}$ - and $\mathrm{H}$-Area statistical analysis of water quality and water elevation data.

\section{Statistical Procedure}

The statistical procedure for constructing and interpreting combined Shewhart-CUSUM control charts for use at F\&H is described below. The procedure should be followed for each Constituent of Interest (COI), at each Well of Interest (WOI). The procedure has been modified from the control chart procedure recommended by the EPA and ASTM to accommodate the prior existence of contamination, and to detect both increasing and decreasing changes.

The procedure is composed of four major tasks: (1) coliect monitoring data and select parameters, (2) adjust the data to fit the data randomness, independence, and normality assumptions required by the control chart statistics, (3) calculate the control chart values, and (4) plot the results. By far, the most complex statistical processes will occur in task (2) - adjustment of the data. Without proper data adjustment, the control chart results would be meaningless.

For each COI, for each WOI, do the following: 


\section{Task (1): Collect Monitoring Data}

(1)-1. Collect Analytical Data: From the Geochemical Information Management System (GIMS), extract the COl sample data for the WOls. As per recent GIMS data guidance for compliance decisions, do not extract data with a result qualifier that contains an " $R$ ", " $J$ ", or " $L$ ". Non-detect flags (a result qualifier of "U") for each sample data shouid be retained.

(1)-2. Select Parameters: Select the background time-period. For F\&H, the background time-period is defined as $1 / 1 / 91$ through $12 / 31 / 95$. Select the control chart parameters and control limits. For F\&H, the following control chart parameters/limits should be used: $k=1 ; h=5.0$; and $\mathrm{SCL}=4.5$, as per $\mathrm{EPA}$ guidance.

Task (2): Adjust Data

(2)-1. Average Samples by Quarter: Control charts require data to be statistically independent. To ensure this independence, sampling is recommended at intervals no shorter than quarterly. As F\&H monitoring data is regularly collected on a quarterly basis (some constituents are sampled less frequently), most wells will only be sampled once per quarter. However, for those instances when a well is sampled more than once per quarter, quarterly average concentration values need to be calculated. The quarterly arithmetic average, $x_{i}$ is to be calculated and stored for each quarter for all the data (the All Dataset). The background time-period data becomes the background time-period quarterly averaged dataset (the BG Dataset). Non-detects (NDs) should be handled as follows: If only NDs exist for a quarter, then no

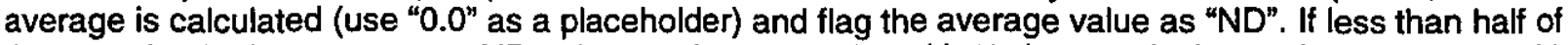
the samples in the quarter are NDs, then replace the NDs with $1 / 2$ the practical quantitation limit (PQL) (or the method detection limit (MDL) if the PQL is not available) and proceed with the averaging calculation. If half or more of the samples are NDs (but not all), then do not use the NDs in the average calculation.

(2)-2. Non-Detect Quantity: If the number of NDs in the entire BG Dataset exceeds $50 \%$, then the control chart analysis should not be continued.

(2)-3. Handling of Non-Detects: If the number of NDs in the BG Dataset is less than $15 \%$, then replace the NDs with $1 / 2$ the practical quantification limit (PQL) (or the method detection limit (MDL) if the PQL is not available). If the number of NDs is greater than $15 \%$ (but less than $50 \%$ ), then Cohen's adjustment to the BG Dataset mean and variance will have to be made in a subsequent step.

(2)-4. Detection and Removal of Outliers: The detection and removal of outliers is still subject to expert disagreement. The method described here, the Dixon test, is consistent with that recommended by the EPA, the ASTM, and others. To simplify the analysis, a test for a maximum of one high outlier will be performed for each set of observations in the All Dataset.

(2)-4.1. Log-transform the data. Assume ND values are $1 / 2$ PQL (or MDL).

(2)-4.2. Calculate the mean, $\bar{x}$, and the standard deviation, $s$, of the log-transformed data.

(2)-4.3. Calculate the $T$ statistic, $T=\left(x_{h}-\bar{x}\right) / s$, where $x_{h}$ represents the sample with the largest positive difference from the mean, $\bar{x}$.

(2)-4.4. Compare the $T$ statistic to the critical value given the sample size, $n$, in Table 1. If a $T$ statistic exceeds the critical value from the table, this is evidence that the suspect observation is a statistical outlier. Remove the outlier from the All Dataset and repeat the test on the next highest value.

(2)-5. Minimum number of Background Samples: If the BG Dataset contains less than 8 samples, then the control chart analysis should not be continued.

(2)-6. Normalize the Data: Determine if the BG Dataset data fits a normal distribution by performing the WilkShapiro test. [The Wilk-Shapiro test (sometimes referred to as Shapiro-Wilk test) is valid for sample 
sizes less than or equal to 50 . If the sample size is greater than 50 , then perform the Shapiro-Francia test.] If the data is not normal, perform a logarithm transform and re-check as discussed below. For BG Dataset sample sizes of $\mathbf{5 0}$ or less, the Wilk-Shapiro test procedure is:

(2)-6.1. Calculate the denominator of the $W$ test statistic, as follows:

$$
d=\sum_{i=1}^{n}\left(y_{i}-\bar{Y}\right)^{2}
$$

where $y_{i}$ represents each data value in the BG Dataset and $\bar{Y}$ is the mean of all BG Dataset values.

(2)-6.2. Order the data from smallest to largest value then compute $W$ :

$$
W=\frac{1}{d}\left[\sum_{i=1}^{k} a_{i}\left(y_{[n-i+1]}-y_{i}\right)\right]^{2}
$$

where $k$ is the greatest integer less than or equal to $n / 2$. The $a_{i}$ coefficients are found in Table 2 .

(2)-6.3. The test statistic, $W$, is then checked against associated probabilities, which at the significance level of 0.05 results in the following decisions. If the $W$ value is greater than that shown in Table 3 , then a normal data distribution is indicated and no transformation is necessary. If the $W$ value is less than that shown in Table 3, then non-normally distributed data is indicated. The All Dataset and BG Dataset data (including PQLs) should be transformed via $y=\ln (y)$, and the new BG Dataset should be rechecked for normality. If non-normality is indicated after the in() transformation, then the control chart analysis should not be continued.

(2)-7. Trend Detection and Removal: Control charts require the data to be random, i.e. data that does not exhibit a trend. For F\&H, many of the monitoring well data will exhibit trends and these trends will need to be detected and removed. The Mann-Kendall test is used to determine if a trend exists. If so, the best-fit trend (either linear or exponential) will be determined and removed from the data. As the MannKendall test is non-parametric, remaining NDs in the BG Dataset will be temporarily considered to be equal to $1 / 2$ PQL (or MDL) for this step.

(2)-7.1. The Mann-Kendall test has two possible procedures, depending on the number of samples in the dataset. Typically if the BG Dataset contains less than 40 values, then an "exact test" is used. For F\&H, however, we can use the "normal approximation test" (normally used for datasets with greater than 40 values) for any BG Dataset as the difference in results will be minor. In addition, determination of the test statistic results is more straight-forward using the "normal approximation test."

(2)-7.1.1. Samples from the BG Dataset, ordered by time, are compared in a nested way to determine the non-parametric "sign" for each comparison:

$$
\operatorname{sgn}\left(x_{j}-x_{i}\right)=\left\{\begin{array}{ccc}
1 & \text { if } & x_{j}-x_{i}>0 \\
0 & \text { if } & x_{j}-x_{i}=0 \\
-1 & \text { if } & x_{j}-x_{i}<0
\end{array}\right\} .
$$

(2)-7.1.2. Then, the Mann-Kendall statistic is calculated as:

$$
S=\sum_{i=1}^{n-1} \sum_{j=i+1}^{n} \operatorname{sgn}\left(x_{j}-x_{i}\right)
$$

(2)-7.1.3. The test statistic is then calculated as: 


$$
\begin{gathered}
Z=\frac{S-1}{\sqrt{\operatorname{var}(S)}} \quad \text { if } \quad S>0 \\
Z=0 \quad \text { if } \quad S=0 \\
Z=\frac{S+1}{\sqrt{\operatorname{var}(S)}} \text { if } \quad S<0
\end{gathered}
$$

where:

$$
\operatorname{var}(S)=\frac{n(n-1)(2 n+5)}{18}
$$

(2)-7.1.4. The test statistic, $Z$, is then checked against associated probabilities, which at the significance level of 0.05 results in the following decisions: If the $Z$ value is greater than 1.96, then a positive trend is indicated. If the $Z$ value is less than -1.96 , then a negative trend is indicated. Otherwise, no trend in the data is likely.

(2)-7.2. If a trend was indicated by the Mann-Kendall test, then the best-fit trend function should be determined. Both linear and exponential trends will be tested, and the optimum will be chosen by comparing the coefficients of determination. It is expected at F\&H that a majority of the trends will be exponential. (Note that an exponential trend on non-transformed data will be equal to a linear trend on (natural) log-transformed data.)

(2)-7.2.1. Linear regression is used on the BG Dataset to calculate the slope $(\beta)$, intercept $(\alpha)$, and the coefficient of determination $\left(R^{2}\right)$ value, as follows:

$$
\begin{gathered}
\beta=\frac{\sum \tilde{x} \tilde{y}}{\sum \tilde{x}^{2}} \\
\alpha=\bar{y}-\beta \bar{x} \\
R^{2}=\frac{\left(\sum \tilde{x} \tilde{y}\right)^{2}}{\sum \tilde{x}^{2} \sum \bar{y}^{2}}
\end{gathered}
$$

where $\tilde{x}$ is the mean difference of the date, $x_{i},\left(x_{i}-\bar{x}\right)$, and $\tilde{y}$ is the mean difference of the sample value, $y_{i},\left(y_{i}-\bar{y}\right)$. The date can be in any "continuous" framework, i.e., decimal years, cumulative quarters, or any other "real" time frame.

(2)-7.2.2. To calculate the exponential trend parameters (if the data has not been aiready logtransformed), the BG Dataset data should be transformed by $\gamma=\ln (y)$, and the linear regression outlined above is to be used to calculate the slope, intercept and coefficient of determination for the $(x, y)$ data pairs.

(2)-7.2.3. The best-fit line would therefore be indicated by the greatest coefficient of determination. For a linear trend, the resulting equation is: $y=\alpha+\beta x$. For an exponential trend, the resulting equation is: $y=\alpha e^{\beta x}$.

(2)-7.3. If applicable, de-trend the All Dataset and the BG Dataset data (including PQLs) as follows: 


$$
\begin{aligned}
& \dot{y}=y-\alpha-\beta x \quad \text { for linear trend } \\
& \dot{y}=y-\alpha e^{\beta x} \quad \text { for exponential trend }
\end{aligned}
$$

(2)-8. Seasonality Detection and Removal: Seasonality in the BG Dataset can be detected using the Kruskal-Wallis test. However, this test requires at least 4 data points in each season. Thus, if the BG Dataset does not contain 4 data points for each season (defined as each quarter), then seasonality detection and removal will not be performed.

(2)-8.1. All the data is ranked according to value (lowest being 1 , highest being $n$ ). Ties are ranked at the average of the tied ranks. (For example, if the ordered dataset was [ND, ND, 1, 2, 2, 2, 3], then the rankings would be $[1.5,1.5,3,5,5,5,7]$.) The Kruskal-Wallis statistic, $H$, is then calculated as follows:

$$
H=\left[\frac{12}{n(n+1)} \sum_{i=1}^{2} \frac{R_{i}^{2}}{N_{i}}\right]-3(n+1)
$$

where $n$ is the total number of samples, $i$ indicates the season, $N_{i}$ is the number of samples in each season, and $R_{i}$ is the sum of the ranks for each season. For the F\&H data, each quarter represents a different season.

(2)-8.2. If there were ranking ties, then the $H^{\prime}$ 'statistic is calculated as follows:

$$
H^{\prime}=\frac{H}{1-\left(\sum_{i=1}^{8} T_{i} /\left(n^{3}-n\right)\right)}
$$

where $g$ is the total number of tied groups, and $T_{i}$ is computed for each tied group as: $T_{i}=\left(t_{i}{ }^{3}-t_{i}\right)$, where $t_{i}$ are the number of ties in each tied group.

(2)-8.3.If either the $H$ or $H^{\prime}$ statistic exceeds 7.815 , then seasonality is indicated and should be removed per EPA guidelines as follows:

(2)-8.3.1. Calculate the arithmetic average concentration for each season, $\bar{Y}_{i}$, in the BG Dataset.

(2)-8.3.2. Calculate the grand mean arithmetic average for all BG Dataset data, $\bar{Y}$.

(2)-8.3.3. Adjust the All Dataset and BG Dataset values, $y_{i j}$ (including PQLs), using: $Z=y_{i j}-\bar{Y}_{i}+\bar{Y}$, where $j$ is the data index, and $i$ represents the corresponding season/quarter.

Task (3): Compute Control Chart Parameters and Values

(3)-1. Background Statistics Calculation: The creation of a control chart requires the calculation of background statistical moments, as follows:

(3)-1.1. Calculate the arithmetic mean, $\bar{Y}$, and standard deviation, $S$, of the (de-trended, seasonally adjusted, transformed) BG Dataset.

(3)-1.2. If the ND test in step (2)-3. indicated the need for Cohen's adjustment, perform the adjustment as follows: 
(3)-1.2.1. Compute the arithmetic mean, $\bar{Y}_{d}$, and variance, $S_{d}{ }^{2}$, for the BG Dataset data that are not NDs.

(3)-1.2.2. Compute the following parameters:

$$
\begin{aligned}
& h=(n-m) / n \\
& \gamma={S_{d}}^{2} /\left(\bar{Y}_{d}-D L\right)^{2}
\end{aligned}
$$

where $m$ is the number of samples above the detection limit, $D L$, and $n$ is the total number of samples in the BG dataset. The value for $D L$ in this equation will be the arithmetic average of $1 / 2$ the PQL's (transformed, detrended, and/or adjusted for seasonality, as appropriate) for the ND samples.

(3)-1.2.3. Determine the parameter $\bar{\lambda}$, from Tabie 4, and estimate the corrected mean and standard deviation by using:

$$
\begin{aligned}
& \bar{Y}=\bar{Y}_{d}-\bar{\lambda}\left(\bar{Y}_{d}-D L\right) \\
& S=\sqrt{S_{d}{ }^{2}+\bar{\lambda}\left(\bar{Y}_{d}-D L\right)}
\end{aligned}
$$

(3)-2. Compute the Control Chart Values: For each non-BG value in the All Dataset, compute the following:

(3)-2.1. Calculate the standardized value, $z_{i}$, for each sample, $y_{i}$ :

$$
z_{i}=\left(y_{i}-\bar{Y}\right) \sqrt{n_{i}} / S
$$

where $n_{i}$ is the number of sample values averaged for the quarter.

(3)-2.2. Calculate the Positive CUSUM value, $S_{i}^{+}$:

$$
S_{i}^{+}=\max \left(0,\left(z_{i}-k\right)+S_{i-1}^{+}\right)
$$

(3)-2.3. Calculate the Negative CUSUM value, $S_{i}{ }^{-}$:

$$
S_{i}^{-}=\min \left(0,\left(z_{i}+k\right)+S_{i-1}^{-}\right)
$$

Task (4): Plot the Control Chart

(4)-1. Plot the results: Plot $z_{i}, S_{i}^{+}$, and $S_{i}^{-}$vs. $t_{i}$ with the control limits. 
Tables

Table 1. Dixon Test Critical Values

\begin{tabular}{|c|c|c|c|c|c|c|c|}
\hline $\begin{array}{c}\text { Number of } \\
\text { Observations } \\
\text { (n) }\end{array}$ & $\begin{array}{c}\text { Upper 1\% } \\
\text { Significance } \\
\text { Level }\end{array}$ & $\begin{array}{c}\text { Number of } \\
\text { Observations } \\
\text { (n) }\end{array}$ & $\begin{array}{c}\text { Upper } 1 \% \\
\text { Significance } \\
\text { Level }\end{array}$ & $\begin{array}{c}\text { Number of } \\
\text { Observations } \\
\text { (n) }\end{array}$ & $\begin{array}{c}\text { Upper } 1 \% \\
\text { Significance } \\
\text { Level }\end{array}$ & $\begin{array}{c}\text { Number of } \\
\text { Observations } \\
\text { (n) }\end{array}$ & $\begin{array}{c}\text { Upper } 1 \% \\
\text { Significance } \\
\text { Level }\end{array}$ \\
\hline 3 & 1.155 & 47 & 3.310 & 91 & 3.567 & 135 & 3.700 \\
\hline 4 & 1.492 & 48 & 3.319 & 92 & 3.570 & 136 & 3.702 \\
\hline 5 & 1.749 & 49 & 3.329 & 93 & 3.575 & 137 & 3.704 \\
\hline 6 & 1.944 & 50 & 3.336 & 94 & 3.579 & 138 & 3.707 \\
\hline 7 & 2.097 & 51 & 3.345 & 95 & 3.582 & 139 & 3.710 \\
\hline 8 & 2.221 & 52 & 3.353 & 96 & 3.586 & 140 & 3.712 \\
\hline 9 & 2.323 & 53 & 3.361 & 97 & 3.589 & 141 & 3.714 \\
\hline 10 & 2.410 & 54 & 3.368 & 98 & 3.593 & 142 & 3.716 \\
\hline 11 & 2.485 & 55 & 3.376 & 99 & 3.597 & 143 & 3.719 \\
\hline 12 & 2.550 & 56 & 3.383 & 100 & 3.600 & 144 & 3.721 \\
\hline 13 & 2.607 & 57 & 3.391 & 101 & 3.603 & 145 & 3.723 \\
\hline 14 & 2.659 & 58 & 3.397 & 102 & 3.607 & 146 & 3.725 \\
\hline 15 & 2.705 & 59 & 3.405 & 103 & 3.610 & 147 & 3.727 \\
\hline 16 & 2.747 & 60 & 3.411 & 104 & 3.614 & & \\
\hline 17 & 2.785 & 61 & 3.418 & 105 & 3.617 & & \\
\hline 18 & 2.821 & 62 & 3.424 & 106 & 3.620 & & \\
\hline 19 & 2.854 & 63 & 3.430 & 107 & 3.623 & & \\
\hline 20 & 2.884 & 64 & 3.437 & 108 & 3.626 & & \\
\hline 21 & 2.912 & 65 & 3.442 & 109 & 3.629 & & \\
\hline 22 & 2.939 & 66 & 3.449 & 110 & 3.632 & & \\
\hline 23 & 2.963 & 67 & 3.454 & 111 & 3.636 & & \\
\hline 24 & 2.987 & 68 & 3.460 & 112 & 3.639 & & \\
\hline 25 & 3.009 & 69 & 3.466 & 113 & 3.642 & & \\
\hline 26 & 3.029 & 70 & 3.471 & 114 & 3.645 & & \\
\hline 27 & 3.049 & 71 & 3.476 & 115 & 3.647 & & \\
\hline 28 & 3.066 & 72 & 3.482 & 116 & 3.650 & & \\
\hline 29 & 3.085 & 73 & 3.487 & 117 & 3.653 & & \\
\hline 30 & 3.103 & 74 & 3.492 & 118 & 3.656 & & \\
\hline 31 & 3.119 & 75 & 3.496 & 119 & 3.659 & & \\
\hline 32 & 3.135 & 76 & 3.502 & 120 & 3.662 & & \\
\hline 33 & 3.150 & 77 & 3.507 & 121 & 3.665 & & \\
\hline 34 & 3.164 & 78 & 3.511 & 122 & 3.667 & & \\
\hline 35 & 3.178 & 79 & 3.516 & 123 & 3.670 & & \\
\hline 36 & 3.191 & 80 & 3.521 & 124 & 3.672 & & \\
\hline 37 & 3.204 & 81 & 3.525 & 125 & 3.675 & & \\
\hline 38 & 3.216 & 82 & 3.529 & 126 & 3.677 & & \\
\hline 39 & 3.228 & 83 & 3.534 & 127 & 3.680 & & \\
\hline 40 & 3.240 & 84 & 3.539 & 128 & 3.683 & & \\
\hline 41 & 3.251 & 85 & 3.543 & 129 & 3.686 & & \\
\hline 42 & 3.261 & 86 & 3.547 & 130 & 3.688 & & \\
\hline 43 & 3.271 & 87 & 3.551 & 131 & 3.690 & & \\
\hline 44 & 3.282 & 88 & 3.555 & 132 & 3.693 & & \\
\hline 45 & 3.292 & 89 & 3.559 & 133 & 3.695 & & \\
\hline 46 & 3.302 & 90 & 3.563 & 134 & 3.697 & & \\
\hline
\end{tabular}


Table 2. a Coefficients for the Wilk-Shapiro Test

\begin{tabular}{|c|c|c|c|c|c|c|c|c|c|c|c|c|c|c|c|c|c|c|c|c|c|c|c|c|}
\hline & 2 & 3 & 4 & 5 & 6 & 7 & 8 & 9 & 10 & 11 & 12 & 13 & 14 & 15 & 16 & 17 & 18 & 19 & 20 & 21 & 22 & 23 & 24 & 25 \\
\hline 1 & .7071 & .7071 & .6872 & .6646 & .6431 & .6233 & .6052 & .5889 & .5739 & .5601 & .5475 & .5359 & .5251 & .5150 & .5056 & .4968 & .4886 & .4808 & .4734 & .4643 & .4590 & .4542 & .493 & .450 \\
\hline 2 & & .0000 & .1677 & .2413 & .2806 & .3031 & .3164 & .3244 & .3291 & .3315 & .32025 & .3325 & .3318 & .3306 & .3290 & .3273 & .3253 & .3232 & .3211 & .3185 & .3156 & .3126 & .3098 & .3069 \\
\hline $\mathbf{3}$ & & & & .0000 & .0875 & .1401 & .1743 & 1976 & .2141 & .2260 & .2347 & .2412 & .2460 & .2495 & .2521 & .2540 & .2553 & .2561 & .2565 & .2578 & .2571 & 2560 & 2554 & 2543 \\
\hline 4 & & & & & & .0000 & .0561 & .0947 & .1224 & .1429 & .1586 & .1707 & .1802 & .1878 & .1939 & .1988 & .2027 & .2059 & .2085 & .2119 & .2131 & .2139 & .2145 & .2148 \\
\hline 5 & & & & & & & & .0000 & .0399 & .0695 & .0822 & .1099 & .1240 & 1353 & .147 & .1524 & .1587 & .1641 & .1686 & .1736 & .1764 & .1787 & .1807 & .1822 \\
\hline c & & & & & & & & & & .0000 & .0303 & .0539 & .0727 & .0880 & .1005 & .1100 & .1197 & .1271 & .1334 & 1399 & .1443 & .1480 & .1512 & 1539 \\
\hline 7 & & & & & & & & & & & & .0000 & .0240 & .0433 & .0593 & .0725 & .0837 & .0932 & .1013 & .1092 & .1150 & 1201 & .1245 & .1283 \\
\hline a & & & & & & & & & & & & & & .0000 & .0196 & .0359 & .0496 & .0612 & .0711 & .0804 & .0878 & .0941 & .0997 & .1046 \\
\hline 9 & & & & & & & & & & & & & & & & .0000 & .0163 & .0300 & .0422 & .0530 & .0618 & .0696 & .0764 & .0823 \\
\hline 10 & & & & & & & & & & & & & & & & & & .0000 & .1040 & .0263 & .0368 & .0459 & .0530 & .0610 \\
\hline 11 & & & & & & & & & & & & & & & & & & & & .0000 & .0122 & .0228 & .0321 & .0408 \\
\hline 12 & & & & & & & & & & & & & & & & & & & & & & .0000 & .1007 & .0200 \\
\hline 13 & & & & & & & & & & & & & & & & & & & & & & & & .0000 \\
\hline
\end{tabular}

\begin{tabular}{|c|c|c|c|c|c|c|c|c|c|c|c|c|c|c|c|c|c|c|c|c|c|c|c|c|c|}
\hline & 26 & 27 & 20 & 29 & 30 & 31 & 32 & 33 & 34 & 35 & 36 & 37 & 38 & 39 & 40 & 41 & 42 & 43 & 4 & 45 & 46 & 47 & 40 & 49 & 50 \\
\hline 1 & .407 & .4366 & .4328 & .4291 & .4254 & .1220 & .4188 & .4456 & 1127 & .4096 & .068 & .4010 & .0015 & .3989 & .3964 & .3960 & .3817 & .3694 & .3872 & .3850 & .3830 & .3806 & .3789 & .3770 & $.375 t$ \\
\hline 2 & .3043 & .3018 & .2992 & .2968 & .2004 & .2921 & .2890 & .2876 & .2854 & .2034 & .2813 & .2794 & .2774 & .2755 & .2737 & .2710 & .2701 & .2684 & 2667 & $.265 t$ & .2635 & .2620 & .2604 & .2589 & .2574 \\
\hline 3 & .2533 & .2522 & .2510 & .2499 & .2487 & .2475 & .2463 & .2451 & .2439 & .2427 & .2415 & .2403 & .2391 & .2380 & .2368 & .2357 & .2345 & .2334 & .2323 & .2313 & .2302 & .2291 & .2281 & .2271 & .2260 \\
\hline 4 & .2151 & 2152 & .2151 & .2150 & .2148 & .2145 & $.214:$ & 2137 & .2132 & .2127 & .2121 & .2116 & .2110 & .2104 & 2098 & .2091 & .2085 & .2078 & .2072 & .2065 & .2058 & .2052 & .2045 & .2038 & .2032 \\
\hline 5 & .1836 & .1848 & .1857 & .1864 & .1870 & .1874 & .1878 & .1830 & .1882 & .1883 & .1880 & .1883 & .1881 & .1880 & .1878 & .1876 & .1874 & .1871 & .1868 & .1865 & .1862 & .1859 & .1855 & .1851 & .1847 \\
\hline 6 & .1563 & .1584 & .1601 & .1616 & 1630 & .1641 & .1651 & .1660 & .1667 & .1673 & .1678 & .1683 & .1686 & .1689 & 1691 & .1693 & .1694 & .1695 & .1695 & .1695 & .1695 & .1695 & .1693 & .1692 & .1691 \\
\hline 7 & .1316 & .1346 & .1372 & .1395 & .1415 & .1433 & 1449 & .1463 & .1475 & .1487 & .1496 & .1505 & .1513 & .1520 & .1526 & .1531 & .1535 & .1539 & .1542 & .1545 & .1548 & .1550 & .1551 & .1553 & .1554 \\
\hline 8 & .1089 & .1128 & .1162 & .1192 & .1219 & .1243 & .1265 & .1284 & .1301 & .1317 & .1331 & .1344 & .1356 & .1366 & 1376 & .1384 & .1390 & .1398 & .1405 & .1410 & .1415 & .1420 & .1423 & .1427 & .1430 \\
\hline 9 & .0876 & .0923 & .0065 & .1002 & .1036 & .1066 & .1003 & . 1118 & .1140 & .1160 & .1179 & .1196 & $.12+1$ & .1225 & .1237 & .1249 & 1259 & .1269 & .1278 & .1286 & .1293 & .1300 & .1306 & .1312 & .1317 \\
\hline 10 & .0672 & .0728 & .0778 & .0822 & .0862 & .0899 & .0931 & .0861 & .0988 & .1013 & .1036 & .1058 & .075 & .1082 & $.1+08$ & .1123 & .1136 & .1149 & .1160 & .1170 & .1180 & 1189 & .1197 & .1205 & .1212 \\
\hline 11 & .0476 & .0540 & .0598 & .0650 & .0697 & .0739 & .077 & .0812 & .0844 & .0873 & .0900 & .0924 & .0947 & .0967 & .0986 & .1004 & .1020 & .1035 & 1049 & .1062 & .1073 & .1085 & .1095 & .1105 & .1113 \\
\hline 12 & .0284 & .0358 & .0424 & .0483 & .0537 & .0585 & .0629 & .0669 & .0706 & .0739 & .0770 & .0798 & .0824 & .0848 & .0870 & .0891 & .0909 & .0927 & .0013 & .0959 & .0972 & .0986 & .0998 & .1010 & .1020 \\
\hline 13 & .0094 & .0178 & .0253 & .0320 & .0381 & .0435 & .0485 & .0530 & .0572 & .0610 & .0645 & .067 & .0706 & .0733 & .0759 & .0782 & .0804 & .0824 & .0842 & .0860 & .0876 & .0692 & .0806 & .0919 & \\
\hline 14 & & .0000 & .0084 & .0159 & .0227 & .0289 & .0344 & .0395 & .0441 & .0484 & .0523 & .0559 & .0592 & .0622 & .0651 & .0677 & .0701 & .0724 & .0745 & .0765 & .0783 & .0801 & .0817 & .0832 & \\
\hline 15 & & & & .0000 & .0076 & .0144 & .0206 & .0262 & .0314 & .0361 & .0404 & .0444 & .0481 & .0515 & .0546 & .0575 & .0602 & .0628 & .0651 & .0673 & .0694 & .0713 & .0731 & .0748 & \\
\hline 16 & & & & & & .0000 & .0068 & .0131 & .0187 & .0239 & .0287 & .0331 & .0972 & .0409 & .0444 & .0476 & .0506 & .0534 & .0560 & .0584 & .0607 & .0628 & .0648 & .0667 & .0685 \\
\hline 17 & & & & . & & & & .0000 & .0062 & .0119 & .0172 & .0220 & .0264 & .0305 & .0343 & .0979 & .0411 & .0442 & .0471 & .0497 & .0522 & .0546 & .0568 & .0588 & .0608 \\
\hline 18 & & & & & & & & & & .0000 & .0057 & .0110 & .0158 & .0203 & .0244 & .0283 & .0318 & .0352 & .0383 & .0412 & .0439 & .0465 & .0489 & .0511 & .0532 \\
\hline 19 & & & & & & & & & & & & .0000 & .0053 & .0101 & .0146 & .0188 & .0227 & .0263 & .0296 & .0328 & .0357 & .0385 & .0411 & .0436 & .0459 \\
\hline 20 & & & & & & & & & & & & & & .0000 & .0049 & .0094 & .0136 & .0175 & .0211 & .0245 & .0277 & .0307 & .0335 & .0361 & .0386 \\
\hline 21 & & & & & & & & & & & & & & & & .0000 & .0045 & .0007 & .0126 & .0163 & .0197 & .0229 & .0259 & .0288 & .0314 \\
\hline 22 & & & & & & & & & & & & & & & & & & .0000 & .0042 & .0081 & .0118 & .0153 & .0185 & .0215 & .0244 \\
\hline 23 & & & & & & & & & & & & & & & & & & & & .0000 & .0039 & .0076 & .0111 & .0143 & .0174 \\
\hline 24 & & & & & & & & & & & & & & & & & & & & & & .0000 & .0037 & .0071 & .0104 \\
\hline 25 & & & & & & & & & & & & & & & & & & & & & & & & .0000 & .0035 \\
\hline
\end{tabular}


Table 3. Wilk-Shapiro Test Critical Values

\begin{tabular}{|cccc|}
\hline Sample Sizo & Value of W 0.05 & Samplo Size & Value of W e 0.05 \\
\hline 3 & 0.767 & 27 & 0.923 \\
4 & 0.748 & 28 & 0.924 \\
5 & 0.762 & 29 & 0.926 \\
6 & 0.788 & 30 & 0.927 \\
7 & 0.803 & 31 & 0.929 \\
8 & 0.818 & 32 & 0.930 \\
9 & 0.829 & 33 & 0.931 \\
10 & 0.842 & 34 & 0.933 \\
11 & 0.850 & 35 & 0.934 \\
12 & 0.859 & 36 & 0.935 \\
13 & 0.866 & 37 & 0.936 \\
14 & 0.874 & 38 & 0.938 \\
15 & 0.881 & 39 & 0.939 \\
16 & 0.887 & 40 & 0.940 \\
17 & 0.892 & 41 & 0.941 \\
18 & 0.897 & 42 & 0.942 \\
19 & 0.901 & 43 & 0.943 \\
20 & 0.905 & 44 & 0.944 \\
21 & 0.908 & 45 & 0.945 \\
22 & 0.911 & 46 & 0.945 \\
23 & 0.914 & 47 & 0.946 \\
24 & 0.916 & 48 & 0.947 \\
25 & 0.918 & 49 & 0.947 \\
26 & 0.920 & 50 & 0.947 \\
\hline
\end{tabular}


Table 4. Cohen's Method $\bar{\lambda}$ Parameters

\begin{tabular}{|c|c|c|c|c|c|c|c|c|c|c|c|}
\hline \multirow[b]{2}{*}{$\boldsymbol{\gamma}$} & \multicolumn{11}{|c|}{ Percantages of Non-detects } \\
\hline & .01 & .05 & .10 & .15 & .20 & .25 & .30 & .35 & .40 & .45 & .50 \\
\hline .01 & .0102 & .0530 & .1111 & .1747 & .2443 & .3205 & .4043 & .4967 & .5989 & .7128 & .8403 \\
\hline .05 & .0105 & .0547 & .1143 & .1793 & .2503 & .3279 & .4130 & .5066 & .6101 & .7252 & .8540 \\
\hline .10 & .0110 & .0566 & .1180 & .1848 & .2574 & .3366 & .4233 & .5184 & .6234 & .7400 & .8703 \\
\hline .15 & .0113 & .0584 & .1215 & .1898 & .2640 & .3448 & .4330 & .5296 & .6361 & .7542 & .8860 \\
\hline .20 & .0116 & .0600 & .1247 & .1946 & .2703 & .3525 & .4422 & .5403 & .6483 & .7678 & .9012 \\
\hline .25 & .0120 & .0615 & .1277 & 1991 & .2763 & .3599 & .4510 & .5506 & .6600 & .7810 & .9158 \\
\hline .30 & .012z & .0630 & .1306 & .2034 & $2 \theta i \dot{y}$ & .3670 & $.45 \$ 5$ & .5604 & .6713 & .7937 & .9500 \\
\hline .35 & .0125 & .0643 & .1333 & .2075 & 2874 & .3738 & .4676 & .5699 & .6821 & .8060 & .9437 \\
\hline .40 & .0128 & .0657 & .1360 & .2114 & .2926 & .3803 & .4755 & .5791 & .6927 & .8179 & .9570 \\
\hline .45 & .0130 & .0669 & .1385 & .2152 & .2976 & .3866 & .4831 & .5880 & .7029 & .8295 & .9700 \\
\hline .50 & .0133 & .0681 & .1409 & .2188 & .3025 & .3928 & .4904 & .5967 & .7129 & .8408 & .9826 \\
\hline .55 & .0135 & .0693 & .1432 & .2224 & .3073 & .3987 & .4976 & .6051 & .7225 & .8517 & .9950 \\
\hline .60 & .0137 & .0704 & .1455 & .2258 & .3118 & .4045 & .5046 & .6133 & .7320 & .8625 & 1.0070 \\
\hline .65 & .0140 & .0715 & .1477 & .2291 & .3163 & .4101 & .5114 & .6213 & .7412 & .8729 & 1.0188 \\
\hline .70 & .0142 & .0726 & .1499 & .2323 & .3206 & .4156 & .5180 & .6291 & .7502 & .8832 & 1.0303 \\
\hline .75 & .0144 & .0736 & .1520 & .2355 & .3249 & .4209 & .5245 & .6367 & .7590 & .8932 & 1.0416 \\
\hline .80 & .0146 & .0747 & .1540 & .2386 & .3290 & .4261 & .5308 & .6441 & .7676 & .9031 & 1.0527 \\
\hline .65 & .0148 & .0756 & .1560 & .2416 & .3331 & .4312 & .5370 & .6515 & .7761 & .9127 & 1.0636 \\
\hline .90 & .0150 & .0766 & .1579 & .2445 & .3370 & .4362 & .5430 & .6586 & .7844 & .9222 & 1.0743 \\
\hline .95 & .0152 & .0755 & .1598 & .2474 & .3409 & .4411 & .5480 & .6656 & .7925 & .9314 & 1.0847 \\
\hline 1.00 & .0153 & .0785 & .1617 & .2502 & .3447 & .4459 & .5548 & .6725 & .8005 & .9406 & 1.0951 \\
\hline 1.05 & .0155 & .0794 & .1635 & .2530 & .3484 & .4506 & .5605 & .6793 & .8084 & .9496 & 1.1052 \\
\hline 1.10 & .0157 & .0803 & .1653 & .2557 & .3521 & .4553 & .5662 & .6860 & .8161 & .9584 & 1.1152 \\
\hline 1.15 & .0159 & .0811 & .1671 & .2584 & .3557 & .4598 & .5717 & .6925 & .8237 & .9671 & 1.1250 \\
\hline 1.20 & .0160 & .0820 & .1688 & .2610 & .3592 & .4643 & .5771 & 6990 & .8312 & .9756 & 1.1347 \\
\hline 1.25 & .0162 & .0828 & .1705 & .2636 & .3627 & .4687 & .5825 & .7053 & .8385 & .9841 & 1.1443 \\
\hline 1.30 & .0164 & .0836 & .1722 & .2661 & $.366 t$ & .4730 & .5878 & .7115 & .8458 & .9924 & 1.1537 \\
\hline 1.35 & .0165 & .0845 & .1738 & .2686 & .3695 & .4773 & .5930 & .7177 & .8529 & 1.0006 & 1.1629 \\
\hline 1.40 & .0167 & .0853 & .1754 & .2710 & .3728 & .4815 & .5981 & .7238 & .8600 & 1.0087 & 1.1721 \\
\hline 1.45 & .0168 & .0860 & .1770 & .2735 & .3761 & .4856 & .6081 & .7298 & .8670 & 1.0166 & 1.1812 \\
\hline 1.50 & .0170 & .0868 & .1786 & .2758 & .3793 & .4897 & 6081 & .7357 & .8738 & 1.0245 & 1.1901 \\
\hline 1.55 & .0171 & .0876 & .1801 & .2782 & .3825 & .4938 & .6130 & .7415 & 8806 & 1.0323 & 1.1989 \\
\hline 1.60 & .0173 & .0883 & .1817 & .2805 & .3856 & .4977 & .6179 & .7472 & .8873 & 1.0400 & 1.2076 \\
\hline 1.65 & .0174 & .0891 & .1832 & .2828 & .3887 & .5017 & .6227 & .7529 & .8939 & 1.0476 & 1.2162 \\
\hline 1.70 & .0176 & .0898 & .1846 & .2851 & .3918 & .5055 & .6274 & .7585 & .9005 & 1.0551 & 1.2240 \\
\hline 1.75 & .0177 & .0905 & .1861 & .2873 & .3948 & .5094 & .6321 & .7641 & .9069 & 1.0625 & 1.2332 \\
\hline 1.80 & .0179 & .0913 & .1876 & .2895 & .3978 & .5132 & .6367 & .7696 & .9133 & 1.0698 & 1.2415 \\
\hline 1.85 & .0180 & .0920 & .1890 & .2917 & .4007 & .5167 & .6413 & .7750 & .9169 & 1.0771 & 1.2497 \\
\hline 1.90 & .0181 & .0927 & .1904 & .2938 & .4036 & .5206 & .6458 & .7804 & .9259 & 1.0842 & 1.2579 \\
\hline 1.95 & .0183 & .0933 & .1918 & .2960 & .4065 & .5243 & .6502 & .7857 & .9321 & 1.0913 & 1.2660 \\
\hline 2.00 & .0184 & .0940 & .1932 & .2981 & .4093 & .5279 & .6547 & .7909 & .9382 & 1.0984 & 1.2739 \\
\hline 2.05 & .0186 & .0947 & 1945 & .3001 & .4122 & .5315 & .6590 & .7961 & .9442 & 1.1053 & 1.2819 \\
\hline 2.10 & .0187 & .0954 & .1959 & .3022 & .4149 & .5350 & .6634 & .8013 & .9502 & 1.1122 & 1.2897 \\
\hline 2.15 & .0188 & .0960 & .1972 & .3042 & .4177 & .5385 & .6676 & .8063 & .9562 & t.t190 & 1.2974 \\
\hline 2.20 & .0189 & .0967 & 1986 & .3062 & .4204 & .5420 & 6719 & .8114 & .9620 & 1.1258 & 1.3051 \\
\hline 2.25 & .0191 & .0973 & 1999 & .3082 & .4231 & .5454 & .6761 & .8164 & .9679 & 1.1325 & 1.3127 \\
\hline 2.30 & .0192 & .0980 & .2012 & .3102 & .4258 & .5488 & 6802 & .8213 & .9736 & 1.1391 & 1.3203 \\
\hline 2.35 & .0193 & .0986 & .2025 & .3122 & .4285 & .5522 & .6844 & .8262 & .9794 & 1.1457 & 1.3278 \\
\hline 2.40 & .0194 & .0992 & .2097 & .3141 & .4311 & .5555 & .6884 & .8311 & .9850 & 1.1522 & 1.3352 \\
\hline 2.45 & .0196 & .0998 & .2050 & .3160 & .4337 & .5588 & .6925 & .8359 & .9906 & 1.1587 & 1.3425 \\
\hline 2.50 & .0197 & .1005 & .2062 & .3179 & .4363 & .5621 & .6965 & .8407 & .9962 & 1.1651 & 1.3498 \\
\hline 2.55 & .0198 & 1011 & .2075 & .3198 & .4388 & .5654 & .7005 & .8454 & 1.0017 & 1.1714 & 1.3571 \\
\hline 2.60 & .0199 & .1017 & .2087 & .3217 & .4414 & .5686 & .7044 & .8501 & 1.0072 & 1.1777 & 1.3642 \\
\hline 2.65 & .0201 & .1023 & .2099 & .3236 & .4439 & .5718 & .7083 & .8548 & 1.0126 & 1.1840 & 1.3714 \\
\hline 2.70 & .0202 & .1029 & .2111 & .3254 & .4464 & .5750 & .7122 & .8594 & 1.0180 & 1.1902 & 1.3784 \\
\hline 2.75 & .0203 & .1035 & .2123 & .3272 & .4489 & .5781 & .7161 & .8639 & 1.0234 & 1.1963 & 1.3854 \\
\hline 2.80 & .0204 & .1040 & $.2+35$ & .3290 & .4513 & .5812 & .7199 & .8685 & 1.0287 & 1.2024 & 1.3924 \\
\hline 2.85 & .0205 & .1046 & .2147 & .3308 & .4537 & .5843 & .7237 & .8730 & 1.0339 & 1.2085 & 1.3993 \\
\hline 2.90 & .0206 & .1052 & .2158 & .3326 & .4562 & .5874 & .7274 & .8775 & 1.0392 & 1.2145 & 1.4061 \\
\hline 2.95 & .0207 & .1058 & .2170 & .3344 & .4585 & .5905 & .7311 & 8819 & 1.0443 & 1.2205 & 1.4129 \\
\hline 3.00 & .0209 & .1063 & .2182 & .3361 & .4609 & .5935 & .7348 & .8863 & 1.0495 & 1.2264 & 1.4197 \\
\hline 3.05 & .0210 & .1069 & .2193 & .3378 & .4633 & .5965 & .7385 & 8907 & 1.0546 & 1.2323 & 1.4264 \\
\hline 3.10 & .0211 & .1074 & .2204 & .3396 & .4656 & .5995 & .7422 & .8950 & 1.0597 & 1.2381 & 1.4330 \\
\hline 3.15 & .0212 & .1080 & .2216 & .3413 & .4679 & .6024 & .7458 & .8993 & 1.0647 & 1.2439 & 1.4396 \\
\hline 3.20 & .0213 & .1085 & .2227 & .3430 & .4703 & .6054 & .7494 & .9036 & 1.0697 & 1.2497 & 1.4462 \\
\hline 3.25 & .0214 & $.109 t$ & .2238 & .3447 & .4725 & .6083 & .7529 & .9079 & 1.0747 & 1.2554 & 1.4527 \\
\hline 3.30 & .0215 & 1096 & .2249 & .3464 & .4748 & .6112 & .7565 & .9121 & 1.0796 & 1.2611 & 1.4592 \\
\hline 3.35 & .0216 & .1102 & .2260 & .3480 & .4771 & $.614 t$ & .7600 & .9163 & 1.0845 & 1.2668 & 1.4657 \\
\hline 3.40 & .0217 & .1107 & .2270 & .3497 & .4793 & 6169 & .7635 & .9205 & 1.0894 & 1.2724 & 1.4720 \\
\hline 3.45 & .0218 & .1112 & .2281 & .3513 & .4816 & .6197 & .7670 & .9246 & 1.0942 & 1.2779 & 1.4784 \\
\hline
\end{tabular}


Table 4 (continued). Cohen's Method $\bar{\lambda}$ Parameters

\begin{tabular}{|c|c|c|c|c|c|c|c|c|c|c|c|}
\hline \multirow[b]{2}{*}{$\gamma$} & \multicolumn{11}{|c|}{ Percentages of Non-detects } \\
\hline & .01 & .05 & .10 & .15 & .20 & .25 & .30 & .35 & .40 & .45 & .50 \\
\hline 3.50 & .0219 & .1118 & .2292 & .3529 & .4838 & .6226 & .7704 & .9287 & 1.0990 & 1.2835 & 1.4847 \\
\hline 3.55 & .0220 & .1123 & .2303 & .3546 & .4860 & .6254 & .7739 & .9328 & 1.1038 & 1.2890 & 1.4910 \\
\hline 3.60 & .0221 & .1128 & .2313 & .3562 & .4882 & .6282 & .7773 & .9369 & 1.1086 & 1.2945 & 1.4972 \\
\hline 3.65 & .0222 & .1133 & .2324 & .3578 & .4903 & .6309 & .7807 & .9409 & 1.1133 & 1.2999 & 1.5034 \\
\hline 3.70 & .0223 & .1138 & .2334 & .3594 & .4925 & .6337 & .7840 & .9449 & 1.1180 & 1.3053 & 1.5096 \\
\hline 3.75 & .0224 & .1143 & .2344 & .3609 & .4946 & .6364 & .7874 & .9489 & 1.1226 & 1.3107 & 1.5157 \\
\hline 3.80 & .0225 & .1148 & .2355 & .3625 & .4968 & .6391 & .7907 & .9529 & 1.1273 & 1.3160 & 1.5218 \\
\hline 3.85 & .0226 & .1153 & .2365 & .3641 & .4989 & .6418 & .7940 & .9568 & 1.1319 & 1.3213 & 1.5279 \\
\hline 3.90 & .0227 & .1158 & .2375 & .3656 & .5010 & .6445 & .7973 & .9607 & 1.1364 & 1.3266 & 1.5339 \\
\hline 3.95 & .0228 & .1163 & .2385 & .3672 & .5031 & .6472 & .8006 & .9646 & 1.1410 & 1.3318 & 1.5399 \\
\hline 4.00 & .0229 & .1168 & .2395 & .3687 & .5052 & .6498 & .8038 & .9685 & 1.1455 & 1.3337 & 1.5458 \\
\hline 4.05 & .0230 & .1173 & .2405 & .3702 & .5072 & .6525 & .8070 & .9723 & 1.1500 & 1.3423 & 1.5518 \\
\hline 4.10 & .0231 & .1178 & .2415 & .3717 & .5093 & .6551 & .8102 & .9976 & 1.1545 & 1.3474 & 1.5577 \\
\hline 4.15 & .0232 & .1183 & .2425 & .3732 & .5113 & .6577 & .8134 & .9800 & 1.1590 & 1.3526 & 1.5635 \\
\hline 4.20 & .0233 & .1188 & .2435 & .3747 & .5134 & .6603 & .8166 & .9837 & 1.1634 & 1.3577 & 1.5693 \\
\hline 4.25 & .0234 & .1193 & .2444 & .3762 & .5154 & .6629 & .8198 & .9875 & 1.1678 & 1.3627 & 1.5751 \\
\hline 4.30 & .0235 & .1197 & .2454 & .3777 & .5174 & .6654 & .8229 & .9913 & 1.1722 & 1.3678 & 1.5809 \\
\hline 4.35 & .0236 & .1202 & .2464 & .3792 & .5194 & .6680 & .8260 & .9950 & 1.1765 & 1.3728 & 1.5866 \\
\hline 4.40 & .0237 & .1207 & .2473 & .3806 & .5214 & .6705 & .8291 & .9987 & 1.1809 & 1.3778 & 1.5924 \\
\hline 4.45 & .0238 & .1212 & .2483 & .3821 & .5234 & .6730 & .8322 & 1.0024 & 1.1852 & 1.3828 & 1.5980 \\
\hline 4.50 & .0239 & .1216 & .2492 & .3836 & .5253 & .6755 & .8353 & 1.0060 & 1.1895 & 1.3878 & 1.6037 \\
\hline 4.55 & .0240 & .1221 & .2502 & .3850 & .5273 & .6780 & .8384 & 1.0097 & 1.1937 & 1.3927 & 1.6093 \\
\hline 4.60 & .0241 & .1225 & 2511 & .3864 & .5292 & .6805 & .8414 & 1.0133 & 1.1980 & 1.3976 & 1.6149 \\
\hline 4.65 & .0241 & .1230 & .2521 & .3879 & .5312 & .6830 & .8445 & 1.0169 & 1.2022 & 1.4024 & 1.6205 \\
\hline 4.70 & .0242 & .1235 & .2530 & .3893 & .5331 & .6855 & .8475 & 1,0205 & 1.2064 & 1.4073 & 1.6260 \\
\hline 4.75 & .0243 & .1239 & .2539 & .3907 & .5350 & .6879 & .8505 & 1.0241 & 1.2106 & 1.4121 & 1.6305 \\
\hline 4.80 & .0244 & .1244 & .2548 & .3921 & .5370 & .6903 & .8535 & 1.0277 & 1.2148 & 1.4169 & 1.6370 \\
\hline 4.85 & .0245 & .1248 & .2558 & .3935 & .5389 & .6928 & .8564 & 1.0312 & 1.2189 & 1.4217 & 1.6425 \\
\hline 4.90 & .0246 & .1253 & .2567 & .3949 & .5407 & .6952 & .8594 & 1.0348 & 1.2230 & 1.4265 & 1.6479 \\
\hline 4.95 & .0247 & .1257 & .2576 & .3963 & .5426 & .6976 & .8623 & 1.0383 & 1.2272 & 1.4312 & 1.6533 \\
\hline 5.00 & .0248 & .1262 & .2585 & .3977 & .5445 & .7000 & 8653 & 1.0418 & 1.2312 & 1.4359 & 1.6587 \\
\hline 5.05 & .0249 & .1266 & .2594 & .3990 & .5464 & .7024 & .8682 & 1.0452 & 1.2353 & 1.4406 & 1.6641 \\
\hline 5.10 & .0249 & .1270 & .2603 & .4004 & .5482 & .7047 & .8711 & 1.0487 & 1.2394 & 1.4453 & 1.6694 \\
\hline 5.15 & .0250 & .1275 & .2612 & .4018 & .5501 & .7071 & .8740 & 1.0521 & 1.2434 & 1.4500 & 1.6747 \\
\hline 5.20 & .0251 & .1279 & .2621 & .4031 & .5519 & .7094 & .0768 & 1.0556 & 1.2474 & 1.4546 & 1.6800 \\
\hline 5.25 & .0252 & .1284 & .2629 & .4045 & .5537 & .7118 & .8797 & 1.0590 & 1.2514 & 1.4592 & 1.6853 \\
\hline 5.30 & .0253 & .1288 & .2638 & .4058 & .5556 & .7141 & .8825 & 1.0624 & 1.2554 & 1.4638 & 1.6905 \\
\hline 5.35 & .0254 & .1292 & .2647 & .4071 & .5574 & .7164 & .8854 & 1.0658 & 1.2594 & 1,4684 & 1.6958 \\
\hline 5.40 & .0255 & .1296 & .2656 & .4085 & .5592 & .7187 & .8882 & $1.069 t$ & 1.2633 & 1.4729 & 1.7010 \\
\hline 5.45 & .0255 & .1301 & .2664 & .4098 & .5610 & .7210 & .8910 & 1.0725 & 1.2672 & 1.4775 & 1.7061 \\
\hline 5.50 & .0256 & .1305 & .2673 & .4111 & .5628 & .7233 & .8938 & 1.0758 & $1.27+1$ & 1.4820 & 5.7113 \\
\hline 5.55 & .0257 & .1309 & .2682 & .4124 & .5646 & .7256 & .8966 & 1.0792 & 1.2750 & 1.4865 & 1.7164 \\
\hline 5.60 & .0258 & .1313 & .2690 & .4137 & .5663 & .7278 & .8994 & 1.0825 & 1.2789 & 1.4910 & 1.7215 \\
\hline 5.65 & .0259 & .1318 & .2699 & .4150 & .5681 & .7301 & .9022 & 1.0858 & 1.2828 & 1.4954 & 1.7266 \\
\hline 5.70 & .0260 & .1322 & .2707 & .4163 & .5699 & .7323 & .9049 & 1.0891 & 1.2866 & 1.4999 & 1.7317 \\
\hline 5.75 & .0260 & .1326 & .2716 & .4176 & .5716 & .7346 & .9077 & 1.0924 & 1.2905 & 1.5043 & 1.7368 \\
\hline 5.80 & .0261 & .1330 & .2724 & .4189 & .5734 & .7368 & .9104 & 1.0956 & 1.2943 & 1.5087 & 1.7418 \\
\hline 5.85 & .0262 & .1334 & .2732 & .4202 & .5751 & .7390 & .9131 & 1.0989 & 1.2981 & 1.5131 & 1.7468 \\
\hline 5.90 & .0263 & .1338 & .2741 & .4215 & .5769 & .7412 & .9158 & 1.1021 & 1.3019 & 1.5175 & 1.7518 \\
\hline 5.95 & .0264 & .1342 & .2749 & .4227 & .5786 & .7434 & .9185 & 1.1053 & 1.3057 & 1.5218 & 1.7568 \\
\hline 6.00 & .0264 & .1346 & .2757 & .4240 & .5803 & .7456 & .9212 & 1.1085 & 1.3094 & 1.5262 & 1.7617 \\
\hline
\end{tabular}




\section{Control Charts}

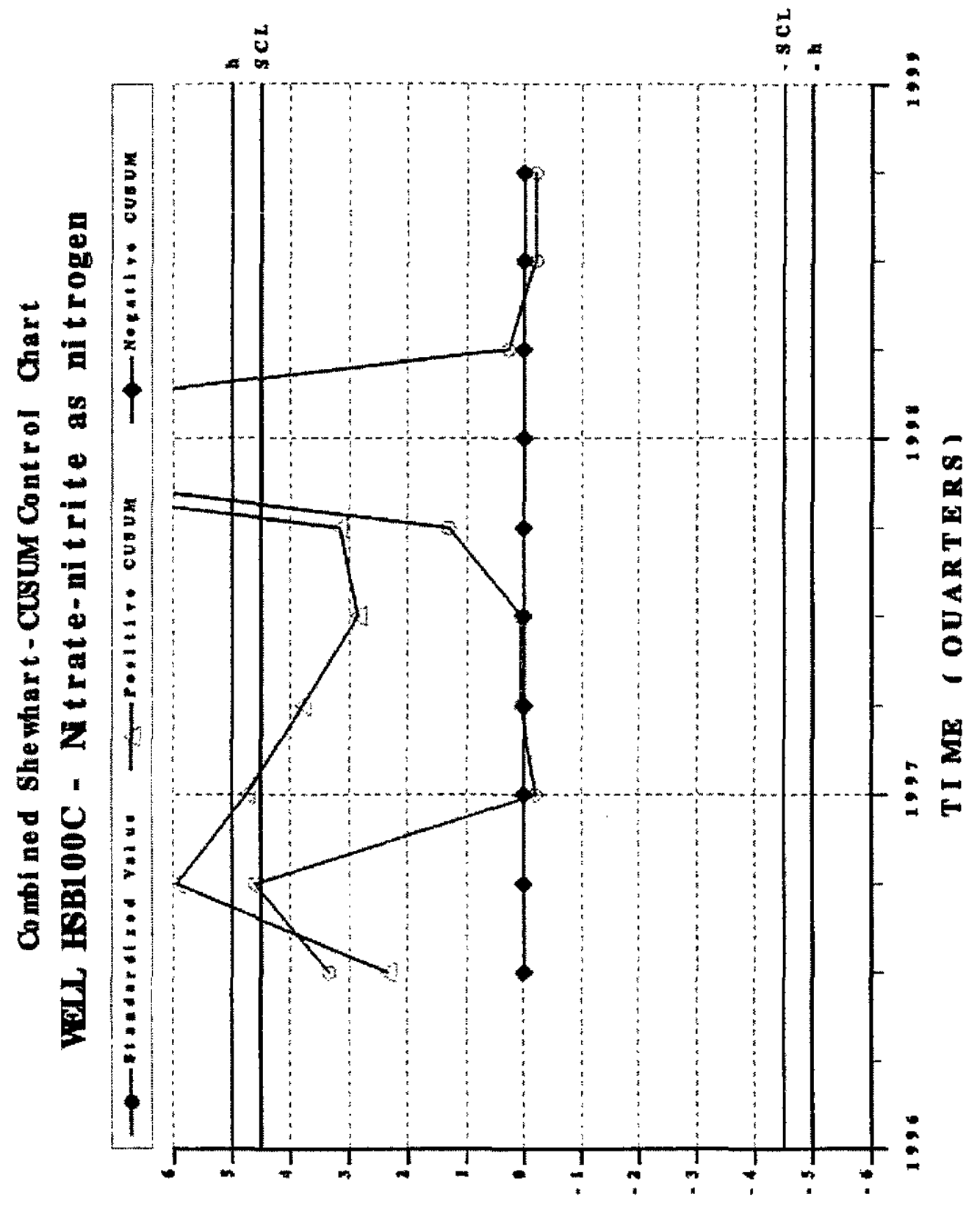

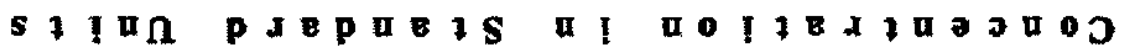



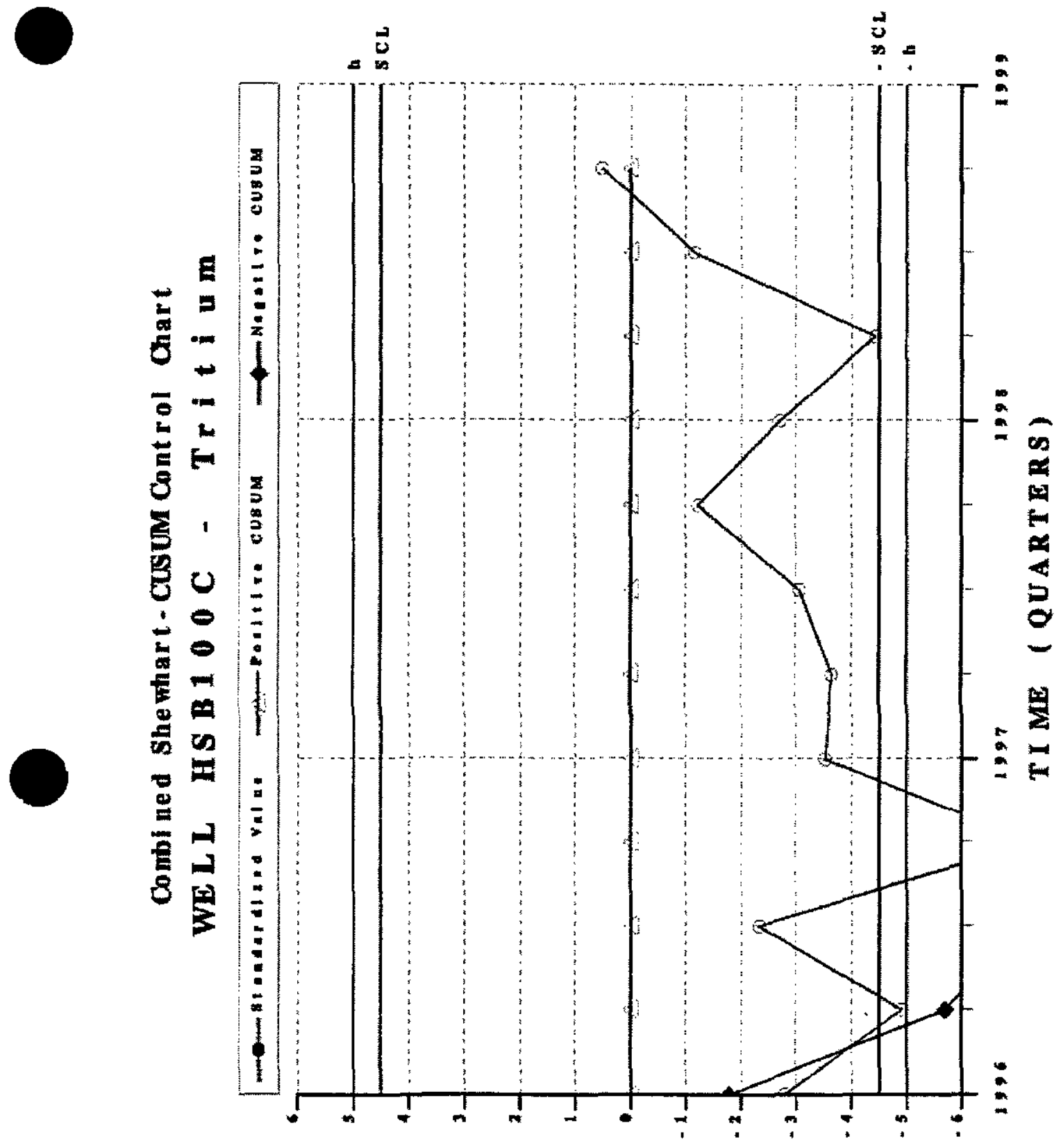

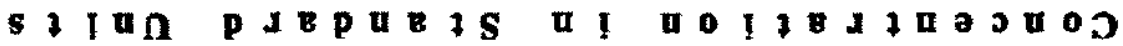



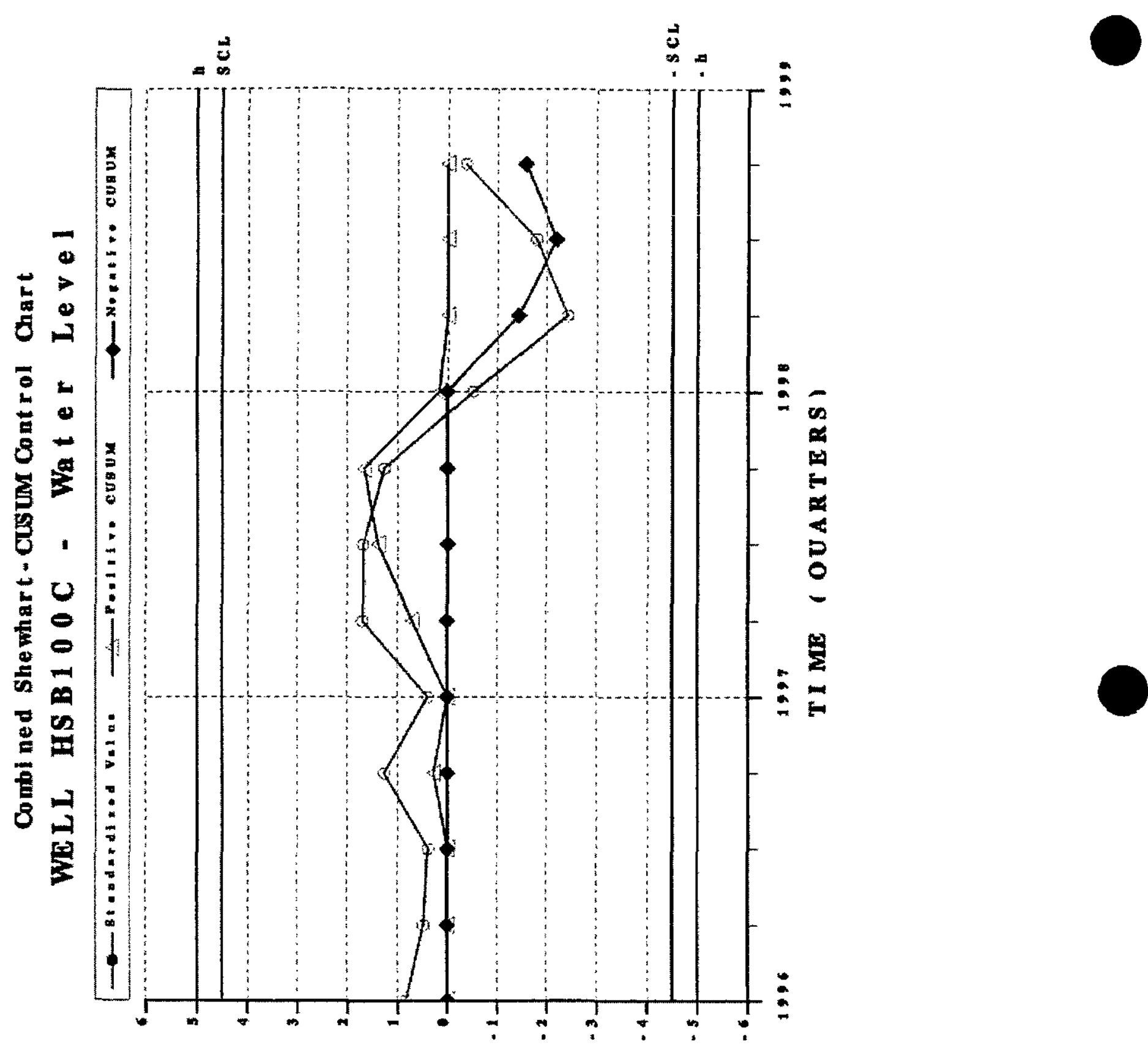


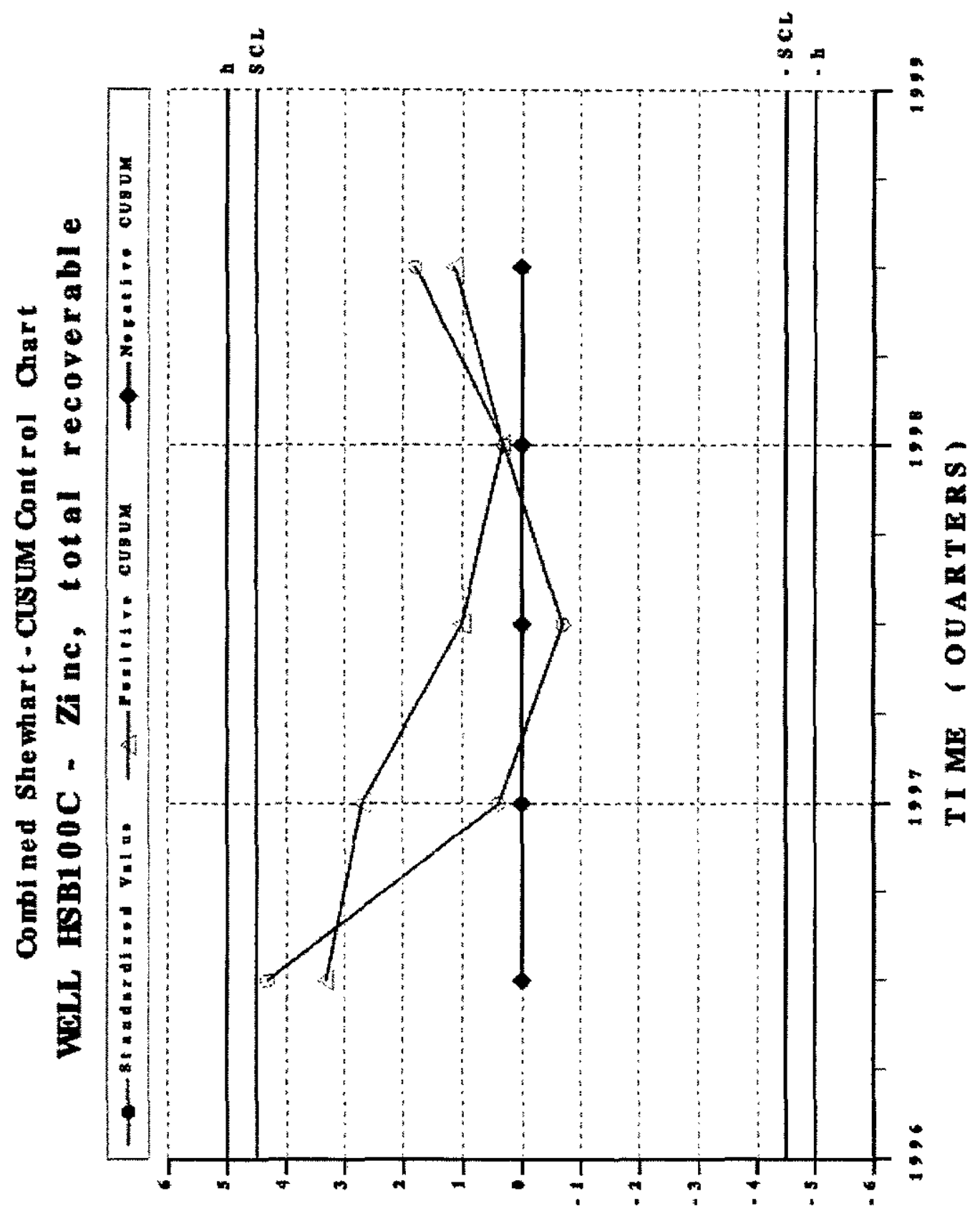

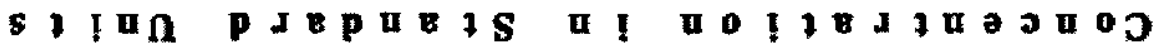




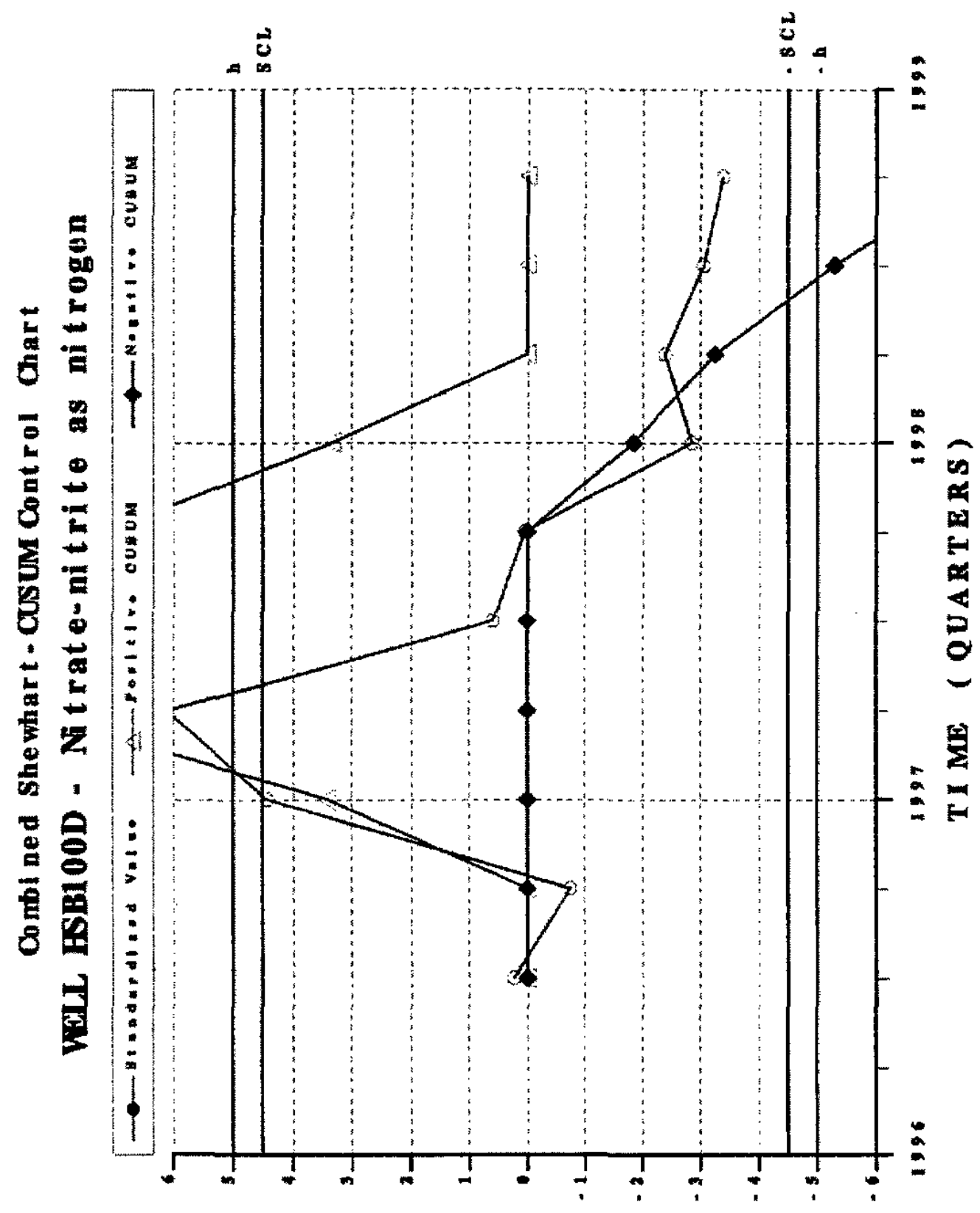

s I un 


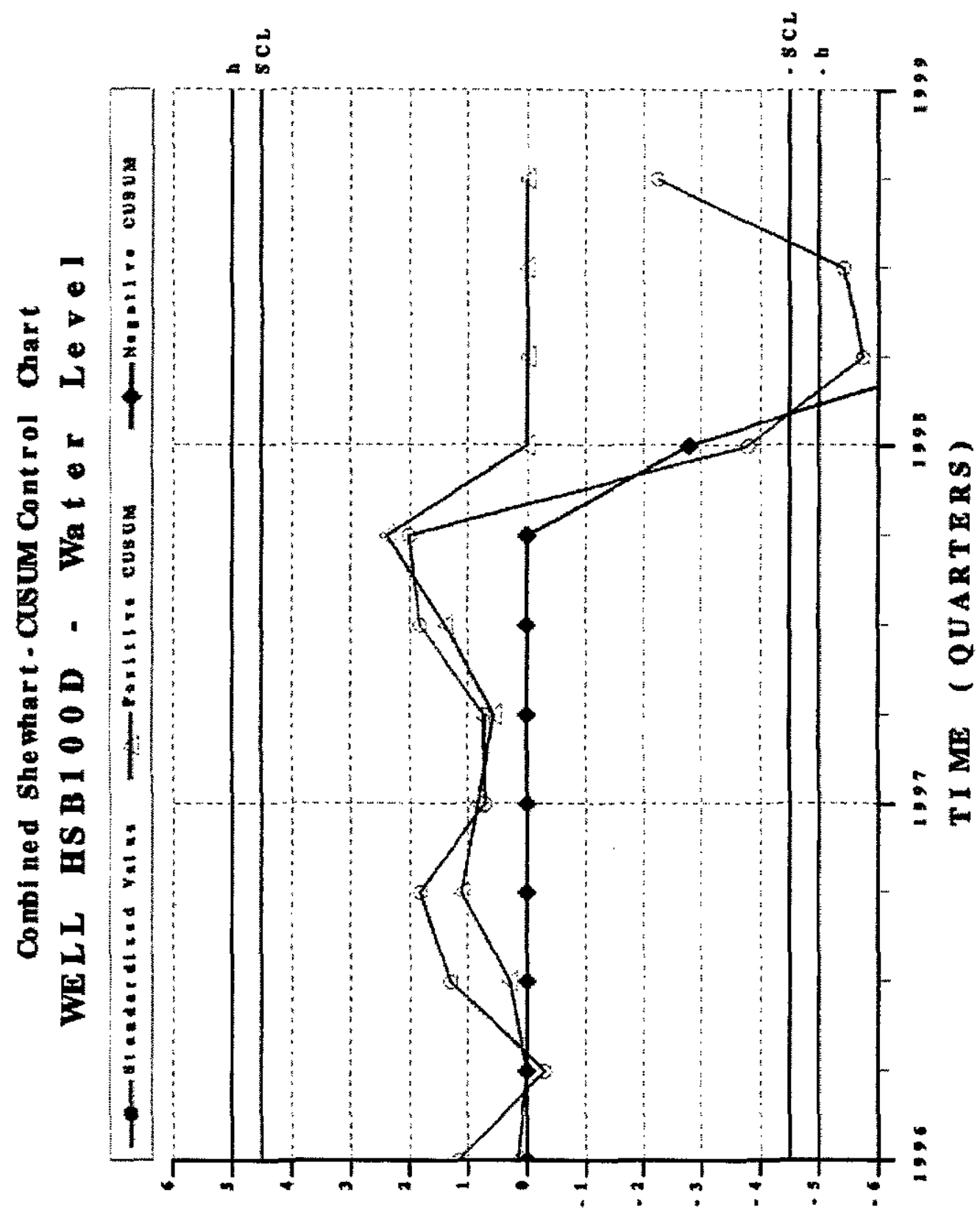

s I I 


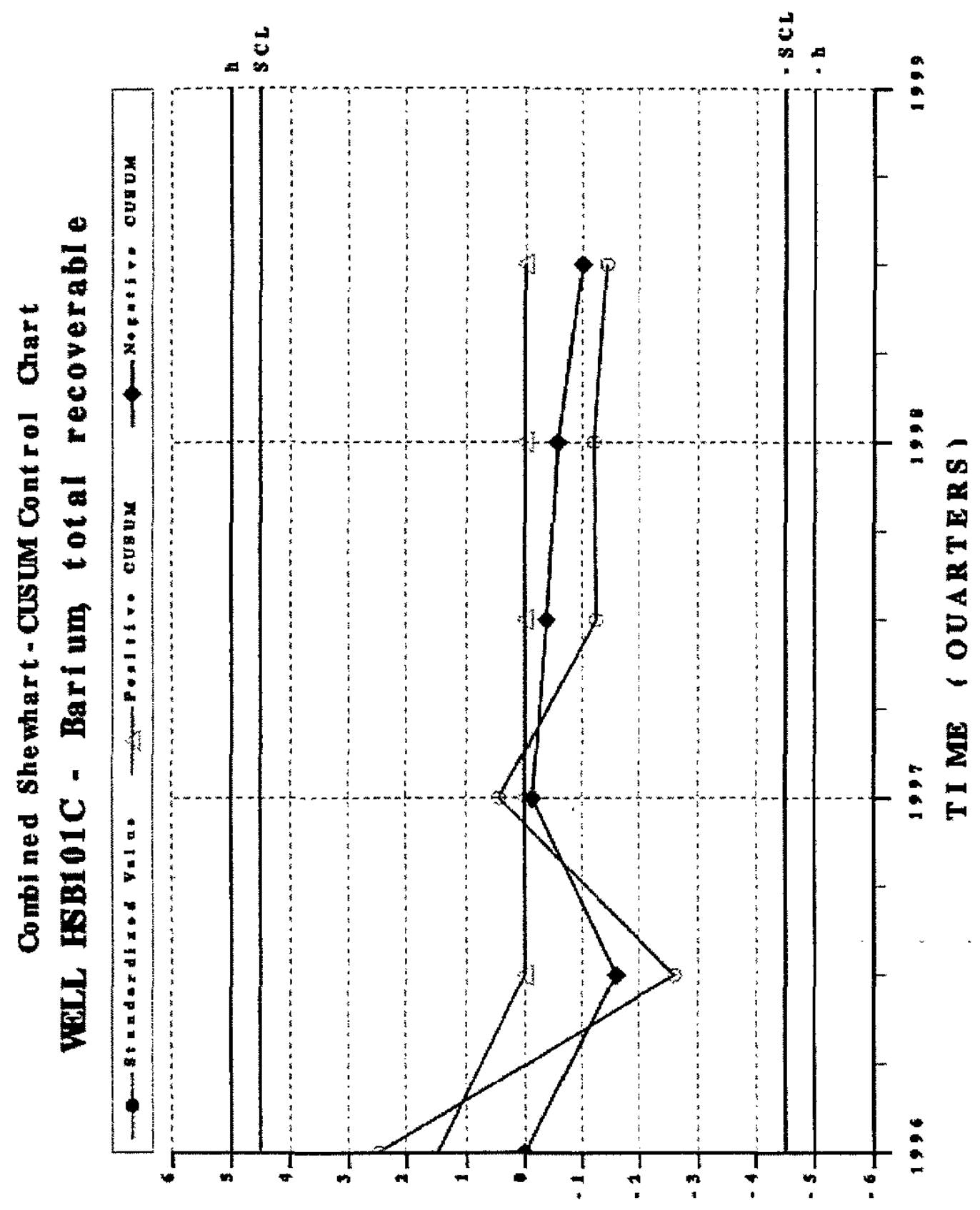

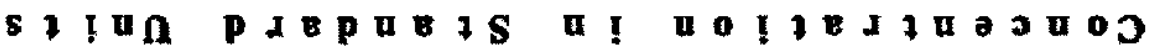



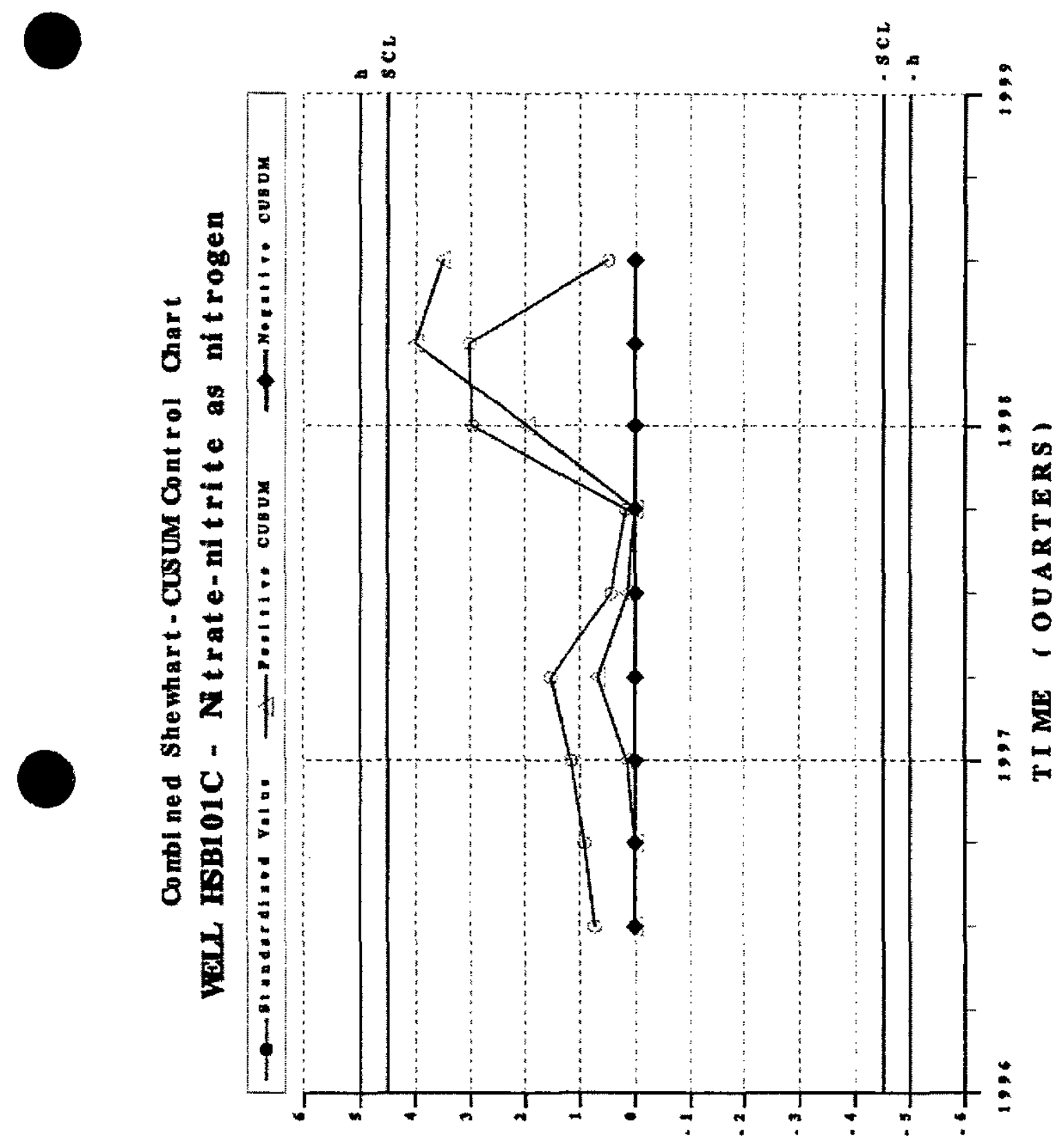

s 1 I 

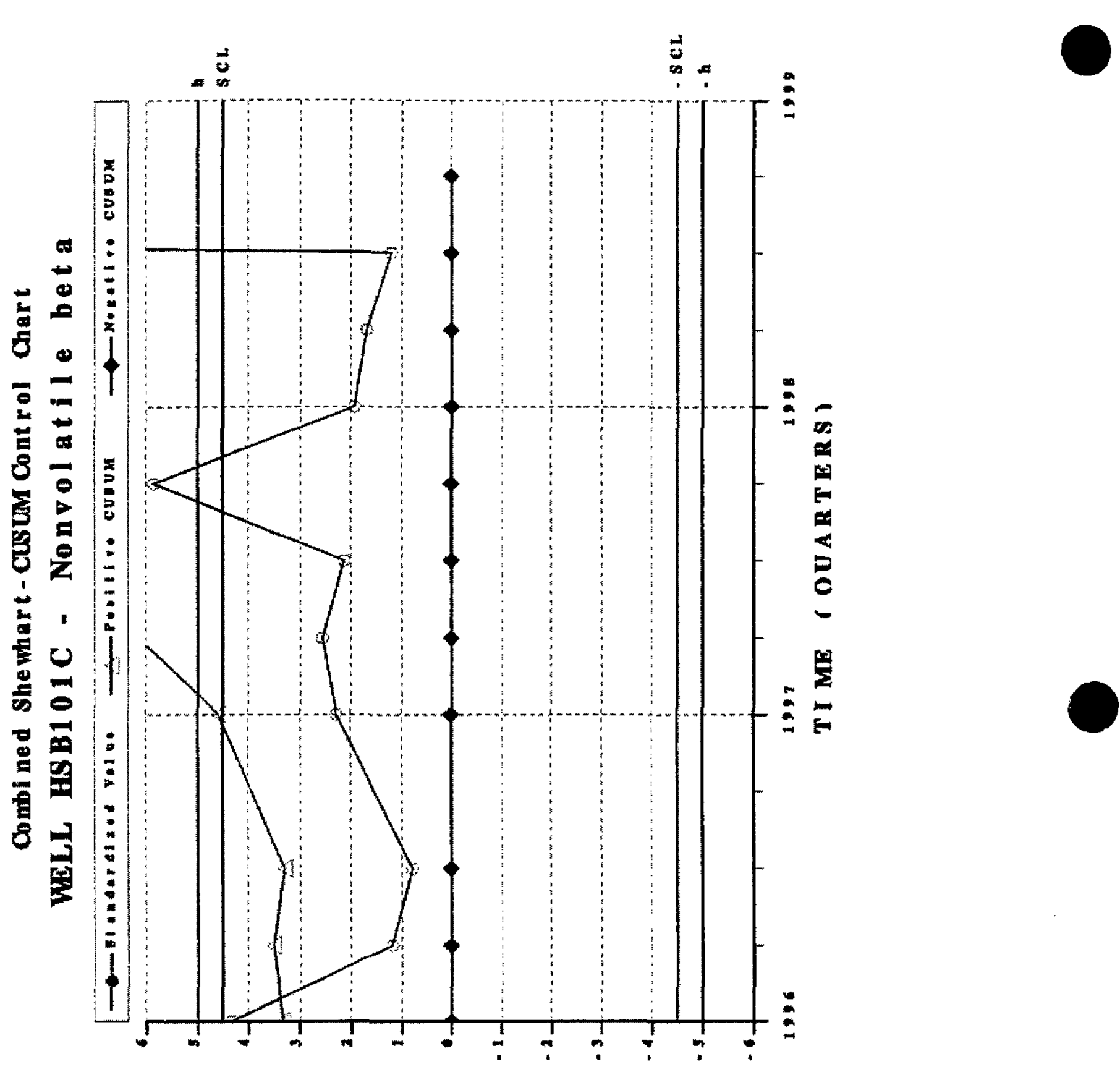

8

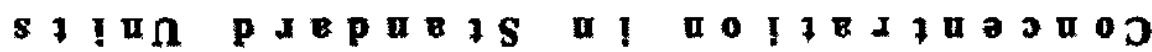



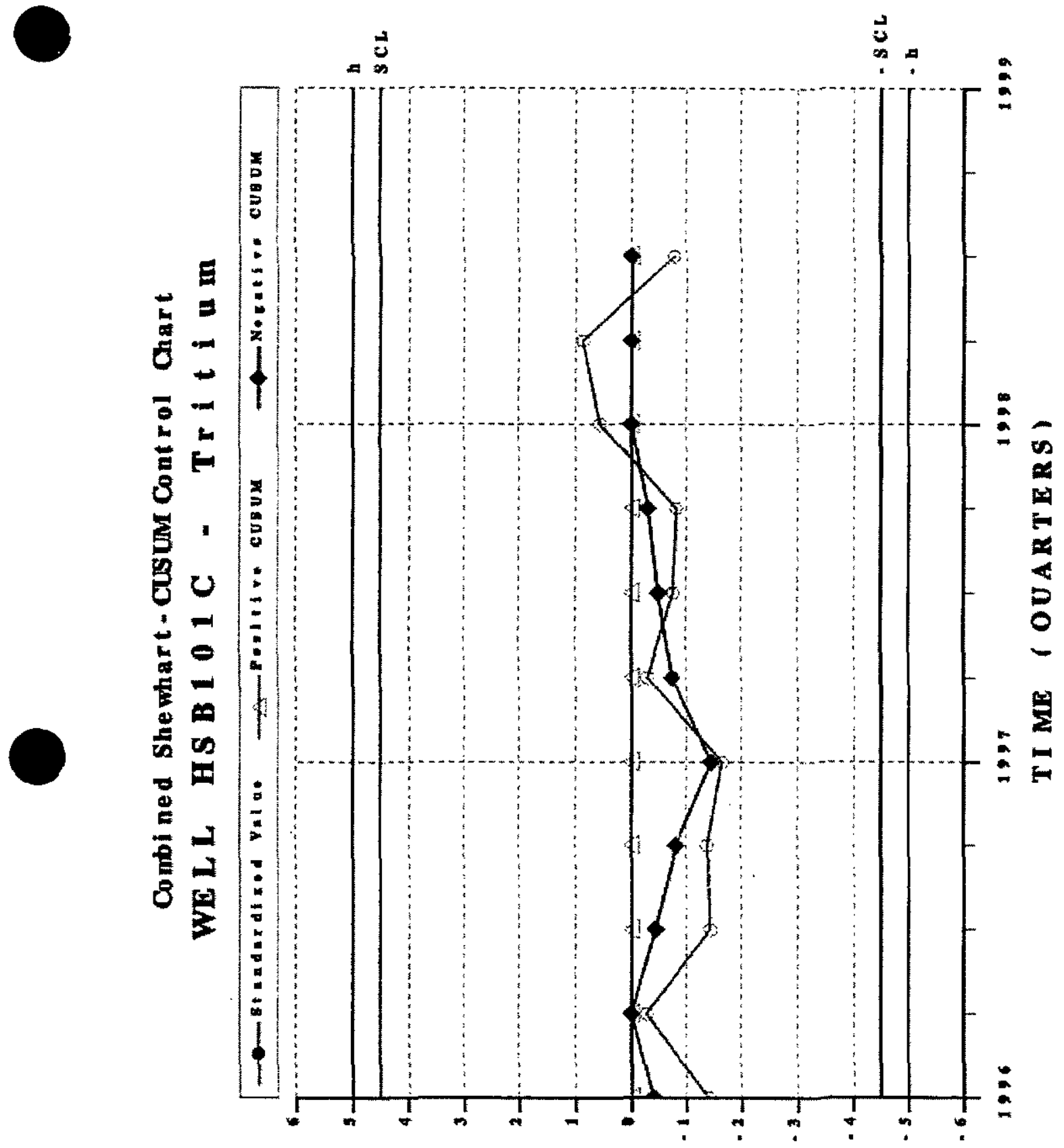

s) I 


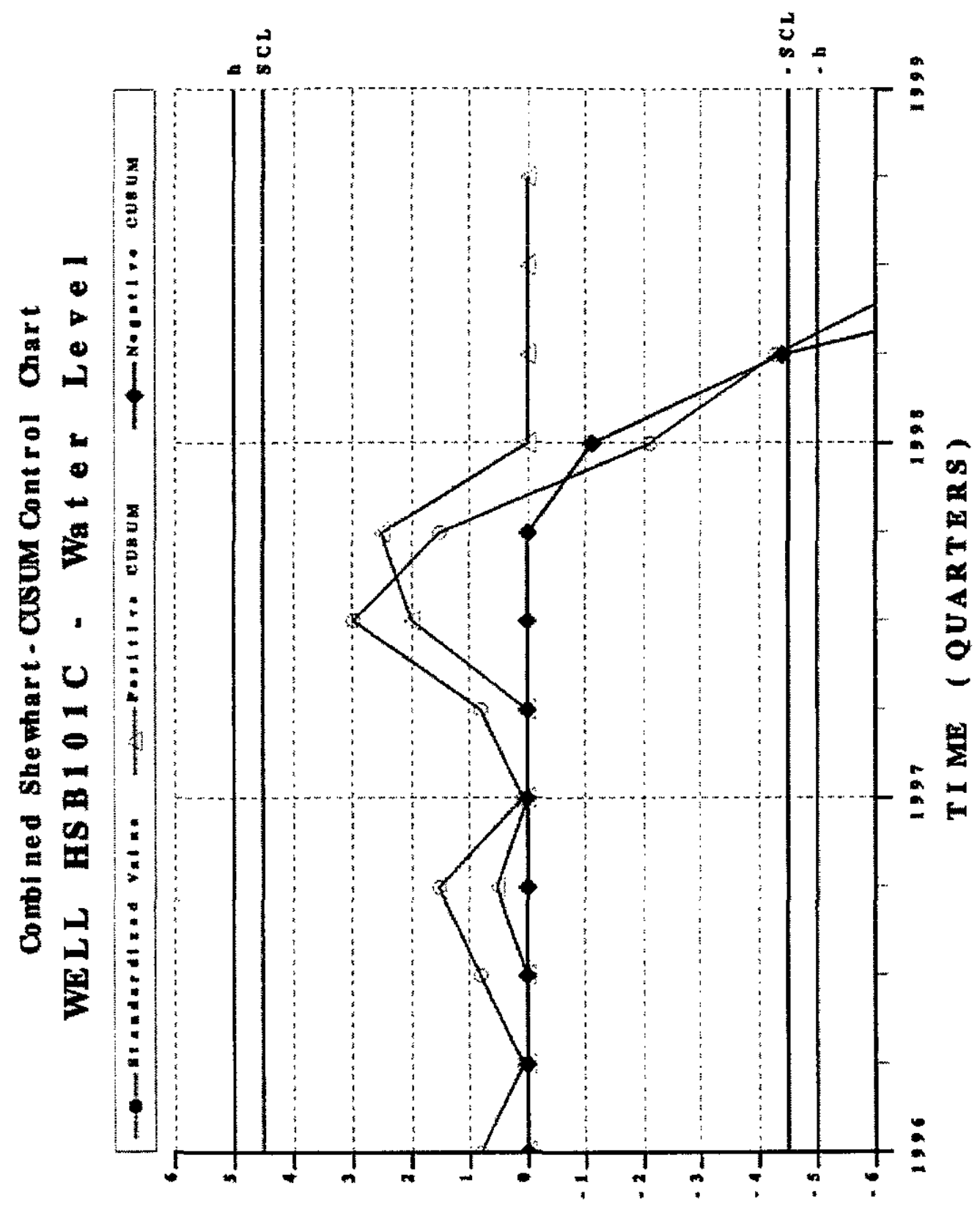

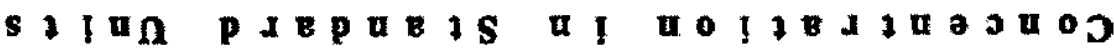


Combi ned Shewhart - Cusum Cont rol Chart WELL HSB101C - Zi nc, total recoverable

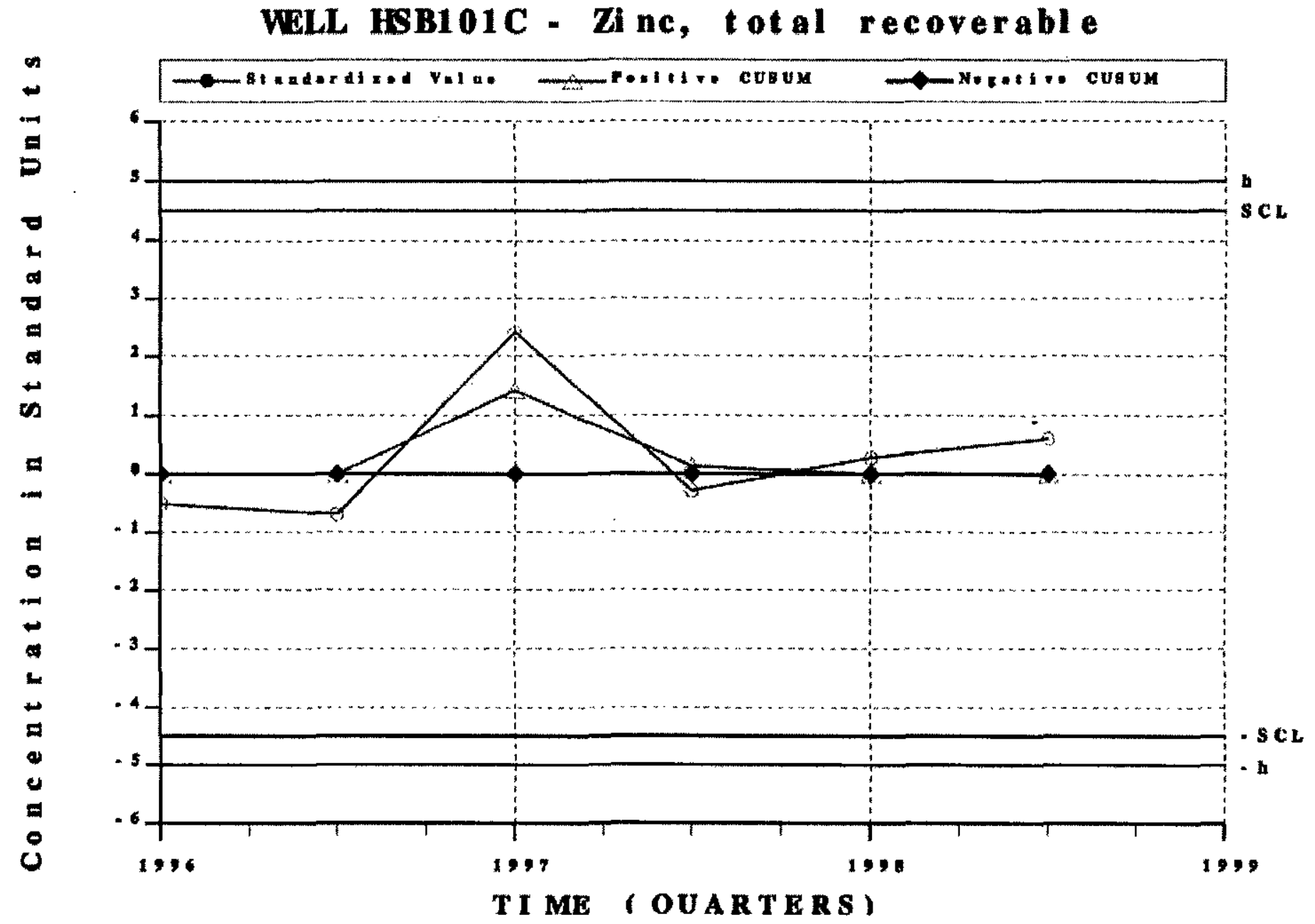


Combi ned Shewhart-Cusum Cont rol Chart

WuL HSB101D - Arsenic, total recoverable

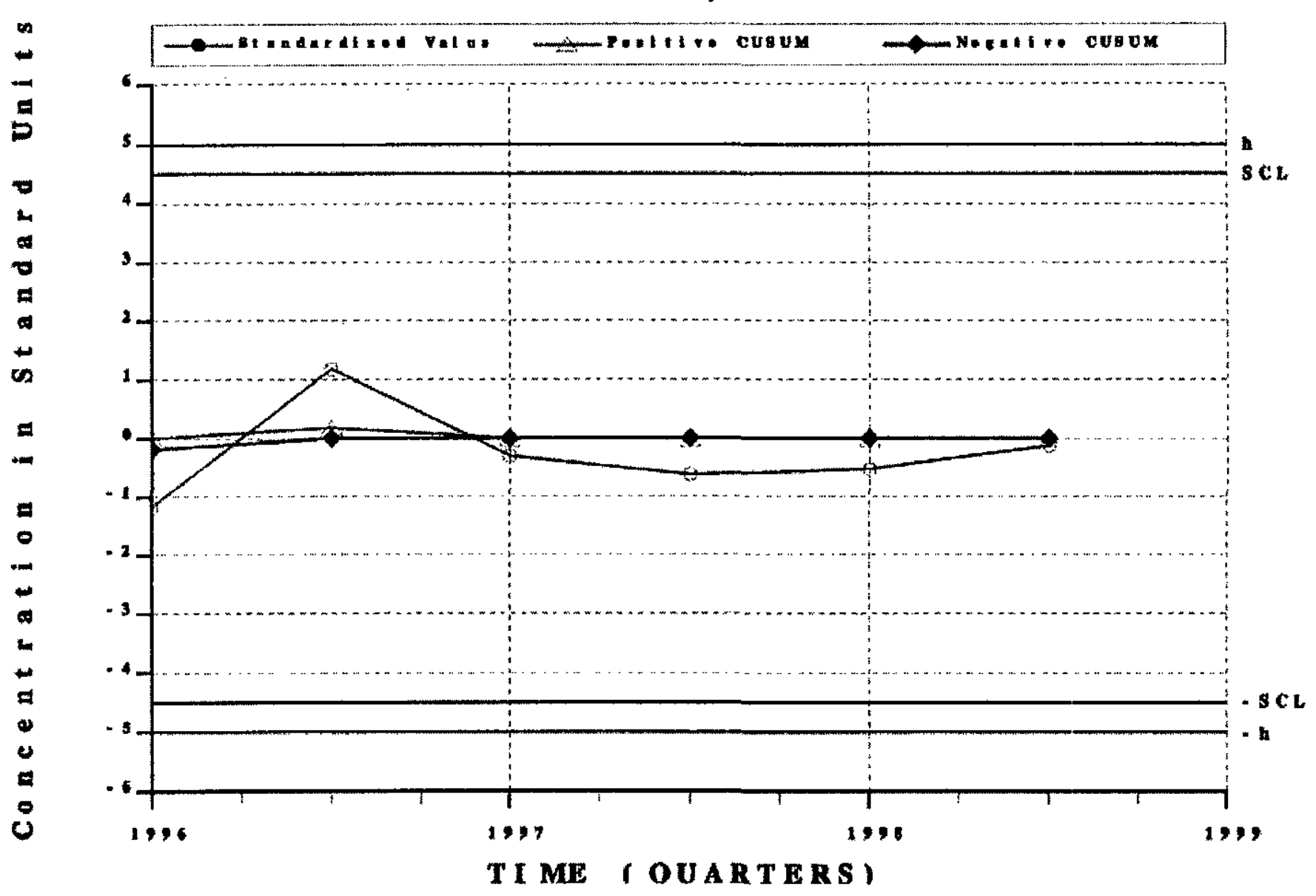

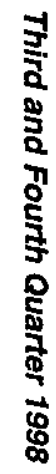



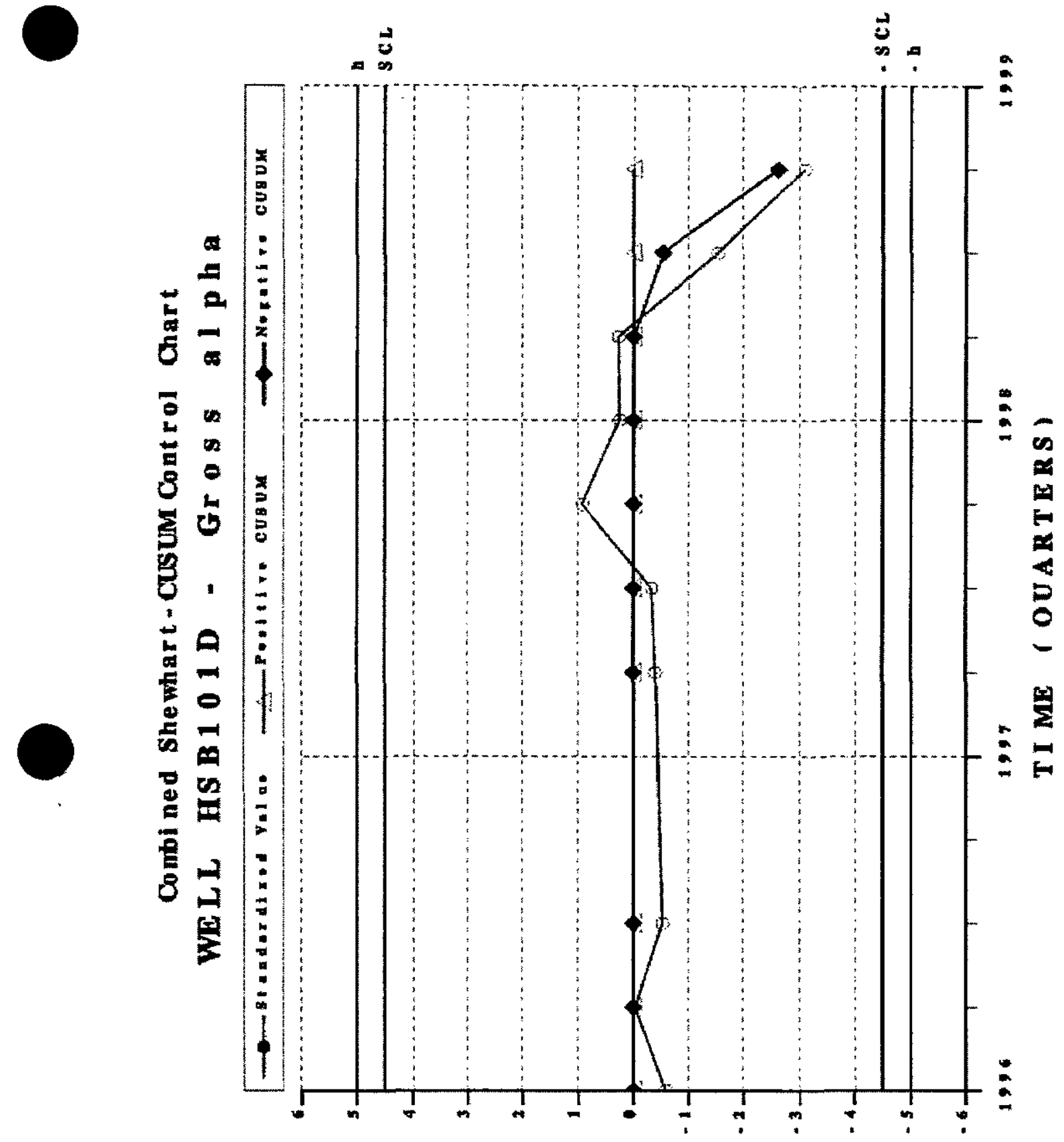

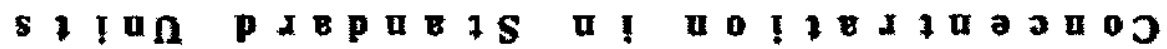


Combl ned Shewhart-Cusum Cont rol Chart

WELL HS B 101 D - I odi ne - 129

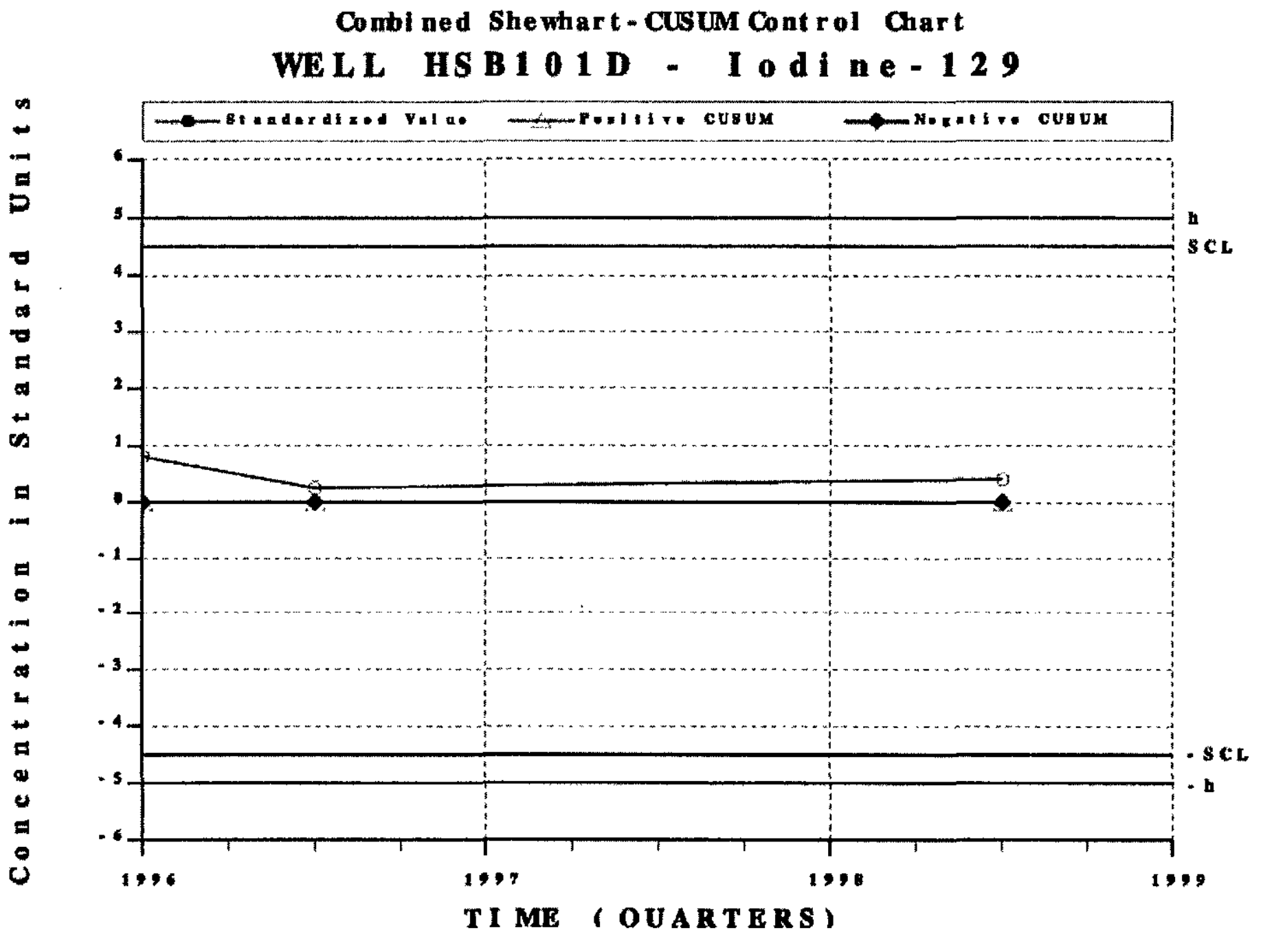




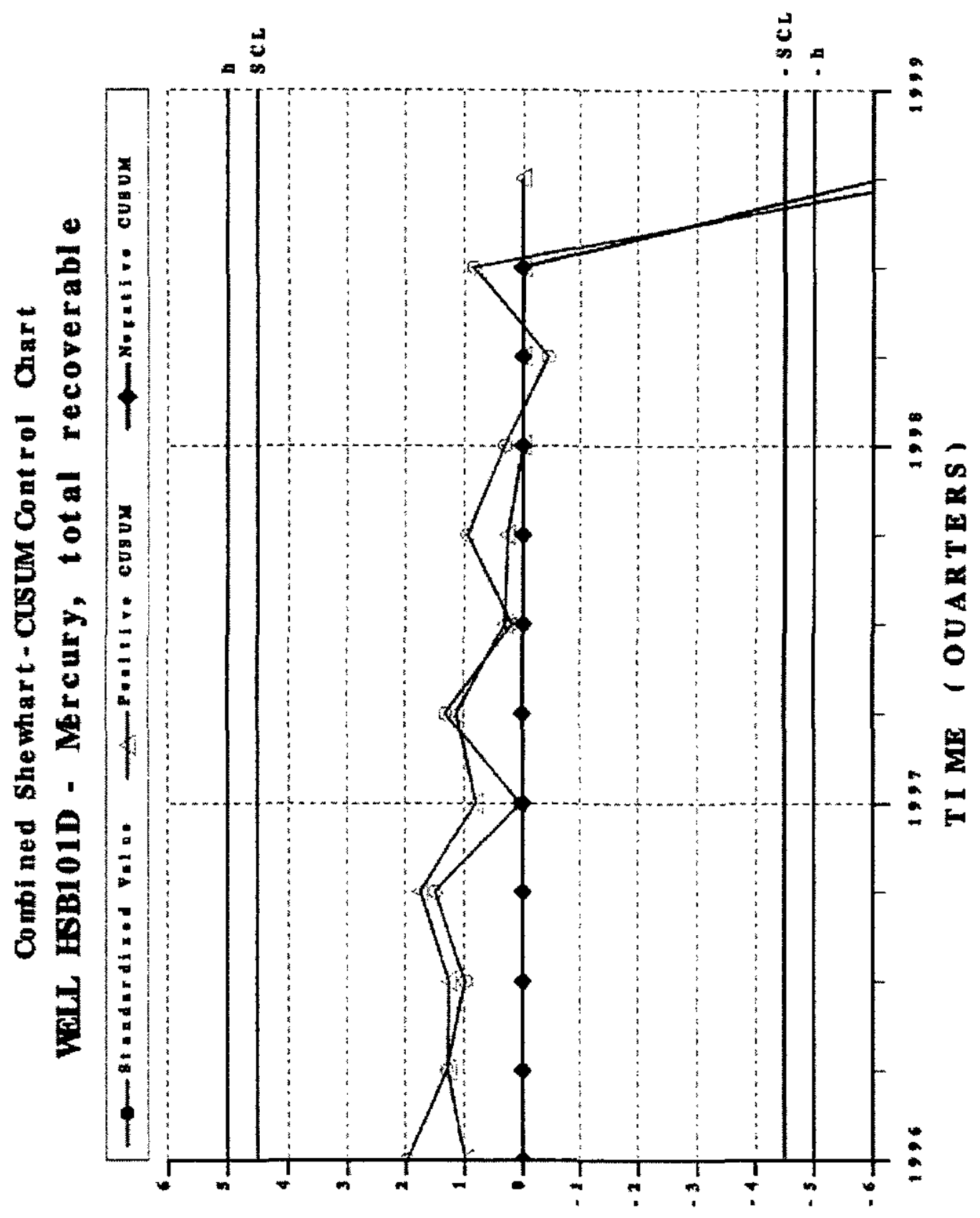

8 I 
Combi ned She whart - CUSUM Control Chart

VELL HBB101D - Nitrate-nitrite as nitrogen

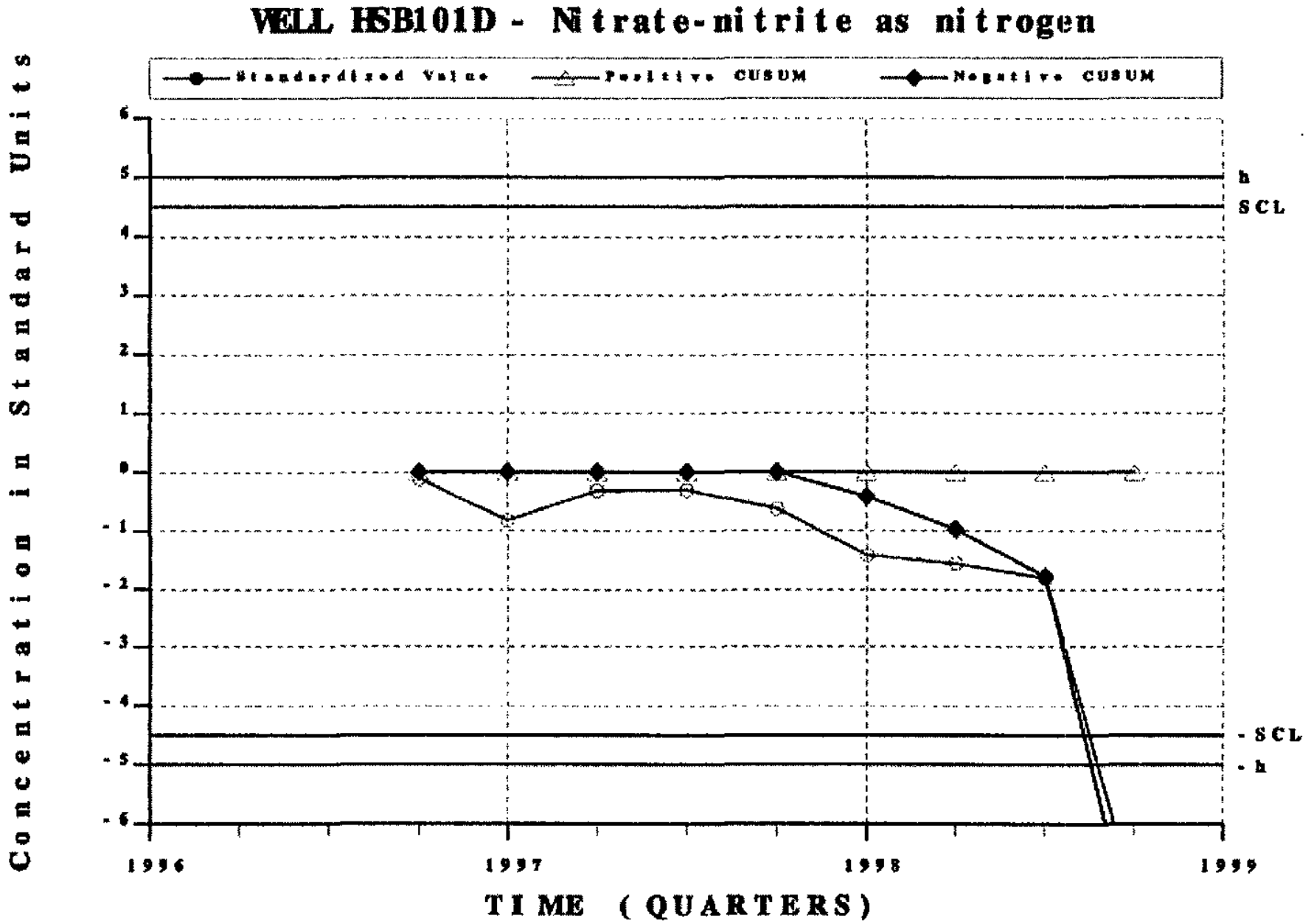


Combi ned Shewhart - Cusum Control Chart

WELL HSB101D - Nonvolatile beta

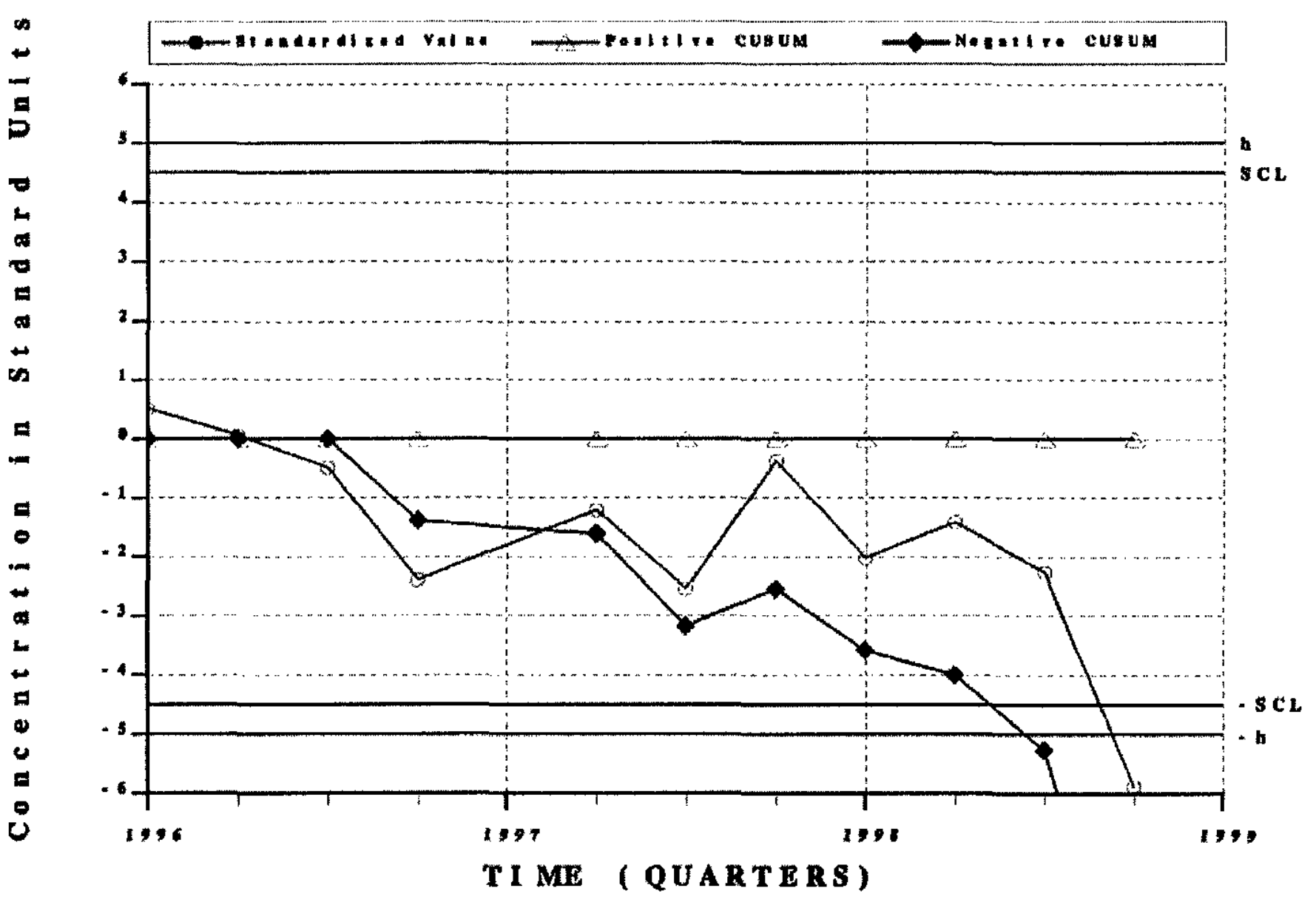


Combi ned She whart - CuSUM Cont rol Chart

WELL HS B 101 D - Ra di um- 228

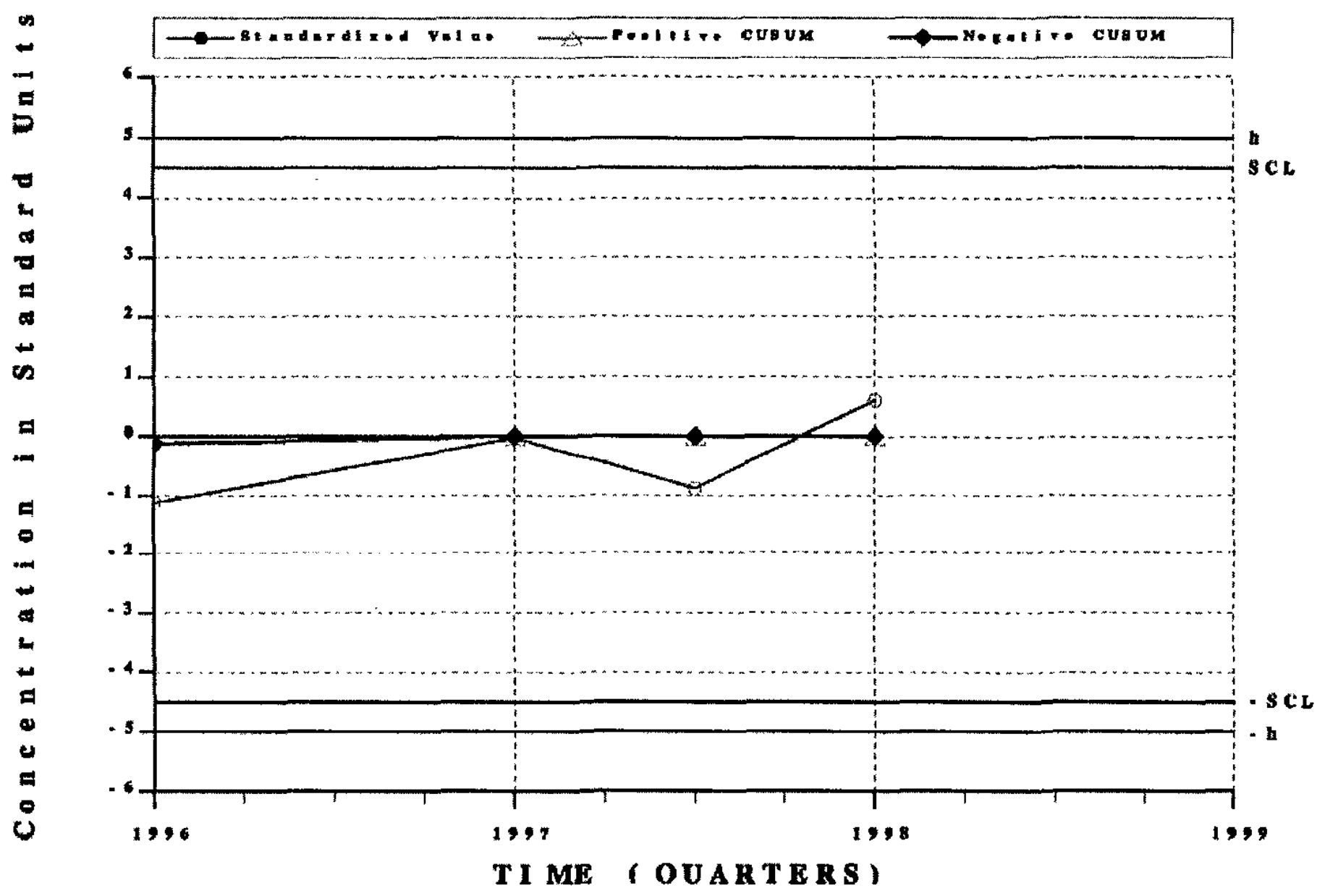

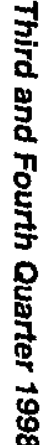

T I ME (OUART ERS ) 
Combi ned She whart - CuSUM Cont rol Chart WELL HS B 101 D - St r Ont i m- 90

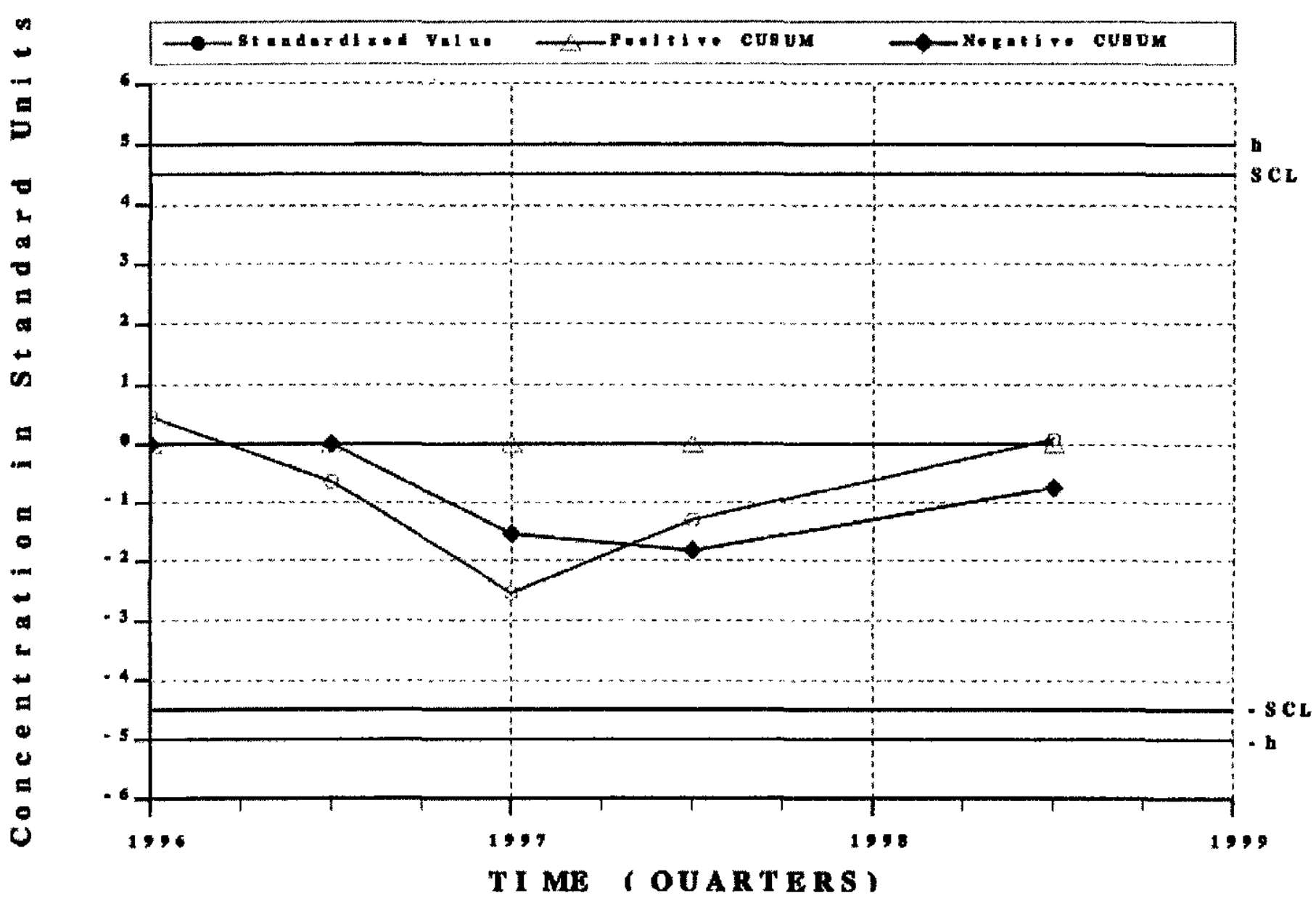




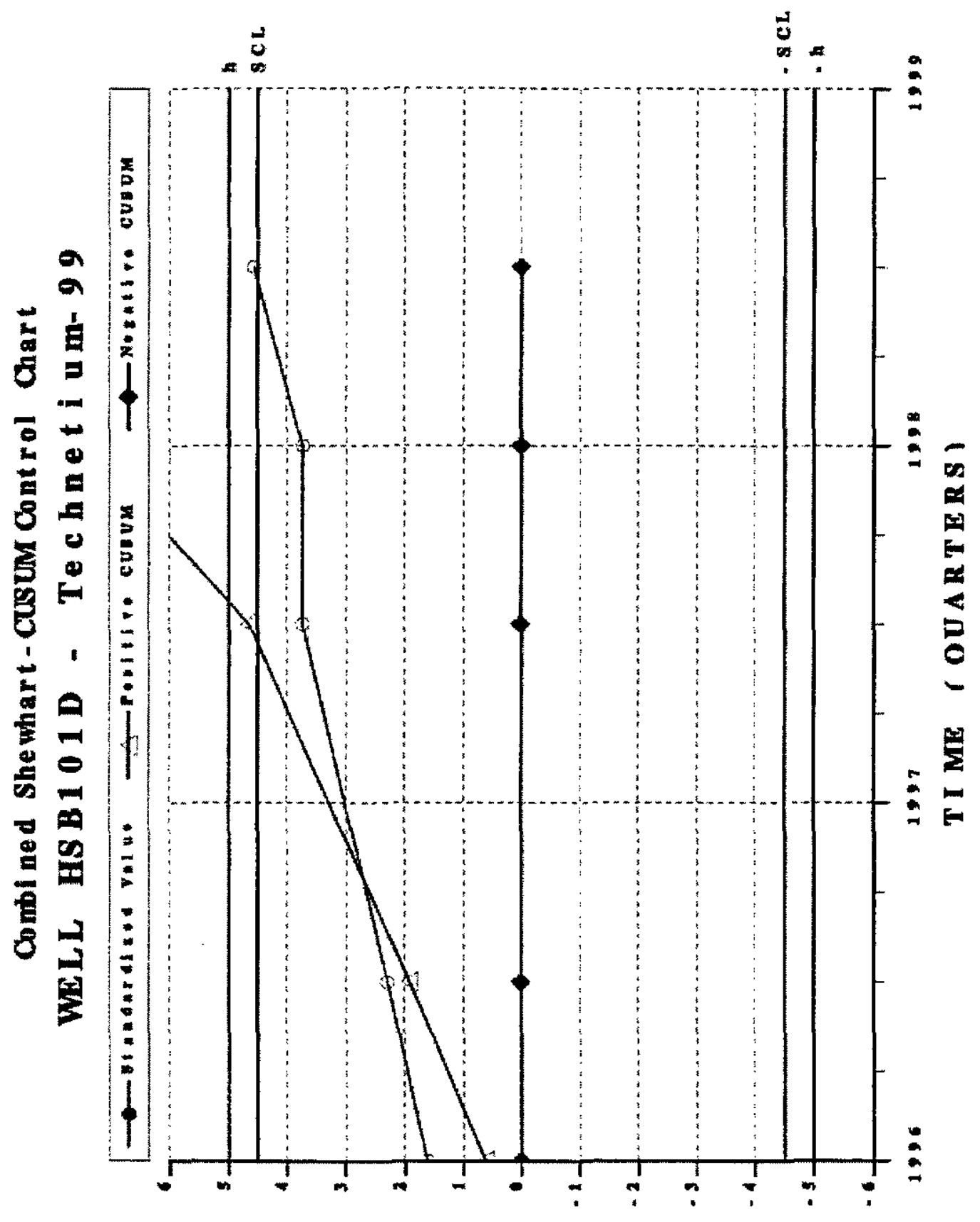

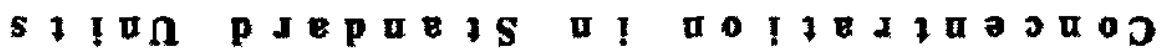




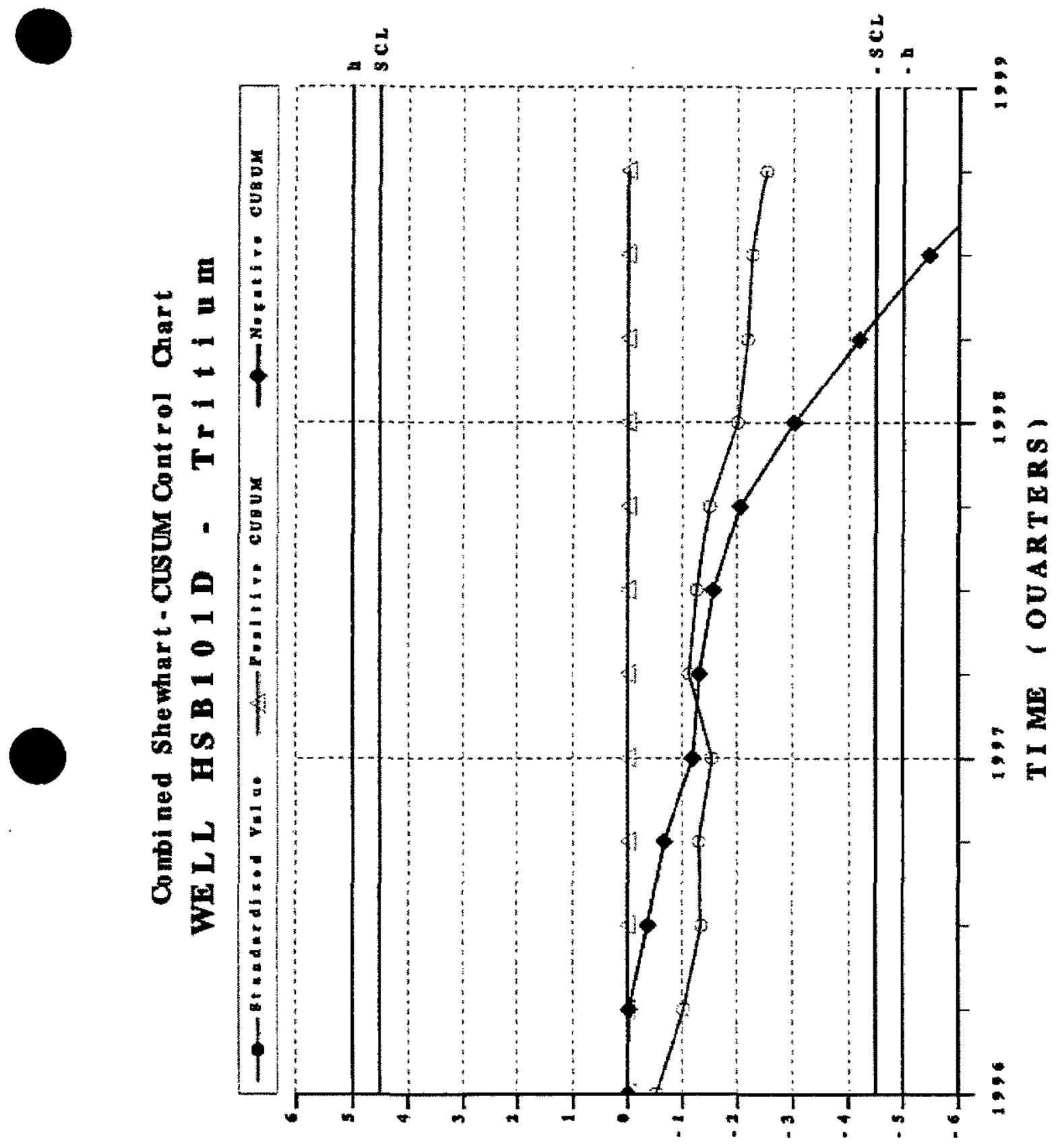

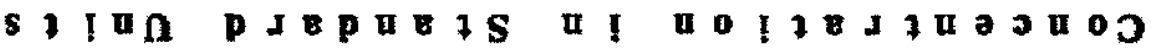



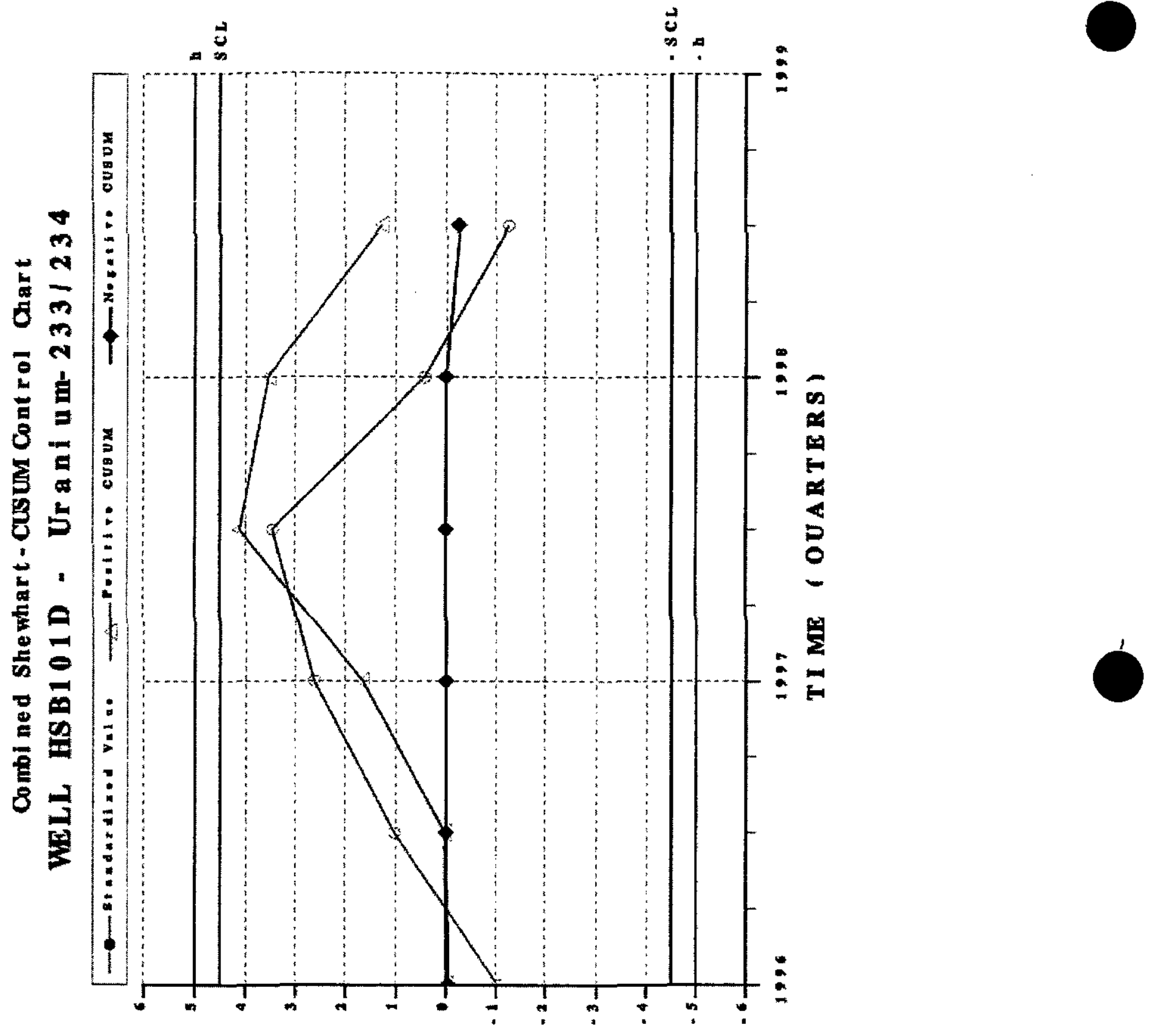

s! เก px 


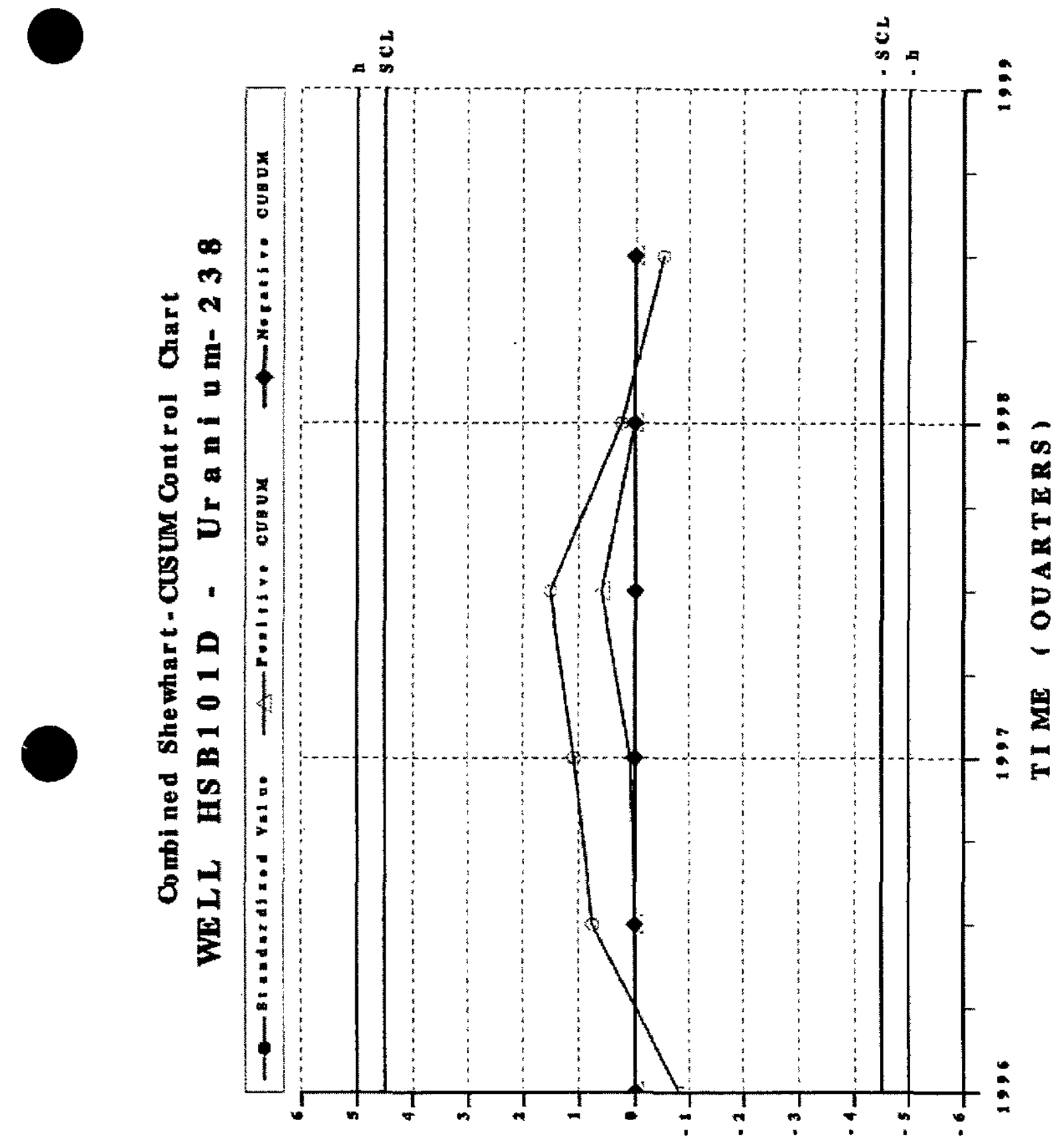

s t I 


\section{Combi ned She whart-CusuM Cont rol Chart} VoLL HSB101D - Vanadi um t ot al recoverable

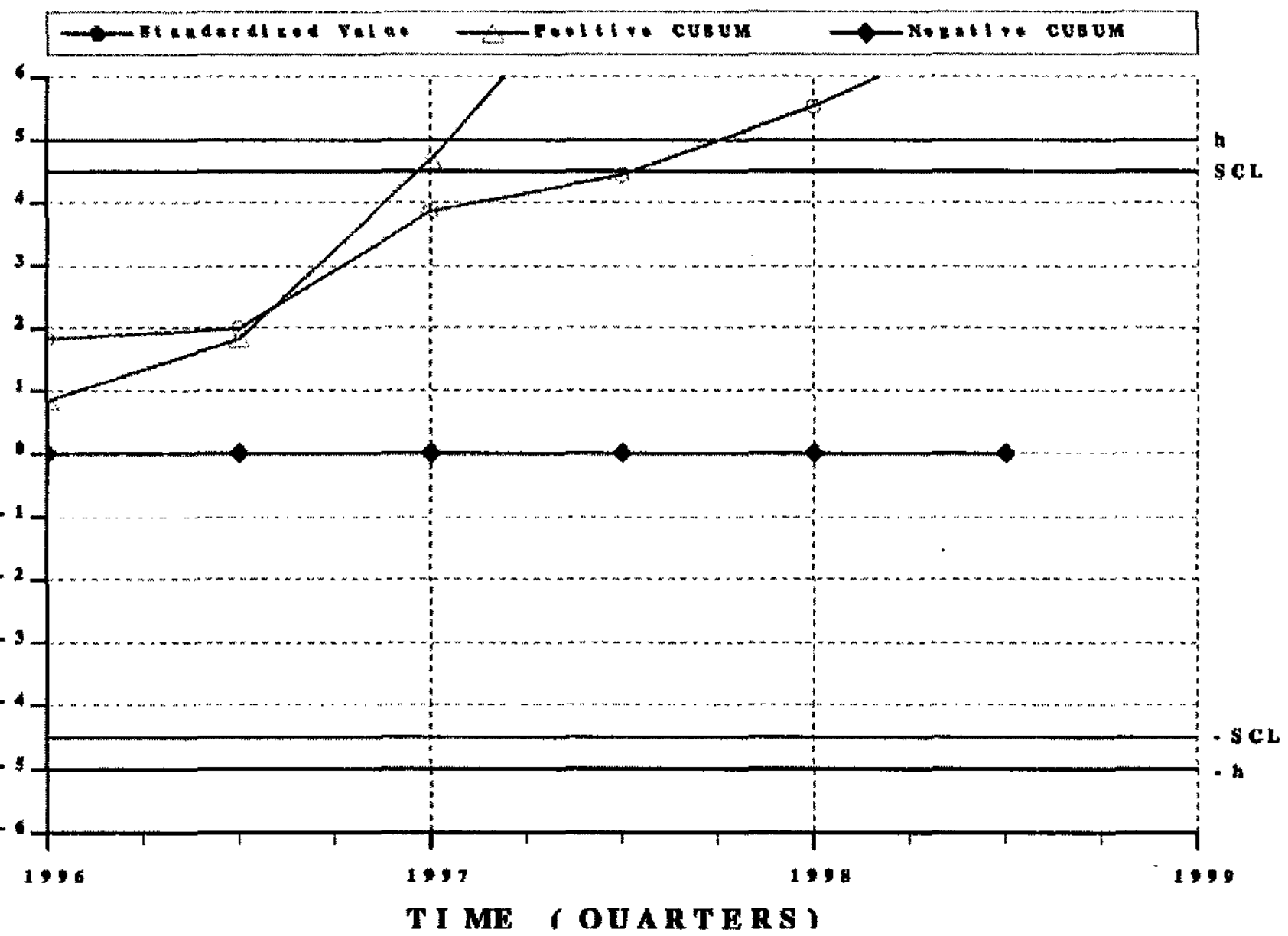



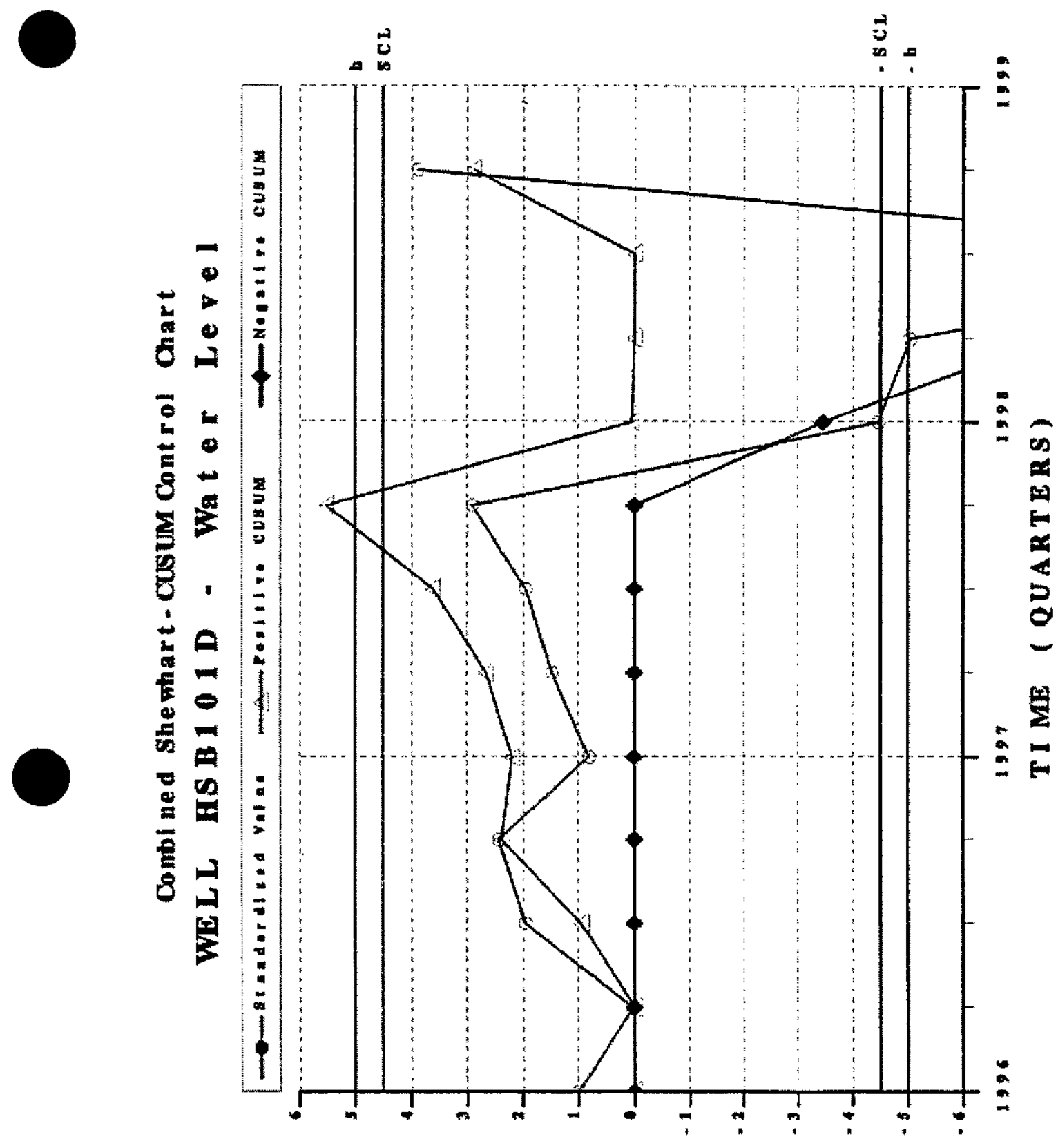

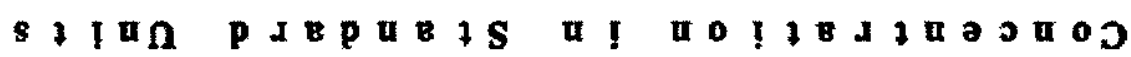




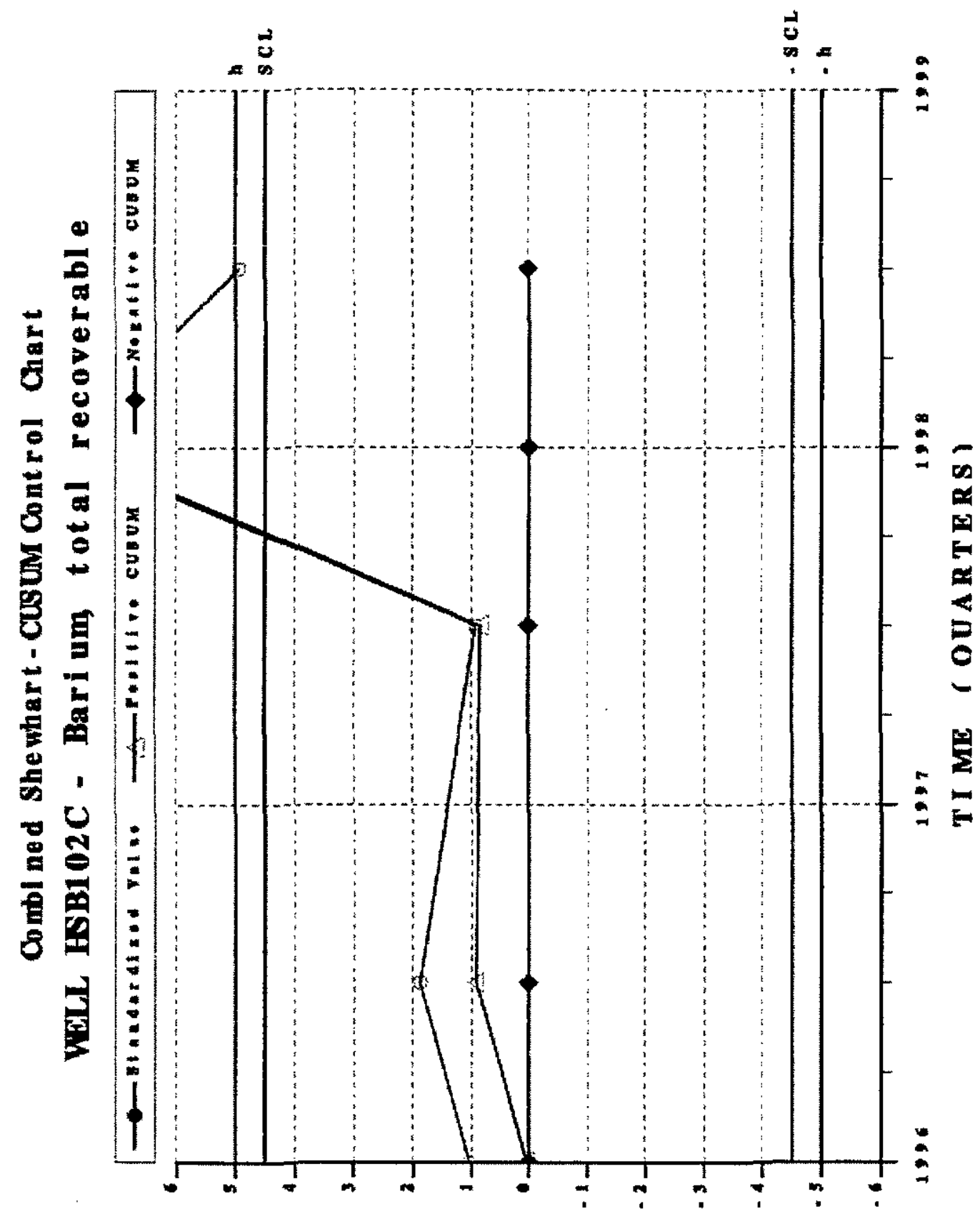

s 1 a $\mathbf{p}$ J E p 

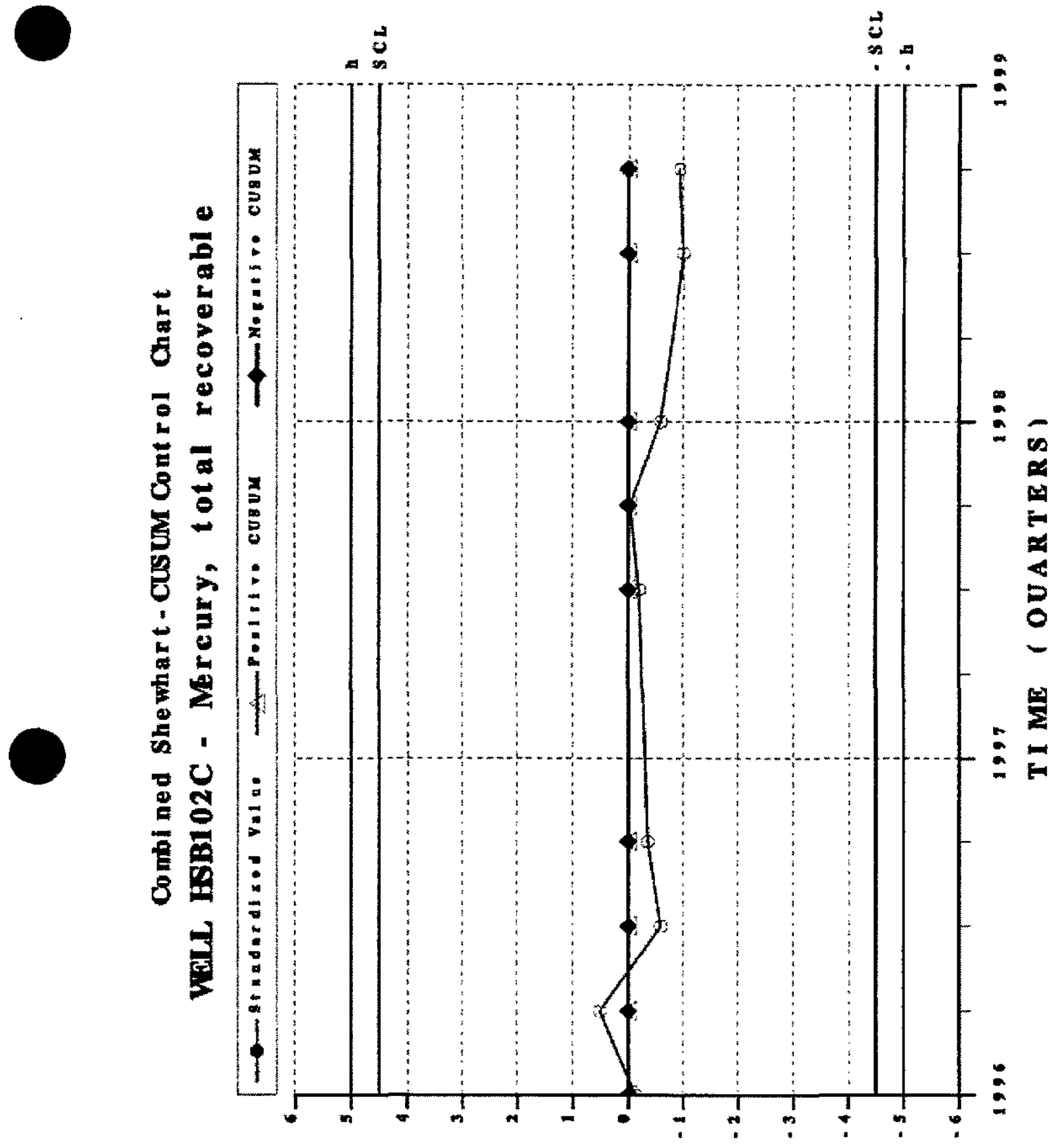

sitan pa 


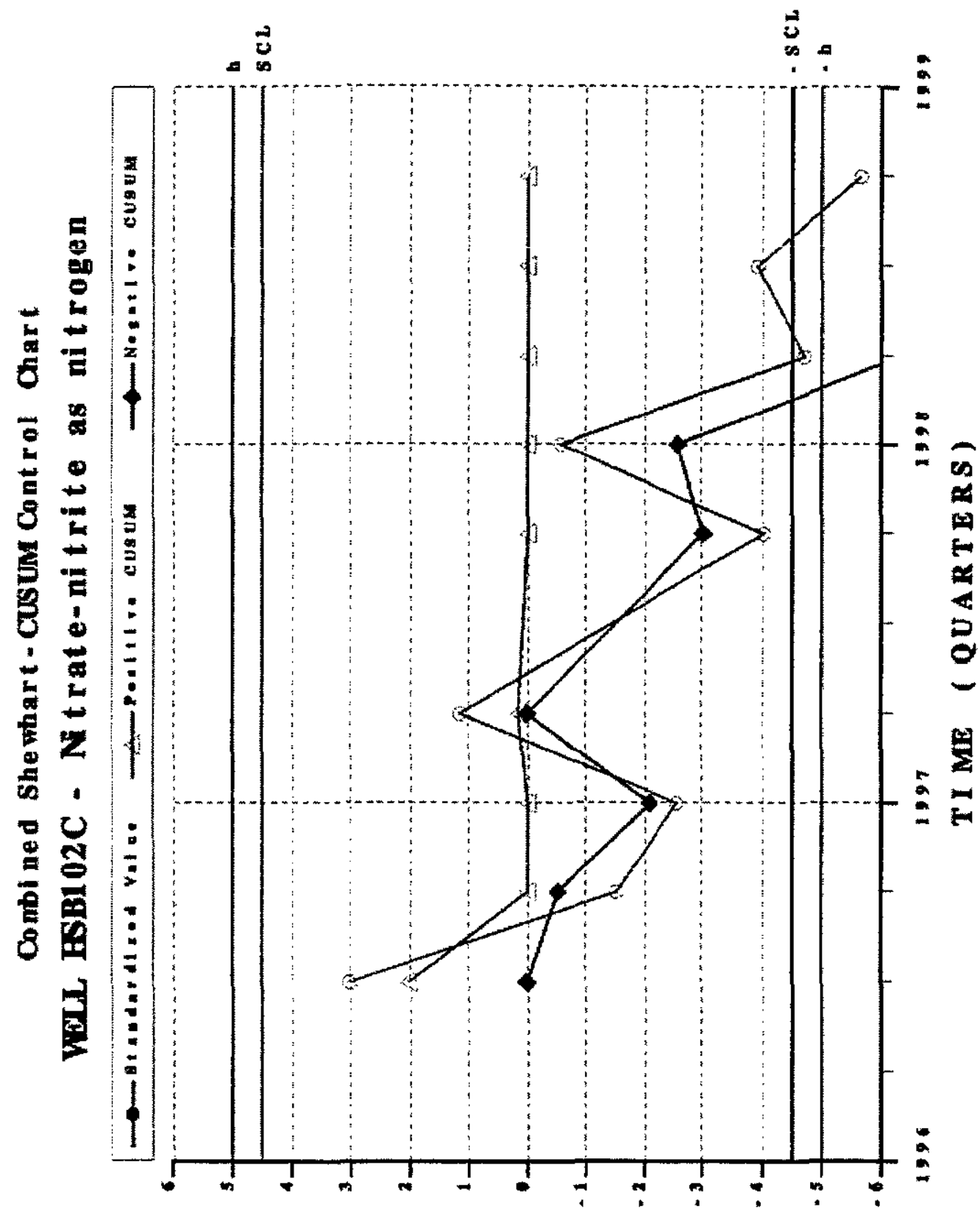

s I I I p 
Combined She whart-Cusum Cont rol Chart WELL HSB1 02 - Nonvol atile beta

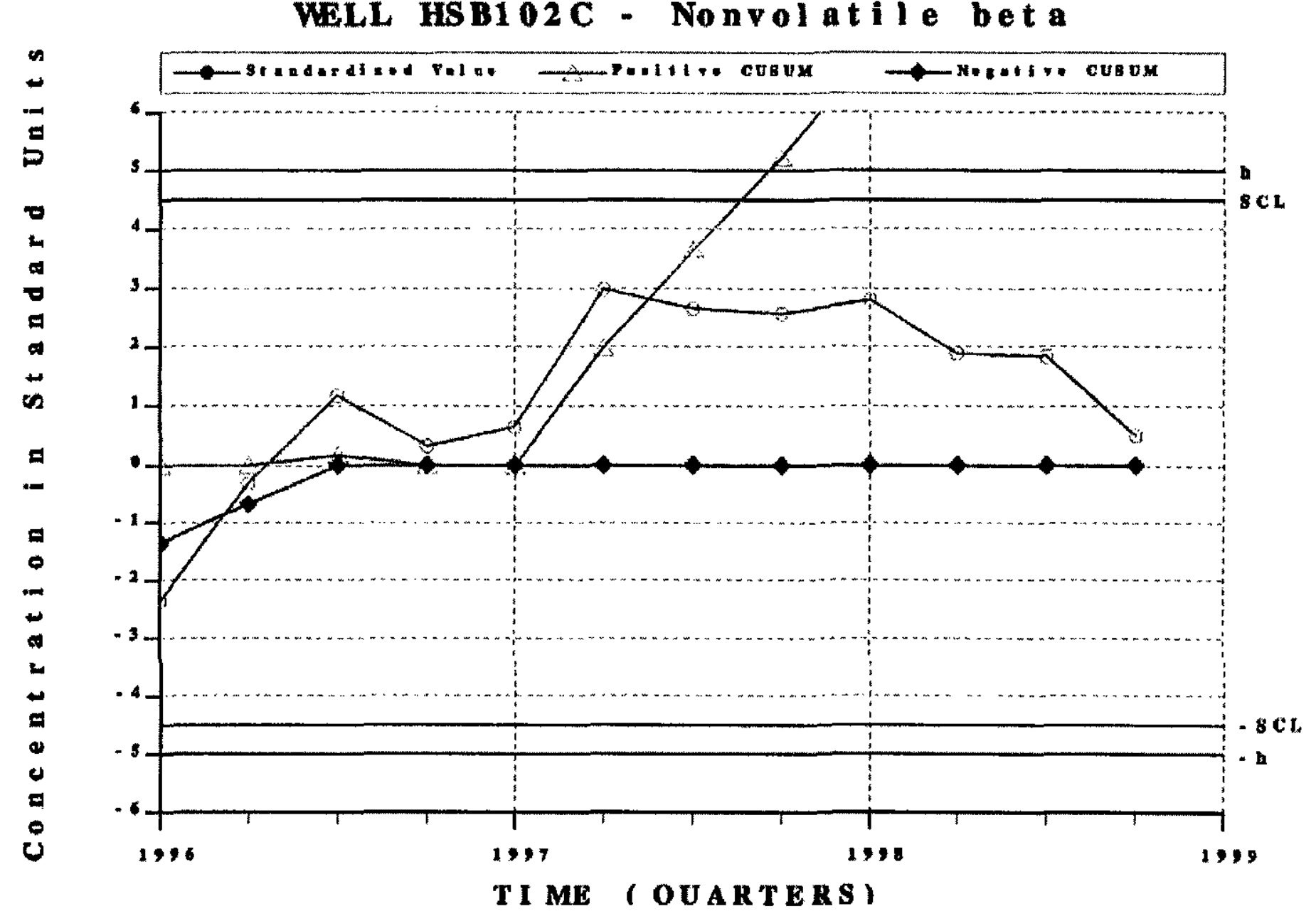




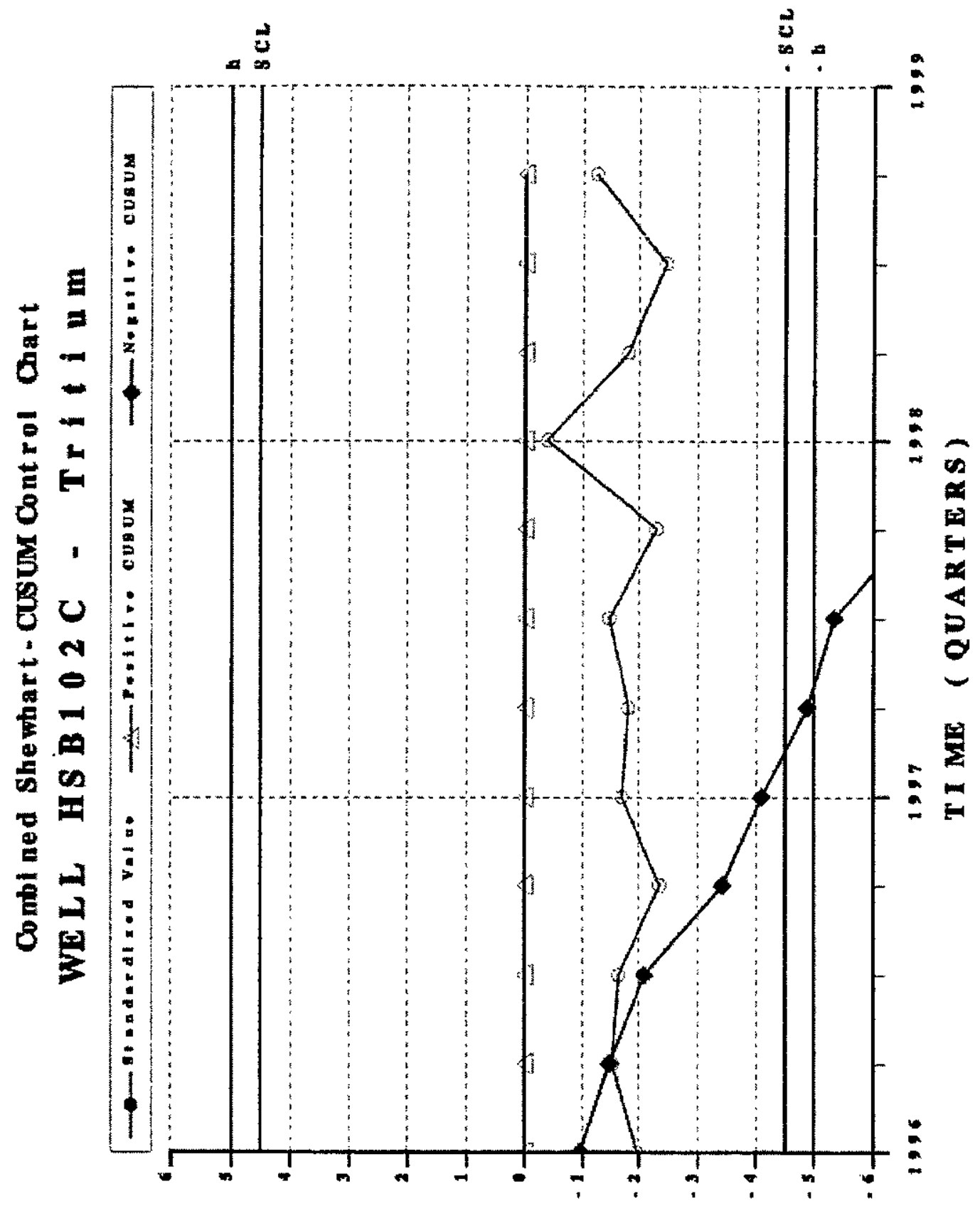

s† ตn pג 
Combi ned Shewhart - Cusum Cont rol Chart

WELL HS B 102 C - Water Level

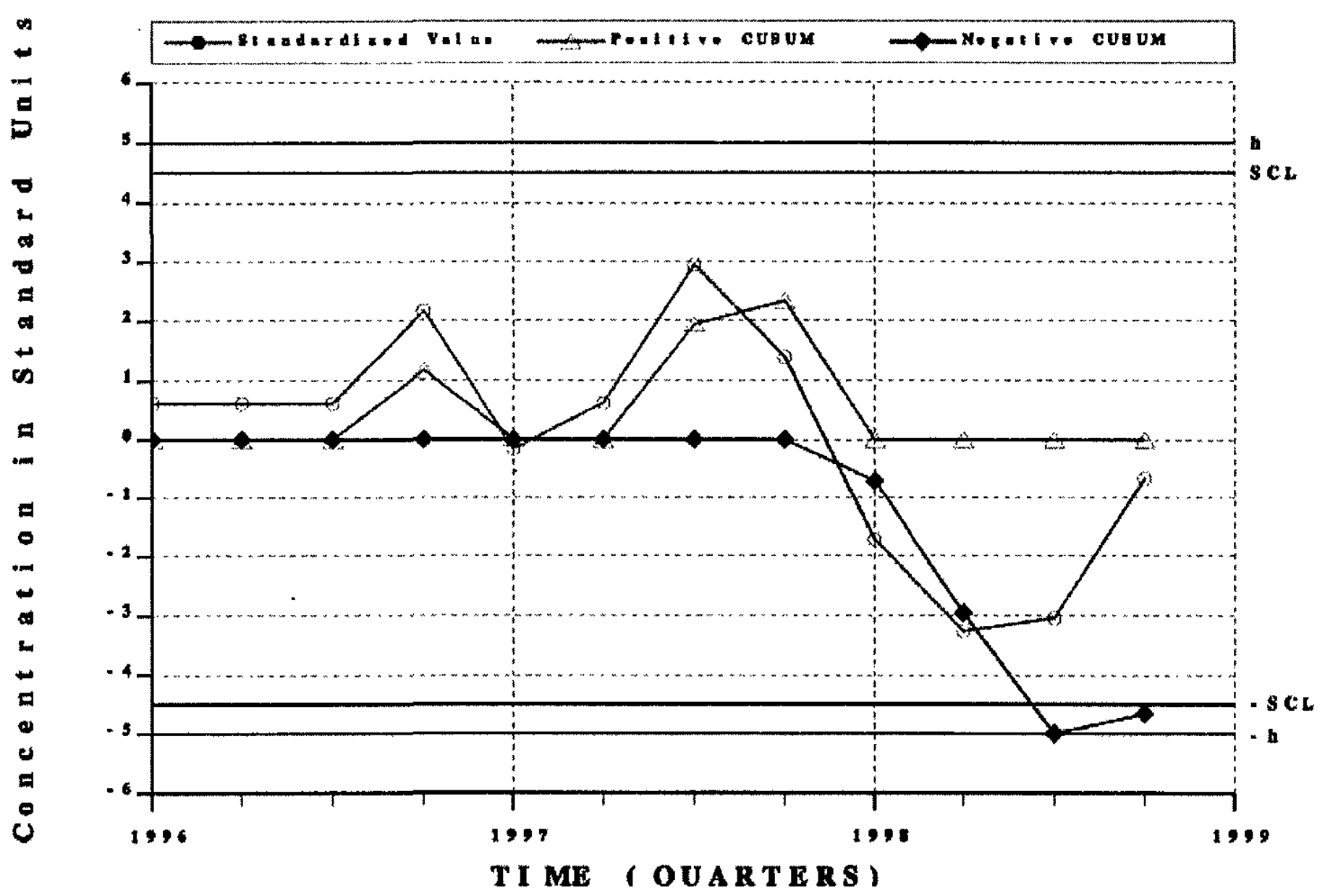


Combined Shewhart-Cusum Cont rol Chart WLLL HSB102C - Zi nc, total recoverable

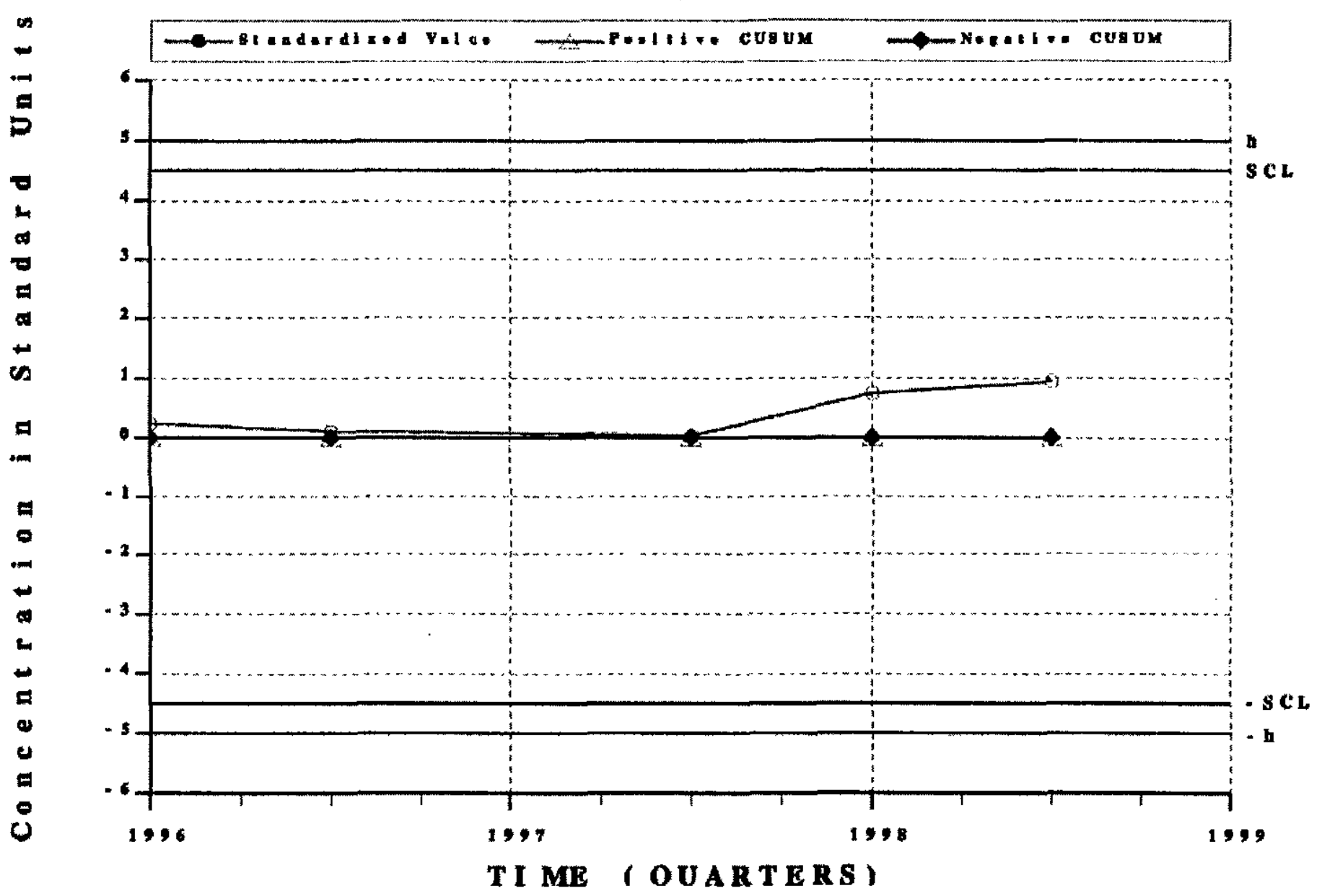

ME OUARTERS 
Combined Shewhart - CUSUM Cont rol Chart

WELL HSB 102 D Gros a 1 pha

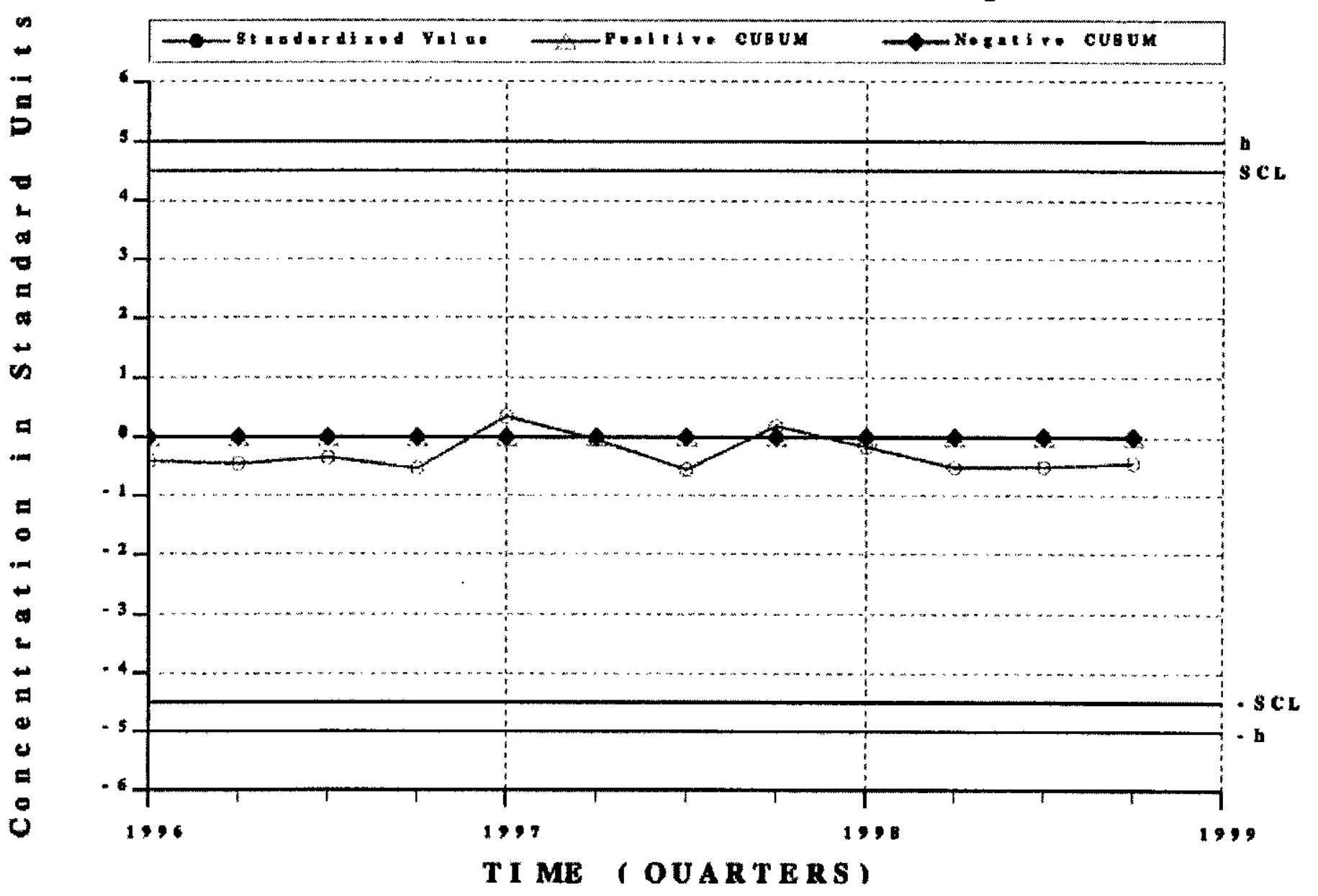




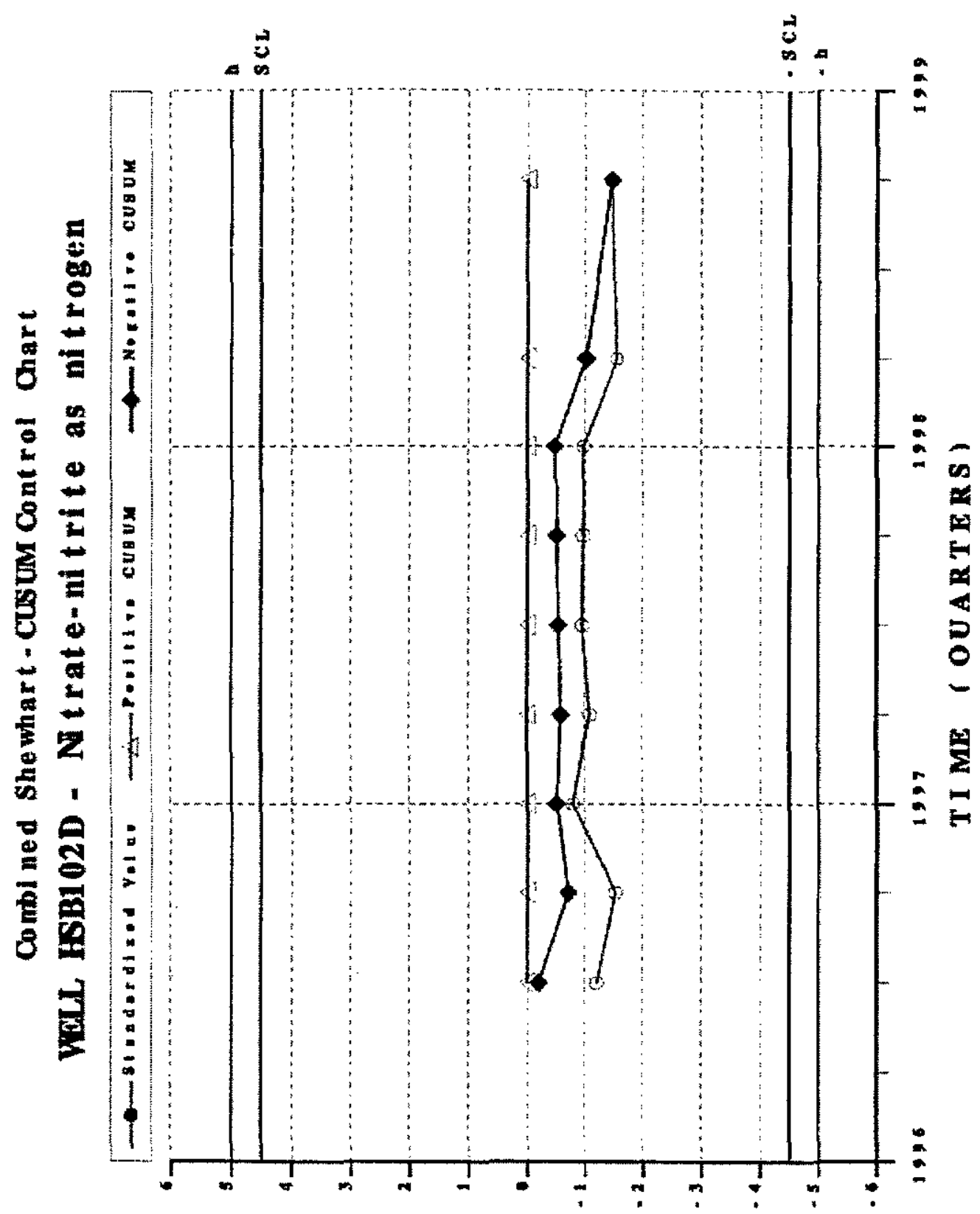

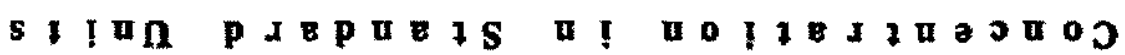



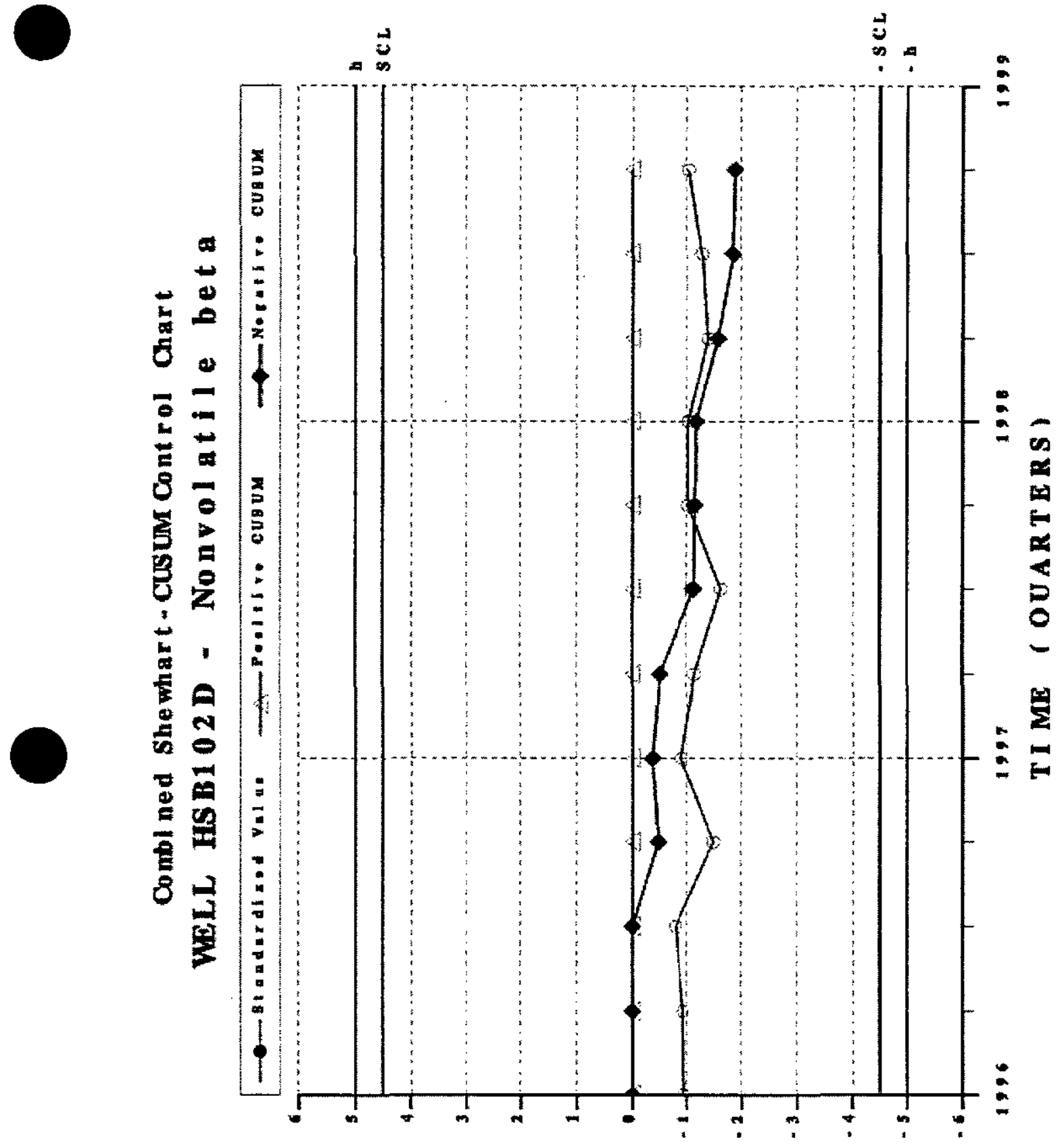

s) I Un pa 


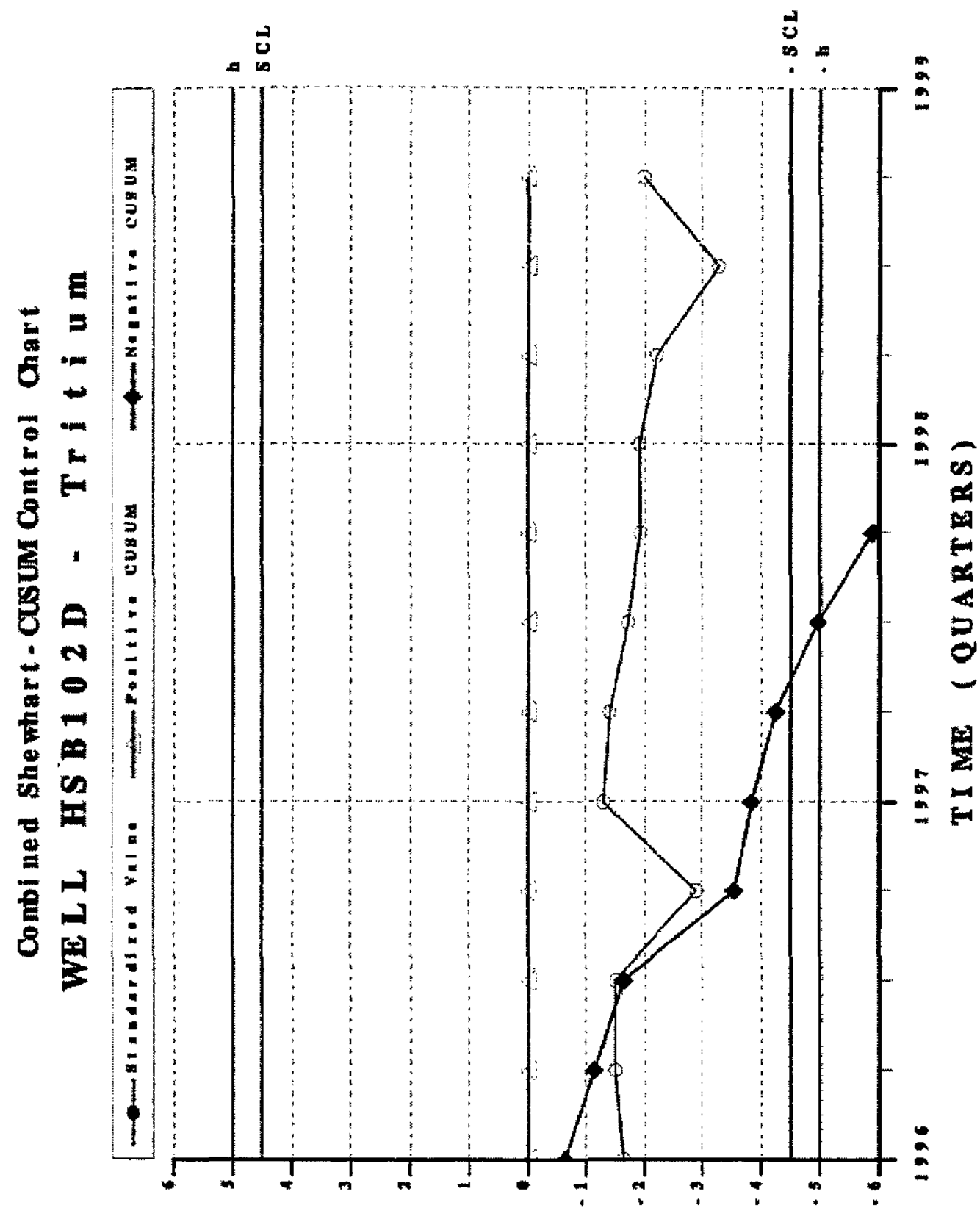

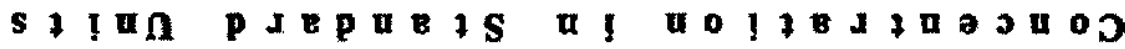


Combl ned Shewhart - CuSUM Control Chart

WELL HSB $102 \mathrm{D}$ - Water Level

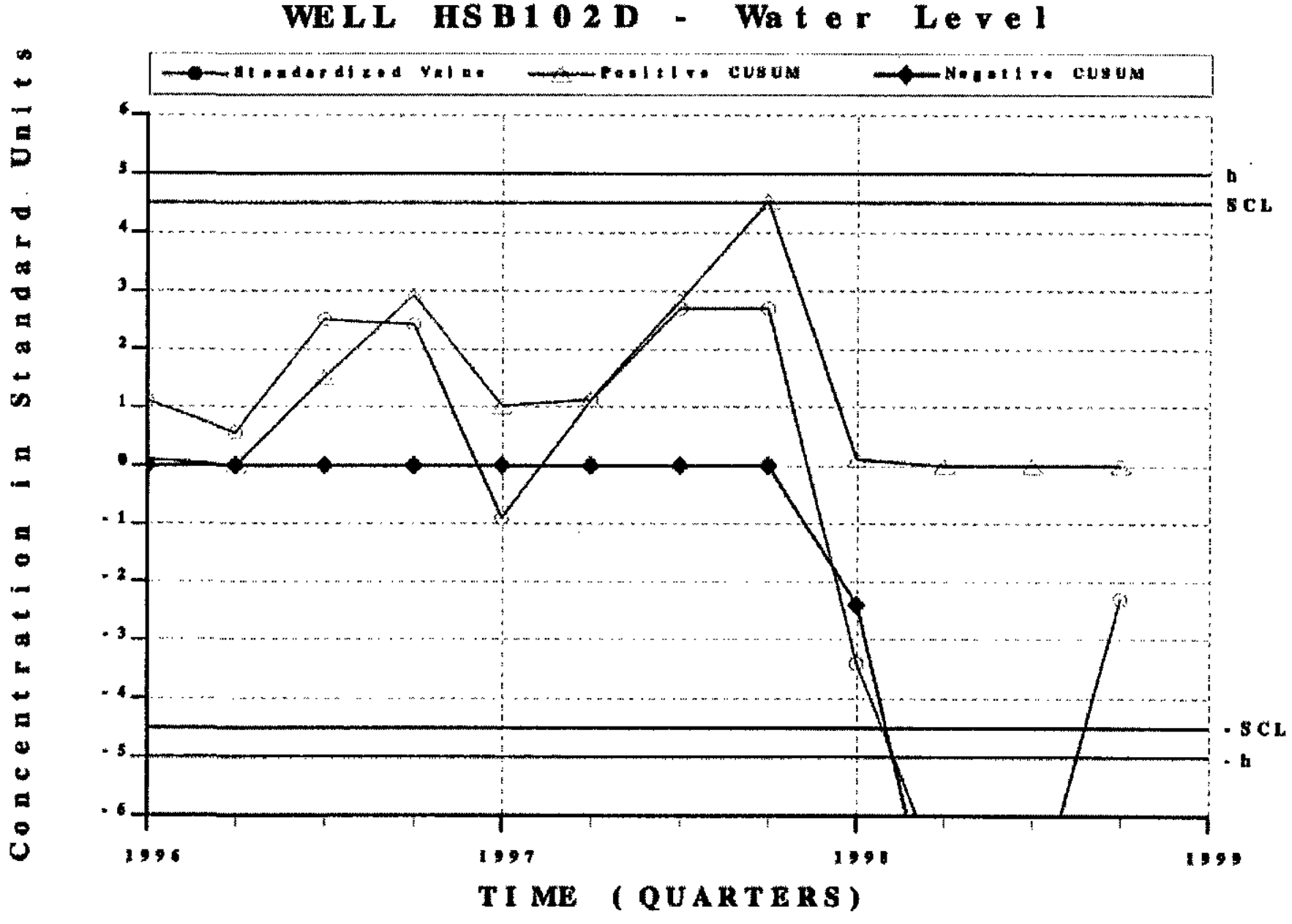


Combi ned Shewhart-Cusum Cont rol Chart

WELL HSB103C - Bari um total recoverable

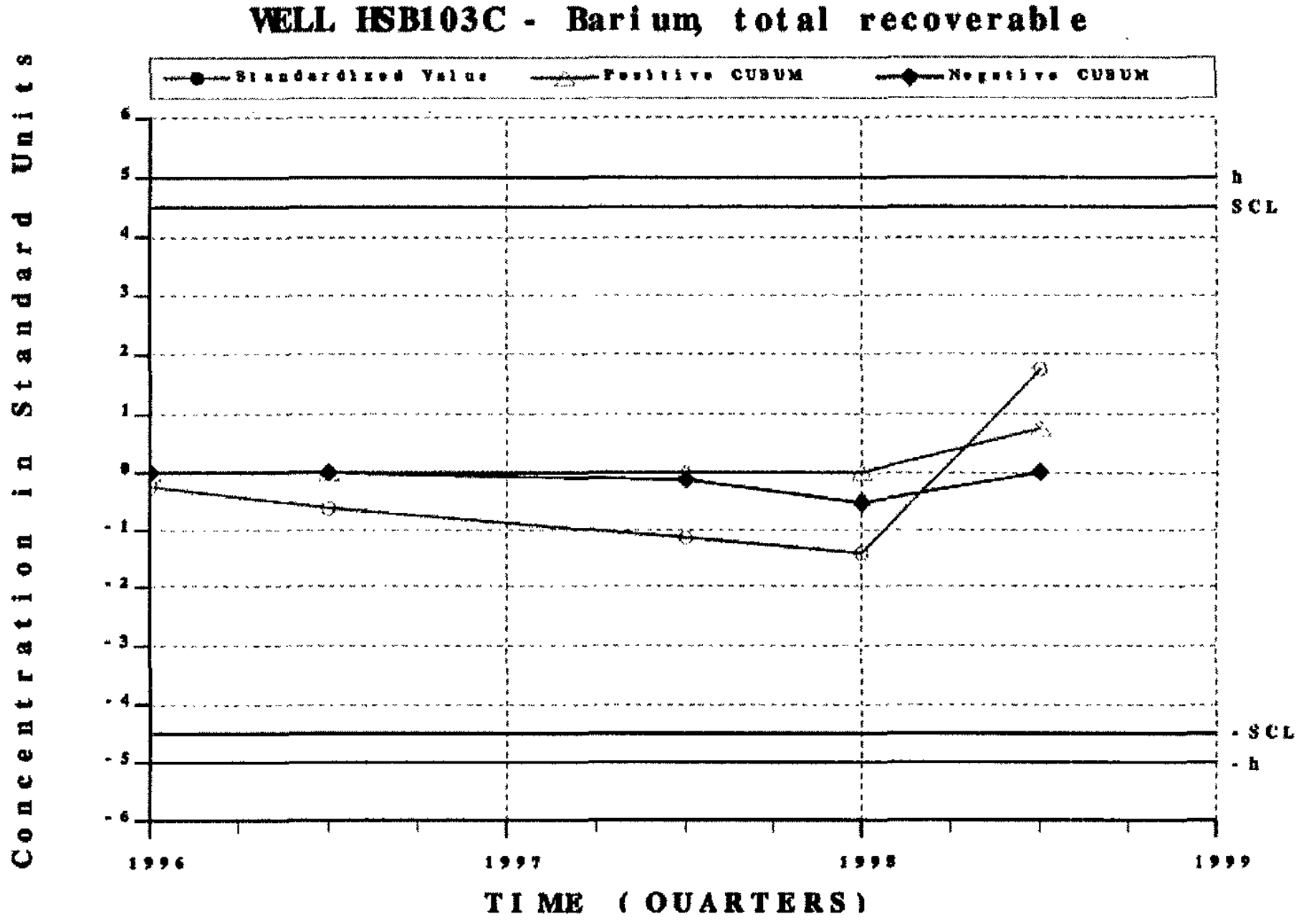

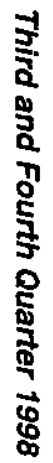

T I ME (OUARTERS ) 
Combi ned She whart - CuSUM Cont rol Chart WELL HSB103C - Cobal t, tot al recoverable

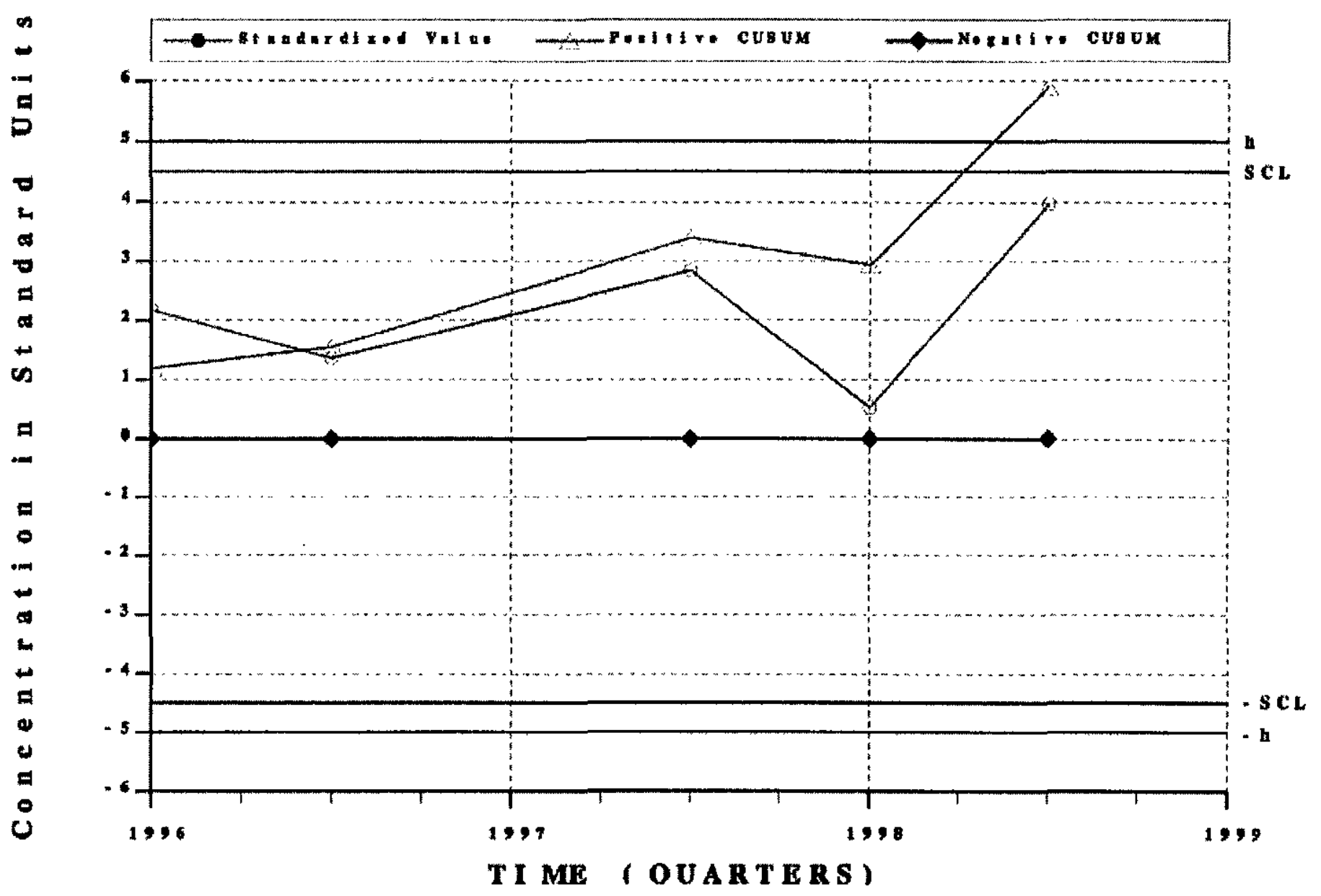




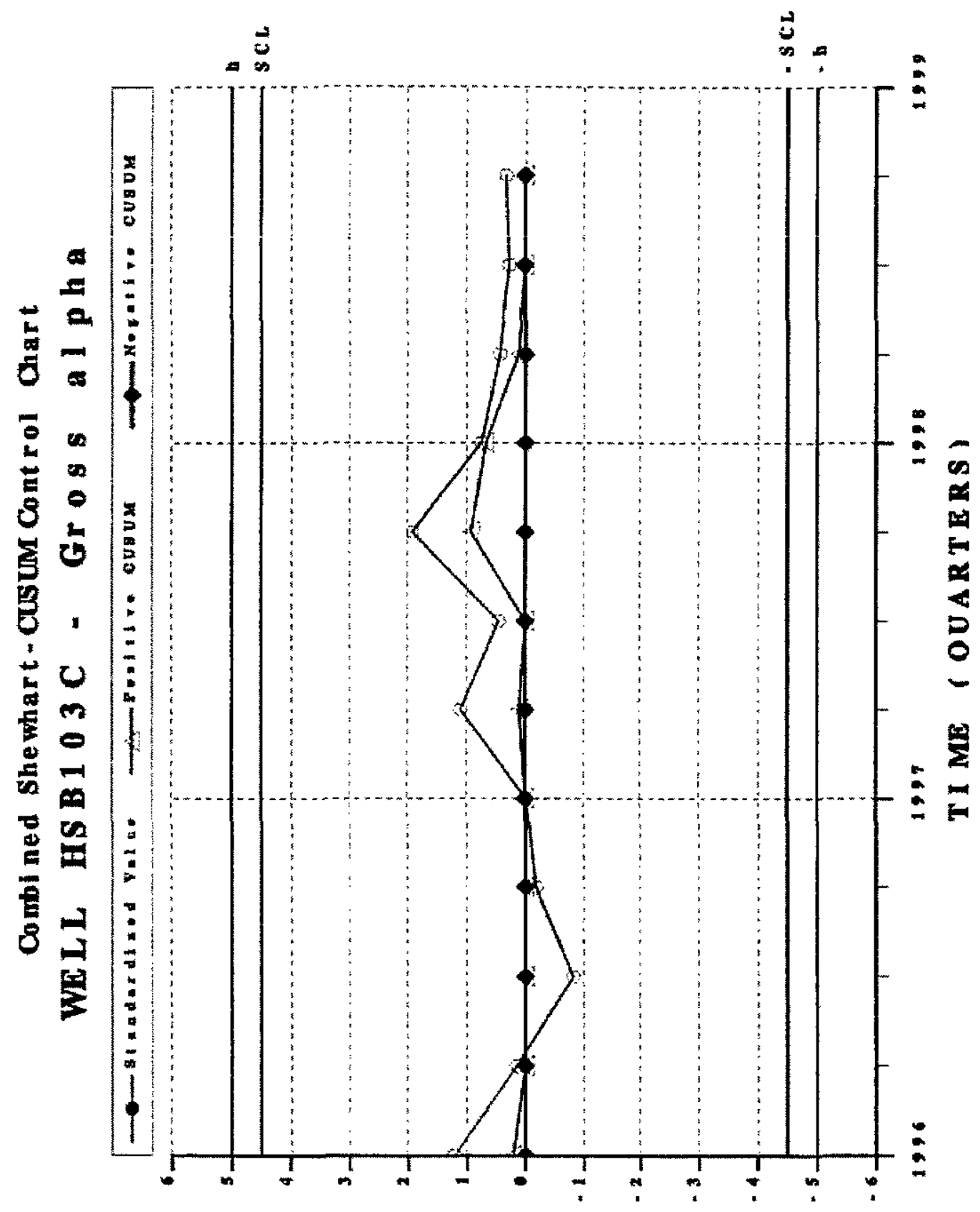

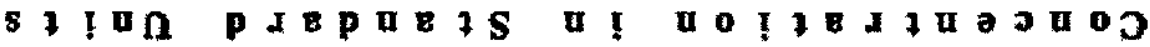



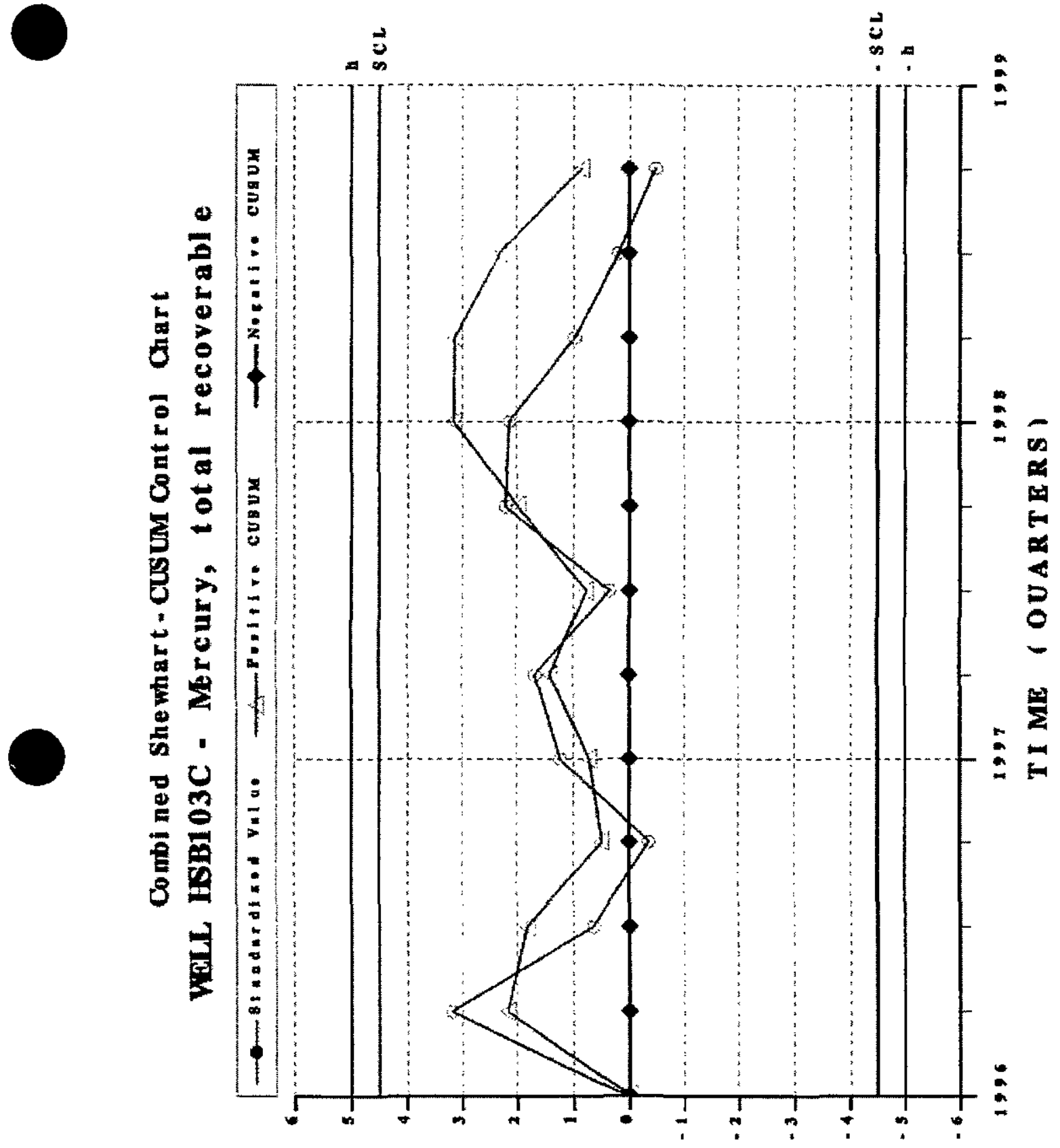

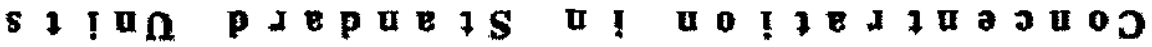




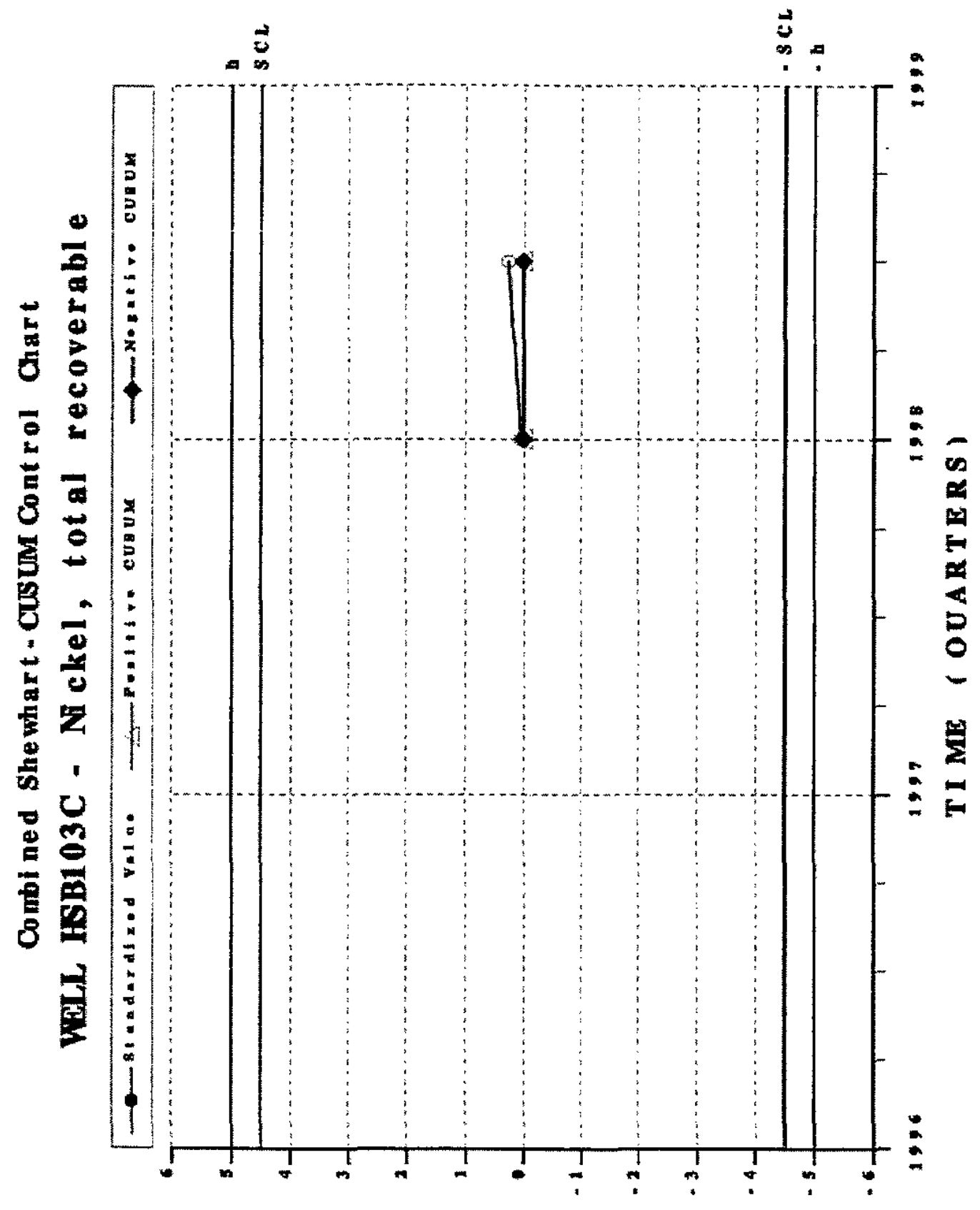

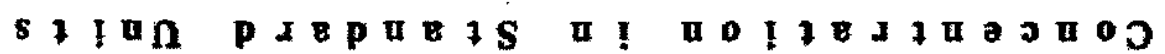


Conbi ned She whart-Cusum Cont rol Chart

Verd HSB103C - N trate-nitrite as nitrogen

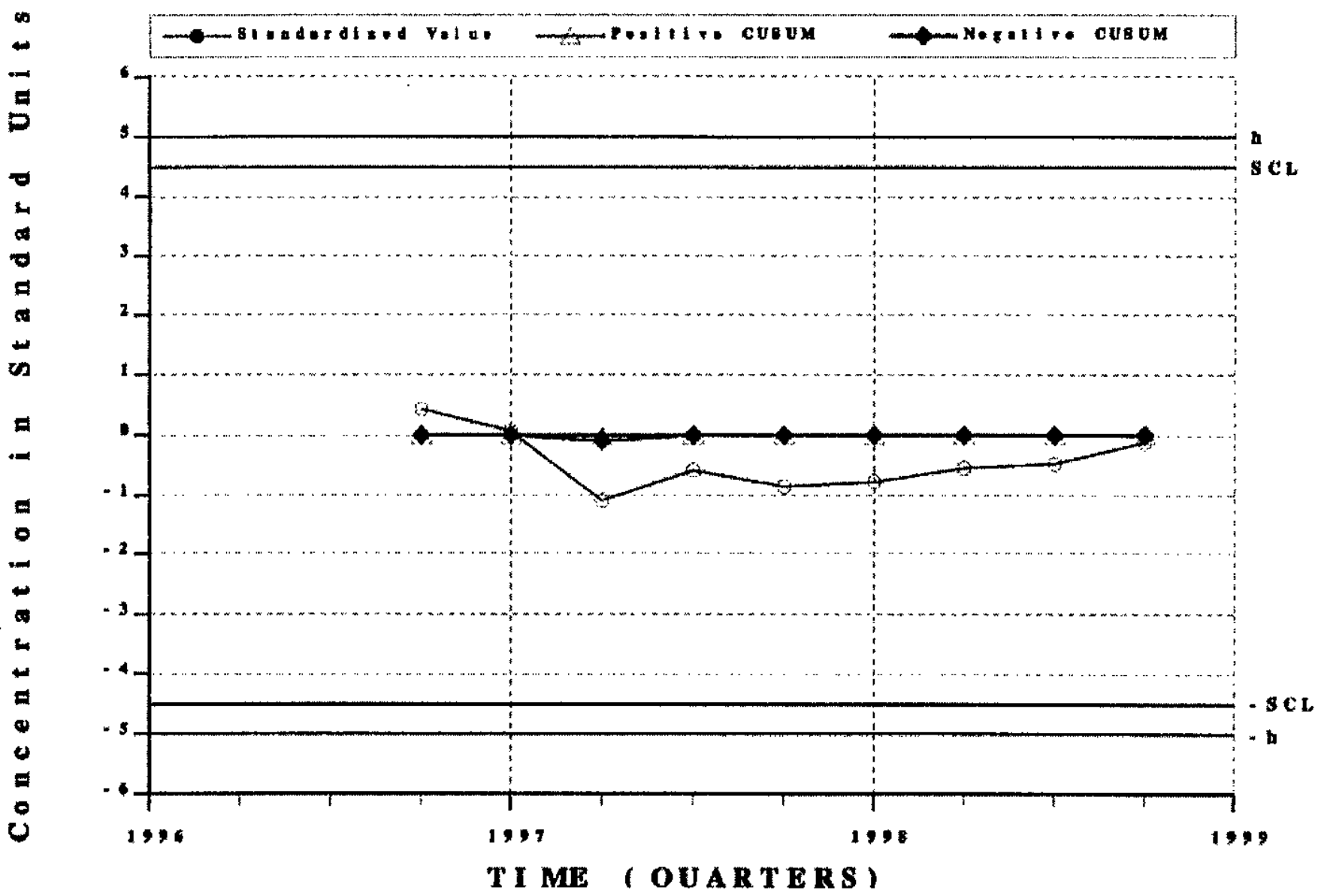




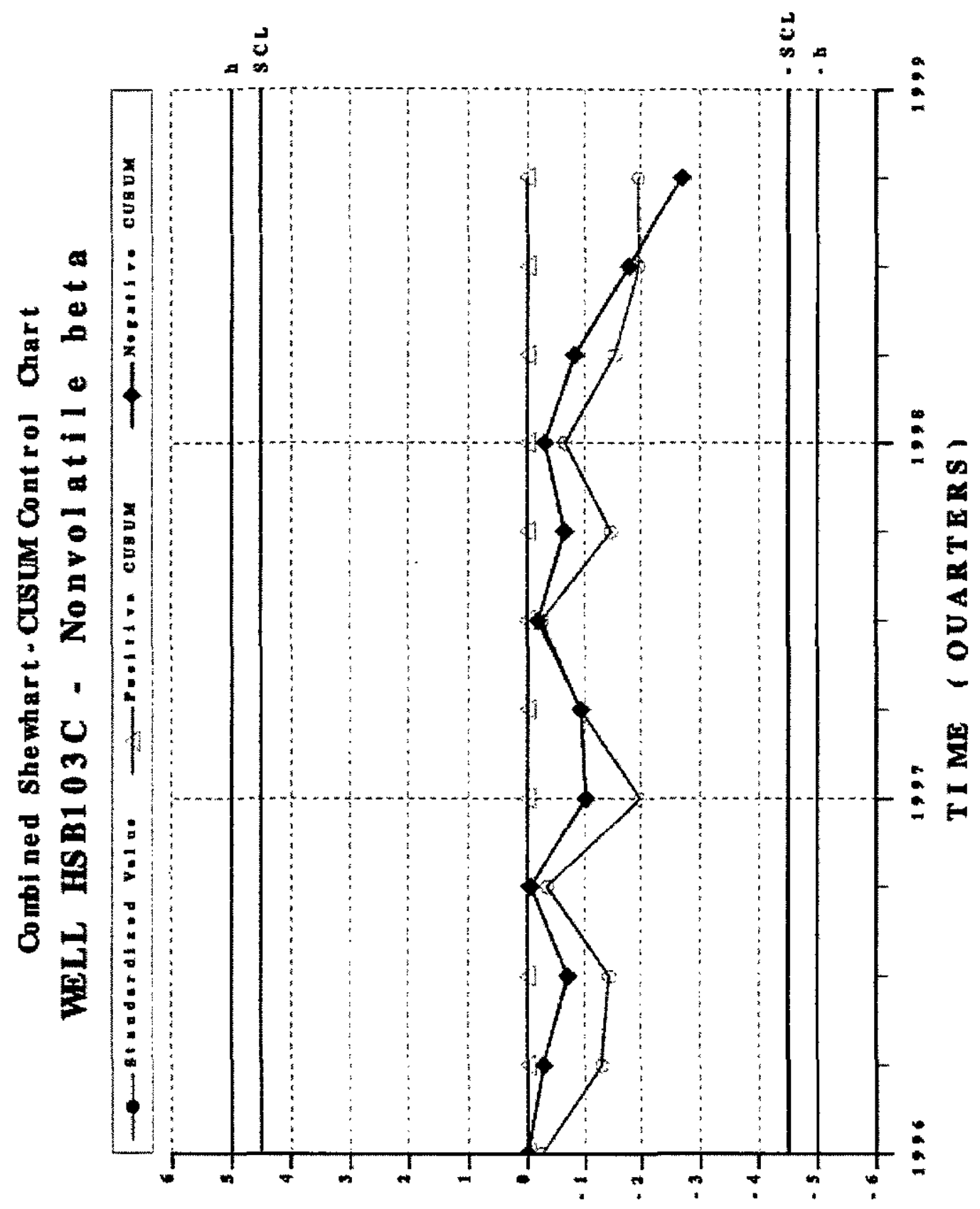

s! I 


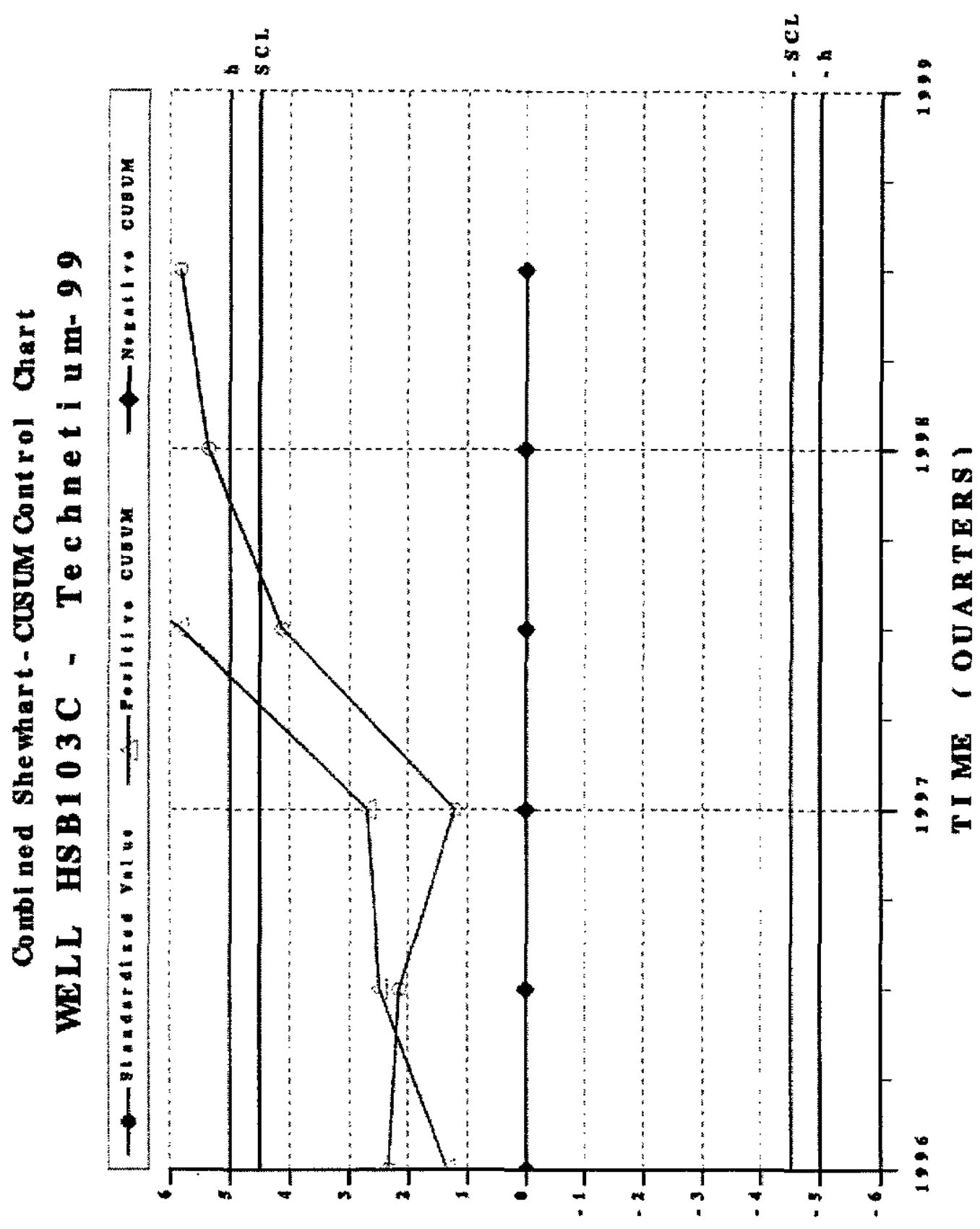

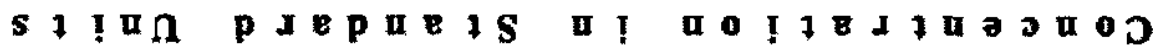


Combi ned Shewhart-Cusum Cont rol Chart

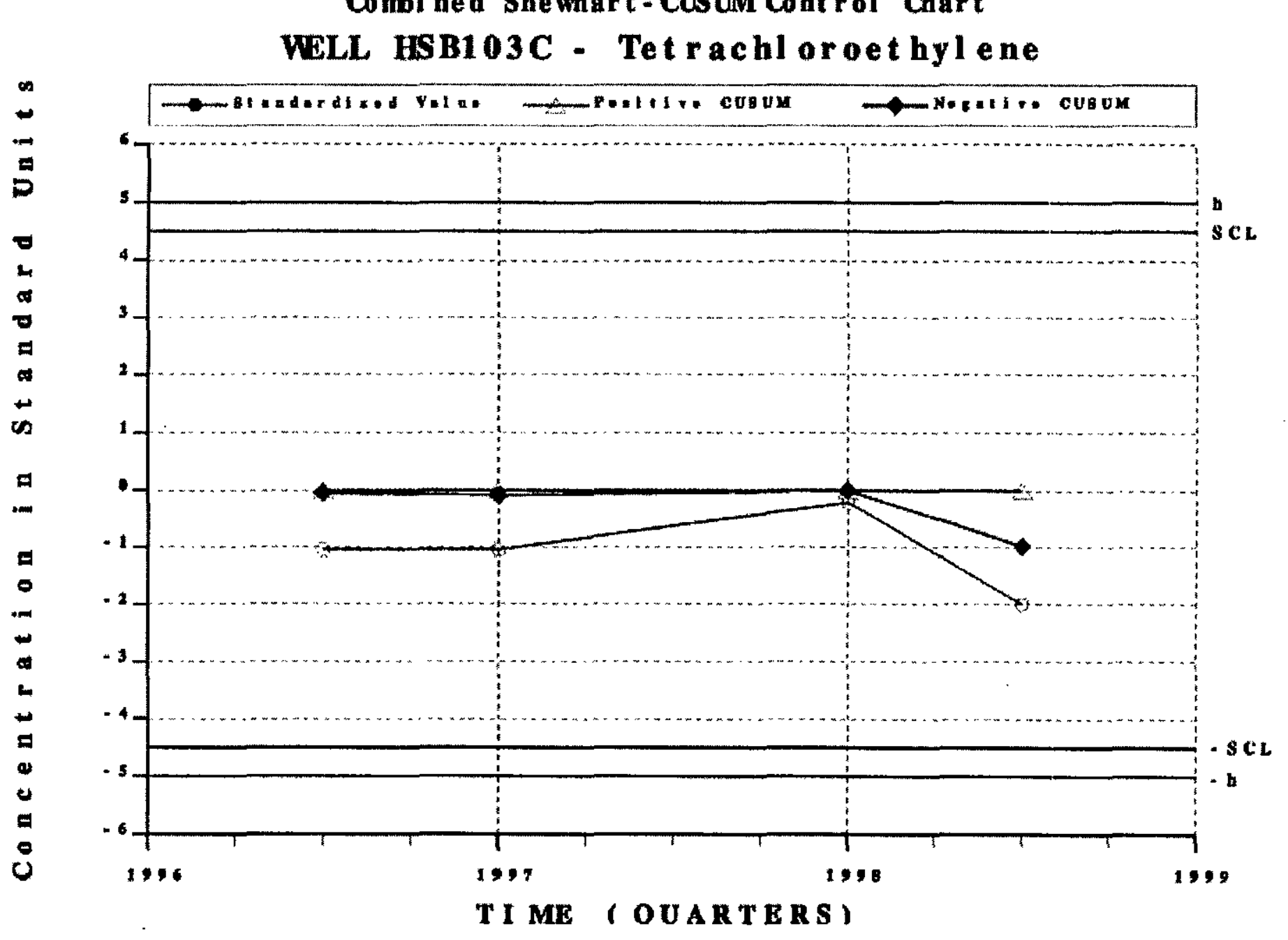

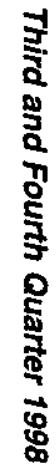

T I ME ( OUARTERS ) 

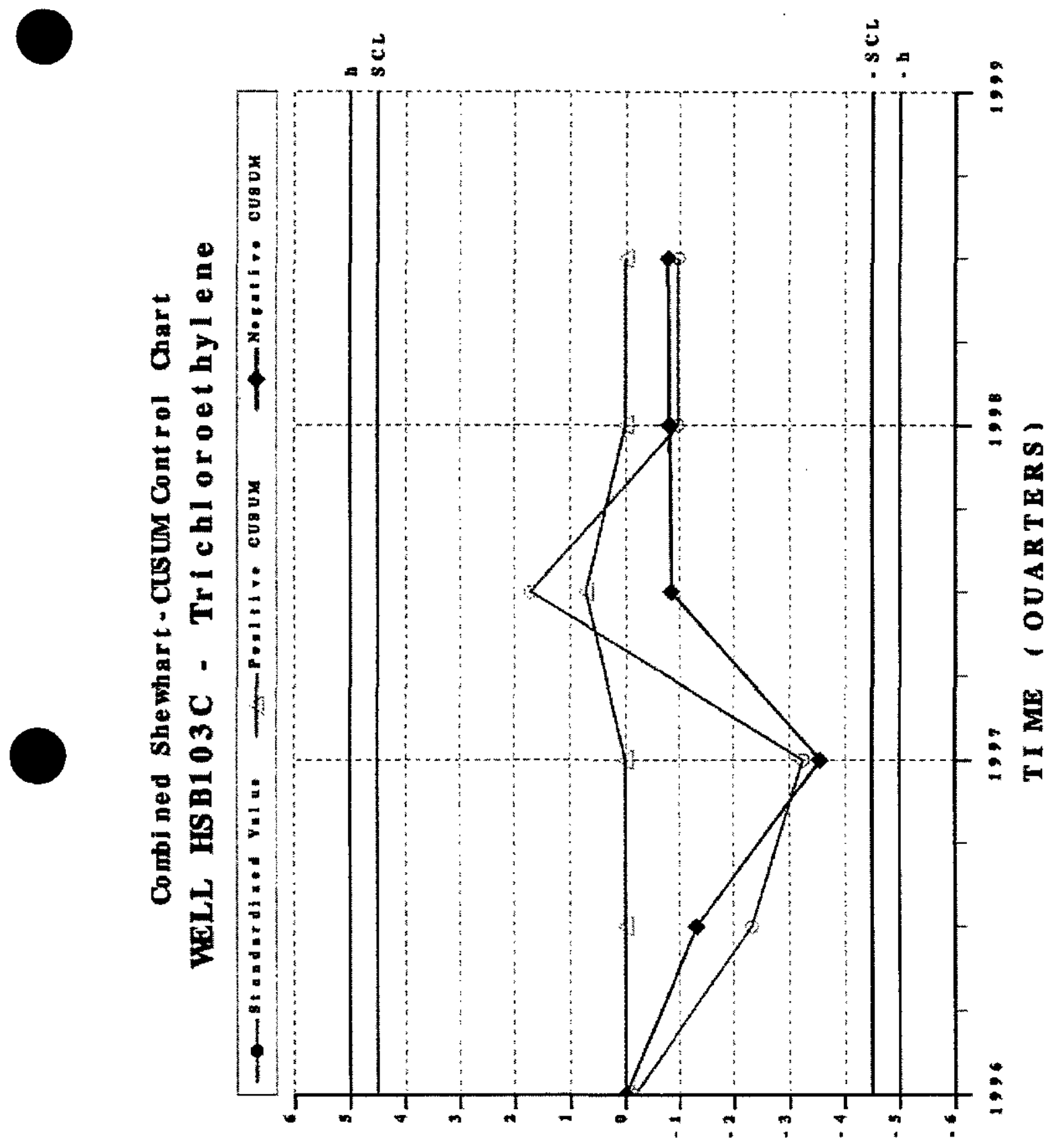

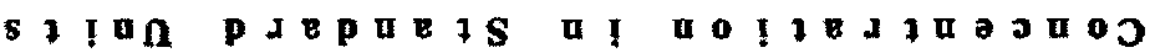




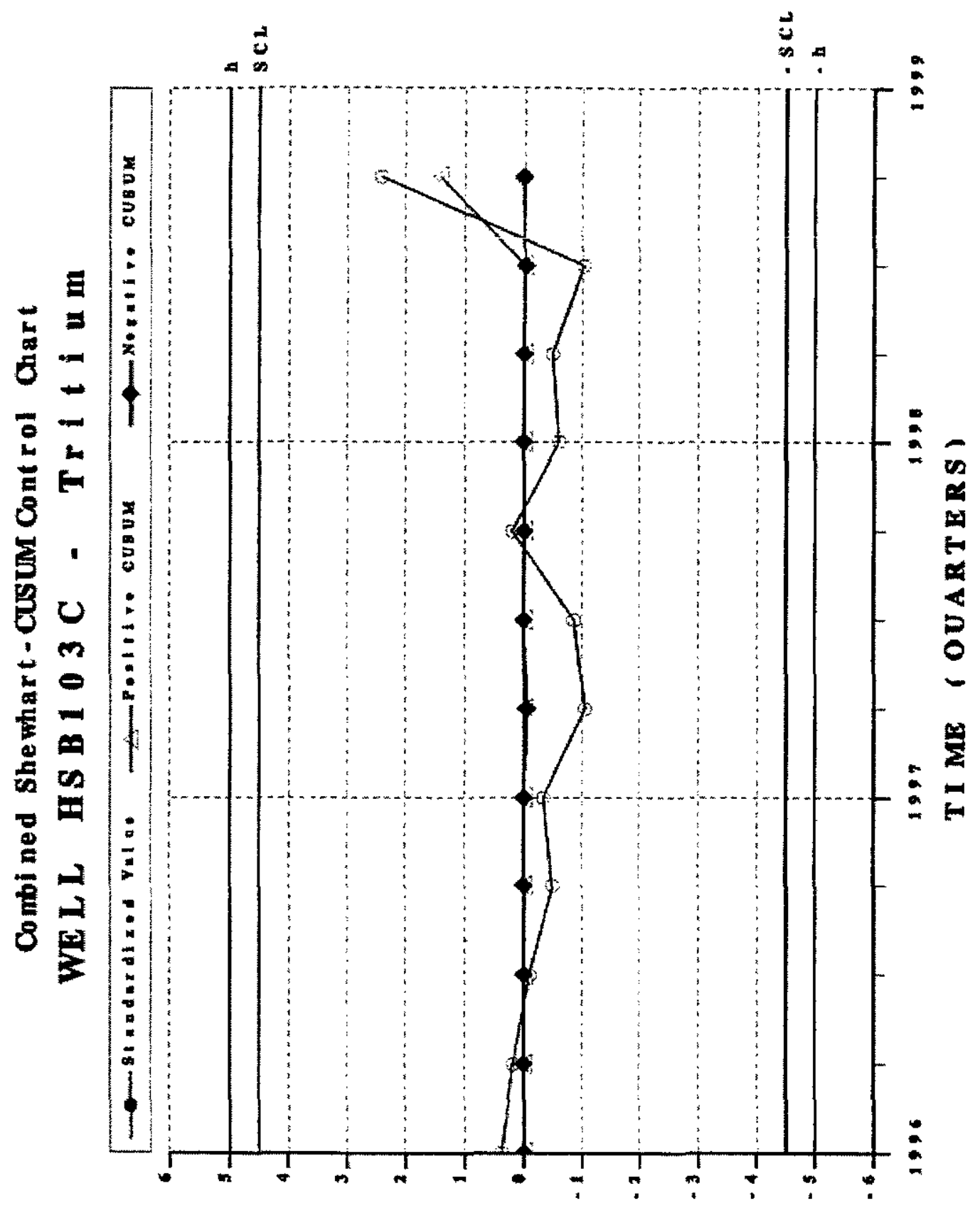

s! I 
Combi ned Shewhart-Cusum Cont rol Chart

WELL HSB $103 \mathrm{C}$ - Wa t e r Le ve 1

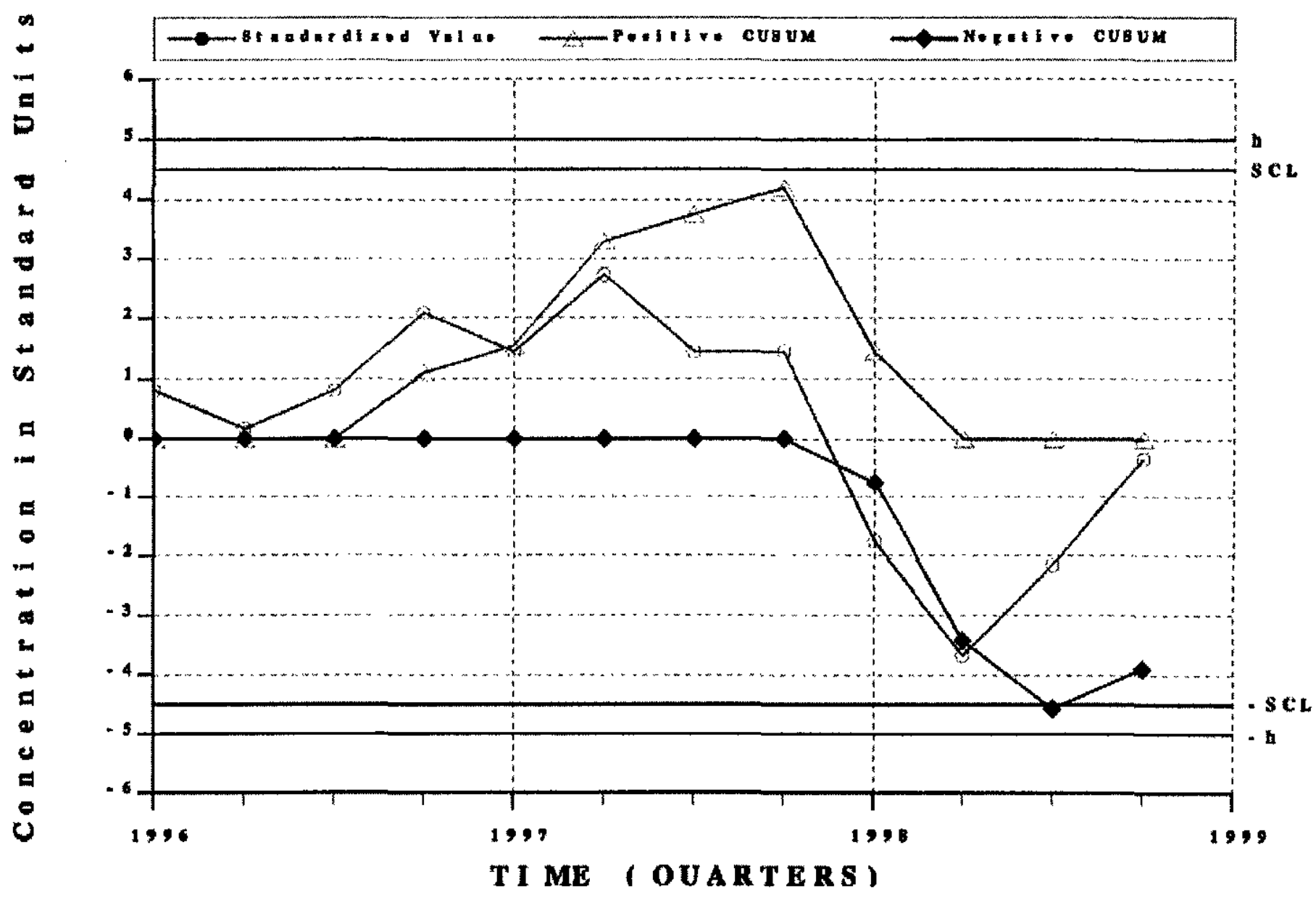


Combi ned Shewhart-Cusum Cont rol Chart WeLL HSB103C - Zi ne, total recoverable

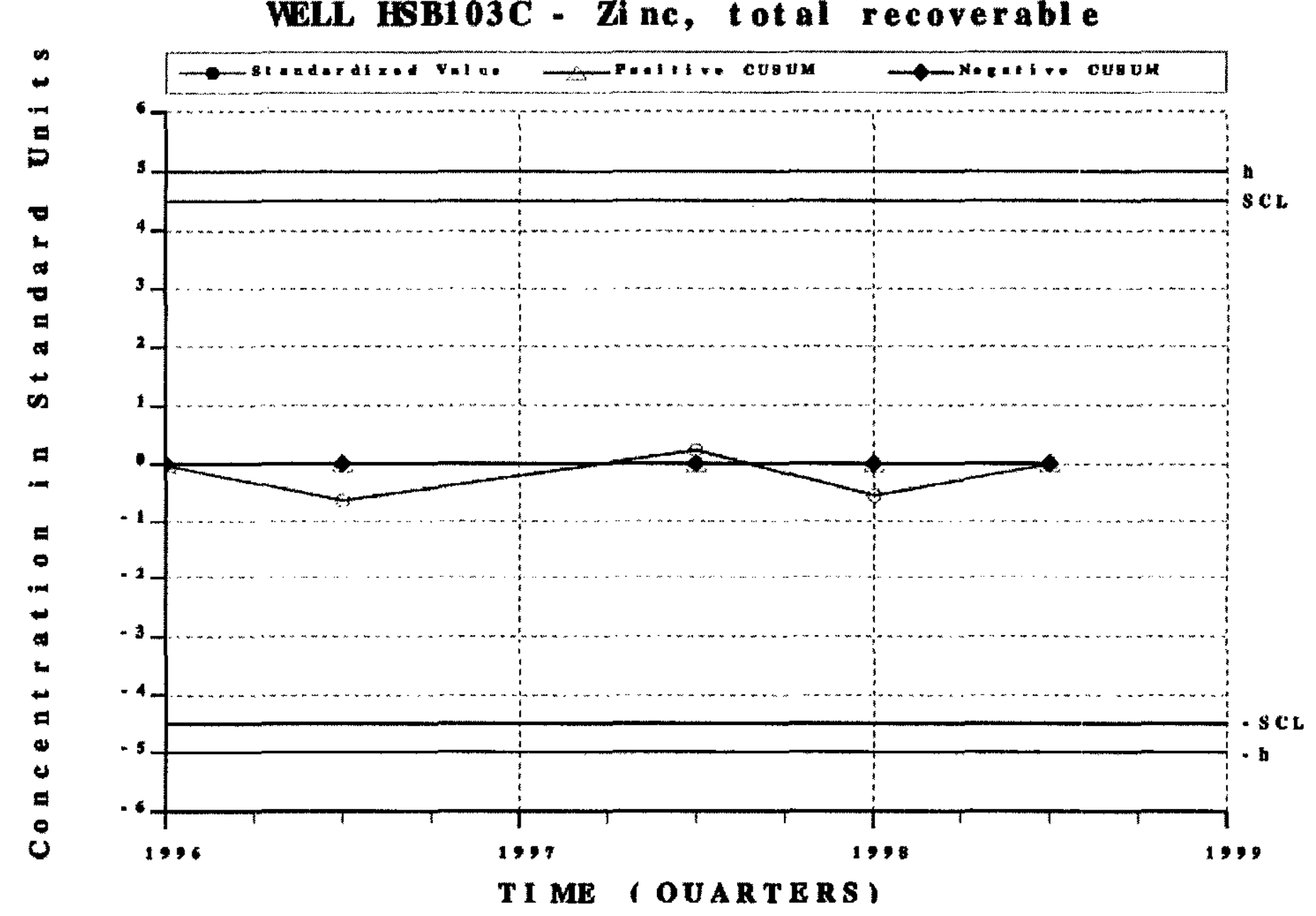


Combi ned Shewhart-CUSUM Cont rol Chart WELL HSB103D - Bari um total recoverable

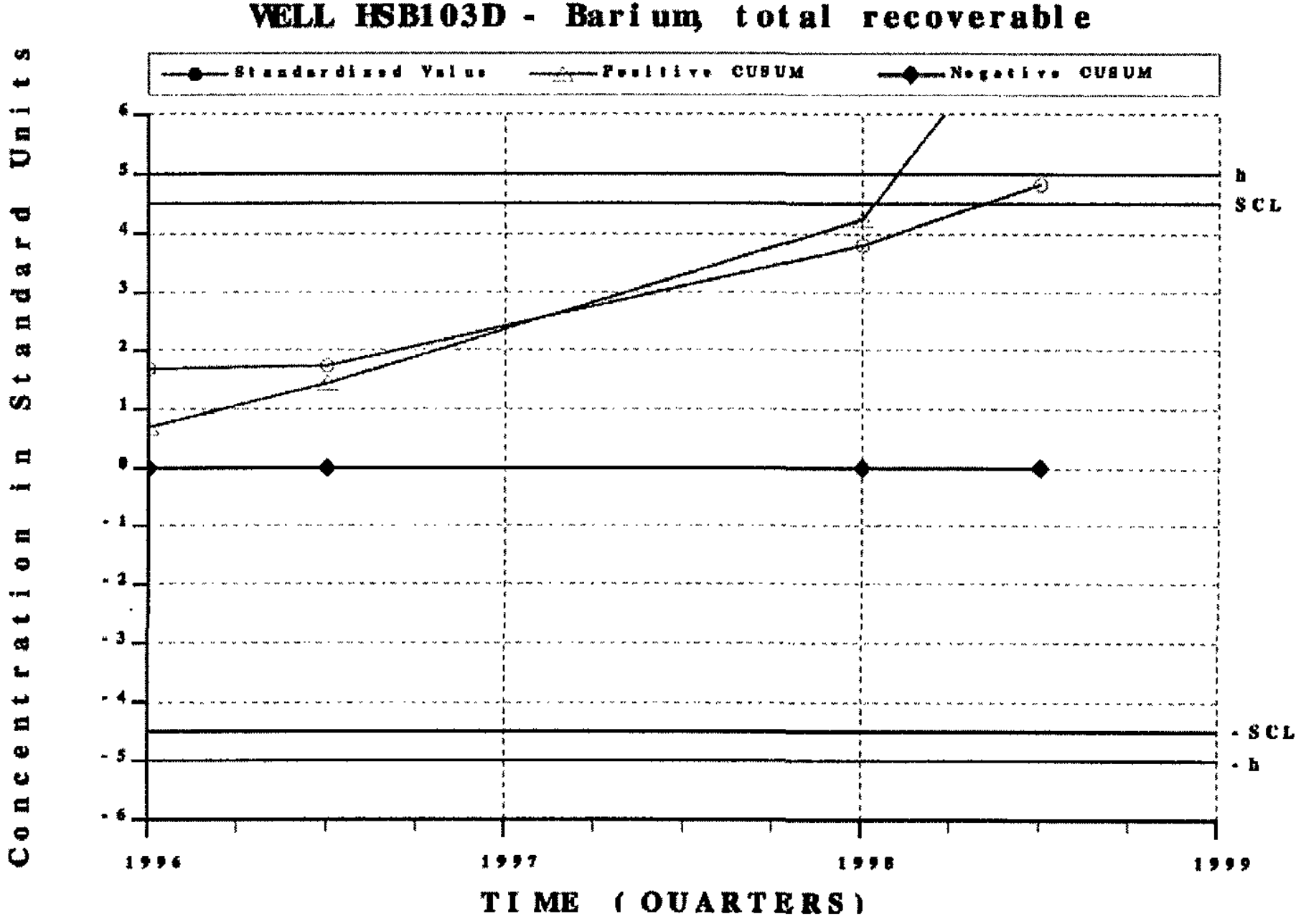




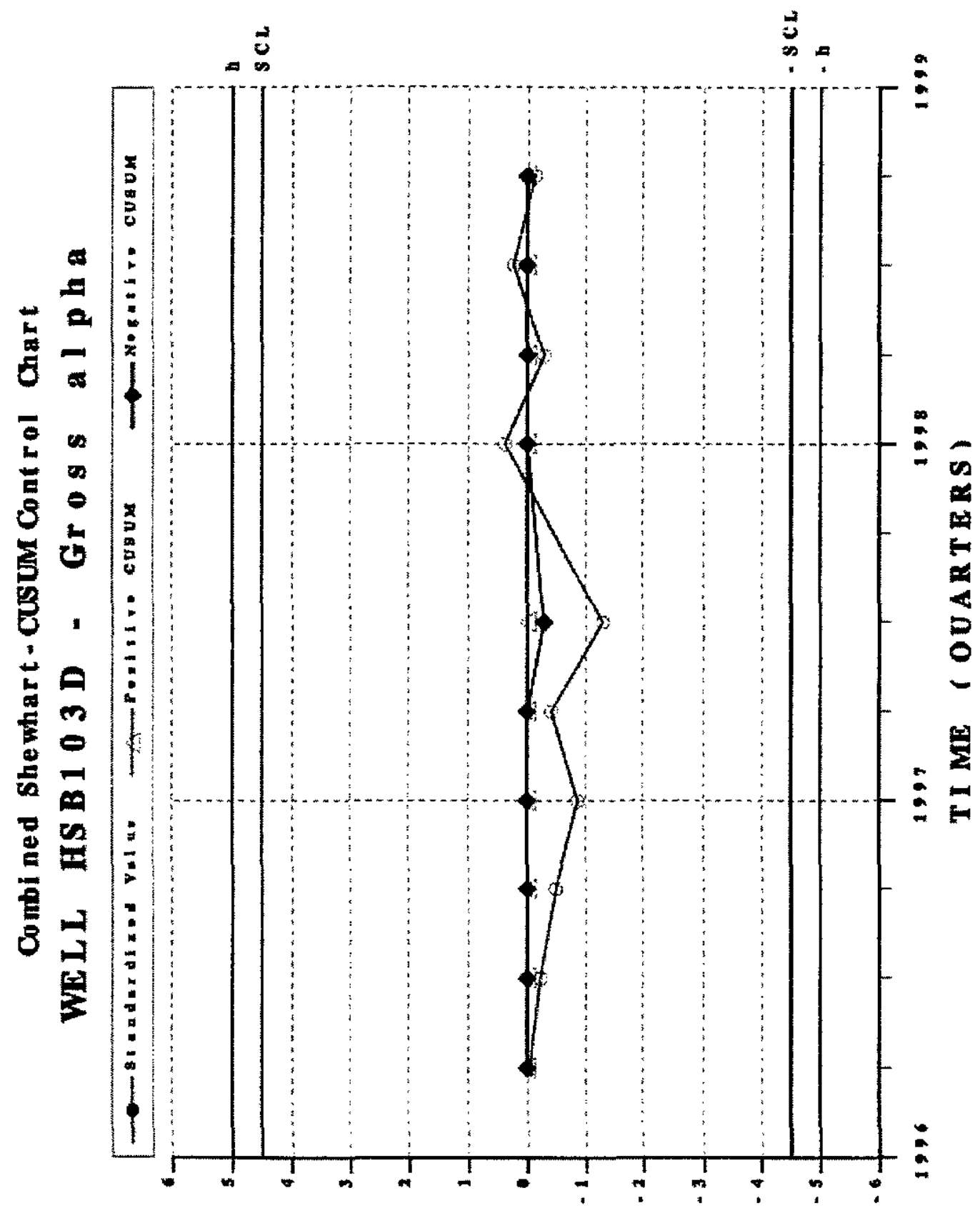

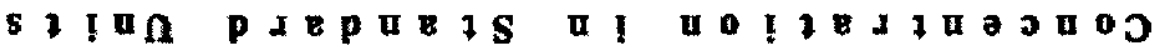



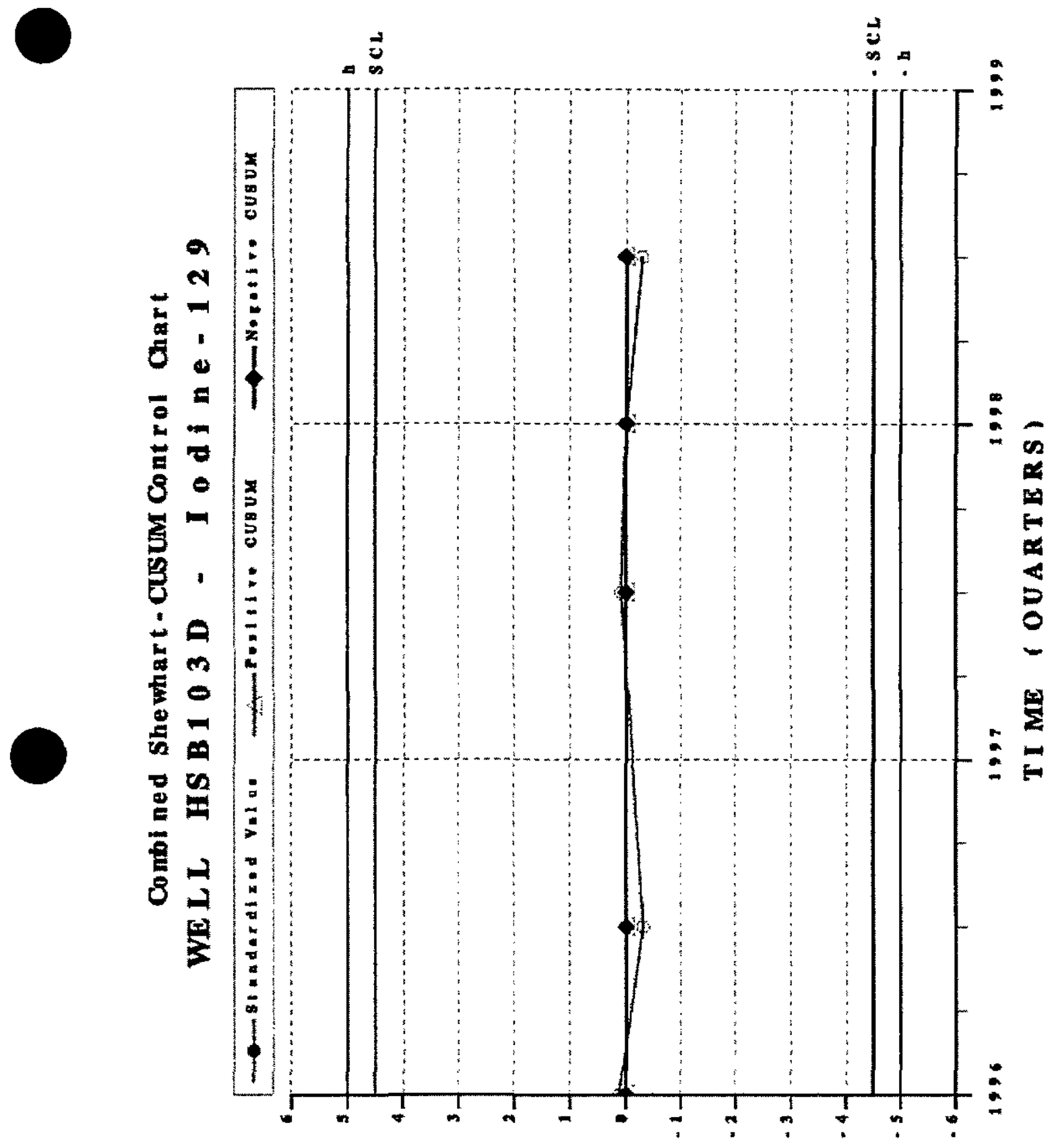

S1! 1 I 


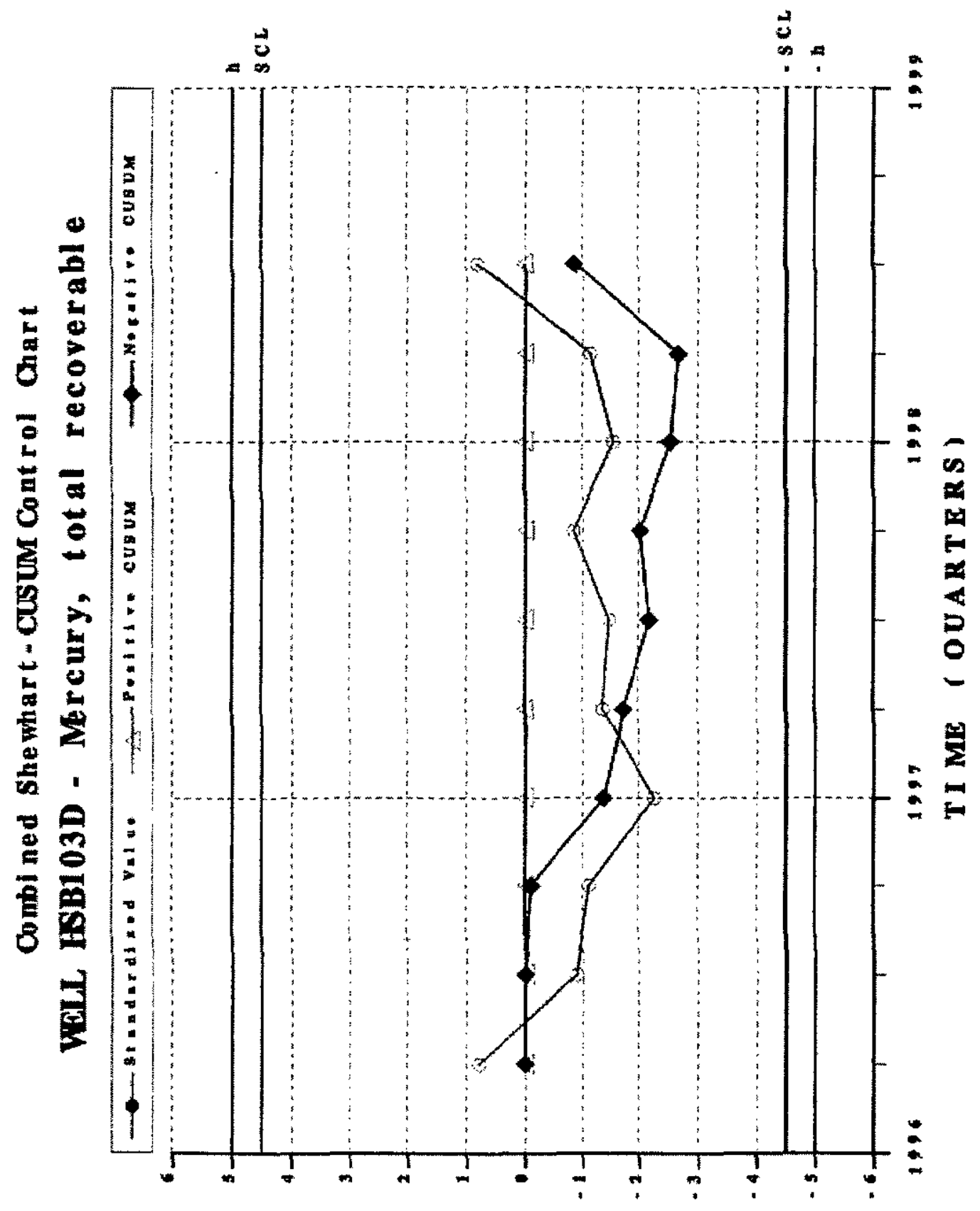

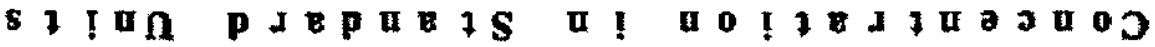



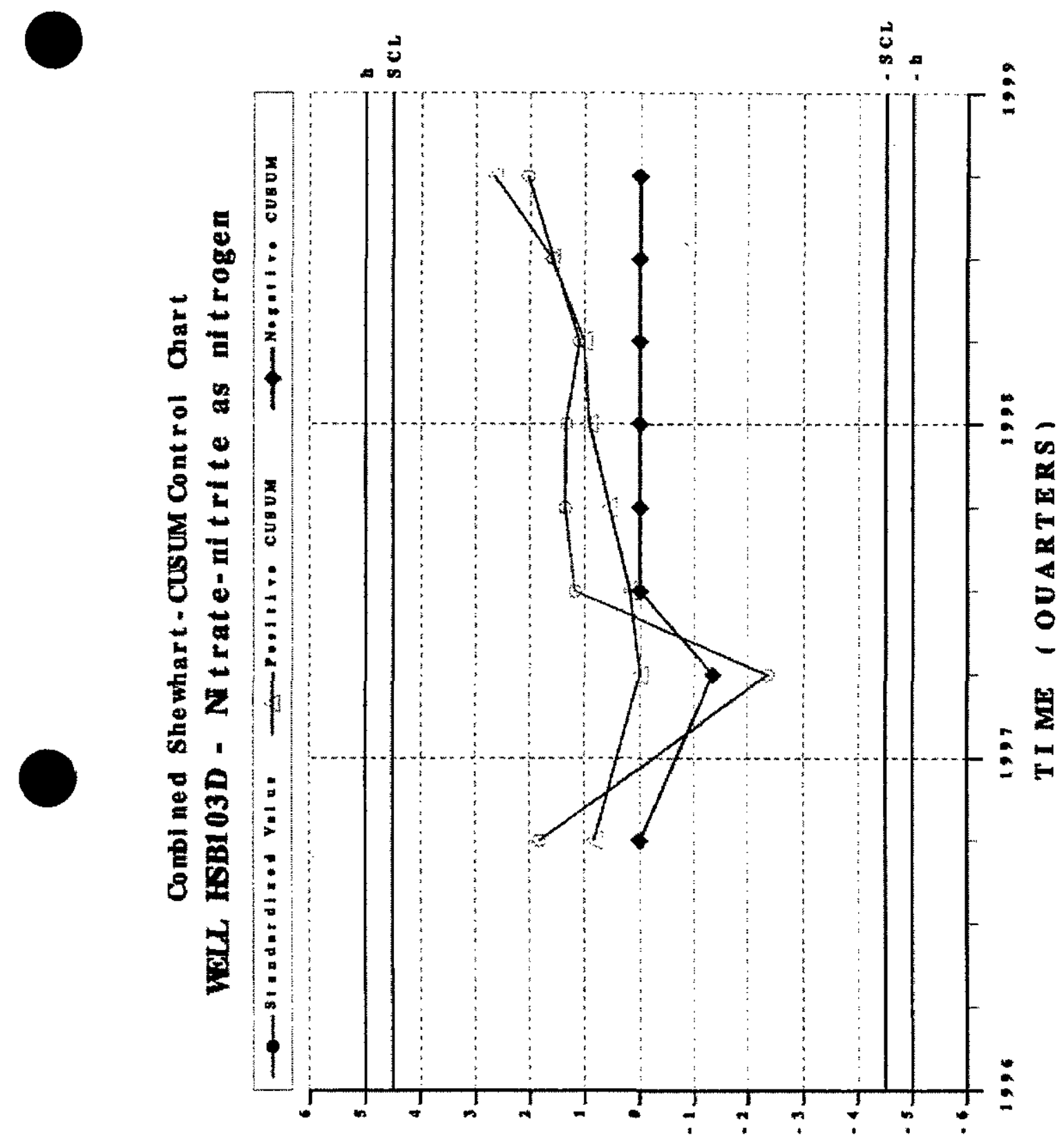

s I I I p I B 
Combi ned Shewhart-Cusum Control Chart WELL HSB103D - Nonvolatile beta

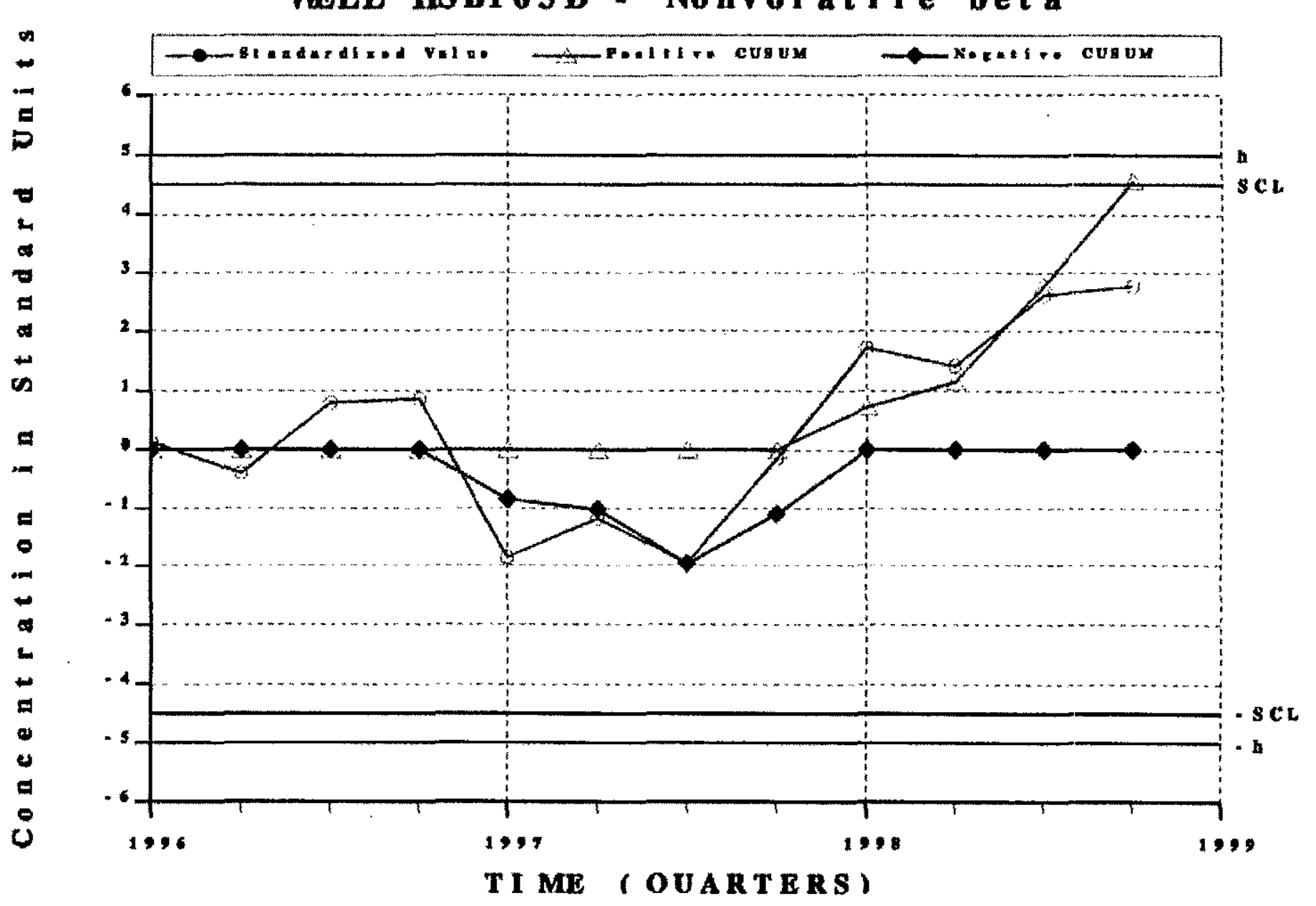

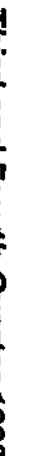



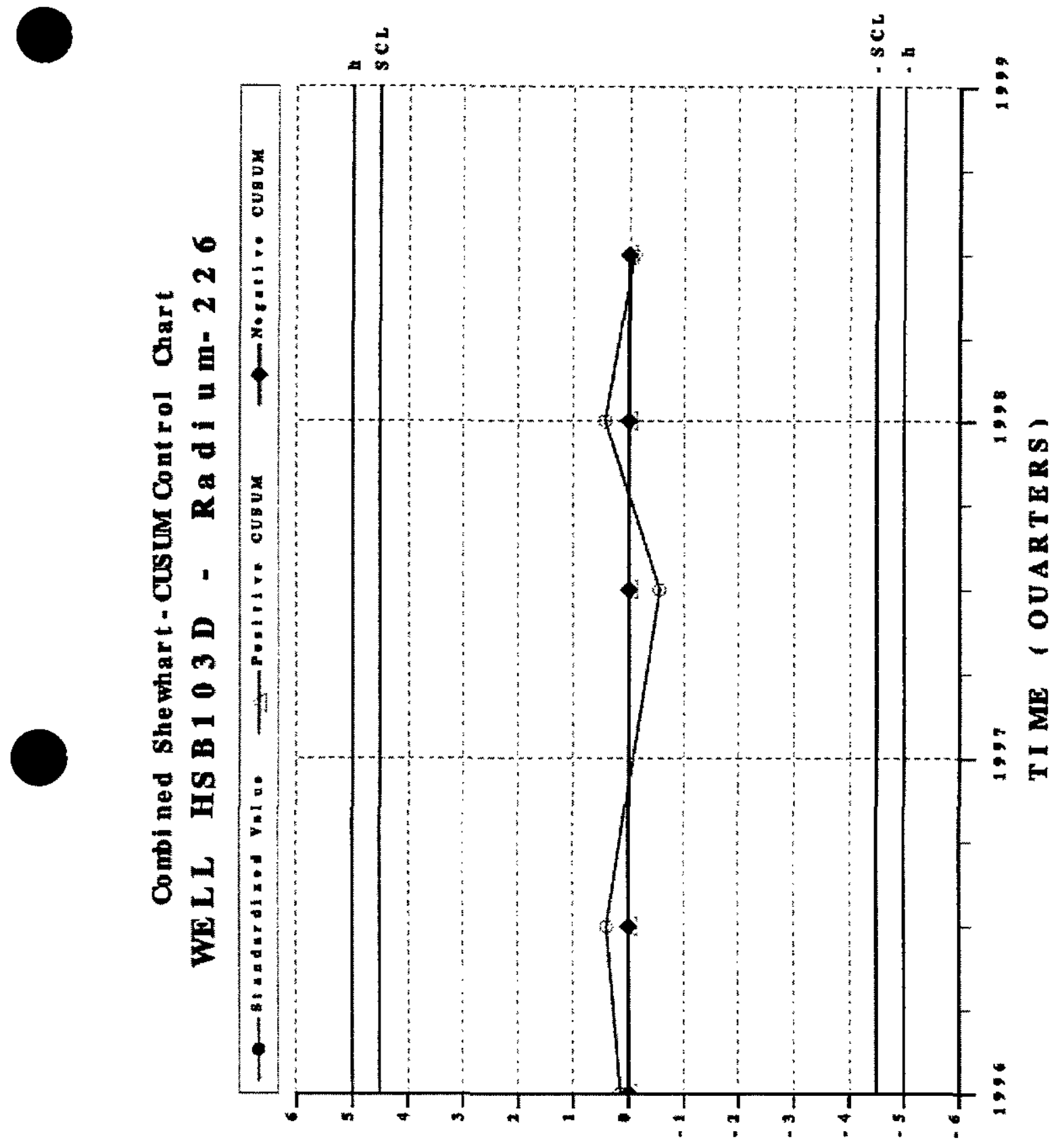

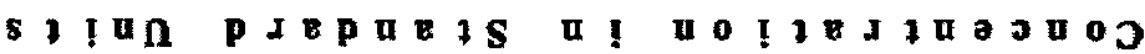


Combi ned She whart-Cusum Control Chart

WELL HS B 103 D - Radi um- 228

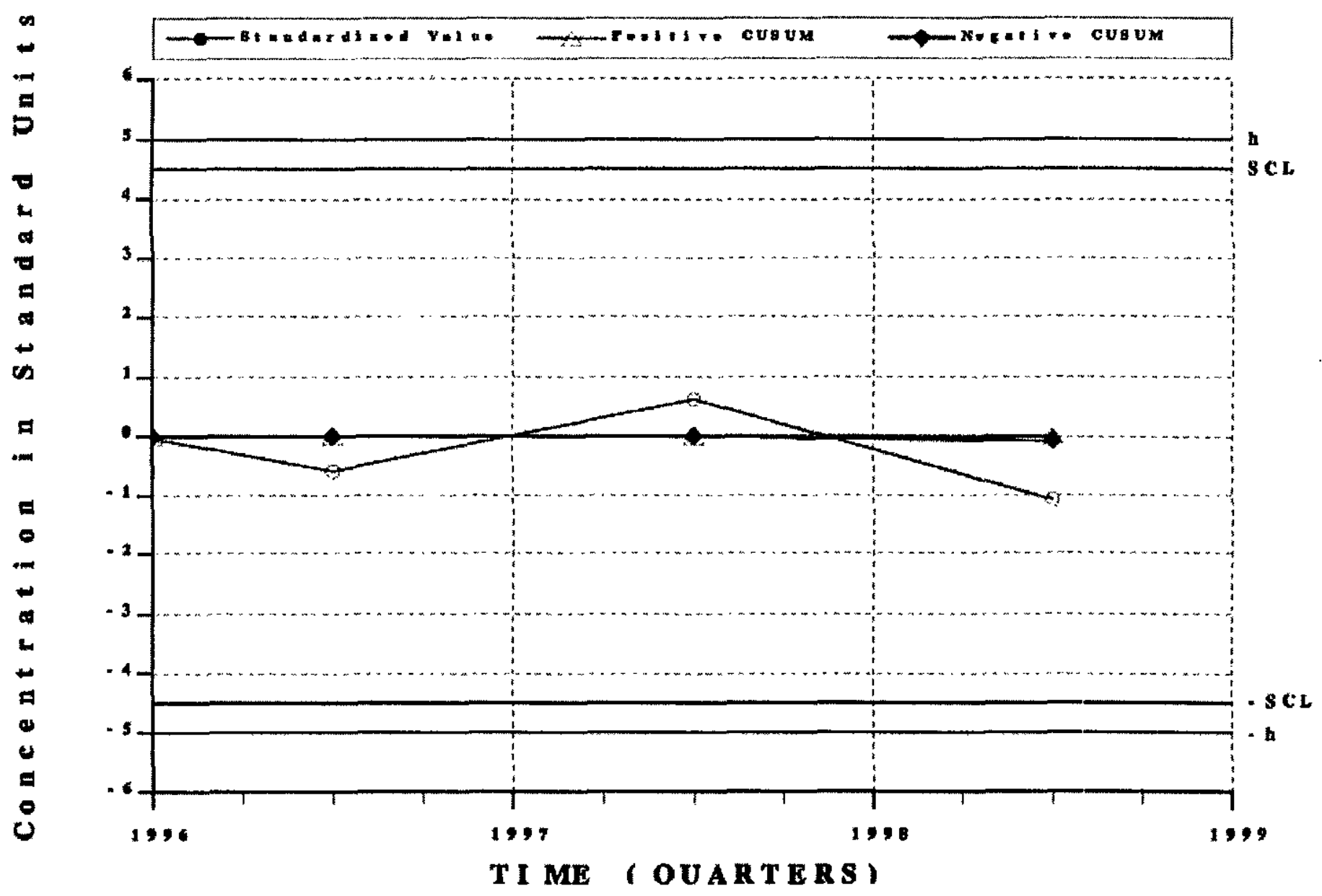


Combi ned Shewhart-CUSUM Cont rol Chart

WELL HSB103 D - Stronti um-90

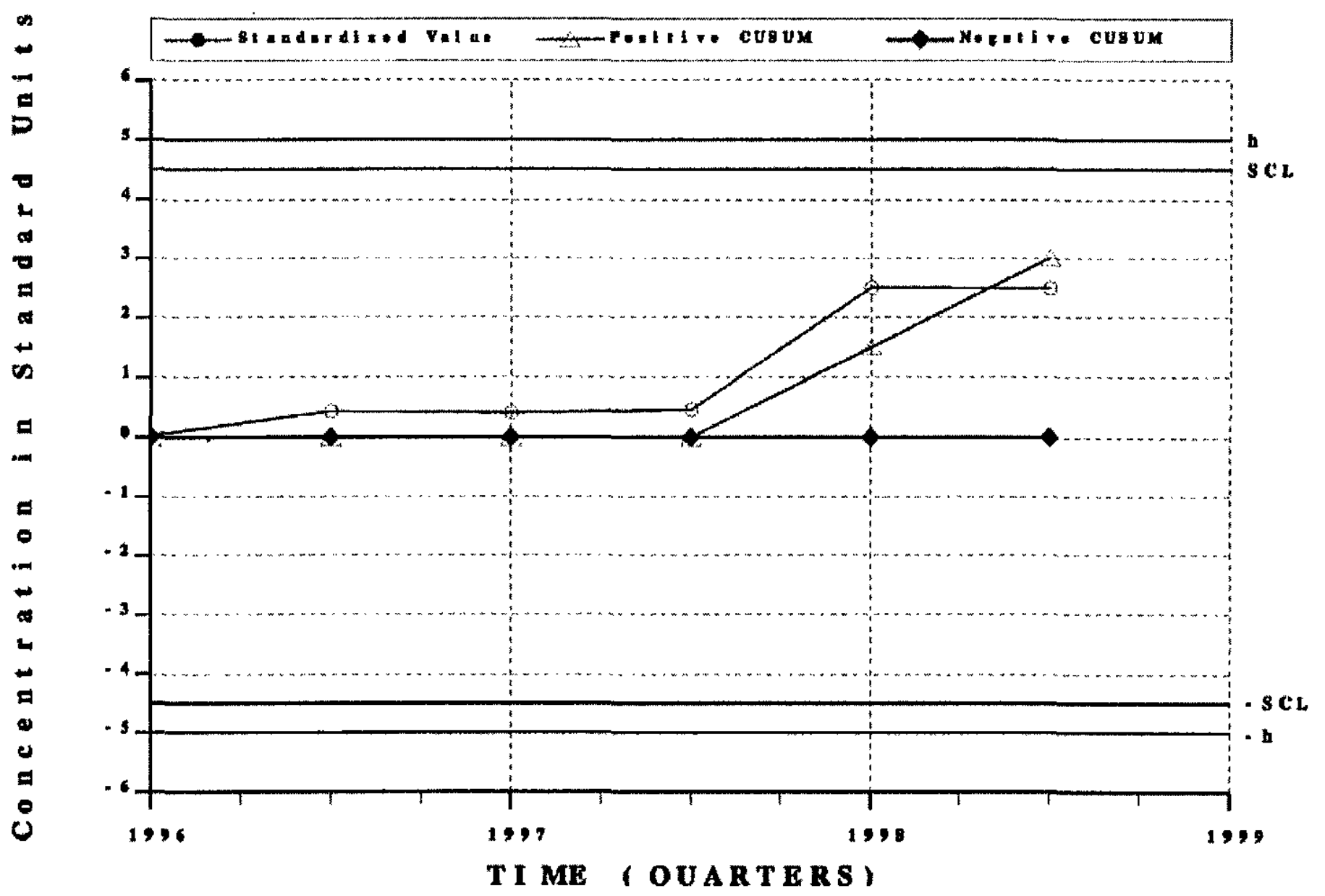




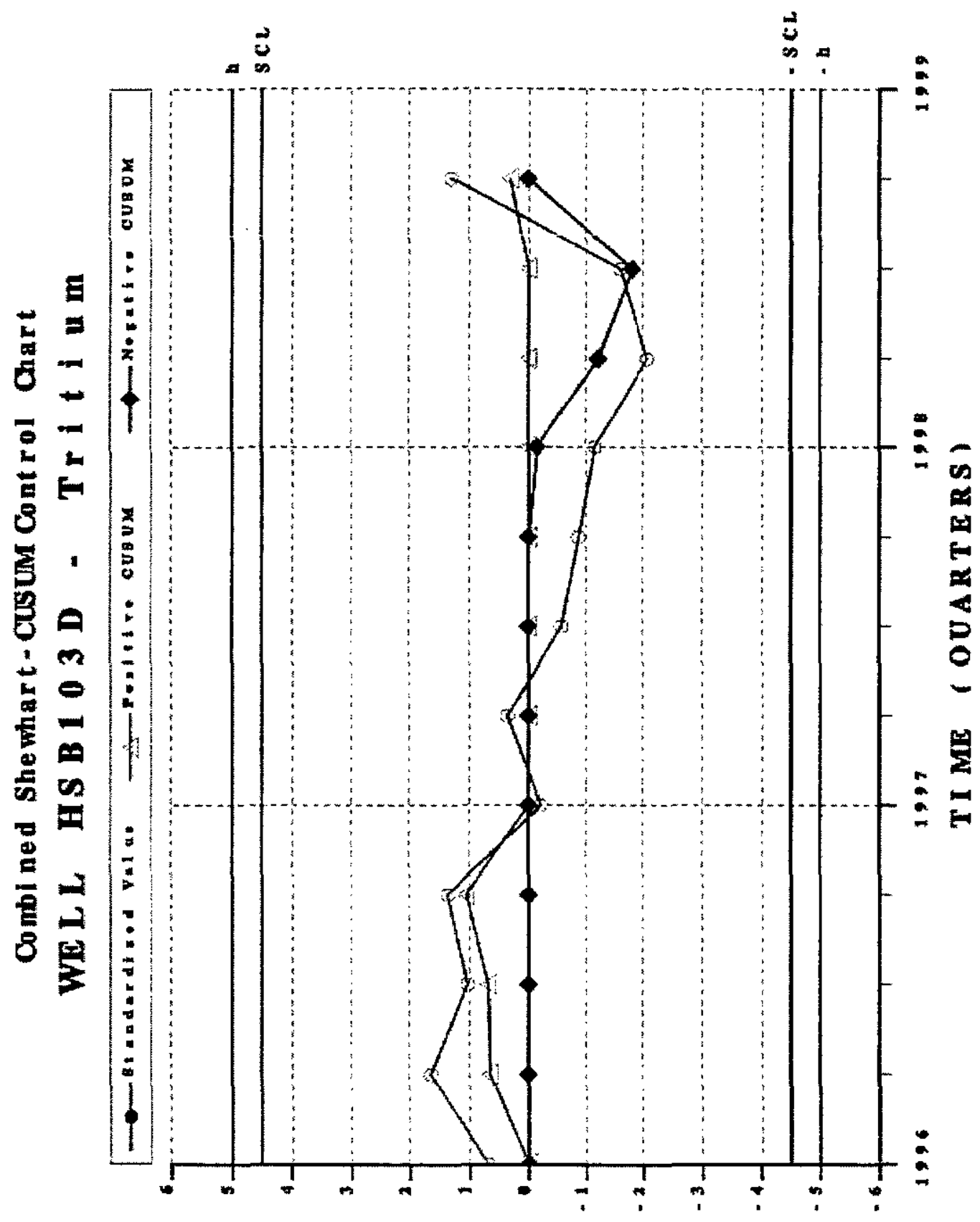

s t I 

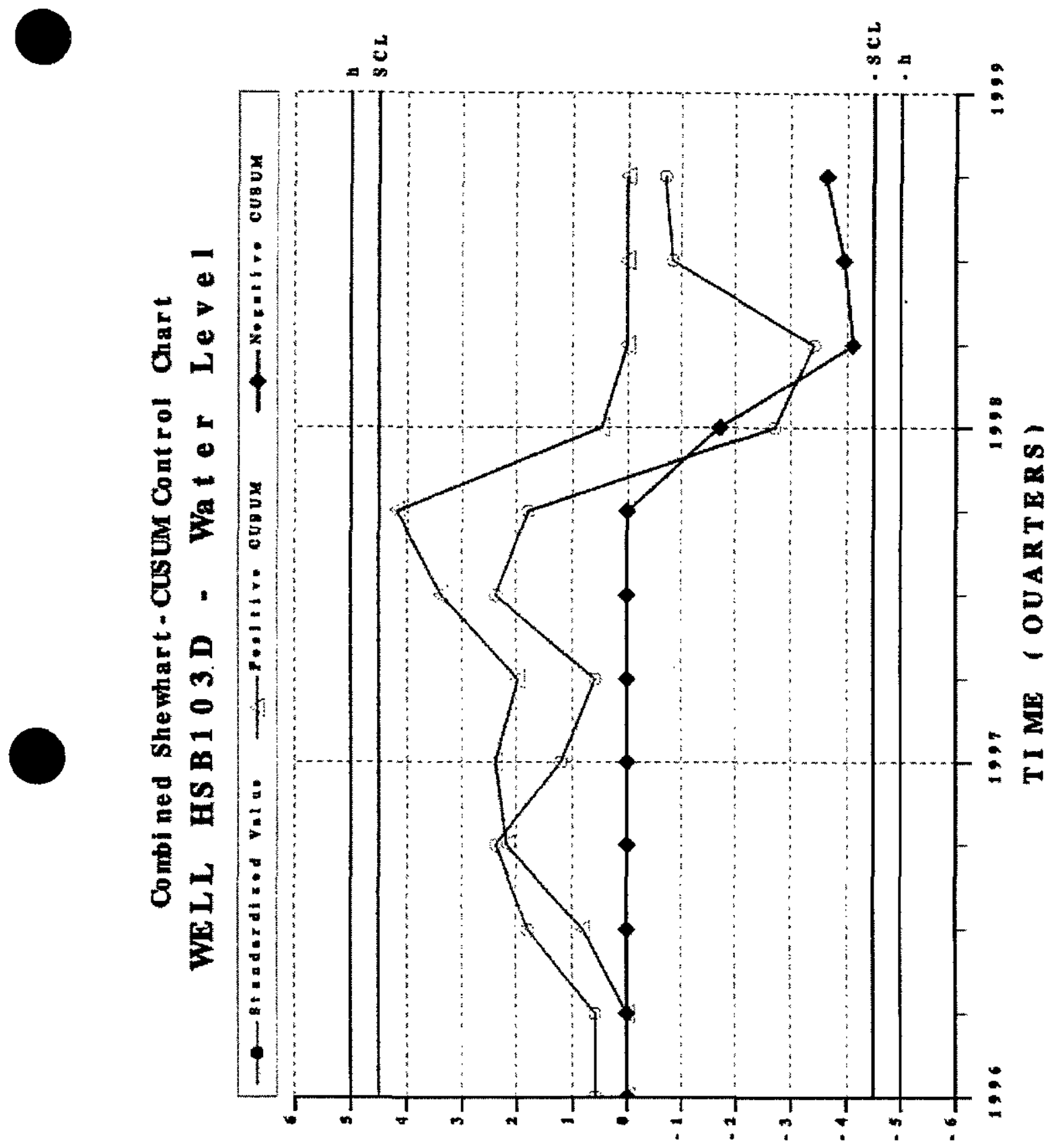

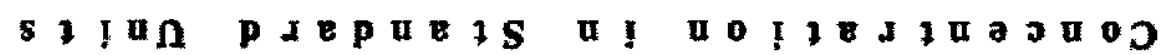


Combi ned Shewhart - CuSuM Cont rol Chart

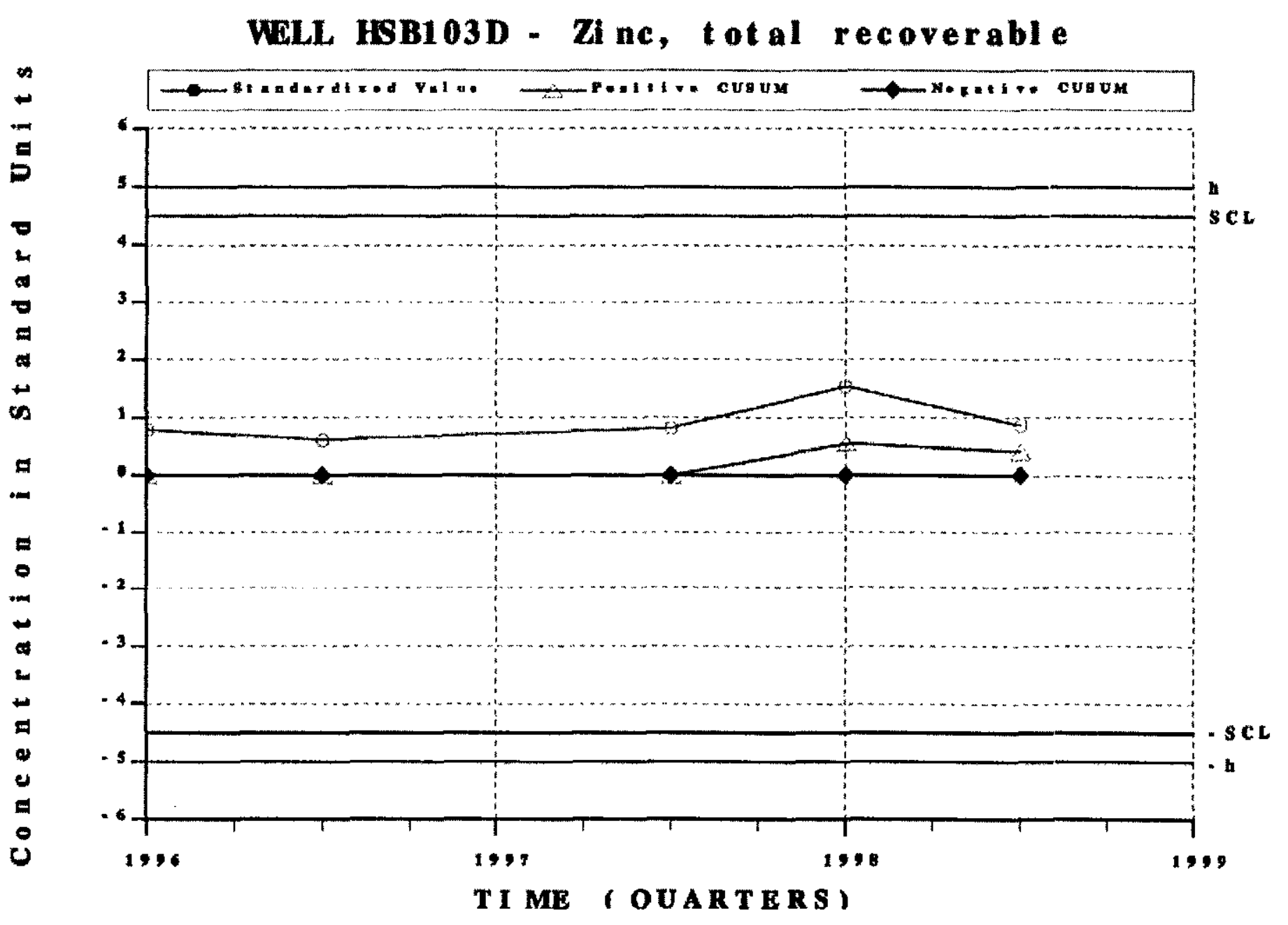

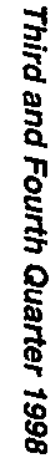

T I ME (OUARTERS ) 

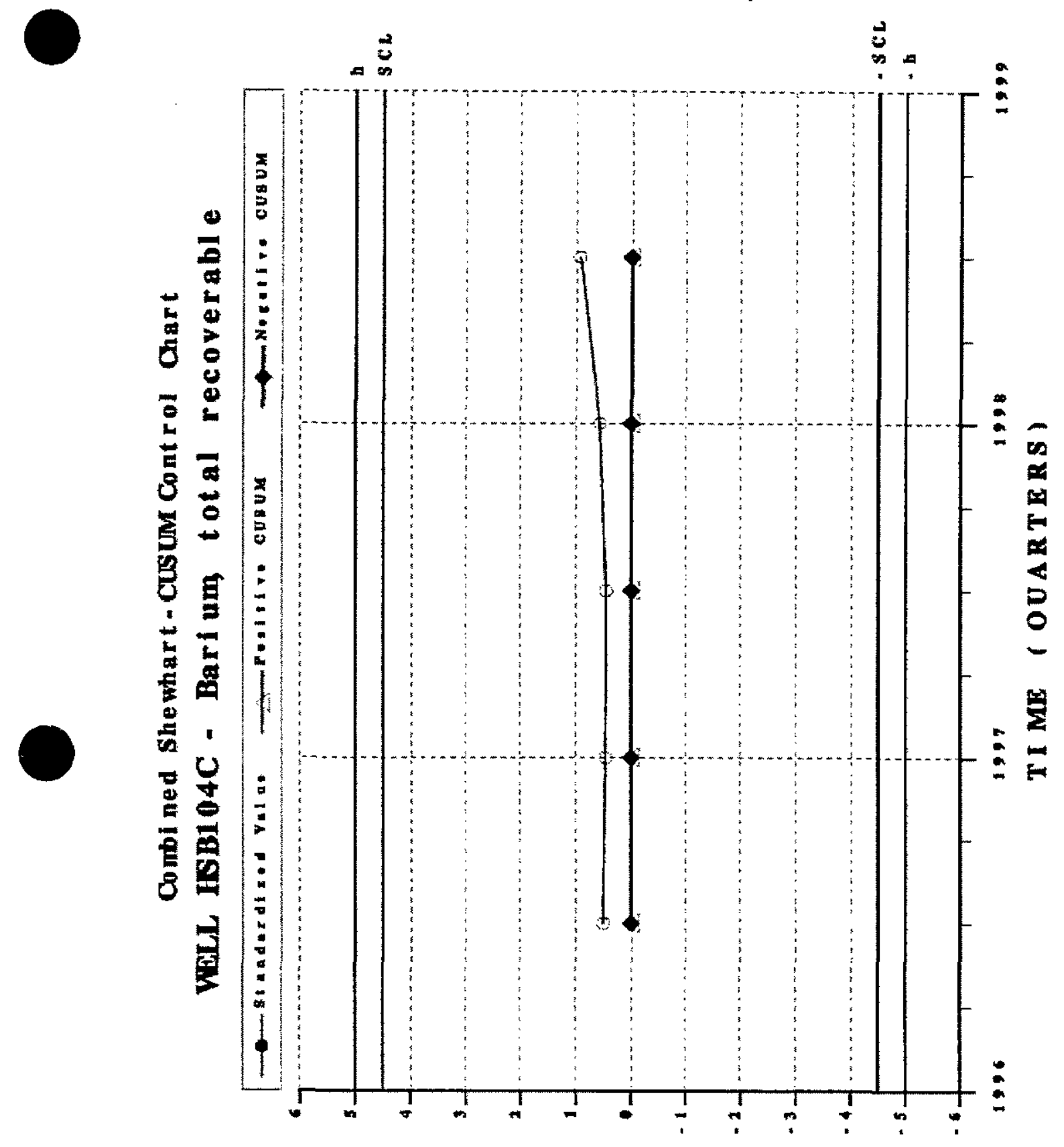

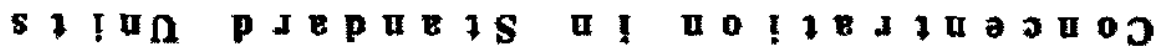




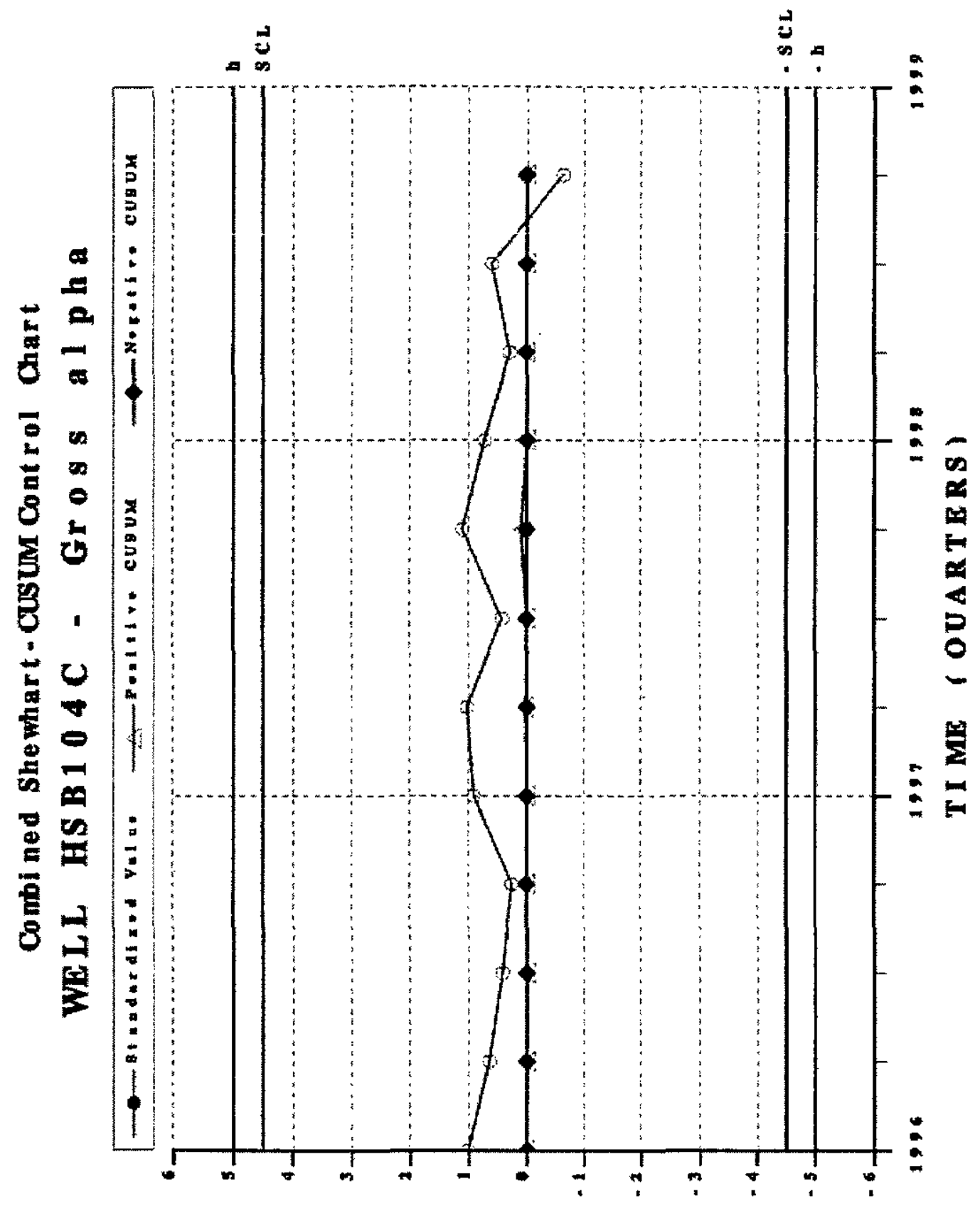

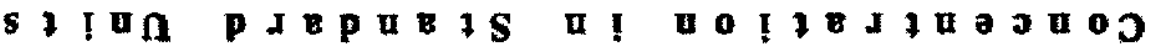


Combi ned Shewhart - CuSUM Cont rol Chart

WoIX HSB104C - Nitrate-nitrite as nitrogen

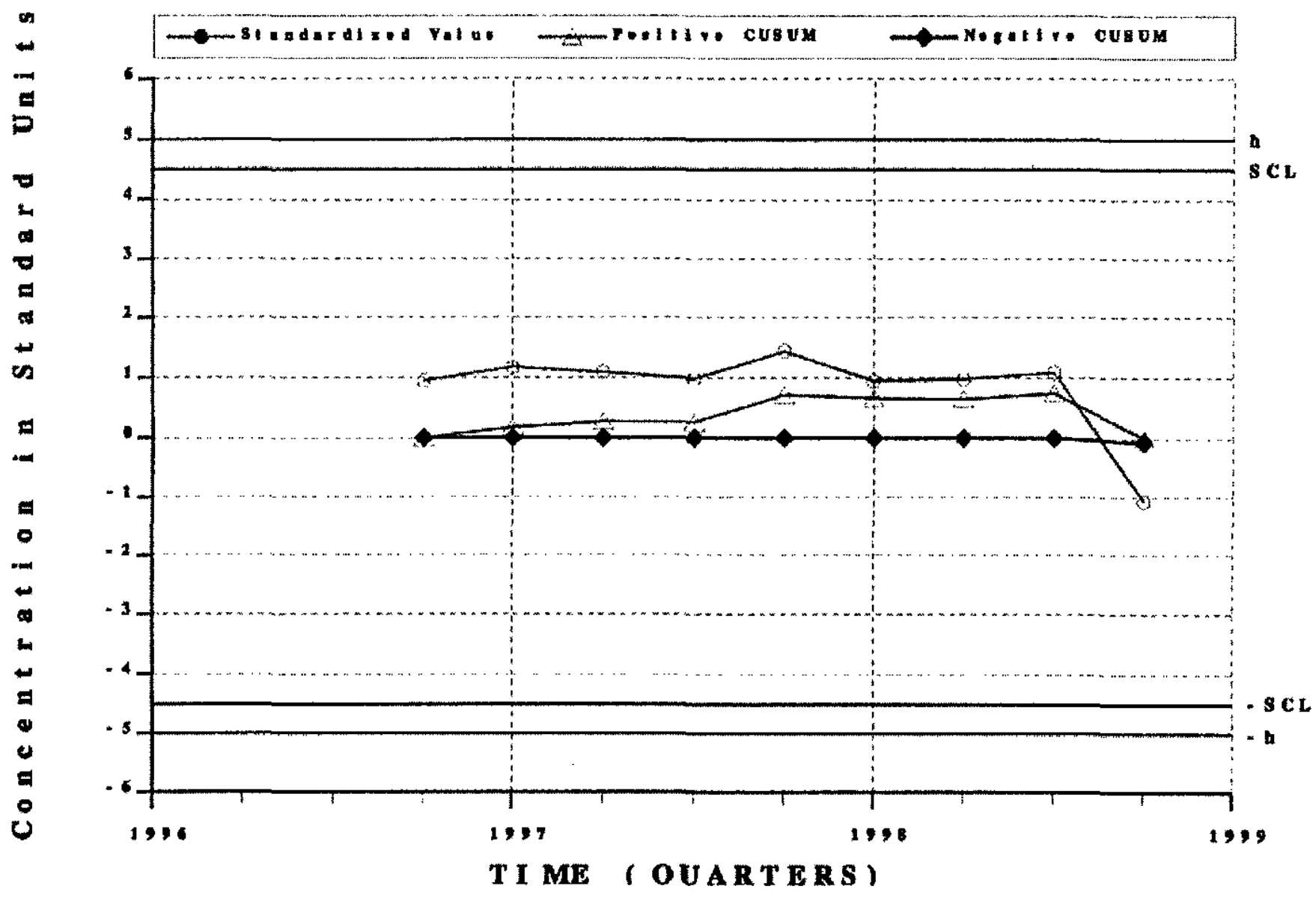




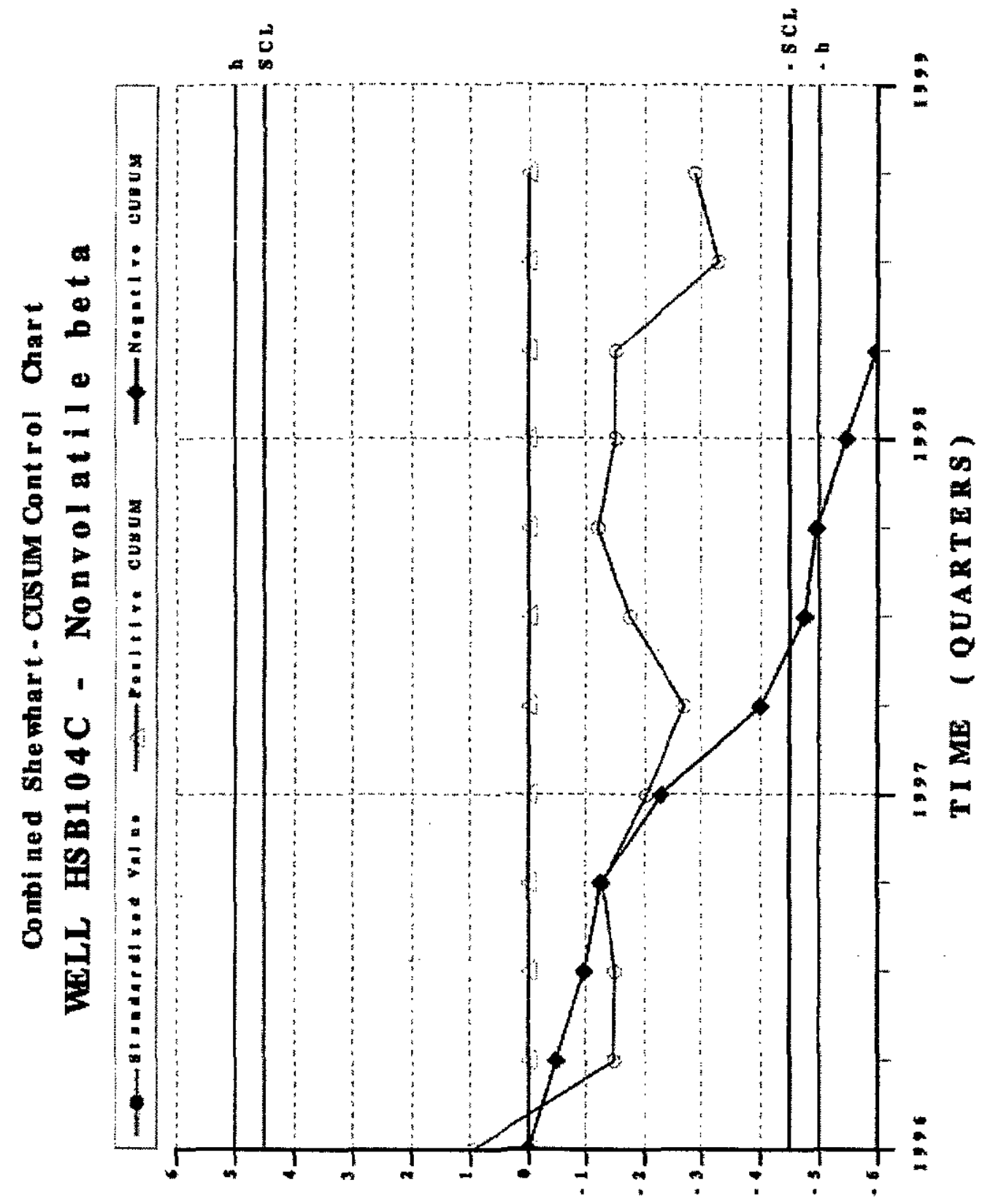

s I I 

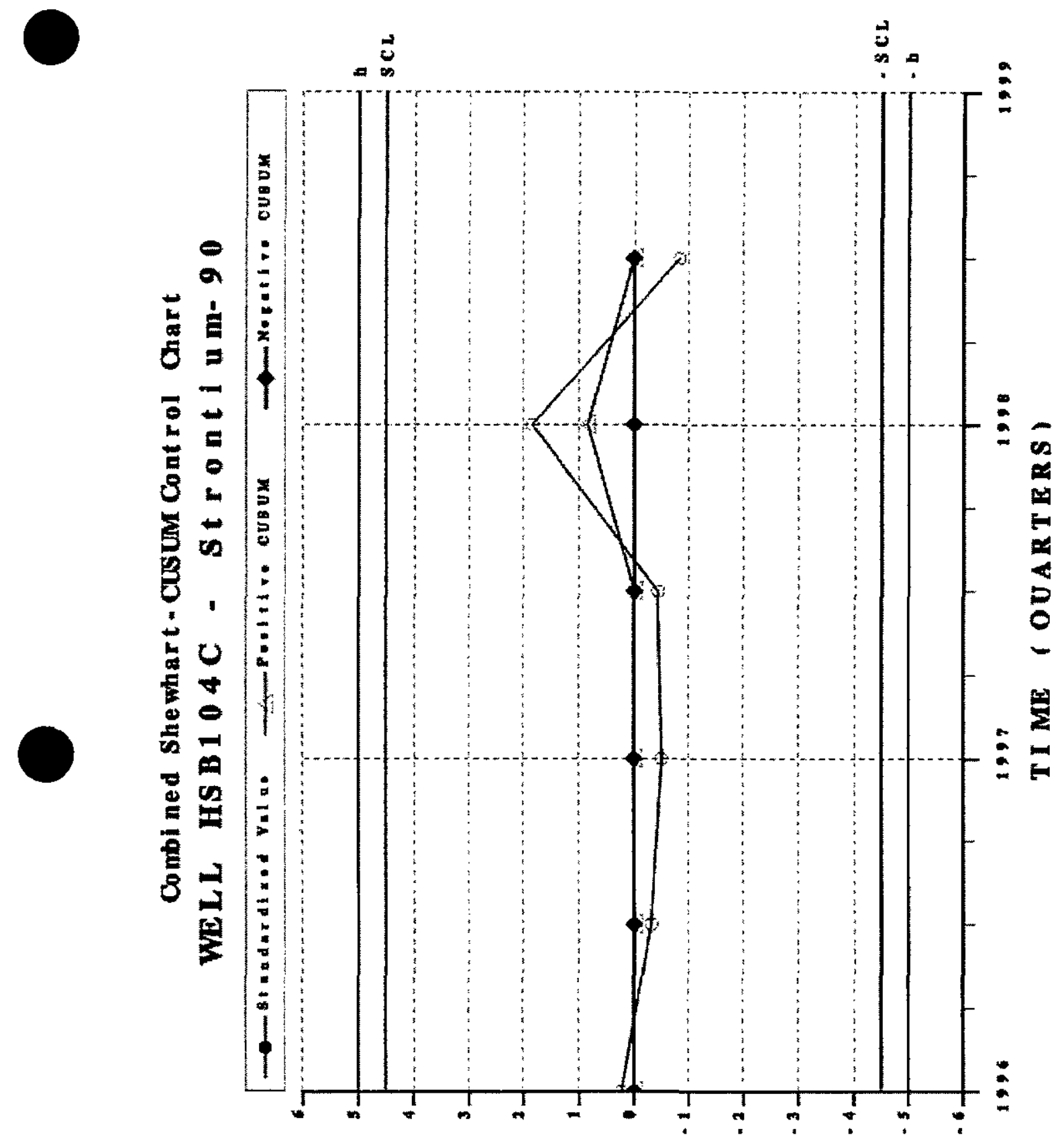

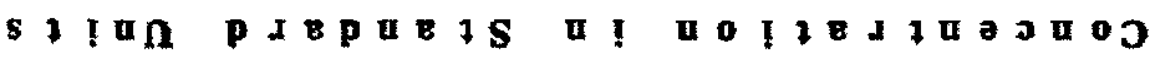




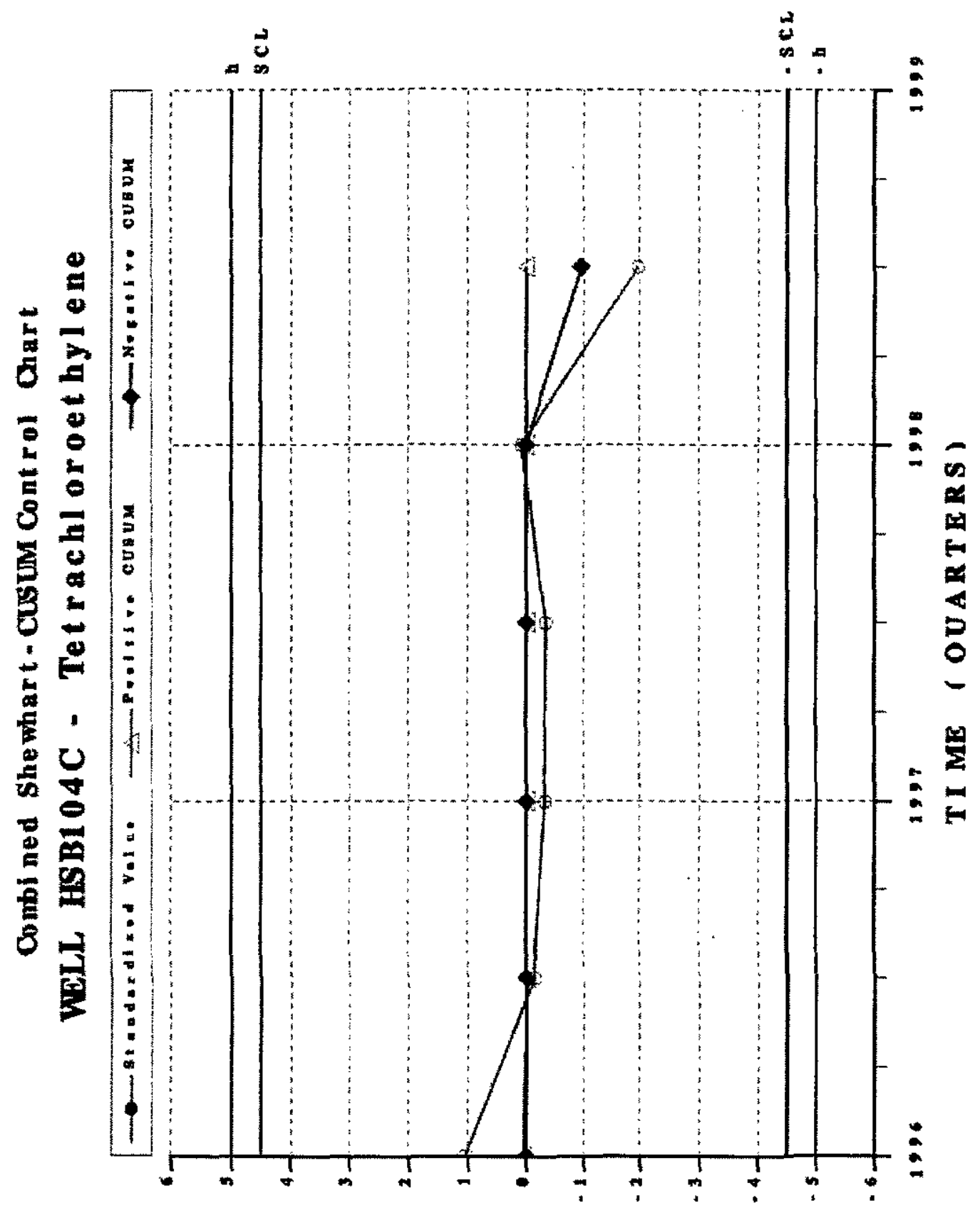

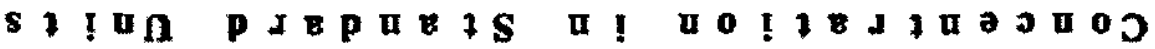



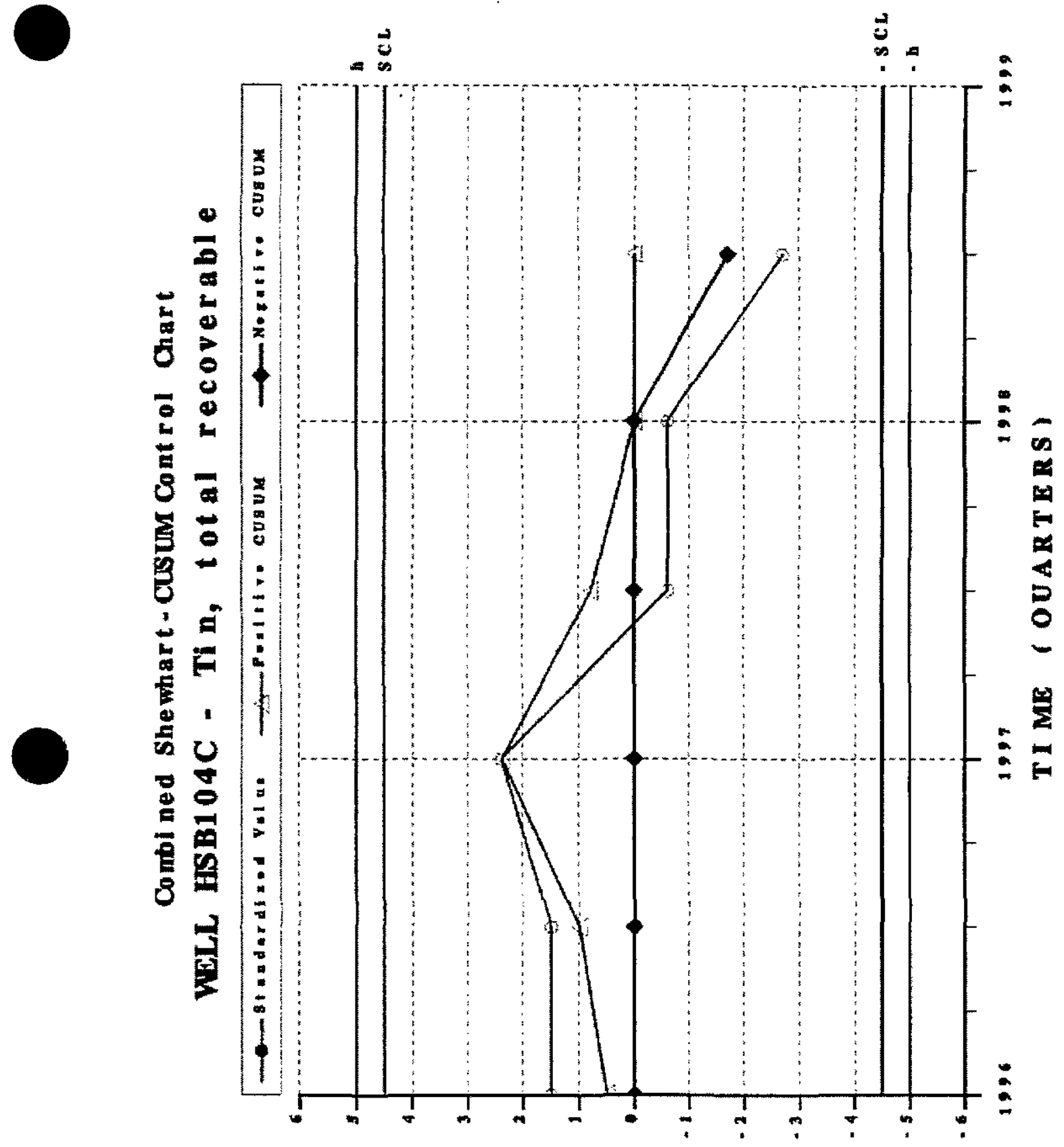

s I 


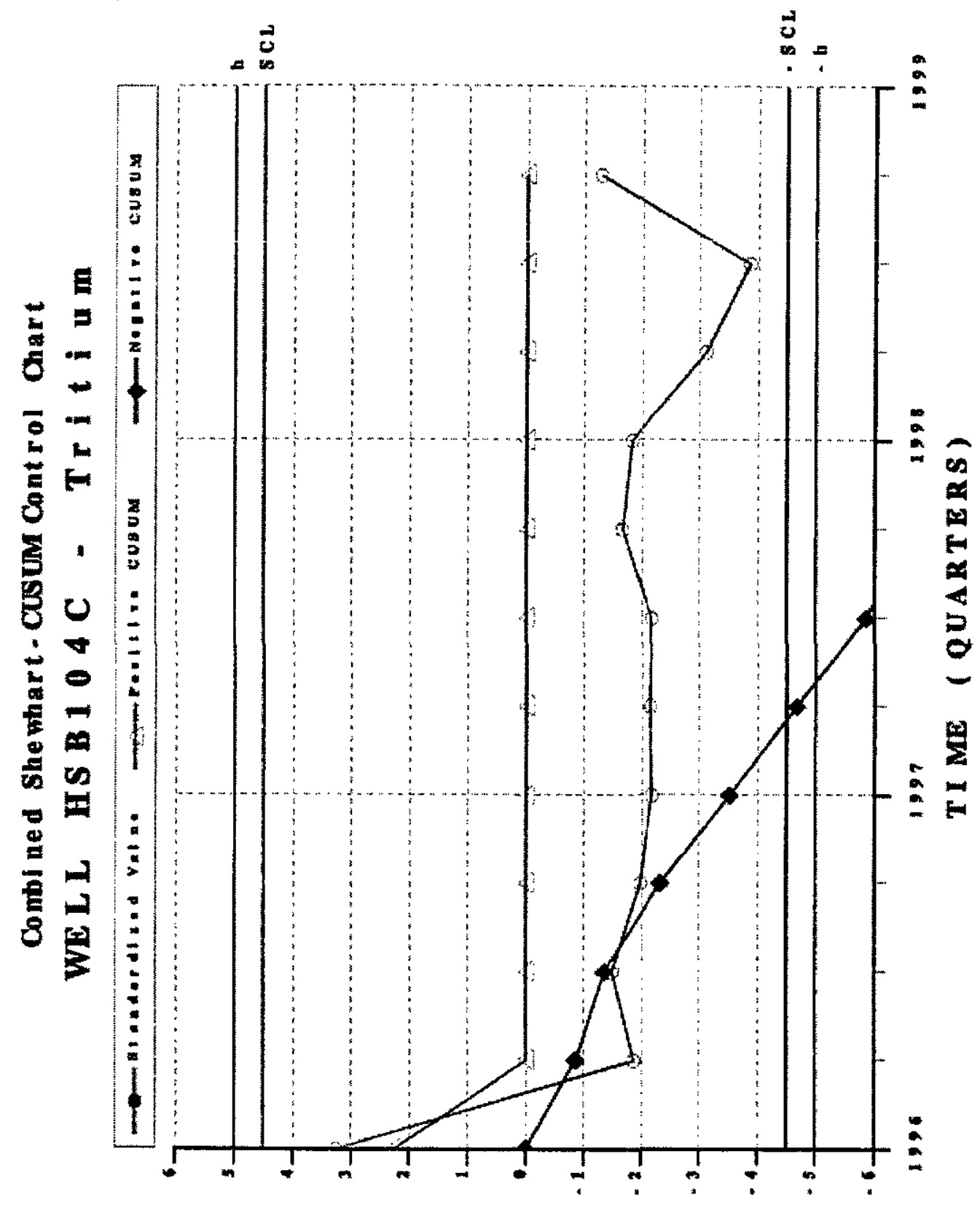

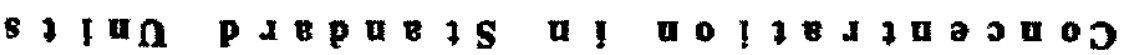



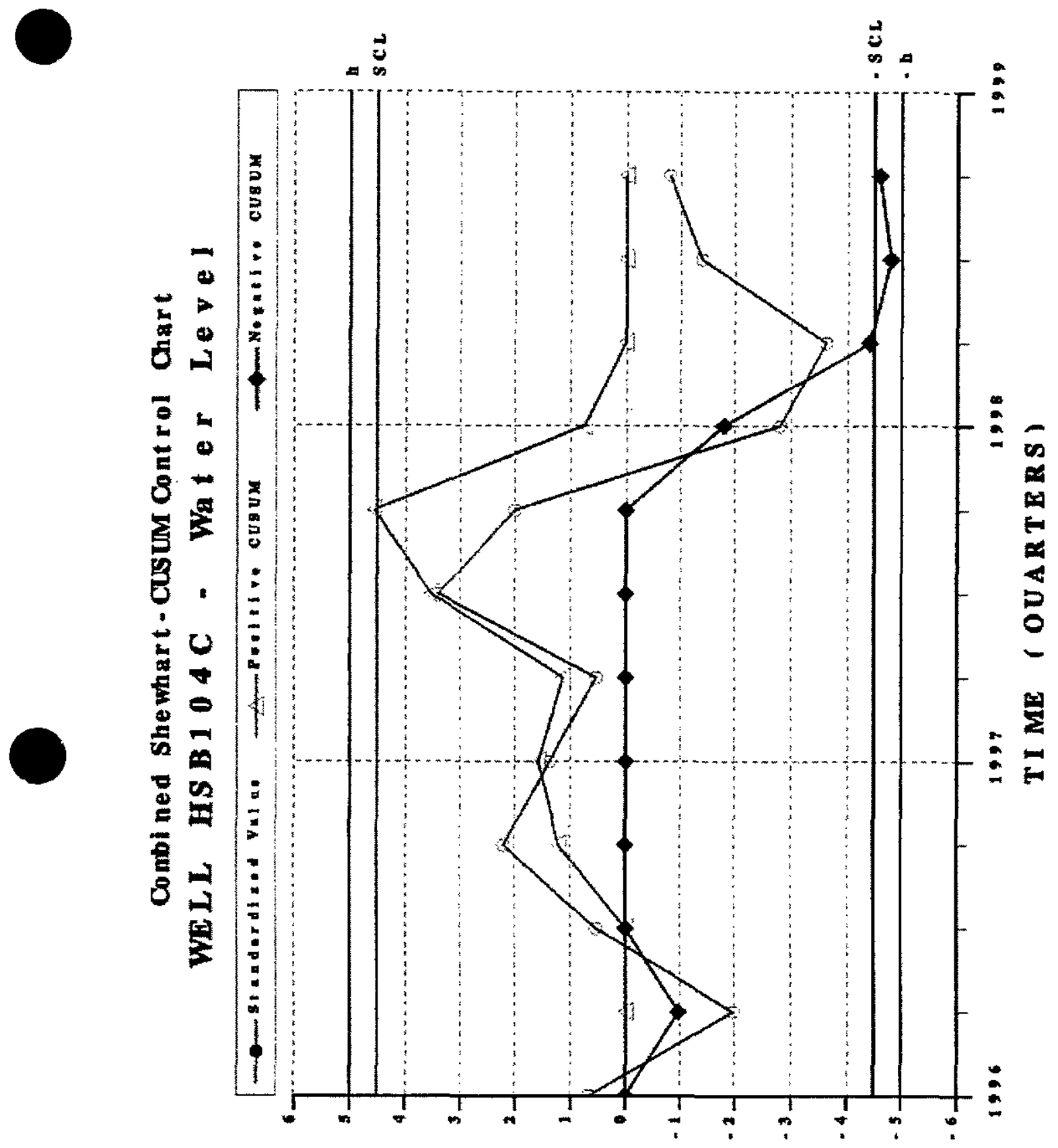

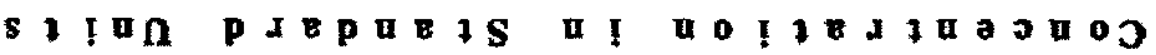


Combi ned She whart-CuSUM Cont rol Chart

WeL HSB104D - Bari um total recoverable

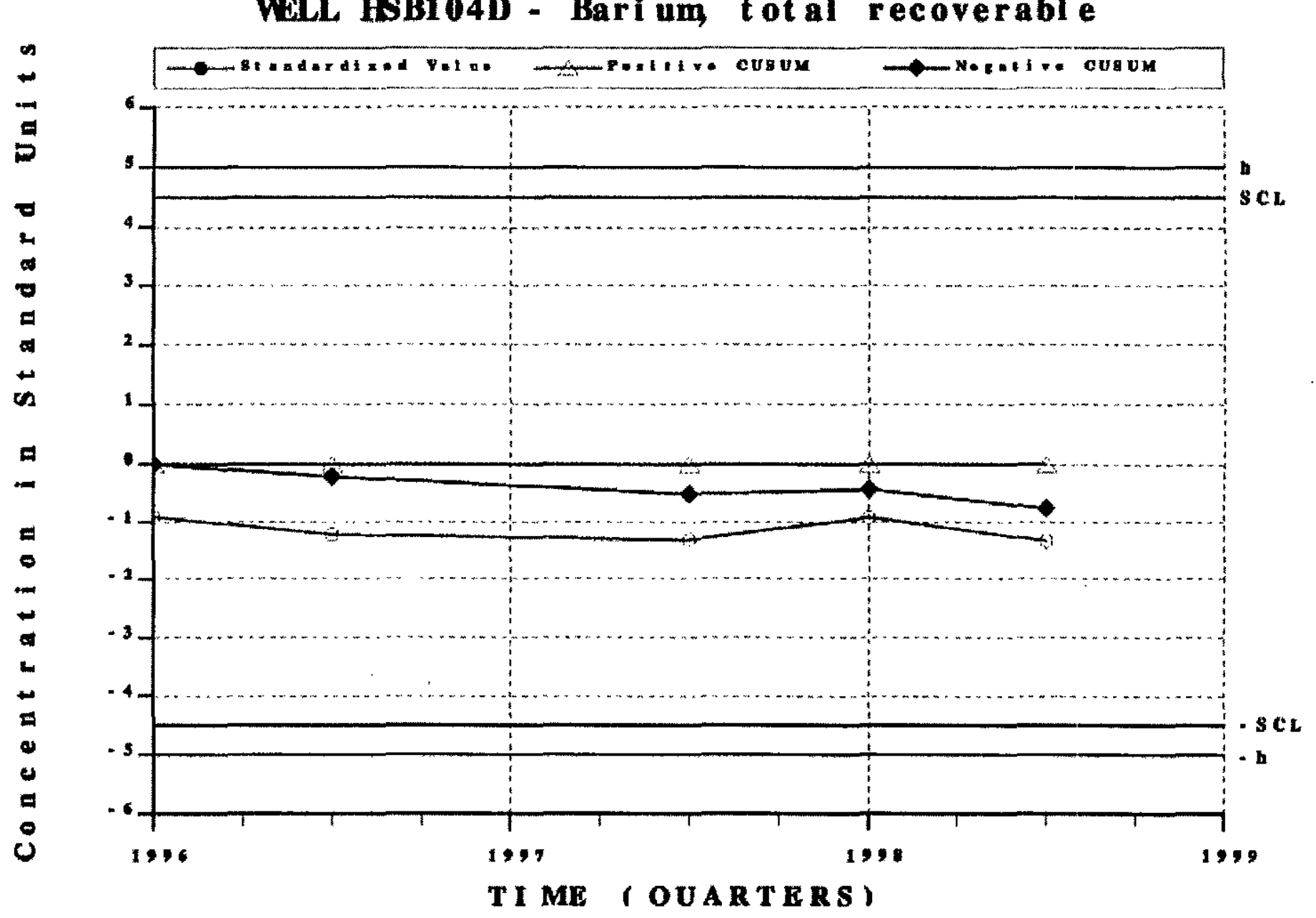

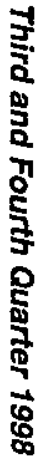



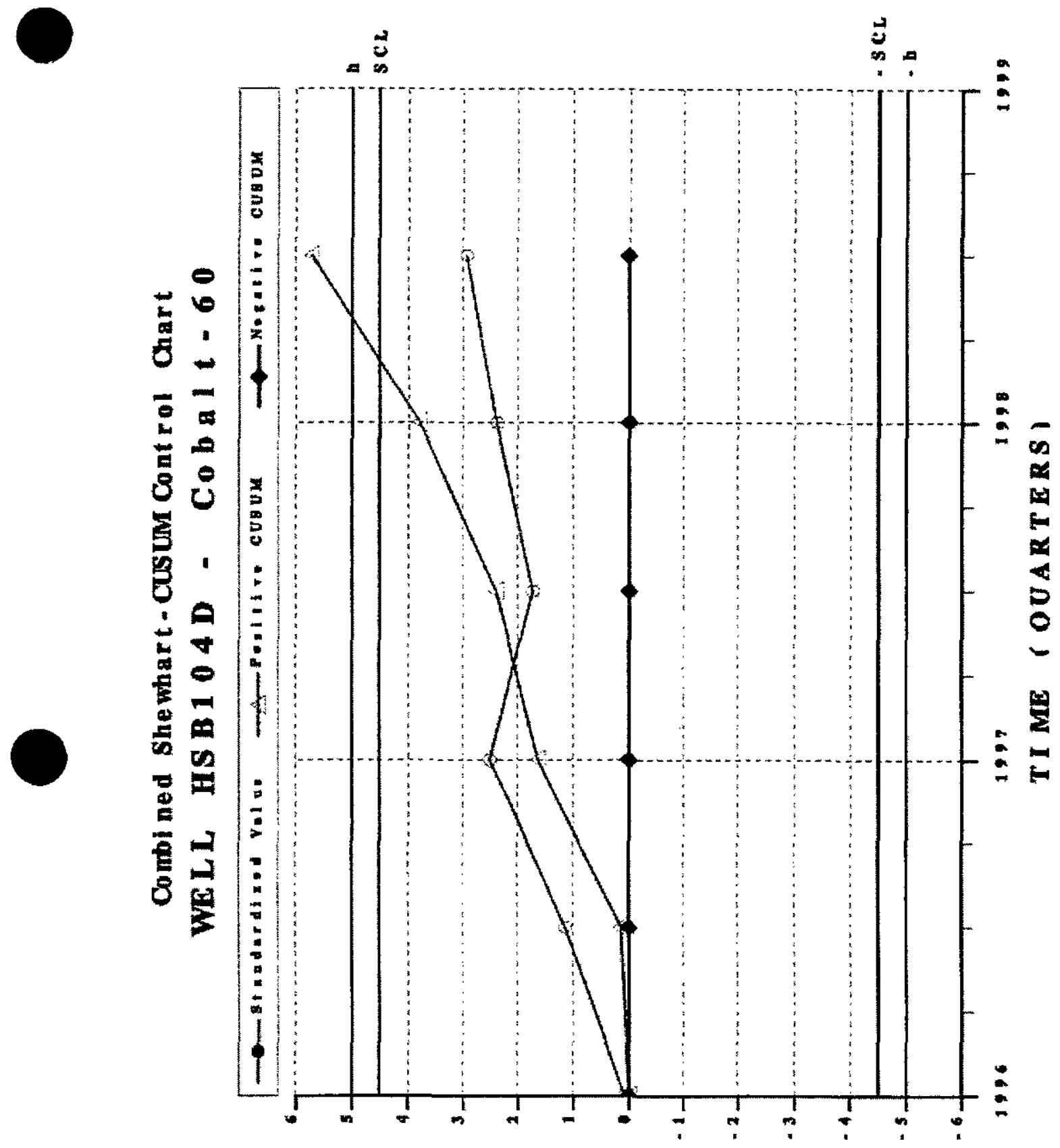

s I I 

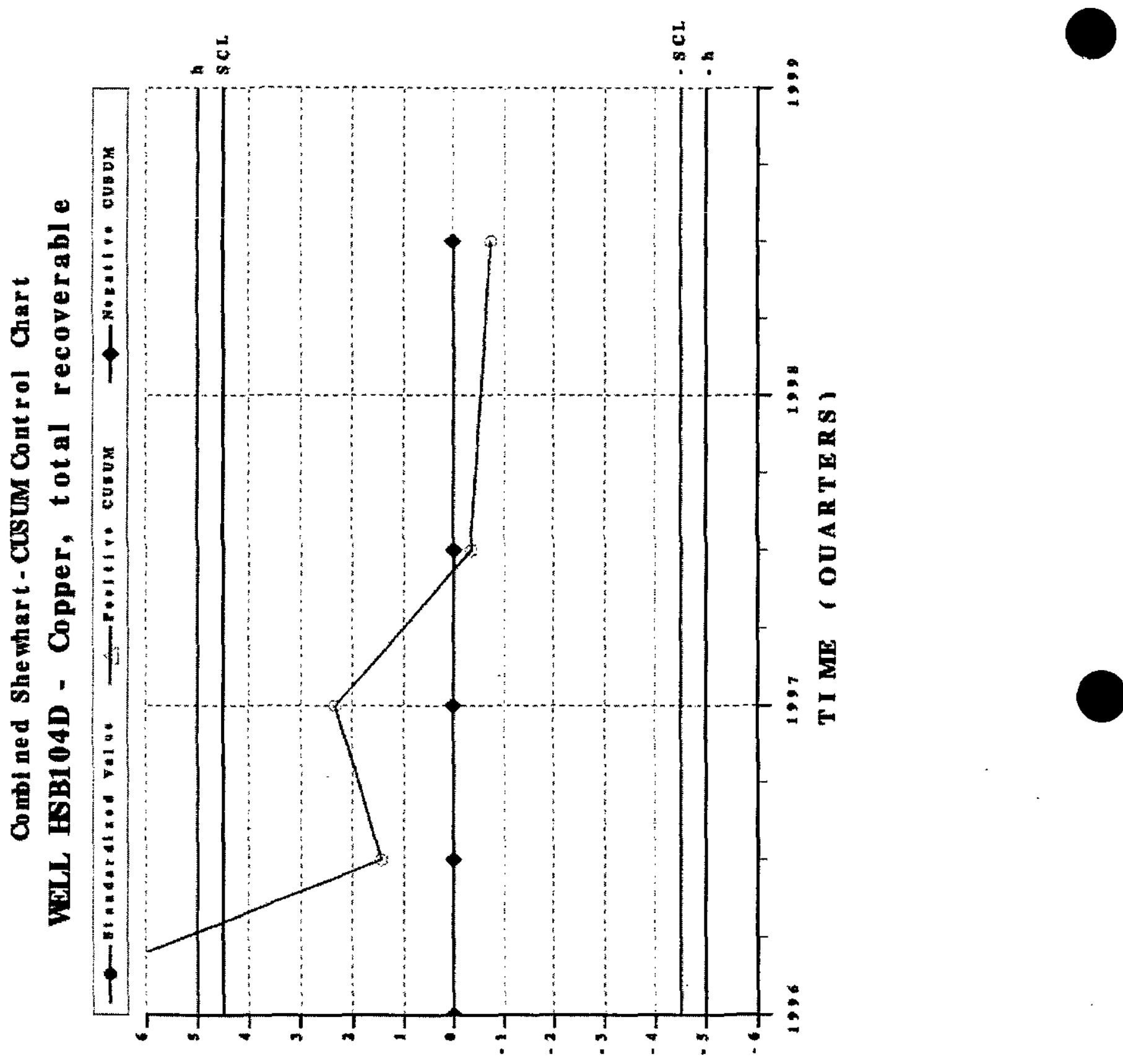
Combi ned She whart - CUSUM Cont rol Chart WELL HSB104D - Gross alpha

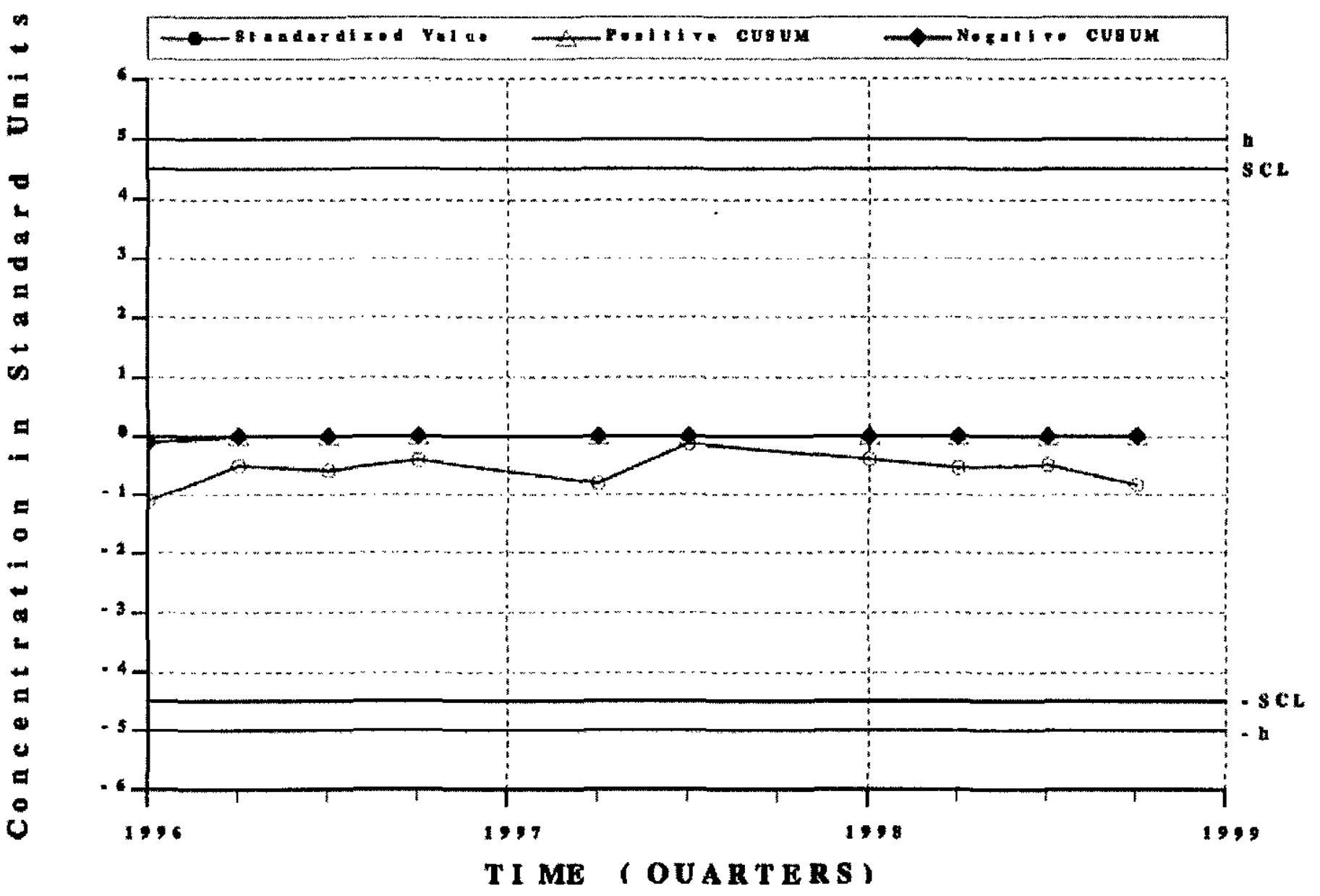




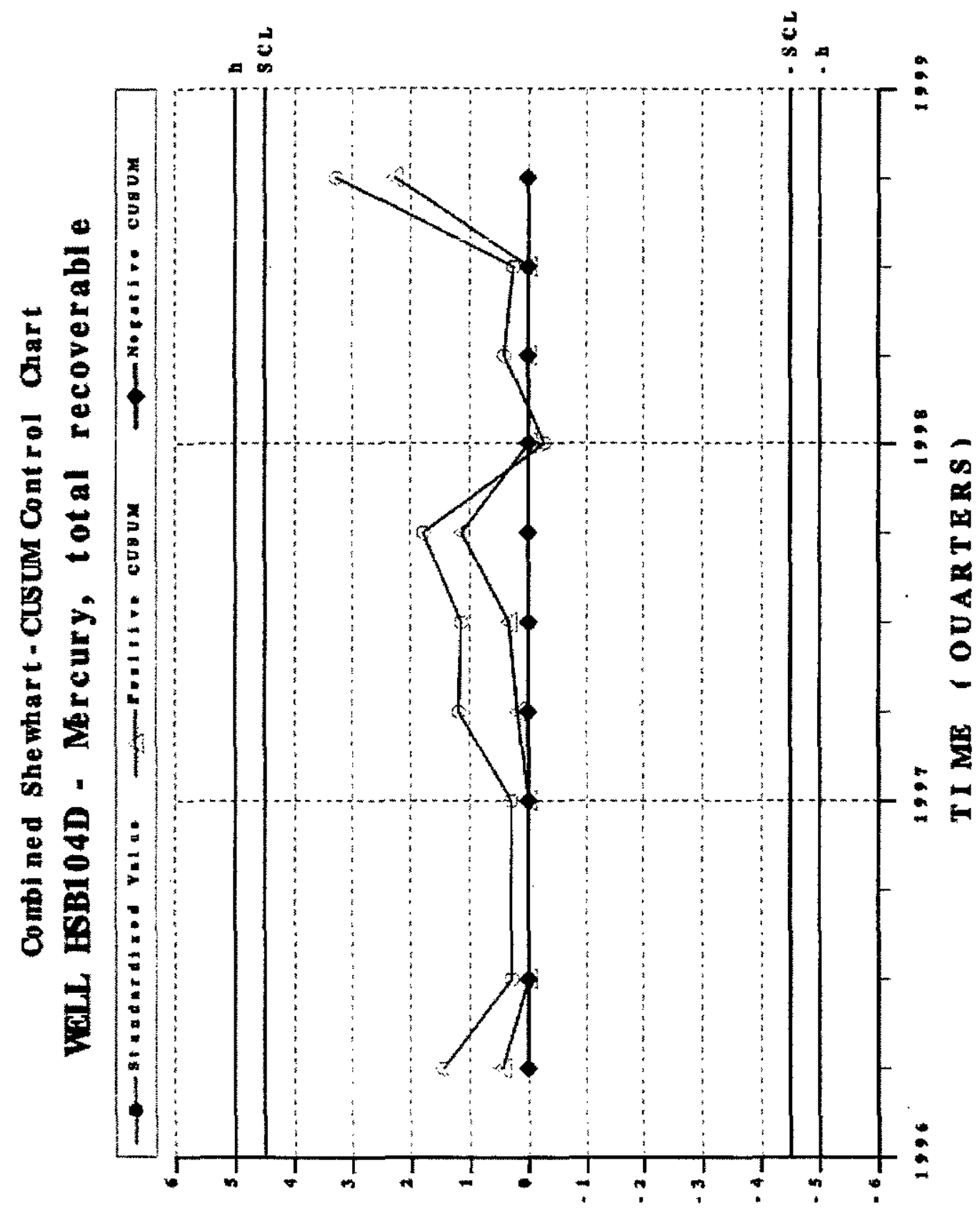

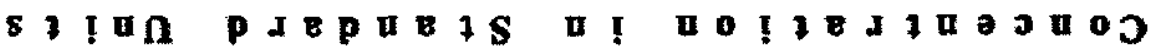



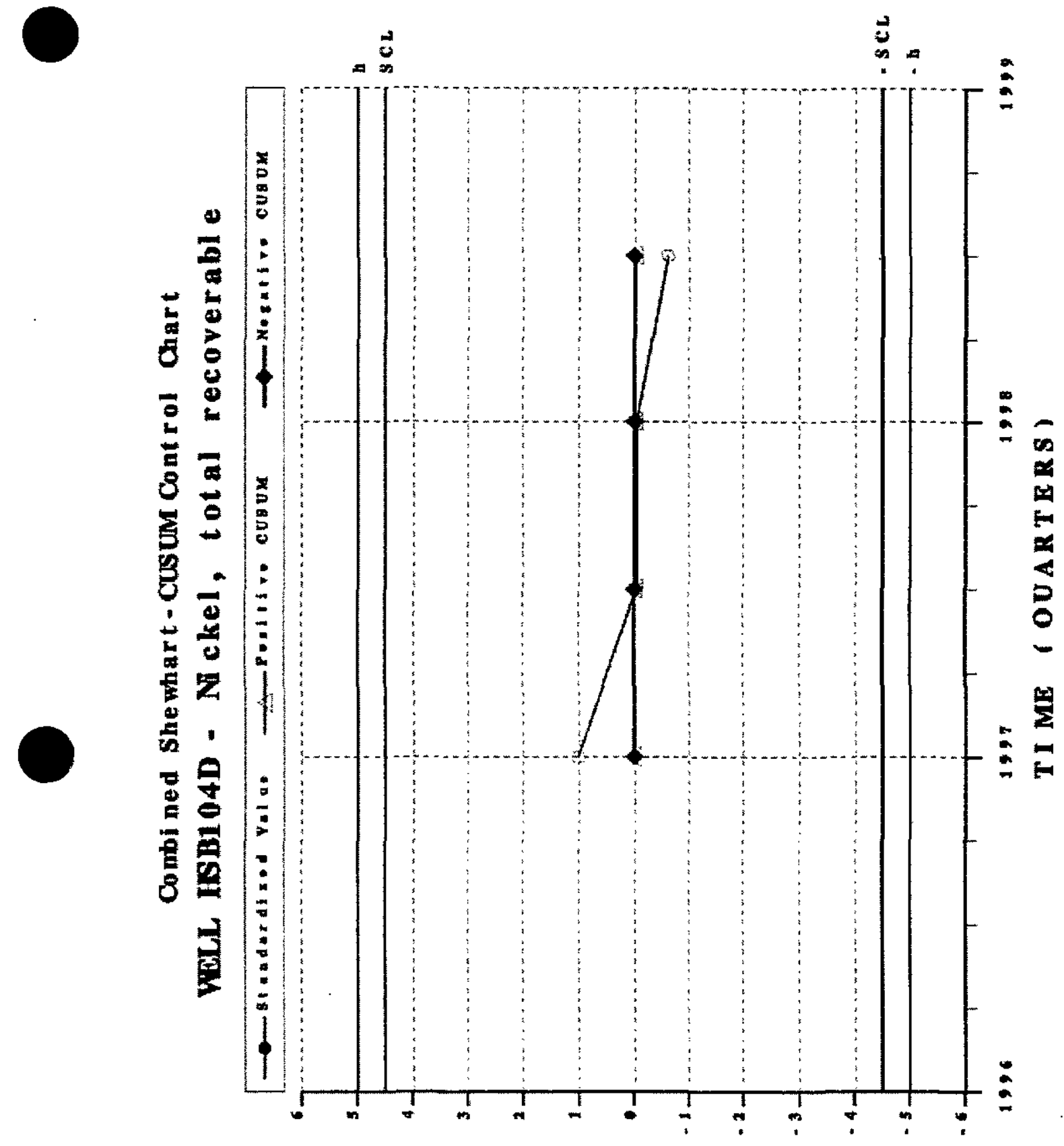

ร) บ 


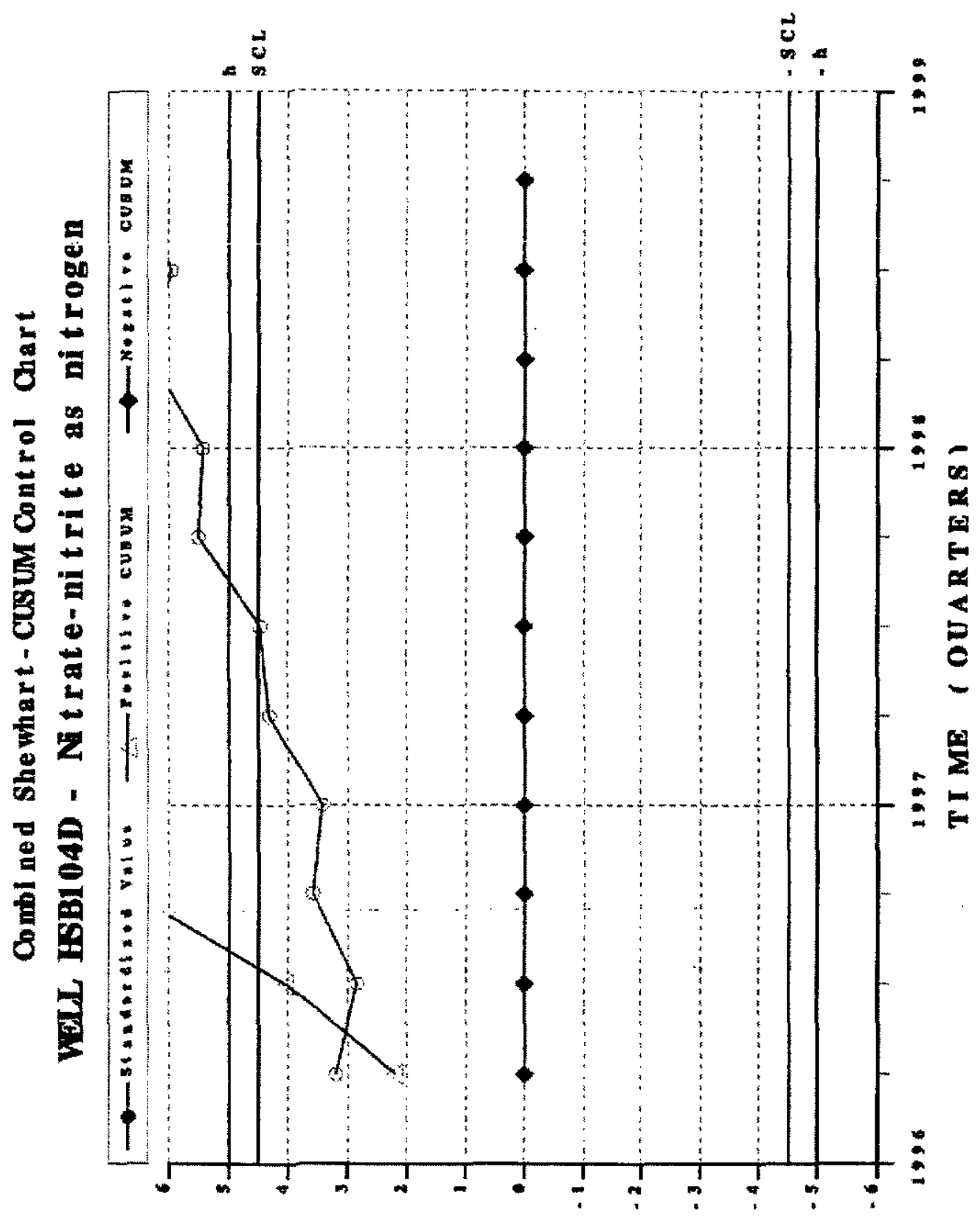

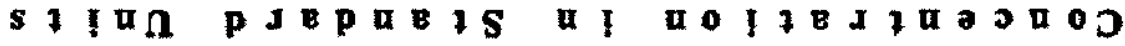


Combi ned Shewhart - Cusum Control Chart

MELL HSB1 $04 \mathrm{D}$ - Nonvolatile beta

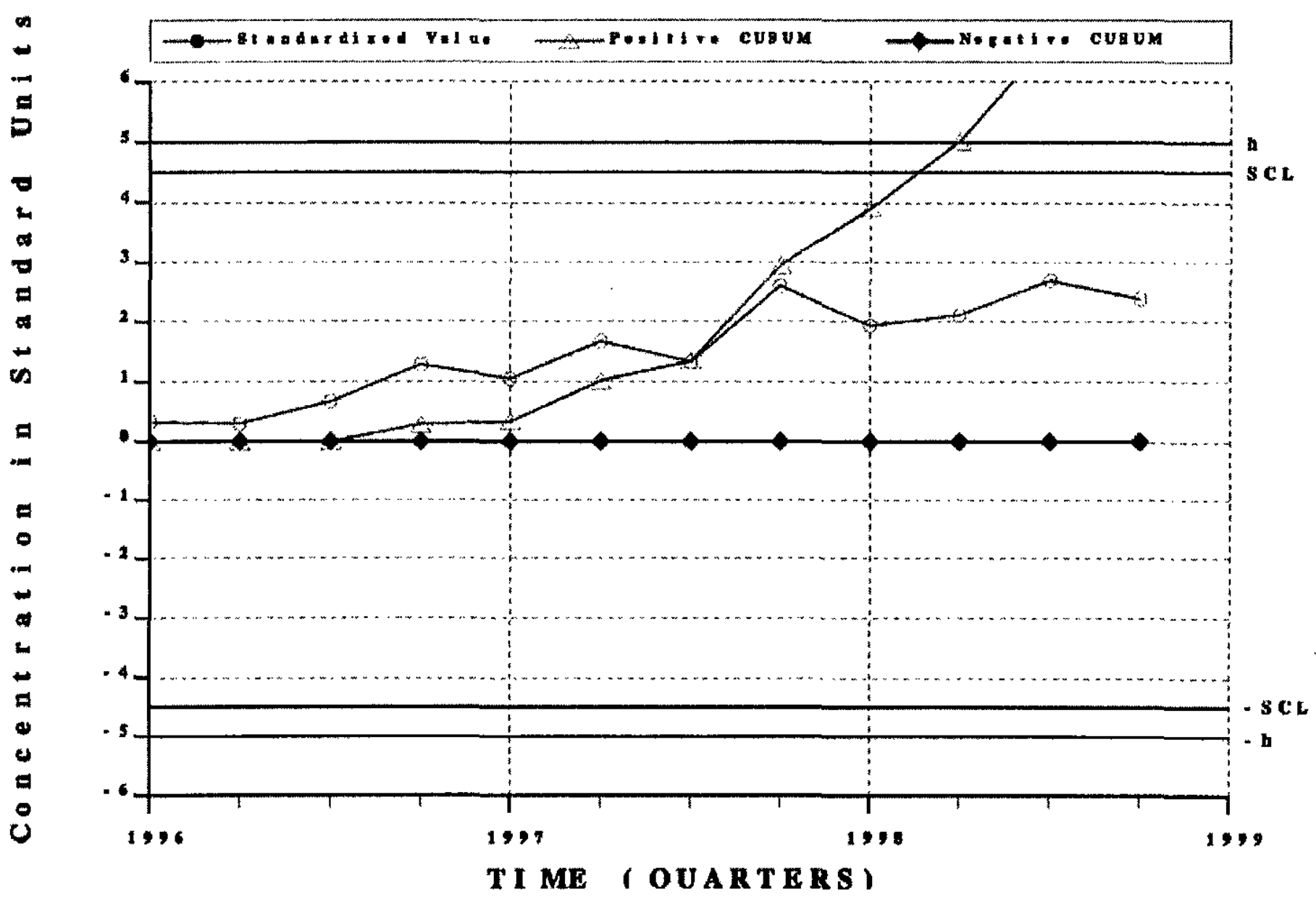

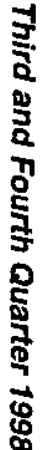


Combi ned Shewhart - Cusum Cont rol Chart

WELL HS B 1040 - Radi um- 226

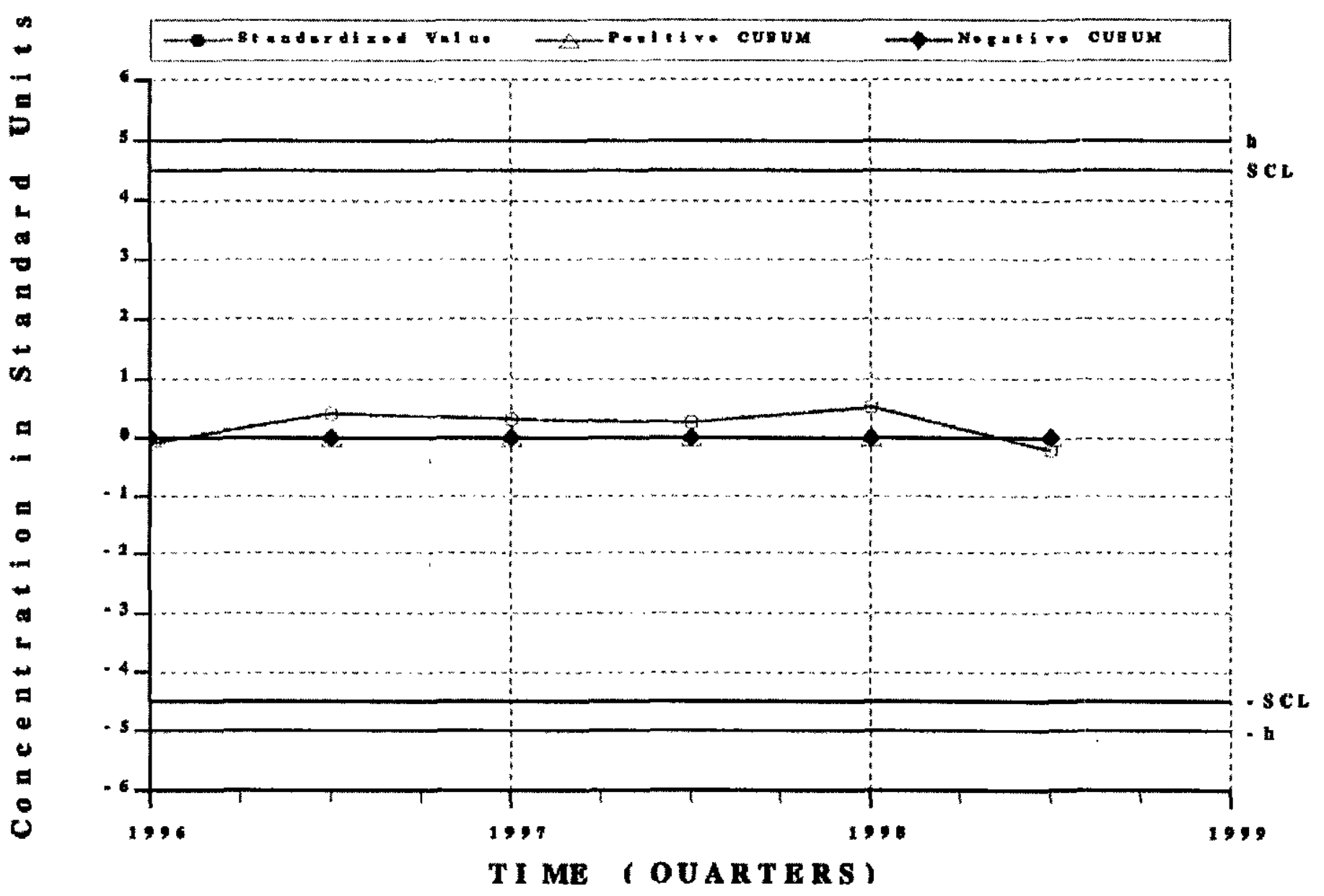

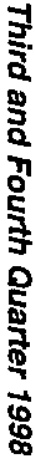



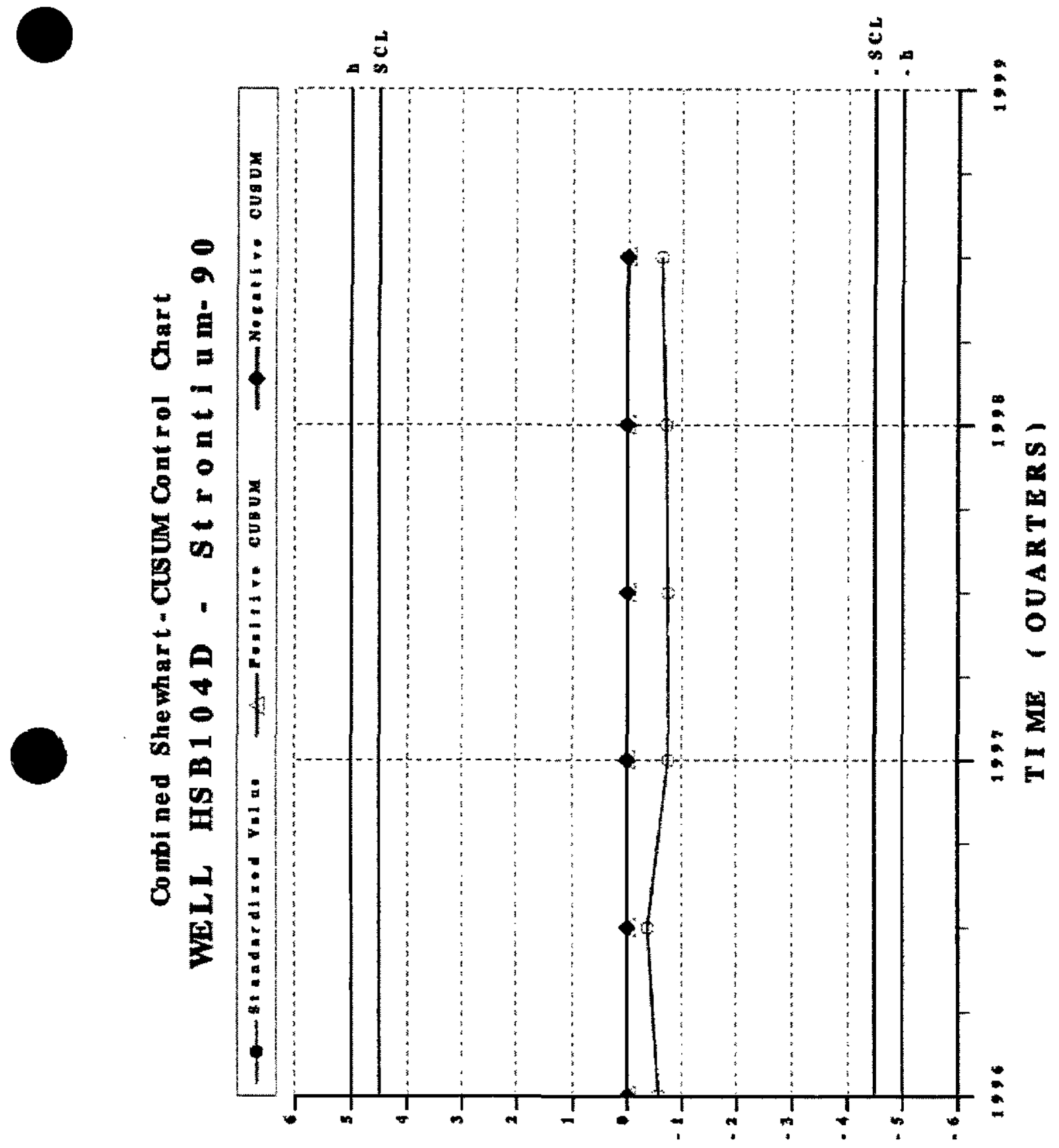

s I $\mathbf{L}$ p 1 E 


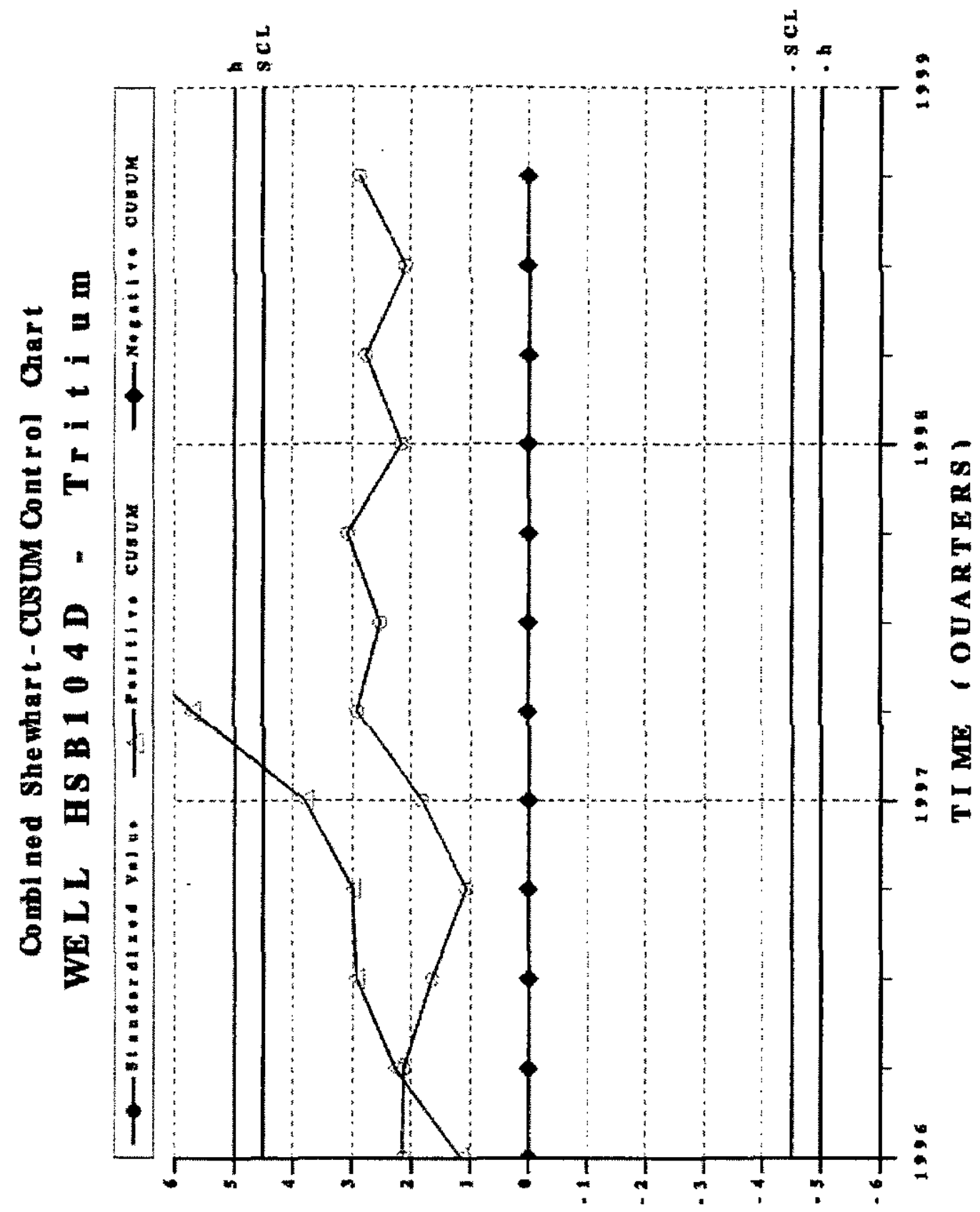

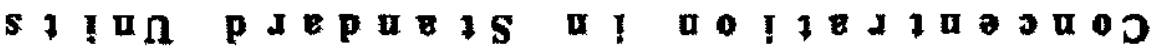



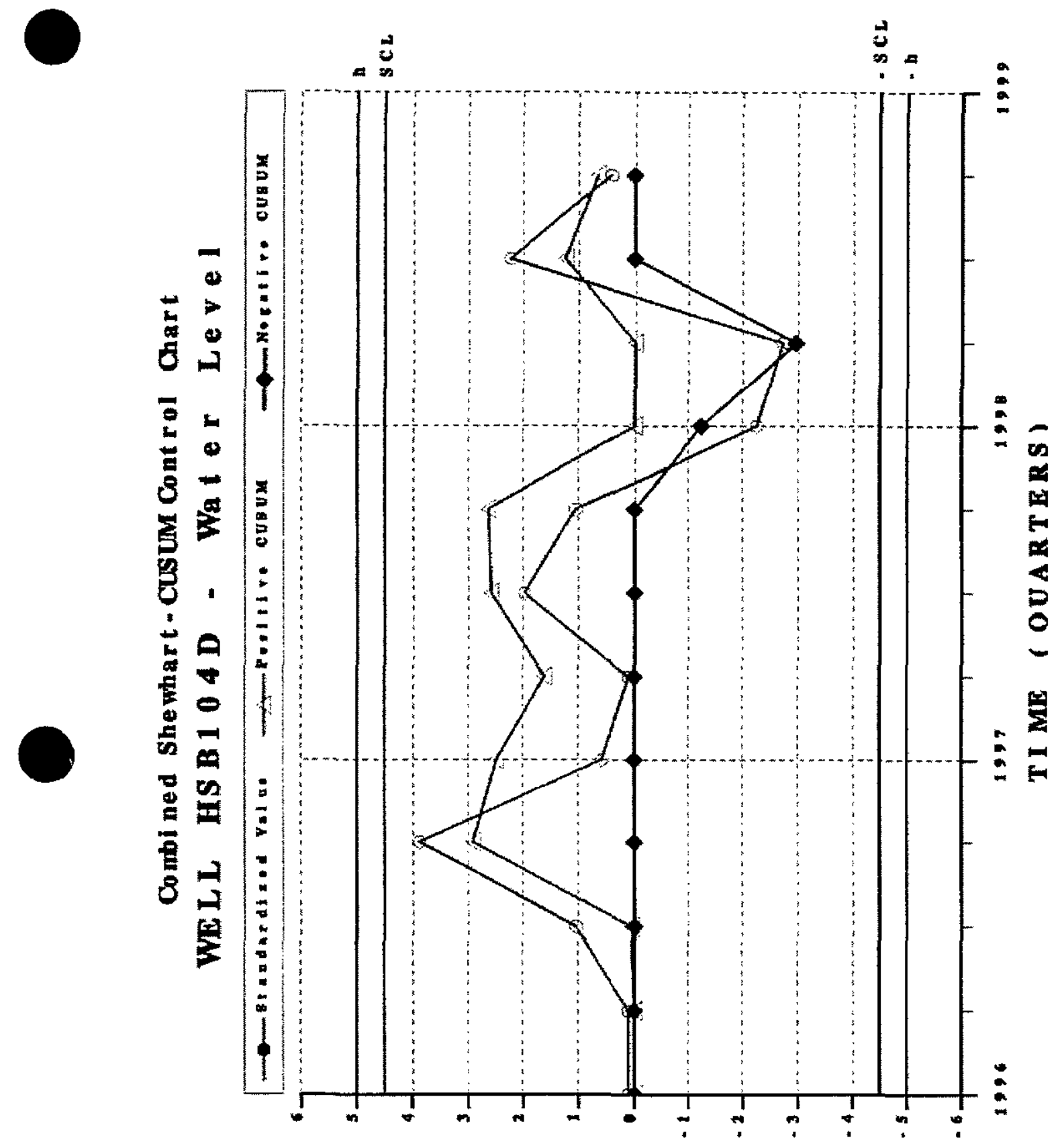

s) I 


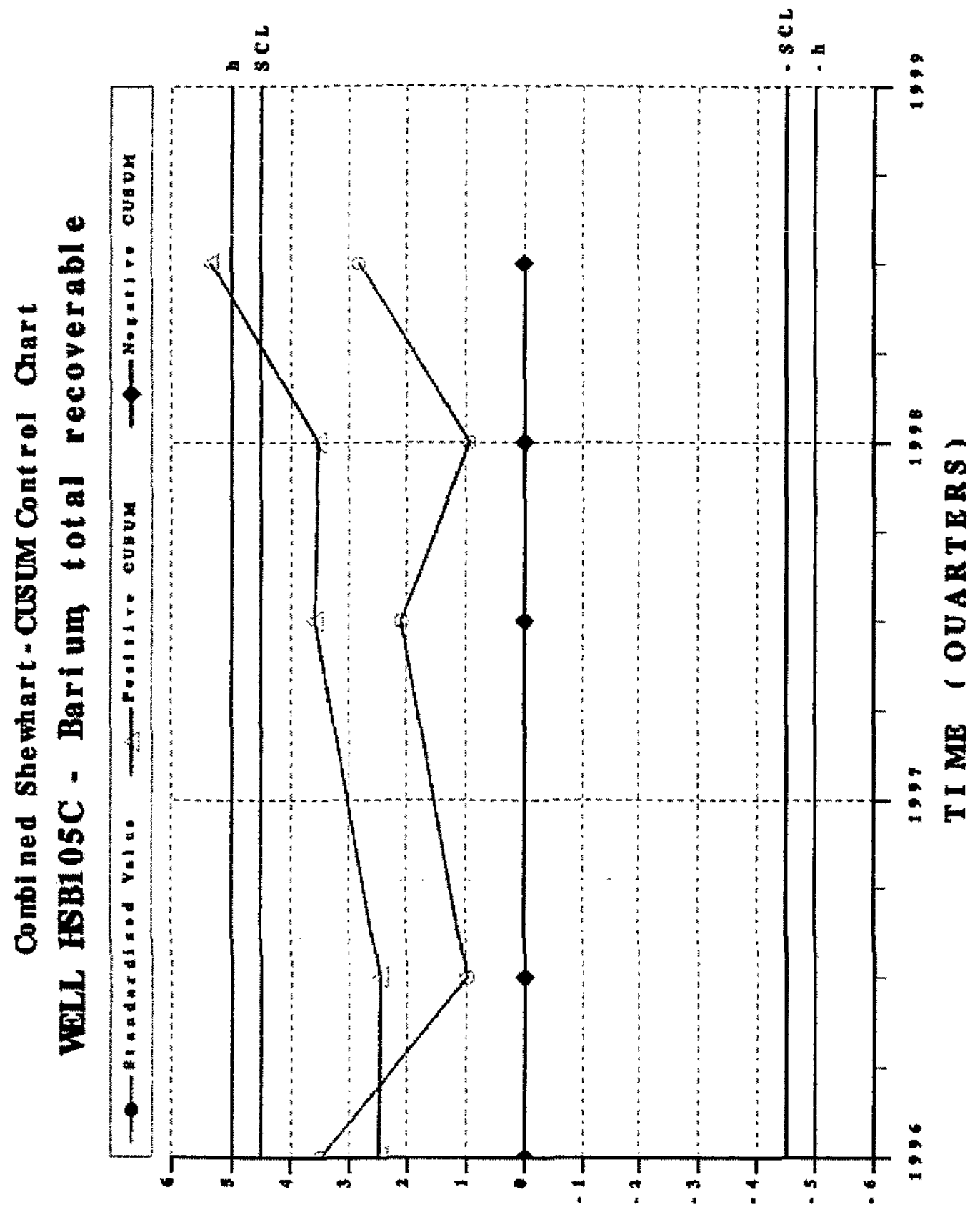

s) โ⿺ 

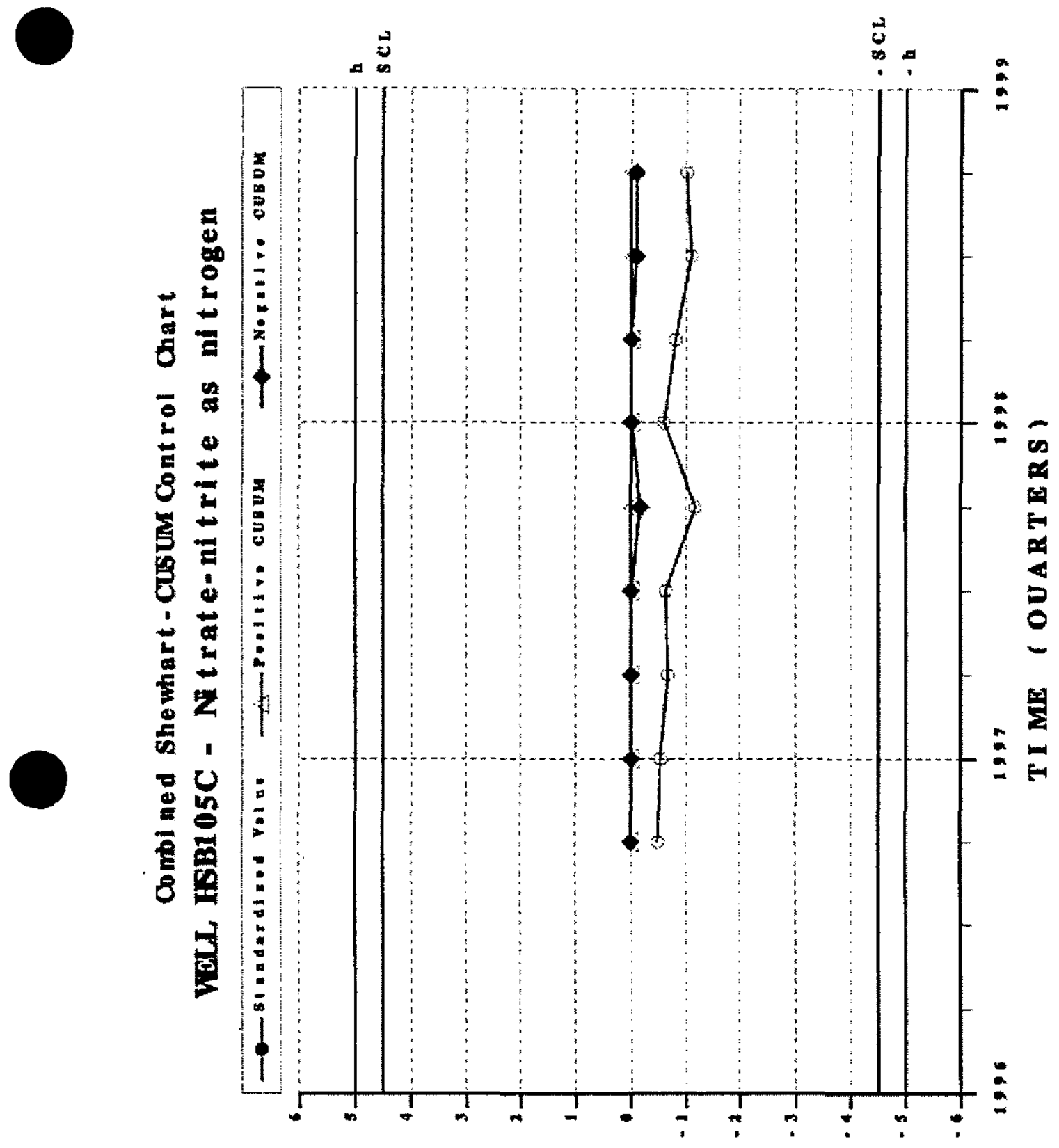

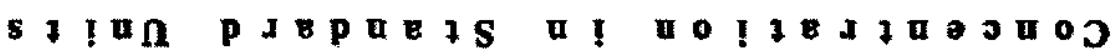




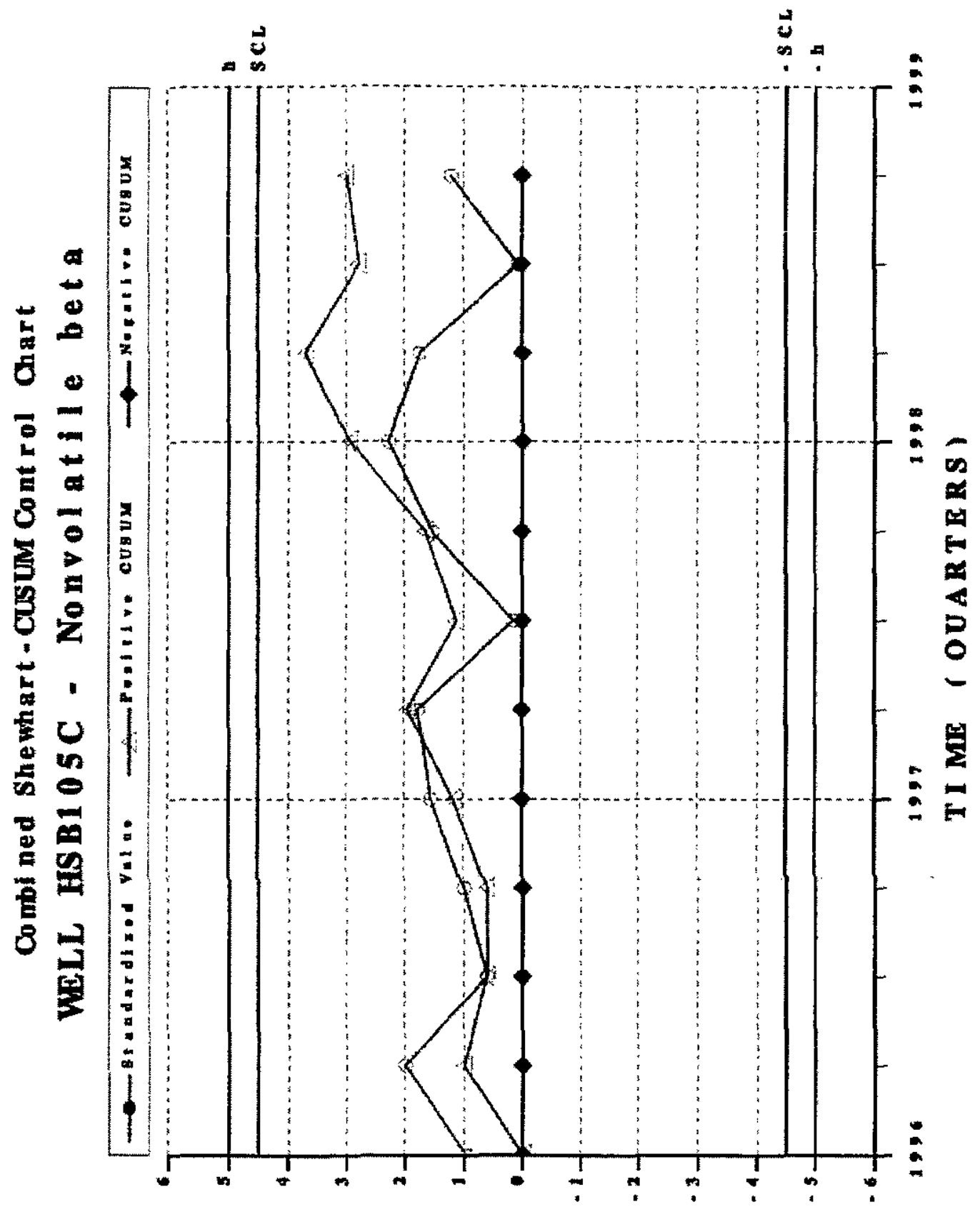

s! ta pa 

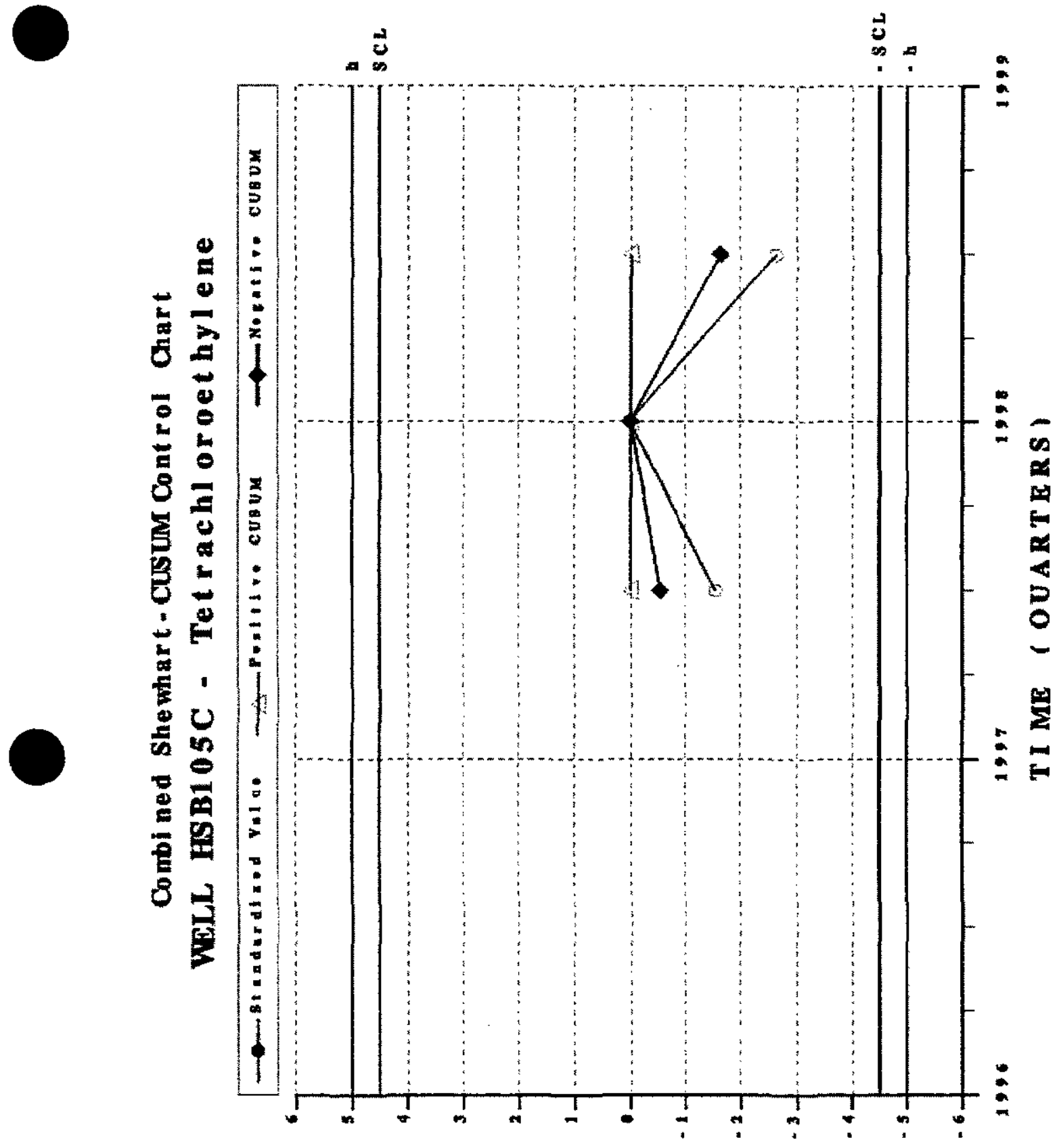

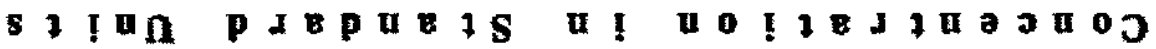




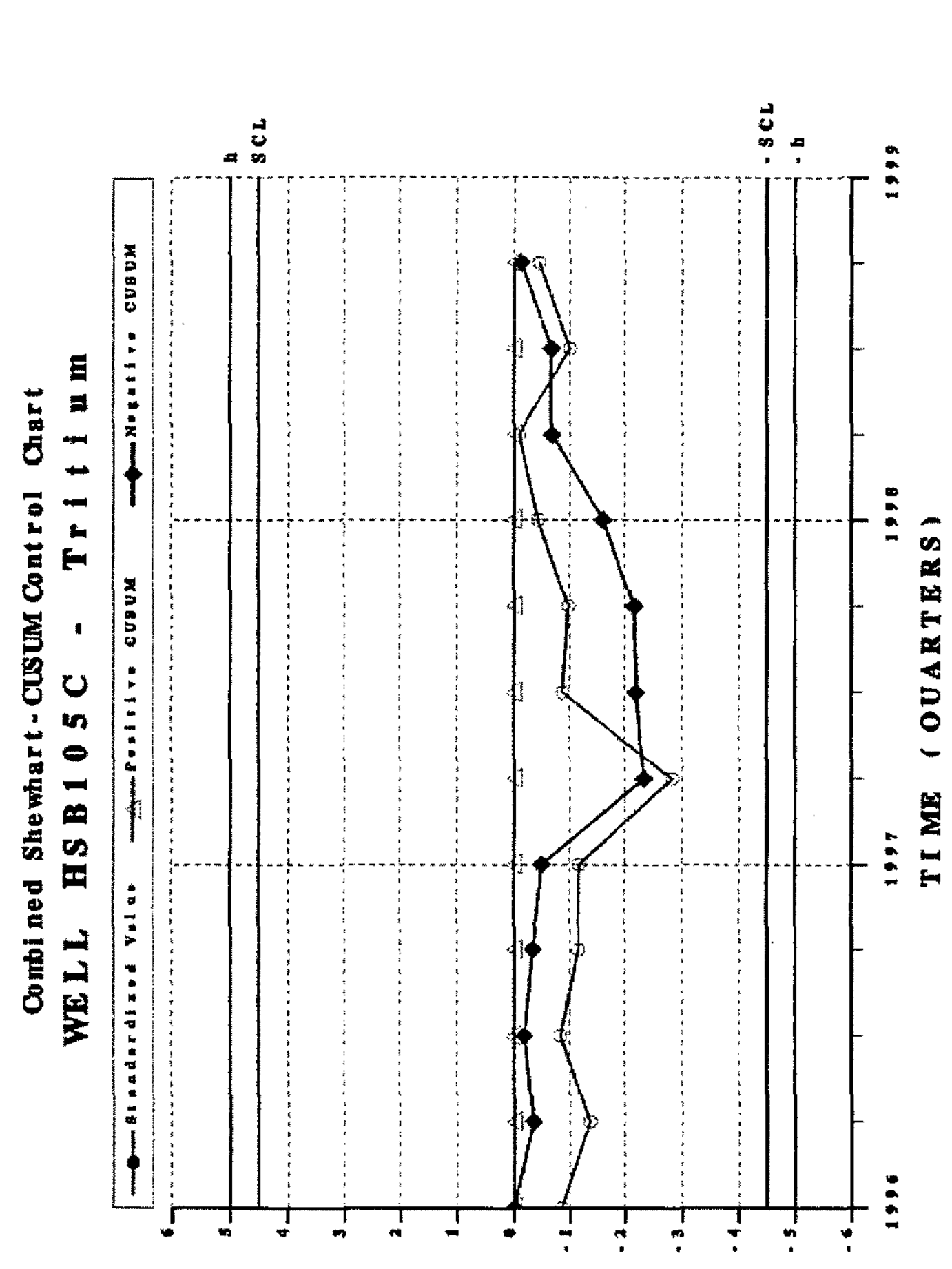

WSRC-TR-99-00013

Unclassified

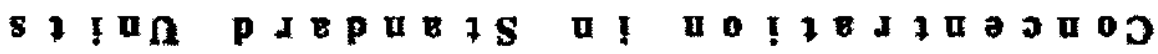


Combi ned She whart - CusuM Cont rol Chart

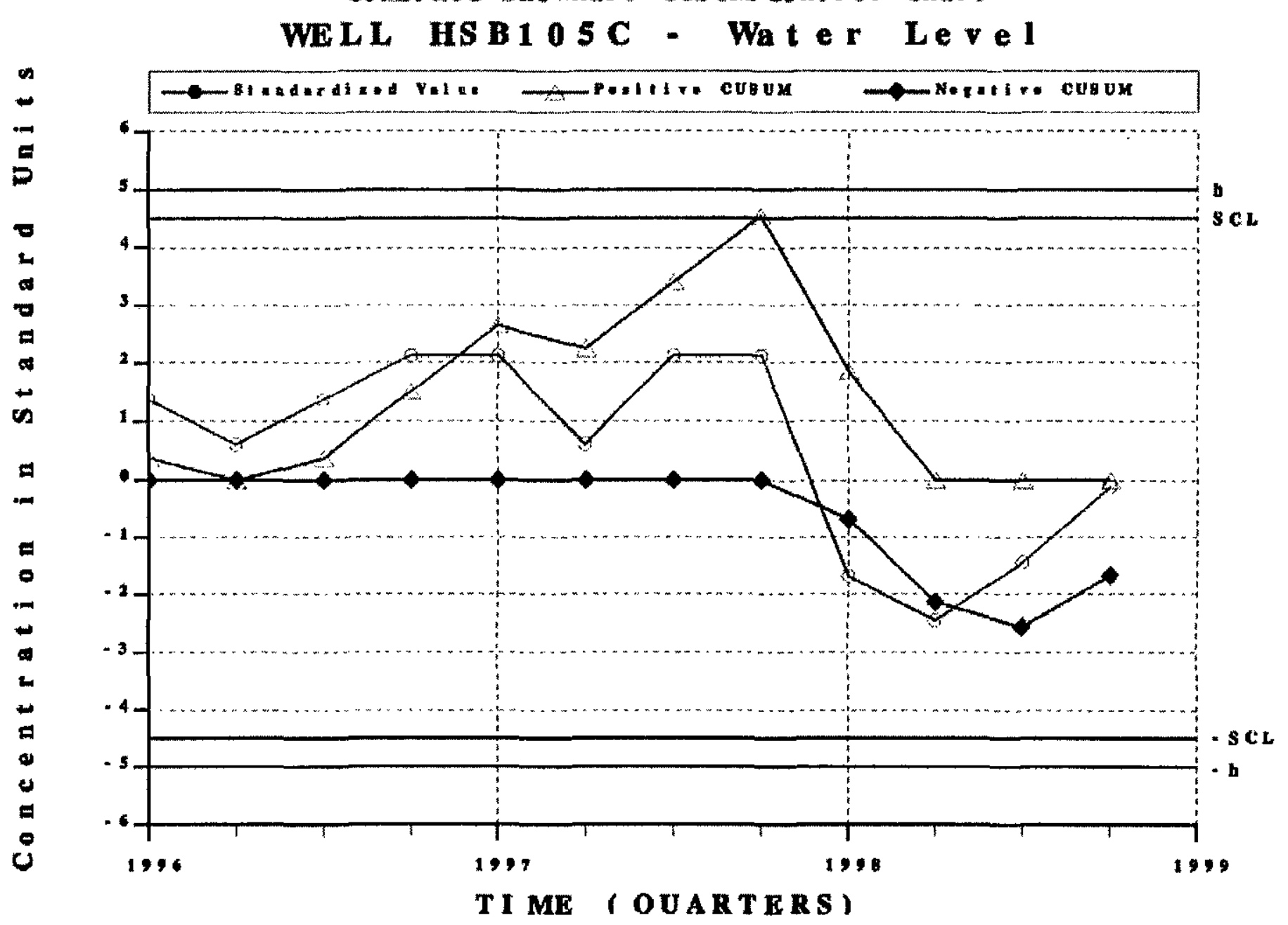



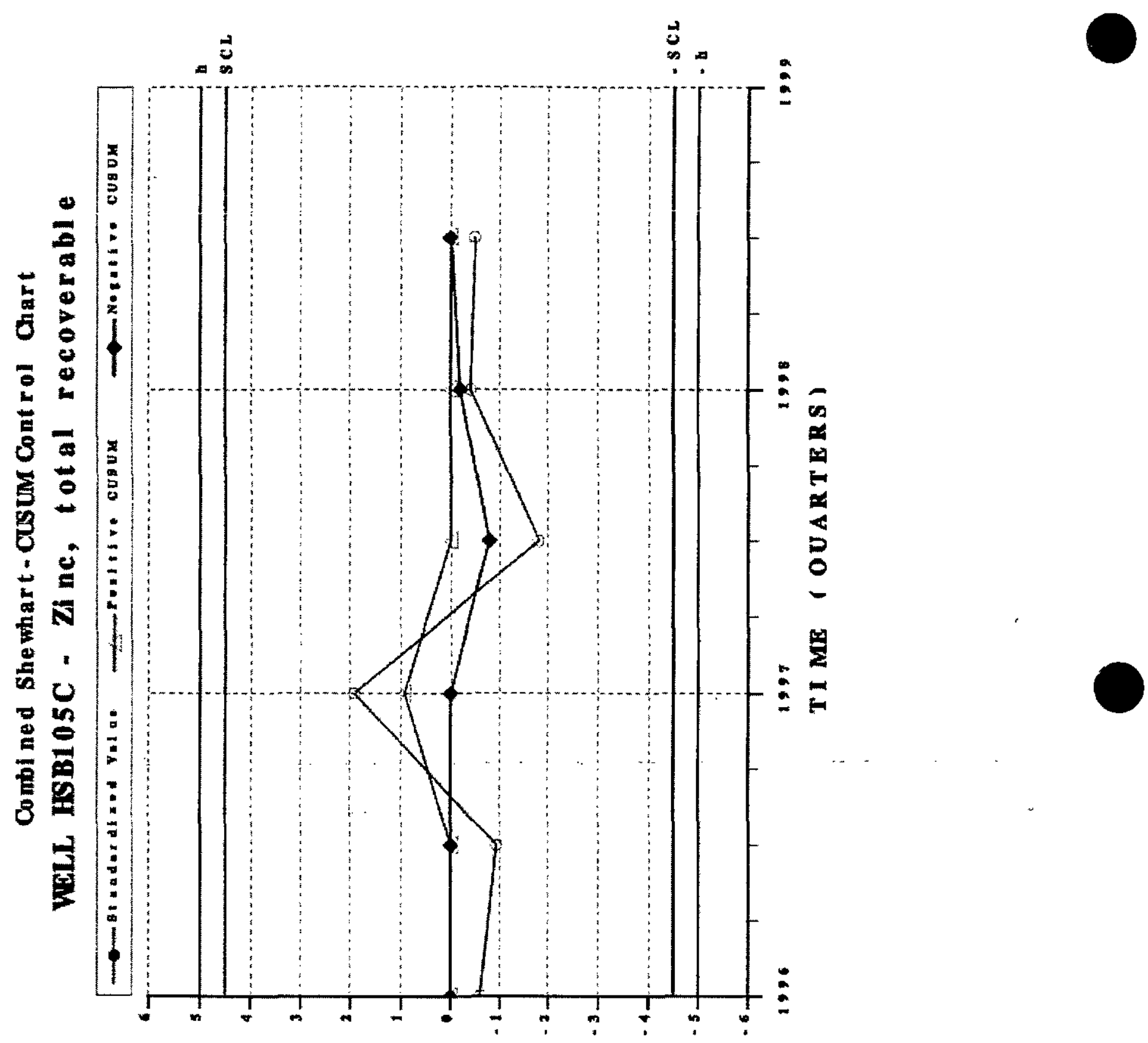

s tun pa 

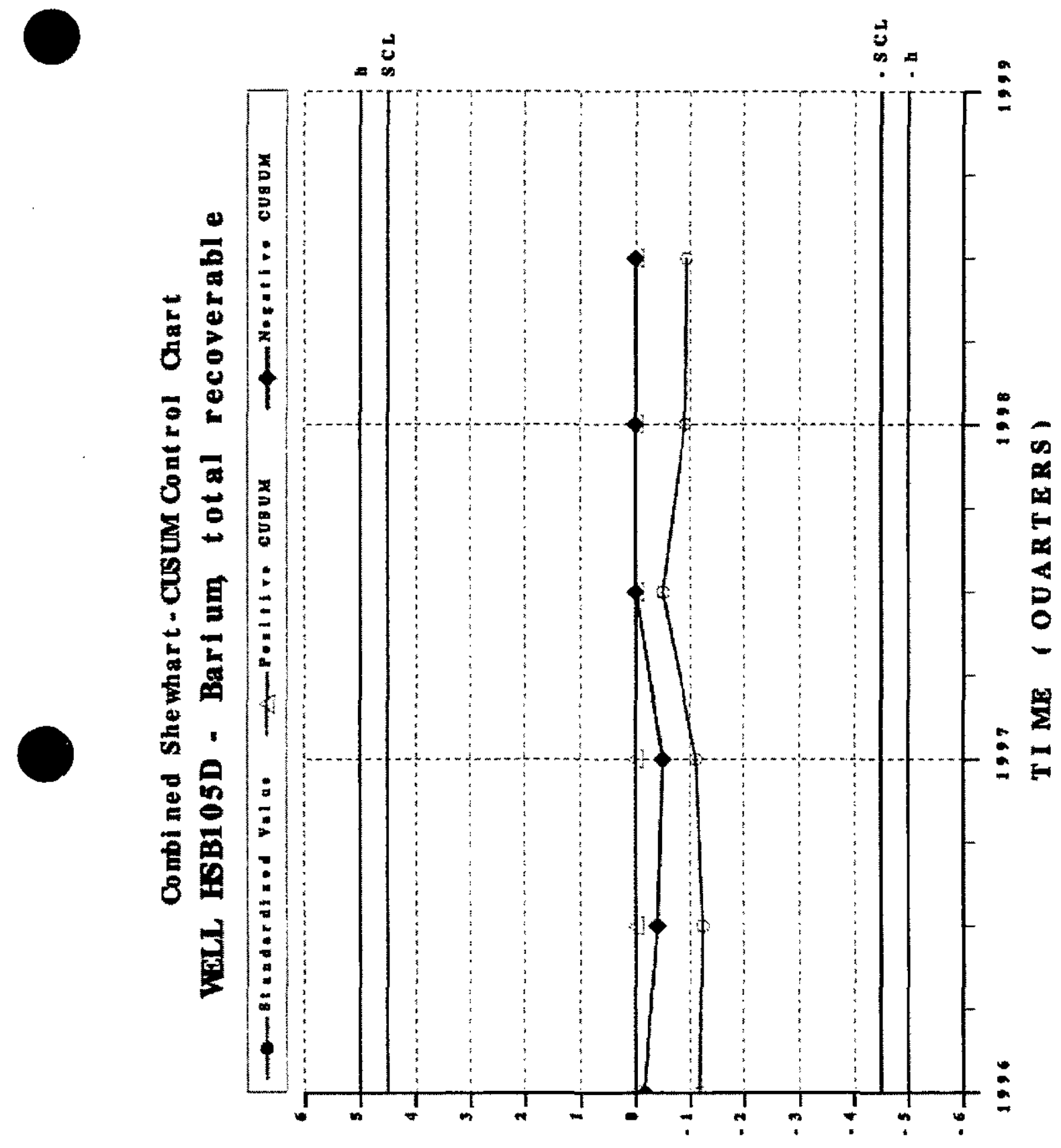

s I 


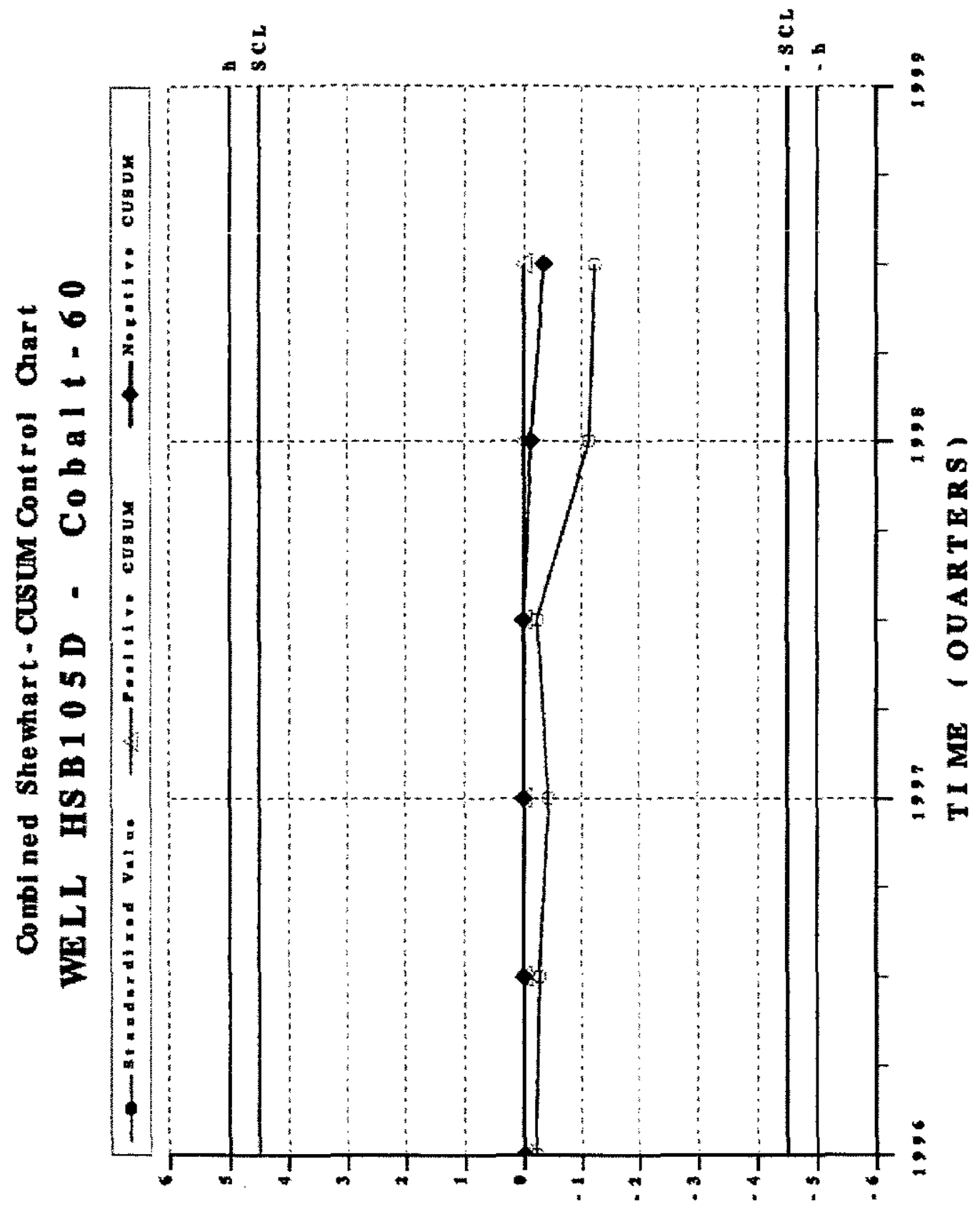

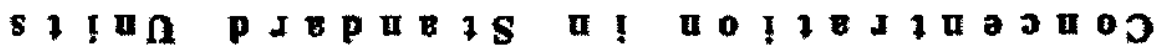



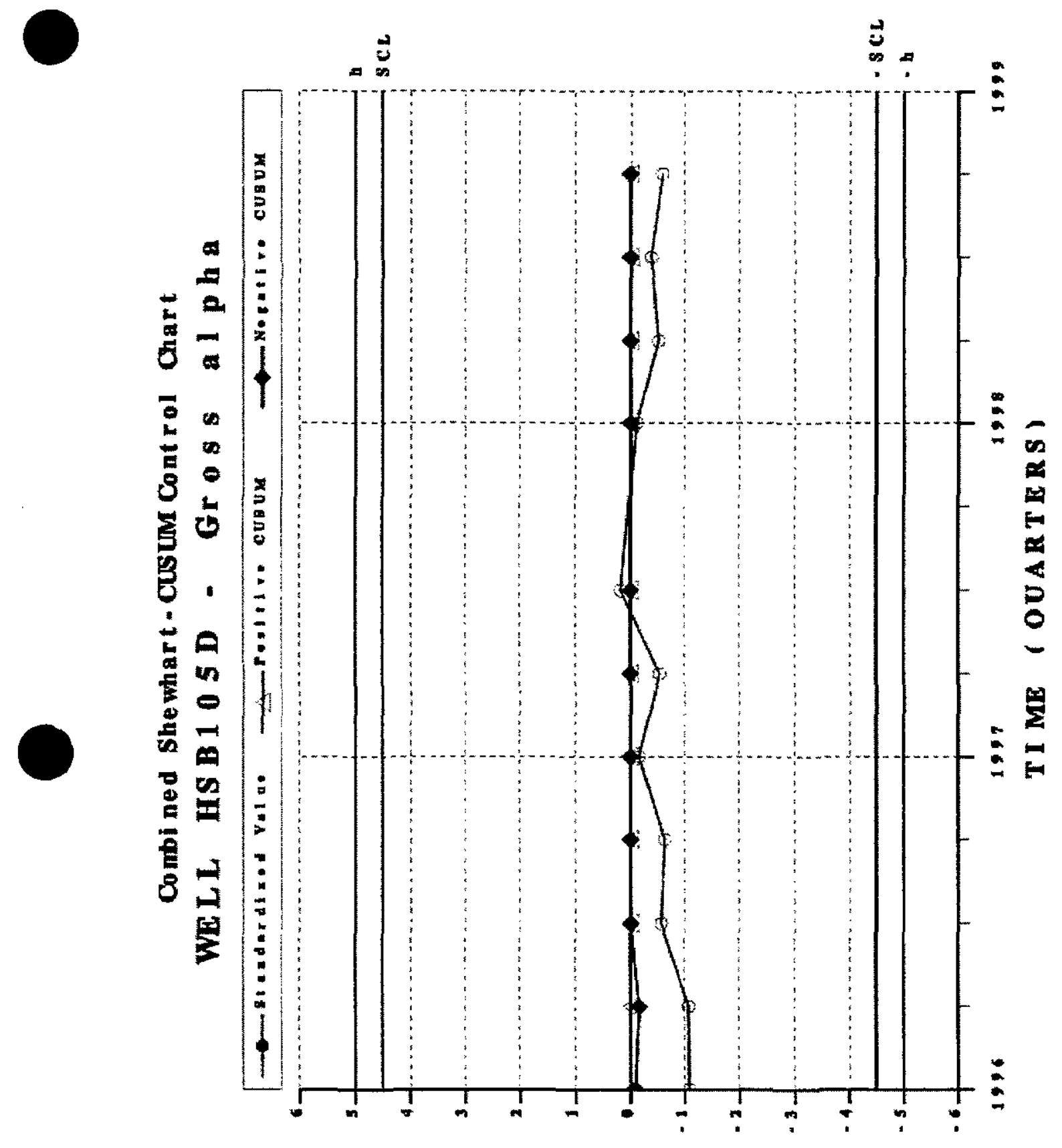

s) I U $\mathrm{p}$ I E 


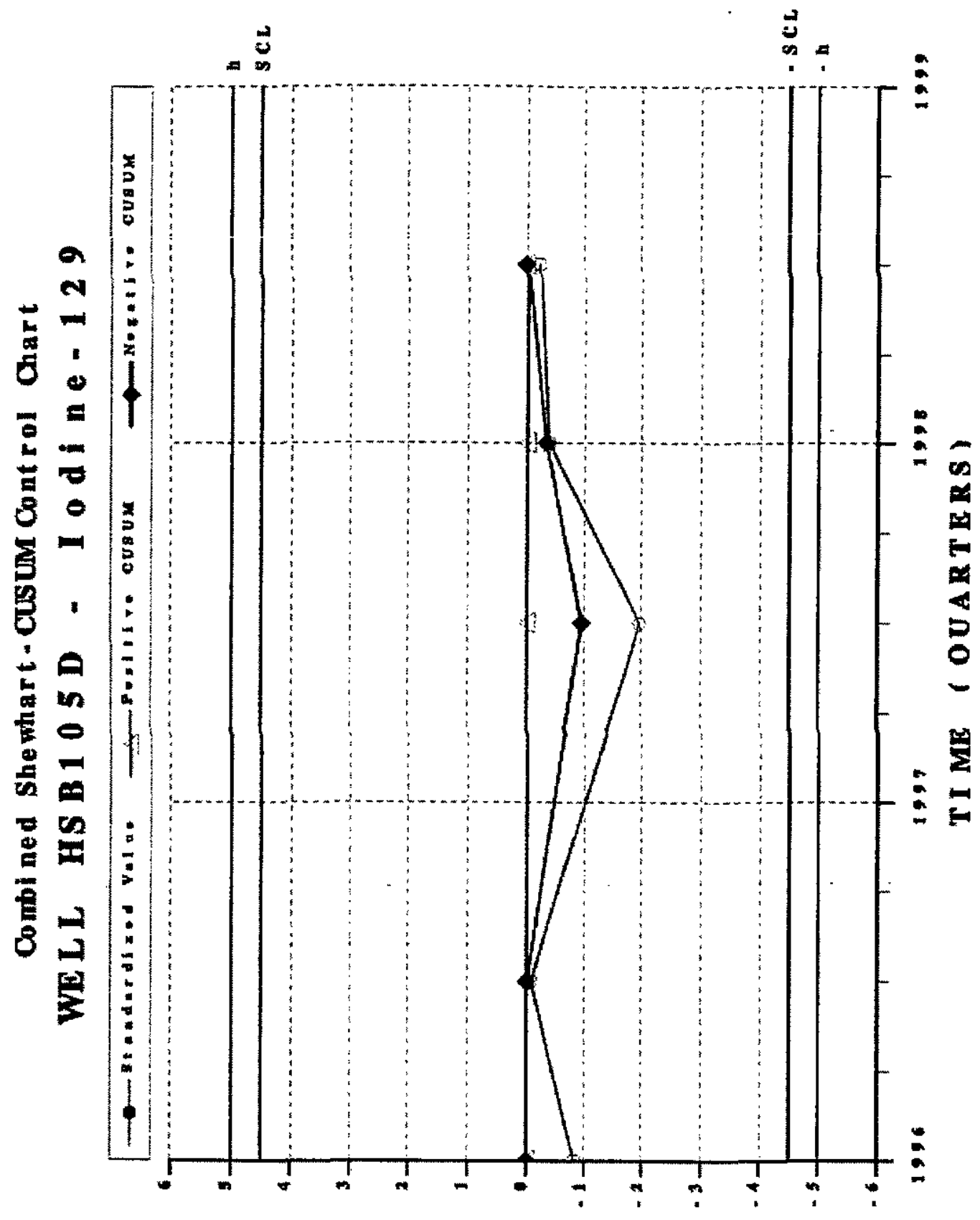

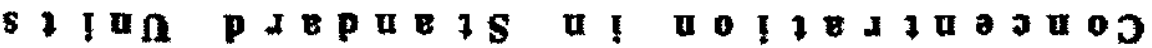



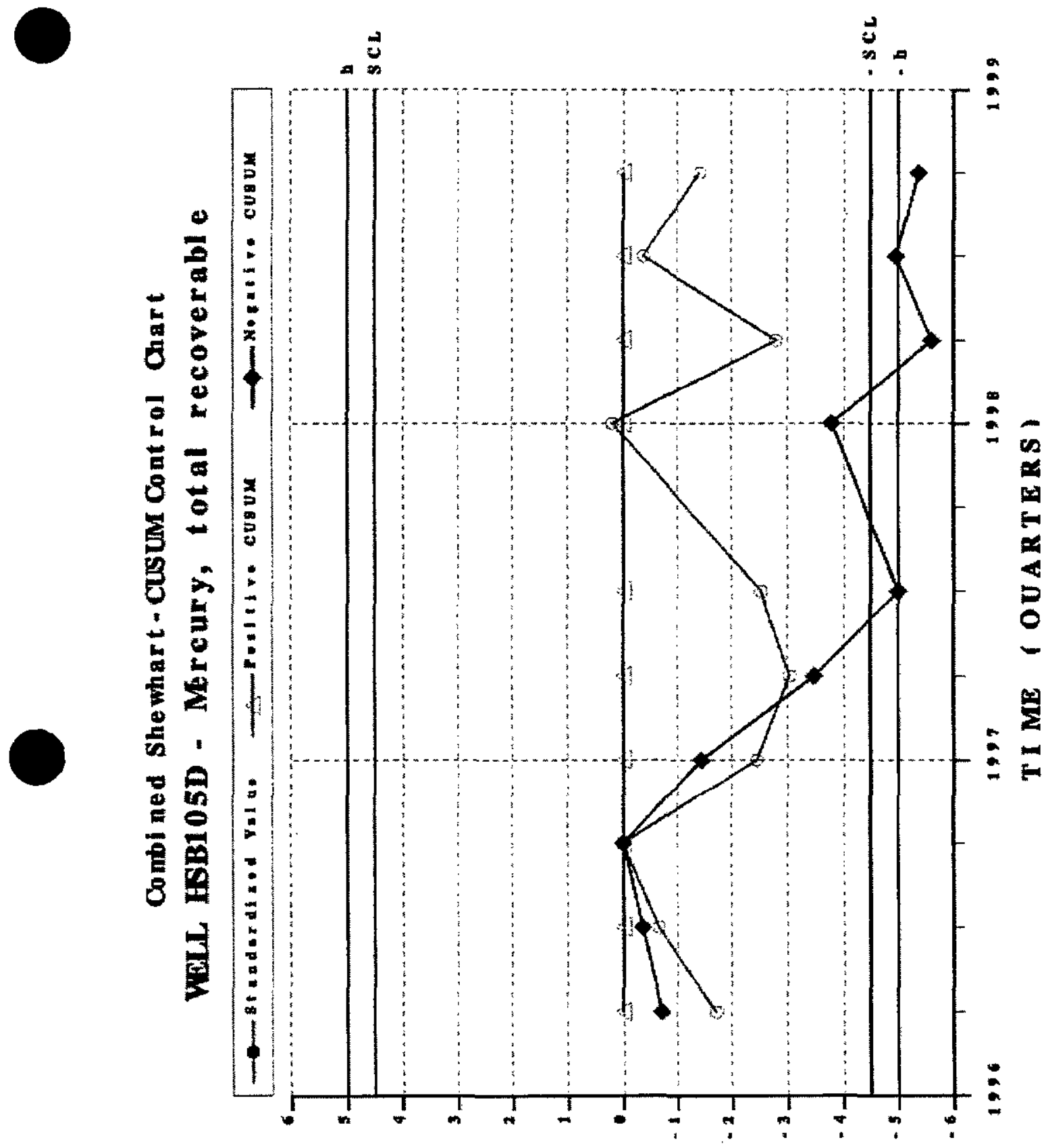

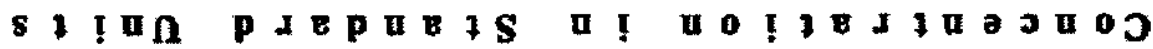




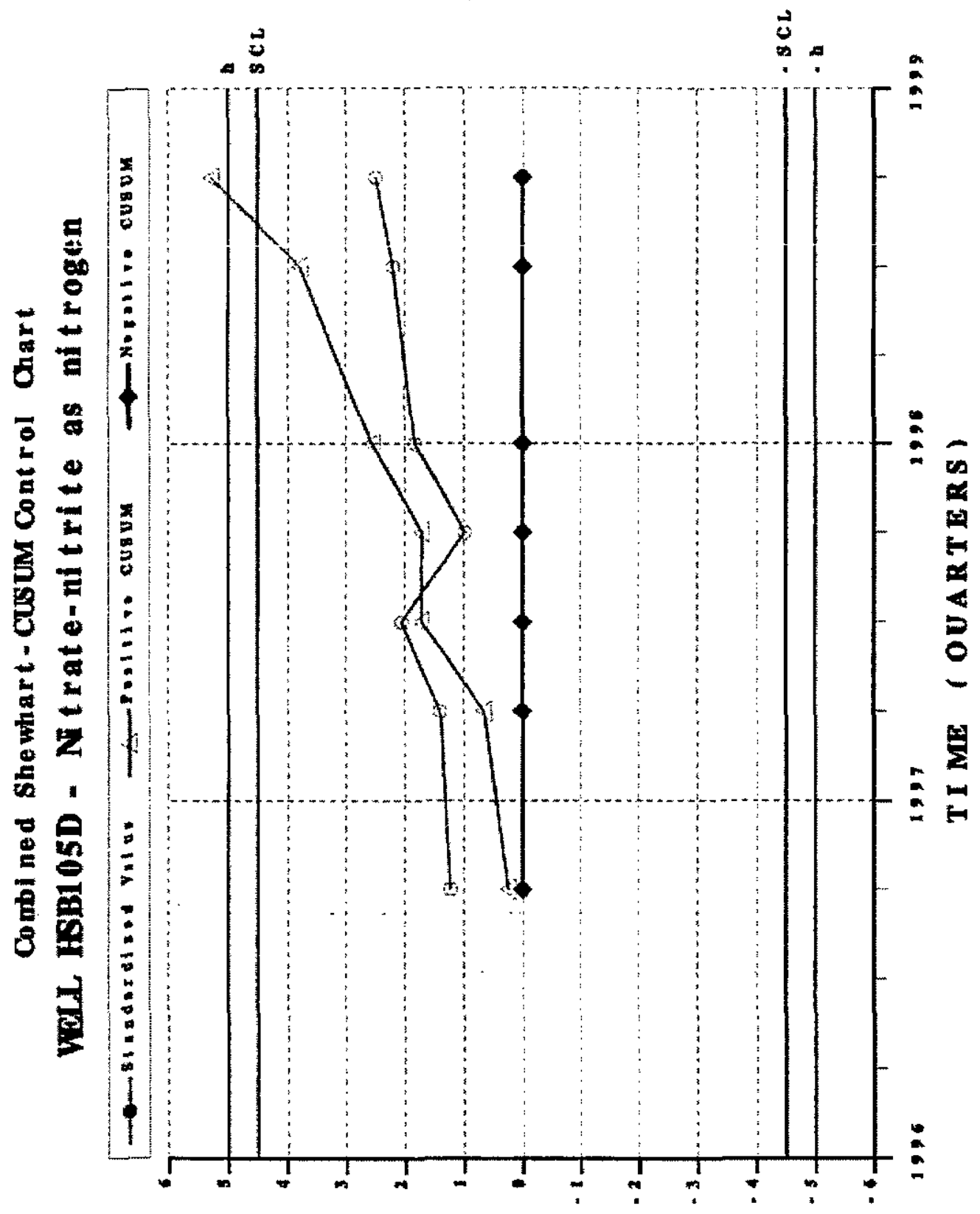

s I 

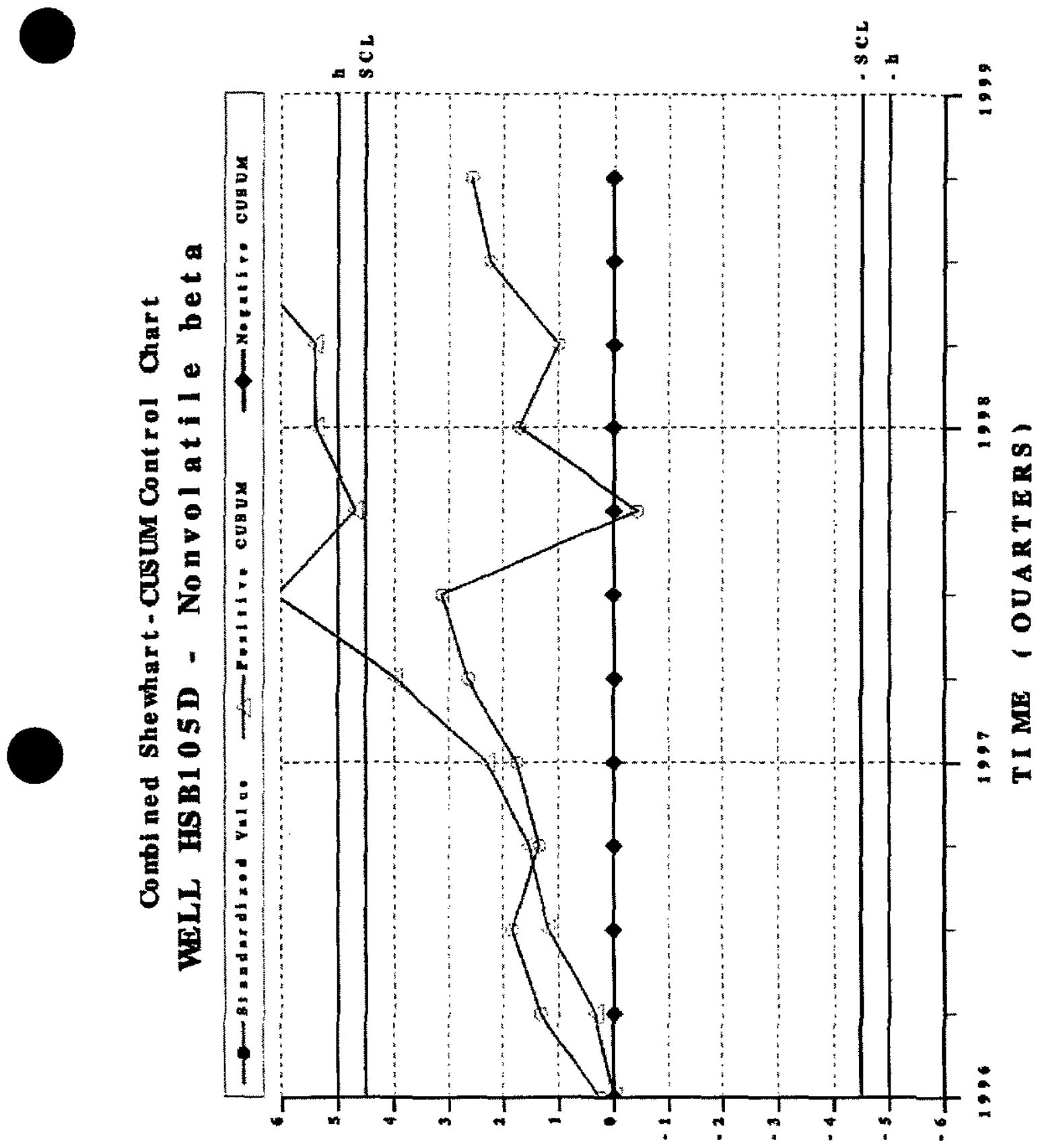

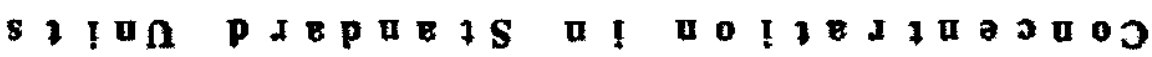




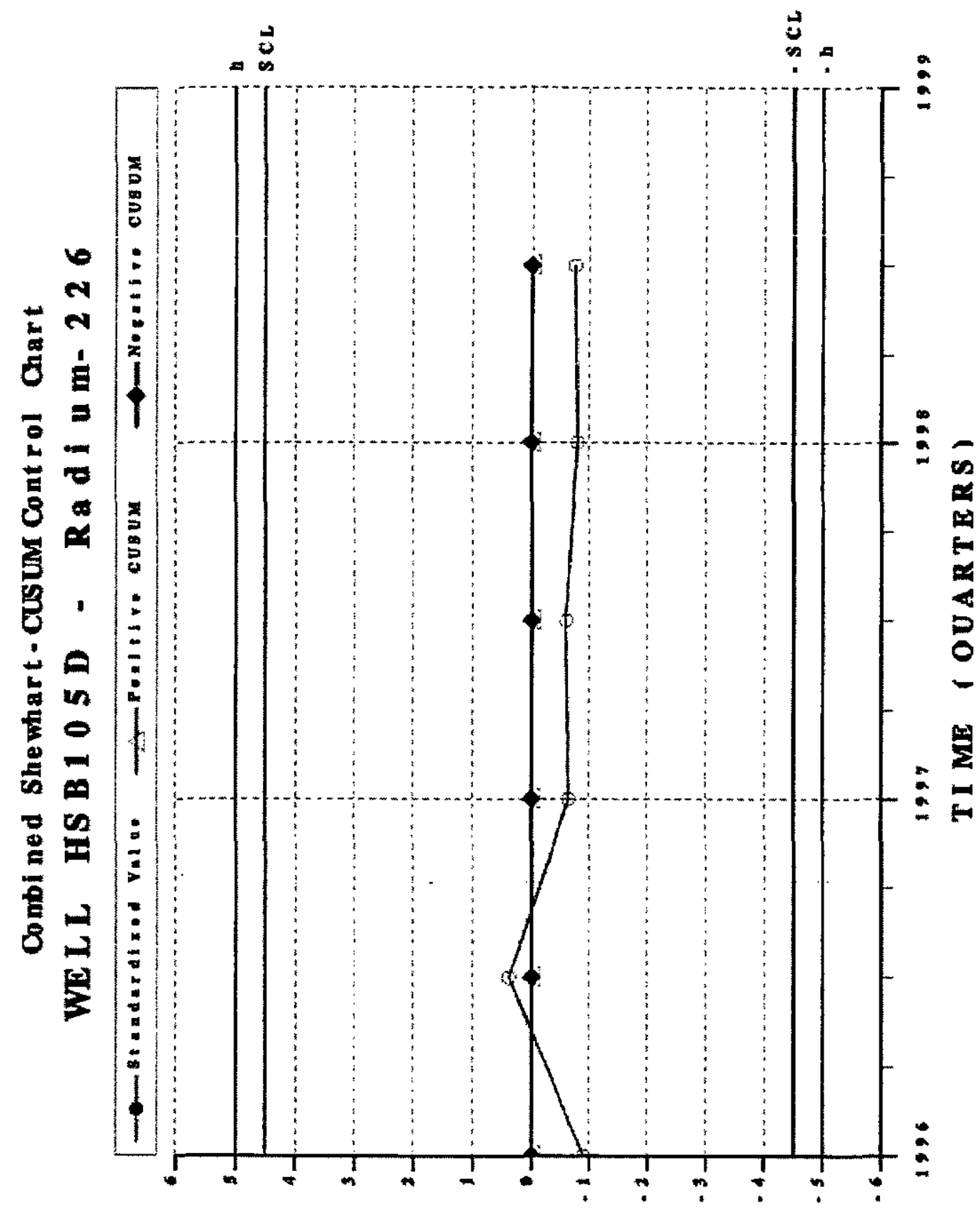

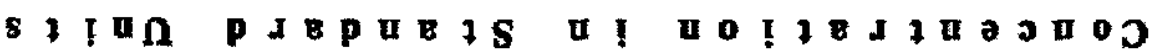



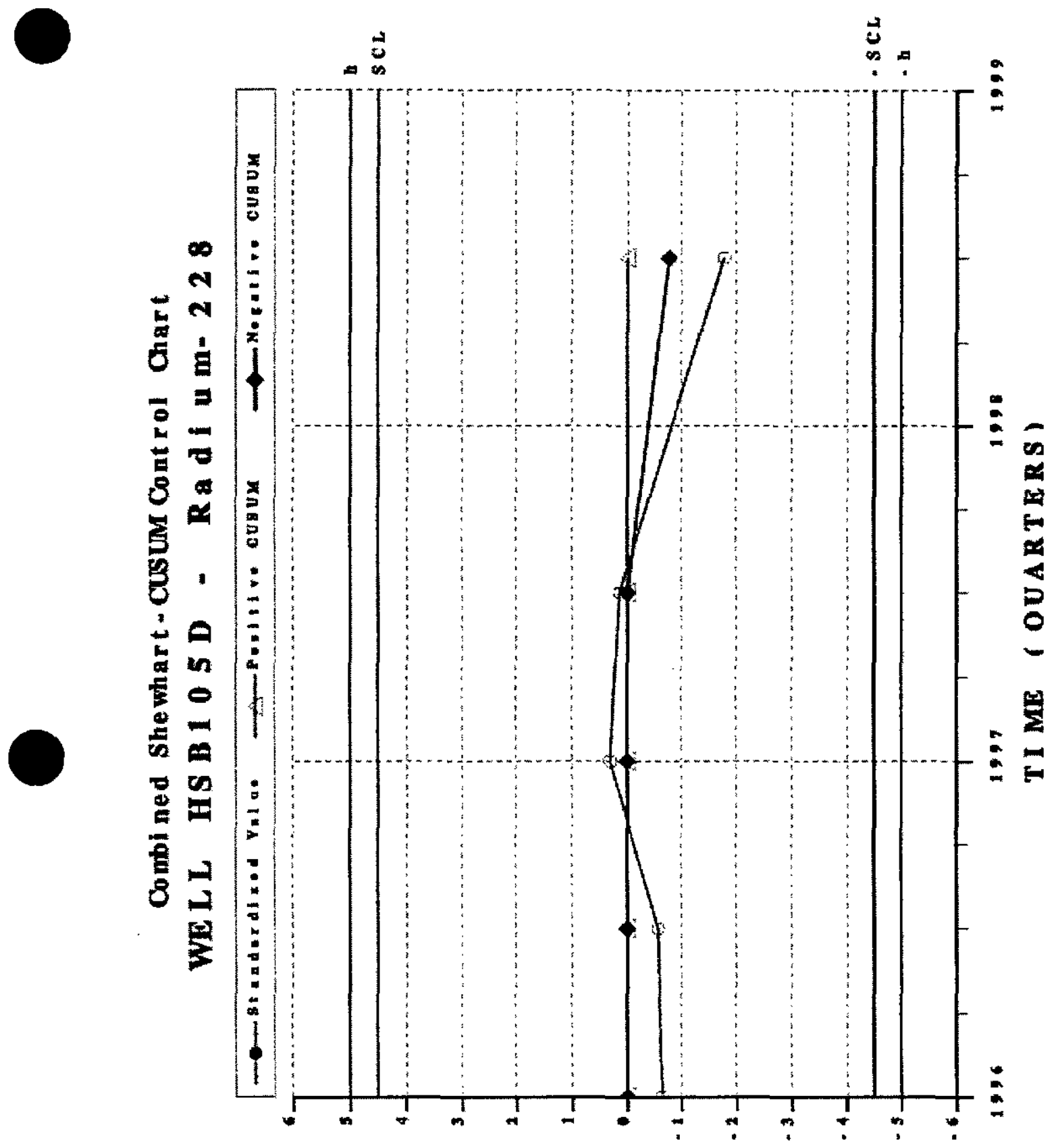

s I I 1 p I E 


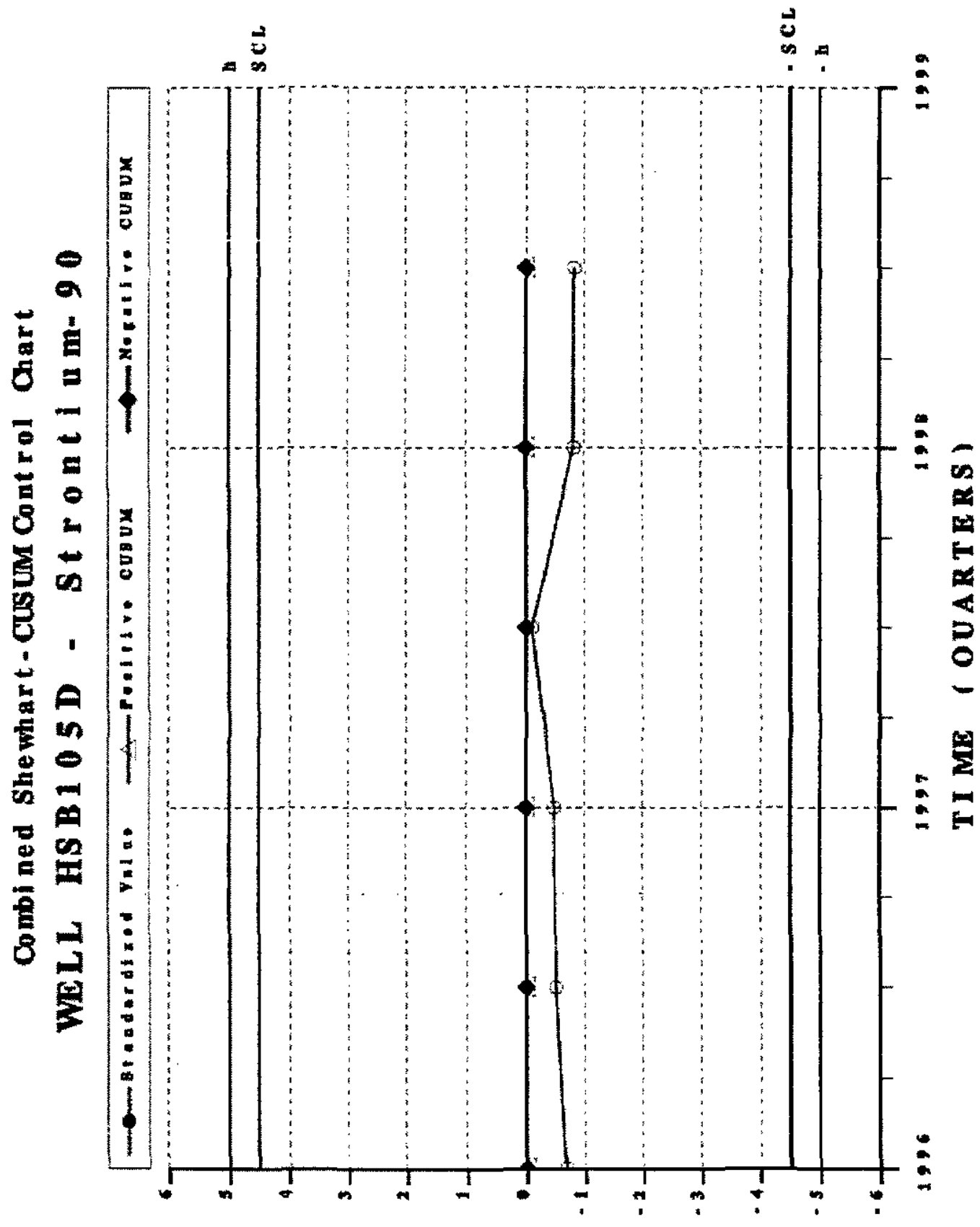

s I In $\mathbf{1}$ I 


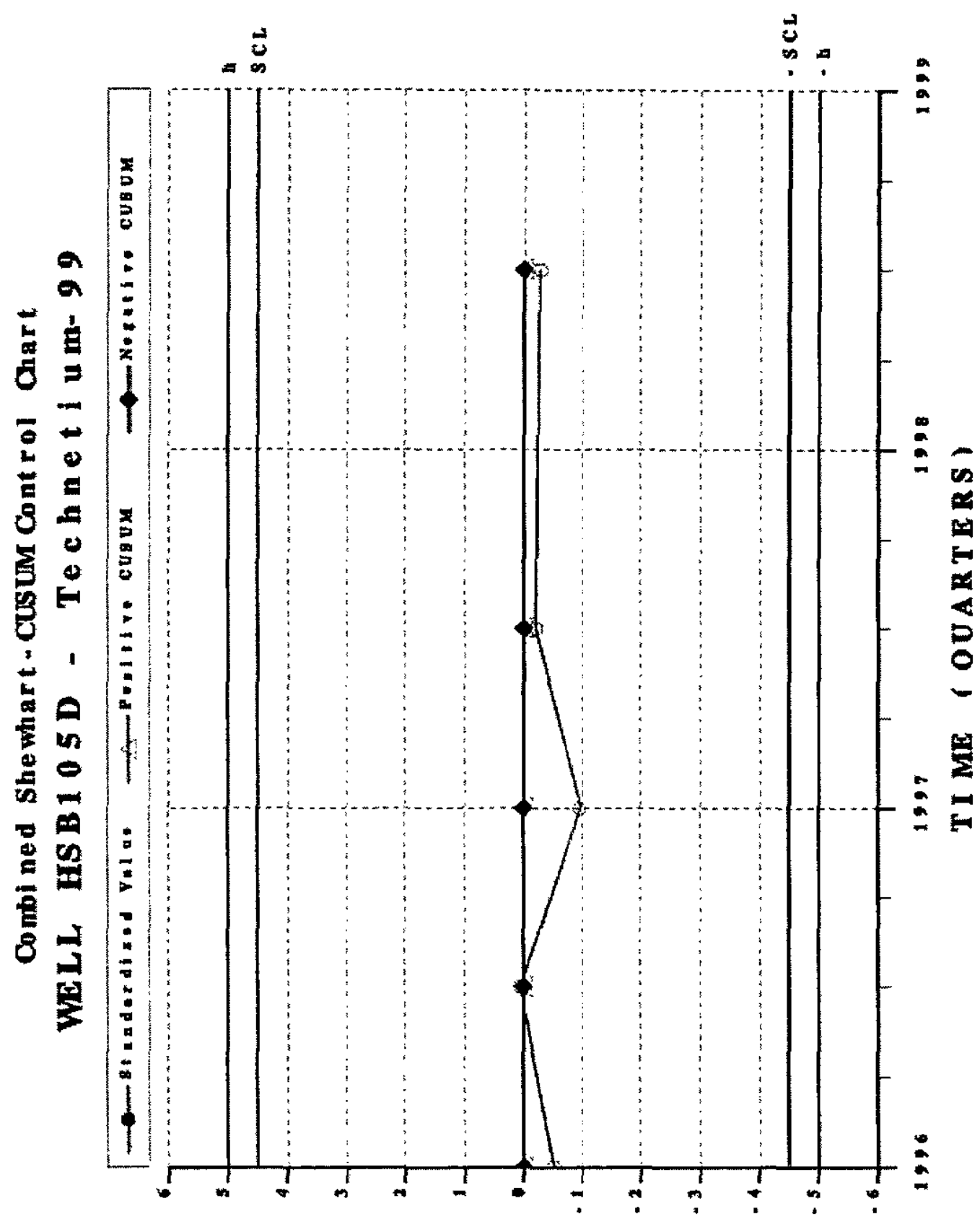

s I In pIEpuE 


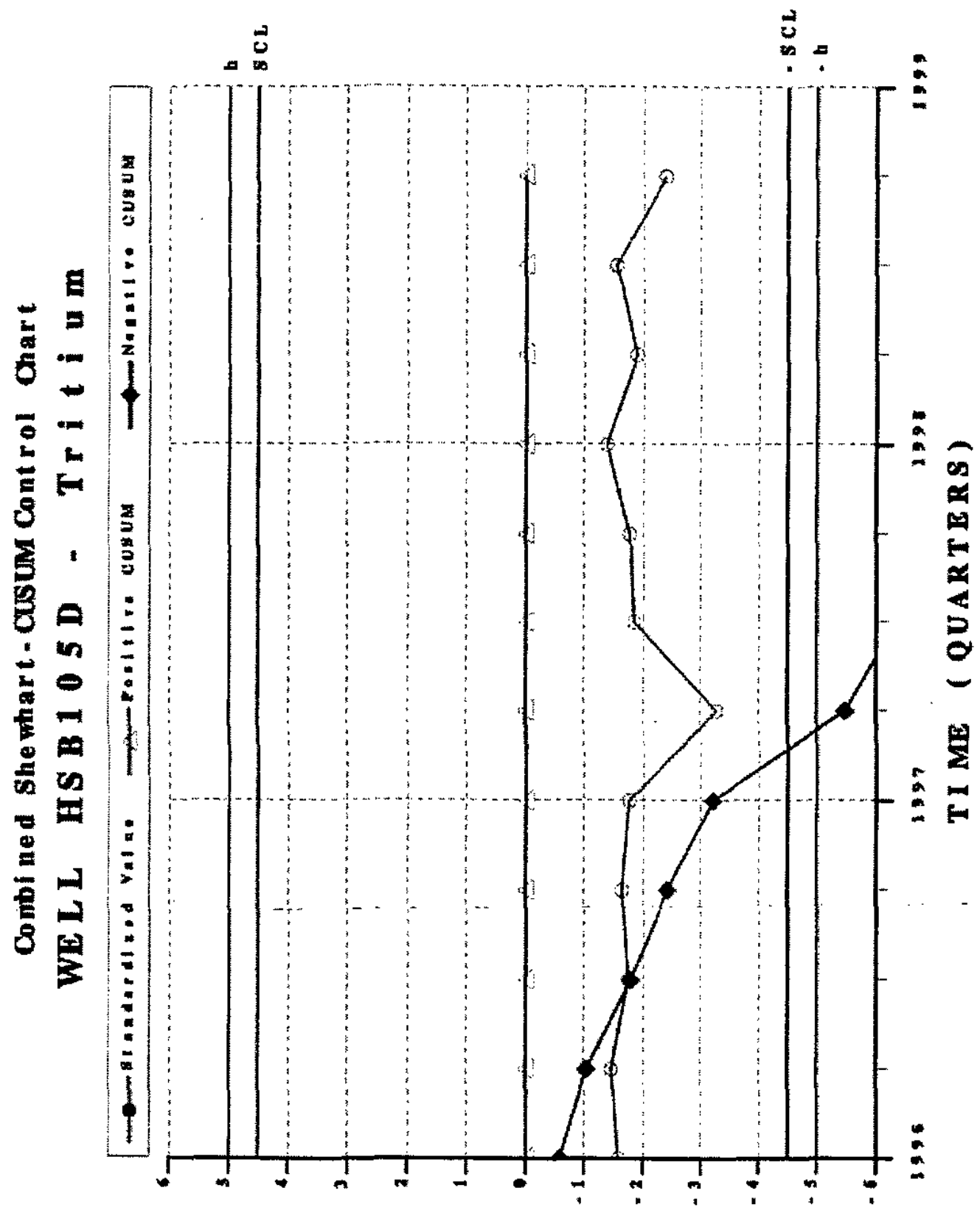

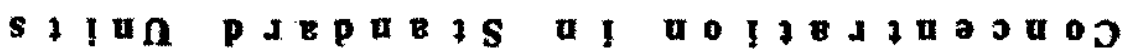



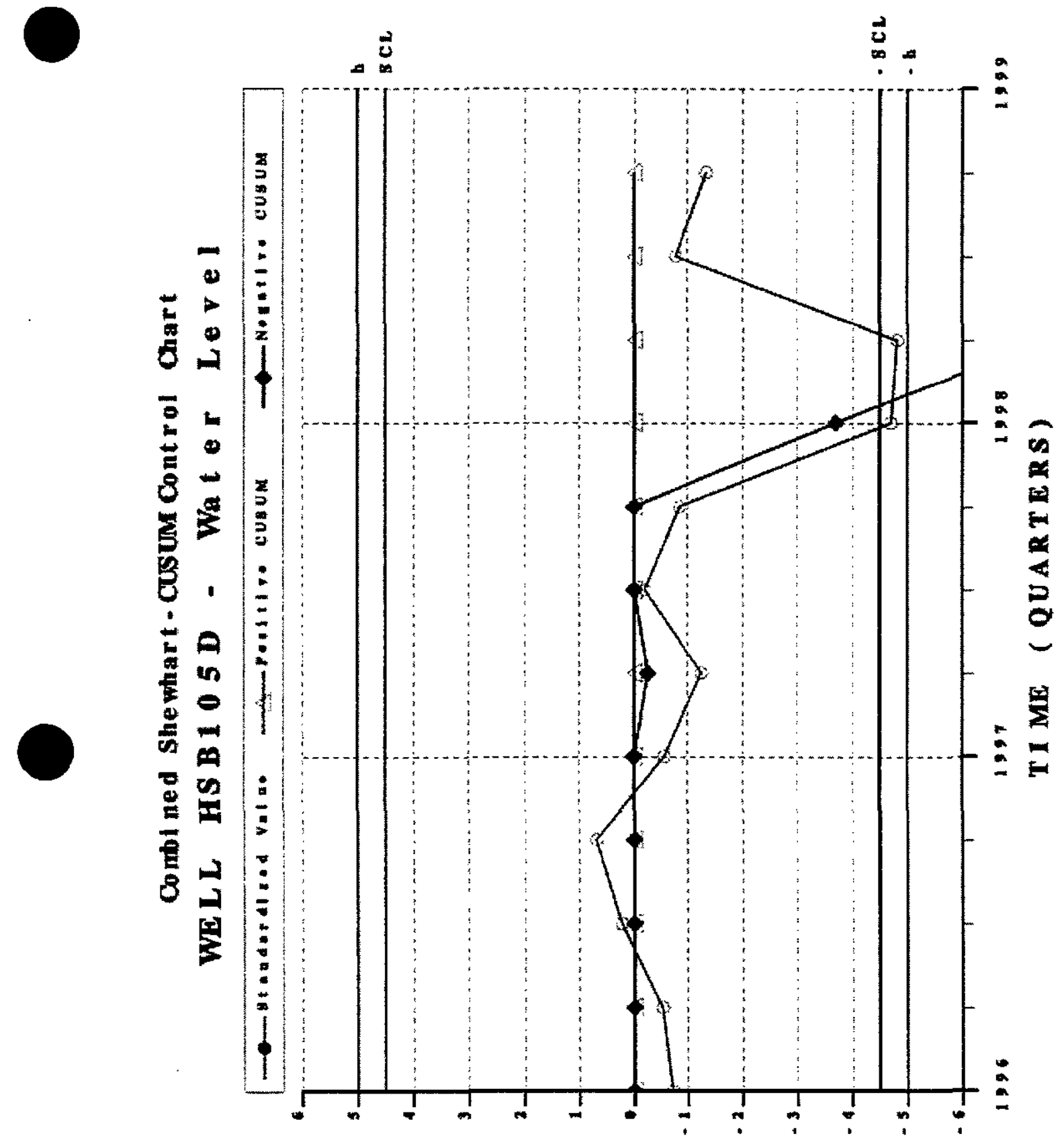

s! I 


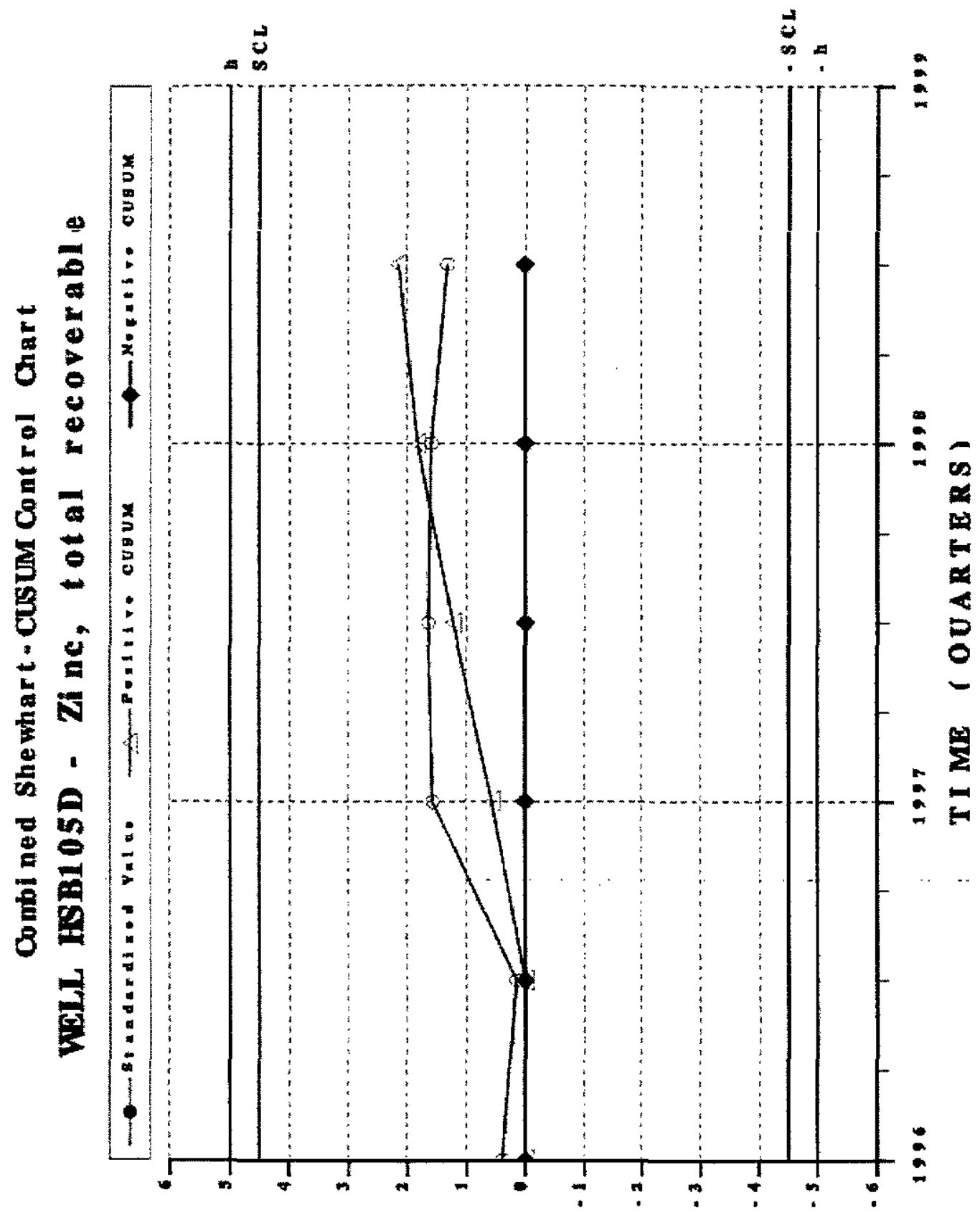

s! I 

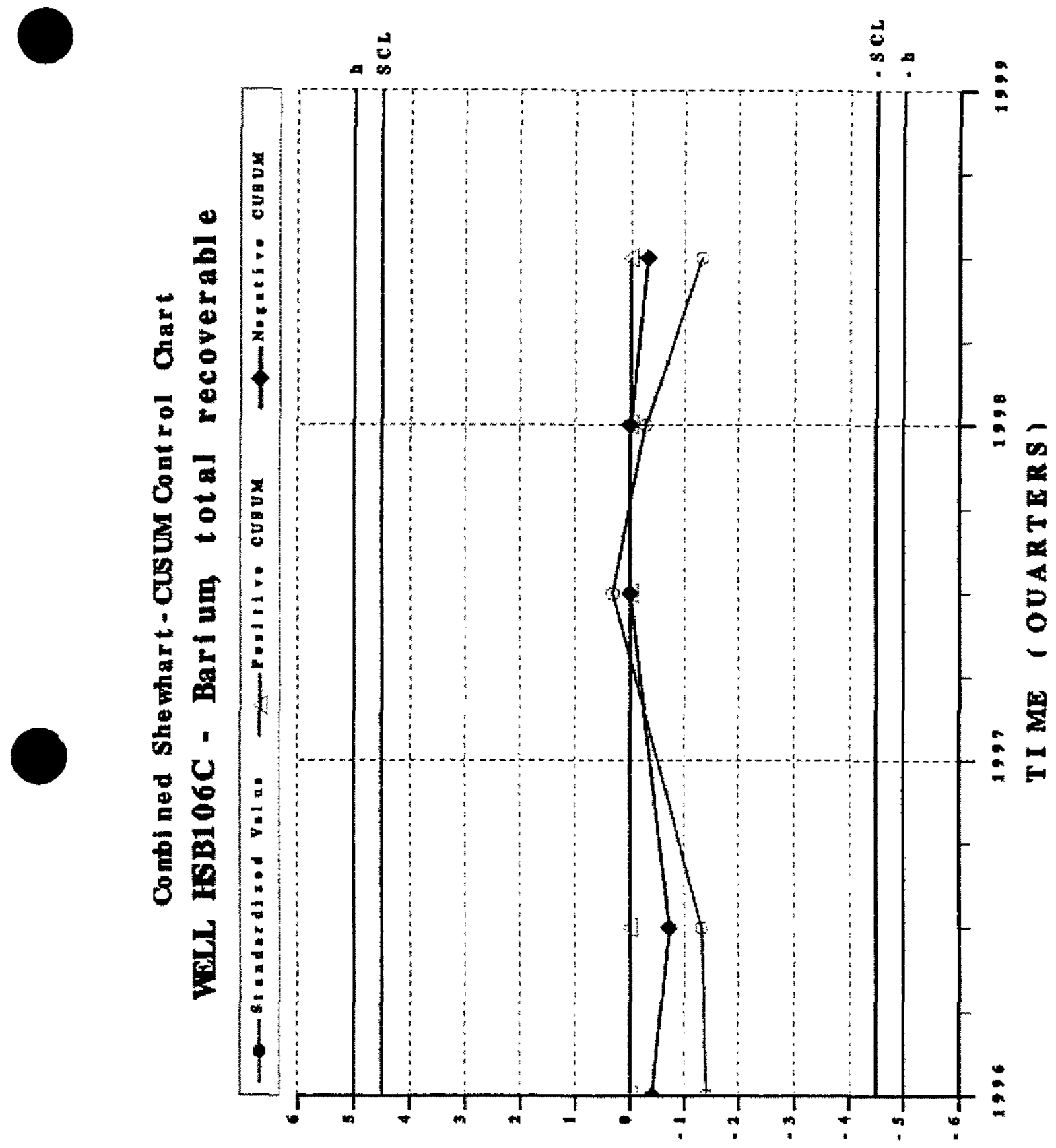

s I In pa $\mathbf{p}$ a 


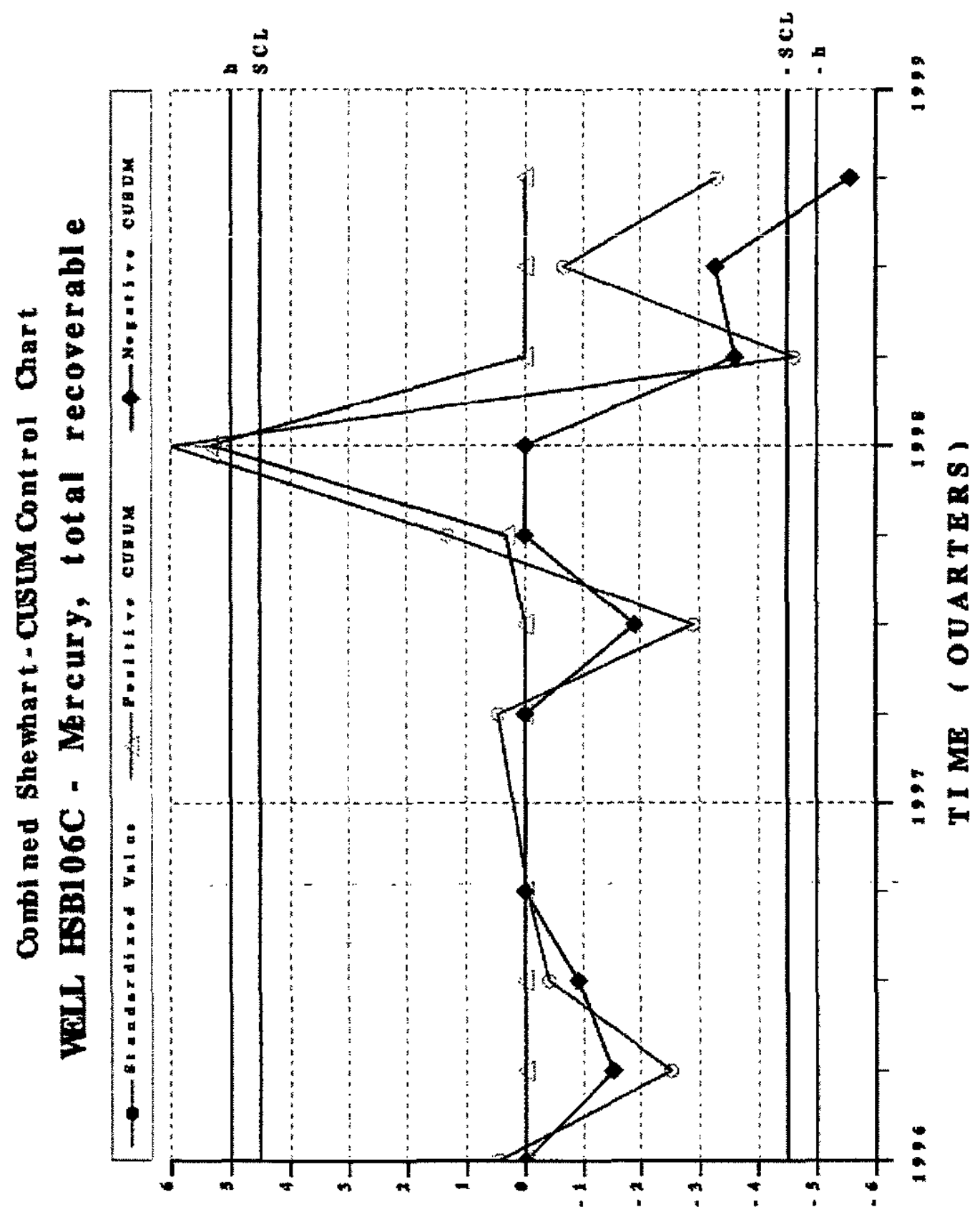

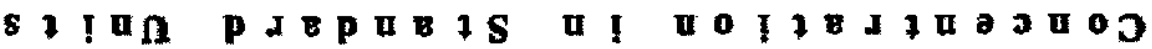





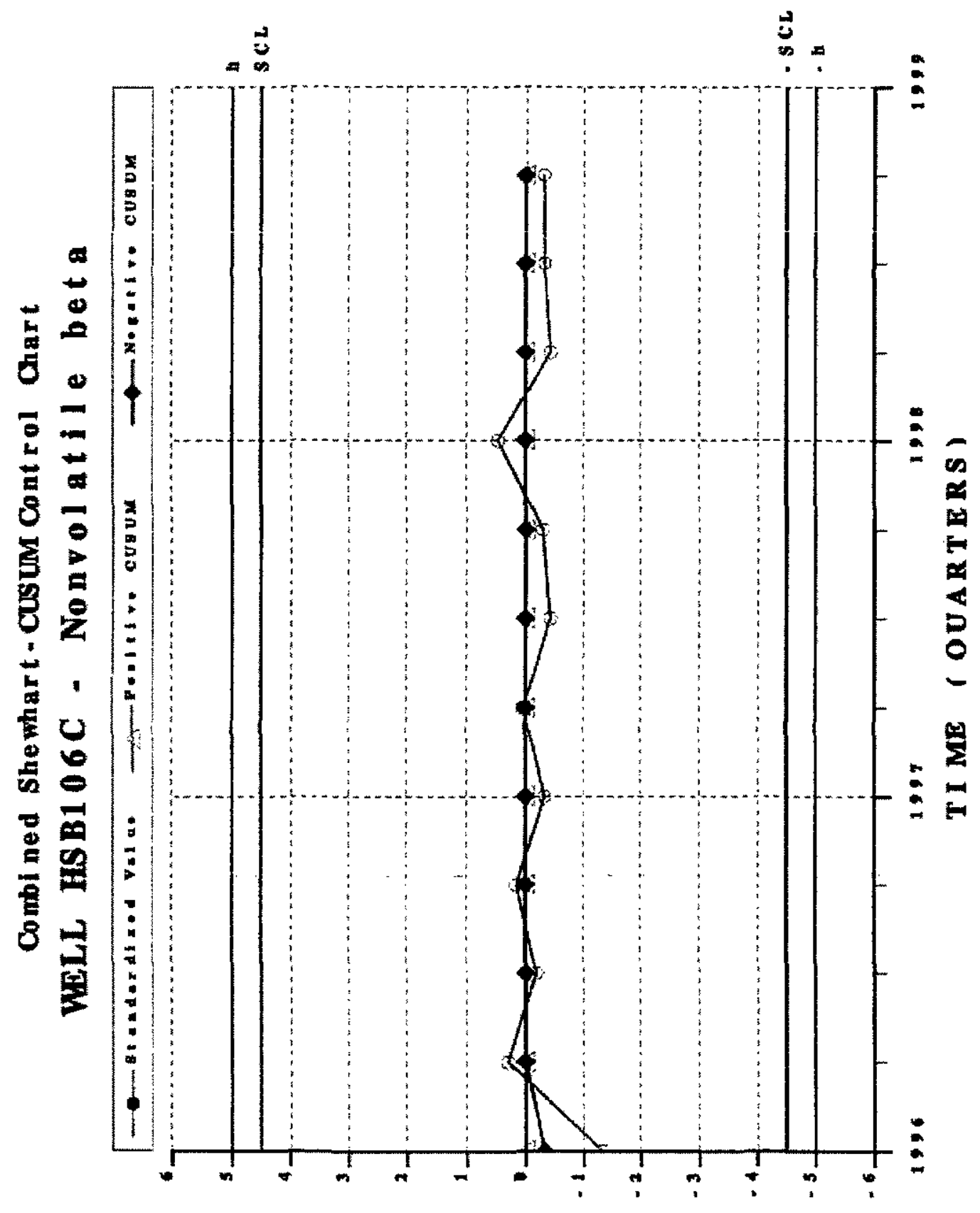

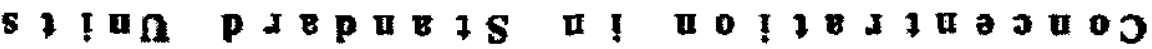




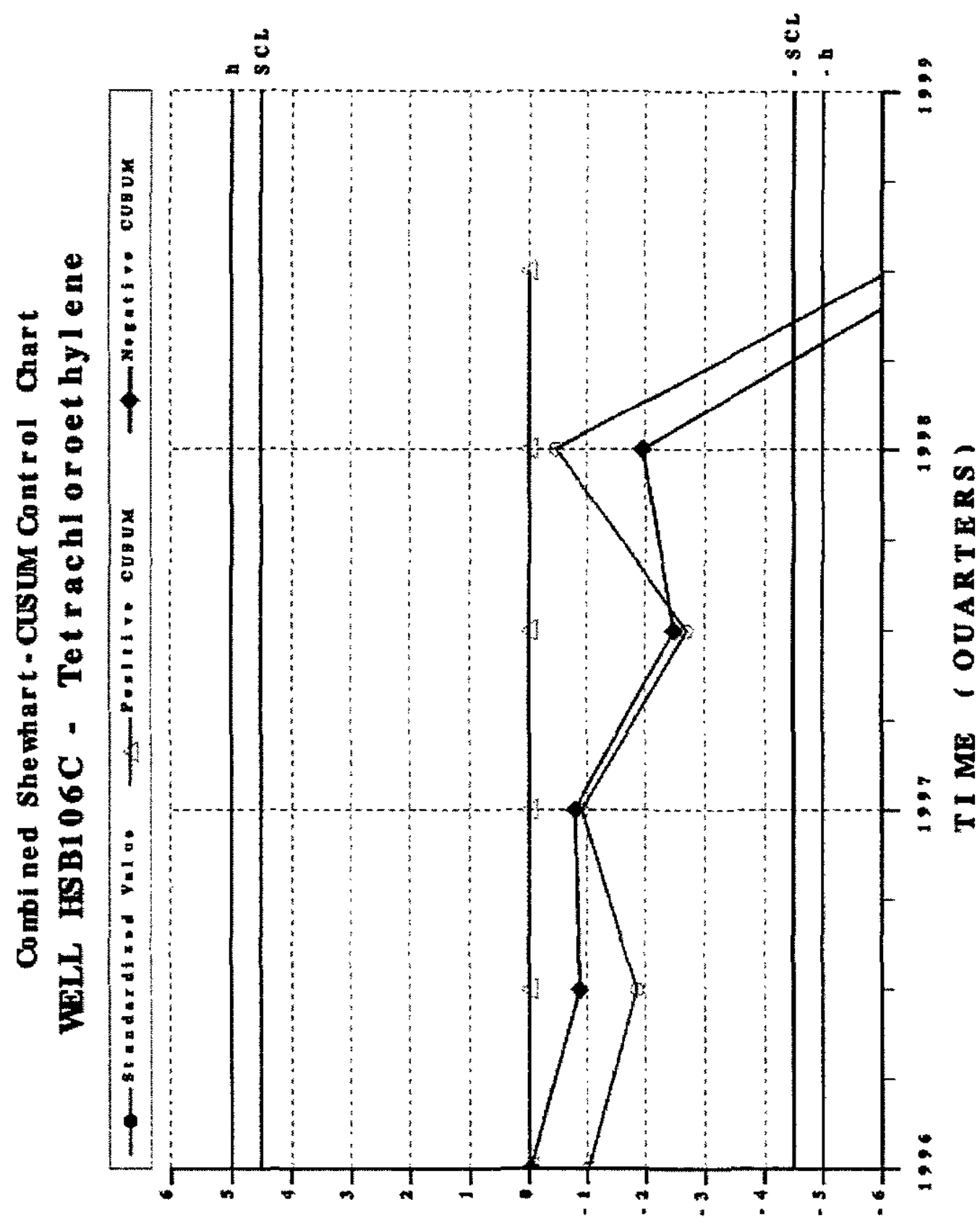

s I I I 


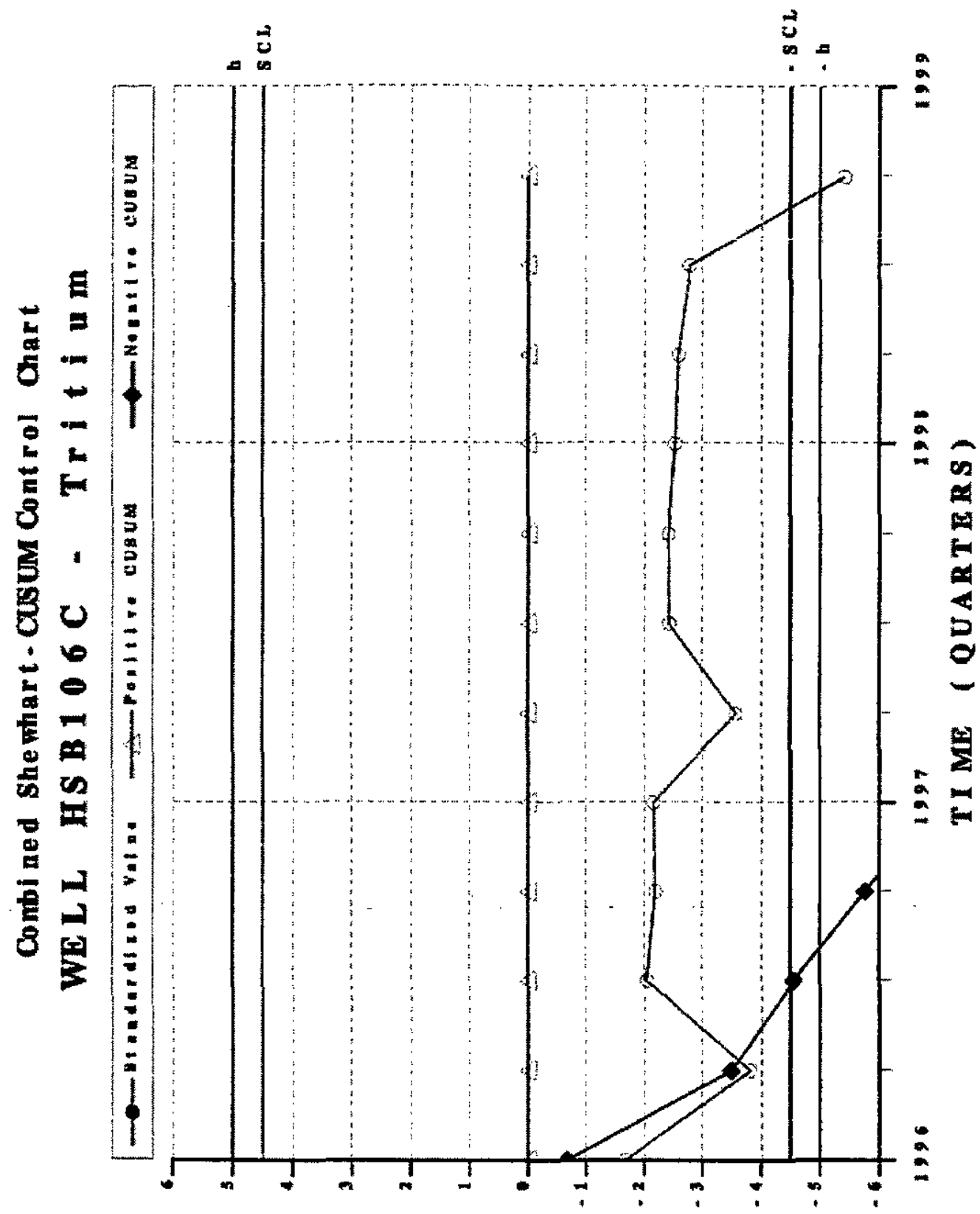


Combi ned Shewhart - CuSUM Control Chart

WELL HSB $106 \mathrm{C}$ - Wat e r Leve 1

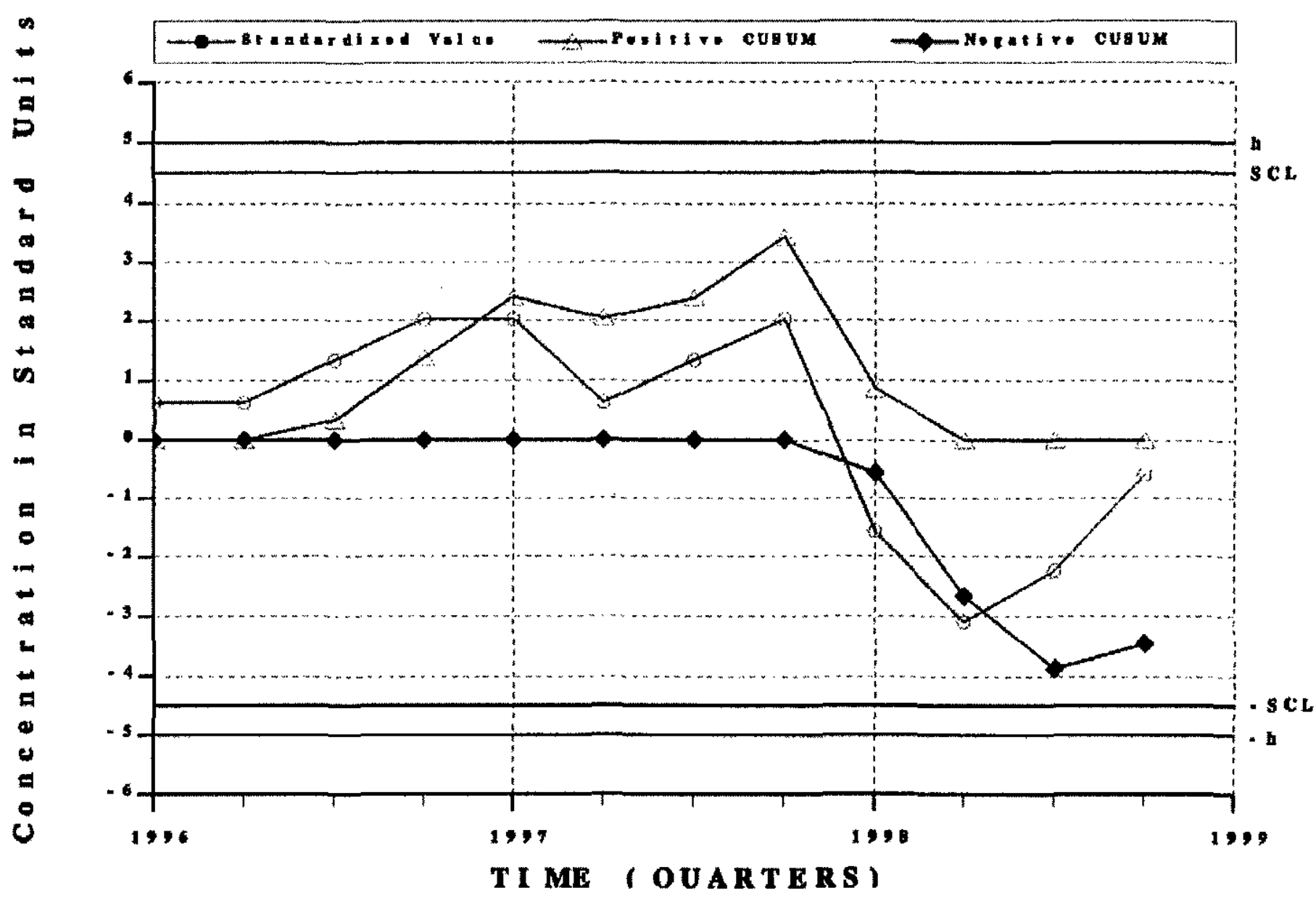




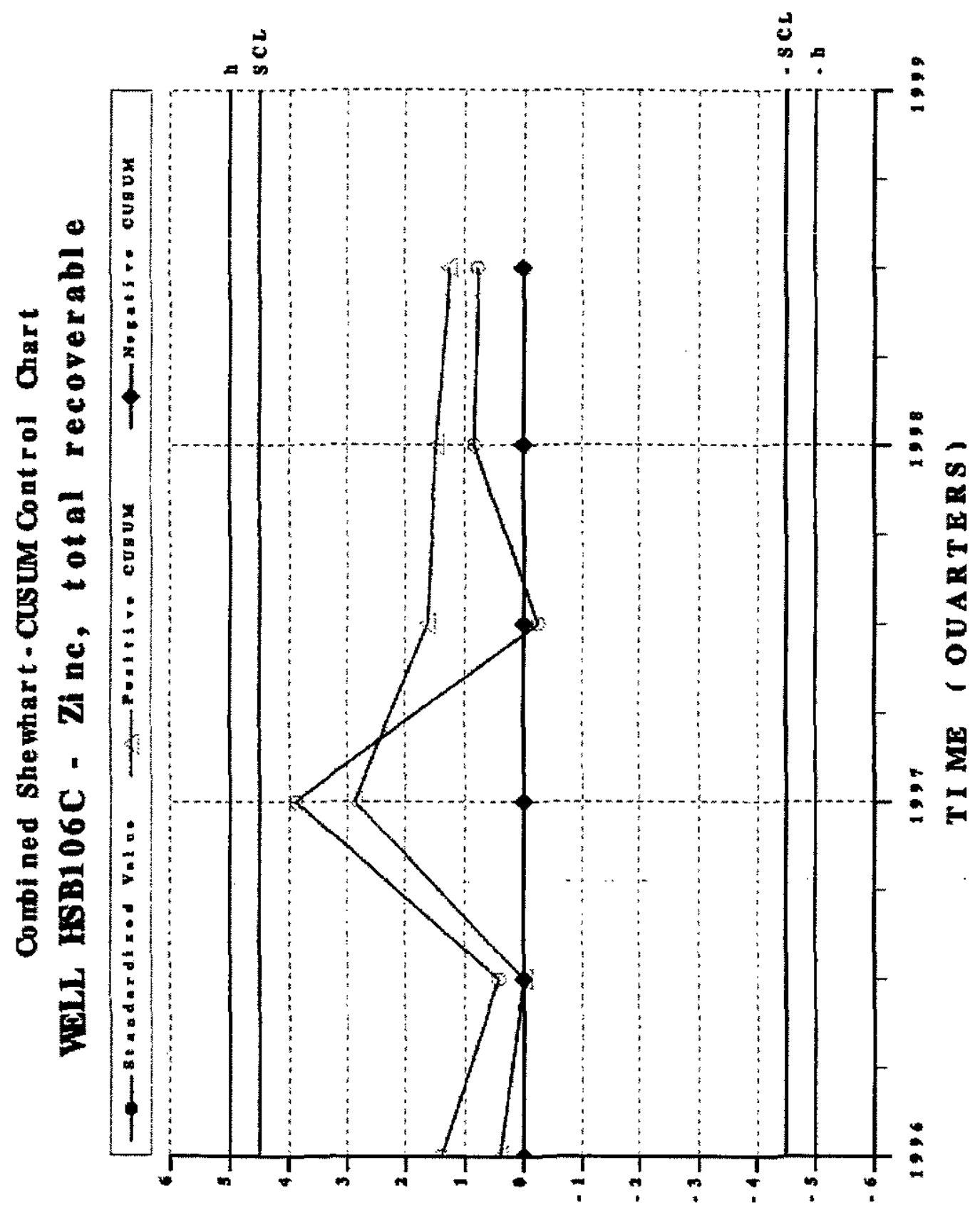

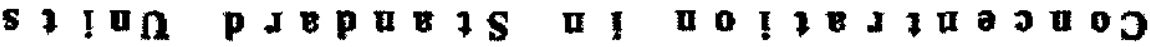



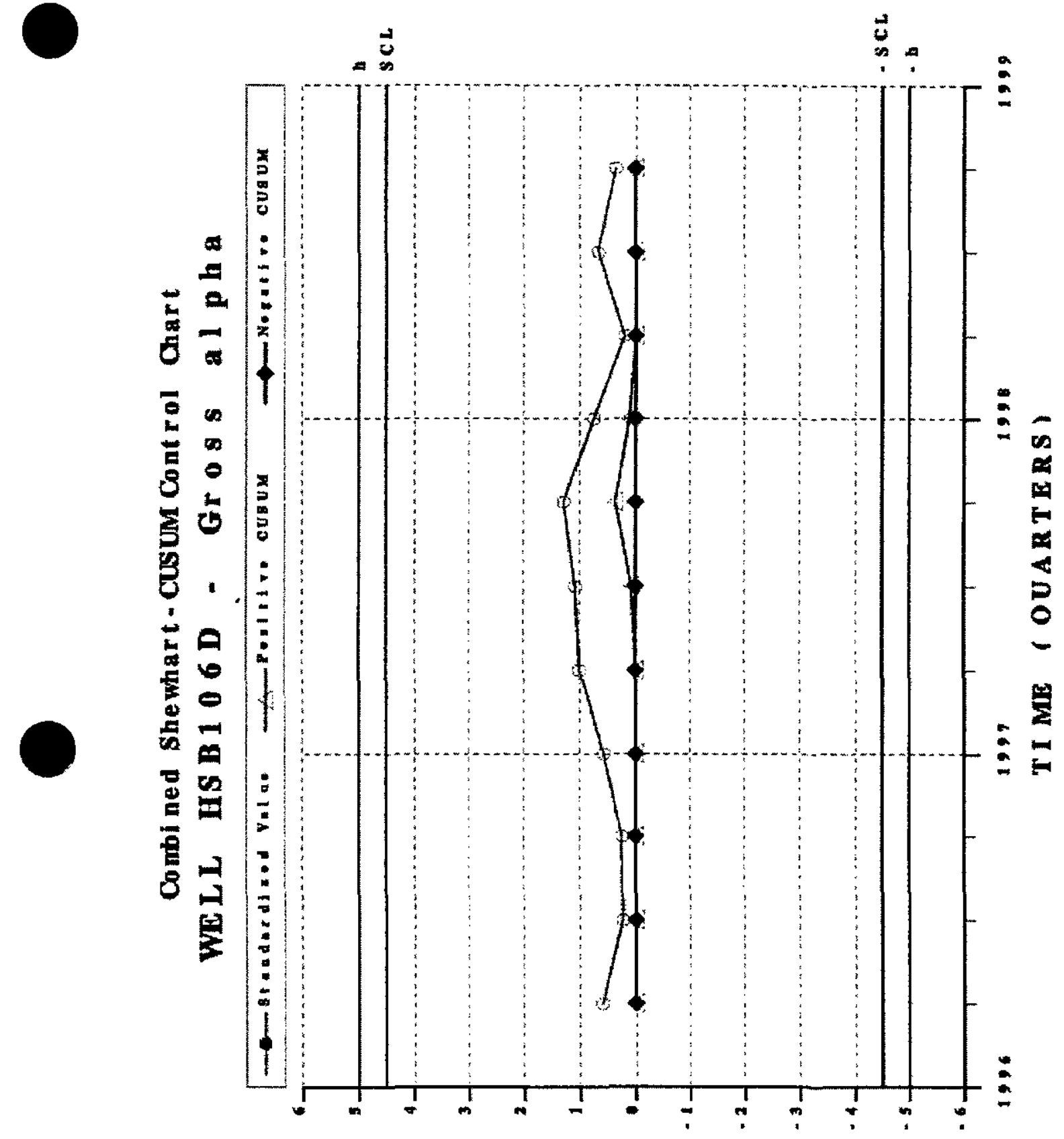

st I 
Conbi ned Shewhart - CuSUM Cont rol Chart VaL HSB106D - Netrate-nitrite as nitrogen

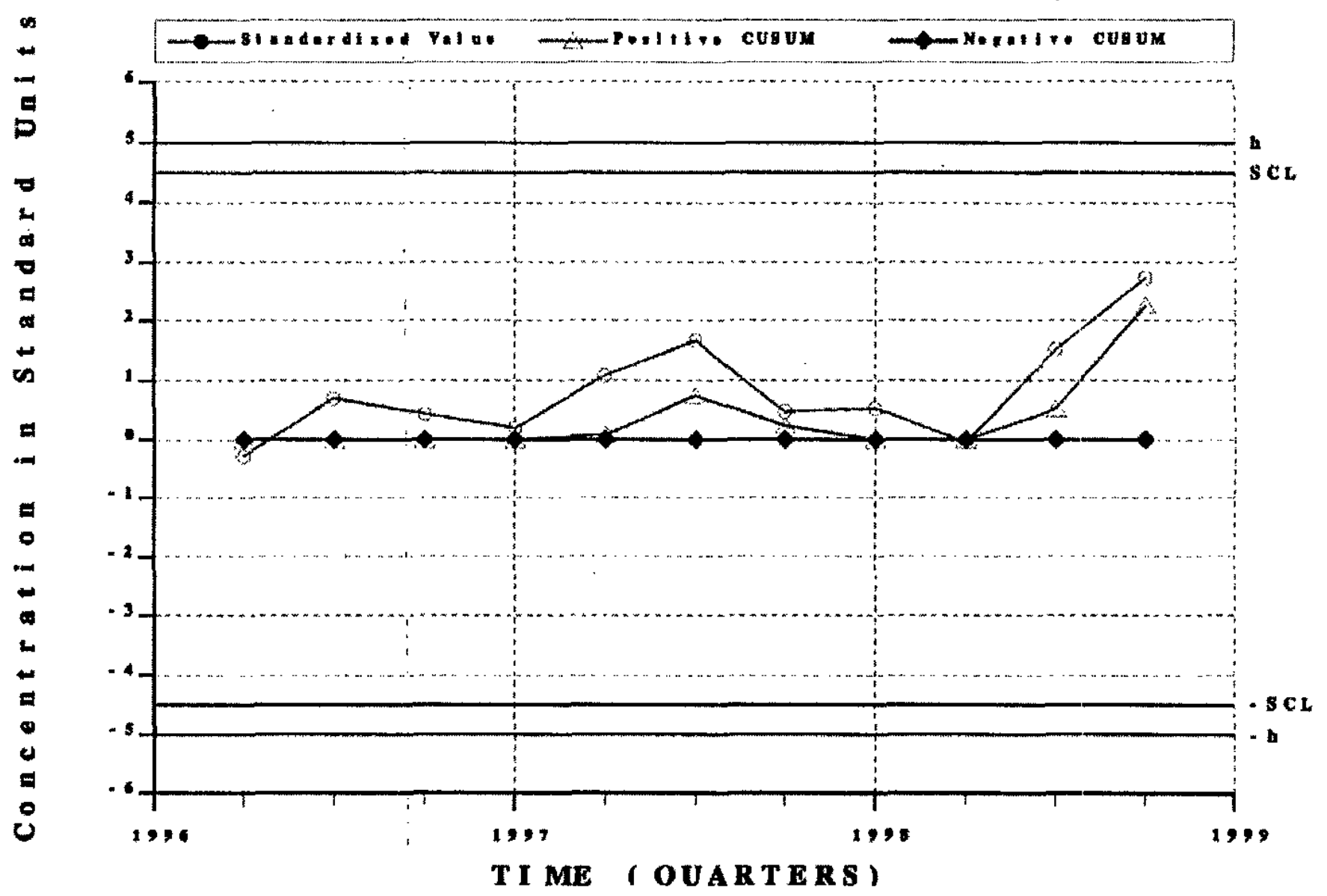




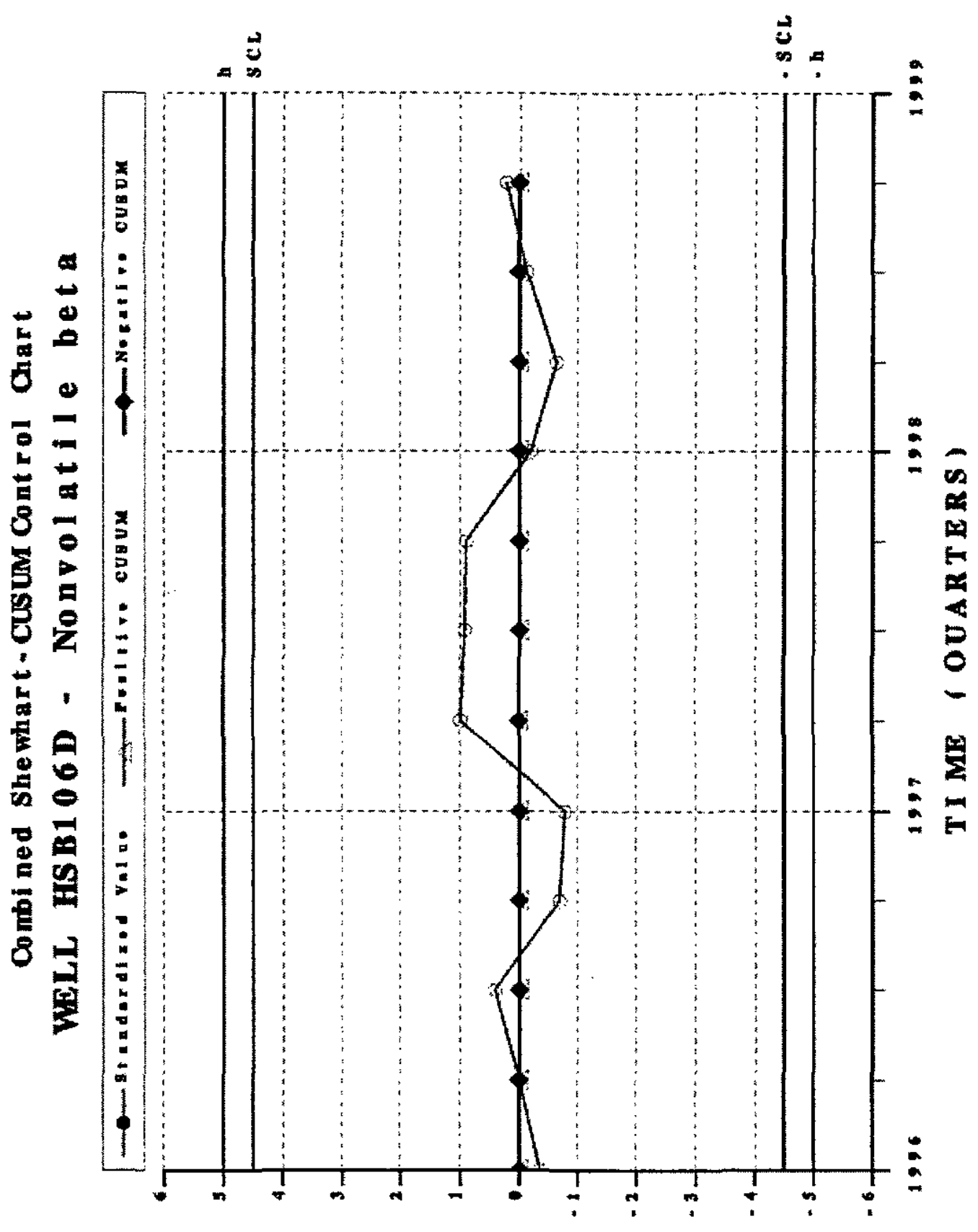

s I U $\mathbf{p}$ I 


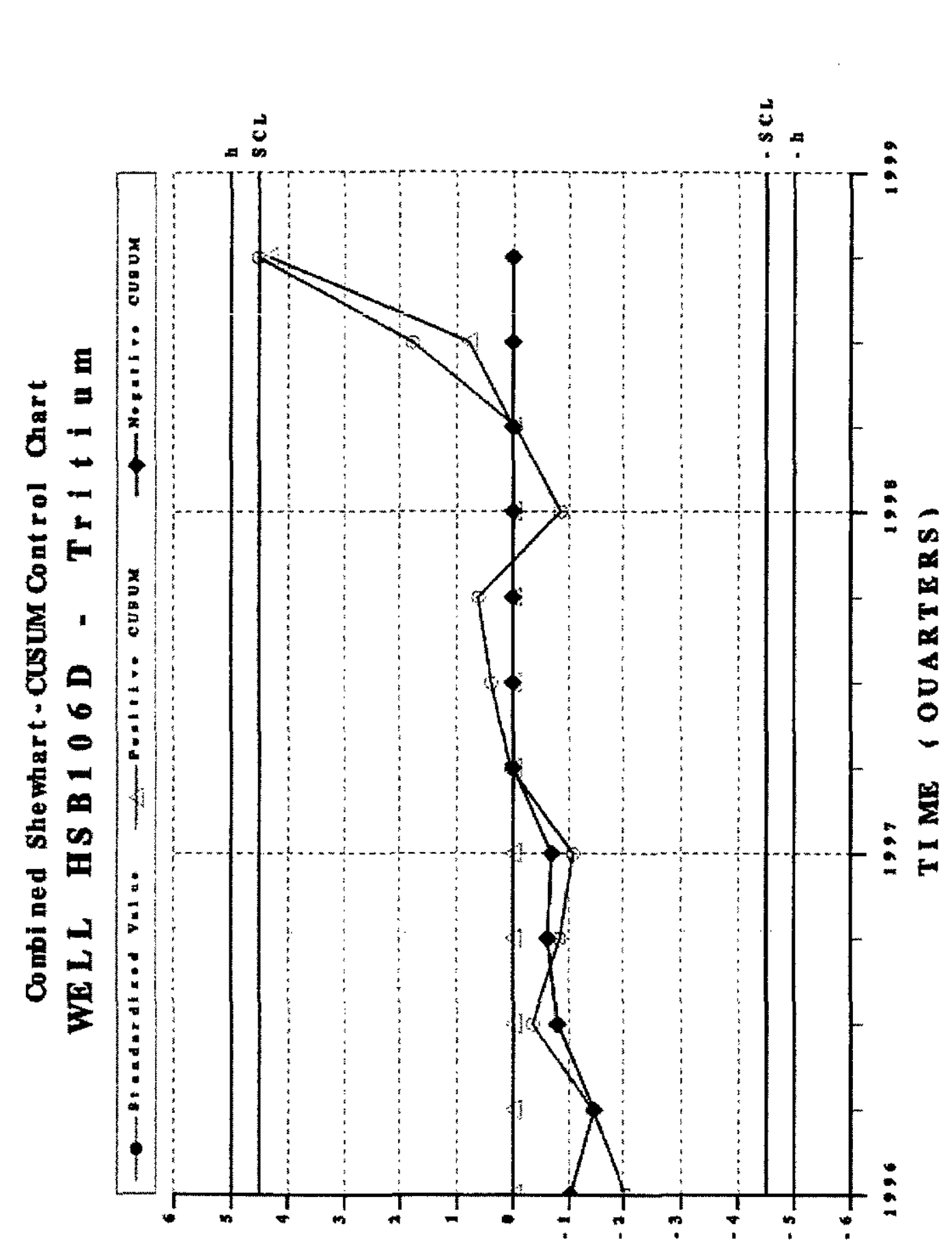

WSRC-TR-99-00013

Unclassified

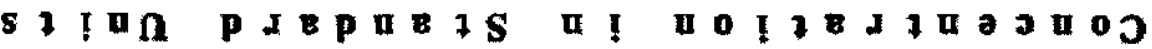




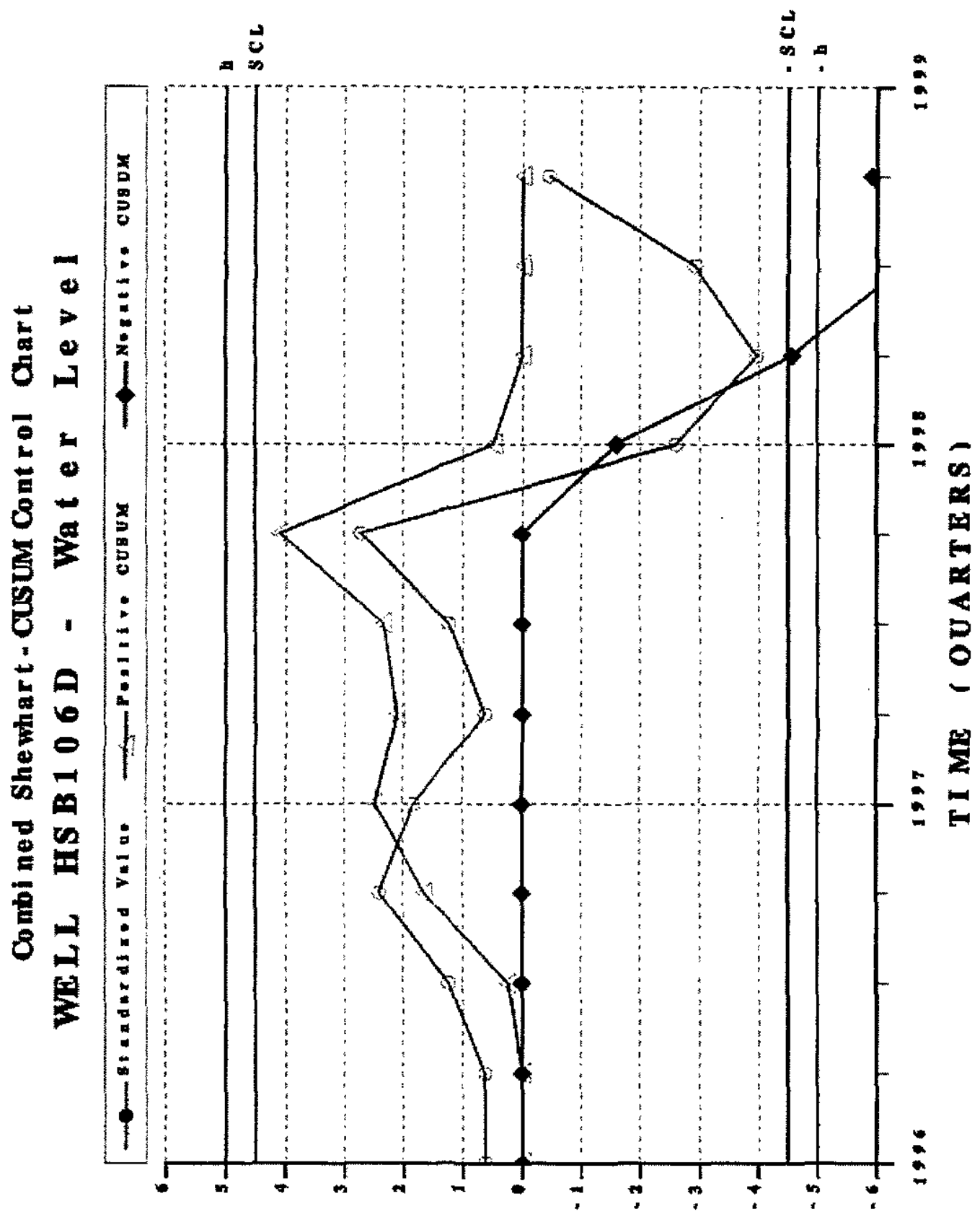

st tan papats 


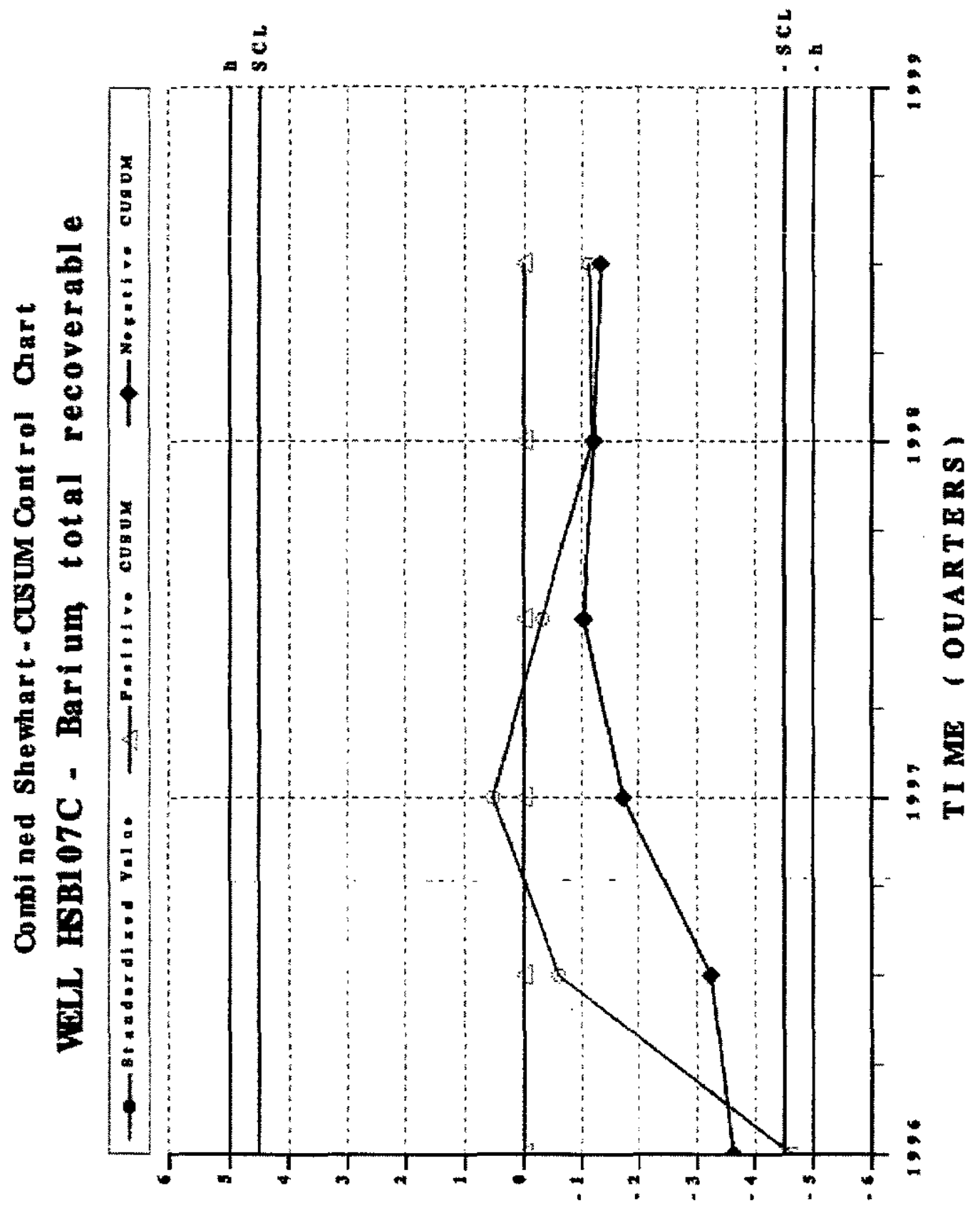

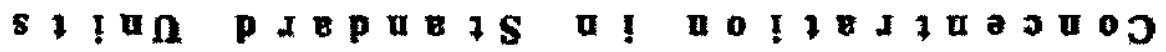




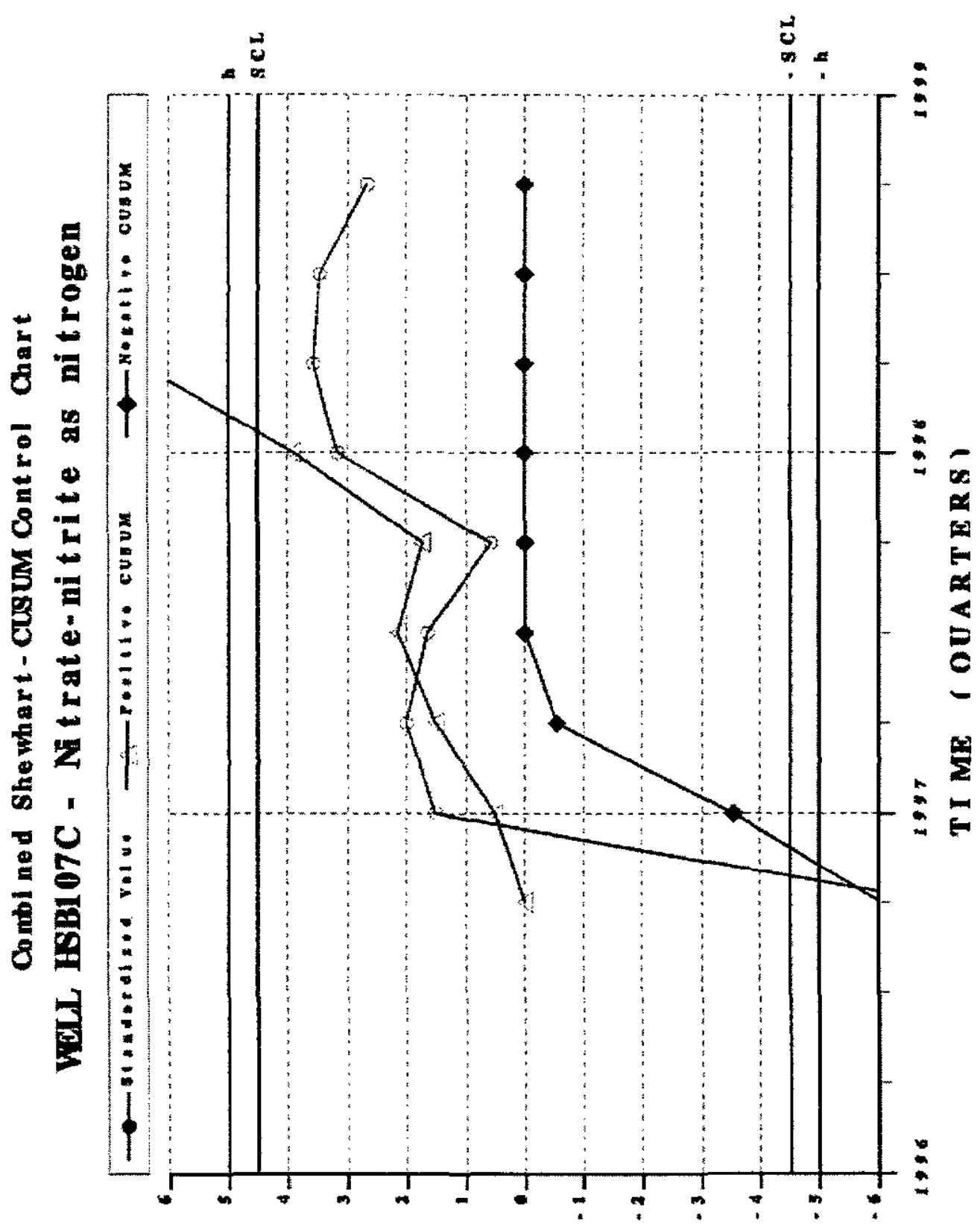

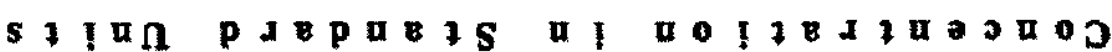


Combined She whart-Cusum Cont rol Chart

WELL HSB1 07C - Nonvolatile beta

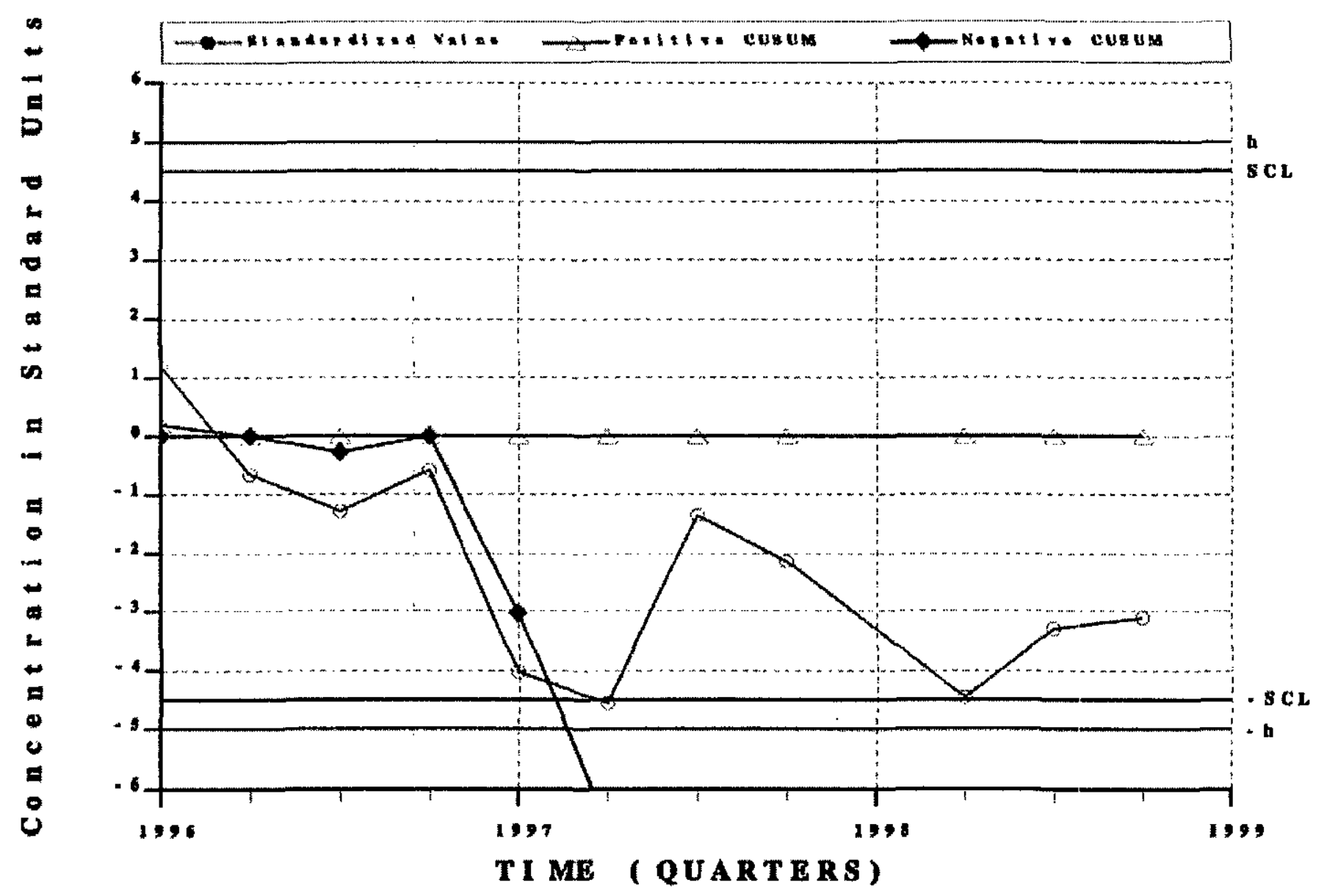

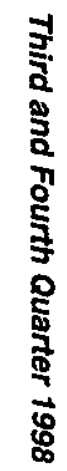


Combi ned She whart - Cusum Cont rol Chart

WELL HSB1 07C - Tet rachl oroet hyl ene

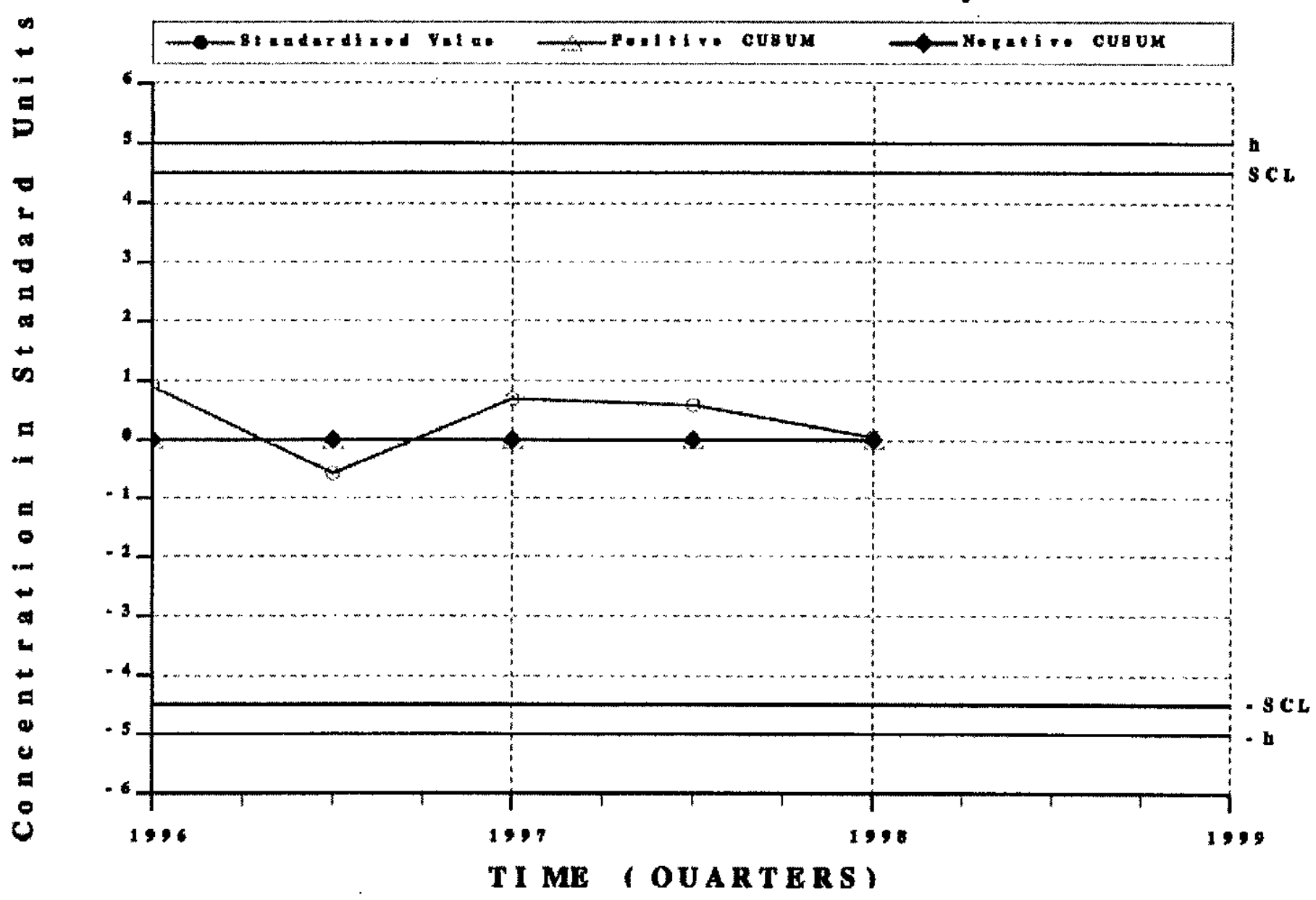


WELL HSB107C - Ti n, t ot al recoverable

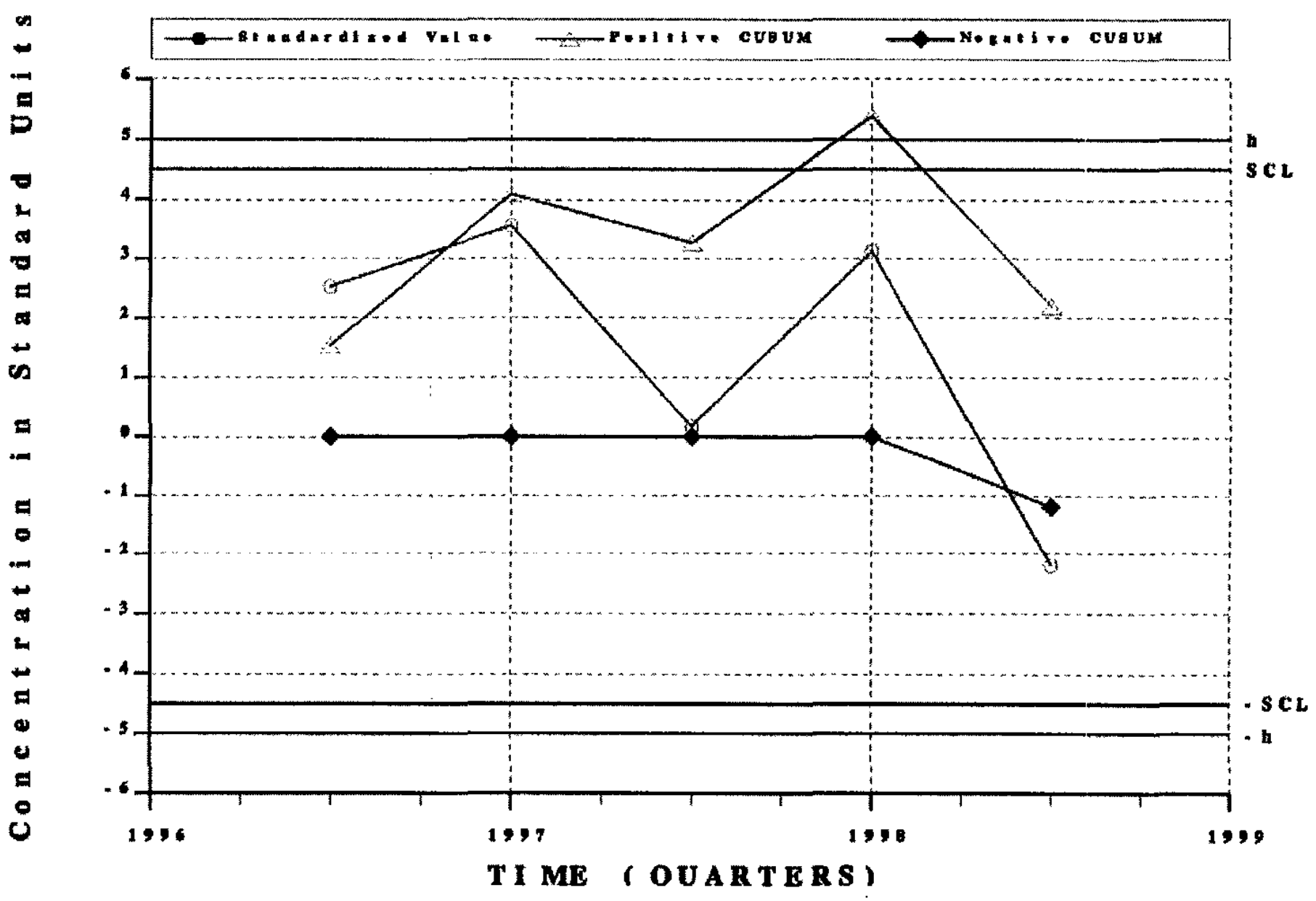

T I ME (OUARTERS) 


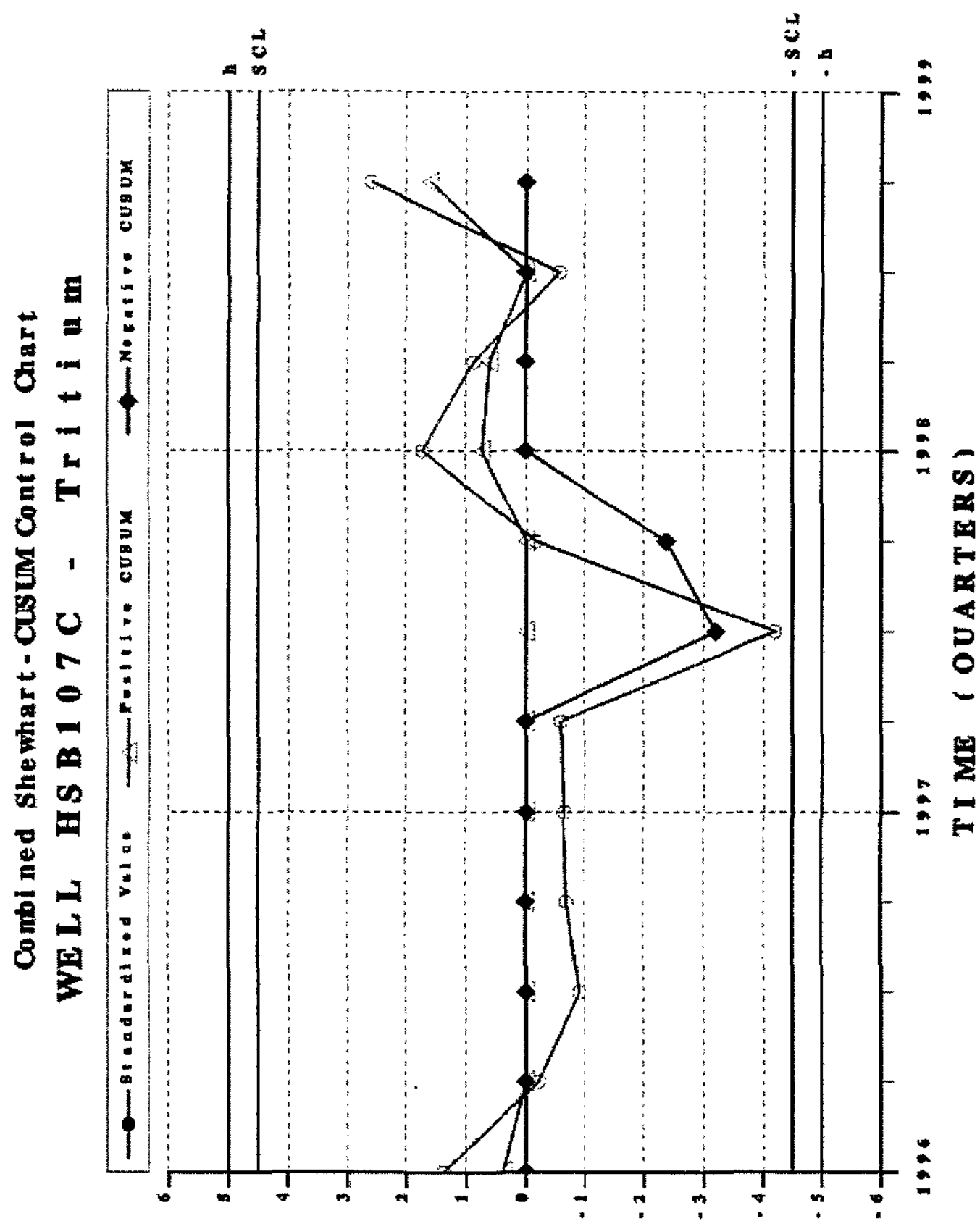

s! Un pa 


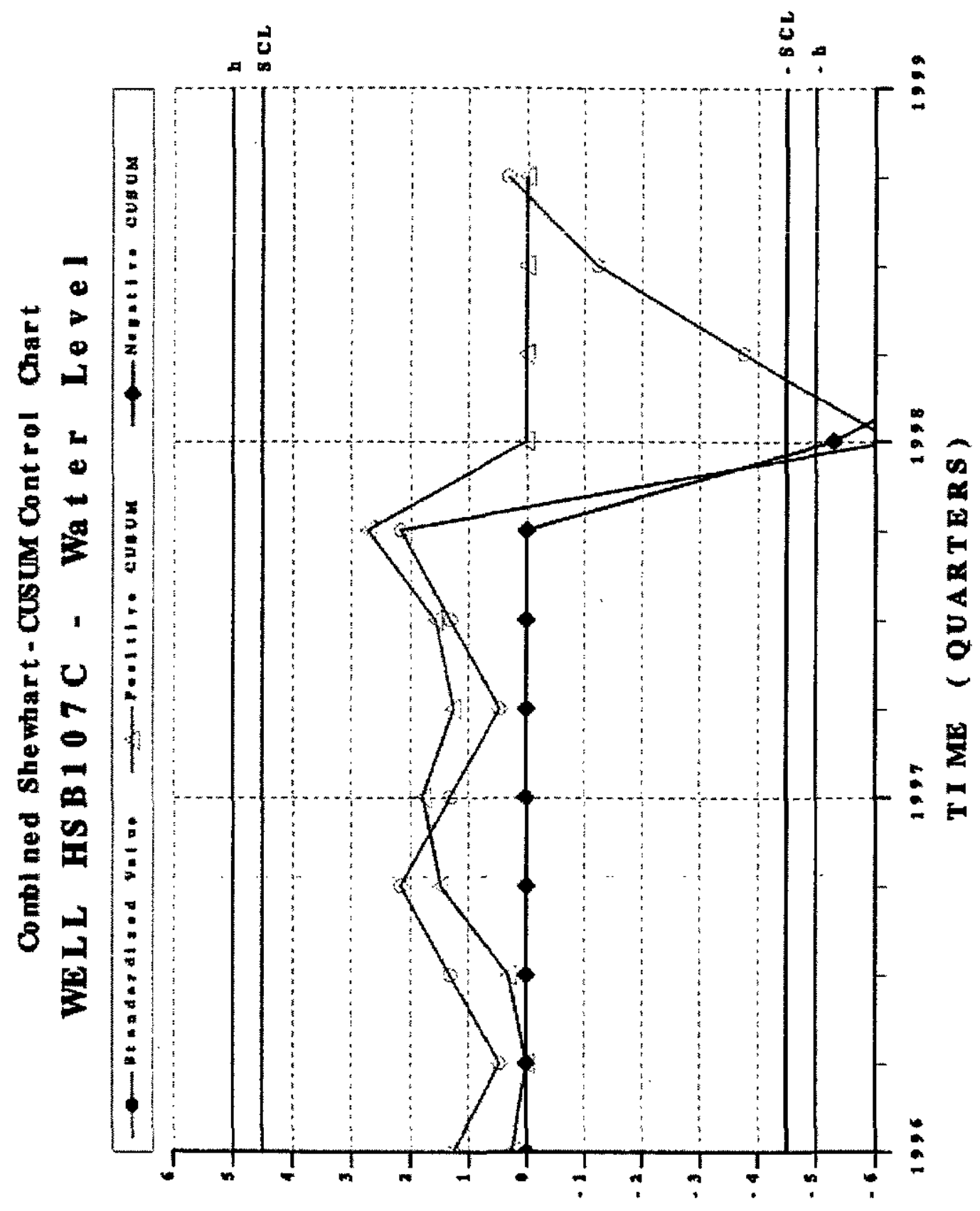

s) I п 


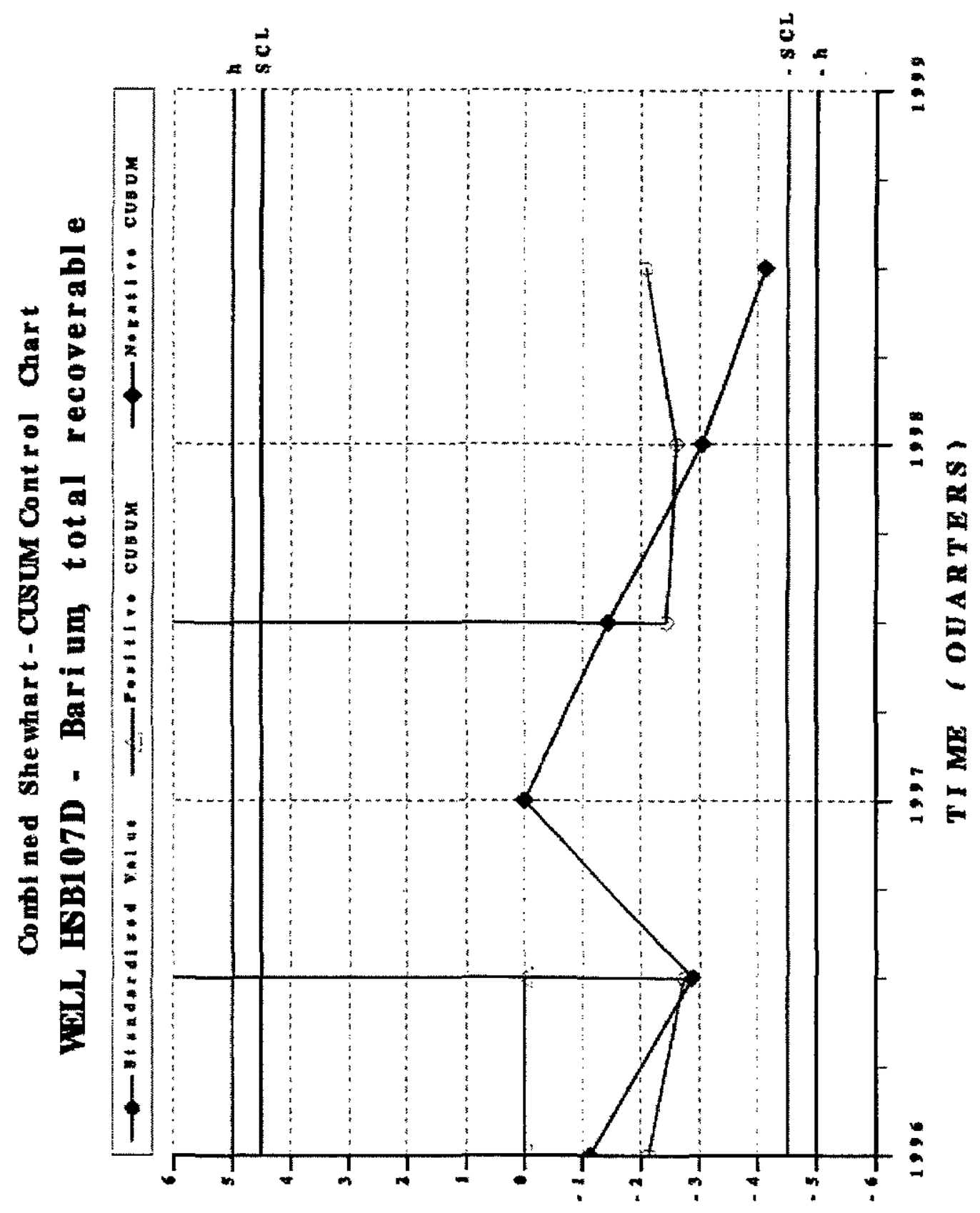

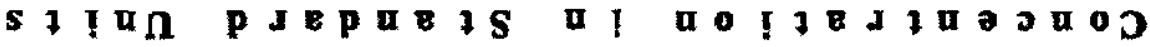



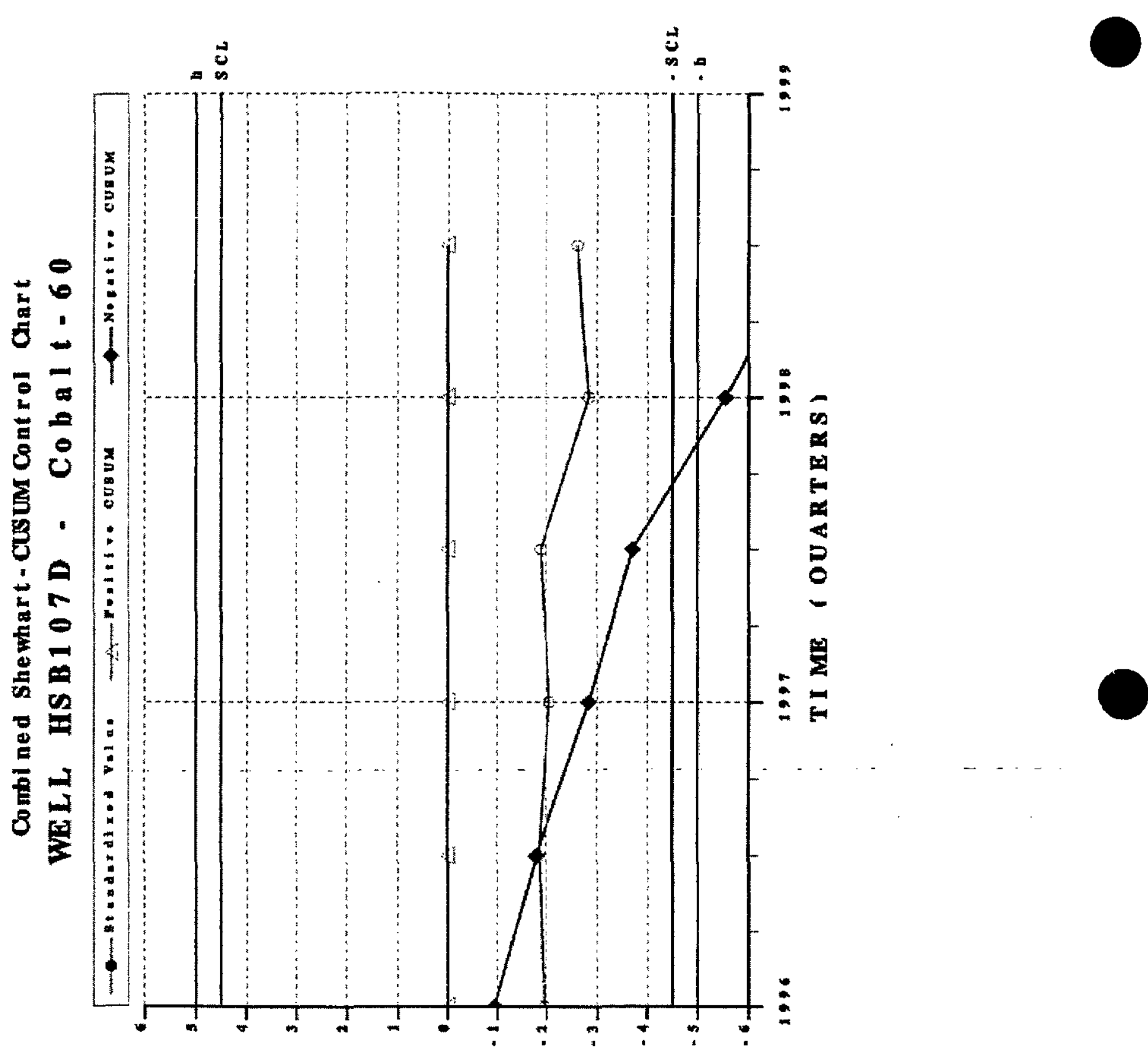
Combi ned She whart-Cusum Cont rol Chart

WELL HSB 107 - Gross a 1 pha

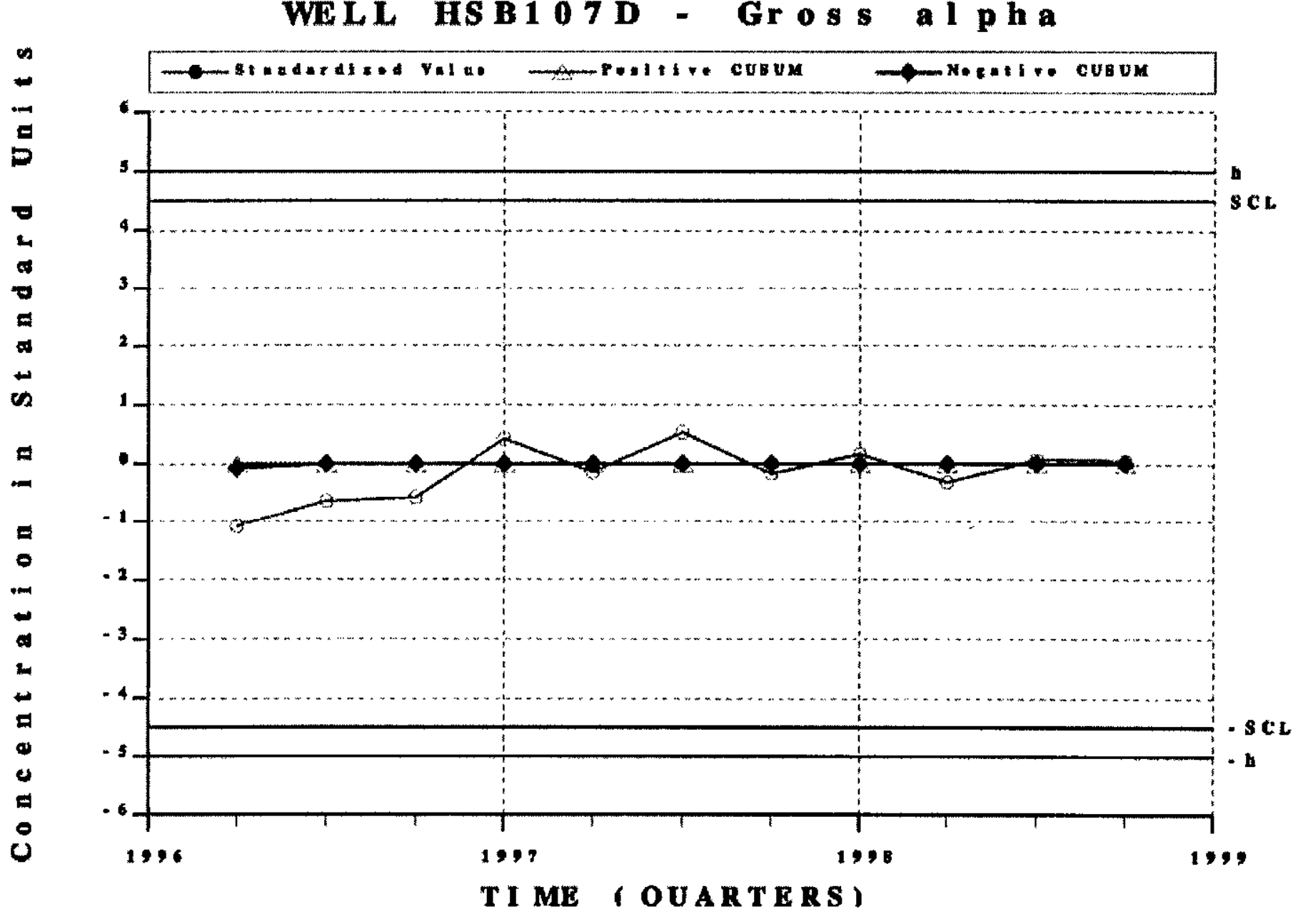



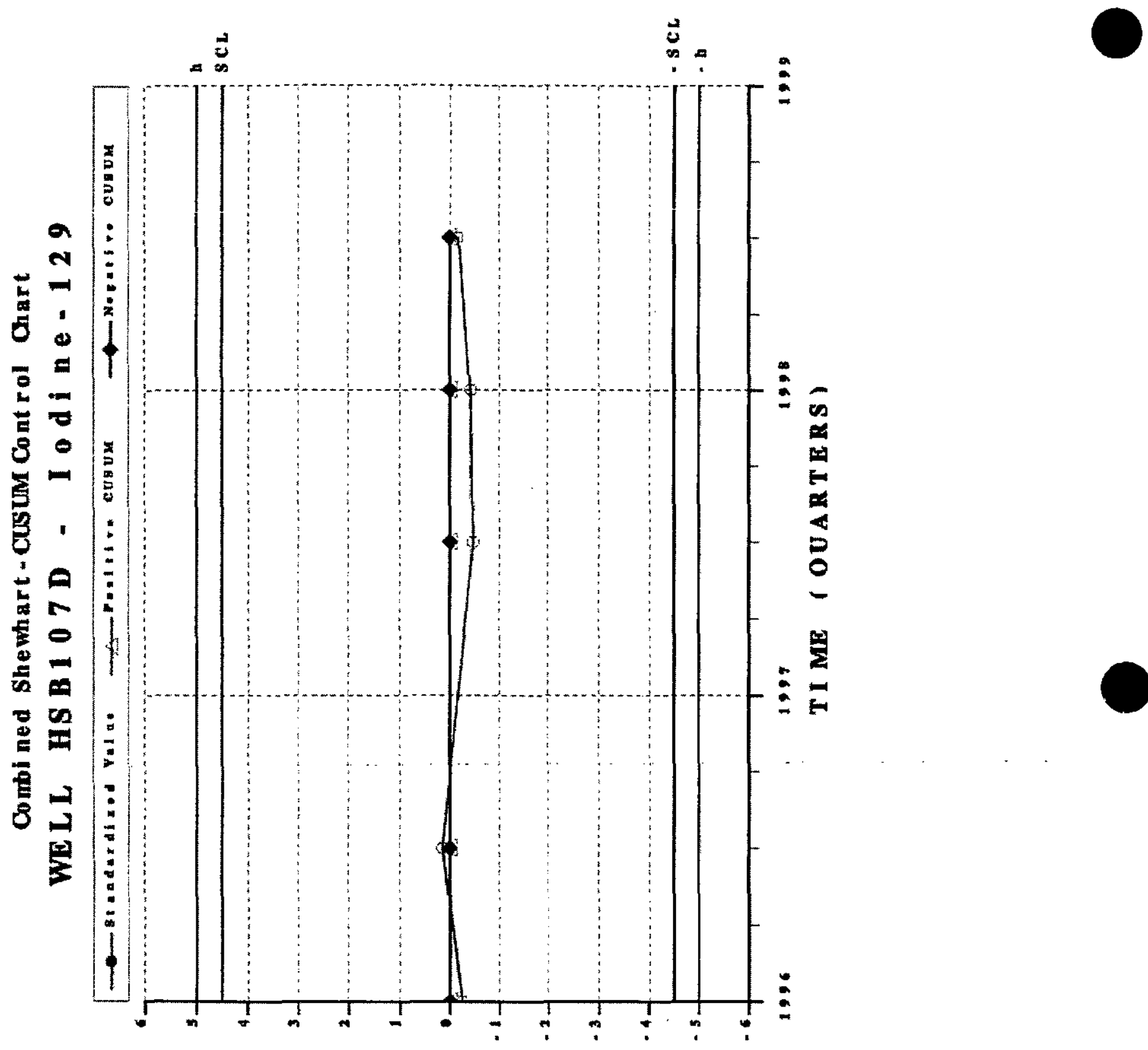
Combi ned Shewhart - CuSUM Cont rol Chart WaL HSB107D - Mercury, total recoverable

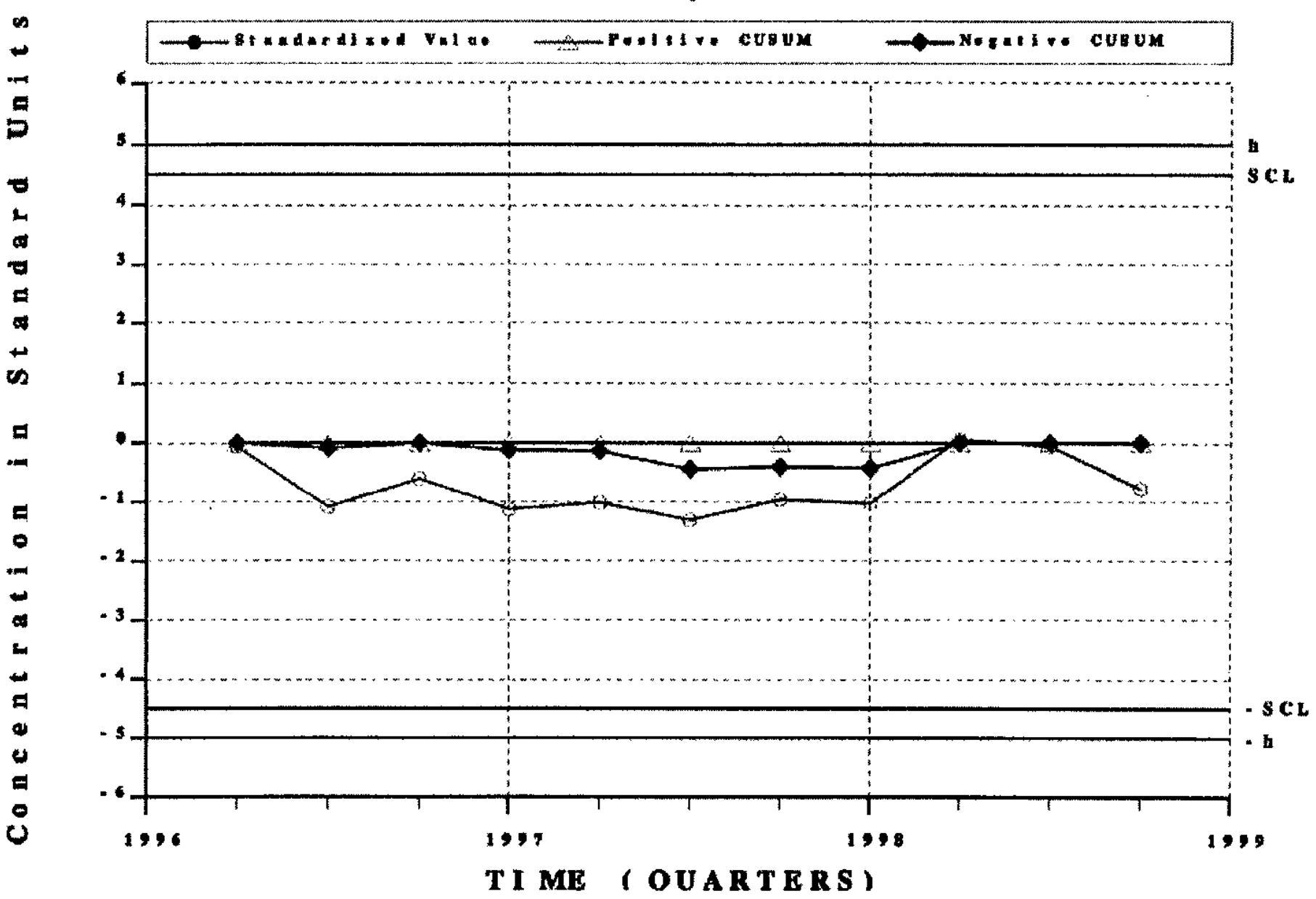




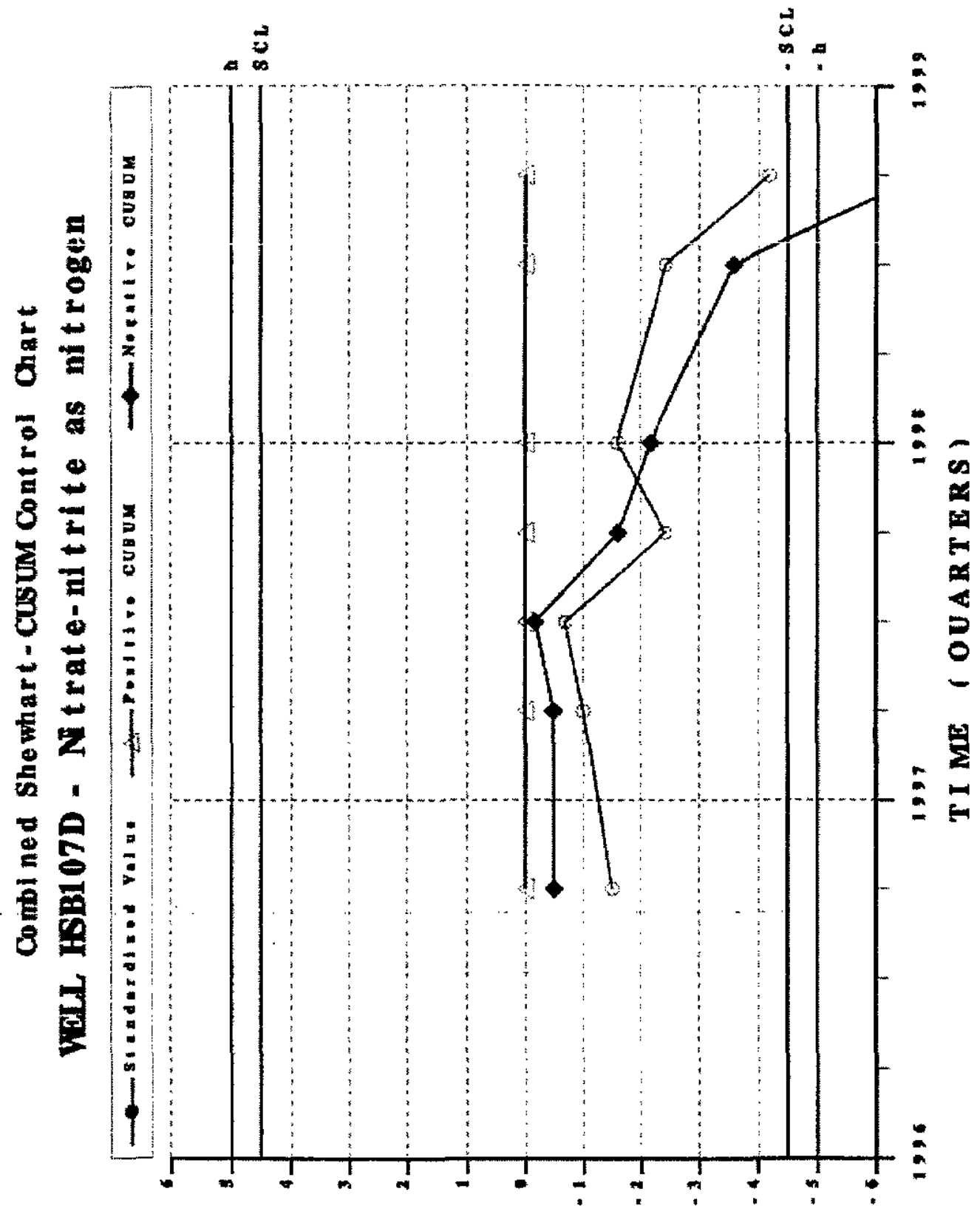

s I In p I E 


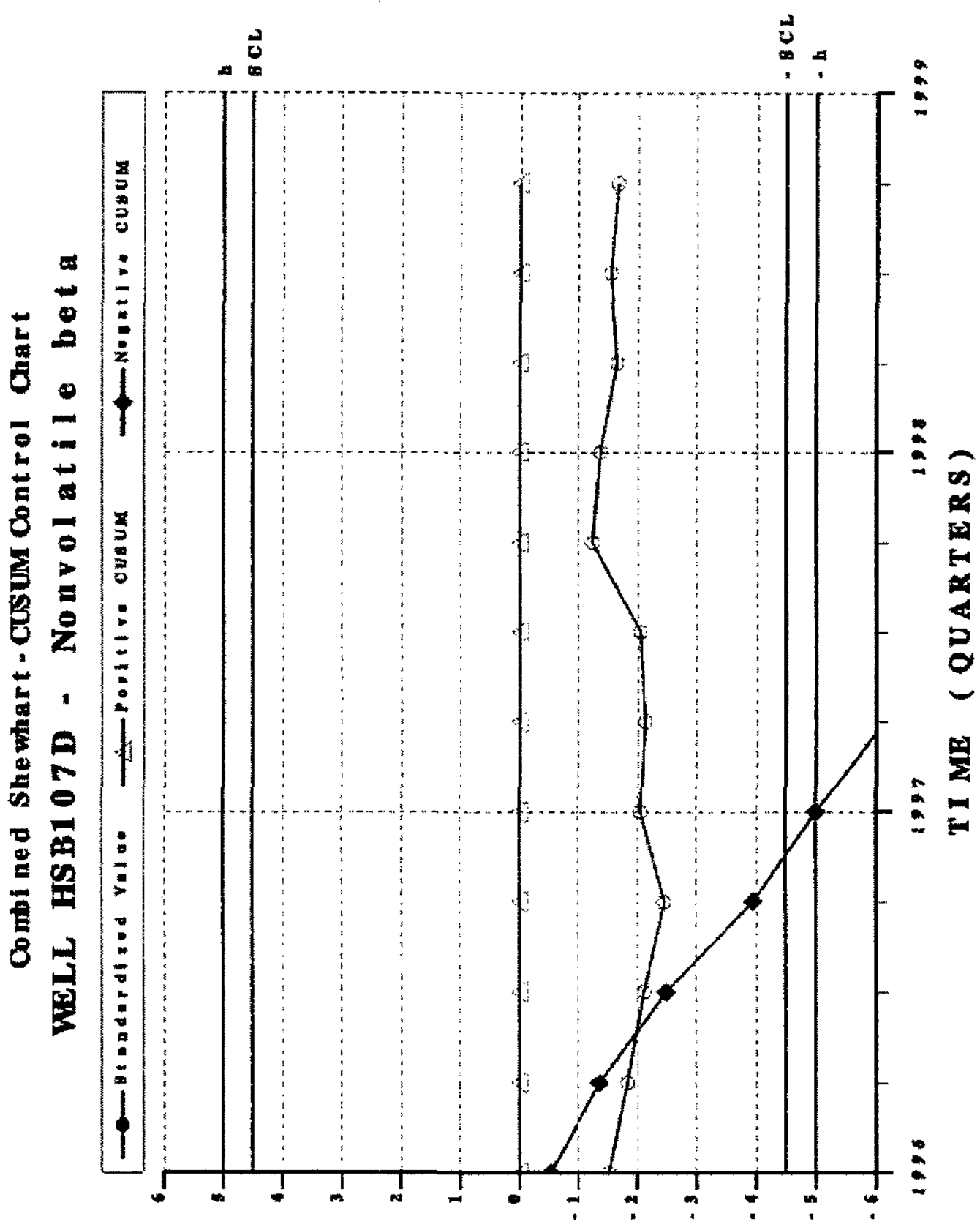

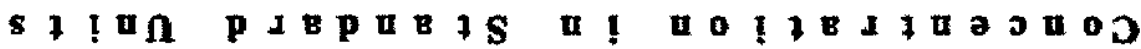




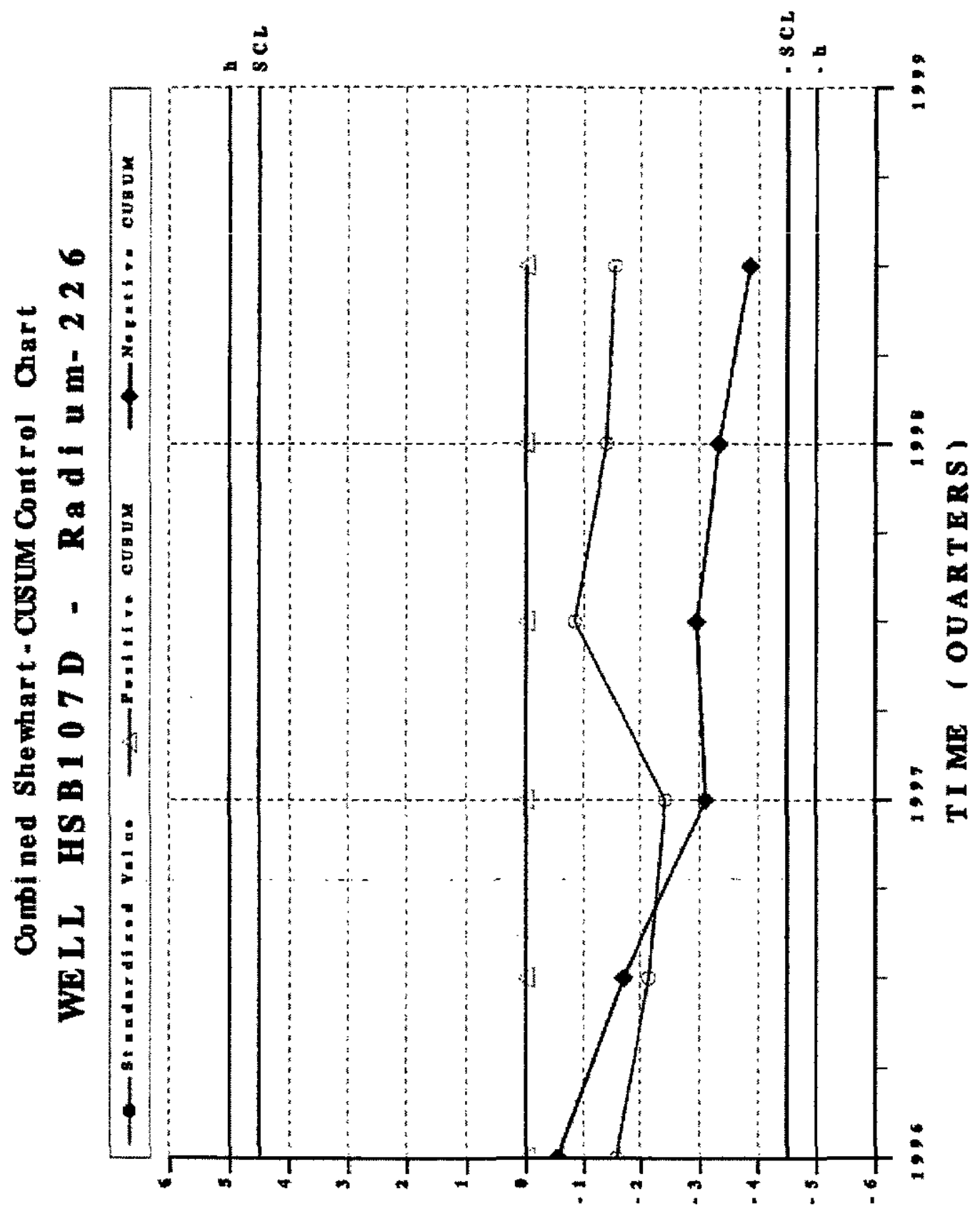

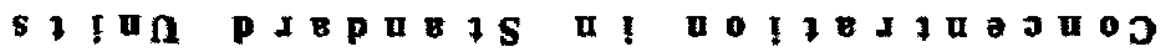




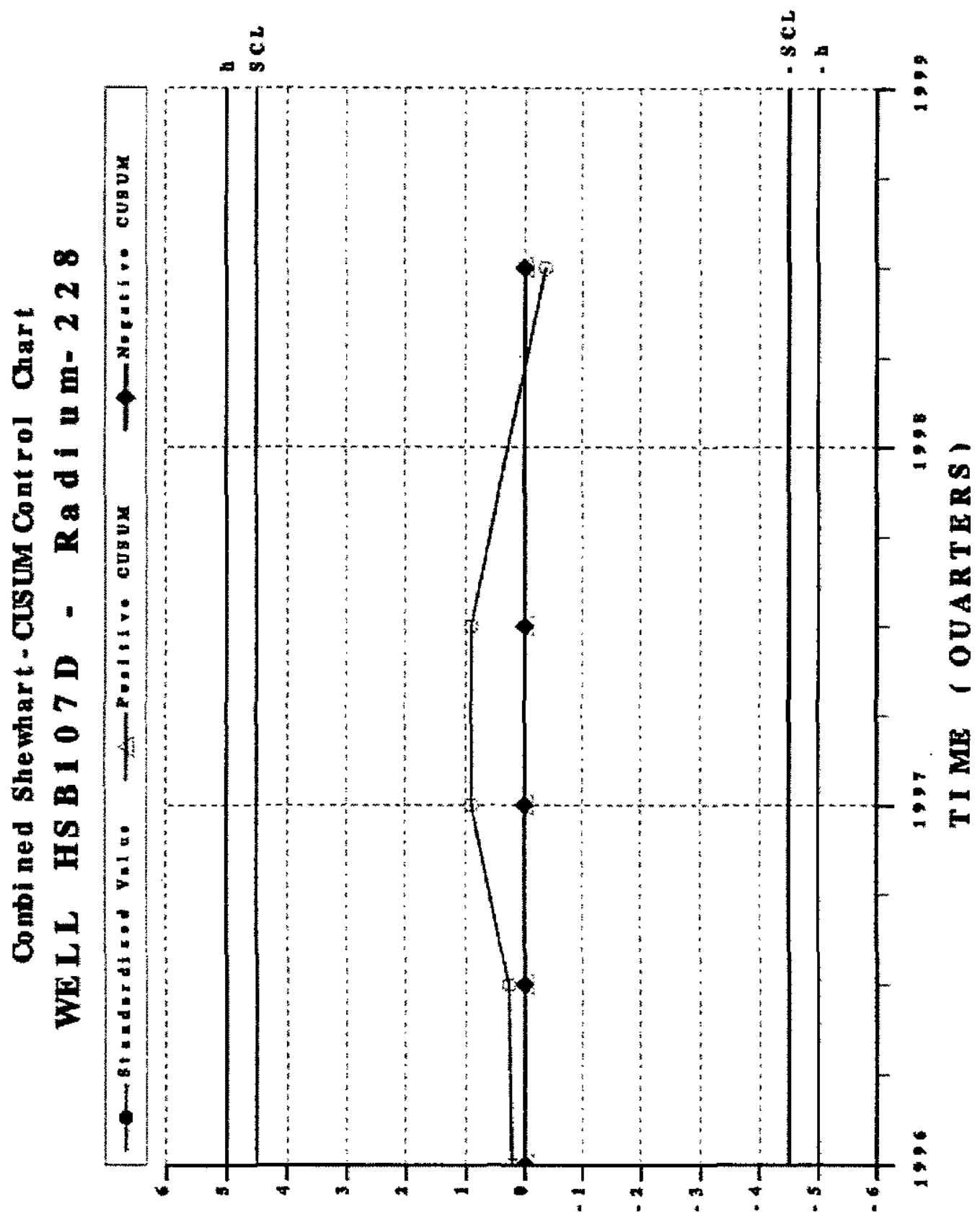

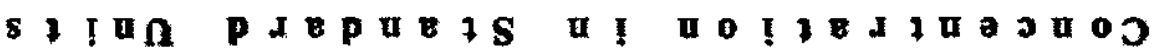




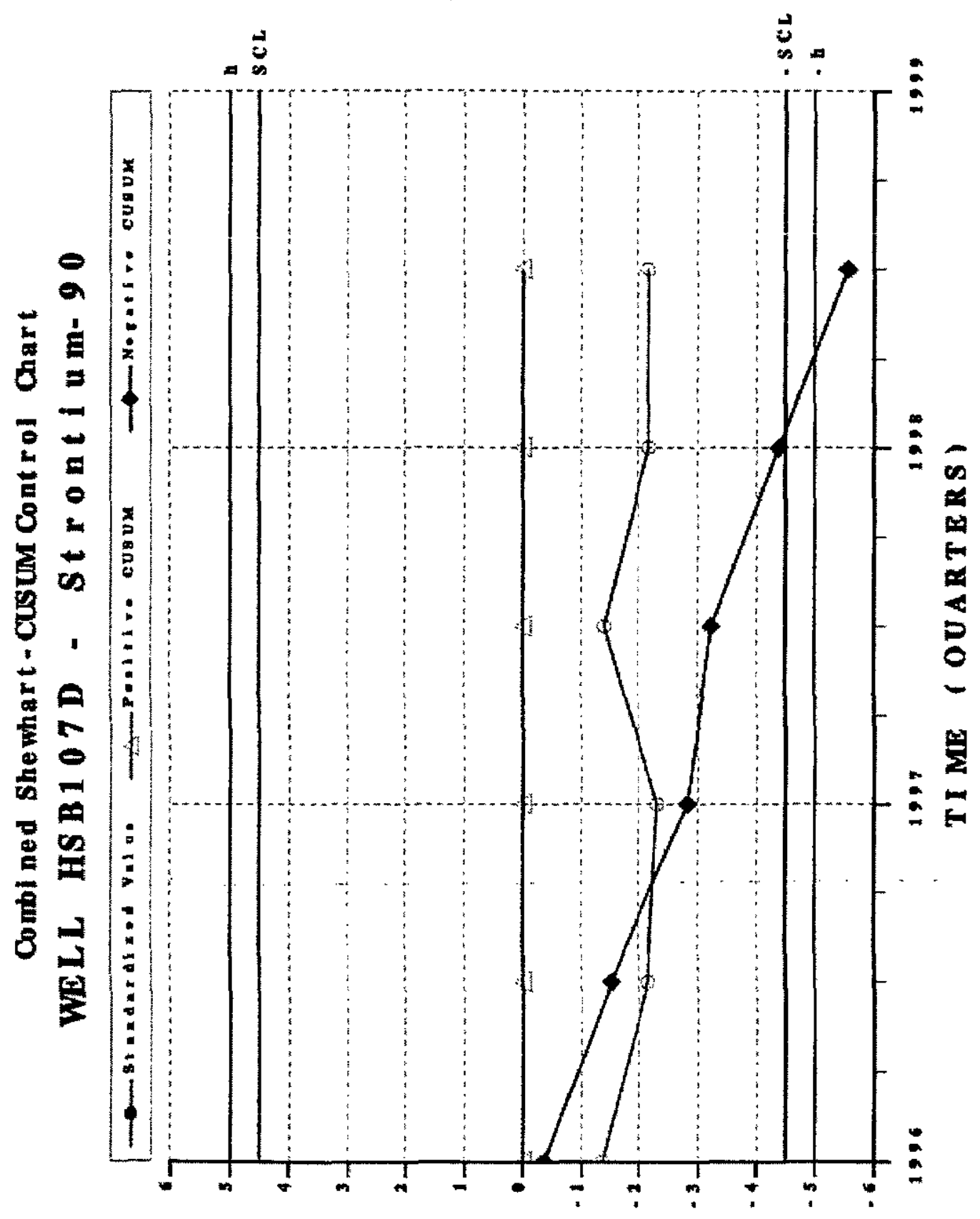

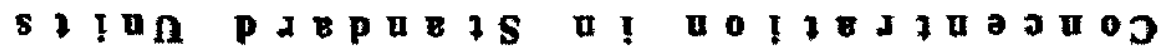




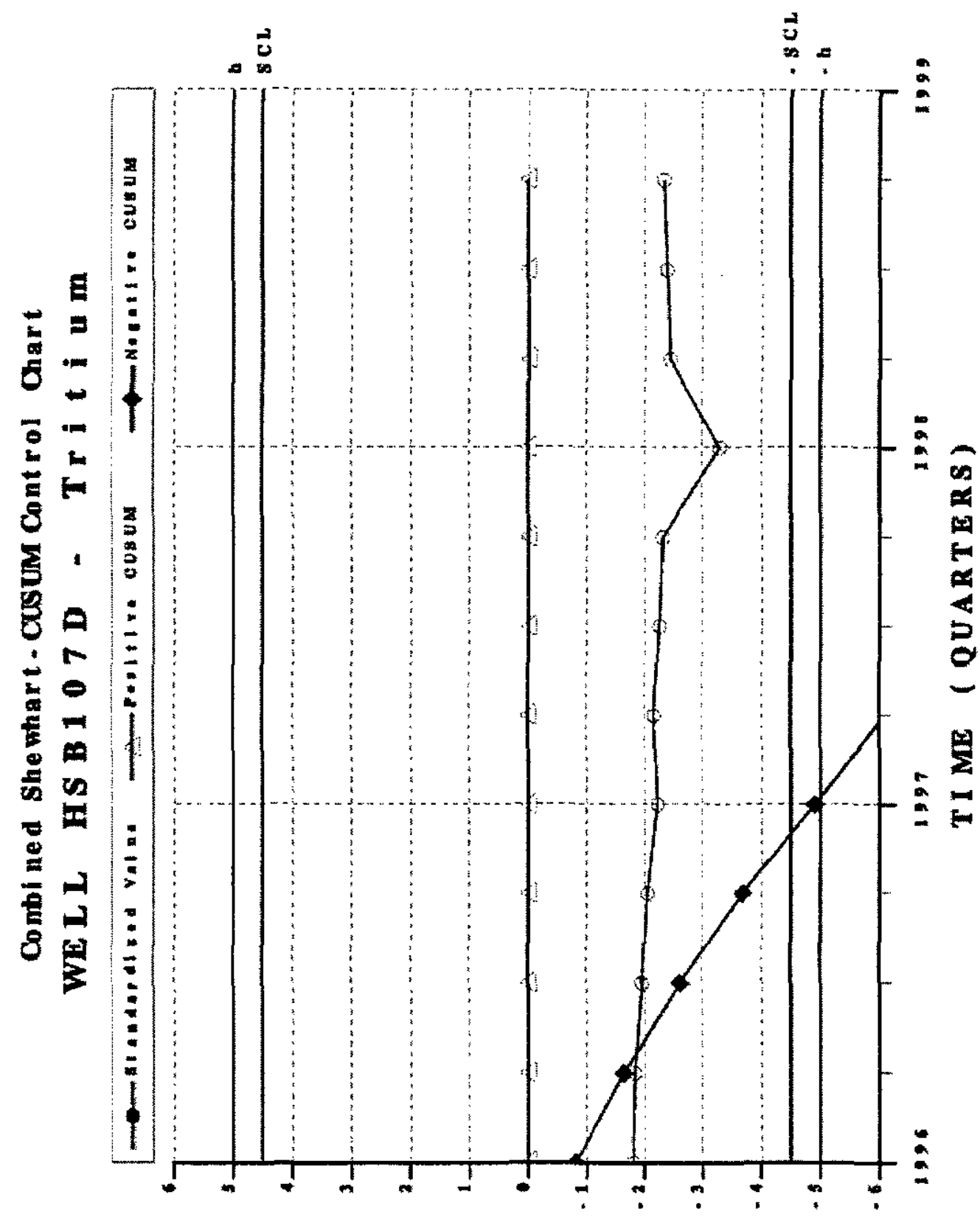

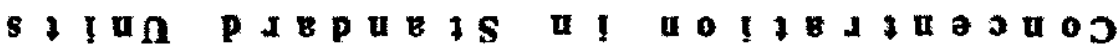


Combined Shewhart-Cusum Cont rol Chart

WELL HSB 107 - Water Level

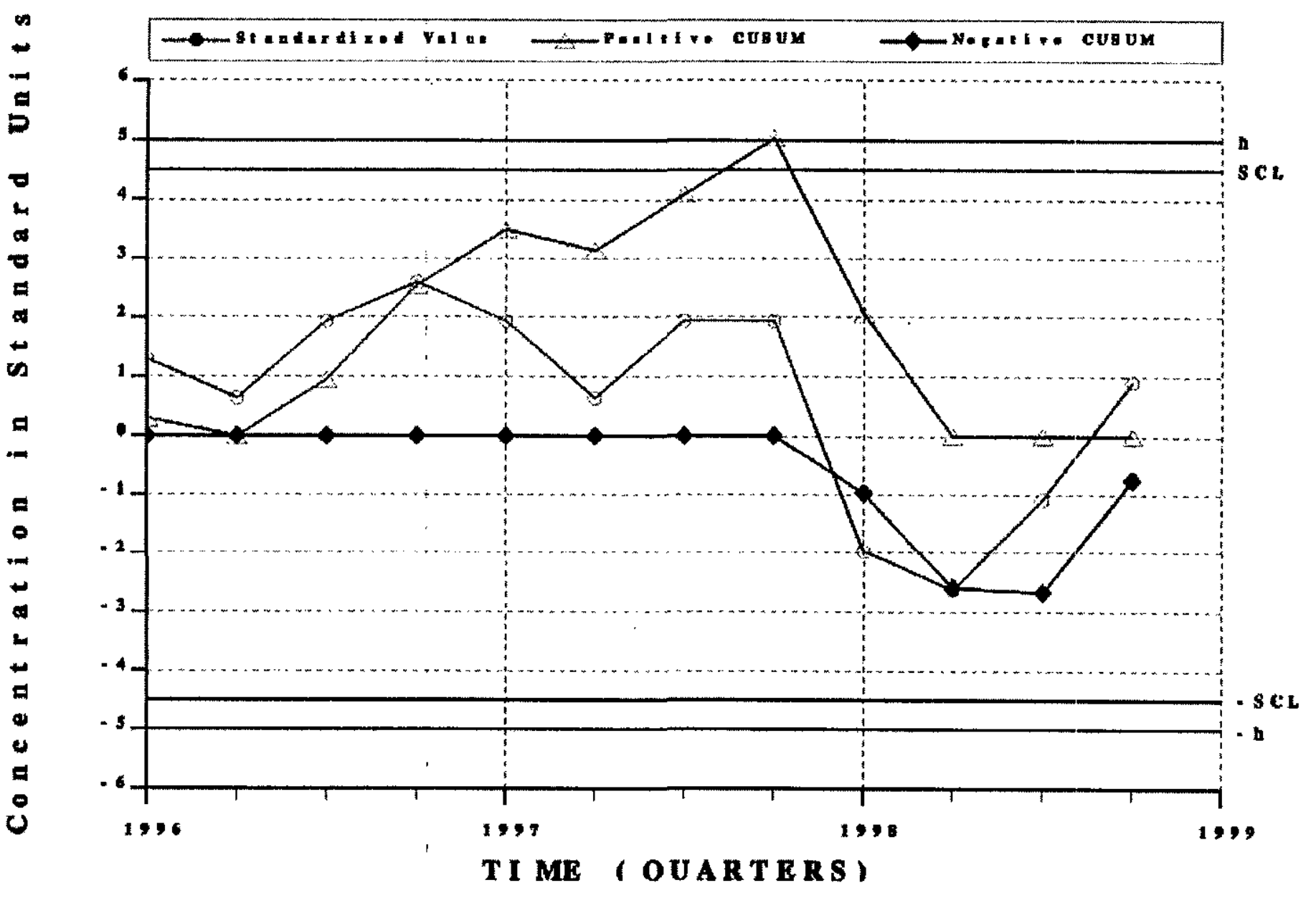

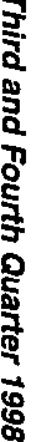


Combi ned Shewhart - Cusum Cont rol Chart

WEL HSB107D - Zi nc, total recoverable

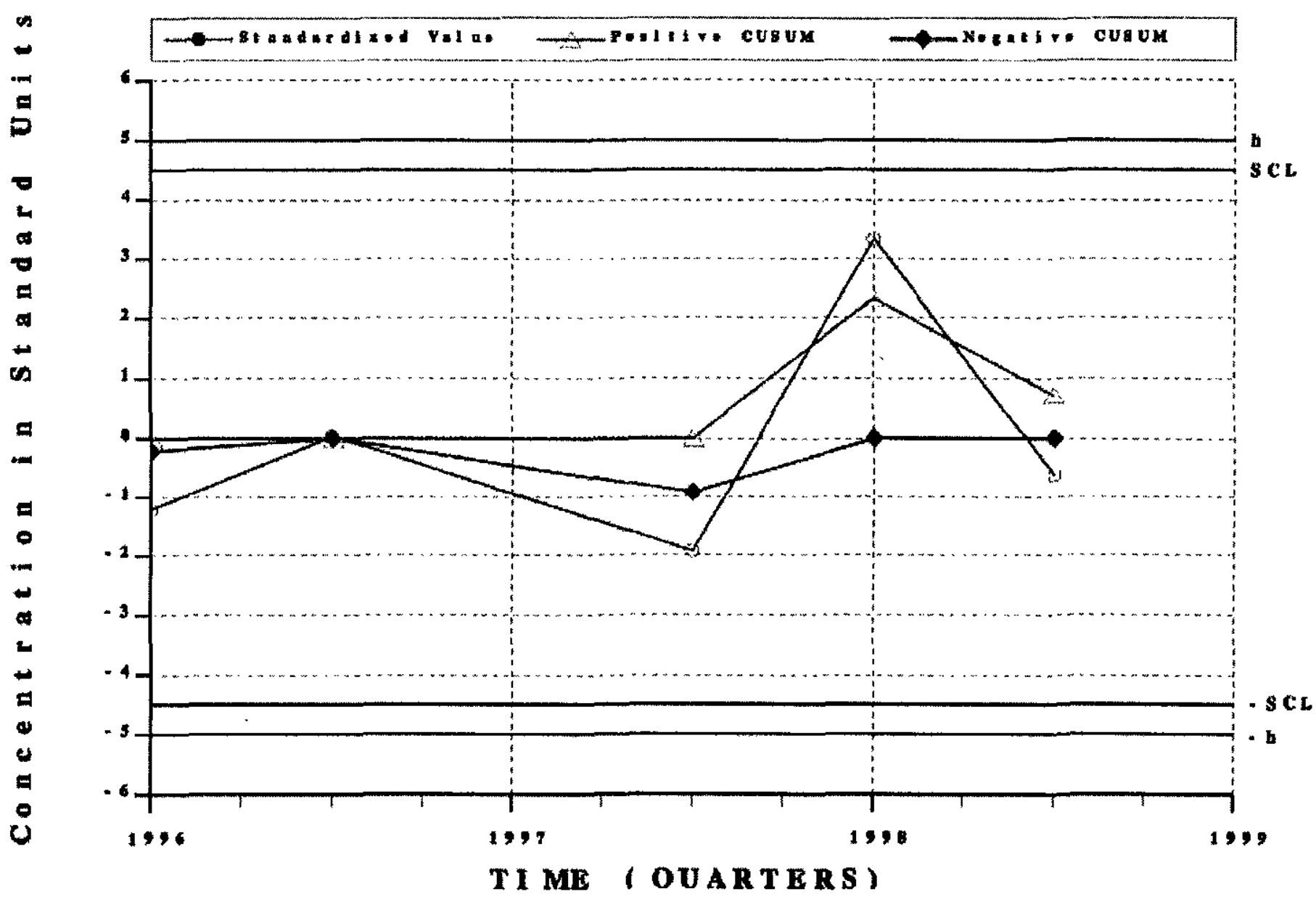

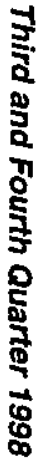




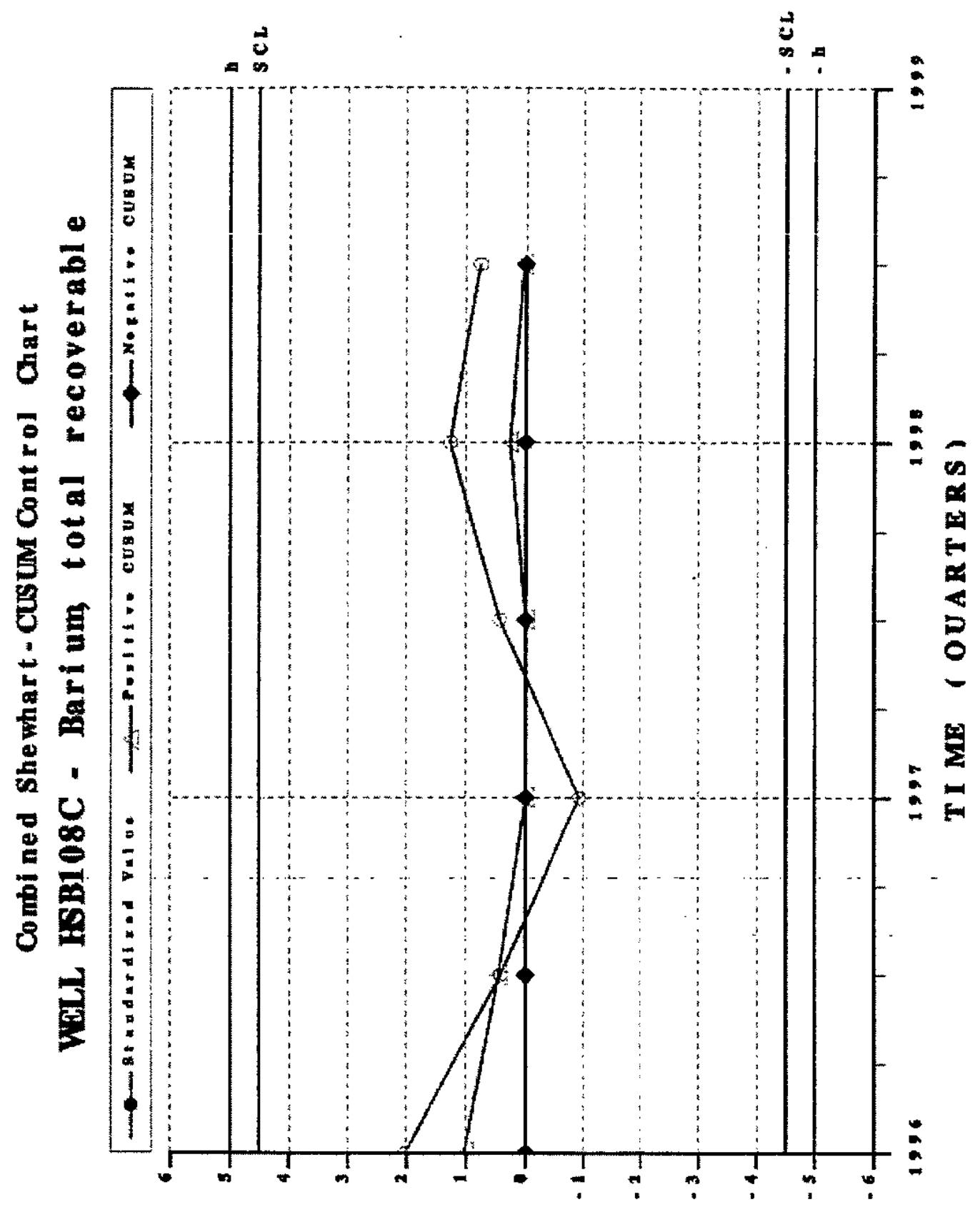

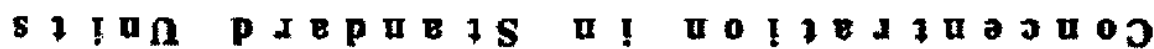


Combl ned Shewhart-Cusum Cont rol Chart

KoLL HSB108C - Nitrate-nitrite as nitrogen

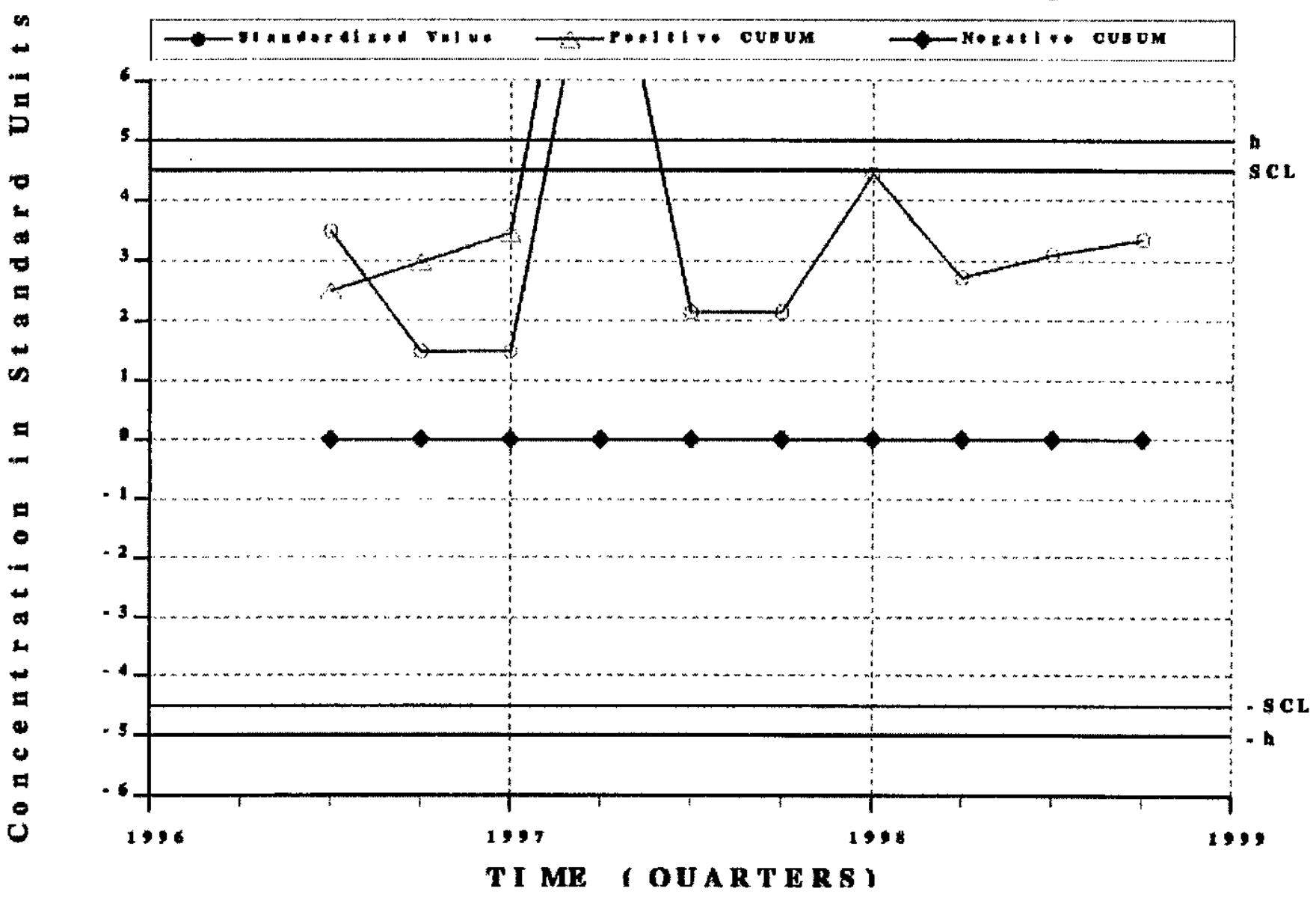

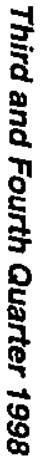


Combi ned Shewhart - CuSUM Cont rol Chart WELL HSB1 $08 \mathrm{C}$ - Nonvolatile beta

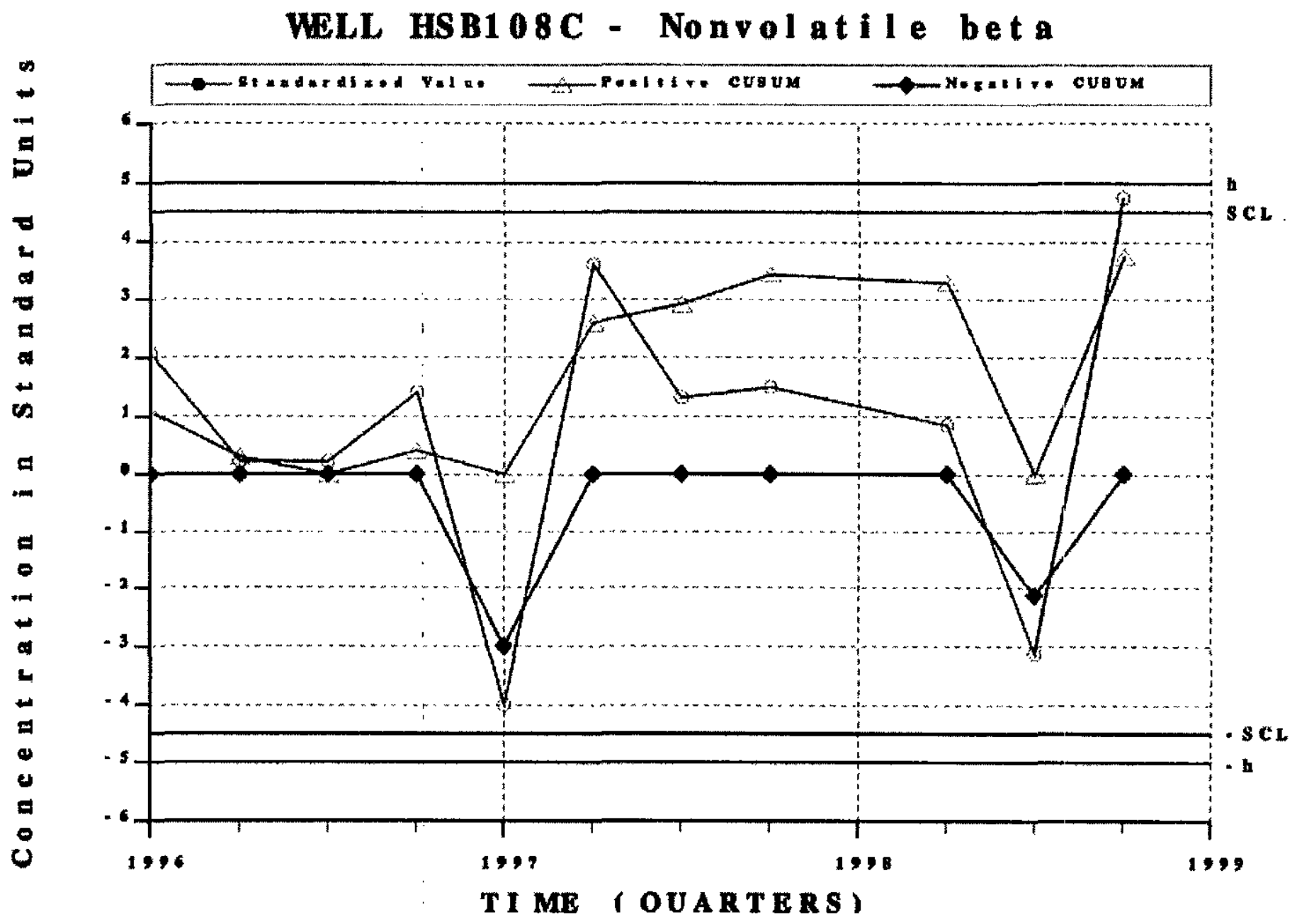




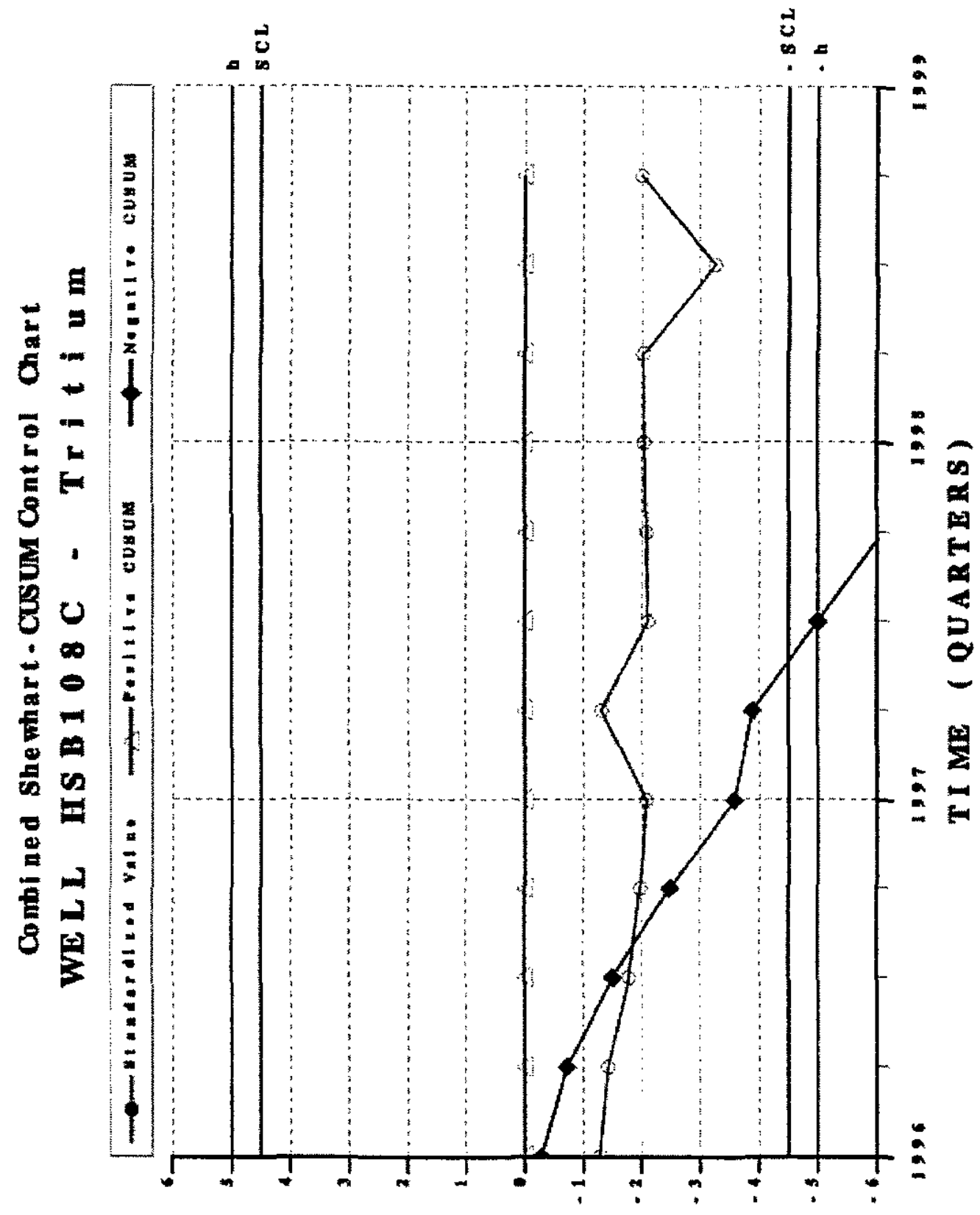

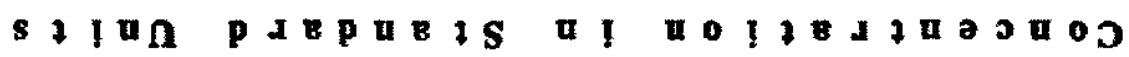




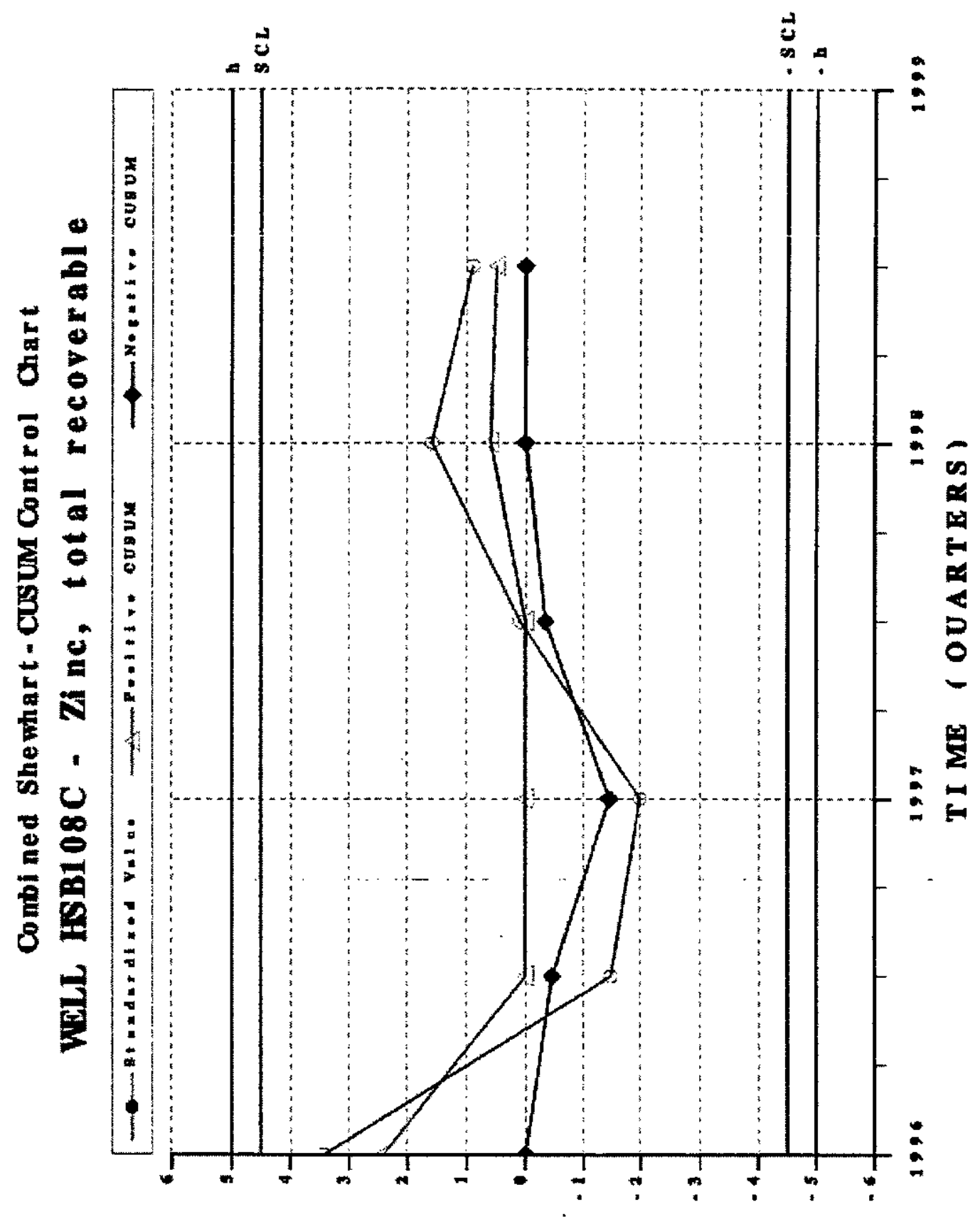

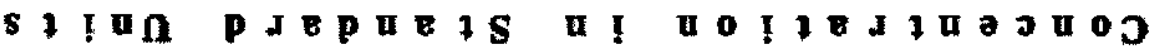



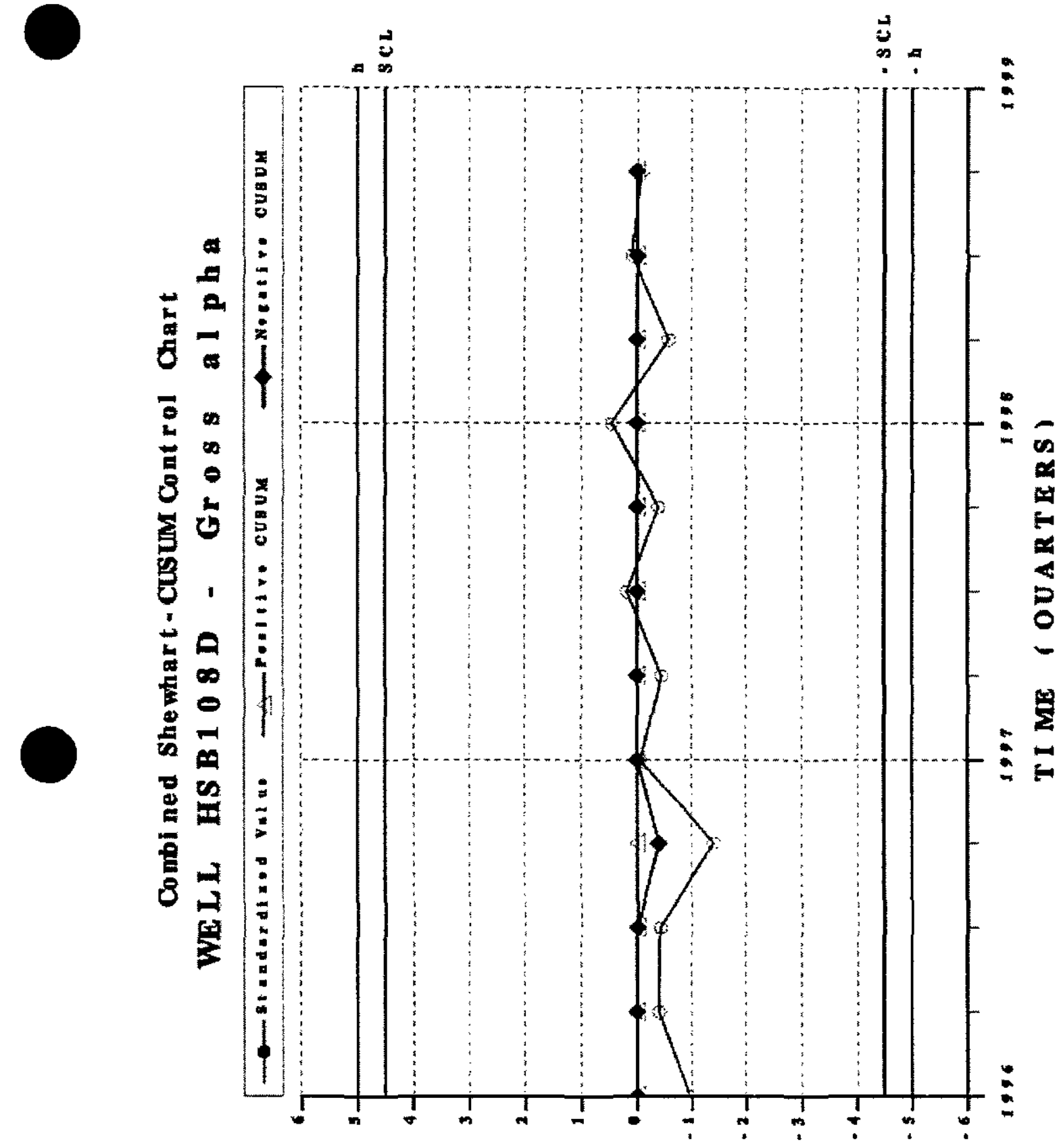

s! I 


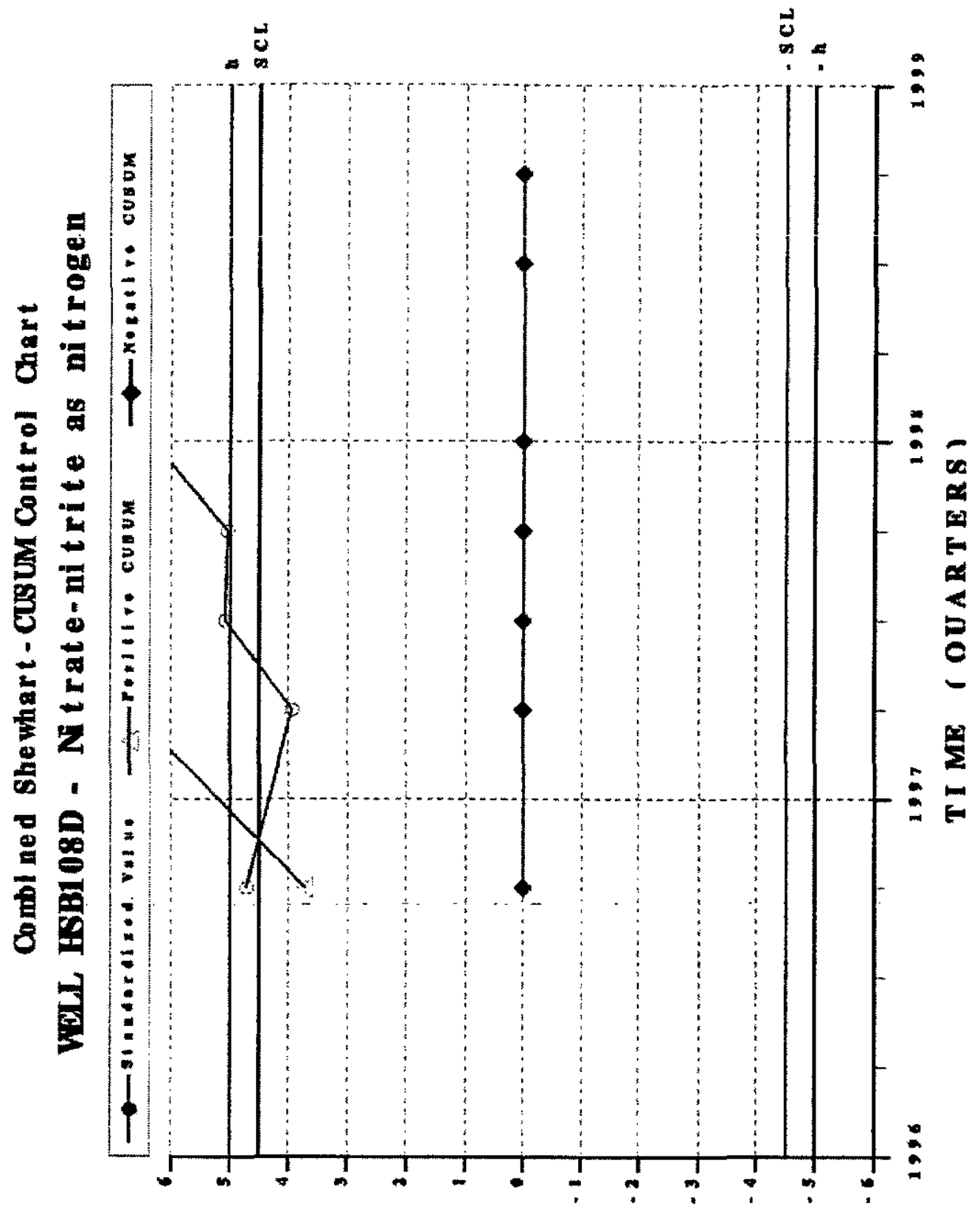

s I 1 I 
Combl ned She whart - Cusum Cont rol Chart

WELL HSB108D - Nonvolatile beta

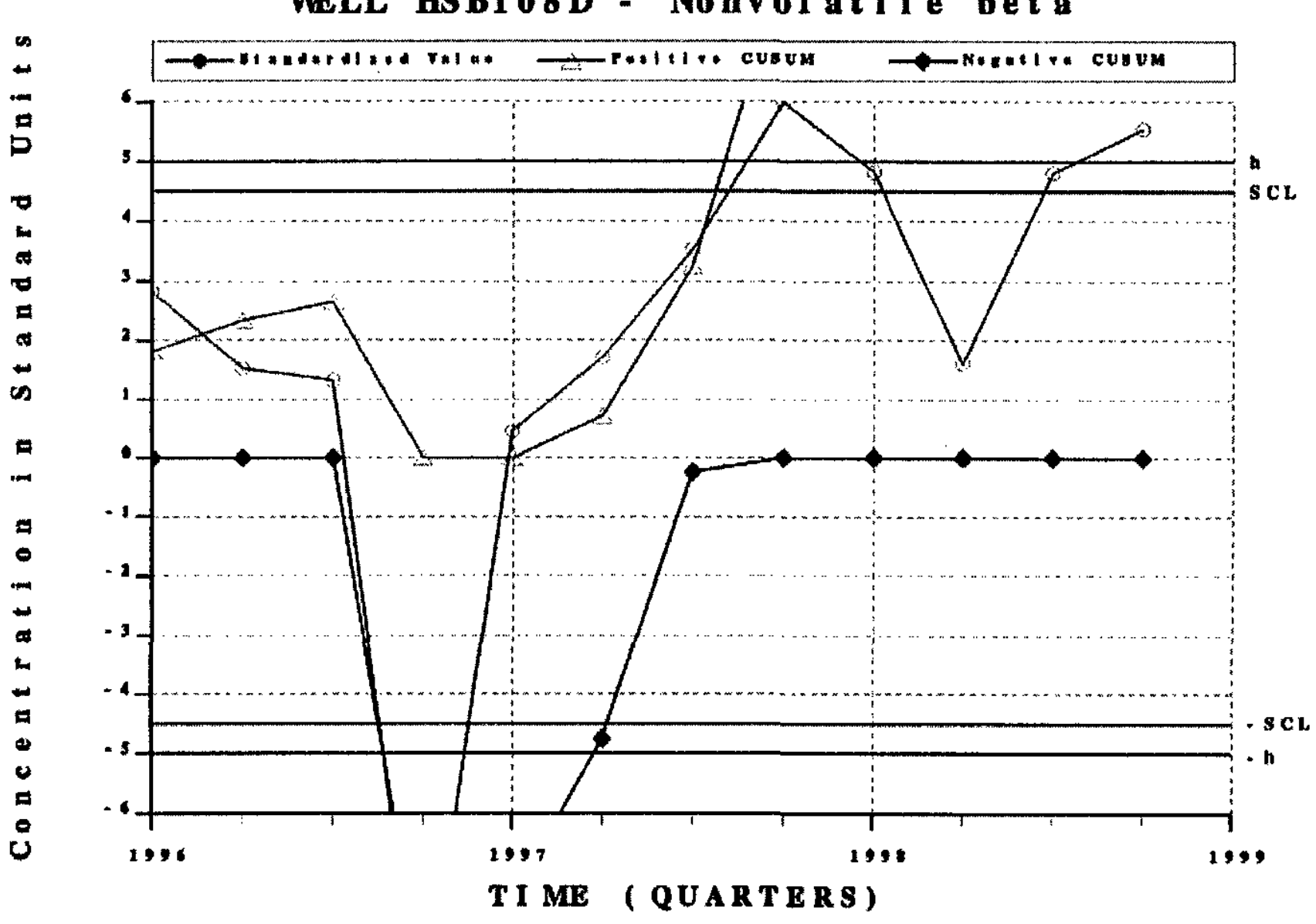

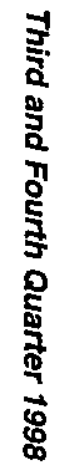




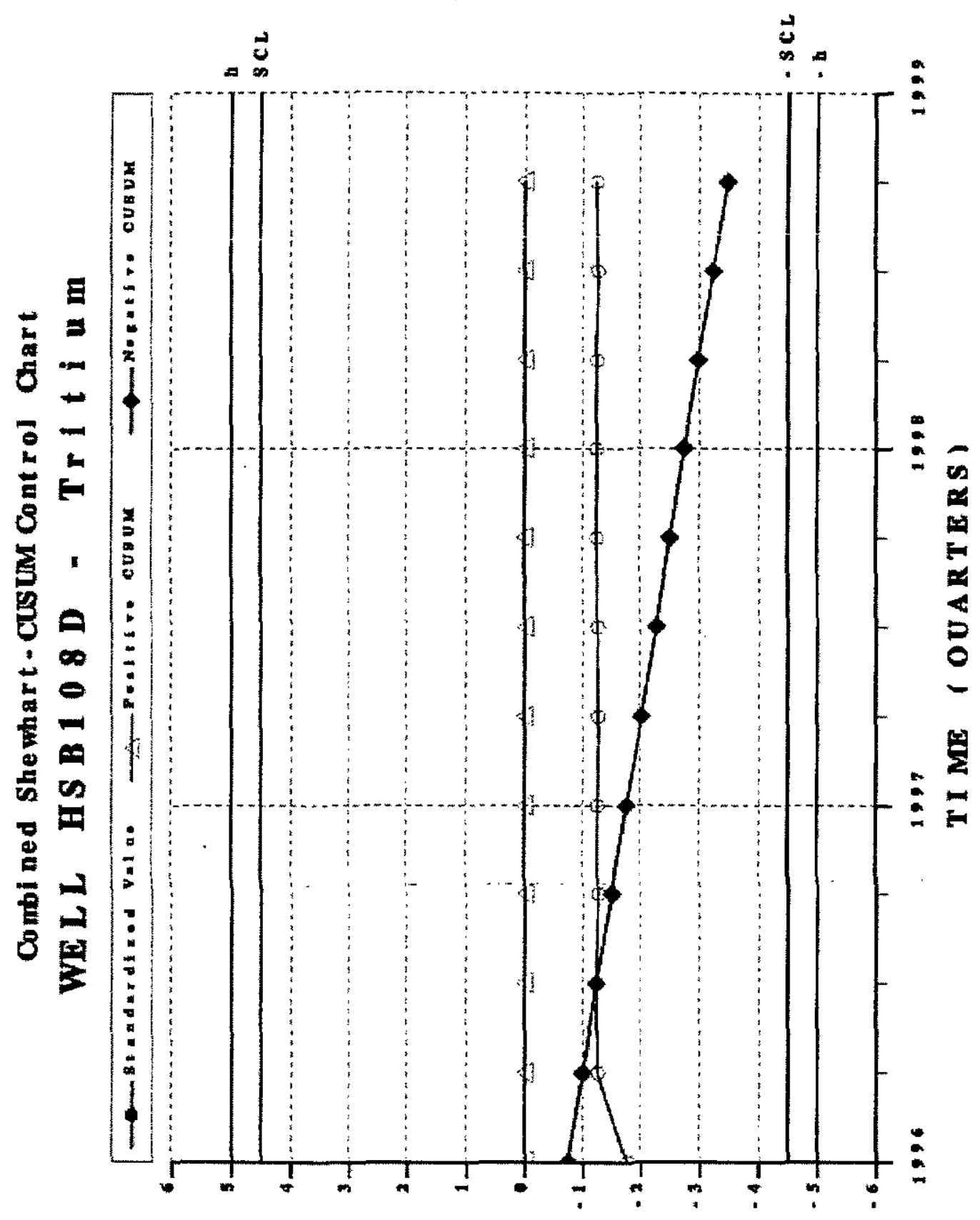

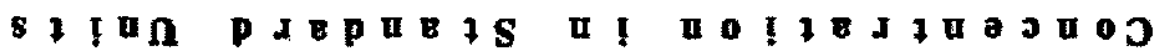


Combi ned She whart-Cusum Cont rol Chart VELL HSB1 09C - Bari um tot al recoverable

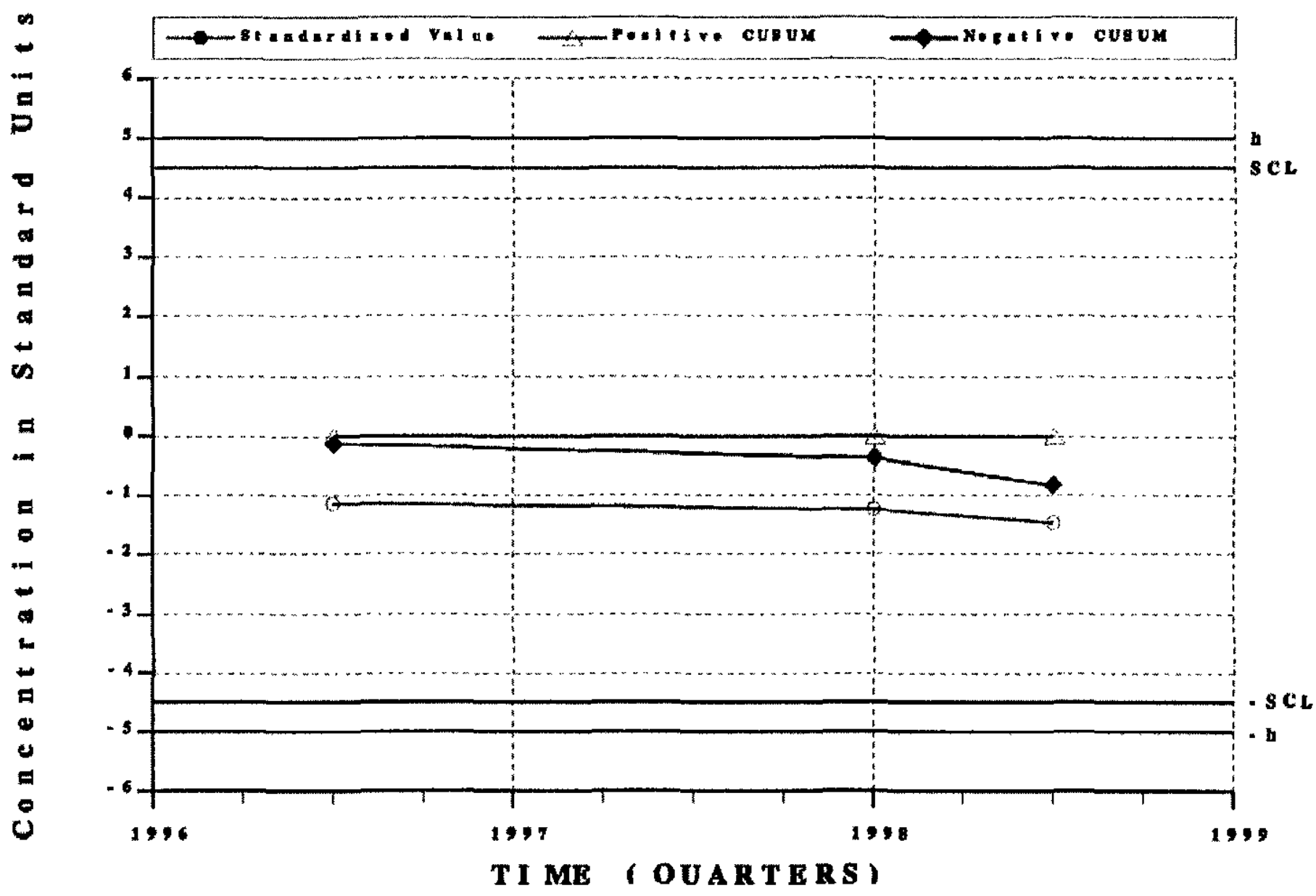


Combi ned She whart-CUSUM Cont rol Chart

VELL HSB109C - Nitrate-nitrite as nitrogen

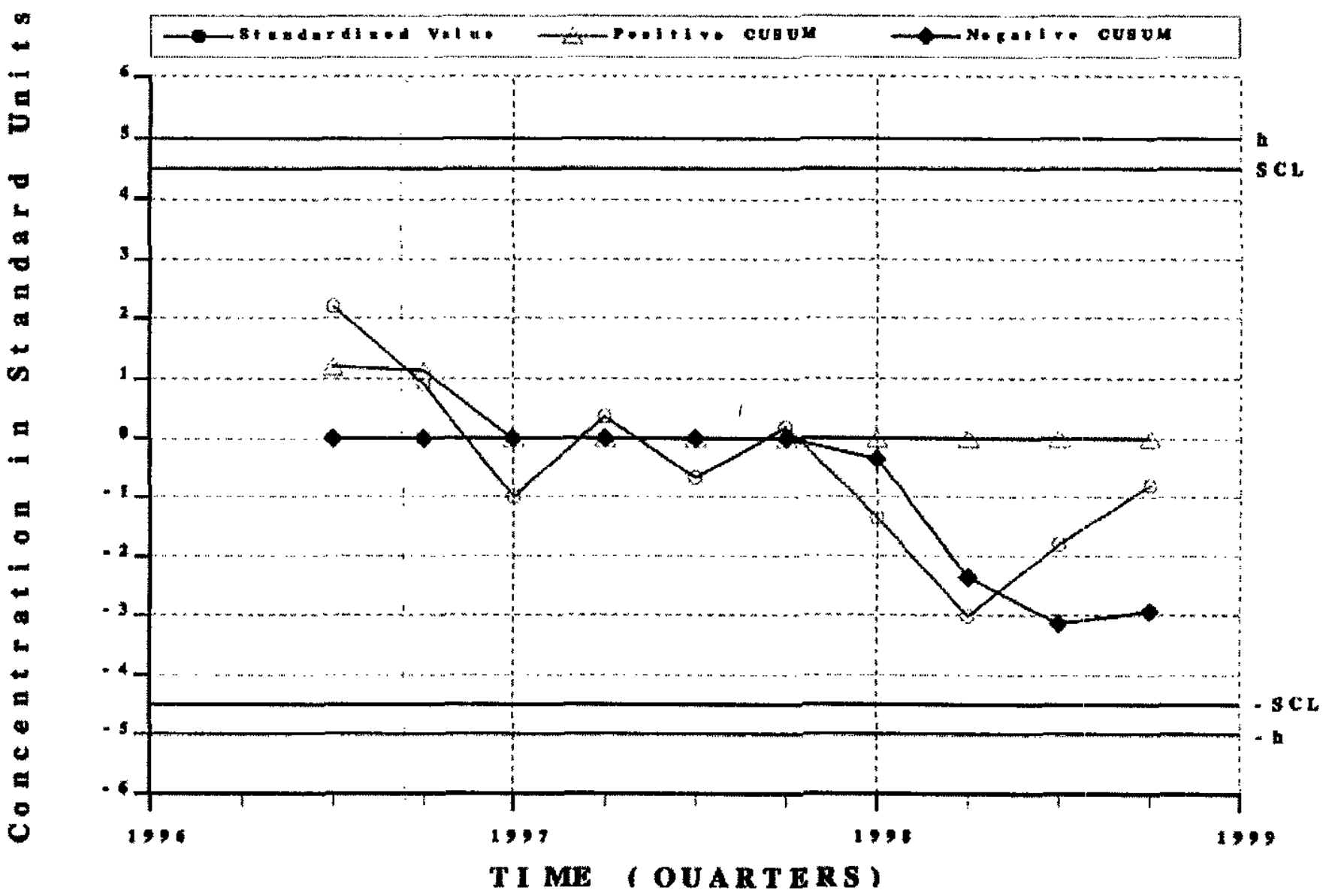


Combi ned Shewhart - CuSuM Cont rol Chart

WELL HSB1 09C - Nonvolatile beta

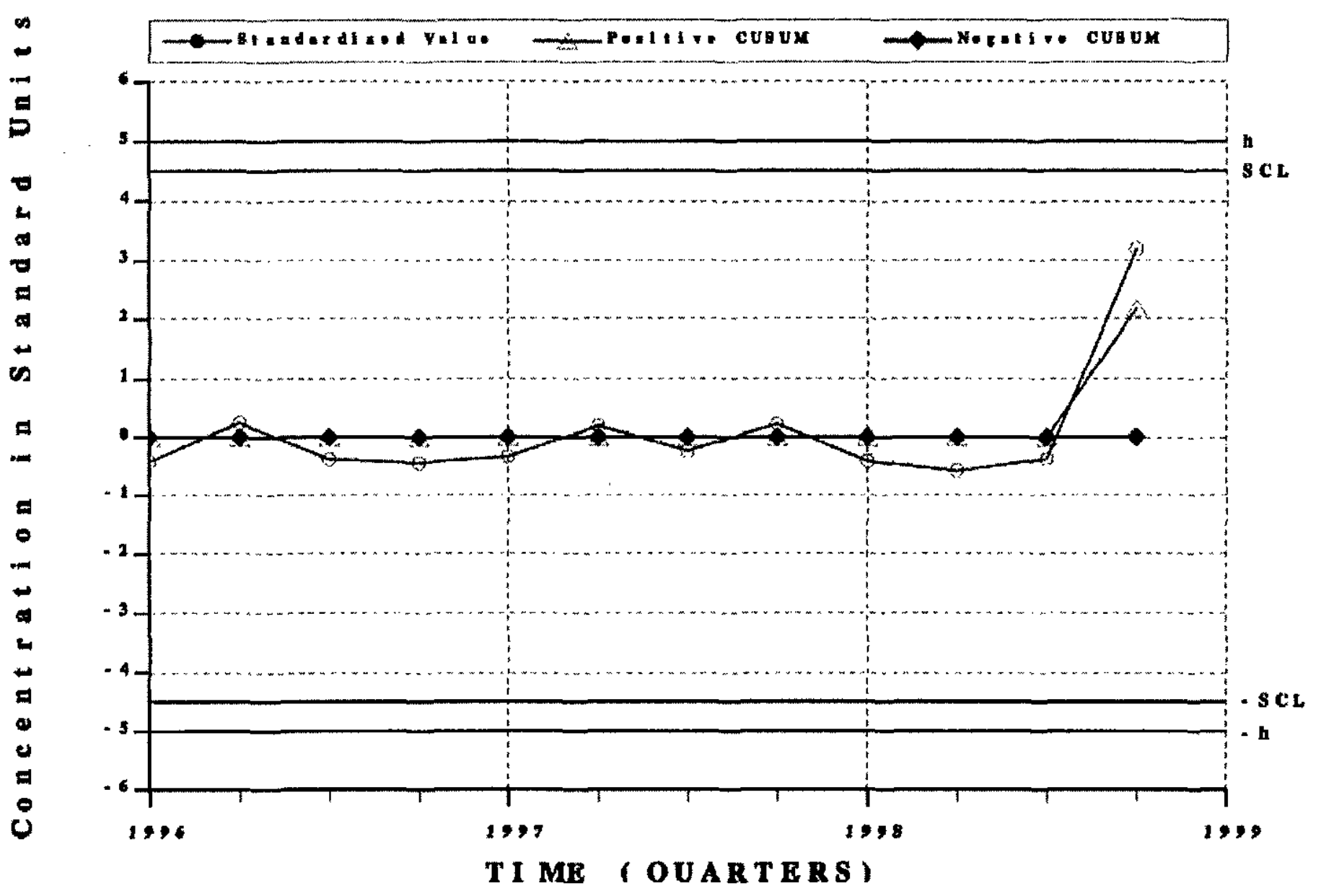




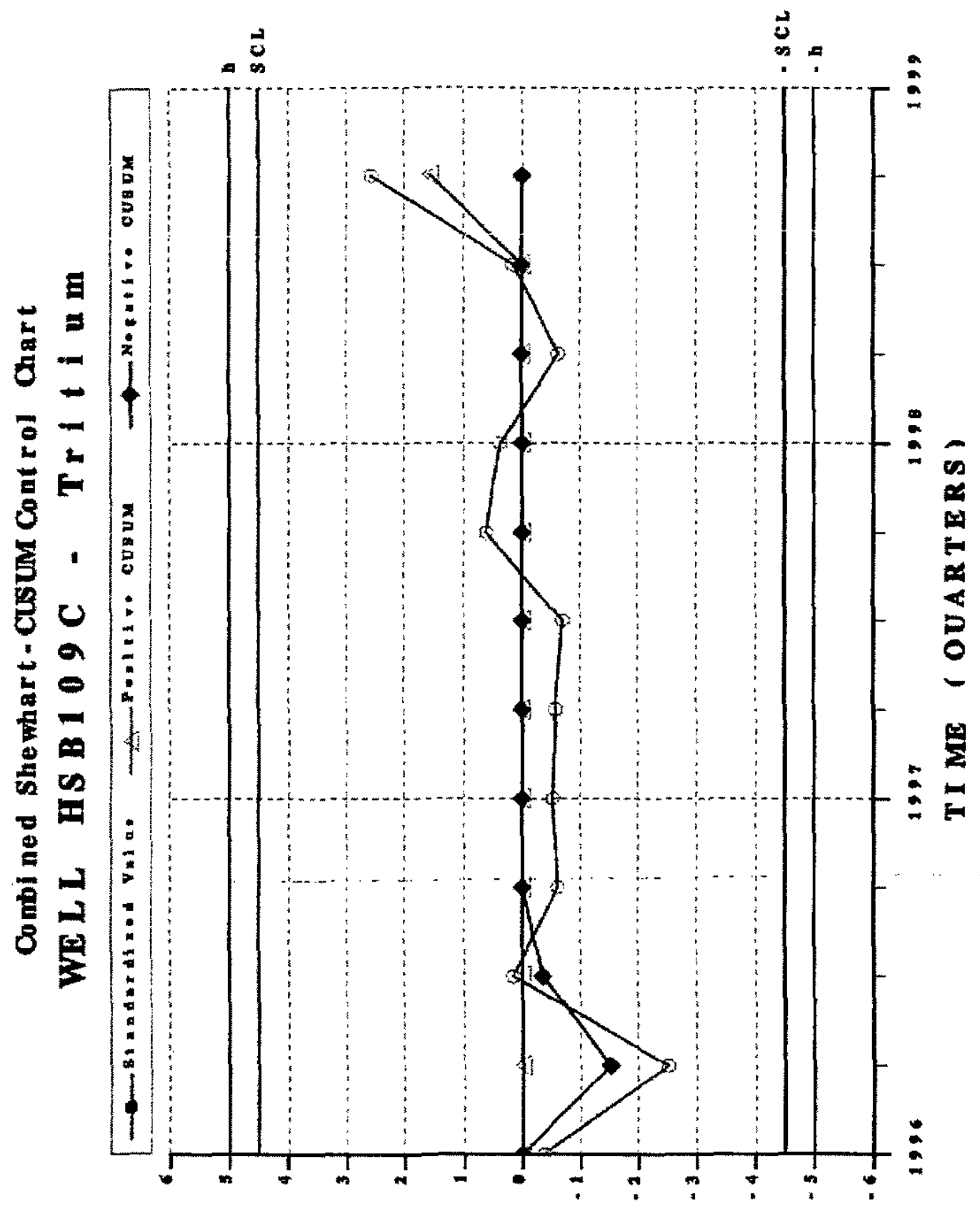

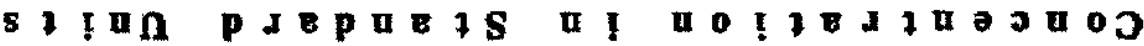




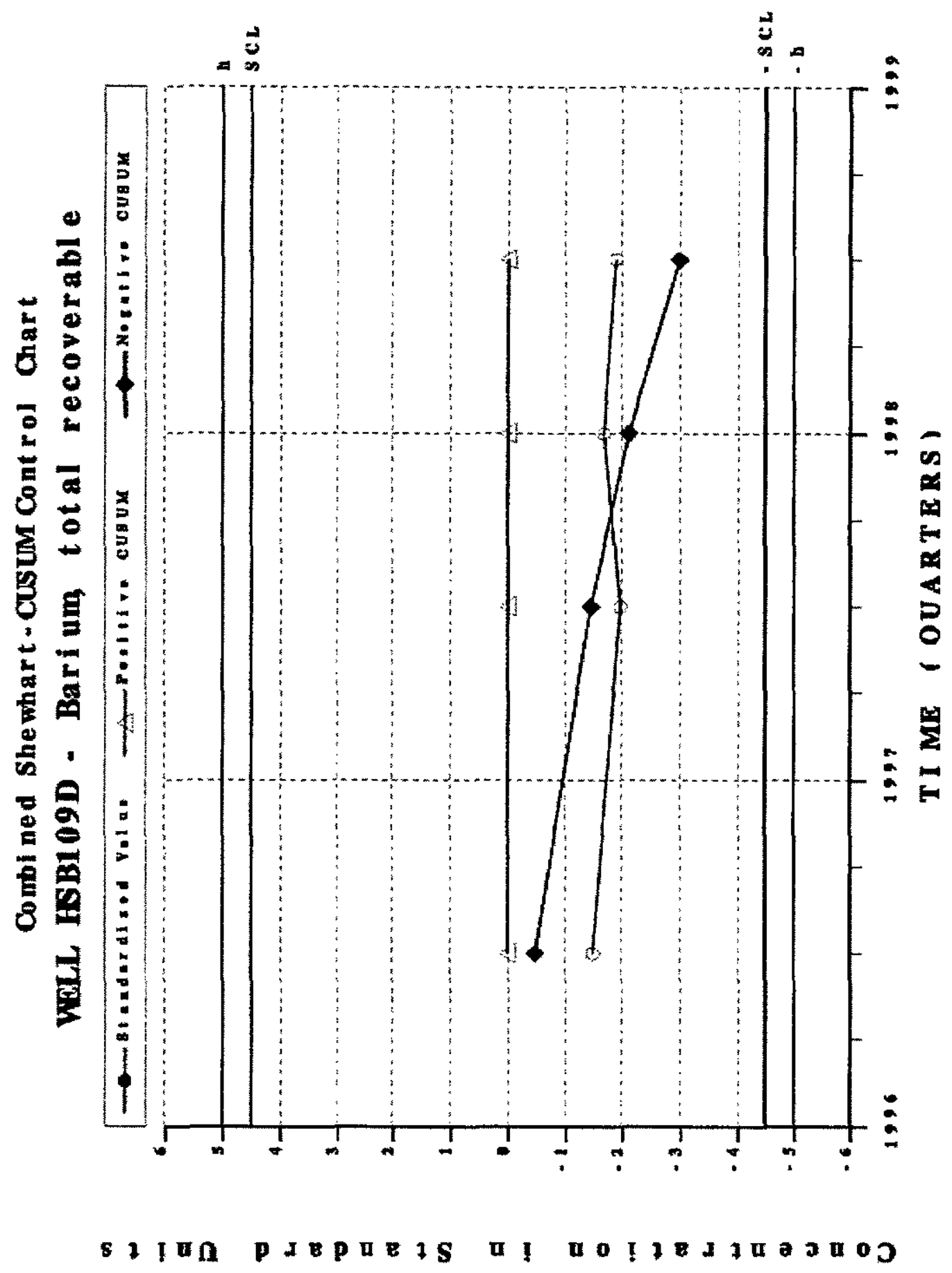




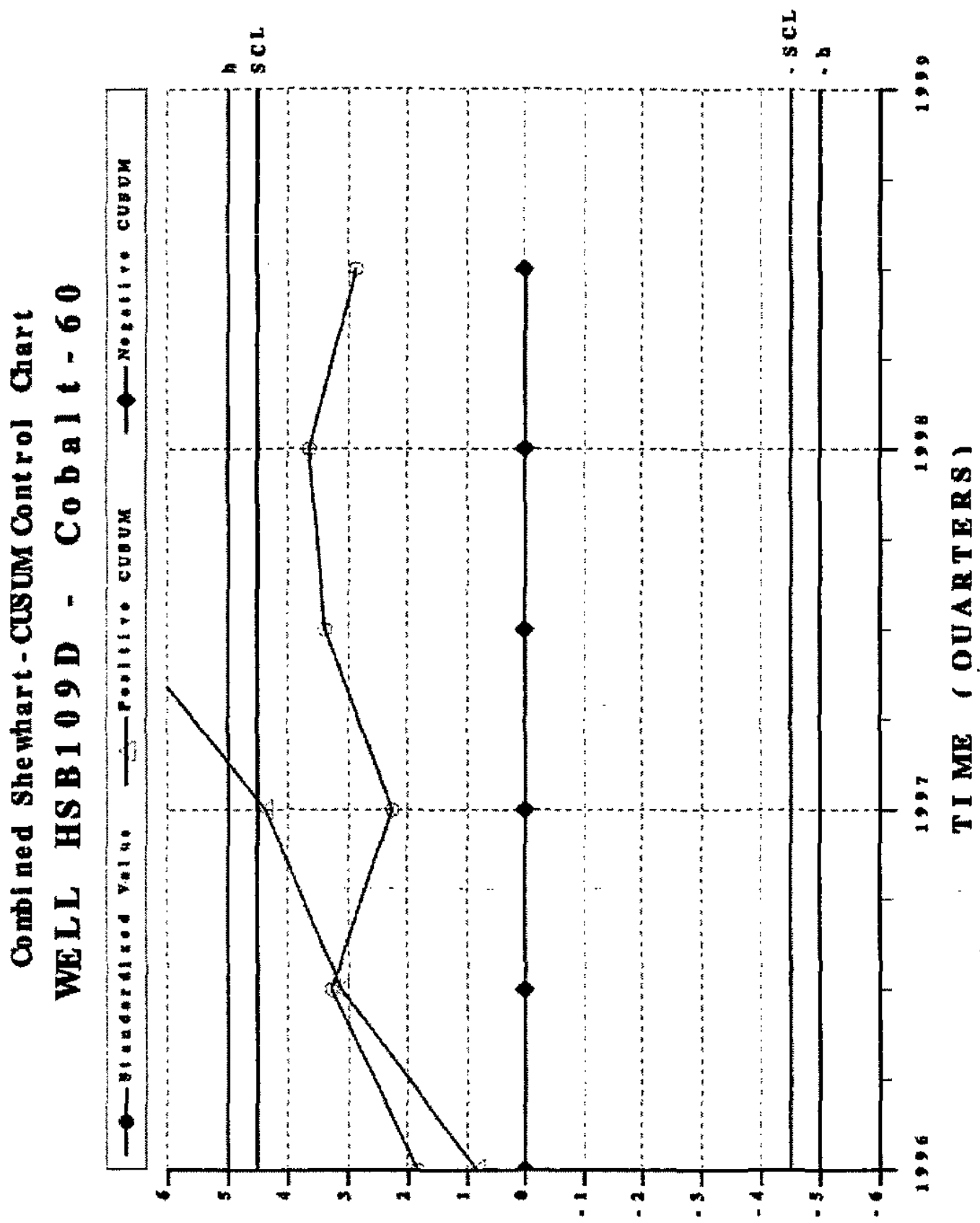

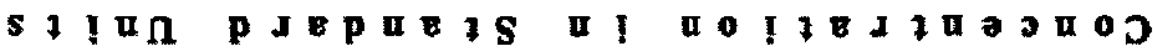



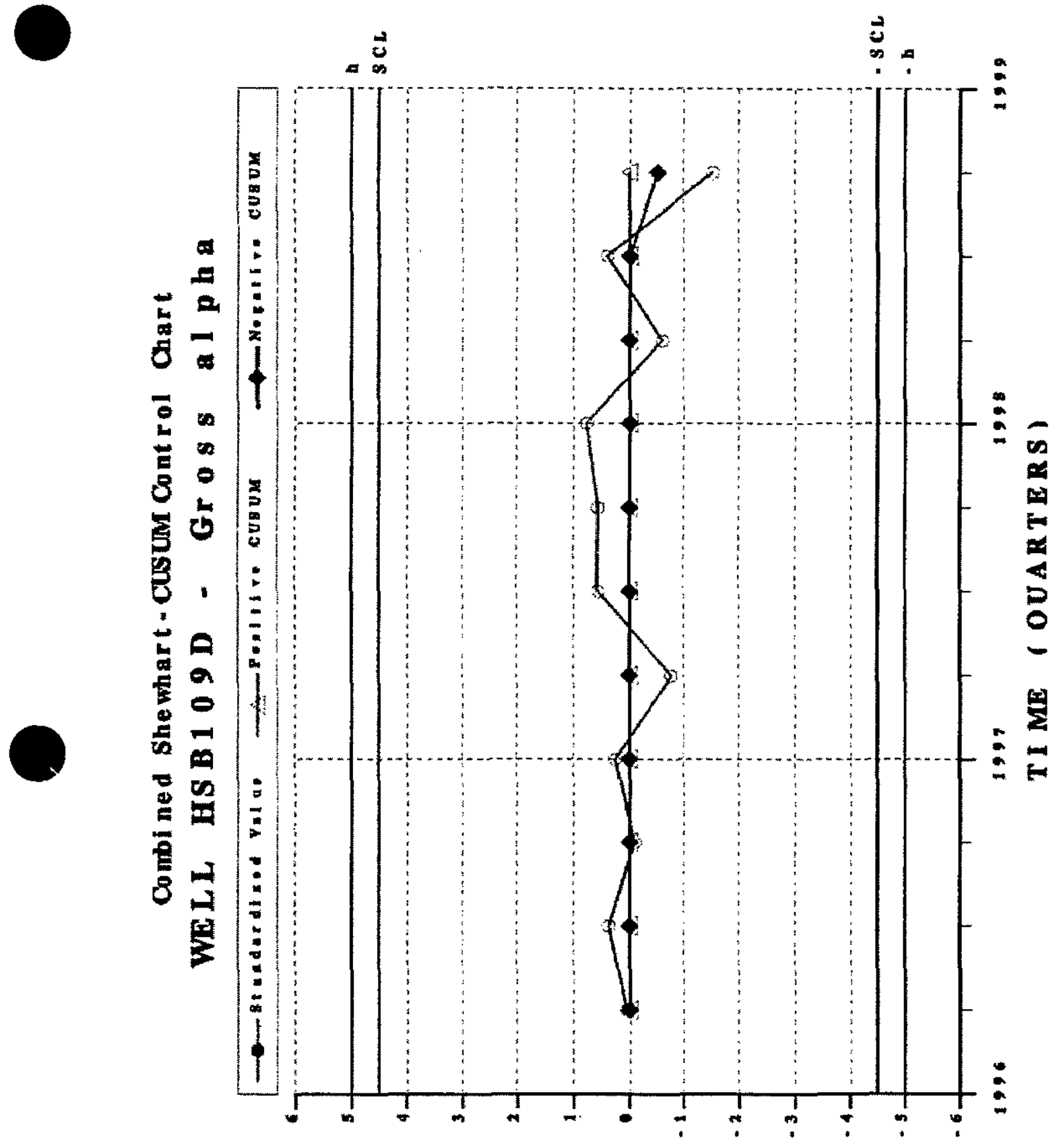

s 1 In pa 


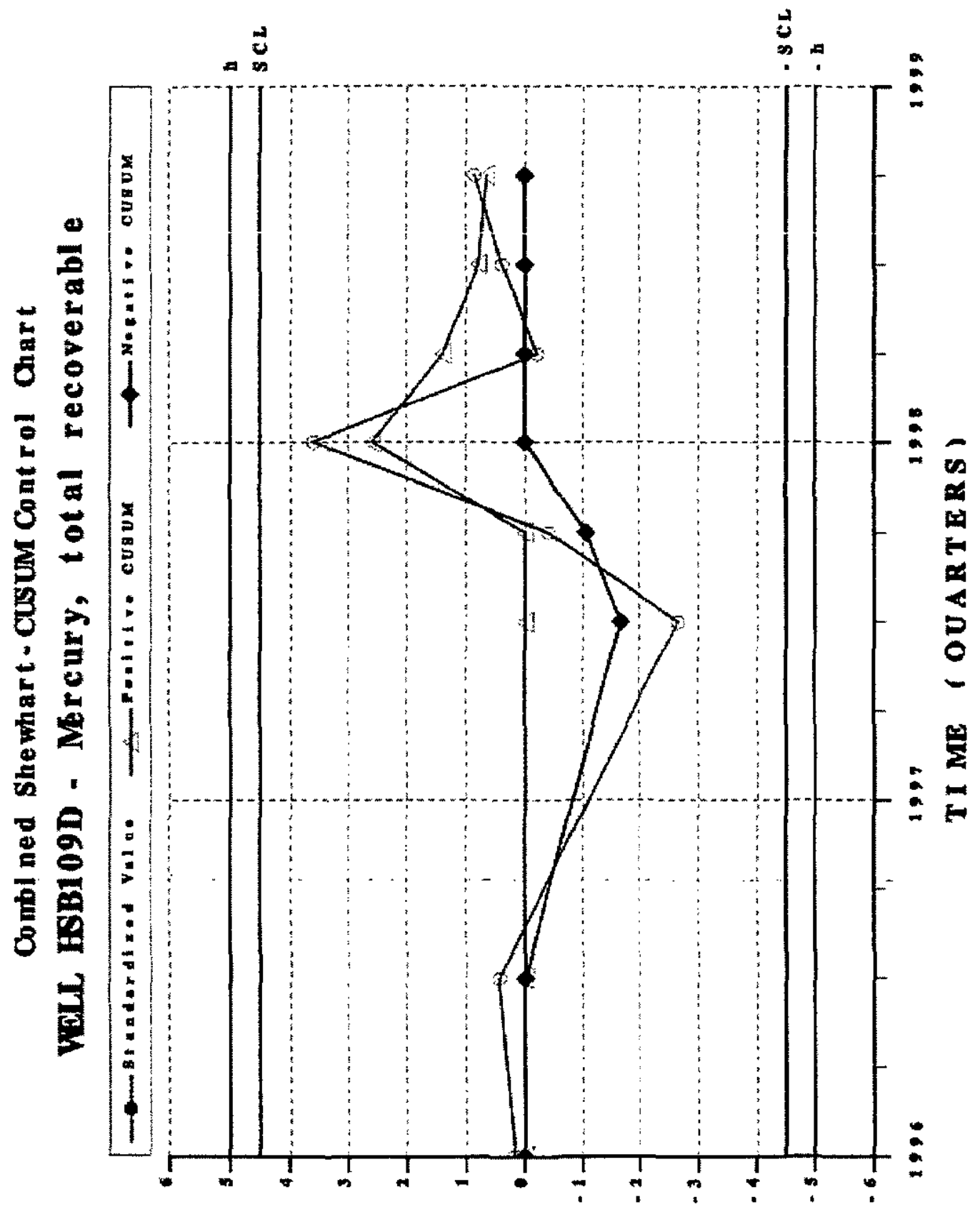

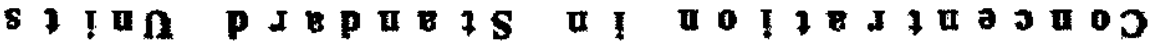



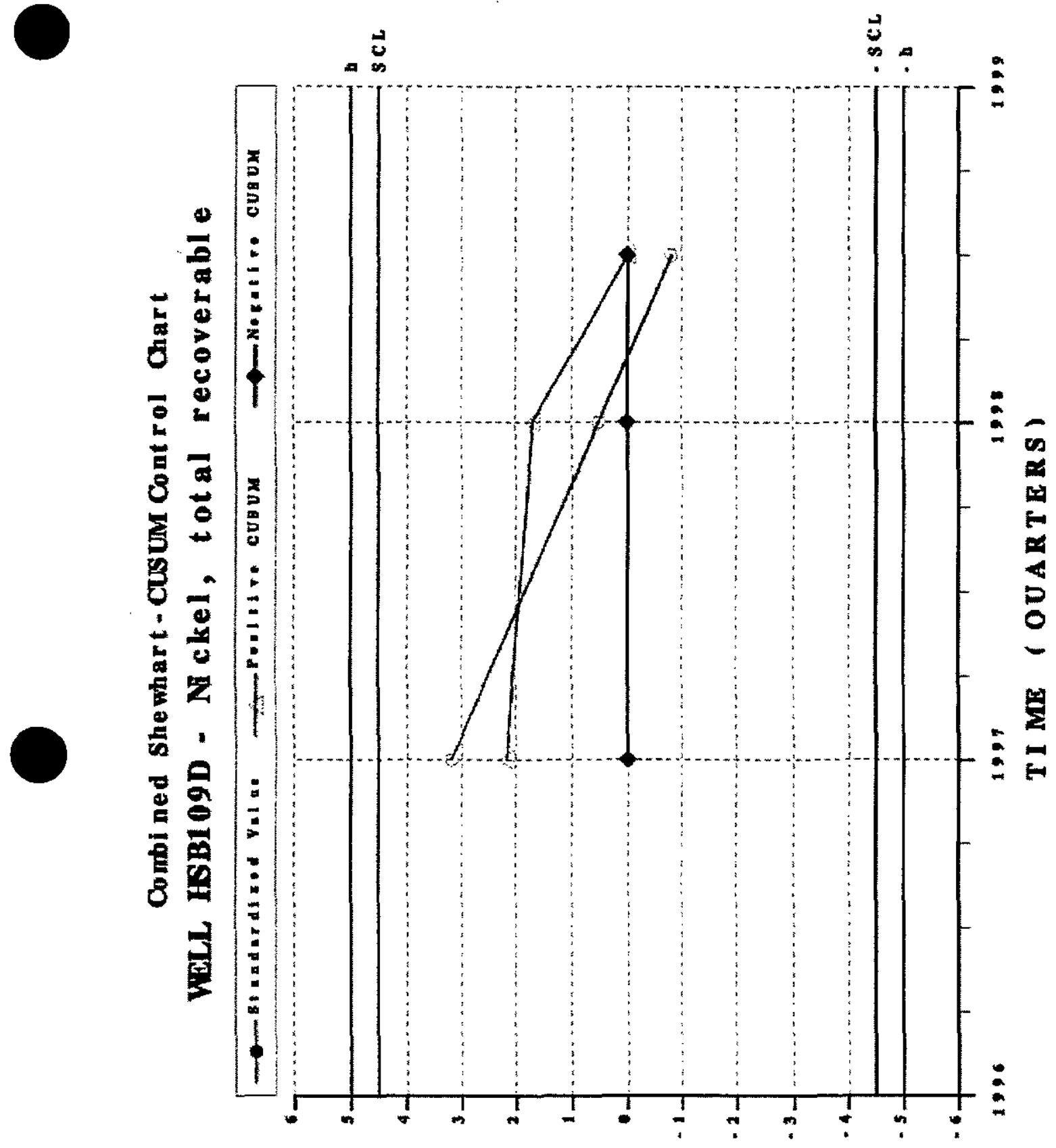

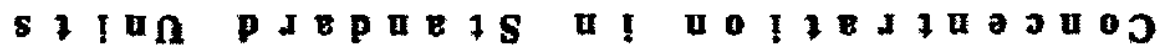


Combi ned Shewhart-Cusum Control Chart

VOL HSB109D - N trate-nitrite as nitrogen

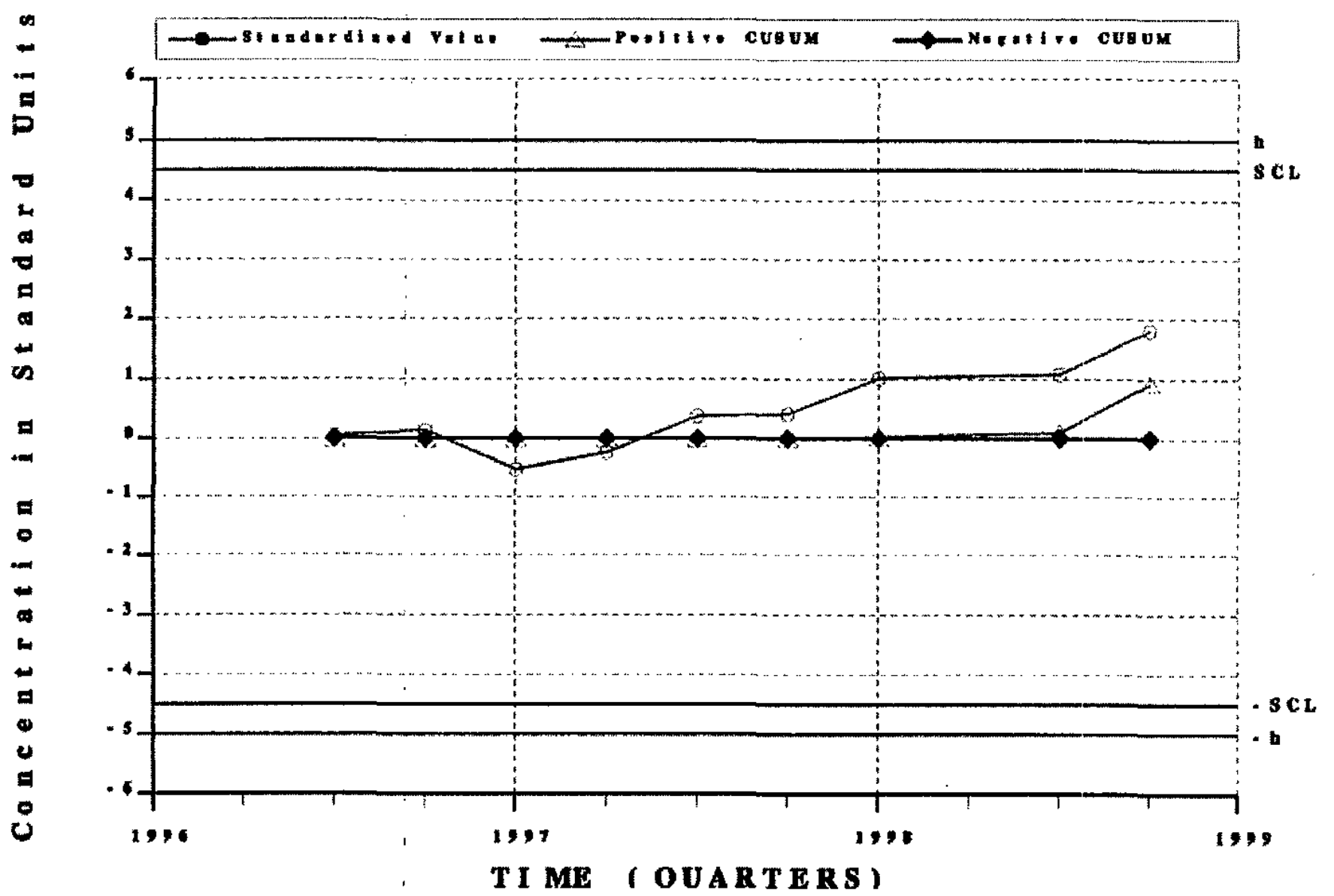

T I ME ( OUARTERS) 
Combined Shewhart-cusum Cont rol Chart

WeLL HSB109D - Nonvolatile beta

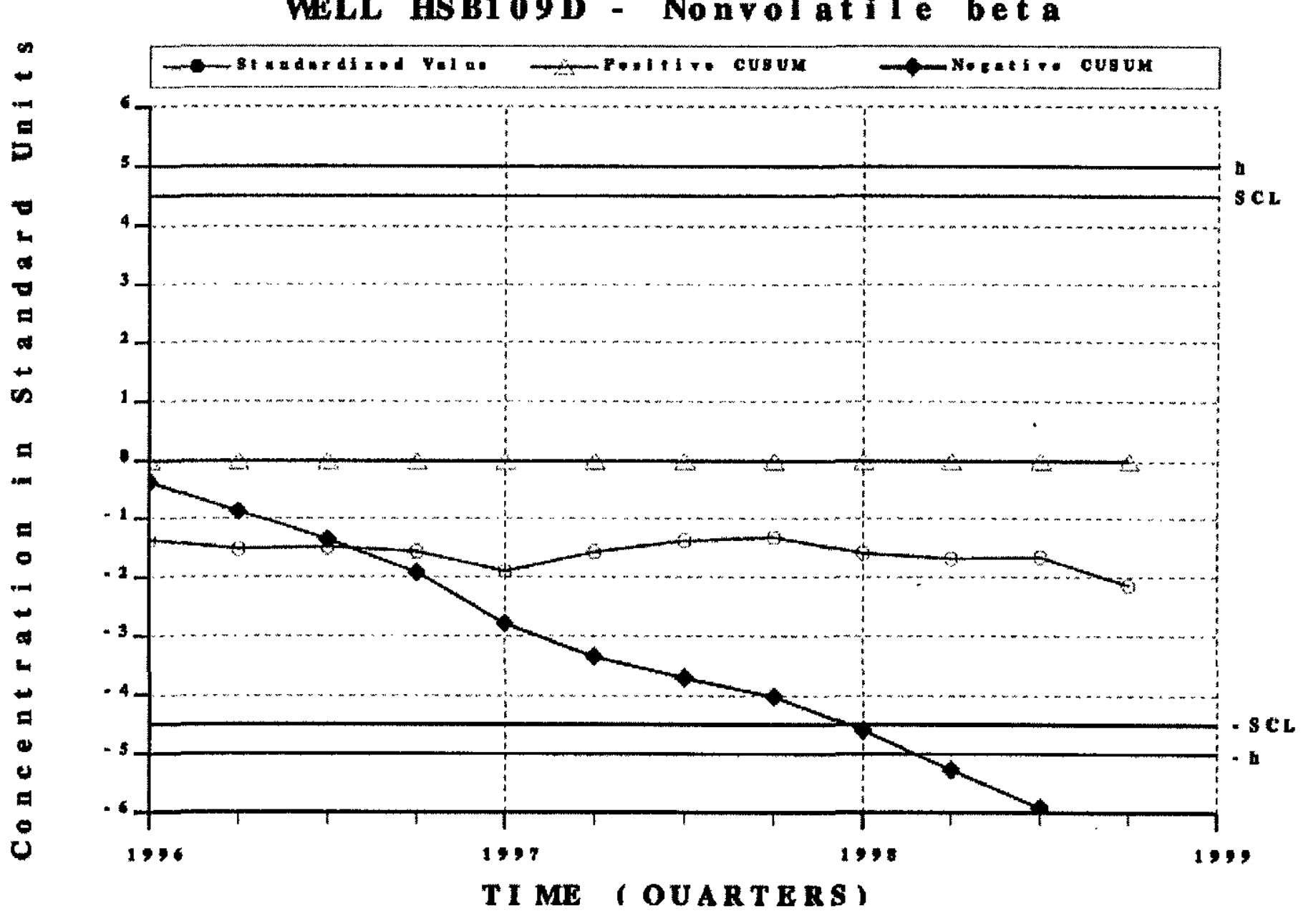




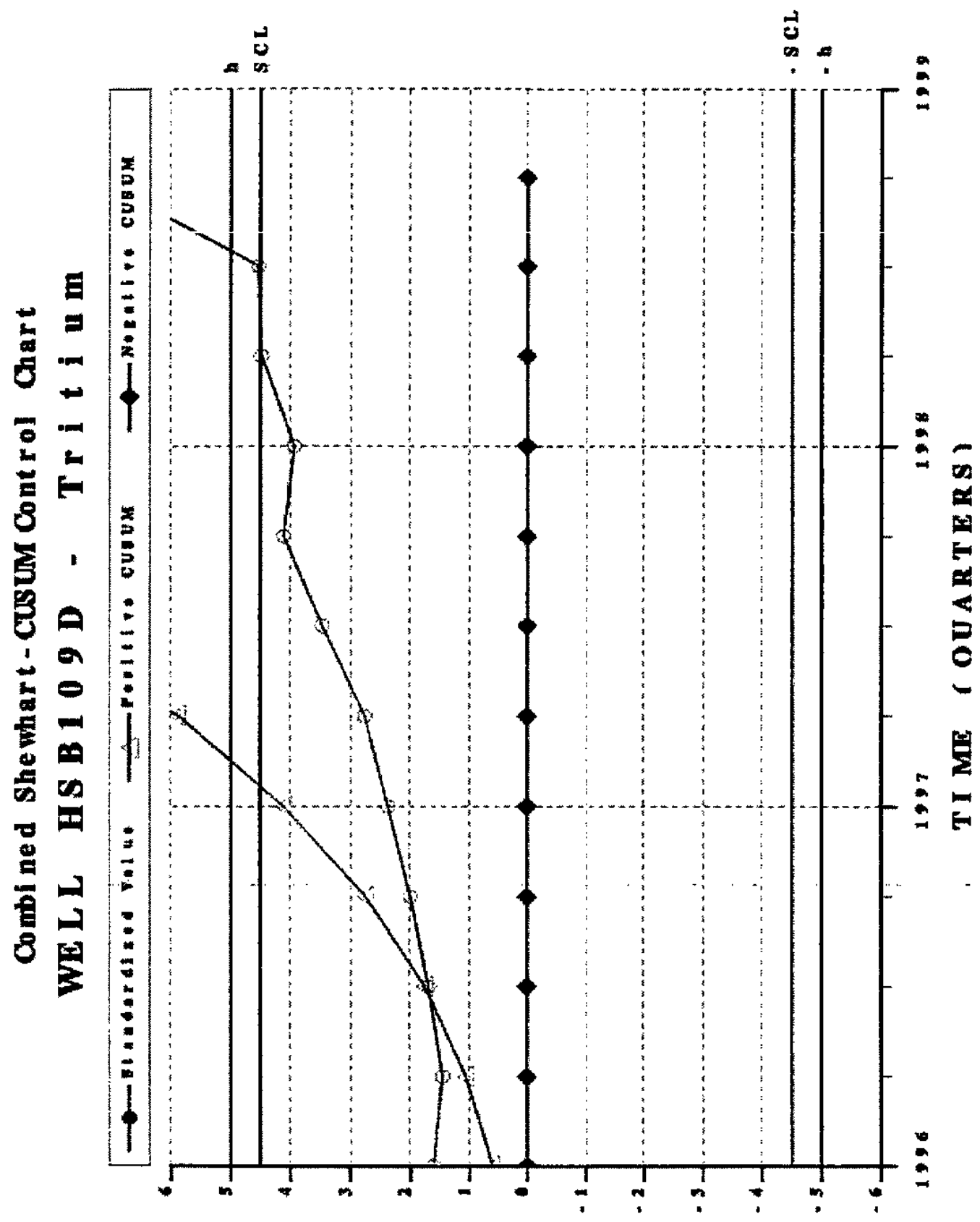

S I 


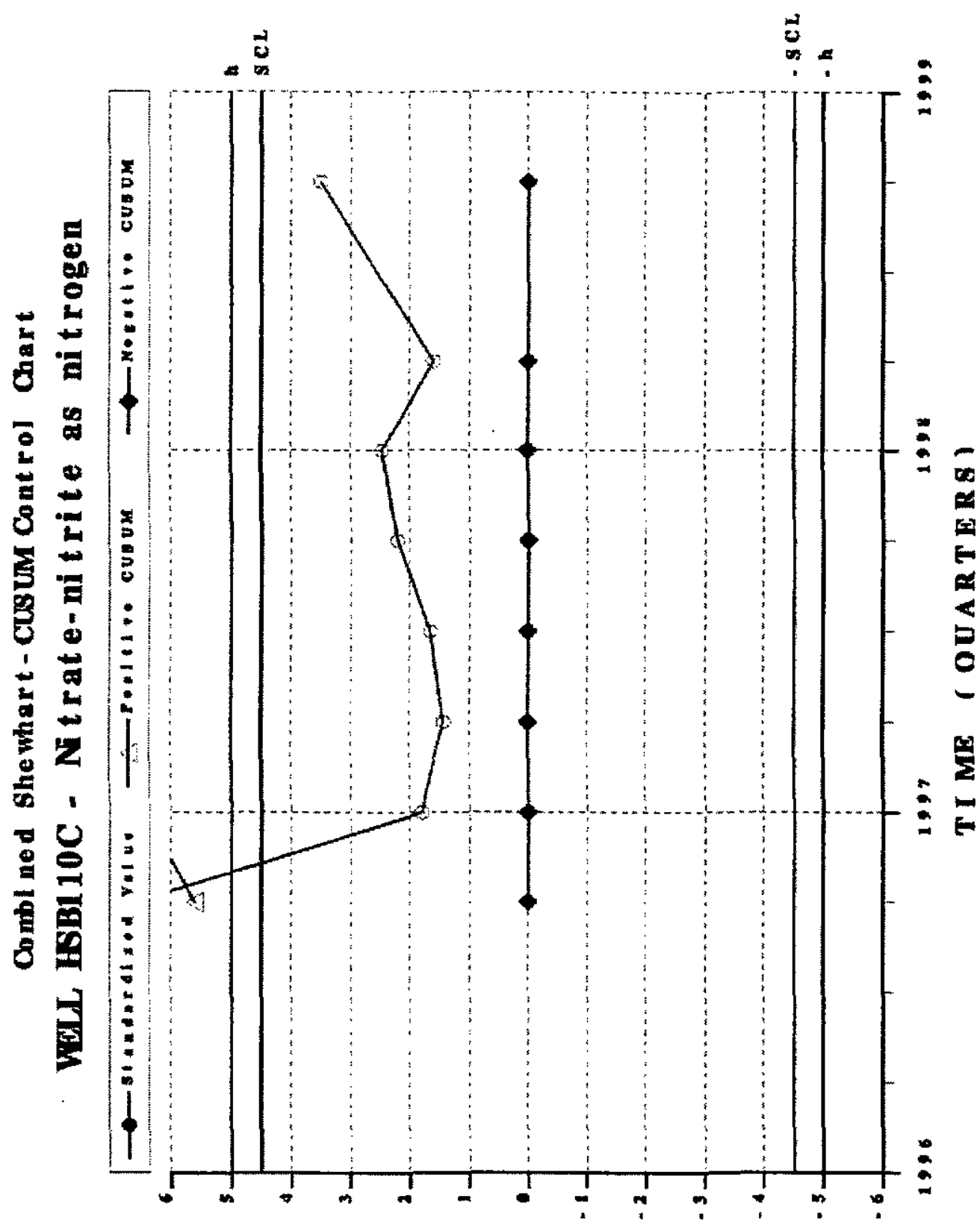

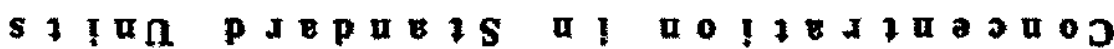




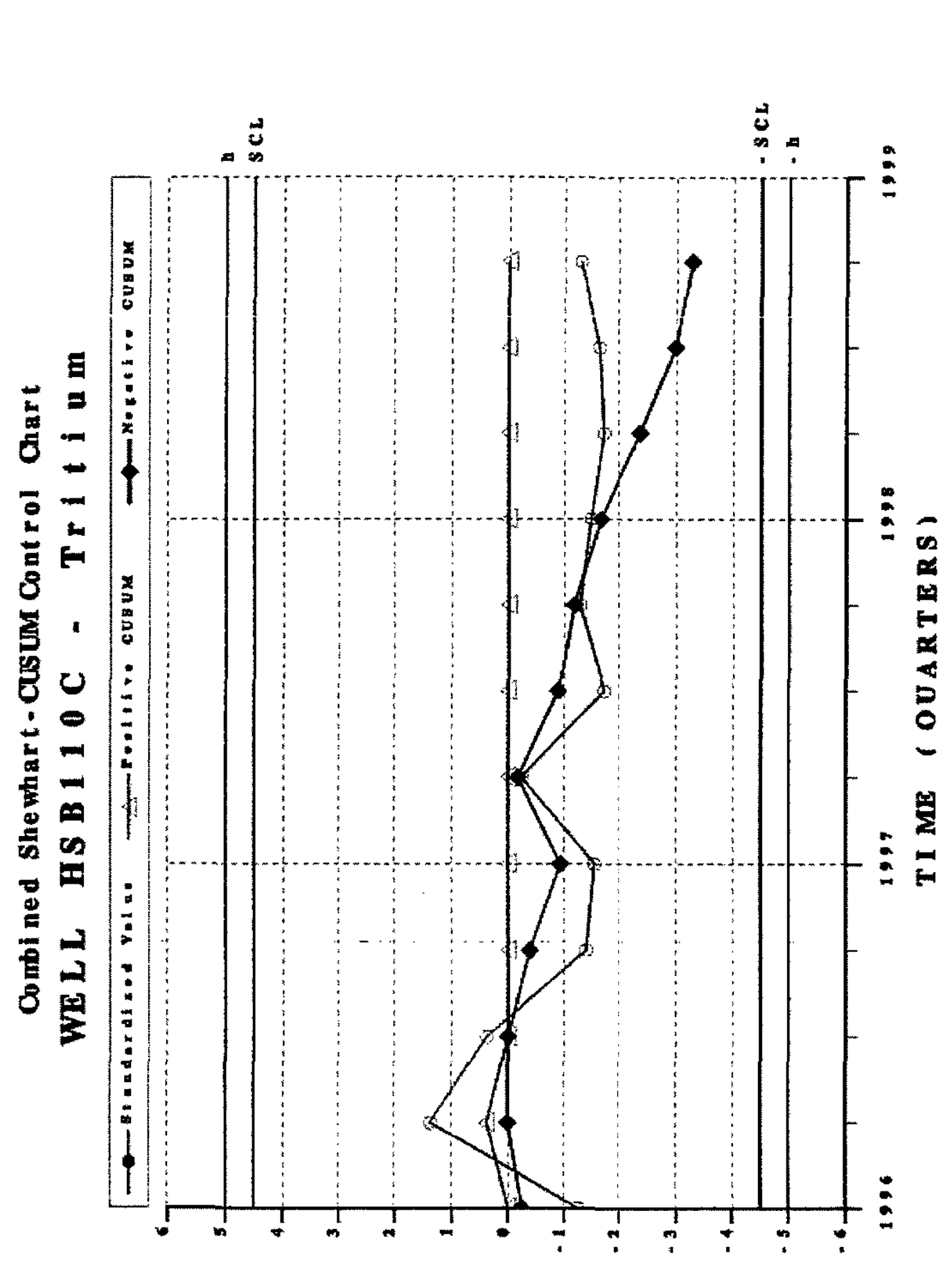

WSRC-TR-99-00013

Unclassified

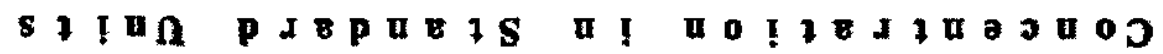



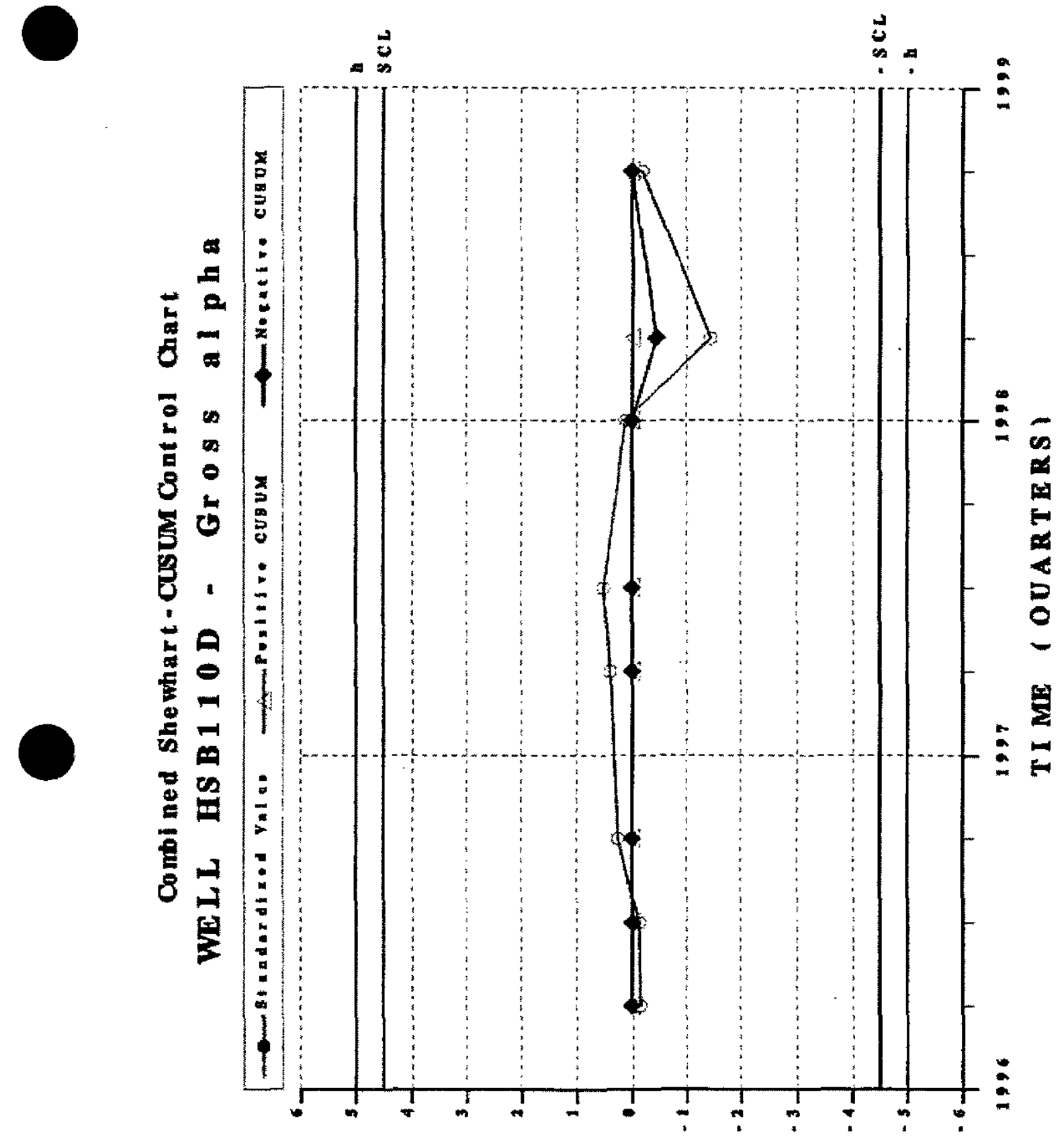

S I I A P E 


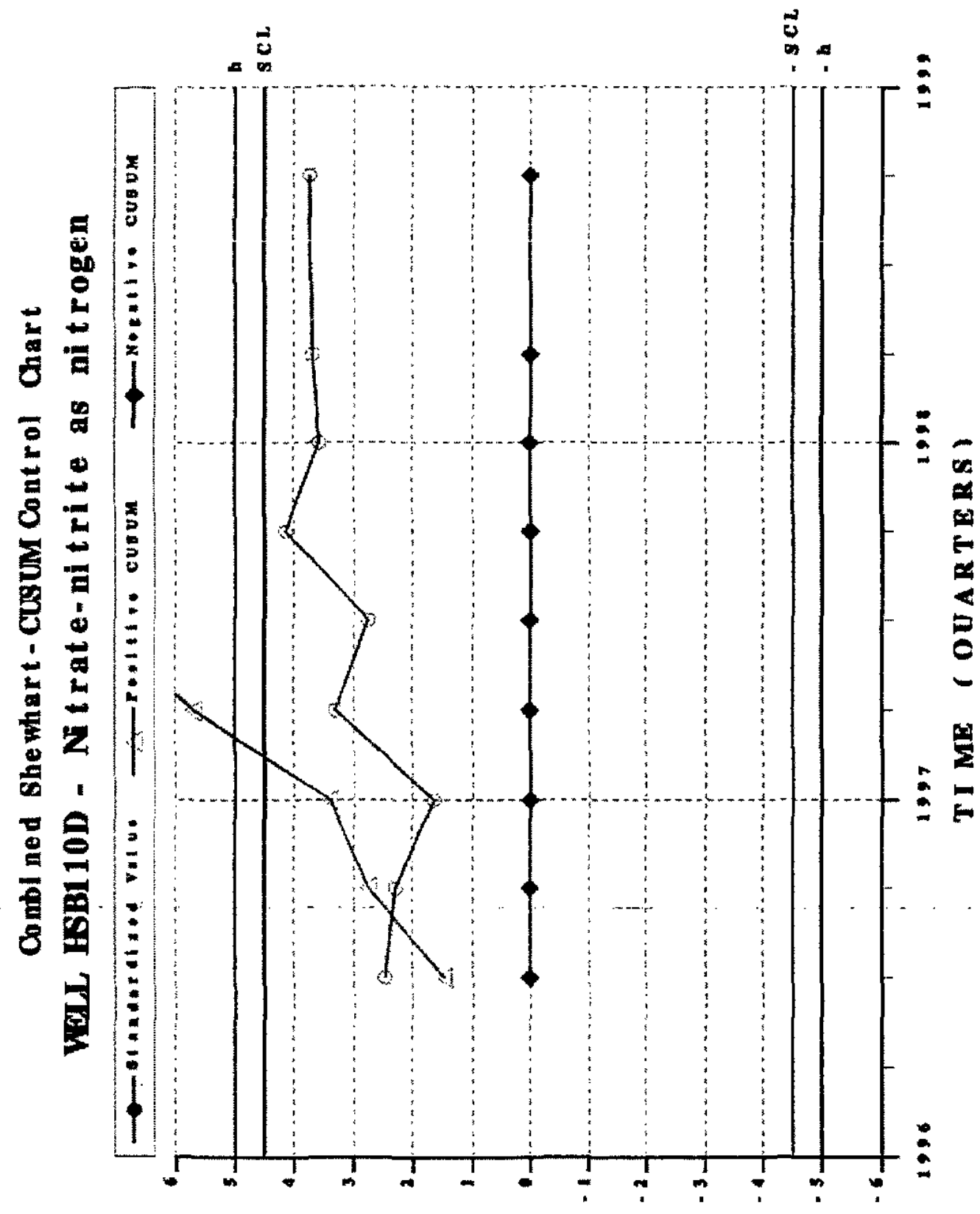

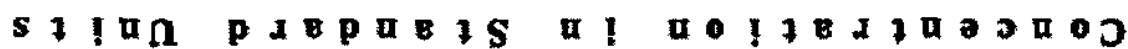




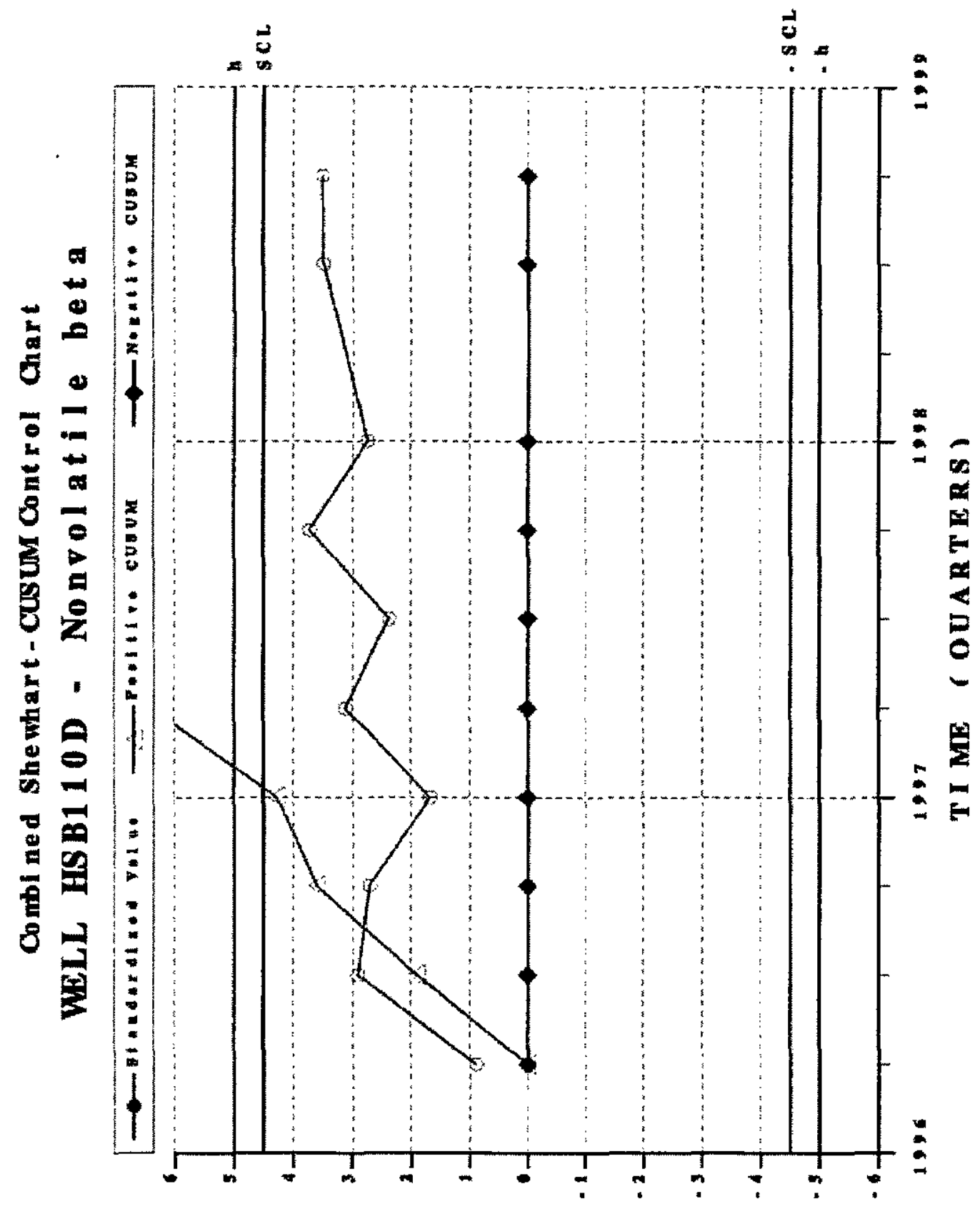

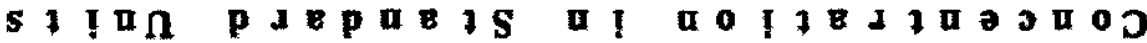




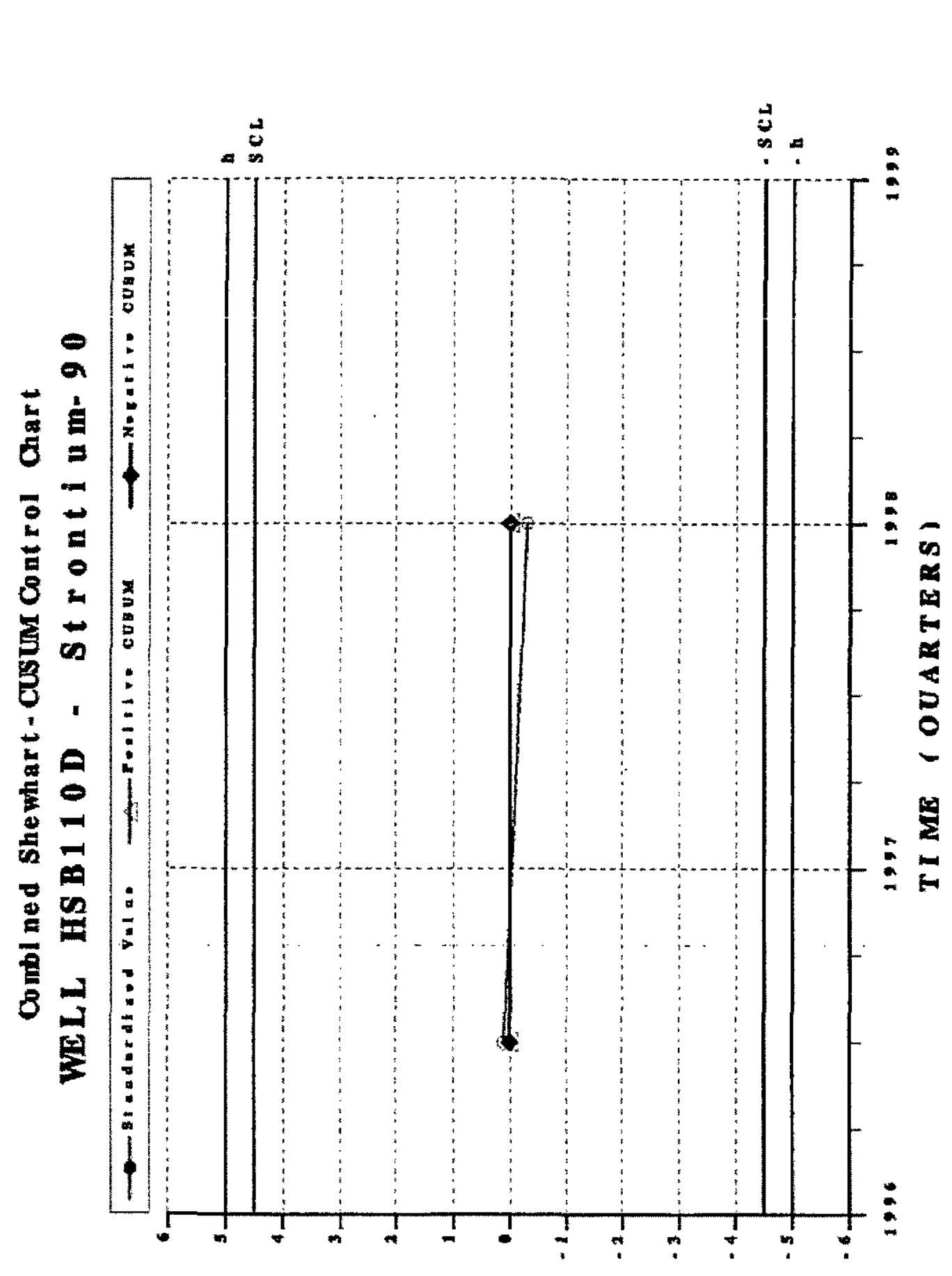

WSRC-TR-99-00013

Unclassified

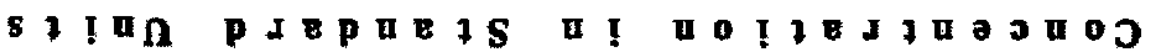


Combl ned She whart-CusuM Cont rol Chart WELL HSB1 10D - Ti n, tot al recoverable

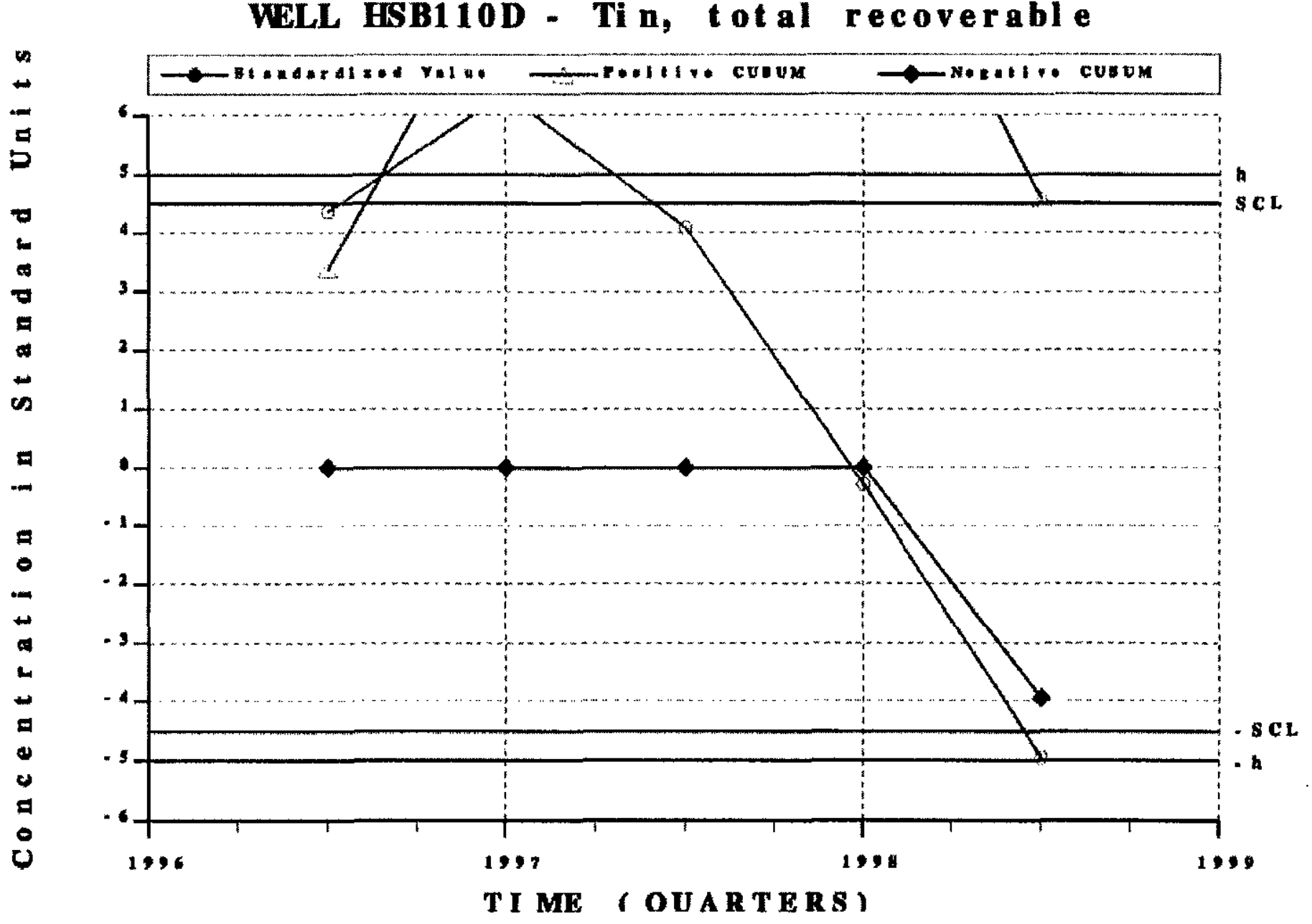




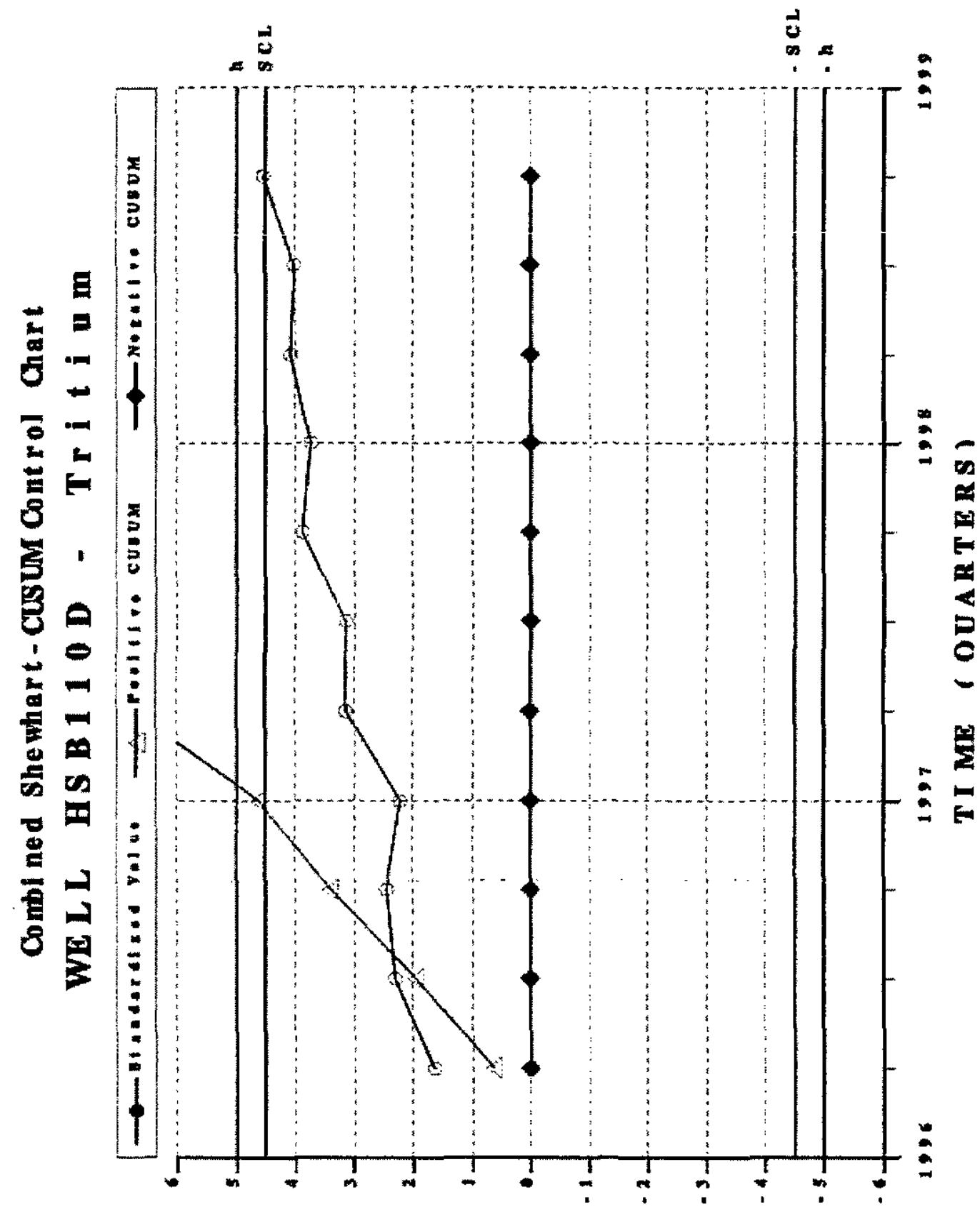

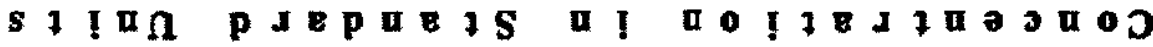



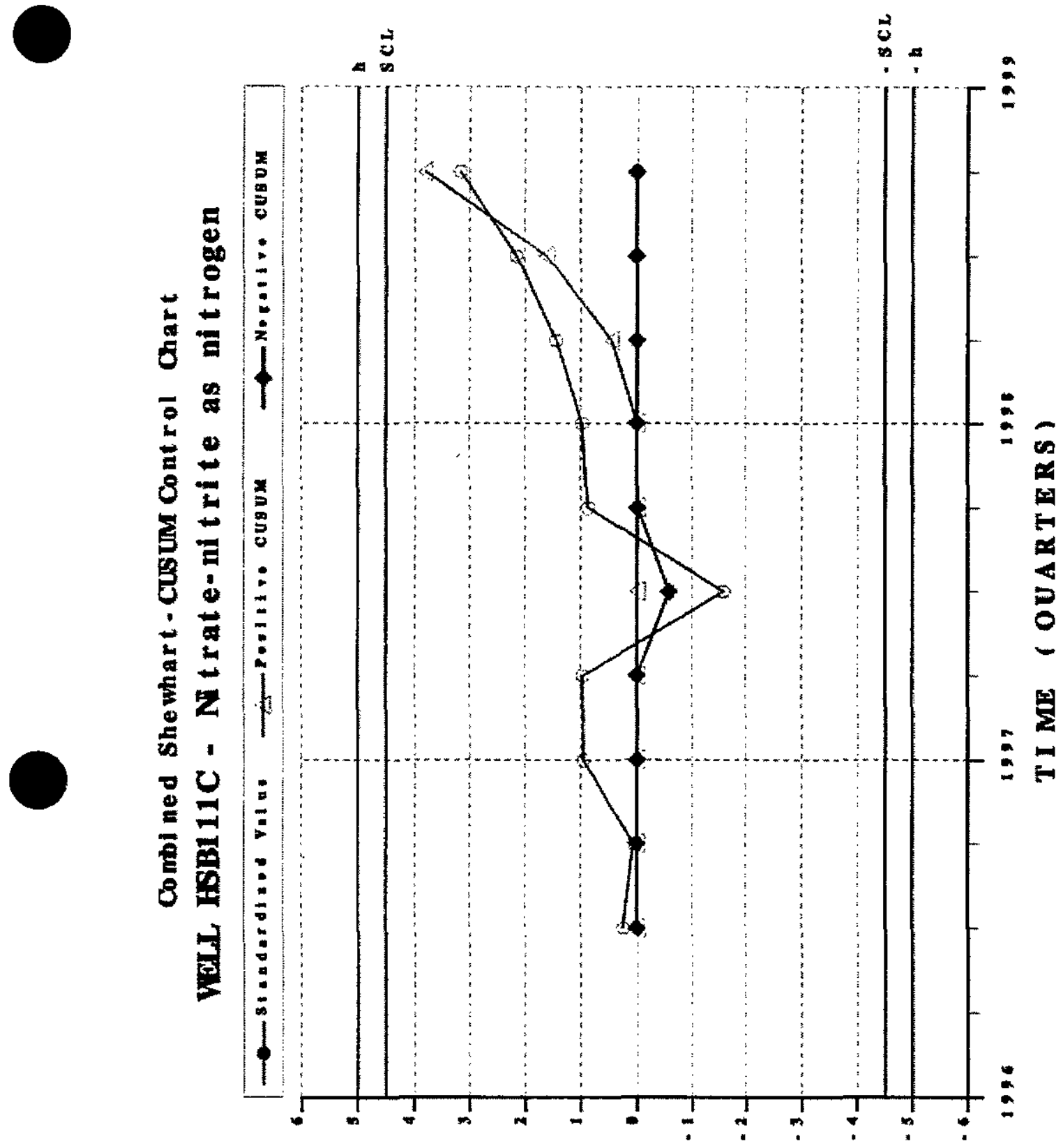

s 1 I I p 
Combi ned Shewhart - CuSUM Cont rol Chart

WELL HSB1 $11 \mathrm{C}$ - Nonvol atile bet a

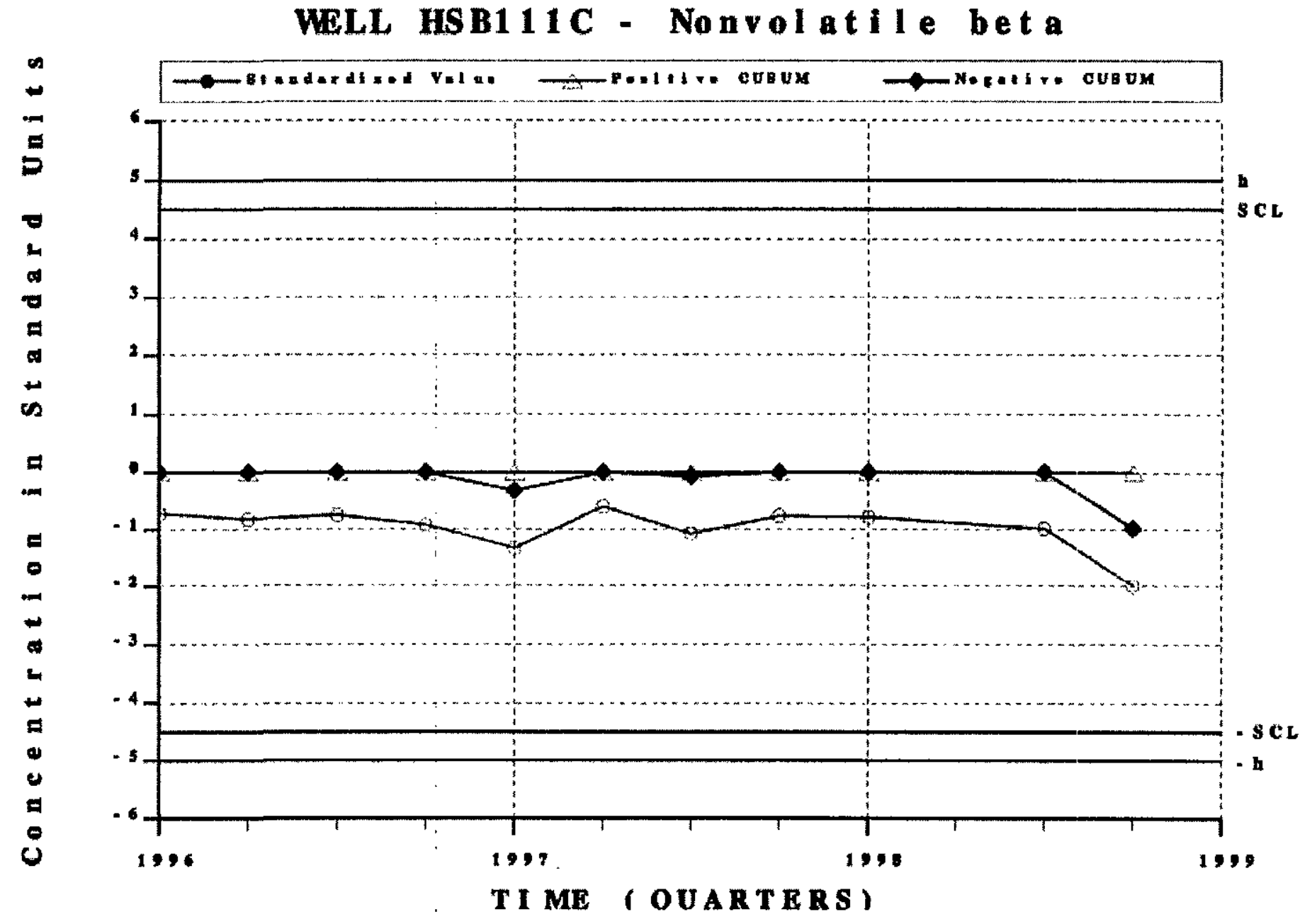

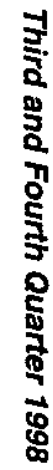



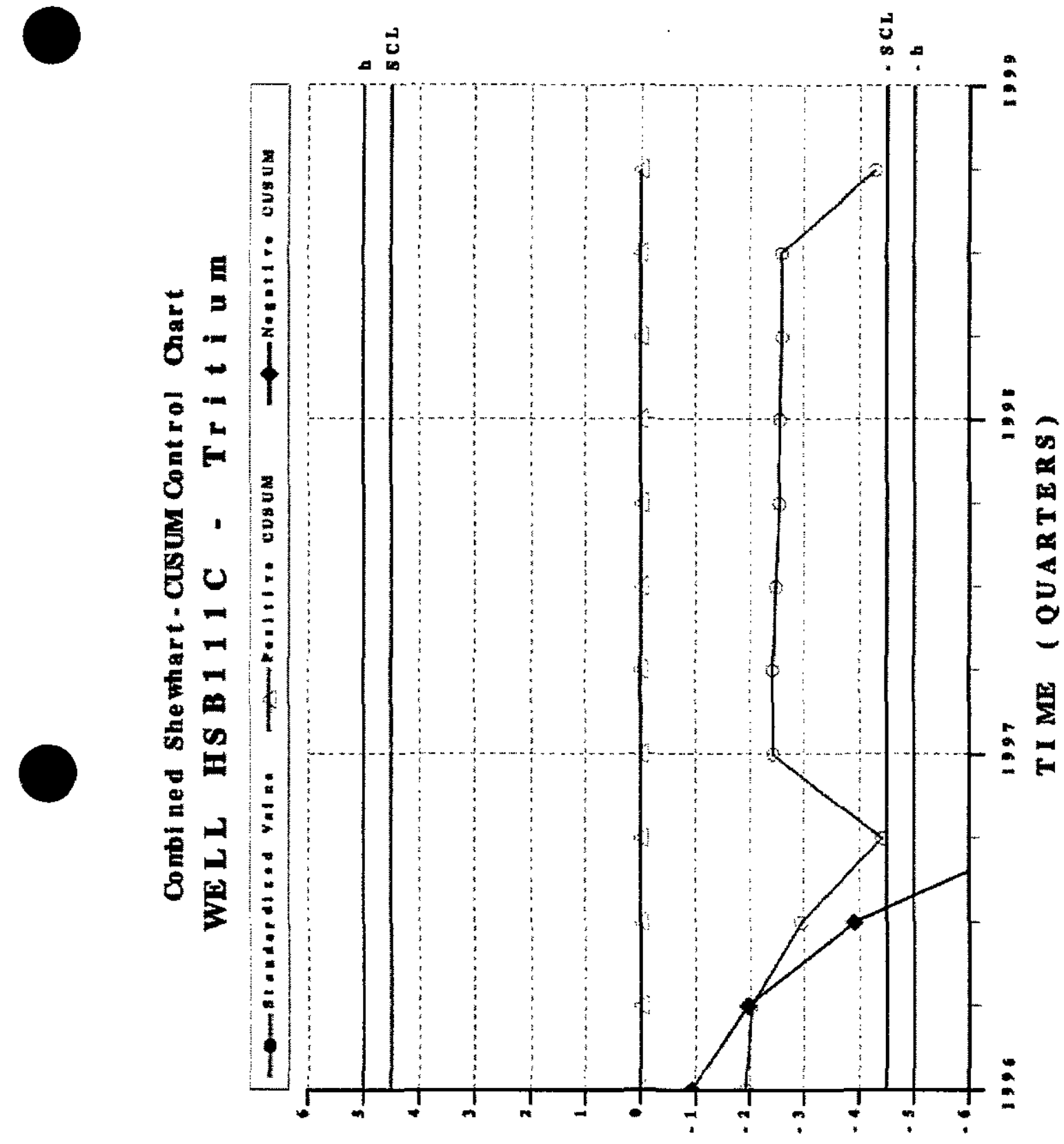

s I In patp a 


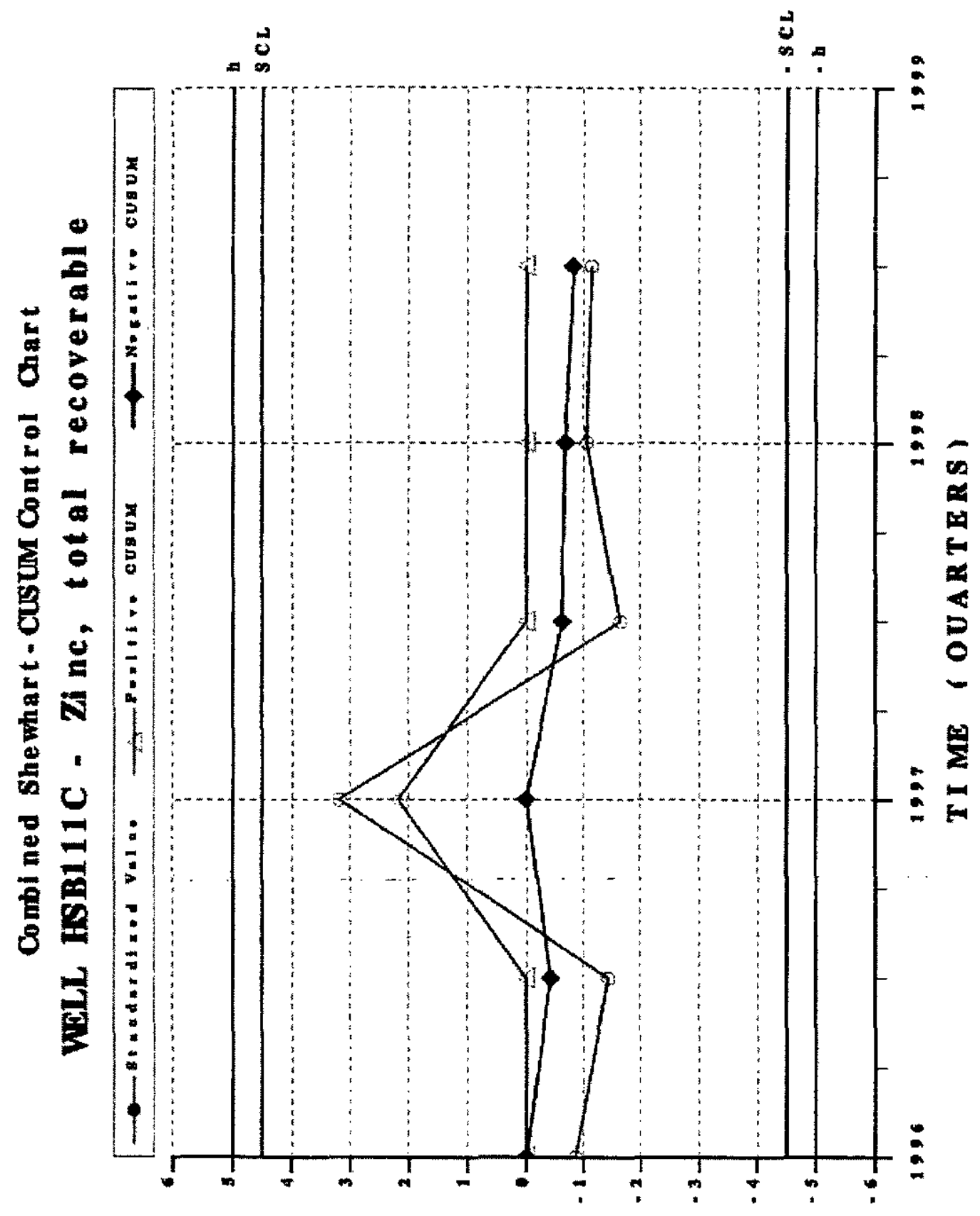

st ta 

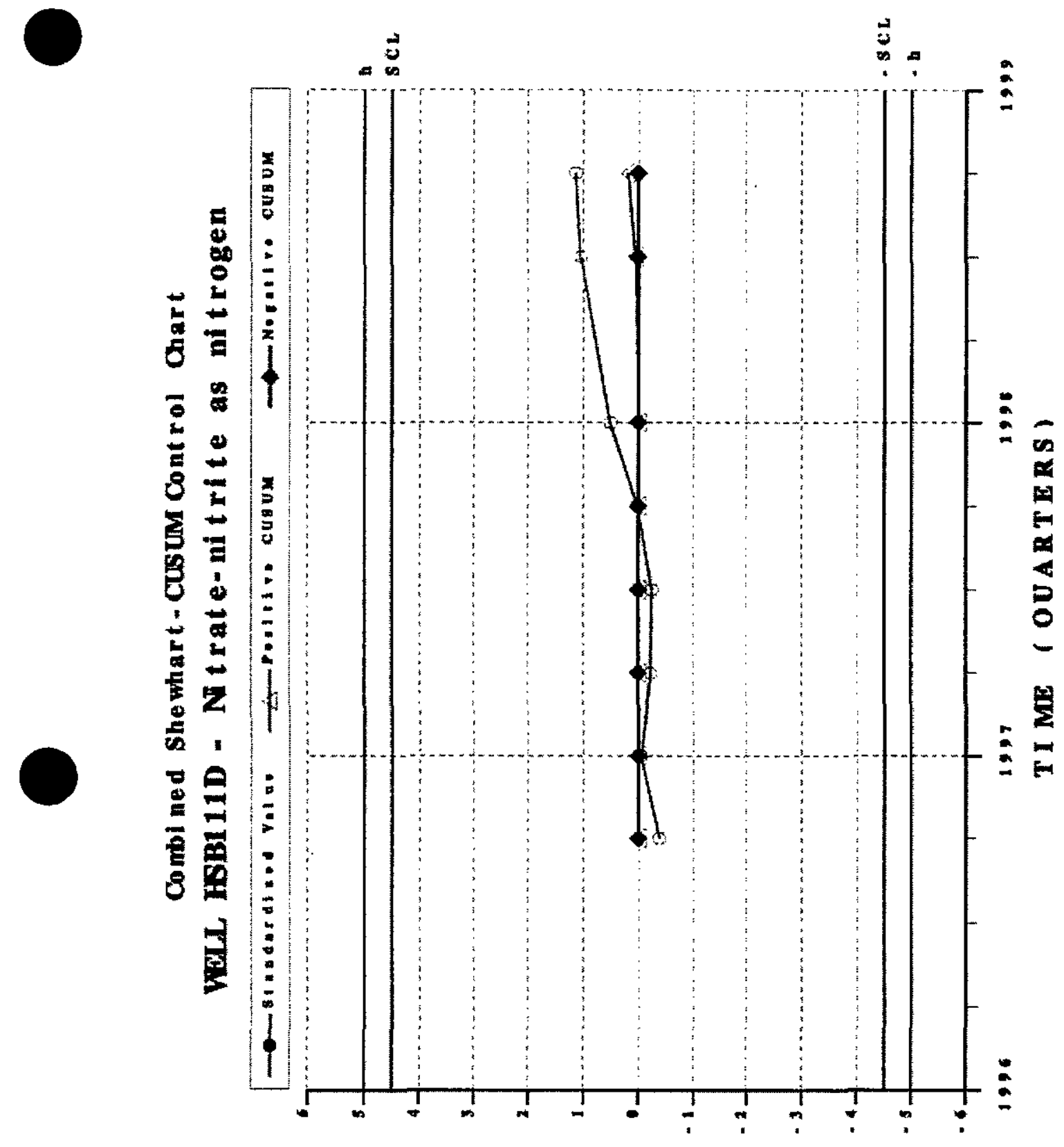

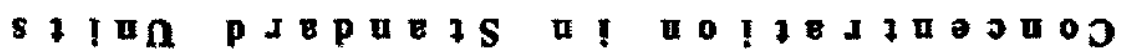


Combi ned Shewhart - Cusum Control Chart WELL HSB111E - Bari um total recoverable

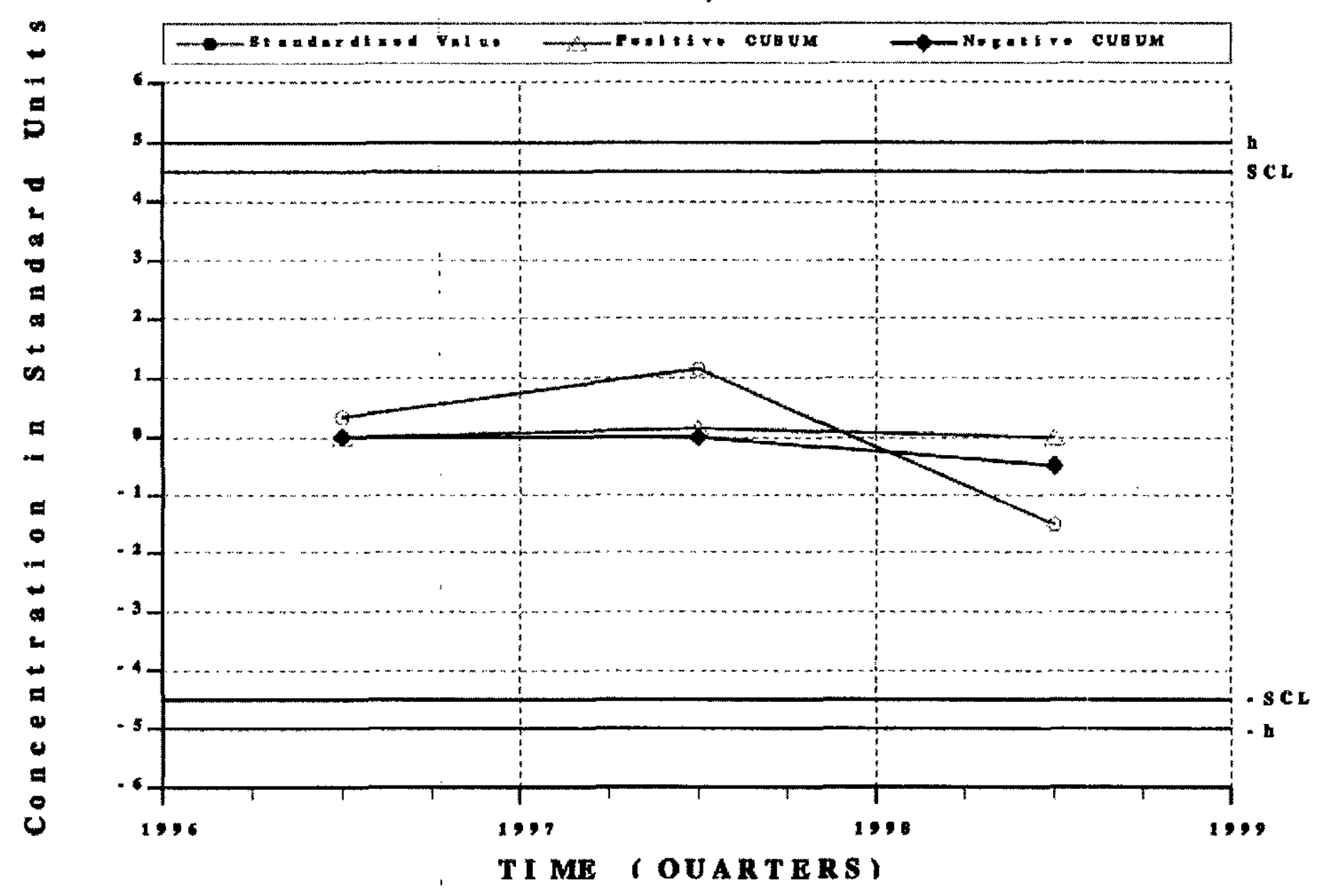

T ME ( OUARTERS 
Combi ned She whart - CuSuM Cont rol Chart

WELL HSB111 1 - Gross a l pha

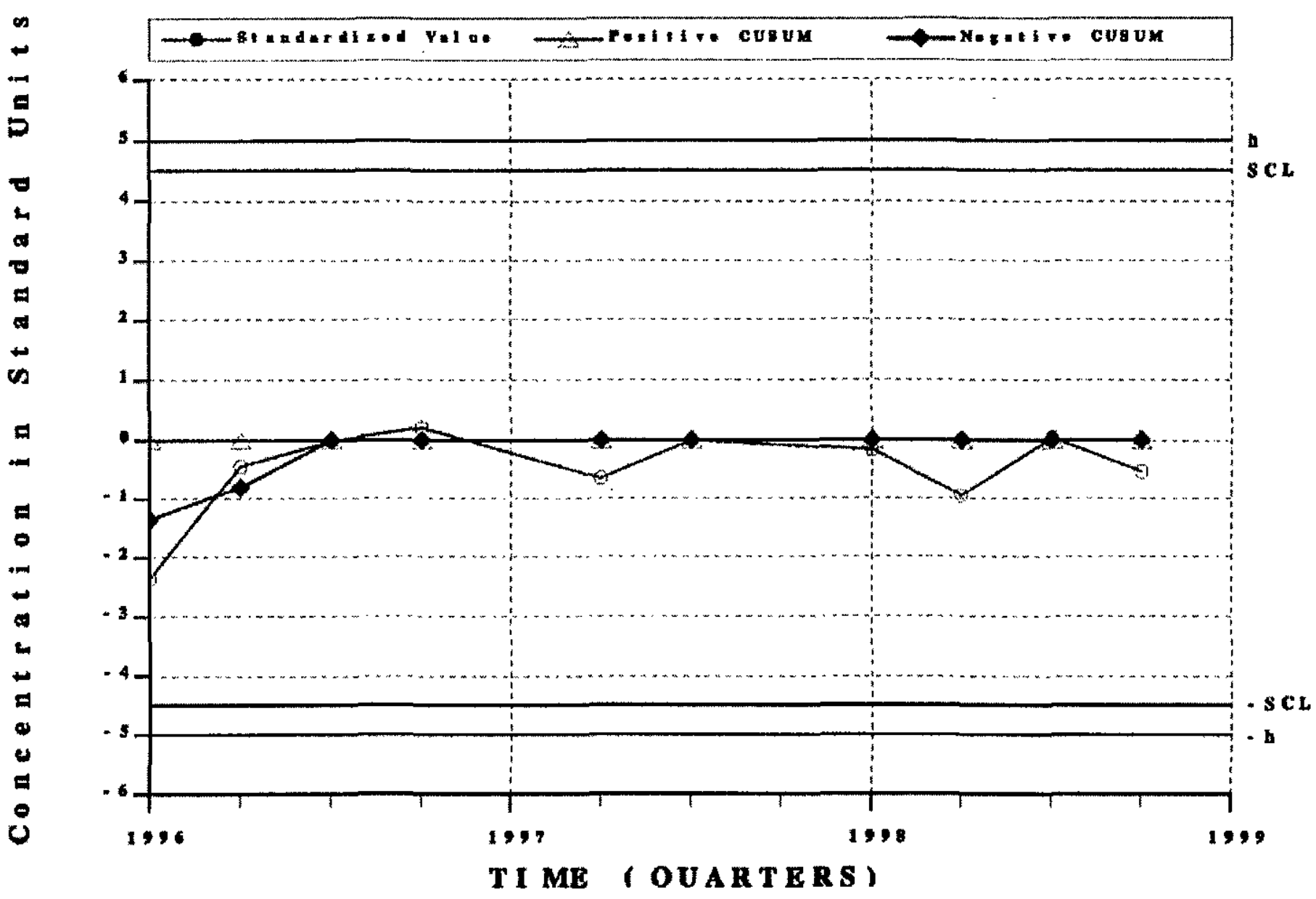




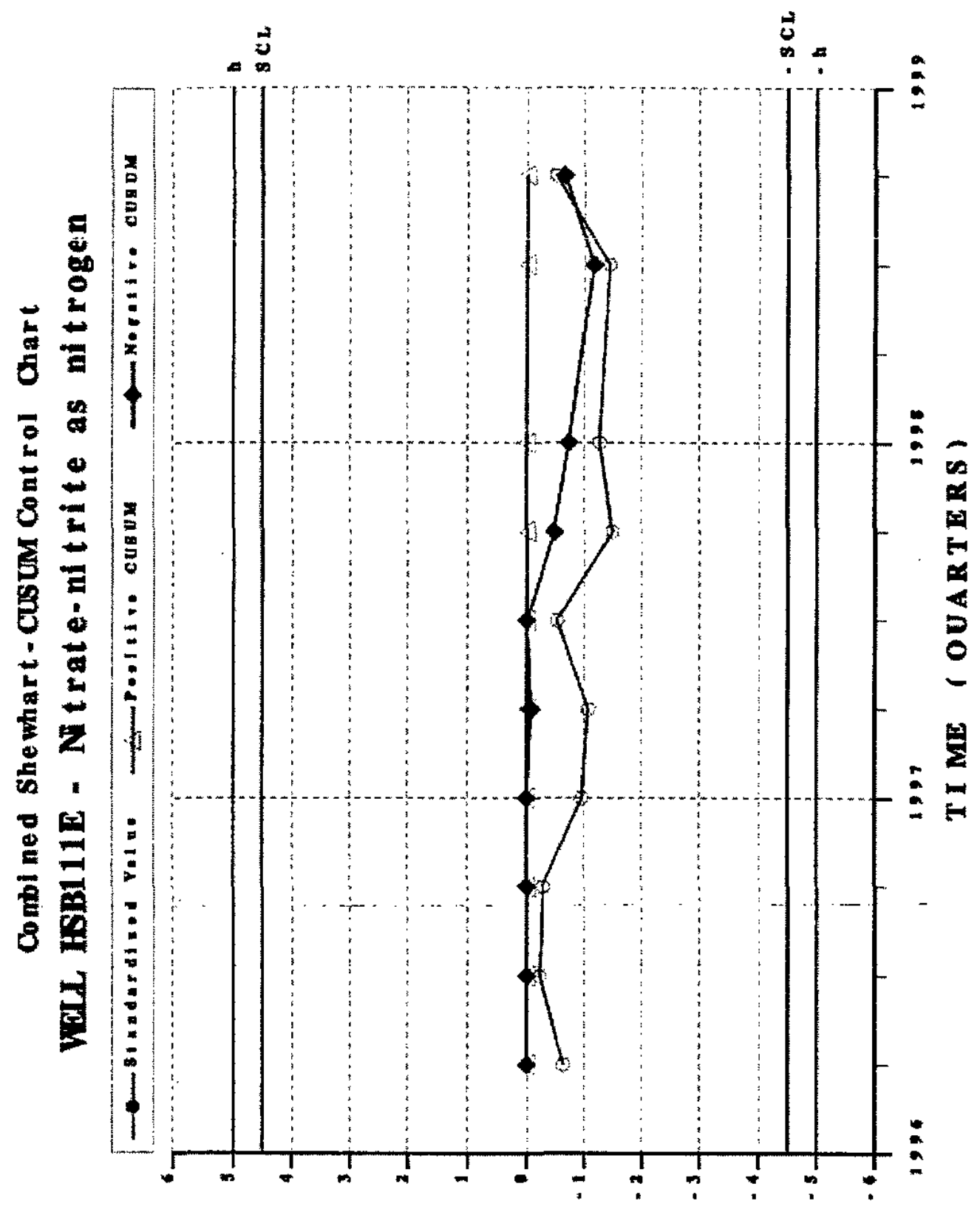

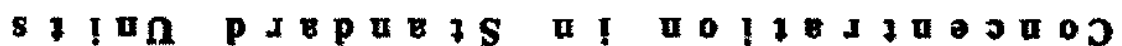




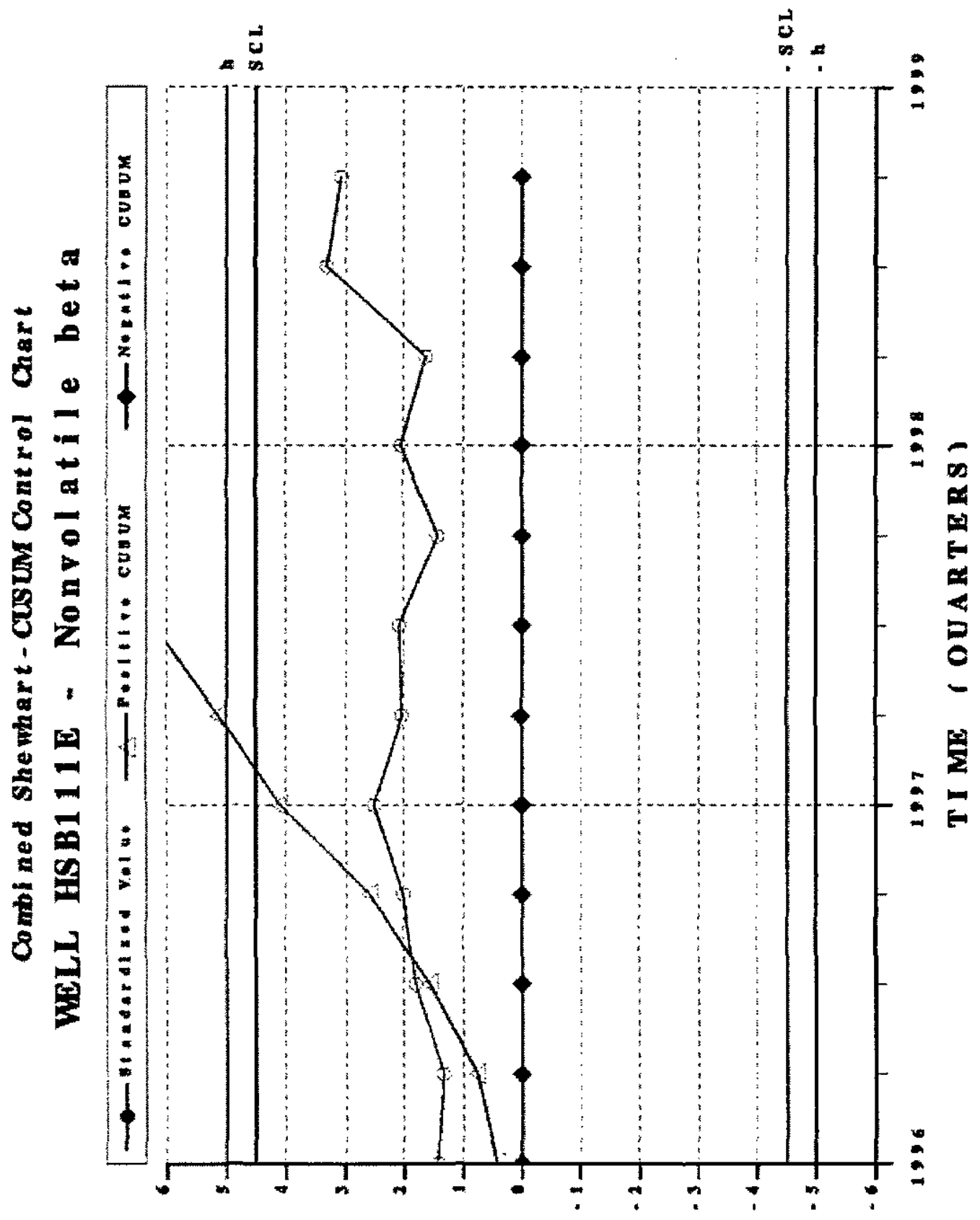

st 


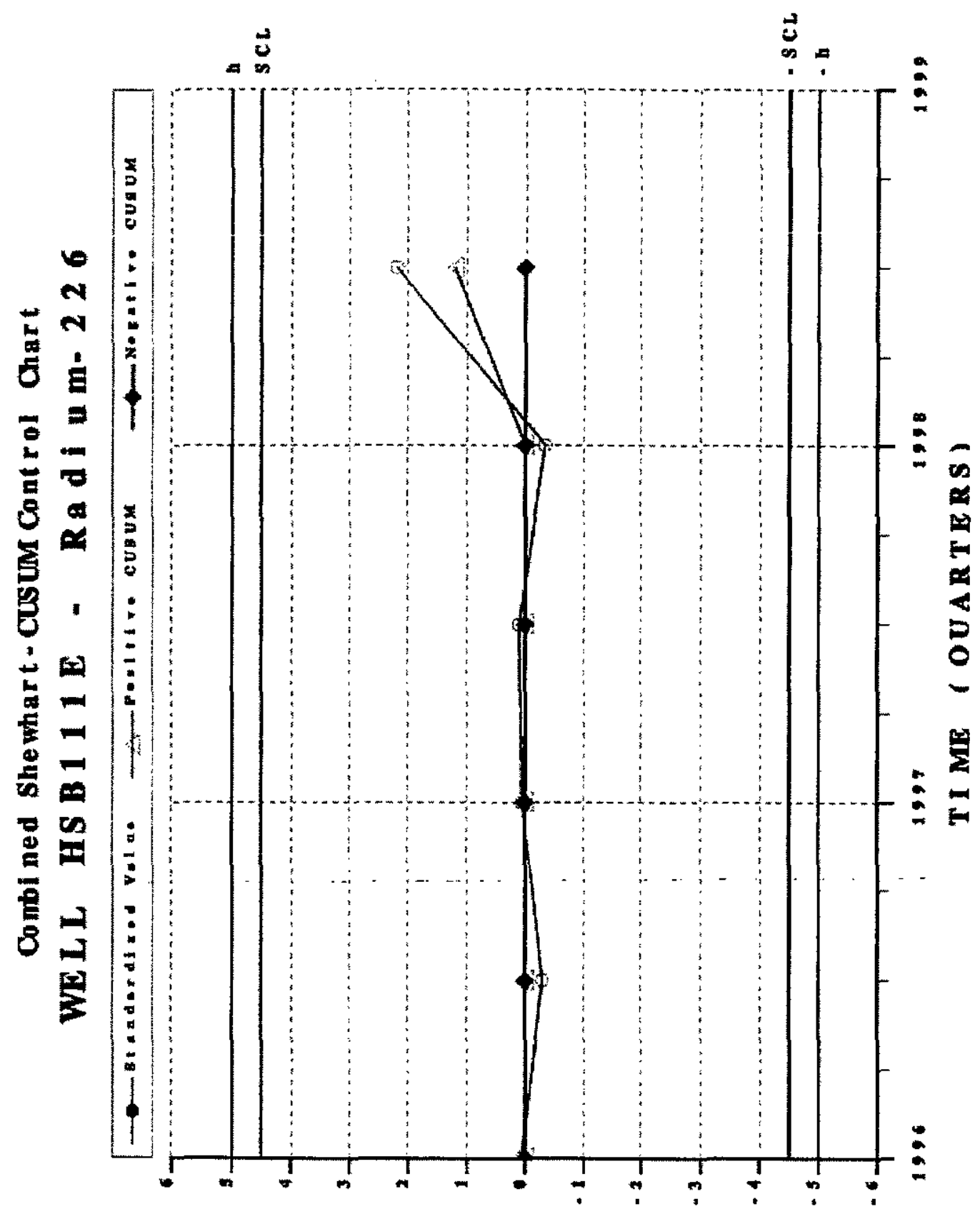

s I I 

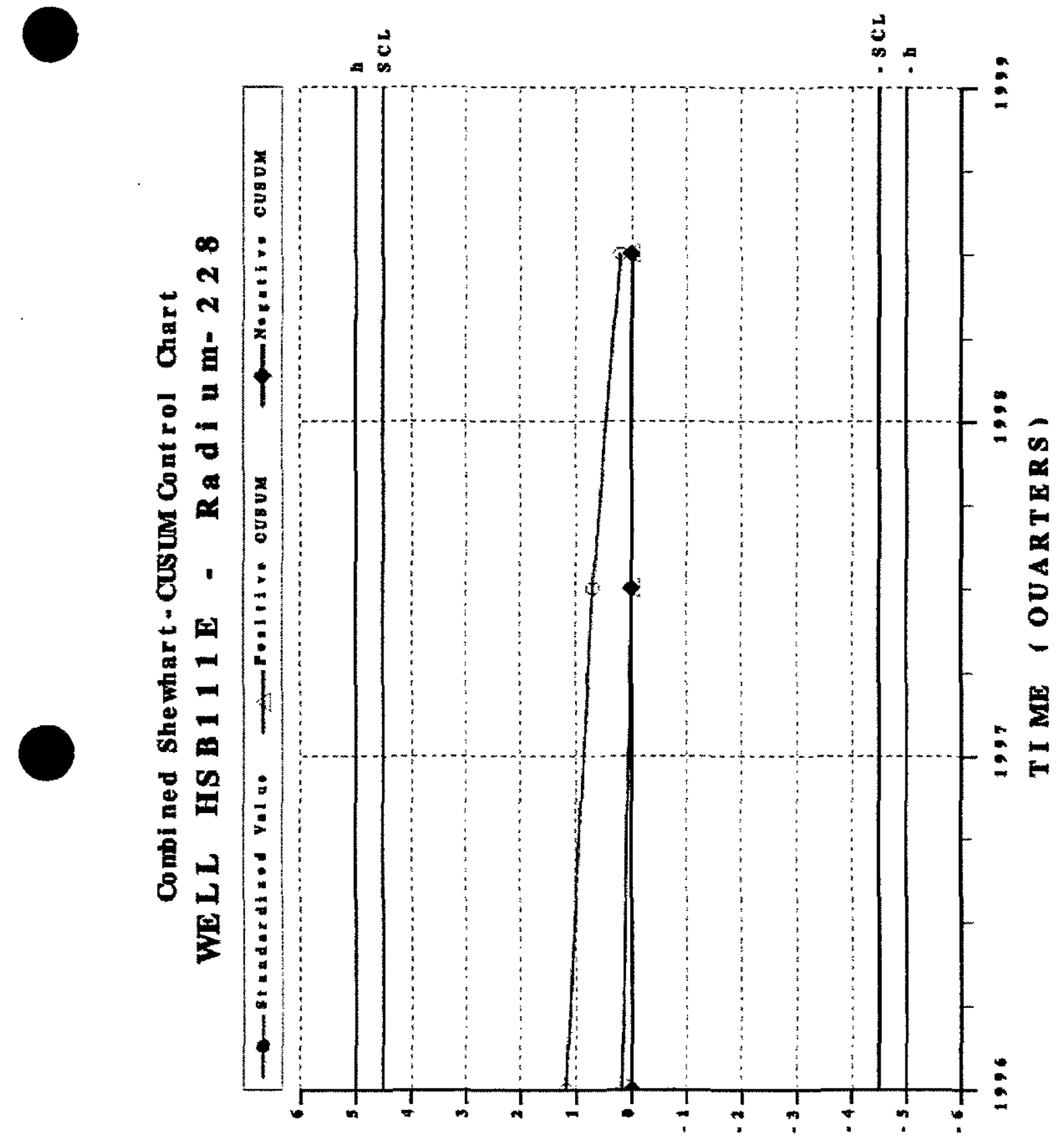

รI I 


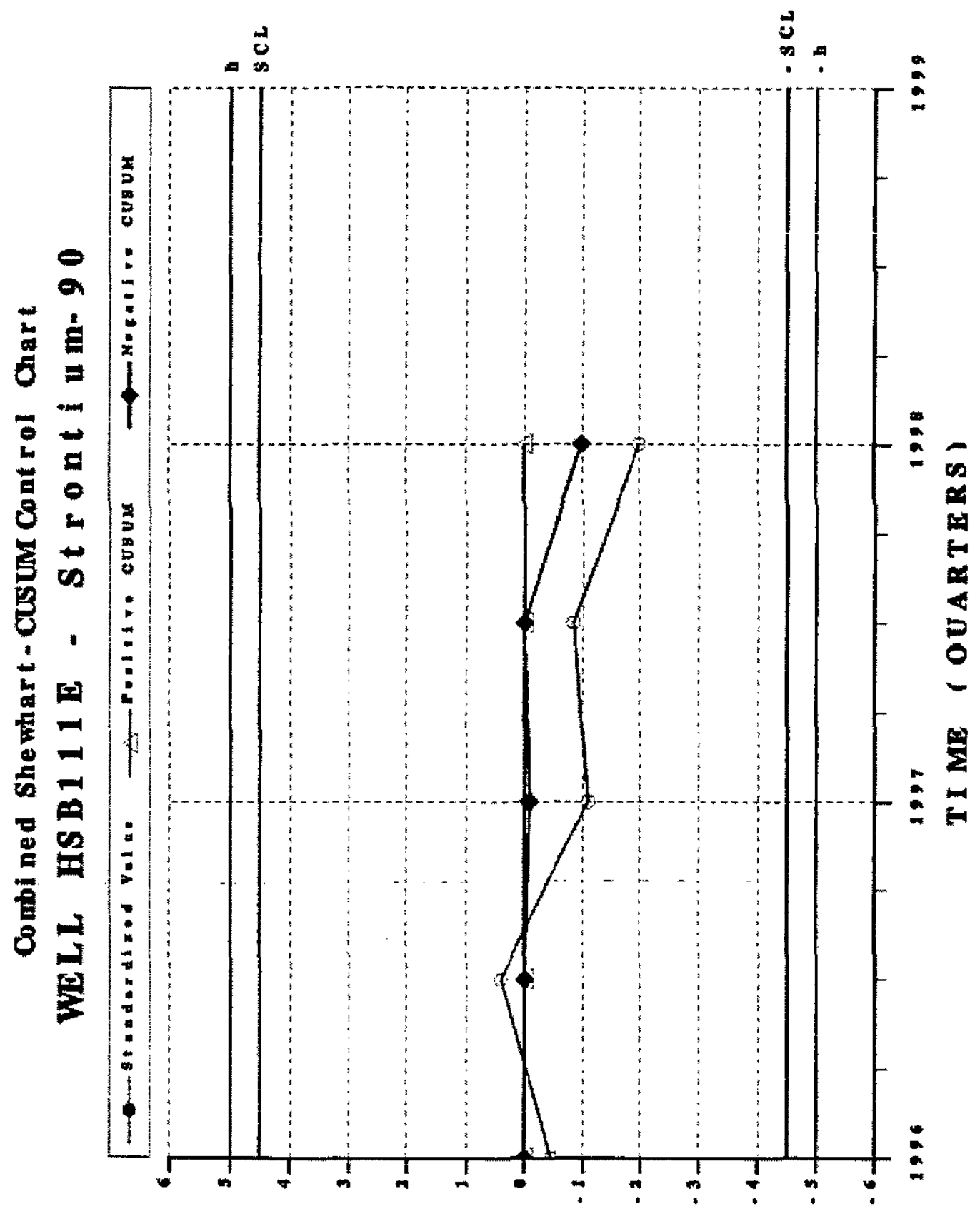

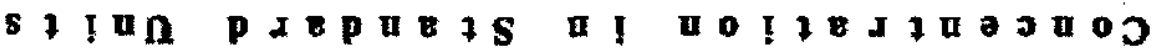



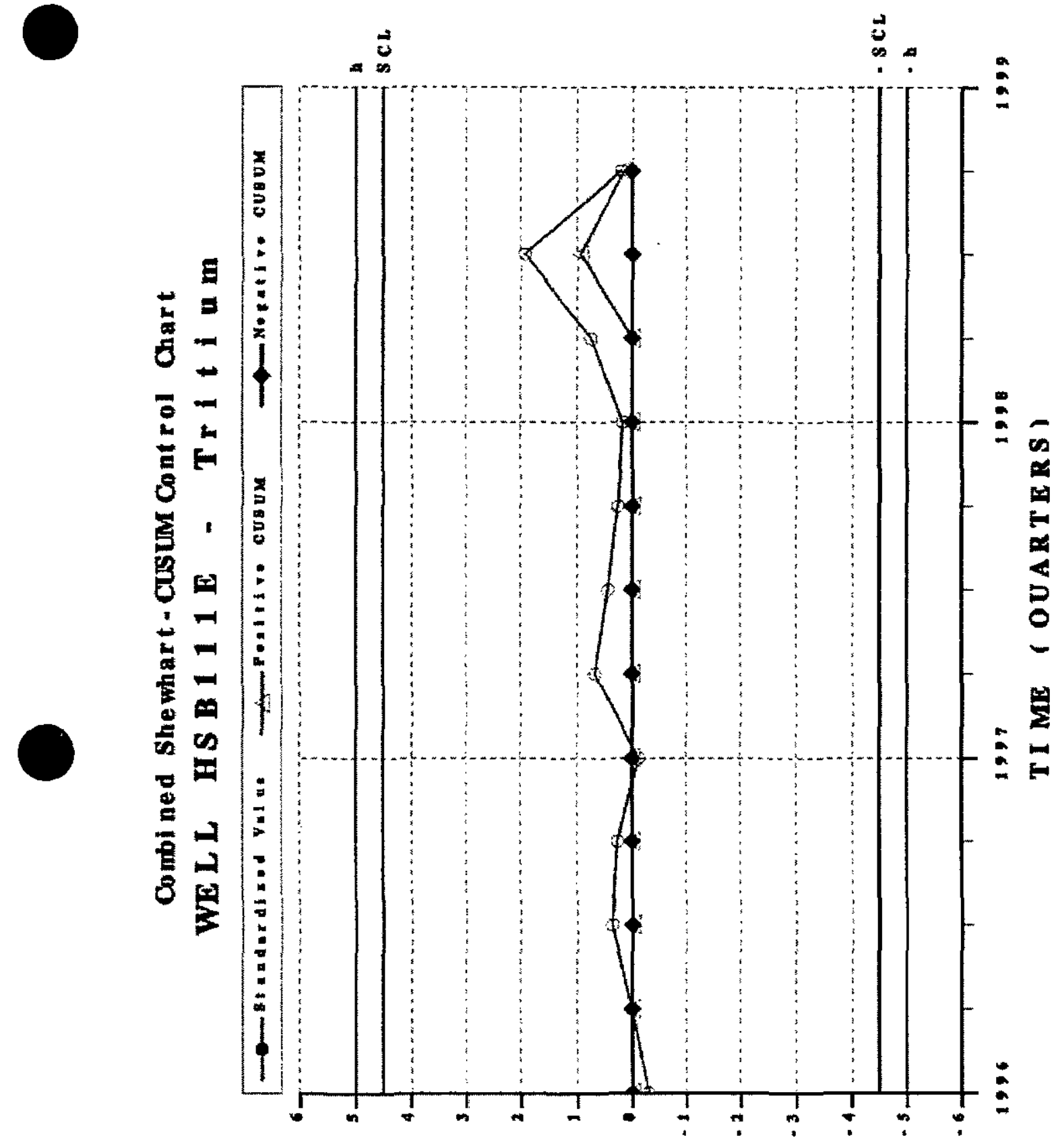

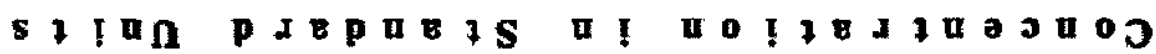


Combi ned Shewhart-CuSuM Cont rol Chart

WELL HSB111E - Zi nc, tot al recoverable

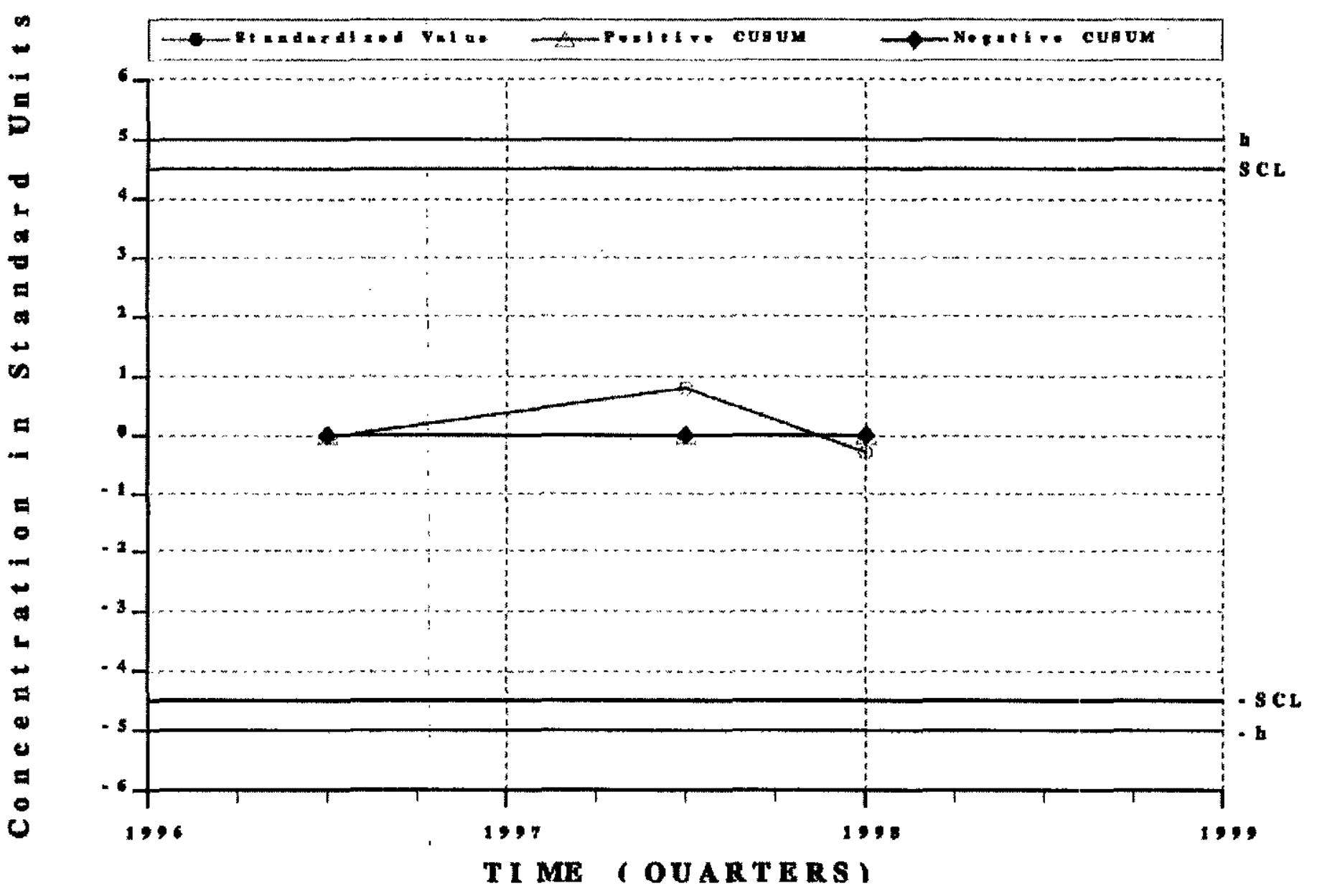

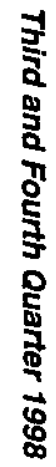

TIME (OUARTERS 
Combi ned Shewhart - Cusum Control Chart

VuL HSB112C - Barium total recoverable

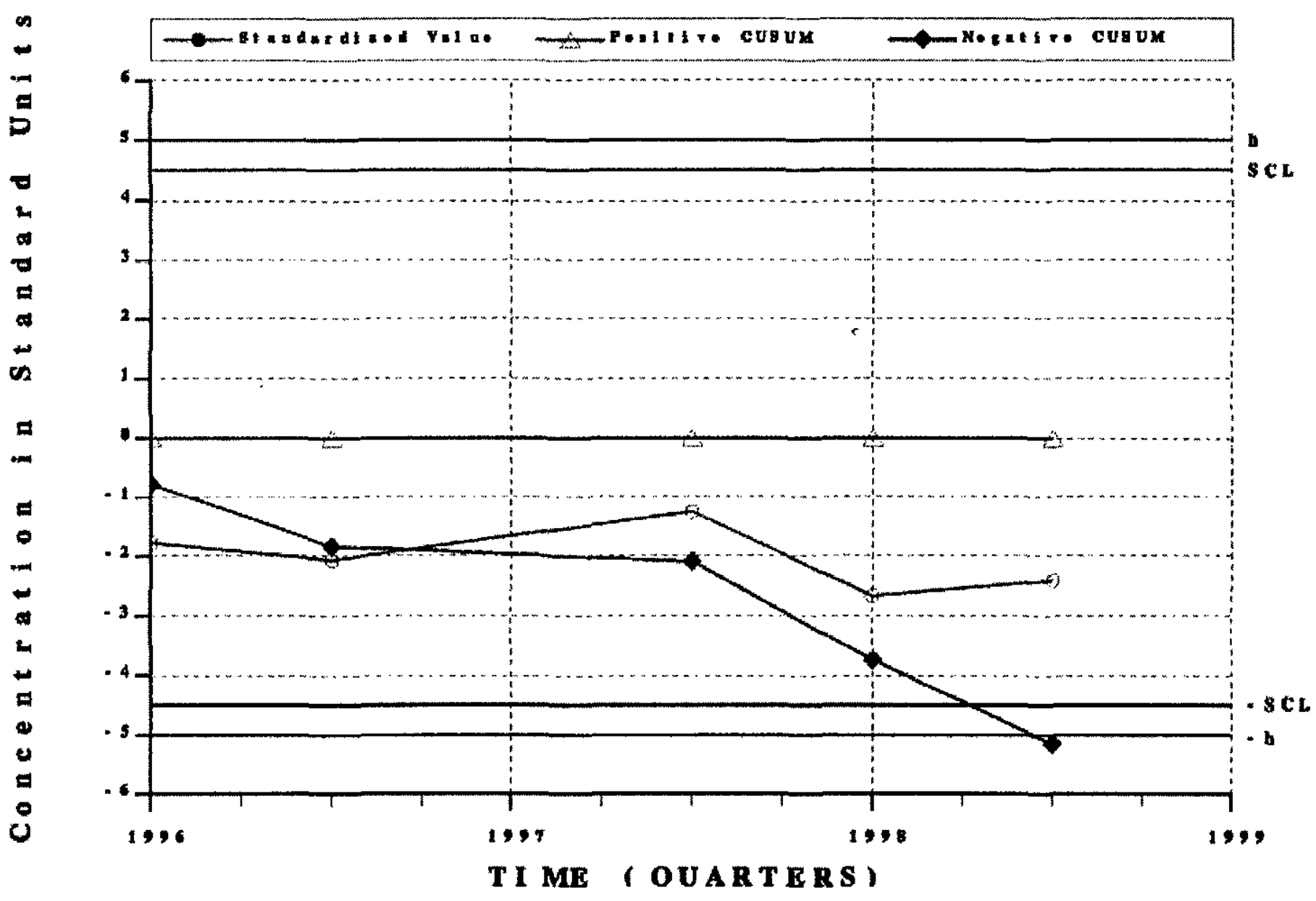




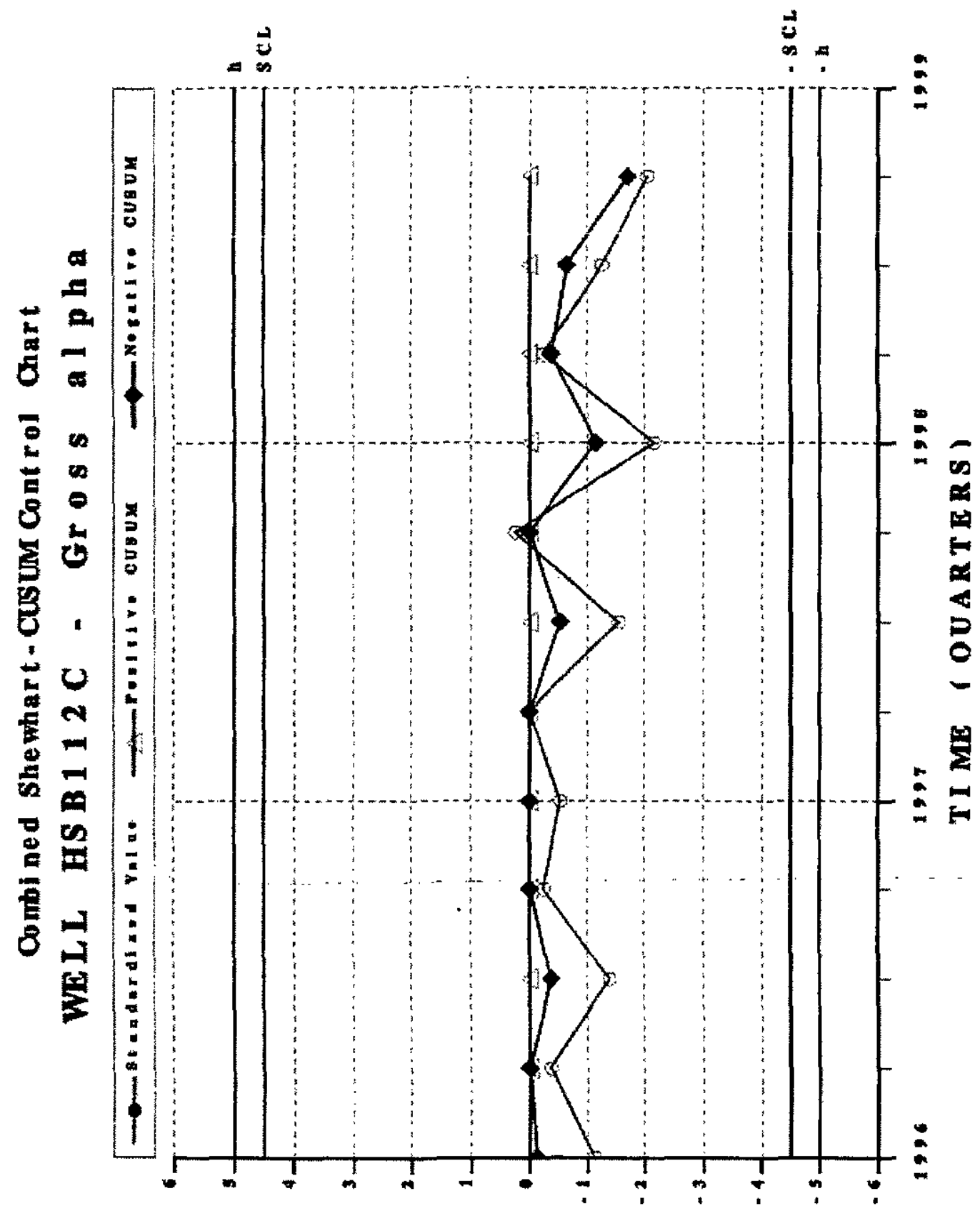

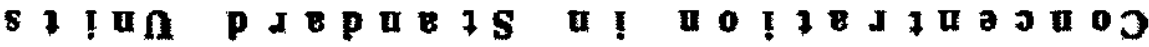




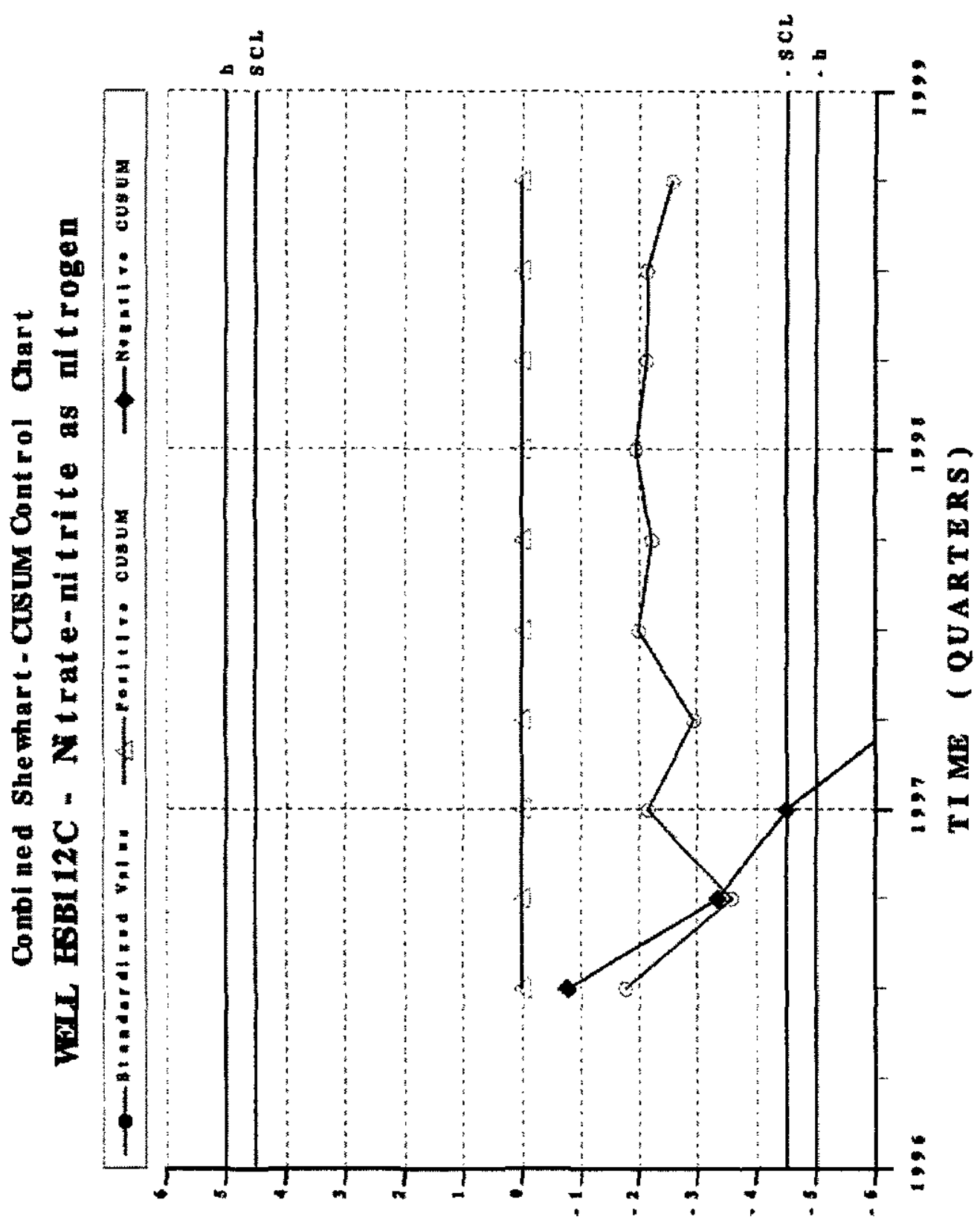

st I I pa 

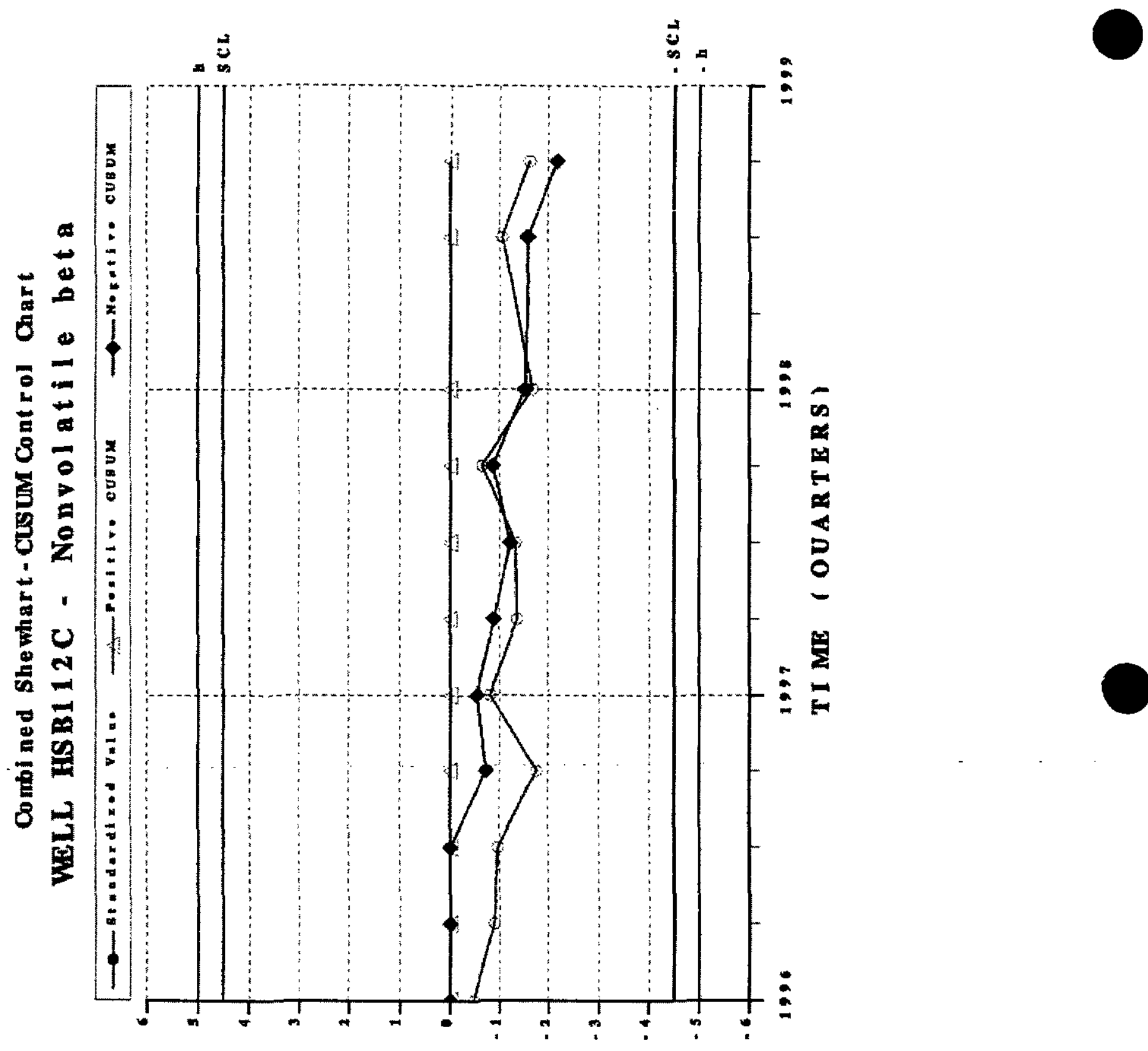


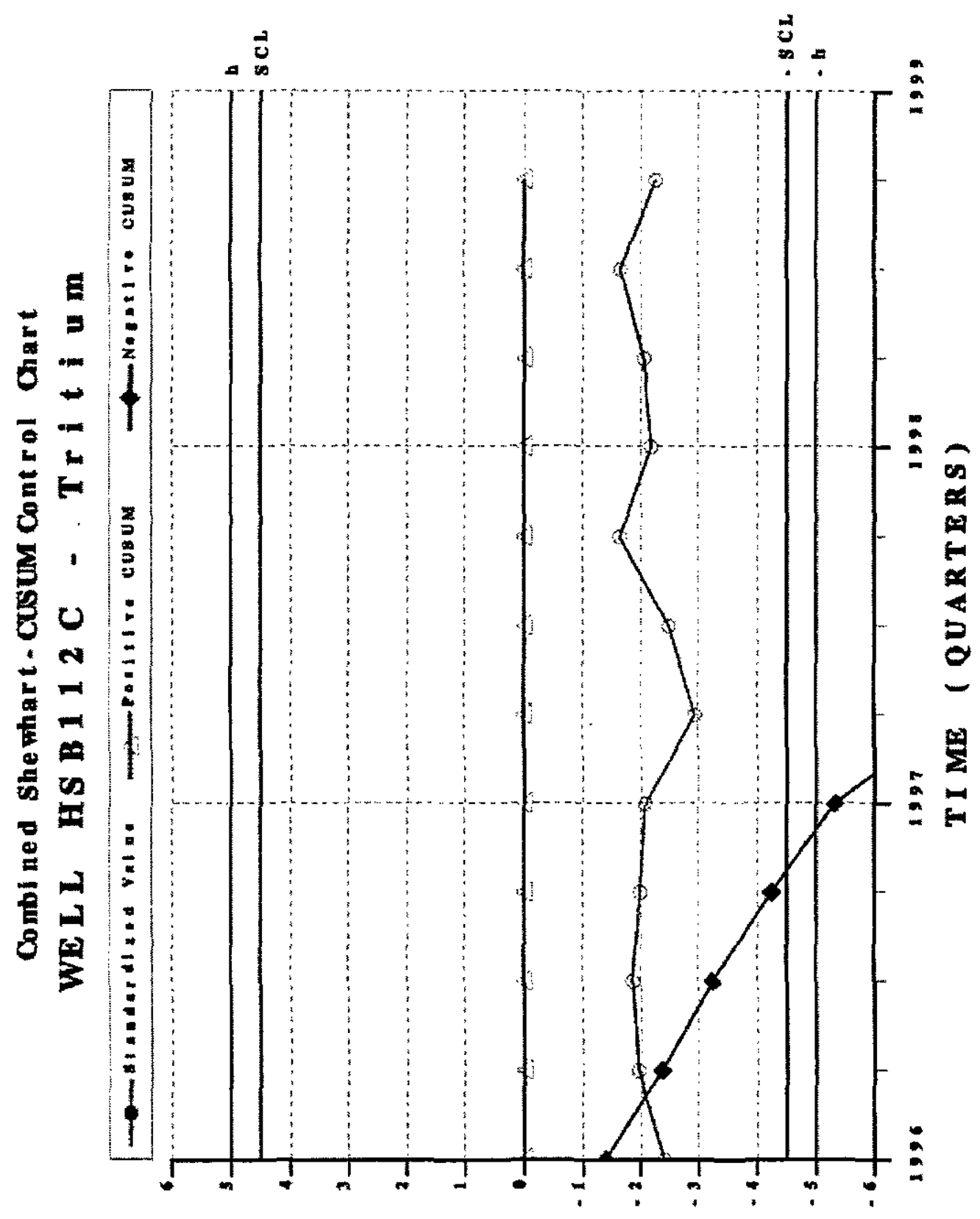

st I 


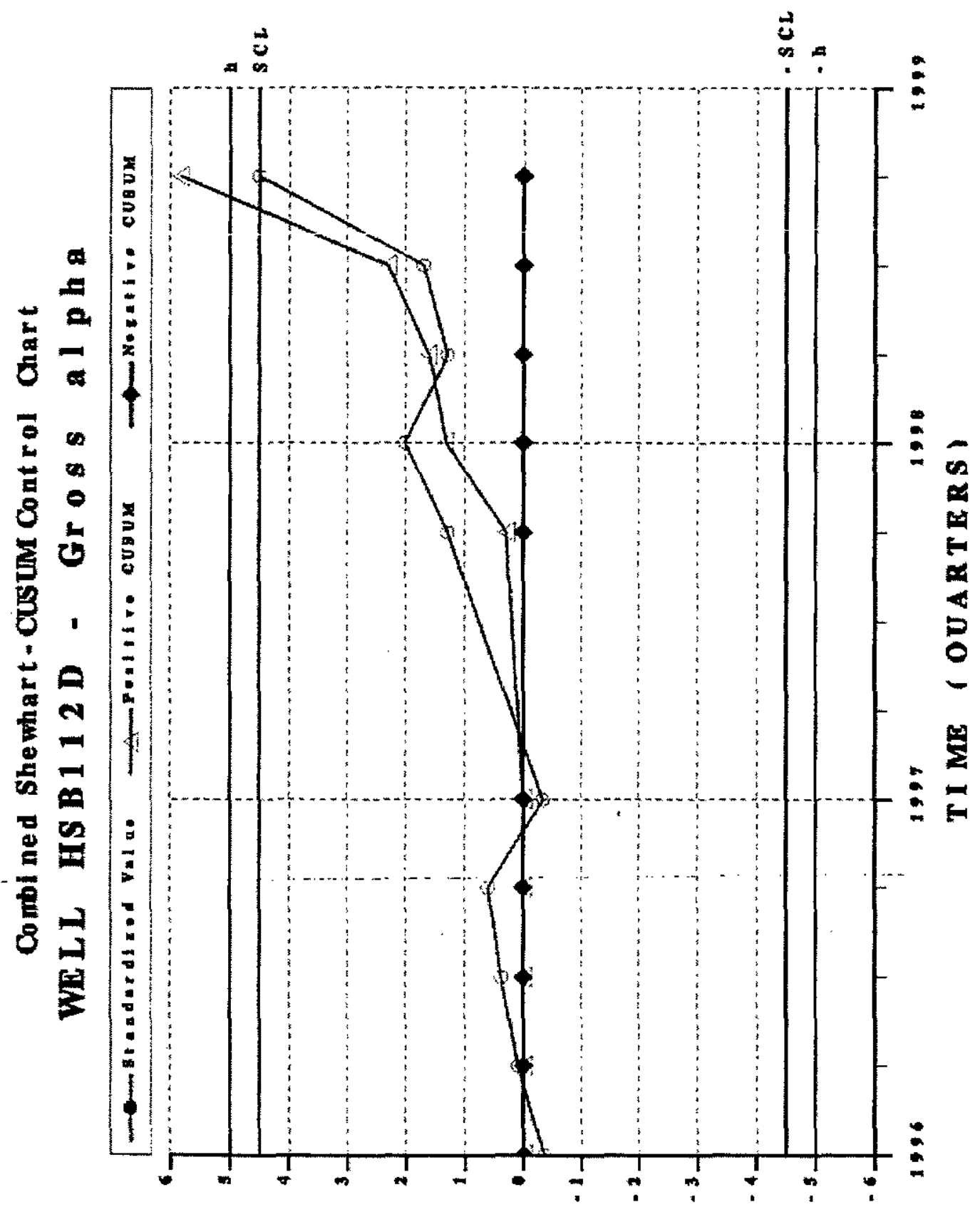

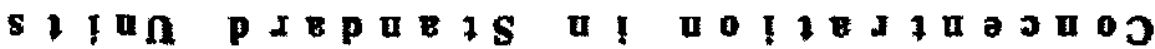




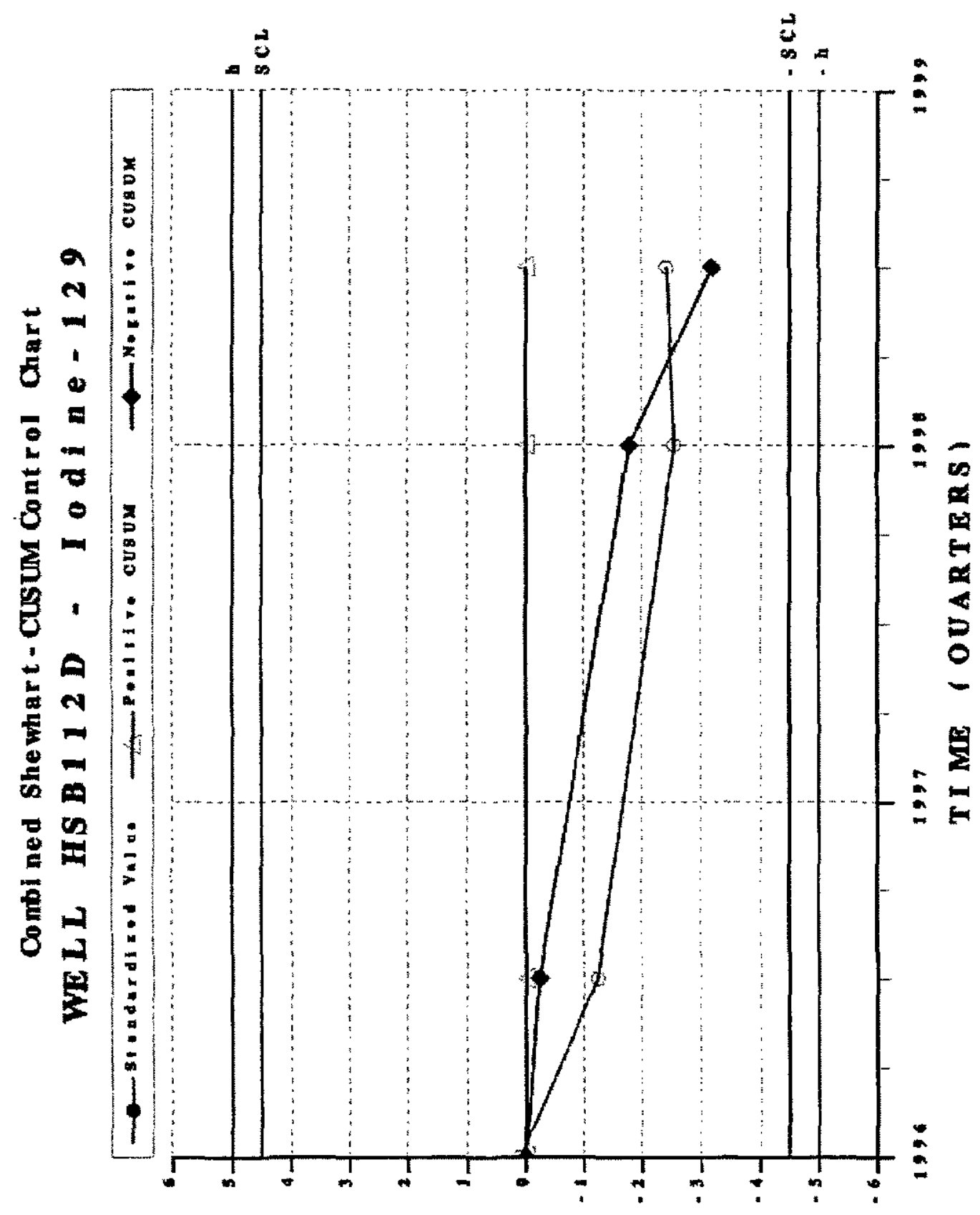

sI I 


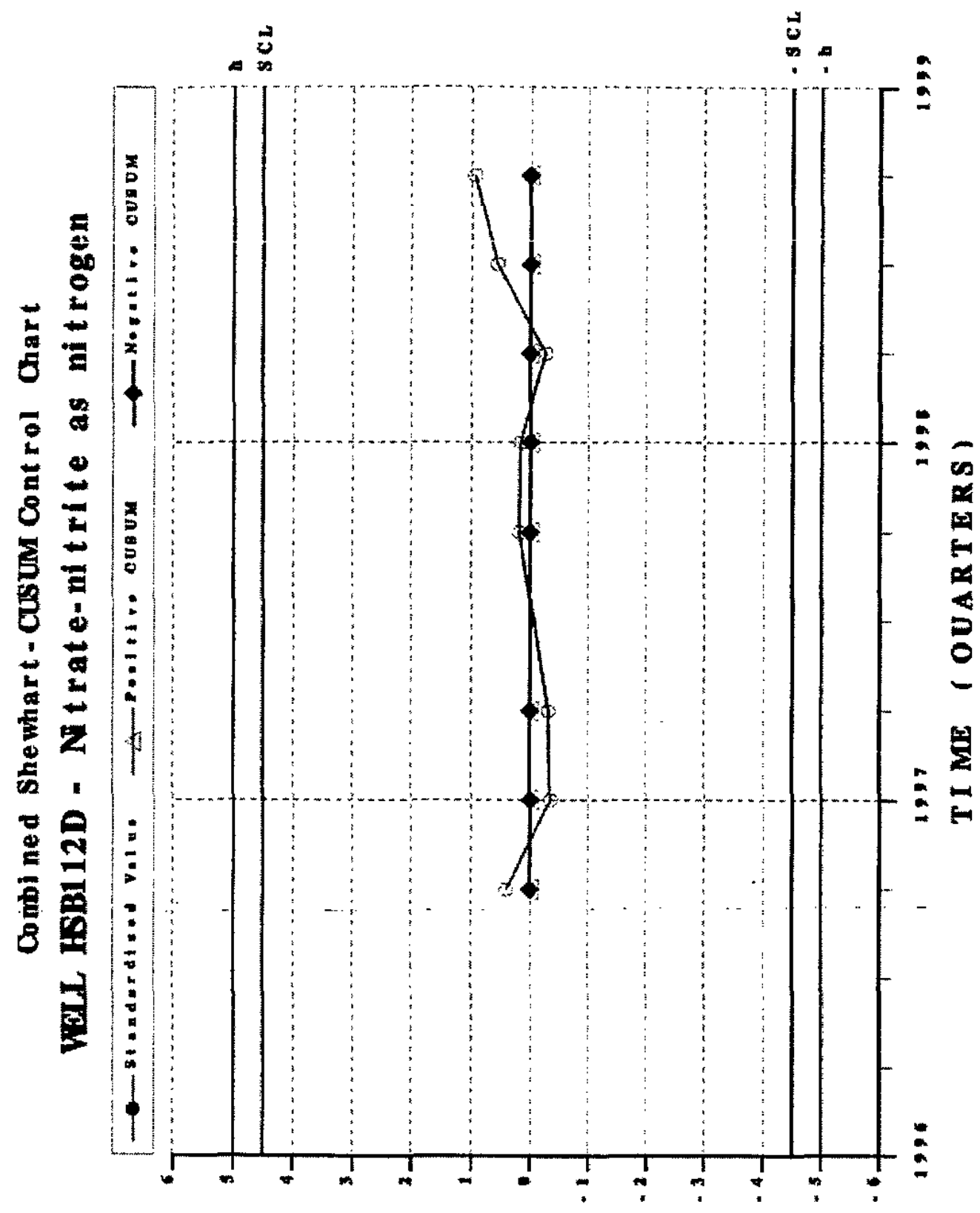

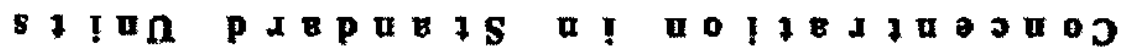




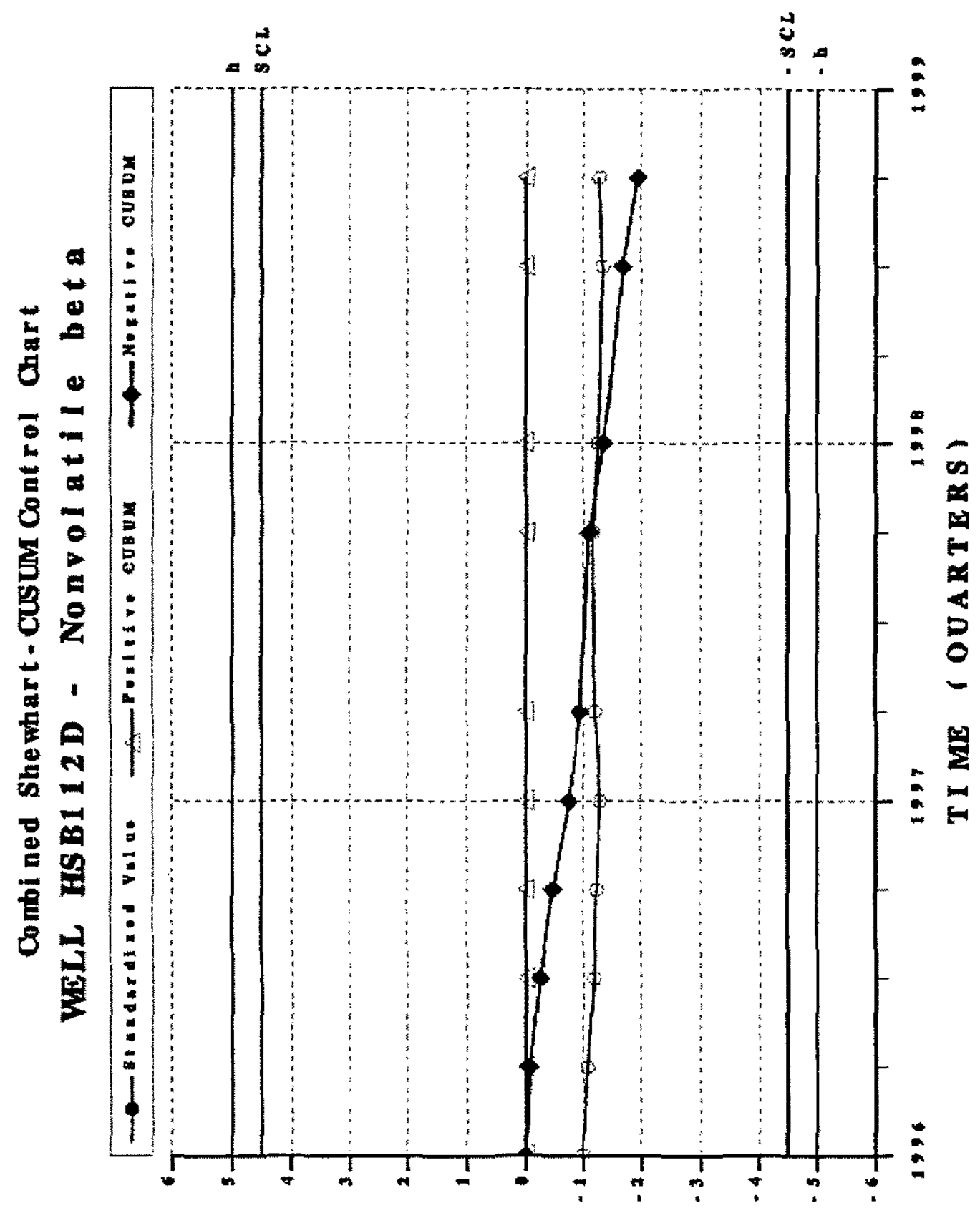

s I I 


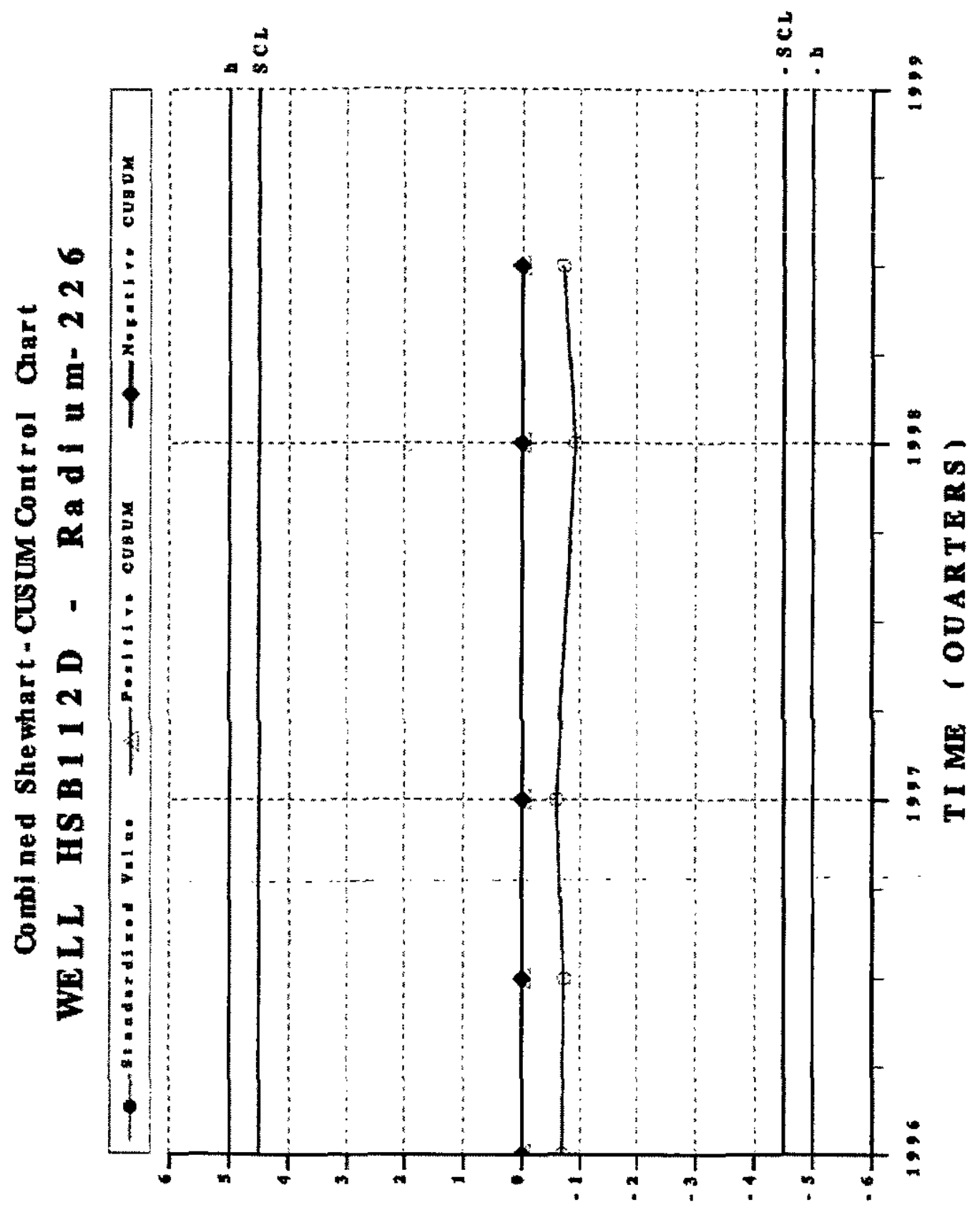

s I In pa E 

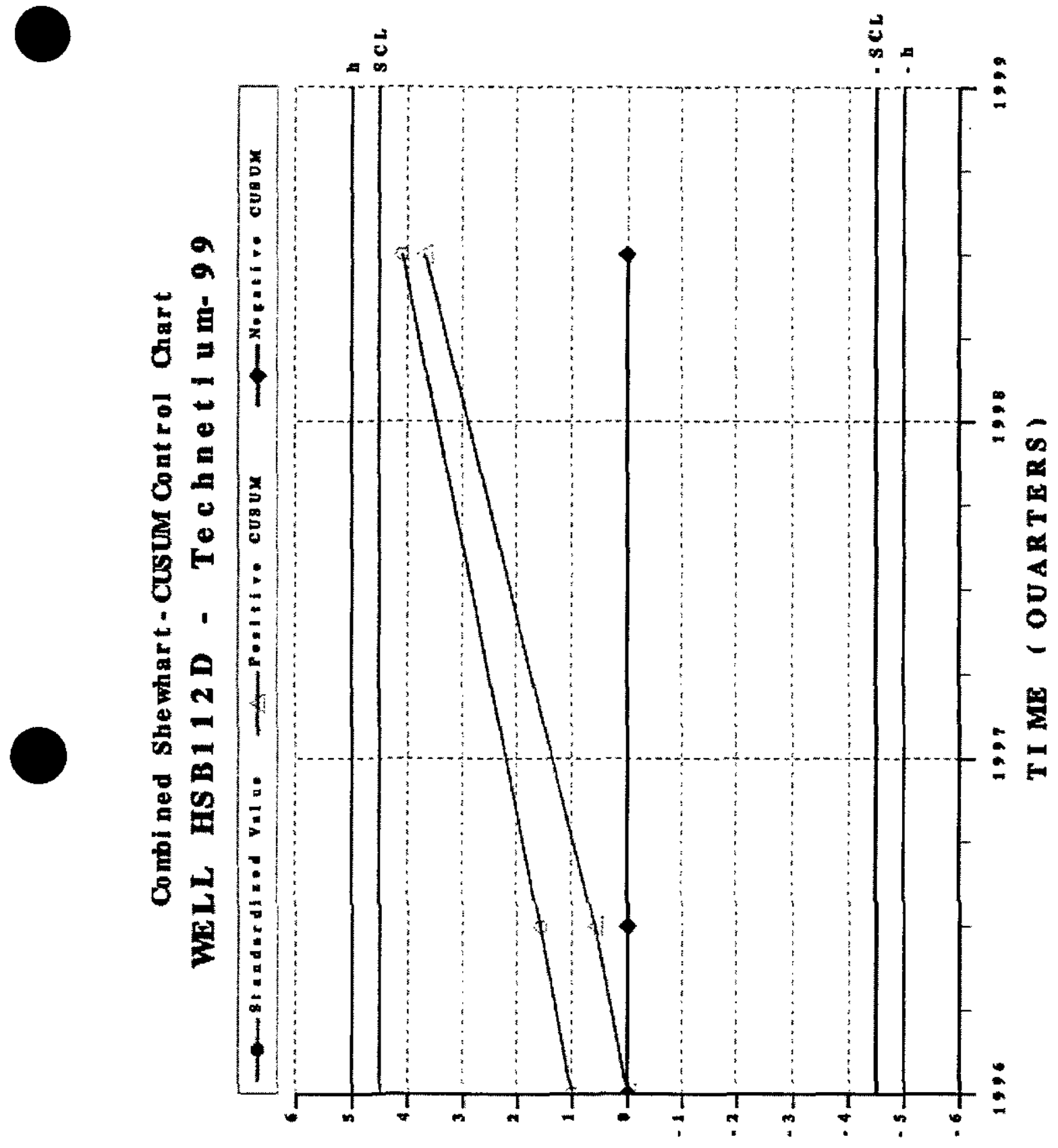

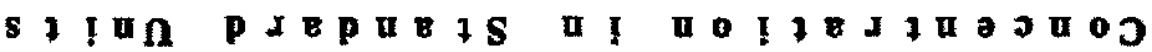




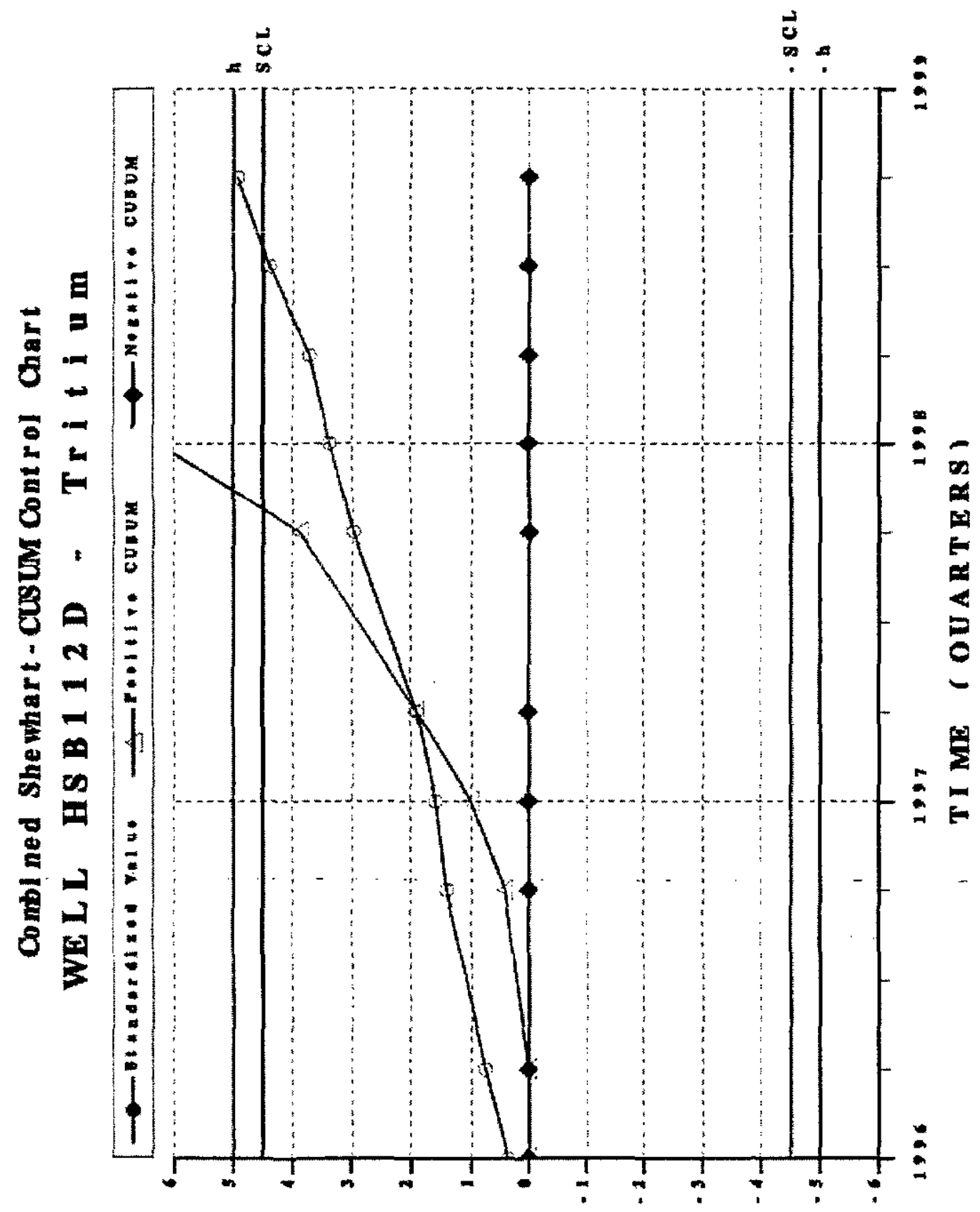

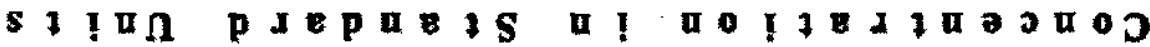



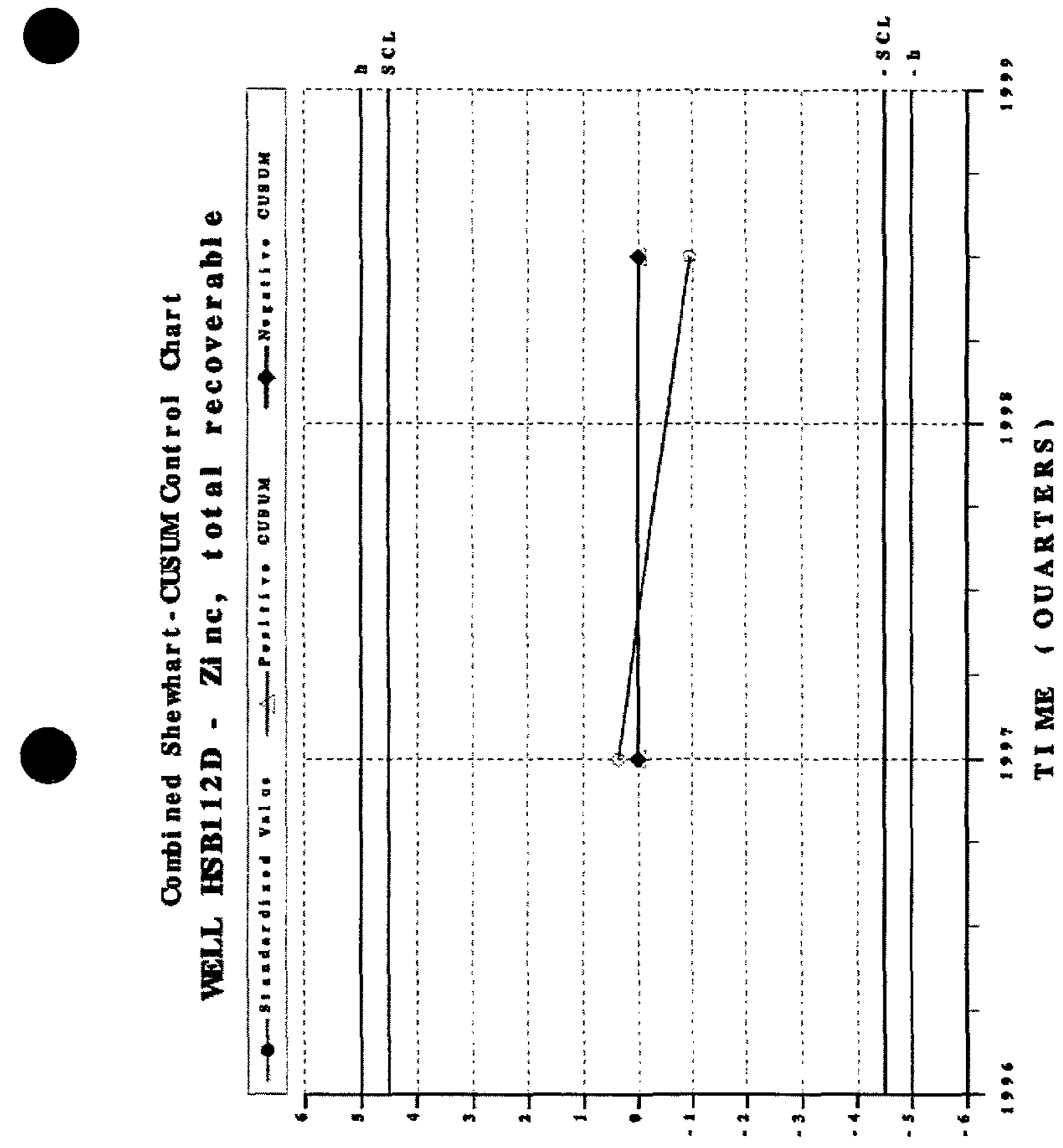

s I I I 


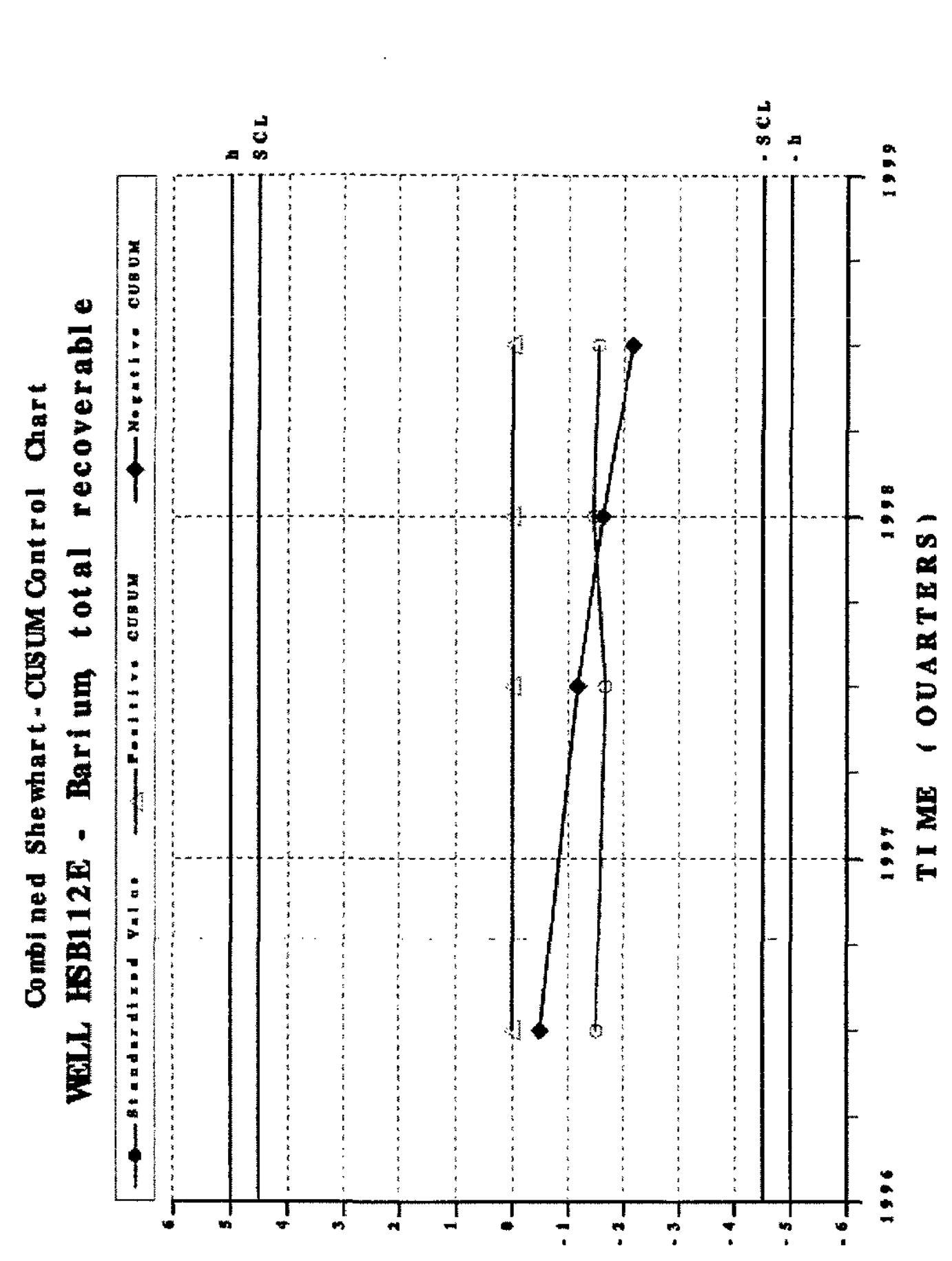

WSRC-TR-99-00013

Unclassified

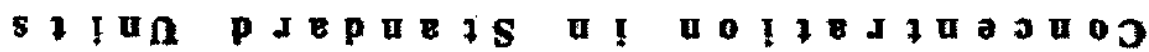



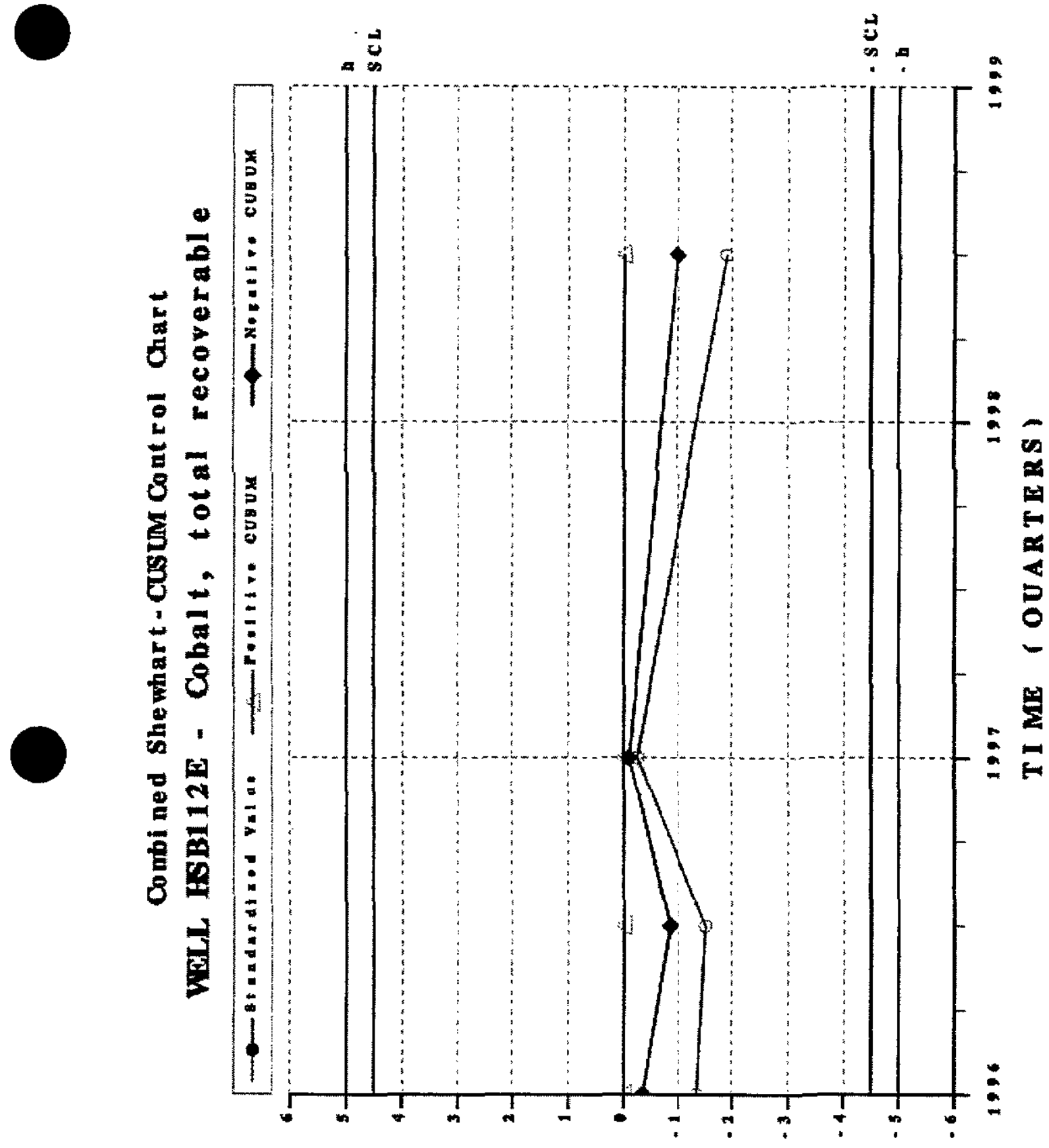

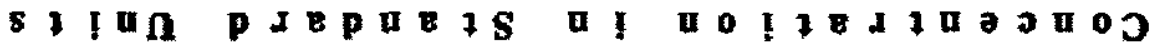




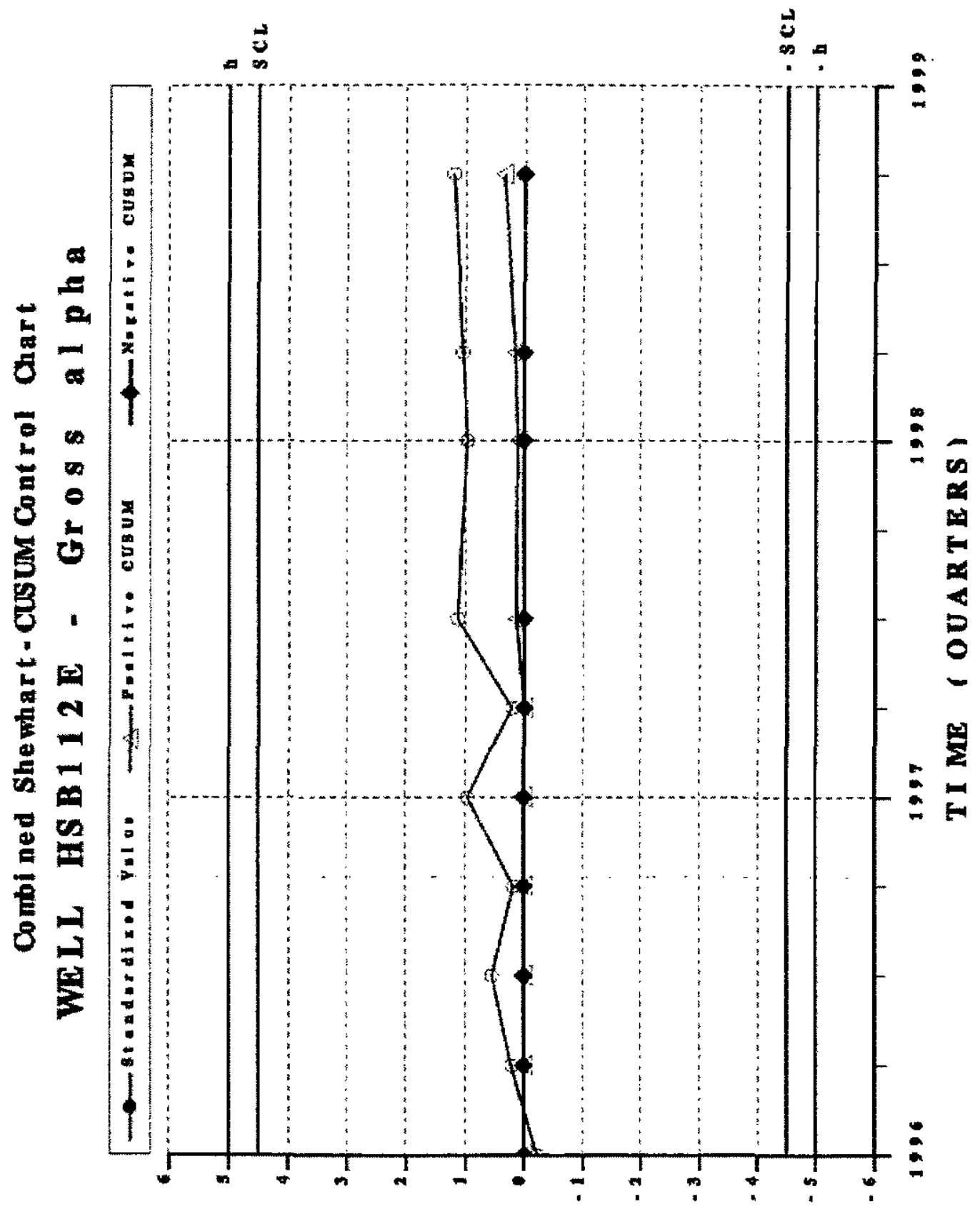

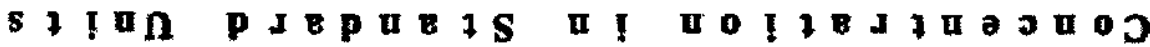


Combi ned Shewhart-CusuM Control Chart WELL HSB112E - Nickel, total recoverable

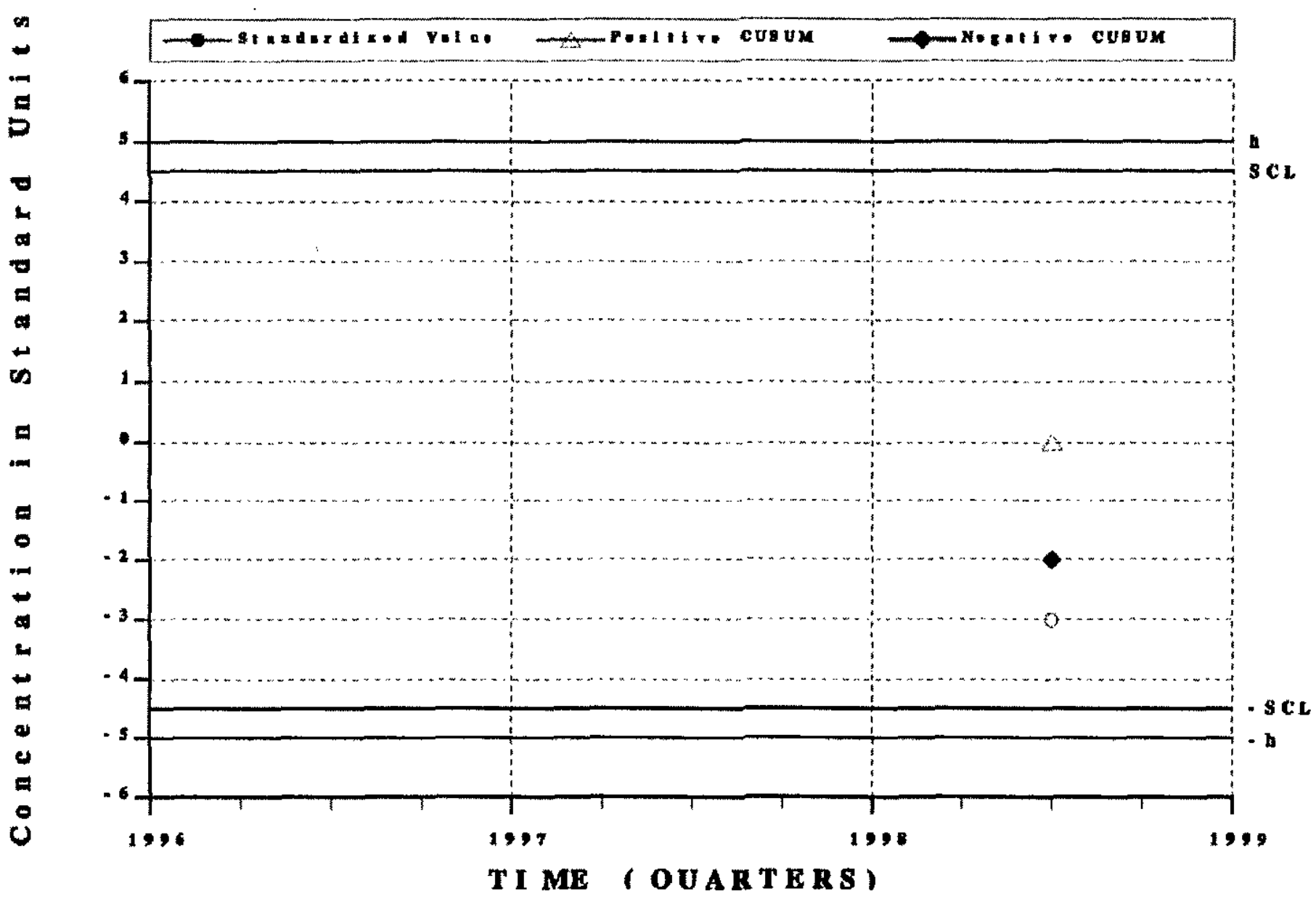




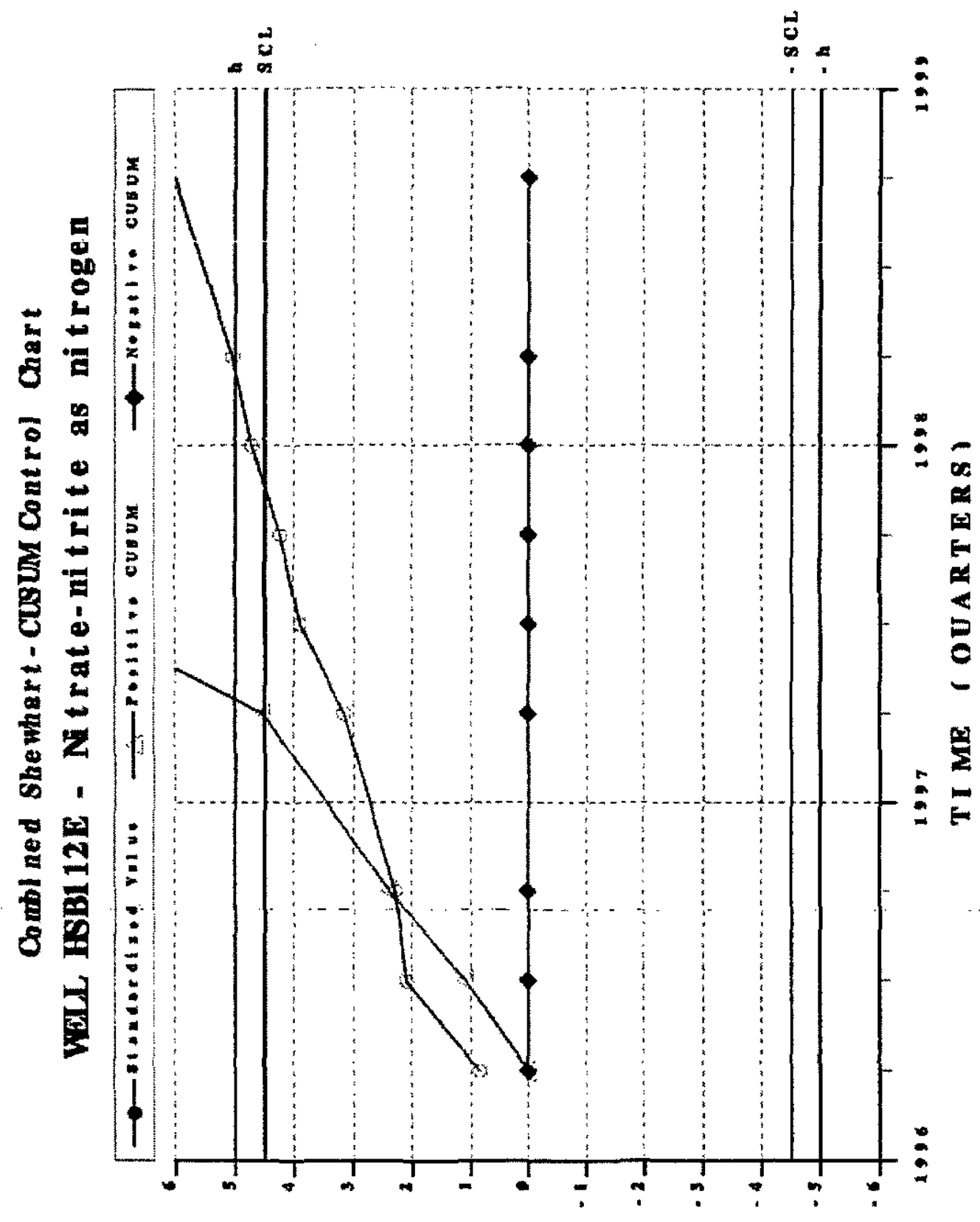

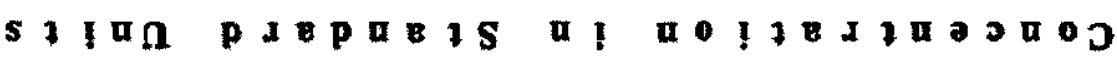


Combi ned Shewhart-CUSUM Control Chart WELL HSB112E - Nonvolatile beta

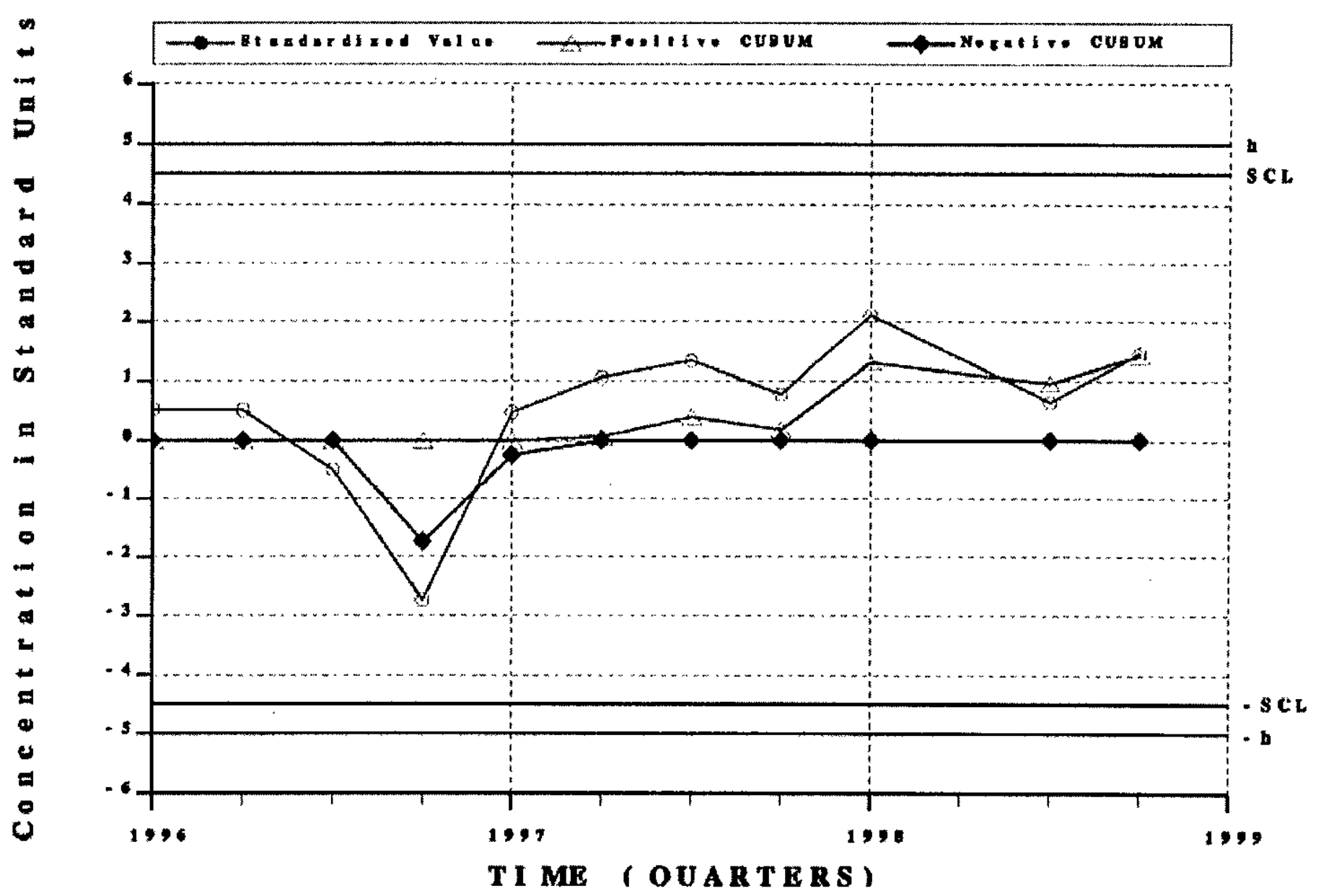




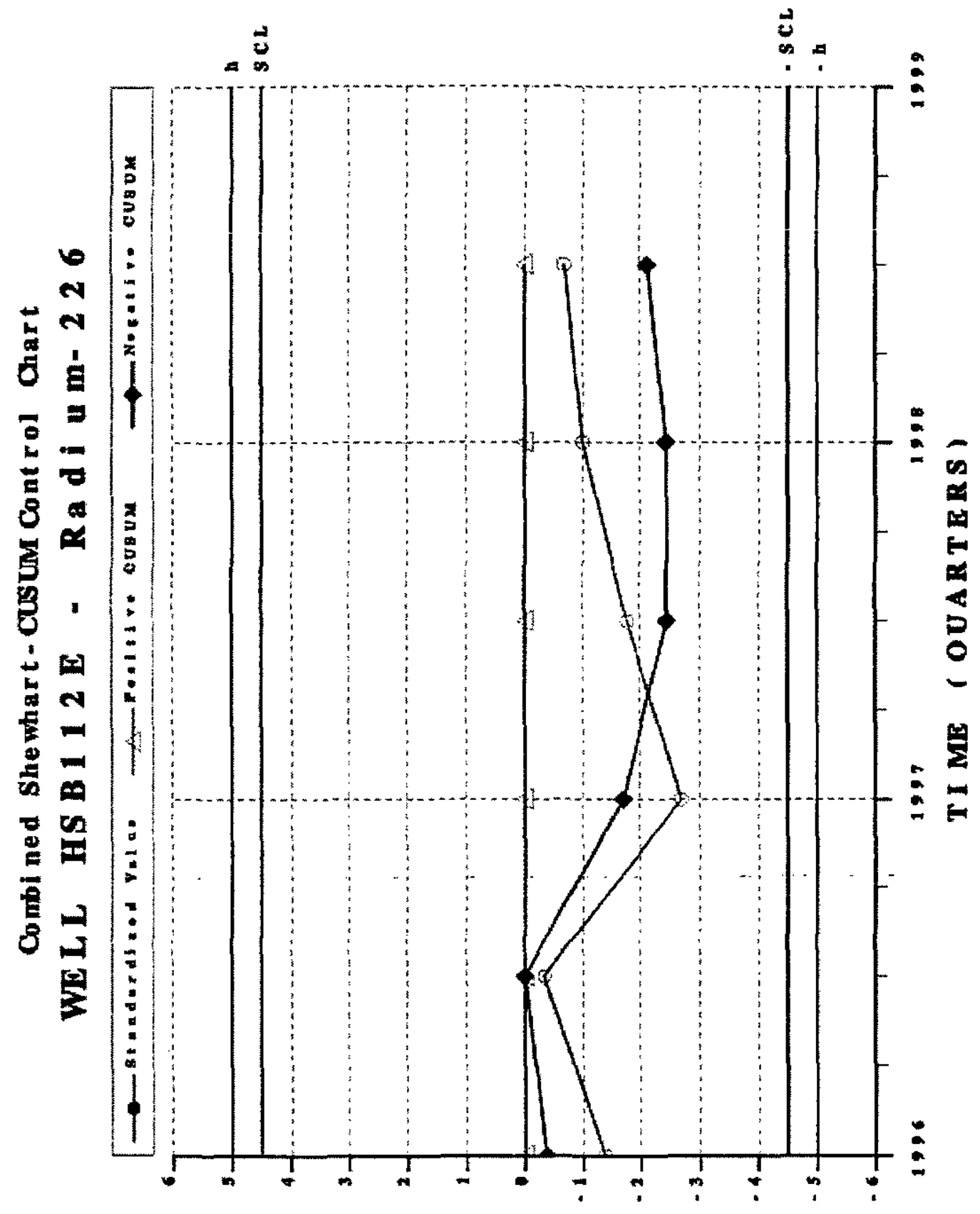

s I In p 

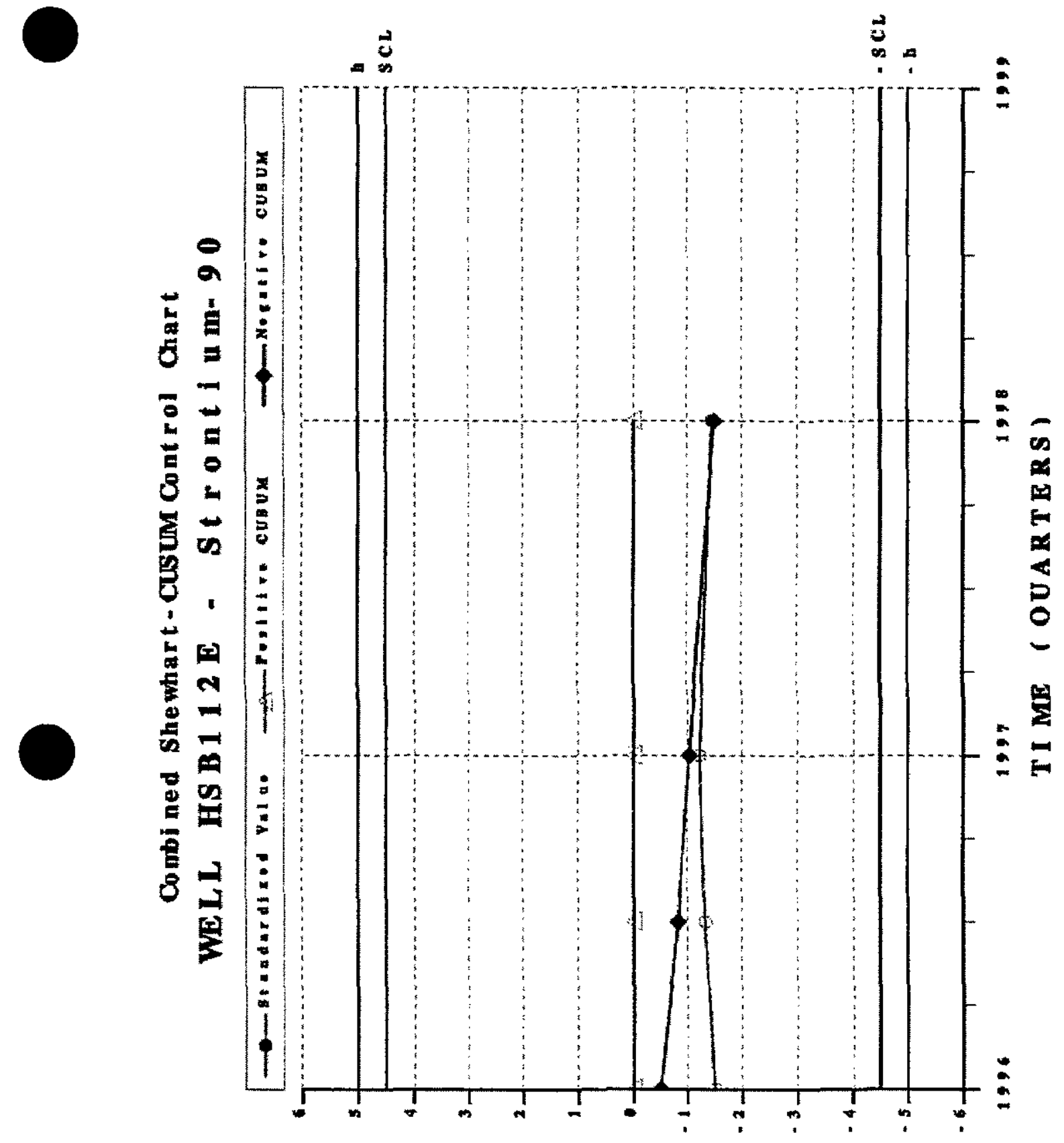

8 I 


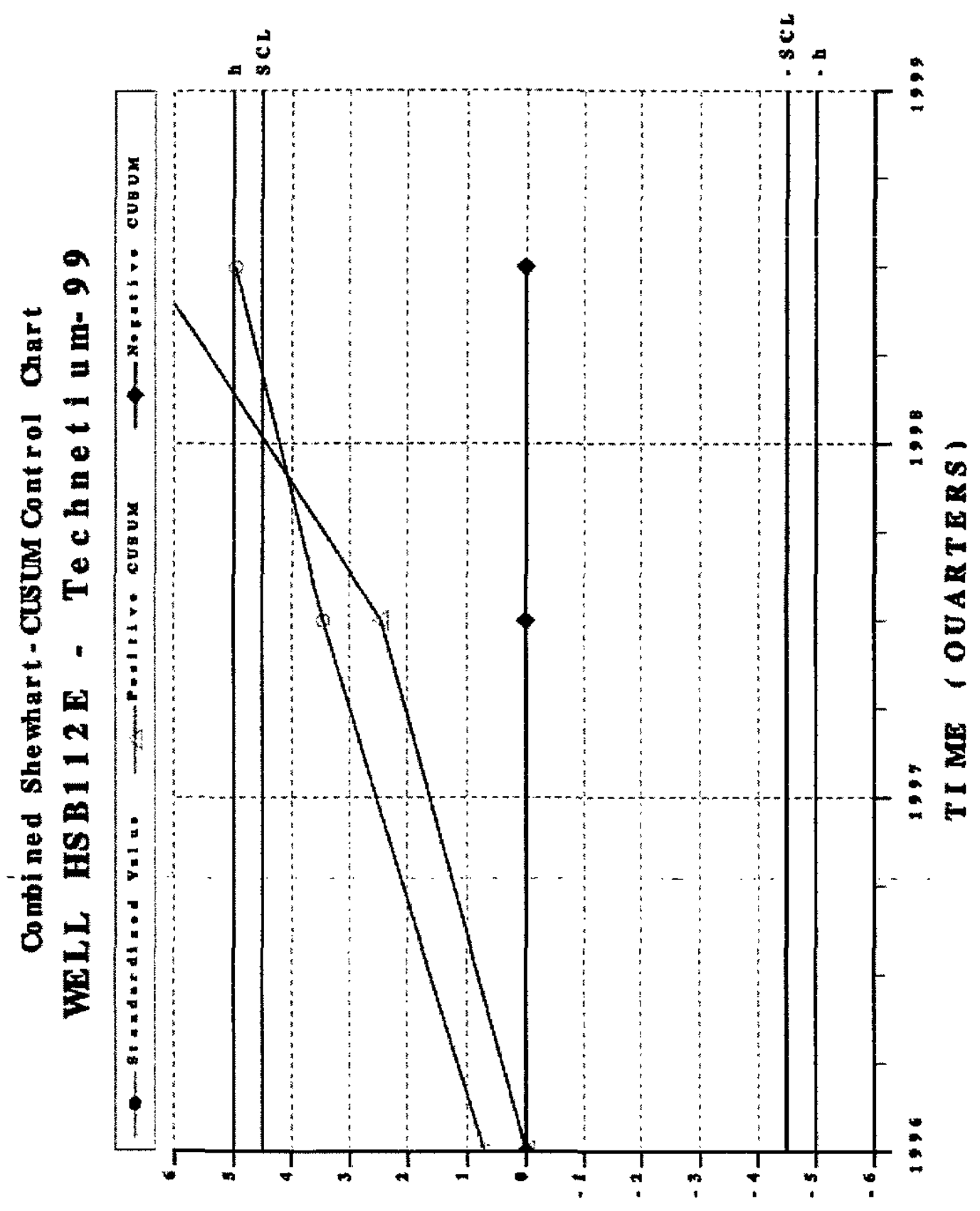

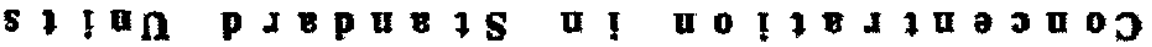



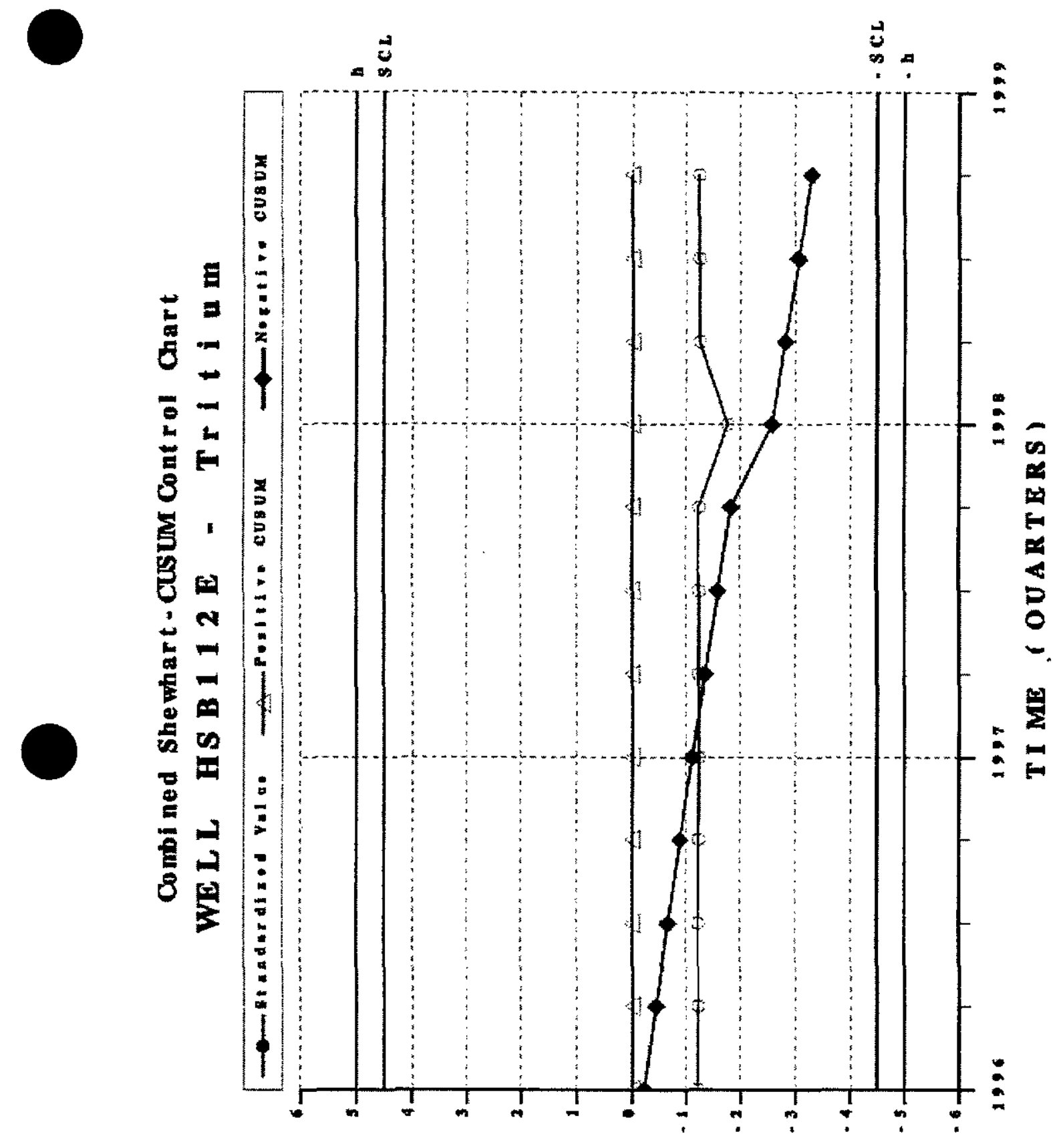

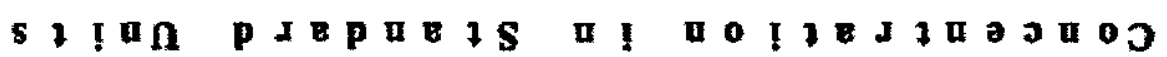




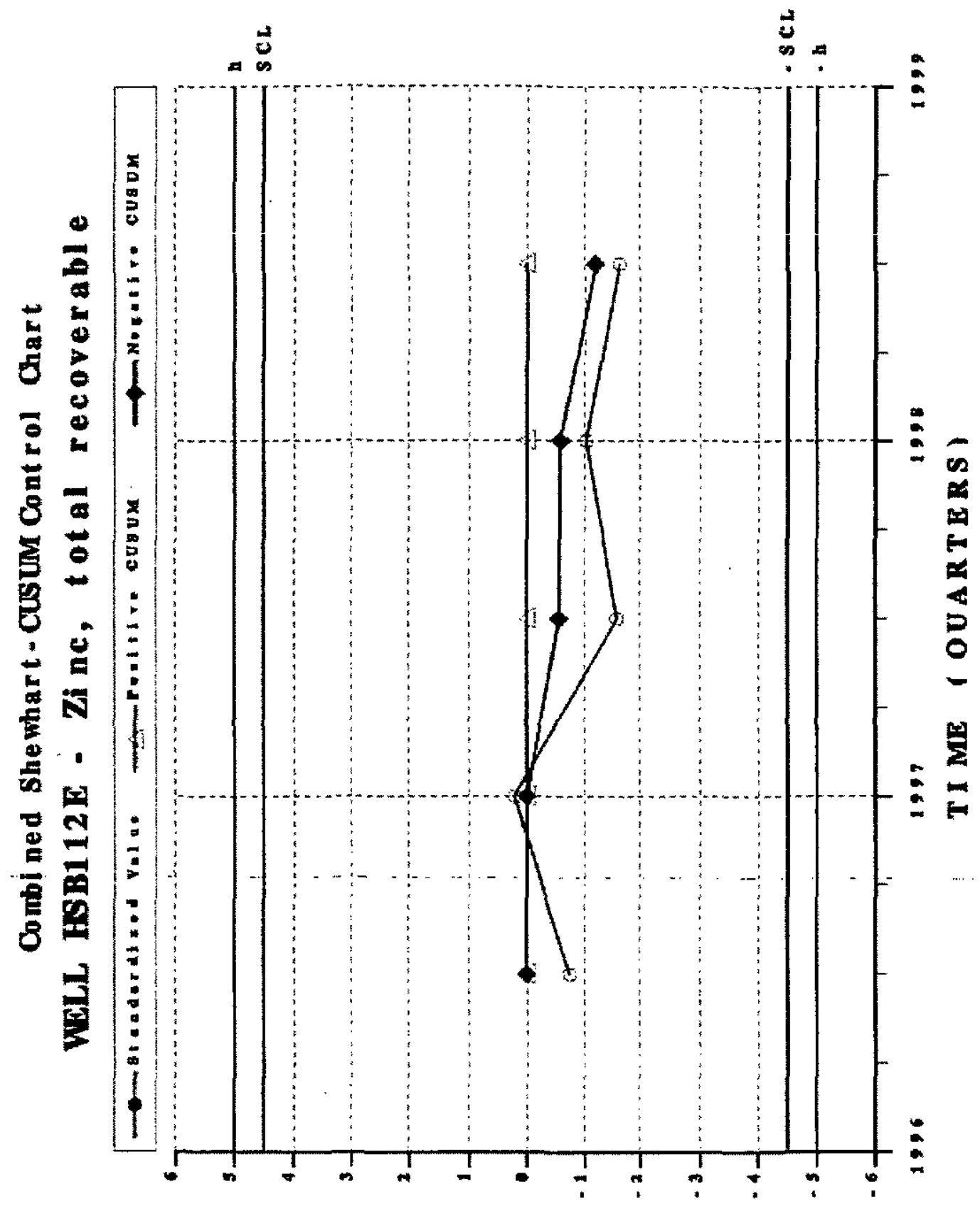

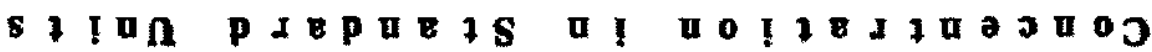




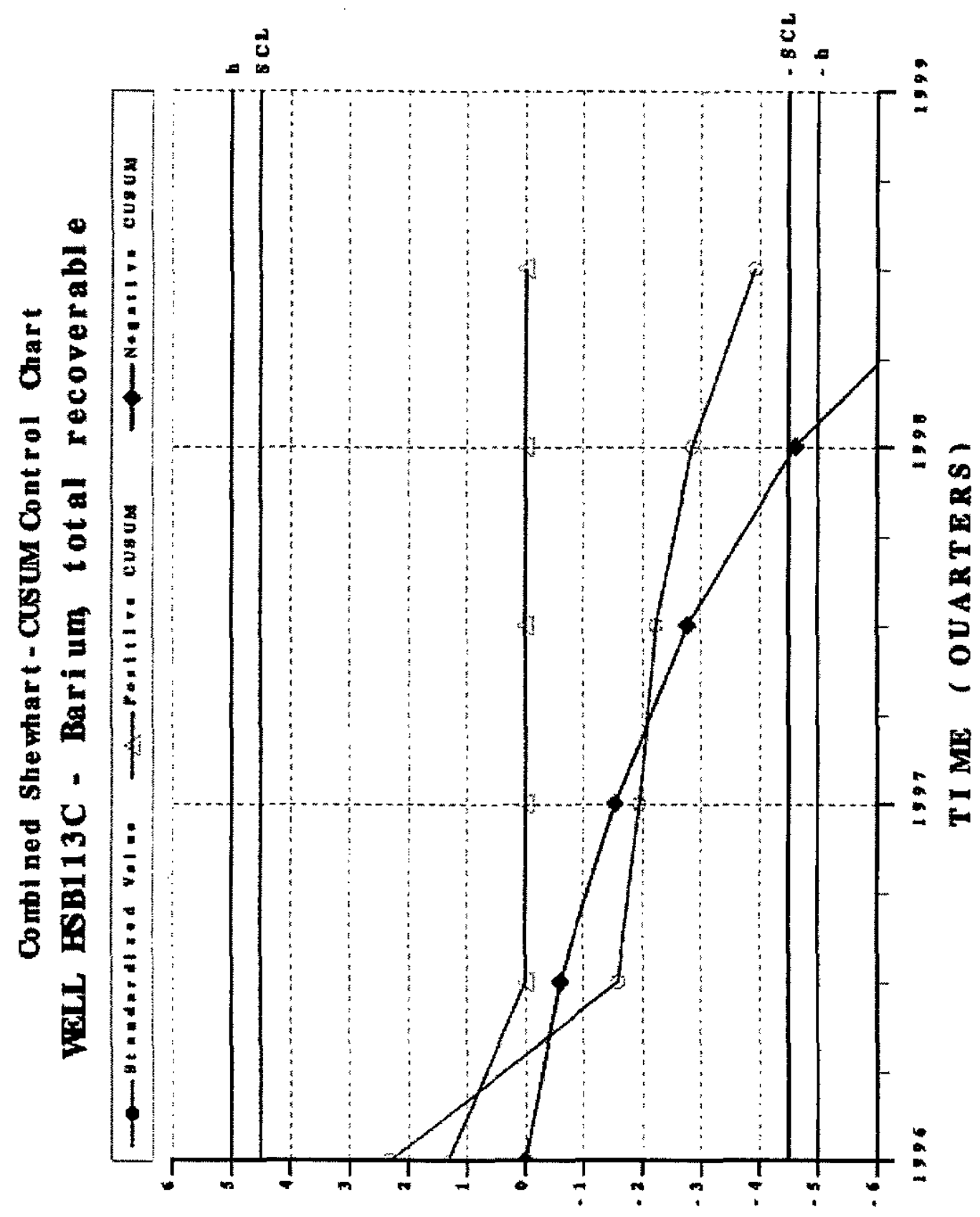

s t 1 n 


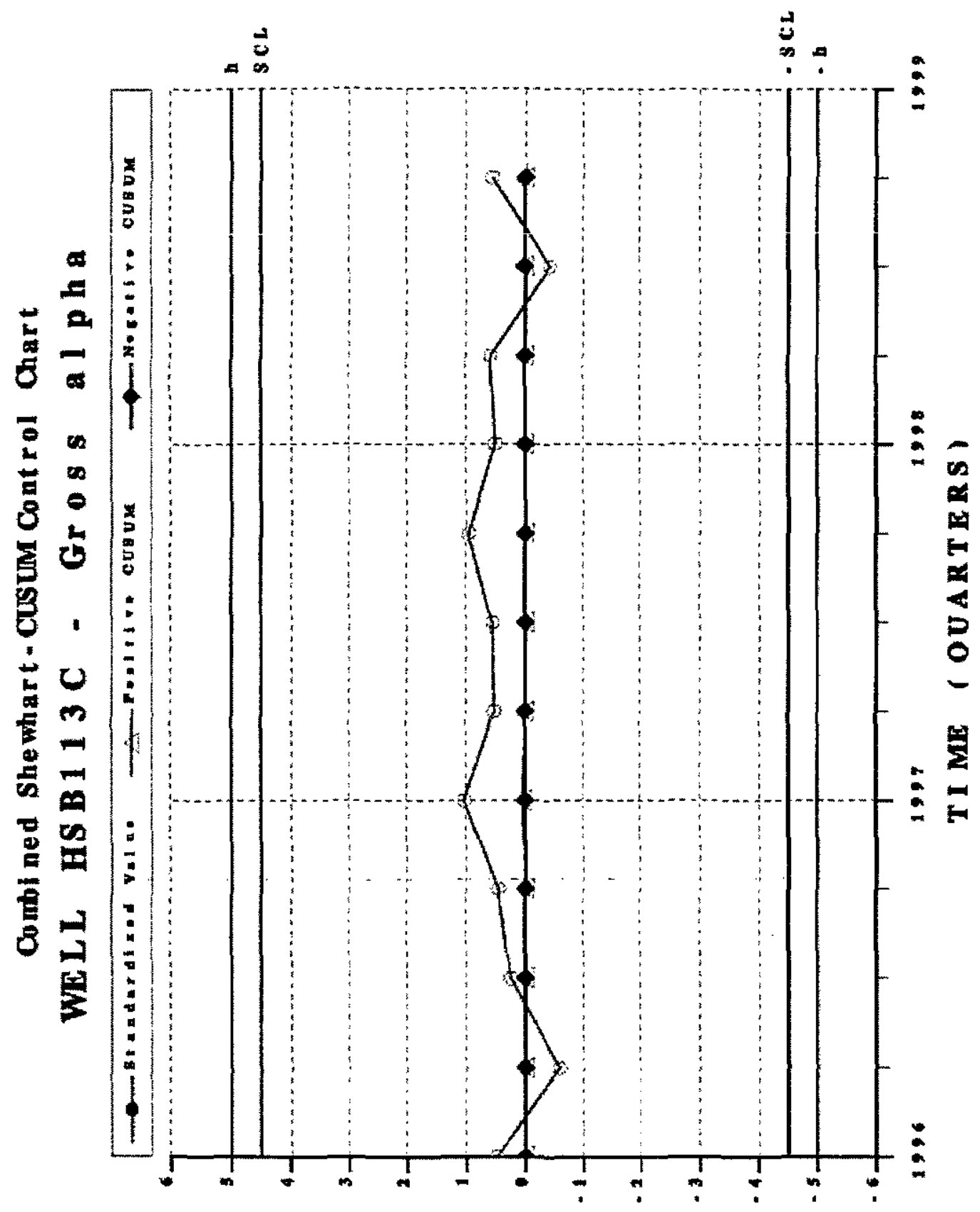

s! tan p I 
Combi ned Shewhart-Cusum Cont rol Chart

VuL HSB1 13C - Nitrate-nitrite as nitrogen

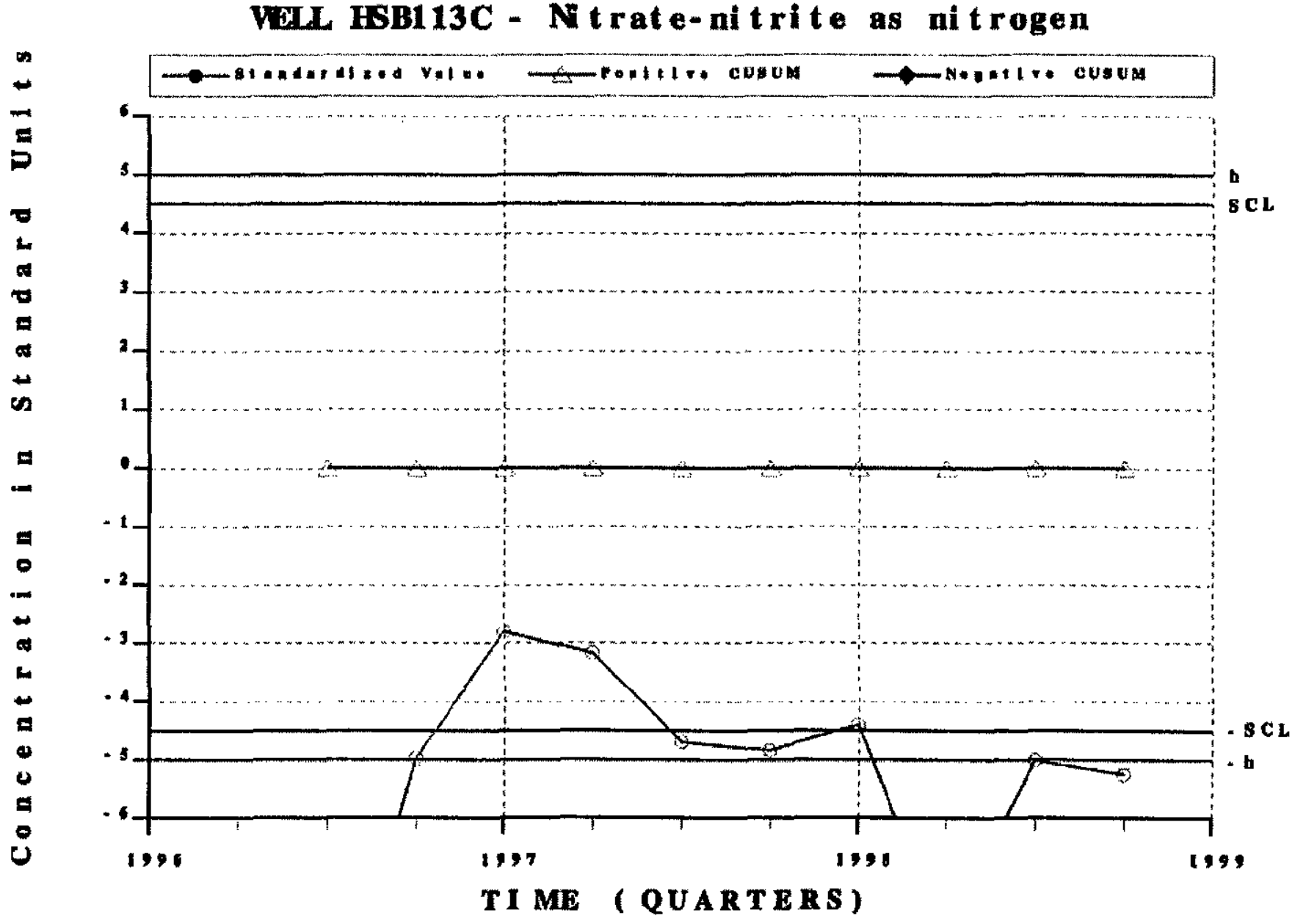




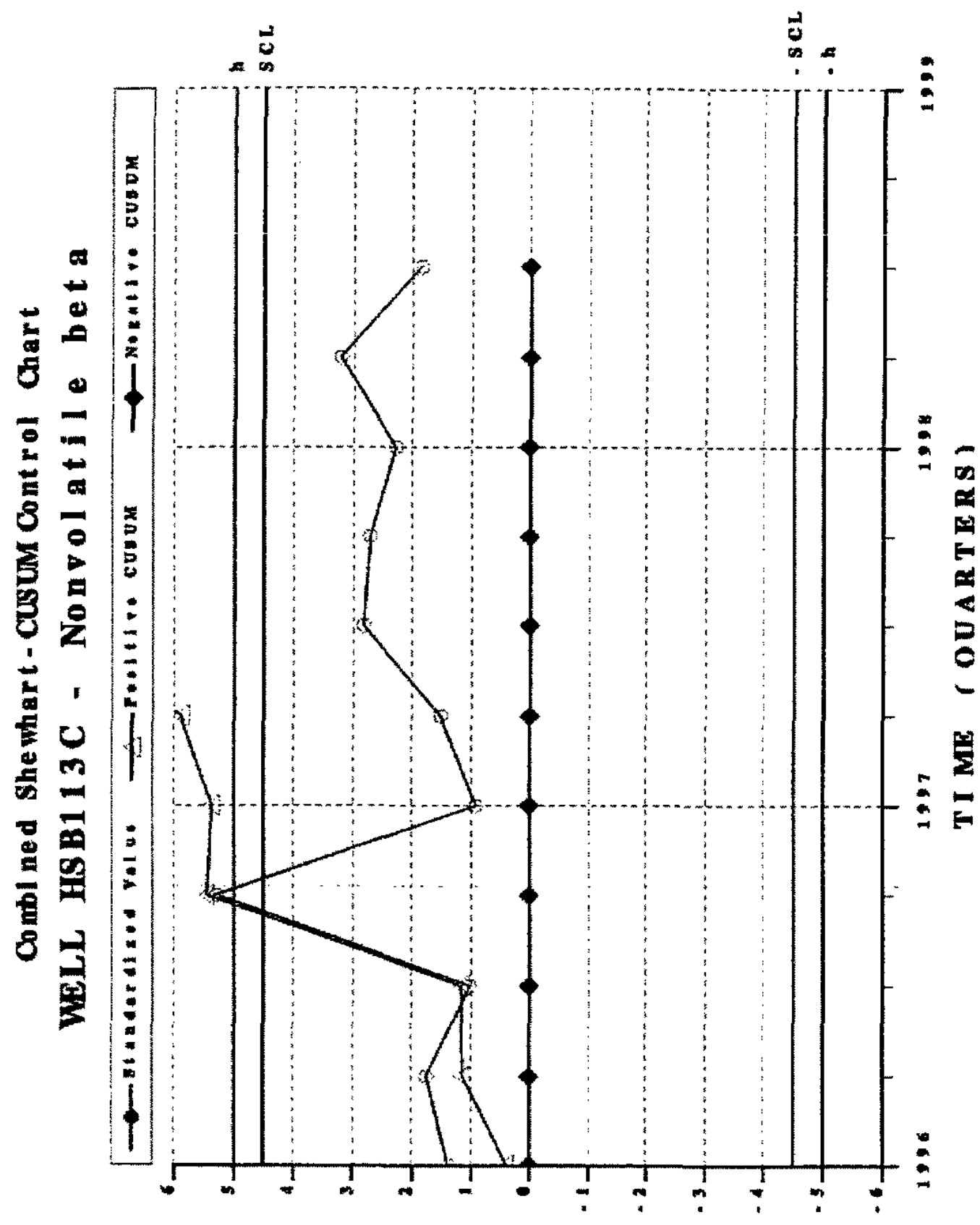

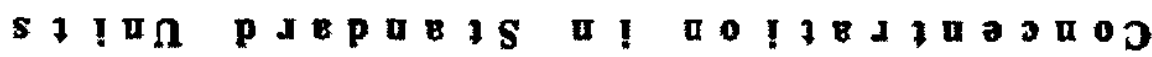


Combi ned She whart - Cusum Cont rol Chart WeLL HSB113C - Zi ne, total recoverable

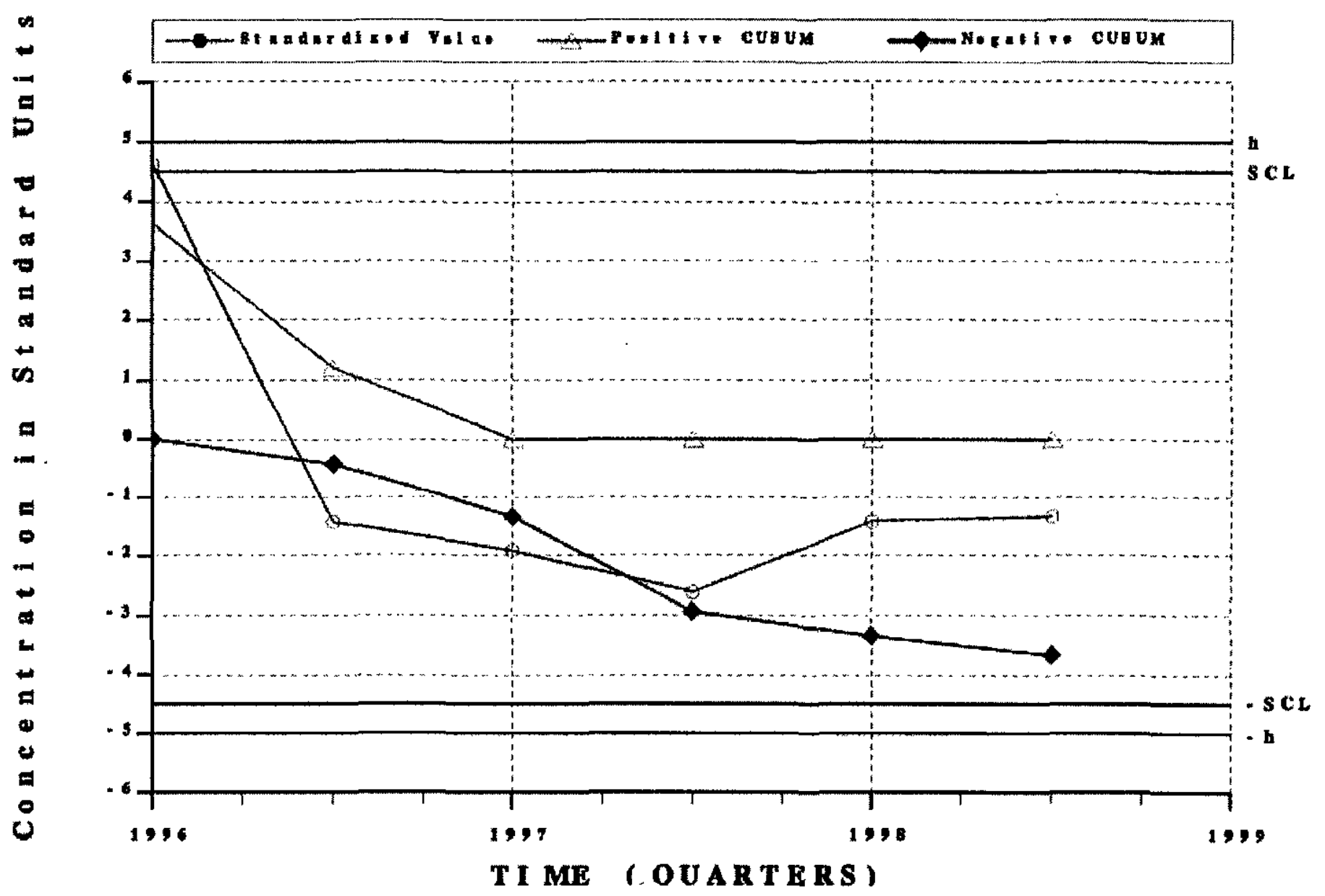




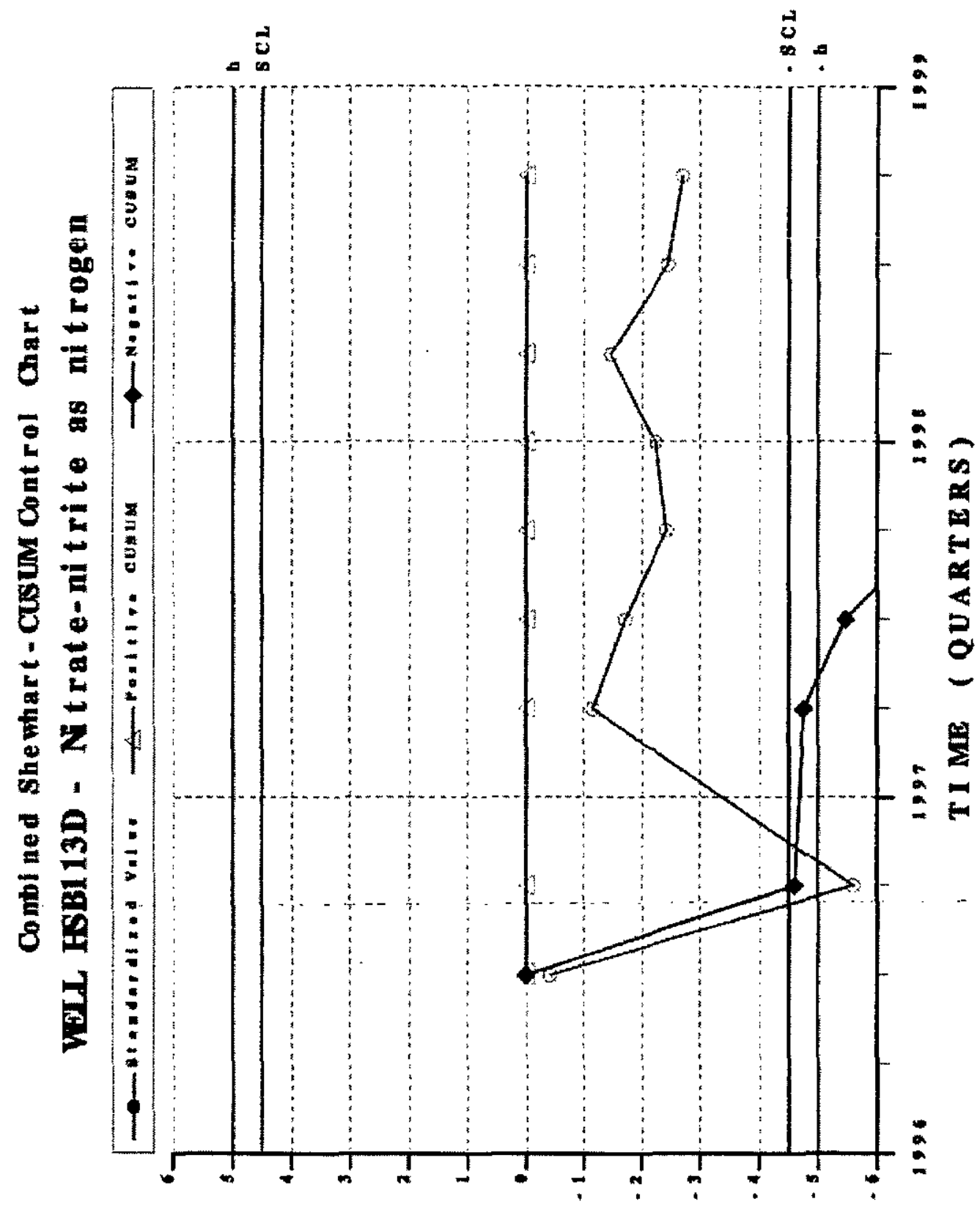

s I I 


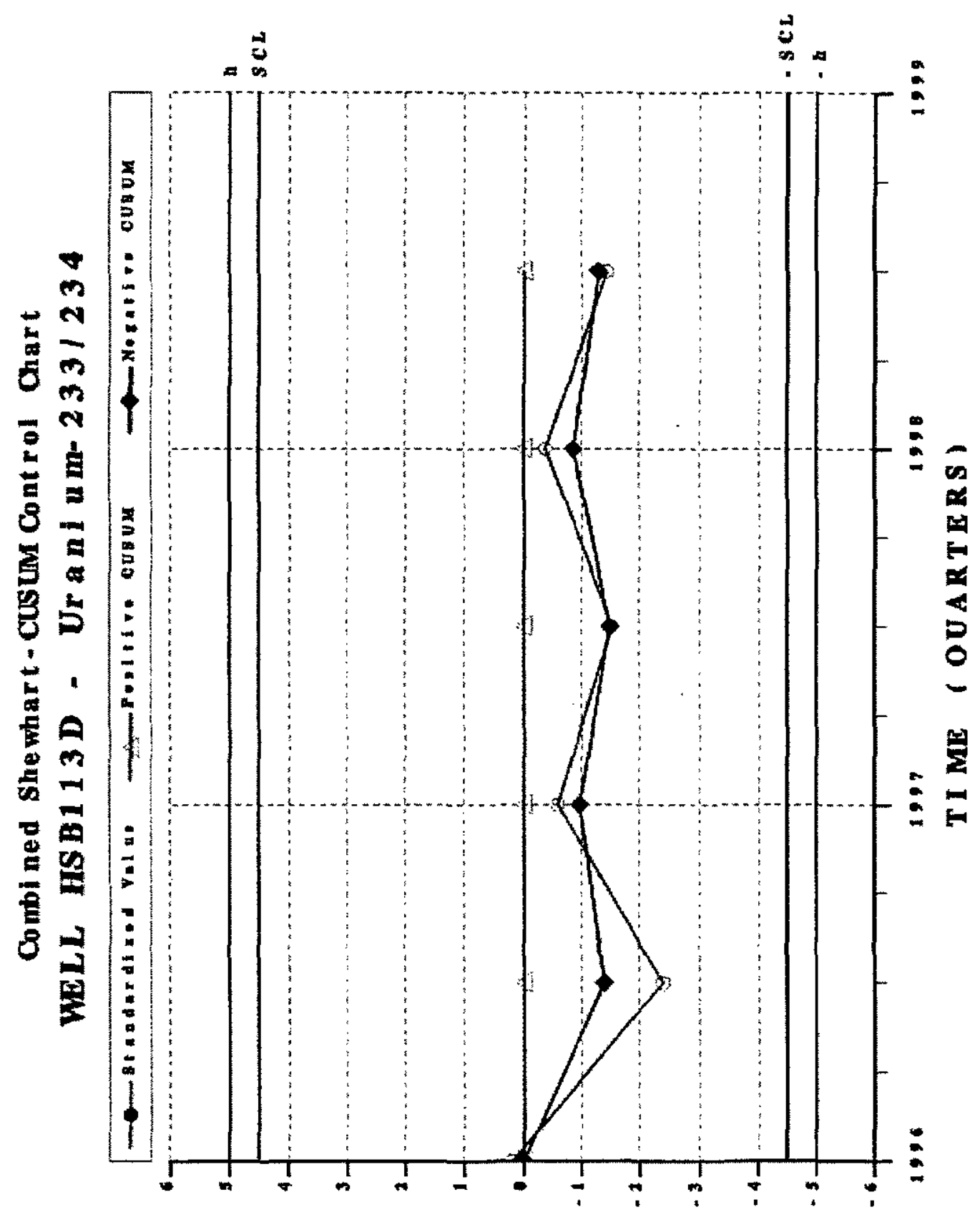

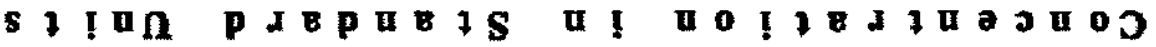


Combi ned Shewhart-cusum Control Chart WEL HSB113D - Zi nc, total recoverable

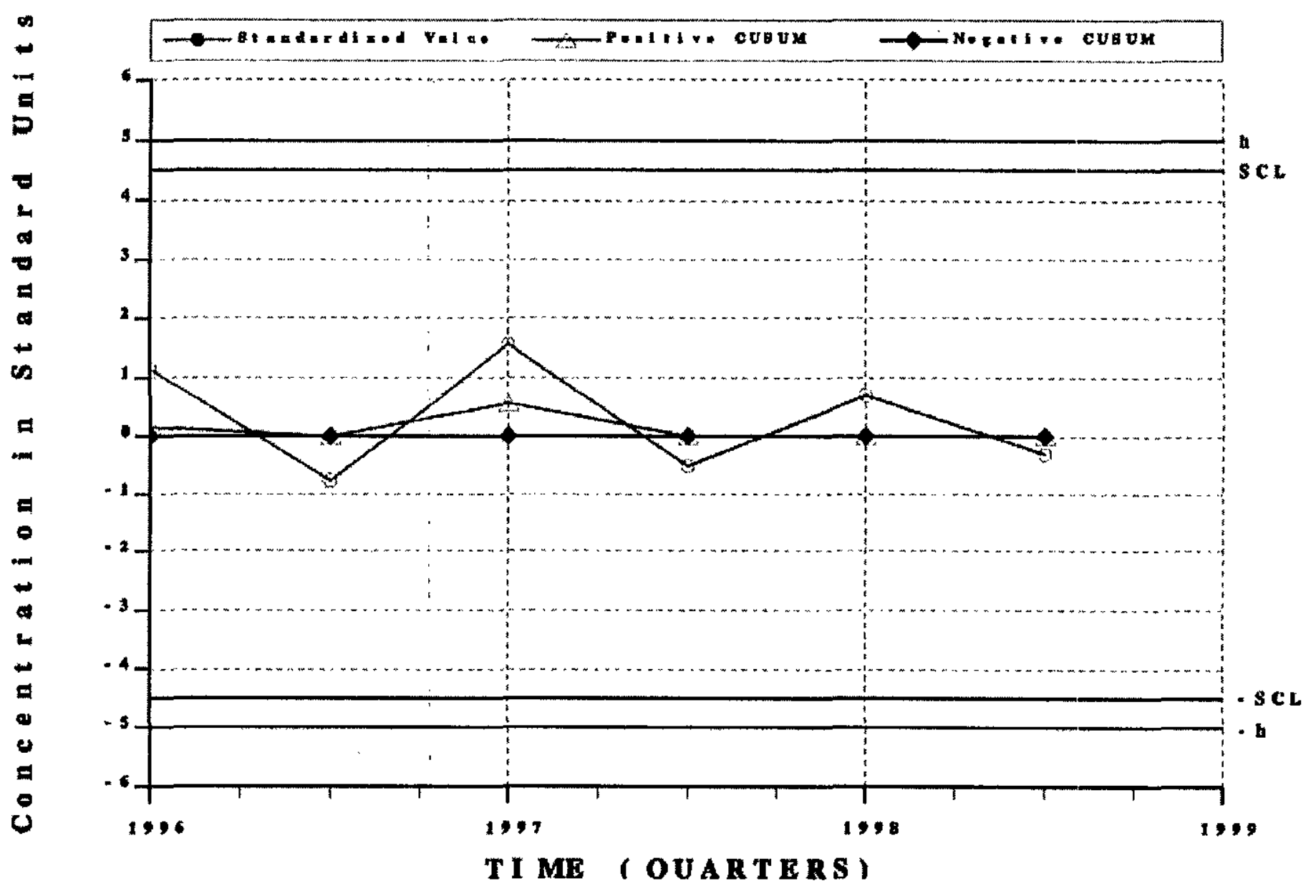




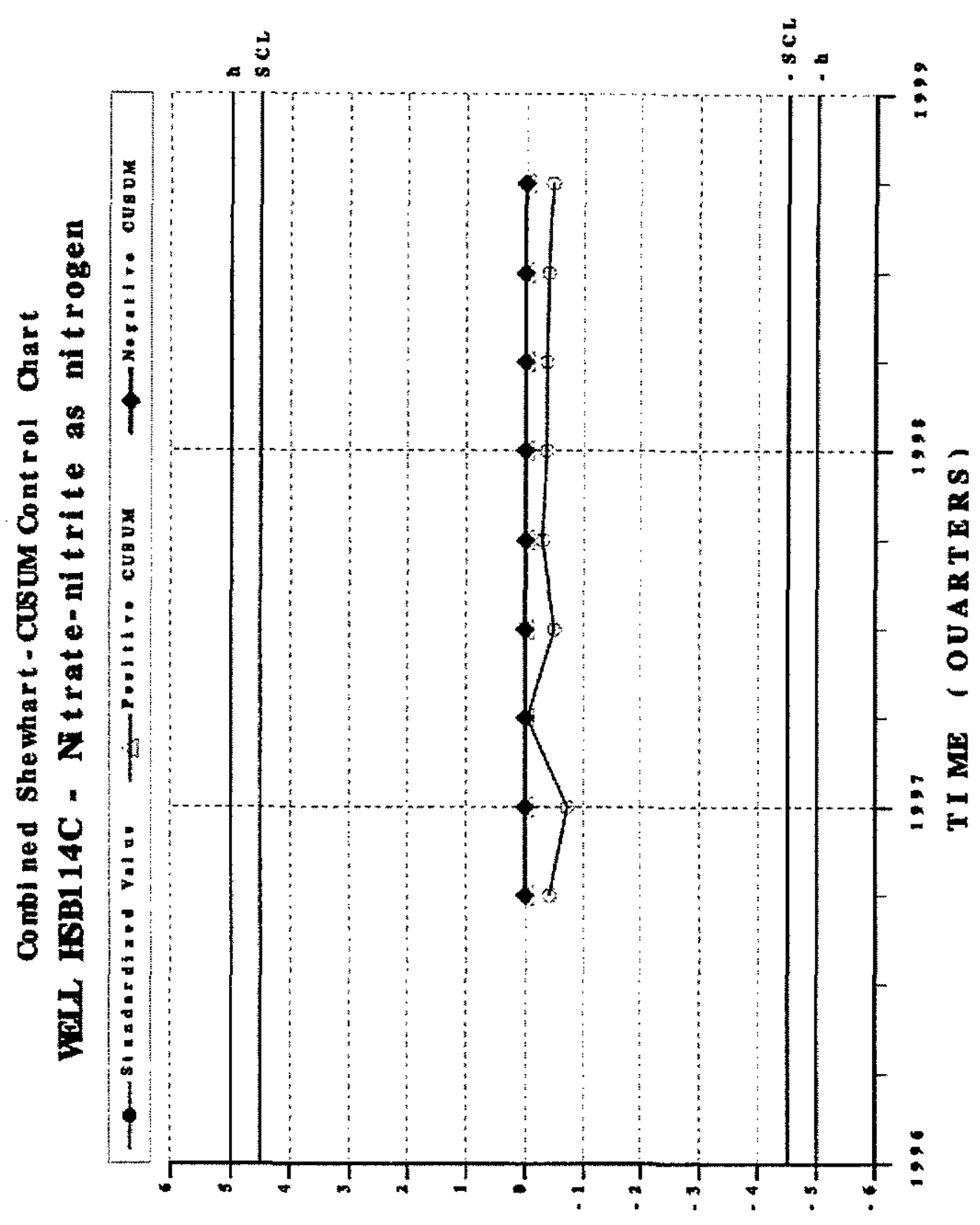

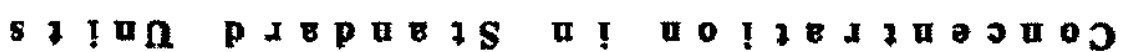


Combi ned Shewhart - CuSUM Cont rol Chart WeLL HSB1 14C - Zi nc, total recoverable

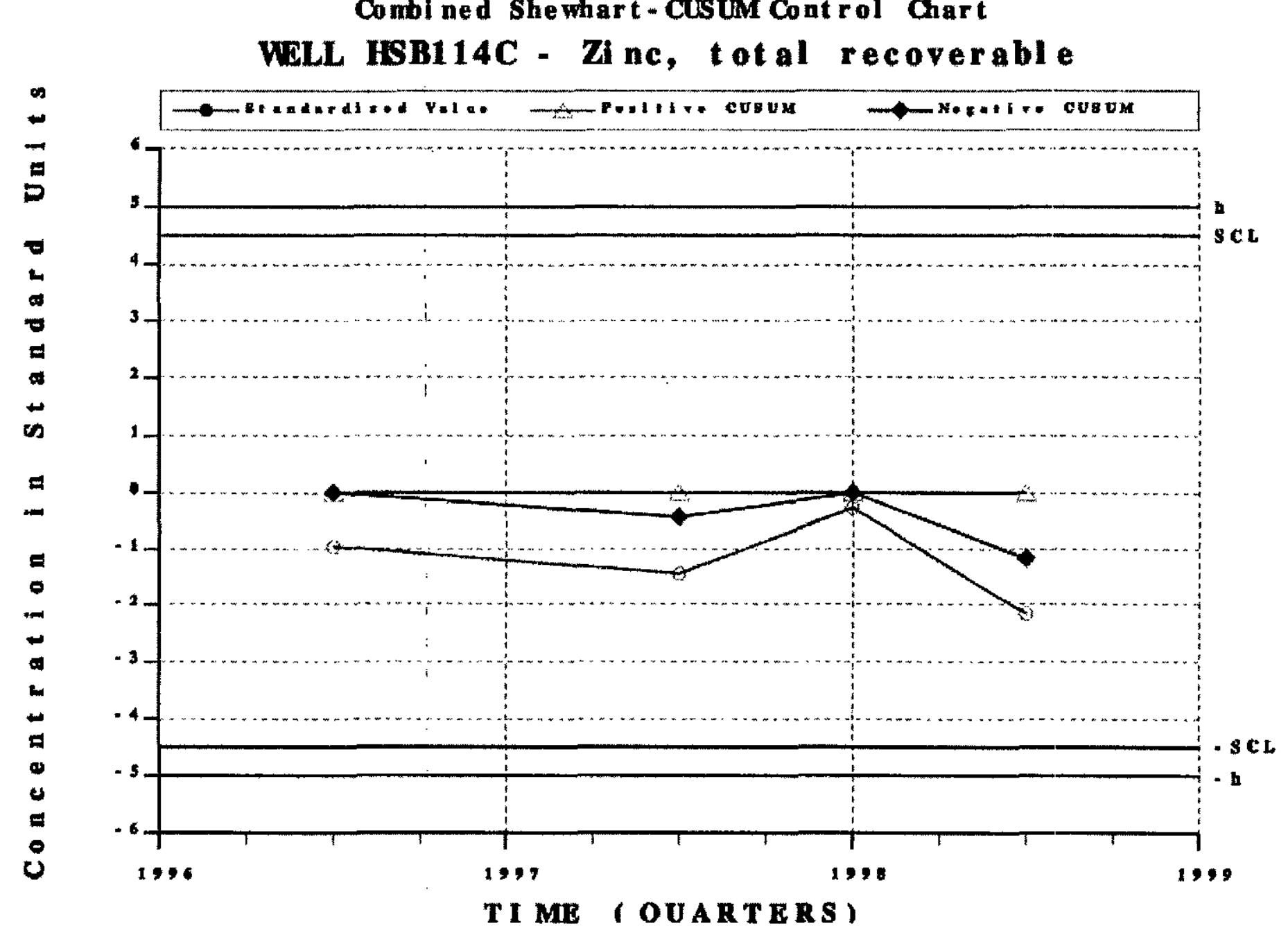




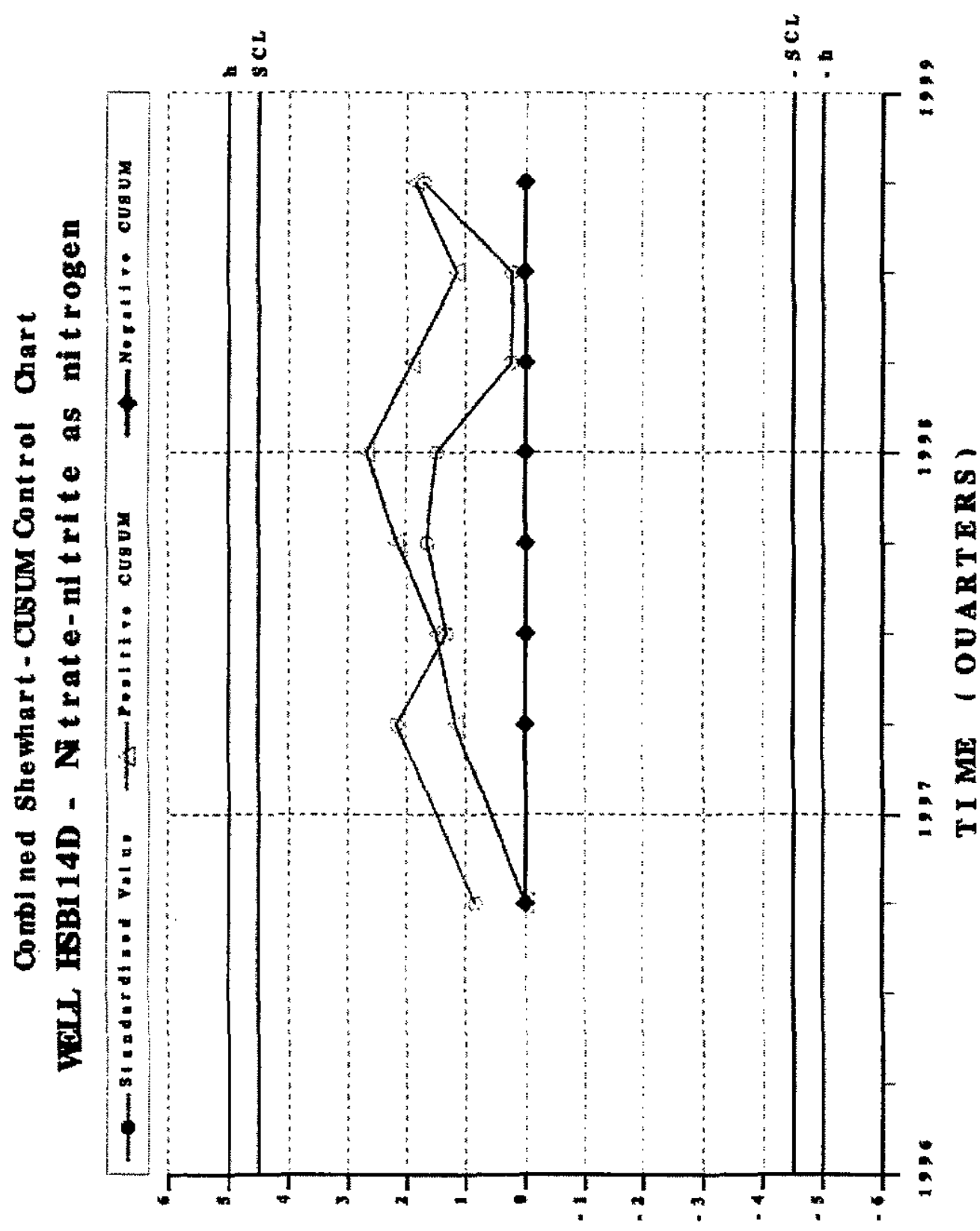

s t เ 
Combi ned Shewhart - Cusum Cont rol Chart

WELL HSB 1 14 D - Ur ani um- 233 / 234

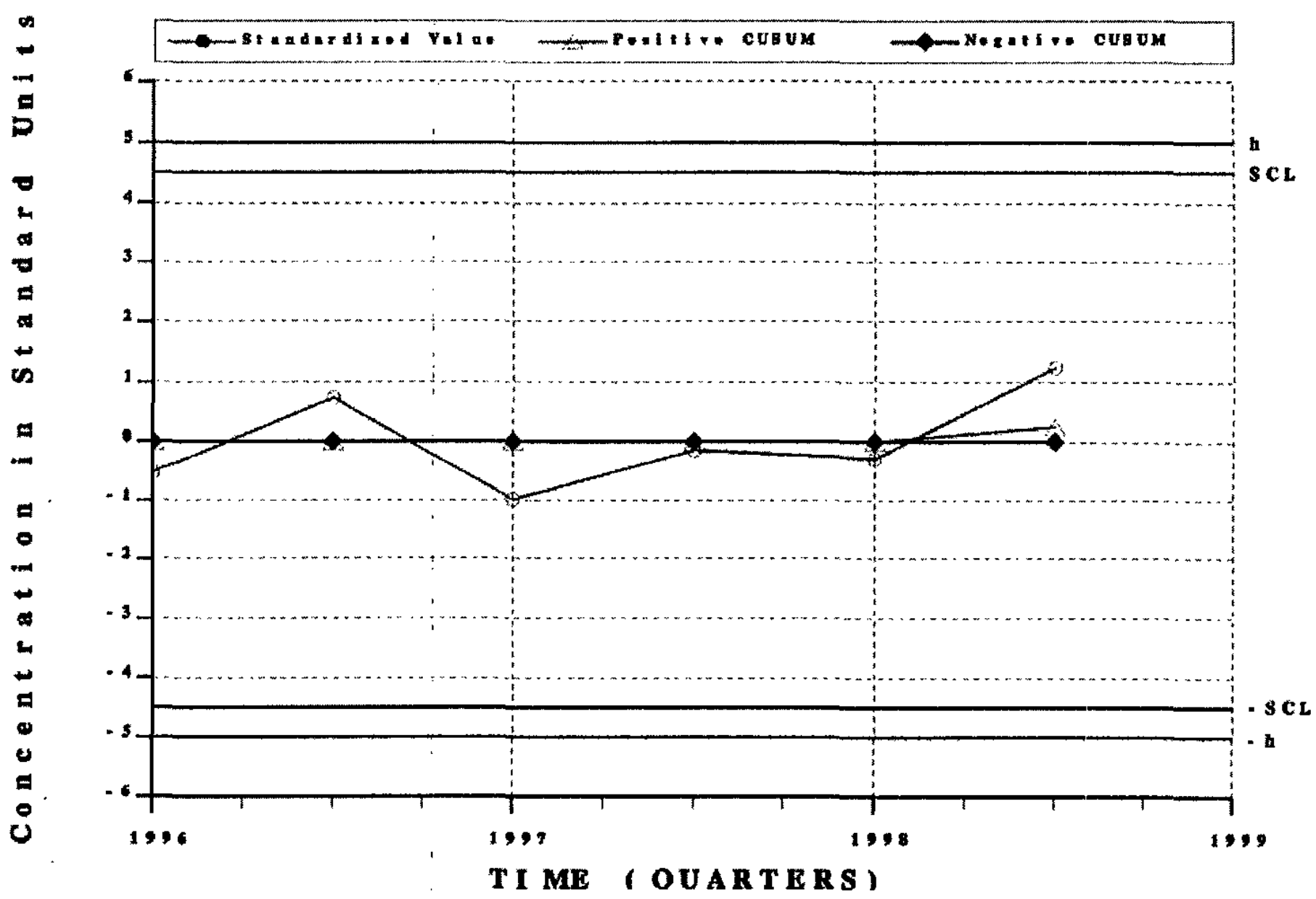

TI ME (OUARTRRS) 


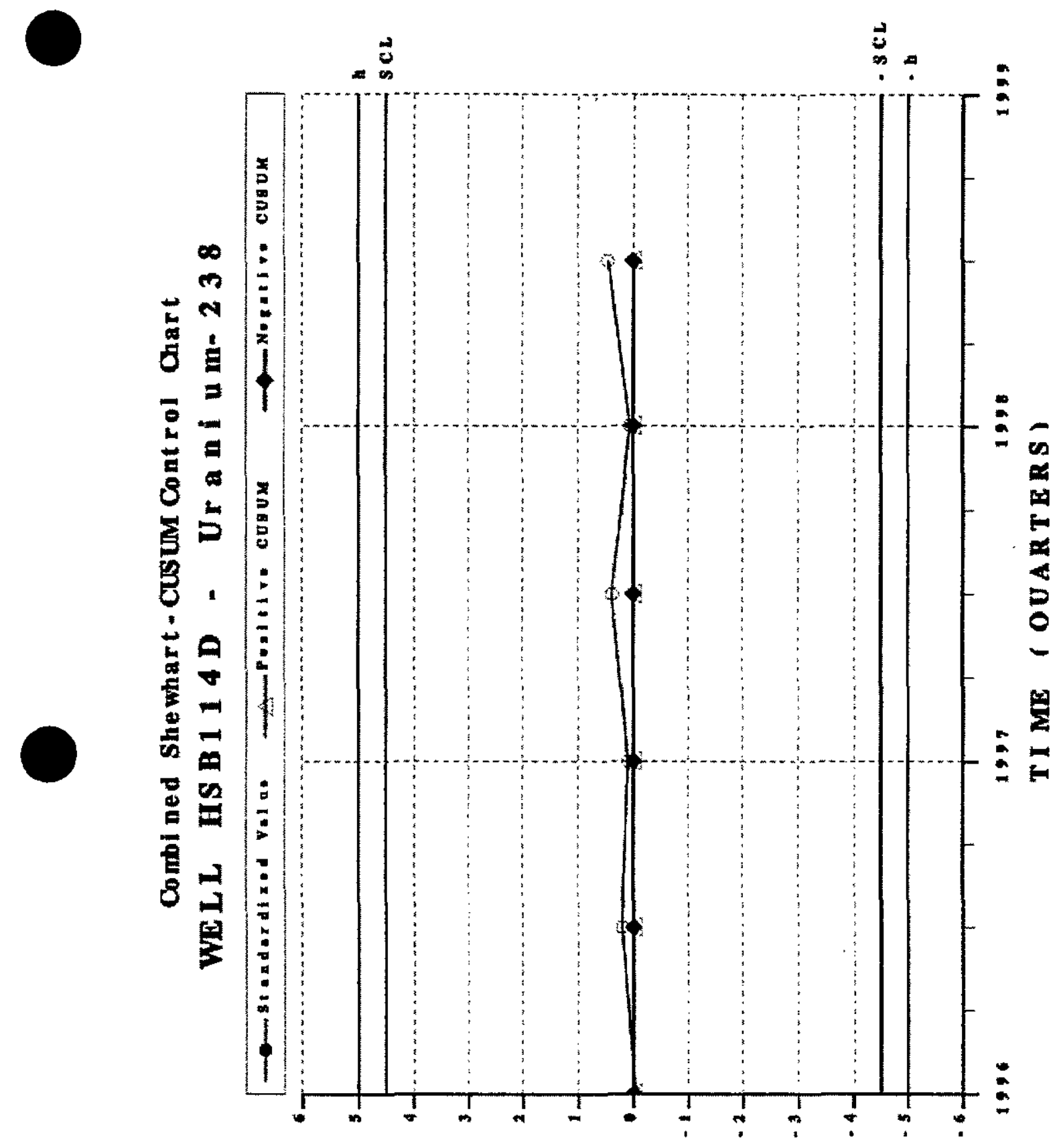

s I I $\mathbf{p}$ a 


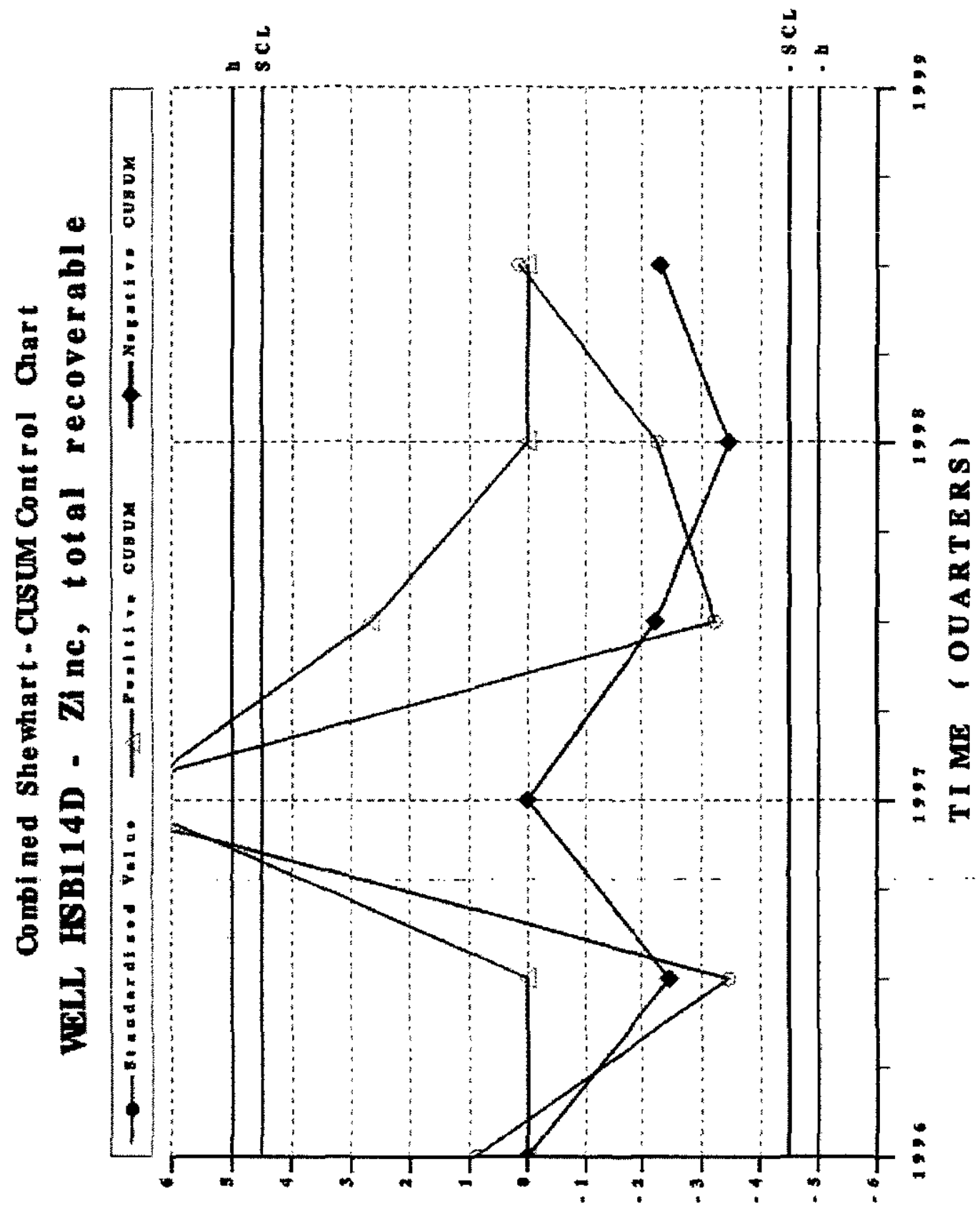

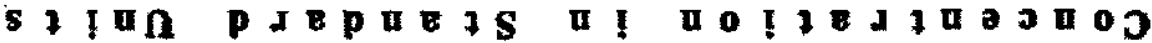


Combi ned She whart-Cusum Cont rol Chart

VoL HSB115C - N trate-nitrite as nitrogen

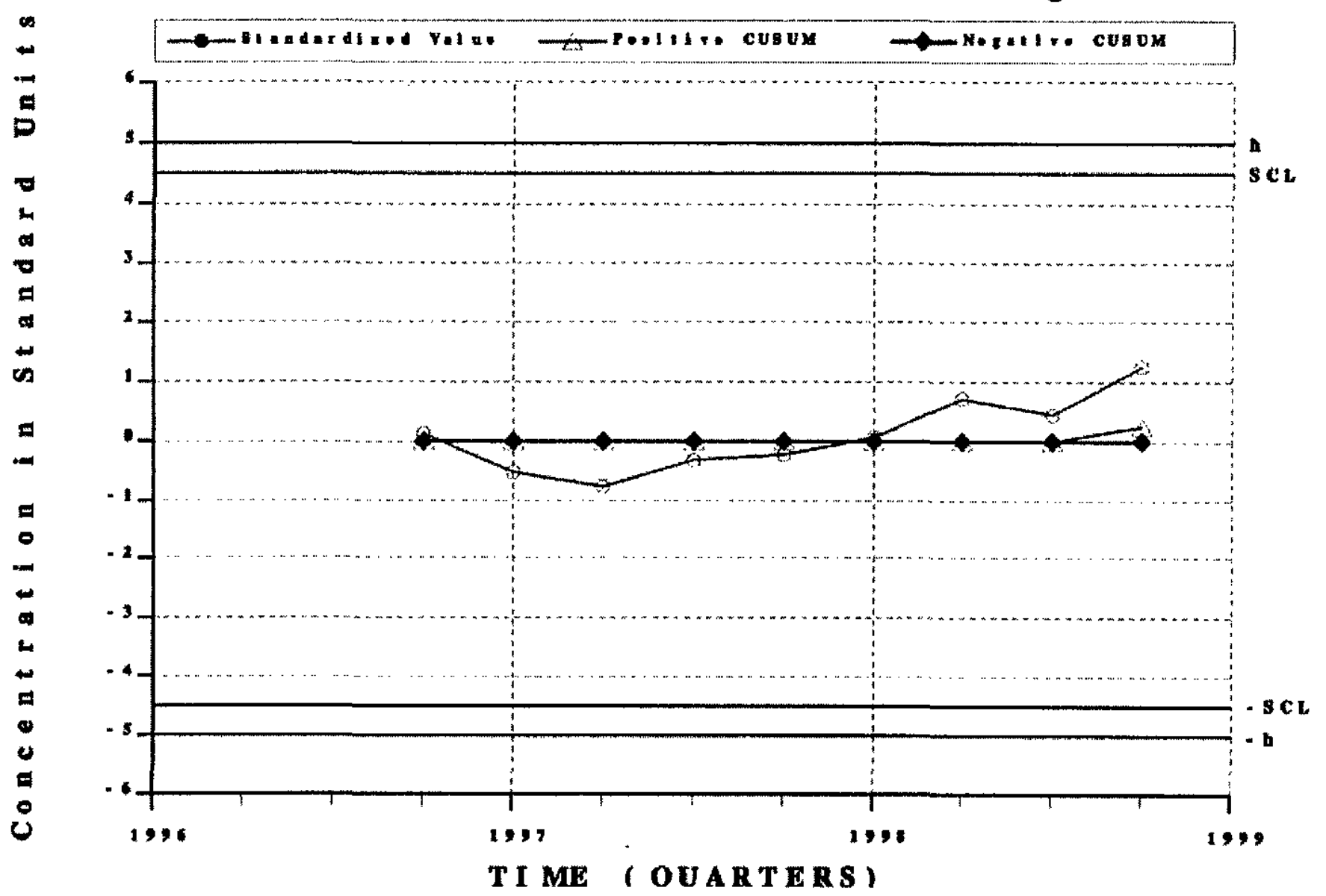




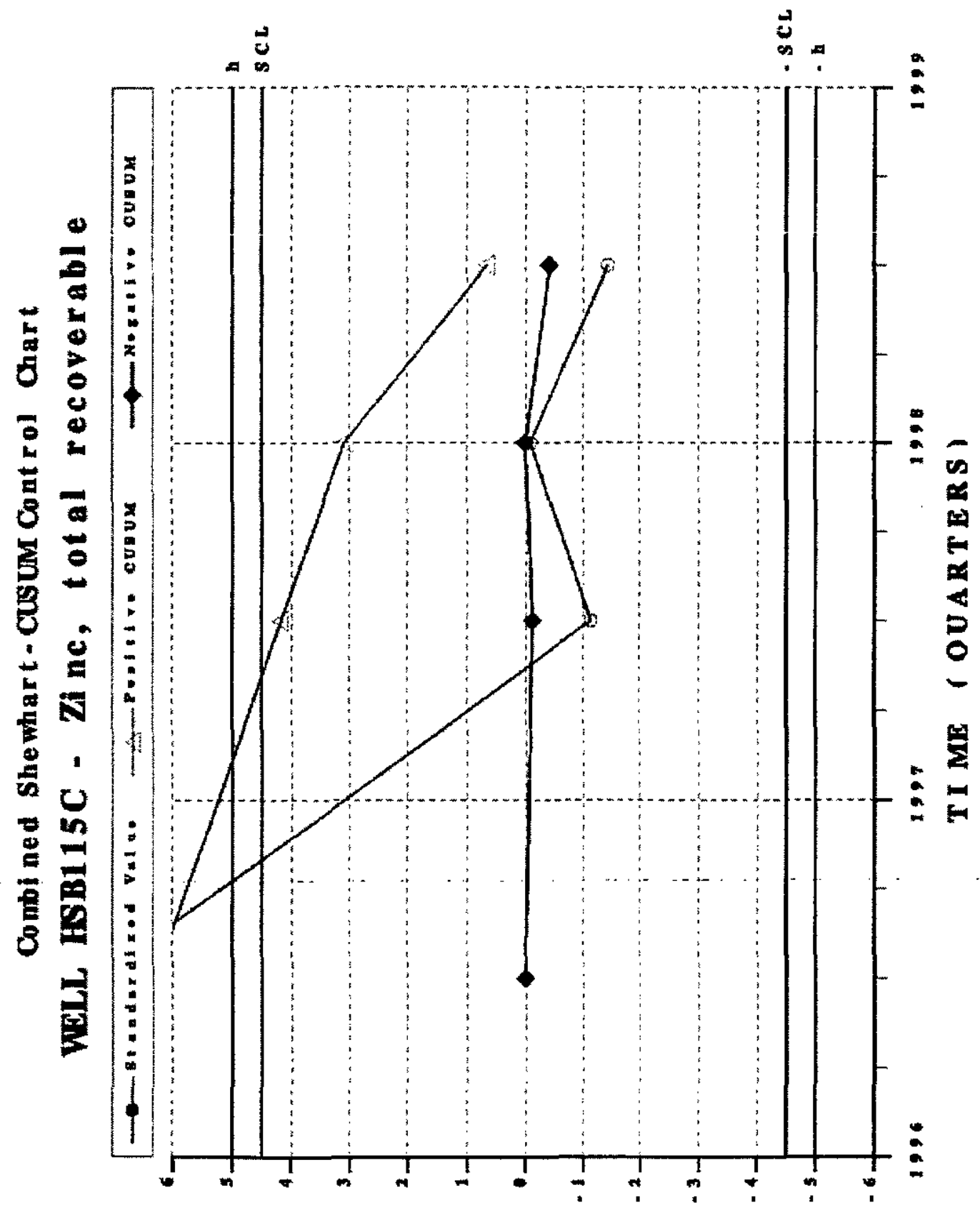

s I I 


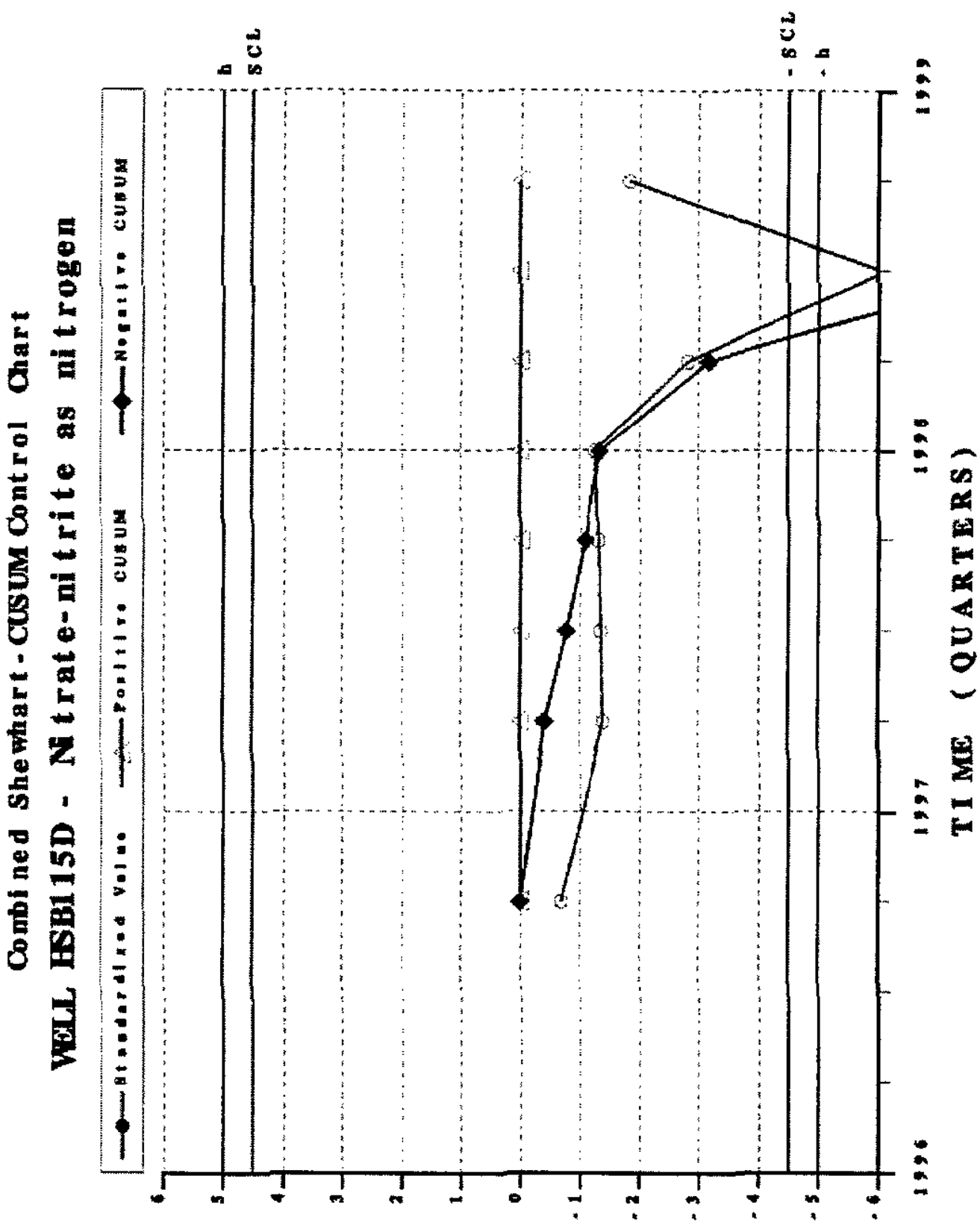

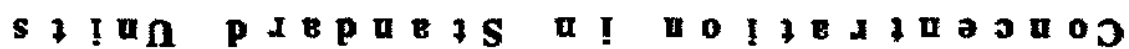




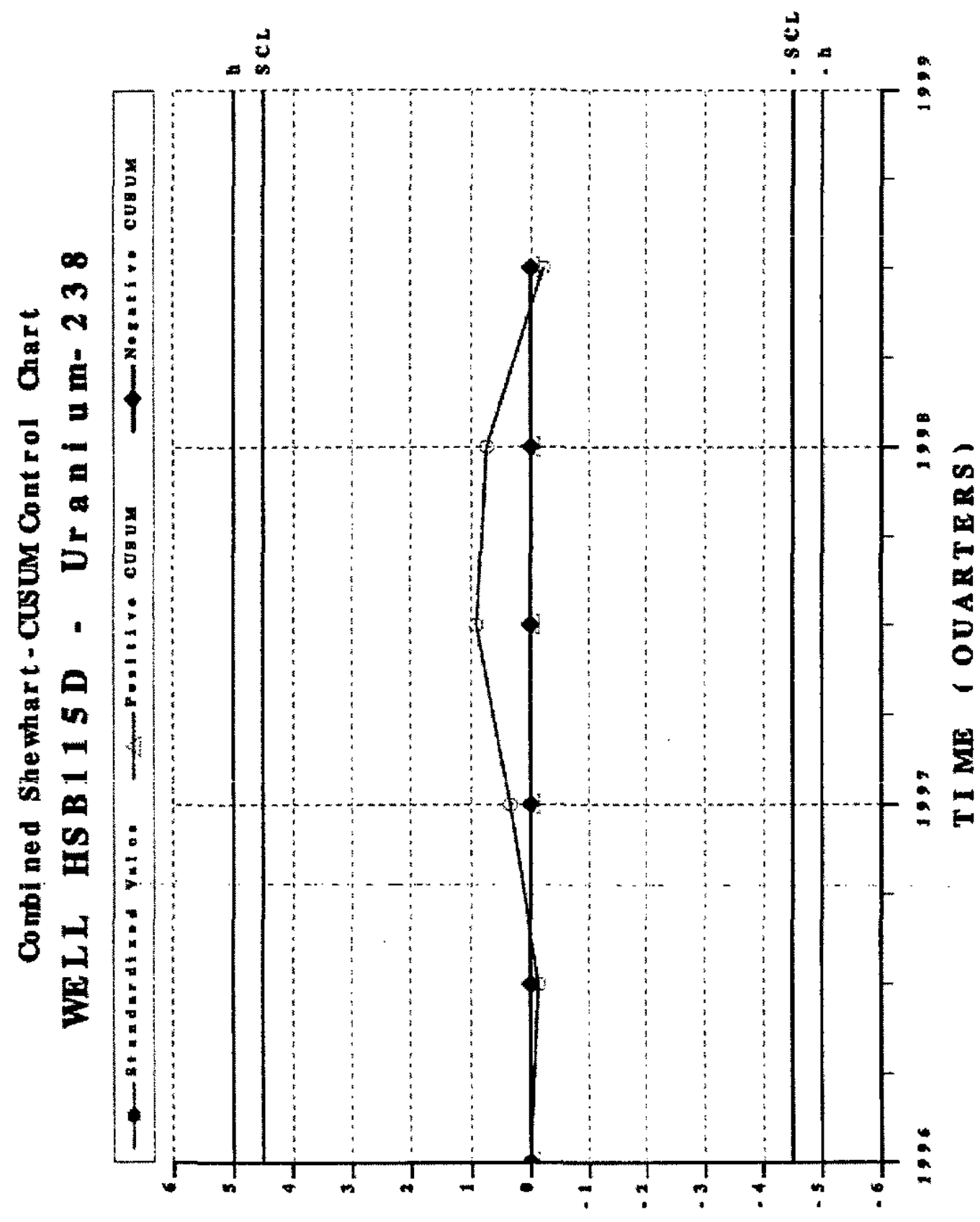

s t I 
Combi ned Shewhart - Cusum Cont rol Chart WLL HSB1 15D - Zi nc, total recoverable

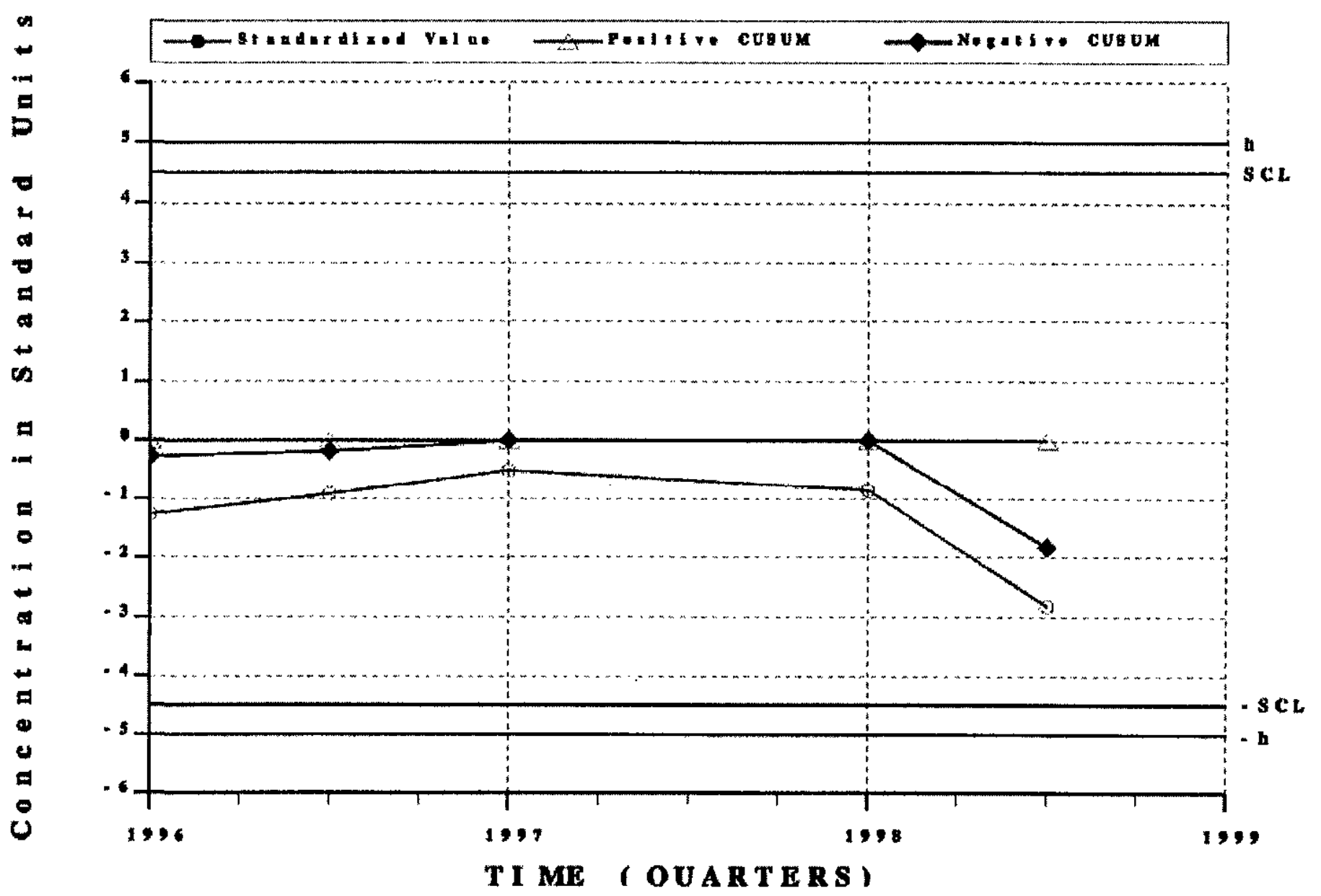




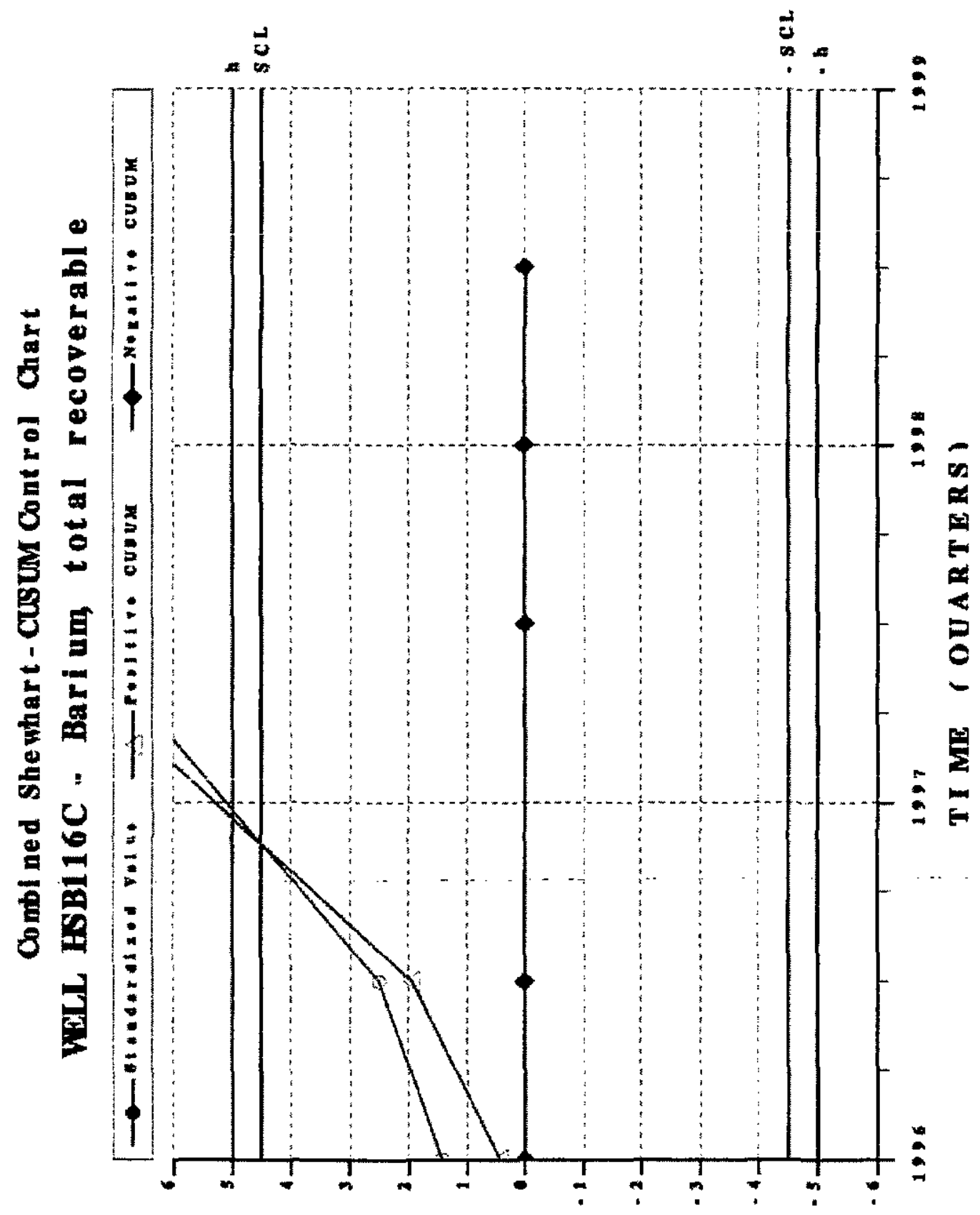

st!un pJepuets u! 
Combi ned She whart - cusum Cont rol Chart WELL HSB116C - Cobalt, total recoverable

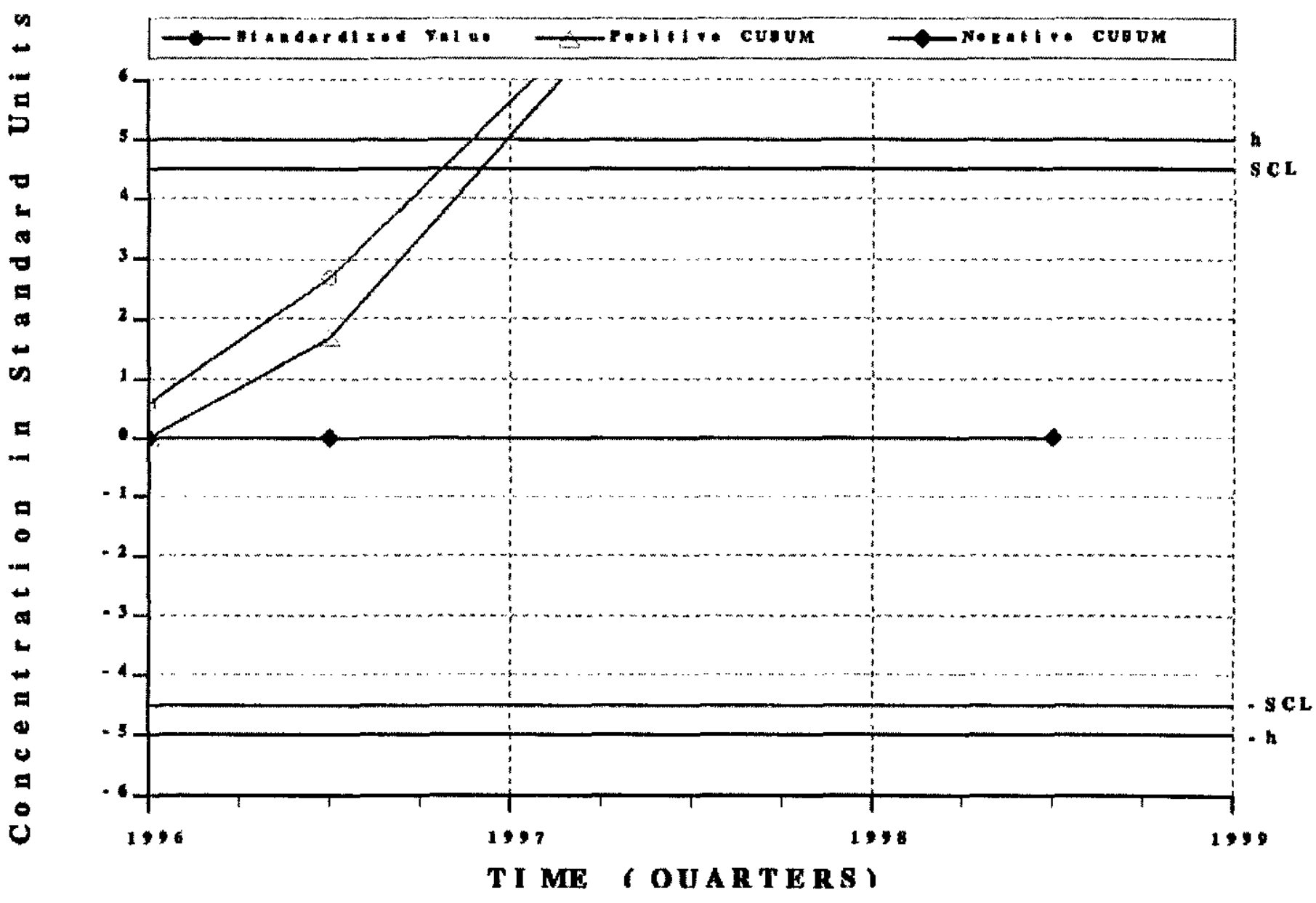




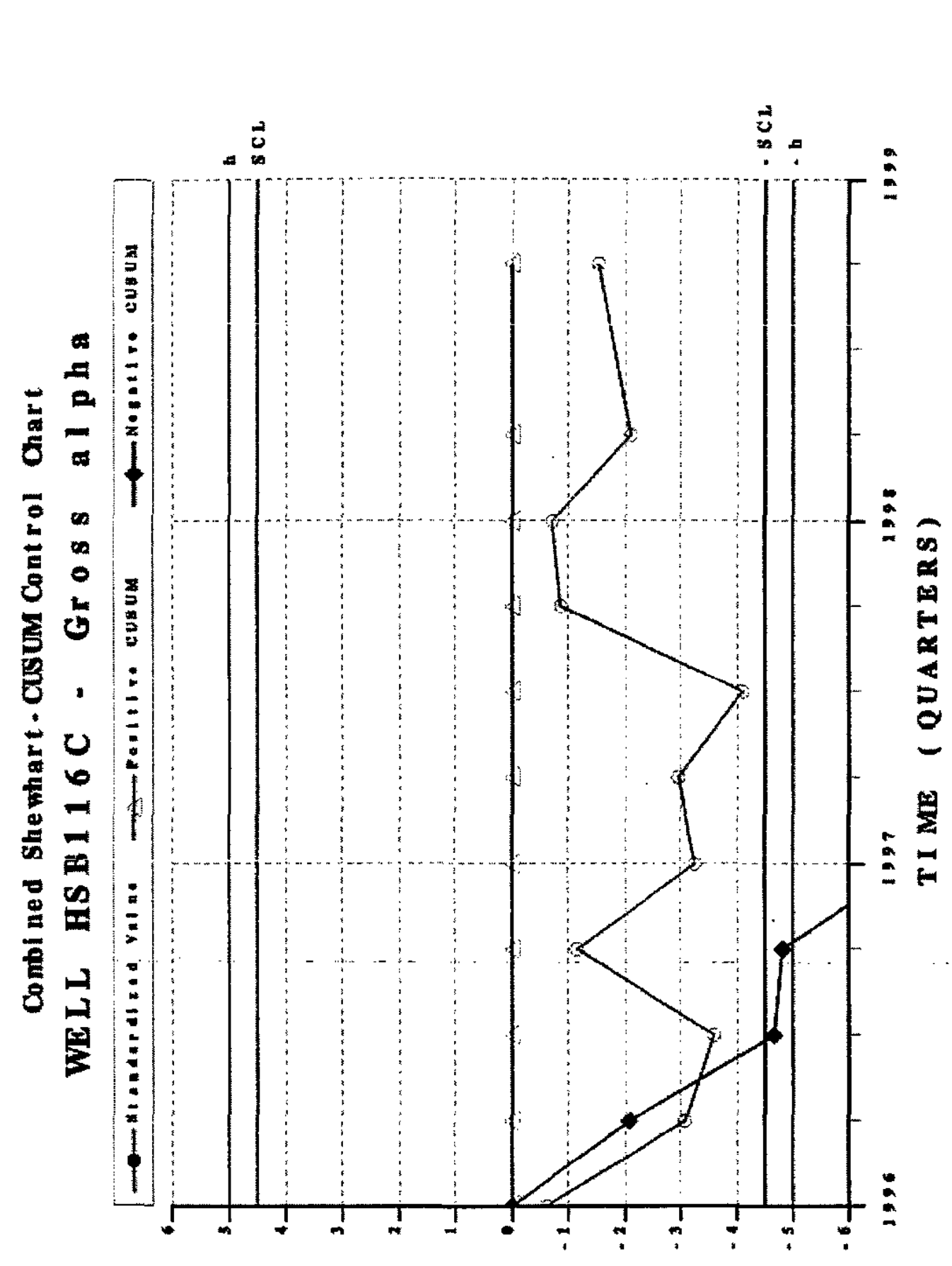

WSRC-TR-99-00013

Unclassified

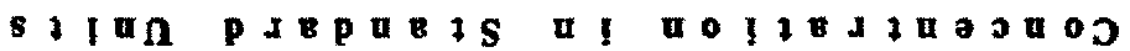



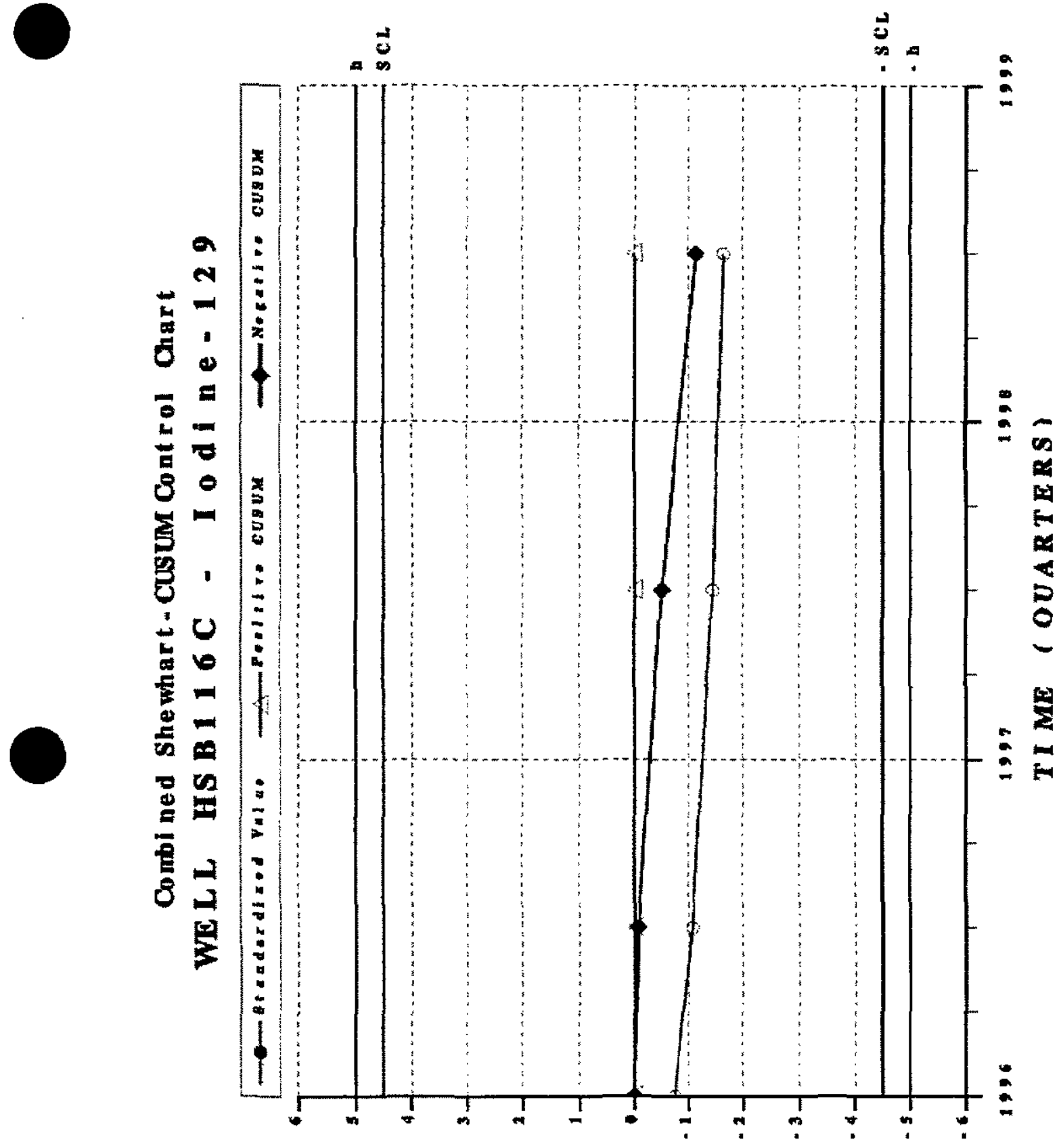

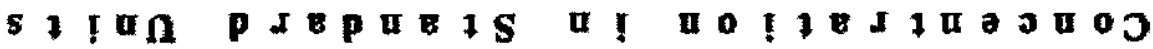




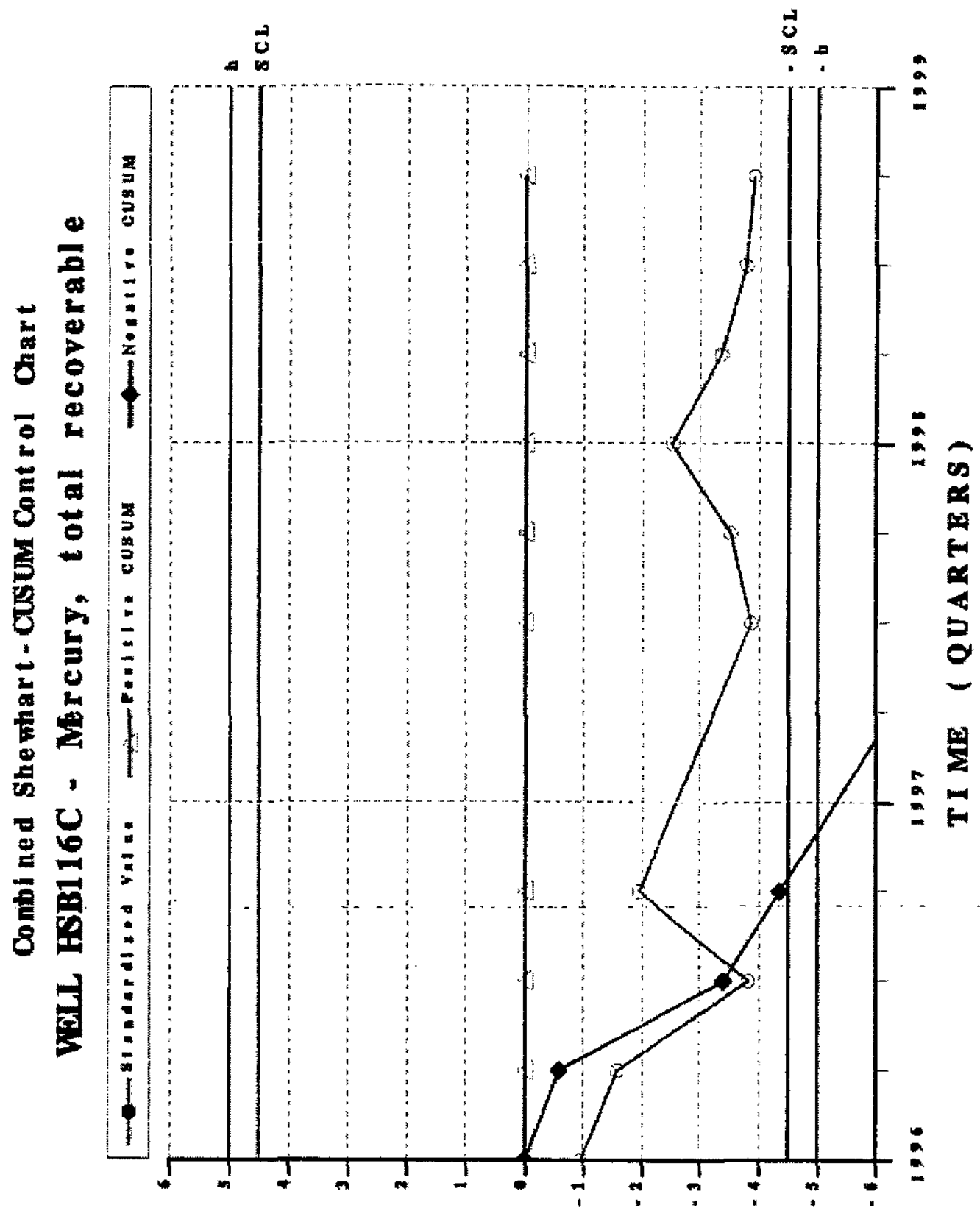

stiun pa 
Combl ned Shewhart - Cusum Cont rol Chart

WoLl HSB116C - Nitrate-nitrite as nitrogen

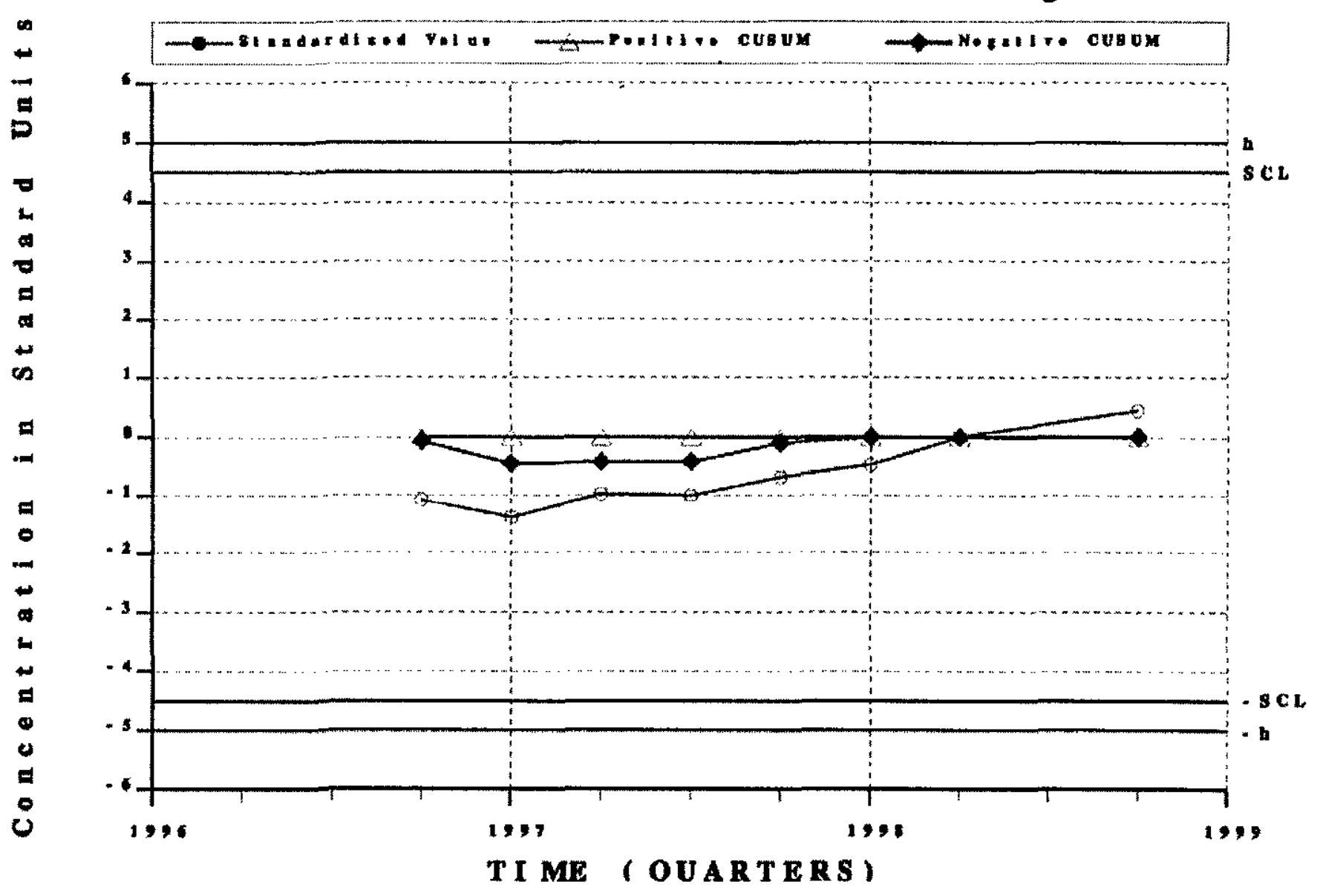


Combi ned She whart - CuSUM Cont rol Chart

WELL HSB1 16C - Nonvolatile beta

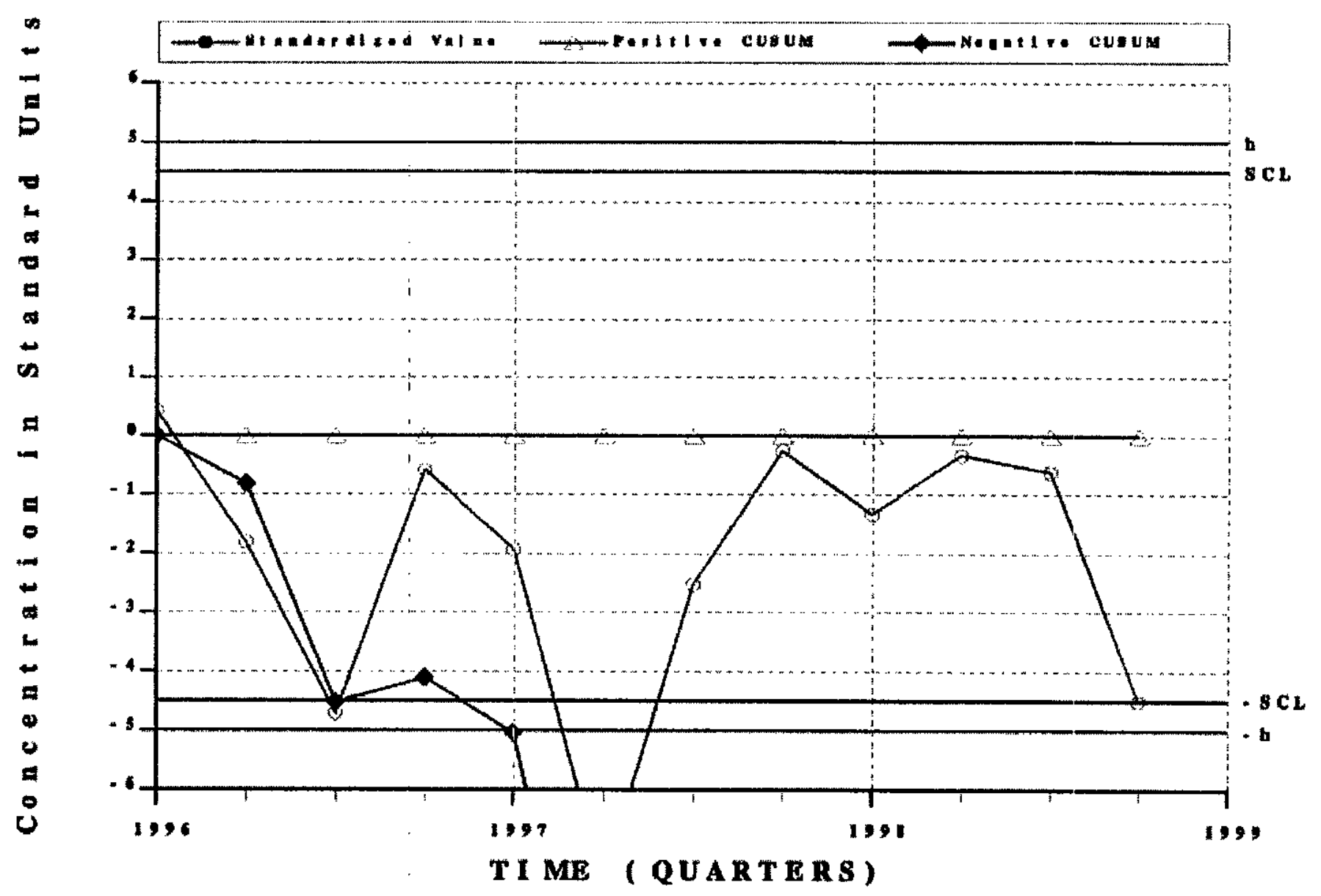

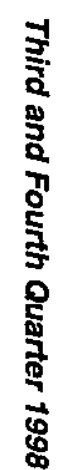



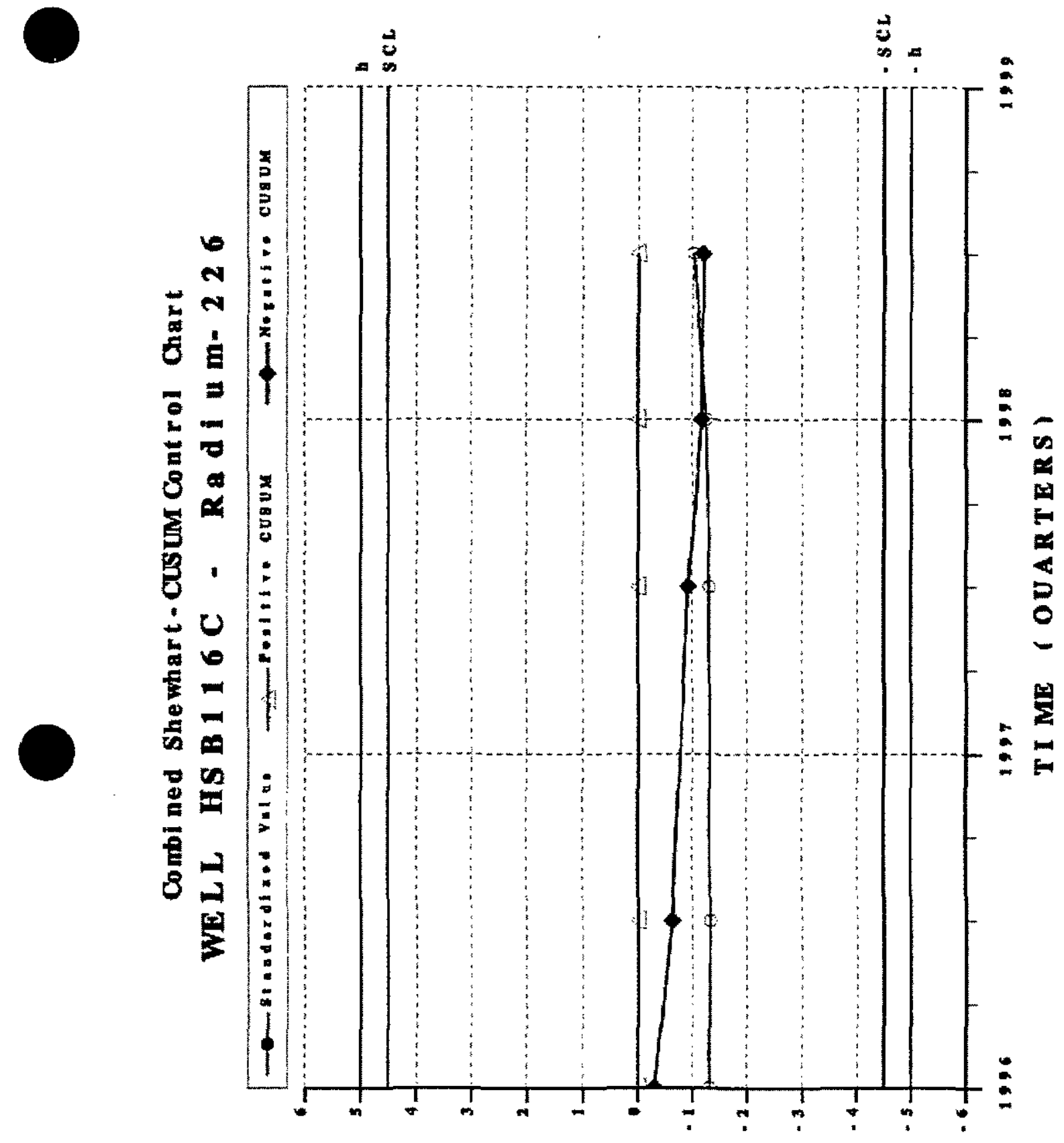

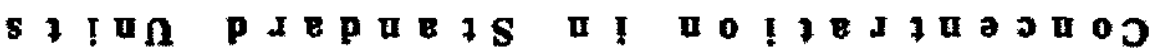




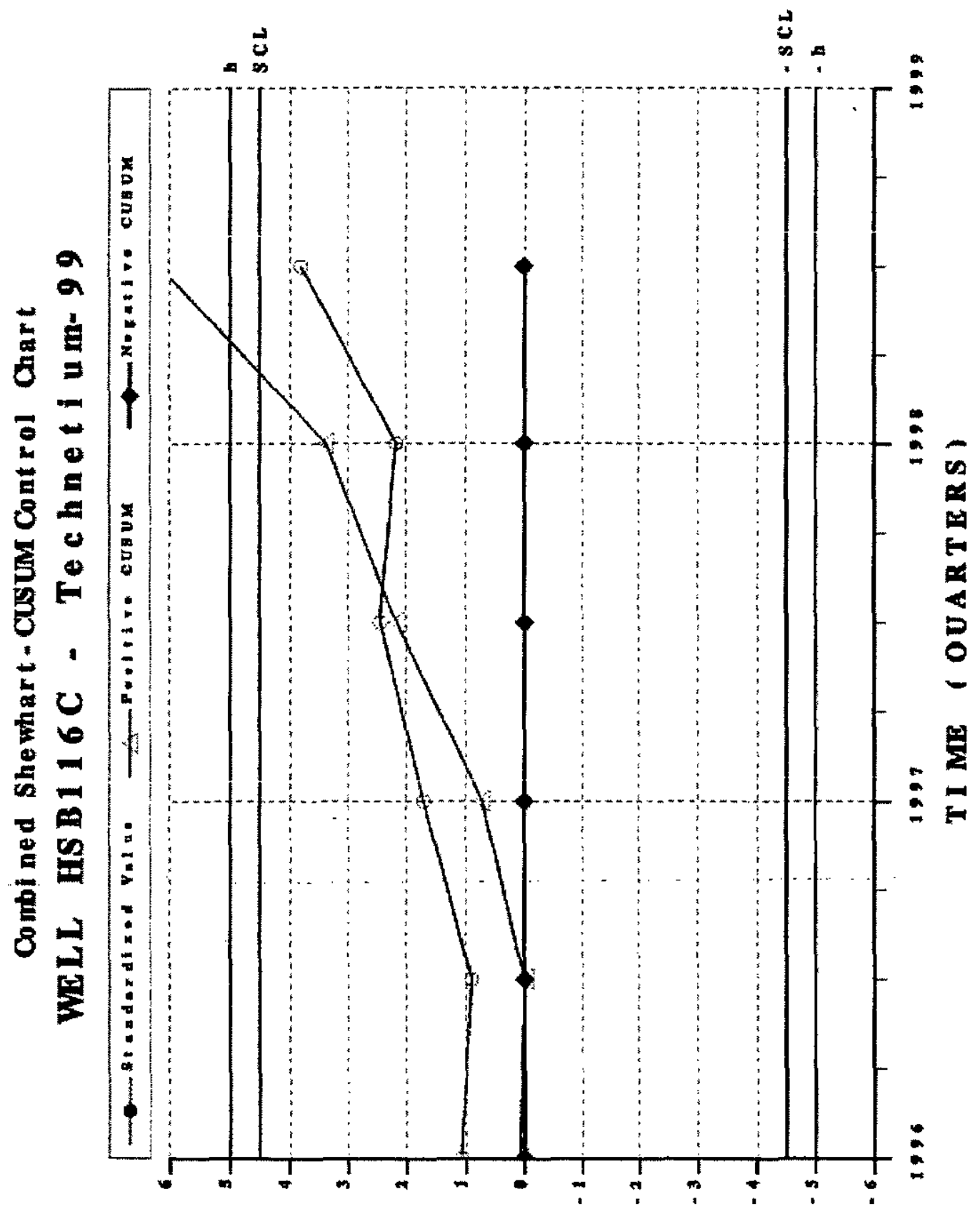

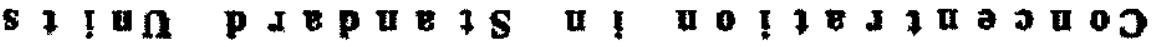




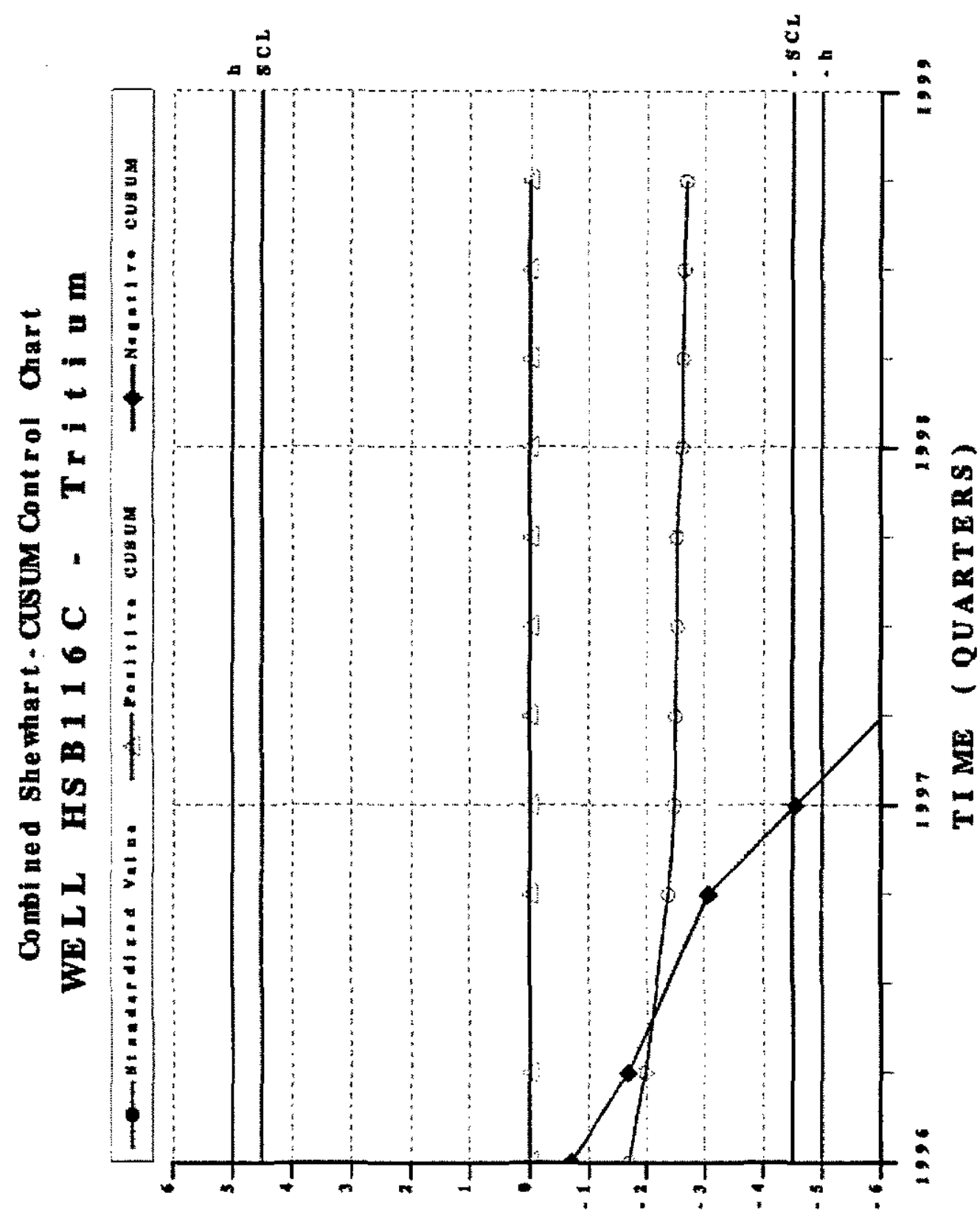

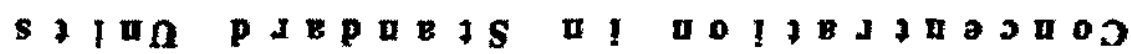




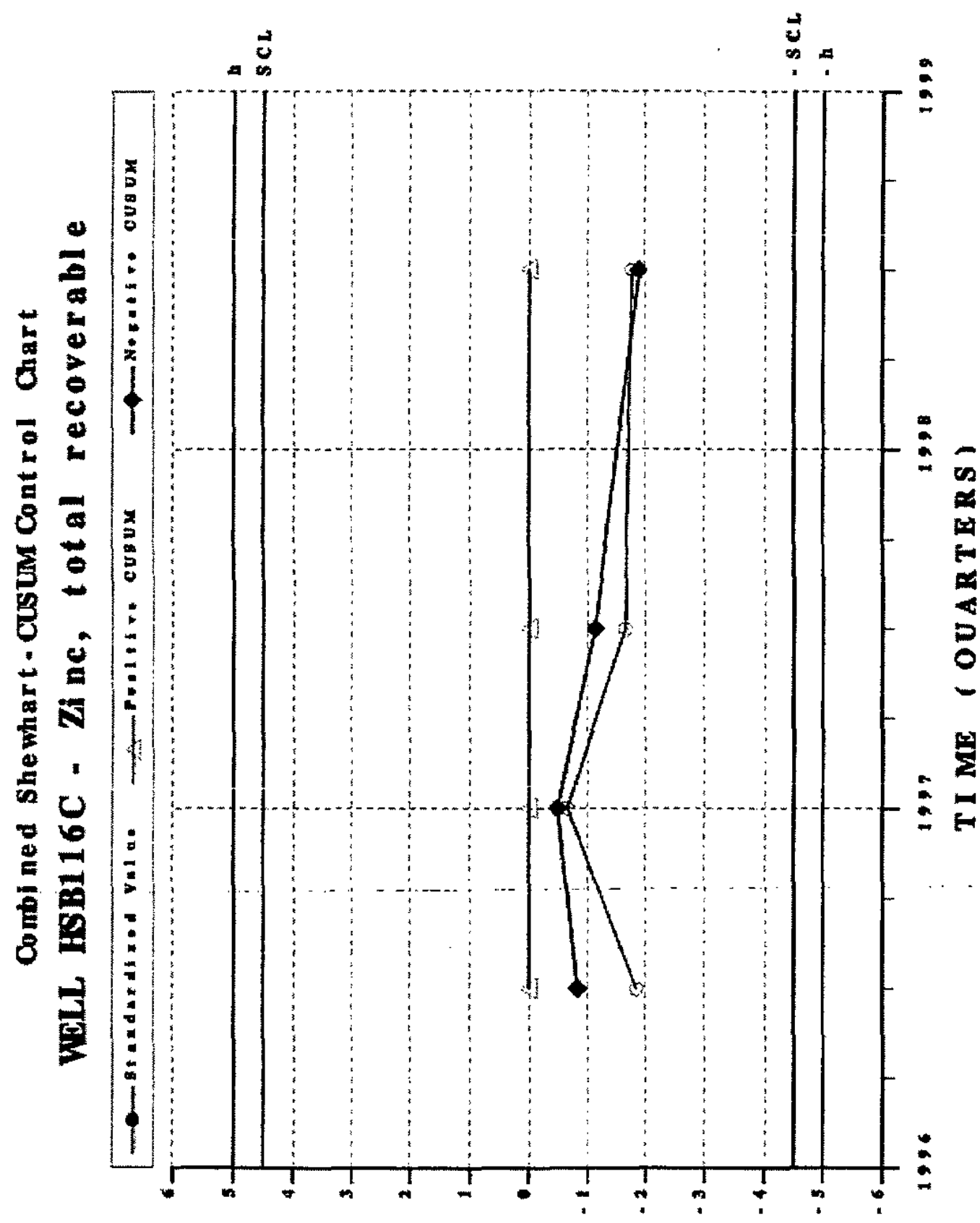

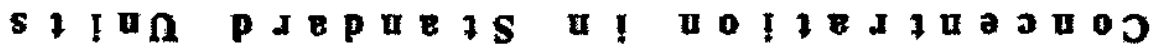


Combi ned Shewhart - CuSUM Cont rol Chart

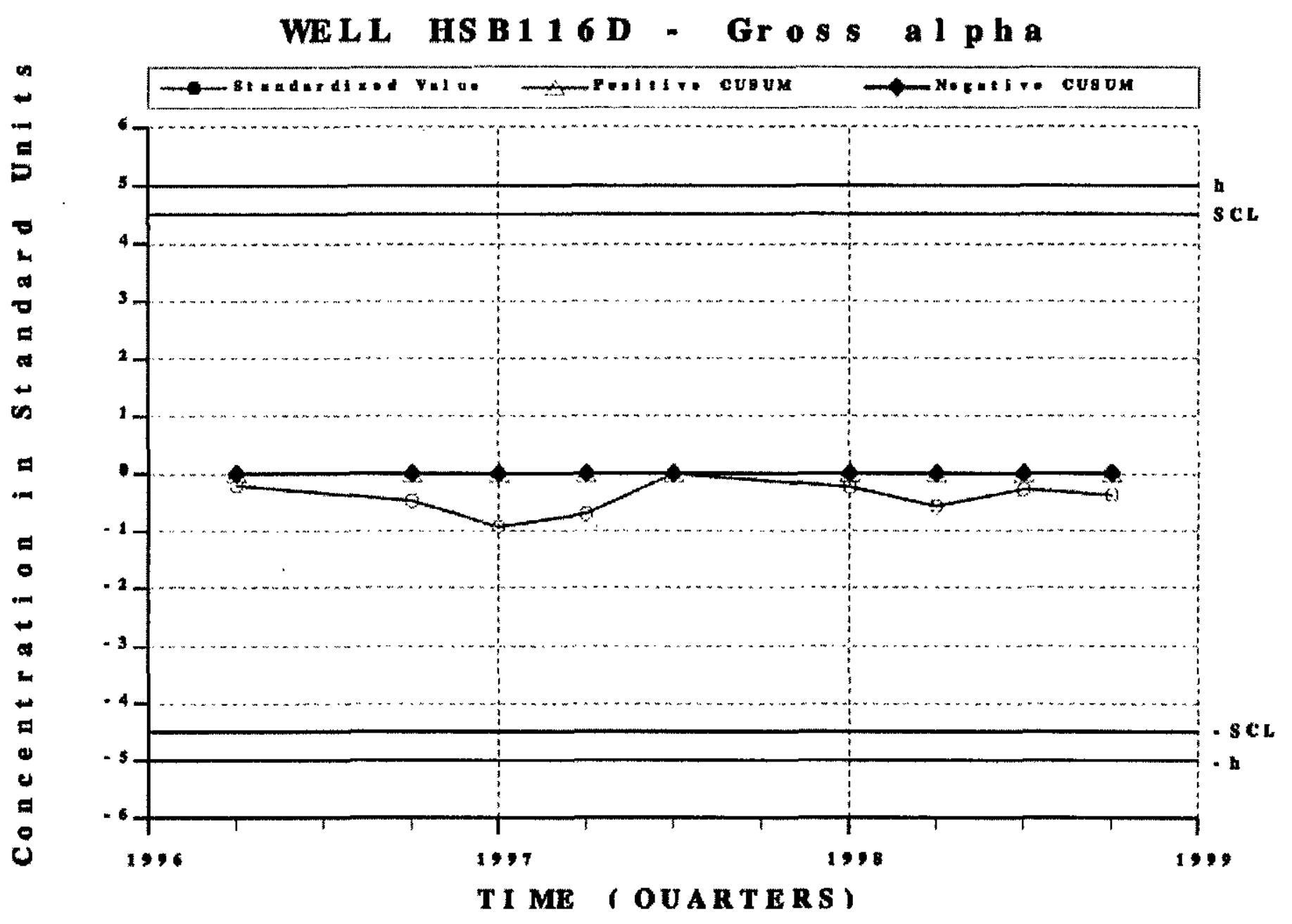




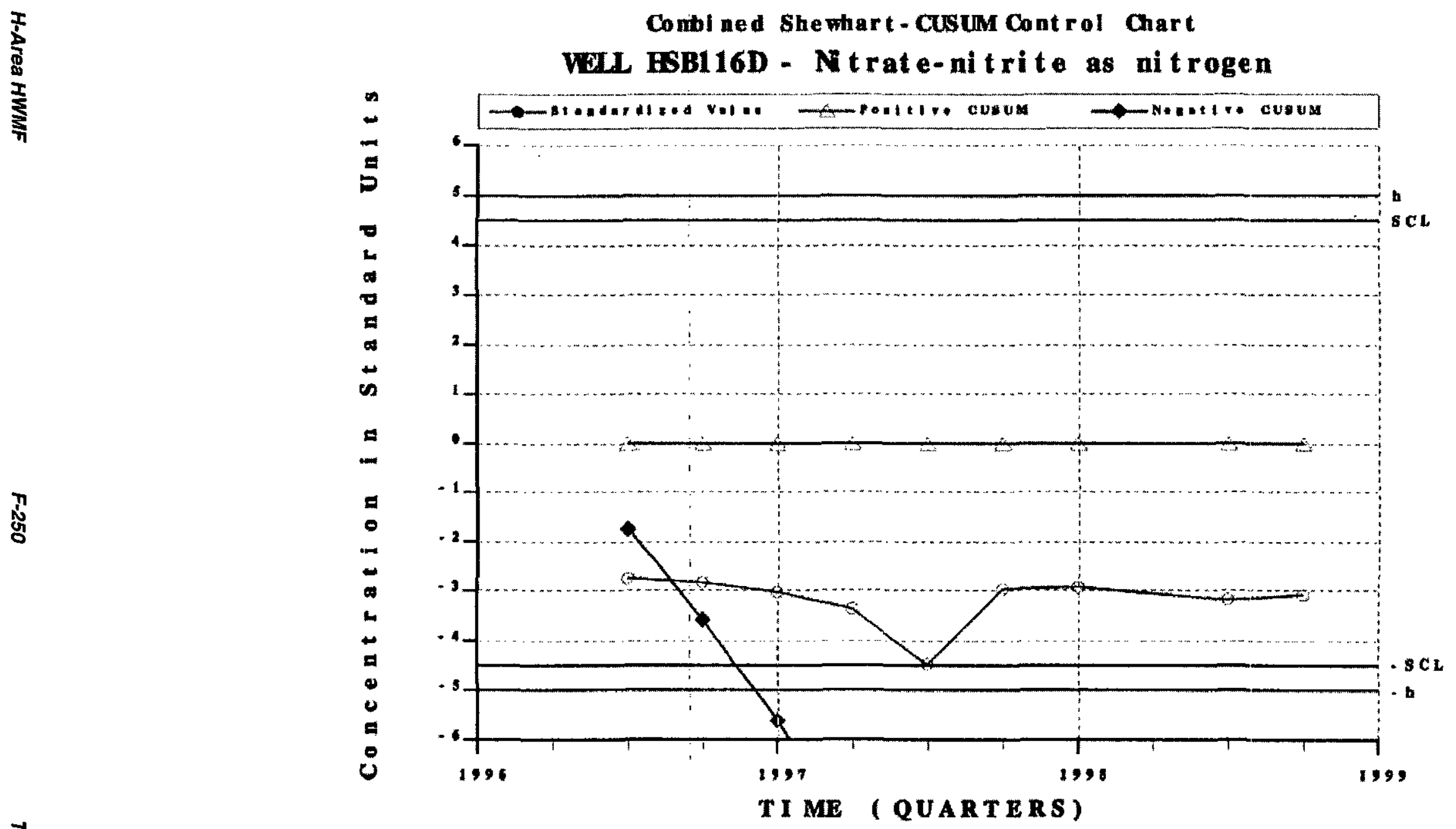

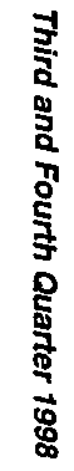

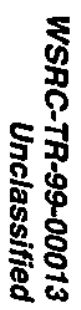



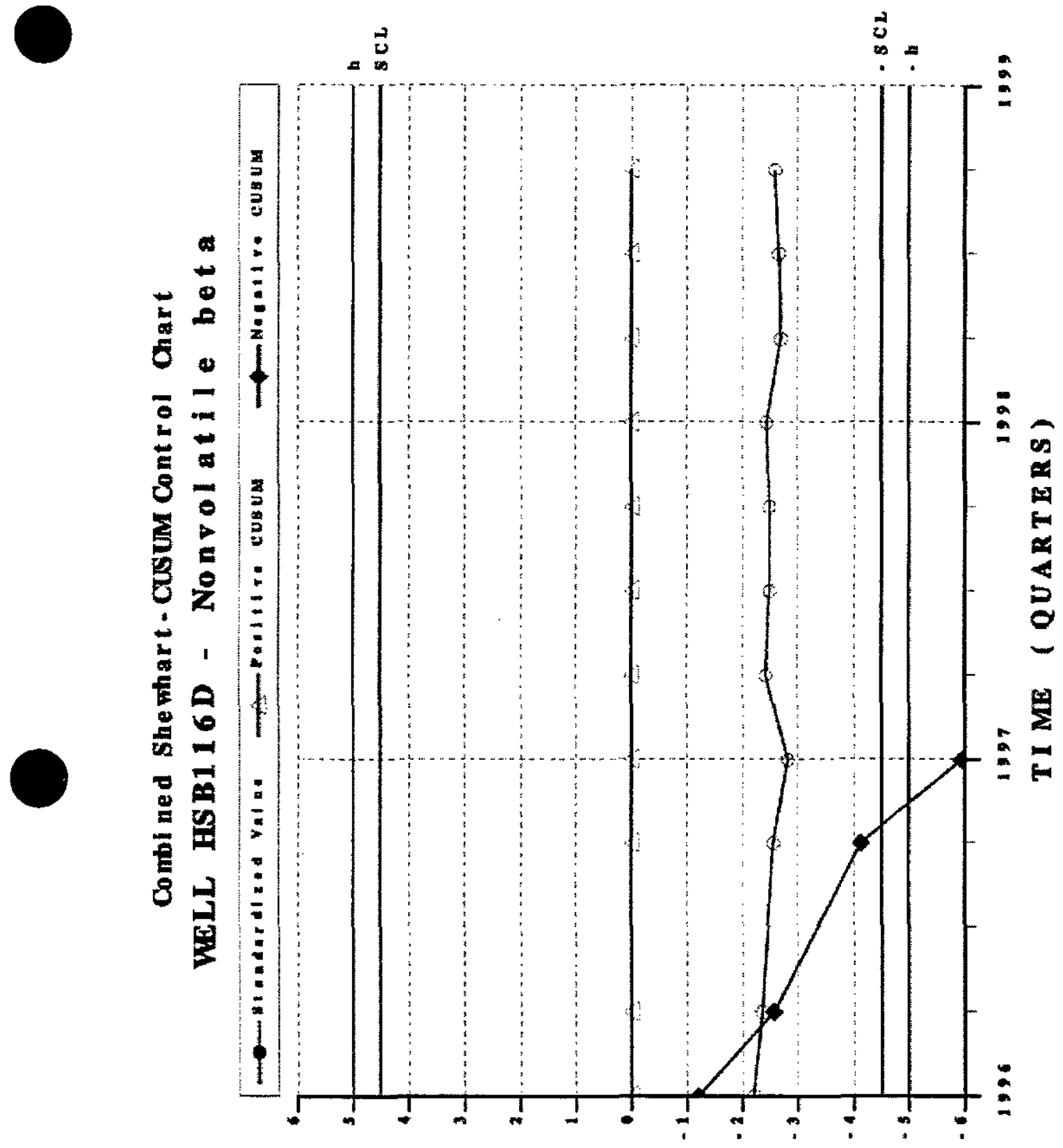

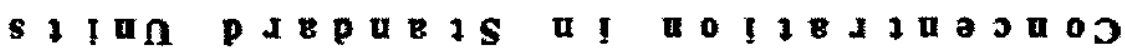




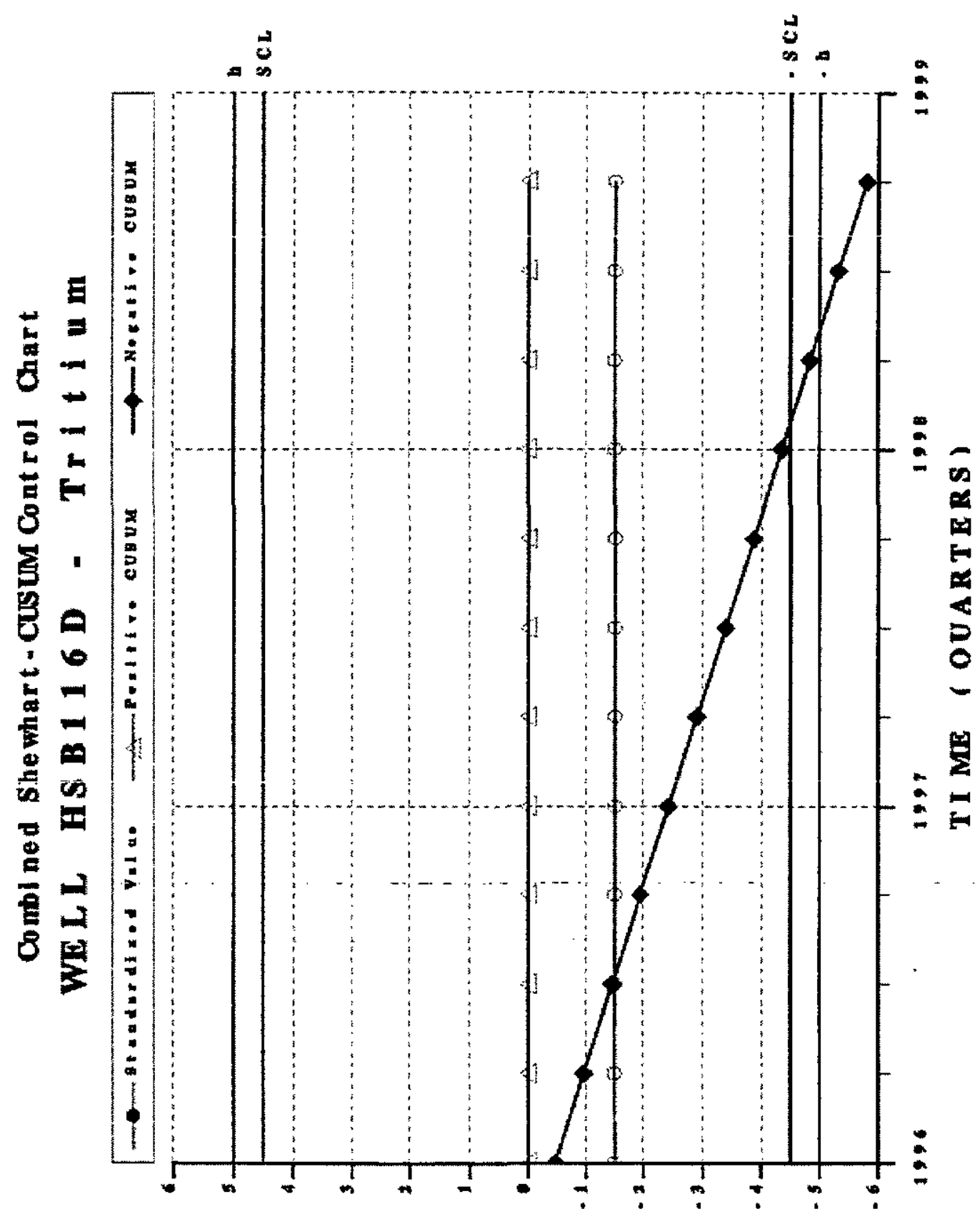

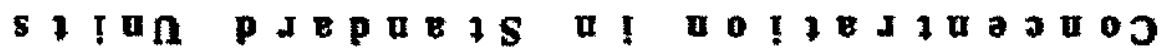



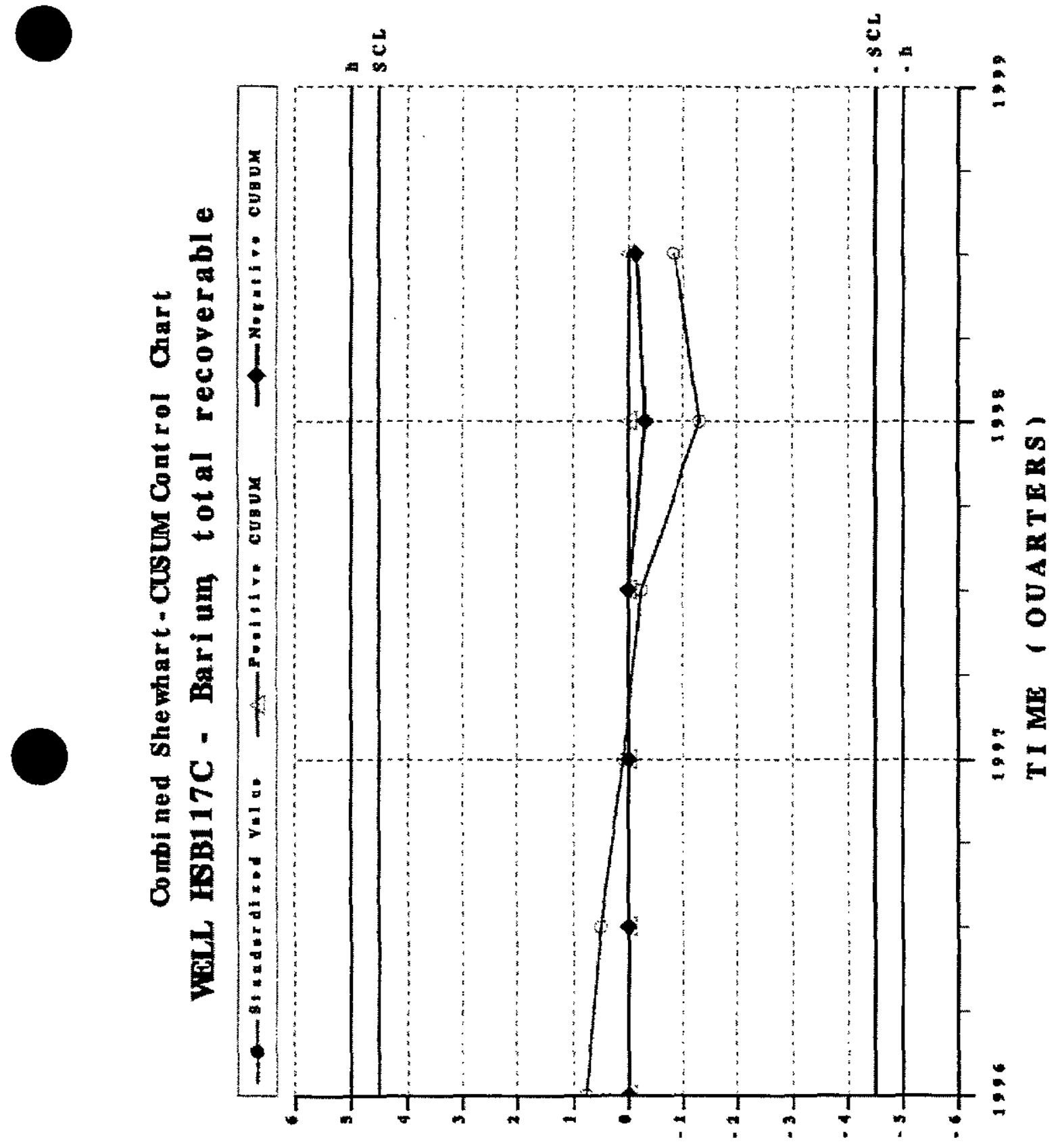

s I 


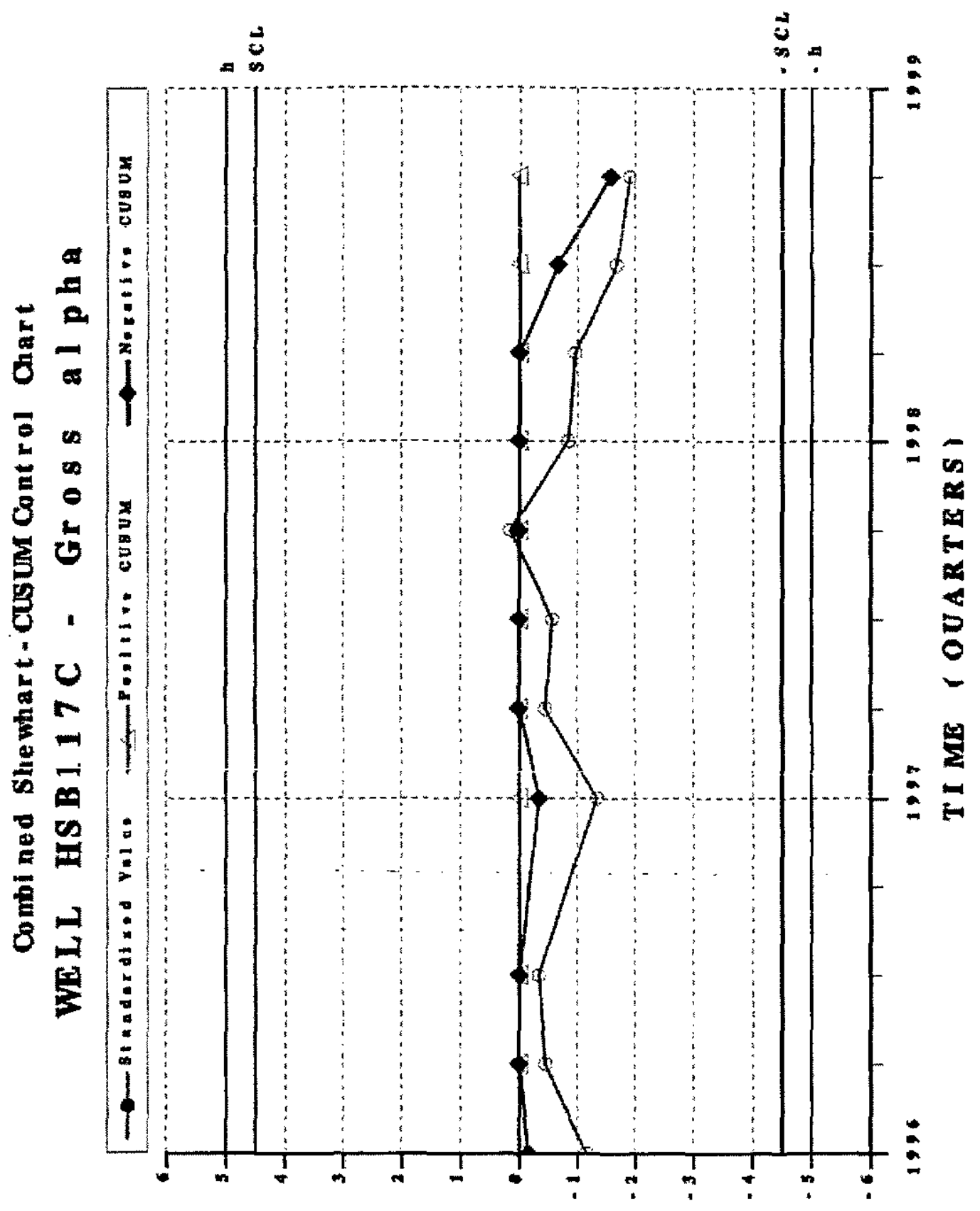

s) I 

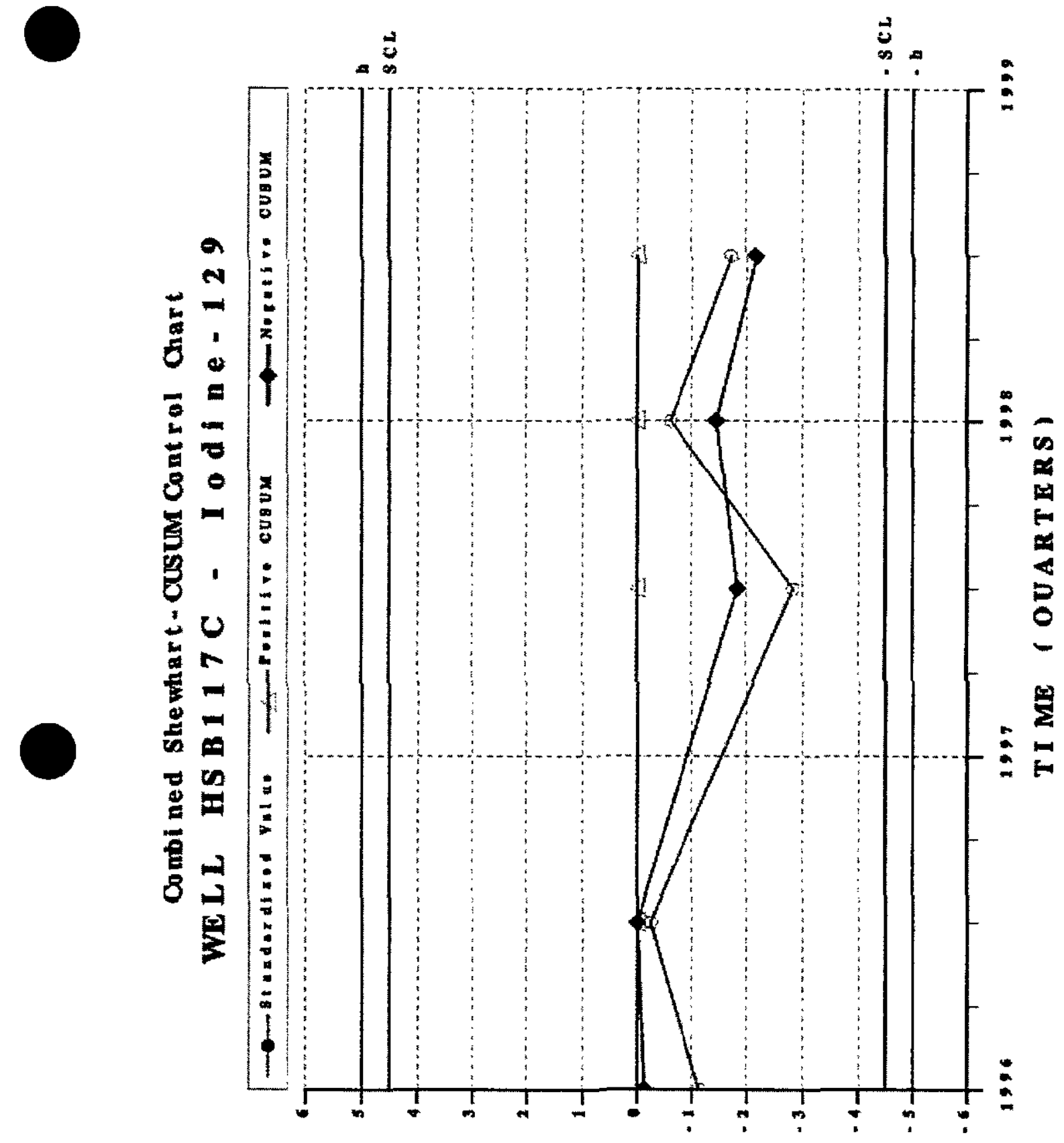

s I I 


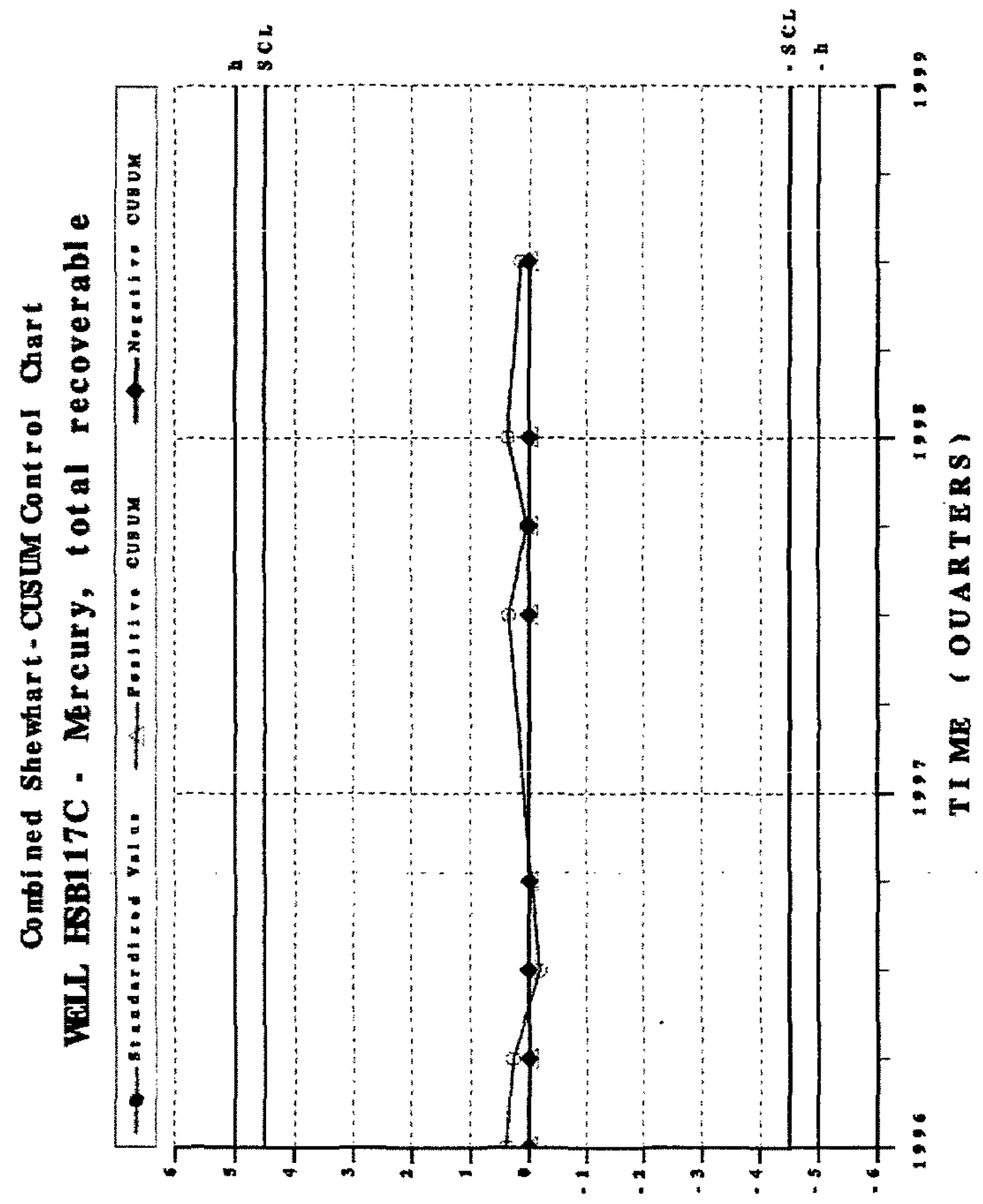

S) I $\Omega$ PI B 


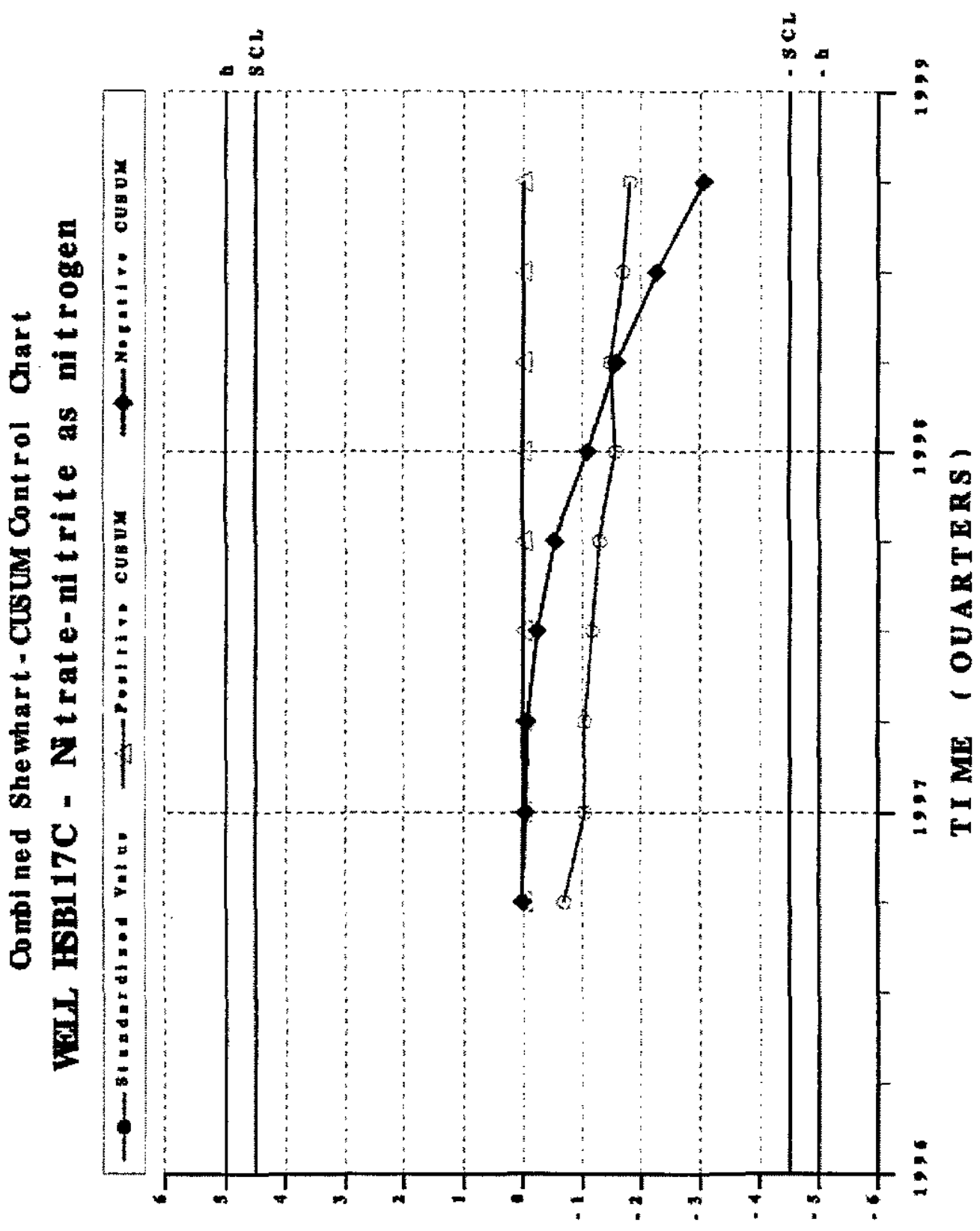

s 1 In p I 
Combined Shewhart-Cusum Control Chart WELL HSB1 17C - Nonvolatile beta

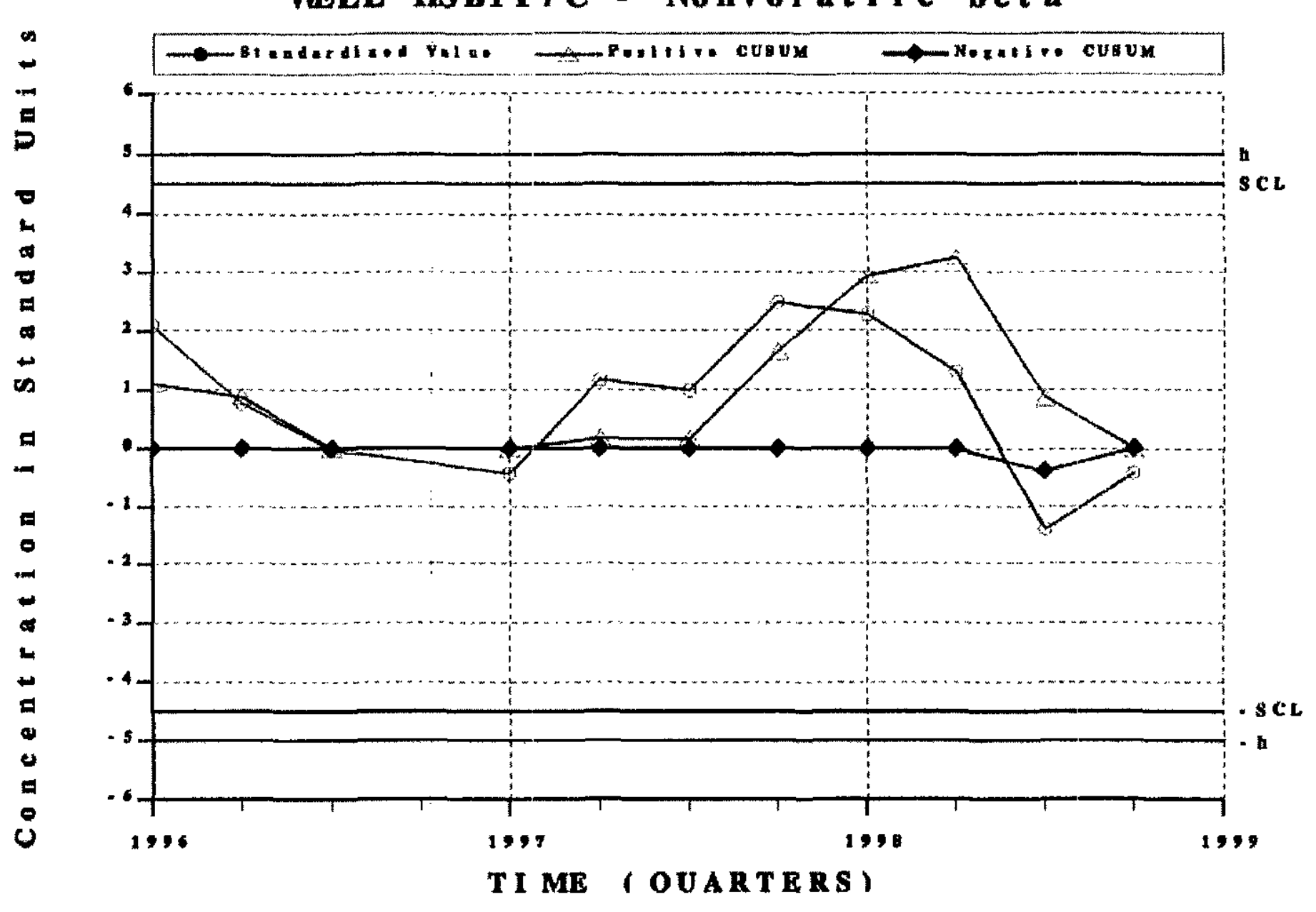

T I ME (OUARTRRS) 


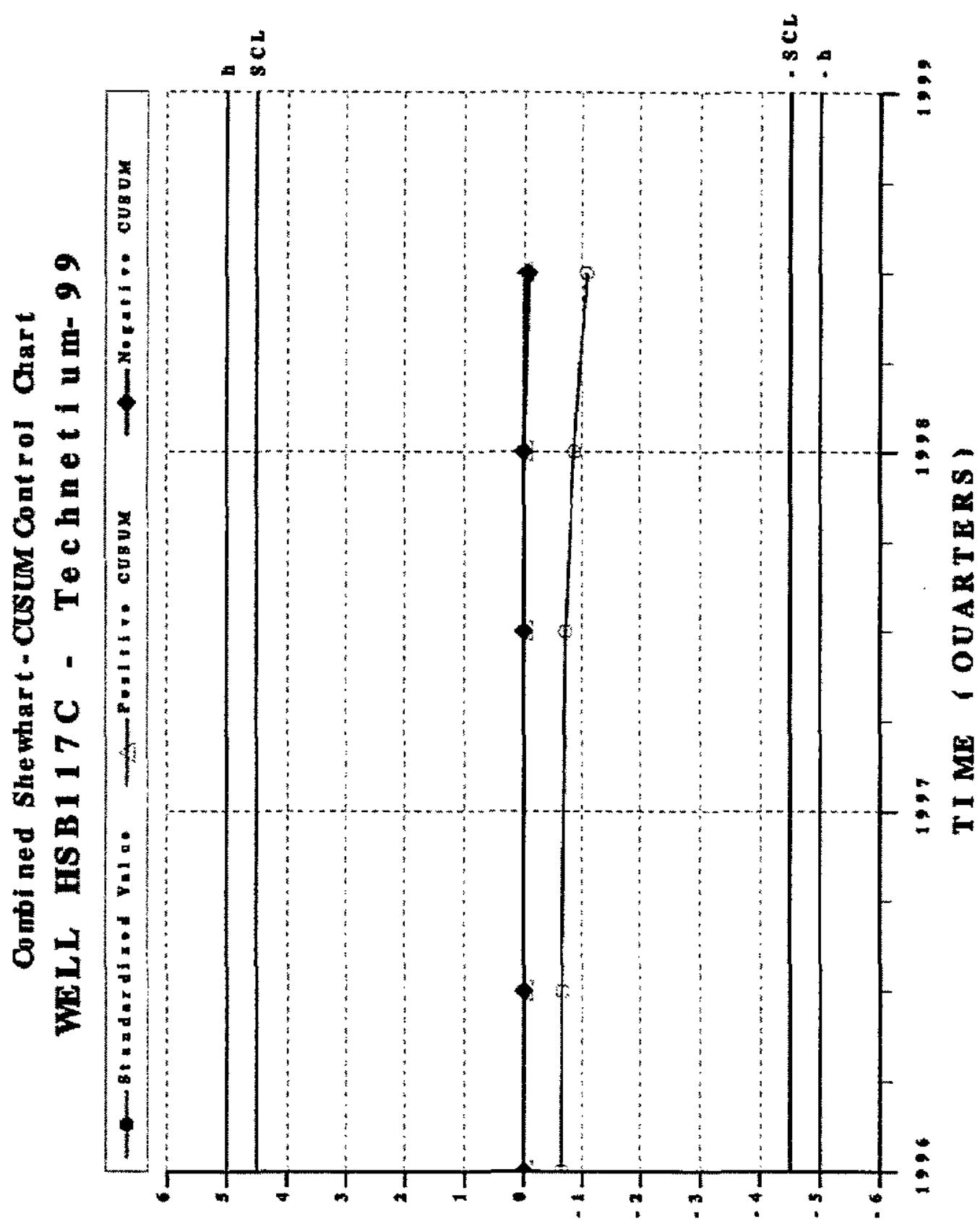

s) I 

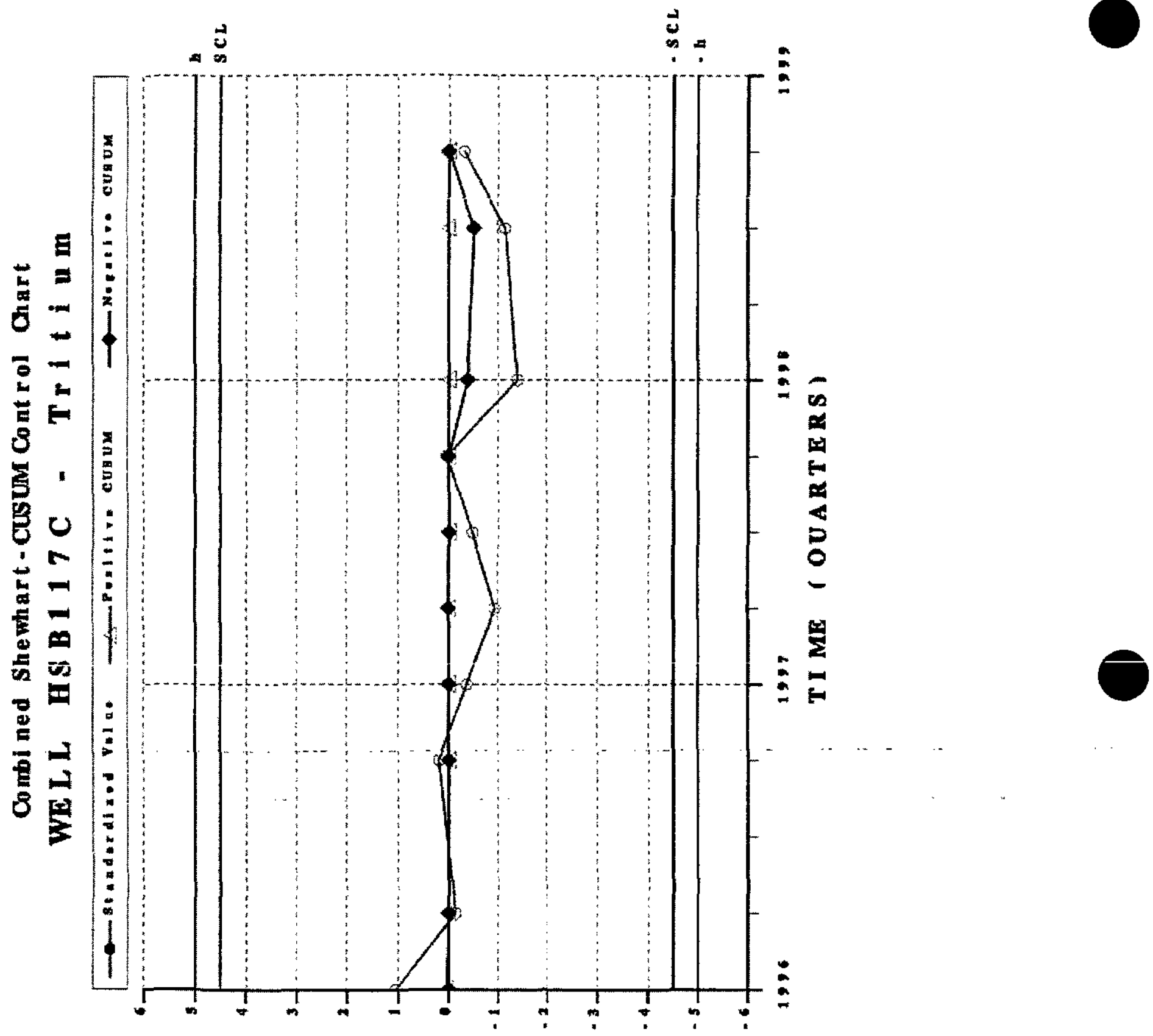

st tan pa 

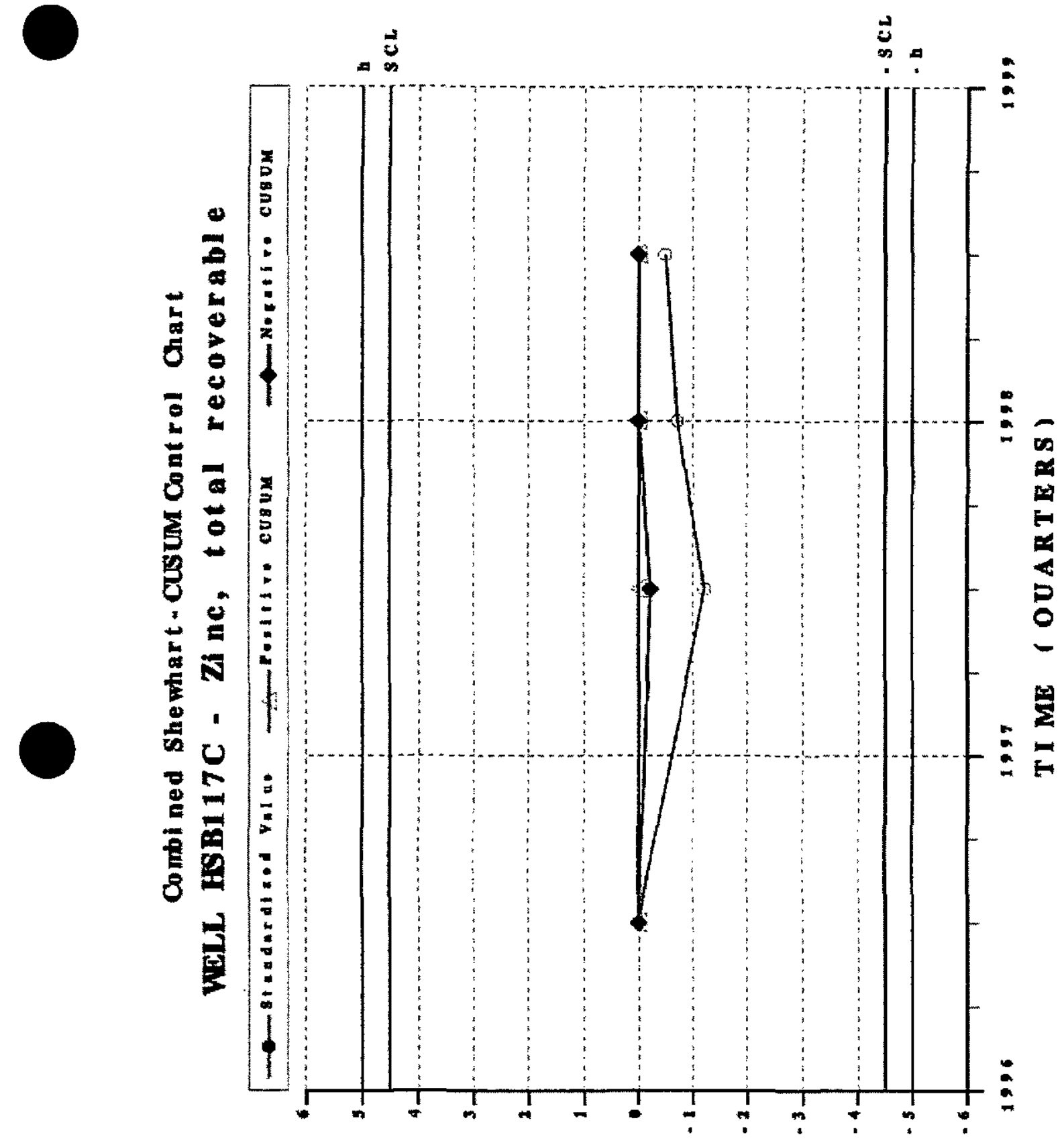

s I I 


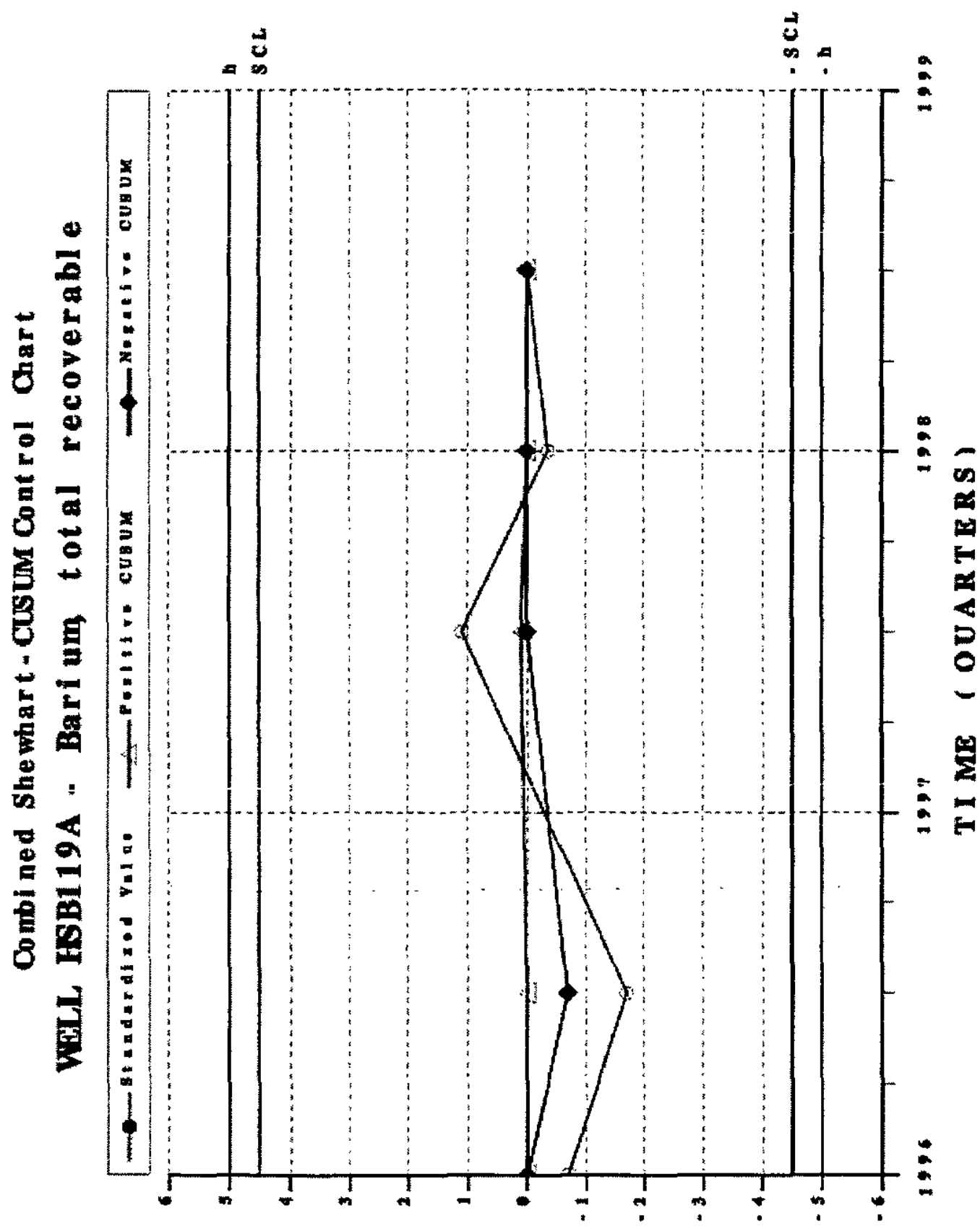

s? I 

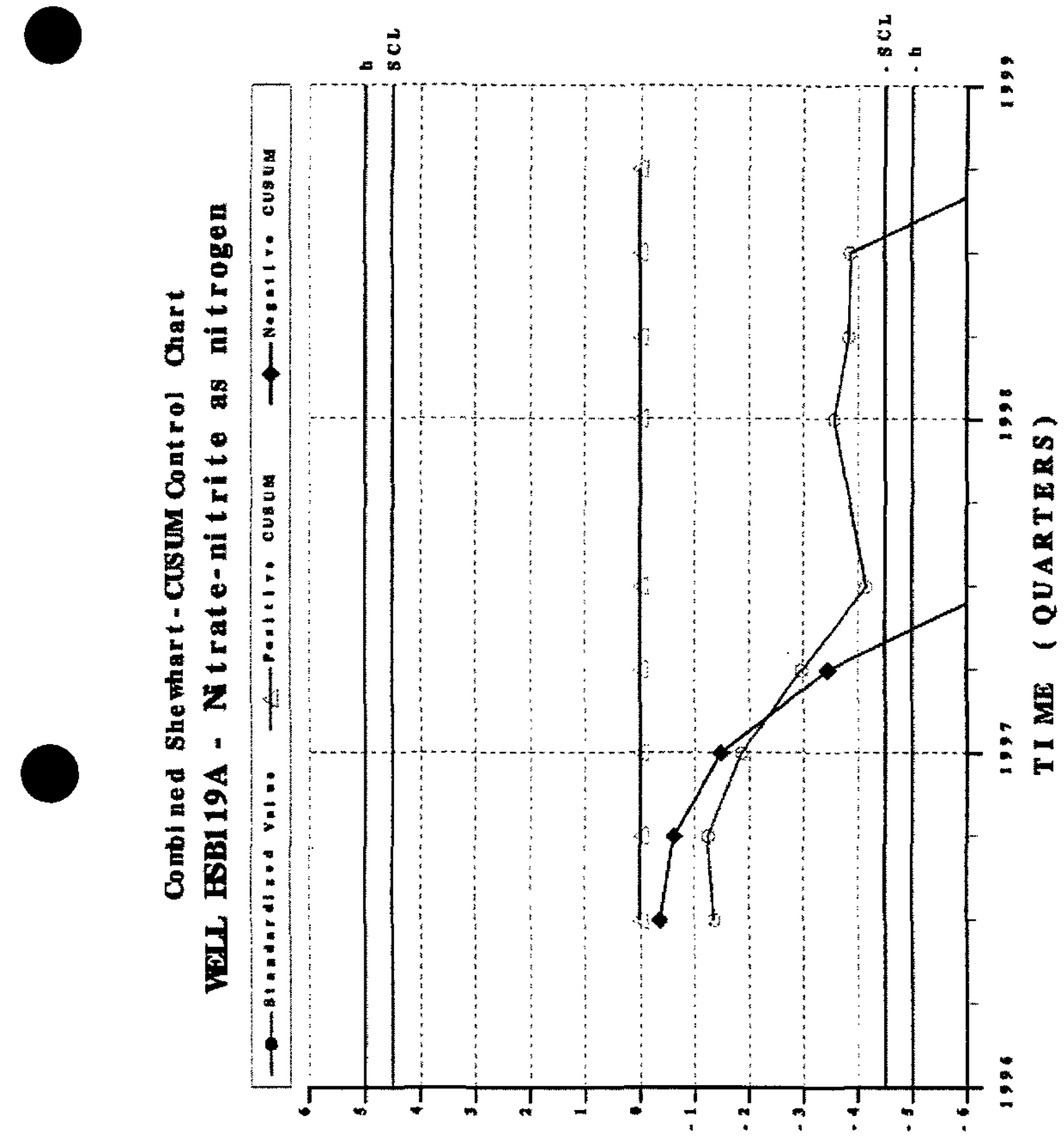

st I I p I E 


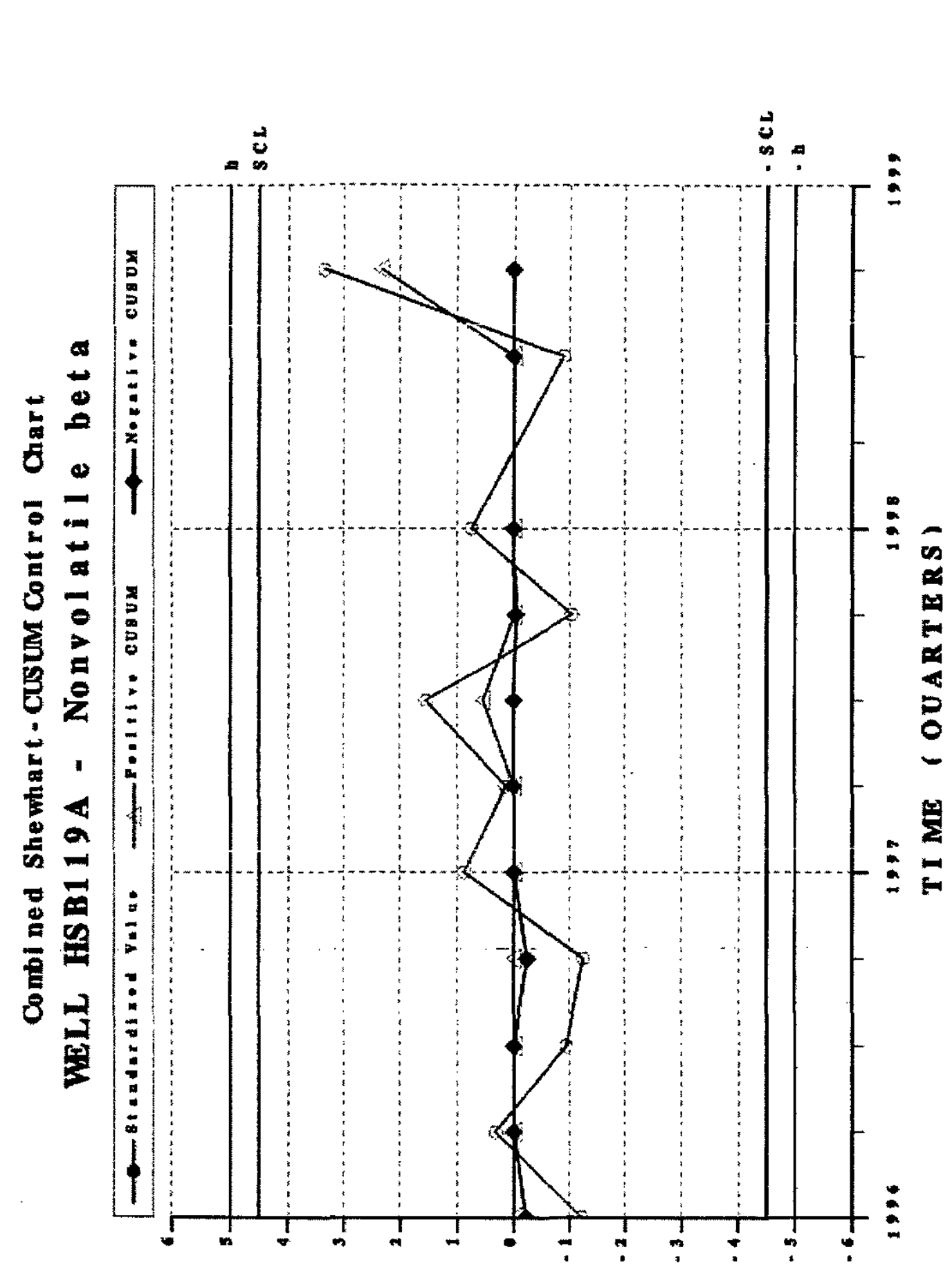

WSRC-TR-99-00013

Unclassified

s I I 

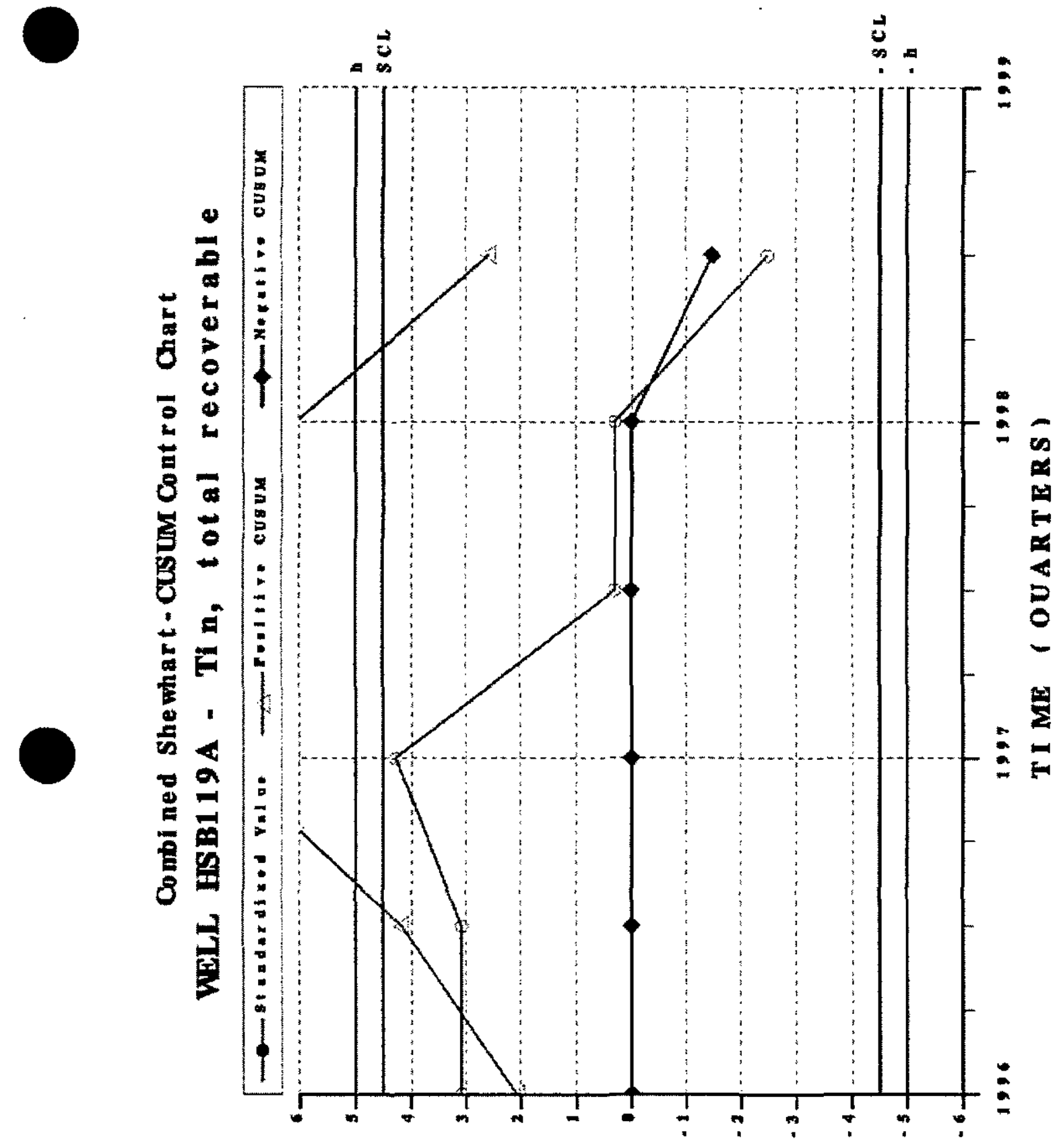

s I 


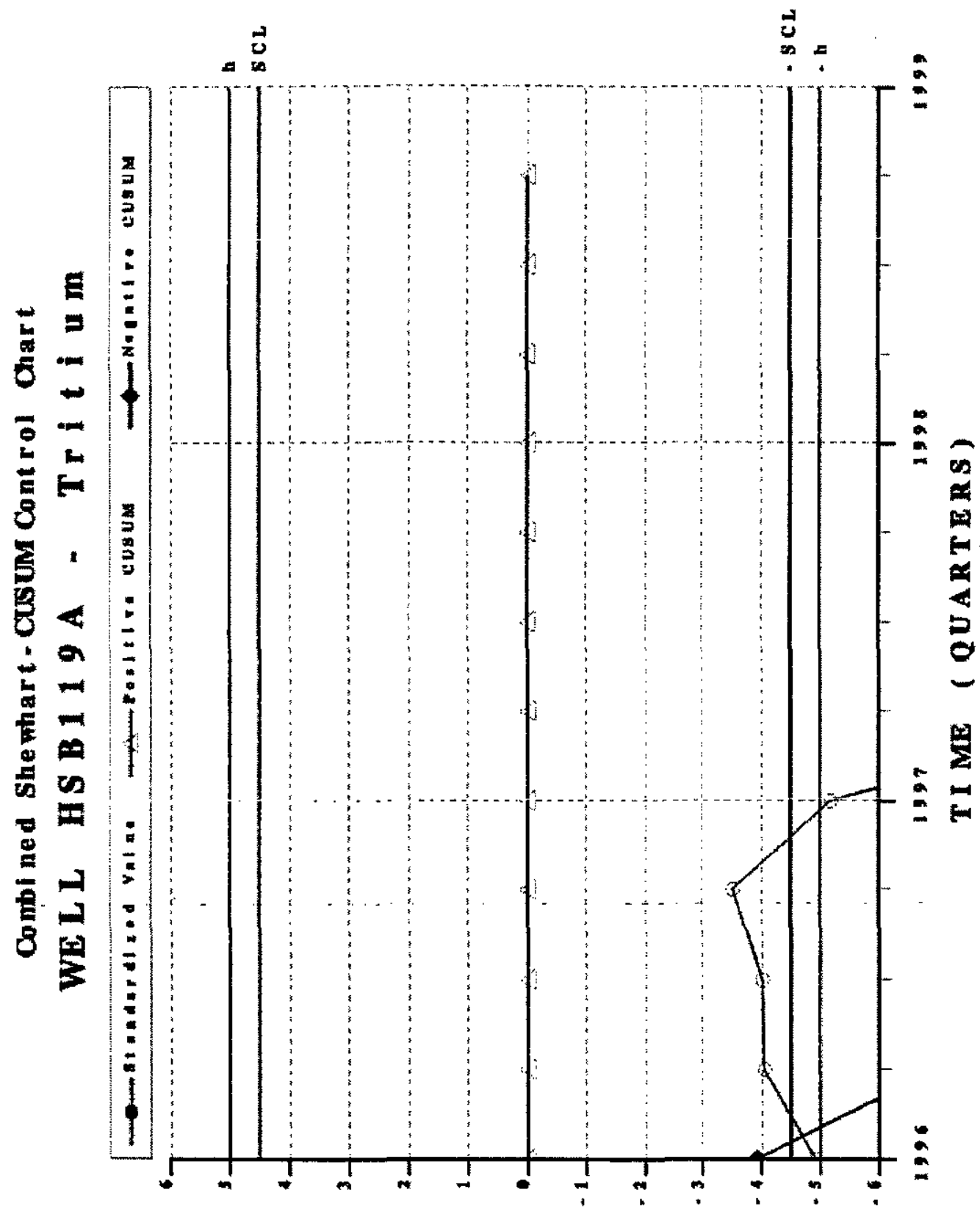

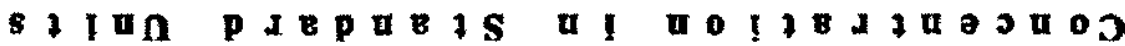



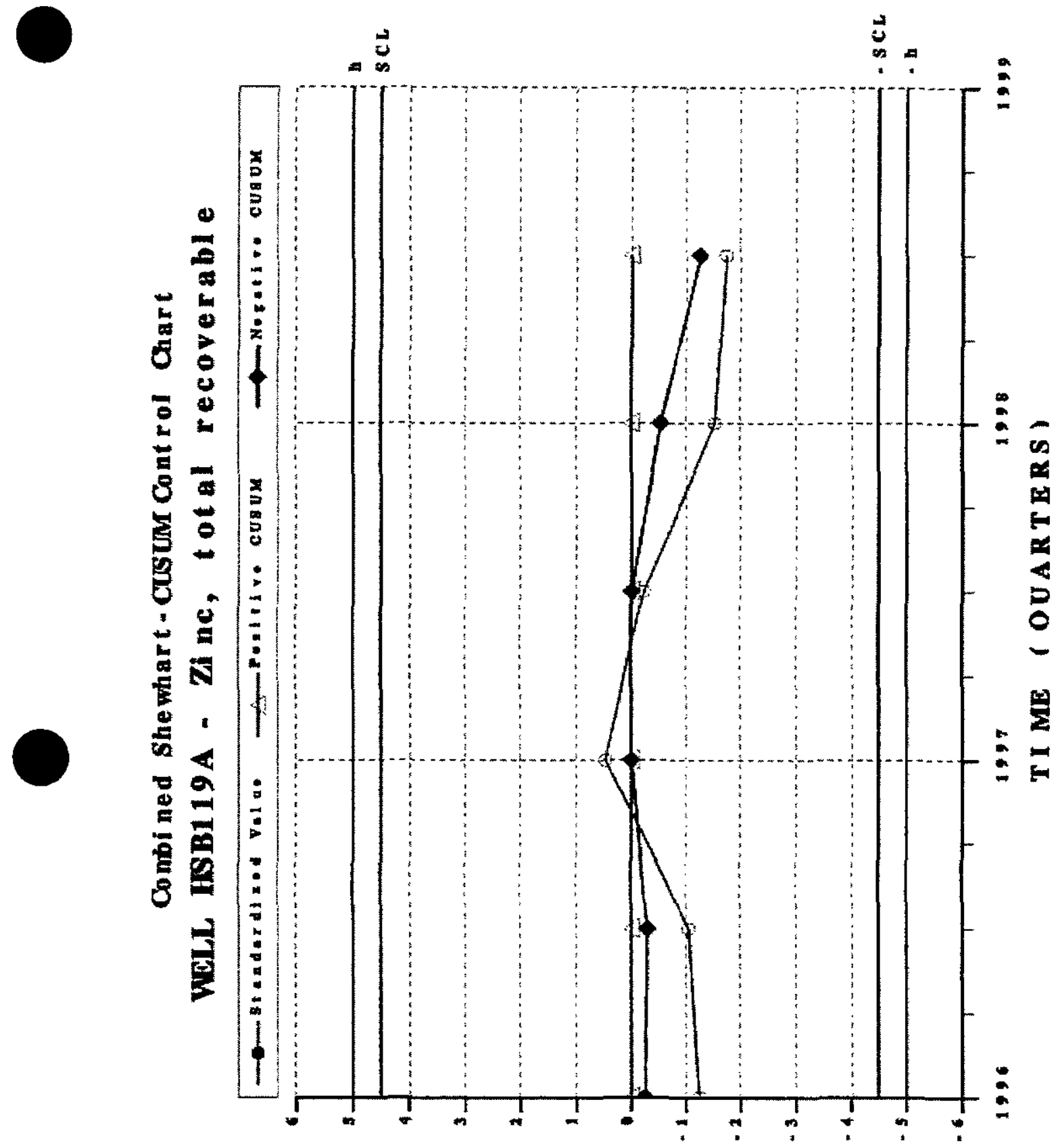

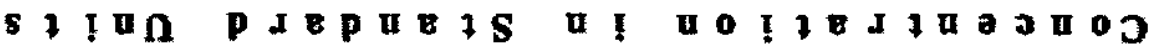


Conbl ned Shewhart - CusuM Cont rol Chart WeLL HSB120A - Bari um total recoverable

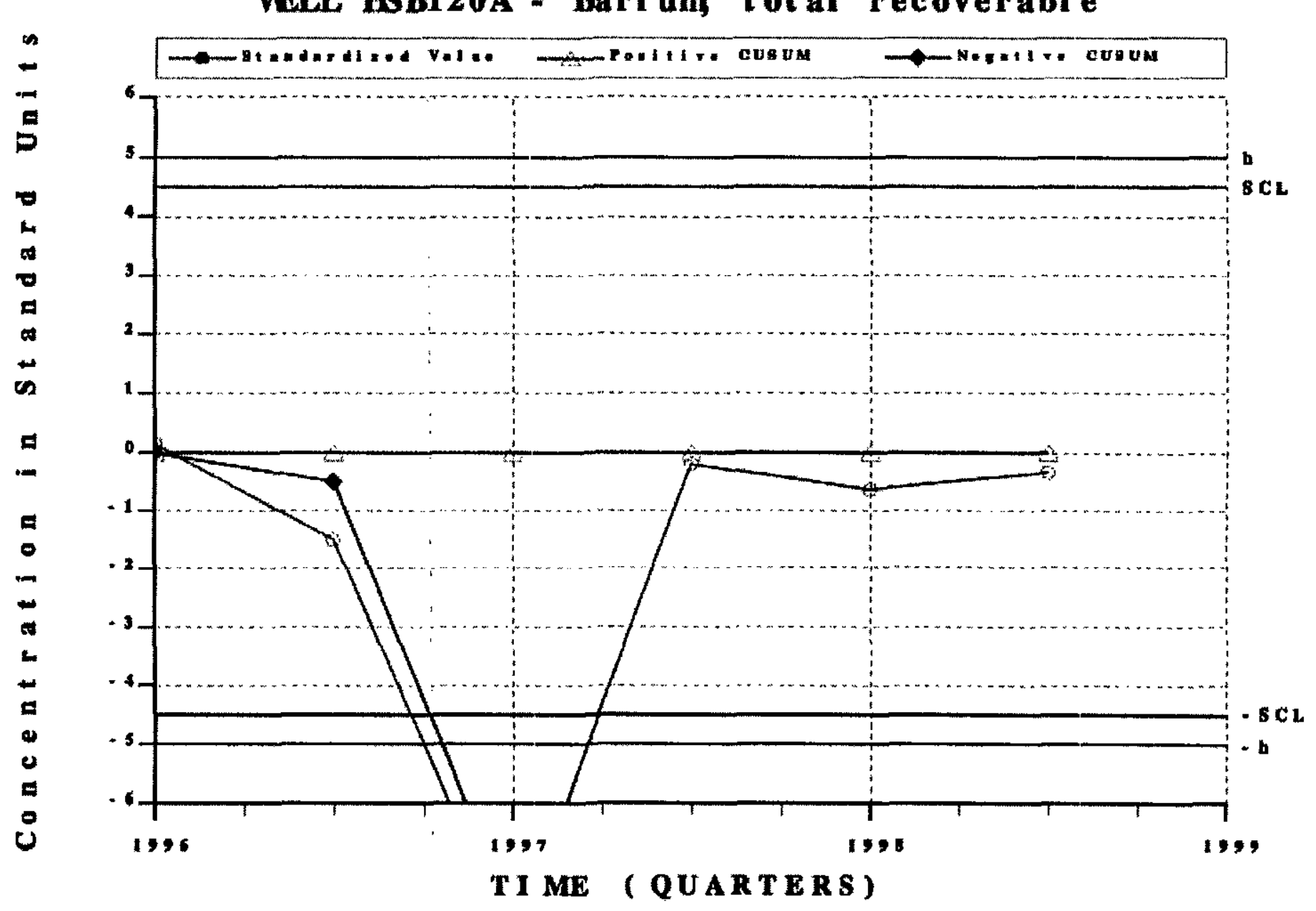

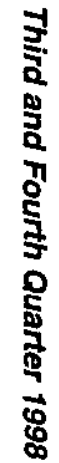


Combi ned Shewhart-cusum Cont rol Chart WELL HSB1 20 - Nonvolatile beta

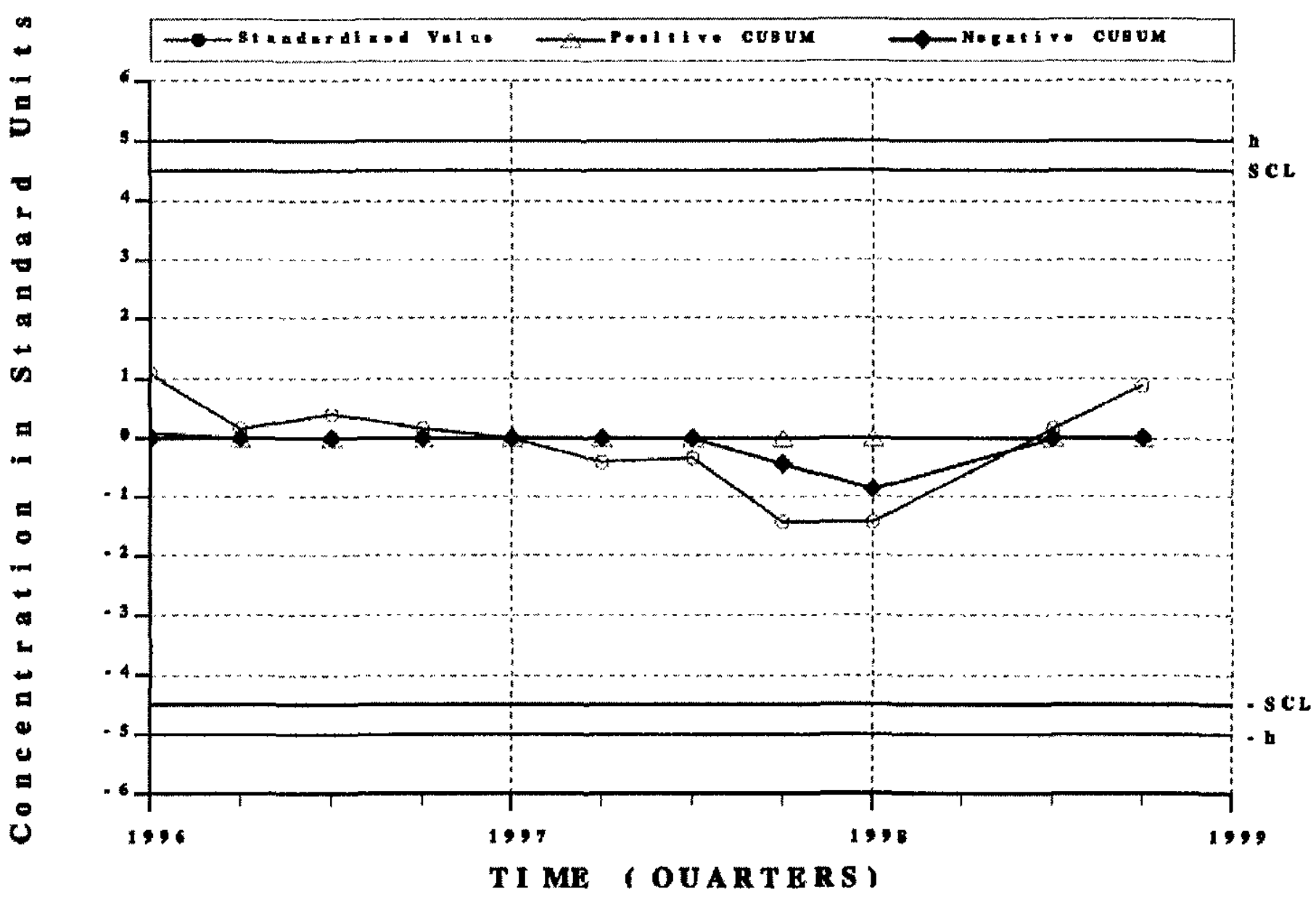


Combi ned Shewhart - Cusum Control Chart

VELL HSB121A - Bari um total recoverable

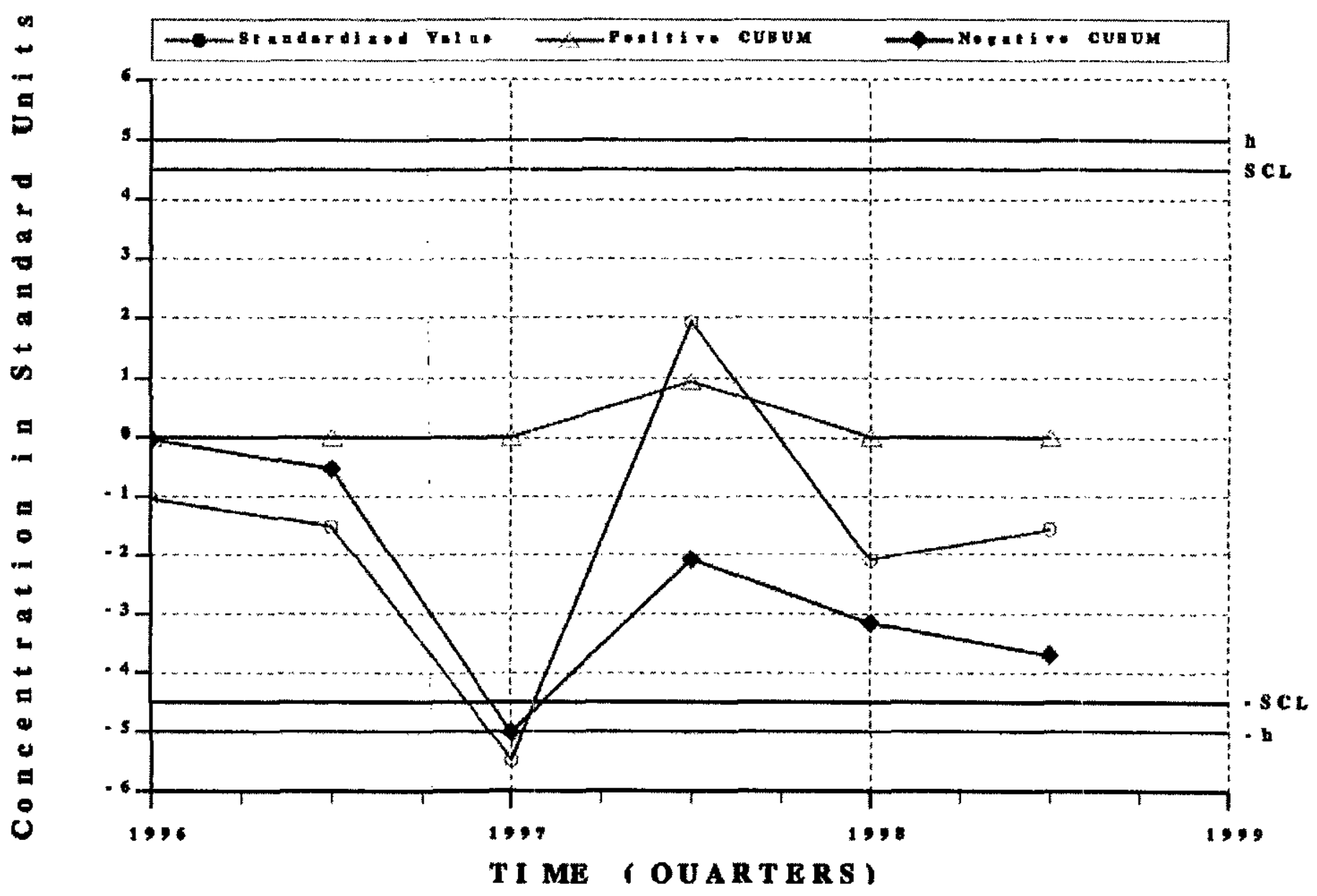




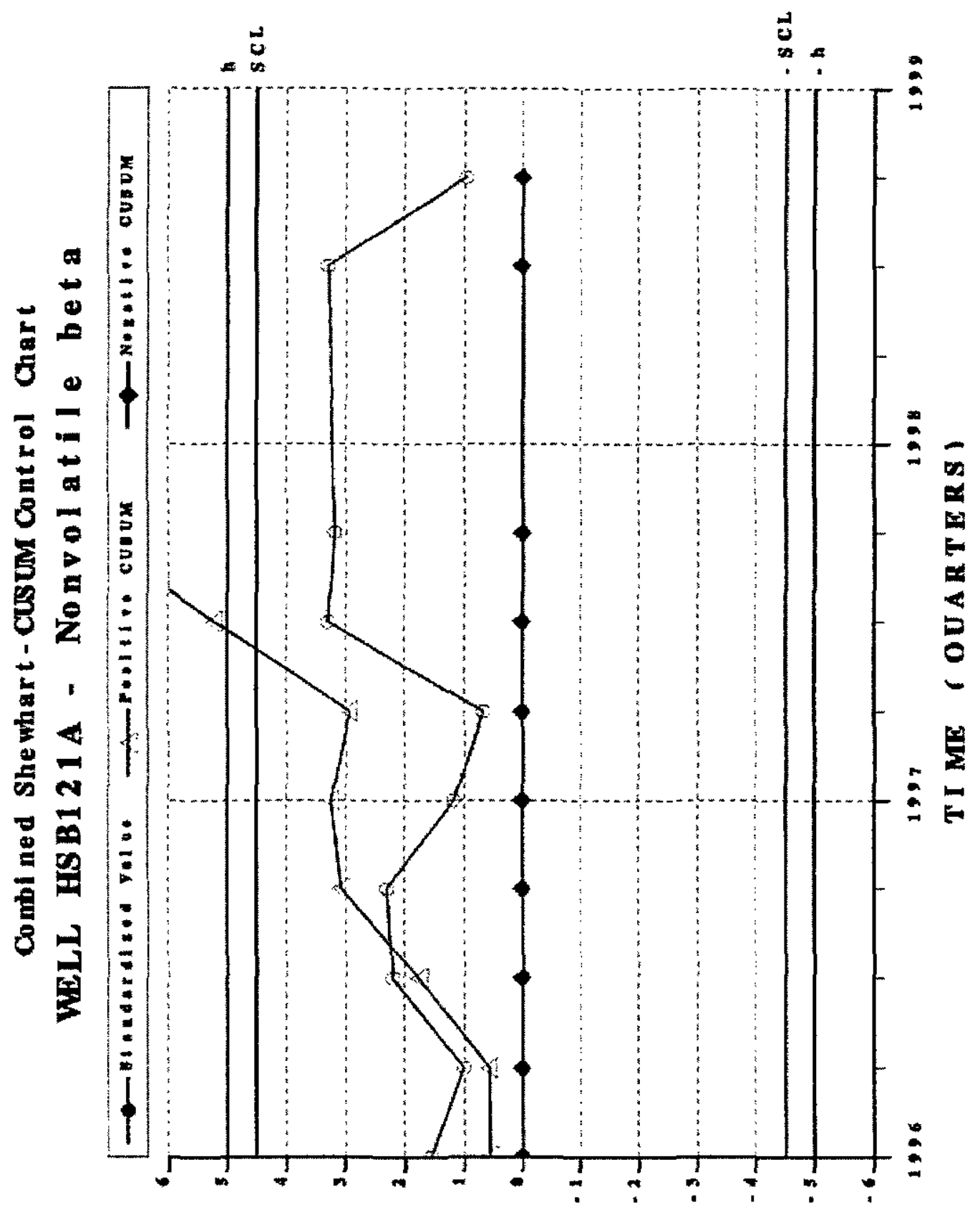

s 1 เ 


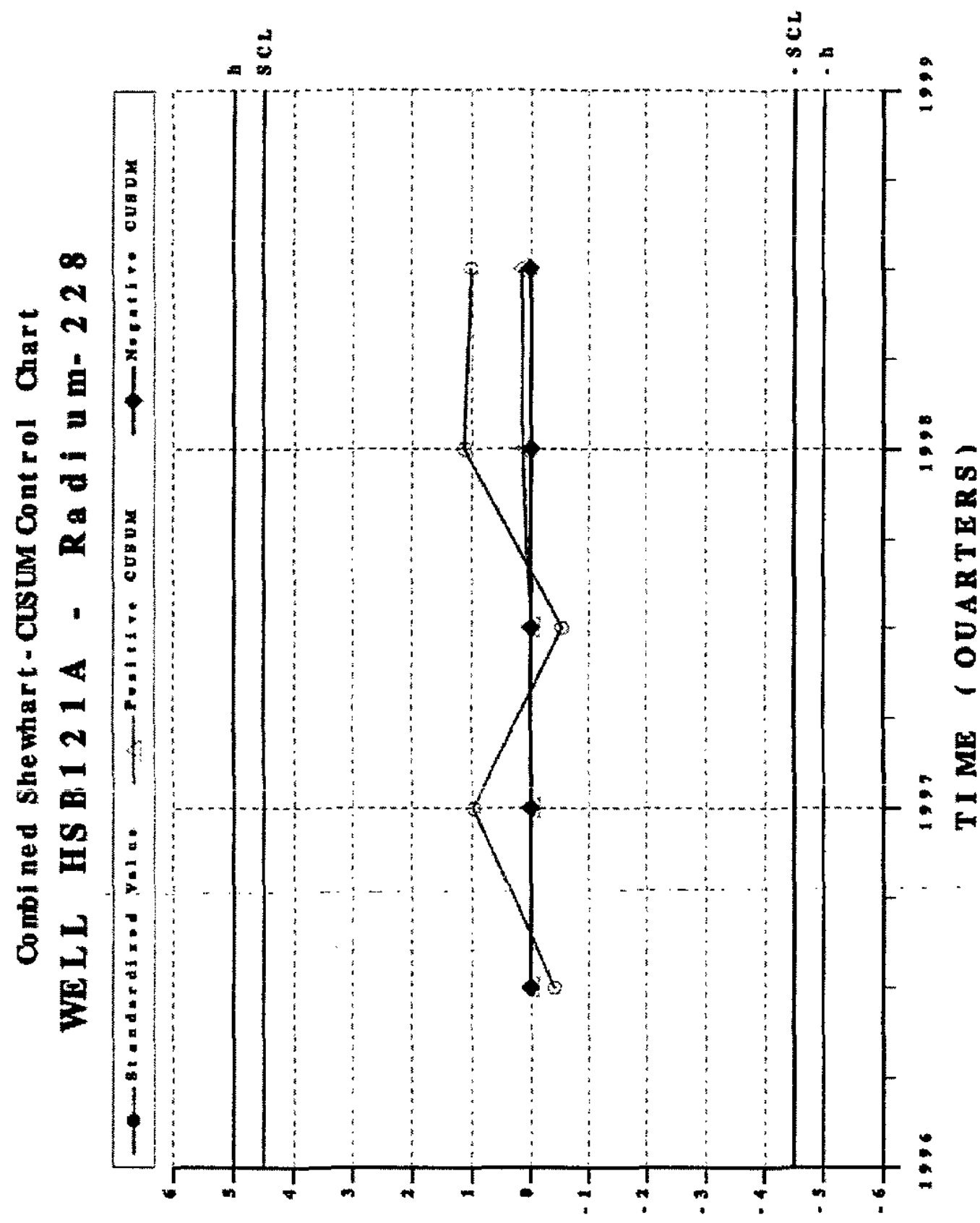

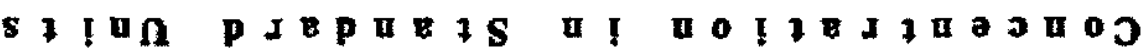




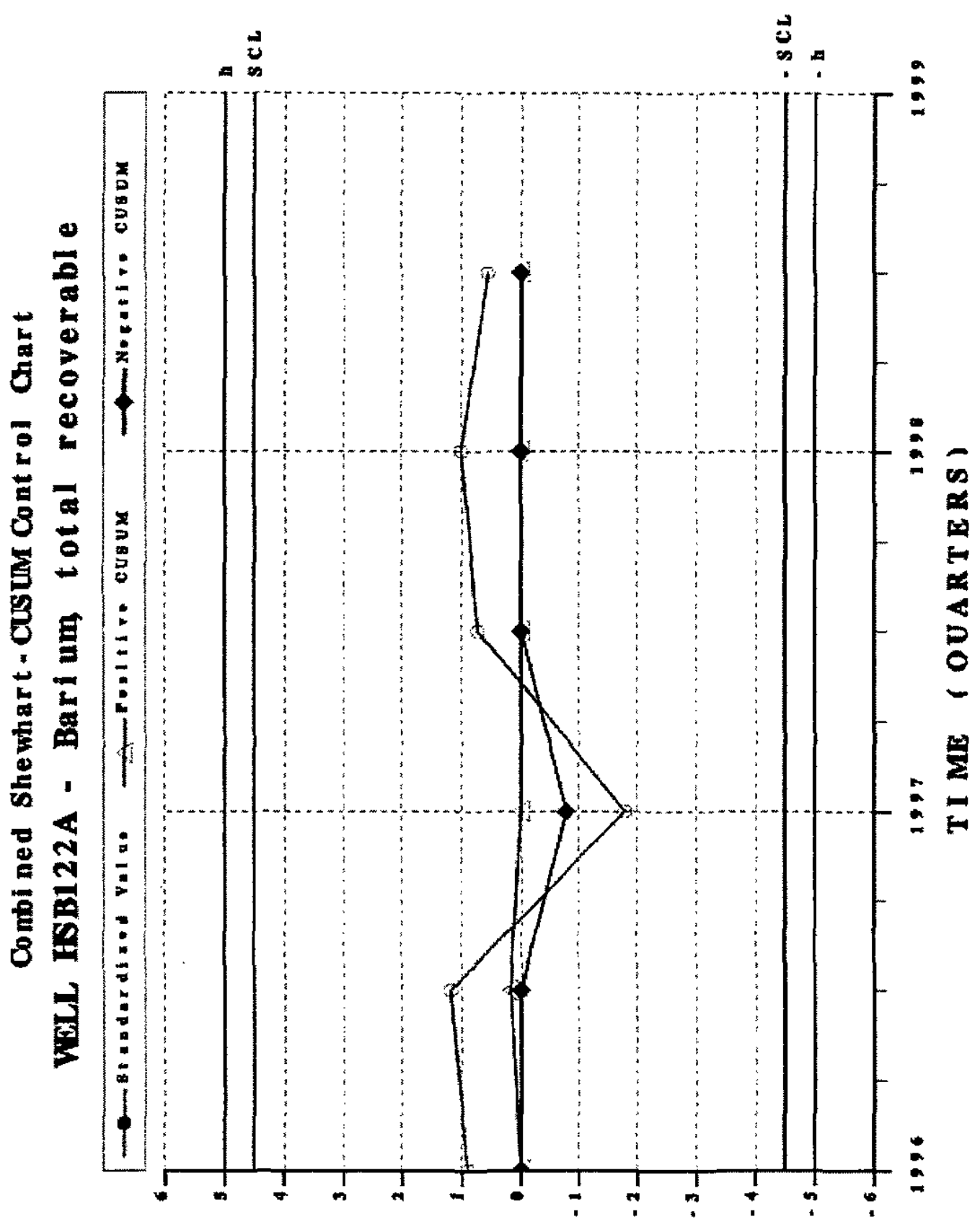

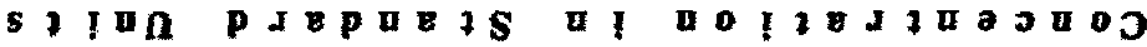


Combl ned Shewhart-Cusum Cont rol Chart

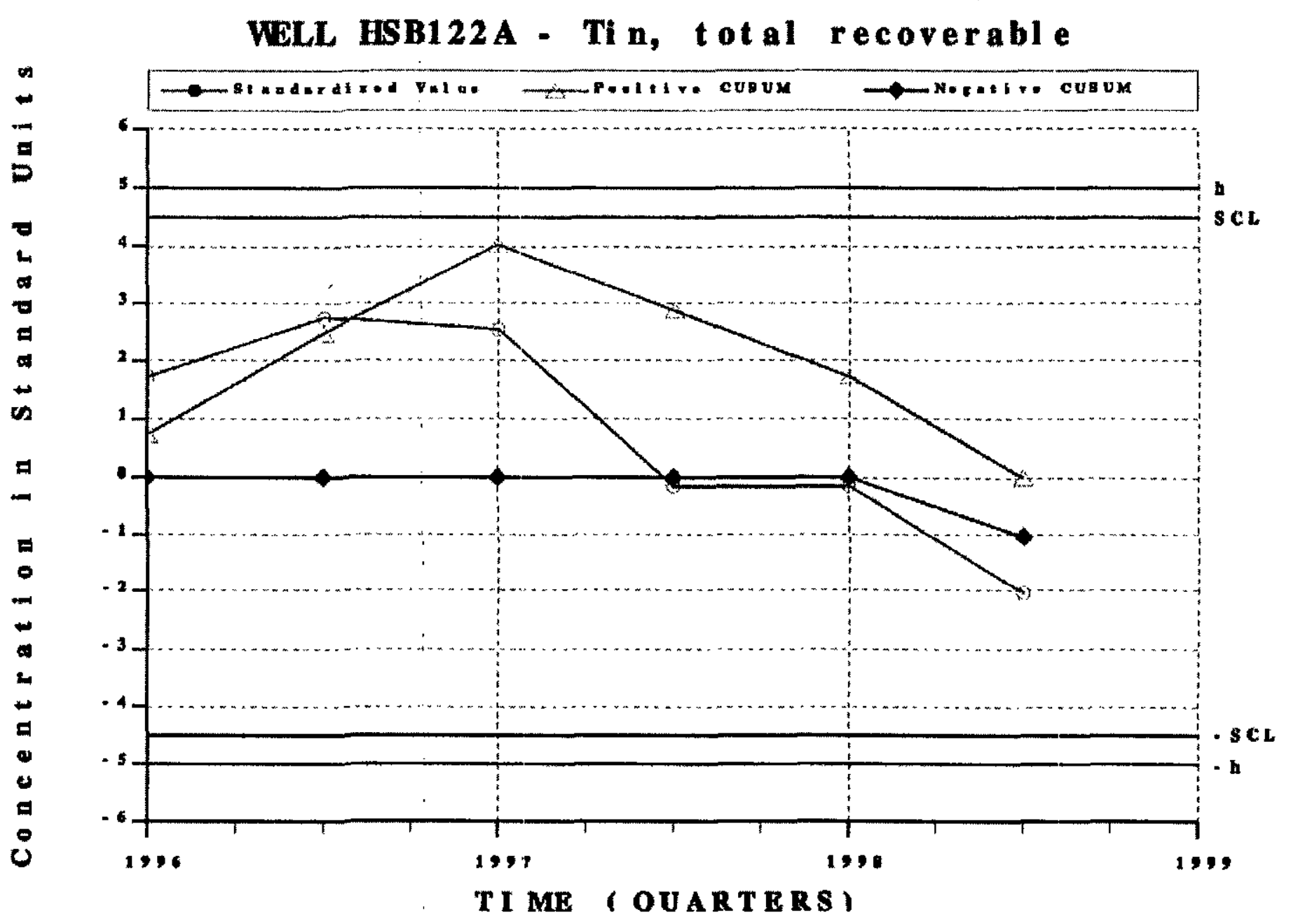

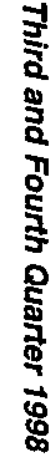

T ME (OUART RRS) 
Combi ned Shewhart - Cusum Cont rol Chart WELL HSB122A - Zi nc, tot al recoverable

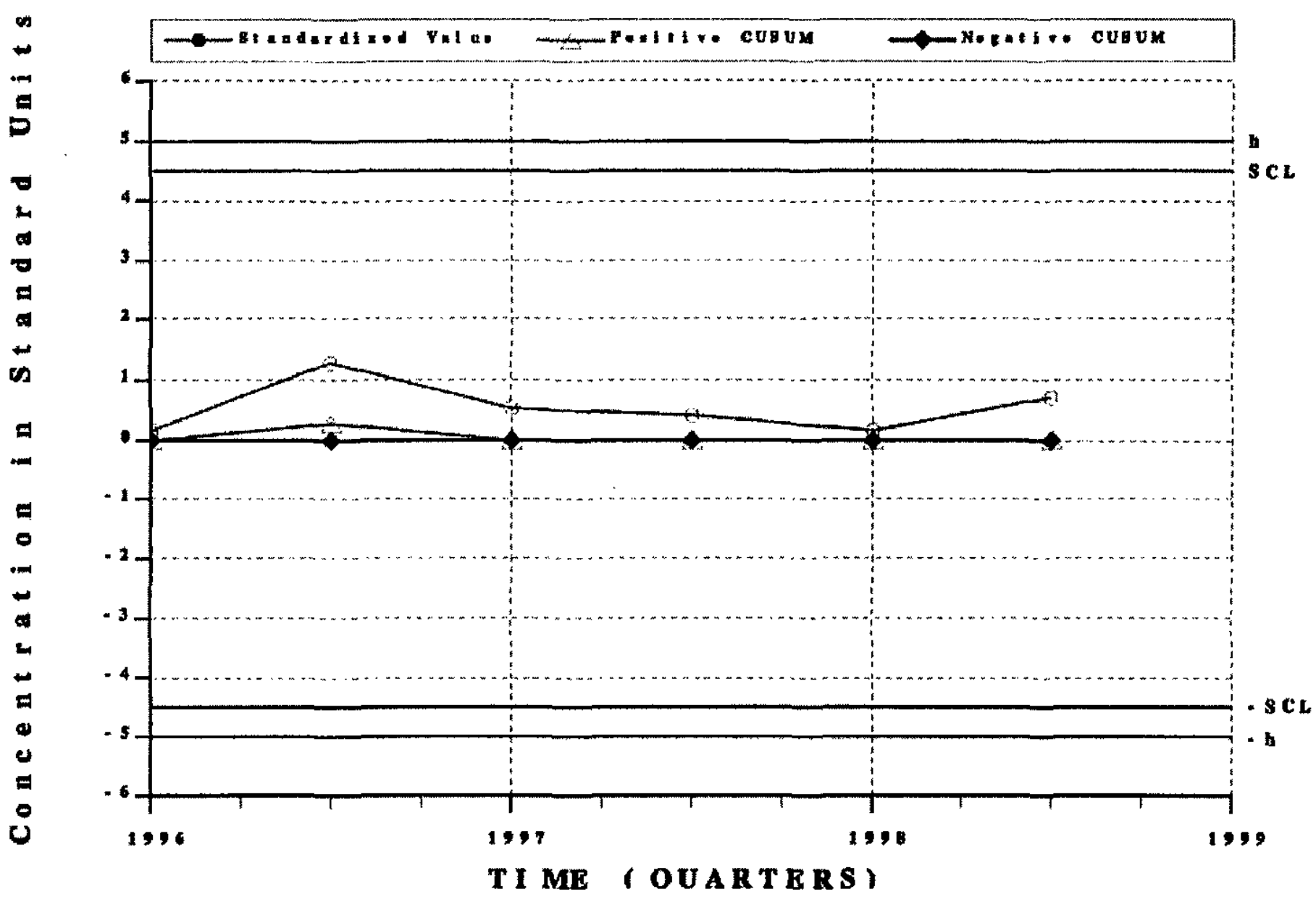




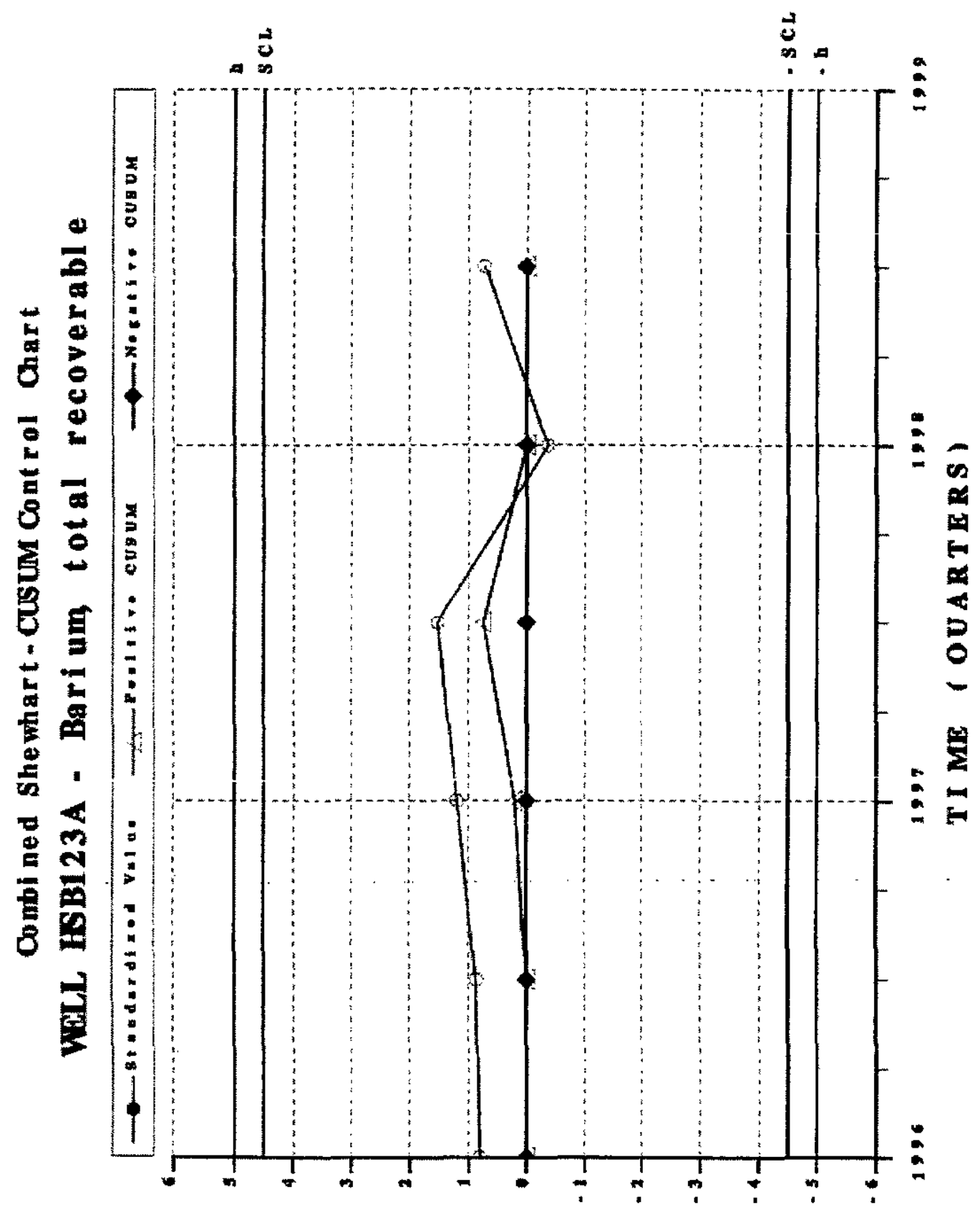

รI I 
Combl ned She whart-Cusum Cont rol Chart WaL HSB125D - N trate-nitrite as nitrogen

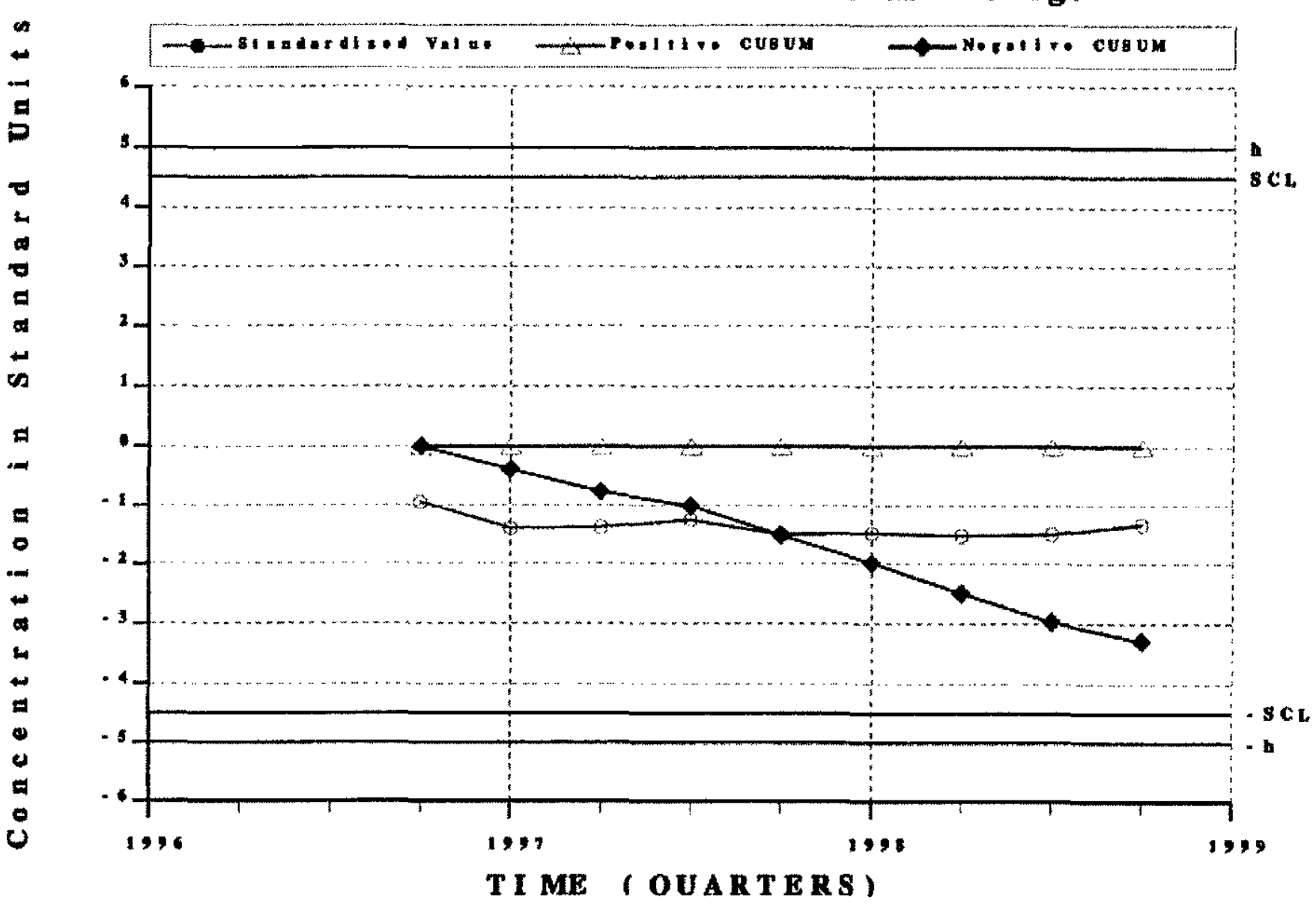




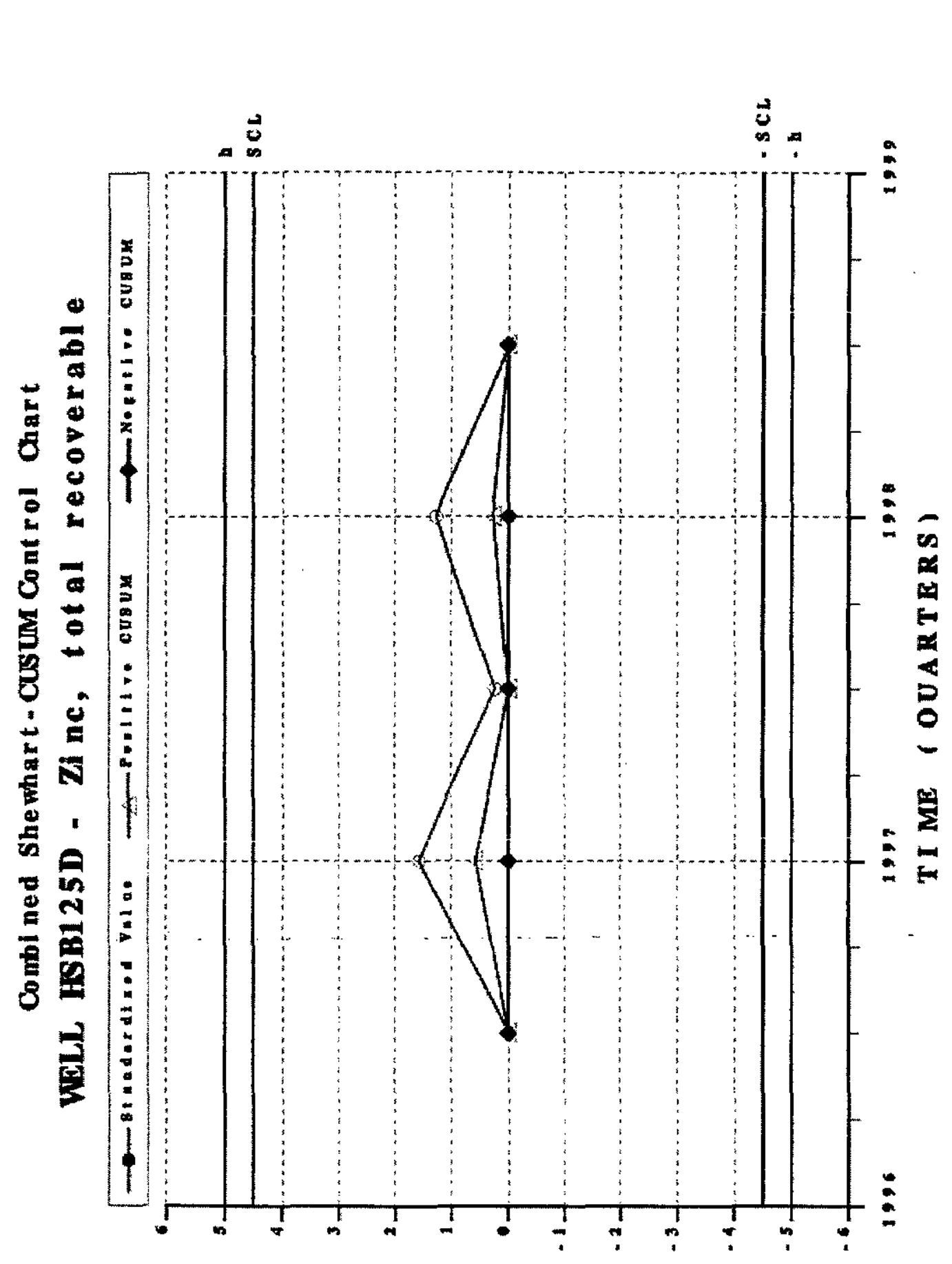

WSRC-TR-99-00013

Unclassified

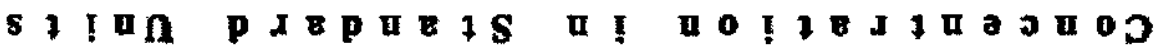




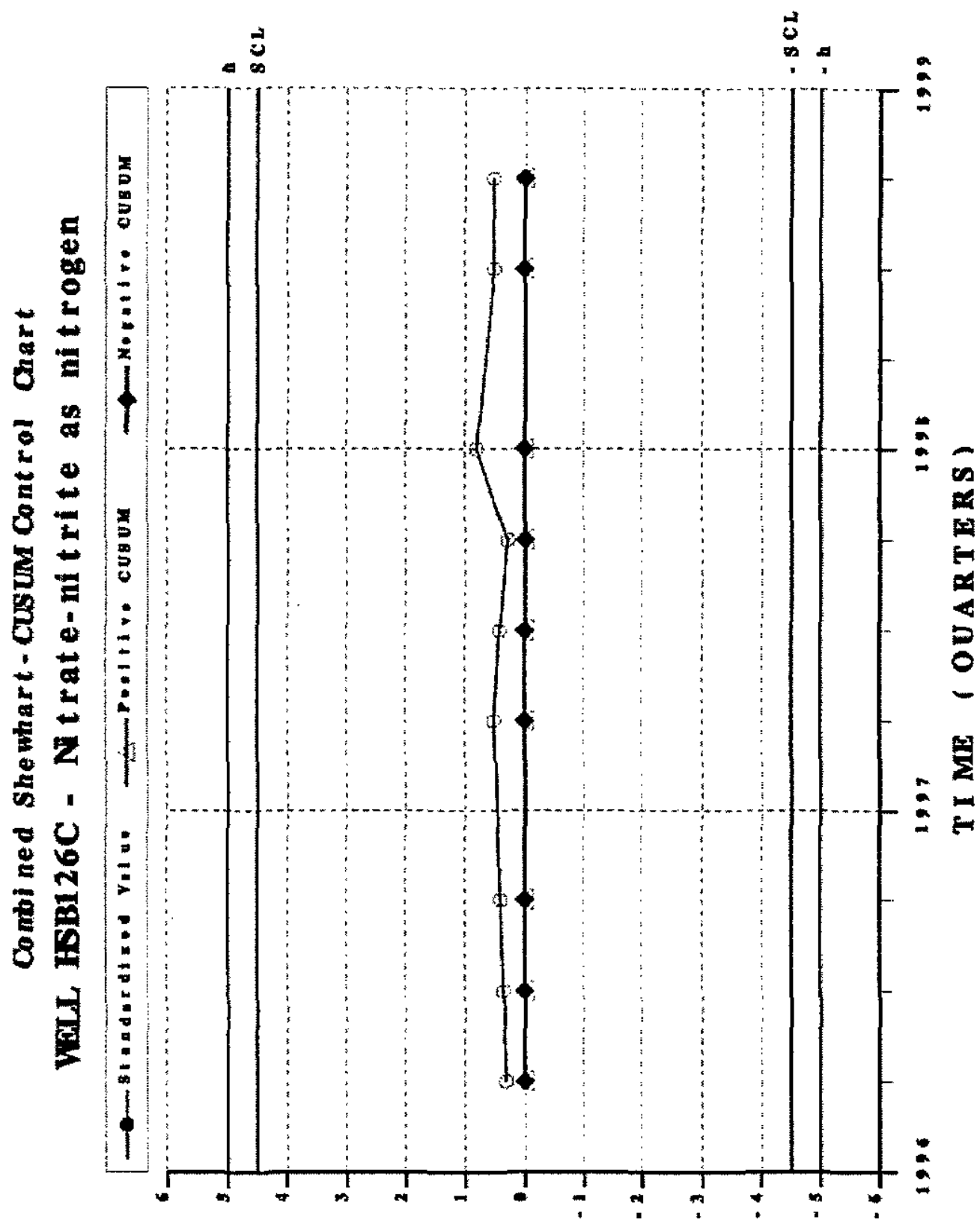

s 1 เ 


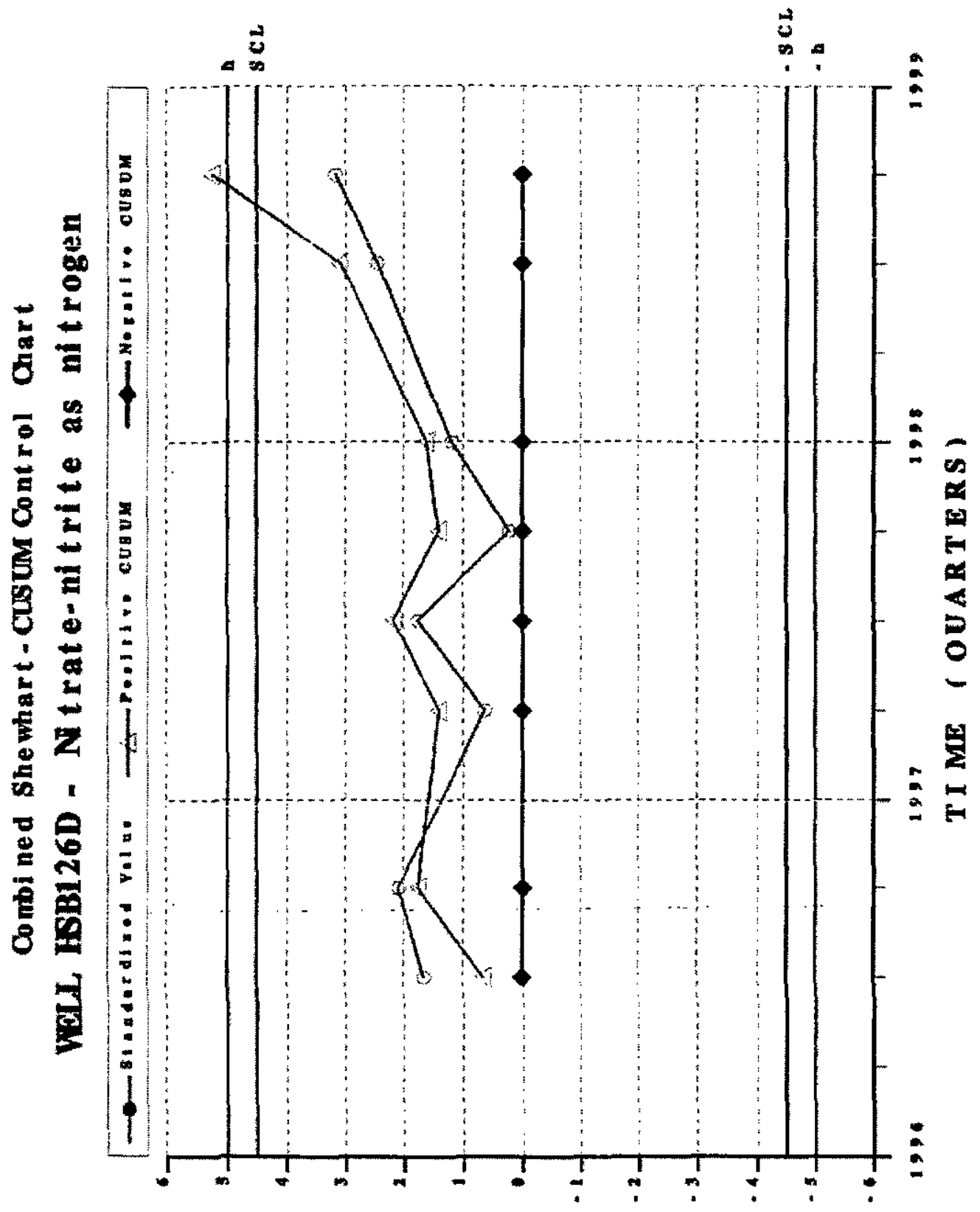

s I I I p I 


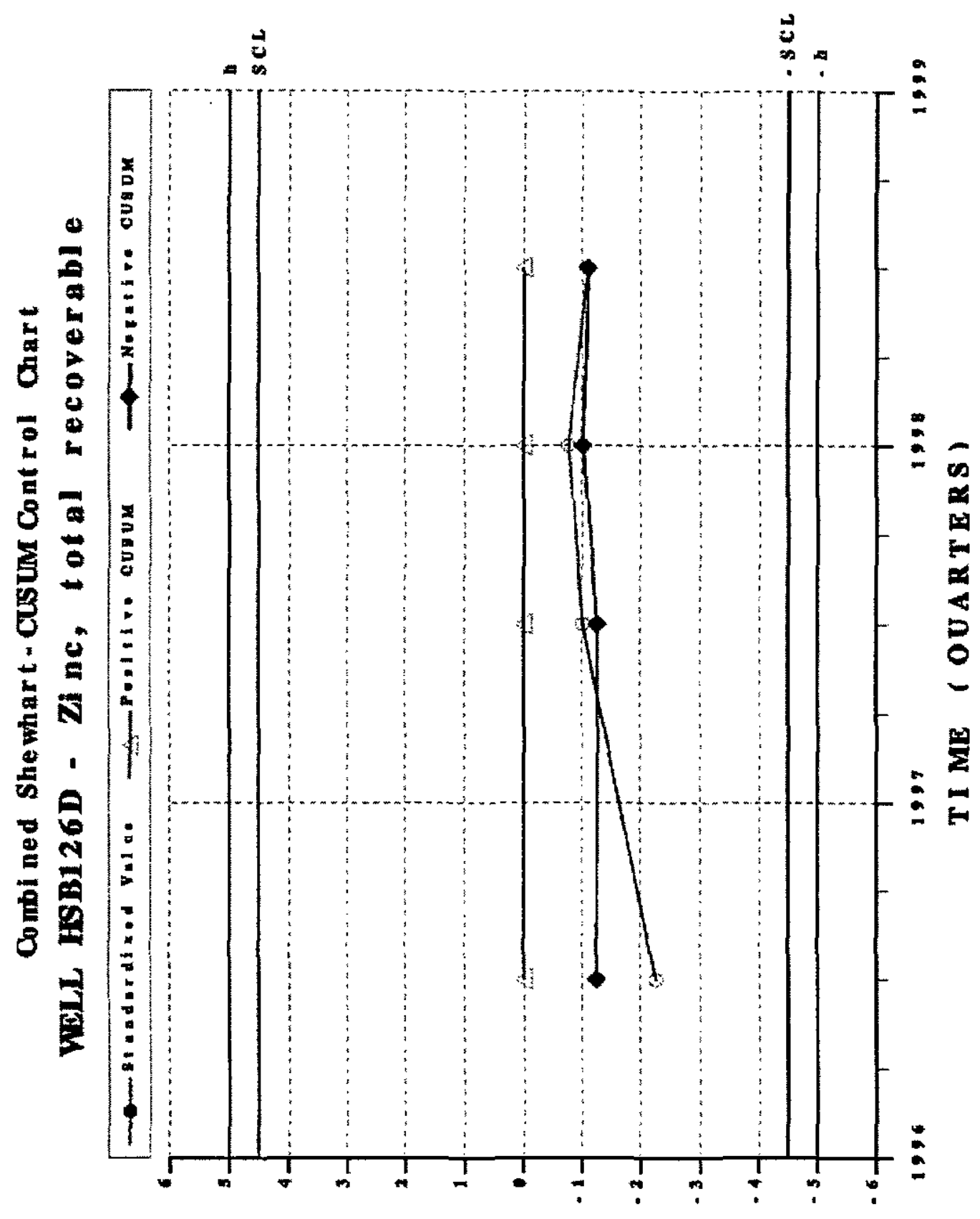

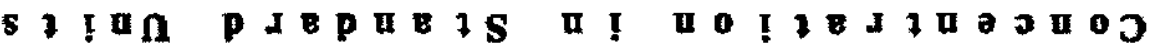




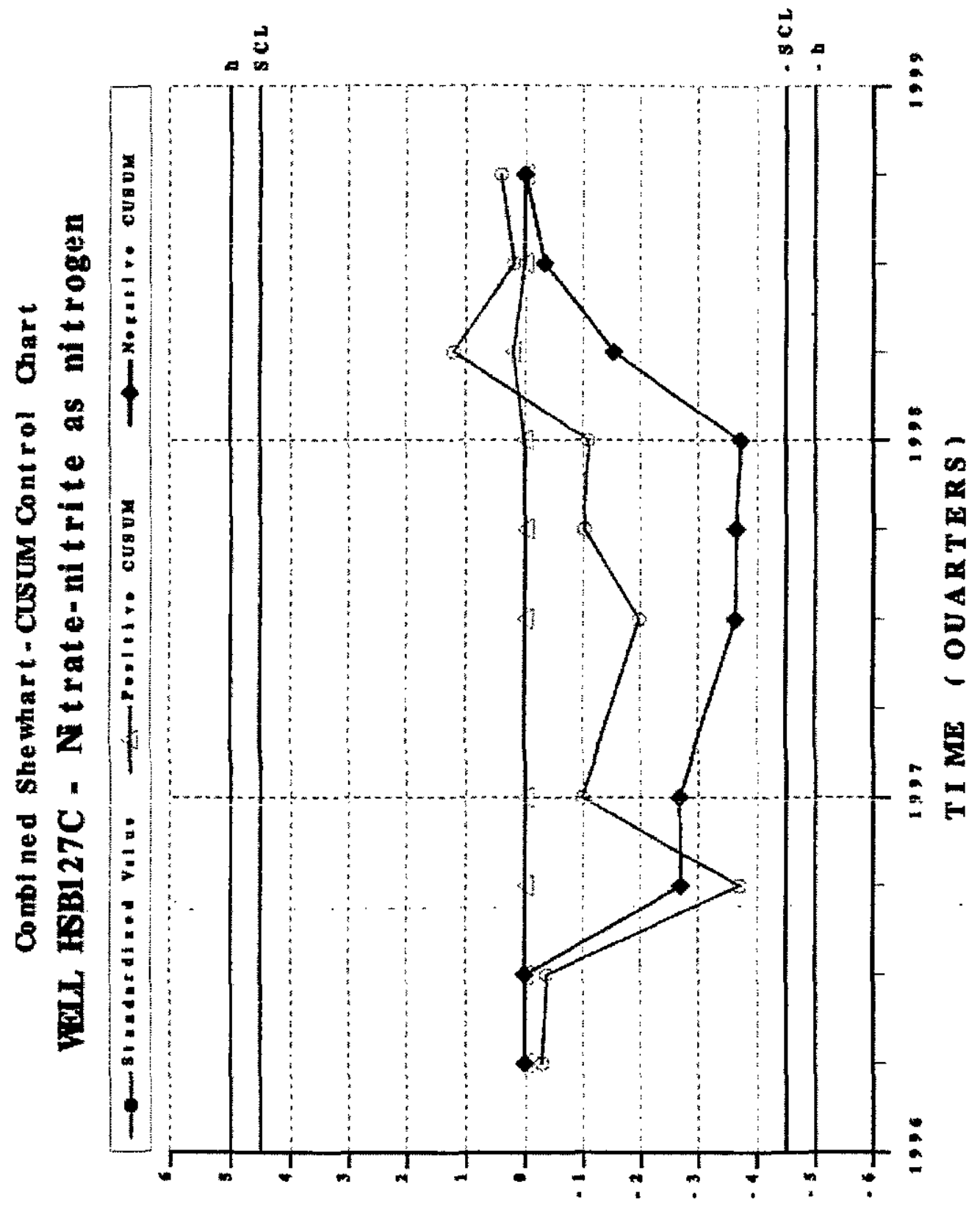

s I 


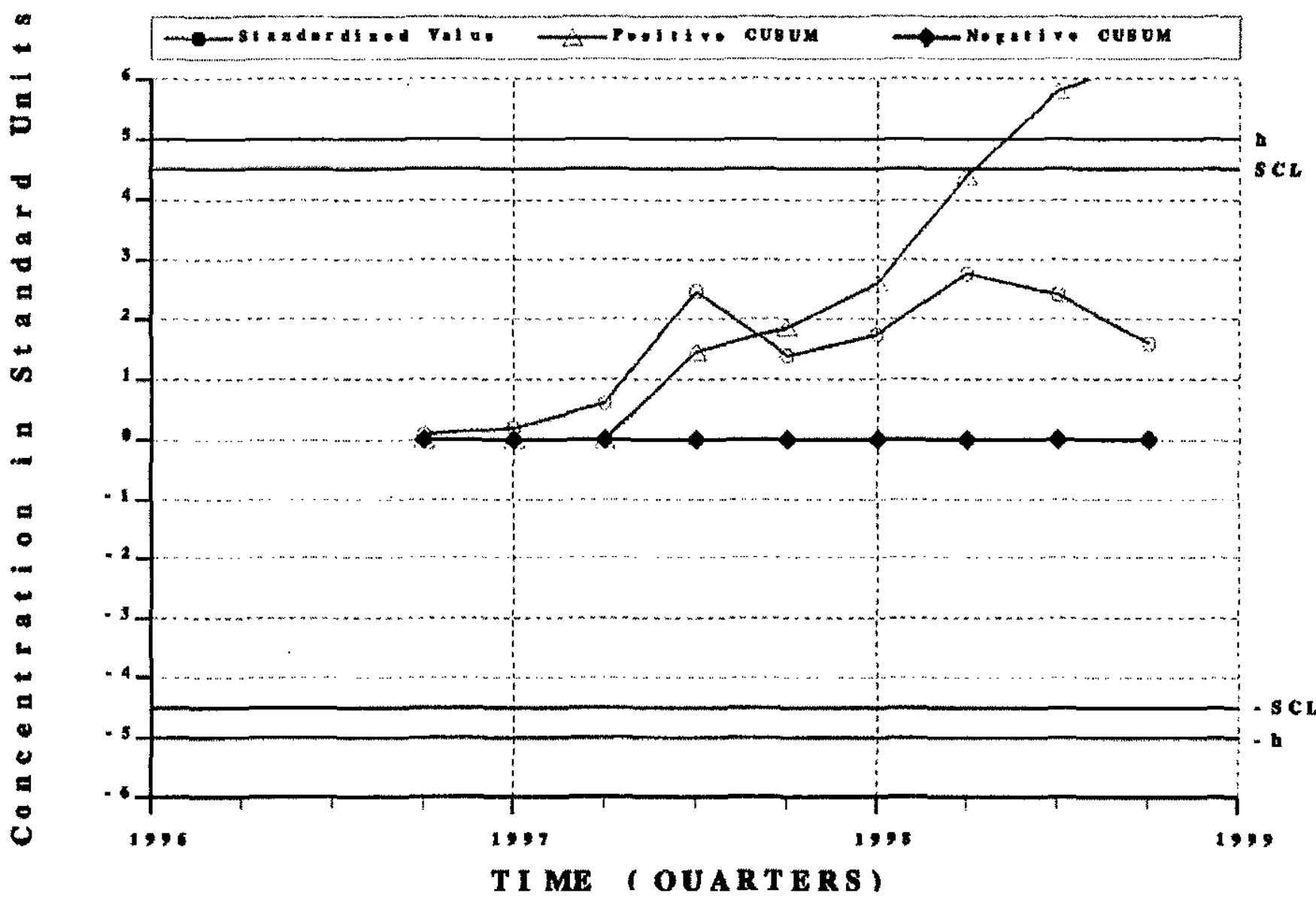




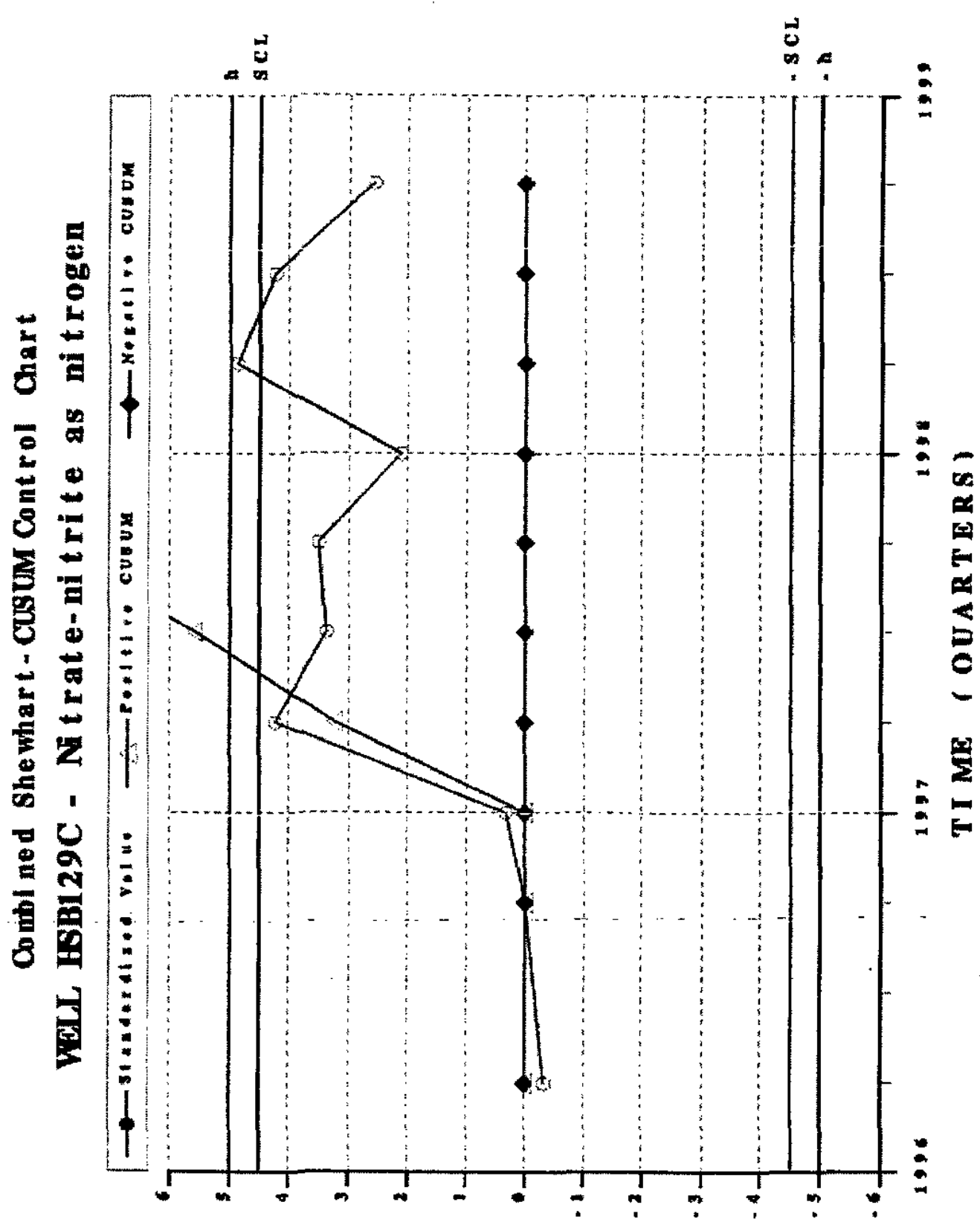

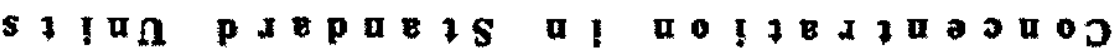




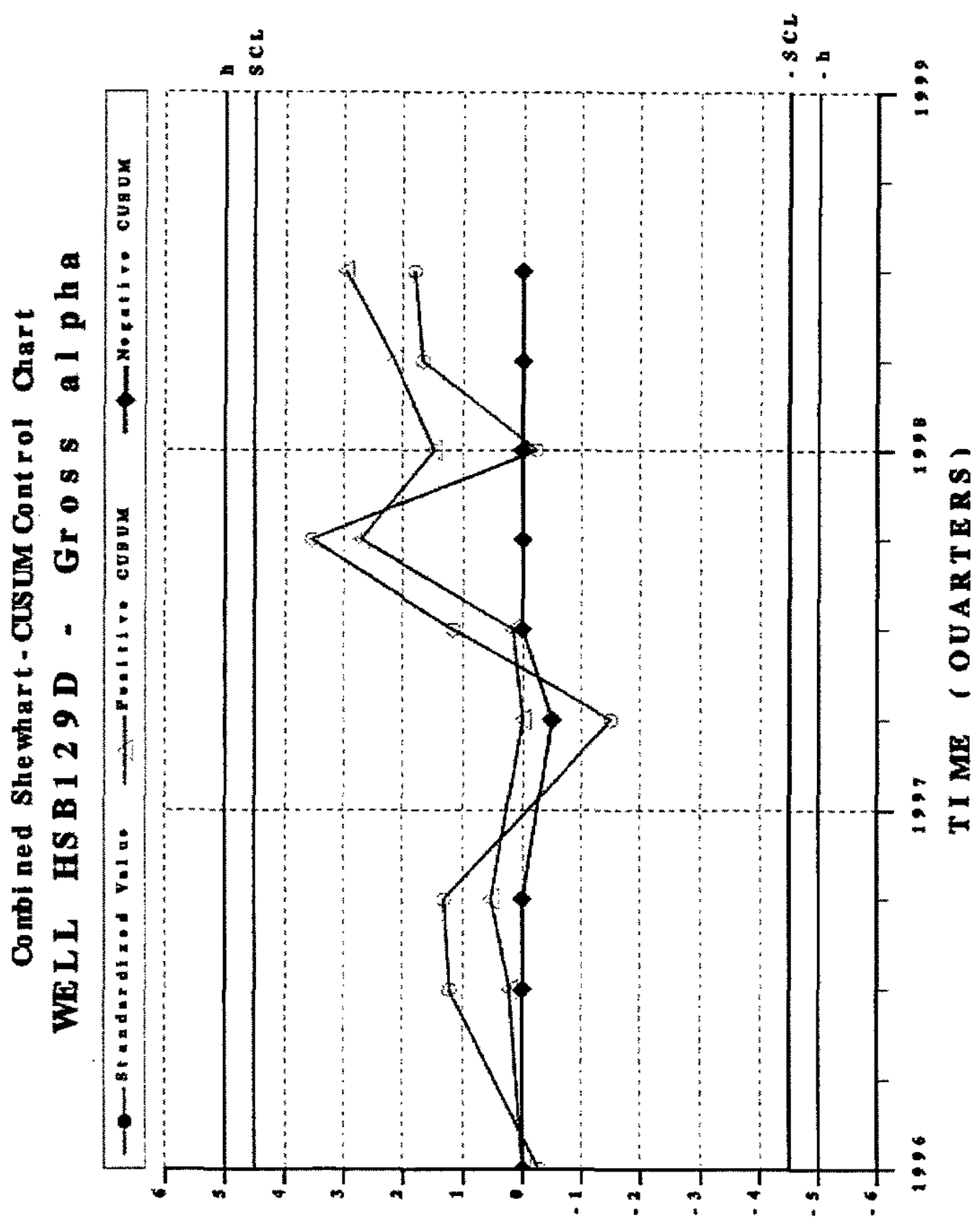

ร) บ แ 
Combl ned She whart-Cusum Cont rol Chart VuL HSB129D " Mrcury, total recoverable

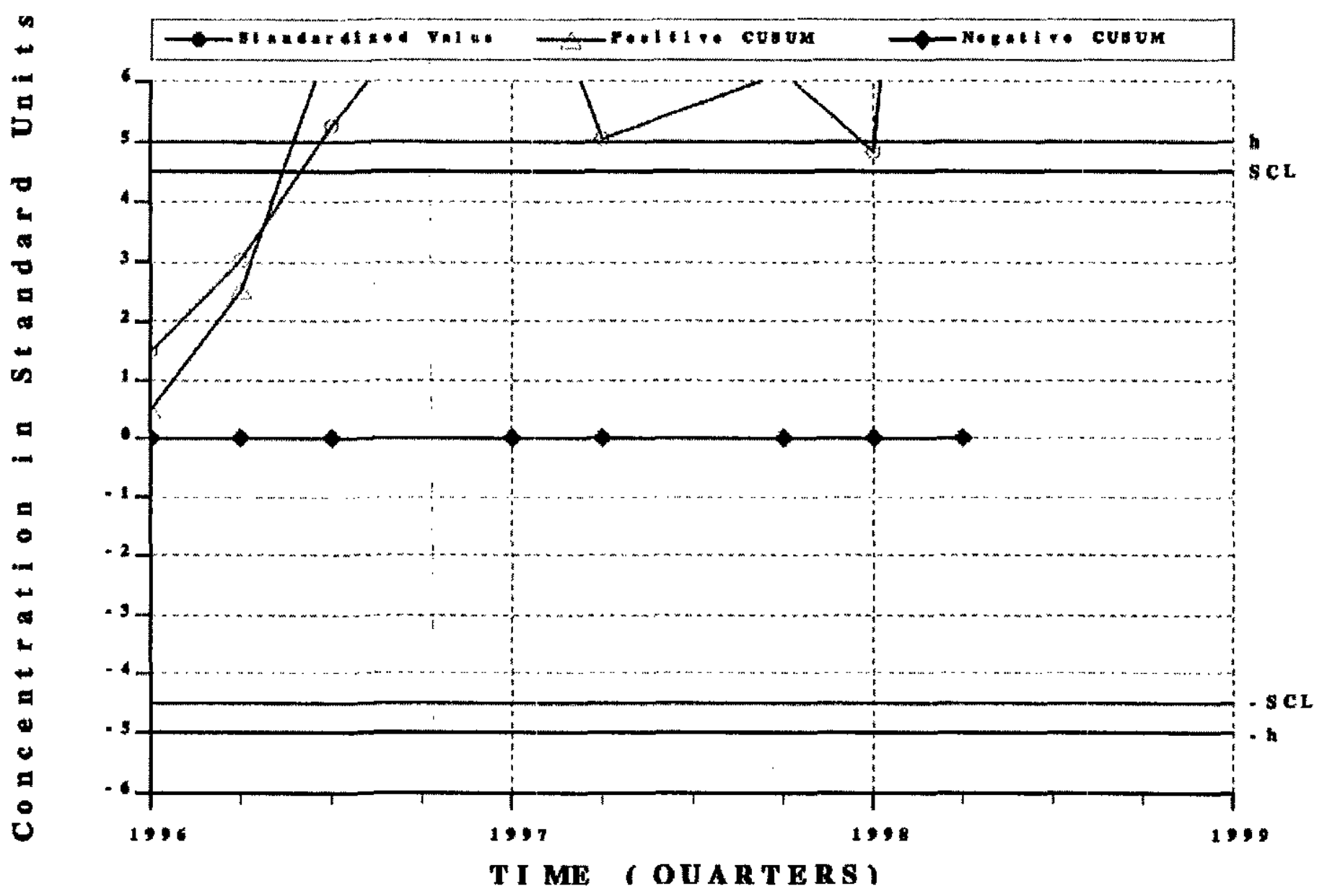




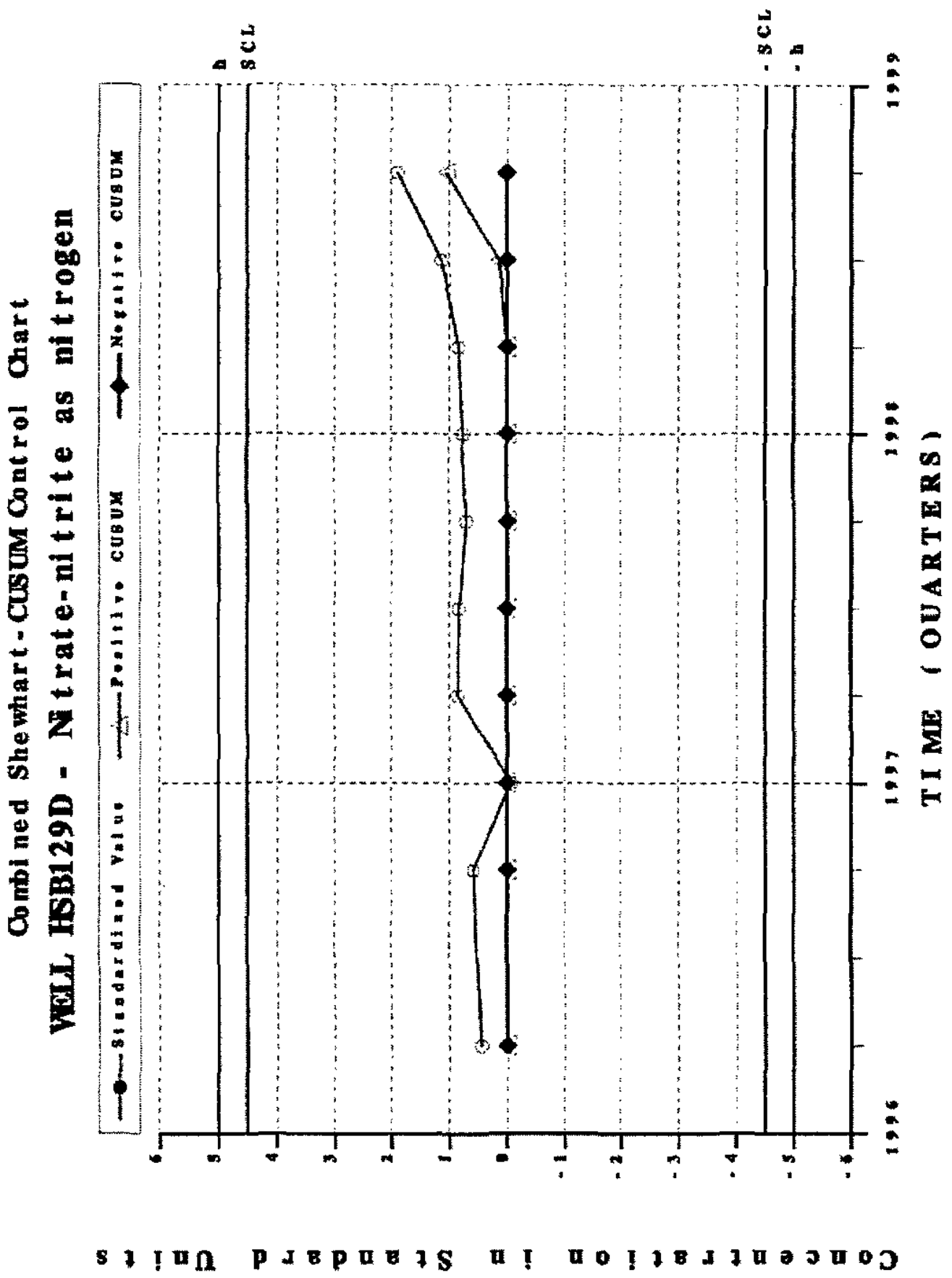




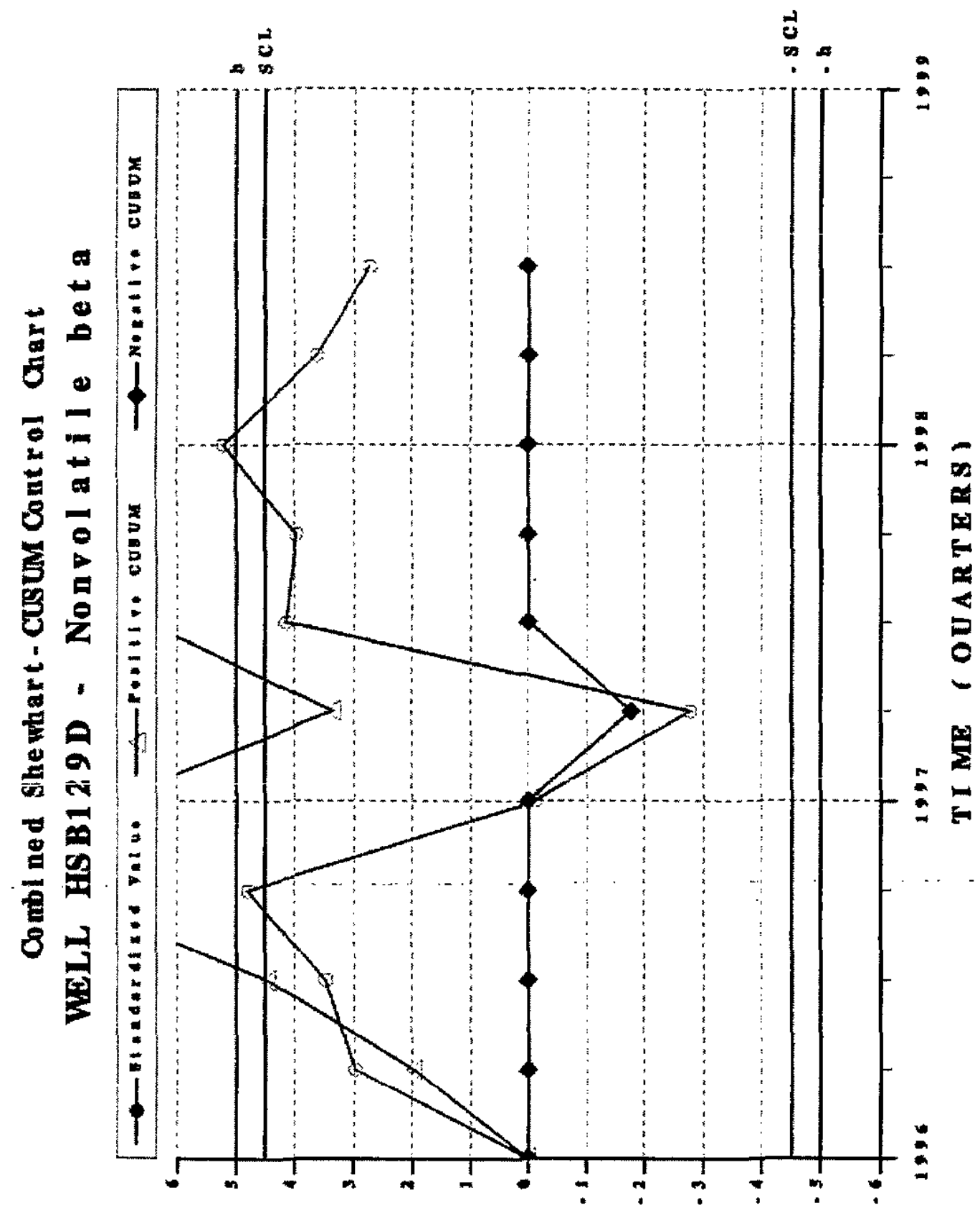

st!n 
Combi ned Shewhart - CUSUM Cont rol Chart

WELL HS B 129 D - Ra di um- 226

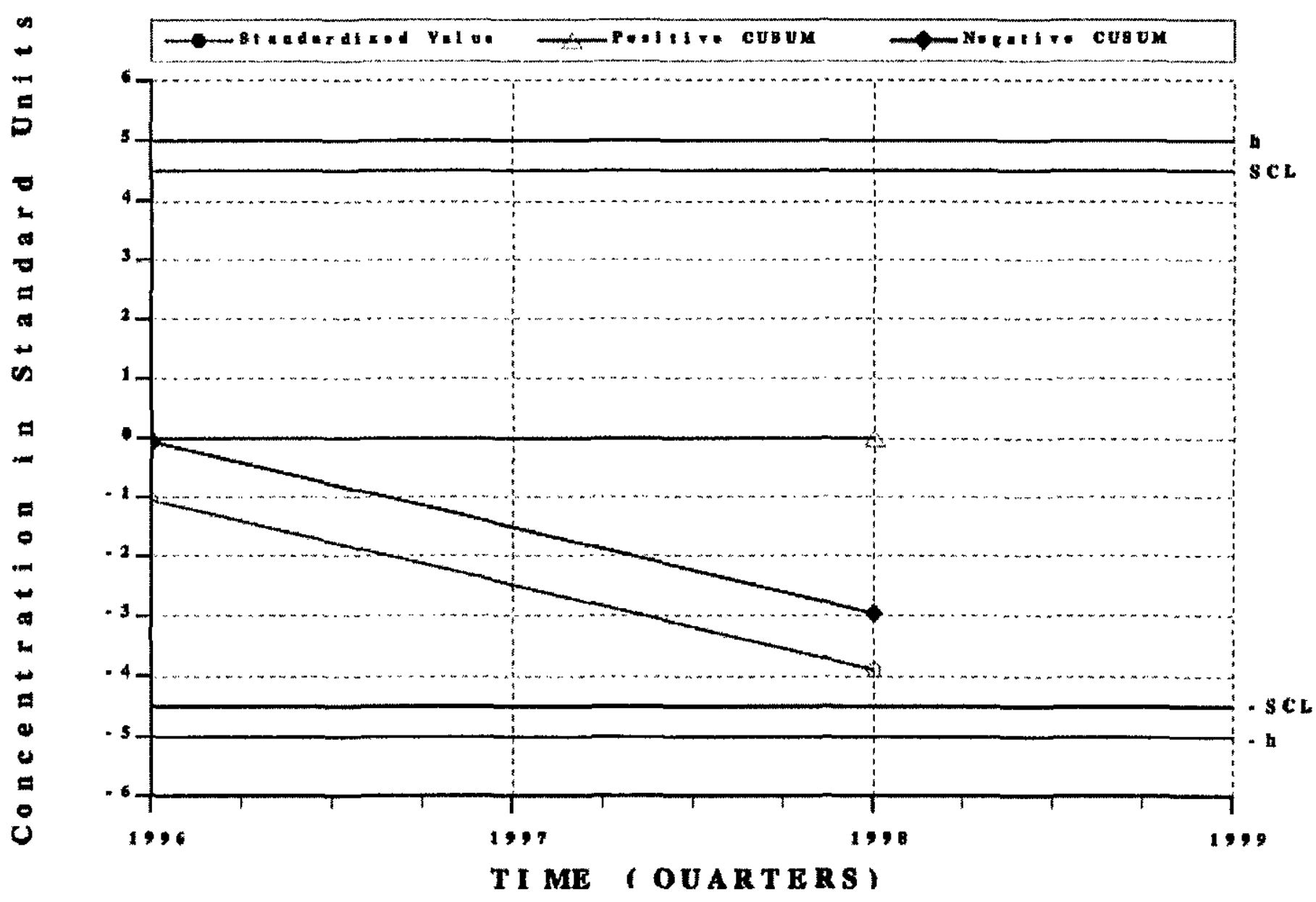




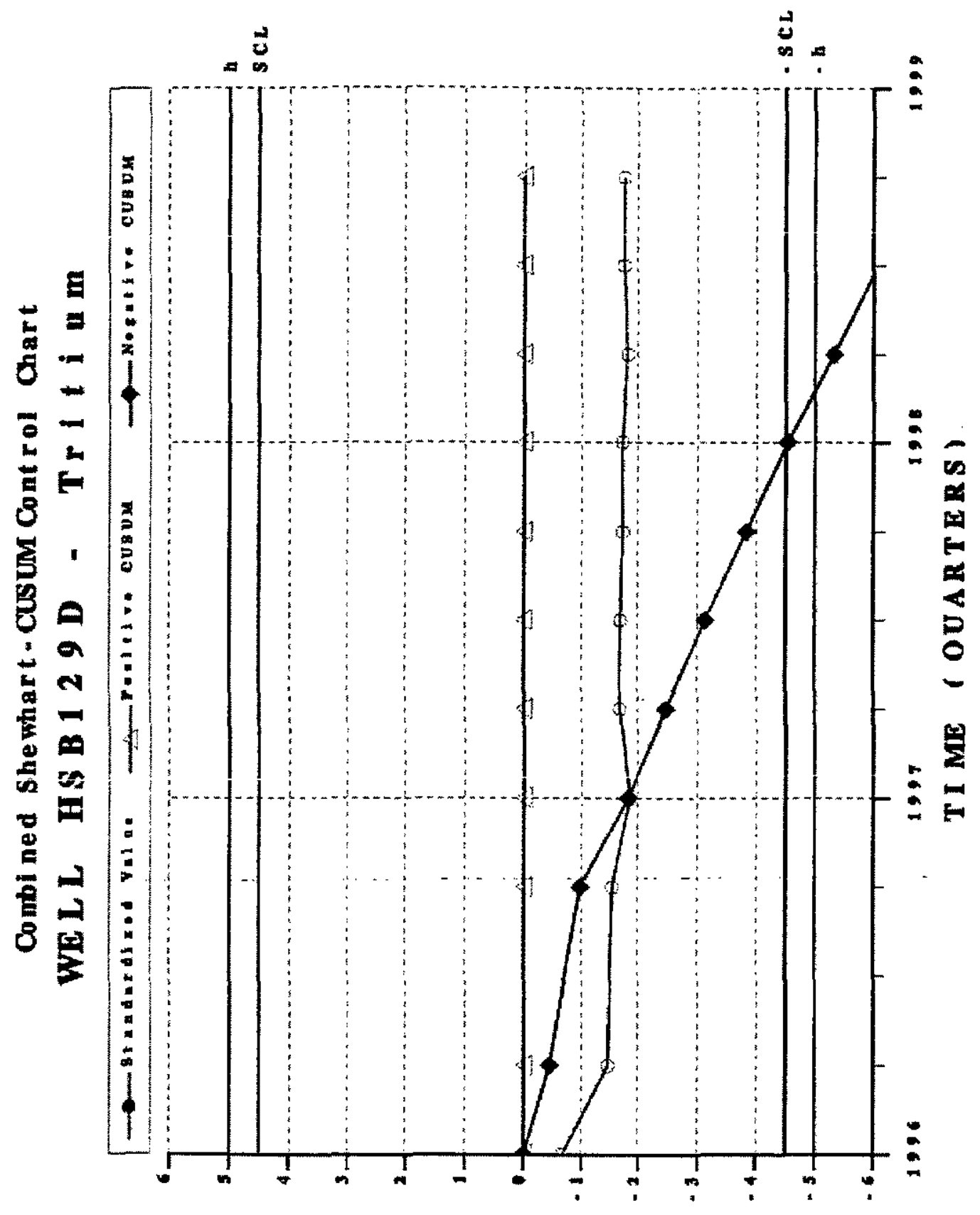

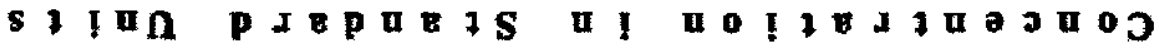


Conbi ned Shewhart-Cusum Cont rol Chart

VaL HSB135C - N trate-nitrite as nitrogen

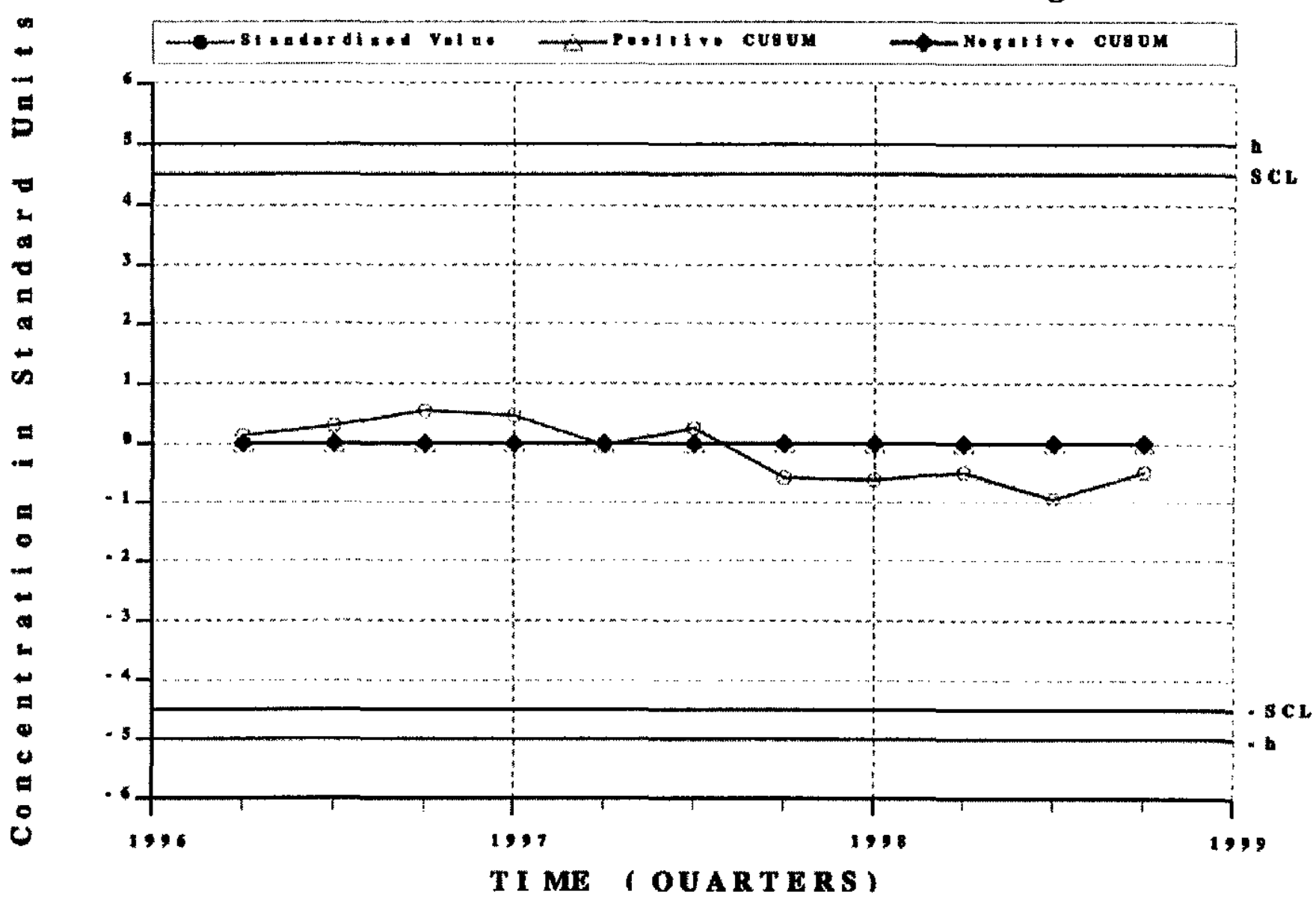




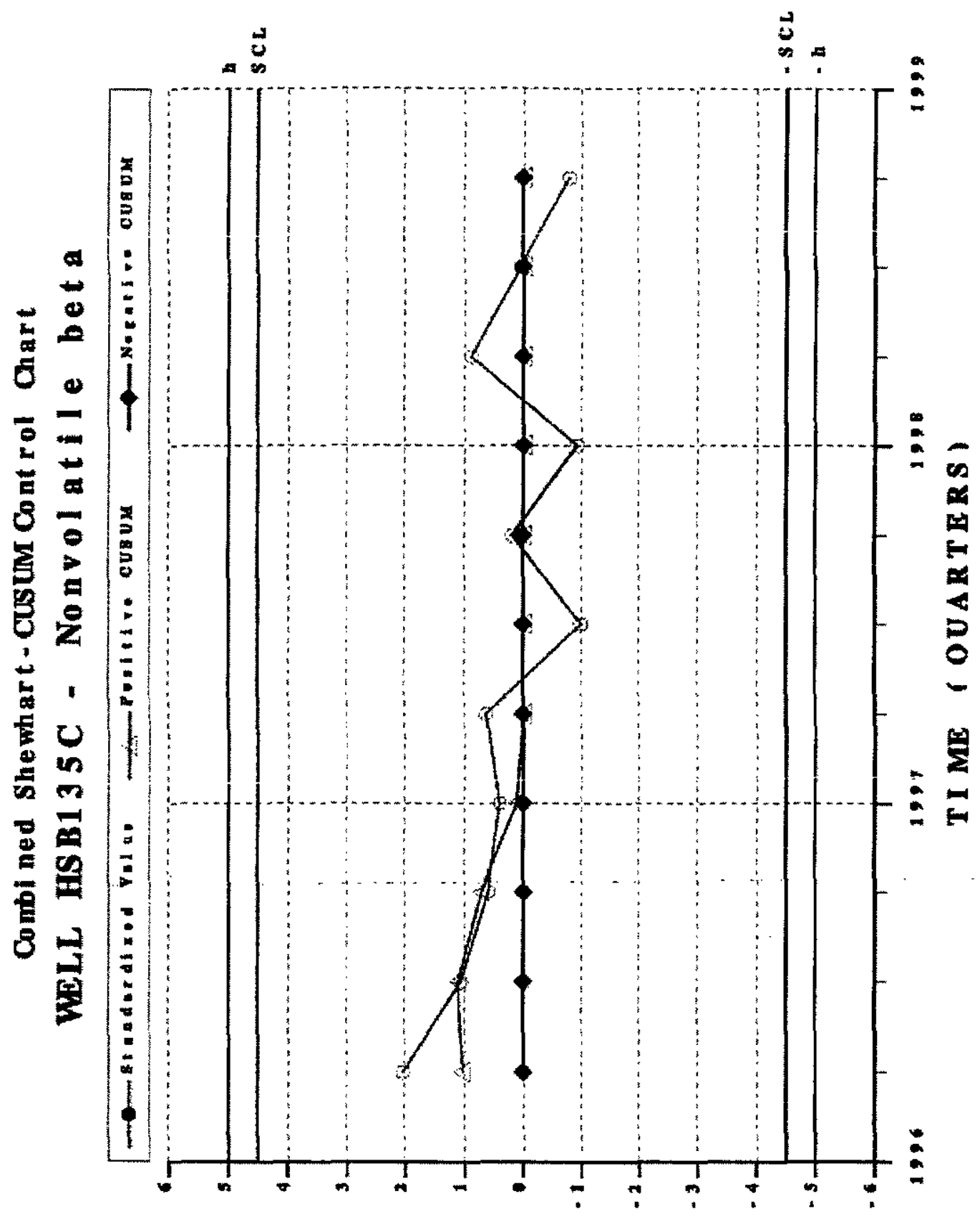

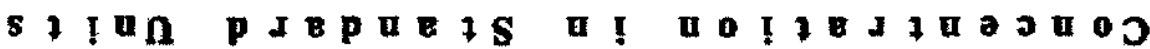




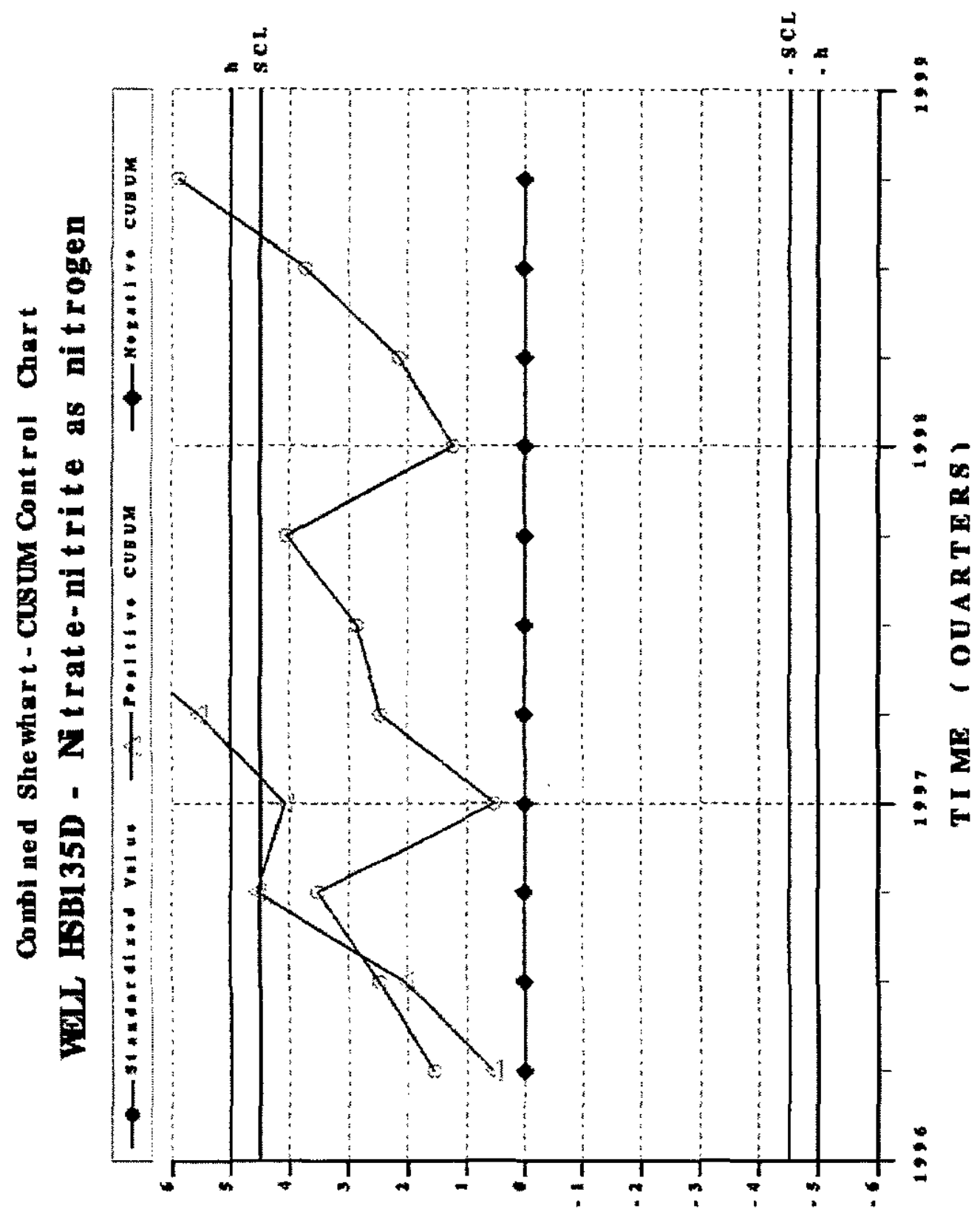

s 1 1 a 


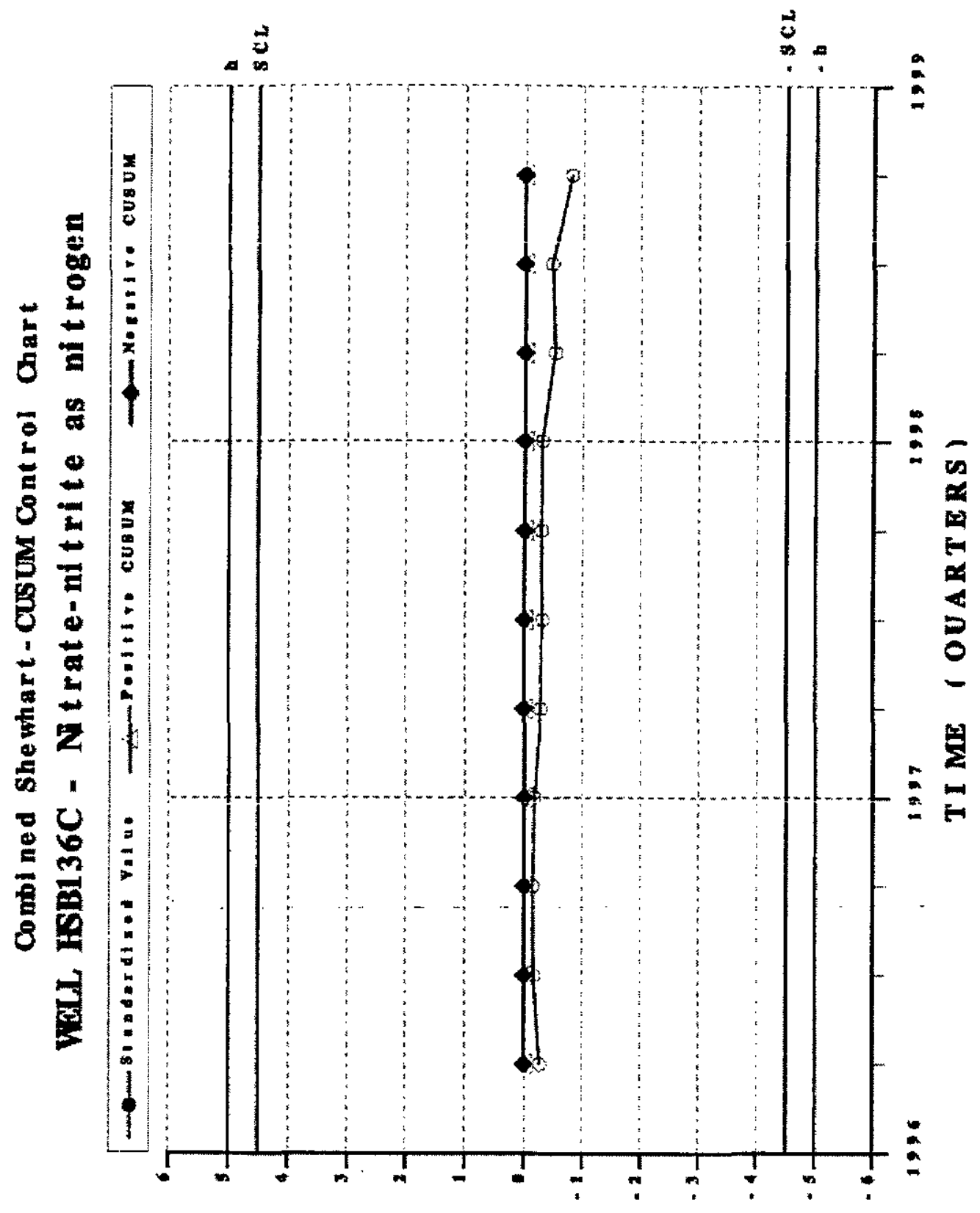

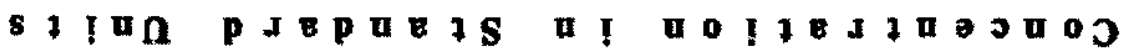



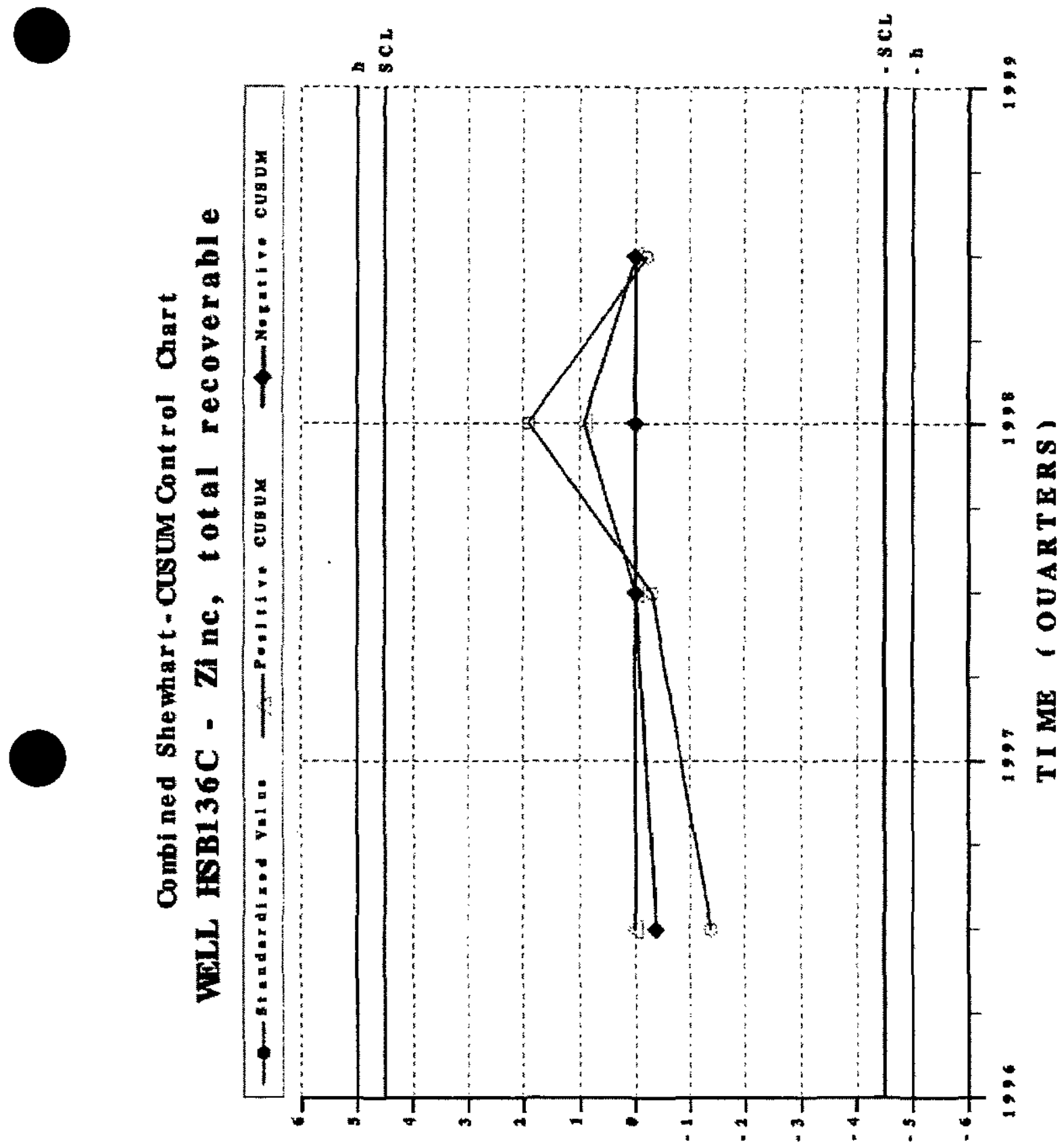

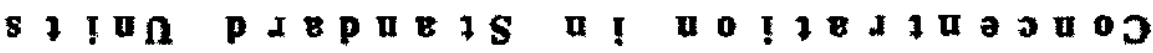


Combi ned Shewhart-Cusum Cont rol Chart Wal HSB136D - Ntrate-nitrite as nitrogen

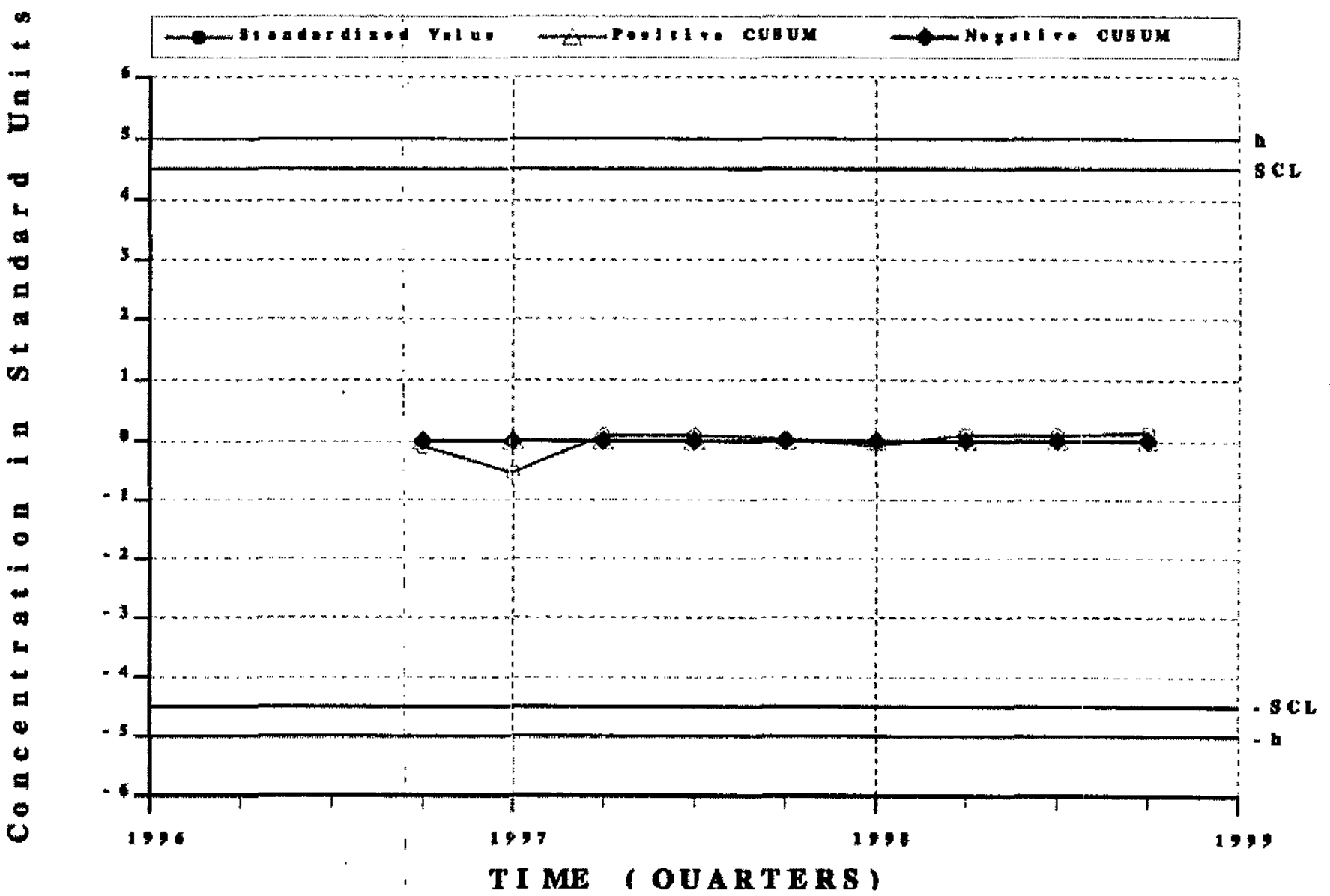

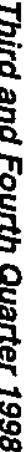


Combi ned She whart - Cusum Cont rol Chart WeLL HSB136D - Zinc, total recoverable

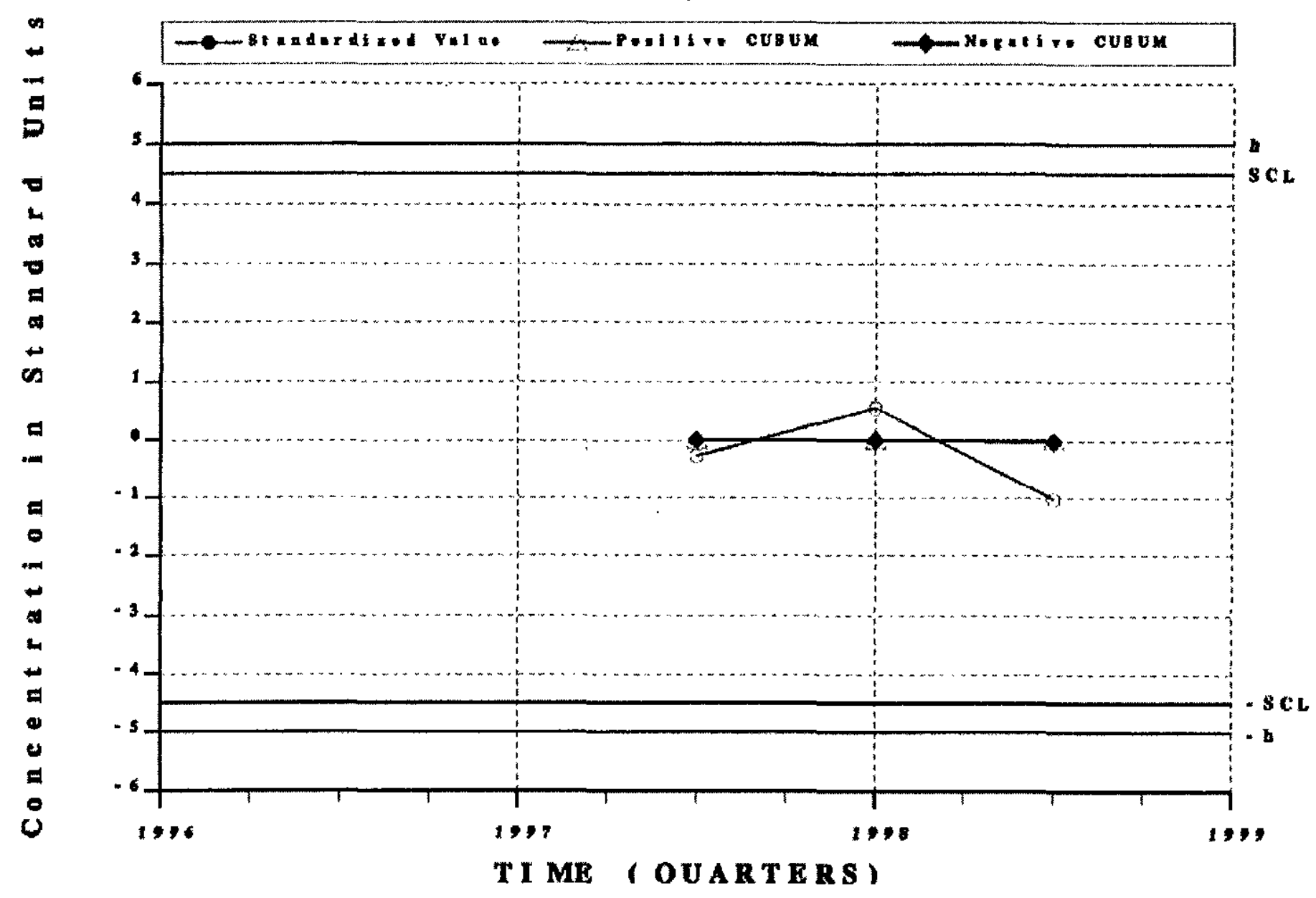


Combined Shewhart-Cusum Cont rol Chart

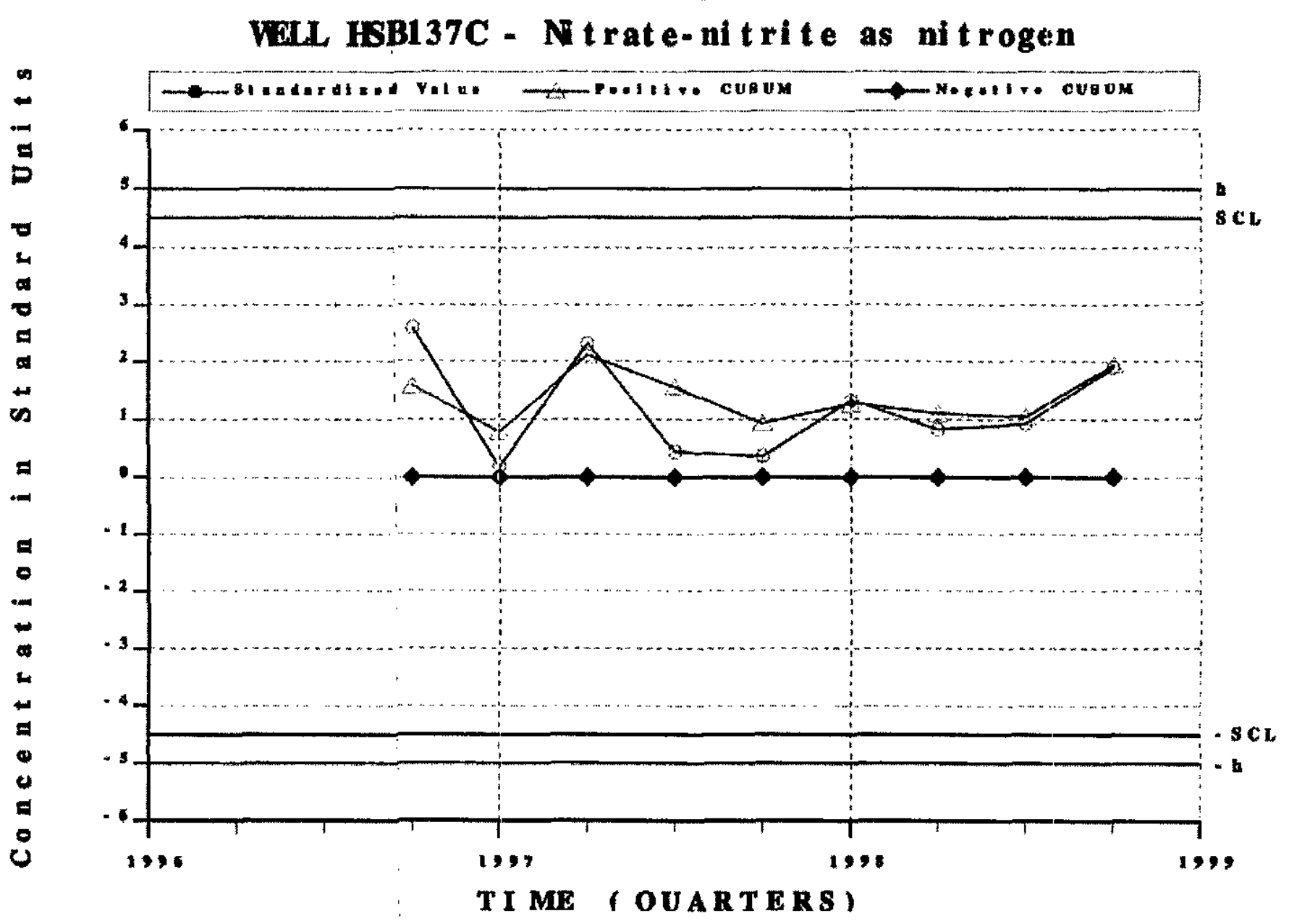

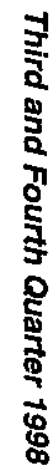



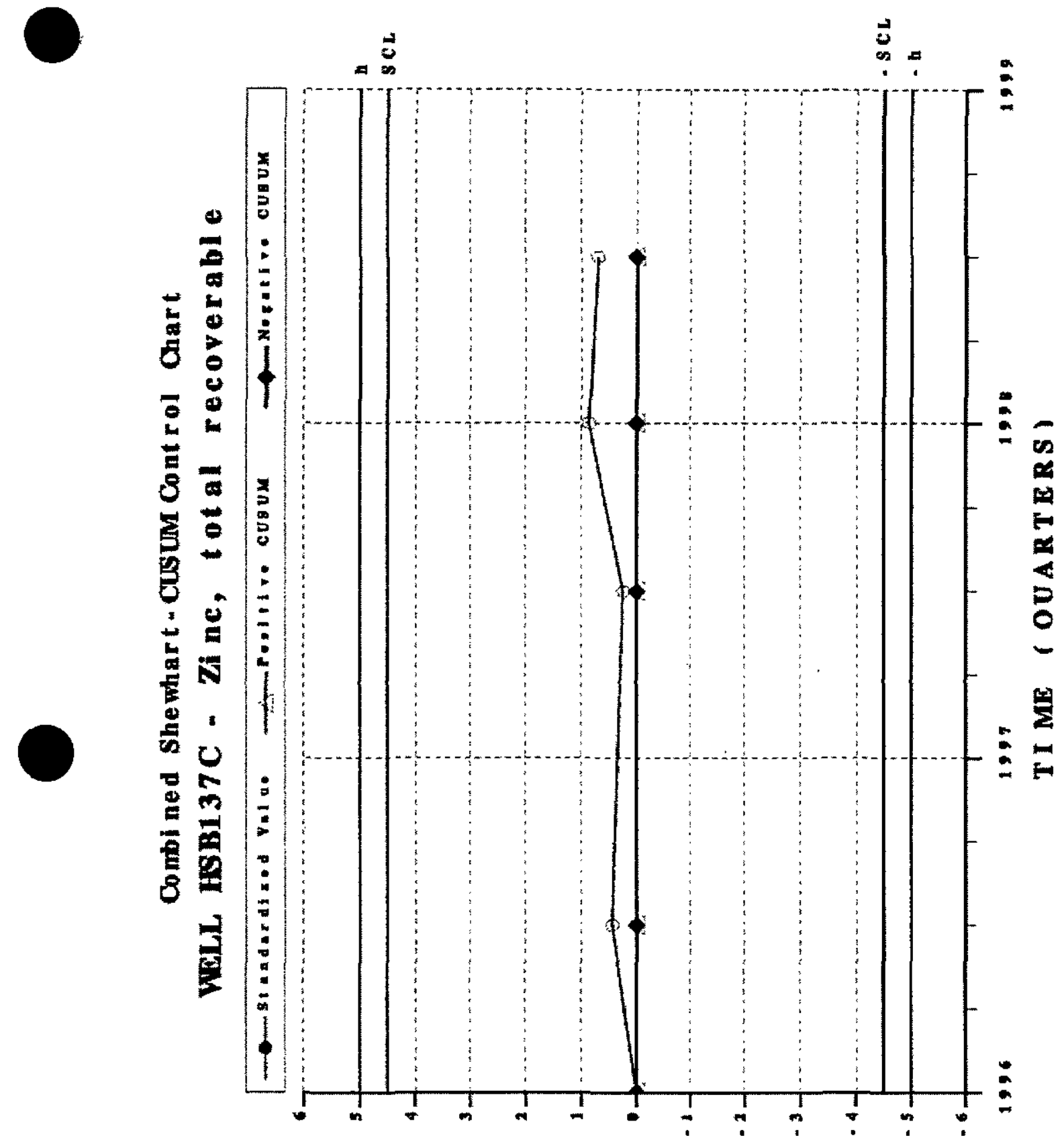

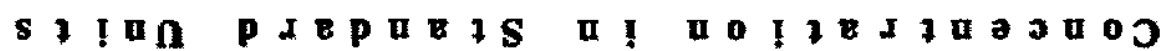


Combi ned Shewhart-Cusum Cont rol Chart WuL HSB137D - N trate-nitrite as nitrogen

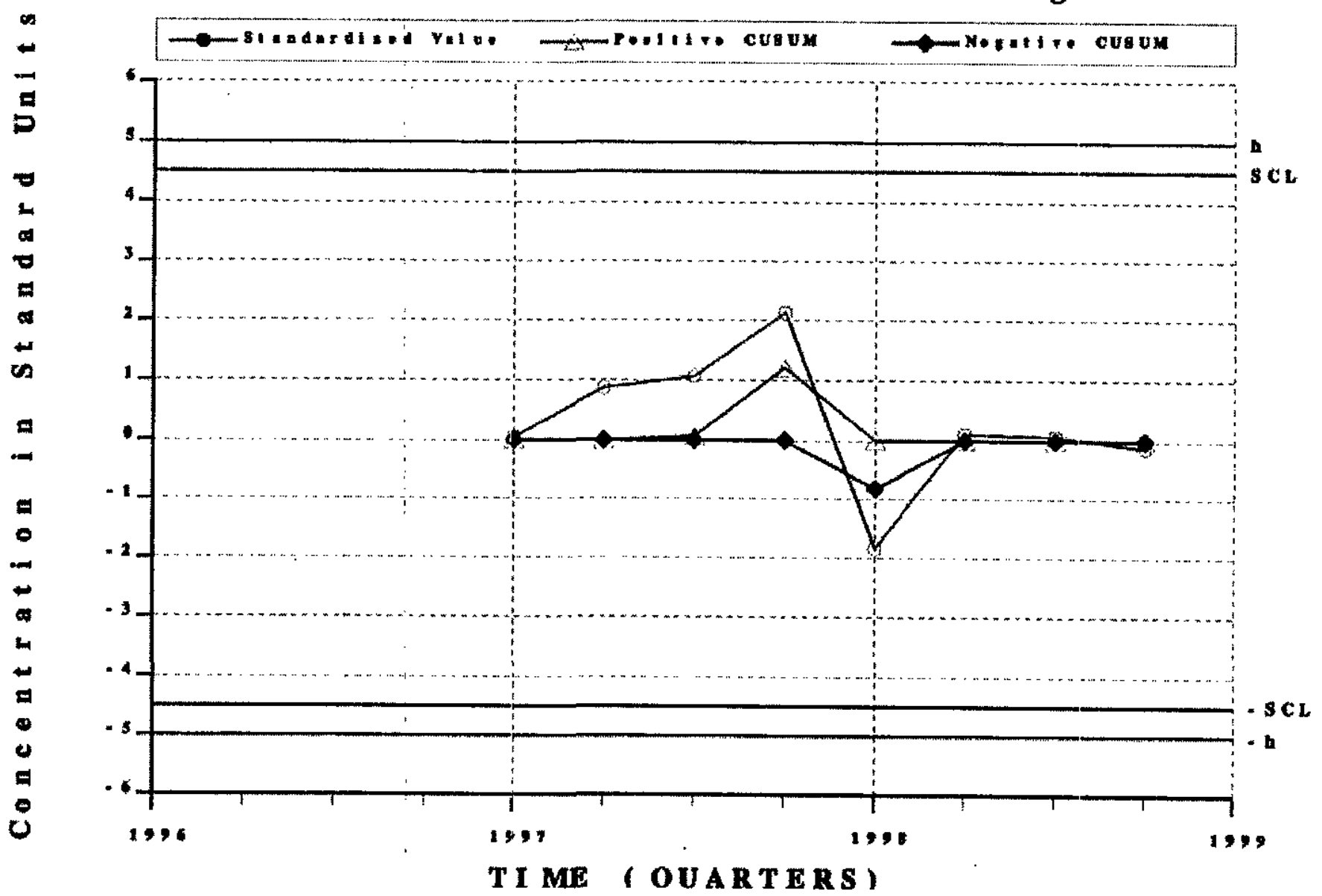


Combl ned She whart-Cusum Cont rol Chart

val HSB139C - Nitrate-nitrite as nitrogen

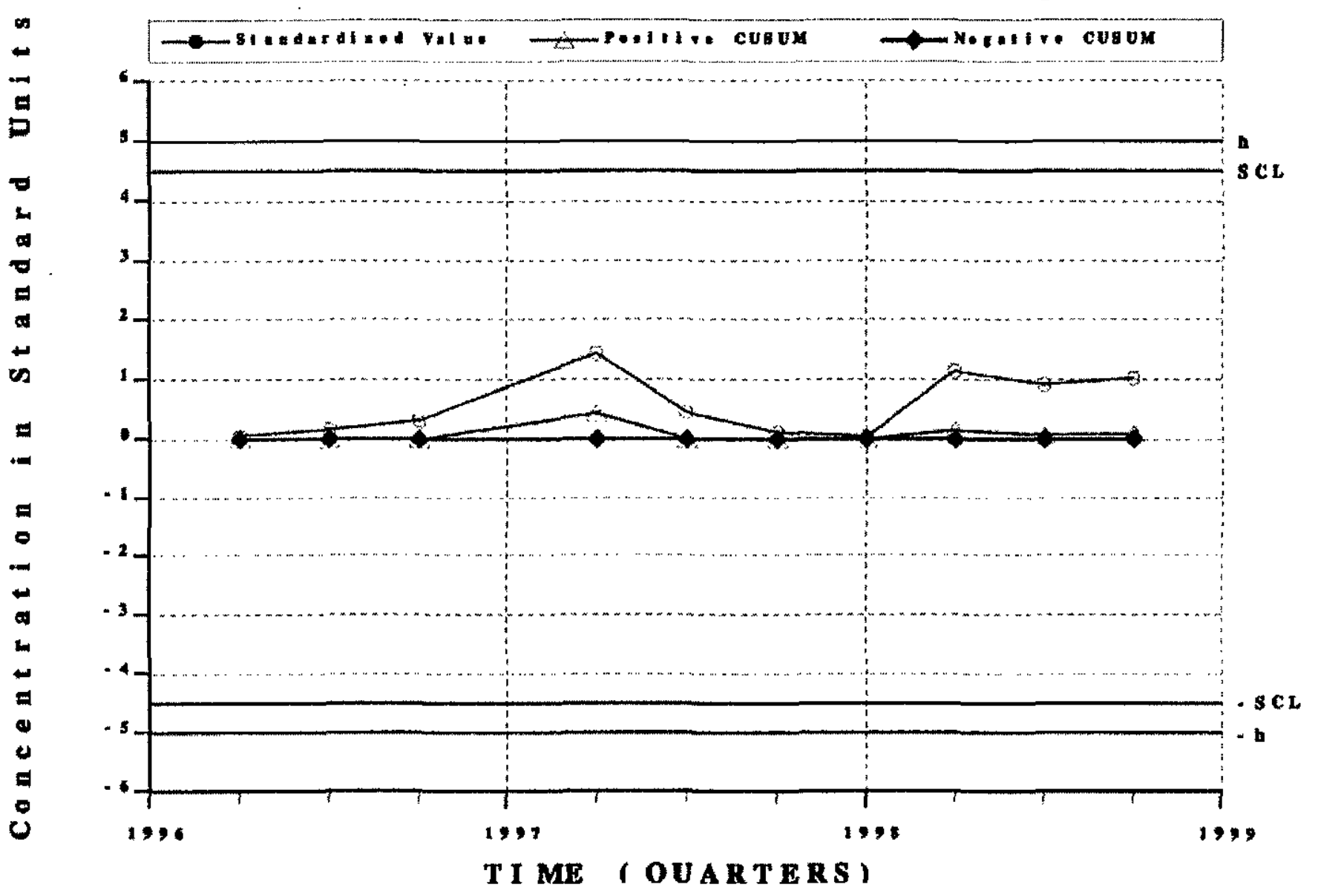




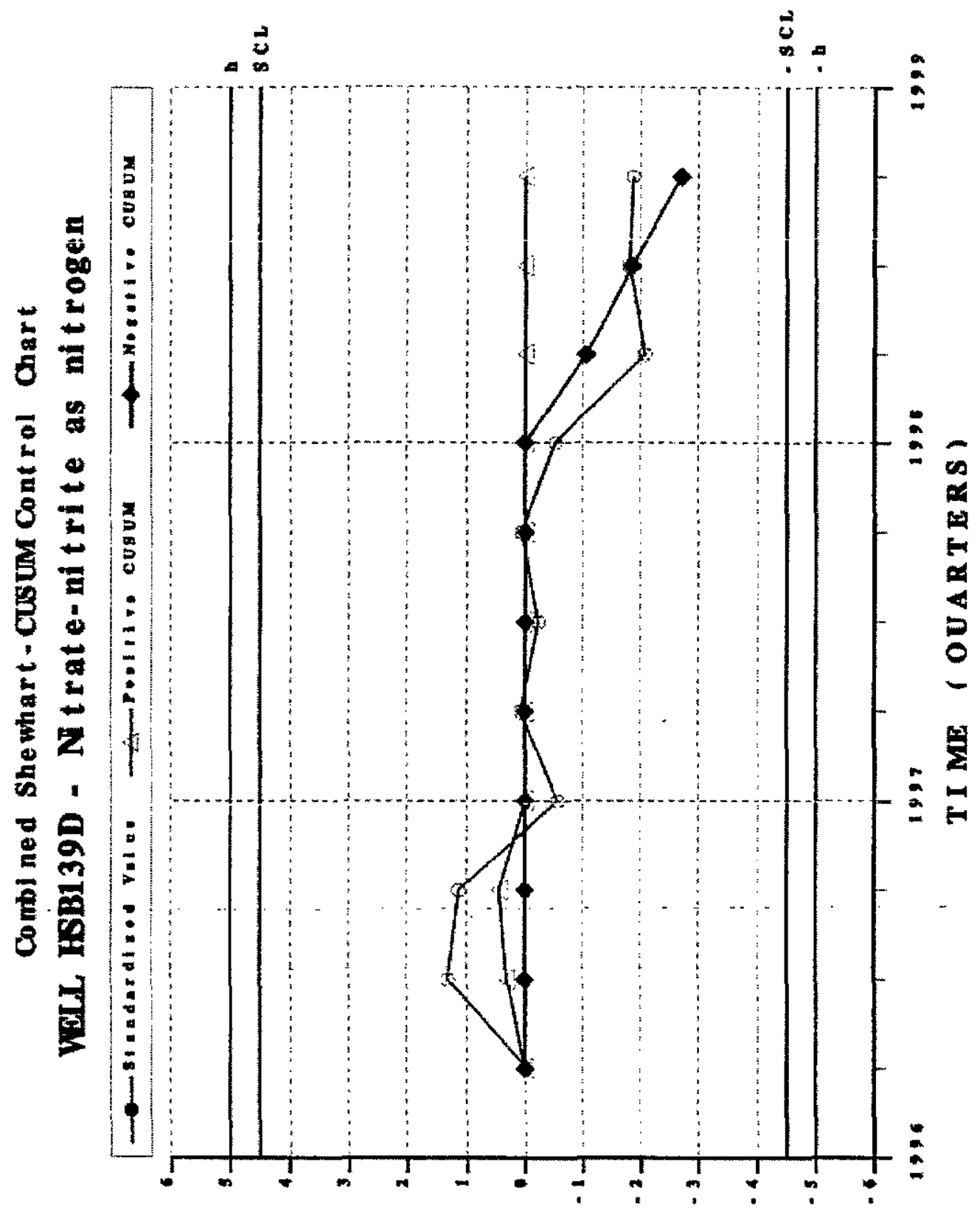

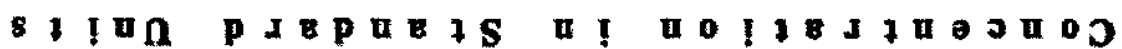



KoLL HSB145C - Nitratenitrite as nitrogen

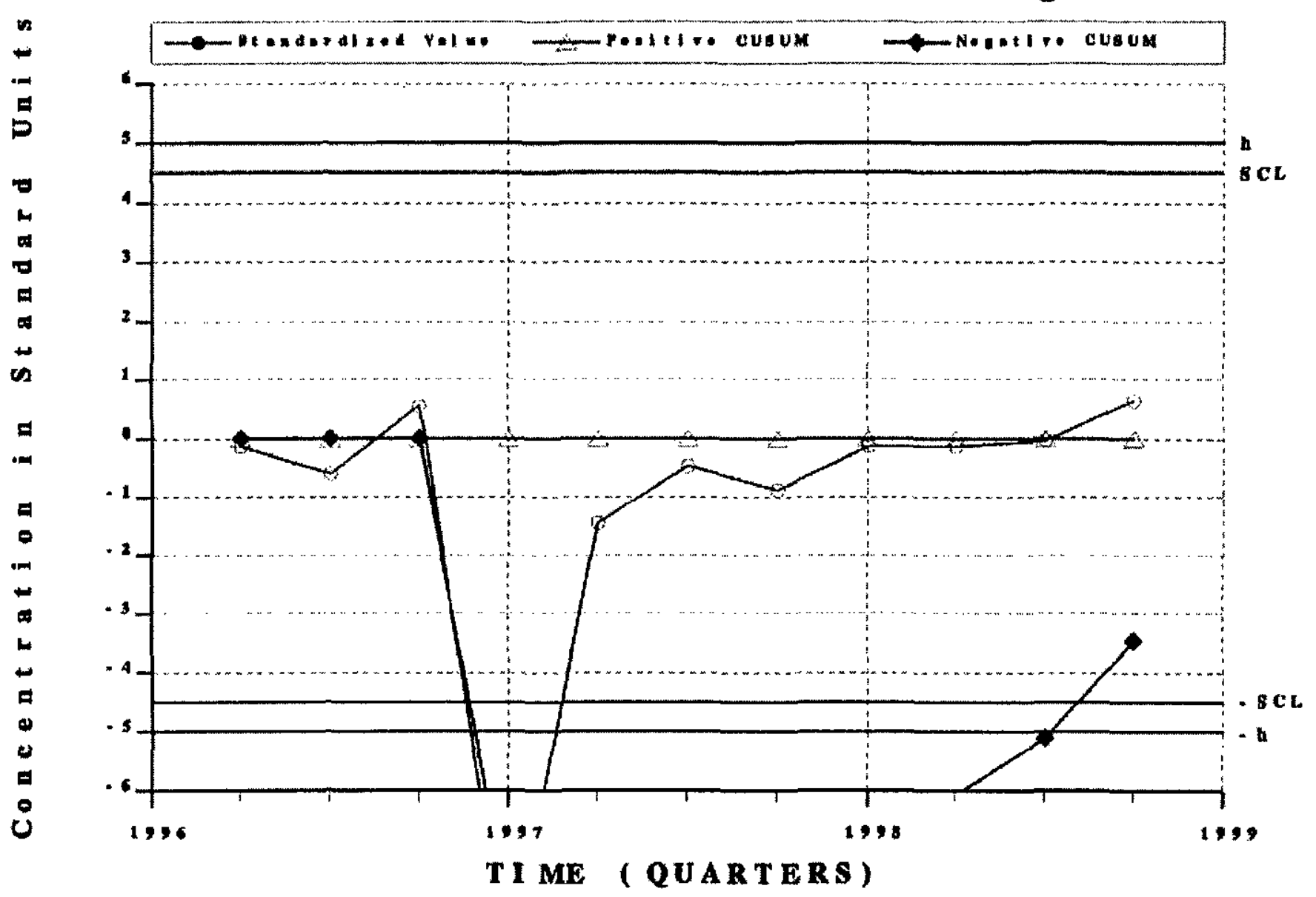




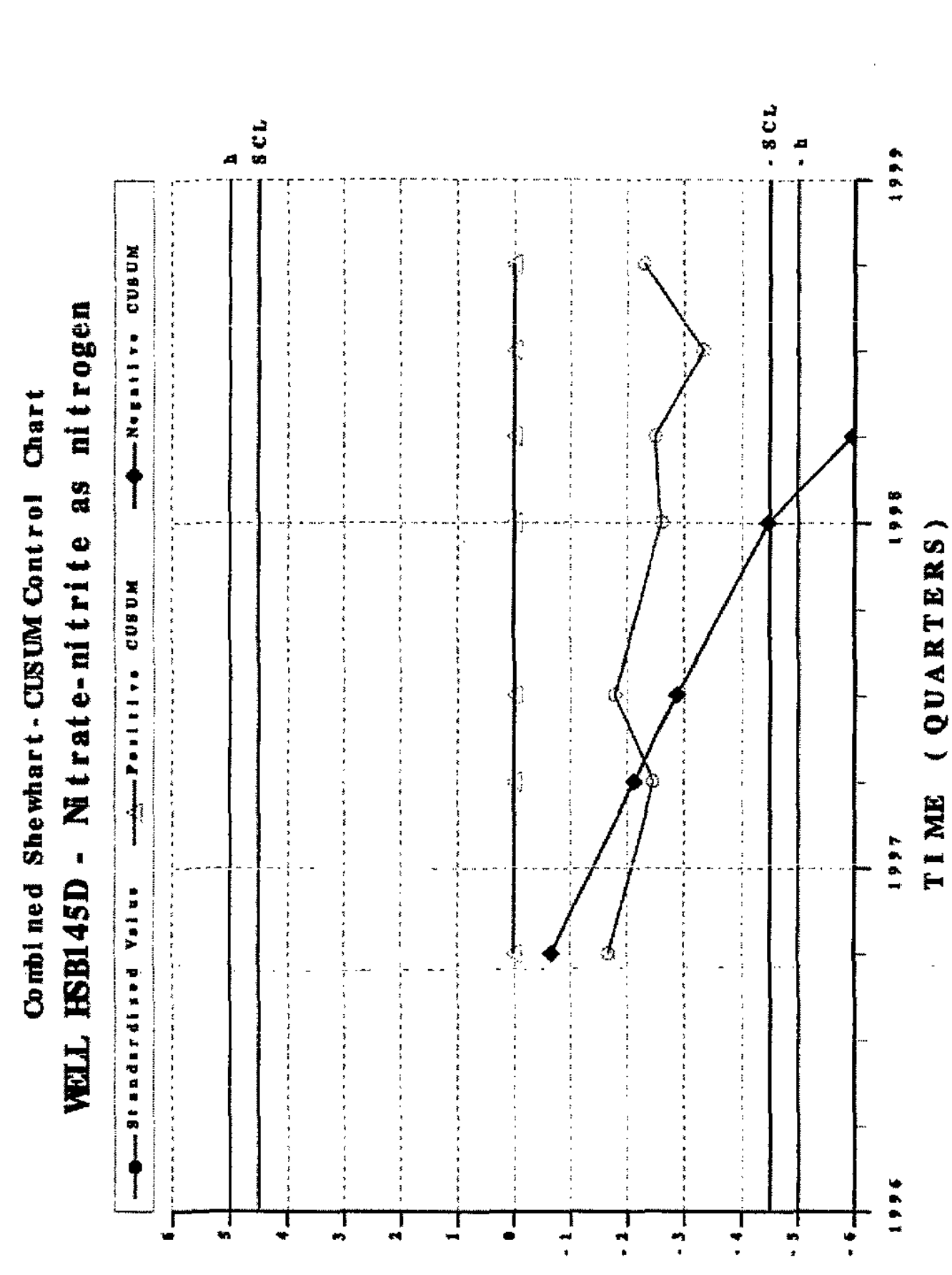

WSRC-TR-99-00013

Unclassified

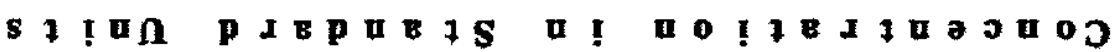


Combi ned Shewhart-CusuM Cont rol Chart WeLL HSB145D - Zi nc, total recoverable

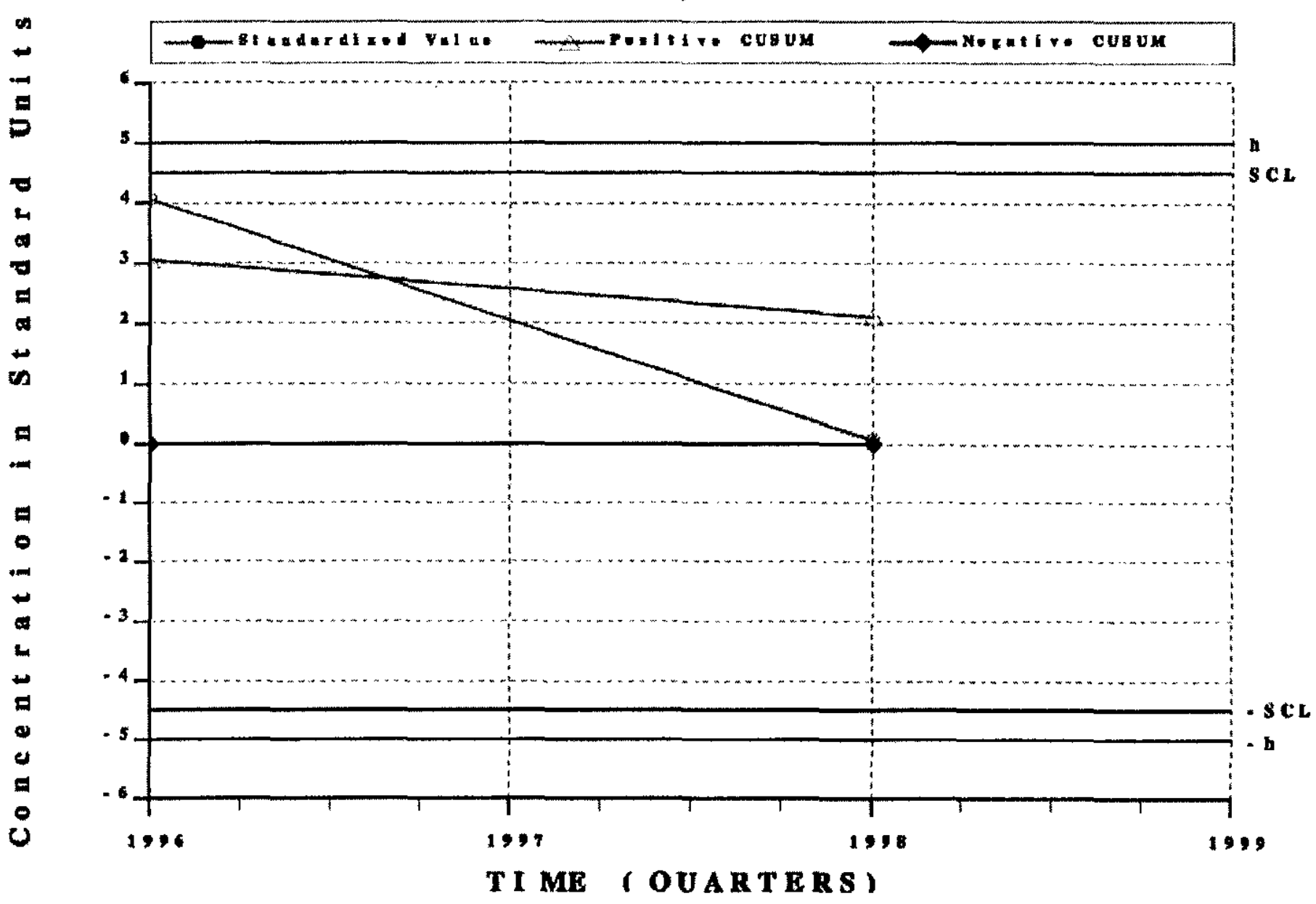


WSRC-TR-99-00013

Unclassified

\section{Appendix G}

\section{Operational Activities and Downtime}


WSRC-TR-99-00013

Unclassified

THIS PAGE LEFT BLANK INTENTIONALLY. 


\section{Operations Activities and Downtime during the Second Half of 1998}

\begin{tabular}{|c|c|c|c|c|c|}
\hline Month & $\begin{array}{c}\text { Run } \\
\text { Percent }\end{array}$ & $\begin{array}{l}\text { Hours } \\
\text { Up }\end{array}$ & $\begin{array}{l}\text { Hours } \\
\text { Down }\end{array}$ & Dates & Explanation \\
\hline \multirow[t]{17}{*}{ July } & 100 & 24 & 0 & 1 & $\begin{array}{l}\text { WTU operated for } 24 \text { hours with no } \\
\text { downtime. }\end{array}$ \\
\hline & $\overline{58}$ & $\overline{14}$ & 10 & 2 & $\begin{array}{l}\text { WTU 100-hour test complete as of } 1930 . \\
\text { WTU shut down for system desludging and } \\
\text { completion of minor maintenance items. }\end{array}$ \\
\hline & 0 & 0 & 24 & 3 & $\begin{array}{l}\text { WTU down for minor modifications including } \\
\text { replacement of filter press sequence switch. }\end{array}$ \\
\hline & 79 & 19 & 5 & 4 & $\begin{array}{l}\text { WTU operated for } 19 \text { hours after completion } \\
\text { of maintenance items. }\end{array}$ \\
\hline & 100 & $\overline{24}$ & 0 & 5 & $\begin{array}{l}\text { WTU operated for } 24 \text { hours with no } \\
\text { downtime. }\end{array}$ \\
\hline & 100 & 24 & 0 & 6 & $\begin{array}{l}\text { WTU operated for } 24 \text { hours with no } \\
\text { downtime. }\end{array}$ \\
\hline & 73 & 17.5 & 6.5 & 7 & $\begin{array}{l}\text { WTU down due to caustic motor pump } \\
\text { failure. }\end{array}$ \\
\hline & 96 & 23 & 1 & 8 & $\begin{array}{l}\text { WTU down to make final electric tie-ins to } \\
\text { new injection wells and repair the injection } \\
\text { and extraction tank. }\end{array}$ \\
\hline & 40 & 9.5 & 14.5 & 9 & WTU down for injection well tie-ins. \\
\hline & 94 & 22.5 & 1.5 & 10 & $\begin{array}{l}\text { WTU down due to flow transmitter problems } \\
\text { on } R O \text { trains } A \& B \text {. }\end{array}$ \\
\hline & 100 & 48 & 0 & $11-12$ & $\begin{array}{l}\text { WTU operated for } 24 \text { hours with no } \\
\text { downtime. }\end{array}$ \\
\hline & 88 & 21 & 3 & 13 & $\begin{array}{l}\text { WTU down to repair leak on RO train B } \\
\text { pressure transmitter and to calibrate and } \\
\text { clean pH probe. }\end{array}$ \\
\hline & 100 & 24 & 0 & 14 & $\begin{array}{l}\text { WTU operated for } 24 \text { hours with no } \\
\text { downtime. }\end{array}$ \\
\hline & 63 & 15 & 9 & 15 & $\begin{array}{l}\text { WTU down due to bag filter change out and } \\
\text { partial sluicing of roughing filters. A lightning } \\
\text { strike disabled the extraction and injection } \\
\text { wells. }\end{array}$ \\
\hline & 35 & 8.5 & 15.5 & 16 & $\begin{array}{l}\text { WTU down due to lightning strikes disabling } \\
\text { the extraction and injection wells. }\end{array}$ \\
\hline & 67 & 16 & 8 & 17 & $\begin{array}{l}\text { WTU down due to maintenance trouble- } \\
\text { shooting extraction and injection wells. }\end{array}$ \\
\hline & 71 & 17 & 7 & 18 & $\begin{array}{l}\text { WTU down to backflush temporary roughing } \\
\text { filter } A \text {. }\end{array}$ \\
\hline
\end{tabular}




\begin{tabular}{|c|c|c|c|c|c|}
\hline Month & $\begin{array}{c}\text { Run } \\
\text { Percent }\end{array}$ & $\begin{array}{l}\text { Hours } \\
\text { Up }\end{array}$ & $\begin{array}{l}\text { Hours } \\
\text { Down }\end{array}$ & Dates & Explanation \\
\hline \multirow[t]{13}{*}{ July, cont. } & 0 & 0 & 24 & 19 & $\begin{array}{l}\text { WTU down to modify the temporary roughing } \\
\text { filters and to replace clearwater control } \\
\text { valve. }\end{array}$ \\
\hline & 0 & 0 & 24 & 20 & $\begin{array}{l}\text { WTU down to modify the temporary roughing } \\
\text { filters. }\end{array}$ \\
\hline & 23 & 5.5 & 18.5 & 21 & $\begin{array}{l}\text { WTU down to modify the temporary roughing } \\
\text { filters. }\end{array}$ \\
\hline & 96 & 23 & 1 & 22 & WTU down to change out post filter. \\
\hline & 83 & 20 & 4 & 23 & $\begin{array}{l}\text { WTU down due to lightning strikes disabling } \\
\text { the injection and extraction wells. }\end{array}$ \\
\hline & 71 & 17 & 7 & 24 & $\begin{array}{l}\text { WTU down to repair damage caused by } \\
\text { lightning strikes and change out bag filter. }\end{array}$ \\
\hline & 100 & 24 & 0 & 25 & $\begin{array}{l}\text { WTU operated for } 24 \text { hours with no } \\
\text { downtime. }\end{array}$ \\
\hline & 67 & 16 & 8 & 26 & $\begin{array}{l}\text { WTU down due to a clog in the Polyblend } \\
\text { system. }\end{array}$ \\
\hline & 98 & 23.5 & .5 & 27 & WTU down due to power interruption. \\
\hline & 0 & 0 & 24 & 28 & $\begin{array}{l}\text { WTU down due to pluggage in the temporary } \\
\text { roughing filters. }\end{array}$ \\
\hline & 0 & 0 & 24 & 29 & $\begin{array}{l}\text { WTU down to remove and replace zeolite in } \\
\text { temporary roughing filters. }\end{array}$ \\
\hline & 0 & 0 & 24 & 30 & $\begin{array}{l}\text { WTU down to remove scum from the top of } \\
\text { the zeolite column and charge the column } \\
\text { with an additional } 20 \mathrm{cu} \mathrm{ft} \text { of zeolite. }\end{array}$ \\
\hline & 0 & 0 & 24 & 31 & $\begin{array}{l}\text { WTU down due to pluggage in the temporary } \\
\text { roughing filters encountered on the } \\
\text { attempted restart of the system. }\end{array}$ \\
\hline July Totals & 61 & $\overline{456}$ & $2 \overline{\overline{288}}$ & & \\
\hline \multirow[t]{4}{*}{ August } & $\overline{11}$ & 2.75 & 21.25 & 1 & $\begin{array}{l}\text { WTU down to remove pluggage of the } \\
\text { temporary roughing filters and a Polyblend } \\
\text { pump malfunction. }\end{array}$ \\
\hline & 4 & 1 & 23 & 2 & $\begin{array}{l}\text { WTU down due to Polyblend pump } \\
\text { malfunction. }\end{array}$ \\
\hline & 8 & 2 & 22 & 3 & $\begin{array}{l}\text { WTU down due to problems with the } \\
\text { Polyblend injection. }\end{array}$ \\
\hline & 39 & 9.3 & 14.7 & 4 & $\begin{array}{l}\text { WTU down due to problems with the } \\
\text { Polyblend injection. }\end{array}$ \\
\hline
\end{tabular}




\begin{tabular}{|c|c|c|c|c|c|}
\hline Month & $\begin{array}{c}\text { Run } \\
\text { Percent }\end{array}$ & $\begin{array}{l}\text { Hours } \\
\text { Up }\end{array}$ & $\begin{array}{l}\text { Hours } \\
\text { Down }\end{array}$ & Dates & Explanation \\
\hline \multirow[t]{20}{*}{$\begin{array}{l}\text { August, } \\
\text { cont. }\end{array}$} & 6 & 1.5 & 22.5 & 5 & $\begin{array}{l}\text { WTU down due to Polyblend overdose } \\
\text { requiring desludging of the flocculation tank } \\
\text { and clarifier. }\end{array}$ \\
\hline & 0 & 0 & 24 & 6 & WTU down to desludge the flocculation tank. \\
\hline & 0 & 0 & $\overline{24}$ & 7 & $\begin{array}{l}\text { WTU down to clean the filter press and } \\
\text { desludge the fitter press and clarifier. }\end{array}$ \\
\hline & $\overline{65}$ & 15.5 & 8.5 & 8 & $\begin{array}{l}\text { WTU down due to lightning strikes disabling } \\
\text { the extraction and injection wells, and } \\
\text { polymer flow problems. }\end{array}$ \\
\hline & 100 & 96 & 0 & $9-12$ & $\begin{array}{l}\text { WTU operated for } 24 \text { hours with no } \\
\text { downtime. }\end{array}$ \\
\hline & 92 & 22 & 2 & 13 & $\begin{array}{l}\text { WTU down to backwash the zeolite, dowex, } \\
\text { and carbon columns. }\end{array}$ \\
\hline & 100 & 24 & 0 & 14 & $\begin{array}{l}\text { WTU operated for } 24 \text { hours with no } \\
\text { downtime. }\end{array}$ \\
\hline & 52 & 12.5 & 11.5 & 15 & $\begin{array}{l}\text { WTU down due to high sludge level in } \\
\text { flocculation tank. }\end{array}$ \\
\hline & $\overline{0}$ & 0 & 24 & 16 & $\begin{array}{l}\text { WTU down due to complete desludging, to } \\
\text { repair leak at turbidity meter, and to prepare } \\
\text { for change out of zeolite in the main column. }\end{array}$ \\
\hline & 6 & 1.5 & 22.5 & 17 & $\begin{array}{l}\text { WTU down due to change out of zeolite in } \\
\text { the main columns and system flush. }\end{array}$ \\
\hline & $\overline{71}$ & 17 & 7 & 18 & $\begin{array}{l}\text { WTU down due to lightning strikes disabling } \\
\text { the extraction and injection wells. }\end{array}$ \\
\hline & 96 & 23 & $\overline{1}$ & 19 & $\begin{array}{l}\text { WTU down due to repairs on extraction and } \\
\text { injection wells. }\end{array}$ \\
\hline & $\overline{94}$ & 22.5 & 1.5 & 20 & $\begin{array}{l}\text { WTU down due to low level in the injection } \\
\text { tank while changing out the bag filter. }\end{array}$ \\
\hline & 85 & 20.5 & 3.5 & 21 & $\begin{array}{l}\text { WTU down due to sludge carryover in the } \\
\text { clarifier. }\end{array}$ \\
\hline & 50 & 12 & 12 & 22 & WTU down to desludge. \\
\hline & 0 & 0 & 24 & 23 & WTU down to desludge. \\
\hline & $\overline{13}$ & 3.2 & 20.8 & 24 & WTU down to desludge. \\
\hline & 100 & 48 & 0 & $25-26$ & $\begin{array}{l}\text { WTU operated for } 24 \text { hours with no } \\
\text { downtime. }\end{array}$ \\
\hline & 88 & 21 & 3 & 27 & WTU down due to $\mathrm{pH}$ control on inline mixer. \\
\hline & 100 & 24 & 0 & 28 & $\begin{array}{l}\text { WTU operated for } 24 \text { hours with no } \\
\text { downtime. }\end{array}$ \\
\hline
\end{tabular}




\begin{tabular}{|c|c|c|c|c|c|}
\hline Month & $\begin{array}{c}\text { Run } \\
\text { Percent }\end{array}$ & $\begin{array}{l}\text { Hours } \\
\text { Up }\end{array}$ & $\begin{array}{l}\text { Hours } \\
\text { Down }\end{array}$ & Dates & Explanation \\
\hline \multirow[t]{3}{*}{$\begin{array}{l}\text { August, } \\
\text { cont. }\end{array}$} & 50 & 12 & 12 & 29 & $\begin{array}{l}\text { WTU down due to desludging the clarifier } \\
\text { and flocculation tank. }\end{array}$ \\
\hline & 54 & 13 & 11 & 30 & $\begin{array}{l}\text { WTU down due to desludging the clarifier } \\
\text { and flocculation tank. }\end{array}$ \\
\hline & 58 & 14 & 10 & $\overline{31}$ & $\begin{array}{l}\text { WTU down due to lightning strikes disabling } \\
\text { the extraction and injection wells. }\end{array}$ \\
\hline $\begin{array}{l}\text { August } \\
\text { Totals }\end{array}$ & 56 & 418.25 & 325.75 & & \\
\hline \multirow[t]{18}{*}{ September } & 60 & 14.5 & 9.5 & 1 & $\begin{array}{l}\text { WTU down to repair extraction and injection } \\
\text { wells. }\end{array}$ \\
\hline & 100 & 24 & 0 & 2 & WTU operated for 24 hours. \\
\hline & 60 & 14.5 & 9.5 & 3 & $\begin{array}{l}\text { WTU down for scheduled maintenance } \\
\text { outage to clean inline mixers and to } \\
\text { complete tie-ins of the flocculation tank pH } \\
\text { probe to the PLC. }\end{array}$ \\
\hline & 100 & 24 & 0 & 4 & WTU operated for 24 hours. \\
\hline & 88 & 21 & 3 & 5 & $\begin{array}{l}\text { WTU down due to excessive air bubbles in } \\
\text { flocculation tank and carryover to clearwell. }\end{array}$ \\
\hline & 100 & 48 & 0 & $6-7$ & WTU operated for 24 hours. \\
\hline & 94 & 22.5 & 1.5 & 8 & $\begin{array}{l}\text { WTU down due to lightning strikes disabling } \\
\text { the extraction and injection wells: }\end{array}$ \\
\hline & 100 & 24 & 0 & $\overline{9}$ & WTU operated for 24 hours. \\
\hline & 63 & 15 & 9 & 10 & WTU outage to repair transformer grounding. \\
\hline & 100 & 72 & 0 & $11-13$ & WTU operated for 24 hours. \\
\hline & 63 & 15 & 9 & 14 & $\begin{array}{l}\text { WTU down for maintenance to clean the } \\
\text { screens on the temporary roughing filters. }\end{array}$ \\
\hline & 100 & 48 & 0 & $15-16$ & WTU operated for 24 hours. \\
\hline & 98 & 23.5 & .5 & 17 & $\begin{array}{l}\text { WTU down to backwash the zeolite column } \\
\text { and temporary roughing filters. }\end{array}$ \\
\hline & 100 & 48 & 0 & $18-19$ & WTU operated for 24 hours. \\
\hline & 45 & 10.7 & 13.3 & 20 & $\begin{array}{l}\text { WTU down due to the leaking flange on the } \\
\text { flocculation tank and leak on acid pump. }\end{array}$ \\
\hline & 58 & 14 & 10 & 21 & WTU down to resolve the acid pump leak. \\
\hline & 100 & 48 & 0 & $22-23$ & WTU operated for 24 hours. \\
\hline & 96 & 23 & 1 & 24 & WTU down due to acid pump failure. \\
\hline
\end{tabular}




\begin{tabular}{|c|c|c|c|c|c|}
\hline Month & $\begin{array}{c}\text { Run } \\
\text { Percent }\end{array}$ & $\begin{array}{l}\text { Hours } \\
\text { Up }\end{array}$ & $\begin{array}{l}\text { Hours } \\
\text { Down }\end{array}$ & Dates & Explanation \\
\hline $\begin{array}{l}\text { September, } \\
\text { cont. }\end{array}$ & 100 & 144 & 0 & $25-30$ & WTU operated for 24 hours. \\
\hline $\begin{array}{l}\text { September } \\
\text { Totals }\end{array}$ & 91 & 653.7 & 66.3 & & \\
\hline \multirow[t]{15}{*}{ October } & 100 & 24 & 0 & 1 & WTU operated for 24 hours. \\
\hline & 50 & 12 & 12 & 2 & $\begin{array}{l}\text { WTU down to change out zeolite in the main } \\
\text { column and to clean the strainer on the ferric } \\
\text { chloride addition. }\end{array}$ \\
\hline & 100 & 72 & 0 & $3-5$ & WTU operated for 24 hours. \\
\hline & 96 & 23 & 1 & 6 & $\begin{array}{l}\text { WTU down due to malfunction of acid drum } \\
\text { pump. }\end{array}$ \\
\hline & 100 & 72 & 0 & $7-9$ & WTU operated for 24 hours. \\
\hline & 83 & $\overline{19.8}$ & 4.2 & 10 & $\begin{array}{l}\text { WTU down due to cracked fitting on the } \\
\text { discharge line from the caustic tank. }\end{array}$ \\
\hline & 83 & 20 & 4 & 11 & $\begin{array}{l}\text { WTU down to repair leak on the discharge } \\
\text { line from the caustic tank. }\end{array}$ \\
\hline & 94 & 22.5 & 1.5 & 12 & $\begin{array}{l}\text { WTU down to repair a gasket leak on the } \\
\text { carbon column manway. }\end{array}$ \\
\hline & 92 & 22 & 2 & 13 & $\begin{array}{l}\text { WTU down to repair a leak on the suction } \\
\text { line to caustic pump. }\end{array}$ \\
\hline & 72 & 17.3 & 6.7 & 14 & $\begin{array}{l}\text { WTU down to change zeolite in temporary } \\
\text { roughing filter } A\end{array}$ \\
\hline & 100 & 120 & 0 & $15-19$ & WTU operated for 24 hours. \\
\hline & 90 & 21.5 & 2.5 & 20 & $\begin{array}{l}\text { WTU down to repair leak on the carbon } \\
\text { column vent line and a leak in temporary } \\
\text { roughing filter } B \text {. }\end{array}$ \\
\hline & 100 & 192 & 0 & $21-28$ & WTU operated for 24 hours. \\
\hline & 85 & 20.5 & 3.5 & 29 & $\begin{array}{l}\text { WTU down due to high sludge levels in the } \\
\text { flocculation tank. }\end{array}$ \\
\hline & 100 & 48 & 0 & $30-31$ & WTU operated for 24 hours. \\
\hline $\begin{array}{l}\text { October } \\
\text { Totals }\end{array}$ & $\overline{95}$ & $\overline{706.6}$ & $\overline{37.4}$ & & \\
\hline \multirow[t]{2}{*}{ November } & 0 & 0 & 48 & $1-2$ & $\begin{array}{l}\text { WTU down due to high differential pressure } \\
\text { on temporary roughing filters } A \& B \text {. }\end{array}$ \\
\hline & 0 & 0 & 24 & 3 & $\begin{array}{l}\text { WTU down to replace zeolite in the } \\
\text { temporary roughing filters. }\end{array}$ \\
\hline
\end{tabular}




\begin{tabular}{|c|c|c|c|c|c|}
\hline Month & $\begin{array}{c}\text { Run } \\
\text { Percent }\end{array}$ & $\begin{array}{l}\text { Hours } \\
\text { Up }\end{array}$ & $\begin{array}{l}\text { Hours } \\
\text { Down }\end{array}$ & Dates & Explanation \\
\hline $\begin{array}{l}\text { November, } \\
\text { cont. }\end{array}$ & 0 & 0 & 24 & 4 & $\begin{array}{l}\text { WTU down to replace zeolite and dowex in } \\
\text { the main columns. }\end{array}$ \\
\hline & 0 & 0 & 24 & 5 & $\begin{array}{l}\text { WTU down to desludge the flocculation tank } \\
\text { for inspection of the dispersal ring. } \\
\text { Maintenance clearing out the effluent tank } \\
\text { lines. Repair of RO piping continues. }\end{array}$ \\
\hline & 0 & 0 & 72 & $6-8$ & $\begin{array}{l}\text { WTU down to soak dispersal ring in low } \mathrm{pH} \\
\text { water. }\end{array}$ \\
\hline & 0 & 0 & 24 & 9 & $\begin{array}{l}\text { WTU down to desludge the flocculation tank } \\
\text { and clarifier. }\end{array}$ \\
\hline & 0 & 0 & 24 & 10 & $\begin{array}{l}\text { WTU down to soak the dispersal ring in low } \\
\text { pH water and desludge the flocculation tank. }\end{array}$ \\
\hline & 0 & 0 & 24 & 11 & WTU down to install RO B piping. \\
\hline & 0 & 0 & 24 & 12 & $\begin{array}{l}\text { WTU down due to pluggage in the charcoal, } \\
\text { dowex, and zeolite backwash piping. }\end{array}$ \\
\hline & 4 & 1 & 23 & 13 & $\begin{array}{l}\text { WTU down due to pluggage in the dowex } \\
\text { and zeolite backwash piping. }\end{array}$ \\
\hline & 0 & 0 & 24 & 14 & $\begin{array}{l}\text { WTU down due to pluggage in the zeolite } \\
\text { dowex backwash piping. }\end{array}$ \\
\hline & 0 & 0 & 24 & 15 & $\begin{array}{l}\text { WTU down due to pluggage in the dowex } \\
\text { zeolite backwash piping. }\end{array}$ \\
\hline & 69 & 16.5 & 7.5 & 16 & $\begin{array}{l}\text { WTU down due to pluggage in the dowex } \\
\text { zeolite backwash piping. }\end{array}$ \\
\hline & 46 & 11 & 13 & 17 & $\begin{array}{l}\text { WTU down due to carryover in the clarifier } \\
\text { and flocculation tank. }\end{array}$ \\
\hline & 0 & 0 & 168 & $18-24$ & $\begin{array}{l}\text { WTU down due to pluggage in the acid } \\
\text { addition line on the effluent tank and failed } \\
\text { diaphram in sludge pump A. Lockout of } \\
\text { equipment required to repair the system not } \\
\text { possible due to a contract dispute between } \\
\text { WSRC and the subcontractor. }\end{array}$ \\
\hline & & & 144 & $25-30$ & $\begin{array}{l}\text { WTU down due to pluggage in the acid } \\
\text { addition line on the effluent tank and failed } \\
\text { diaphragm in sludge pump A. }\end{array}$ \\
\hline $\begin{array}{l}\text { November } \\
\text { Totals }\end{array}$ & 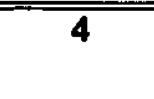 & 28.5 & 691.5 & & \\
\hline
\end{tabular}




\begin{tabular}{|l|c|c|c|c|l|}
\hline Month & $\begin{array}{c}\text { Run } \\
\text { Percent }\end{array}$ & $\begin{array}{c}\text { Hours } \\
\text { Up }\end{array}$ & $\begin{array}{c}\text { Hours } \\
\text { Down }\end{array}$ & Dates & Explanation \\
\hline December & 0 & 0 & 24 & 1 & $\begin{array}{l}\text { WTU down for maintenance to unplug the } \\
\text { acid addition line on the effient tank. }\end{array}$ \\
\hline & 0 & 0 & 24 & 2 & $\begin{array}{l}\text { WTU down for maintenance to charge RO } \\
\text { pump accumulator. Completed valve line- } \\
\text { up. }\end{array}$ \\
\hline & 27 & 6.5 & 17.5 & 3 & $\begin{array}{l}\text { WTU down for maintenance on RO pump } \\
\text { accumulator. }\end{array}$ \\
\hline & 85 & 20.5 & 3.5 & 4 & WTU down due to a leak on the RO Train B. \\
\hline $\begin{array}{l}\text { Wotals, } \\
\text { July- } \\
\text { December } \\
\text { 1998 }\end{array}$ & 100 & 360 & 0 & $6-20$ & WTU operated for 24 hours. \\
\hline \hline
\end{tabular}


WSRC-TR-99-00013

Unclassified

THIS PAGE LEFT BLANK INTENTIONALLY 